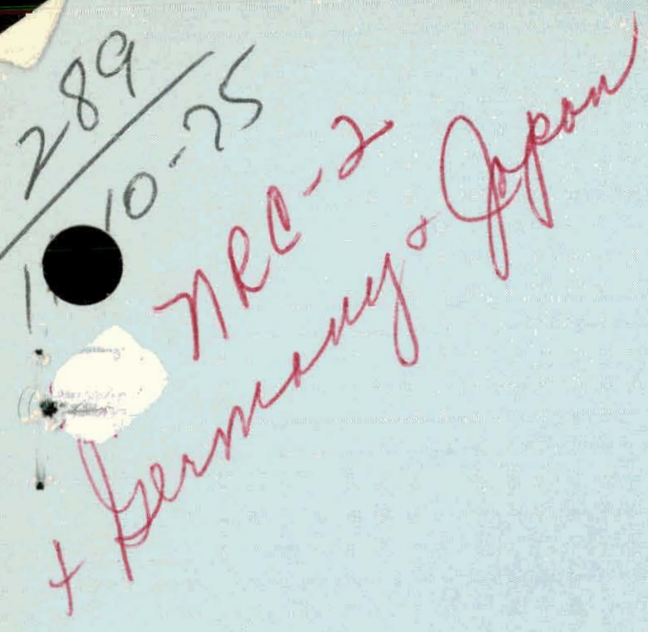

Dri: 1782

ANCR-1163

NRC-2

\title{
EXPERIMENT DATA REPORT FOR SEMISCALE TRANSPARENT VESSEL COUNTERCURRENT FLOW TESTS
}

NOTICE

PORTIONS OF THIS REPORT ARE IIIFGIRTG. It has been reproduced from the best availeble ability. w

\section{Aerojet nuclear Company}

IDAHO NATIONAL ENGINEERING LABORATORY Idaho Falls, Idaho - 83401

\author{
DATE PUBLISHED - OCTOBER 1975
}

PREPARED FOR THE 


\section{DISCLAIMER}

This report was prepared as an account of work sponsored by an agency of the United States Government. Neither the United States Government nor any agency Thereof, nor any of their employees, makes any warranty, express or implied, or assumes any legal liability or responsibility for the accuracy, completeness, or usefulness of any information, apparatus, product, or process disclosed, or represents that its use would not infringe privately owned rights. Reference herein to any specific commercial product, process, or service by trade name, trademark, manufacturer, or otherwise does not necessarily constitute or imply its endorsement, recommendation, or favoring by the United States Government or any agency thereof. The views and opinions of authors expressed herein do not necessarily state or reflect those of the United States Government or any agency thereof. 


\section{DISCLAIMER}

Portions of this document may be illegible in electronic image products. Images are produced from the best available original document. 


\section{DISCLAIMER}

This report was prepared as an account of work sponsored by an agency of the United States Government. Neither the United States Government nor any agency Thereof, nor any of their employees, makes any warranty, express or implied, or assumes any legal liability or responsibility for the accuracy, completeness, or usefulness of any information, apparatus, product, or process disclosed, or represents that its use would not infringe privately owned rights. Reference herein to any specific commercial product, process, or service by trade name, trademark, manufacturer, or otherwise does not necessarily constitute or imply its endorsement, recommendation, or favoring by the United States Government or any agency thereof. The views and opinions of authors expressed herein do not necessarily state or reflect those of the United States Government or any agency thereof. 


\section{DISCLAIMER}

Portions of this document may be illegible in electronic image products. Images are produced from the best available original document. 
Printed in the United States of America Available from

National Technical Information Service

U. S. Department of Commerce

5285 Port Roya1 Road

Springfield, Virginia 2216

Price: Printed Copy $\$ 7.60$; Microfiche $\$ 2.25$

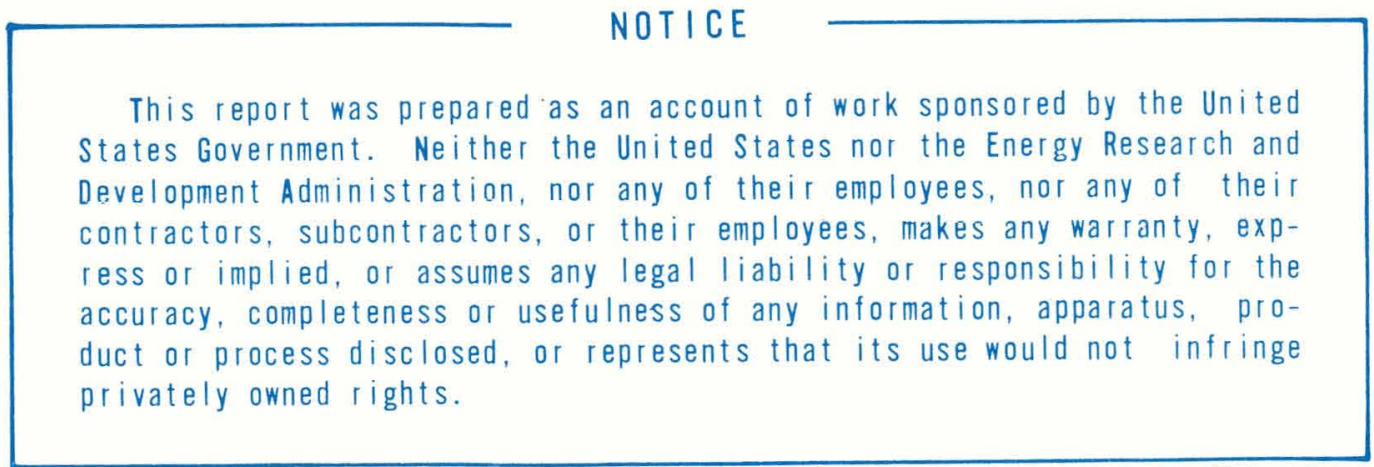


This report was prepared as an account of work sponsored by the United States Covernment. Neither the United States nor the United States Energy Research and Development Administration, nor any of their empluyees. nor any of, their contractors, subcontractors, or their employees, makes any liabily, express or ily for the accuracy, completeness or usefulness of any information, apparatus, product or process disclosed, or represents that its use would not infringe privately owned tights.

\section{EXPERIMENT DATA REPORT}

\section{FOR SEMISCALE TRANSPARENT VESSEL}

COUNTERCURRENT FLOW TESTS

by

D. J. Hanson

AEKOJET NUCLEAR COMPANY

Date Published - October 1975

PREPARED FOR THE

U.S. ENERGY RESEARCH AND DEVELOPMENT ADMINISTRATION

IDAHO OPERATIONS OFFICE

UNDER CONTRACT NO. E(10-1)-1375 


\section{ACKNOWLEDGMENTS}

The author gratefully acknowledges the contributions of many of the personnel of the Fluids Laboratory Division for their work in the performance of the transparent vessel tests. In particular, the efforts of L. T. Cartee, R. W. Gould, P. L. Chase, and G. E. Casper are appreciated. In addition, thanks are extended to J. F. Whitbeck for his work in developing and directing the initial stages of the test program and to S. A. Naff for his work in

conducting the first half of the tests. The.work of R.S. Alder for the error analysis presented in Appendix A is also appreciated. 


\begin{abstract}
Steady state air-water tests were performed as part of the Semiscale Blowdown and Emergency Core Cooling (ECC) Project to investigate downcomer countercurrent flow and downcomer bypass flow phenomena. These tests were performed in a plexiglass representation of the Semiscale pressure vessel which allowed changes to be made in the geometry of the upper annulus and downcomer for the purpose of investigating the sensitivity of downcomer and bypass flow to changes in system geometry. Tests were also performed to investigate the effects of two-phase inlet flows and different initial system pressures on countercurrent and bypass flow.
\end{abstract}

Results for each test are presented in the form of computer printout of the measurements and of a summary of the pertinent calculated flow rates, pressures, and dimensionless volumetric fluxes. Descriptions of the test faciiity, instrumentation, operating procedures, and test conditions are also presented. An error analysis is presented to identify maximum and probable errors for selected volumetric flux calculations. 


\section{SUMMARY}

Steady state air-water countercurrent flow and bypass flow tests were performed as part of the Semiscale Blowdown and Emergency Core Cooling (ECC) Project. These tests were the initial part of a more extensive experimental program conducted to investigate countercurrent flow and other ECC related phenomena in the Semiscale geometry. The objectives of these initial tests, reported herein, were to:

(1) Investigate the countercurrent flow phenomena that occur in an annulus with dimensions similar to the Semiscale downcomcr to determine whether commonly used countercurrent flow correlations describe the effect of the countercurrent air flow on the delivery of water (ECC) to the lower plenum.

(2) Investigate the relationship between flow bypassing the downcomer and countercurrent flow in the downcomer.

(3) Investigate the effect on countercurrent flow and bypass flow of changing the geometry of the upper annulus.

(4) Investigate the effect on countercurrent flow of changing the downcomer length by changing the length of the core barrel.

(5) Investigate the effect on countercurrent and bypass flow of a two-phase air-water mixture entering the vessel rather than water only.

(6) Establish a relationship between the countercurrent flow tests in the transparent vessel and the metal Semiscale vessel.

These objectives were fulfilled using the data from the reported tests. The data provided information which aided in understanding the results from steam-water counterclirrent flow tests performed in the metal Semiscale vessel.

The tests described were performed in a transparent plexiglass vessel with dimensions and internal parts similar to those of the Semiscale pressure vessel. The vessel was designed to facilitate geometric changes in the downcomer and upper annulus regions. The vessel was connected to air and water supply systems and to a drain system for the water. Volumetric flow, pressure, and temperature instrumentation and weigh tanks were used to measure the system parameters of interest.

This report presents the experimental data from the tests performed in the transparent vessel test program and provides the supportive information necessary to make the data usable. Results for each test are presented in the form of computer printout of the measurements and of a summary of the pertinent calculated flow rates, pressures, and

dimensionless volumetric fluxes. An error analysis is presented to identify maximum and probable errors for selected volumetric flux calculations. 


\section{CONTENTS}

ACKNOWLEDGMENTS $\ldots \ldots \ldots \ldots \ldots \ldots \ldots$ ii

ABSTRACT $\ldots \ldots \ldots \ldots \ldots \ldots \ldots \ldots \ldots \ldots \ldots \ldots \ldots \ldots$ iii

SUMMARY $\ldots \ldots \ldots \ldots \ldots \ldots \ldots \ldots \ldots \ldots \ldots \ldots \ldots \ldots \ldots \ldots$

EXPERIMENT DATA REPORT FOR SEMISCALE

TRANSPARENT VESSEL COUNTERCURRENT

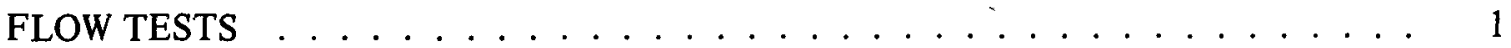

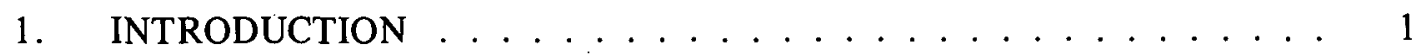

II. EXPERIMENTAL SYSTEM AND INSTRUMENTATION $\ldots \ldots \ldots$

1. EXPERIMENTAL SYSTEM $\ldots \ldots \ldots \ldots \ldots \ldots$

2. SYSTEM INSTRUMENTATION $\ldots \ldots \ldots \ldots \ldots \ldots$

III. TEST PROCEDURES $\ldots \ldots \ldots \ldots \ldots \ldots \ldots \ldots \ldots \ldots$

IV. TEST DATA PRESENTATION $\ldots \ldots \ldots \ldots \ldots \ldots$

1. BASELINE COUNTERCURRENT

FLOW TESTS $\ldots \ldots \ldots \ldots \ldots \ldots \ldots \ldots$

2. BYPASS FLOW TESTS $\ldots \ldots \ldots \ldots \ldots \ldots$

3. MODIFIED UPPER ANNULUS GEOMETRY TESTS . . . . . . . . . . 94

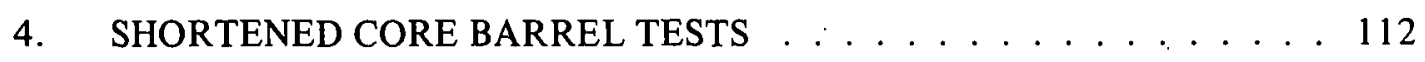

5. TWO-PHASE COLD LEG MIXTURE TESTS $\ldots \ldots \ldots \ldots \ldots$

6. COMBINED EFFECTS TESTS $\ldots \ldots \ldots \ldots \ldots \ldots$

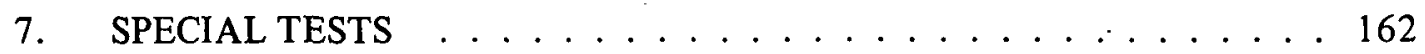

7.1 One-Dimensional Inlet Test Group $\ldots \ldots \ldots$. . . . . . . 162

7.2 Increasing and Decreasing Air Flow at Constant Water Flow . . . . . . . . . . . . . . . . 196

7.3 Double Annulus Tests . . . . . . . . . . . . . . . . . 196

7.4 Longitudinal Flow Restrictor Tests . . . . . . . . . . . 208

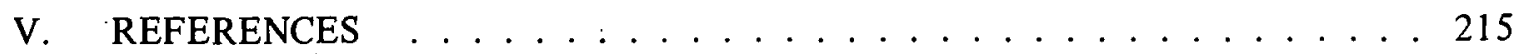

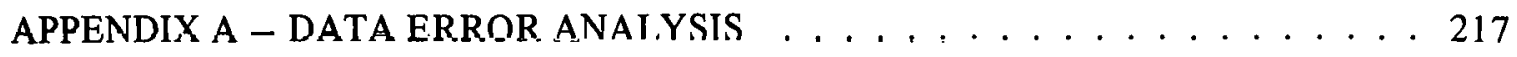




\section{FIGURES}

1. Transparent vessel and internals ................... 5

2. Upper annulus uniform inlet flow distributor $\ldots \ldots \ldots$

3. Isometric diagram of the low capacity air flow system . . . . . . . . . . . 9

4. Isometric diagram of the high capacity air flow system . . . . . . . . 10

5. Air and water flow paths in the transparent vessel . . . . . . . . . . . 14

6. Filler piece flange configuration for double annulus tests . . . . . . . . 197

7. Location and dimensions of longitudinal flow restrictors . . . . . . . . 208

\section{TABLES}

I. Transparent Vessel Countercurrent Flow Tests _. . . . . . . . . . 3

II. Possible Downcomer Gap Geometries for Transparent Vessel Tests . . . . . . . 6

III. Instrumentation for Transparent Vessel Tests . . . . . . . . . . . . . 12

IV. Baseline Countercurrent Flow Test Group $1.1 \ldots \ldots$. . . . . . . . . . . 19

V. Baseline Countercurrent Flow Test Group 1.2 . . . . . . . . . . . . . 23

VI. Baseline Countercurrent Flow Test Group $2.1 \ldots \ldots$. . . . . . . . . . 27

VII. Baseline Countercurrent Flow Test Group $2.2 \ldots \ldots$. . . . . . . . . . . 29

VIII. Baseline Countercurrent Flow Test Group $2.3 \ldots \ldots$. . . . . . . . . . . . 35

IX. Baseline Countercurrent Flow Test Group $4.1 \ldots \ldots$. . . . . . . . . . 39

X. Baseline Countercurrent Flow Test Group $4.2 \ldots \ldots$. . . . . . . . . . 46

XI. Baseline Countercurrent Flow Test Group 5:1 . . . . . . . . . . . . 52

XII. Baseline Countercurrent Flow Test Group 6.1 . . . . . . . . . . . . . 56

XIII. Baseline Countercurrent Flow Test Group $7.1 \ldots \ldots$. . . . . . . . . . . . 59

XIV. Baseline Countercurrent Flow Test Group $9.1 \ldots \ldots 6$ 
XV. Bypass Flow Test Group $5.5 \ldots \ldots \ldots \ldots \ldots$

XVI. Bypass Flow Test Group $5.7 \ldots \ldots \ldots \ldots \ldots$

XVII. Bypass Flow Test Group $5.12 \ldots \ldots \ldots \ldots$

XVIII. Bypass Flow Test Group $5.15 \ldots \ldots \ldots \ldots$

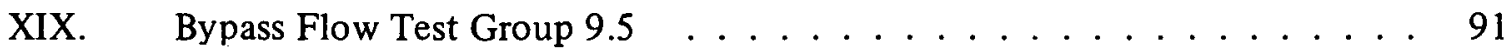

XX. Modified Upper Annulus Geometry Test Group $5.4 \ldots \ldots \ldots$

XXI. Modified Upper Annulus Geometry Test Group 5.8 . . . . . . . . . . . . 98

XXII. Modified Upper Annulus Geometry Test Group $5.10 \ldots \ldots$

XXIII. Modified Upper Annulus Geometry Test Group 5.13 . . . . . . . . . . 102

XXIV. Modified Upper Annulus Geometry Test Group $6.2 \ldots \ldots$. . . . . . 105

XXV. Modified Upper Annulus Geometry Test Group $9.2 \ldots \ldots \ldots$

XXVI. Modified Upper Annulus Geometry Test Group $9.6 \ldots \ldots$. . . . . . . . 110

XXVII. Shortened Core Barrel Test Group $3.1 \quad \ldots \ldots \ldots \ldots$. . . . . . . 113

XXVIII. Shortened Core Barrel Test Group $4.4 \ldots \ldots$. . . . . . . . 117

XXIX. Shortened Core Barrel Test Group $4.5 \ldots \ldots \ldots$

XXX. Shortened Core Barrel Test Group $4.6 \ldots \ldots \ldots \ldots$

XXXI. Shortened Core Barrel Test Group 8.1 . . . . . . . . . . . . . . 129

XXXXII. Two-Phase Cold Leg Mixture Test Group $1.3 \ldots \ldots$

XXXIII. Two-Phase Cold Leg Mixture Test Group $1.4 \ldots \ldots$. . . . . . . . . . 145

XXXIV. Two-Phase Cold Leg Mixture Test Group $5.6 \ldots \ldots$. . . . . . 150

XXXV. Two-Phase Cold Leg Mixture Test Group $7.2 \ldots \ldots$. . . . . . . 154

XXXVI. Two-Phase Cold Leg Mixture Test Group $9.4 \ldots \ldots \ldots$

XXXVII. Combined Effects Test Group $3.2 \ldots \ldots \ldots$. . . . . . . . . 163

XXXVIII.Combined Effects Test Group $4.3 \ldots \ldots$. . . . . . . . . . . 167

XXXIX. Combined Effects Test Group $4.7 \ldots \ldots \ldots$ 
XL. Combined Effects Test Group $4.8 \ldots \ldots \ldots$. . . . . . . . 173

XLI. Combined Effects Test Group $4.9 \ldots \ldots \ldots \ldots$. . . . . . . . . . . .

XLII. Combined Effects Test Group $5.9 \ldots \ldots \ldots \ldots$

XLIII. Combined Effects Test Group $5.11 \ldots \ldots \ldots$. . . . . . . . 184

XLIV. Combined Effects Test Group $5.14 \ldots \ldots \ldots \ldots 7$

LXV. Combined Effects Test Group $9.3 \ldots \ldots$. . . . . . . . . 190

XLVI. Combined Effects Test Group $9.7 \ldots \ldots$. . . . . . . . . . . 193

XLVII. Special Test Group 4.10-One-dimensional Inlet . . . . . . . . . . 198

XLVIII. Special Test Group 5.2 - Increasing Air Flow . . . . . . . . . . . . 200

XLIX. Special Test Group 5.3 - Decreasing Air Flow . . . . . . . . . . . 203

L. Special Test Group 7.3 - Double Annulus . . . . . . . . . . 205

LI. Special Test Group 9.8 - Longitudinal Flow Restrictors . . . . . . . . 209

LII. Special Test Group 9.9 - Shortened Longitudinal Flow Restrictors . . . . . 212 


\section{EXPERIMENT DATA REPORT \\ FOR SEMISCALE TRAN SPARENT VESSEL COUNTERCURRENT FLOW TESTS}

\section{INTRODUCTION}

Steady state air-water tests were performed in a transparent vessel to investigate downcomer countercurrent flow and downcomer bypass flow phenomena. These tests are part of an extensive experimental program conducted by Aerojet Nuclear Company to investigate countercurrent flow and emergency core coolant (ECC) related phenomena in the Semiscale geometry. The results from this experimental program are part of the Nuclear Regulatory Commission (NRC) data base which is used for evaluating the adequacy and improving the predictive capability of analytical models developed to predict system response during a loss-of-coolant accident (LOCA). In addition, the countercurrent flow data from the Semiscale geometry are also valuable for comparison with countercurrent flow data from similar investigations in larger geometries to aid in evaluating the effects of physical scale.

The experimental program conducted to investigate countercurrent flow and ECC related phenomena was made up of three different test programs which were coordinated to provide initially a basic understanding of certain ECC related phenomena and then to investigate progressively these phenomena as well as other ECC related phenomena in greater detail. The transparent vessel tests, which are reported herein, were conducted initially to investigate countercurrent flow and flow bypass phenomena in a Semiscale-sized downcomer and to determine whether these phenomena could be described in terms of existing countercurrent flow correlations. Air and water were used as the fluids for the transparent vessel tests because existing correlations were based on data limited almost exclusively to these fluids. The Semiscale system countercurrent flow tests ${ }^{[1]}$ were subsequently conducted to investigate steady state and transient countercurrent flow phenomena in a steam-water medium in which condensation and evaporation could affect the countercurrent flow phenomena. The concluding test program, the isothermal system test program ${ }^{[2-9]}$, was conducted to investigate transient ECC related phenomena and the interaction between these phenomena as well as the phenomena that occur during the blowdown portion of a simulated LOCA.

The transparent vessel test program was initiated when a literature search indicated that the majority of the countercurrent flow data was limited to data taken in small diameter tubes or packed beds with inlet geometries that distribute the flow uniformly around the top of the test section. Since the Semiscale vessel downcomer region is annular with a much larger diameter than most tubes tested and with an inlet geometry which would not necessarily distribute the fluid uniformly around the annulus, the applicability of prior countercurrent flow data to the Semiscale downcomer was uncertain. Therefore, a test program was conducted to supply countercurrent flow test data in a geometry similar to the Semiscale vessel. The objectives of the transparent vessel tests were to: 
(1) Investigate the countercurrent flow phenomena that occur in an annulus with dimensions similar to the Semiscale downcomer to determine whether commonly used countercurrent flow correlations describe the effect of the countercurrent air flow on the delivery of water (ECC) to the lower plenum.

(2) Investigate the relationship between flow bypassing the downcomer and countercurrent flow in the downcomer.

(3) Investigate the effect on countercurrent flow and bypass flow of changing the geometry of the upper annulus.

(4) Investigate the effect on countercurrent flow of changing the downcomer length by changing the length of the core barrel.

(5) Investigate the effect on countercurrent and bypass flow of a two-phase air-water mixture entering the vessel rather than water only.

(6) Establish a relationship between the countercurrent flow tests in the transparent vessel and the metal Semiscale vessel.

In addition to the tests performed to meet the outlined objectives, a limited number of tests were performed to investigate the effect on countercurrent flow of specialized system geometries. Table I summarizes the tests performed during the transparent vessel test program to meet the objectives outlined. The data presentation table column lists the report table number of Section IV in which the data are presented. Comments on the test groups are included to aid the reader in locating test groups of interest.

The intent of this document is to present the experimental data from the transparent vessel test program and to provide the necessary supportive information to make those data usable. Section II of the report provides a description of the experimental apparatus and instrumentation. Section III describes the test procedures and the data acquisition process. A complete listing of all collected data is presented in Section IV. 


\begin{tabular}{|c|c|c|c|c|c|}
\hline $\begin{array}{l}\text { Test } \\
\text { Group }\end{array}$ & $\begin{array}{l}\text { Downcomer } \\
\text { Radial } \\
\text { Gap (1n.) }\end{array}$ & $\begin{array}{c}\text { Lower Plenum } \\
\text { Pressure } \\
\text { (psig) }\end{array}$ & $\begin{array}{l}\text { Cold Leg } \\
\text { Air Flow } \\
\text { (scfm) }\end{array}$ & $\begin{array}{c}\text { Data } \\
\text { Presentation } \\
\text { Table }\end{array}$ & Coments \\
\hline
\end{tabular}
BASELINE COUNTERCURRENT FLOW TESTS

$\begin{array}{lccll}1.1 & 0.35 & 10 & 0 & \text { IV } \\ 1.2 & 0.35 & 30 & 0 & \text { V } \\ 2.1 & 0.37 & 7.3 & 0 & \text { VI } \\ 2.2 & 0.37 & 9 & 0 & \text { VII } \\ 2.3 & 0.37 & 30 & 0 & \text { VIII } \\ 4.1 & 0.49 & 7.3 & 0 & \text { IX } \\ 4.2 & 0.49 & 30 & 0 & \text { X } \\ 5.1 & 0.53 & 10 & 0 & \text { XI } \\ 6.1 & 0.63 & 10 & 0 & \text { XII } \\ 7.1 & 0.70 & 10 & 0 & \text { XIII } \\ 9.1 & 1.58 & 210 & 0 & \text { XIV }\end{array}$

BYPASS FLOW TESTS

$\begin{array}{llrrll}5.5 & 0.53 & 0,10 & 20-160 & \text { XV } & \\ 5.7 & 0.53 & 0 & 0-100 & \text { XVI } & \text { Hot leg simulators removed } \\ 5.12 & 0.53 & 0 & 0-200 & \text { XVII } & \text { Extended upper annulus } \\ 5.15 & 0.53 & 0 & 0-200 & \text { XVIII } & \text { Nozzle Inserts removed } \\ 9.5 & 1.58 & 0 & 100-165 & \text { XIX } & \end{array}$

MODIFIED UPPER ANNULUS GEOMETRY TESTS

\begin{tabular}{|c|c|c|c|c|c|}
\hline 5.4 & 0.53 & in & 0 & $x x$ & Filler piece lowered \\
\hline 5.8 & 0.53 & 10 & 0 & $\mathrm{XXI}$ & Hot leg simulators removed \\
\hline 5.10 & 0.53 & 10 & 0 & XXII & Extended upper annulus \\
\hline 5.13 & 0.53 & 10 & 0 & XXIII & Nozzle Inserts removed \\
\hline $6.2^{\prime}$ & 0.63 & 10 & 0 & XXIV & Filler plece lowered \\
\hline 9.2 & 1.58 & 10 & 0 & $\mathrm{xxV}$ & Nozzle Inserts removed \\
\hline 9.6 & 1.58 & 10 & 0 & XXvI & Hot leg simulators removed \\
\hline
\end{tabular}

SHORTENED CORE BARREL TESTS

$\begin{array}{llllll}3.1 & 0.40 & 10 & 0 & \text { XXVII } & \text { Downcomer length } 6 \text { in. } \\ 4.4 & 0.49 & 10 & 0 & \text { XXVIII } & \text { Downcomer length } 34 \text { in. } \\ 4.5 & 0.49 & 10 & 0 & \text { XXIX } & \text { Downcomer length } 24 \text { in. } \\ 4.6 & 0.49 & 10 & 0 & \text { XXX } & \text { Downcomer length } 6 \text { in. } \\ 8.1 & 1.45 & 10 & 0 & \text { XXXI } & \text { Downcomer length } 12 \text { in. }\end{array}$

TWO-PHASE COLD LEG MIXTURE TESTS

$\begin{array}{lllcl}.1 .3 & 0.35 & 10 & 30,80 & \text { XXXII } \\ 1.4 & 0.35 & 30 & 50 & \text { XXXIII } \\ 5.6 & 0.53 & 10 & 50-200 & \text { XXXIV } \\ 7.2 & 0.70 & 10 & 53 & \text { XXXV } \\ 9.4 & 1.58 & 10 & 50-200 & \text { XXXVI }\end{array}$

COMBINED EFFECTS TESTS

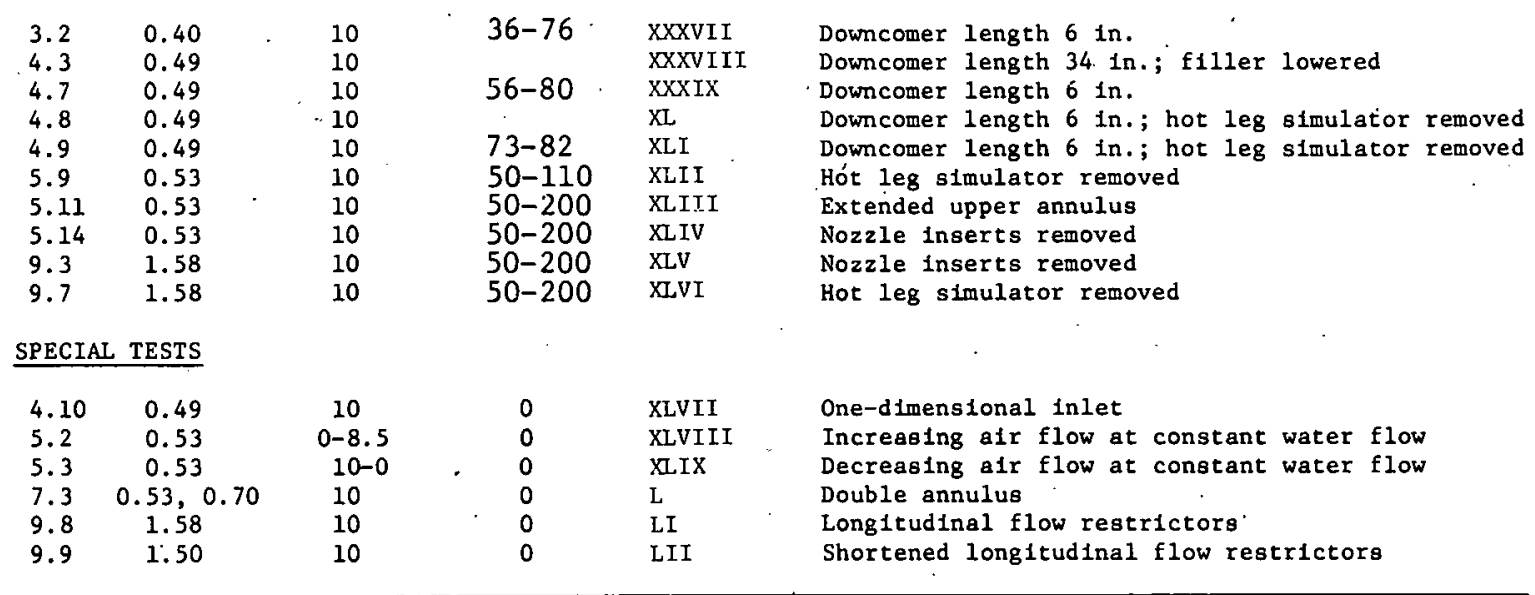




\section{EXXPERIMENTAL SYSTEM AND INSTRUMENTATION}

The transparent vessel experimental system consists of a transparent pressure vessel connected to an air and water supply system and to a drain system for the water. Two different air and water supply systems were used during the test program; a low air capacity system was used initially and a high air capacity system was used for subsequent tests.

The system instrumentation used to monitor the test conditions included flow, pressure, and temperature measuring devices.

\section{EXPERIMENTAL SYSTEM}

The pressure vessel is shown in the normal operating configuration in Figure 1 along with the nomenclature used to identify the different parts of the vessel. The pressure vessel was designed so that the core barrels and filler pieces could be changed to obtain different downcomer gap widths. Table II lists the radial downcomer gap widths as a function of the core barrel outside diameter (OD) and the filler piece inside diameter (ID). Since the inside diameter of the vessel is 8.4 inches, gaps using Filler D in Table II do not actually incorporate a filler piece. The effective length of the downcomer was varied by shortening the length of the core barrel. The upper annulus region is also designed to allow a change to be made in the diameter of the inlet pipe by removal of the nozzle liner and to allow changes to be made in the flow area of the upper annulus by removal of the hot leg simulators or by extension upward of the baffle.

The exterior portion of the transparent vessel is constructed of three sections of plexiglass tube each with an ID of 8.4 inches and an OD of 9.25 inches. The uppermost section of the vessel includes the nozzles for the cold leg inlet and the cold leg outlet which attach to the water supply system and the cold leg outlet drain system, respectively. Water or a two-phase air-water mix.ture enters the vessel through the cold leg inlet. All of the air that is forced into the vessel plus any water that bypasses the downcomer exits the vessel through the cold leg vessel outlet. The middle section of the vessel spans most of the distance of the downcomer, and the bottom section of the vessel, in conjunction with the lower head, forms the lower plenum region of the vessel. The lower head contains a 3 -inch drain for removal of the water that falls down the downcomer and into the lower plenum.

Three different plexiglass filler pieces were designed to change the downcomer dimensions by reducing the inside diameter of the vessel. The inside diameters of these filler pieces, which are the outside diameters of the corresponding downcomer, are listed in Table II. A somewhat different upper annulus geometry existed during tests with a filler piece installed than during tests with no filler piece. When a filler piece is installed to form the downcomer gap, a step occurs and reduces the flow area at the transition between the upper annulus and the downcomer. This step is 1.05 inches for Filler Piece A, 0.96 inch for Filler Piece B, and 0.34 inch for Filler Piece C. When the vessel wall, rather than one of the 


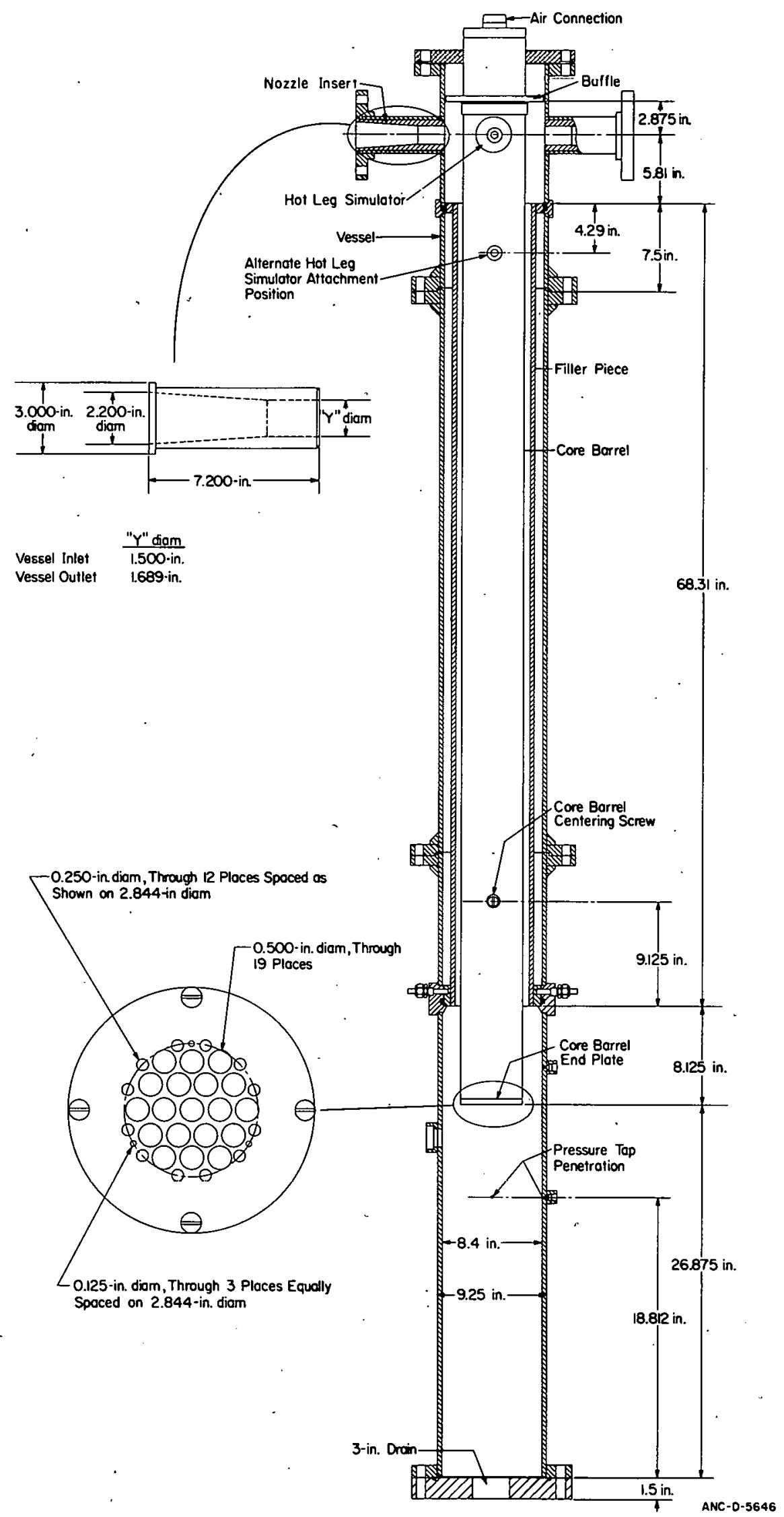

Fig. 1 Transparent vessel and internals. 
TABLE II

POSSIBLE DOWNCOMER GAP GEOMETRIES FOR TRANSPARENT VESSEL TESTS

\begin{tabular}{|c|r|r|c|c|}
\hline $\begin{array}{c}\text { Core } \\
\text { Filler } \\
\text { Piece }\end{array}$ & 1 & 2 & 3 & 4 \\
\hline A & $\begin{array}{r}0.53 \\
9.57\end{array}$ & $\begin{array}{r}0.40 \\
7.31\end{array}$ & & \\
\hline B & 0.63 & 0.49 & & \\
\hline C & 11.53 & 9.27 & & \\
\hline D & 1.25 & 1.11 & 0.37 & \\
& 25.43 & 23.17 & 8.48 & \\
\hline
\end{tabular}

Annular gap (in.)

Annulus flow area (in. ${ }^{2}$ )
Corè Barrel Dimensions

\begin{tabular}{lc} 
Outside & Associated \\
Diameter & Cross-Sectional \\
(in.) & Area (in.2) \\
\hline
\end{tabular}

1

5.24

5.51

7.00

7.69
21.55

23.81

38.50

$46.49^{\prime}$
Filler Piece Dimensions

Inside

Diameter

(in.)

$6: 30$

6.49

B

7.73

46.98

8.40

33.08

46.98

55.42
Associated

Cross-Sectional Area (in. 2)

filler pieces, is used to form the downcomer, no step occurs and the upper annulus and downcomer have the same flow area. Leakage of air and water between the filler piece and the vessel wall is prohibited by an 0-ring seal at the top and bottom of the filler piece. The groove in the vessel which holds the 0-ring seal in place was filled flush with the vessel surface for all tests in which a filler piece was not installed.

Four different aluminum core barrels were designed to allow changes to be made in the dimensions of the downcomer gap. The outside diameters of these core barrels, which form the inside diameters of the downcomer, are listed in Table II. The outer surface of each core barrel is anodized a blue color to enhance the clarity of visual and photographic observations of two-phase flow patterns in the downcomer region. 
The nozzle at the top of the core barrel is used to attach the core barrel to the air supply system. Air that enters the pressure vessel through the core barrel is forced to flow down through the core barrel, up the downcomer, and from the vessel through the vessel cold leg outlet. A multiholed plate is attached to the bottom of the core to decrease the flow area to a value that is similar to the flow area at the bottom of the Semiscale core.

A baffle ring attached near the top of the core barrel is used to simulate the seal ring which separates the upper annulus and upper plenum regions in the Semiscale vessel. Also attached to the core barrel in the upper annulus region are obstructions which have dimensions similar to those of the hot leg nozzles simulated in the Semiscale system. These hot leg simulators can be either removed completely or removed and placed at a lower position on the core barrel for tests with special upper annulus geometry configurations. These simulators are located such that their centerline is in the same axial plane as the centerline of the cold leg and vessel outlet nozzles.

Two different fixtures were designed so that geometry changes can be made in the upper annulus region of the pressure vessel. A 10-inch-long cylindrical plexiglass section with the same inside diameter as the vessel is designed to set directly on top of the vessel to act as a vessel extension. This section raises the core barrel and effectively increases the height of the upper annulus region. When this section is used, the hot leg simulators are removed from their normal position on the core barrel and moved down to a position where their centerline matches the centerline of the cold leg and bypass leg.

The second fixture is designed to replace the entire upper section of the vessel including the cold leg vessel iniet and outlet nozzles. This fixture, shown in Figure 2, is used to distribute the water uniformly around the top of the downcomer and to induce flow parallel to the downcomer axis. The perforated plate provides an even distribution of the incoming water, and the weir-type entrance to the downcomer induces the initial water flow to be parallel to the downcomer axis.

Two different air and water supply systems were used during the course of the transparent vessel tests. The initial system, shown in Figure 3, was designed for relatively low air flow rates and was used for performing tests with downcomer gap sizes of 0.70 inch or smaller. The subsequent system, shown in Figure 4, was designed for much larger air flows and was used for performing tests with both large and small downcomer gap sizes. For both systems, air was supplied from multistage compressors, and water was supplied from the building water supply system. The drain system from the lower plenum and the vessel cold leg outlet remained the same for all tests.

\section{SYSTEM INSTRUMENTATION}

Measurements of temperature, pressure, and volumetric flow were made by instrumentation located in the vessel throughout both the low capacity and the high capacity supply systems. A list of the instrumentation and a description of each detector is 

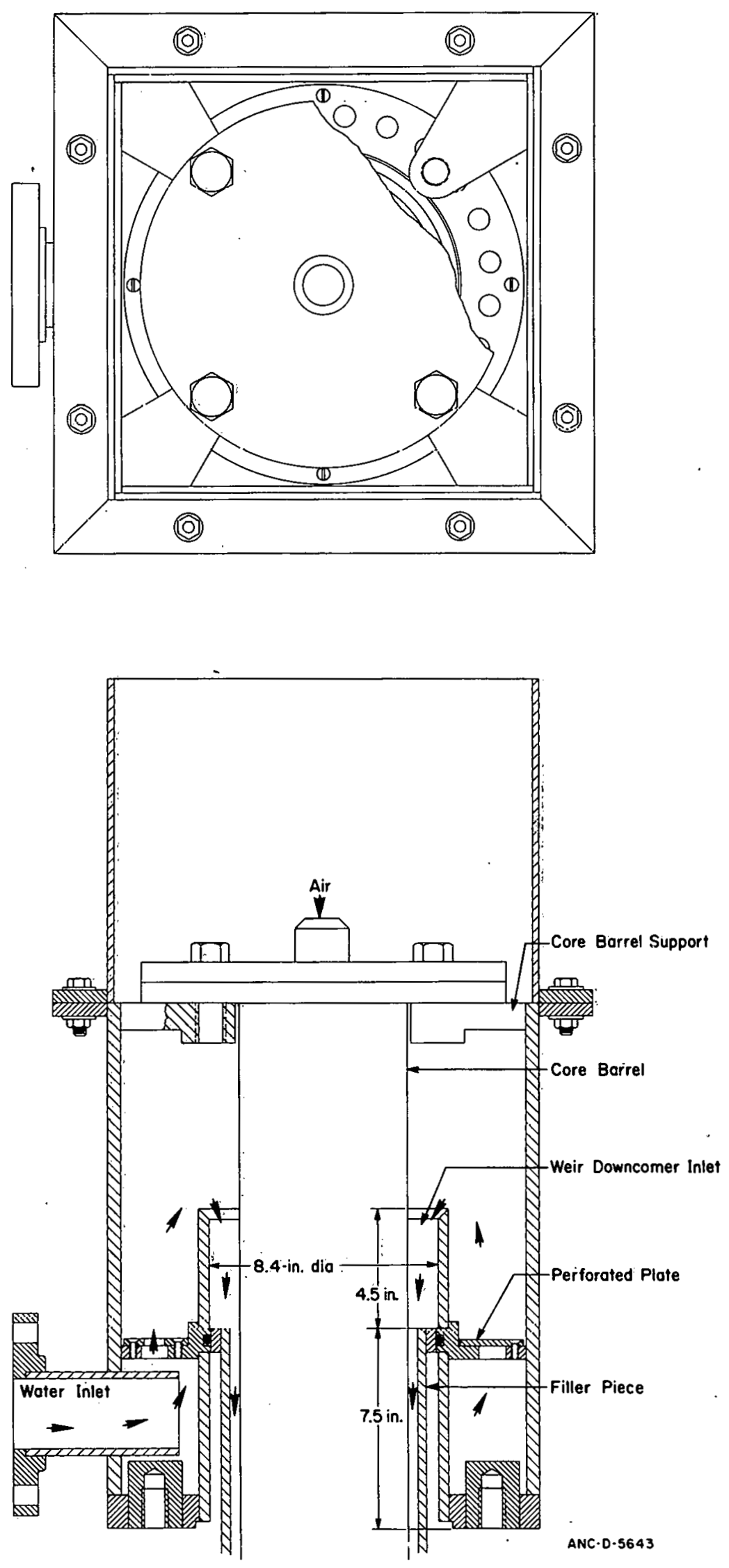

Fig. 2 Upper annulus uniform inlet flow distributor . 


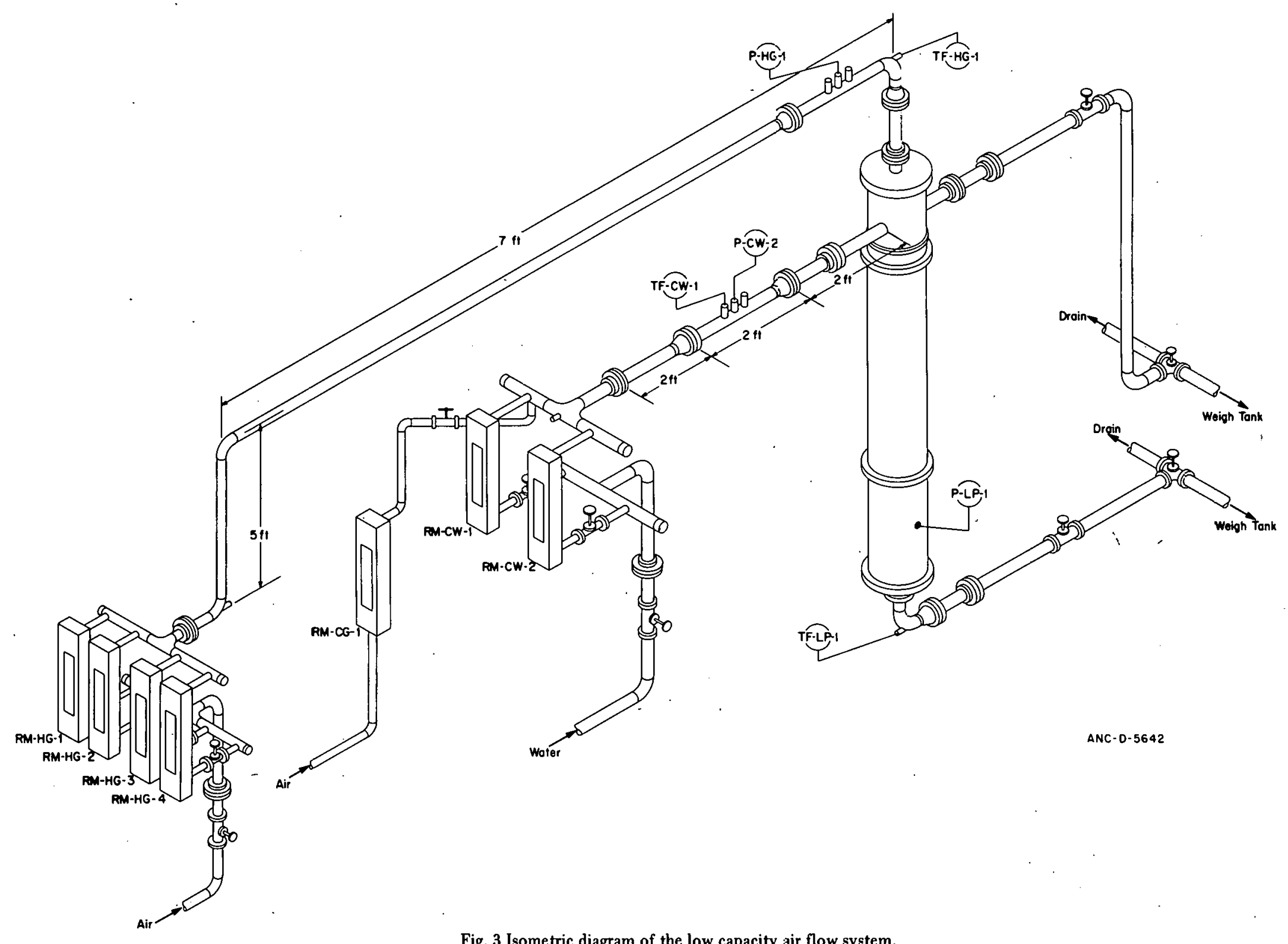




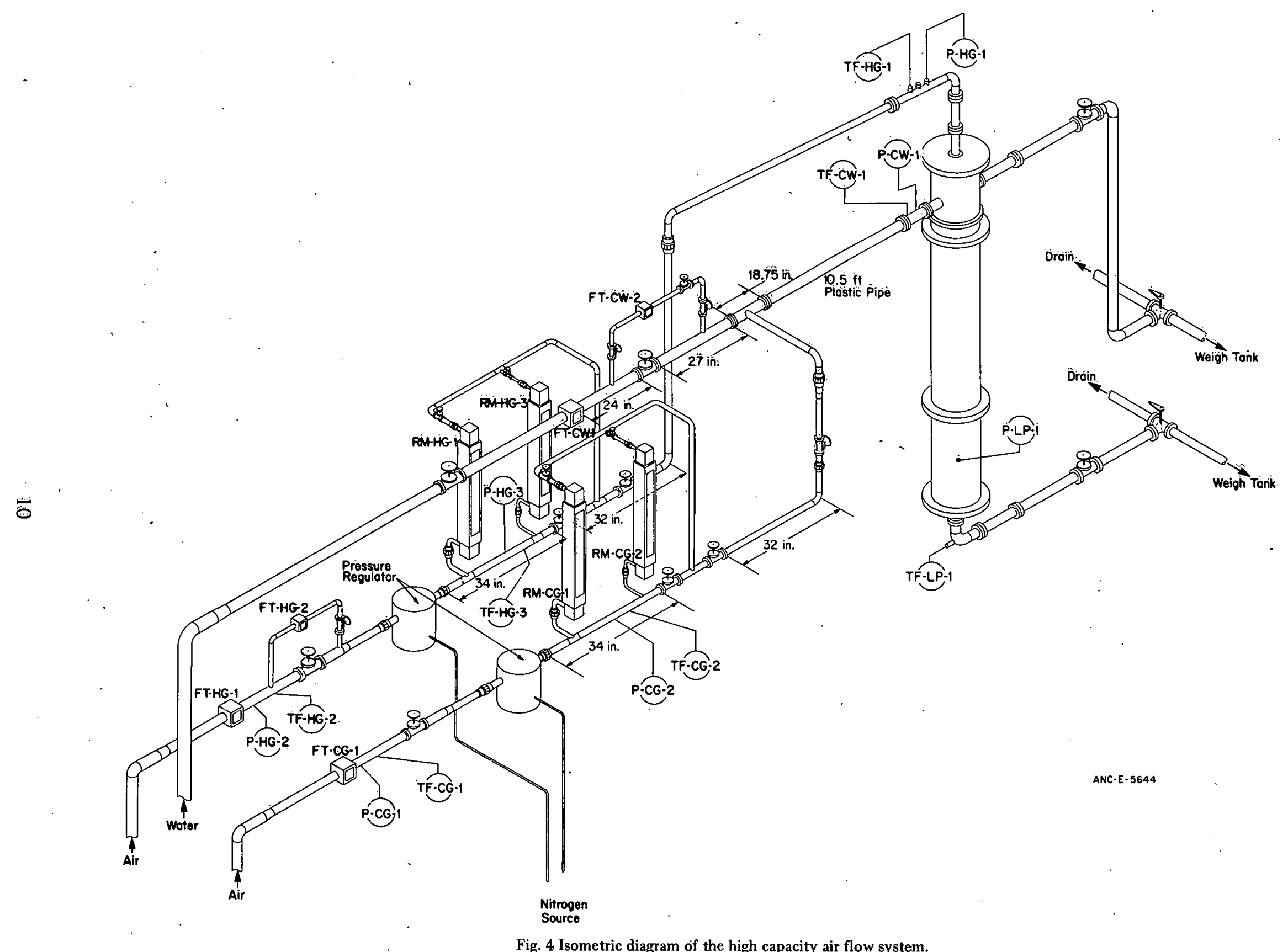

Fig. 4 Isometric diagram of the high capacity air flow system. 
included in Table III. The maximum error includes instrumentation error, signal conditioner error, and errors in reading the scales of the direct reading instrumentation. Figure 3 and 4 show the detector locations for the low and high capacity systems, respectively.

In addition to the instrumentation listed in Table III, weigh tanks were used in conjunction with a timing device to measure the mass flow of water from the lower plenum and from the vessel cold leg outlet. The two-phase air-water mixture flowing from the cold leg outlet was separated by directing the mixture downward into a large weigh tank with sides that were sufficiently high to prevent water from being blown out of the tank. Lower plenum liquid level was measured using ruled indicators scribed on the plexiglass. This measurement was made from the bottom of the core barrel to the liquid surface.

The measurements made for each test are presented in the section on data presentation (Section IV). Those instruments used for each test can be distinguished because the results are shown as nonzero values. An error analysis for typical tests is presented in Appendix A. The analysis has been restricted to a consideration of the probable and the maximum errors that would exist for the parameters of the Wallis ${ }^{[10]}$ countercurrent flow correlation if the data were put into this form. The Wallis correlation was used for this error analysis because it is a commonly used countercurrent flow correlation that was expected to correlate the transparent vessel test data. 


\section{TABLE III}

INSTRUMENTATION FOR TRANSPARENT VESSEL TESTS [a]

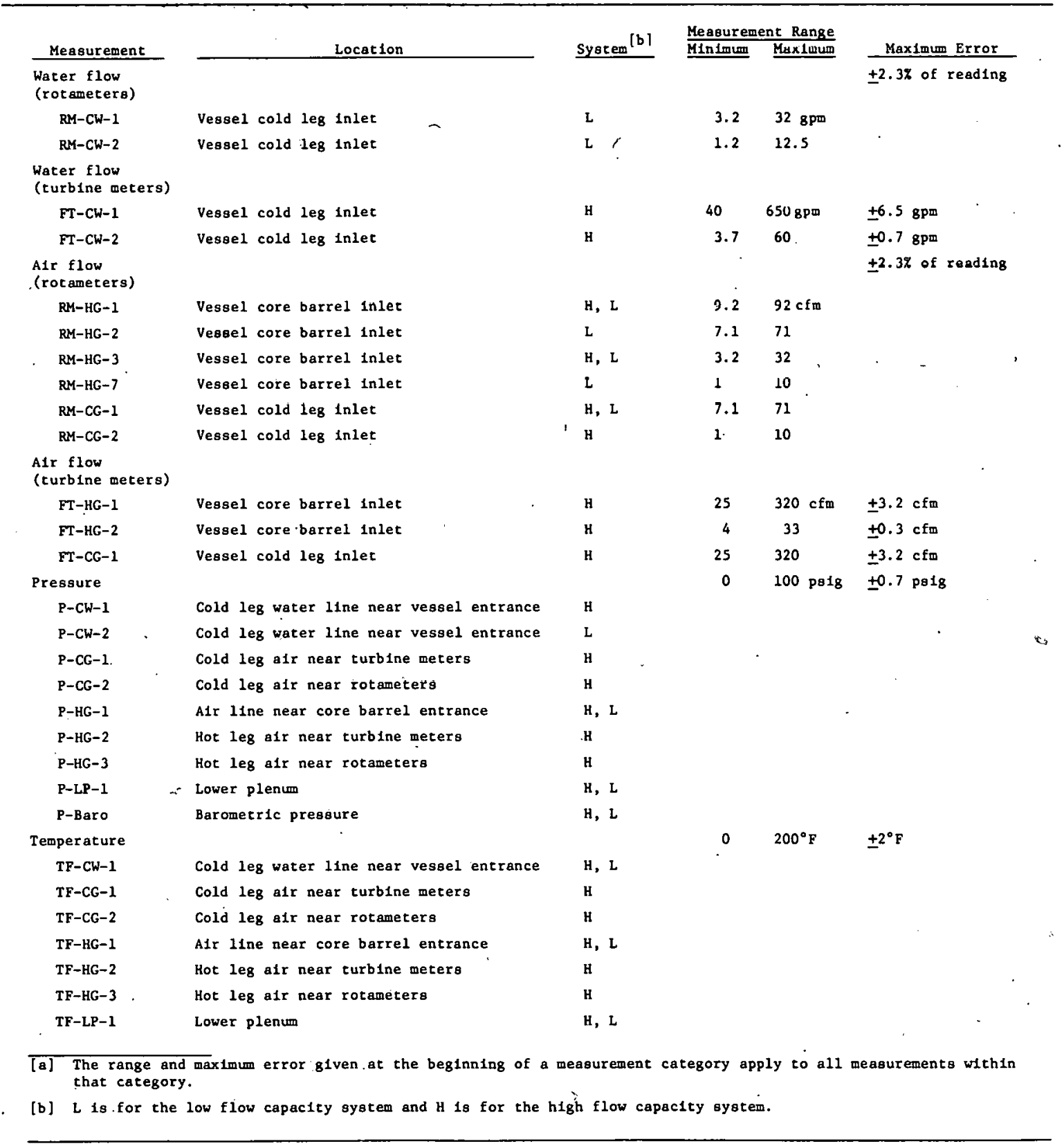




\section{TEST PROCEDURES}

The experimental system was used to obtain data for several different types of tests including countercurrent flow tests, bypass flow tests, and a number of tests with specialized objectives. The test procedures for the countercurrent flow tests and the bypass flow tests were different and, therefore, a separate discussion for these two types of tests follows. Some of the test procedures for the specialized tests were different from the other test procedures. However, these procedures apply to a limited number of tests and, therefore, are included in the description of the test groups documented in Section IV.

The paths of the air and water flowing in the transparent vessel are shown in Figure 5. Either water or an air-water mixture entered the transparent vessel through the vessel cold leg inlet nozzle. In either case, the entering water flowed either down the downcomer to the lower plenum or around the core barrel and out the cold leg vessel outlet. The air from the air-water mixture flowed around the core barrel and out the vessel cold leg outlet. Water reaching the lower plenum was removed at a rate consistent with maintaining a constant water level. Air entered the vessel through the top of the core barrel, flowed downward through the core barrel to the lower plenum, and then upward through the downcomer gap formed by the core barrel and the vessel wall or filler piece. Air could leave the vessel only through the vessel cold leg outlet because the only other outlet from the vessel, through the lower plenum, was sealed by the water collected therein.

Prior to the conduction of any countercurrent or flow bypass tests, several preliminary tests were conducted to establish a lower plenum liquid level that was far enough below the core barrel that no entrainment of lower plenum liquid occurred. These tests were accomplished by filling the lower plenum to a level above the bottom of the core barrel and then establishing a large air flow rate down through the core barrel. The air flow was continued until the lower plenum liquid level was stabilized. By using several different large air flows, a level was established below which lower plenum liquid entrainment would not occur. No formalized data were taken during these preliminary tests.

The majority of the tests conducted in the transparent vessel had countercurrent flow

in the downcomer. Operational testing procedures for these countercurrent flow tests included the following steps:

(1) The desired downcomer countercurrent air flow was established through the appropriate flow measuring device(s).

(2) The cold leg water flow along with the cold leg air flow, when used, was established through the appropriate flow measuring device(s).

(3) A constant lower plenum water level was established and maintained by throttling the drain valve. Water drained from the lower plenum was diverted to the building drainage system whenever flow measurements were not being made. 


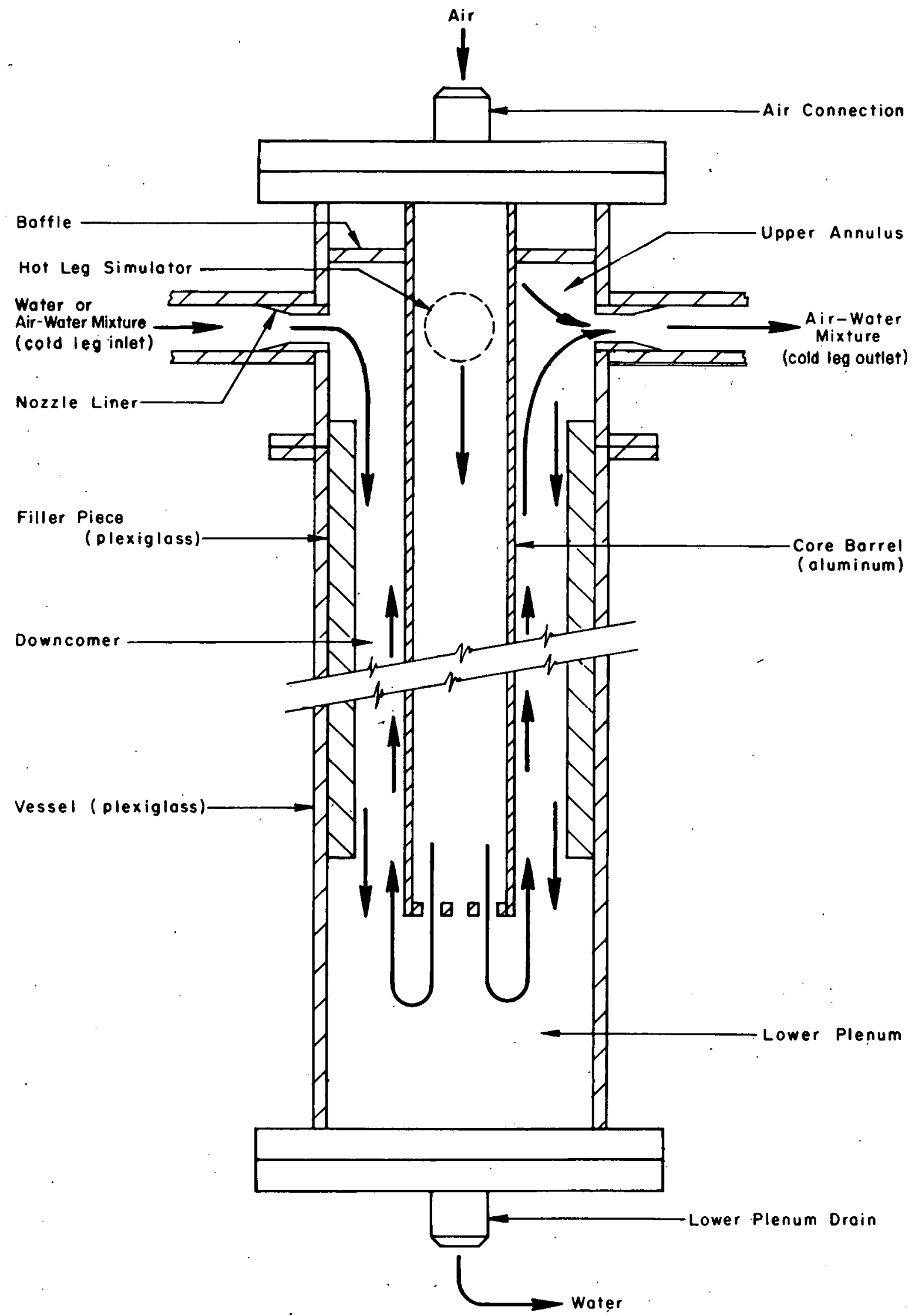

ANC-A-964-H

Fig. 5 Air and water flow paths in the transparent vessel. 
(4) The selected system pressure was established by throttling the valve on the vessel cold leg outlet line. The water flowing through this line was also dumped in to the building drainage system prior to measurement.

(5) The flows were continuously adjusted until the desired steady state operating point was.established.

(6) Appropriate data were hand recorded from the flow, temperature, and pressure instrumentation. The flows from the lower plenum and the vessel outlet were measured by directing these flows into weigh tanks for a measured period of time.

Several of the initial countercurrent flow tests were conducted with Steps 1 and 2 reversed to determine whether the order in which air and water flows were established influenced the countercurrent flow results. Since the order of establishment did not significantly influence the results, the listed procedure was used for the bulk of the countercurrent flow tests.

A limited number of bypass flow tests were performed to determine, in the absence of countercurrent air flow, the maximum cold leg water and air flow rates below which no bypass flow would occur. The operational test procedure for these bypass flow tests included the following steps:

(1) The desired air flow in the cold leg was established for those tests in which cold air flow was used.

(2) The water flow in the cold leg was increased slowly until water began to flow out the vessel'cold leg outlet. All bypass tests were conducted at or above this water flow rate.

(3) During tests in which cold leg air flow was used, the pressure in the system was adjusted to the desired value by establishing a lower plenum liquid level and subsequently throttling the valve in the vessel outlet line.

(4) Appropriate data were recorded from flow, temperature, and pressure instrumentation. The flows from the lower plenum and the vessel outlet were measured by directing these flows into weigh tanks for a measured period of time. 


\section{TEST DATA PRESENTATION}

The transparent vessel tests have been categorized into test groups according to the purposes of the tests and the system geometry. The test data for each group of tests have been organized into a series of tables for the purpose of data presentation. Correspondence between the test groups and the tables containing the data was shown in Table I.

Each data table contains a summary page listing pertinent variables calculated from the experimental data. Following this summary page are up to four sets of tabulated experimental data. The first set contains air and water volumetric flow measurements, and the second set contains pressure and temperature measurements associated with the flow measurements. The third set of data contains the weigh tank measurements for the lower plenum and vessel cold leg bypass as well as lower plenum pressure, liquid level, and temperature measurements. The barometric pressure is also included in this set of measurements. The fourth data set contains flow, pressure, and temperature measurements associated with air flow in the cold leg. This fourth data set is not included in the tables for tests which did not have air flow in the cold leg.

A brief description of the variables included on the summary page is provided to make those variables usable for further calculations. The downcomer air flow was obtained by summing the mass flow rates calculated at each separate air flow measurement in the core barrel air supply. The mass flow at each meter was calculated using the measured volumetric flow, and a density which was calculated using the perfect gas law with pressure and temperature measurements located near the volumetric flow measurement. At locations where a significant pressure drop occurred between the flow and pressure measurements, the pressure at the flow measurement location was determined by subtracting the pressure drop calculated to occur between the flow and pressure instrumentation from the measured pressure. The process of calculating mass flow using a pressure and a pressure drop was iterative because the magnitude of the pressure drop was influenced by the mass flow which in turn was influenced through a density change by the magnitude of the pressure drop. The cold leg air flow was calculated similarly using the cold leg flow, temperature, and pressure measurements. The downcomer air density was calculated using the perfect gas law with pressure and temperature measurements in the lower plenum.

Downcomer water flow was calculated from the measurement of the mass being drained from the lower plenum. Water flow continuity was obtained by adding the calculated water mass flow from the lower plenum to the calculated water mass flow leaving the vessel through the vessel cold leg outlet and dividing this sum by the measured water mass flow into the vessel. This ratio can be used as an indicator of the exactness of the water mass balance. Even though the water mass balance may not be exact, the lower plenum mass flow used in the countercurrent flow calculations may be valid. The vessel inlet water flow was calculated using the water. volumetric flow measurements and a fluid density of 62.4 $\mathrm{lb}_{\mathrm{m}} / \mathrm{ft}^{3}$. 
The parameters $\mathrm{J}$-air downcomer and $\mathrm{J}$-water downcomer are, respectively, the superficial velocities of the air and the water in the downcomer. The superficial air velocity was calculated by dividing the downcomer air flow by the downcomer air density and the total flow area of the downcomer, and the water superficial velocity was calculated similarly using a water density of $62.4 \mathrm{lb}_{\mathrm{m}} / \mathrm{ft}^{3}$.

The last two columns of the summary page contain parameters from the Wallis $[10]$ countercurrent flow correlation. The parameters for the Wallis countercurrent flow correlation are presented because this correlation is universally accepted as providing good correlation of countercurrent flow data. The form of the Wallis correlation

where

$$
\mathrm{J}_{\mathrm{g}}^{* 1 / 2}+\mathrm{m} \mathrm{J}_{1}^{* 1 / 2}=\mathrm{C}
$$

$$
\begin{aligned}
& \mathrm{J}_{g}^{*}=\frac{J_{g}}{\sqrt{g D}} \cdot\left(\frac{\rho_{g}}{\rho_{1}^{-\rho} g}\right)^{1 / 2} \\
& J_{1}^{*}=\frac{J_{1}}{\sqrt{g D}}\left(\frac{\rho_{1}}{\rho_{1}-\rho_{g}}\right)^{1 / 2}
\end{aligned}
$$

and

$$
\begin{aligned}
& \mathrm{J}_{\mathrm{g}}=\text { superficial gas velocity } \\
& \mathrm{J}_{1}=\text { superficial liquid velocity } \\
& \mathrm{D}=\text { downcomer hydraulic diameter } \\
& \rho_{\mathrm{g}}=\text { gas density } \\
& \rho_{\cdot 1}=\text { liquid density } \\
& \mathrm{g}=\text { gravitational constant }
\end{aligned}
$$

allows countercurrent flow data to be presented as a straight line with slope $\mathrm{m}$ and ordinate intercept $\mathrm{C}$.

A brief description of the test classifications, shown in Table I and certain of the test groups, is provided in the following paragraphs to aid in understanding the test data. Test groups were conducted with different radial downcomer gap widths in each different test classification to provide data for a range of downcomer geometries. 


\section{BASELINE COUNTERCURRENT FLOW TESTS .}

The baseline countercurrent flow tests were performed to investigate countercurrent phenomena in an annulus with dimensions and an inlet geometry similar to those of the Semiscale downcomer. The main objective of these tests was to provide data that could be used to determine whether commonly used countercurrent flow correlations are applicable to a downcomer the size of the Semiscale downcomer. Results from these baseline tests are presented in Tables IV through XIV.

During the early tests in which the smaller size downcomer gaps were used, several different cold leg water flow rates were used for each downcomer air flow rate. The results from these early tests showed that the amount of water reaching the lower plenum was relatively insensitive to the amount of water entering the vessel from the cold leg at high and intermediate downcomer air flows. However, at very low downcomer air flow rates, the results of the early tests showed that an increase in the inlel water flow would usually result in an increase in delivery to the lower plenum indicating some sensitivity in the amount of water reaching the lower plenum to the vessel inlet (cold leg) water flow. Usually, some maximum inlet water flow occurred above which no increase in lower plenum delivery rate was observed for an increase in the inlet flow, and a minimum inlet flow occurred below which all the water fell to the lower plenum. The difference between the maximum and minimum delivery rates to the lower plenum increased rapidly as the air flow approached zero.

Tests performed with the larger downcomer gap sizes, especially with the 1.58 -inch downcomer gap, showed that the amount of water reaching the lower plenum was sensitive to the inlet water flow rate even at relatively high downcomer air flow rates. The difference in vessel inlet water flow was large between the minimum flow for which all inlet flow fell to the lower plenum and the maximum flow for which delivery to the lower plenum was no longer sensitive to an increase in the inlet flow rate.

Several different groups of tests were conducted with different lower plenum pressures. The purpose of these tests was to provide data that could be used to check the sensitivity of the countercurrent flow correlations to changes in the operating pressure.

\section{BYPASS FLOW TESTS}

The objectives of the bypass flow tests were to determine, in the absence of countercurrent air flow, the minimum cold leg flow above which flow would bypass the downcomer and leave the vessel through the vessel cold leg outlet and to investigate the relationship between the amount of flow that bypassed for different cold leg flows above the minimum: The results from these tests are presented in Tables XV through XIX. Bypass flow tests were conducted using both single- and two-phase cold leg flows so that the results from both types of tests could be compared to determine the effect of a two-phase cold leg 
TABLE. IV

BASELINE COUNTERCURRENT FLOW TEST GROUP 1.1

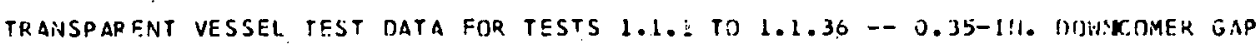
SUMMARY UF CALCILATEO P.:SULTS

\begin{tabular}{|c|c|c|c|c|c|c|c|c|c|c|}
\hline $\begin{array}{l}\text { TEST } \\
\text { NO }\end{array}$ & $\begin{array}{l}\text { OJWNCOMMER } \\
\text { AIR FLOW } \\
\text { (LB/SEC.) }\end{array}$ & $\begin{array}{l}\text { UJWNCOHEP } \\
\text { AIK DE:ISIIY } \\
\text { (LU/FT } \$ 3)\end{array}$ & $\begin{array}{c}\text { LOWER PLENUM } \\
\text { PRESSIJPE } \\
\text { (PSIA) }\end{array}$ & $\begin{array}{l}\text { COLD LEG } \\
\text { ALR FLNH } \\
\text { (LB/SEC) }\end{array}$ & $\begin{array}{l}\text { DOANCS:HEP. } \\
\text { WATEF FLCW } \\
\text { (LS/SEC) }\end{array}$ & $\begin{array}{l}\text { WATFR FLOIW } \\
\text { CONTENUITY }\end{array}$ & $\begin{array}{c}\text { J-AIF } \\
\text { DUWIICDMEP } \\
\text { (FT/SEC) }\end{array}$ & $\begin{array}{l}\text { J-HATER } \\
\text { OOHHITIMER } \\
\text { (FTSECI }\end{array}$ & $(J G *) * \# 1 / 2$ & $(\mathrm{Jl} *) * 1 / 2$ \\
\hline 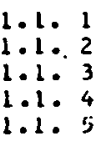 & $\begin{array}{l}0.0155 \\
0.0155 \\
0.0152 \\
0.0152 \\
0.0475\end{array}$ & $\begin{array}{l}0.1174 \\
0.1174 \\
0.1161 \\
0.11101 \\
0.1174\end{array}$ & $\begin{array}{l}22.45 \\
22.45 \\
22.45 \\
22.45 \\
22.45\end{array}$ & $\begin{array}{l}0.0 \\
0.0 \\
0.0 \\
0.0 \\
0.0\end{array}$ & $\begin{array}{l}4.783 \\
.3 .702 \\
4.633 \\
4.467 \\
1.842\end{array}$ & $\begin{array}{l}0.934 \\
0.957 \\
0.949 \\
1.008 \\
1.0053\end{array}$ & $\begin{array}{l}2.13 \\
2.13 \\
2.12 \\
2.12 \\
6.32\end{array}$ & $\begin{array}{l}1.237 \\
1.0457 \\
1.198 \\
1.155 \\
0.476\end{array}$ & $\begin{array}{l}0.2593 \\
0.2595 \\
0.2579 \\
0.2578 \\
0.4535\end{array}$ & $\begin{array}{l}0.9484 \\
0.8343 \\
0.9334 \\
0.9165 \\
0.5885\end{array}$ \\
\hline $\begin{array}{l}1.1 .6 \\
1.1: 7 \\
1.1: 9 \\
1.1 .99 \\
1.1 .10\end{array}$ & $\begin{array}{l}0.0472 \\
0.0470 \\
0.0470 \\
0.0793 \\
0.0789\end{array}$ & $\begin{array}{l}0.1174 \\
0.1174 \\
0.1174 \\
0.1164 \\
0.1164\end{array}$ & $\begin{array}{l}22.45 \\
22.45 \\
22.45 \\
22.33 \\
22.33\end{array}$ & $\begin{array}{l}0.0 \\
0.0 \\
0.0 \\
0.0 \\
0.0\end{array}$ & $\begin{array}{l}1.830 \\
2.017 \\
1.959 \\
3.755 \\
0.78 .\end{array}$ & $\begin{array}{l}1.009 \\
0.099 \\
0.995 \\
0.975 \\
1.111\end{array}$ & $\begin{array}{r}6.49 \\
6.45 \\
6.45 \\
11.00 \\
10.95\end{array}$ & $\begin{array}{l}0.475 \\
0.521 \\
0.396 \\
0.190 \\
0.2103\end{array}$ & $\begin{array}{l}0.4524 \\
0.4512 \\
0.4512 \\
0.5378 \\
0.5960\end{array}$ & $\begin{array}{l}0.5876 \\
0.6158 \\
0.6269 \\
0.3718 \\
0.3940\end{array}$ \\
\hline $\begin{array}{l}1.1 .11 \\
1.1 .12 \\
1.1 .13 \\
1.1 .14 \\
1.1 .15\end{array}$ & $\begin{array}{l}0.0743 \\
0.0793 \\
0.1129 \\
0.1123 \\
0.1116\end{array}$ & $\begin{array}{l}0.1164 \\
0.1104 \\
0.1164 \\
0.11164 \\
0.1163\end{array}$ & $\begin{array}{l}22.33 \\
22.33 \\
22.33 \\
22.33 \\
22.33\end{array}$ & $\begin{array}{l}0.0 \\
0.0 \\
0.0 \\
0.0 \\
0.0\end{array}$ & $\begin{array}{l}0.794 \\
0.784 \\
0.254 \\
0.250 \\
0.243\end{array}$ & $\begin{array}{l}J .993 \\
1.02 \mathrm{~J} \\
1.023 \\
1.011 \\
1.004\end{array}$ & $\begin{array}{l}11.010 \\
11.00 \\
12.55 \\
15.60 \\
13.62\end{array}$ & $\begin{array}{l}0.205 \\
0.204 \\
0.066 \\
0.065 \\
0.063\end{array}$ & $\begin{array}{l}0.5378 \\
0.5378 \\
0.7011 \\
0.7000 \\
0.6900\end{array}$ & $\begin{array}{l}0.3865 \\
0.3952 \\
0.2146 \\
0.2168 \\
0.2137\end{array}$ \\
\hline $\begin{array}{l}1.1 .16 \\
1.1 .17 \\
1.1 .18 \\
1.1 .19 \\
1.1 .20\end{array}$ & $\begin{array}{l}0.1126 \\
0.1420 \\
0.1428 \\
0.1267 \\
0.1200\end{array}$ & $\begin{array}{l}0.11160 \\
0.11131 \\
0.1137 \\
0.11130 \\
0.11250\end{array}$ & $\begin{array}{l}22.33 \\
22.33 \\
22.33 \\
22.33 \\
22.33\end{array}$ & $\begin{array}{l}0.0 \\
0.0 \\
0.0 \\
0.0 \\
0.0\end{array}$ & $\begin{array}{l}0.242 \\
0.067 \\
0.007 \\
0.117 \\
0.117\end{array}$ & $\begin{array}{l}1.031 \\
0.981 \\
1.011 \\
1.012 \\
0.976\end{array}$ & $\begin{array}{l}15.50 \\
20.15 \\
20.25 \\
11.79 \\
17.68\end{array}$ & $\begin{array}{l}0.062 \\
0.01 ? \\
0.01 ? \\
0.0313 \\
0.030\end{array}$ & $\begin{array}{l}0.5596 \\
0.7910 \\
j .7930 \\
0.7449 \\
0.7430\end{array}$ & $\begin{array}{l}0.2132 \\
0.1120 \\
0.1120 \\
0.1481 \\
0.14 H 1\end{array}$ \\
\hline $\begin{array}{l}1.1 .21 \\
1.1 .22 \\
1.1 .23 \\
1.1 .24 \\
1.1 .25\end{array}$ & $\begin{array}{l}0.1260 \\
0.1275 \\
0.1280 \\
0.0954 \\
0.0944\end{array}$ & $\begin{array}{l}0.1120 \\
1.1160 \\
0.11613 \\
0.1167 \\
0.1167\end{array}$ & $\begin{array}{l}22.33 \\
22.40 \\
22.40 \\
22.90 \\
22.40\end{array}$ & $\begin{array}{l}0.0 \\
0.0 \\
0.0 \\
0.0 \\
0.0\end{array}$ & $\begin{array}{l}0.111 \\
0.112 \\
0.133 \\
0.423 \\
0.371\end{array}$ & $\begin{array}{l}0.904 \\
1.904 \\
1.970 \\
0.905 \\
1.007\end{array}$ & $\begin{array}{l}11.65 \\
17.73 \\
17.80 \\
13.19 \\
13.05\end{array}$ & $\begin{array}{l}0.030 \\
0.027 \\
0.034 \\
0.125 \\
0.096\end{array}$ & $\begin{array}{l}0.7430 \\
0.7456 \\
0.7470 \\
0.6440 \\
0.6406\end{array}$ & $\begin{array}{l}0.1481 \\
0.1455 \\
0.1583 \\
0.3015 \\
0.2641\end{array}$ \\
\hline $\begin{array}{l}1.1 .26 \\
1.1 .27 \\
1.1 .28 \\
1.1 .29 \\
1.1 .30\end{array}$ & $\begin{array}{l}0.0951 \\
0.0940 \\
0.0451 \\
0.0946 \\
0.0622\end{array}$ & $\begin{array}{l}0.1167 \\
0.1172 \\
0.11172 \\
0.11172 \\
0.1174\end{array}$ & $\begin{array}{l}22.40 \\
22.40 \\
22.40 \\
22.40 \\
22.40\end{array}$ & $\begin{array}{l}0.0 \\
0.0 \\
c .0 \\
0.0 \\
0.0\end{array}$ & $\begin{array}{l}0.425 \\
0.452 \\
0.446 \\
0.371 \\
1.225\end{array}$ & $\begin{array}{l}1.013 \\
1.1115 \\
0.046 \\
0.980 \\
0.995\end{array}$ & $\begin{array}{r}13.14 \\
13.04 \\
13.10 \\
13.02 \\
8.54\end{array}$ & $\begin{array}{l}0.1120 \\
0.112 \\
0.115 \\
0.201 \\
0.311\end{array}$ & $\begin{array}{l}0.6429 \\
0.6409 \\
0.6426 \\
0.6406 \\
0.5191\end{array}$ & $\begin{array}{l}0.2827 \\
0.225 \mathrm{C} \\
0.2846 \\
0.2711 \\
0.4800\end{array}$ \\
\hline $\begin{array}{l}1.1 .31 \\
1.1 .32 \\
1.1 .33 \\
1.1 .34 \\
1.1 .35\end{array}$ & $\begin{array}{l}0.0024 \\
0.0620 \\
0.0621 \\
0.0300 \\
0.0307\end{array}$ & $\begin{array}{l}0.1174 \\
0.1174 \\
0.1176 \\
0.1113 \\
0.1174\end{array}$ & $\begin{array}{l}22.40 \\
22.40 \\
22.40 \\
22.40 \\
22.40\end{array}$ & $\begin{array}{l}0.0 \\
0.0 \\
0.0 \\
0.0 \\
0.0\end{array}$ & $\begin{array}{l}1.209 \\
1.319 \\
1.275 \\
2.707 \\
2.957\end{array}$ & $\begin{array}{l}1.01 ? \\
1.005 \\
1.045 \\
0.993 \\
0.938\end{array}$ & $\begin{array}{l}9.53 \\
8.63 \\
4.53 \\
4.16 \\
4.25\end{array}$ & $\begin{array}{l}0.323 \\
0.314 \\
0.339 \\
0.721 \\
0.765\end{array}$ & $\begin{array}{l}0.5203 . \\
0.5217 \\
0.5186 \\
0.3612 \\
0.3551\end{array}$ & $\begin{array}{l}0.4985 \\
0.4980 \\
0.4857 \\
0.7240 \\
0.7457\end{array}$ \\
\hline 1.1 .36 & 0.0312 & 0.1170 & 22.40 & 0.0 & 3.093 & 0.970 & 4.27 & $0.300^{\circ}$ & 0.3679 & 0.7627 \\
\hline
\end{tabular}


TABLE IV (Contd.)

BASELINE COUNTERCURRENT FLOW TEST GROUP $1: 1$

EXPERIMENTAL MEASUPMENTS FOR TESTS 1.1 .1 TO $1.1 .36-0.35-I N$. DOWNCOMER GA?

\begin{tabular}{|c|c|}
\hline $\begin{array}{c}R M-C H-1 \\
\{G P M\}\end{array}$ & $\begin{array}{c}R M-C W-2 \\
(G P M)\end{array}$ \\
\hline $\begin{array}{l}30.0 \\
20.0 \\
30.0 \\
25.0 \\
30.0\end{array}$ & $\begin{array}{l}10.0 \\
10.0 \\
10.0 \\
10.0 \\
10.0\end{array}$ \\
\hline $\begin{array}{l}20.0 \\
20.0 \\
25.0 \\
30.0 \\
20.0\end{array}$ & $\begin{array}{r}10.0 \\
0.0 \\
0.0 \\
10.0 \\
10.0\end{array}$ \\
\hline $\begin{array}{r}2.0 .0 \\
0.0 \\
30.0 \\
20.0 \\
20.0\end{array}$ & $\begin{array}{r}0.0 \\
8.0 \\
10.0 \\
10.0 \\
0.0\end{array}$ \\
\hline $\begin{array}{r}0.0 \\
30.0 \\
0.0 \\
30.0 \\
2.0 .0\end{array}$ & $\begin{array}{r}5.0 \\
10.0 \\
10.0 \\
10.0 \\
10.0\end{array}$ \\
\hline $\begin{array}{r}20.0 \\
3.0 \\
3.0 \\
30.0 \\
20.0\end{array}$ & $\begin{array}{r}0.0 \\
10.0 \\
4.0 \\
10.0 \\
10.0\end{array}$ \\
\hline $\begin{array}{r}20.0 \\
20.0 \\
0.0 \\
0.0 \\
30.0\end{array}$ & $\begin{array}{r}0.0 \\
10.0 \\
10.0 \\
5.0 \\
10.0\end{array}$ \\
\hline $\begin{array}{l}20.0 \\
20.0 \\
12.0 \\
30.0 \\
20.0\end{array}$ & $\begin{array}{r}10.0 \\
0.0 \\
0.0 \\
10.0 \\
10.0\end{array}$ \\
\hline 23.0 & 0.0 \\
\hline
\end{tabular}

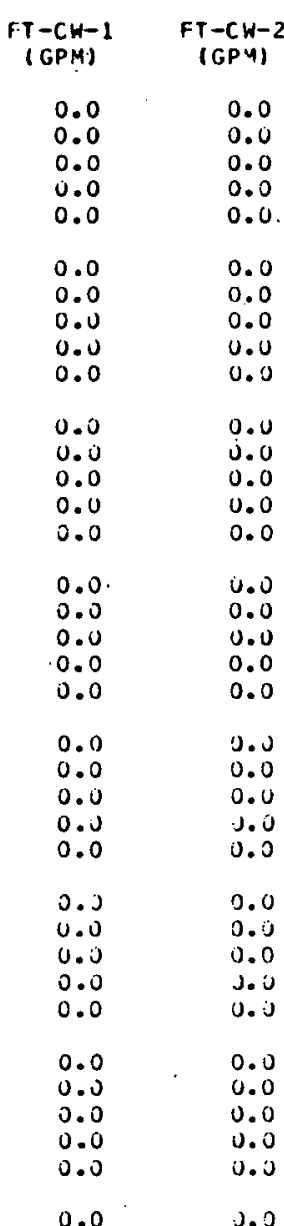

T

1.1.

1.1 .3

1.1. 4

1.1. 6

1.1. 7

1.1 .9

1.1 .11

1.1 .12

1.1 .14
1.1 .15

1.1 .1

1.1 .17
1.1 .18
1.1 .19

$$
1.1 .1
$$

1.1 .21

$$
\begin{aligned}
& 1.1 .22 \\
& 1.1 .23 \\
& 1.1 .24 \\
& 1.1 .25
\end{aligned}
$$$$
\text { 1.1.2 }
$$

$$
\begin{aligned}
& 1.1 .28 \\
& 1.1 .29
\end{aligned}
$$$$
1.1 .30
$$

1.1 .31

$$
\begin{array}{lll}
1.1 .33 \\
1.1 .34
\end{array}
$$$$
1.1 .35
$$

23.0

0.0

$R M-H G-1$
$(C F+1)$

$$
\begin{aligned}
& 50.00 \\
& 50.00 \\
& 50.00 \\
& 0.0
\end{aligned}
$$$$
0.0
$$

0.0
0.0
0.0

(CFM)

RM-HC, 3 (C,FM)

RM-11G-4 (CFM)

$F T-H G-1$ [CFM]

FT $-H C,-2$
(CFM)

$0.0 \quad 10.00$

$0.0 \quad 10.00$

$\begin{array}{ll}0.0 & 10.000 \\ 0.0 & 10.00\end{array}$

0.0

$0.0 \quad 0.0$

$0.0 \quad 0.0 \quad 10.00$

0.0

0.0
0.0
0.0

$\begin{array}{ll}0.0 & 0.0 \\ 0.0 & 0.0\end{array}$

$\begin{array}{lll}0.0 & 30.00 & 0.0 \\ 0.0 & 30.000 & 0.00\end{array}$

$0.0 \quad 30.00 \quad 0.0$

$0.0 \quad 30.00$

50.00
50.00

0.0

50.00

30.00

70.00

70.00

70.00

70.00

50.00
50.00

50.00

50.00
50.00

0.0

0.0
.0 .0

0.0
0.0
0.0

0.0
0.0

0.0

0.0

40.00

20.00

30.00

30.02

31.000
30.00

6.0.00

60.00

00.00

60.00
60.00

40.010

10.00
40.00

40.00
40.00

0.0

0.0

0.0

U. 0
0.0

0.0
0.0
0.0

0.0

3.j

0.0
0.0
0.0

0.0

0.0

0.0
0.0
0.0

0.0

0.0
0.0
0.3

0.0

0.0

0.0
0.0
0.0

0.0
0.2
0.0

0.0

0.0

20.00

20.00

20.00

0.0
0.0

0.0
0.0

0.0

0.0
0.0

0.0

$\begin{array}{ll}0.0 & 0.0 \\ 0.0 & 0.0\end{array}$

$\begin{array}{ll}0.0 & 0.0 \\ 0.0 & 0.0\end{array}$

0.0

c. 0
a. 0

0.0
0.0

0.0
0.0

$\begin{array}{ll}0.0 & 0.0 \\ 0.0 & 0.0\end{array}$

$0.0 \quad 0.0$

$\begin{array}{ll}0.0 & 0.0 \\ 0.0 & 0.0\end{array}$

$0.0 \quad 0.0 \quad 0.0$

$\begin{array}{lll}1.0 & 0.0 & 0 \\ 0.0 & 0.0 & 0.0\end{array}$

3.0

13.0

0.0

0.0
3.0
3.0

0.0

0.0

0.0
0.0

0.0
0.0

0.0

0.0
0.0
13.0
0.0

0.0

0.0
0.0
0.0

0.0

0.0

3.0
0.0
0.0

0.0 
TABLE IV (Contd.)

BASELINE COUNTERCURRENT FLOW TEST GROUP 1.1

EXPEF IMENTAL IAEASURMENTS FOR TESTS 1.1.1 TO $1.1 .36-0.35-1 N$. DUWINCOMER TAP

\begin{tabular}{|c|c|c|c|c|c|c|c|c|c|c|}
\hline $\begin{array}{l}\text { TEST } \\
\text { NC }\end{array}$ & $\begin{array}{l}P-C H-1 \\
\text { (PSIC) }\end{array}$ & $\begin{array}{l}P-C H-2 \\
(P S I G)\end{array}$ & $\begin{array}{l}P-H G-1 \\
(P S I G)\end{array}$ & $\begin{array}{l}\mathrm{P}-\mathrm{H} C_{2}-2 \\
\left(P S \mid C_{3}\right)\end{array}$ & $\begin{array}{l}P-H G G=3 \\
\text { (PSIG) }\end{array}$ & $\begin{array}{l}T F-C(W-1 \\
(D F G F)\end{array}$ & $\begin{array}{l}\mid F-11 G=1 \\
(D E G F)\end{array}$ & $\begin{array}{l}Y F-H>-2 \\
(D E G \quad F)\end{array}$ & $\begin{array}{l}T F-H G-3 \\
(J E F, F)\end{array}$ & $\begin{array}{l}\text { P-BAE? } \\
\text { (PSIAI) }\end{array}$ \\
\hline $\begin{array}{lll}1.1 .1 & 1 \\
1.1 .1 & 2 \\
1.1 . & 3 \\
1.1 . & 4 \\
1.1 . & 5\end{array}$ & $\begin{array}{l}0.0 \\
0.0 \\
0.0 \\
0.0 \\
0.0\end{array}$ & $\begin{array}{l}10.5 \\
10.7 \\
10.0 \\
10.2 \\
11.0\end{array}$ & $\begin{array}{l}10.7 \\
10.7 \\
10.0 \\
10.0 \\
11.5\end{array}$ & $\begin{array}{l}0.0 \\
0.0 \\
0.0 \\
0.0 \\
0.0\end{array}$ & $\begin{array}{l}0.0 \\
0.0 \\
0.0 \\
0.0 \\
0.0\end{array}$ & $\begin{array}{l}53.0 \\
53.0 \\
53.0 \\
53.0 \\
53.0\end{array}$ & $\begin{array}{l}81.0 \\
80.0 \\
82.0 \\
82.0 \\
82.0\end{array}$ & $\begin{array}{l}0.0 \\
0.0 \\
0.0 \\
0.0 \\
0.0\end{array}$ & $\begin{array}{l}1.0 \\
1.0 \\
0.0 \\
0.0 \\
0.0\end{array}$ & $\begin{array}{l}12.5 \\
12.5 \\
12.5 \\
12.5 \\
12.5\end{array}$ \\
\hline $\begin{array}{ll}1.1 .1 & 6 \\
1.1 . & 7 \\
1.1 .1 & 8 \\
1.1 . & 9 \\
1.1 .1 & 0\end{array}$ & $\begin{array}{l}J .0 \\
0.0 \\
0.0 \\
1.0 \\
0.0\end{array}$ & $\begin{array}{l}10.7 \\
10.7 \\
10.7 \\
11.2 \\
11.2\end{array}$ & $\begin{array}{l}11.2 \\
11.0 \\
11.0 \\
11.5 \\
11.2\end{array}$ & $\begin{array}{l}0.0 \\
0.0 \\
0.0 \\
0.0 \\
0.0\end{array}$ & $\begin{array}{l}0.0 \\
0.0 \\
0.0 \\
0.0 \\
0.0\end{array}$ & $\begin{array}{l}53.0 \\
53.0 \\
53.0 \\
53.0 \\
55.0\end{array}$ & $\begin{array}{l}82.0 \\
82.0 \\
82.0 \\
84.0 \\
85.0\end{array}$ & $\begin{array}{l}0.0 \\
0.0 \\
0.0 \\
0.0 \\
0.0\end{array}$ & $\begin{array}{l}0.0 \\
0.0 \\
0.0 \\
0.0 \\
0.0\end{array}$ & $\begin{array}{l}12.5 \\
12.5 \\
12.5 \\
12.3 \\
12.3\end{array}$ \\
\hline $\begin{array}{l}1.1 .11 \\
1.1 .12 \\
1.1 .13 \\
1.1 .14 \\
1.1 .15\end{array}$ & $\begin{array}{l}0.0 \\
0.0 \\
0.0 \\
0.0 \\
0.0\end{array}$ & $\begin{array}{l}11.2 \\
11.2 \\
11.5 \\
11.0 \\
11.5\end{array}$ & $\begin{array}{l}11.5 \\
11.5 \\
11.7 \\
11.5 \\
11.2\end{array}$ & $\begin{array}{l}0.0 \\
0.0 \\
0.0 \\
0.0 \\
0.0\end{array}$ & $\begin{array}{l}0.0 \\
0.0 \\
0.0 \\
0.0 \\
0.0\end{array}$ & $\begin{array}{l}54.0 \\
54.0 \\
53.0 \\
53.0 \\
53.0\end{array}$ & $\begin{array}{l}84.0 \\
84.0 \\
84.0 \\
82.3 \\
85.0\end{array}$ & $\begin{array}{l}0.0 \\
0.0 \\
0.0 \\
0.0 \\
0.0\end{array}$ & $\begin{array}{l}0.0 \\
0.0 \\
0.0 \\
0.0 \\
0.0\end{array}$ & $\begin{array}{l}12.3 \\
12.3 \\
12.3 \\
12.3 \\
12.3\end{array}$ \\
\hline $\begin{array}{l}1.1 .16 \\
1.1 .17 \\
1.1 .18 \\
1.1 .19 \\
1.1 .20\end{array}$ & $\begin{array}{l}0.0 \\
0.0 \\
0.0 \\
0.0 \\
0.0\end{array}$ & $\begin{array}{l}11.0 \\
11.0 \\
11.2 \\
11.5 \\
11.2\end{array}$ & $\begin{array}{l}11.5 \\
11.5 \\
11.7 \\
11.7 \\
11.3\end{array}$ & $\begin{array}{l}0.0 \\
0.0 \\
j .0 \\
0.0 \\
0.0\end{array}$ & $\begin{array}{l}0.0 \\
0.0 \\
0.0 \\
0.0 \\
0.0\end{array}$ & $\begin{array}{l}53.0 \\
52.0 \\
53.2 \\
53.0 \\
53.0\end{array}$ & $\begin{array}{l}81.0 \\
91.0 \\
91.0 \\
41.0 \\
31.0\end{array}$ & $\begin{array}{l}1.0 \\
0.0 \\
0.0 \\
0.0 \\
0.0\end{array}$ & $\begin{array}{l}0.0 \\
0.0 \\
0.0 \\
3.0 \\
0.0\end{array}$ & $\begin{array}{l}12.3 \\
12.3 \\
12.3 \\
12.3 \\
12.3\end{array}$ \\
\hline $\begin{array}{l}1.1 .21 \\
1.1 .22 \\
1.1 .23 \\
1.1 .24 \\
1.1 .25\end{array}$ & $\begin{array}{l}0.0 \\
3.0 \\
0.0 \\
0.0 \\
0.0\end{array}$ & $\begin{array}{l}11.2 \\
11.2 \\
11.2 \\
10.7 \\
10.7\end{array}$ & $\begin{array}{l}11.5 \\
11.5 \\
11.5 \\
11.0 \\
10.5\end{array}$ & $\begin{array}{l}0.0 \\
0.0 \\
0.0 \\
0.0 \\
0.0\end{array}$ & $\begin{array}{l}u . u \\
0.0 \\
0.0 \\
0.0 \\
0.0\end{array}$ & $\begin{array}{l}53.0 \\
56.0 \\
53.0 \\
52.0 \\
51.0\end{array}$ & $\begin{array}{l}91.0 \\
90.0 \\
70.0 \\
70.0 \\
70.0\end{array}$ & $\begin{array}{l}0.0 \\
0.0 \\
j .0 \\
0.0 \\
9.0\end{array}$ & $\begin{array}{l}0.0 \\
0.0 \\
0.0 \\
0.0 \\
0.0\end{array}$ & $\begin{array}{l}12.3 \\
12.4 \\
12.4 \\
12.4 \\
12.4\end{array}$ \\
\hline $\begin{array}{l}1.1 .20 \\
1.1 .27 \\
1.1 .28 \\
1.1 .29 \\
1.1 .30\end{array}$ & $\begin{array}{l}j .0 \\
0.0 \\
1.0 \\
0.0 \\
0.0\end{array}$ & $\begin{array}{l}10.5 \\
10.5 \\
10.2 \\
10.2 \\
10.0\end{array}$ & $\begin{array}{l}10.7 \\
10.5 \\
10.7 \\
10.5 \\
10.2\end{array}$ & $\begin{array}{l}0.0 \\
0.0 \\
0.0 \\
0.0 \\
0.0\end{array}$ & $\begin{array}{l}0.0 \\
0.0 \\
0.0 \\
0.0 \\
0.0\end{array}$ & $\begin{array}{l}51.0 \\
51.0 \\
52.0 \\
52.0 \\
51.0\end{array}$ & $\begin{array}{l}76.0 \\
75.0 \\
75.0 \\
75.0 \\
75.0\end{array}$ & $\begin{array}{l}0.0 \\
0.0 \\
0.0 \\
0.0 \\
0.0\end{array}$ & $\begin{array}{l}3.0 \\
0.0 \\
0.0 \\
0.0 \\
0.0\end{array}$ & $\begin{array}{l}12.4 . \\
12.4 \\
12.4 \\
12.4 \\
12.4\end{array}$ \\
\hline $\begin{array}{l}1.1 .31 \\
1.1 .32 \\
1.1 .33 \\
1.1 .34 \\
1.1 .15\end{array}$ & $\begin{array}{l}נ .0 \\
j .0 \\
j .0 \\
j .0 \\
j .0\end{array}$ & $\begin{array}{r}10.5 \\
11.0 \\
9.7 \\
7.2 \\
10.0\end{array}$ & $\begin{array}{r}10.5 \\
10.7 \\
10.2 \\
9.5 \\
10.2\end{array}$ & $\begin{array}{l}0.0 \\
0.0 \\
0.0 \\
0.0 \\
0.0\end{array}$ & $\begin{array}{l}0.0 \\
0.0 \\
0.0 \\
0.0 \\
0.0\end{array}$ & $\begin{array}{l}52.0 \\
52.0 \\
52.1 \\
53.0 \\
52.0\end{array}$ & $\begin{array}{l}76.1 \\
76.0 \\
76.0 \\
88.0 \\
7.0 .0\end{array}$ & $\begin{array}{l}0.0 \\
0.0 \\
0.0 \\
0.0 \\
0.0\end{array}$ & $\begin{array}{l}0.3 \\
0.3 \\
.03 \\
0.0 \\
0.0\end{array}$ & $\begin{array}{l}12.4 \\
12.4 \\
12.4 \\
12.4 \\
12.4\end{array}$ \\
\hline 1.1 .36 & נ. נ. & 10.5 & 11.0 & 0.0 & u.j & 52.0 & TU.J & 0.0 & 0.1 & 12.4 \\
\hline
\end{tabular}


TABLE IV (Contd.)

BASELINE COUNTERCURRENT FLOW TEST GROUP 1.1

EXPER IMENTAL MEASURMENTS FOR TESTS 1.1 .1 TO $1.1 .36-0.35-1 \mathrm{~N}$. OOHNCOMER GAP

\begin{tabular}{|c|c|c|c|c|c|c|c|c|c|}
\hline \multirow[b]{2}{*}{$\begin{array}{l}\text { TEST.T } \\
\text { NO }\end{array}$} & \multicolumn{2}{|c|}{ LOWEP. PLENUM } & FLOW & \multicolumn{3}{|c|}{ BYPASS FLOW } & \multirow[b]{2}{*}{$\begin{array}{l}\text { LOWER } \\
\text { PLENUM } \\
\text { LEVEL } \\
\text { (INI) }\end{array}$} & \multirow[b]{2}{*}{$\begin{array}{l}P-(P-1) \\
(P S I G)\end{array}$} & \multirow[b]{2}{*}{$\begin{array}{l}\text { TF-LP-1 } \\
\text { (OES F) }\end{array}$} \\
\hline & $\begin{array}{l}\text { INITIAL } \\
\text { WE I GHT } \\
\text { (LB) }\end{array}$ & $\begin{array}{l}\text { FINAL } \\
\text { WEIGHT } \\
\text { (LB) }\end{array}$ & $\begin{array}{l}\text { TIME OF } \\
\text { COLLECTION } \\
\text { ( SEC) }\end{array}$ & $\begin{array}{l}\text { INI I I AL } \\
\text { WE ICHT } \\
\text { (LB) }\end{array}$ & $\begin{array}{l}\text { FINAL } \\
\text { WEIGHT } \\
\text { (LB) }\end{array}$ & $\begin{array}{l}\text { TIME DF } \\
\text { COLLECTION } \\
\text { (SEC) }\end{array}$ & & & \\
\hline 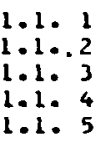 & $\begin{array}{l}219.0 \\
362.5 \\
133.5 \\
272.5 \\
406.5\end{array}$ & $\begin{array}{l}362.5 \\
477.3 \\
272.5 \\
406.5 \\
461.8\end{array}$ & $\begin{array}{l}30.0 \\
31.0 \\
30.0 \\
30.0 \\
30.0\end{array}$ & $\begin{array}{r}90.0 \\
117.5 \\
90.5 \\
119.5 \\
143.8\end{array}$ & $\begin{array}{l}117.5 \\
132.0 \\
119.5 \\
143.8 \\
349.8\end{array}$ & $\begin{array}{l}40.0 \\
50.0 \\
45.0 \\
55.0 \\
55.1\end{array}$ & $\begin{array}{l}12.0 \\
12.0 \\
12.0 \\
12.0 \\
12.0\end{array}$ & $\begin{array}{l}10.0 \\
10.0 \\
10.0 \\
10.0 \\
10.0\end{array}$ & $\begin{array}{l}56.0 \\
56.0 \\
62.0 \\
62.0 \\
56.0\end{array}$ \\
\hline $\begin{array}{l}1.1 .9 \\
1.1 .97 \\
1.1 .99 \\
1.1 .99 \\
1.1 .10\end{array}$ & $\begin{array}{l}461.8 \\
369.5 \\
456.0 \\
132.8 \\
169.5\end{array}$ & $\begin{array}{l}526.0 \\
430.0 \\
514.8 \\
169.5 \\
201.3\end{array}$ & $\begin{array}{l}35.0 \\
30.0 \\
30.0 \\
50.0 \\
40.5\end{array}$ & $\begin{array}{r}349.8 \\
241.5 \\
274.5 \\
82.0 \\
250.0\end{array}$ & $\begin{array}{l}444.8 \\
274.5 \\
334.5 \\
250.0 \\
390.0\end{array}$ & $\begin{array}{l}40.0 \\
45.0 \\
40.0 \\
35.0 \\
40.5\end{array}$ & $\begin{array}{l}12.0 \\
12.0 \\
12.0 \\
12.0 \\
12.0\end{array}$ & $\begin{array}{l}10.0 \\
10.0 \\
10.0 \\
10.0 \\
10.0\end{array}$ & $\begin{array}{l}56.0 \\
56.0 \\
56.0 \\
58.0 \\
58.0\end{array}$ \\
\hline $\begin{array}{l}1.1 .11 \\
1.1 .12 \\
1.1 .13 \\
1.1 .14 \\
1.1 .15\end{array}$ & $\begin{array}{l}201.3 \\
237.0 \\
272.5 \\
287.5 \\
302.5\end{array}$ & $\begin{array}{l}237.0 \\
272.5 \\
237.0 \\
302.5 \\
317.1\end{array}$ & $\begin{array}{l}45.0 \\
45.0 \\
60.0 \\
60.0 \\
60.0\end{array}$ & $\begin{array}{l}390.0 \\
463.3 \\
490.5 \\
653.5 \\
660.0\end{array}$ & $\begin{array}{l}469.3 \\
490.5 \\
653.5 \\
172.5 \\
736.5\end{array}$ & $\begin{array}{l}40.0 \\
60.0 \\
30.0 \\
30.0 \\
30.0\end{array}$ & $\begin{array}{l}12.0 \\
12.0 \\
12.0 \\
12.0 \\
12.0\end{array}$ & $\begin{array}{l}10.0 \\
10.0 \\
10.0 \\
10.0 \\
10.0\end{array}$ & $\begin{array}{l}59.0 \\
58.0 \\
53.0 \\
58.0 \\
56.0\end{array}$ \\
\hline $\begin{array}{l}1.1 .16 \\
1.1 .17 \\
1.1 .18 \\
1.1 .19 \\
1.1 .20\end{array}$ & $\begin{array}{l}317.8 \\
133.0 \\
137.0 \\
141.0 \\
148.0\end{array}$ & $\begin{array}{l}332.3 \\
137.0 \\
141.0 \\
148.0 \\
155.0\end{array}$ & $\begin{array}{l}60.0 \\
60.0 \\
60.0 \\
60.0 \\
60.0\end{array}$ & $\begin{array}{r}736.5 \\
89.5 \\
224.3 \\
278.3 \\
388.5\end{array}$ & $\begin{array}{l}765.0 \\
224.3 \\
276.3 \\
398.5 \\
523.0\end{array}$ & $\begin{array}{l}60.0 \\
25.0 \\
40.0 \\
20.0 \\
34.0\end{array}$ & $\begin{array}{l}12.0 \\
12.0 \\
12.0 \\
12.0 \\
12.0\end{array}$ & $\begin{array}{l}10.0 \\
10.0 \\
10.0 \\
10.0 \\
10.0\end{array}$ & $\begin{array}{l}55.0 \\
70.0 \\
70.0 \\
64.0 \\
64.0\end{array}$ \\
\hline $\begin{array}{l}1.1 .21 \\
1.1 .22 \\
1.1 .23 \\
1.1 .24 \\
1.1 .25\end{array}$ & $\begin{array}{l}155.0 \\
133.0 \\
139.0 \\
133.3 \\
162.3\end{array}$ & $\begin{array}{l}162.0 \\
139.8 \\
147.3 \\
162.3 \\
184.5\end{array}$ & $\begin{array}{l}60.0 \\
60.0 \\
60.0 \\
60.0 \\
60.0\end{array}$ & $\begin{array}{r}523.0 \\
37.0 \\
125.5 \\
39.0 \\
190.0\end{array}$ & $\begin{array}{l}012.5 \\
125.5 \\
144.0 \\
190.0 \\
266.8\end{array}$ & $\begin{array}{l}34.0 \\
30.0 \\
40.0 \\
20.03 \\
20.0\end{array}$ & $\begin{array}{l}12.0 \\
12.0 \\
12.0 \\
12.0 \\
12.0\end{array}$ & $\begin{array}{l}10.3 \\
10.0 \\
10.0 \\
10.0 \\
10.0\end{array}$ & $\begin{array}{l}64.0 \\
51.0 \\
61.0 \\
53.0 \\
59.0\end{array}$ \\
\hline $\begin{array}{l}1.1 .28 \\
1.1 .27 \\
1.1 .28 \\
1.1 .29 \\
1.1 .30\end{array}$ & $\begin{array}{l}184.5 \\
210.0 \\
233.8 \\
26: 5.5 \\
282.0\end{array}$ & $\begin{array}{l}210.0 \\
233.9 \\
260.5 \\
282.0 \\
343.3\end{array}$ & $\begin{array}{l}60.0 \\
55.0 \\
60.0 \\
35.0 \\
50.0\end{array}$ & $\begin{array}{l}266.8 \\
350.5 \\
420.5 \\
454.3 \\
467.5\end{array}$ & $\begin{array}{l}350.5 \\
426.5 \\
454.3 \\
467.5 \\
575.3\end{array}$ & $\begin{array}{l}35.0 \\
20.0 \\
30.0 \\
45.0 \\
25.0\end{array}$ & $\begin{array}{l}12.0 \\
12.0 \\
12.0 \\
12.0 \\
12.0\end{array}$ & $\begin{array}{l}10.0 \\
10.0 \\
10.0 \\
10.0 \\
10.0\end{array}$ & $\begin{array}{l}53.0 \\
56.0 \\
50.0 \\
55.0 \\
55.0\end{array}$ \\
\hline $\begin{array}{l}1.1 .31 \\
1.1 .32 \\
1.1 .33 \\
1.1 .34 \\
1.1 .35\end{array}$ & $\begin{array}{l}343.3 \\
39.4 .0 \\
446.8 \\
135.5 \\
247.0\end{array}$ & $\begin{array}{l}394.0 \\
446.8 \\
497.8 \\
247.0 \\
350.5\end{array}$ & $\begin{array}{l}40.0 \\
40.0 \\
40.0 \\
40.0 \\
35.0\end{array}$ & $\begin{array}{r}575.3 \\
664.5 \\
723.5 \\
99.3 \\
193.0\end{array}$ & $\begin{array}{l}564.5 \\
723.5 \\
742.5 \\
193.0 \\
235.5\end{array}$ & $\begin{array}{l}30.0 \\
40.0 \\
40.0 \\
35.0 \\
40.0\end{array}$ & $\begin{array}{l}12.0 \\
12.0 \\
12.0 \\
12.0 \\
12.0\end{array}$ & $\begin{array}{l}10.0 \\
10.0 \\
10.0 \\
10.0 \\
10.0\end{array}$ & $\begin{array}{l}55.0 \\
55.0 \\
51.0 \\
50.0 \\
55.0\end{array}$ \\
\hline 1.1 .36 & 350.5 & 453.8 & 35.0 & 239.5 & 246.5 & 75.0 & 12.0 & 10.0 & 54.0 \\
\hline
\end{tabular}


TABLE V

BASELINE COUNTERCURRENT FLOW TEST GROUP 1.2

TRANSPARENT VESSEL TEST DATA FIR TESTS 1.2.1 TO 1.2.34 -- U.35-ti.. DOWNC OMER GAP

SUMMAPY OF CALCULATED RESULTS

\begin{tabular}{|c|c|c|c|c|c|c|c|c|c|c|}
\hline $\begin{array}{l}\text { TEST } \\
\text { NO }\end{array}$ & $\begin{array}{l}\text { DOHNCOMER } \\
\text { AIR FLOW. } \\
\text { (LB/SEC) }\end{array}$ & $\begin{array}{l}\text { DOWNCOMER } \\
\text { AIR DE INSITY } \\
\text { (LB/FT*3) }\end{array}$ & $\begin{array}{c}\text { LOWER PLENUM } \\
\text { PRESSURE } \\
\text { (PSIA) }\end{array}$ & $\begin{array}{l}\text { CCLD LEG } \\
\text { AIR FLUH } \\
\text { (LB/SEC) }\end{array}$ & $\begin{array}{l}\text { OOHNCUMER } \\
\text { HATER FL(WW } \\
\text { (LB/SEC) }\end{array}$ & $\begin{array}{l}\text { WATER FLUW } \\
\text { CONTINU:TY }\end{array}$ & $\begin{array}{c}\text { J-AIR } \\
\text { DOWNCUMER } \\
\text { (FT/SEC) }\end{array}$ & $\begin{array}{l}\text { J-HATER } \\
\text { DOMNCOMER } \\
\text { (F T/SEC) }\end{array}$ & $(J G *) * * 1 / 2$ & $(J l * 1 * 1)$ \\
\hline $\begin{array}{l}1.2 \cdot 1 \\
1.2 \cdot 2 \\
1.2 .3 \\
1.2 .4 \\
1.2 .5\end{array}$ & $\begin{array}{l}0.0214 \\
0.0321 \\
0.0323 \\
0.0640 \\
0.0641\end{array}$ & $\begin{array}{l}0.2226 \\
0.2226 \\
3.2218 \\
0.2218 \\
0.2214\end{array}$ & $\begin{array}{l}42.40 \\
42.40 \\
42.40 \\
42.40 \\
42.10\end{array}$ & $\begin{array}{l}0.0 \\
0.0 \\
0.0 \\
0.0 \\
0.0\end{array}$ & $\begin{array}{l}4.767 \\
3.692 \\
3.592 \\
2.097 \\
2.236\end{array}$ & $\begin{array}{l}0.959 \\
0.984 \\
1.001 \\
1.006 \\
1.036\end{array}$ & $\begin{array}{l}1.55 \\
2.33 \\
2.35 \\
4.65 \\
4.67\end{array}$ & $\begin{array}{l}1.233 \\
0.955 \\
0.924 \\
0.542 \\
0.578\end{array}$ & $\begin{array}{l}0.2596 \\
0.3180 \\
0.3196 \\
0.4494 \\
0.4500\end{array}$ & $\begin{array}{l}0.9472 . \\
0.8336 \\
0.8222 \\
0.6282 \\
0.6487\end{array}$ \\
\hline $\begin{array}{l}1.2 \cdot 6 \\
.2 \cdot 7 \\
1.2 \cdot 8 \\
1.2 \cdot 9 \\
1.2 .10\end{array}$ & $\begin{array}{l}0.0641 \\
0.0644 \\
0.1074 \\
0.1065 \\
0.1072\end{array}$ & $\begin{array}{l}0.2222 \\
0.2218 \\
0.2218 \\
0.2218 \\
0.2192\end{array}$ & $\begin{array}{l}42.40 \\
42.40 \\
42.40 \\
42.40 \\
42.40\end{array}$ & $\begin{array}{l}0.0 \\
0.0 \\
0.0 \\
0.0 \\
0.0\end{array}$ & $\begin{array}{l}2.250 \\
2.267 \\
0.939 \\
1.061 \\
1.017\end{array}$ & $\begin{array}{l}1.034 \\
1.001 \\
1.012 \\
1.061 \\
0.984\end{array}$ & $\begin{array}{l}4.66 \\
4.68 \\
7.81 \\
7.75 \\
7.89\end{array}$ & $\begin{array}{l}0.582 \\
0.586 \\
0.242 \\
0.274 \\
0.263\end{array}$ & $\begin{array}{l}0.4498 \\
0.4509 \\
0.5823 \\
0.5798 \\
0.5834\end{array}$ & $\begin{array}{l}0.6508 \\
0.6532 \\
0.4201 \\
0.4469 \\
0.4374\end{array}$ \\
\hline $\begin{array}{l}1.2 .11 \\
1.2 .12 \\
1.2 .13 \\
1.2 .14 \\
1.2 .15\end{array}$ & $\begin{array}{l}0.1073 \\
0.1486 \\
0.1477 \\
0.1482 \\
0.1192\end{array}$ & $\begin{array}{l}0.2192 \\
0.2218 \\
0.2214 \\
0.2218 \\
0.2218\end{array}$ & $\begin{array}{l}42.40 \\
42.40 \\
42.40 \\
42.40 \\
42.40\end{array}$ & $\begin{array}{l}0.0 \\
0.0 \\
0.0 \\
c .0 \\
0.0\end{array}$ & $\begin{array}{l}1.061 \\
0.361 \\
0.355 \\
0.405 \\
0.378\end{array}$ & $\begin{array}{l}1.031 \\
0.995 \\
1.014 \\
0.945 \\
1.049\end{array}$ & $\begin{array}{r}7.90 \\
10.81 \\
10.77 \\
10.78 \\
10.85\end{array}$ & $\begin{array}{l}0.274 \\
0.093 \\
0.092 \\
0.105 \\
0.098\end{array}$ & $\begin{array}{l}0.5837 \\
0.6849 \\
0.6833 \\
0.6840 \\
0.6863\end{array}$ & $\begin{array}{l}0.4469 \\
0.2607 \\
0.2585 \\
0.2759 \\
0.2667\end{array}$ \\
\hline $\begin{array}{l}1.2 .16 \\
1.2 .17 \\
1.2 .18 \\
1.2 .19 \\
1.2 .20\end{array}$ & $\begin{array}{l}0.1918 \\
0.1423 \\
0.1438 \\
0.1924 \\
0.1714\end{array}$ & $\begin{array}{l}0.2214 \\
0.2214 \\
0.2201 \\
0.2201 \\
0.2201\end{array}$ & $\begin{array}{l}42.40 \\
42.40 \\
42.32 \\
42.32 \\
42.32\end{array}$ & $\begin{array}{l}0.0 \\
0.0 \\
0.0 \\
0.0 \\
0.0\end{array}$ & $\begin{array}{l}0.104 \\
0.142 \\
0.144 \\
0.136 \\
0.282\end{array}$ & $\begin{array}{l}0.996 \\
0.943 \\
1.023 \\
1.004 \\
0.997\end{array}$ & $\begin{array}{l}13.98 \\
14.02 \\
14.21 \\
14.14 \\
12.51\end{array}$ & $\begin{array}{l}0.027 \\
0.037 \\
0.037 \\
0.035 \\
0.073\end{array}$ & $\begin{array}{l}0.7785 \\
0.7796 \\
0.7837 \\
0.7818 \\
0.7371\end{array}$ & $\begin{array}{l}0.1400 \\
0.1633 \\
0.1649 \\
0.1602 \\
0.2303\end{array}$ \\
\hline $\begin{array}{l}1.2 .21 \\
1.2 .22 \\
1.2 .23 \\
1.2 .24 \\
1.2 .25\end{array}$ & $\begin{array}{l}0.1721 \\
0.1711 \\
0.1111 \\
0.1299 \\
0.1295\end{array}$ & $\begin{array}{l}0.2210 \\
0.2214 \\
0.2214 \\
0.2214 \\
0.2218\end{array}$ & $\begin{array}{l}42.32 \\
42.32 \\
42.32 \\
42.32 \\
42.32\end{array}$ & $\begin{array}{l}0.0 \\
0.0 \\
0.0 \\
0.0 \\
0.0\end{array}$ & $\begin{array}{l}0.225 \\
0.186 \\
0.155 \\
0.577 \\
0.575\end{array}$ & $\begin{array}{l}1.023 \\
1.020 \\
0.937 \\
0.991 \\
0.987\end{array}$ & $\begin{array}{r}12.57 \\
12.47 \\
12.47 \\
9.46 \\
9.42\end{array}$ & $\begin{array}{l}0.058 \\
0.048 \\
0.040 \\
0.149 \\
0.149\end{array}$ & $\begin{array}{l}0.7378 \\
0.7353 \\
0.7353 \\
0.6406 \\
0.6394\end{array}$ & $\begin{array}{l}0.2058 \\
0.1873 \\
0.1706 \\
0.3296 \\
0.3290\end{array}$ \\
\hline $\begin{array}{l}1.2 .26 \\
1.2 .27 \\
1.2 .28 \\
1.2 .29 \\
1.2 .30\end{array}$ & $\begin{array}{l}0.1284 \\
0.1291 \\
0.0862 \\
0.0861 \\
0.0852\end{array}$ & $\begin{array}{l}0.2218 \\
0.2223 \\
0.2193 \\
0.2218 \\
0.2218\end{array}$ & $\begin{array}{l}42.32 \\
42.32 \\
42.32 \\
42.32 \\
42.32\end{array}$ & $\begin{array}{l}0.0 \\
0.0 \\
0.0 \\
0.0 \\
0.0\end{array}$ & $\begin{array}{l}0.569 \\
0.627 \\
1.400 \\
1.503 \\
1.582\end{array}$ & $\begin{array}{l}0.960 \\
1.005 \\
1.004 \\
1.001 \\
1.010\end{array}$ & $\begin{array}{l}9.34 \\
9.37 \\
6.34 \\
6.27 \\
6.20\end{array}$ & $\begin{array}{l}0.147 \\
0.162 \\
0.362 \\
0.404 \\
0.409\end{array}$ & $\begin{array}{l}0.6366 \\
0.6381 \\
0.5232 \\
0.5214 \\
0.5187\end{array}$ & $\begin{array}{l}0.3273 \\
0.3436 \\
0.5133 \\
0.5423 \\
0.5456\end{array}$ \\
\hline $\begin{array}{l}1.2 .31 \\
1.2 .32 \\
1.2 .33 \\
1.2 .34\end{array}$ & $\begin{array}{l}0.0960 \\
0.0430 \\
0.0428 \\
0.0429\end{array}$ & $\begin{array}{l}0.2218 \\
0.2223 \\
0.2223 \\
0.2223\end{array}$ & $\begin{array}{r}42.32 \\
42.32 \\
42.32 \\
42.32\end{array}$ & $\begin{array}{l}\text { c.0 } \\
0.0 \\
0.0 \\
0.0\end{array}$ & $\begin{array}{l}1.619 \\
2.950 \\
3.114 \\
3.175\end{array}$ & $\begin{array}{l}1.022 \\
1.007 \\
1.003 \\
0.988\end{array}$ & $\begin{array}{l}6.25 \\
3.12 \\
3.11 \\
3.11\end{array}$ & $\begin{array}{l}0.419 \\
0.763 \\
0.805 \\
0.821\end{array}$ & $\begin{array}{l}0.5209 \\
0.3683 \\
0.3674 \\
0.3678\end{array}$ & $\begin{array}{l}0.5520 \\
0.7451 \\
0.7656 \\
0.7730\end{array}$ \\
\hline
\end{tabular}


TABLE V (Contd.)

BASELINE COUNTERCURRENT FLOW. TEST GROUP 1.2

EXPERIIAENTAL MEASURMENTS FOR TESTS 1.2 .1 TO $1.2 .34 \ldots .35-1$. 0.3 . DOWNCOMER GAP

\begin{tabular}{|c|c|c|c|c|c|c|c|c|c|c|}
\hline $\begin{array}{l}\text { TEST } \\
\text { NO. }\end{array}$ & $\begin{array}{c}R M-C H-1 \\
(G P M)\end{array}$ & $\begin{array}{c}\text { RH-CH-2 } \\
\text { (GPM) }\end{array}$ & $\begin{array}{c}F T-C H-1 \\
(G P M)\end{array}$ & $\begin{array}{c}F T-C H-2 \\
(G P M)\end{array}$ & $\begin{array}{c}\text { RM-HIG-1 } \\
\text { (CFMI) }\end{array}$ & $\begin{array}{c}R M-H G-2 \\
(C F M)\end{array}$ & $\begin{array}{c}R M=H G-3 \\
(C F \cdot M)^{-3}\end{array}$ & $\begin{array}{c}R: 4-H G-4 \\
\text { (CFM) }\end{array}$ & $\begin{array}{l}F T-H G-1 \\
(C F M) .\end{array}$ & $\begin{array}{c}F T-H G-Z \\
\text { (CFM) }\end{array}$ \\
\hline $\begin{array}{l}\text { l.2. } 1 \\
\text { l.2. } \\
1.2 .3 \\
1.2 .4 \\
1.2 .5\end{array}$ & $\begin{array}{l}29.0 \\
28.0 \\
20.0 \\
30.0 \\
20.0\end{array}$ & $\begin{array}{l}11.0 \\
11.5 \\
10.0 \\
10.0 \\
10.0\end{array}$ & $\begin{array}{l}0.0 \\
0.0 \\
0.0 \\
0.0 \\
0.0\end{array}$ & $\begin{array}{l}0.0 \\
0.0 \\
0.0 \\
0.0 \\
0.0\end{array}$ & $\begin{array}{l}0.0 \\
0.0 \\
0.0 \\
0.0 \\
0.0\end{array}$ & $\begin{array}{c}0.0 \\
0.0 \\
0.0 \\
30.00 \\
30.00\end{array}$ & $\begin{array}{c}10.00 \\
15.00 \\
15.00 \\
0.0 \\
0.0\end{array}$ & $\begin{array}{l}0.0 \\
0.0 \\
0.0 \\
0.0 \\
0.0\end{array}$ & $\begin{array}{l}0.0 \\
0.0 \\
0.0 \\
0.0 \\
0.0\end{array}$ & $\begin{array}{l}0.0 \\
0.0 \\
0.0 \\
0.0 \\
0.0\end{array}$ \\
\hline $\begin{array}{l}1.2 .6 \\
1.2 .7 \\
1.2 .8 \\
1.2 .4 \\
1.2 .10\end{array}$ & $\begin{array}{l}25.0 \\
17.0 \\
29.0 \\
20.0 \\
20.0\end{array}$ & $\begin{array}{r}0.0 \\
0.0 \\
11.0 \\
10.0 \\
0.0\end{array}$ & $\begin{array}{l}0.0 \\
0.0 \\
0.0 \\
0.0 \\
0.0\end{array}$ & $\begin{array}{l}0.0 \\
0.0 \\
0.0 \\
0.0 \\
0.0\end{array}$ & $\begin{array}{l}u .0 \\
0.0 \\
0.0 \\
0.0 \\
0.0\end{array}$ & $\begin{array}{l}30.00 \\
30.00 \\
50.00 \\
50.00 \\
50.00\end{array}$ & $\begin{array}{l}0.0 \\
0.0 \\
0.0 \\
0.0 \\
0.0\end{array}$ & $\begin{array}{l}0.0 \\
0.0 \\
0.0 \\
0.0 \\
0.0\end{array}$ & $\begin{array}{l}0.0 \\
0.0 \\
0.0 \\
0.0 \\
0.0\end{array}$ & $\begin{array}{l}0.0 \\
0.0 \\
0.0 \\
0.0 \\
0.0\end{array}$ \\
\hline $\begin{array}{l}1.2 .11 \\
1.2 .12 \\
1.2 .13 \\
1.2 .14 \\
1.2 .15\end{array}$ & $\begin{array}{r}0.0 \\
30.0 \\
20.0 \\
20.0 \\
0.0\end{array}$ & $\begin{array}{r}10.0 \\
10.0 \\
10.0 \\
0.0 \\
8.0\end{array}$ & $\begin{array}{l}0.0 \\
0.0 \\
0.0 \\
0.0 \\
0.0\end{array}$ & $\begin{array}{l}0.0 \\
0.0 \\
0.0 \\
0.0 \\
0.0\end{array}$ & $\begin{array}{c}0.0 \\
40.00 \\
40.00 \\
40.00 \\
40.00\end{array}$ & $\begin{array}{l}50.00 \\
30.00 \\
30.00 \\
30.00 \\
30.00\end{array}$ & $\begin{array}{l}0.0 \\
0.0 \\
0.0 \\
0.0 \\
0.0\end{array}$ & $\begin{array}{l}0.0 \\
0.0 \\
0.0 \\
0.0 \\
0.0\end{array}$ & $\begin{array}{l}0.0 \\
0.0 \\
0.0 \\
0.0 \\
0.0\end{array}$ & $\begin{array}{l}0.0 \\
0.0 \\
0.0 \\
0.0 \\
0.0\end{array}$ \\
\hline $\begin{array}{l}1.2 .16 \\
1.2 .17 \\
1.2 .18 \\
1.2 .17 \\
1.2 .20\end{array}$ & $\begin{array}{r}30.0 \\
20.0 \\
20.0 \\
0.0 \\
21.0\end{array}$ & $\begin{array}{r}10.0 \\
10.0 \\
0.0 \\
4.0 \\
12.0\end{array}$ & $\begin{array}{l}0.0 \\
0.0 \\
0.0 \\
0.0 \\
0.0\end{array}$ & $\begin{array}{l}0.0 \\
0.0 \\
0.0 \\
0.0 \\
0.0\end{array}$ & $\begin{array}{l}50.00 \\
50.00 \\
50.00 \\
50.00 \\
50.00\end{array}$ & $\begin{array}{l}40.00 \\
40.00 \\
40.00 \\
40.00 \\
30.00\end{array}$ & $\begin{array}{l}0.0 \\
0.0 \\
0.0 \\
0.0 \\
0.0\end{array}$ & $\begin{array}{l}0.0 \\
0.0 \\
0.0 \\
0.0 \\
0.0\end{array}$ & $\begin{array}{l}0.0 \\
0.0 \\
0.0 \\
0.0 \\
0.0\end{array}$ & $\begin{array}{l}0.0 \\
0.0 \\
0.0 \\
0.0 \\
0.0\end{array}$ \\
\hline $\begin{array}{l}1.2 .21 \\
1.2 .22 \\
1.2 .23 \\
1.2 .24 \\
1.2 .25\end{array}$ & $\begin{array}{r}20.0 \\
20.0 \\
0.0 \\
28.0 \\
20.0\end{array}$ & $\begin{array}{r}10.0 \\
0.0 \\
4.0 \\
12.0 \\
10.0\end{array}$ & $\begin{array}{l}0.0 \\
0.0 \\
0.0 \\
0.0 \\
0.0\end{array}$ & $\begin{array}{l}0.0 \\
0.0 \\
0.0 \\
0.0 \\
0.0\end{array}$ & $\begin{array}{c}50.00 \\
50.00 \\
50.00 \\
0.0 \\
0.0\end{array}$ & $\begin{array}{l}30.00 \\
30.00 \\
30.00 \\
60.00 \\
60.00\end{array}$ & $\begin{array}{l}0.0 \\
0.0 \\
0.0 \\
0.0 \\
0.0\end{array}$ & $\begin{array}{l}0.0 \\
0.0 \\
0.0 \\
0.0 \\
0.0\end{array}$ & $\begin{array}{l}0.0 \\
0.0 \\
0.0 \\
0.0 \\
0.0\end{array}$ & $\begin{array}{l}0.0 \\
0.0 \\
0.0 \\
0.0 \\
0.0\end{array}$ \\
\hline $\begin{array}{l}1.2 .26 \\
1.2 .27 \\
1.2 .28 \\
1.2 .29 \\
1.2 .30\end{array}$ & $\begin{array}{r}2 \mathrm{~J} .0 \\
0.0 \\
20.0 \\
20.0 \\
2.00\end{array}$ & $\begin{array}{r}0.0 \\
6.0 \\
12.0 \\
10.0 \\
0.0\end{array}$ & $\begin{array}{l}0.0 \\
0.0 \\
0.0 \\
0.0 \\
0.0\end{array}$ & $\begin{array}{l}0.0 \\
0.0 \\
0.0 \\
0.0 \\
0.0\end{array}$ & $\begin{array}{l}0.0 \\
0.0 \\
0.0 \\
0.0 \\
0.0\end{array}$ & $\begin{array}{l}60.00 \\
60.00 \\
40.00 \\
40.00 \\
40.00\end{array}$ & $\begin{array}{l}0.0 \\
0.0 \\
0.0 \\
0.0 \\
0.0\end{array}$ & $\begin{array}{l}0.0 \\
0.0 \\
0.0 \\
0.0 \\
0.0\end{array}$ & $\begin{array}{l}0.0 \\
0.0 \\
0.0 \\
0.0 \\
0.0\end{array}$ & $\begin{array}{l}0.0 \\
0.0 \\
0.0 \\
0.0 \\
0.0\end{array}$ \\
\hline $\begin{array}{l}1.2 .31 \\
1.2 .32 \\
1.2 .33 \\
1.2 .34\end{array}$ & $\begin{array}{l}13.0 \\
25.0 \\
22.0 \\
24.0\end{array}$ & $\begin{array}{r}0.0 \\
12.5 \\
10.0 \\
0.0\end{array}$ & $\begin{array}{l}0.0 \\
0.0 \\
0.0 \\
0.0\end{array}$ & $\begin{array}{l}0.0 \\
0.0 \\
0.0 \\
0.0\end{array}$ & $\begin{array}{l}0.0 \\
0.0 \\
0.0 \\
0.0\end{array}$ & $\begin{array}{c}40.00 \\
0.0 \\
0.0 \\
0.0\end{array}$ & $\begin{array}{c}0 . G \\
20.00 \\
20.00 \\
20.00\end{array}$ & $\begin{array}{l}0.0 \\
0.0 \\
0.0 \\
0.0\end{array}$ & $\begin{array}{l}0.0 \\
0.0 \\
0.0 \\
0.0\end{array}$ & $\begin{array}{l}0.0 \\
0.0 \\
0.0 \\
0.0\end{array}$ \\
\hline
\end{tabular}


TABLE V (Contd.)

BASELINE COUNTERCURRENT FLOW TEST GROUP 1.2

EXPERIMENTAL MEASURMENTS FOR TESTS 1.2 .1 TO 1.2.34 -- 0.35-1N. UOWNCOMER GAP

\begin{tabular}{|c|c|c|c|c|c|c|c|c|c|c|}
\hline $\begin{array}{l}\text { TEST } \\
\text { NO }\end{array}$ & $\begin{array}{l}P-C W-1 \\
(P S I G)\end{array}$ & $\begin{array}{l}P-(H-2 \\
(P S I G)\end{array}$ & $\begin{array}{l}P-H G-i \\
(P S I G) .\end{array}$ & $\begin{array}{l}\text { P-HG -2 } \\
\text { (PSIG) }\end{array}$ & $\begin{array}{l}P-H G-3 \\
(P S I G)\end{array}$ & $\begin{array}{l}T F-C H-1 \\
(D E G F)\end{array}$ & $\begin{array}{l}\text { TF-HG-I } \\
\text { (DEG F) }\end{array}$ & $\begin{array}{l}\text { TF-HG-2 } \\
\text { (DEG FI) }\end{array}$ & $\begin{array}{l}\text { TF-HG-3 } \\
\text { (DEG F) }\end{array}$ & $\begin{array}{l}\text { P-BARO } \\
\text { (PSIA) }\end{array}$ \\
\hline $\begin{array}{l}1.2 .1 \\
1.2 . \\
1.2 .3 \\
1.2 .4 \\
1.2 .5\end{array}$ & $\begin{array}{l}0.0 \\
0.0 \\
0.0 \\
0.0 \\
0.0\end{array}$ & $\begin{array}{l}31.7 \\
31.7 \\
31.7 \\
31.0 \\
31.2\end{array}$ & $\begin{array}{l}31.2 \\
31.5 \\
32.2 \\
31.2 \\
31.5\end{array}$ & $\begin{array}{l}0.0 \\
0.0 \\
0.0 \\
0.0 \\
0.0\end{array}$ & $\begin{array}{l}0.0 \\
0.0 \\
0.0 \\
0.0 \\
0.0\end{array}$ & $\begin{array}{l}51.0 \\
52.0 \\
53.0 \\
52.0 \\
53.0\end{array}$ & $\begin{array}{l}76.0 \\
78.0 \\
74.0 \\
79.0 \\
80.0\end{array}$ & $\begin{array}{l}0.0 \\
0.0 \\
0.0 \\
0.0 \\
0.0\end{array}$ & $\begin{array}{l}0.0 \\
0.0 \\
0.0 \\
0.0 \\
0.0\end{array}$ & $\begin{array}{l}12.4 \\
12.4 \\
12.4 \\
12.4 \\
12.4\end{array}$ \\
\hline $\begin{array}{ll}1.2 .6 \\
1.2 .7 \\
1.2 .8 \\
1.2 .9 \\
1.2 .10\end{array}$ & $\begin{array}{l}0.0 \\
0.0 \\
0.0 \\
0.0 \\
0.0\end{array}$ & $\begin{array}{l}31.0 \\
31.02 \\
31.0 \\
30.2 \\
31.0\end{array}$ & $\begin{array}{l}31.5 \\
31.7 \\
31.2 \\
30.5 \\
31.2\end{array}$ & $\begin{array}{l}0.0 \\
0.0 \\
0.0 \\
0.0 \\
0.0\end{array}$ & $\begin{array}{l}0.0 \\
0.0 \\
0.0 \\
0.0 \\
0.0\end{array}$ & $\begin{array}{l}53.0 \\
53.0 \\
52.0 \\
51.0 \\
55.0\end{array}$ & $\begin{array}{l}79.0 \\
78.0 \\
78.0 \\
76.0 \\
80.0\end{array}$ & $\begin{array}{l}0.0 \\
0.0 \\
0.0 \\
0.0 \\
0.0\end{array}$ & $\begin{array}{l}0.0 \\
0.0 \\
0.0 \\
0.0 \\
0.0\end{array}$ & $\begin{array}{l}12.4 \\
12.4 \\
12.4 \\
12.4 \\
12.4\end{array}$ \\
\hline $\begin{array}{l}1.2 .11 \\
1.2 .12 \\
1.2 .13 \\
1.2 .14 \\
1.2 .15\end{array}$ & $\begin{array}{l}u .0 \\
0.0 \\
0.0 \\
0.0 \\
0.0\end{array}$ & $\begin{array}{l}30.5 \\
30.2 \\
30.0 \\
30.7 \\
30.2\end{array}$ & $\begin{array}{l}31.2 \\
30.5 \\
30.0 \\
30.2 \\
30.7\end{array}$ & $\begin{array}{l}0.0 \\
0.0 \\
0.0 \\
0.0 \\
0.0\end{array}$ & $\begin{array}{l}0.0 \\
0.0 \\
0.0 \\
0.0 \\
0.0\end{array}$ & $\begin{array}{l}54.0 \\
53.0 \\
53.0 \\
54.0 \\
54.0\end{array}$ & $\begin{array}{l}79.0 \\
79.0 \\
79.0 \\
79.0 \\
78.0\end{array}$ & $\begin{array}{l}0.0 \\
0.0 \\
0.0 \\
0.0 \\
0.0\end{array}$ & $\begin{array}{l}0.0 \\
0.0 \\
0.0 \\
0.0 \\
0.0\end{array}$ & $\begin{array}{l}12.4 \\
12.4 \\
12.4 \\
12.4 \\
12.4\end{array}$ \\
\hline $\begin{array}{l}1.2 .16 \\
1.2 .17 \\
1.2 .18 \\
1.2 .19 \\
1.2 .20\end{array}$ & $\begin{array}{l}0.0 \\
0.0 \\
0.0 \\
0.0 \\
0.0\end{array}$ & $\begin{array}{l}30.2 \\
30.5 \\
30.7 \\
30.2 \\
30.2\end{array}$ & $\begin{array}{l}30.5 \\
30.7 \\
31.2 \\
30.7 \\
30.1\end{array}$ & $\begin{array}{l}0.0 \\
0.0 \\
0.0 \\
0.0 \\
0.0\end{array}$ & $\begin{array}{l}0.0 \\
0.0 \\
0.0 \\
0.0 \\
0.0\end{array}$ & $\begin{array}{l}53.0 \\
52.0 \\
53.0 \\
54.0 \\
52.0\end{array}$ & $\begin{array}{l}78.0 \\
78.0 \\
75.0 \\
74.0 \\
73.0\end{array}$ & $\begin{array}{l}0.0 \\
0.0 \\
0.0 \\
0.0 \\
0.0\end{array}$ & $\begin{array}{l}0.0 \\
0.0 \\
0.0 \\
0.0 \\
0.0\end{array}$ & $\begin{array}{l}12.4 \\
12.4 \\
12.3 \\
12.3 \\
12.3\end{array}$ \\
\hline $\begin{array}{l}1.2 .21 \\
1.2 .22 \\
1.2 .23 \\
1.2224 \\
1.2 .25\end{array}$ & $\begin{array}{l}0.0 \\
0.0 \\
0.0 \\
0.0 \\
0.0\end{array}$ & $\begin{array}{l}30.7 \\
30.0 \\
30.2 \\
31.0 \\
30.7\end{array}$ & $\begin{array}{l}31.0 \\
30.5 \\
30.5 \\
31.2 \\
31.0\end{array}$ & $\begin{array}{l}0.0 \\
0.0 \\
0.0 \\
0.0 \\
0.0\end{array}$ & $\begin{array}{l}0.0 \\
0.0 \\
0.0 \\
0.0 \\
0.0\end{array}$ & $\begin{array}{l}52.0 \\
53.0 \\
53.0 \\
52.0 \\
51.0\end{array}$ & $\begin{array}{l}72.0 \\
72.0 \\
72.0 \\
72.0 \\
72.0\end{array}$ & $\begin{array}{l}0.0 \\
0.0 \\
0.0 \\
0.0 \\
0.0\end{array}$ & $\begin{array}{l}0.0 \\
0.0 \\
0.0 \\
0.0 \\
0.0\end{array}$ & $\begin{array}{l}12.3 \\
12.3 \\
12.3 \\
12.3 \\
12.3\end{array}$ \\
\hline $\begin{array}{l}1.2 .26 \\
1.2 .27 \\
1.228 \\
1.2 .29 \\
1.2 .30\end{array}$ & $\begin{array}{l}0.0 \\
0.0 \\
0.0 \\
. .0 \\
0.0\end{array}$ & $\begin{array}{l}30.0 \\
30.5 \\
31.0 \\
31.2 \\
30.0\end{array}$ & $\begin{array}{l}30.2 \\
30.7 \\
31.5 \\
31.5 \\
30.5\end{array}$ & $\begin{array}{l}0.0 \\
0.0 \\
0.0 \\
0.0 \\
0.0\end{array}$ & $\begin{array}{l}0.0 \\
0.0 \\
0.0 \\
0.0 \\
0.0\end{array}$ & $\begin{array}{l}51.0 \\
52.0 \\
53.0 \\
52.0 \\
52.0\end{array}$ & $\begin{array}{l}72.0 \\
72.0 \\
74.0 \\
75.0 \\
74.0\end{array}$ & $\begin{array}{l}0.0 \\
0.0 \\
0.0 \\
0.0 \\
0.0\end{array}$ & $\begin{array}{l}0.0 \\
0.0 \\
0.0 \\
0.0 \\
0.0\end{array}$ & $\begin{array}{l}12.3 \\
12.3 \\
12.3 \\
12.3 \\
12.3\end{array}$ \\
\hline $\begin{array}{l}1.2 .31 \\
1.2 .32 \\
1.2 .33 \\
1.2 .34\end{array}$ & $\begin{array}{l}0.0 \\
0.0 \\
0.0 \\
0.0\end{array}$ & $\begin{array}{l}31.0 \\
31.5 \\
30.7 \\
31.5\end{array}$ & $\begin{array}{l}31.2 \\
31.7 \\
31.2 \\
31.5\end{array}$ & $\begin{array}{l}0.0 \\
0.0 \\
0.0 \\
0.0\end{array}$ & $\begin{array}{l}0.0 \\
0.0 \\
0.0 \\
0.0\end{array}$ & $\begin{array}{l}52.0 \\
52.0 \\
51.0 \\
52.0\end{array}$ & $\begin{array}{l}7 \% .0 \\
15.0 \\
74.0 \\
75.0\end{array}$ & $\begin{array}{l}0.0 \\
0.0 \\
0.0 \\
0.0\end{array}$ & $\begin{array}{l}0.0 \\
0.0 \\
0.0 \\
0.0\end{array}$ & $\begin{array}{l}12.3 \\
12.3 \\
12.3 \\
12.3\end{array}$ \\
\hline
\end{tabular}


TABLE V (Contd.)

BASELINE COUNTERCURRENT FLOW TEST GROUP 1.2

EXPPERIMENTAL MEASURMENTS FIR TESTS 1.2 .1 TO $1.2 .34--0.35-$ IN. DOHNCOMER GAP

\begin{tabular}{|c|c|c|c|c|c|c|c|c|}
\hline \multicolumn{2}{|c|}{ LOWER PLENUM } & FL.OW & \multicolumn{3}{|c|}{ BYPASS FLOW } & \multirow[b]{2}{*}{$\begin{array}{l}\text { LOWER } \\
\text { PLENUM } \\
\text { LFVEL } \\
\text { (IN) }\end{array}$} & \multirow[b]{2}{*}{$\begin{array}{l}P-L P-1 \\
\text { PPSIGS }\end{array}$} & \multirow[b]{2}{*}{$\begin{array}{l}\text { TF-LP-I } \\
\text { IDEG FI }\end{array}$} \\
\hline $\begin{array}{l}\text { INITIAL } \\
\text { WEISHT } \\
\text { (LB) }\end{array}$ & $\begin{array}{l}\text { FINAL } \\
\text { WEIGHT } \\
\text { (LBI }\end{array}$ & $\begin{array}{l}\text { TIME OF } \\
\text { COLLECTION } \\
\text { (SEC) }\end{array}$ & $\begin{array}{l}\text { INITIAL } \\
\text { ME!GHT } \\
\text { (LBI }\end{array}$ & $\begin{array}{l}\text { FINAL } \\
\text { WEIGHT } \\
\text { ILBI }\end{array}$ & $\begin{array}{l}\text { TIME OF } \\
\text { COLLECTION } \\
\text { (SEC) }\end{array}$ & & & \\
\hline $\begin{array}{l}253.8 \\
396.8 \\
24.1 .5 \\
348.3 \\
136.3\end{array}$ & $\begin{array}{l}396.8 \\
507.5 \\
348.3 \\
413.3 \\
214.5\end{array}$ & $\begin{array}{l}30.0 \\
30.0 \\
30.0 \\
31.0 \\
35.0\end{array}$ & $\begin{array}{r}107.0 \\
141.0 \\
98.0 \\
136.0 \\
261.0\end{array}$ & $\begin{array}{l}141.0 \\
209.5 \\
136.0 \\
241.0 \\
324.5\end{array}$ & $\begin{array}{l}60.0 \\
40.0 \\
65.0 \\
30.0 \\
40.0\end{array}$ & $\begin{array}{l}12.0 \\
12.0 \\
12.0 \\
12.0 \\
12.0\end{array}$ & $\begin{array}{l}30.0 \\
30.0 \\
30.0 \\
30.0 \\
30.0\end{array}$ & $\begin{array}{l}54.0 \\
54.0 \\
56.0 \\
56.0 \\
57.0\end{array}$ \\
\hline $\begin{array}{l}214.5 \\
293.3 \\
361.3 \\
344.0 \\
133.0\end{array}$ & $\begin{array}{l}293.3 \\
361.3 \\
398.8 \\
391.8 \\
194.0\end{array}$ & $\begin{array}{l}35.0 \\
30.0 \\
40.0 \\
45.0 \\
60.0\end{array}$ & $\begin{array}{r}324.5 \\
371.5 \\
379.0 \\
430.0 \\
89.8\end{array}$ & $\begin{array}{l}371.5 \\
379.0 \\
543.3 \\
564.5 \\
158.5\end{array}$ & $\begin{array}{l}35.0 \\
75.0 \\
35.0 \\
40.0 \\
40.0\end{array}$ & $\begin{array}{l}12.0 \\
12.0 \\
12.0 \\
12.0 \\
12.0\end{array}$ & $\begin{array}{l}30.0 \\
30.0 \\
30.0 \\
30.0 \\
30.0\end{array}$ & $\begin{array}{l}55.0 \\
56.0 \\
56.0 \\
56.0 \\
62.0\end{array}$ \\
\hline $\begin{array}{l}194.0 \\
241.8 \\
258.0 \\
275.0 \\
298.0\end{array}$ & $\begin{array}{l}241.8 \\
258.0 \\
275.8 \\
238.0 \\
315.0\end{array}$ & $\begin{array}{l}45.0 \\
45.0 \\
50.0 \\
55.0 \\
45.0\end{array}$ & $\begin{array}{l}158.5 \\
179.0 \\
368.3 \\
424.5 \\
494.5\end{array}$ & $\begin{array}{l}179.0 \\
308.3 \\
424.5 \\
494.5 \\
530.0\end{array}$ & $\begin{array}{l}55.0 \\
25.0 \\
30.0 \\
30.0 \\
45.0\end{array}$ & $\begin{array}{l}12.0 \\
12.0 \\
12.0 \\
12.0 \\
12.0\end{array}$ & $\begin{array}{l}30.0 \\
30.0 \\
30.0 \\
30.0 \\
30.0\end{array}$ & $\begin{array}{l}62.0 \\
56.0 \\
57.0 \\
56.0 \\
56.0\end{array}$ \\
\hline $\begin{array}{l}315.0 \\
321.3 \\
133.0 \\
139.5 \\
147.0\end{array}$ & $\begin{array}{l}321.3 \\
329.8 \\
139.5 \\
147.0 \\
162.5\end{array}$ & $\begin{array}{l}60.0 \\
60.0 \\
45.0 \\
55.0 \\
55.0\end{array}$ & $\begin{array}{r}530.0 \\
693.0 \\
86.5 \\
140.5 \\
159.5\end{array}$ & $\begin{array}{l}693.0 \\
833.0 \\
140.5 \\
159.5 \\
364.5\end{array}$ & $\begin{array}{l}30.0 \\
30.0 \\
20.0 \\
43.0 \\
40.0\end{array}$ & $\begin{array}{l}12.0 \\
12.0 \\
12.0 \\
12.0 \\
12.0\end{array}$ & $\begin{array}{l}30.0 \\
30.0 \\
30.0 \\
30.0 \\
30.0\end{array}$ & $\begin{array}{l}57.0 \\
57.0 \\
59.0 \\
59.0 \\
59.0\end{array}$ \\
\hline $\begin{array}{l}162.5 \\
173.8 \\
194.0 \\
192.5 \\
224.3\end{array}$ & $\begin{array}{l}173.8 \\
184.0 \\
192.5 \\
224.3 \\
258.0\end{array}$ & $\begin{array}{l}50.0 \\
55.0 \\
55.0 \\
55.0 \\
60.0\end{array}$ & $\begin{array}{l}364.5 \\
465.5 \\
545.0 \\
561.5 \\
709.5\end{array}$ & $\begin{array}{l}465.5 \\
345.0 \\
561.5 \\
709.5 \\
833.5\end{array}$ & $\begin{array}{l}25.0 \\
30.0 \\
45.0 \\
30.0 \\
35.0\end{array}$ & $\begin{array}{l}12.0 \\
12.0 \\
12.0 \\
12.0 \\
12.0\end{array}$ & $\begin{array}{l}30.0 \\
30.0 \\
30.0 \\
30.0 \\
30.0\end{array}$ & $\begin{array}{l}57.0 \\
56.0 \\
56.0 \\
56.0 \\
55.0\end{array}$ \\
\hline $\begin{array}{l}258.8 \\
295.8 \\
133.0 \\
203.0 \\
265.5\end{array}$ & $\begin{array}{l}295.8 \\
330.3 \\
203.0 \\
265.5 \\
352.5\end{array}$ & $\begin{array}{l}65.0 \\
55.0 \\
50.0 \\
40.0 \\
55.0\end{array}$ & $\begin{array}{r}833.5 \\
907.0 \\
89.5 \\
215.0 \\
319.5\end{array}$ & $\begin{array}{l}907.0 \\
916.5 \\
215.0 \\
319.5 \\
374.8\end{array}$ & $\begin{array}{l}35.0 \\
45.0 \\
30.0 \\
44.0 \\
45.0\end{array}$ & $\begin{array}{l}12.0 \\
12.0 \\
12.0 \\
12.0 \\
12.0\end{array}$ & $\begin{array}{l}30.0 \\
30.0 \\
30.0 \\
30.0 \\
30.0\end{array}$ & $\begin{array}{l}55.0 \\
54.0 \\
61.0 \\
55.0 \\
55.0\end{array}$ \\
\hline $\begin{array}{l}352.5 \\
417.3 \\
435.5 \\
390.0\end{array}$ & $\begin{array}{l}417.3 \\
491.0 \\
542.5 \\
485.3\end{array}$ & $\begin{array}{l}40.0 \\
25.0 \\
35.0 \\
30.0\end{array}$ & $\begin{array}{l}374.8 \\
385.0 \\
488.5 \\
536.0\end{array}$ & $\begin{array}{l}385.0 \\
488.5 \\
556.0 \\
563.3\end{array}$ & $\begin{array}{l}45.0 \\
45.0 \\
50.0 \\
63.0\end{array}$ & $\begin{array}{l}12.0 \\
12.0 \\
12.0 \\
12.0\end{array}$ & $\begin{array}{l}30.0 \\
50.0 \\
30.0 \\
30.0\end{array}$ & $\begin{array}{l}55.0 \\
54.0 \\
54.0 \\
54.0\end{array}$ \\
\hline
\end{tabular}




\section{TABLE VI}

BASEIINE COUNTERCURRENT FLOW TEST GROUP 2.1

THANSPAFENT VESEEL TEST DATA FUR TESTS 2.1 .1 TO $2.1 .3-0.37-I N$. JONNCOMER GAP SUMMARY JF CALCULATED RE:SULTS

\begin{tabular}{|c|c|c|c|c|c|c|c|c|c|c|}
\hline $\begin{array}{l}\text { TEST } \\
\text { NO }\end{array}$ & $\begin{array}{l}\text { DOWNCOMER } \\
\text { AIR FLOW } \\
\text { ILB/SEC) }\end{array}$ & $\begin{array}{l}\text { DUWNCOMER } \\
\text { AIR DE INSITY } \\
\text { (LB/FT } \$ 3 \text { I }\end{array}$ & $\begin{array}{c}\text { LOWER PLENUM } \\
\text { PZESSURE } \\
\text { (PSIA) }\end{array}$ & $\begin{array}{l}\text { CQLD LES } \\
\text { AIR FLDW } \\
\text { (LB/SECI }\end{array}$ & $\begin{array}{l}\text { DOWIICOIMEF. } \\
\text { WATER FLOIV } \\
\text { (LB/SEC) }\end{array}$ & $\begin{array}{l}\text { WATER FLON } \\
\text { CONTIVUTYY }\end{array}$ & $\begin{array}{c}J-A I F \\
\text { JUANEJUED } \\
\text { (FT/SEC) }\end{array}$ & $\begin{array}{l}\text { J-ANTFR } \\
\text { OWWOZYSE } \\
\text { (FT/SEC) }\end{array}$ & $\left.\left.(\mathrm{J} ;)^{\star}\right)+1\right)$ & $(g L *) * 1 / 2$ \\
\hline $\begin{array}{l}2.1 \cdot 1 \\
2.1 \cdot 2 \\
2.1 .3\end{array}$ & $\begin{array}{l}0.0296 \\
0.0293 \\
0.0293\end{array}$ & $\begin{array}{l}0.1023 \\
0.1023 \\
0.1023\end{array}$ & $\begin{array}{l}19.67 \\
19.67 \\
19.67\end{array}$ & $\begin{array}{l}0.0 \\
0.0 \\
0.0\end{array}$ & $\begin{array}{l}2.757 \\
2.950 \\
2.933\end{array}$ & $\begin{array}{l}1.013 \\
0.933 \\
1.031\end{array}$ & $\begin{array}{l}4.9 ? \\
4.86 \\
4.36\end{array}$ & $\begin{array}{l}0.753 \\
0.803 \\
0.799\end{array}$ & $\begin{array}{l}0.3768 \\
0.3745 \\
0.3745\end{array}$ & $\begin{array}{l}0.7329 \\
0.7568 \\
0.7546\end{array}$ \\
\hline
\end{tabular}


TABLE VI (Contd.)

BASELINE COUNTERCURRENT FLOW TEST-GROUP 2.1

EXPER IMENTAL MEASURMENTS FOR TESTS 2.1 .1 TO $2.1 .3--0.37-1$ - 0 . DOWNCOMER GAP

\begin{tabular}{|c|c|c|c|c|c|c|c|c|c|c|}
\hline $\begin{array}{l}\text { TEST } \\
\text { NO }\end{array}$ & $\begin{array}{c}R M-C W-1 \\
(G P M)\end{array}$ & $\begin{array}{c}R \cdot M-C W-2 \\
(G P M)\end{array}$ & $\begin{array}{c}F T-C W-1 \\
(G P M)\end{array}$ & $\begin{array}{c}F T-C W-2 \\
(G P M)\end{array}$ & $\begin{array}{c}R M-H G-1 \\
(C F M)\end{array}$ & $\begin{array}{c}R M-H G-2 \\
(C F M)\end{array}$ & $\begin{array}{c}R M-H G-3 \\
(C F M)\end{array}$ & $\begin{array}{c}R: Y-H G-4 \\
(C F M)\end{array}$ & $\begin{array}{c}F T-H G-1 \\
(C F M)\end{array}$ & $\begin{array}{c}F T-H G-2 \\
(C F M)\end{array}$ \\
\hline $\begin{array}{l}2.1 \cdot 1 \\
2 \cdot 1 \cdot 2 \\
2.1 .3\end{array}$ & $\begin{array}{l}30.0 \\
30.0 \\
22.0\end{array}$ & $\begin{array}{r}10.0 \\
0.0 \\
0.0\end{array}$ & $\begin{array}{l}0.0 \\
0.0 \\
0.0\end{array}$ & $\begin{array}{l}0.0 \\
0.0 \\
0.0\end{array}$ & $\begin{array}{l}0.0 \\
0.0 \\
0.0\end{array}$ & $\begin{array}{c}20.00 \\
20.00 \\
0.0\end{array}$ & $\begin{array}{c}0.0 \\
0.0 \\
20.00\end{array}$ & $\begin{array}{l}0.0 \\
0.0 \\
0.0\end{array}$ & $\begin{array}{l}0.0 \\
0.0 \\
0.0\end{array}$ & $\begin{array}{l}0.0 \\
0.00 \\
0.0\end{array}$ \\
\hline & & & & & & & & & & . \\
\hline $\begin{array}{l}\text { TEST } \\
\text { NO }\end{array}$ & $\begin{array}{l}P-C W-1 \\
\text { (PS IG I }\end{array}$ & $\begin{array}{l}P-C W-2 \\
(P S I G)\end{array}$ & $\begin{array}{l}P-H G-1 \\
(P S I G)\end{array}$ & $\begin{array}{l}P \cdot-H G-2 \\
(P S I G)\end{array}$ & $\begin{array}{l}P-H G-3 \\
\text { (PSIG) }\end{array}$ & $\begin{array}{l}T F-C N-1 \\
(D E G F \mid\end{array}$ & $\begin{array}{l}\text { TF-HF-1 } \\
\text { (UEG F }\end{array}$ & $\begin{array}{l}T F-4 T-2 \\
(D E r, E)\end{array}$ & $\begin{array}{l}T C-H G-3 \\
(D S G F)\end{array}$ & $\begin{array}{l}\text { P-BAQn } \\
\text { (PSIA) }\end{array}$ \\
\hline $\begin{array}{l}2 \cdot 1 \cdot 1 \\
2 \cdot 1 \cdot 2 \\
2 \cdot 1 \cdot 3\end{array}$ & $\begin{array}{l}0.0 \\
0.0 \\
0.0\end{array}$ & $\begin{array}{l}3.2 \\
7.7 \\
8.2\end{array}$ & $\begin{array}{l}8.2 \\
7.7 \\
7.7\end{array}$ & $\begin{array}{l}0.0 \\
0.0 \\
0.0\end{array}$ & $\begin{array}{l}0.0 \\
0.0 \\
0.0\end{array}$ & $\begin{array}{l}57.0 \\
56.0 \\
56.0\end{array}$ & $\begin{array}{l}69.0 \\
69.0 \\
69.0\end{array}$ & $\begin{array}{l}3.0 \\
0.0 \\
1.0\end{array}$ & $\begin{array}{l}0.0 \\
0.0 \\
0.0\end{array}$ & $\begin{array}{l}12.4 \\
12.4 \\
12.4\end{array}$ \\
\hline
\end{tabular}

LUWER PLENUH FLOW

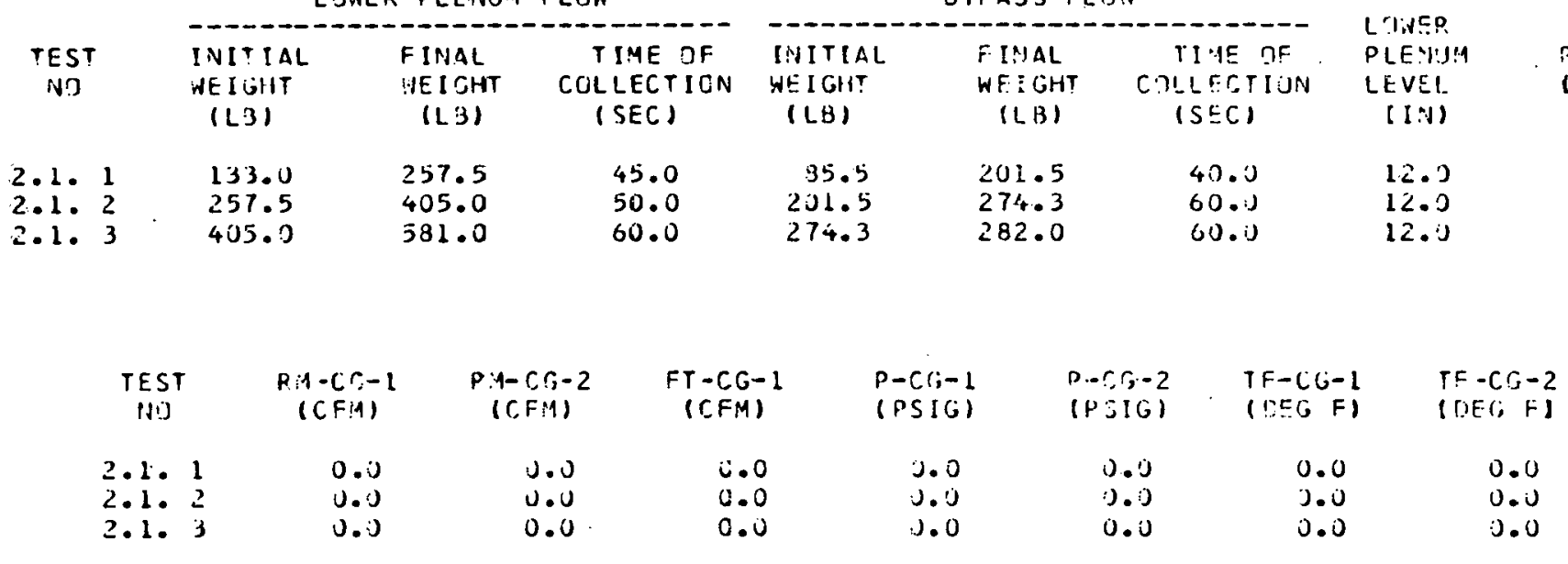


TABLE VII

BASELINE COUNTERCURRENT FLOW TEST GROUP 2.2

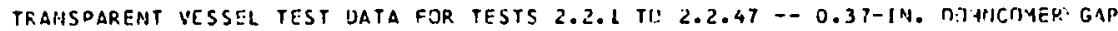

SUMMARY JF CALCULATES RESULTS

\begin{tabular}{|c|c|c|c|c|c|c|c|c|c|c|}
\hline $\begin{array}{l}\text { TEST } \\
\text { NO }\end{array}$ & $\begin{array}{l}\text { POWNCDMER } \\
\text { AIR FLOHA } \\
\text { ILR/SECI }\end{array}$ & $\begin{array}{l}\text { OSINAC DMER } \\
\text { AIR DENSITY } \\
\text { (LB/TT*\#3) }\end{array}$ & $\begin{array}{l}\text { LOWER PLENU' } \\
\text { PUESSUFE } \\
\text { (PSIA) }\end{array}$ & $\begin{array}{l}\text { COLD LES } \\
\text { AIR FLITH } \\
\text { (LIISTEC) }\end{array}$ & 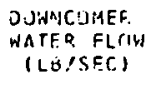 & 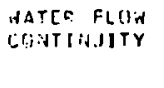 & 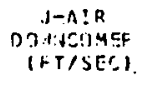 & 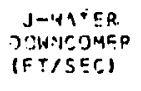 & $(1.0 \%)+01 / 2$ & $(J \|) \notin t$ \\
\hline $\begin{array}{ll}2.2 .2 & 1 \\
2.2 .2 & 2 \\
2.2 .3 \\
2.2 .4 \\
2.2 .5\end{array}$ & $\begin{array}{l}0.1141 \\
0.1421 \\
0.1426 \\
0.1423 \\
0.1423\end{array}$ & $\begin{array}{l}J .1101 \\
\text { J.11 J1 } \\
\text { J.1110 } \\
\text { J.1110 } \\
\text { J.1112 }\end{array}$ & $\begin{array}{l}21.29 \\
21.29 \\
21.29 \\
21.30 \\
21.30\end{array}$ & $\begin{array}{l}0.0 \\
0.0 \\
c .0 \\
0.0 \\
0.0\end{array}$ & $\begin{array}{l}0.040 \\
0.147 \\
0.115 \\
0.033 \\
0.142\end{array}$ & $\begin{array}{l}1.011 \\
1.035 \\
1.01 \% \\
1.013 \\
1.008\end{array}$ & $\begin{array}{l}26.94 \\
21.30 \\
21.82 \\
21.76 \\
21.72\end{array}$ & $\begin{array}{l}0.011^{1} \\
0.040 \\
0.031 \\
0.019 \\
0.032\end{array}$ & $\begin{array}{l}0.8986 \\
0.9103 \\
0.3103 \\
0.91093 \\
0.8009\end{array}$ & $\begin{array}{l}0.0881 \\
0.1687 \\
0.1492 \\
0.0864 \\
0.1658\end{array}$ \\
\hline $\begin{array}{l}2.2 .6 \\
2.2 .7 \\
2.2 .8 \\
2.2 .99 \\
2.2 .10\end{array}$ & $\begin{array}{l}0.1427 \\
0.1423 \\
0.1427 \\
0.1414 \\
0.1121\end{array}$ & $\begin{array}{l}J .1114 \\
0.1114 \\
J .11114 \\
0.1114 \\
0.1114\end{array}$ & $\begin{array}{l}21.30 \\
21.30 \\
21.30 \\
21.30 \\
21.30\end{array}$ & $\begin{array}{l}0.0 \\
0.0 \\
c .0 \\
0.0 \\
0.0\end{array}$ & $\begin{array}{l}0.146 \\
0.142 \\
0.153 \\
0.175 \\
0.325\end{array}$ & $\begin{array}{l}1.010 \\
0.99 .2 \\
3.971 \\
1.119 \\
1.014\end{array}$ & $\begin{array}{l}21.7 \% \\
21.0 \% \\
21.7 \% \\
21.85 \\
17.0 \%\end{array}$ & $\begin{array}{l}0.040 \\
0.039 \\
0.042 \\
0.019 \\
0.033\end{array}$ & $\begin{array}{l}0.3996 \\
0.3035 \\
0.9096 \\
0.3118 \\
0.7178\end{array}$ & $\begin{array}{l}0.1693 \\
0.1654 \\
0.1721 \\
0.1043 \\
0.2512\end{array}$ \\
\hline $\begin{array}{l}2.2 .11 \\
2.2 .12 \\
2.2 .13 \\
2.2214 \\
2.2 .15\end{array}$ & $\begin{array}{l}0.1126 \\
0.1114 \\
0.1113 \\
0.1131 \\
0.0790\end{array}$ & $\begin{array}{l}J .1114 \\
.11114 \\
\text { J.1119 } \\
\text { J.1119 } \\
0.1119\end{array}$ & $\begin{array}{l}21.30 \\
21.30 \\
21.30 \\
21.30 \\
21.30\end{array}$ & $\begin{array}{l}0.0 \\
0.0 \\
0.0 \\
0.0 \\
0.0\end{array}$ & $\begin{array}{l}0.344 \\
0.330 \\
10.337 \\
0.287 \\
0.805\end{array}$ & $\begin{array}{l}1.028 \\
1.018 \\
1.031 \\
1.391 \\
1.017\end{array}$ & $\begin{array}{l}17.15 \\
17.09 \\
16.9 .3 \\
17.15 \\
12.00\end{array}$ & $\begin{array}{l}0.474 \\
0.082 \\
0.992 \\
4.173 \\
0.210\end{array}$ & $\begin{array}{l}J .7193 \\
0.7169 . \\
0.1146 \\
0.77202 \\
0.702 .0\end{array}$ & $\begin{array}{l}0.2585 \\
0.2413 \\
0.2560 \\
0.2363 \\
0.3453\end{array}$ \\
\hline $\begin{array}{l}2.2 .16 \\
2.2 .17 \\
2.2 .18 \\
2.219 \\
2.2 .20\end{array}$ & $\begin{array}{l}0.0780 \\
0.0790 \\
0.0793 \\
.04781 \\
0.0781\end{array}$ & $\begin{array}{l}J .1116 \\
0.1116 \\
0.1119 \\
0.1119 \\
0.1119\end{array}$ & $\begin{array}{l}21.30 \\
21.30 \\
21.30 \\
21.30 \\
21.30\end{array}$ & $\begin{array}{l}0.0 \\
0.0 \\
0.0 \\
0.0 \\
c .0\end{array}$ & $\begin{array}{l}0.973 \\
0.765 \\
0.767 \\
0.836 \\
0.350\end{array}$ & $\begin{array}{l}1.0344 \\
1.023 \\
0.4: 45 \\
0.364 \\
0.1774\end{array}$ & $\begin{array}{l}11.95 \\
12.01 \\
11.99 \\
11.35 \\
11.85\end{array}$ & $\begin{array}{l}0.230 \\
0.239 \\
0.21 \% \\
0.227 \\
0.231\end{array}$ & $\begin{array}{l}0.61007 \\
0.6020 \\
0.6017 \\
0.5935 \\
0.5985\end{array}$ & $\begin{array}{l}0.4131 \\
0.3853 \\
0.3910 \\
0.4026 \\
0.4002\end{array}$ \\
\hline $\begin{array}{l}2.2 .21 \\
2.22 .22 \\
2.2 .23 \\
2.2 .24 \\
2.22 .25\end{array}$ & $\begin{array}{l}J .0460 j \\
0.0467 \\
0.0467 \\
0.0310 \\
0.0310\end{array}$ & $\begin{array}{l}J .1110 \\
.11116 \\
.11119 \\
.11119 \\
0.1114\end{array}$ & $\begin{array}{l}21.30 \\
21.30 \\
21.30 \\
21.30 \\
21.30\end{array}$ & $\begin{array}{l}0.0 \\
0.0 \\
0.0 \\
0.0 \\
0.0\end{array}$ & $\begin{array}{l}2.003 \\
2.044 \\
2.214 \\
2.325 \\
3.042\end{array}$ & $\begin{array}{l}1.186 \\
0.003 \\
1.021 \\
1.005 \\
1.014\end{array}$ & $\begin{array}{l}1.9 .3 \\
7.11 \\
7.1 .0 \\
4.71 \\
4.11\end{array}$ & $\begin{array}{l}9.543 \\
0.556 \\
9.302 \\
3.169 \\
0.9233\end{array}$ & $\begin{array}{l}0.4022 \\
3.4632 \\
0.1630 \\
0.3770 \\
0.3772\end{array}$ & $\begin{array}{l}0.6244 \\
0.6300 \\
0.6557 \\
0.7406 \\
0.76,45\end{array}$ \\
\hline $\begin{array}{l}2.2 .26 \\
2.22 .27 \\
2.2 .28 \\
2.2 .29 \\
2.2 .30\end{array}$ & $\begin{array}{l}0.0311 \\
0.0156 \\
J .0155 \\
0.0620 \\
0.063 j\end{array}$ & $\begin{array}{l}0.1110 \\
0.11116 \\
0.1114 \\
0.1114 \\
0.11114\end{array}$ & $\begin{array}{l}21.30 \\
21.30 \\
21.30 \\
21.30 \\
21.30\end{array}$ & $\begin{array}{l}0.0 \\
0.0 \\
0 . j \\
: .0 \\
0.0\end{array}$ & $\begin{array}{l}2.012 \\
4.053 \\
4.143 \\
1.214 \\
1.342\end{array}$ & $\begin{array}{l}1.003 \\
0.591 . \\
1.017 \\
1.018 \\
1.031\end{array}$ & $\begin{array}{l}4.73 \\
2.37 \\
2.35 \\
5.44 \\
9.65\end{array}$ & $\begin{array}{l}3.722 \\
1.193 \\
1.127 \\
0.330 \\
0.355\end{array}$ & $\begin{array}{l}0.3779 \\
0.2572 \\
0.2660 \\
0.5335 \\
0.5390\end{array}$ & $\begin{array}{l}0.7520 \\
0.8473 \\
0.9968 \\
0.9455 \\
0.5104\end{array}$ \\
\hline $\begin{array}{l}2.2 .31 \\
2.2 .32 \\
2.2 .33 \\
2.22 .34 \\
2.2 .35\end{array}$ & $\begin{array}{l}0.0623 \\
0.0623 \\
0.0627 \\
0.0955 \\
0.0945\end{array}$ & $\begin{array}{l}0.11114 \\
0.11114 \\
0.1114 \\
0.11114 \\
0.11114\end{array}$ & $\begin{array}{l}21.30 \\
21.30 \\
21.30 \\
21.30 \\
21.30\end{array}$ & $\begin{array}{l}u .0 \\
0.0 \\
0.0 \\
0.0 \\
0.0\end{array}$ & $\begin{array}{l}1.300 \\
1.450 \\
1.340 \\
0.430 \\
0.492\end{array}$ & $\begin{array}{l}1.023 \\
1.000 \\
2.0113 \\
1.021 \\
1.203\end{array}$ & $\begin{array}{r}3.49 \\
7.411 \\
9.55 \\
14.53 \\
14.94\end{array}$ & $\begin{array}{l}0.154 \\
3.394 \\
3.370 \\
0.131 \\
3.134\end{array}$ & $\begin{array}{l}0.5351 \\
0.5351 \\
0.5369 \\
0.6024 \\
0.6599\end{array}$ & $\begin{array}{l}0.5074 \\
0.5306 \\
0.5139 \\
0.3053 \\
0.3000\end{array}$ \\
\hline $\begin{array}{l}2.2 .36 \\
2.2 .37 \\
2.2 .38 \\
2.2 .39 \\
2.2 .40\end{array}$ & $\begin{array}{l}0.0945 \\
0.0960 \\
0.0953 \\
0.1284 \\
0.1289\end{array}$ & $\begin{array}{l}0.1114 \\
0.1250 \\
0.1113 \\
0.1113 \\
0.1117\end{array}$ & $\begin{array}{l}21.30 \\
21.30 \\
21.28 \\
21.28 \\
21.28\end{array}$ & $\begin{array}{l}0.0 \\
0.0 \\
0.0 \\
0.0 \\
0.0\end{array}$ & $\begin{array}{l}0.524^{\circ} \\
\text { U.j\$J } \\
0.570 \\
0.182 \\
0.173\end{array}$ & $\begin{array}{l}0.1068 \\
0.011 \\
1.037 \\
1.010 \\
0.762\end{array}$ & $\begin{array}{l}14.4 \mathrm{~J} \\
13.5 \% \\
19.61 \\
19.6 \% \\
19.56\end{array}$ & $\begin{array}{l}0.194 \\
0.150 . \\
0.155 \\
0.21 \% \\
0.194\end{array}$ & $\begin{array}{l}0.0504 \\
0.6453 \\
0.61 .37 \\
0.76,34 \\
0.7636\end{array}$ & $\begin{array}{l}0.3205 \\
0.3204 \\
0.3327 \\
0.1379 \\
0.1833\end{array}$ \\
\hline
\end{tabular}


TABLE VII (Contd.)

BASELINE COUNTERCURRENT FLOW TEST GROUP 2.2

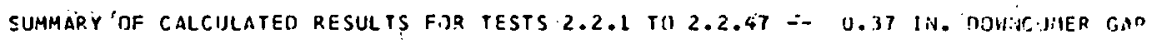

\begin{tabular}{|c|c|c|c|c|c|c|c|c|c|c|}
\hline $\begin{array}{l}\text { TEST } \\
\text { NO }\end{array}$ & $\begin{array}{l}\text { DONINCUMERR } \\
\text { AIR. FLTHW } \\
\text { (LE/SEC) }\end{array}$ & $\begin{array}{l}\text { COHNC OMER } \\
\text { ALR DENSITY } \\
\left(L B / F T * 3^{\prime}\right)\end{array}$ & $\begin{array}{l}\text { LOHER PLENUM } \\
\text { PRESSURE } \\
\text { (PSIA) }\end{array}$ & $\begin{array}{l}\text { COLO LEG } \\
\text { AIR FLOH } \\
\text { (LB/SEC) }\end{array}$ & $\begin{array}{l}\text { DOWIACOHER } \\
\text { TATER FLOH } \\
\text { (LI/SEC) }\end{array}$ & $\begin{array}{l}\text { WATER FLIJW } \\
\text { CONTINIJITY }\end{array}$ & $\begin{array}{c}\text { J-11k } \\
\text { OOSIIDHEN } \\
\text { (FT/SECI }\end{array}$ & $\begin{array}{l}\text { J-NATER } \\
\text { OTWHCDMEP. } \\
\text { IFT/SECI }\end{array}$ & $(J \div)=1 / 2$ & $i J l * 1 * \approx 1 / 2$ \\
\hline $\begin{array}{l}2.2 .41 \\
2.2 .42 \\
2.2 .43 \\
2.2 .44 \\
2.2 .45\end{array}$ & $\begin{array}{r}0.1294 \\
0.1280 \\
0.1280 \\
-0.1383 \\
0.1596\end{array}$ & $\begin{array}{l}0.1117 \\
0.11 .13 \\
0.1117 \\
0.1117 \\
0.1117\end{array}$ & $\begin{array}{l}21.28 \\
21.28 \\
21.28 \\
21.28 \\
21.28\end{array}$ & $\begin{array}{l}0.0 \\
0.0 \\
0.0 \\
0.0 \\
0.0\end{array}$ & $\begin{array}{l}0.244 \\
0.2115 \\
0.225 \\
0.047 \\
0.092\end{array}$ & $\begin{array}{l}1.014 \\
0.032 \\
1.064 \\
1.013 \\
1.007\end{array}$ & $\begin{array}{l}19.64 \\
19.64 \\
19.44 \\
24.12 \\
24.25\end{array}$ & $\begin{array}{l}0.055 \\
0.059 \\
0.031 \\
0.021 \\
0.025\end{array}$ & $\begin{array}{l}0.7796 \\
0.7694 \\
0.7663 \\
0.0535 \\
0.3557\end{array}$ & $\begin{array}{l}0.1945 \\
0.2045 \\
0.2090 \\
0.1303 \\
0.1334\end{array}$ \\
\hline $\begin{array}{l}2.2 .46 \\
2.2 .47\end{array}$ & $\begin{array}{l}0.1585 \\
0.1578\end{array}$ & $\begin{array}{l}0.1111 \\
0.1111\end{array}$ & $\begin{array}{r}21.28 \\
21.28\end{array}$ & $\begin{array}{l}0.0 \\
0.0\end{array}$ & $\begin{array}{l}0.084 \\
0.079\end{array}$ & $\begin{array}{l}0.901 \\
1.312\end{array}$ & $\begin{array}{l}24.2 .2 \\
24.11\end{array}$ & $\begin{array}{l}0.02 i \\
0.021\end{array}$ & $\begin{array}{l}0.3535 \\
0.8521\end{array}$ & $\begin{array}{l}0.1316 \\
0.1224\end{array}$ \\
\hline
\end{tabular}


TABLE VII (Contd.)

BASELINE COUNTERCURRENT FLOW TEST GROUP 2.2

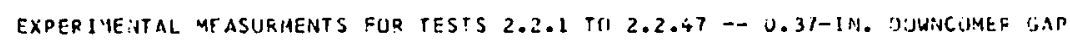

\begin{tabular}{|c|c|c|c|c|c|c|c|c|c|c|}
\hline $\begin{array}{l}\text { TEST } \\
\mathrm{NIT}\end{array}$ & $\begin{array}{c}R M-C W-1 \\
(G P M)\end{array}$ & $\begin{array}{c}K: 1-C H-2 \\
(r, P(H)\end{array}$ & $\begin{array}{l}F T-C H-1 \\
(\text { (LPM) }\end{array}$ & $\begin{array}{l}F T-C W-2 \\
(G P 4)\end{array}$ & $\begin{array}{l}R: 4-H C,-1 \\
(C F Y)\end{array}$ & $\begin{array}{l}F: A-H G=2 \\
(C F M A)\end{array}$ & $\begin{array}{l}(C F M) \\
P C-H / 3-3\end{array}$ & $\begin{array}{c}P M-H(j-4) \\
(C, Y Y)\end{array}$ & $\begin{array}{l}r .-4 i s-1 \\
(C F \div 9)\end{array}$ & $\begin{array}{l}F T-H G-2 \\
(C F M)\end{array}$ \\
\hline $\begin{array}{ll}2.2 .2 & 1 \\
2.2 . & 2 \\
2.2 . & 3 \\
2.2 . & 4 \\
2.2 . & 5\end{array}$ & $\begin{array}{l}30.0 \\
30.0 \\
30.0 \\
30.0 \\
30.0\end{array}$ & $\begin{array}{r}10.0 \\
10.0 \\
0.0 \\
10.0 \\
10.0\end{array}$ & $\begin{array}{l}0.0 \\
0.0 \\
0.0 \\
0.0 \\
0.0\end{array}$ & $\begin{array}{l}0.0 \\
0.0 \\
0.0 \\
0.01 \\
0.0\end{array}$ & $\begin{array}{l}70.00 \\
60.00 \\
60.00 \\
50.00 \\
30.00\end{array}$ & $\begin{array}{l}40.00 \\
30.00 \\
3.0 .00 \\
40.00 \\
40.010\end{array}$ & $\begin{array}{l}0.0 \\
0.0 \\
0.0 \\
.0 .0 \\
0.0\end{array}$ & $\begin{array}{l}0.0 \\
0.0 \\
u .0 \\
u . j \\
0.0\end{array}$ & $\begin{array}{l}0.0 \\
0.0 \\
0.01 \\
0.0 \\
0.0\end{array}$ & $\begin{array}{l}3.0 \\
0.0 \\
0.0 \\
0.0 \\
0.0\end{array}$ \\
\hline $\begin{array}{l}2.2 .6 \\
2.2 .7 \\
2.2 .9 \\
2.2 .97 \\
2.2 .10\end{array}$ & $\begin{array}{r}30.0 \\
20.0 \\
0.0 \\
0.0 \\
.30 .0\end{array}$ & $\begin{array}{r}0.0 \\
0.0 \\
10.0 \\
3.0 \\
10.0\end{array}$ & $\begin{array}{l}0.0 \\
0.0 \\
0.0 \\
0.0 \\
0.0\end{array}$ & $\begin{array}{l}0.0 \\
0.0 \\
0.0 \\
0.0 \\
0.0\end{array}$ & $\begin{array}{c}50.00 \\
50.00 \\
50.00 \\
50.00 \\
0.0\end{array}$ & $\begin{array}{l}40.00 \\
40.001 \\
40.00 \\
4.0 .00 \\
70.00\end{array}$ & $\begin{array}{l}j . j \\
0.0 \\
j .0 \\
0.0 \\
0.0\end{array}$ & $\begin{array}{l}3.0 \\
0.0 \\
0.0 \\
0.0 \\
0.0\end{array}$ & $\begin{array}{l}0.0 \\
0.0 \\
0.0 \\
0.0 \\
0.0\end{array}$ & $\begin{array}{l}0.0 \\
0.0 \\
0.0 \\
0.0 \\
0.0\end{array}$ \\
\hline $\begin{array}{l}2.2 .11 \\
2.2 .12 \\
2.2 .13 \\
2.214 \\
2.2 .15\end{array}$ & $\begin{array}{r}3.0 .0 \\
2.0 .0 \\
j .0 \\
.00 \\
3.0\end{array}$ & $\begin{array}{r}0.0 \\
0.00 \\
10.0 \\
10.0 \\
10.0\end{array}$ & $\begin{array}{l}0.0 \\
0.0 \\
0.0 \\
0.0 \\
0.0\end{array}$ & $\begin{array}{l}0.1 \\
0.0 \\
0.0 \\
0.0 \\
0.0\end{array}$ & $\begin{array}{l}0.0 \\
0.00 \\
0.0 \\
0.0 \\
0.0\end{array}$ & $\begin{array}{l}70.05 \\
70.019 \\
70.00 \\
70.00 \\
50.010\end{array}$ & $\begin{array}{l}\text { J.J } \\
\text { J.J } \\
0 . J \\
0.0 \\
0.0\end{array}$ & $\begin{array}{l}0.0 \\
0.0 \\
0.0 \\
0.0 \\
0.0\end{array}$ & $\begin{array}{l}0.0 \\
j .0 \\
0.0 \\
.00 \\
.00\end{array}$ & $\begin{array}{l}0.0 \\
0.0 \\
0.0 \\
0.2 \\
0.0\end{array}$ \\
\hline $\begin{array}{l}2.2 .16 \\
2.2 .17 \\
2.2 .19 \\
2.213 \\
2.2 .20\end{array}$ & $\begin{array}{r}30.0 \\
30.0 \\
2.0 .0 \\
3.0 \\
3.0\end{array}$ & $\begin{array}{l}0.0 \\
0.0 \\
0.0 \\
7.0 \\
8.0\end{array}$ & $\begin{array}{l}0.0 \\
0.0 \\
0.0 \\
0.0 \\
0.0\end{array}$ & $\begin{array}{l}0.0 \\
0.0 \\
0.0 \\
0.0 \\
0.0\end{array}$ & $\begin{array}{l}0.0 \\
0.0 \\
0.0 \\
0.0 \\
0.0\end{array}$ & $\begin{array}{l}50.030 \\
50.0 .0) \\
50.000 \\
50.00 \\
50.00\end{array}$ & $\begin{array}{l}0.0 \\
0.0 \\
0.0 \\
0.0 \\
0.0\end{array}$ & $\begin{array}{l}0.0 \\
0.0 \\
6.0 \\
0.0 \\
n .0\end{array}$ & $\begin{array}{l}0.0 \\
0.0 \\
0.0 \\
0.0 \\
0.0\end{array}$ & $\begin{array}{l}0.0 \\
0.0 \\
0.0 \\
0.1 \\
0.0\end{array}$ \\
\hline $\begin{array}{l}2.2 .21 \\
2.22 .22 \\
2.2 .23 \\
2.2 .24 \\
2.22 .25\end{array}$ & $\begin{array}{l}30.0 \\
30.0 \\
20.0 \\
30.0 \\
30.0\end{array}$ & $\begin{array}{r}10.0 \\
0.0 \\
0.0 \\
10.0 \\
0.0\end{array}$ & $\begin{array}{l}0.0 \\
0.0 \\
0.0 \\
0.0 \\
0.0\end{array}$ & $\begin{array}{l}0.0 \\
0.0 \\
0.0 \\
0.0 \\
0.0\end{array}$ & $\begin{array}{l}0.0 \\
0.0 \\
0.0 \\
0.0 \\
0.0\end{array}$ & $\begin{array}{l}0 . .1 \\
0.0 \\
u .0 \\
0.0 \\
0.0\end{array}$ & $\begin{array}{l}30.00 \\
30.00 \\
30.00 \\
20.010 \\
20.00\end{array}$ & $\begin{array}{l}u .0 \\
0.0 \\
0.0 \\
9.0 \\
0.0\end{array}$ & $\begin{array}{l}0.0 \\
0.0 \\
0.0 \\
.0 .0 \\
0.0\end{array}$ & $\begin{array}{l}0.0 \\
0.0 \\
0.0 \\
0.0 \\
0.0\end{array}$ \\
\hline $\begin{array}{l}2.2 .26 \\
2.22 .27 \\
2.22 .28 \\
2.2229 \\
2.22 .30\end{array}$ & $\begin{array}{l}24.0 \\
3.0 .0 \\
32.0 \\
3 \% .0 \\
20.0\end{array}$ & $\begin{array}{r}0.0 \\
10.0 \\
0.0 \\
10.0 \\
10.0\end{array}$ & $\begin{array}{l}0.0 \\
0.0 \\
0.0 \\
0.0 \\
0.0\end{array}$ & $\begin{array}{l}0.0 \\
0.0 \\
0.0 \\
u .0 \\
0.0\end{array}$ & $\begin{array}{l}0.0 \\
0.0 \\
0.0 \\
0.0 \\
0.0\end{array}$ & $\begin{array}{c}0.0 \\
0.0 \\
0.0 \\
40.00 \\
40.030\end{array}$ & $\begin{array}{c}2 . J . j 0 \\
10.30 \\
10.00 \\
\text { J.J } \\
0.0\end{array}$ & $\begin{array}{l}2.0 \\
0.0 \\
0.0 \\
0.0 \\
0.0\end{array}$ & $\begin{array}{l}0.0 \\
0.0 \\
0.0 \\
0.0 \\
0.0\end{array}$ & $\begin{array}{l}0.0 \\
0.0 \\
0.0 \\
0.0 \\
0.0\end{array}$ \\
\hline $\begin{array}{l}2.2 .31 \\
2.2 .32 \\
2.2 .33 \\
2.2 .34 \\
2.2 .35\end{array}$ & $\begin{array}{l}2.3 .0 \\
213.0 \\
12.0 \\
3 \mathrm{~J} .0 \\
20.0\end{array}$ & $\begin{array}{r}1,3.0 \\
0.0 \\
0.0 \\
10.0 \\
10.0\end{array}$ & $\begin{array}{l}0.0 \\
0.0 \\
0.0 \\
0.0 \\
0.0\end{array}$ & $\begin{array}{l}J . J \\
0.0 \\
.00 \\
.00 \\
0.0\end{array}$ & $\begin{array}{l}0.0 \\
0.0 \\
0.0 \\
. .0 \\
0.0\end{array}$ & $\begin{array}{l}40.05 \\
40.00 \\
40.00 \\
60.00 \\
60.000\end{array}$ & 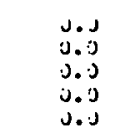 & $\begin{array}{l}0.5 \\
0.0 \\
0.0 \\
0.0 \\
0.0\end{array}$ & $\begin{array}{l}0.0 \\
0.0 \\
0.0 \\
0.0 \\
0.0\end{array}$ & $\begin{array}{l}0.0 \\
0.0 \\
0.0 \\
0.0 \\
0.0\end{array}$ \\
\hline $\begin{array}{l}2.2 .36 \\
2.2231 \\
2.2 .38 \\
2.2 .39 \\
2.2 .430\end{array}$ & $\begin{array}{r}3.0 .0 \\
0.0 \\
0.0 \\
3 \% .0 \\
3 \% .0\end{array}$ & $\begin{array}{r}10.0 \\
10.0 \\
5.0 \\
10.0 \\
10.0\end{array}$ & $\begin{array}{l}0.0 \\
0.0 \\
0.0 \\
0.0 \\
0.0\end{array}$ & $\begin{array}{l}0.0 \\
0.0 \\
0.0 \\
0.0 \\
0.0\end{array}$ & $\begin{array}{l}0.0 \\
0.00 \\
0.00 \\
80.00 \\
80.00\end{array}$ & $\begin{array}{l}61.000 \\
10.000 \\
0.000 \\
0.00 \\
0.0\end{array}$ & $\begin{array}{l}0.0 \\
0.0 \\
0.0 \\
0.0 \\
0.0 \\
0.0\end{array}$ & $\begin{array}{l}0.1 \\
0.0 \\
0.0 \\
0.0 \\
2.0 \\
0.0\end{array}$ & $\begin{array}{l}1.0 \\
0.0 \\
0.0 \\
9.0 \\
0.0\end{array}$ & $\begin{array}{l}0.0 \\
0.0 \\
\dot{0.0} \\
0.0 \\
\text { J.0 }\end{array}$ \\
\hline
\end{tabular}


TABLE VII (Contd.)

BASELINE COUNTERCURRENT FLOW TEST GROUP 2.2

EXPERI IENTAL MEASURMENTS FOR TESTS 2.2 .1 TO $2.2 .47,0-0.37-1 \mathrm{~N}$. DOWNCOMER GAP

\begin{tabular}{|c|c|c|c|c|c|c|c|c|c|c|}
\hline$\underset{N D S}{T E S T}$ & $\begin{array}{c}R M-C N-1 \\
(G P: A)\end{array}$ & $\begin{array}{l}\mathrm{K} H-\mathrm{CH}-2 \\
(G P M)\end{array}$ & $\begin{array}{l}F T-(\mathcal{L})-1 \\
(\in P M)\end{array}$ & $\begin{array}{l}F Y-C H-2 \\
(G P M)\end{array}$ & $\begin{array}{l}R M-H(G-1 \\
(C F M)\end{array}$ & 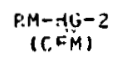 & 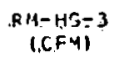 & $\begin{array}{c}P M-! 15,-4 \\
(C-4)\end{array}$ & $\begin{array}{c}F T-H ?,-1 \\
\text { ICFM! }\end{array}$ & $\begin{array}{l}F T-H F_{,}-2 \\
\text { ICF!:I }\end{array}$ \\
\hline $\begin{array}{l}2.2 .41 \\
2.2 .42 \\
2.2 .43 \\
2.2 .44 \\
2.2 .45\end{array}$ & $\begin{array}{r}23.0 \\
0.0 \\
3.0 \\
3: 0.0 \\
20.0\end{array}$ & $\begin{array}{r}0.0 \\
10.0 \\
4.0 \\
10.0 \\
10.0\end{array}$ & $\begin{array}{l}0.0 \\
0.0 \\
0 . J \\
0.0 \\
0.0\end{array}$ & $\begin{array}{l}0.0 \\
0.0 \\
0.0 \\
0.0 \\
0.0\end{array}$ & $\begin{array}{l}80.00 \\
80.0 j \\
80.00 \\
60.00 \\
60.0 j\end{array}$ & $\begin{array}{c}0.0 \\
0.0 \\
0.0 \\
40.00 \\
40.00\end{array}$ & $\begin{array}{l}0.0 \\
j .0 \\
0.0 \\
0.0 \\
u . j\end{array}$ & $\begin{array}{l}0.0 \\
0.0 \\
0.0 \\
0.0 \\
0.0\end{array}$ & $\begin{array}{l}0.0 \\
0.0 \\
0.0 \\
0.0 \\
0.0\end{array}$ & $\begin{array}{l}0.0 \\
0.0 \\
0.0 \\
0.0 \\
0.0\end{array}$ \\
\hline $\begin{array}{l}2.2 .46 \\
2.2 .47\end{array}$ & $\begin{array}{r}20.0 \\
3.0\end{array}$ & $\begin{array}{l}0.00 \\
10.0\end{array}$ & $\begin{array}{l}0.0 \\
0.0\end{array}$ & $\begin{array}{l}0.0 \\
0.0\end{array}$ & $\begin{array}{l}60.00 \\
60.000\end{array}$ & $\begin{array}{l}40.00 \\
40.00\end{array}$ & $\begin{array}{l}0.0 \\
0.0\end{array}$ & 0.0 & $\begin{array}{l}0.3 \\
0.0\end{array}$ & $\begin{array}{l}0.0 \\
0.0\end{array}$ \\
\hline $\begin{array}{c}\text { TEST } \\
\text { NO }\end{array}$ & $\begin{array}{l}P-C: 1-1 \\
\mid P S I G)\end{array}$ & $\begin{array}{l}P-C H-2 \\
\text { PSIG }\end{array}$ & $\begin{array}{l}P-H G-1 \\
(P S I G)\end{array}$ & $\begin{array}{l}P-H G-2 \\
(P S) G)\end{array}$ & $\begin{array}{l}\mathrm{D}-\mathrm{HG}-3 \\
\text { (PSIS) }\end{array}$ & $\begin{array}{l}\{F-C h-1 \\
(0) G(G)\end{array}$ & $\begin{array}{l}\text { Tr. } H(T,-1 \\
\text { (OEG F.) }\end{array}$ & 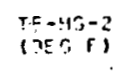 & $\begin{array}{l}\text { IF-HC-3 } \\
(\mathrm{CEST})\end{array}$ & $\begin{array}{l}\text { o-japr } \\
\text { irsial }\end{array}$ \\
\hline $\begin{array}{ll}2.2 . & 1 \\
2.2 .2 & 2 \\
2.2 .3 \\
2.2 .4 \\
2.2 .5\end{array}$ & $\begin{array}{l}3.0 \\
\text { J.0 } \\
\text { J.0 } \\
\text { J.0 }\end{array}$ & $\begin{array}{l}10.7 \\
10.7 \\
10.7 \\
10.7 \\
10.7\end{array}$ & $\begin{array}{l}10.5 \\
10.5 \\
10.5 \\
10.5 \\
10.5\end{array}$ & $\begin{array}{l}0.0 \\
0.0 \\
0.0 \\
0.0 \\
0.0\end{array}$ & $\begin{array}{l}0.0 \\
0.0 \\
0.0 \\
0.0 \\
0.0\end{array}$ & $\begin{array}{l}51.1 \\
34.0 \\
55.0 \\
55.0 \\
55.0\end{array}$ & $\begin{array}{l}70.0 \\
70.3 \\
66.3 \\
00.0 \\
66.0\end{array}$ & $\begin{array}{l}0.0 \\
.0 .0 \\
0.0 \\
0.0 \\
3.0\end{array}$ & $\begin{array}{l}3.0 \\
3.0 \\
0.0 \\
0.0 \\
0.0\end{array}$ & $\begin{array}{l}12.3 \\
12.3 \\
12.3 \\
12.3 \\
12.3\end{array}$ \\
\hline $\begin{array}{l}2.2 .6 \\
2.2 .7 \\
2.2 .9 \\
2.209 \\
2.2 .10\end{array}$ & $\begin{array}{l}3.0 \\
נ .0 \\
j .0 \\
j .0 \\
. .0\end{array}$ & $\begin{array}{l}10.7 \\
10.5 \\
10.7 \\
10.7 \\
10.1\end{array}$ & $\begin{array}{l}10.5 \\
10.5 \\
10.5 \\
10.7 \\
10.5\end{array}$ & $\begin{array}{l}0.0 \\
0.0 \\
0.0 \\
0.0 \\
0.0\end{array}$ & $\begin{array}{l}u .0 \\
\dot{u} .0 \\
u . j \\
\dot{u} . j \\
\dot{0} . j\end{array}$ & $\begin{array}{l}55.3 \\
50.0 \\
53.0 \\
57.0 \\
35.0\end{array}$ & $\begin{array}{l}63.3 \\
60.3 \\
63.0 \\
63.0 \\
63.0\end{array}$ & $\begin{array}{l}3.0 \\
3.0 \\
0.0 \\
0.0 \\
0.0\end{array}$ & $\begin{array}{l}0.0 \\
0.0 \\
0.0 \\
0.0 \\
0.0\end{array}$ & $\begin{array}{l}12.3 \\
12.3 \\
12.3 \\
12.3 \\
12.3\end{array}$ \\
\hline $\begin{array}{l}2.2 .11 \\
2.2 .12 \\
2.2 .13 \\
2.2 .14 \\
2.2 .15\end{array}$ & $\begin{array}{l}0.0 \\
0.0 \\
.00 \\
0.0 \\
.00\end{array}$ & $\begin{array}{l}10.7 \\
10.5 \\
10.5 \\
11.0 \\
10.7\end{array}$ & $\begin{array}{l}10.7 \\
10.5 \\
10.2 \\
11.0 \\
10.5\end{array}$ & $\begin{array}{l}0.0 \\
0.0 \\
0.3 \\
0.0 \\
0.00\end{array}$ & $\begin{array}{l}0.0 \\
0.0 \\
0.0 \\
0 . j \\
0.0\end{array}$ & $\begin{array}{l}29.3 \\
54.0 \\
52.3 \\
52.5 \\
53.5\end{array}$ & $\begin{array}{l}0.1 .5 \\
60.0 \\
65.0 \\
65.0 \\
65.0 \\
61.0\end{array}$ & $\begin{array}{l}1.3 \\
\because: 3 \\
0.1 \\
3.3 \\
1.0\end{array}$ & $\begin{array}{l}0.0 \\
0.0 \\
0.0 \\
0.0 \\
0.0\end{array}$ & $\begin{array}{l}12.3 \\
12.3 \\
12.3 \\
12.3 \\
12.3\end{array}$ \\
\hline $\begin{array}{l}2.2 .10 \\
2.211 \\
2.2 .18 \\
2.2219 \\
2.2 .20\end{array}$ & $\begin{array}{l}.00 \\
0.0 \\
j .0 \\
0.0 \\
0.0\end{array}$ & $\begin{array}{l}10.5 \\
10.7 \\
10.5 \\
10.5 \\
10.0\end{array}$ & $\begin{array}{l}10.2 \\
10.5 \\
10.5 \\
10.0 \\
10.0\end{array}$ & $\begin{array}{l}0.0 \\
0.0 \\
0.0 \\
0.0 \\
0.0 \\
0.0\end{array}$ & $\begin{array}{l}0.0 \\
0.0 \\
0.0 \\
0.0 \\
0.0\end{array}$ & $\begin{array}{l}133.0 \\
53.0 \\
53.0 \\
53.0 \\
53.0\end{array}$ & $\begin{array}{l}05.2 \\
60.0 \\
60.0 \\
66.3 \\
66.2\end{array}$ & $\begin{array}{l}0.0 \\
0.0 \\
0.0 \\
0.0 \\
0.0\end{array}$ & $\begin{array}{l}0.0 \\
.00 \\
2.0 \\
0.0 \\
0.0\end{array}$ & $\begin{array}{l}12.3 \\
1<.3 \\
12.3 \\
12.3 \\
12.3\end{array}$ \\
\hline $\begin{array}{l}2.2 .21 \\
2.2 .22 \\
2.2 .23 \\
2.2 .24 \\
2.2 .25\end{array}$ & $\begin{array}{l}0.0 \\
0.0 \\
.00 \\
.00 \\
0.0\end{array}$ & $\begin{array}{l}10.5 \\
10.5 \\
10.5 \\
10.5 \\
10.5\end{array}$ & $\begin{array}{l}10.2 \\
10.5 \\
10.5 \\
10.2 \\
10.2\end{array}$ & $\begin{array}{l}0.0 \\
0.0 \\
0.0 \\
0.0 \\
0.0\end{array}$ & $\begin{array}{l}0.0 \\
0.0 \\
0.0 \\
6.0 \\
0.0\end{array}$ & $\begin{array}{l}53.0 \\
53.0 \\
53.0 \\
53.0 \\
51.0\end{array}$ & $\begin{array}{l}66.0 \\
67.5 \\
67.2 \\
67.3 \\
67.9\end{array}$ & $\begin{array}{l}3.0 \\
3.0 \\
0.3 \\
0.0 \\
0.0\end{array}$ & $\begin{array}{l}0.0 \\
0.0 \\
0.0 \\
0.0 \\
0.0\end{array}$ & $\begin{array}{l}12.3 \\
12.3 \\
12.3 \\
12.3 \\
12.3\end{array}$ \\
\hline
\end{tabular}




\section{TABLE VII (Contd.)}

BASELINE COUNTERCURRENT FLOW TEST GROUP 2.2

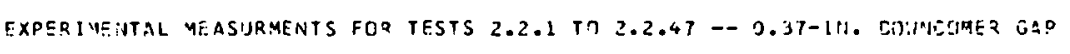

\begin{tabular}{|c|c|c|c|c|c|c|c|c|c|c|}
\hline $\begin{array}{c}\text { TEST } \\
\text { NO }\end{array}$ & $\begin{array}{l}\text { P-CW-1 } \\
\text { (DSig) }\end{array}$ & $\begin{array}{l}\text { P-cis-z } \\
\text { ipsicis }\end{array}$ & $\begin{array}{l}P-H 1 G-1 \\
\text { |PSIGI }\end{array}$ & $\begin{array}{l}P=H(-2 \\
\text { (PS!G) }\end{array}$ & $\begin{array}{l}\mathrm{O}-\mathrm{HG}-3 \\
|P S| G \mid\end{array}$ & $\begin{array}{l}T r-C h-1 \\
(נ J E F)\end{array}$ & $\begin{array}{l}\text { TF-H';-1 } \\
\text { IOEO }=\text { I }\end{array}$ & $\begin{array}{l}\text { TC }-H(j-2-2 \\
\text { (i)E } T, \text { C. }\end{array}$ & $\begin{array}{l}T F-! 15-\hat{3} \\
10 \leq G \mathrm{~F}\end{array}$ & $\begin{array}{l}P-38 Q 7 \\
(P 5 \mid 0)\end{array}$ \\
\hline $\begin{array}{l}2.2 .26 \\
2.2 .27 \\
2.22 .29 \\
2.2 .29 \\
2.2 .310\end{array}$ & $\begin{array}{l}3.0 \\
3.0 \\
3.0 \\
3.0 \\
.00\end{array}$ & $\begin{array}{l}10.7 \\
10.7 \\
10.7 \\
10.2 \\
10.7\end{array}$ & $\begin{array}{l}10.5 \\
10.5 \\
10.2 \\
10.3 \\
10.7\end{array}$ & $\begin{array}{l}3.10 \\
0.0 \\
0.0 \\
0.0 \\
0.0\end{array}$ & $\begin{array}{l}0.0 \\
0.0 \\
0.0 \\
0.0 \\
2.0\end{array}$ & $\begin{array}{l}54.0 \\
54.0 \\
54.0 \\
53.3 \\
55.0\end{array}$ & $\begin{array}{l}69.0^{\circ} \\
69.05 \\
69.0 \\
70.0 \\
10.0\end{array}$ & $\begin{array}{l}0.0 \\
0.0 \\
0.0 \\
0.0 \\
0.0\end{array}$ & $\begin{array}{l}0.0 \\
0.0 \\
0.0 \\
0.0 \\
0.0\end{array}$ & $\begin{array}{l}12.3 \\
12.3 \\
12.3 \\
12.3 \\
12.3\end{array}$ \\
\hline $\begin{array}{l}2.2 .31 \\
2.2 .32 \\
2.22 .33 \\
2.2234 \\
2.2 .35\end{array}$ & $\begin{array}{l}\jmath .0 \\
.0 .0 \\
.00 \\
.00 \\
\text { J.j }\end{array}$ & $\begin{array}{l}10.2 \\
10.2 \\
10.5 \\
10.7 \\
10.5\end{array}$ & $\begin{array}{l}10.2 \\
10.2 \\
10.5 \\
10.7 \\
10.2\end{array}$ & $\begin{array}{l}0.0 \\
0.0 \\
0.0 \\
0.0 \\
0.0\end{array}$ & $\begin{array}{l}0.0 \\
0.0 \\
0.0 \\
0.0 \\
0.0\end{array}$ & $\begin{array}{l}53.0 \\
3 \% .0 \\
5 \% .0 \\
5,11.0 \\
35.0\end{array}$ & $\begin{array}{l}70.0 \\
7 \% .0 \\
62.0 \\
69.3 \\
69.0\end{array}$ & $\begin{array}{l}0.0 \\
0.0 \\
0.0 \\
0.0 \\
0.0\end{array}$ & $\begin{array}{l}2.0 \\
0.0 \\
0.0 \\
3.0 \\
0: 0 .\end{array}$ & $\begin{array}{l}12.3 \\
12.2 \\
12.3 \\
12.3 \\
12.3\end{array}$ \\
\hline $\begin{array}{l}2.2 .36 \\
2.2 .37 \\
2.2 .38 \\
2.2234 \\
2.2 .40\end{array}$ & $\begin{array}{l}3.0 \\
3.0 \\
2.0 \\
1.0 \\
3.0\end{array}$ & $\begin{array}{l}10.5 \\
10.7 \\
10.7 \\
10.7 \\
10.7\end{array}$ & $\begin{array}{l}10.2 \\
11.0 \\
10.7 \\
10.5 \\
10.5\end{array}$ & $\begin{array}{l}0.0 \\
0.0 \\
0.0 \\
0.0 \\
0.0\end{array}$ & $\begin{array}{l}0.0 \\
0.0 \\
0.0 \\
0.0 \\
0.0\end{array}$ & $\begin{array}{l}55.0 \\
55.0 \\
50.0 \\
53.0 \\
53.0\end{array}$ & $\begin{array}{l}69.0 \\
69.3 \\
65.0 \\
65.0 \\
65.0\end{array}$ & $\begin{array}{l}0.0 \\
0.0 \\
0.0 \\
0.2 \\
0.0\end{array}$ & $\begin{array}{l}0.0 \\
0.0 \\
0.0 \\
.00 \\
0.0\end{array}$ & $\begin{array}{l}12.3 \\
12.3 \\
12.3 \\
12.3 \\
12.3\end{array}$ \\
\hline $\begin{array}{l}2.2 .41 \\
2.2 .42 \\
2.2 .43 \\
2.22 .44 \\
2.2 .45\end{array}$ & $\begin{array}{l}\jmath .0 \\
.00 \\
j .0 \\
. .0 \\
j .0\end{array}$ & $\begin{array}{l}10.5 \\
10.5 \\
10.2 \\
10.7 \\
10.7\end{array}$ & $\begin{array}{l}10.7 \\
10.5 \\
10.2 \\
10.5 \\
10.7\end{array}$ & $\begin{array}{l}0.0 \\
0 . u \\
0.0 \\
0 . j \\
0.0\end{array}$ & $\begin{array}{l}0.0 \\
0.0 \\
0.0 \\
0.0 \\
0.0\end{array}$ & $\begin{array}{l}53.0 \\
52.0 \\
5: 00 \\
53.0 \\
53.0\end{array}$ & $\begin{array}{l}65.3 \\
65.5 \\
60.0 \\
65.0 \\
05.0\end{array}$ & $\begin{array}{l}0.0 \\
0.0 \\
0.0 \\
0.0 \\
0.0\end{array}$ & $\begin{array}{l}0.0 \\
0.0 \\
0.0 \\
0.0 \\
0.0\end{array}$ & $\begin{array}{l}12.3 \\
12.3 \\
12.3 \\
12.3 \\
12.3\end{array}$ \\
\hline \multirow[t]{2}{*}{$\begin{array}{l}2.2 .46 \\
2.2 .47\end{array}$} & $\begin{array}{l}3.0 \\
j .0\end{array}$ & $\begin{array}{l}10.5 \\
10.5\end{array}$ & $\begin{array}{l}10.5 \\
10.2\end{array}$ & $\begin{array}{l}3.0 \\
0.0\end{array}$ & $\begin{array}{l}0.0 \\
0.0\end{array}$ & $\begin{array}{l}53.0 \\
5.60\end{array}$ & $\begin{array}{l}67.0 \\
60.7\end{array}$ & $\begin{array}{l}0.0 \\
0.3\end{array}$ & $\begin{array}{l}0.0 \\
0.0\end{array}$ & $\begin{array}{l}12.3 \\
12.3\end{array}$ \\
\hline & \multicolumn{3}{|c|}{ LOWEP. PLENUM FLOW } & \multicolumn{3}{|c|}{ BYMASS FL!JW } & & & & \\
\hline $\begin{array}{c}\text { TEST } \\
\text { ido }\end{array}$ & $\begin{array}{l}\text { INITIAL } \\
\text { WEIGHT } \\
\text { ILBI }\end{array}$ & $\begin{array}{l}\text { FINAL } \\
\text { :HE ILGHT } \\
\text { ILGI }\end{array}$ & $\begin{array}{l}\text { TIME NF } \\
\text { COLLECTICN } \\
\text { (SEC) }\end{array}$ & $\begin{array}{l}\text { INITIALL } \\
\text { WEICHT } \\
\text { ILBI }\end{array}$ & $\begin{array}{l}\text { FINAL } \\
\text { WEIGHT } \\
\text { IL } 31\end{array}$ & $\begin{array}{l}\text { TIME OF } \\
\text { COLLECTION } \\
\text { (SFE) }\end{array}$ & $\begin{array}{l}\text { DLE:VU: } \\
\text { LEVEL } \\
\text { IIVI }\end{array}$ & $\begin{array}{l}n-L^{n}-1 \\
\cos \left(\cos ^{2}\right)\end{array}$ & $\begin{array}{l}n F-(P-1 \\
(n \leq G) \leq 1\end{array}$ & \\
\hline $\begin{array}{ll}2.2 . & 1 \\
2.2 . & 2 \\
2.2 . & 3 \\
2.2 . & 4 \\
2.2 .5\end{array}$ & $\begin{array}{l}132.5 \\
135.5 \\
145.5 \\
132.0 \\
134.0\end{array}$ & $\begin{array}{l}135.5 \\
146.5 \\
156.3 \\
134.0 \\
142.5\end{array}$ & $\begin{array}{l}75.0 \\
75.0 \\
95.0 \\
60.0 \\
60.0\end{array}$ & $\begin{array}{r}86.3 \\
254.0 \\
417.3 \\
42.0 \\
166.1\end{array}$ & $\begin{array}{l}234.0 \\
417.5 \\
341.0 \\
166.0 \\
330.0\end{array}$ & $\begin{array}{l}30.0 \\
30.0 \\
30.0 \\
15.0 \\
30.0\end{array}$ & $\begin{array}{l}12.0 \\
12.0 \\
12.0 \\
12.0 \\
12.0\end{array}$ & $\begin{array}{l}9.0 \\
9.0 \\
7.0 \\
9.0 \\
7.0\end{array}$ & $\begin{array}{l}52.0 \\
62.0 \\
53.0 \\
58.0 \\
57.0\end{array}$ & \\
\hline $\begin{array}{l}2.2 .0 \\
2.2 .7 \\
2.2 .8 \\
2.2 .9 \\
2.2 .10\end{array}$ & $\begin{array}{r}142.5 \\
151.3 \\
13 \% .0 \\
171.5 \\
112.0\end{array}$ & $\begin{array}{l}151.3 \\
159.8 \\
171.5 \\
192.0 \\
201.5\end{array}$ & $\begin{array}{l}60.0 \\
60.0 \\
77.0 \\
60.0 \\
60.0\end{array}$ & $\begin{array}{l}330.0 \\
452.0 \\
530.5 \\
304.0 \\
121.5\end{array}$ & $\begin{array}{l}432.0 \\
330.5 \\
304.0 \\
321.5 \\
281.5\end{array}$ & $\begin{array}{l}30.0 \\
30.0 \\
100.0 \\
60.0 \\
30.0\end{array}$ & $\begin{array}{l}1<.2 \\
12.0 \\
12.0 \\
12.0 \\
12.0\end{array}$ & $\begin{array}{r}3.0 \\
.01 \\
9.0 \\
9.4 \\
9.3\end{array}$ & $\begin{array}{l}56.0 \\
36.0 \\
56.0 \\
50.0 \\
50.0\end{array}$ & \\
\hline $\begin{array}{l}2.2 .11 \\
2.2 .12 \\
2.2 .13 \\
2.2 .24 \\
2.2 .13\end{array}$ & $\begin{array}{l}201.3 \\
222.5 \\
24.1 .5 \\
26,3.9 \\
27 y .5\end{array}$ & $\begin{array}{l}222.5 \\
240.5 \\
260.9 \\
270.9 \\
313.3\end{array}$ & $\begin{array}{l}61.0 \\
60.0 \\
60.0 \\
60.0 \\
30.1\end{array}$ & $\begin{array}{r}261.0 \\
89.5 \\
190.3 \\
256.0 \\
271.5\end{array}$ & $\begin{array}{l}399.3 \\
190.8 \\
256.0 \\
271.5 \\
417.0\end{array}$ & $\begin{array}{l}30.0 \\
40.0 \\
0.0 .0 \\
60.00 \\
35.0\end{array}$ & $\begin{array}{l}12.0 \\
12.0 \\
12.0 \\
12.0 \\
12.0\end{array}$ & $\begin{array}{l}9.0 \\
3.0 \\
9.0 \\
9.0 \\
11.3\end{array}$ & $\begin{array}{l}56.0 \\
56.0 \\
51.0 \\
5 \% .0 \\
5 \% .0\end{array}$ & \\
\hline
\end{tabular}




\section{TABLE VII . (Contd.)}

BASELINE COUNTERCURRENT FLOW TEST GROUP 2.2

EXPERIMENTAL MEASURMENTS FOP TSSTS 2.2 .1 in 2.2 .47 - 0.37 -IN. DOHNCOMER GAP

LCHER PLENUM FLOH TFST INITIAL FIHAL TIME WEIGHT

2.2 .16
2.2 .11
2.2 .13

318.39371 .0

$\begin{array}{ll}316.3 & 371.0 \\ 371.0 & 436.0\end{array}$

$436.0 \quad 483.3$

$483.5 \quad 512.5$

2.2 .20

2.2 .21
2.2 .22
2.2 .22

$\begin{array}{ll}191.5 & 312.0 \\ 312.0 & 404.0\end{array}$

312.0

2.2 .24

$348.8 \quad 546.5$

-2.2 .24
2.2 .27
2.2 .25

13.3.3

3.07 .8
433.8

241.5
34400
387.5
347.3

489.0

330.4

2.2 .35

482.0
267.3

2.2 .31
2.2 .32

2.2 .3
2.2 .3

43...)

$157.5 \quad 181.5$

$\begin{array}{lll}2.2 .36 & 211.9 & 242.8 \\ 2.2 .37 & 242.3 & 275.8\end{array}$

$\begin{array}{lll}2.2 .37 & 242.3 & 275.8 \\ 2.2 .38 & 132.0 & 174.0\end{array}$

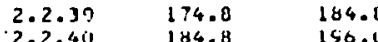

$\begin{array}{lll}2.2 .45 & 136.0 & 209.3 \\ 2.2 .62 & 202.3 & 223.3\end{array}$

$\begin{array}{lll}2.2 .162 & 200.3 & 223.3 \\ 2.2 .43 & 223.3 & 236.8\end{array}$

$2.2 .40 \quad 23.0 .3 . \quad 242.0$

$\begin{array}{lll}2.2 .403 & 132.5 & 137.8 \\ 2.2 .47 & 137.8 & 143.3\end{array}$

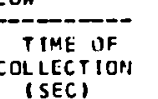

INITIAL
HEISHT

BYPASS FL U. FHAL TIME UUF LONER

$\begin{array}{ll}60.0 & 417.0 \\ 85.0 & 572.5\end{array}$

$60.0 \quad 113.0$

$35.0 \quad 731.5$

$60.0 \quad 329.0$

$45.0 \quad 489.5$

$35.0 \quad 501.5$

$65.0 \quad 444.0$

$\begin{array}{ll}60.0 & 497 . \% \\ 50.0 & 529.3\end{array}$

$\begin{array}{ll}35.0 & 616.5 \\ 35.0 & 461.5\end{array}$

$\begin{array}{ll}40.0 & 295.0 \\ 35.0 & 135.5 \\ 50.0 & 203.1\end{array}$

$\begin{array}{ll}50.0 & 293.1 \\ 50.0 & 427.0\end{array}$

$\begin{array}{ll}60.5 & 130.0 \\ 60.5 & 809.5\end{array}$

$\begin{array}{ll}60.0 & 809.5 \\ 15.0 & 90.5 \\ 55.0 & 101.0\end{array}$

$\begin{array}{ll}55.0 & 101.0 \\ 63.0 & 264.1)\end{array}$

$\begin{array}{ll}65.13 & 336.12 \\ 65.0 & 464.5\end{array}$

$\begin{array}{ll}60.0 & 499.0 \\ 60.0 & 510.0\end{array}$

$\begin{array}{ll}60.0 & 22.5 \\ 70.0 & 110.5\end{array}$

60.0
70.0

$\begin{array}{lll}512.5 & 45.0 & 12.0 \\ 713.0 & 40.0 & 12.0 \\ 741.5 & 40.0 & 12.0\end{array}$

$792.0 \quad 105.0 \quad 120$

489.5
561.5

540.0

in 97.5

629.0
616.5

043.3

575.0
421.5

034.0

0.34 .0
203.0

225.0
503.0

35.3

35.0
35.0
60.0

60.0
55.13

45.0

70.0
60.0
70.0

7.03
3.0 .0
30.0

30.0
30.1
00.5
30.0
30.0

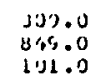

304.0

464.5
494.0

510.0
521.3
555.3



174.5
2.37 .0

12.1
12.0
12.0

12.0
12.0
12.0
12.0

[2.:

12.0
12.5
$1<.5$

12.3

12.05
12.0
12.0
12.0

15.0

30.0
30.0
30.0
30.0

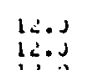

120.0
$12 . j$
$12 . j$

3..2 1:.0

250
30.0
30.0
20.0

12.0
12.0
12.0
12.0
12.0

3.ग.

12.0
12.0

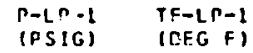

$\begin{array}{ll}9.0 & 55.0 \\ 9.3 & 55.0\end{array}$

54.0
7.0 $\quad 5 \% .0$

55.0

9.0
7.0 $\quad 55.0$

$9.0 \quad 54.0$

$9.3 \quad 55.0$

?.) 55.0

$\begin{array}{ll}9.0 & 56.0 \\ 4.0 & 56.0\end{array}$

$\begin{array}{ll}9.3 & 55.0 \\ 9.0 & 50.0\end{array}$

$\begin{array}{ll}9.0 & 55.0 \\ 9.0 & 55.0 \\ 9.0 & 56.0\end{array}$

3.1 56.0

$\begin{array}{ll}7.7 & 0.0 \\ 7.0 & 50.0 \\ 1.0 & 56.0\end{array}$

.') 54.0

$\because 254.0$

$\begin{array}{ll}9.0 & 50.0 \\ 9.0 & 54.0 \\ 9.0 & 54.0 \\ 9.0 & 54.0\end{array}$

5.0
7.0
7.5 
TABLE VIII

BASELINE COUNTERCURRENT FLOW TEST GROUP 2.3

TRANSPARENT VESSEL TEST DATA FUR TESTS 2.3 .1 TO $2.3 .36-2$ O.37-IN. DONNCUMER GAP

SUMMARY UF CALCULATED RESULTS$$
\begin{aligned}
& \text { TEST } \\
& \text { NO }
\end{aligned}
$$

0.0212

0.2216

12.28
42.28
4.208

$2.3 .2 \quad 0.0217$

$\begin{array}{ll}2.3 .3 & 0.0666 \\ 2.3 .4 & 0.0045\end{array}$

0.2216

$\begin{array}{ll}0.2216 & 42.28 \\ 0.2210 & 42.28\end{array}$

2.3 .50 .0639

J.2216

2.3. $0 \quad 0.1009$

2.3. 8 3.1072

2.3 .590 .1015

$0.2216 \quad 42.28$

0.2211

0.2211

42.28

0.1069

$\begin{array}{ll}2.3 .11 & 0.1514 \\ 2.3 .12 & 0.1504\end{array}$

$2.3 .13 \quad 0.1509$

$\begin{array}{ll}2.3 .14 & 0.1508 \\ 2.3 .15 & 0.1955\end{array}$

J. 2199

0.2199

0.2203

0.2203

0.1955

3.2203

0.0
0.0

0.0
0.0
0.0
0.0

0.0

0.0

0.0

0.0
0.0
0.0

42.28
42.28

$2.3 .16 \quad 0.1939$

2.3 .170 .1932

0.2207

0.2168
0.2168

2.3 .190 .1930

0.2168

$2.3 .20 \quad 0.1739$

$J .2184$
$J .2193$

$\begin{array}{ll}2.3 .21 & 0.1737 \\ 2.3 .22 & 0.1742\end{array}$

2.3 .23 0.1731

$\begin{array}{ll}2.3 .24 & 0.1750 \\ 2.3 .25 & 0.1301\end{array}$

0.2197

.02147
.3 .2197

.2197
0.2197

0.2197
0.2197

$2.3 .26 \quad 0.1289$

2.3 .27 J. 1286

2.3 .280 .1200

ง. 2201

J.2201

0.2193
0.2210

42.28

42.28

42.28
42.28

42.28

2.3 .30 J.0857

J.221a

$2.3 .31 \quad 0.0853$

$2.3 .32 \quad 0.1063$

2.3 .33

2.3 .35

0.0433

0.0431

0.2213

0.2218

0.2213

0.2213

42.28

42.24
42.24

42.24
42.24

c.o

0.0
0.0
0.0

c.u

0.0

0.0
0.0
0.0

0.0
0.0
0.0
0.0

42.24

42.24

42.24
42.24

42.24
42.24

12.24

42.24

42.24
42.24
42.24

42.24
42.24

42.24

42.24
42.24
2.34

12.24
+2.24

12.24
42.24

$2.3 .36 \quad 0.0432$

J. 2210

42.24

$\begin{array}{ll}3.908 & 0.974 \\ 4.133 & 1.012 \\ 1.994 & 1.031 \\ 2.027 & 1.025 \\ 2.028 & 1.021 \\ 2.071 & 1.02 \% \\ 0.004 & 1.003 \\ 0.867 & 0.997 \\ 0.944 & 1.005 \\ 0.962 & 1.037\end{array}$

0.392

0.432

0.427
0.208

0.21 .4

0.107

0.188
0.211
0.273

0.265
0.262

0.268

0.295

0.565

0.000

0.073
0.646

$0.6+6$

1.339
1.350

1.427

1.427
1.355
2.775

1.4275
2.775
2.789

2.789
1.050

2.378

0.991
1.011

$0.97 \%$

1. 022

1.020
1.004

0.950
1.004

1.000

1.007

1.075
0.975
1.015
1.035

1.015
1.030

$1.03 i 1$
$j .978$

$1.039^{\circ}$

$1.01 \%$

1.01 's

1.0115
0.9 .13

3.9 .19

1.012

1.031
1.026

1.026
1.020

1.055
0.0
$J-j \mid k$ IFT/SEC) (FT/SEC)

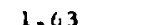

$1.66 \quad 1.063$

$4.94 \quad 0.551$

0.552

8.19

8.19

$8.26 \quad 0.219$

$\begin{array}{ll}8.23 & 0.236 \\ 8.26 & 0.257\end{array}$

$0.21 \quad 0.262$

11.69

11.01

11.02
11.62

11.62

14.91

15.13

15.12
15.00

$\$ 3.46$

13.43

13.43
13.46
13.37

13.53

10.05

9.94

9.12

9.96
6.53

$6.5 ?$
6.50

6.57

6.57
6.03
3.31

3.31

3.30

3.32

0.107

0.117
0.120

0.120
0.116

0.057

0.053

0.045

0.059

0.074

0.072

0.071

$0.0 ? 3$

0.030
0.154

3.163

0.133

0.176

0.367

0.393

0.367

0.313

0.331

0.810
$\left(J \sigma^{*}\right) * * 1 / 2 \quad(J L *) * * 1 / 2$

$\begin{array}{ll}0.2632 & 0.8715 \\ 0.2661 & 0.8962 \\ 0.4660 & 0.6224 \\ 0.4585 & 0.6276 \\ 0.4566 & 0.6277 \\ 0.5905 & 0.6344 \\ 0.5925 & 0.3953 \\ 0.5916 & 0.4104 \\ 0.5925 & 0.4284 \\ 0.5908 & 0.4325 \\ 0.7040 & 0.2761 \\ 0.7016 & 0.2897 \\ 0.7022 & 0.2927 \\ 0.7022 & 0.2881 \\ 0.7996 & 0.2009 \\ 0.7959 & 0.2037 \\ 0.7942 & 0.1800 \\ 0.7978 & 0.1914 \\ 0.7963 & 0.2052 \\ 0.7550 & 0.2302 \\ 0.7543 & 0.2271 \\ 0.7554 & 0.2254 \\ 0.7529 & 0.2283 \\ 0.7571 & 0.2396 \\ 0.6529 & 0.3313 \\ 0.6494 & 0.3415 \\ 0.6497 & 0.3616 \\ 0.6494 & 0.3543 \\ 0.5293 & 0.5195 \\ 0.5295 & 0.5122 \\ 0.5298 & 0.5266 \\ 0.5303 & 0.5131 \\ 0.3757 & 0.7343 \\ 0.3757 & 0.7621 \\ 0.3747 & 0.7706 \\ 0.3755 & 0.7607 \\ 0.375 & \end{array}$


TABLE VIII (Contd.)

BASELINE COUNTERCURRENT FLOW TEST GROUP 2.3

EXPERIHENTAL MEASURMENTS FOR TESTS 2.3.1 TO 2.3.36 - 0.37 -IN. DOWNCOMER GAP

\begin{tabular}{|c|c|c|c|c|c|c|c|c|c|c|}
\hline $\begin{array}{c}\text { TEST } \\
\text { NO }\end{array}$ & $\begin{array}{c}R M-(H-1 \\
(G P M)\end{array}$ & $\begin{array}{c}R M-C H-2 \\
(G P M)\end{array}$ & $\begin{array}{c}F T-C H-1 \\
(G P, Y)\end{array}$ & $\begin{array}{c}F T-(W-2 \\
(G P M)\end{array}$ & $\begin{array}{c}R M-H G-1 \\
(E F M)\end{array}$ & $\begin{array}{c}\text { RM-HG-2 } \\
\text { (CFM) }\end{array}$ & $\begin{array}{c}R M-H G-3 \\
(C F M)\end{array}$ & $\begin{array}{c}\text { RM- }-\mathrm{HG}-4 \\
(C F M)\end{array}$ & $\begin{array}{l}=T-H G-1 \\
\text { (CFM) }\end{array}$ & $\begin{array}{l}F T-H G-2 \\
\text { [CFM] }\end{array}$ \\
\hline $\begin{array}{ll}2.3 .3 & 1 \\
2.3 .3 & 2 \\
2.3 .3 & 3 \\
2.3 . & 4 \\
2.3 . & 5\end{array}$ & $\begin{array}{l}30.0 \\
22.0 \\
30.0 \\
20.0 \\
20.0\end{array}$ & $\begin{array}{r}10.0 \\
10.0 \\
10.0 \\
10.0 \\
0.0\end{array}$ & $\begin{array}{l}0.0 \\
0.0 \\
0.0 \\
0.0 \\
0.0\end{array}$ & $\begin{array}{l}0.0 \\
0.0 \\
0.0 \\
0.0 \\
0.0\end{array}$ & $\begin{array}{c}0.0 \\
0.0 \\
30.00 \\
30.00 \\
30.00\end{array}$ & $\begin{array}{l}0.0 \\
0.0 \\
0.0 \\
0.0 \\
0.0\end{array}$ & $\begin{array}{l}0.0 \\
0.0 \\
0.0 \\
0.0 \\
0.0\end{array}$ & $\begin{array}{c}10.00 \\
10.00 \\
0.0 \\
0.0 \\
0.0\end{array}$ & $\begin{array}{l}0.0 \\
0.0 \\
0.0 \\
0.0 \\
0.0\end{array}$ & $\begin{array}{l}0.0 \\
0.0 \\
0.0 \\
0.0 \\
0.0\end{array}$ \\
\hline $\begin{array}{l}2.3 .6 \\
2.3 .7 \\
2.3 .8 \\
2.3 .9 \\
2.3 .10\end{array}$ & $\begin{array}{r}16.0 \\
30.0 \\
20.0 \\
20.0 \\
0.0\end{array}$ & $\begin{array}{r}0.0 \\
10.0 \\
10.0 \\
0.0 \\
10.0\end{array}$ & $\begin{array}{l}0.0 \\
0.0 \\
0.0 \\
0.0 \\
0.0\end{array}$ & $\begin{array}{l}0.0 \\
0.0 \\
0.0 \\
0.0 \\
0.0\end{array}$ & $\begin{array}{l}50.00 \\
50.00 \\
50.00 \\
50.00 \\
50.00\end{array}$ & $\begin{array}{l}0.0 \\
0.0 \\
0.0 \\
0.0 \\
0.0\end{array}$ & $\begin{array}{l}0.0 \\
0.0 \\
0.0 \\
0.0 \\
0.0\end{array}$ & $\begin{array}{l}0.0 \\
0.0 \\
0.0 \\
0.0 \\
0.0\end{array}$ & $\begin{array}{l}0.0 \\
0.0 \\
0.0 \\
0.0 \\
0.0\end{array}$ & $\begin{array}{l}0.0 \\
0.0 \\
0.0 \\
0.0 \\
0.0\end{array}$ \\
\hline $\begin{array}{l}2.3 .11 \\
2.3 .12 \\
2.3 .13 \\
2.3 .14 \\
2.3 .15\end{array}$ & $\begin{array}{r}30.0 \\
20.0 \\
20.0 \\
0.0 \\
310.0\end{array}$ & $\begin{array}{r}10.0 \\
10.0 \\
0.0 \\
6.0 \\
10.0\end{array}$ & $\begin{array}{l}0.0 \\
0.0 \\
0.0 \\
0.0 \\
0.0\end{array}$ & $\begin{array}{l}0.0 \\
0.0 \\
0.0 \\
0.0 \\
0.0\end{array}$ & $\begin{array}{l}70.00 \\
70.00 \\
70.00 \\
70.00 \\
50.00\end{array}$ & $\begin{array}{c}0.0 \\
0.0 \\
0.0 \\
0.0 \\
40.00\end{array}$ & $\begin{array}{l}0.0 \\
0.0 \\
0.0 \\
0.0 \\
0.0\end{array}$ & $\begin{array}{l}0.0 \\
0.0 \\
0.0 \\
0.0 \\
0.0\end{array}$ & $\begin{array}{r}0.0 \\
0.0 \\
0.0 \\
0.0 \\
0.0\end{array}$ & $\begin{array}{l}0.0 \\
0.0 \\
0.0 \\
0.0 \\
0.0\end{array}$ \\
\hline $\begin{array}{l}2.3 .16 \\
2.3 .17 \\
2.3 .19 \\
2.3 .19 \\
2.3 .20\end{array}$ & $\begin{array}{r}20.0 \\
2.0 .0 \\
0.0 \\
0.0 \\
3.0 .0\end{array}$ & $\begin{array}{r}10.0 \\
0.0 \\
10.0 \\
4.0 \\
10.0\end{array}$ & $\begin{array}{l}0.0 \\
0.0 \\
0.0 \\
0.0 \\
0.0\end{array}$ & $\begin{array}{l}0.0 \\
0.0 \\
u .0 \\
0.0 \\
0.0\end{array}$ & $\begin{array}{l}50.00 \\
50.00 \\
50.00 \\
50.00 \\
80.00\end{array}$ & $\begin{array}{c}40.00 \\
40.00 \\
40.00 \\
40.00 \\
j .0\end{array}$ & $\begin{array}{l}0.0 \\
0.0 \\
0 . j \\
0.0 \\
0.0\end{array}$ & $\begin{array}{l}0.0 \\
0.0 \\
0.0 \\
0.0 \\
0.0\end{array}$ & $\begin{array}{l}0.0 \\
0.0 \\
0.0 \\
0.0 \\
0.0\end{array}$ & $\begin{array}{l}0.0 \\
0.0 \\
0.0 \\
0.0 \\
0.0\end{array}$ \\
\hline $\begin{array}{l}2.3 .21 \\
2.3 .22 \\
2.3 .23 \\
2.3 .24 \\
2.3 .25\end{array}$ & $\begin{array}{r}20.0 \\
20.0 \\
0.0 \\
0.0 \\
30.0\end{array}$ & $\begin{array}{r}10.0 \\
0.0 \\
10.0 \\
4.0 \\
10.0\end{array}$ & $\begin{array}{l}0.0 \\
0.0 \\
0.0 \\
0.0 \\
0.0\end{array}$ & $\begin{array}{l}0.0 \\
0.0 \\
0.0 \\
0.0 \\
0.0\end{array}$ & $\begin{array}{c}80.00 \\
80.00 \\
80.00 \\
80.00 \\
0.0\end{array}$ & $\begin{array}{c}0.0 \\
0.0 \\
0.0 \\
0.0 \\
0.0 .00\end{array}$ & $\begin{array}{l}0.0 \\
0.0 \\
0.0 \\
u .0 \\
0.0\end{array}$ & $\begin{array}{l}0.0 \\
0.0 \\
0.0 \\
0.0 \\
0.0\end{array}$ & $\begin{array}{l}0.0 \\
0.0 \\
0.0 \\
0.0 \\
0.0\end{array}$ & $\begin{array}{l}0.0 \\
0.0 \\
0.0 \\
0.0 \\
0.0\end{array}$ \\
\hline $\begin{array}{l}2.3 .26 \\
2.3 .27 \\
2.3 .28 \\
2.3 .29 \\
2.3 .30\end{array}$ & $\begin{array}{r}20.0 \\
2 J .0 \\
0.0 \\
30.0 \\
20.0\end{array}$ & $\begin{array}{r}10.0 \\
0.0 \\
6.0 \\
10.0 \\
10.0\end{array}$ & $\begin{array}{l}0.0 \\
0.0 \\
0.0 \\
0.0 \\
0.0\end{array}$ & $\begin{array}{l}0.0 \\
0.0 \\
0.0 \\
0.0 \\
0.0\end{array}$ & $\begin{array}{l}0.0 \\
0.0 \\
0.0 \\
0.0 \\
0.0\end{array}$ & $\begin{array}{l}60.00 \\
60.00 \\
60.00 \\
42.00 \\
40.00\end{array}$ & $\begin{array}{l}0.0 \\
0.0 \\
0.0 \\
0.0 \\
0.0\end{array}$ & $\begin{array}{l}0.0 \\
0.0 \\
0.0 \\
0.0 \\
0.0\end{array}$ & $\begin{array}{l}0.0 \\
0.0 \\
0.0 \\
0.0 \\
0.0\end{array}$ & $\begin{array}{l}0.0 \\
0.0 \\
0.0 \\
u .0 \\
0.0\end{array}$ \\
\hline $\begin{array}{l}2.3 .31 \\
2.3 .32 \\
2.3 .33 \\
2.3 .34 \\
2.3 .35\end{array}$ & $\begin{array}{l}20.0 \\
12.0 \\
30.0 \\
20.0 \\
20.0\end{array}$ & $\begin{array}{r}0.0 \\
0.0 \\
10.0 \\
10.0 \\
10.0\end{array}$ & $\begin{array}{l}c .0 \\
0.0 \\
0.0 \\
0.0 \\
0.0\end{array}$ & $\begin{array}{l}0.0 \\
0.0 \\
0.0 \\
0.0 \\
0.0\end{array}$ & $\begin{array}{l}0.0 \\
0.0 \\
0.0 \\
0.0 \\
0.0\end{array}$ & $\begin{array}{c}40.00 \\
40.00 \\
0.0 \\
0.0 \\
0.0\end{array}$ & $\begin{array}{c}0.0 \\
0.0 \\
20.00 \\
20.00 \\
20.00\end{array}$ & $\begin{array}{l}0.0 \\
0.0 \\
0.0 \\
0.1 \\
0.0\end{array}$ & $\begin{array}{l}0.0 \\
0.0 \\
0.0 \\
0.0 \\
0.0\end{array}$ & $\begin{array}{l}0.0 \\
0.0 \\
0.0 \\
0.0 \\
0.0\end{array}$ \\
\hline 2.3 .36 & 24.0 & 0.0 & 0.0 & 0.0 & 0.0 & 0.0 & 20.00 & 0.0 & 0.0 & 0.0 \\
\hline
\end{tabular}


TABLE VIII (Contd.)

BASELINE COUNTERCURRENT FLOW TEST GROUP 2.3

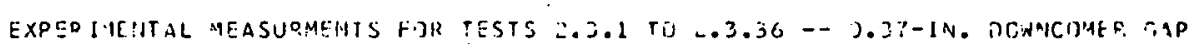

\begin{tabular}{|c|c|c|c|c|c|c|c|c|c|c|}
\hline סוס & $\begin{array}{l}P-C H-1 \\
1051 B)\end{array}$ & $\begin{array}{l}P-\mathrm{CW}-2 \\
(P S I G)\end{array}$ & $\begin{array}{l}P-H G-1 \\
(P S I G)\end{array}$ & $\begin{array}{l}\text { P-Hi;-2 } \\
\text { (OSI;i) }\end{array}$ & $\begin{array}{l}p-11(;-3 \\
(r \leq 1 \leq)\end{array}$ & 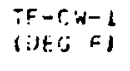 & $\begin{array}{l}T F-1 C-1 \\
(0: 0 ;-1)\end{array}$ & $\begin{array}{l}T E-: H r,-2 \\
\{D E F F\}\end{array}$ & $\begin{array}{l}\text { IF-145-3 } \\
\text { (IOEG } F)\end{array}$ & $\begin{array}{l}\text { P-BAPח } \\
\text { POSIAI }\end{array}$ \\
\hline $\begin{array}{l}2.3 \cdot 1 \\
2.3 \cdot \frac{1}{2} \\
2.3 \cdot 3 \\
2.3 \cdot 3 \\
2.3 \cdot 5\end{array}$ & $\begin{array}{l}J .0 \\
.0 . \\
0.0 \\
.0 \\
0.0\end{array}$ & $\begin{array}{l}32.5 \\
32.0 \\
33.0 \\
31.5 \\
30.5\end{array}$ & $\begin{array}{l}30.1 \\
32.0 \\
34.0 \\
11.5 \\
30.7\end{array}$ & $\begin{array}{l}1.0 \\
j .0 \\
0.0 \\
0.0 \\
0.0\end{array}$ & $\begin{array}{l}1.0 \\
u .0 \\
u .0 \\
0.0 \\
0.0\end{array}$ & $\begin{array}{l}59.03 \\
5.1 .0 \\
55.0 \\
54.0 \\
54.0\end{array}$ & $\begin{array}{l}67.0 \\
67.0 \\
67.0 \\
72.0 \\
72.0\end{array}$ & $\begin{array}{l}0.0 \\
.0 .0 \\
0.0 \\
9.0 \\
0.0\end{array}$ & $\begin{array}{l}0.0 \\
0.0 \\
0.0 \\
0.0 \\
0.0\end{array}$ & $\begin{array}{l}12.3 \\
12.3 \\
12.3 \\
12.3 \\
12.3\end{array}$ \\
\hline $\begin{array}{l}2.3 .0 \\
2.3 .7 \\
2.3 .0 \\
2.3 .9 \\
2.3 .13\end{array}$ & $\begin{array}{l}J . u \\
. . J \\
0.0 \\
\jmath . J \\
\jmath . J\end{array}$ & $\begin{array}{r}30.5 \\
31.5 \\
31.5 \\
31.0 \\
30.2\end{array}$ & $\begin{array}{r}30.5 \\
21.0 \\
30.7 \\
31.9 \\
30.5\end{array}$ & $\begin{array}{l}0.0 \\
0.0 \\
0.0 \\
0.0 \\
0.0\end{array}$ & $\begin{array}{l}0.0 \\
0.0 \\
0.0 \\
0.0 \\
0.0\end{array}$ & $\begin{array}{l}54.03 \\
34.0 \\
54.03 \\
55.0 \\
55.0\end{array}$ & $\begin{array}{l}72.5 \\
72.0 \\
72.0 \\
72.0 \\
72.0\end{array}$ & $\begin{array}{l}j .0 \\
9.0 \\
0.0 \\
.00 \\
.0 .0\end{array}$ & $\begin{array}{l}0.0 \\
2.0 \\
0.0 \\
0.0 \\
0.0\end{array}$ & $\begin{array}{l}12.3 \\
12.3 \\
12.3 \\
12.3 \\
12.3\end{array}$ \\
\hline $\begin{array}{l}2.3 .11 \\
2.3 .12 \\
2.3 .13 \\
2.3 .14 \\
2.3 .15\end{array}$ & $\begin{array}{l}3.0 \\
0.1 \\
3.0 \\
3.0 \\
0.0\end{array}$ & $\begin{array}{l}31.5 \\
30.7 \\
30.7 \\
50.5 \\
31.7\end{array}$ & $\begin{array}{l}31.1 \\
30.5 \\
30.7 \\
00.5 \\
31.7\end{array}$ & $\begin{array}{l}0.0 \\
0.0 \\
0.0 \\
0.0 \\
0.0\end{array}$ & $\begin{array}{l}j .0 \\
0.0 \\
0.0 \\
0.0 \\
.0 .3\end{array}$ & $\begin{array}{l}53.0 \\
55.0 \\
55.0 \\
35.0 \\
50.0\end{array}$ & $\begin{array}{l}74.0 \\
74.0 \\
74.0 \\
71.0 \\
71.0\end{array}$ & $\begin{array}{l}0.0 \\
0.2 \\
3.0 \\
0.0 \\
1.0\end{array}$ & $\begin{array}{l}0.0 \\
0.0 \\
0.0 \\
0.0 \\
0.0\end{array}$ & $\begin{array}{l}12.3 \\
12.3 \\
12.3 \\
12.3 \\
12.3\end{array}$ \\
\hline $\begin{array}{l}2.3 .16 \\
2.3 .117 \\
2.3 .13 \\
2.3 .19 \\
2.3 .20\end{array}$ & $\begin{array}{l}\jmath . j \\
3.0 \\
j .0 \\
j .0 \\
.00\end{array}$ & $\begin{array}{l}31.0 \\
30.7 \\
30.7 \\
31.0 \\
30.5\end{array}$ & $\begin{array}{l}21.0 \\
31.5 \\
30.7 \\
30.7 \\
30.5\end{array}$ & $\begin{array}{l}0.0 \\
0.0 \\
0.0 \\
0.0 \\
0.0\end{array}$ & $\begin{array}{l}0.0 \\
0.0 \\
0.0 \\
0.0 \\
0.0\end{array}$ & $\begin{array}{l}54.0 \\
59.0 \\
51.0 \\
59.1 \\
50.1\end{array}$ & $\begin{array}{l}71.0 \\
71.0 \\
72.0 \\
72.0 \\
05.0\end{array}$ & $\begin{array}{l}3.0 \\
0.0 \\
3.0 \\
3.0 \\
3.0\end{array}$ & $\begin{array}{l}1.0 \\
0.0 \\
0.0 \\
0.0 \\
0.0\end{array}$ & $\begin{array}{l}12.3 \\
12.2 \\
12.2 \\
12.2 \\
12.2\end{array}$ \\
\hline $\begin{array}{l}2.3 .01 \\
2.3 .22 \\
2.3 .23 \\
2.3 .24 \\
2.3 .25\end{array}$ & $\begin{array}{l}3.0 \\
3.0 \\
3.0 \\
3.0 \\
.0\end{array}$ & $\begin{array}{l}30.7 \\
31.0 \\
30.7 \\
31.2 \\
31.5\end{array}$ & $\begin{array}{l}30.5 \\
31.0 \\
30.5 \\
31.5 \\
31.5\end{array}$ & $\begin{array}{l}0.0 \\
0.0 \\
0.0 \\
0.0 \\
0.0\end{array}$ & $\begin{array}{l}0.0 \\
0.0 \\
0.0 \\
0.0 \\
0.0\end{array}$ & $\begin{array}{l}50.0 \\
56.0 \\
50.0 \\
53.0 \\
50.0\end{array}$ & $\begin{array}{l}66.0 \\
69.0 \\
7 \mathrm{~J} .0 \\
7 \mathrm{~J} . \mathrm{J} \\
72.0\end{array}$ & $\begin{array}{l}0.0 \\
0.0 \\
0.0 \\
0.0 \\
0.0\end{array}$ & $\begin{array}{l}0.0 \\
0.0 \\
0.0 \\
0.0 \\
0.0\end{array}$ & $\begin{array}{l}12.2 \\
12 . ? \\
12.2 \\
12.2 \\
12.2\end{array}$ \\
\hline $\begin{array}{l}2.3 .24 \\
2.3 .27 \\
2.3 .24 \\
2.3 .29 \\
2.3 .30\end{array}$ & $\begin{array}{l}. .0 \\
0.0 \\
\text { J.u } \\
j .0 \\
0.0\end{array}$ & $\begin{array}{l}0.5 \\
30.7 \\
30.7 \\
31.0 \\
30.7\end{array}$ & $\begin{array}{l}30.5 \\
30.5 \\
30.5 \\
31.0 \\
30.5\end{array}$ & $\begin{array}{l}0.0 \\
0.0 \\
0.0 \\
0.0 \\
0.0\end{array}$ & $\begin{array}{l}0.0 \\
0.0 \\
0.0 \\
0.0 \\
0.0\end{array}$ & $\begin{array}{l}125.0 \\
56.0 \\
00.0 \\
52.1 \\
32.0\end{array}$ & $\begin{array}{l}7.0 .0 \\
72.0 \\
72.1 \\
72.0 \\
67.0\end{array}$ & $\begin{array}{l}0.0 \\
3.0 \\
.0 .0 \\
3.0 \\
0.0\end{array}$ & $\begin{array}{l}0.0 \\
0.0 \\
0.0 \\
0.0 \\
0.0\end{array}$ & $\begin{array}{l}12.2 \\
12.2 \\
12.2 \\
12.2 \\
12.2\end{array}$ \\
\hline $\begin{array}{l}2.3 .31 \\
2.3 .32 \\
2.3 .33 \\
2.3 .34 \\
2.3 .31\end{array}$ & $\begin{array}{l}0.0 \\
0.0 \\
.00 \\
.00 \\
0.0\end{array}$ & $\begin{array}{l}30.5 \\
30.7 \\
31.5 \\
31.5 \\
31.7\end{array}$ & $\begin{array}{l}30.5 \\
31.0 \\
31.8 \\
31.7 \\
31.7\end{array}$ & $\begin{array}{l}0 . u \\
0.0 \\
0.0 \\
0.0 . \\
u .0\end{array}$ & $\begin{array}{l}0.0 \\
0.0 \\
0.0 \\
0.0 \\
0.0\end{array}$ & $\begin{array}{l}52.1 \\
5.03 \\
53.0 \\
53.0 \\
53.0\end{array}$ & $\begin{array}{l}\text { 6e.j } \\
60.0 \\
00.0 \\
66.0 \\
12.0\end{array}$ & $\begin{array}{l}0.0 \\
0.3 \\
0.0 \\
0.0 \\
.3 .3\end{array}$ & $\begin{array}{l}0.0 \\
0.0 \\
1.0 \\
0.0 \\
0.0\end{array}$ & $\begin{array}{l}12.2 \\
12.2 \\
12.2 \\
12.2 \\
12.2\end{array}$ \\
\hline 2.3 .30 & J.J & 31.7 & 31.7 & 0 & 0.0 & 53.3 & 70.0 & 0.9 & 0.0 & 12.2 \\
\hline
\end{tabular}


TABLE VIII (Contd.)

BASELINE COUNTERCURRENT FLOW TEST GROUP 2.3

EXPERIMENTAL MEASURMENTS FDR TESTS 2.3 .1 TO 2.3.36-- U.37-IN. DUHNCOMER GAP

\begin{tabular}{|c|c|c|c|c|c|c|c|c|}
\hline \multicolumn{3}{|c|}{ LOWEK PLENUM FLOH } & \multicolumn{3}{|c|}{ BYPASS FLOW } & \multirow{2}{*}{$\begin{array}{l}\text { LOWEO } \\
\text { PLENIJM } \\
\text { LEVEI. } \\
\text { (IN) }\end{array}$} & \multirow[b]{2}{*}{$\begin{array}{l}P-t P-1 \\
(n S I S)\end{array}$} & \multirow[b]{2}{*}{$\begin{array}{l}\text { IE-LP-1 } \\
(D F F, F)\end{array}$} \\
\hline $\begin{array}{l}\text { IH!I TIAL } \\
\text { WEI:HTT } \\
\text { (LE) }\end{array}$ & $\begin{array}{l}\text { FINAL } \\
\text { HEIGHIT } \\
\text { (LB) }\end{array}$ & $\begin{array}{l}\text { TIME DF } \\
\text { COLLECT ICN } \\
\text { (SEC) }\end{array}$ & $\begin{array}{l}\text { INITIAL } \\
\text { WEIGHT } \\
\text { (LU) }\end{array}$ & $\begin{array}{l}\text { FINAL } \\
\text { HEIGHT } \\
\text { (LB) }\end{array}$ & $\begin{array}{l}\text { TIME JF } \\
\text { COLLECTION } \\
\text { ISECI }\end{array}$ & & & \\
\hline $\begin{array}{l}143.3 \\
20.5 .5 \\
384.5 \\
132.5 \\
263.5\end{array}$ & $\begin{array}{l}260.5 \\
384.5 \\
464.3 \\
263.8 \\
353.0\end{array}$ & $\begin{array}{l}30.0 \\
30.0 \\
40.0 \\
65.0 \\
45.0\end{array}$ & $\begin{array}{l}237.0 \\
327.5 \\
353.5 \\
+14.0 \\
504.0\end{array}$ & $\begin{array}{l}327.5 \\
353.5 \\
521.8 \\
504.5 \\
540.5\end{array}$ & $\begin{array}{l}60.0 \\
7.0 .0 \\
45.0 \\
40.0 \\
45.0\end{array}$ & $\begin{array}{l}12.0 \\
12.0 \\
12.3 \\
12.0 \\
12.0\end{array}$ & $\begin{array}{l}30.0 \\
30.0 \\
3.0 .0 \\
30.0 \\
30.0\end{array}$ & $\begin{array}{l}55.0 \\
55.0 \\
55.0 \\
55.0 \\
55.0\end{array}$ \\
\hline $\begin{array}{l}155.0 \\
350.0 \\
393.3 \\
437.3 \\
473.8\end{array}$ & $\begin{array}{l}479.3 \\
398.3 \\
437.3 \\
479.8 \\
537.1\end{array}$ & $\begin{array}{l}60.0 \\
60.0 \\
45.3 \\
45.0 \\
60.0\end{array}$ & $\begin{array}{l}540.5 \\
553.0 \\
744.0 \\
874.0 \\
943.0\end{array}$ & $\begin{array}{l}553.0 \\
744.0 \\
874.0 \\
348.0 \\
972.0\end{array}$ & $\begin{array}{l}60.0 \\
40.0 \\
40.0 \\
40.0 \\
30.0\end{array}$ & $\begin{array}{l}12.3 \\
12.0 \\
12.0 \\
12.0 \\
12.0\end{array}$ & $\begin{array}{l}3.0 .0 \\
3.1 .0 \\
30.0 \\
33.0 \\
30.0\end{array}$ & $\begin{array}{l}55.0 \\
56.0 \\
56.0 \\
56.0 \\
56.0\end{array}$ \\
\hline $\begin{array}{l}132.5 \\
153.0 \\
191.8 \\
206.5 \\
229.5\end{array}$ & $\begin{array}{l}153.0 \\
181.3 \\
200.0 \\
223.5 \\
243.0\end{array}$ & $\begin{array}{l}65.0 \\
55.0 \\
55.0 \\
35.0 \\
65.0\end{array}$ & $\begin{array}{r}90.0 \\
243.5 \\
310.5 \\
444.5 \\
464.5\end{array}$ & $\begin{array}{l}243.5 \\
376.0 \\
444.0 \\
469.5 \\
577.0\end{array}$ & $\begin{array}{l}3 J .0 \\
315.0 \\
3.0 .1 \\
60.0 \\
2 J .0\end{array}$ & $\begin{array}{l}12.0 \\
12.0 \\
12.0 \\
22.0 \\
12.0\end{array}$ & $\begin{array}{l}30.0 \\
30.0 \\
30.0 \\
30.0 \\
30.0\end{array}$ & $\begin{array}{l}59.0 \\
59.0 \\
59.0 \\
53.0 \\
53.0\end{array}$ \\
\hline $\begin{array}{l}243.13 \\
132.5 \\
142.5 \\
15 \% .3 \\
161.3\end{array}$ & $\begin{array}{l}25.4 .3 \\
142.5 \\
154.3 \\
107.3 \\
102.3\end{array}$ & $\begin{array}{l}55.0 \\
60.0 \\
65.0 \\
60.0 \\
55.0\end{array}$ & $\begin{array}{r}444.3 \\
87.3 \\
106.3 \\
21.32 .0 \\
212.3\end{array}$ & $\begin{array}{l}545.5 \\
160.0 \\
202.0 \\
212.3 \\
344.5\end{array}$ & $\begin{array}{l}25.0 \\
30.0 \\
30.3 \\
310.1 \\
25.0\end{array}$ & $\begin{array}{l}12.0 \\
12.0 \\
12.0 \\
12.0 \\
12.0\end{array}$ & $\begin{array}{l}3.0 .0 \\
30.0 \\
30.0 \\
30.0 \\
3.00\end{array}$ & $\begin{array}{l}57.0 \\
66.0 \\
66.0 \\
62.0 \\
60.0\end{array}$ \\
\hline $\begin{array}{l}23 \% .3 \\
197.0 \\
216.5 \\
231.3 \\
23 \% .0\end{array}$ & $\begin{array}{l}199.5 \\
210.5 \\
231.3 \\
247.5 \\
237.3\end{array}$ & $\begin{array}{l}05.0 \\
65.0 \\
55.0 \\
55.0 \\
50.0\end{array}$ & $\begin{array}{l}344.3 \\
602.3 \\
536.5 \\
376.5 \\
202.3\end{array}$ & $\begin{array}{l}462.5 \\
530.0 \\
576.0 \\
511.5 \\
3152.0\end{array}$ & $\begin{array}{l}30.10 \\
30.0 \\
30 . .1 \\
23.03 \\
30.0\end{array}$ & $\begin{array}{l}12.01 \\
12.0 \\
12.0 \\
12.0 \\
12.0\end{array}$ & $\begin{array}{l}31.00 \\
30.0 \\
311.0 \\
30.0 \\
3.0 .0\end{array}$ & $\begin{array}{l}59.0 \\
59.0 \\
57.0 \\
59.0 \\
57.0\end{array}$ \\
\hline $\begin{array}{l}23 ! .3 \\
21.3 .3 \\
133.3 \\
172.0 \\
234.5\end{array}$ & $\begin{array}{l}270.3 \\
307.3 \\
172.0 \\
23.05 \\
315.5\end{array}$ & $\begin{array}{l}55.0 \\
55.0 \\
00.0 \\
45.0 \\
60.0\end{array}$ & $\begin{array}{r}352.0 \\
464.0 \\
70.5 \\
107.3 \\
277.3\end{array}$ & $\begin{array}{l}+64.0 \\
555.0 \\
107.3 \\
277.5 \\
390.0\end{array}$ & $\begin{array}{l}30.0 \\
40.0 \\
40.0 \\
40.0 \\
40.0\end{array}$ & $\begin{array}{l}12.0 \\
12.0 \\
12.0 \\
12.0 \\
12.0\end{array}$ & $\begin{array}{l}30.0 \\
3.3 .0 \\
3.0 .0 \\
3.10 .0 \\
30.0\end{array}$ & $\begin{array}{l}53.0 \\
58.0 \\
60.0 \\
56.0 \\
54.0\end{array}$ \\
\hline $\begin{array}{l}313.5 \\
394.0 \\
241.3 \\
325.0 \\
441.5\end{array}$ & $\begin{array}{l}39 \% .0 \\
461.0 \\
325.0 \\
45 \% .5 \\
563.8\end{array}$ & $\begin{array}{l}53.0 \\
50.0 \\
30.0 \\
15.0 \\
40.0\end{array}$ & $\begin{array}{l}390.3 \\
445.3 \\
121.5 \\
265.5 \\
327.5\end{array}$ & $\begin{array}{l}4.44 .0 \\
+65.0 \\
269.5 \\
327.5 \\
382.5\end{array}$ & $\begin{array}{l}4.1 .0 \\
63.0 \\
5 j .0 \\
45.0 \\
43.0\end{array}$ & $\begin{array}{l}12.01 \\
12.0 \\
12.0 \\
12.0 \\
12.0\end{array}$ & $\begin{array}{l}31.00 \\
3.1 .0 \\
3.5 .0 \\
30.0 \\
311.0\end{array}$ & $\begin{array}{l}54.03 \\
514.0 \\
54.0 \\
54.0 \\
54.0\end{array}$ \\
\hline ל.33 & $5\left(6^{\prime}\right) .5$ & 45.0 & 382.5 & $42 J . J$ & 65.5 & $12 .{ }^{\circ}$ & $3 \% .0$ & 56.0 \\
\hline
\end{tabular}


TABLE IX

BASELINE COUNTERCURRENT FLOW TEST GROUP 4.1

TRAIASPARENT VESSEL TEST DATA FOR TESTS 4.1.1 TO $4.1 .53-0.49-1 \%$. DOWNCJMER GAP SIMMAARY UF CALCULATED RESULTS

\begin{tabular}{|c|c|c|c|c|c|c|c|c|c|c|}
\hline $\begin{array}{c}\text { TEST } \\
\text { NO }\end{array}$ & $\begin{array}{l}\text { UUWNCOUER } \\
\text { AIR FLOH } \\
\text { (LA/SEC) }\end{array}$ & $\begin{array}{l}\text { DOHNCOMER } \\
\text { AIR DERISITY } \\
\text { (L.3/FT**3) }\end{array}$ & $\begin{array}{l}\text { LOWER PLENUM } \\
\text { PRESSURF } \\
\text { (PSIA) }\end{array}$ & $\begin{array}{l}\text { CQLD LES } \\
\text { AIR FLOH } \\
\text { (LB/SEC) }\end{array}$ & $\begin{array}{l}\text { DUWNCLIMEP } \\
\text {-WATER FLUN } \\
\text { (LB/SEC) }\end{array}$ & $\begin{array}{l}\text { WATER FLOW } \\
\text { CINTINIIITY }\end{array}$ & $\begin{array}{c}J-A(R \\
\text { OUWNCIRMEP. } \\
\text { (FT/SEC) }\end{array}$ & 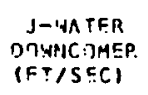 & $(J G *) * 1 / 2$ & $(J L * 1 * * 1)$ \\
\hline $\begin{array}{l}4.1 .1 \\
4.1 .2 \\
4.1 .5 \\
4.1 .1 \\
4.1 .5\end{array}$ & $\begin{array}{l}0.1672 \\
0.1672 \\
0.1672 \\
0.0146 \\
0.0146\end{array}$ & $\begin{array}{l}0.1034 \\
0.1036 \\
0.1036 \\
0.103 i \\
0.1030\end{array}$ & $\begin{array}{l}19.70 \\
19.70 \\
19.70 \\
19.70 \\
19.70\end{array}$ & $\begin{array}{l}0.0 \\
0.0 \\
0.0 \\
0.0 \\
0.0\end{array}$ & $\begin{array}{l}0.075 \\
0.075 \\
0.079 \\
4.717 \\
4.500\end{array}$ & $\begin{array}{r}1.005 \\
-1.033 \\
0.799 \\
0.991 \\
1.009\end{array}$ & $\begin{array}{r}25.10 \\
25.05 \\
25.05 \\
2.20 \\
2.20\end{array}$ & $\begin{array}{l}0.019 \\
0.1919 \\
0.019 \\
1.174 \\
1.120\end{array}$ & $\begin{array}{l}0.7934 \\
0.7930 \\
0.7930 \\
0.2347 \\
0.2348\end{array}$ & $\begin{array}{l}0.1072 \\
0.1072 \\
0.1096 \\
0.8504 \\
0.8306\end{array}$ \\
\hline $\begin{array}{l}4.1 .106 \\
4.1: 7 \\
4.1 .1 .9 \\
4.1 .19 \\
4.1 .10\end{array}$ & $\begin{array}{l}0.0147 \\
0.1667 \\
0.1668 \\
0.1390 \\
0.1390\end{array}$ & $\begin{array}{l}0.1030 \\
0.1020 \\
J .1029 \\
0.1020 \\
.1030\end{array}$ & $\begin{array}{l}19.70 \\
19.70 \\
19.70 \\
19.70 \\
19.70\end{array}$ & $\begin{array}{l}0.0 \\
0.0 \\
0.0 \\
0.0 \\
0.0\end{array}$ & $\begin{array}{l}4.650 \\
0.086 \\
0.075 \\
0.229 \\
0.240\end{array}$ & $\begin{array}{l}1.115 \\
0.993 \\
0.247 \\
0.995 \\
1.003\end{array}$ & $\begin{array}{l}2.22 \\
25.18 \\
25.20 \\
21.00 \\
20.90\end{array}$ & $\begin{array}{l}1.157 \\
0.021 \\
0.017 \\
0.057 \\
0.000\end{array}$ & $\begin{array}{l}0.2355 \\
0.7934 \\
0.7938 \\
0.7246 \\
0.7242\end{array}$ & $\begin{array}{l}0.8443 \\
0.1149 \\
0.1072 \\
0.1874 \\
0.1917\end{array}$ \\
\hline $\begin{array}{l}4.1 .11 \\
4.1 .12 \\
4.1 .13 \\
4.1 .14 \\
4.1 .15\end{array}$ & $\begin{array}{l}0.1390 \\
0.1390 \\
0.1398 \\
0.1389 \\
0.1063\end{array}$ & $\begin{array}{l}0.1032 \\
0.1032 \\
0.1032 \\
0.1030 \\
0.1030\end{array}$ & $\begin{array}{l}19.70 \\
19.70 \\
19.70 \\
19.70 \\
19.70\end{array}$ & $\begin{array}{l}c .0 \\
0.0 \\
0.0 \\
c .0 \\
0.0\end{array}$ & $\begin{array}{l}0.237 \\
0.250 \\
0.242 \\
0.623 \\
0.642\end{array}$ & $\begin{array}{l}0.982 \\
0.994 \\
0.932 \\
0.993 \\
1.000\end{array}$ & $\begin{array}{l}20.92 \\
20.92 \\
21.03 \\
20.94 \\
16.02\end{array}$ & $\begin{array}{l}0.059 \\
0.052 \\
0.060 \\
3.155 \\
0.160\end{array}$ & $\begin{array}{l}0.7239 \\
0.7239 \\
0.7259 \\
0.72 .39 \\
0.6333\end{array}$ & $\begin{array}{l}0.1908 \\
0.1958 \\
0.1925 \\
0.3090 \\
0.3137\end{array}$ \\
\hline $\begin{array}{l}4.1 .16 \\
4.1 .17 \\
4.1 .18 \\
4.1 .15 \\
4.1 .20\end{array}$ & $\begin{array}{l}0.1063 \\
0.1057 \\
0.1063 \\
0.1060 \\
0.0751\end{array}$ & $\begin{array}{l}0.1032 \\
0.1032 \\
0.103 .0 \\
0.1024 \\
0.102 ?\end{array}$ & $\begin{array}{l}19.70 \\
19.70 \\
19.70 \\
19.70 \\
19.67\end{array}$ & $\begin{array}{l}0.0 \\
0.0 \\
0.0 \\
0.0 \\
0.0\end{array}$ & $\begin{array}{l}0.617 \\
0.708 \\
0.742 \\
0.683 \\
1.462\end{array}$ & $\begin{array}{l}0.994 \\
1.017 \\
1.436 \\
1.012 \\
0.992\end{array}$ & $\begin{array}{l}15.99 \\
15.09 \\
16.02 \\
16.07 \\
11.35\end{array}$ & $\begin{array}{l}0.153 \\
0.176 \\
0.185 \\
0.170 \\
0.364\end{array}$ & $\begin{array}{l}0.6330 \\
0.6311 \\
0.6333 \\
0.6333 \\
0.5326\end{array}$ & $\begin{array}{l}0.3075 \\
0.3295 \\
0.3372 \\
0.3237 \\
0.4735\end{array}$ \\
\hline $\begin{array}{l}4.1 .21 \\
4.1 .22 \\
4.1 .23 \\
4.1 .24 \\
4.1 .25\end{array}$ & $\begin{array}{l}0.0751 \\
0.0751 \\
0.0746 \\
0.0446 \\
0.0449\end{array}$ & $\begin{array}{l}0.1027 \\
0.1027 \\
0.1027 \\
0.1029 \\
0.11029\end{array}$ & $\begin{array}{l}19.67 \\
19.67 \\
19.67 \\
19.67 \\
19.67\end{array}$ & $\begin{array}{l}0.0 \\
0.0 \\
0.0 \\
0.0 \\
0.0\end{array}$ & $\begin{array}{l}1.525 \\
1.583 \\
1.496 \\
2.583 \\
2.563\end{array}$ & $\begin{array}{l}1.032 \\
1.045 \\
1.079 \\
0.9115 \\
0.989\end{array}$ & $\begin{array}{r}11.35 \\
11.35 \\
11.29 \\
6.73 \\
6.77\end{array}$ & $\begin{array}{l}0.380 \\
0.394 \\
0.372 \\
0.643 \\
0.638\end{array}$ & $\begin{array}{l}0.5326 \\
0.5326 \\
0.5310 \\
0.41 .04 \\
0.4116\end{array}$ & $\begin{array}{l}0.4835 \\
0.4927 \\
0.4789 \\
0.6293 \\
0.6268\end{array}$ \\
\hline $\begin{array}{l}4.1 .26 \\
4.1 .27 \\
4.1 .28 \\
4.1 .29 \\
4.1 .30\end{array}$ & $\begin{array}{l}0.0449 \\
0.0433 \\
0.0295 \\
0.0293 \\
0.0294\end{array}$ & $\begin{array}{l}0.1029 \\
0.1023 \\
0.1023 \\
0.1031 \\
0.1030\end{array}$ & $\begin{array}{l}19.67 \\
19.67 \\
19.67 \\
19.67 \\
19.60\end{array}$ & $\begin{array}{l}0.0 \\
0.0 \\
0.0 \\
0.0 \\
0.0\end{array}$ & $\begin{array}{l}2.512 \\
1.375 \\
3.552 \\
3.313 \\
2.758\end{array}$ & $\begin{array}{l}0.939 \\
0.989 \\
1.010 \\
1.001 \\
0.949\end{array}$ & $\begin{array}{l}6.7 ? \\
6.5 ? \\
4.48 \\
4.45 \\
4.43\end{array}$ & $\begin{array}{l}0.825 \\
0.31+2 \\
3.894 \\
0.825 \\
0.687\end{array}$ & $\begin{array}{l}0.4116 \\
0.4047 \\
0.3343 \\
0.3338 \\
0.3329\end{array}$ & $\begin{array}{l}0.6206 \\
0.4591 \\
0.7421 \\
0.7126 \\
0.6503\end{array}$ \\
\hline $\begin{array}{l}4.1 .31 \\
4.1 .32 \\
4.1 .33 \\
4.1 .34 \\
4.1 .35\end{array}$ & $\begin{array}{l}J .0295 \\
0.0597 \\
j .0599 \\
j .0596 \\
0.0589\end{array}$ & $\begin{array}{l}0.1029 \\
0.1027 \\
0.1027 \\
0.1027 \\
0.1027\end{array}$ & $\begin{array}{l}19.67 \\
19.67 \\
19.67 \\
19.67 \\
19.67\end{array}$ & $\begin{array}{l}0.0 \\
0.0 \\
0.0 \\
0.0 \\
c .0\end{array}$ & $\begin{array}{l}3.212 \\
2.046 \\
2.079 \\
2.037 \\
2.354\end{array}$ & $\begin{array}{l}0.944 \\
1.012 \\
1.003 \\
0.995 \\
0.996\end{array}$ & $\begin{array}{l}4.106 \\
9.07 \\
3.07 \\
9.01 \\
0.90\end{array}$ & $\begin{array}{l}0.990 \\
0.507 \\
.5189 \\
.0 .507 \\
7.586\end{array}$ & $\begin{array}{l}0.3339 \\
0.4760 \\
0.4760 \\
0.4746 \\
0.4717\end{array}$ & $\begin{array}{l}0.7018 \\
0.5601 \\
0.5646 \\
0.5589 \\
0.6008\end{array}$ \\
\hline $\begin{array}{l}4.1 .36 \\
4.1 .37 \\
4.1 .38 \\
4.1 .39 \\
4.1 .40\end{array}$ & $\begin{array}{l}0.0592 \\
0.0909 \\
0.0898 \\
0.0898 \\
0.0910\end{array}$ & $\begin{array}{l}0.1027 \\
0.1927 \\
0.1029 \\
0.1029 \\
0.1025\end{array}$ & $\begin{array}{l}19.07 \\
19.07 \\
19.67 \\
19.67 \\
19.67\end{array}$ & $\begin{array}{l}\text { c.0 } \\
0.0 \\
0.0 \\
0.0 \\
0.0\end{array}$ & $\begin{array}{l}1.947 \\
1.053 \\
1.017 \\
1.050 \\
0.433\end{array}$ & $\begin{array}{l}1.021 \\
1.012 \\
1.001 \\
0.993 \\
0.996\end{array}$ & $\begin{array}{l}0.36 \\
13.74 \\
13.56 \\
13.56 \\
13.80\end{array}$ & $\begin{array}{l}3.435 . \\
0.263 \\
0.253 \\
0.261 \\
0.232\end{array}$ & $\begin{array}{l}0.4731 \\
0.5860 \\
0.5823 \\
0.5823 \\
0.5869\end{array}$ & $\begin{array}{l}0.5520 \\
0.4028 \\
0.3948 \\
0.4012 \\
0.3783\end{array}$ \\
\hline
\end{tabular}




\section{TABLE IX (Contd.)}

BASELINE COUNTERCURRENT FLOW TEST GROUP 4.1

SUMMARY DF CALCJLATED RESULTS FOR TESTS 4.1 .1 TO $4.1 .53-0.49$ IN. DOWIAC JMER GAP

\begin{tabular}{|c|c|c|c|}
\hline $\begin{array}{l}\text { TEST } \\
\text { NO }\end{array}$ & $\begin{array}{l}\text { DOHNCOMER } \\
\text { AIR FLOW } \\
(L \cdot B / \text { SEC })\end{array}$ & $\begin{array}{l}\text { DOWNE JMER } \\
\text { AIR DENSITY } \\
\text { (LQ BET } * * 3)\end{array}$ & $\begin{array}{l}\text { LOHER PLENUY } \\
\text { PRESSURE } \\
\text { (PSIA) }\end{array}$ \\
\hline $\begin{array}{l}4.1 .41 \\
4.1 .42 \\
4.1 .43 \\
4.1 .44 \\
4.1 .45\end{array}$ & $\begin{array}{l}0.0920 \\
0.1218 \\
0.1231 \\
0.1223 \\
0.1229\end{array}$ & $\begin{array}{l}0.1025 \\
0.1027 \\
0.1029 \\
0.1023 \\
0.1021\end{array}$ & $\begin{array}{l}19.67 \\
19.67 \\
19.67 \\
19.63 \\
19.63\end{array}$ \\
\hline $\begin{array}{l}4.1 .46 \\
4.1 .47 \\
4.1 .48 \\
4.1 .49 \\
4.1 .50\end{array}$ & $\begin{array}{l}0.1223 \\
0.1510 \\
0.1522 \\
0.1513 \\
0.1512\end{array}$ & $\begin{array}{l}0.1025 \\
0.1025 \\
0.1027 \\
. .1027 \\
0.1029\end{array}$ & $\begin{array}{l}19.63 \\
19.63 \\
19.63 \\
19.63 \\
19.63\end{array}$ \\
\hline $\begin{array}{l}4.1 .51 \\
4.1 .52 \\
4.1 .53\end{array}$ & $\begin{array}{l}0.1301 \\
0.0030 \\
0.0030\end{array}$ & $\begin{array}{l}0.1023 \\
u .1029 \\
0.1029\end{array}$ & $\begin{array}{l}19.63 \\
19.63 \\
19.63\end{array}$ \\
\hline
\end{tabular}

\begin{tabular}{|c|c|c|c|c|c|c|}
\hline $\begin{array}{l}\text { OLD LEG } \\
\text { IR FLOW } \\
\text { LB/SECI }\end{array}$ & $\begin{array}{l}\text { DOWNCOMER } \\
\text { WATER FLOH } \\
\text { (LH/SEC) }\end{array}$ & $\begin{array}{l}\text { WATER FLOH } \\
\text { CONTI VUITY }\end{array}$ & $\begin{array}{c}J-A I R \\
\text { DOWNCOMER } \\
\text { (FT/SEC) }\end{array}$ & $\begin{array}{l}\text { J-WATER } \\
\text { DOWNCOMER } \\
\text { IFT/SECI }\end{array}$ & $(J G *) * * 1 / 2$ & $(J t * 1 * * 1)$ \\
\hline & $\begin{array}{l}1.008 \\
0.325 \\
0.321 \\
0.308 \\
0.333\end{array}$ & $\begin{array}{l}1.031 \\
1.003 \\
1.010 \\
1.013 \\
1.003\end{array}$ & $\begin{array}{l}14.53 \\
18.41 \\
18.59 \\
18.65 \\
18.51\end{array}$ & $\begin{array}{l}0.251 \\
0.081 \\
0.080 \\
0.077 \\
0.093\end{array}$ & $\begin{array}{l}0.5919 \\
0.6783 \\
0.6919 \\
3.6919 \\
0.6316\end{array}$ & $\begin{array}{l}0.3932 \\
0.2232 \\
0.2218 \\
0.2174 \\
0.2261\end{array}$ \\
\hline ). & $\begin{array}{l}0.367 \\
0.140 \\
0.138 \\
0.150 \\
0.143\end{array}$ & $\begin{array}{l}1.040 \\
1.004 \\
1.010 \\
1.014 \\
0.996\end{array}$ & $\begin{array}{l}18.61 \\
22.88 \\
23.01 \\
22.89 \\
22.02\end{array}$ & $\begin{array}{l}0.091 \\
0.035 \\
0.034 \\
0.037 \\
0.035\end{array}$ & $\begin{array}{l}0.6816 \\
0.7558 \\
0.7583 \\
0.7561 \\
0.7554\end{array}$ & $\begin{array}{l}0.2371 \\
0.1465 \\
0.1456 \\
0.1516 \\
0.1478\end{array}$ \\
\hline & $\begin{array}{l}0.121 \\
5.149 \\
0.150\end{array}$ & $\begin{array}{l}0.050 \\
0.926 \\
0.990\end{array}$ & $\begin{array}{r}22.79 \\
0.45 \\
0.45\end{array}$ & $\begin{array}{l}0.030 \\
1.282 \\
1.531\end{array}$ & $\begin{array}{l}0.7540 \\
0.1059 \\
0.1067\end{array}$ & $\begin{array}{l}0.1361 \\
0.8885 \\
0.9710\end{array}$ \\
\hline
\end{tabular}




\section{TABLE IX (Contd.)}

BASELINE COUNTERCURRENT FLOW TEST GROUP 4.1

EXPERIMENTAL MEASURMENTS FOR TESTS 4.1 .1 TO $4.1 .53-0.49-1 \mathrm{~N}$. NONNCOMER GAP

\begin{tabular}{|c|c|c|c|c|c|c|c|c|c|c|}
\hline $\begin{array}{l}\text { TEST } \\
\text { NO }\end{array}$ & $\begin{array}{l}R: 1-C W-1 \\
(G P M I)\end{array}$ & $\begin{array}{l}F M-C H-2 \\
(G P M)\end{array}$ & $\begin{array}{l}F T-C(1-1 \\
(\in P: M)\end{array}$ & $\begin{array}{c}F T-C H-2 \\
(1, P+1)\end{array}$ & $\begin{array}{l}F M-H C-1 \\
(C F=-1)\end{array}$ & $\begin{array}{l}K M-H C_{i}-2 \\
(C, F M)\end{array}$ & $\begin{array}{c}R M-H C_{2}-3 \\
\left(C F^{4}()^{\prime}\right)\end{array}$ & $\begin{array}{c}\text { RM- M-HG-4 } \\
([\subset \bar{C})\end{array}$ & $\begin{array}{l}f T-(15-1 \\
(C F Y y)\end{array}$ & $\begin{array}{c}F T-H G-2 \\
(C F M)\end{array}$ \\
\hline $\begin{array}{l}4.1 \cdot 1 \\
4.1 .2 \\
4.1 . \\
4.1 .4 \\
4.1 .5\end{array}$ & $\begin{array}{l}25.0 \\
25.0 \\
20.0 \\
33.0 \\
25.0\end{array}$ & $\begin{array}{r}10.0 \\
: 1.0 \\
0.0 \\
1.0 .0 \\
10.0\end{array}$ & $\begin{array}{l}0.0 \\
0.0 \\
0.0 \\
0.0 \\
0.0\end{array}$ & $\begin{array}{l}0.0 \\
0.0 \\
0.0 \\
0.0 \\
0.0\end{array}$ & $\begin{array}{c}70.00 \\
70.00 \\
70.00 \\
0.0 \\
0.0\end{array}$ & $\begin{array}{c}40.00 \\
40.00 \\
40.00 \\
0.0 \\
0.0\end{array}$ & $\begin{array}{l}j . j \\
j .0 \\
0.0 \\
0.0 \\
u .0\end{array}$ & $\begin{array}{c}0.0 \\
0.0 \\
0.0 \\
10.00 \\
10.00\end{array}$ & $\begin{array}{l}0.0 \\
0.0 \\
1.0 \\
0.0 \\
0.0\end{array}$ & $\begin{array}{l}0.0 \\
0.0 \\
0.0 \\
0.0 \\
0.0\end{array}$ \\
\hline $\begin{array}{l}4.1 .1 \\
4.1 .7 \\
4.2 .28 \\
4.1 .9 \\
4.1 .10\end{array}$ & $\begin{array}{l}3.0 .0 \\
3 \% .0 \\
33.0 \\
3 \% .0 \\
3.0 .0\end{array}$ & $\begin{array}{r}0.0 \\
10.0 \\
10.0 \\
10.0 \\
0.0\end{array}$ & $\begin{array}{l}0.0 \\
0.0 \\
0.0 \\
0.0 \\
0.0\end{array}$ & $\begin{array}{l}0.0 \\
0.0 \\
j .0 \\
0.0 \\
0.0\end{array}$ & $\begin{array}{l}0.0 \\
70.00 \\
70.00 \\
90.00 \\
90.00\end{array}$ & $\begin{array}{c}0.0 \\
40.00 \\
40.00 \\
0.00 \\
0.0\end{array}$ & $\begin{array}{l}0.0 \\
0.0 \\
0.0 \\
0.0 \\
0.0\end{array}$ & $\begin{array}{c}10.001 \\
0.0 \\
0.0 \\
0.0 \\
0.0\end{array}$ & $\begin{array}{l}0.0 \\
0.0 \\
0.0 \\
0.0 \\
0.0\end{array}$ & $\begin{array}{l}0.0 \\
0.0 \\
0.0 \\
0.0 \\
0.0\end{array}$ \\
\hline $\begin{array}{l}4.1 .11 \\
4.1 .12 \\
4.1 .13 \\
4.1 .14 \\
4.1 .15\end{array}$ & $\begin{array}{l}2.0 .0 \\
10.0 \\
3.0 \\
30.0 \\
33.0\end{array}$ & $\begin{array}{r}J .0 \\
0.0 \\
2.0 \\
10.0 \\
0.0\end{array}$ & $\begin{array}{l}0.0 \\
0.0 \\
0.0 \\
0.0 \\
0.0\end{array}$ & $\begin{array}{l}0.0 \\
.00 \\
0.0 \\
0.0 \\
0.0\end{array}$ & $\begin{array}{l}90.00 \\
90.00 \\
9.0 .00 \\
90.00 \\
70.00\end{array}$ & $\begin{array}{l}3.0 \\
0.0 \\
0.0 \\
0.0 \\
0.0\end{array}$ & $\begin{array}{l}0.0 \\
0.0 \\
0.0 \\
0.0 \\
0.0\end{array}$ & $\begin{array}{l}0.0 \\
0.0 \\
0.0 \\
3.0 \\
0.0\end{array}$ & $\begin{array}{l}0.0 \\
0.0 \\
0.0 \\
0.0 \\
0.0\end{array}$ & $\begin{array}{l}0.0 \\
0.0 \\
0.0 \\
0.0 \\
0.0\end{array}$ \\
\hline $\begin{array}{l}4.1 .10 \\
4.1 .17 \\
4.1 .10 \\
4.1 .19 \\
4.1 .20\end{array}$ & $\begin{array}{r}3.0 .0 \\
1 . j . j \\
\jmath . J \\
\jmath .0 \\
3.0\end{array}$ & $\begin{array}{r}11.0 \\
0.0 \\
3.0 \\
5.0 \\
10.0\end{array}$ & $\begin{array}{l}0.0 \\
0.0 \\
0.0 \\
0.0 \\
0.0\end{array}$ & $\begin{array}{l}0.0 \\
0.0 \\
0.0 \\
0.0 \\
0.0\end{array}$ & $\begin{array}{c}70.00 \\
70.00 \\
75.00 \\
70.00 \\
0.0\end{array}$ & $\begin{array}{c}0.0 \\
. .0 \\
J .0 \\
j .0 \\
51.00\end{array}$ & $\begin{array}{l}0.0 \\
0.0 \\
0.0 \\
0.0 \\
0.0\end{array}$ & $\begin{array}{l}2.0 \\
2.3 \\
0.0 \\
j .0 \\
0.3\end{array}$ & $\begin{array}{l}0.0 \\
3.0 \\
0.0 \\
0.0 \\
0.0\end{array}$ & $\begin{array}{l}0.0 \\
0.0 \\
0.0 \\
0.0 \\
0.0\end{array}$ \\
\hline $\begin{array}{l}4.1 .21 \\
4.1 .22 \\
4.1 .23 \\
4.1 .24 \\
4.1 .25\end{array}$ & $\begin{array}{l}30.0 \\
20.0 \\
1 \mathrm{~J} .0 \\
4 \mathrm{~J} .0 \\
3 \mathrm{~J} .0\end{array}$ & $\begin{array}{l}0.0 \\
0.0 \\
0.0 \\
0.0 \\
0.0\end{array}$ & $\begin{array}{l}0.2 \\
0.0 \\
0.0 \\
0.0 \\
0.0\end{array}$ & $\begin{array}{l}0.0 \\
j .0 \\
0.0 \\
0.0 \\
0.0\end{array}$ & $\begin{array}{l}0.0 \\
0.0 \\
0.0 \\
0.0 \\
0.0\end{array}$ & $\begin{array}{l}50.00 \\
50.00 \\
50.00 \\
30.00 \\
30.00\end{array}$ & $\begin{array}{l}0.0 \\
0.0 \\
0.0 \\
0.0 \\
0.0\end{array}$ & $\begin{array}{l}0.0 \\
0.0 \\
3.0 \\
0.0 \\
0.0\end{array}$ & $\begin{array}{l}0.0 \\
0.0 \\
0.0 \\
0.0 \\
0.0\end{array}$ & $\begin{array}{l}0.0 \\
0.0 \\
0.0 \\
0.0 \\
0.0\end{array}$ \\
\hline $\begin{array}{l}4.1 .26 \\
4.1 .27 \\
4.1 .20 \\
4.1 .29 \\
4.1 .30\end{array}$ & $\begin{array}{l}20.0 \\
13.0 \\
30.0 \\
3 j .0 \\
20.0\end{array}$ & $\begin{array}{r}u .0 \\
0.0 \\
10.0 \\
j .0 \\
. .0\end{array}$ & $\begin{array}{l}0.0 \\
0.0 \\
0.0 \\
0.0 \\
0.3\end{array}$ & $\begin{array}{l}0.0 \\
0.0 \\
0.0 \\
0.0 \\
0.0\end{array}$ & $\begin{array}{l}0.0 \\
0.0 \\
0.0 \\
0.0 \\
0.3\end{array}$ & $\begin{array}{c}j u . v 0 \\
3 u . j u \\
0.01 \\
0.0 \\
0.0\end{array}$ & $\begin{array}{c}0.0 \\
0.0 \\
20.00 \\
20.00 \\
20.00\end{array}$ & $\begin{array}{l}0.0 \\
0.0 \\
0.0 \\
0.0 \\
0.0\end{array}$ & $\begin{array}{l}0.0 \\
0.0 \\
0.0 \\
0.0 \\
0.0\end{array}$ & $\begin{array}{l}0.0 \\
0.0 \\
0.0 \\
0.0 \\
0.0\end{array}$ \\
\hline $\begin{array}{l}4.1 .31 \\
4.1 .32 \\
4.1 .33 \\
4.11 .34 \\
4.1 .33\end{array}$ & $\begin{array}{l}21.0 \\
33.0 \\
31.0 \\
2.0 .0 \\
17.0\end{array}$ & $\begin{array}{r}0.0 \\
1 J .0 \\
0.0 \\
0.0 \\
0.0\end{array}$ & $\begin{array}{l}0.0 \\
0.0 \\
0.0 \\
0.0 \\
0.0\end{array}$ & $\begin{array}{l}0.0 \\
0.0 \\
0.0 \\
0.0 \\
0.0\end{array}$ & $\begin{array}{l}0.0 \\
0.0 \\
0.0 \\
0.0 \\
0.0\end{array}$ & $\begin{array}{c}0.0 \\
41.010 \\
40.00 \\
40.00 \\
40.00\end{array}$ & $\begin{array}{c}20.00 \\
\text { J.0 } \\
u .0 \\
\text { j. } \\
0.0\end{array}$ & $\begin{array}{l}0.0 \\
0.0 \\
0.0 \\
0.0 \\
0.0\end{array}$ & $\begin{array}{l}0.0 \\
0.0 \\
0.0 \\
0.0 \\
0.0\end{array}$ & $\begin{array}{l}0.0 \\
0.0 \\
0.0 \\
0.0 \\
0.0\end{array}$ \\
\hline $\begin{array}{l}4.1 .36 \\
4.1 .37 \\
4.1 .30 \\
4.1 .19 \\
4.1 .40\end{array}$ & $\begin{array}{l}21.0 \\
3 j .0 \\
3.0 .0 \\
2 j .0 \\
0.0\end{array}$ & $\begin{array}{r}0.0 \\
1.0 .0 \\
0.0 \\
0.0 \\
1.0 .0\end{array}$ & $\begin{array}{l}0.0 \\
0.0 \\
0.0 \\
0.0 \\
0.0\end{array}$ & $\begin{array}{l}0.0 \\
0.0 \\
0.0 \\
0.0 \\
0.0\end{array}$ & $\begin{array}{l}0.0 \\
0.0 \\
0.0 \\
0.0 \\
0.0\end{array}$ & $\begin{array}{l}40.00 \\
60.00 \\
60.00 \\
60.00 \\
60.00\end{array}$ & $\begin{array}{l}0.0 \\
0.0 \\
0.3 \\
0.3 \\
0.0\end{array}$ & $\begin{array}{l}0.1 \\
.00 \\
0.0 \\
.00 \\
0.1\end{array}$ & $\begin{array}{l}J .0 \\
0.0 \\
0.0 \\
0.0 \\
0.0\end{array}$ & $\begin{array}{l}0.0 \\
0.0 \\
0.0 \\
0.0 \\
j .0\end{array}$ \\
\hline
\end{tabular}


TABLE IX (Contd.)

BASELINE COUNTERCURRENT FLOW TEST GROUP 4.1

EXPERI MENT AL YEASURMENTS FOR TESTS 4.1 .1 TO $4.1 .53=0.49-1 \mathrm{~N}$. DONNCOMER G.JP

\begin{tabular}{|c|c|c|c|c|c|c|c|c|c|c|}
\hline $\begin{array}{l}\text { TEST } \\
\text { NO }\end{array}$ & $\begin{array}{c}R M-E H-1 \\
(G M M)\end{array}$ & $\begin{array}{l}F \cdot M-C W-2 \\
(G P M)\end{array}$ & $\begin{array}{c}F T-C(H-1 \\
(G P M)\end{array}$ & $\begin{array}{c}F T-C H-2 \\
(G P M)\end{array}$ & $\begin{array}{c}R M-H G-1 \\
(C F M)\end{array}$ & $\begin{array}{c}R M-\mid H G-2 \\
(C F M)\end{array}$ & $\begin{array}{c}R M-H G-3 \\
(C F M)\end{array}$ & $\begin{array}{c}P . M-H F-4 \\
(C F . Y)\end{array}$ & $\begin{array}{c}F Y-H G-1 \\
(C F M)\end{array}$ & $\begin{array}{c}F T-H G-2 \\
\text { (CFM) }\end{array}$ \\
\hline $\begin{array}{l}4.1 .41 \\
4.1 .42 \\
4.1 .43 \\
4.1 .44 \\
4.1 .45\end{array}$ & $\begin{array}{r}3.0 \\
30.0 \\
30.0 \\
2.0 .0 \\
.0\end{array}$ & $\begin{array}{r}3.0 \\
10.0 \\
0.0 \\
0.0 \\
10.0\end{array}$ & $\begin{array}{l}0.0 \\
0.0 \\
0.0 \\
0.0 \\
0.0\end{array}$ & $\begin{array}{r}0.0 \\
0.0 \\
0.0 \\
0.0 \\
j .0\end{array}$ & $\begin{array}{l}0.0 \\
80.00 \\
80.00 \\
80.00 \\
80.00\end{array}$ & $\begin{array}{c}6.000 \\
0.0 \\
0.0 \\
0.0 \\
0.0\end{array}$ & $\begin{array}{l}0.3 \\
0.0 \\
0.3 \\
0.0 \\
0.0\end{array}$ & $\begin{array}{l}0.0 \\
9.0 \\
0.0 \\
0.0 \\
0.0\end{array}$ & $\begin{array}{l}0.0 \\
0.0 \\
0.0 \\
0.0 \\
0.0\end{array}$ & $\begin{array}{l}0.0 \\
0.0 \\
0.0 \\
0.0 \\
0.0\end{array}$ \\
\hline $\begin{array}{l}4.1 .46 \\
4.1 .47 \\
4.11 .48 \\
4.1 .49 \\
4.1 .50\end{array}$ & $\begin{array}{r}3.0 \\
30.0 \\
30.0 \\
20.0 \\
0.0\end{array}$ & $\begin{array}{r}5.0 \\
10.0 \\
0.0 \\
0.0 \\
10.0\end{array}$ & $\begin{array}{l}0.0 \\
0.0 \\
0.0 \\
0.0 \\
0.0\end{array}$ & $\begin{array}{l}0.0 \\
0.0 \\
0.0 \\
0.0 \\
0.0\end{array}$ & $\begin{array}{l}80.00 \\
70.00 \\
70.00 \\
70.00 \\
70.00\end{array}$ & $\begin{array}{c}0.0 \\
30.00 \\
30.00 \\
30.00 \\
30.00\end{array}$ & $\begin{array}{l}0.0 \\
0.0 \\
0.0 \\
0.0 \\
0.0\end{array}$ & $\begin{array}{l}0.0 \\
0.0 \\
0.0 \\
0.0 \\
0.0\end{array}$ & $\begin{array}{l}0.0 \\
0.0 \\
0.0 \\
0.0 \\
0.0\end{array}$ & $\begin{array}{l}0.0 \\
0.0 \\
0.0 \\
\therefore .0 \\
j .0\end{array}$ \\
\hline $\begin{array}{l}4.1 .51 \\
4.1 .52 \\
4.1 .53\end{array}$ & $\begin{array}{l}3.0 .0 \\
3 \mathrm{~J} .0 \\
32.0\end{array}$ & $\begin{array}{l}10.0 \\
10.0 \\
12.5\end{array}$ & $\begin{array}{l}0.0 \\
0.0 \\
0.0\end{array}$ & $\begin{array}{l}0.0 \\
0.0 \\
0.0\end{array}$ & $\begin{array}{c}70.00 \\
0.0 \\
0.0\end{array}$ & $\begin{array}{c}30.00 \\
0.0 \\
0.0\end{array}$ & $\begin{array}{l}0.0 \\
0.3 \\
0.0\end{array}$ & $\begin{array}{l}1.0 \\
2.00 \\
2.00\end{array}$ & $\begin{array}{l}0.0 \\
0.0 \\
0.0\end{array}$ & $\begin{array}{l}0.0 \\
0.0 \\
0.0\end{array}$ \\
\hline $\begin{array}{c}T E S T \\
N O\end{array}$ & $\begin{array}{l}P-C W-1 \\
(P S I G)\end{array}$ & $\begin{array}{l}r-c y-2 \\
(P S I G)\end{array}$ & $\begin{array}{l}P-H G,-1 \\
\text { PSI I I I }\end{array}$ & $\begin{array}{l}\mathrm{P}-\mathrm{HS}-2 \\
\mid \mathrm{PS} S \mathrm{SI}\end{array}$ & $\begin{array}{l}\text { p-rkis-3 } \\
\text { (psic) }\end{array}$ & $\begin{array}{l}\text { TF-CWi-1 } \\
\text { IDES FI }\end{array}$ & $\begin{array}{l}T f-H 1 F_{5}-1 \\
(D \leq G F)\end{array}$ & $\begin{array}{l}T F-H F,-2 \\
\text { IDEF, FI }\end{array}$ & $\begin{array}{l}T F-1 H G-3 \\
(r, E G F)\end{array}$ & $\begin{array}{l}\text { P-BARD } \\
\text { (PSIA) }\end{array}$ \\
\hline $\begin{array}{l}4.1 \cdot 1 \\
4.1 .2 \\
4.1 .13 \\
4.1 .4 \\
4.1 .5\end{array}$ & $\begin{array}{l}0.0 \\
0.0 \\
0.0 \\
0.0 \\
0.0\end{array}$ & $\begin{array}{l}3.2 \\
8.2 \\
8.2 \\
9.0 \\
8.0\end{array}$ & $\begin{array}{l}8.2 \\
3.2 \\
8.2 \\
7.7 \\
7.7\end{array}$ & $\begin{array}{l}0.0 \\
0.0 \\
0.0 \\
0.0 \\
0.0\end{array}$ & $\begin{array}{l}0.0 \\
0.0 \\
0.0 \\
0.0 \\
0.0\end{array}$ & $\begin{array}{l}54 . J \\
54.0 \\
54.0 \\
54.0 \\
54.0\end{array}$ & $\begin{array}{l}60 . J \\
60.0 \\
69.3 \\
72.0 \\
71.0\end{array}$ & $\begin{array}{l}0.0 \\
0.0 \\
0.0 \\
0.0 \\
0.0\end{array}$ & $\begin{array}{l}0.0 \\
0.0 \\
0.0 \\
0.0 \\
0.0\end{array}$ & $\begin{array}{l}12.4 \\
12.4 \\
12.4 \\
12.4 \\
12.4\end{array}$ \\
\hline $\begin{array}{l}4.1 .4 \\
4.1 .7 \\
4.1 .3 \\
4.1 . \\
4.1 .10\end{array}$ & $\begin{array}{l}J . u \\
\jmath .0 \\
0.0 \\
0.0 \\
0.0\end{array}$ & $\begin{array}{l}9.2 \\
0.2 \\
8.0 \\
3.2 \\
8.2\end{array}$ & $\begin{array}{l}8.2 \\
8.2 \\
8.2 \\
8.2 \\
8.2\end{array}$ & $\begin{array}{l}0.0 \\
j .0 \\
. .0 \\
0.0 \\
0.0\end{array}$ & $\begin{array}{l}0.0 \\
0.0 \\
0.0 \\
0.0 \\
0.0\end{array}$ & $\begin{array}{l}34.0 \\
55.0 \\
57.0 \\
55.0 \\
34.0\end{array}$ & $\begin{array}{l}71.0 \\
71.0 \\
70.0 \\
70.0 \\
70.0\end{array}$ & $\begin{array}{l}0.0 \\
0.0 \\
1.00 \\
0.0 \\
. .0\end{array}$ & $\begin{array}{l}0.0 \\
0.0 \\
0.0 \\
0.0 \\
0.0\end{array}$ & $\begin{array}{l}12.4 \\
12.4 \\
12.4 \\
12.4 \\
12.4\end{array}$ \\
\hline $\begin{array}{l}4.1 .11 \\
4.1 .12 \\
4.1 .13 \\
4.1 .14 \\
4.1 .15\end{array}$ & $\begin{array}{l}\jmath .0 \\
j .00 \\
j .0 \\
0.0 \\
j .0\end{array}$ & $\begin{array}{l}8.2 \\
3.0 \\
3.0 \\
8.2 \\
8.0\end{array}$ & $\begin{array}{l}9.2 \\
8.2 \\
8.5 \\
8.2 \\
8.2\end{array}$ & $\begin{array}{l}0.0 \\
0.0 \\
0.0 \\
0.0 \\
0.0\end{array}$ & $\begin{array}{l}0.0 \\
0.0 \\
0.0 \\
0.0 \\
0.0\end{array}$ & $\begin{array}{l}52.0 \\
52.0 \\
53.0 \\
54.0 \\
54.0\end{array}$ & $\begin{array}{l}70.1 \\
70.0 \\
7 \mathrm{J.0} \\
71.0 \\
71.0\end{array}$ & $\begin{array}{l}0.0 \\
0.0 \\
0.0 \\
0.0 \\
0.0\end{array}$ & $\begin{array}{l}0.0 \\
0.0 \\
0.0 \\
0.0 \\
0.0\end{array}$ & $\begin{array}{l}12.4 \\
12.4 \\
12.4 \\
12.4 \\
12.4\end{array}$ \\
\hline $\begin{array}{l}4.1 .16 \\
4.1 .17 \\
4.1 .10 \\
4.1 .19 \\
4.1 .20\end{array}$ & $\begin{array}{l}0.0 \\
0.0 \\
0.0 \\
0.0 \\
0.0\end{array}$ & $\begin{array}{l}9.0 \\
8.0 \\
8.0 \\
8.0 \\
3.2\end{array}$ & $\begin{array}{l}8.2 \\
8.0 \\
8.2 \\
8.0 \\
8.2\end{array}$ & $\begin{array}{l}0.0 \\
0.0 \\
0.0 \\
0.0 \\
0.0\end{array}$ & $\begin{array}{l}0.0 \\
0.0 \\
0.0 \\
0.0 \\
0.0\end{array}$ & $\begin{array}{l}54.0 \\
54.0 \\
54.0 \\
54.0 \\
55 . J\end{array}$ & $\begin{array}{l}71.0 \\
71.0 \\
71.0 \\
63.0 \\
69.0\end{array}$ & $\begin{array}{l}0.0 \\
0.0 \\
0.0 \\
0.0 \\
0.0\end{array}$ & $\begin{array}{l}0.0 \\
0.0 \\
0.0 \\
0.0 \\
0.0\end{array}$ & $\begin{array}{l}12.4 \\
12.4 \\
12.4 \\
12.4 \\
12.4\end{array}$ \\
\hline
\end{tabular}


TABLE IX (Contd.)

BASELINE COUNTERCURRENT FLOW TEST GROUP 4.1

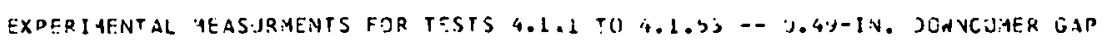

\begin{tabular}{|c|c|c|c|c|c|c|c|c|c|c|}
\hline $\begin{array}{c}\text { TEST } \\
\text { NJ }\end{array}$ & $\begin{array}{l}D-C: 1-1 \\
|P S| G \mid\end{array}$ & $\begin{array}{l}n-C W-2 \\
(P S I G)\end{array}$ & $\begin{array}{l}P-H G-1 \\
(P S I G)\end{array}$ & $\begin{array}{l}p-1(G-2 \\
(p \leq 10)\end{array}$ & $\begin{array}{l}P-11 G-3 \\
|P S 1 G|\end{array}$ & $\begin{array}{l}\text { TF-CH-1 } \\
\text { (UEG }=1\end{array}$ & $\begin{array}{l}\text { TF-itS- } \\
\text { (DES F) }\end{array}$ & $\begin{array}{l}\text { TF-Hr-2 } \\
\text { (nes of }\end{array}$ & $\begin{array}{l}T F-H F-3 \\
(D E S F)\end{array}$ & $\begin{array}{l}\text { P-HART } \\
\text { (PSIA) }\end{array}$ \\
\hline $\begin{array}{l}4.1 .21 \\
4.1 .22 \\
4.1 .23 \\
4.1 .24 \\
4.1 .25\end{array}$ & $\begin{array}{l}j .0 \\
0.0 \\
0.0 \\
0.0 \\
0.0\end{array}$ & $\begin{array}{l}8.5 \\
3.0 \\
3.2 \\
3.5 \\
3.7\end{array}$ & $\begin{array}{l}8.2 \\
8.2 \\
8.3 \\
8.2 \\
3.5\end{array}$ & $\begin{array}{l}0.0 \\
0.0 \\
0.0 \\
2.0 \\
0.0\end{array}$ & $\begin{array}{l}0.0 \\
0.0 \\
0.0 \\
0.0 \\
0.0\end{array}$ & $\begin{array}{l}51.0 \\
54.0 \\
55.1 \\
34.0 \\
54.0\end{array}$ & $\begin{array}{l}69.0 \\
69.0 \\
69.0 \\
70.0 \\
70.0\end{array}$ & $\begin{array}{l}0.0 \\
0.0 \\
0.0 \\
0.0 \\
0.0\end{array}$ & $\begin{array}{l}0.0 \\
0.0 \\
0.0 \\
0.0 \\
0.0\end{array}$ & $\begin{array}{l}12.4 \\
12.4 \\
12.4 \\
12.4 \\
12.4\end{array}$ \\
\hline $\begin{array}{l}4.1 .20 \\
4.1 .27 \\
4.1 .23 \\
4.1 .29 \\
4.1 .23\end{array}$ & $\begin{array}{l}0.0 \\
0.0 \\
3.0 \\
.00 \\
0.0\end{array}$ & $\begin{array}{l}3.7 \\
7.7 \\
8.2 \\
3.7 \\
8.2\end{array}$ & $\begin{array}{l}8.5 \\
7.5 \\
8.2 \\
8.2 \\
8.5\end{array}$ & $\begin{array}{l}0.0 \\
0.0 \\
0.0 \\
1.0 \\
0.0\end{array}$ & $\begin{array}{l}0.0 \\
0.0 \\
0.0 \\
0.0 \\
0.0\end{array}$ & $\begin{array}{l}54.0 \\
55.0 \\
55.0 \\
52.0 \\
154.0\end{array}$ & $\begin{array}{l}70.0 \\
70.0 \\
73.0 \\
72.0 \\
72.0\end{array}$ & $\begin{array}{l}0.0 \\
0.0 \\
0.0 \\
0.0 \\
0.0\end{array}$ & $\begin{array}{l}0.0 \\
0.0 \\
0.0 \\
0.0 \\
0.0\end{array}$ & $\begin{array}{l}12.4 \\
12.4 \\
12.4 \\
12.4 \\
12.4\end{array}$ \\
\hline $\begin{array}{l}4.1 .31 \\
4.1 .32 \\
4.1 .33 \\
4.1 .34 \\
4.1 .35\end{array}$ & $\begin{array}{l}J .0 \\
u .0 \\
j .0 \\
j .0 \\
3.0\end{array}$ & $\begin{array}{l}3.2 \\
0.5 \\
8.7 \\
3.5 \\
3.0\end{array}$ & $\begin{array}{l}3.2 \\
3.5 \\
8.5 \\
3.2 \\
7.7\end{array}$ & $\begin{array}{l}0.0 \\
0.0 \\
0.0 \\
0.0 \\
0.0\end{array}$ & $\begin{array}{l}0.0 \\
0.0 \\
0.0 \\
0.0 \\
0.0\end{array}$ & $\begin{array}{l}54.0 \\
54.0 \\
15 \% .0 \\
54.0 \\
54.0\end{array}$ & $\begin{array}{l}72.0 \\
72.0 \\
72.0 \\
72.0 \\
72.0\end{array}$ & $\begin{array}{l}0.0 \\
0.0 \\
.00 \\
0.0 \\
0.0\end{array}$ & $\begin{array}{l}0.0 \\
0.0 \\
0.0 \\
0.0 \\
0.0\end{array}$ & $\begin{array}{l}12.4 \\
12.4 \\
12.4 \\
12.4 \\
12.4\end{array}$ \\
\hline $\begin{array}{l}4.1 .36 \\
4.1 .37 \\
4.1 .39 \\
4.1 .39 \\
4.1 .40\end{array}$ & $\begin{array}{l}3.3 \\
0.0 \\
.00 \\
1.0 \\
3.0\end{array}$ & $\begin{array}{l}3.2 \\
0.7 \\
3.0 \\
8.2 \\
3.2\end{array}$ & $\begin{array}{l}8.0 \\
3.3 \\
8.1 \\
8.0 \\
3.5\end{array}$ & $\begin{array}{l}0.0 \\
0.0 \\
0.0 \\
0.0 \\
0.0\end{array}$ & $\begin{array}{l}0.0 \\
0.0 \\
0.0 \\
0.0 \\
0.0\end{array}$ & $\begin{array}{l}55.0 \\
33.0 \\
52.0 \\
51.0 \\
50.0\end{array}$ & $\begin{array}{l}72.0 \\
72.0 \\
72.0 \\
72.0 \\
70.0\end{array}$ & $\begin{array}{l}0.0 \\
0.0 \\
0.0 \\
0.0 \\
0.0\end{array}$ & $\begin{array}{l}0.0 \\
0.0 \\
0.0 \\
0.0 \\
0.0\end{array}$ & $\begin{array}{l}12.4 \\
12.4 \\
! 2.4 \\
12.4 \\
12.4\end{array}$ \\
\hline $\begin{array}{l}4.1 .41 \\
4.1 .42 \\
4.1 .43 \\
4.1 .44 \\
4.1 .45\end{array}$ & $\begin{array}{l}3 . j \\
0.0 \\
0.0 \\
0.0 \\
0.0\end{array}$ & $\begin{array}{l}9.2 \\
3.2 \\
8.5 \\
8.5 \\
0.5\end{array}$ & $\begin{array}{l}9.2 \\
9.5 \\
3.5 \\
8.5 \\
9.5\end{array}$ & $\begin{array}{l}0.0 \\
0.0 \\
0.0 \\
0.0 \\
0.0\end{array}$ & $\begin{array}{l}0.0 \\
0.0 \\
0.0 \\
0.0 \\
0.0\end{array}$ & $\begin{array}{l}35.0 \\
52.0 \\
52.0 \\
55.0 \\
55.0\end{array}$ & $\begin{array}{l}70.0 \\
70.0 \\
70.0 \\
72.0 \\
72.0\end{array}$ & $\begin{array}{l}0.0 \\
0.0 \\
0.0 \\
0.0 \\
0.0\end{array}$ & $\begin{array}{l}0.0 \\
0.0 \\
0.0 \\
0.0 \\
0.0\end{array}$ & $\begin{array}{l}12.4 \\
12.4 \\
12.4 \\
12.4 \\
12.4\end{array}$ \\
\hline $\begin{array}{l}4.1 .46 \\
4.1 .47 \\
4.1 .45 \\
4.1 .49 \\
4.1 .50\end{array}$ & $\begin{array}{l}j .0 \\
. .0 \\
0.0 .0 \\
j .0 \\
1.0\end{array}$ & $\begin{array}{l}8.2 \\
9.2 \\
8.2 \\
3.2 \\
8.2\end{array}$ & $\begin{array}{l}8.5 \\
8.2 \\
8.5 \\
8.2 \\
9.2\end{array}$ & $\begin{array}{l}0.0 \\
0.0 \\
0.0 \\
0.0 \\
0.0\end{array}$ & $\begin{array}{l}0.0 \\
0.0 \\
0.0 \\
0.0 \\
0.0\end{array}$ & $\begin{array}{l}55.0 \\
54.0 \\
54.0 \\
54.0 \\
54.0\end{array}$ & $\begin{array}{l}72.0 \\
72.0 \\
70.0 \\
70.0 \\
71.0\end{array}$ & $\begin{array}{l}0.0 \\
0.0 \\
3.0 \\
0.0 \\
2.0\end{array}$ & $\begin{array}{l}0.0 \\
1.0 \\
0.0 \\
0.0 \\
0.0\end{array}$ & $\begin{array}{l}12.4 \\
12.4 \\
12.4 \\
12.4 \\
12.4\end{array}$ \\
\hline $\begin{array}{l}4.1 .51 \\
4.1 .52 \\
4.1 .53\end{array}$ & $\begin{array}{l}0.0 \\
j .0 \\
\text { J.u }\end{array}$ & $\begin{array}{l}8.0 \\
3.7 \\
3.5\end{array}$ & $\begin{array}{l}8.5 \\
8.5 \\
9.2\end{array}$ & $\begin{array}{l}0.0 \\
0.0 \\
0.0\end{array}$ & $\begin{array}{l}0.0 \\
0.0 \\
0.0\end{array}$ & $\begin{array}{l}58.0 \\
53.0 \\
33.0\end{array}$ & $\begin{array}{l}72.0 \\
71.0 \\
75.0\end{array}$ & $\begin{array}{l}0.3 \\
0.0 \\
0.0\end{array}$ & $\begin{array}{l}0.0 \\
0.0 \\
0.0\end{array}$ & $\begin{array}{l}12.4 \\
12.4 \\
12.4\end{array}$ \\
\hline
\end{tabular}


TABLE IX (Contd.)

BASELINE COUNTERCURRENT FLOW TEST GROUP 4.1

EXPERIMENTAL MEASULMENTS FOR TESTS 4.1 .1 TO $4.1: 53$-- 0.49-IN. DOHNCOMER GAP

\begin{tabular}{|c|c|c|c|c|c|c|c|c|c|}
\hline \multirow[b]{2}{*}{$\underset{N O S T}{\text { TEST }}$} & \multicolumn{3}{|c|}{ LOHEP. PLENUM FLOH } & \multicolumn{3}{|c|}{ BYPASS FLIIW } & \multirow[b]{2}{*}{$\begin{array}{l}\text { LOWER } \\
\text { PLENUM } \\
\text { LEVEL } \\
\text { (IIN) }\end{array}$} & \multirow[b]{2}{*}{$\begin{array}{l}\text { P-LO-1) } \\
\text { IPSIGI }\end{array}$} & \multirow[b]{2}{*}{$\begin{array}{l}\text { TF- }(P-1) \\
(\mathrm{DEG} F)\end{array}$} \\
\hline & $\begin{array}{l}\text { INI YIAAL } \\
\text { WE IIHHT } \\
\text { (Ly) }\end{array}$ & $\begin{array}{l}\text { FINAL } \\
\text { WEI GHI } \\
\text { (LU) }\end{array}$ & $\begin{array}{l}\text { TIME OF } \\
\text { COLLECTICN } \\
\text { ISEC }\end{array}$ & $\begin{array}{l}\text { INITIAL } \\
\text { HEIGHY } \\
\text { ILB) }\end{array}$ & $\begin{array}{l}\text { FINAL } \\
\text { HEIGHT } \\
\text { II } 31\end{array}$ & $\begin{array}{l}\text { TIME OF } \\
\text { COLLECTION } \\
\text { (SEC) }\end{array}$ & & & \\
\hline $\begin{array}{l}4.1: 1 \\
4.1 .2 \\
4.1 .3 \\
4.1 .5 \\
4.1 .5\end{array}$ & $\begin{array}{l}151.0 \\
181.5 \\
204.0 \\
134.5 \\
285.0\end{array}$ & $\begin{array}{l}181.5 \\
204.0 \\
227.5 \\
417.5 \\
555.0\end{array}$ & $\begin{array}{r}300.0 \\
300.0 \\
300.0 \\
60.0 \\
60.0\end{array}$ & $\begin{array}{r}250.0 \\
394.5 \\
500.5 \\
86.5 \\
184.0\end{array}$ & $\begin{array}{l}394.5 \\
500.5 \\
662.5 \\
184.0 \\
241.0\end{array}$ & $\begin{array}{r}30.0 \\
30.0 \\
60.0 \\
122.3 \\
140.3\end{array}$ & $\begin{array}{l}12.0 \\
12.0 \\
12.0 \\
12.0 \\
12.0\end{array}$ & $\begin{array}{l}7.2 \\
7.2 \\
7.2 \\
7.2 \\
7.2\end{array}$ & $\begin{array}{l}54.0 \\
53.0 \\
53.0 \\
56.0 \\
56.0\end{array}$ \\
\hline $\begin{array}{l}4.1 .8 \\
4.1 .7 \\
4.1 .8 \\
4.1 .9 \\
4.1 .1 \mathrm{~J}\end{array}$ & $\begin{array}{l}257.0 \\
143.0 \\
165.5 \\
172.5 \\
20.0 .0\end{array}$ & $\begin{array}{l}396.5 \\
163.5 \\
172.5 \\
200.0 \\
229.3\end{array}$ & $\begin{array}{r}30.0 \\
130.0 \\
120.0 \\
120.0 \\
120.0\end{array}$ & $\begin{array}{l}241.0 \\
241.0 \\
572.5 \\
102.0 \\
550.5\end{array}$ & $\begin{array}{r}241.0 \\
572.5 \\
702.0 \\
1043.0 \\
777.0\end{array}$ & $\begin{array}{r}0.0 \\
61.0 \\
97.0 \\
64.3 \\
57.4\end{array}$ & $\begin{array}{l}12.0 \\
12.0 \\
12.0 \\
12.0 \\
12.0\end{array}$ & $\begin{array}{l}7.2 \\
7.2 \\
7.2 \\
7.2 \\
7.2\end{array}$ & $\begin{array}{l}56.0 \\
57.0 \\
57.0 \\
57.0 \\
56.0\end{array}$ \\
\hline $\begin{array}{l}4.1 .11 \\
4.1 .12 \\
4.1 .13 \\
4.1 .14 \\
4.1 .15\end{array}$ & $\begin{array}{l}223.8 \\
257.3 \\
227.3 \\
316.3 \\
391.0\end{array}$ & $\begin{array}{l}257.3 \\
287.3 \\
316.3 \\
391.0 \\
429.5\end{array}$ & $\begin{array}{r}120.0 \\
120.0 \\
120.0 \\
120.0 \\
60.0\end{array}$ & $\begin{array}{l}777.5 \\
761.5 \\
252.5 \\
365.5 \\
689.0\end{array}$ & $\begin{array}{l}848.5 \\
852.0 \\
854.7 \\
684.0 \\
925.5\end{array}$ & $\begin{array}{r}68.8 \\
79.9 \\
134.6 \\
60.0 \\
66.5\end{array}$ & $\begin{array}{l}12.0 \\
12.0 \\
12.0 \\
12.0 \\
12.0\end{array}$ & $\begin{array}{l}7 \cdot 2 \\
7: 2 \\
7.2 \\
7.2 \\
7.2\end{array}$ & $\begin{array}{l}55.0 \\
55.0 \\
55.0 \\
56.1 \\
56.0\end{array}$ \\
\hline $\begin{array}{l}4.1 .10 \\
4.1 .17 \\
4.1 .18 \\
4.1 .19 \\
4.1 .20\end{array}$ & $\begin{array}{l}423.5 \\
451.0 \\
432.5 \\
132.0 \\
173.0\end{array}$ & $\begin{array}{l}468.5 \\
492.5 \\
537.0 \\
173.0 \\
261.3\end{array}$ & $\begin{array}{l}60.3 \\
60.3 \\
60.0 \\
60.5 \\
60.5\end{array}$ & $\begin{array}{r}817.0 \\
350.5 \\
901.5 \\
85.3 \\
134.0\end{array}$ & $\begin{array}{r}949.0 \\
101.5 \\
731.0 \\
08.0 \\
474.4\end{array}$ & $\begin{array}{r}61.5 \\
72.2 \\
101.3 \\
122.4 \\
13.3\end{array}$ & $\begin{array}{l}12.0 \\
12.0 \\
12.0 \\
12.0 \\
12.0\end{array}$ & $\begin{array}{l}?: 2 \\
?: 2 \\
?: 2 \\
7: \frac{2}{2} \\
? ?\end{array}$ & $\begin{array}{l}55.0 \\
55.0 \\
56.0 \\
59.0 \\
57.0\end{array}$ \\
\hline $\begin{array}{l}4.1 .21 \\
4.1 .22 \\
4.1 .23 \\
4.1 .24 \\
4.1 .25\end{array}$ & $\begin{array}{l}201.5 \\
353.0 \\
443.0 \\
133.5 \\
231.5\end{array}$ & $\begin{array}{l}333.0 \\
441.0 \\
537.0 \\
273.5 \\
.42 .3\end{array}$ & $\begin{array}{l}60.0 \\
60.0 \\
60.0 \\
60.0 \\
60.0\end{array}$ & $\begin{array}{r}474.4 \\
651.5 \\
732.0 \\
97.5 \\
274.5\end{array}$ & $\begin{array}{l}653.5 \\
732.0 \\
732.5 \\
274.5 \\
368.5\end{array}$ & $\begin{array}{r}04.4 \\
60.0 \\
120.0 \\
60.0 \\
60.0\end{array}$ & $\begin{array}{l}12.0 \\
12.0 \\
12.0 \\
12.0 \\
12.0\end{array}$ & $\begin{array}{l}7.2 \\
7: 2 \\
7: \frac{2}{3} \\
7: \frac{2}{2}\end{array}$ & $\begin{array}{l}57.0 \\
57.0 \\
57.0 \\
56.0 \\
56.0\end{array}$ \\
\hline $\begin{array}{l}4.1 .26 \\
4.1 .27 \\
4.1 .20 \\
4.1 .23 \\
4.1 .30\end{array}$ & $\begin{array}{l}442.3 \\
354.5 \\
369.3 \\
335.0 \\
421.0\end{array}$ & $\begin{array}{l}593.0 \\
437.0 \\
534.0 \\
533.8 \\
537.3\end{array}$ & $\begin{array}{l}60.0 \\
60.0 \\
60.5 \\
60.0 \\
60.0\end{array}$ & $\begin{array}{l}369.3 \\
139.5 \\
139.1 \\
315.5 \\
367.3\end{array}$ & $\begin{array}{l}390.0 \\
131.00 \\
315.5 \\
367.3 \\
369.0\end{array}$ & $\begin{array}{r}120.0 \\
0.0 \\
87.1 \\
00.0 \\
91.0\end{array}$ & $\begin{array}{l}12.0 \\
12.0 \\
12.0 \\
12.0 \\
12.0\end{array}$ & $\begin{array}{l}7.2 \\
7.2 \\
7.2 \\
7.2 \\
7.3\end{array}$ & $\begin{array}{l}56.0 \\
59.0 \\
59.0 \\
55.0 \\
56.0\end{array}$ \\
\hline $\begin{array}{l}4.1 .31 \\
4.1 .32 \\
4.1 .33 \\
4.1 .34 \\
4.1 .35\end{array}$ & $\begin{array}{l}367.3 \\
23.3 .3 \\
353.0 \\
450.5 \\
631.0\end{array}$ & $\begin{array}{l}580.0 \\
353.0 \\
471.9 \\
313.0 \\
572.3\end{array}$ & $\begin{array}{l}60.0 \\
60.0 \\
60.0 \\
00.0 \\
60.0\end{array}$ & $\begin{array}{l}36.1 .0 \\
107.0 \\
622.0 \\
636.0 \\
471.5\end{array}$ & $\begin{array}{l}407.0 \\
522.0 \\
752.0 \\
678.5 \\
471.5\end{array}$ & $\begin{array}{r}70.3 \\
6.0 .13 \\
611.3 \\
19.5 \\
0.0\end{array}$ & $\begin{array}{l}12.0 \\
12.0 \\
12.0 \\
12.0 \\
12.0\end{array}$ & $\begin{array}{l}7.2 \\
7.2 \\
7.2 \\
7.2 \\
7.2\end{array}$ & $\begin{array}{l}50.0 \\
57.0 \\
57.0 \\
57.0 \\
57.0\end{array}$ \\
\hline $\begin{array}{l}4.1 .36 \\
4.1 .37 \\
4.1 .38 \\
4.1 .319 \\
4.1 .40\end{array}$ & $\begin{array}{l}351.5 \\
32 \% 4.3 \\
401.03 \\
3.33 .0 \\
132.8\end{array}$ & $\begin{array}{l}470.8 \\
431.8 \\
463.8 \\
456.0 \\
188.8\end{array}$ & $\begin{array}{l}60.0 \\
60.3 \\
60.0 \\
60.0 \\
60.0\end{array}$ & $\begin{array}{r}159.0 \\
526.5 \\
922.0 \\
364.0 \\
00.0\end{array}$ & $\begin{array}{r}493.0 \\
322.0 \\
1311.3 \\
1772.0 \\
126.5\end{array}$ & $\begin{array}{l}85 . ? \\
64.1 \\
6.0 .0 \\
63.4 \\
89.7\end{array}$ & $\begin{array}{l}12.0 \\
12.0 \\
12.0 \\
12.0 \\
12.0\end{array}$ & $\begin{array}{l}7.2 \\
7.2 \\
7.2 \\
7.2 \\
7.2\end{array}$ & $\begin{array}{l}57.0 \\
57.0 \\
56.0 \\
51.0 \\
58.0\end{array}$ \\
\hline
\end{tabular}




\section{$\underline{\text { TABLE IX (Contd.) }}$}

BASELINE COUNTERCURRENT FLOW TEST GROUP 4.1

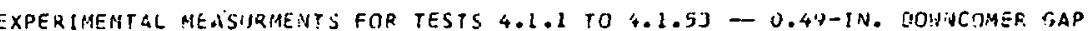

\begin{tabular}{|c|c|c|c|c|c|c|c|c|c|}
\hline \multirow[b]{2}{*}{$\begin{array}{l}\text { TEST } \\
\text { ivo }\end{array}$} & \multicolumn{2}{|r|}{ LUNEP OLENUM } & $\mathrm{FLCH}$ & \multicolumn{3}{|c|}{ BYPASS FL'Sir } & \multirow[b]{2}{*}{$\begin{array}{l}\text { LIJWER } \\
\text { PLEVUM } \\
\text { LEVEL } \\
\text { IINI }\end{array}$} & \multirow[b]{2}{*}{$\begin{array}{l}n-2 n-1 \\
\text { (PSISI) }\end{array}$} & \multirow[b]{2}{*}{$\begin{array}{l}T F-L P-1 \\
(D E G F)\end{array}$} \\
\hline & $\begin{array}{l}\text { INITIAL } \\
\text { WEIGHT } \\
\text { (L3) }\end{array}$ & $\begin{array}{l}\text { TINAL } \\
\text { ALIGHT } \\
\text { (LLY) }\end{array}$ & $\begin{array}{l}\text { TIME IF } \\
\text { COLLECTICN } \\
\text { (SECI }\end{array}$ & $\begin{array}{l}\text { INI I IAL } \\
\text { NEIGHT } \\
\text { IL } 3 \text { I }\end{array}$ & $\begin{array}{l}\text { FINAL } \\
\text { W:GHT } \\
(L \forall)\end{array}$ & $\begin{array}{l}\text { TI:AE OF } \\
\text { COULECTINA } \\
\text { ISECI }\end{array}$ & & & \\
\hline $\begin{array}{l}1.41 \\
1.1 .42 \\
1.43 \\
01.44 \\
01.41\end{array}$ & $\begin{array}{l}183.8 \\
249.3 \\
203.8 \\
139.5 \\
203.5\end{array}$ & $\begin{array}{l}249.3 \\
268.8 \\
233.0 \\
200.0 \\
229.0\end{array}$ & $\begin{array}{l}60.0 \\
60.0 \\
60.0 \\
60.3 \\
60.5\end{array}$ & $\begin{array}{r}120.5 \\
140.0 \\
i 54.0 \\
91.3 \\
238.5\end{array}$ & $\begin{array}{l}140.0 \\
454.0 \\
687.5 \\
238.5 \\
120.0\end{array}$ & $\begin{array}{l}97 . \overline{1} \\
59 . \varepsilon \\
60.0 \\
58.0 \\
70.8\end{array}$ & $\begin{array}{l}12.0 \\
12.0 \\
12.0 \\
12.0 \\
12.0\end{array}$ & $\begin{array}{l}7.2 \\
7.2 \\
7.2 \\
7.2 \\
7.2\end{array}$ & $\begin{array}{l}58.0 \\
57.0 \\
56.0 \\
58.0 \\
57.0\end{array}$ \\
\hline $\begin{array}{l}1.46 \\
1.47 \\
1.40 \\
1.49 \\
.1 .50\end{array}$ & $\begin{array}{l}223.0 \\
250.4 \\
253.8 \\
266.8 \\
27 \% .8\end{array}$ & $\begin{array}{l}250.0 \\
250.4 \\
266.7 \\
275.7 \\
234.3\end{array}$ & $\begin{array}{l}60 . J \\
60.0 \\
60.0 \\
60.0 \\
60.0\end{array}$ & $\begin{array}{l}320.0 \\
346.5 \\
159.0 \\
190.0 \\
105.0\end{array}$ & $\begin{array}{r}346.5 \\
659.0 \\
686.0 \\
106.4 .0 \\
984.0\end{array}$ & $\begin{array}{l}73.2 \\
57.2 \\
55.2 \\
60.0 \\
63.6\end{array}$ & $\begin{array}{l}12.0 \\
12.0 \\
12.0 \\
12.3 \\
12.0\end{array}$ & $\begin{array}{l}7.2 \\
7.2 \\
7.2 \\
7.2 \\
7.2\end{array}$ & $\begin{array}{l}57.0 \\
57.0 \\
56.0 \\
56.0 \\
55.0\end{array}$ \\
\hline $\begin{array}{l}1.51 \\
1.52 \\
01.53\end{array}$ & $\begin{array}{l}231.8 \\
24 j .0 \\
34 j .3\end{array}$ & $\begin{array}{l}245.0 \\
201.0 \\
524.5\end{array}$ & $\begin{array}{l}00.3 \\
30.3 \\
30.0\end{array}$ & $\begin{array}{r}9 \% .0 \\
110.3 \\
110.0\end{array}$ & $\begin{array}{l}110.0 \\
110.0 \\
111.0\end{array}$ & $\begin{array}{r}101.0 \\
0.6 \\
102.5\end{array}$ & $\begin{array}{l}12.0 \\
12.0 \\
12.0\end{array}$ & $\begin{array}{l}7: 2 \\
7 \because 2 \\
7: 2\end{array}$ & $\begin{array}{l}53.0 \\
55.0 \\
55.0\end{array}$ \\
\hline
\end{tabular}

110.3
110.3

111.0

102.5

12.0

55.0 
TABLE X

BASELINE COUNTERCURRENT FLOW TEST GROUP 4.2

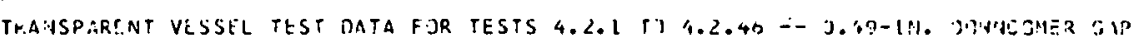
SUYYARY JF CALCIJLATEO RESULTS

\begin{tabular}{|c|c|c|c|c|c|c|c|c|c|c|}
\hline $\begin{array}{l}\text { TEST } \\
\text { NU }\end{array}$ & 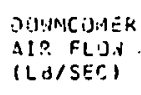 & 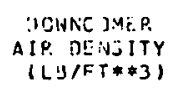 & $\begin{array}{l}\text { LOPEF. PLENUY } \\
\text { PAESSURE } \\
\text { (PSIA) }\end{array}$ & $\begin{array}{l}C O L) L E ; \\
A I R \text { FLINA } \\
(L B / S E C)\end{array}$ & 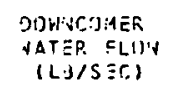 & $\begin{array}{l}\text { WATER FLOA. } \\
\text { STITINJITY }\end{array}$ & 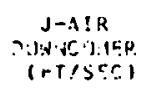 & 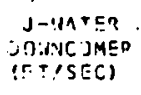 & $(.1 ; *) * 1 / 2$ & $1 \mathrm{JL} * 1 * * 1 / 2$ \\
\hline 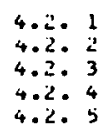 & $\begin{array}{l}0.0217 \\
0.0213 \\
0.0217 \\
2.0430 \\
0.0433\end{array}$ & $\begin{array}{l}0.2214 \\
0.2212 \\
0.2217 \\
.2212 \\
3.2212\end{array}$ & $\begin{array}{l}12.29 \\
42.29 \\
42.29 \\
42.29 \\
42.27\end{array}$ & $\begin{array}{l}0.0 \\
0.0 \\
0.0 \\
0.0 \\
i .0\end{array}$ & $\begin{array}{l}4.383 \\
4.183 \\
4.008 \\
3.267 \\
3.117\end{array}$ & $\begin{array}{l}1.017 \\
1.000 \\
1.111 \\
1.017 \\
0.949\end{array}$ & $\begin{array}{l}1.53 \\
1.53 \\
1.52 \\
3.02 \\
3.04\end{array}$ & $\begin{array}{l}1.991 \\
1.04 ! \\
1.299 \\
3.913 \\
0.776\end{array}$ & $\begin{array}{l}0.2360 \\
0.2370 \\
0.2353 \\
0.3329 \\
0.3340\end{array}$ & $\begin{array}{l}0.8202 \\
0.8012 \\
0.7843 \\
0.7080 \\
0.6916\end{array}$ \\
\hline $\begin{array}{l}4.2 .6 \\
4.207 \\
4.208 \\
4.2 .89 \\
4.2 .10\end{array}$ & $\begin{array}{l}J .0421 \\
0.0036 \\
0.0634 \\
J .0036 \\
0.003 .1\end{array}$ & $\begin{array}{l}3.220 .0 \\
0.2200 \\
3.2200 \\
0.2230 \\
0.2504\end{array}$ & $\begin{array}{l}42.29 \\
42.29 \\
12.29 \\
42.29 \\
12.29\end{array}$ & $\begin{array}{l}0.0 \\
0.0 \\
0.0 \\
0.0 \\
0.0\end{array}$ & $\begin{array}{l}2.460 \\
2.503 \\
2.412 \\
2.433 \\
2.533\end{array}$ & $\begin{array}{l}1.000 \\
1.000 \\
1.003 \\
1.001 \\
1.010\end{array}$ & $\begin{array}{l}3.52 \\
4.47 \\
4.40 \\
4.410 \\
4.4 .6\end{array}$ & $\begin{array}{l}0.714 \\
0.624 \\
0.621 \\
0.536 \\
0.231\end{array}$ & $\begin{array}{l}0.3322 \\
0.4053 \\
0.4049 \\
0.4053 \\
0.4034\end{array}$ & $\begin{array}{l}0.6635 \\
0.6204 \\
0.6085 \\
0.6111 \\
0.0235\end{array}$ \\
\hline $\begin{array}{l}4.2 .11 \\
4.2 .12 \\
4.2 .19 \\
4.2 .14 \\
4.2 .15\end{array}$ & $\begin{array}{l}0.0145 \\
0.0043 \\
0.0 .35 .3 \\
0.034 .3 \\
0.1069\end{array}$ & $\begin{array}{l}1.2212 \\
1.2203 \\
0.2208 \\
0.2201 \\
.0 .2204\end{array}$ & $\begin{array}{l}42.24 \\
+2.29 \\
42.29 \\
42.29 \\
62.29\end{array}$ & $\begin{array}{l}c . j \\
c .0 \\
0.0 \\
c .0 \\
c .0\end{array}$ & $\begin{array}{l}1.973 \\
1.230 \\
2.017 \\
2.020 \\
1.381\end{array}$ & $\begin{array}{l}1.034 \\
0.760 \\
0.992 \\
0.907 \\
1.022\end{array}$ & $\begin{array}{l}3.94 \\
5.76 \\
5.71 \\
5.76 \\
7.53\end{array}$ & $\begin{array}{l}1.192 \\
0.492 \\
0.592 \\
0.503 \\
0.344\end{array}$ & $\begin{array}{l}0.4669 \\
0.4677 \\
0.4684 \\
0.4677 \\
0.5253\end{array}$ & $\begin{array}{l}0.5505 \\
0.5453 \\
0.556 .3 \\
0.5568 \\
0.4604\end{array}$ \\
\hline $\begin{array}{l}4.2 .16 \\
6.2 .17 \\
4.2 .18 \\
4.2 .14 \\
4.2 .20\end{array}$ & $\begin{array}{l}0.1004 \\
0.106 .3 \\
0.1 .062 \\
0.1500 \\
0.1503\end{array}$ & $\begin{array}{l}3.2204 \\
3.2200 \\
0.2200 \\
j .2195 \\
.2201\end{array}$ & $\begin{array}{l}2.29 \\
12.29 \\
42.29 \\
42.29 \\
42.29\end{array}$ & $\begin{array}{l}0.0 \\
0.0 \\
0.0 \\
0.0 \\
0.0\end{array}$ & $\begin{array}{l}1.413 \\
1.428 \\
1.544 \\
0.592 \\
0.623\end{array}$ & $\begin{array}{l}0.990 \\
0.914 \\
1.075 \\
0.993 \\
0.995\end{array}$ & $\begin{array}{r}1.53 \\
7.53 \\
7.52 \\
10.01 \\
10.35\end{array}$ & $\begin{array}{l}0.357 \\
0.355 \\
3.394 \\
0.147 \\
0.155\end{array}$ & $\begin{array}{l}0.5253 \\
0.5255 \\
0.521 .5 \\
0.6229 \\
0.6226\end{array}$ & $\begin{array}{l}0.4690 \\
0.4681 \\
0.4867 \\
0.3015 \\
0.3092\end{array}$ \\
\hline $\begin{array}{l}4.2 .21 \\
4.2 .22 \\
4.2 .23 \\
4.2 .24 \\
6.2 .25\end{array}$ & $\begin{array}{l}0.130 .0 \\
0.1493 \\
0.1311 \\
0.1553 \\
0.1341\end{array}$ & $\begin{array}{l}0.2207 \\
1.2202 \\
0.21,90 \\
0.2203 \\
3.2202\end{array}$ & $\begin{array}{l}42.27 \\
42.21 \\
42.27 \\
42.27 \\
42.27\end{array}$ & $\begin{array}{l}c .0 \\
0.0 \\
c .0 \\
c . j \\
c .0\end{array}$ & $\begin{array}{l}0.637 \\
0.614 \\
0.619 \\
j .348 \\
0.258\end{array}$ & $\begin{array}{l}1.005 \\
0.906 \\
0.536 \\
1.00 .17 \\
1.0 .17\end{array}$ & $\begin{array}{l}10.65 \\
10.53 \\
10.57 \\
13.91 \\
13.73\end{array}$ & $\begin{array}{l}0.159 \\
0.153 \\
0.167 \\
0.077 \\
0.034\end{array}$ & $\begin{array}{l}0.6235 \\
0.3211 \\
0.6250 \\
0.7112 \\
0.7092\end{array}$ & $\begin{array}{l}0.3128 \\
0.3070 \\
0.3205 \\
0.2175 \\
0.1991\end{array}$ \\
\hline $\begin{array}{l}6.2 .20 \\
4.2 .27 \\
4.2 .24 \\
4.2 .24 \\
4.2 .30\end{array}$ & $\begin{array}{l}0.15333 \\
0.1974 \\
0.2371 \\
0.2391 \\
0.2303\end{array}$ & 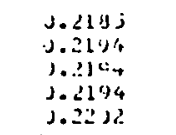 & $\begin{array}{l}42.27 \\
22.27 \\
42.27 \\
42.27 \\
42.27\end{array}$ & $\begin{array}{l}0.0 \\
0.0 \\
c .0 \\
c .0 \\
c .0\end{array}$ & $\begin{array}{l}0.205 \\
0.321 \\
0.130 \\
0.167 \\
0.137\end{array}$ & $\begin{array}{l}1.985 \\
1.059 \\
1.015 \\
1.011 \\
1.026\end{array}$ & $\begin{array}{l}13.330 \\
13.93 \\
10.99 \\
10.93 \\
16.80\end{array}$ & $\begin{array}{l}0.074 \\
0.090 \\
0.037 \\
0.041 \\
0.034\end{array}$ & $\begin{array}{l}0.7115 \\
0.7148 \\
0.7934 \\
0.7967 \\
0.7445\end{array}$ & $\begin{array}{l}0.2129 \\
0.2219 \\
0.1517 \\
0.1599 \\
0.1453\end{array}$ \\
\hline $\begin{array}{l}4.2 .31 \\
4.2 .32 \\
4.2 .33 \\
4.2 .34 \\
4.2 .35\end{array}$ & $\begin{array}{l}0.21 d 7 \\
0.2353 \\
0.2181 \\
0.2131 \\
0.2151\end{array}$ & $\begin{array}{l}0.219 d \\
0.2198 \\
0.2194 \\
1.21 \% 4 \\
0.219 \%\end{array}$ & $\begin{array}{l}42.27 \\
42.27 \\
42.27 \\
42.27 \\
42.27\end{array}$ & $\begin{array}{l}c . c \\
0.0 \\
c .0 \\
0.0 \\
0.0\end{array}$ & $\begin{array}{l}0.125 \\
0.142 \\
0.181 \\
0.212 \\
0.177\end{array}$ & $\begin{array}{l}0.976 \\
1.077 \\
1.0229 \\
1.026 \\
1.017\end{array}$ & $\begin{array}{l}15.47 \\
16.0 .3 \\
15.41 \\
15.44 \\
15.23\end{array}$ & $\begin{array}{l}0.031 \\
0.035 \\
0.045 \\
0.053 \\
0.044\end{array}$ & $\begin{array}{l}0.7523 \\
1.7800 \\
3.7510 \\
0.7513 \\
3.7463\end{array}$ & $\begin{array}{l}0.1385 \\
0.1474 \\
0.1666 \\
0.1806 \\
0.1647\end{array}$ \\
\hline $\begin{array}{l}4.2 .36 \\
4.2 .37 \\
4.2 .38 \\
4.2 .39 \\
\because .2 .4 C\end{array}$ & $\begin{array}{l}0.2151 \\
0.25 j 0 \\
0.1734 \\
0.175 . \\
0.1749\end{array}$ & $\begin{array}{l}J . ? 194 \\
1.2104 \\
3 . ? 176 \\
0.2185 \\
. .2105\end{array}$ & $\begin{array}{l}42.27 \\
41.43 \\
41.93 \\
41.93 \\
41.93\end{array}$ & $\begin{array}{l}0.0 \\
0.0 \\
\therefore .0 \\
0.0 \\
c .0\end{array}$ & $\begin{array}{l}0.214 \\
0.167 \\
0.318 \\
0.418 \\
0.431\end{array}$ & $\begin{array}{l}1.009 \\
1.039 \\
1.020 \\
1.003 \\
0.993\end{array}$ & $\begin{array}{l}15.23 \\
13.41 \\
12.39 \\
12.47 \\
12.94\end{array}$ & $\begin{array}{l}0.053 \\
0.041 \\
0.021 \\
0.194 \\
0.107\end{array}$ & $\begin{array}{l}0.7460 \\
0.71882 \\
0.6713 \\
0.67155 \\
0.6735\end{array}$ & $\begin{array}{l}0.1813 \\
0.1599 \\
0.2442 \\
0.2532 \\
0.2571\end{array}$ \\
\hline
\end{tabular}




\section{TABLE X (Contd.)}

BASELINE COUNTERCURRENT FLOW TEST GFOUP 4.2

SUMMAPYY IJF CALCULATEJ RESULTS FOR TESTS 4.2 .1 TU 4.2 .46 - 0.49 IM. DOWIVOMER GAF

\begin{tabular}{|c|c|c|c|c|c|c|c|c|c|c|}
\hline $\begin{array}{l}\text { TEST } \\
\text { NC }\end{array}$ & $\begin{array}{l}\text { MOHNCI)MER } \\
\text { AIF FLIOH } \\
\text { (LA/SEC) }\end{array}$ & $\begin{array}{l}\text { DU:NNCUMER } \\
\text { AIF. SFNSITY } \\
\text { (LJ/FT*3) }\end{array}$ & $\begin{array}{l}\text { L(IWER PLENUJiA } \\
\text { PRESSURE } \\
\text { (PSIA) }\end{array}$ & $\begin{array}{l}\text { COLO LEG } \\
\text { AR FL'JW } \\
\text { ILE/SECI }\end{array}$ & $\begin{array}{l}\text { DOWNCOMEP } \\
\text { WATER FLOW } \\
\text { (L:3/SEC) }\end{array}$ & $\begin{array}{l}\text { MATER FLOW } \\
\text { CONTINISITY }\end{array}$ & $\begin{array}{c}J-A I R \\
\text { DOWNSIUMEP } \\
\text { (FT/SEC) }\end{array}$ & $\begin{array}{l}\text { J-IATER } \\
\text { OCNAT,OMER } \\
\text { (FT/SEC) }\end{array}$ & $(J G=)=* 1,2$ & $\begin{array}{l}1 \mathrm{JL} * 1 * * 1 / 2 \\
\text {. }\end{array}$ \\
\hline $\begin{array}{l}4.2 .41 \\
4.2 .42 \\
4.2 .43 \\
4.2 .44 \\
4.22 .45\end{array}$ & $\begin{array}{l}0.1730 \\
0.1739 \\
0.13 .16 \\
0.1294 \\
0.1291\end{array}$ & $\begin{array}{l}0.2135 \\
0.2185 \\
3.2176 \\
3.2176 \\
3.2170\end{array}$ & $\begin{array}{l}41.93 \\
41.93 \\
41.93 \\
41.93 \\
41.93\end{array}$ & $\begin{array}{l}0.0 \\
0.0 \\
c .0 \\
c .0 \\
c .0\end{array}$ & $\begin{array}{l}0.396 \\
0.429 \\
0.846 \\
0.915 \\
0.938\end{array}$ & $\begin{array}{l}0.986 \\
1.035 \\
1.000 \\
1.019 \\
0.990\end{array}$ & $\begin{array}{r}12.34 \\
12.37 \\
9.32 \\
9.16 \\
9.22\end{array}$ & $\begin{array}{l}0.099 \\
0.107 \\
0.211 \\
0.228 \\
0.234\end{array}$ & $\begin{array}{l}0.6710 \\
0.6717 \\
0.5926 \\
0.5776 \\
0.5793\end{array}$ & $\begin{array}{l}0.2465 \\
0.2566 \\
0.3604 \\
0.3748 \\
0.3795\end{array}$ \\
\hline 0.2 .40 & 0.1291 & 0.2185 & 41.93 & c.u & 0.971 & 1.032 & 9.18 & 0.212 & 0.5797 & 0.3860 \\
\hline
\end{tabular}


TABLE X (Contd.)

BASELINE COUNTERCURRENT FLOW TEST GROUP 4.2

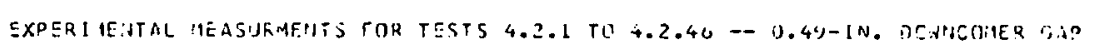

\begin{tabular}{|c|c|c|c|c|c|c|c|c|c|c|}
\hline $\begin{array}{l}\text { TEST } \\
\text { נ11 }\end{array}$ & $\begin{array}{l}R M-C: 1-1-1 \\
(G P: T)\end{array}$ & $\begin{array}{c}R M-C ! 1-2 \\
(G P: 1)\end{array}$ & $\begin{array}{l}r(-C H-1 \\
(\in P: 4)\end{array}$ & $\begin{array}{c}F T-C \cdot A-2 \\
(G P: A)\end{array}$ & $\begin{array}{c}R: A-H G-1 \\
(C F: 1)\end{array}$ & $\begin{array}{c}F M-1 G-2 \\
(C F=4 \mid\end{array}$ & $\begin{array}{c}R M-H ;-3-3 \\
(C F \cdot 1)\end{array}$ & $\begin{array}{c}P M-1 H(S-4 \\
{[C<+1)}\end{array}$ & $\begin{array}{l}{[r-H S-1} \\
(C F M)\end{array}$ & $\begin{array}{l}F ?-H C_{j}-2 \\
(C F M)^{(C)}\end{array}$ \\
\hline $\begin{array}{l}4.2 \cdot 1 \\
4.2 .2 \\
4.2 .3 \\
4.2 .4 \\
4.2 .5\end{array}$ & 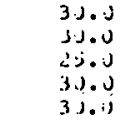 & $\begin{array}{r}10.0 \\
j .0 \\
11.0 \\
19.0 \\
0.0\end{array}$ & $\begin{array}{l}0.0 \\
0.5 \\
0.3 \\
0.0 \\
0.0\end{array}$ & $\begin{array}{l}0.0 \\
j .0 \\
0.0 \\
0.0 \\
0.0\end{array}$ & $\begin{array}{l}0.0 \\
0.0 \\
0.0 \\
0.0 \\
0.0\end{array}$ & $\begin{array}{l}0.0 \\
3.0 \\
0.0 \\
1.0 \\
0.0\end{array}$ & $\begin{array}{c}0.0 \\
0.0 \\
j .0 \\
2.0 .00 \\
20.00\end{array}$ & $\begin{array}{c}10.00 \\
10.00 \\
10.00 \\
0.0 \\
0.0\end{array}$ & $\begin{array}{l}0.0 \\
0.3 \\
0.0 \\
0.0 \\
0.0\end{array}$ & $\begin{array}{l}0.0 \\
0.0 \\
0.0 \\
0.0 \\
0.0\end{array}$ \\
\hline $\begin{array}{l}4.20 \\
4.207 \\
4.2 .3 \\
4.209 \\
4.2 .1 .0\end{array}$ & $\begin{array}{l}23.0 \\
33 . j \\
3 j .0 \\
23.0 \\
13.0\end{array}$ & $\begin{array}{r}.1 . J \\
1 . J .0 \\
j .0 \\
0.0 \\
.0 .0\end{array}$ & $\begin{array}{l}0.0 \\
0.0 \\
0.0 \\
0.0 \\
0.0\end{array}$ & $\begin{array}{l}0.0 \\
0.0 \\
0.0 \\
0.0 \\
0.0\end{array}$ & $\begin{array}{l}0.0 \\
0.0 \\
0.0 \\
0.0 \\
0.0\end{array}$ & $\begin{array}{l}J .0 \\
0.0 \\
0 . j \\
u . j \\
0 . n 1\end{array}$ & $\begin{array}{l}20.00 \\
30.30 \\
30.00 \\
30.50 \\
30.00\end{array}$ & $\begin{array}{l}0.0 \\
0.0 \\
0.0 \\
0.0 \\
0.0\end{array}$ & $\begin{array}{l}0.0 \\
3.0 \\
0.0 \\
0.0 \\
0.0\end{array}$ & $\begin{array}{l}0.0 \\
0.0 \\
0.0 \\
0.0 \\
0.0\end{array}$ \\
\hline $\begin{array}{l}4.2 .11 \\
4.2 .12 \\
4.2 .13 \\
4.2 .14 \\
4.2 .13\end{array}$ & $\begin{array}{l}30.0 \\
30.0 \\
21.0 \\
13.0 \\
31.0\end{array}$ & $\begin{array}{r}10.0 \\
10.0 \\
0.0 \\
j .0 \\
10.0\end{array}$ & $\begin{array}{l}0.0 \\
0.0 \\
0.0 \\
0.0 \\
0.0\end{array}$ & $\begin{array}{l}0.0 \\
3.0 \\
0.0 \\
3.0 \\
0.0\end{array}$ & $\begin{array}{l}u .0 \\
0.0 \\
0.0 \\
0.0 \\
0.0\end{array}$ & $\begin{array}{l}40.00 \\
40.01 \\
4.0 .10 \\
40.010 \\
5.0 .00\end{array}$ & $\begin{array}{l}0.0 \\
0.0 \\
0.0 \\
0 . j \\
0.0\end{array}$ & $\begin{array}{l}0.0 \\
3.0 \\
3.0 \\
0.0 \\
0.3\end{array}$ & $\begin{array}{l}0.0 \\
0.0 \\
0.0 \\
0.0 \\
0.0\end{array}$ & $\begin{array}{l}0.0 \\
0.0 \\
0.0 \\
0.0 \\
0.0\end{array}$ \\
\hline $\begin{array}{l}4.2 .10 \\
4.2 .17 \\
4.22 .13 \\
4.2 .11 \\
4.2 .20\end{array}$ & $\begin{array}{l}3 נ .0 \\
2 \jmath .0 \\
j .0 \\
3.0 \\
3 ! 1.3\end{array}$ & $\begin{array}{r}1.0 \\
0.0 \\
12.0 \\
10.0 \\
0.0\end{array}$ & $\begin{array}{l}0.0 \\
0.0 \\
0.0 \\
0.0 \\
0.0\end{array}$ & $\begin{array}{l}0.0 \\
0.0 \\
0.0 \\
0.0 \\
0.3\end{array}$ & $\begin{array}{c}u . u \\
u .0 \\
u .0 \\
70.00 \\
70.0 .0\end{array}$ & $\begin{array}{c}30.0 J u \\
5.0 .00 \\
5.0 .00 \\
0.0 \\
1.00\end{array}$ & $\begin{array}{l}0.3 \\
0.0 \\
0.0 \\
u . j \\
0.0\end{array}$ & $\begin{array}{l}3.0 \\
0.0 \\
3.0 \\
10.0 \\
3.0\end{array}$ & $\begin{array}{l}0.0 \\
0.0 \\
0.0 \\
1.0 \\
., 0\end{array}$ & $\begin{array}{l}0.0 \\
0.0 \\
0.0 \\
0.0 \\
0.0\end{array}$ \\
\hline $\begin{array}{l}4.2 .21 \\
4.2 .22 \\
4.22 .23 \\
4.2 .24 \\
4.25\end{array}$ & $\begin{array}{r}2 נ . J \\
3.5 \\
3.3 \\
30.3 \\
3 . .3\end{array}$ & $\begin{array}{r}J .0 \\
10.0 \\
6.0 \\
10.0 \\
1.0\end{array}$ & $\begin{array}{l}0.0 \\
0.0 \\
0.0 \\
0.0 \\
0.0\end{array}$ & $\begin{array}{l}j .0 \\
0.0 \\
0.0 \\
0.0 \\
0.0\end{array}$ & $\begin{array}{l}70.00 \\
70.00 \\
70.00 \\
90.00 \\
90.00\end{array}$ & $\begin{array}{l}u . J \\
j . j \\
0 . j \\
0.0 \\
0 . j\end{array}$ & $\begin{array}{l}0.0 \\
3.0 \\
0.0 \\
0.0 \\
0.0\end{array}$ & $\begin{array}{l}3.0 \\
0.0 \\
0.0 \\
1.0 \\
0.0\end{array}$ & $\begin{array}{l}3.0 \\
0.0 \\
0.0 \\
0.0 \\
1.0\end{array}$ & $\begin{array}{l}0.0 \\
0.0 \\
0.0 \\
0.0 \\
0.0\end{array}$ \\
\hline $\begin{array}{l}4.2 .20 \\
4.2 .27 \\
4.2200 \\
4.2 .20 \\
4.2 .30\end{array}$ & $\begin{array}{l}2.0 .0 \\
0.0 \\
3.0 .0 \\
30.0 \\
2.0 .0\end{array}$ & $\begin{array}{r}\text { J.j } \\
3.0 \\
10.0 \\
1.0 \\
0.0\end{array}$ & $\begin{array}{l}0.0 \\
0.0 \\
0.0 \\
0.0 \\
0.0\end{array}$ & $\begin{array}{l}0.1 \\
0.0 \\
0.0 \\
0.0 \\
0.0\end{array}$ & $\begin{array}{l}90.00 \\
90.00 \\
70.00 \\
70.00 \\
70.00\end{array}$ & $\begin{array}{r}0.0 \\
11.0 \\
40.00 \\
40.00 \\
+3.00\end{array}$ & $\begin{array}{l}0.0 \\
0.0 \\
0.0 \\
0.0 \\
0.0\end{array}$ & $\begin{array}{l}1.0 \\
0.0 \\
0.0 \\
3.0 \\
0.0\end{array}$ & $\begin{array}{l}3.0 \\
1.0 \\
0.0 \\
3.0 \\
3.0\end{array}$ & $\begin{array}{l}0.0 \\
0.0 \\
0.0 \\
0.0 \\
0.0\end{array}$ \\
\hline $\begin{array}{l}4.2 .31 \\
4.2 .32 \\
4.22 .33 \\
4.2 .34 \\
4.2 .35\end{array}$ & $\begin{array}{l}. .0 \\
j .0 \\
3 \mathrm{~J} .0 \\
3.00 \\
20.0\end{array}$ & $\begin{array}{r}10.0 \\
3.0 \\
10.0 \\
0.0 \\
0.0\end{array}$ & $\begin{array}{l}0.0 \\
0.0 \\
j .0 \\
j .0 \\
0.0\end{array}$ & $\begin{array}{l}0.0 \\
0.0 \\
0.0 \\
J .0 \\
1.0\end{array}$ & $\begin{array}{l}70.100 \\
70.00 \\
70.00 \\
70.00 \\
70.00\end{array}$ & $\begin{array}{l}3.3 .00 \\
40.00 \\
30.010 \\
30.00 \\
30.0 .0\end{array}$ & $\begin{array}{l}0.0 \\
0.0 \\
0.1 \\
j .0 \\
0.0\end{array}$ & $\begin{array}{l}3.0 \\
0.0 \\
3.0 \\
3.0 \\
0.1\end{array}$ & $\begin{array}{l}3.0 \\
3.0 \\
3.0 \\
0.0 \\
0.0\end{array}$ & $\begin{array}{l}0.0 \\
0.0 \\
0.0 \\
0.0 \\
0.1\end{array}$ \\
\hline $\begin{array}{l}4.2 .36 \\
4.2 .37 \\
4.2 .39 \\
4.2 .39 \\
4.2 .40\end{array}$ & $\begin{array}{l}0.0 \\
j .0 \\
3.0 .0 \\
3.0 .0 \\
2.0 .1\end{array}$ & $\begin{array}{r}10.0 \\
3.0 \\
10.0 \\
0.0 \\
0.0\end{array}$ & $\begin{array}{l}0.0 \\
0.0 \\
0.0 \\
0.0 \\
0.0\end{array}$ & $\begin{array}{l}0.0 \\
0.0 \\
0.0 \\
j .0 \\
\text { u. }\end{array}$ & $\begin{array}{l}70.00 \\
71.00 \\
80.00 \\
80.00 \\
80.00\end{array}$ & $\begin{array}{c}30.00 \\
30.00 \\
0.0 \\
0.0 \\
0.0\end{array}$ & $\begin{array}{l}0.1 \\
0.0 \\
0.0 \\
0.0 \\
0.5\end{array}$ & $\begin{array}{l}0.0 \\
0.0 \\
0.0 \\
0.0 \\
0.0\end{array}$ & $\begin{array}{l}0.0 \\
3.0 \\
0.0 \\
0.0 \\
0.0\end{array}$ & $\begin{array}{l}0.0 \\
0.0 \\
0.0 \\
0.0 \\
0.0\end{array}$ \\
\hline
\end{tabular}




\section{TABLE X (Contd.)}

BASELINE COUNTERCURRENT FLOW TEST GROUP 4.2

EXPERI'IENTAL MEASURMENTS FOR TESTS 4.2 .1 TO $4.2 .46-0.49-1 \mathrm{~N}$. UCWNCUMER SAP

\begin{tabular}{|c|c|c|c|c|c|c|c|c|c|c|}
\hline$\underset{M O}{T C S T}$ & $\begin{array}{l}R, M-C \cdot d-1 \\
(G \cup M)\end{array}$ & $\begin{array}{c}R \cdot 1-C W-2 \\
(G F M)\end{array}$ & $\begin{array}{l}F T-C W-1 \\
(G P M)\end{array}$ & $\begin{array}{c}f T-C H-2 \\
(6 P: 1)\end{array}$ & $\begin{array}{c}\text { RM- } \|(G-1 \\
(C F M)\end{array}$ & $\begin{array}{c}R ! 1-H G-2 \\
(C, F Y)\end{array}$ & $\begin{array}{c}\text { R.M-1LC-3 } \\
\left.(C F)^{\prime}\right)\end{array}$ & $\begin{array}{c}P . M-H F-4 \\
(I, F+1)\end{array}$ & $\begin{array}{c}F ?-H G-1 \\
(C F M)\end{array}$ & $\begin{array}{c}F T-H G-2 \\
(C F M)\end{array}$ \\
\hline $\begin{array}{l}4.2 .41 \\
4.2 .482 \\
4.2 .43 \\
4.2 .44 \\
4.22 .45\end{array}$ & $\begin{array}{r}1 \mathrm{J.0} \\
1.0 \\
3 \mathrm{~J} .0 \\
3 \mathrm{~J} .0 \\
2 \mathrm{~J} .0\end{array}$ & $\begin{array}{r}0.0 \\
.0 .0 \\
10.0 \\
0.0 \\
0.0\end{array}$ & $\begin{array}{l}0.0 \\
0.0 \\
0.0 \\
0.0 \\
0.0\end{array}$ & $\begin{array}{l}0.0 \\
0.0 \\
0.0 \\
0.0 \\
0.0\end{array}$ & $\begin{array}{c}80.00 \\
80.00 \\
0.0 \\
0.0 \\
0.0\end{array}$ & $\begin{array}{c}0.0 \\
0.0 \\
60.00 \\
60.00 \\
611.00\end{array}$ & $\begin{array}{l}0.0 \\
0.0 \\
0.0 \\
0.0 \\
0.0\end{array}$ & $\begin{array}{l}0.0 \\
0.0 \\
0.0 \\
0.0 \\
0.0\end{array}$ & $\begin{array}{l}0.0 \\
0.0 \\
0.0 \\
0.0 \\
0.0\end{array}$ & $\begin{array}{l}0.0 \\
0.0 \\
0.0 \\
0.0 \\
0.0\end{array}$ \\
\hline 4.2 .40 & J.J & 8.0 & 0.0 & 0.0 & 0.0 & 60.00 & J.J & 0.0 & 0.0 & $0.0^{\prime}$ \\
\hline $\begin{array}{c}\text { TEST } \\
\text { UIJ }\end{array}$ & $\begin{array}{l}p-s y-1 \\
\text { (PSig) }\end{array}$ & $\begin{array}{l}P-C W-2 \\
(P S I G)\end{array}$ & $\begin{array}{l}\text { P-H:j-1 } \\
\text { (PSiC) }\end{array}$ & $\begin{array}{l}P-H G-2 \\
\text { (PSIC) }\end{array}$ & $\begin{array}{l}P-H G-3 \\
\text { PSIGI }\end{array}$ & $\begin{array}{l}T F-(h-1 \\
(D F, F)\end{array}$ & $\begin{array}{l}\text { TF-IHS-1 } \\
\text { IDESFIT }\end{array}$ & $\begin{array}{l}\text { TF. HS }-2 \\
(D=G \mathrm{FI}\end{array}$ & $\begin{array}{l}\text { IF-HG-3 } \\
\text { (DEG FI }\end{array}$ & $\begin{array}{l}\text { P-BARO } \\
\text { (PSIAI }\end{array}$ \\
\hline $\begin{array}{l}4.2 .1 \\
4.2 .2 \\
4.2 .3 \\
4.2 .4 \\
4.2 .1\end{array}$ & $\begin{array}{l}3.0 \\
3.0 \\
3.0 \\
3.0 \\
.00\end{array}$ & $\begin{array}{l}32.5 \\
33.2 \\
32.5 \\
31.5 \\
32.0\end{array}$ & $\begin{array}{l}22.2 \\
32.7 \\
22.2 \\
31.5 \\
32.0\end{array}$ & $\begin{array}{l}0.0 \\
j .0 \\
j .0 \\
.00 \\
0.0\end{array}$ & $\begin{array}{l}0.0 \\
0.0 \\
0.0 \\
0.0 \\
0.0\end{array}$ & $\begin{array}{l}57.0 \\
53.0 \\
53.0 \\
24.0 \\
55.0\end{array}$ & $\begin{array}{l}72.0 \\
72.0 \\
72.0 \\
72.0 \\
71.0\end{array}$ & $\begin{array}{l}0.0 \\
0.0 \\
0.0 \\
0.0 \\
0.0\end{array}$ & $\begin{array}{l}0.0 \\
0.0 \\
0.0 \\
0.0 \\
0.0\end{array}$ & $\begin{array}{l}12.3 \\
12.3 \\
12.3 \\
12.3 \\
12.3\end{array}$ \\
\hline $\begin{array}{l}4.2 \cdot 0 \\
4.2 . \\
4.20 \\
4.2 .13 \\
4.2 .10\end{array}$ & $\begin{array}{l}3.0 \\
.00 \\
3.0 \\
3.0 \\
0.0\end{array}$ & $\begin{array}{l}31.5 \\
3 . .50 \\
30.0 \\
30.2 \\
30.0\end{array}$ & $\begin{array}{l}31.2 \\
30.5 \\
30.2 \\
30.5 \\
34.7\end{array}$ & $\begin{array}{l}0.0 \\
0.0 \\
1.00 \\
0.0 \\
0.0\end{array}$ & $\begin{array}{l}0.0 \\
0.0 \\
0.0 \\
0.0 \\
0.0\end{array}$ & $\begin{array}{l}50.0 \\
56.0 \\
56.0 \\
56.0 \\
53.0\end{array}$ & $\begin{array}{l}76.0 \\
75.0 \\
75.3 \\
75.0 \\
75.0\end{array}$ & $\begin{array}{l}0.0 \\
.0 .0 \\
0.3 \\
3.0 \\
0.0\end{array}$ & $\begin{array}{l}0.0 \\
0.0 \\
0.0 \\
0.0 \\
0.0\end{array}$ & $\begin{array}{l}12.3 \\
12.3 \\
12.3 \\
12.3 \\
12.3\end{array}$ \\
\hline $\begin{array}{l}4.2 .11 \\
4.2 .12 \\
4.2 .13 \\
4.2 .14 \\
4.2 .13\end{array}$ & $\begin{array}{l}1.0 \\
.00 \\
.03 \\
j .0 \\
0.0\end{array}$ & $\begin{array}{l}30.0 \\
30.5 \\
30.2 \\
30.0 \\
31.0\end{array}$ & $\begin{array}{l}30.2 \\
30.2 \\
30.5 \\
30.2 \\
3.1 .1\end{array}$ & $\begin{array}{l}0.0 \\
0.0 \\
0.0 \\
0.0 \\
0.0\end{array}$ & $\begin{array}{l}0.0 \\
0.0 \\
0.0 \\
0.0 \\
0.0\end{array}$ & $\begin{array}{l}55.0 \\
35.0 \\
55.0 \\
51.0 \\
56.0\end{array}$ & $\begin{array}{l}76.5 \\
76.5 \\
76.0 \\
76.3 \\
70.1\end{array}$ & $\begin{array}{l}0.0 \\
.0 .0 \\
0.0 \\
0.0 \\
0.0\end{array}$ & $\begin{array}{l}0.0 \\
0.0 \\
0.0 \\
0.0 \\
0.0\end{array}$ & $\begin{array}{l}12.3 \\
12.3 \\
12.3 \\
12.3 \\
12.3\end{array}$ \\
\hline $\begin{array}{l}4.2 .10 \\
4.2 .11 \\
4.2 .19 \\
4.2 .19 \\
4.22 .20\end{array}$ & $\begin{array}{l}3.0 \\
j .0 \\
0.0 \\
j .0 \\
. .0\end{array}$ & $\begin{array}{l}30.5 \\
30.2 \\
30.2 \\
30.5 \\
30.5\end{array}$ & $\begin{array}{l}20.7 \\
3.1 .7 \\
20.2 \\
30.2 \\
30.5\end{array}$ & $\begin{array}{l}0 . J \\
. .0 \\
0 . J \\
j .0 \\
j .0\end{array}$ & $\begin{array}{l}0.0 \\
0.0 \\
0.0 \\
0.0 \\
0.0\end{array}$ & $\begin{array}{l}56 . J \\
56.0 \\
30.0 \\
54.0 \\
j 3 . J\end{array}$ & $\begin{array}{l}76.0 \\
76.0 \\
70.0 \\
70.0 \\
70.0 . \\
75.0 .5\end{array}$ & $\begin{array}{l}0.0 \\
0.0 \\
0.0 \\
j .0 \\
0.0\end{array}$ & $\begin{array}{l}0.0 \\
0.0 \\
0.0 \\
0.0 \\
0.0\end{array}$ & $\begin{array}{l}12.3 \\
12.3 \\
12.3 \\
12.3 \\
12.3\end{array}$ \\
\hline $\begin{array}{l}4.2 .21 \\
4.2 .222 \\
4.2 .23 \\
4.2 .24 \\
4.22 .23\end{array}$ & $\begin{array}{l}3.0 \\
3.0 \\
3.0 \\
0.0 \\
0.0\end{array}$ & $\begin{array}{l}30: 7 \\
30.5 \\
30.7 \\
31.0 \\
30.5\end{array}$ & $\begin{array}{l}30.7 \\
30.0 \\
21.0 \\
31.0 \\
30.5\end{array}$ & $\begin{array}{l}0.0 \\
0.0 \\
0.1 \\
j . j \\
0.0\end{array}$ & $\begin{array}{l}0.0 \\
0.0 \\
0.0 \\
0.0 \\
0.0 \\
0.0\end{array}$ & $\begin{array}{l}5 j .0 \\
\text { Se. } \\
50.0 \\
56 . j \\
\text { sh.j }\end{array}$ & $\begin{array}{l}7.5 .0 \\
75.0 \\
75.0 \\
75.0 \\
75.0\end{array}$ & $\begin{array}{l}0.0 \\
0.0 \\
0.0 \\
0.0 \\
0.0\end{array}$ & $\begin{array}{l}0.0 \\
0.0 \\
0.0 \\
0.0 \\
0.0\end{array}$ & $\begin{array}{l}12.3 \\
12.3 \\
12.3 \\
12.3 \\
12.3\end{array}$ \\
\hline
\end{tabular}


TABLE X (Contd.)

BASELINE COUNTERCURRENT FLOW TEST GROUP 4.2

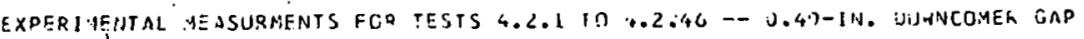

\begin{tabular}{|c|c|c|c|c|c|c|c|c|c|c|}
\hline $\begin{array}{l}\text { TEST } \\
\text { NU }\end{array}$ & $\begin{array}{l}P-C N-1 \\
(10516)\end{array}$ & $\begin{array}{l}p-c(d-2 \\
\text { psigit }\end{array}$ & $\begin{array}{l}P-H S-1 \\
(H S(G)\end{array}$ & $\begin{array}{l}\text { P-HO-2 } \\
\text { (PS:G) }\end{array}$ & $\begin{array}{l}P-1 C_{1}-3 \\
\text { (PSI:G) }\end{array}$ & 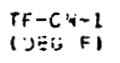 & 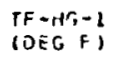 & 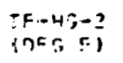 & $\begin{array}{l}\text { CE - HG -3 } \\
\text { (CSTF) }\end{array}$ & $\begin{array}{l}P-B A P D \\
\text { PSIAI }\end{array}$ \\
\hline $\begin{array}{l}4.2 .06 \\
4.2 .27 \\
4.2 .23 \\
4.2 .24 \\
4.2 .37\end{array}$ & $\begin{array}{l}J .0 \\
\jmath .0 \\
\jmath . J \\
j .0 \\
\text { J.J }\end{array}$ & $\begin{array}{l}31 . ? \\
32.0 \\
31.5 \\
32.0 \\
31 . ?\end{array}$ & $\begin{array}{l}30.7 \\
31.7 \\
21.0 \\
31.7 \\
31.0\end{array}$ & $\begin{array}{l}0.0 \\
0.0 \\
0.0 \\
0.0 \\
0.0\end{array}$ & $\begin{array}{l}0.0 \\
0.0 \\
0.0 \\
0.0 \\
.0 .0\end{array}$ & $\begin{array}{l}33.0 \\
57.0 \\
55.0 \\
54.0 \\
55.0\end{array}$ & $\begin{array}{l}73.0 \\
75.0 \\
75.0 \\
73.0 \\
73.0\end{array}$ & $\begin{array}{l}0.0 \\
2.0 \\
0.0 \\
0.0 \\
0.0\end{array}$ & $\begin{array}{l}0.0 \\
0.0 \\
0.0 \\
0.0 \\
.2 .0\end{array}$ & $\begin{array}{l}12.3 \\
12.3 \\
12.3 \\
12.3 \\
12.3\end{array}$ \\
\hline $\begin{array}{l}4.2 .31 \\
4.2 .32 \\
4.2 .33 \\
4.2 .34 \\
4.2 .35\end{array}$ & $\begin{array}{l}9.0 \\
100 \\
1.0 \\
.00 \\
1.0\end{array}$ & $\begin{array}{l}32.0 \\
30.0 \\
30.5 \\
31.2 \\
30.5\end{array}$ & $\begin{array}{l}32.0 \\
30.0 \\
31.7 \\
31.7 \\
30.5\end{array}$ & $\begin{array}{l}0.0 \\
0.3 \\
0.0 \\
0.0 \\
0.0\end{array}$ & $\begin{array}{l}0.0 \\
0.0 \\
0.0 \\
0.0 \\
0.0\end{array}$ & $\begin{array}{l}51.0 \\
50.3 \\
57.0 \\
5 \% .0 \\
5 \% .2\end{array}$ & $\begin{array}{l}70.0 \\
71.0 \\
71.0 \\
71.0 \\
71.0\end{array}$ & $\begin{array}{l}0.0 \\
0.0 \\
0.0 \\
0.0 \\
0.0\end{array}$ & $\begin{array}{l}0.0 \\
0.0 \\
0.0 \\
0.0 \\
0.0\end{array}$ & $\begin{array}{l}12.3 \\
12.3 \\
12.3 \\
12.3 \\
12.3\end{array}$ \\
\hline $\begin{array}{l}4.2 .36 \\
4.2 .37 \\
4.2 .38 \\
4.2 .39 \\
4.2 .40\end{array}$ & $\begin{array}{l}3.0 \\
j .0 \\
j .0 \\
j .0\end{array}$ & $\begin{array}{l}30.5 \\
30.5 \\
31.0 \\
31.5 \\
31.5\end{array}$ & $\begin{array}{l}30.5 \\
30.7 \\
31.0 \\
31.7 \\
11.5\end{array}$ & $\begin{array}{l}0.0 \\
. .0 \\
0.0 \\
j .0 \\
0.0\end{array}$ & $\begin{array}{l}0.0 \\
0.0 \\
0.0 \\
0.0 \\
0.0\end{array}$ & $\begin{array}{l}57.0 \\
59.0 \\
51.0 \\
50.0 \\
51,0.0\end{array}$ & $\begin{array}{l}71.0 \\
70.0 \\
70.0 \\
67.0 \\
67.0\end{array}$ & $\begin{array}{l}0.0 \\
0.0 \\
0.0 \\
0.0 \\
0.0\end{array}$ & $\begin{array}{l}3.0 \\
0.0 \\
0.0 \\
0.0 \\
0.0\end{array}$ & $\begin{array}{l}12.3 \\
11.9 \\
11.9 \\
11.9 \\
11.9\end{array}$ \\
\hline $\begin{array}{l}4.2 .41 \\
4.2 .42 \\
4.2 .43 \\
4.2 .44 \\
4.2 .45\end{array}$ & $\begin{array}{l}3.0 \\
., 0 \\
.00 \\
j .0\end{array}$ & $\begin{array}{l}31.0 \\
31.0 \\
31.5 \\
31.0 \\
30.5\end{array}$ & $\begin{array}{l}31.0 \\
31.0 \\
31.1 \\
30.2 \\
10.7\end{array}$ & $\begin{array}{l}0.0 \\
0.0 \\
0.0 \\
0.0 \\
0.0\end{array}$ & $\begin{array}{l}0.0 \\
0.0 \\
0.0 \\
0.0 \\
0.0\end{array}$ & $\begin{array}{l}50.0 \\
56.0 \\
56.0 \\
50.0 \\
\text { 5is.j }\end{array}$ & $\begin{array}{l}69.5 \\
67.5 \\
67.5 \\
67.5 \\
67.0\end{array}$ & $\begin{array}{l}0.0 \\
0.0 \\
0.0 \\
0.0 \\
0.0\end{array}$ & $\begin{array}{l}0.0 \\
0.0 \\
0.3 \\
0.0 \\
0.0\end{array}$ & $\begin{array}{l}11.9 \\
11.9 \\
11.9 \\
11.9 \\
11.9\end{array}$ \\
\hline 4.2 .46 & 1.11 & 31.2 & 30.7 & 0.J) & 0.0 & j6. & 67.J & 1.0 & 0.0 & 11.9 \\
\hline
\end{tabular}

\begin{tabular}{|c|c|c|c|c|c|c|c|c|c|}
\hline \multirow[b]{2}{*}{$\begin{array}{l}T=5 \mathrm{~T} \\
\text { inis }\end{array}$} & \multicolumn{3}{|c|}{ LCATFF PLENUAA FLON } & \multicolumn{3}{|c|}{ BYPASS FLDW } & \multirow[b]{2}{*}{$\begin{array}{l}\text { LONEP } \\
\text { PLENAM } \\
\text { LEVEL } \\
\text { IINS }\end{array}$} & \multirow[b]{2}{*}{$\begin{array}{l}-L P-1 \\
\text { ins } s\left(S_{1}\right)\end{array}$} & \multirow[b]{2}{*}{$\begin{array}{l}\text { TE. LPF-1 } \\
\text { TDES Fi }\end{array}$} \\
\hline & $\begin{array}{l}\text { INIFIAL } \\
\text { ICLM1T } \\
\text { ILd) }\end{array}$ & $\begin{array}{l}\text { FI:YAL } \\
\text { WII:SHT } \\
\text { IL:31 }\end{array}$ & $\begin{array}{l}\text { Tidt (JF } \\
\text { rolectire } \\
\text { (ses) }\end{array}$ & $\begin{array}{l}\text { IHITIAL } \\
\text { Wr:GITT } \\
\text { WLI }\end{array}$ & 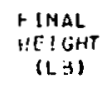 & 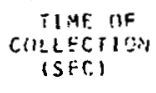 & & & \\
\hline $\begin{array}{l}4 \cdot 2 \cdot 1 \\
4.2 \cdot 2 \\
4.2 \cdot 3 \\
4.2 . \\
4.2 .5\end{array}$ & $\begin{array}{l}13.0 .0 \\
2050.5 \\
321.0 \\
351.5 \\
+31.5\end{array}$ & $\begin{array}{l}205.3 \\
131.0 \\
511.3 \\
655.5 \\
53.0\end{array}$ & $\begin{array}{l}30.0 \\
30.0 \\
30.0 \\
30.0 \\
30.0\end{array}$ & $\begin{array}{l}131.0 \\
1517.5 \\
193.3 \\
173.5 \\
247.3\end{array}$ & $\begin{array}{l}157.5 \\
158.3 \\
201.0 \\
247.3 \\
310.3\end{array}$ & $\begin{array}{l}011.01 \\
00.0 \\
30.0 \\
31,00 \\
00.0\end{array}$ & $\begin{array}{l}12.3 \\
12.0 \\
12.0 \\
12.0 \\
12.0\end{array}$ & $\begin{array}{l}30.0 \\
30.0 \\
39.0 \\
30.0 \\
30.0\end{array}$ & $\begin{array}{l}53.0 \\
56.0 \\
55.0 \\
56.0 \\
56.0\end{array}$ \\
\hline 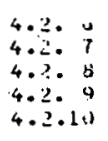 & $\begin{array}{l}133.5 \\
2 \% 1.5 \\
323.5 \\
119.5 \\
492.5\end{array}$ & $\begin{array}{l}247.0 \\
323.0 \\
41 \% .5 \\
472.5 \\
36.5\end{array}$ & $\begin{array}{l}40.0 \\
30.0 \\
40.0 \\
31.0 \\
30.0\end{array}$ & $\begin{array}{r}93.0 \\
139.5 \\
321.5 \\
429.0 \\
449.0\end{array}$ & $\begin{array}{l}120.5 \\
321.9 \\
424.0 \\
447.0 \\
430.3\end{array}$ & $\begin{array}{l}60.0 \\
60.0 \\
0.0 .3 \\
60.0 \\
B J .0\end{array}$ & $\begin{array}{l}12.01 \\
12.0 \\
12.0 \\
12.0 \\
12.0\end{array}$ & $\begin{array}{l}30.0 \\
30.0 \\
310.0 \\
31.0 \\
30.0\end{array}$ & $\begin{array}{l}59.0 \\
59.0 \\
59.0 \\
59.0 \\
59.0\end{array}$ \\
\hline $\begin{array}{l}4.2 .11 \\
4.2 .12 \\
4.2 .11 \\
4.2 .11 \\
4.815\end{array}$ & $\begin{array}{l}13.3 .0 \\
262.3 \\
231.5 \\
351.0 \\
24 ? .0\end{array}$ & $\begin{array}{l}212.0 \\
233.3 \\
350.0 \\
451.0 \\
297.3\end{array}$ & $\begin{array}{l}40.0 \\
40.0 \\
30.0 \\
5.0 .0 \\
40.0\end{array}$ & $\begin{array}{l}70.8 \\
232.1 \\
380.0 \\
424.5 \\
155.5\end{array}$ & $\begin{array}{l}242.1 \\
330.0 \\
124.0 \\
134.0 \\
344.0\end{array}$ & $\begin{array}{l}40.0 \\
6.0 .0 \\
0 J .0 \\
7.1 .1 \\
.650\end{array}$ & $\begin{array}{l}12.0 \\
12.0 \\
12.0 \\
12.0 \\
12.0\end{array}$ & $\begin{array}{l}30.0 \\
30.3 \\
310.0 \\
30.0 \\
31.0\end{array}$ & $\begin{array}{l}56.0 \\
57.0 \\
57.1 \\
57.0 \\
53.0\end{array}$ \\
\hline
\end{tabular}


TABLE X (Contd.)

BASELINE COUNTERCURRENT FLOW TEST GROUP 4.2

EXPERIAENTAL MEASURMENTS FOQ TESTS 4.2 .1 TO $4.2 .46--0.44$-IN. DOHNCOMER G.AP

\begin{tabular}{|c|c|c|c|c|c|c|c|c|c|}
\hline \multirow[b]{2}{*}{$\underset{i=S T}{T+S T}$} & \multicolumn{3}{|c|}{ LOWEP. PLENIM FLCW } & \multicolumn{3}{|c|}{ BYPASS FL!JW } & \multirow[b]{2}{*}{$\begin{array}{l}\text { LIJWER } \\
\text { PLENIIA } \\
\text { LEVEL } \\
\text { II:II }\end{array}$} & \multirow[b]{2}{*}{$\begin{array}{l}P-L P-1 \\
\text { (PSIS) }\end{array}$} & \multirow[b]{2}{*}{$\begin{array}{l}\text { TF-LP-1 } \\
\text { TDEG } F\end{array}$} \\
\hline & $\begin{array}{l}\text { INITIAL } \\
\text { WEISHT } \\
\text { IL } 3\}\end{array}$ & $\begin{array}{l}\text { FIVAL } \\
\text { WEIGHT } \\
\text { 1L31 }\end{array}$ & $\begin{array}{l}\text { TIME UF } \\
\text { COLLECTISN } \\
\text { (SEC) }\end{array}$ & $\begin{array}{l}\text { MITIAL } \\
\text { WFILHT } \\
(L B)\end{array}$ & $\begin{array}{l}\text { FIHAL } \\
\text { NEI I GHT } \\
\text { (L B B }\end{array}$ & $\begin{array}{c}\text { TIHE DF } \\
\text { CHELECTIUN } \\
\text { ISER, }\end{array}$ & & & \\
\hline $\begin{array}{l}4.2 .16 \\
4.2 .17 \\
4.2 .10 \\
4.2 .19 \\
4.2 .20\end{array}$ & $\begin{array}{l}297.3 \\
361.3 \\
420.0 \\
133.0 \\
171.5\end{array}$ & $\begin{array}{l}161.3 \\
126.0 \\
491.8 \\
171.5 \\
212.0\end{array}$ & $\begin{array}{l}45.10 \\
45.0 \\
40.0 \\
: 5.0 \\
65.0\end{array}$ & $\begin{array}{r}347.0 \\
471.5 \\
538.3 \\
36.5 \\
259.0\end{array}$ & $\begin{array}{l}471.5 \\
538.3 \\
352.3 \\
259.0 \\
382.5\end{array}$ & $\begin{array}{l}45.1 \\
53.0 \\
03.0 \\
35.0 \\
35.0\end{array}$ & $\begin{array}{l}12.0 \\
12.0 \\
12.0 \\
12.0 \\
12.0\end{array}$ & $\begin{array}{l}30.9 \\
30.0 \\
30.0 \\
30.0 \\
30.0\end{array}$ & $\begin{array}{l}53.0 \\
59.0 \\
59.0 \\
60.0 \\
57.0\end{array}$ \\
\hline $\begin{array}{l}4.2 .21 \\
4.2 .22 \\
9.2 .23 \\
4.2 .24 \\
4.2 .25\end{array}$ & $\begin{array}{l}212.3 \\
251.3 \\
293.3 \\
313.8 \\
3 \% .3\end{array}$ & $\begin{array}{l}253.3 \\
293.3 \\
336.9 \\
353.3 \\
373.8\end{array}$ & $\begin{array}{l}60.0 \\
10.0 \\
65.0 \\
62.0 \\
60.0\end{array}$ & $\begin{array}{l}3.12 .5 \\
200.0 \\
203.0 \\
255.3 \\
+40.0\end{array}$ & $\begin{array}{l}458.0 \\
243.0 \\
255.3 \\
4 \% 0.5 \\
373.5\end{array}$ & $\begin{array}{l}35.0 \\
63.0 \\
75.0 \\
35.0 \\
3 \% .0\end{array}$ & $\begin{array}{l}12.0 \\
12.0 \\
12.0 \\
12.0 \\
12.0\end{array}$ & $\begin{array}{l}30.0 \\
30.0 \\
30.0 \\
30.0 \\
3 \% .0\end{array}$ & $\begin{array}{l}57.0 \\
58.0 \\
59.0 \\
58.0 \\
59.0\end{array}$ \\
\hline $\begin{array}{l}4.2 .26 \\
4.2 .227 \\
+.2 .25 \\
4.2020 \\
4.2 .3 \mathrm{v}\end{array}$ & $\begin{array}{l}133.3 \\
1+9.3 \\
163.5 \\
173.0 \\
180.5\end{array}$ & $\begin{array}{l}141.3 \\
103.2 \\
171.0 \\
136.5 \\
144.0\end{array}$ & $\begin{array}{l}55.0 \\
60.0 \\
13.11 \\
65.0 \\
60.0\end{array}$ & $\begin{array}{r}95 . J \\
190.3 \\
199.0 \\
352.0 \\
413.5\end{array}$ & $\begin{array}{l}100.0 \\
188.0 \\
352.11 \\
173.5 \\
355.0\end{array}$ & $\begin{array}{l}15.0 \\
60.0 \\
31.0 \\
30.0 \\
30.0\end{array}$ & $\begin{array}{l}12.0 \\
12.0 \\
12.0 \\
12.0 \\
12.0\end{array}$ & $\begin{array}{l}30.0 \\
3 \% .0 \\
30.0 \\
30.0 \\
3.3 .0\end{array}$ & $\begin{array}{l}62.0 \\
63.0 \\
60.0 \\
63.0 \\
53.0\end{array}$ \\
\hline $\begin{array}{l}4.2 .31 \\
4.2 .32 \\
4.2 .33 \\
4.2 .34 \\
4.2 .33\end{array}$ & $\begin{array}{l}194.0 \\
20 . .5 \\
210.3 \\
222.3 \\
23 \% 3\end{array}$ & $\begin{array}{l}202.3 \\
213.3 \\
222.5 \\
231.3 \\
243.5\end{array}$ & $\begin{array}{l}60.0 \\
06.0 \\
65.0 \\
6.0 .3 \\
75.0\end{array}$ & $\begin{array}{l}555.0 \\
j 98.0 \\
616.5 \\
093.5 \\
593.0\end{array}$ & $\begin{array}{l}580.0 \\
\text { i2 } 0.5 \\
310.5 \\
925.05 \\
112.5\end{array}$ & $\begin{array}{l}33.0 \\
63.0 \\
35.0 \\
30.0 \\
30.0\end{array}$ & $\begin{array}{l}12.0 \\
12.0 \\
12.0 \\
12.0 \\
12.0\end{array}$ & $\begin{array}{l}39.0 \\
310.0 \\
310.0 \\
30.0 \\
30.0\end{array}$ & $\begin{array}{l}59.0 \\
59.0 \\
59.0 \\
60.0 \\
60.0\end{array}$ \\
\hline $\begin{array}{l}4.2 .30 \\
+.2 .37 \\
4.2 .39 \\
4.2 .31 \\
4.2 .413\end{array}$ & $\begin{array}{l}29.3 .5 \\
13.0 .0 \\
113.0 \\
163.3 \\
197.5\end{array}$ & $\begin{array}{l}263.5 \\
143.3 \\
100.3 \\
137.5 \\
223.5\end{array}$ & $\begin{array}{l}70.5 \\
60.0 \\
65.0 \\
70.0 \\
65 . J\end{array}$ & $\begin{array}{r}870.5 \\
46.5 \\
106.5 \\
231.5 \\
424.0\end{array}$ & $\begin{array}{l}132.0 \\
105.5 \\
291.5 \\
424.0 \\
517.3\end{array}$ & $\begin{array}{l}4.0 .0 \\
3 i .0 \\
35.03 \\
35.0 \\
4.0 .0\end{array}$ & $\begin{array}{l}12.3 \\
12.3 \\
12.0 \\
12.0 \\
12.0\end{array}$ & $\begin{array}{l}30.0 \\
30.0 \\
30.0 \\
30.0 \\
30.0\end{array}$ & $\begin{array}{l}32.0 \\
52.0 \\
63.0 \\
54.0 \\
53.0\end{array}$ \\
\hline $\begin{array}{l}4.2 .41 \\
4.2 .42 \\
4.2 .43 \\
4.2 .04 \\
4.2 .05\end{array}$ & $\begin{array}{l}223.5 \\
2.7 .3 \\
2: 29.0 \\
203.3 \\
327.3\end{array}$ & $\begin{array}{l}249.3 \\
275.0 \\
263.3 \\
327.0 \\
j 83.0\end{array}$ & $\begin{array}{l}60.0 \\
0.0 .0 \\
70.0 \\
65.0 \\
05.0\end{array}$ & $\begin{array}{l}317.3 \\
104.3 \\
107.5 \\
249.0 \\
349.0\end{array}$ & $\begin{array}{l}560.0 \\
571.0 \\
249.0 \\
349.0 \\
112.5\end{array}$ & $\begin{array}{l}50.0 \\
75.0 \\
310.0 \\
3.00 \\
35.0\end{array}$ & $\begin{array}{l}12.0 \\
12.0 \\
12.01 \\
12.0 \\
12.0\end{array}$ & $\begin{array}{l}3.0 .0 \\
30.0 \\
30.0 \\
30.5 \\
30.0\end{array}$ & $\begin{array}{l}59.0 \\
59.0 \\
60.0 \\
6.3 .3 \\
60.2 .2\end{array}$ \\
\hline 4.2 .400 & 3.13 .4 & 447.0 & 60.0 & 412.5 & 4.240 .0 & ט.jo & 12.0 & 30.0 & 5.3 .0 \\
\hline
\end{tabular}


TABLE XI

BASELINE COUNTERCURRENT FLOW TEST GROUP 5.1

TKANSPARENT VESSEL TEST DATA FOTR TESTS 5.1 .1 TO $5.1 .29-0.53-1 \%$ - COWNCOMER GAP SUMMARY OF CALCULATED RESULTS

\begin{tabular}{|c|c|c|c|c|c|c|c|c|c|c|}
\hline $\begin{array}{c}\text { TEST } \\
N \cdot \operatorname{So}\end{array}$ & $\begin{array}{l}\text { OIWNCOMER } \\
\text { AIR FLTW } \\
(L B / S E C)\end{array}$ & $\begin{array}{l}\text { IISWACTMER } \\
\text { AIP DENSITY } \\
\text { (LG/FT*3) }\end{array}$ & $\begin{array}{c}\text { LOWER PLENISM } \\
\text { PRESSUP.E } \\
\text { (PSIA) }\end{array}$ & $\begin{array}{l}\text { CCLD LEE } \\
\text { AIR FLOW } \\
\text { (L } / S \text { /SEC) }\end{array}$ & $\begin{array}{l}\text { DUWHCU:AER } \\
\text { WATEP. ELOIN } \\
\text { (LY/SEC) }\end{array}$ & $\begin{array}{l}\text { WATER FLOH } \\
\text { CIONT INUITY }\end{array}$ & $\begin{array}{c}\text { J-AIR } \\
\text { DOWNCOMER } \\
\text { (FT/SECI }\end{array}$ & $\begin{array}{l}\text { J-NATER } \\
\text { DDNMITMEP } \\
\text { (FT/SEC) }\end{array}$ & $(J G *) * * 1 / 2$ & $(J L *) * 1 / 2$ \\
\hline $\begin{array}{l}5.1: 1 \\
5.1: 2 \\
5.1: 3 \\
5.1: 4 \\
5.1: 5\end{array}$ & $\begin{array}{l}0.0082 \\
0.0086 \\
0.0079 \\
0.0165 \\
0.024 ?\end{array}$ & $\begin{array}{l}0.1177 \\
0.1184 \\
0.1184 \\
0.1179 \\
0.1151\end{array}$ & $\begin{array}{l}22.37 \\
22.37 \\
22.37 \\
22.37 \\
22.37\end{array}$ & $\begin{array}{l}0.0 \\
0.0 \\
0.0 \\
0.0 \\
0.0\end{array}$ & $\begin{array}{l}4.580 \\
3.588 \\
4.330 \\
3.7 .10 \\
3.442\end{array}$ & $\begin{array}{l}0.457 \\
0.860 \\
0.916 \\
1.003 \\
0.981 .\end{array}$ & $\begin{array}{l}1.14 \\
1.30 \\
1.00 \\
2.10 \\
3.21\end{array}$ & $\begin{array}{l}1.103 \\
0.864 \\
1.343 \\
0.891 \\
0.329\end{array}$ & $\begin{array}{l}0.1639 \\
0.1676 \\
0.1611 \\
0.2329 \\
0.2365\end{array}$ & $\begin{array}{l}0.8055 \\
0.7165 \\
0.7271 \\
0.7276 \\
0.7017\end{array}$ \\
\hline $\begin{array}{l}5.1 \cdot 6 \\
5.1 \cdot 7 \\
5.1 \cdot 8 \\
5.1 \cdot 9 \\
5.1 .10\end{array}$ & $\begin{array}{l}0.0240 \\
0.0247 \\
0.0237 \\
0.0319 \\
0.0473\end{array}$ & $\begin{array}{l}0.1179 \\
0.1177 \\
3.1179 \\
0.1179 \\
0.1179\end{array}$ & $\begin{array}{l}22.37 \\
22.37 \\
22.37 \\
22.37 \\
22.37\end{array}$ & $\begin{array}{l}0.0 \\
0.0 \\
c .0 \\
0.0 \\
c .0\end{array}$ & $\begin{array}{l}3.530 \\
3.080 \\
2.397 \\
2.575 \\
2.390\end{array}$ & $\begin{array}{l}1.008 \\
0.390 \\
0.935 \\
0.971 \\
0.987\end{array}$ & $\begin{array}{l}3.05 \\
3.15 \\
3.02 \\
4.06 \\
3.99\end{array}$ & $\begin{array}{l}0.943 \\
0.742 \\
0.695 \\
0.520 \\
0.576\end{array}$ & $\begin{array}{l}0.2809 \\
0.2851 \\
0.2791 \\
0.3249 \\
0.3934\end{array}$ & $\begin{array}{l}0.7076 \\
0.6638 \\
0.6427 \\
0.6070 \\
0.5847\end{array}$ \\
\hline $\begin{array}{l}5.1 .11 \\
5.1 .12 \\
5.1 .13 \\
5.1 .14 \\
5.1 .15\end{array}$ & $\begin{array}{l}0.0622 \\
0.0792 \\
0.1130 \\
0.1475 \\
0.1751\end{array}$ & $\begin{array}{l}0.1179 \\
0.1119 \\
0.1179 \\
0.1179 \\
0.1179\end{array}$ & $\begin{array}{l}22.37 \\
2.37 \\
22.37 \\
22.37 \\
22.37\end{array}$ & $\begin{array}{l}0.0 \\
0.0 \\
0.0 \\
0.0 \\
0.0\end{array}$ & $\begin{array}{l}1.958 \\
1.745 \\
0.879 \\
0.417 \\
0.150\end{array}$ & $\begin{array}{l}0.978 \\
1.039 \\
1.036 \\
1.038 \\
i .959\end{array}$ & $\begin{array}{r}1.93 \\
9.96 \\
14.40 \\
18.79 \\
22.31\end{array}$ & $\begin{array}{l}3.972 \\
1.423 \\
0.212 \\
0.1 .30 \\
0.036\end{array}$ & $\begin{array}{l}0.4526 \\
0.5072 \\
0.6099 \\
0.6966 \\
0.7590\end{array}$ & $\begin{array}{l}0.5293 \\
0.4998 \\
0.3545 \\
0.2442 \\
0.1465\end{array}$ \\
\hline $\begin{array}{l}5.1 .16 \\
5.1 .17 \\
5.1 .18 \\
5.1 .19 \\
5.1 .20\end{array}$ & $\begin{array}{l}0.1954 \\
0.021 \% \\
0.0437 \\
0.1293 \\
0.1397\end{array}$ & $\begin{array}{l}0.1179 \\
0.1131 \\
0.1133 \\
0.1170 \\
0.1180\end{array}$ & $\begin{array}{l}22.37 \\
22.34 \\
22.34 \\
22.34 \\
22.34\end{array}$ & $\begin{array}{l}0.0 \\
0.0 \\
0.0 \\
0.0 \\
0.0\end{array}$ & $\begin{array}{l}0.106 \\
4.291 \\
2.035 \\
0.500 \\
0.350\end{array}$ & $\begin{array}{l}1.052 \\
1.314 \\
1.910 \\
1.008 \\
1.0140\end{array}$ & $\begin{array}{r}24.99 \\
2.79 \\
3.54 \\
16.50 \\
17.79\end{array}$ & $\begin{array}{l}0.026 \\
1.033 \\
0.635 \\
0.120 \\
0.034\end{array}$ & $\begin{array}{l}0.3018 \\
0.2635 \\
0.3797 \\
0.6525 \\
0.6780\end{array}$ & $\begin{array}{l}0.1233 \\
0.7835 \\
0.6140 \\
0.2675 \\
0.2238\end{array}$ \\
\hline $\begin{array}{l}5.1 .21 \\
5.1 .22 \\
5.1 .23 \\
5.1 .24 \\
5.1 .25\end{array}$ & $\begin{array}{l}0.0295 \\
0.0293 \\
0.2037 \\
0.0289 \\
0.0294\end{array}$ & $\begin{array}{l}0.1111 \\
0.1200 \\
0.1173 \\
0.1170 \\
0.1195\end{array}$ & $\begin{array}{l}22.13 \\
22.63 \\
22.42 \\
22.13 \\
22.63\end{array}$ & $\begin{array}{l}0.0 \\
0.0 \\
0.0 \\
c .0 \\
0.0\end{array}$ & $\begin{array}{l}3.750 \\
3.767 \\
0.008 \\
4.127 \\
3.307\end{array}$ & $\begin{array}{l}-.295 \\
0.900 \\
1.014 \\
0.999 \\
1.003\end{array}$ & $\begin{array}{r}3.74 \\
3.6 ? \\
26.10 \\
3.69 \\
3.70\end{array}$ & $\begin{array}{l}0.323 \\
0.207 \\
0.302 \\
0.274 \\
0.311\end{array}$ & $\begin{array}{l}0.3119 \\
0.3091 \\
0.8199 \\
0.3086 \\
0.3100\end{array}$ & $\begin{array}{l}0.7325 \\
0.7341 \\
0.0345 \\
0.7684 \\
0.6940\end{array}$ \\
\hline $\begin{array}{l}5.1 .26 \\
5.1 .27 \\
5.1 .28 \\
5.1 .29\end{array}$ & $\begin{array}{l}0.0294 \\
0.0295 \\
0.0295 \\
0.0295\end{array}$ & $\begin{array}{l}0.1193 \\
0.1211 \\
0.1200 \\
0.1188\end{array}$ & $\begin{array}{l}22.63 \\
22.88 \\
22.63 \\
22.38\end{array}$ & $\begin{array}{l}c .0 \\
0.0 \\
c .0 \\
c .0\end{array}$ & $\begin{array}{l}2.850 \\
3.150 \\
3.667 \\
3.950\end{array}$ & $\begin{array}{l}1.022 \\
0.932 \\
0.965 \\
0.959\end{array}$ & $\begin{array}{l}3.71 \\
3.56 \\
3.70 \\
3.73\end{array}$ & $\begin{array}{l}0.636 \\
0.759 \\
0.383 \\
0.051\end{array}$ & $\begin{array}{l}0.3104 \\
0.3094 \\
0.3103 \\
0.3111\end{array}$ & $\begin{array}{l}0.6385 \\
0.6713 \\
0.7243 \\
0.7517\end{array}$ \\
\hline
\end{tabular}


TABLE XI (Contd.)

BASELINE COUNTERCURRENT FLOW TEST GROUP 5.1

EXPER IMENTAL MEASURMENTS FOR TESTS 5.1 .1 TO $5.1 .29-0.53-$ IN. DUWNCOMER GAP

\begin{tabular}{|c|c|c|c|c|c|c|c|c|c|c|}
\hline $\begin{array}{c}\text { TEST } \\
\text { NO }\end{array}$ & $\begin{array}{c}R M-C W-1 \\
(G P M)\end{array}$ & $\begin{array}{c}R M-C W-2 \\
(G P M)\end{array}$ & $\begin{array}{c}F T-C H-1 \\
(G P M)\end{array}$ & $\begin{array}{c}F T-C H-2 \\
(G P M)\end{array}$ & $\begin{array}{c}R M-H G-1 \\
(C F M)\end{array}$ & $\begin{array}{c}R M-H G-2 \\
(C F M)\end{array}$ & $\begin{array}{c}R M-H G-3 \\
(C F M)\end{array}$ & $\begin{array}{c}R M-H F,-4 \\
(C F M)\end{array}$ & $\begin{array}{c}F T-H G-1 \\
(C F M)\end{array}$ & $\begin{array}{c}F T-H G-2 \\
(C F M)\end{array}$ \\
\hline  & $\begin{array}{l}29.5 \\
30.0 \\
25.0 \\
30.0 \\
30.0\end{array}$ & $\begin{array}{r}10.0 \\
0.0 \\
10.0 \\
0.0 \\
10.0\end{array}$ & $\begin{array}{l}0.0 \\
0.0 \\
0.0 \\
0.0 \\
0.0\end{array}$ & $\begin{array}{l}0.0 \\
0.0 \\
0.0 \\
0.0 \\
0.0\end{array}$ & $\begin{array}{l}0.0 \\
0.0 \\
0.0 \\
0.0 \\
0.0\end{array}$ & $\begin{array}{l}0.0 \\
0.0 \\
0.0 \\
0.0 \\
0.0\end{array}$ & $\begin{array}{c}0.0 \\
0.0 \\
0.0 \\
10.00 \\
15.00\end{array}$ & $\begin{array}{l}5.00 \\
5.00 \\
5.00 \\
0.0 \\
0.0\end{array}$ & $\begin{array}{l}0.0 \\
0.0 \\
0.0 \\
0.0 \\
0.0\end{array}$ & $\begin{array}{l}0.0 \\
0.0 \\
0.0 \\
0.0 \\
0.0\end{array}$ \\
\hline $\begin{array}{l}5.1 \cdot 6 \\
5.1 \cdot 7 \\
5.1 \cdot 9 \\
5.1 \cdot 9 \\
5.1 .10\end{array}$ & $\begin{array}{l}25.0 \\
28.0 \\
25.0 \\
25.8 \\
20.0\end{array}$ & $\begin{array}{l}0.0 \\
0.0 \\
0.0 \\
0.0 \\
0.0\end{array}$ & $\begin{array}{l}0.0 \\
0.0 \\
0.0 \\
0.0 \\
0.0\end{array}$ & $\begin{array}{l}0.0 \\
0.0 \\
0.0 \\
0.0 \\
0.0\end{array}$ & $\begin{array}{l}0.0 \\
0.0 \\
0.0 \\
0.0 \\
0.0\end{array}$ & $\begin{array}{c}0.0 \\
0.0 \\
0.0 \\
20.00 \\
30.00\end{array}$ & $\begin{array}{c}15.00 \\
15.00 \\
15.00 \\
0.0 \\
0.0\end{array}$ & $\begin{array}{l}0.0 \\
0.0 \\
0.0 \\
0.0 \\
0.0\end{array}$ & $\begin{array}{l}0.0 \\
0.0 \\
0.0 \\
0.0 \\
0.0\end{array}$ & $\begin{array}{l}0.0 \\
0.0 \\
0.0 \\
0.0 \\
0.0\end{array}$ \\
\hline $\begin{array}{l}5.1 .11 \\
5.1 .12 \\
5.1 .13 \\
5.1 .14 \\
5.1 .15\end{array}$ & $\begin{array}{r}20.0 \\
15.0 \\
12.0 \\
0.0 \\
5.0\end{array}$ & $\begin{array}{l}0.0 \\
0.0 \\
0.0 \\
0.0 \\
0.0\end{array}$ & $\begin{array}{l}0.0 \\
0.0 \\
0.0 \\
0.0 \\
\text { J.0 }\end{array}$ & $\begin{array}{l}0.0 \\
0.0 \\
0.0 \\
0.0 \\
0.0\end{array}$ & $\begin{array}{l}0.0 \\
0.0 \\
70.00 \\
90.00 \\
60.00\end{array}$ & $\begin{array}{c}40.00 \\
50.00 \\
0.0 \\
0.0 \\
50.00\end{array}$ & $\begin{array}{l}0.0 \\
0.0 \\
0.0 \\
0.0 \\
0.0\end{array}$ & $\begin{array}{l}0.0 \\
0.0 \\
0.0 \\
0.0 \\
0.0\end{array}$ & $\begin{array}{l}0.0 \\
0.0 \\
0.0 \\
0.0 \\
0.0\end{array}$ & $\begin{array}{l}0.0 \\
0.0 \\
0.0 \\
0.0 \\
0.0\end{array}$ \\
\hline $\begin{array}{l}5.1 .16 \\
5.1 .17 \\
5.1 .13 \\
5.1 .19 \\
5.1 .20\end{array}$ & $\begin{array}{l}5.0 \\
0.0 \\
j .0 \\
0.0 \\
0.0\end{array}$ & $\begin{array}{l}0.0 \\
0.0 \\
0.0 \\
0.0 \\
0.0\end{array}$ & $\begin{array}{l}0.0 \\
0.0 \\
0.0 \\
0.0 \\
0.0\end{array}$ & $\begin{array}{l}0.0 \\
50.0 \\
40.2 \\
24.9 \\
10.5\end{array}$ & $\begin{array}{c}60.00 \\
0.0 \\
0.0 \\
60.00 \\
0.0\end{array}$ & $\begin{array}{l}60.00 \\
0.0 \\
0.0 \\
0.0 \\
0.0\end{array}$ & $\begin{array}{c}0.0 \\
10.00 \\
20.00 \\
0.0 \\
0.0\end{array}$ & $\begin{array}{l}0.0 \\
0.0 \\
0.0 \\
0.0 \\
0.0\end{array}$ & $\begin{array}{c}0.0 \\
0.0 \\
0.0 \\
0.0 \\
15.77\end{array}$ & $\begin{array}{l}0.0 \\
0.0 \\
0.0 \\
0.0 \\
0.0\end{array}$ \\
\hline $\begin{array}{l}5.1 .21 \\
5.1 .22 \\
5.2 .23 \\
5.1 .24 \\
5.1 .25\end{array}$ & $\begin{array}{l}0.0 \\
0.0 \\
0.0 \\
0.0 \\
0.0\end{array}$ & $\begin{array}{l}0.0 \\
0.0 \\
0.0 \\
0.0 \\
0.0\end{array}$ & $\begin{array}{r}80.0 \\
70.0 \\
0.0 \\
59.0 \\
0.0\end{array}$ & $\begin{array}{r}0.0 \\
0.0 \\
10.2 \\
0.0 \\
39.4\end{array}$ & $\begin{array}{l}0.0 \\
0.0 \\
0.0 \\
0.0 \\
0.0\end{array}$ & $\begin{array}{l}0.0 \\
0.0 \\
0.0 \\
0.0 \\
0.0\end{array}$ & $\begin{array}{c}13.40 \\
13.30 \\
0.0 \\
13.40 \\
13.40\end{array}$ & $\begin{array}{l}0.0 \\
0.0 \\
0.0 \\
0.0 \\
0.0\end{array}$ & $\begin{array}{c}0.0 \\
0.0 \\
22.82 \\
0.0 \\
0.0\end{array}$ & $\begin{array}{l}0.0 \\
0.0 \\
0.0 \\
0.0 \\
0.0\end{array}$ \\
\hline $\begin{array}{l}5.1 .26 \\
5.1 .27 \\
5.1 .23 \\
5.1 .29\end{array}$ & $\begin{array}{l}0.0 \\
0.0 \\
0.0 \\
j .0\end{array}$ & $\begin{array}{l}0.0 \\
0.0 \\
0.0 \\
0.0\end{array}$ & $\begin{array}{r}0.0 \\
0.0 \\
90.0 \\
60.0\end{array}$ & $\begin{array}{r}24.3 \\
50.0 \\
0.0 \\
0.0\end{array}$ & $\begin{array}{l}0.0 \\
0.0 \\
0.0 \\
0.0\end{array}$ & $\begin{array}{l}0.0 \\
j .0 \\
0.0 \\
0.0\end{array}$ & $\begin{array}{l}13.40 \\
13.43 \\
13.40 \\
13.43\end{array}$ & $\begin{array}{l}0.0 \\
0.0 \\
0.0 \\
0.0\end{array}$ & $\begin{array}{l}0.0 \\
0.0 \\
0.0 \\
0.0\end{array}$ & $\begin{array}{l}0.0 \\
0.0 \\
0.0 \\
0.0\end{array}$ \\
\hline
\end{tabular}


TABLE XI (Contd.)

BASELINE COUNTERCURRENT FLOW TEST GROUP 5.1

EXPERIIERTTAL MEASUR:AENTS FOR TESTS 5.1.1 TO $5.1 .29-0.53-1$ - OU. DONNCDMER GAP

\begin{tabular}{|c|c|c|c|c|c|c|c|c|c|c|}
\hline $\begin{array}{l}\text { TEST } \\
\text { NIS }\end{array}$ & $\begin{array}{l}P-C(y-1 \\
(P S I G)\end{array}$ & $\begin{array}{l}P-C W-2 \\
(P S I C)\end{array}$ & $\begin{array}{l}P-\mid 16-1 \\
(P S I G)\end{array}$ & $\begin{array}{l}P-H G-2 \\
(P S I G)\end{array}$ & $\begin{array}{l}P-H(G-3 \\
(P S I G)\end{array}$ & $\begin{array}{l}T F-C ! N-1 \\
(D E F, F) .\end{array}$ & $\begin{array}{l}\text { TF-IHS-1 } \\
\text { (DEG F) }\end{array}$ & $\begin{array}{l}T C-40-2 \\
(D E S F)\end{array}$ & $\begin{array}{l}T F-H S-3 \\
(D E G F)\end{array}$ & $\begin{array}{l}\text { P-BARก } \\
\text { (PSIA) }\end{array}$ \\
\hline $\begin{array}{l}5.1 \cdot 1 \\
5.1 \cdot 2 \\
5.1 \cdot 3 \\
5.1 \cdot 4 \\
5.1 \cdot 5\end{array}$ & $\begin{array}{l}0.0 \\
0.0 \\
j .0 \\
0.0 \\
0.0\end{array}$ & $\begin{array}{l}13.0 \\
13.0 \\
11.0 \\
13.0 \\
12.0\end{array}$ & $\begin{array}{l}12.5 \\
15.0 \\
11.0 \\
13.0 \\
13.0\end{array}$ & $\begin{array}{l}0.0 \\
0.0 \\
0.0 \\
0.0 \\
0.0\end{array}$ & $\begin{array}{l}0.0 \\
0.0 \\
0.0 \\
0.0 \\
0.0\end{array}$ & $\begin{array}{l}60.0 \\
59.0 \\
6.1 .3 \\
60.0 \\
61.0\end{array}$ & $\begin{array}{l}65.0 \\
65.0 \\
05.0 \\
65.0 \\
65.0\end{array}$ & $\begin{array}{l}0.0 \\
0.0 \\
0.0 \\
0.0 \\
0.0\end{array}$ & $\begin{array}{l}0.0 \\
0.0 \\
0.0 \\
.00 \\
0.0\end{array}$ & $\begin{array}{l}12.4 \\
12.4 \\
12.4 \\
12.4 \\
12.4\end{array}$ \\
\hline $\begin{array}{l}5.1 .6 \\
5.1 \cdot 7 \\
5.1 \cdot 9 \\
5.1 .9 \\
5.1 .10\end{array}$ & $\begin{array}{l}0.0 \\
0.0 \\
0.0 \\
0.0 \\
0.0\end{array}$ & $\begin{array}{l}12.2 \\
11.5 \\
11.0 \\
11.0 \\
11.0\end{array}$ & $\begin{array}{l}11.5 \\
13.0 \\
11.0 \\
11.5 \\
10.5\end{array}$ & $\begin{array}{l}0.0 \\
0.0 \\
0.0 \\
0.0 \\
0.0\end{array}$ & $\begin{array}{l}0.0 \\
0.0 \\
0.0 \\
0.0 \\
0.0\end{array}$ & $\begin{array}{l}61.0 \\
62.0 \\
61.0 \\
61.0 \\
61.0\end{array}$ & $\begin{array}{l}65.0 \\
66.0 \\
67.0 \\
67.0 \\
67.0\end{array}$ & $\begin{array}{l}0.0 \\
0.0 \\
0.0 \\
0.0 \\
0.0\end{array}$ & $\begin{array}{l}0.0 \\
0.0 \\
0.0 \\
0.0 \\
0.0\end{array}$ & $\begin{array}{l}12.4 \\
12.4 \\
12.4 \\
12.4 \\
12.4\end{array}$ \\
\hline $\begin{array}{l}5.1 .11 \\
5.1 .12 \\
5.1 .13 \\
5.1 .14 \\
5.1 .15\end{array}$ & $\begin{array}{l}i j . j \\
j . j \\
j .0 \\
0.0 \\
0.0\end{array}$ & $\begin{array}{r}10.0 \\
9.5 \\
10.0 \\
10.0 \\
10.0\end{array}$ & $\begin{array}{l}10.0 \\
10.0 \\
11.0 \\
11.0 \\
10.5\end{array}$ & $\begin{array}{l}0.0 \\
0.0 \\
0.0 \\
0.0 \\
0.0\end{array}$ & $\begin{array}{l}0.0 \\
0.0 \\
0.0 \\
0.0 \\
0.0\end{array}$ & $\begin{array}{l}61.0 \\
62.0 \\
61.0 \\
61.0 \\
62.0\end{array}$ & $\begin{array}{l}67.0 \\
67.0 \\
67.0 \\
67.0 \\
67.0\end{array}$ & $\begin{array}{l}0.0 \\
0.0 \\
0.0 \\
0.0 \\
0.0\end{array}$ & $\begin{array}{l}0.0 \\
0.0 \\
0.0 \\
0.0 \\
0.0\end{array}$ & $\begin{array}{l}12.4 \\
12.4 \\
12.4 \\
12.4 \\
12.4\end{array}$ \\
\hline $\begin{array}{l}5.1 .16 \\
5.1 .17 \\
5.1 .18 \\
5.1 .19 \\
5.1 .21\end{array}$ & $\begin{array}{r}0.0 \\
10.2 \\
10.0 \\
0.7 \\
9.5\end{array}$ & $\begin{array}{r}10.5 \\
0.0 \\
j .0 \\
0.0 \\
0.00\end{array}$ & $\begin{array}{l}11.5 \\
10.0 \\
10.0 \\
10.8 \\
10.0\end{array}$ & $\begin{array}{r}0.0 \\
99.5 \\
38.0 \\
95.0 \\
95.0\end{array}$ & $\begin{array}{r}0.0 \\
33.5 \\
33.0 \\
32.0 \\
33.5\end{array}$ & $\begin{array}{l}63.0 \\
50.0 \\
40.5 \\
47.5 \\
49.0\end{array}$ & $\begin{array}{l}68.0 \\
78.5 \\
73.0 \\
76.0 \\
77.1\end{array}$ & $\begin{array}{r}0.0 \\
73.5 \\
73.5 \\
77.0 \\
35.0\end{array}$ & $\begin{array}{l}0.0 \\
75.0 \\
74.0 \\
76.0 \\
79.5\end{array}$ & $\begin{array}{l}12.4 \\
12.3 \\
12.3 \\
12.3 \\
12.3\end{array}$ \\
\hline $\begin{array}{l}5.1 .21 \\
5.1 .22 \\
5.1 .23 \\
5.1 .24 \\
5.1 .25\end{array}$ & $\begin{array}{r}10.2 \\
10.5 \\
2.2 \\
9.7 \\
9.7\end{array}$ & $\begin{array}{l}0.0 \\
0.0 \\
0.0 \\
0.0 \\
0.0\end{array}$ & $\begin{array}{r}9.4 \\
10.0 \\
10.1 \\
9.7 \\
10.0\end{array}$ & $\begin{array}{r}134.0 \\
103.0 \\
58.5 \\
106.0 \\
105.0\end{array}$ & $\begin{array}{l}33.5 \\
33.5 \\
33.5 \\
32.0 \\
33.5\end{array}$ & $\begin{array}{l}49.5 \\
48.5 \\
54.0 \\
48.0 \\
51.0\end{array}$ & $\begin{array}{r}78.0 \\
10.0 .0 \\
87.0 \\
80.0 \\
80.0\end{array}$ & $\begin{array}{r}0.0 \\
0.0 \\
0.0 \\
0.0 \\
0.0\end{array}$ & $\begin{array}{l}72.5 \\
71.0 \\
93.0 \\
76.0 \\
75.0\end{array}$ & $\begin{array}{l}12.4 \\
12.4 \\
12.4 \\
12.4 \\
12.4\end{array}$ \\
\hline $\begin{array}{l}5 \cdot 1 \cdot 26 \\
5 \cdot 1 \cdot 27 \\
5 \cdot 1.23 \\
5 \cdot 1 \cdot 29\end{array}$ & $\begin{array}{l}10.0 \\
1.0 .7 \\
1.0 .1 \\
10.5\end{array}$ & $\begin{array}{l}0.0 \\
0.0 \\
0.0 \\
0.0\end{array}$ & $\begin{array}{l}10.3 \\
10.7 \\
10.3 \\
10.1\end{array}$ & $\begin{array}{r}104.5 \\
104.0 \\
83.5 \\
103.0\end{array}$ & $\begin{array}{l}33.5 \\
33.5 \\
33.5 \\
33.5\end{array}$ & $\begin{array}{l}52.0 \\
49.5 \\
40.5 \\
48.0\end{array}$ & $\begin{array}{l}78.5 \\
78.0 \\
77.0 \\
77.5\end{array}$ & $\begin{array}{l}0.0 \\
0.0 \\
0.0 \\
.0\end{array}$ & $\begin{array}{l}73.5 \\
72.0 \\
71.0 \\
71.0\end{array}$ & $\begin{array}{l}12.4 \\
12.4 \\
12.4 \\
12.4\end{array}$ \\
\hline
\end{tabular}


TABLE XI (Contd.)

BASELINE COUNTERCURRENT FLOW TEST GROUP 5.1

EXPERIMENTAL MEASURMENTS FOR TESTS 5.1.1 TO 5.1.29-- 0.53-IN: DOHNCOMER GAP

\begin{tabular}{|c|c|c|c|c|c|c|c|c|c|}
\hline \multirow{2}{*}{$\begin{array}{l}\text { TEST } \\
\text { NO }\end{array}$} & \multicolumn{2}{|c|}{ LOWER PLENUM } & FLOH & \multicolumn{3}{|c|}{ BYPASS FLOW } & \\
\hline & $\begin{array}{l}\text { INITIAL } \\
\text { WE IGHT } \\
\text { (LB) }\end{array}$ & $\begin{array}{l}\text { FINAL } \\
\text { WEIGHT } \\
\text { (LB) }\end{array}$ & $\begin{array}{l}\text { TIME OF } \\
\text { COLLECTION } \\
\text { (SEC) }\end{array}$ & $\begin{array}{l}\text { INITIAL } \\
\text { WE I GHTT } \\
\text { (LB) }\end{array}$ & $\begin{array}{l}\text { FINAL } \\
\text { WEIGHT } \\
\text { (LB) }\end{array}$ & $\begin{array}{l}\text { TIME OF } \\
\text { COLLECTION } \\
\text { ISECI }\end{array}$ & $\begin{array}{l}\text { PUWER } \\
\text { PLENUM } \\
\text { LEVEL } \\
\text { (IN) }\end{array}$ & $\begin{array}{l}P-L P-1 \\
(P S I G)\end{array}$ & $\begin{array}{l}\text { TF-LP-1 } \\
\text { (CEG F) }\end{array}$ \\
\hline $\begin{array}{l}5.1 \cdot 1 \\
5.1 \cdot 2 \\
5.1 \cdot 3 \\
5.1 \cdot 4 \\
5.1 \cdot 5\end{array}$ & $\begin{array}{l}133.0 \\
247.5 \\
334.0 \\
453.0 \\
284.0\end{array}$ & $\begin{array}{l}247.5 \\
339.0 \\
453.8 \\
546.3 \\
387.3\end{array}$ & $\begin{array}{l}25.0 \\
25.5 \\
26.5 \\
25.0 \\
30.0\end{array}$ & $\begin{array}{r}83.5 \\
114.0 \\
114.0 \\
135.0 \\
164.0\end{array}$ & $\begin{array}{l}114.0 \\
114.0 \\
135.0 \\
164.0 \\
244.5\end{array}$ & $\begin{array}{r}45.0 \\
0.0 \\
50.0 \\
60.0 \\
40.0\end{array}$ & $\begin{array}{l}12.0 \\
12.0 \\
12.0 \\
12.0 \\
12.0\end{array}$ & $\begin{array}{l}10.0 \\
10.0 \\
10.0 \\
10.0 \\
10.0\end{array}$ & $\begin{array}{l}53.0 \\
50.0 \\
50.0 \\
52.0 \\
62.0\end{array}$ \\
\hline $\begin{array}{l}5.1 .11 \\
5.1 .12 \\
5.1 .13 \\
5.1 .14 \\
5.1 .15\end{array}$ & $\begin{array}{l}379.0 \\
437.8 \\
250.5 \\
287.3 \\
299.8\end{array}$ & $\begin{array}{l}437.8 \\
491.0 \\
287.3 \\
299.8 \\
308.0\end{array}$ & $\begin{array}{l}30.0 \\
30.5 \\
35.0 \\
30.0 \\
55.0\end{array}$ & $\begin{array}{l}369.5 \\
400.0 \\
467.5 \\
491.5 \\
521.0\end{array}$ & $\begin{array}{l}400.0 \\
421.0 \\
491.5 \\
521.0 \\
544.3\end{array}$ & $\begin{array}{l}40.0 \\
50.0 \\
30.0 \\
40.0 \\
410.0\end{array}$ & $\begin{array}{l}12.0 \\
12.0 \\
12.0 \\
12.0 \\
12.0\end{array}$ & $\begin{array}{l}10.0 \\
10.0 \\
10.0 \\
10.0 \\
10.0\end{array}$ & $\begin{array}{l}52.0 \\
52.0 \\
52.0 \\
52.0 \\
52.0\end{array}$ \\
\hline $\begin{array}{l}5.1 .21 \\
5.1 .22 \\
5.1 .23 \\
5.1 .24 \\
5.1 .23\end{array}$ & $\begin{array}{l}173.5 \\
154.0 \\
176.2 \\
140.0 \\
22 \% .0\end{array}$ & $\begin{array}{l}236.0 \\
380.0 \\
178.0 \\
262.5 \\
320.0\end{array}$ & $\begin{array}{r}30.0 \\
60.0 \\
180.0 \\
29.0 \\
30.0\end{array}$ & $\begin{array}{l}420.0 \\
434.0 \\
273.0 \\
101.5 \\
200.5\end{array}$ & $\begin{array}{l}205.0 \\
780.0 \\
354.0 \\
223.5 \\
266.5\end{array}$ & $\begin{array}{l}30.0 \\
60.0 \\
10.0 \\
30.0 \\
30.0\end{array}$ & $\begin{array}{l}12.0 \\
12.0 \\
12.0 \\
12.0 \\
12.0\end{array}$ & $\begin{array}{r}9.7 \\
10.2 \\
10.0 \\
9.7 \\
10.2\end{array}$ & $\begin{array}{l}50.0 \\
49.0 \\
56.0 \\
49.0 \\
51.0\end{array}$ \\
\hline $\begin{array}{l}5.1 .26 \\
5.1 .27 \\
5.1 .28 \\
5.1 .29\end{array}$ & $\begin{array}{l}199.5 \\
344.0 \\
177.0 \\
157.0\end{array}$ & $\begin{array}{l}205.0 \\
439.5 \\
287.0 \\
275.5\end{array}$ & $\begin{array}{l}30.0 \\
30.0 \\
30.0 \\
30.0\end{array}$ & $\begin{array}{l}190.0 \\
161.0 \\
369.0 \\
466.5\end{array}$ & $\begin{array}{l}209.0 \\
271.0 \\
531.0 \\
590.0\end{array}$ & $\begin{array}{l}30.0 \\
30.0 \\
30.0 \\
30.0\end{array}$ & $\begin{array}{l}12.0 \\
12.0 \\
12.0 \\
12.0\end{array}$ & $\begin{array}{l}10.2 \\
10.5 \\
10.2 \\
10.0\end{array}$ & $\begin{array}{l}52.0 \\
50.0 \\
49.0 \\
49.5\end{array}$ \\
\hline
\end{tabular}


TABLE XII

BASELINEE COUNTERCURRENT FLOW TEST GROUP 6.1

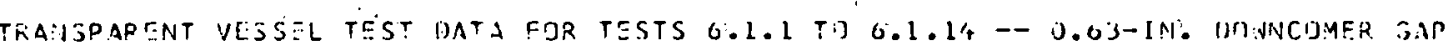
SUMMARY JF CALCIULATER RESULTS

\begin{tabular}{|c|c|c|c|c|c|c|c|c|c|c|}
\hline $\begin{array}{l}\text { TEST } \\
N 0^{-}\end{array}$ & $\begin{array}{l}\text { OMNNCOHEI } \\
\text { ALR FLOU } \\
\text { (LB/SEC) }\end{array}$ & 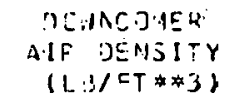 & $\begin{array}{c}\text { LUWER PLENIJH } \\
\text { PU!SSLKE } \\
(P S I A)\end{array}$ & $\begin{array}{l}\text { COLI) LEG } \\
\text { AIR FLIU) } \\
\text { (LU/SES) }\end{array}$ & 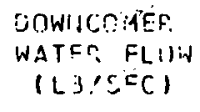 & $\begin{array}{l}\text { AATER FLIJW } \\
\text { DIJTINIJITY }\end{array}$ & $\begin{array}{c}J-1 ! ? \\
\text { DOW:NETMER } \\
\text { (FTISEC) }\end{array}$ & $\begin{array}{l}\text { J-NATEK } \\
\text { OTHNCTMED } \\
\text { (ETASTE) }\end{array}$ & $(J G *) * 1 / 2$ & $(J \mathrm{~L} *)=\mathrm{L}^{\prime} / 2$ \\
\hline $\begin{array}{l}0.1: 1 \\
6.1 \cdot 2 \\
6.1: 3 \\
0.1: 3 \\
0.1: 5\end{array}$ & $\begin{array}{l}0.0233 \\
0.0233 \\
0.0073 \\
0.0163 \\
0.0160\end{array}$ & $\begin{array}{l}0.1160 \\
0.1162 \\
0.1102 \\
0.1104 \\
0.1162\end{array}$ & $\begin{array}{l}22.29 \\
22.29 \\
22.29 \\
22.29 \\
22.29\end{array}$ & $\begin{array}{l}0.0 \\
0.0 \\
0.0 \\
0.0 \\
0.0\end{array}$ & $\begin{array}{l}4.210 \\
3.953 \\
5.397 \\
4.550 \\
4.762\end{array}$ & $\begin{array}{l}1.0199 \\
1.016 \\
0.390 \\
0.946 \\
0.934\end{array}$ & $\begin{array}{l}2.79 \\
2.53 \\
0.84 \\
1.70 \\
1.71\end{array}$ & $\begin{array}{l}0.340 \\
0.797 \\
1.377 \\
0.9110 \\
0.352\end{array}$ & $\begin{array}{l}0.2426 \\
0.2429 \\
0.1405 \\
0.2049 \\
0.2009\end{array}$ & $\begin{array}{l}0.6771 \\
0.6594 \\
0.7669 \\
0.7047 \\
0.7210\end{array}$ \\
\hline 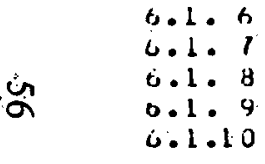 & $\begin{array}{l}0.0312 \\
0.0411 \\
0.0632 \\
0.0625 \\
0.076 .9\end{array}$ & 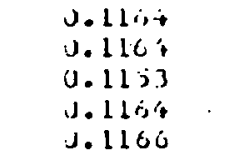 & $\begin{array}{l}22.29 \\
22.29 \\
22.29 \\
22.29 \\
22.29\end{array}$ & $\begin{array}{l}0.0 \\
0.0 \\
0.0 \\
0.0 \\
0.0\end{array}$ & $\begin{array}{l}3.340 \\
2.712 \\
2.300 \\
2.203 \\
2.110\end{array}$ & $\begin{array}{l}1.005 \\
1.016 \\
1.011 \\
1.027 \\
1.024\end{array}$ & $\begin{array}{l}3.35 \\
5.05 \\
6.34 \\
6.713 \\
8.22\end{array}$ & $\begin{array}{l}0.668 \\
0.542 \\
0.560 \\
0.457 \\
0.422\end{array}$ & $\begin{array}{l}0.2510 \\
0.3449 \\
0.4007 \\
0.3973 \\
0.4404\end{array}$ & $\begin{array}{l}0.6038 \\
0.5441 \\
0.5528 \\
0.499 .2 \\
0.4799\end{array}$ \\
\hline $\begin{array}{l}6.1 .11 \\
0.1 .12 \\
6.1 .13 \\
0.1 .14\end{array}$ & $\begin{array}{r}0.1097 \\
1.1414 \\
0.1637 \\
0.2019\end{array}$ & $\begin{array}{l}J .1164 \\
3.1159 \\
J .1155 \\
3.1153\end{array}$ & $\begin{array}{l}22.29 \\
22.29 \\
22.29 \\
22.29\end{array}$ & $\begin{array}{l}0.0 \\
0.0 \\
0.0 \\
0.0\end{array}$ & $\begin{array}{l}1.840 \\
0.925 \\
0.410 \\
0.210\end{array}$ & $\begin{array}{r}1.003 \\
1.037 \\
1.000 \\
1.013\end{array}$ & $\begin{array}{r}11.76 \\
15.22 \\
19.22 \\
21.9 .5\end{array}$ & $\begin{array}{l}0.373 \\
9.135 \\
0.396 \\
0.144 .0\end{array}$ & $\begin{array}{l}0.5265 \\
0.5985 \\
0.6541 \\
0.7161\end{array}$ & $\begin{array}{l}0.4542 \\
0.3178 \\
0.2284 \\
0.1479\end{array}$ \\
\hline
\end{tabular}




\section{TABLE XII (Contd.)}

BASELINE COUNTERCURRENT FLOW TEST GROUP 6.1

EXPER IMENTAL IALASIJRMENTS FOR TESTS 6.1 .1 TO $6.1 .14-0.03-1$. COWNCOMER GAP

\begin{tabular}{|c|c|c|c|c|c|c|c|c|c|c|}
\hline $\begin{array}{c}\text { TEST } \\
\text { HI }\end{array}$ & $\begin{array}{c}R M-C(1-1 \\
(G P: 1)\end{array}$ & $\begin{array}{c}R M-C(y-2 \\
(G P M)\end{array}$ & $\begin{array}{c}F T-C(N-1 \\
\left(C^{\prime} M\right)\end{array}$ & $\begin{array}{c}F T-C W-2 \\
(G P M)\end{array}$ & $\begin{array}{c}R M-H G-1 \\
(C F M)\end{array}$ & $\begin{array}{c}R M-H C-2 \\
(C F M)\end{array}$ & $\begin{array}{c}R M-H G-3 \\
(C F M)\end{array}$ & $\begin{array}{l}R M-H r-4 \\
(C F M)\end{array}$ & $\begin{array}{c}F T-H G-1 \\
(C F M)\end{array}$ & $\begin{array}{c}F T-H G-2 \\
(C F M)\end{array}$ \\
\hline $\begin{array}{l}6.1 \cdot 1 \\
6.1 \cdot 2 \\
6.1 \cdot 5 \\
6.1 .5 \\
6.1 \cdot 5\end{array}$ & $\begin{array}{l}30.0 \\
30.0 \\
3.0 .0 \\
3.0 .0 \\
3.0 .0\end{array}$ & $\begin{array}{r}10.0 \\
5.0 \\
10.0 \\
10.0 \\
10.0\end{array}$ & $\begin{array}{l}0.0 \\
0.0 \\
0.0 \\
0.0 \\
0.0\end{array}$ & $\begin{array}{l}0.0 \\
0.0 \\
0.0 \\
0.0 \\
0.0\end{array}$ & $\begin{array}{l}0.0 \\
0.0 \\
0.0 \\
0.0 \\
0.0\end{array}$ & $\begin{array}{c}15.00 \\
15.00 \\
0.0 \\
0.0 \\
0.0\end{array}$ & $\begin{array}{l}0.0 \\
0.0 \\
5.00 \\
10.00 \\
10.00\end{array}$ & $\begin{array}{l}0.0 \\
0.0 \\
0.0 \\
0.0 \\
0.0\end{array}$ & $\begin{array}{l}0.0 \\
0.0 \\
0.0 \\
0.0 \\
0.0\end{array}$ & $\begin{array}{l}0.0 \\
0.0 \\
0.0 \\
0.0 \\
0.0\end{array}$ \\
\hline $\begin{array}{l}6.1 .6 \\
6.1 .7 \\
6.1 .9 \\
6.1 .9 \\
6.1 .1 .5\end{array}$ & $\begin{array}{l}30.0 \\
25.0 \\
20.0 \\
2.0 .0 \\
20.0\end{array}$ & $\begin{array}{l}0.0 \\
0.0 \\
0.0 \\
0.0 \\
0.0\end{array}$ & $\begin{array}{l}0.0 \\
0.0 \\
0.0 \\
0.0 \\
0.0 .\end{array}$ & $\begin{array}{l}0.0 \\
0.0 \\
0.0 \\
0.0 \\
0.0\end{array}$ & $\begin{array}{l}0.0 \\
0.0 \\
0.0 \\
0.0 \\
0.0\end{array}$ & $\begin{array}{l}20.00 \\
30.00 \\
40.00 \\
40.00 \\
50.00\end{array}$ & $\begin{array}{l}0.0 \\
0.0 \\
0.0 \\
0.0 \\
0.0\end{array}$ & $\begin{array}{l}0.0 \\
0.0 \\
0.0 \\
0.0 \\
0.0\end{array}$ & $\begin{array}{l}0.0 \\
0.0 \\
0.0 \\
0.0 \\
0.0\end{array}$ & $\begin{array}{l}0.0 \\
0.0 \\
0.0 \\
0.0 \\
0.0\end{array}$ \\
\hline $\begin{array}{l}6.1 .11 \\
0.1 .12 \\
6.1 .13 \\
6.1 .14\end{array}$ & $\begin{array}{r}13.0 \\
1.0 .0 \\
3.0 \\
0.0\end{array}$ & $\begin{array}{l}0.0 \\
\dot{j} .0 \\
5.0 \\
5.0\end{array}$ & $\begin{array}{l}0.0 \\
0.0 \\
0.0 \\
0.0\end{array}$ & $\begin{array}{l}0.0 \\
0.0 \\
0.0 \\
0.0\end{array}$ & $\begin{array}{l}0.0 \\
90.00 \\
60.05 \\
70.00\end{array}$ & $\begin{array}{c}7.000 \\
0.00 \\
50.00 \\
6.5 .00\end{array}$ & $\begin{array}{l}0.0 \\
0.0 \\
0.0 \\
0.0\end{array}$ & $\begin{array}{l}0.0 \\
0.0 \\
0.0 \\
0.0\end{array}$ & $\begin{array}{l}0.0 \\
0.0 \\
0.0 \\
0.0\end{array}$ & $\begin{array}{l}0.0 \\
0.0 \\
0.0 \\
0.0\end{array}$ \\
\hline $\begin{array}{l}\text { TEST } \\
\text { III }\end{array}$ & $\begin{array}{l}P-C W-1 \\
(P S I G)\end{array}$ & $\begin{array}{l}P-C N-2 \\
\text { (PSIG) }\end{array}$ & $\begin{array}{l}P-H G-1 \\
(P S I G)\end{array}$ & $\begin{array}{l}P-H(;-2 \\
(P S I G)\end{array}$ & $\begin{array}{l}P-H G-S \\
\text { (PSIG) }\end{array}$ & $\begin{array}{l}T F-C W-1 \\
(U E S G F)\end{array}$ & $\begin{array}{l}T F-H ;-1 \\
(D E G F)\end{array}$ & $\begin{array}{l}T F-H G=2 \\
(O F S F)\end{array}$ & $\begin{array}{l}T F-H G-3 \\
\text { (DEG F) }\end{array}$ & $\begin{array}{l}\text { P-BARO } \\
\text { (PSIA) }\end{array}$ \\
\hline 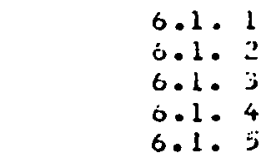 & $\begin{array}{l}1.0 \\
1.0 \\
1.0 \\
.00 \\
1.0\end{array}$ & $\begin{array}{l}11.0 \\
10.1 \\
11.0 \\
14.0 \\
12.5\end{array}$ & $\begin{array}{l}10.7 \\
10.7 \\
11.0 \\
14.0 \\
12.0\end{array}$ & $\begin{array}{l}0.0 \\
0.0 \\
0.0 \\
0.0 \\
0.0\end{array}$ & $\begin{array}{l}0.0 \\
0.0 \\
0.0 \\
0.0 \\
0.0\end{array}$ & $\begin{array}{l}55.3 \\
53.0 \\
53.0 \\
57.0 \\
53.5\end{array}$ & $\begin{array}{l}70.10 \\
76.1 \\
77.5 \\
75.3 \\
76.0\end{array}$ & $\begin{array}{l}0.0 \\
0.0 \\
0.0 \\
0.0 \\
0.0\end{array}$ & $\begin{array}{l}0.0 \\
0.0 \\
0.0 \\
0.0 \\
0.0\end{array}$ & $\begin{array}{l}12.3 \\
12.3 \\
12.3 \\
12.3 \\
12.3\end{array}$ \\
\hline $\begin{array}{l}0.1 .6 \\
0.1 .7 \\
6.1 .1 \\
6.1 .19 \\
6.1 .1 .)\end{array}$ & $\begin{array}{l}j .0 \\
j .0 \\
.00 \\
j .0 \\
. .0\end{array}$ & $\begin{array}{l}1.3 .5 \\
11.0 \\
11.2 \\
11.5 \\
10.7\end{array}$ & $\begin{array}{l}11.0 \\
11.0 \\
11.2 \\
10.7 \\
10.0\end{array}$ & $\begin{array}{l}0.0 \\
0.0 \\
0.0 \\
0.01 \\
3.0\end{array}$ & $\begin{array}{l}0.0 \\
0.0 \\
0.0 \\
0.0 \\
0.0\end{array}$ & $\begin{array}{l}57.0 \\
57.0 \\
61.0 \\
56.0 \\
50.0\end{array}$ & $\begin{array}{l}76.0 \\
76.0 \\
77.0 \\
79.0 \\
84.0\end{array}$ & $\begin{array}{l}0.0 \\
0.0 \\
0.0 \\
0.0 \\
0.0\end{array}$ & $\begin{array}{l}0.0 \\
0.0 \\
0.0 \\
0.0 \\
0.0\end{array}$ & $\begin{array}{l}12.3 \\
12.3 \\
12.3 \\
12.3 \\
12.3\end{array}$ \\
\hline $\begin{array}{l}6.1 .11 \\
6.1 .12 \\
6.1 .1 .3 \\
6.1 .14\end{array}$ & $\begin{array}{l}1.0 \\
j .0 \\
j .0 \\
.00\end{array}$ & $\begin{array}{l}10.7 \\
1.0 .5 \\
10.2 \\
10.1\end{array}$ & $\begin{array}{l}10.1 \\
10.2 \\
10.2 \\
10.7\end{array}$ & $\begin{array}{l}3.0 \\
3.0 \\
0.0 \\
0.0\end{array}$ & $\begin{array}{l}0.0 \\
0.0 \\
0.0 \\
0.0\end{array}$ & $\begin{array}{l}57.0 \\
53.0 \\
5 \% .0 \\
59.0\end{array}$ & $\begin{array}{r}92.5 \\
94.0 \\
100.5 \\
103.5\end{array}$ & $\begin{array}{l}0.0 \\
0.0 \\
0.0 \\
0.0\end{array}$ & $\begin{array}{l}0.0 \\
0.0 \\
0.0 \\
0.0\end{array}$ & $\begin{array}{l}12.3 \\
12.3 \\
12.3 \\
12.3\end{array}$ \\
\hline
\end{tabular}




\section{TABLE XII (Contd.)}

BASELINE COUNTERCURRENT FLOW TEST GROUP 6.1

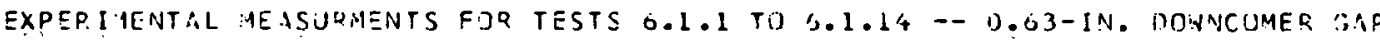

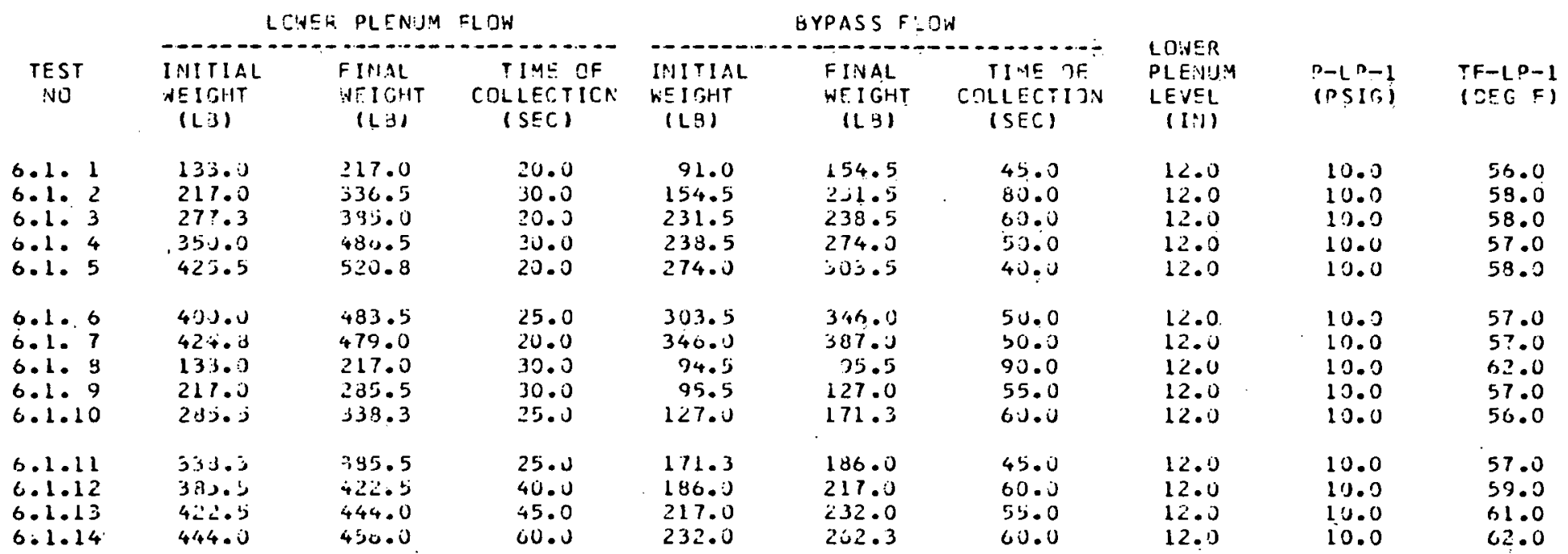


TABLE XIII

BASELINE COUNTERCURRENT FLOW TEST GROUP 7.1

TRANSPARENT VESSEL TEST DATA FIRR TESTS 7.1.1 TU 7.1.60 - - U.70-IN. DOWNCOMER GAP SUMMARY OF CALCULATED RESULTS

\begin{tabular}{|c|c|c|c|c|c|c|c|c|c|c|}
\hline $\begin{array}{l}\text { TEST } \\
\text { NO }\end{array}$ & $\begin{array}{l}\text { UO:ANCOMER } \\
\text { AIR. FLUWH } \\
\text { ILB/SEC, }\end{array}$ & $\begin{array}{l}\text { UDWNCOHER } \\
\text { AIR CENSITY } \\
\text { (LU/FT*3) }\end{array}$ & $\begin{array}{l}\text { LOHER PLENUM } \\
\text { PRESSURS } \\
\text { (PSIA) }\end{array}$ & $\begin{array}{l}\text { CELD LEG } \\
\text { AIR CLN' } \\
\text { (LB/SEC) }\end{array}$ & $\begin{array}{l}\text { POWIVIOMER } \\
\text { WATEP ELIUW } \\
(L H / S \subseteq C)\end{array}$ & $\begin{array}{l}\text { HATEP FLOA } \\
\text { C:JNTINUITY }\end{array}$ & $\begin{array}{c}J-A I R \\
\text { DONF.OMER } \\
\text { (FT/SEC) }\end{array}$ & $\begin{array}{l}\text { J-WHATER } \\
\text { DOWNEOMER } \\
\text { (FT/SEC) }\end{array}$ & $(J G *) * * 1 / 2$ & $(J(*) * * 1 / 2$ \\
\hline $\begin{array}{ll}7.1 .1 & 1 \\
7.1: 2 \\
7.1: 5 \\
7.1: & 4 \\
7.1 . & 5\end{array}$ & $\begin{array}{l}J .0158 \\
0.0236 \\
0.0315 \\
0.0388 \\
0.0467\end{array}$ & $\begin{array}{l}0.1163 \\
0.1163 \\
0.1163 \\
0.1163 \\
0.1163\end{array}$ & $\begin{array}{l}22.24 \\
22.24 \\
22.24 \\
22.24 \\
22.24\end{array}$ & $\begin{array}{l}0.0 \\
0.0 \\
0.0 \\
0.0 \\
0.0\end{array}$ & $\begin{array}{l}5.650 \\
5.487 \\
5.450 \\
5.300 \\
5.188\end{array}$ & $\begin{array}{l}1.013 \\
0.942 \\
1.009 \\
0.993 \\
1.010\end{array}$ & $\begin{array}{l}1.15 \\
1.73 \\
2.30 \\
2.34 \\
3.41\end{array}$ & $\begin{array}{l}0.770 \\
0.749 \\
0.743 \\
0.722 \\
0.707\end{array}$ & $\begin{array}{l}0.1605 \\
0.1962 \\
0.2265 \\
0.2516 \\
0.2758\end{array}$ & $\begin{array}{l}0.6306 \\
0.6215 \\
0.6193 \\
0.6107 \\
0.6042\end{array}$ \\
\hline $\begin{array}{l}7.1 .1 \\
7.1 .9 \\
7.1: 8 \\
7.1 .8 \\
7.1 .10\end{array}$ & $\begin{array}{l}0.0471 \\
0.0551 \\
0.0554 \\
0.065 ; \\
0.0652\end{array}$ & $\begin{array}{l}0.1163 \\
0.1163 \\
0.1133 \\
.01192 \\
.11135\end{array}$ & $\begin{array}{l}22.24 \\
22.24 \\
22.44 \\
22.47 \\
22.47\end{array}$ & $\begin{array}{l}0.0 \\
0.0 \\
0.0 \\
.0 .0 \\
c .0\end{array}$ & $\begin{array}{l}5.070 \\
5.108 \\
4.887 \\
5.020 \\
4.717\end{array}$ & $\begin{array}{l}1.011 \\
1.007 \\
1.007 \\
1.002 \\
1.005\end{array}$ & $\begin{array}{l}3.49 \\
4.03 \\
3.93 \\
4.71 \\
4.68\end{array}$ & $\begin{array}{l}0.671 \\
0.636 \\
0.666 \\
0.684 \\
0.643\end{array}$ & $\begin{array}{l}0.2797 \\
0.2997 \\
0.2993 \\
0.3255 \\
0.3245\end{array}$ & $\begin{array}{l}0.5973 \\
0.5996 \\
0.5865 \\
0.5944 \\
0.5762\end{array}$ \\
\hline $\begin{array}{l}7.1 .11 \\
7.1 .12 \\
7.1 .13 \\
1.1 .14 \\
7.1 .15\end{array}$ & $\begin{array}{l}0.0904 \\
0.0310 \\
0.0794 \\
0.0982 \\
0.0981\end{array}$ & $\begin{array}{l}0.1135 \\
0.1183 \\
3.1175 \\
0.1175 \\
0.1175\end{array}$ & $\begin{array}{l}22.47 \\
22.47 \\
22.47 \\
22.47 \\
22.47\end{array}$ & $\begin{array}{l}0.0 \\
0.0 \\
c .0 \\
0.0 \\
c .0\end{array}$ & $\begin{array}{l}4.054 \\
4.012 \\
4.117 \\
4.730 \\
4.281\end{array}$ & $\begin{array}{l}0.997 \\
0.9132 \\
0.990 \\
0.999 \\
0.976\end{array}$ & $\begin{array}{l}5.77 \\
5.46 \\
5.75 \\
0.51 \\
1.14\end{array}$ & $\begin{array}{l}0.662 \\
0.601 \\
0.561 \\
3.579 \\
0.584\end{array}$ & $\begin{array}{l}0.3604 \\
0.3631 \\
0.3589 \\
0.3777 \\
0.4002\end{array}$ & $\begin{array}{l}0.5848 \\
0.5573 \\
0.5383 \\
0.5461 \\
0.5493\end{array}$ \\
\hline $\begin{array}{l}7.1 .16 \\
7.1 .11 \\
7.1 .18 \\
7.1 .11 \\
7.1 .20\end{array}$ & $\begin{array}{l}J .0973 \\
0.1100 \\
j .1144 \\
0.1143 \\
0.1148\end{array}$ & $\begin{array}{l}0.1175 \\
j .1171 \\
j .1171 \\
0.1171 \\
0.1171\end{array}$ & $\begin{array}{l}22.47 \\
22.38 \\
22.38 \\
22.38 \\
22.38\end{array}$ & $\begin{array}{l}c .0 \\
c .0 \\
c . u \\
c .0 \\
0.0\end{array}$ & $\begin{array}{l}4.100 \\
4.310 \\
3.60 \mathrm{~J} \\
3.9510 \\
3.71 .7\end{array}$ & $\begin{array}{l}1.099 \\
1.047 \\
1.001 \\
1.027 \\
1.010\end{array}$ & $\begin{array}{l}7.1 .3 \\
8.33 \\
3.31 \\
3.31 \\
8.34\end{array}$ & $\begin{array}{l}0.553 \\
0.557 \\
0.502 \\
0.539 \\
0.516\end{array}$ & $\begin{array}{l}0.3983 \\
0.4342 \\
0.4311 \\
0.4309 \\
0.4320\end{array}$ & $\begin{array}{l}0.5372 \\
0.5508 \\
0.5089 \\
0.5273 \\
0.5163\end{array}$ \\
\hline $\begin{array}{l}7.1 .21 \\
7.1 .22 \\
7.1 .23 \\
7.1 .24 \\
7.1 .25\end{array}$ & $\begin{array}{l}0.1305 \\
0.132 .3 \\
0.13 .02 \\
0.1302 \\
0.1448\end{array}$ & $\begin{array}{l}0.1171 \\
3.1171 \\
0.1171 \\
. .1171 \\
.01169\end{array}$ & $\begin{array}{l}22.33 \\
22.313 \\
22.33 \\
22.38 \\
22.35\end{array}$ & $\begin{array}{l}c .0 \\
0.0 \\
c .0 \\
c .0 \\
0.0\end{array}$ & $\begin{array}{l}3.133 \\
3.9110 \\
3.270 \\
3.1003 \\
2.407\end{array}$ & $\begin{array}{l}0.737 \\
0.931 \\
1.002 \\
0.741 \\
1.007\end{array}$ & $\begin{array}{r}9.49 \\
5.65 \\
9.46 \\
9.46 \\
10.53\end{array}$ & $\begin{array}{l}0.413 \\
0.409 \\
0.443 \\
0.410 \\
0.329\end{array}$ & $\begin{array}{l}0.4605 \\
0.4646 \\
0.4600 \\
0.4500 \\
0.4852\end{array}$ & $\begin{array}{l}0.4620 \\
0.4595 \\
0.4797 \\
0.4601 \\
0.4116\end{array}$ \\
\hline $\begin{array}{l}7.1 .26 \\
7.1 .27 \\
7.1 .28 \\
7.1 .29 \\
7.1 .30\end{array}$ & $\begin{array}{l}3.1715 \\
0.1934 \\
0.2103 \\
0.2317 \\
0.2543\end{array}$ & $\begin{array}{l}. .1160 \\
.01160 \\
0.116 .1 \\
0.116 .3 \\
.11600\end{array}$ & $\begin{array}{l}22.35 \\
22.32 \\
22.32 \\
22.32 \\
22.32\end{array}$ & $\begin{array}{l}0.0 \\
c .0 \\
0.0 \\
0.0 \\
0.0\end{array}$ & $\begin{array}{l}1.933 \\
1.393 \\
1.2915 \\
1.015 \\
0.319\end{array}$ & $\begin{array}{l}1.004 \\
1.045 \\
1.031 \\
1.011 \\
0.952\end{array}$ & $\begin{array}{l}12.43 \\
14.39 \\
15.32 \\
10.39 \\
18.52\end{array}$ & $\begin{array}{l}0.263 \\
0.120 \\
0.174 \\
0.147 \\
0.120\end{array}$ & $\begin{array}{l}0.5281 \\
0.5609 \\
0.5453 \\
0.6142 \\
0.6432\end{array}$ & $\begin{array}{l}0.3689 \\
0.3131 \\
0.2996 \\
0.2751 \\
0.2447\end{array}$ \\
\hline $\begin{array}{l}7.1 .31 \\
7.1 .32 \\
7.1 .33 \\
7.1 .34 \\
7.1 .35\end{array}$ & $\begin{array}{l}0.2631 . \\
0.0213 \\
0.0215 \\
0.0219 \\
j .0423\end{array}$ & $\begin{array}{l}0.1163 \\
0.1170 \\
0.1171 \\
0.1195 \\
0.1202\end{array}$ & $\begin{array}{l}2: .32 \\
21.94 \\
21.92 \\
22.12 \\
22.42\end{array}$ & $\begin{array}{l}0.0 \\
0.0 \\
0.0 \\
0.0 \\
0.0\end{array}$ & $\begin{array}{r}3.0 .00 \\
11.9511 \\
9.167 \\
8.000 \\
7.633\end{array}$ & $\begin{array}{l}1.014 \\
0.9199 \\
0.971 \\
0.974 \\
0.936\end{array}$ & $\begin{array}{r}19.10 \\
1.59 \\
1.54 \\
1.59 \\
2.49\end{array}$ & $\begin{array}{l}0.109 \\
1.330 \\
1.250 \\
1.091 \\
1.04 !\end{array}$ & $\begin{array}{l}0.6543 \\
0.1947 \\
0.1871 \\
0.1993 \\
0.2004\end{array}$ & $\begin{array}{l}0.2373 \\
0.3175 \\
0.8036 \\
0.7507 \\
0.7333\end{array}$ \\
\hline $\begin{array}{l}7.1 .30 \\
7.1 .37 \\
7.1 .38 \\
7.1 .39 \\
7.1 .40\end{array}$ & $\begin{array}{l}0.0432 \\
0.0432 \\
0.1073 \\
0.1292 \\
0.1751\end{array}$ & $\begin{array}{l}0.1230 \\
. .1201 \\
j .1190 \\
j .1191 \\
0.1191\end{array}$ & $\begin{array}{l}22.92 \\
22.42 \\
22.42 \\
22.42 \\
22.42\end{array}$ & $\begin{array}{l}0.0 \\
0.0 \\
0.0 \\
c .01 \\
c .0\end{array}$ & $\begin{array}{l}8.430 \\
6.536 \\
5.133 \\
3.733 \\
2.267\end{array}$ & $\begin{array}{l}1.191 \\
3.913 \\
1.3 .03 \\
0.4013 \\
1.010\end{array}$ & $\begin{array}{r}2.27 \\
3.06 \\
7.6 ? \\
9.23 \\
12.51\end{array}$ & $\begin{array}{l}1.1160 \\
0.998 \\
. .731 \\
0.599 \\
0.339\end{array}$ & $\begin{array}{l}0.2619 \\
0.2635 \\
0.4165 \\
0.4564 \\
0.5314\end{array}$ & $\begin{array}{l}0.7693 \\
0.6812 \\
0.6016 \\
0.5129 \\
0.3996\end{array}$ \\
\hline
\end{tabular}


TABLE XIII (Contd.)

BASELINE COUNTERCURRENT FLOW TEST GROUP 7.1

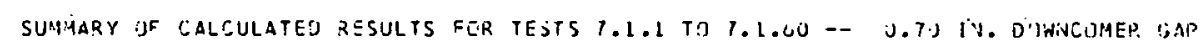

\begin{tabular}{|c|c|c|c|c|c|c|c|c|c|c|}
\hline $\begin{array}{l}\text { TEST } \\
\text { NO }\end{array}$ & 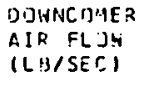 & $\begin{array}{l}\text { DUWNCOMER } \\
\text { MIR CENSITY } \\
\text { ILB/FTEZI }\end{array}$ & $\begin{array}{l}\text { LOWEQ PLENUM } \\
\text { PRESSURE } \\
\text { (PSIA) }\end{array}$ & $\begin{array}{l}\text { CDL" LE' } \\
\text { AIR FLUH } \\
\text { (LQ/SEC) }\end{array}$ & 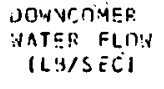 & $\begin{array}{l}\text { WAIER FLON } \\
\text { CHITIMITTY }\end{array}$ & $\begin{array}{l}J-1 I R \\
\text { DOWVEDSP } \\
\text { (FTISECC) }\end{array}$ & 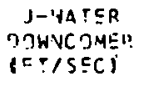 & $\left(J \sigma^{*}\right) * 1 / 2$ & $(J L * 1 * * 1)$ \\
\hline $\begin{array}{l}7.1 .41 \\
7.1 .42 \\
7.1 .43 \\
7.1 .44 \\
7.1 .45\end{array}$ & $\begin{array}{l}0.1295 \\
0.1513 \\
0.2031 \\
0.2020 \\
0.2368\end{array}$ & $\begin{array}{l}J .1170 \\
j .1191 \\
.11188 \\
.11133 \\
0.1201\end{array}$ & $\begin{array}{l}22.17 \\
22.42 \\
22.42 \\
22.42 \\
22.67\end{array}$ & $\begin{array}{l}0.0 \\
0.0 \\
0.0 \\
0.0 \\
0.0\end{array}$ & $\begin{array}{l}4.000 \\
2.900 \\
1.850 \\
1.533 \\
0.350\end{array}$ & $\begin{array}{l}1.039 \\
1.030 \\
1.145 \\
1.060 \\
1.005\end{array}$ & $\begin{array}{r}9.37 \\
10.35 \\
14.55 \\
14.52 \\
16.75\end{array}$ & $\begin{array}{l}0.345 \\
0.395 \\
0.252 \\
0.203 \\
0.103\end{array}$ & $\begin{array}{l}0.4585 \\
0.4948 \\
0.5727 \\
0.5720 \\
0.6167\end{array}$ & $\begin{array}{l}0.5309 \\
0.4520 \\
0.3610 \\
0.3287 \\
0.2374\end{array}$ \\
\hline $\begin{array}{l}7.1 .46 \\
7.1 .47 \\
7.1 .40 \\
7.1 .49 \\
7.1 .50\end{array}$ & $\begin{array}{l}0.3960 \\
0.3441 \\
0.0361 \\
0.04312 \\
0.0570\end{array}$ & $\begin{array}{l}0.1178 \\
0.1179 \\
0.1212 \\
.12113 \\
0.1191\end{array}$ & $\begin{array}{l}22.42 \\
22.67 \\
22.92 \\
22.42 \\
22.42\end{array}$ & $\begin{array}{l}c .0 \\
0.0 \\
c .0 \\
0.0 \\
c .0\end{array}$ & $\begin{array}{r}0.122 \\
0.112 \\
13.933 \\
9.300 \\
9.300\end{array}$ & $\begin{array}{l}1.072 \\
0.994 \\
1.095 \\
0.493 \\
0.963\end{array}$ & $\begin{array}{r}28.6 \mathrm{~J} \\
24.84 \\
2.53 \\
3.512 \\
4.11\end{array}$ & $\begin{array}{l}0.053 \\
0.015 \\
1.899 \\
1.336 \\
1.267\end{array}$ & $\begin{array}{l}0.8013 \\
3.04469 \\
0.2399 \\
0.2624 \\
0.3045\end{array}$ & $\begin{array}{l}0.1725 \\
0.0 .890 \\
0.3903 \\
0.8305 \\
0.8090\end{array}$ \\
\hline $\begin{array}{l}1.1 .51 \\
7.1 .52 \\
7.1 .53 \\
7.1 .54 \\
7.1 .55\end{array}$ & $\begin{array}{l}0.1091 \\
0.1382 \\
0.143 \% \\
0.191 ; \\
0.2708\end{array}$ & $\begin{array}{l}0.1205 \\
.1260 \\
.1238 \\
0.1249 \\
.01141\end{array}$ & $\begin{array}{l}22.67 \\
23.67 \\
23.42 \\
23.67 \\
22.45\end{array}$ & $\begin{array}{l}0.0 \\
0.0 \\
c .0 \\
0.0 \\
0.0\end{array}$ & $\begin{array}{l}4.167 \\
5.567 \\
3.267 \\
1.417 \\
0.417\end{array}$ & $\begin{array}{l}0.570 \\
2.003 \\
1.303 \\
1.004 \\
1.019\end{array}$ & $\begin{array}{r}7.53 \\
7.31 \\
9.33 \\
12.36 \\
19.49\end{array}$ & $\begin{array}{l}0.650 \\
0.759 \\
0.445 \\
0.261 \\
0.057\end{array}$ & $\begin{array}{l}0.4162 \\
0.4116 \\
0.4768 \\
0.5343 \\
0.6619\end{array}$ & $\begin{array}{l}0.5792 \\
0.6259 \\
0.4795 \\
0.3673 \\
0.1712\end{array}$ \\
\hline $\begin{array}{l}7.1 .50 \\
7.1 .57 \\
7.1 .58 \\
7.1 .54 \\
7.1 .60\end{array}$ & $\begin{array}{l}0.2641 \\
0.4094 \\
0.3943 \\
0.3347 \\
0.3312\end{array}$ & $\begin{array}{l}0.1347 \\
0.1403 \\
0.1635 \\
0.1475 \\
0.1242\end{array}$ & $\begin{array}{l}25.45 \\
26.70 \\
31.20 \\
28.20 \\
23.70\end{array}$ & $\begin{array}{l}0.0 \\
0.0 \\
0.0 \\
0.0 \\
0.0\end{array}$ & $\begin{array}{l}0.515 \\
0.021 \\
0.175 \\
0.400 \\
0.130\end{array}$ & $\begin{array}{l}1.010 \\
1.010 \\
1.020 \\
1.035 \\
0.990\end{array}$ & $\begin{array}{l}16.97 \\
24.31 \\
20.51 \\
19.31 \\
22.53\end{array}$ & $\begin{array}{l}0.079 \\
0.003 \\
0.024 \\
0.055 \\
0.014\end{array}$ & $\begin{array}{l}0.5396 \\
0.7735 \\
0.7365 \\
0.6962 \\
0.7229\end{array}$ & $\begin{array}{l}0.2012 \\
0.0383 \\
0.1110 \\
0.1678 \\
0.0839\end{array}$ \\
\hline
\end{tabular}




\section{TABLE XIII (Contd.)}

BASELINE COUNTERCURRENT FLOW TEST GROUP 7.1

EXPERIMENTAL MEASURMENTS FOR TESTS 7.1 .1 TO $7.1 .60-0.70-$ IN. COHNCOMER GAP

\begin{tabular}{|c|c|c|c|c|c|c|c|c|c|c|}
\hline $\begin{array}{l}\text { TEST } \\
\text { NO }\end{array}$ & $\begin{array}{l}R M-C W-1 \\
\text { (GPM) }\end{array}$ & $\begin{array}{c}R M-C H-2 \\
(G P M) .\end{array}$ & $\begin{array}{c}F T-C H-1 \\
(G P M)\end{array}$ & $\begin{array}{c}F P-C H-2 \\
(G P M)\end{array}$ & $\begin{array}{c}K M-\| G G-1 \\
(C F M)\end{array}$ & $\begin{array}{l}\text { RHI-HG-2 } \\
\text { (CFM) }\end{array}$ & $\begin{array}{c}\text { RM-HG-3 } \\
(C F M)^{2}\end{array}$ & $\begin{array}{l}P \text { PN-HG-4 } \\
\text { (CFM) }\end{array}$ & $\begin{array}{c}F T-H G-1 \\
(C F M)\end{array}$ & $\begin{array}{c}F !-H G-2 \\
\text { (CFM) }\end{array}$ \\
\hline $\begin{array}{ll}7.1 . & 1 \\
7.1 . & 2 \\
7.1 . & 3 \\
7.1 . & 4 \\
7.1 . & 5\end{array}$ & $\begin{array}{l}30.0 \\
30.0 \\
30.0 \\
30.0 \\
30.0\end{array}$ & $\begin{array}{l}10.0 \\
10.0 \\
10.0 \\
10.0 \\
10.0\end{array}$ & $\begin{array}{l}0.0 \\
0.0 \\
0.0 \\
0.0 \\
0.0\end{array}$ & $\begin{array}{l}0.0 \\
0.0 \\
0.0 \\
0.0 \\
0.0\end{array}$ & $\begin{array}{l}0.0 \\
0.0 \\
0.0 \\
0.0 \\
0.0\end{array}$ & $\begin{array}{l}10.00 \\
15.000 \\
20.00 \\
25.00 \\
30.00\end{array}$ & $\begin{array}{l}0.0 \\
0.0 \\
0.0 \\
0.0 \\
0.0\end{array}$ & $\begin{array}{l}0.0 \\
0.0 \\
0.0 \\
0.0 \\
0.0\end{array}$ & $\begin{array}{l}0.0 \\
0.0 \\
0.0 \\
0.0 \\
0.0\end{array}$ & $\begin{array}{l}0.0 \\
0.0 \\
0.0 \\
0.0 \\
0.0\end{array}$ \\
\hline $\begin{array}{ll}7.1 . & 0 \\
7.1 .1 .7 \\
7.1 .0 \\
7.1 .0 \\
7.1 .1 .0\end{array}$ & $\begin{array}{l}27.0 \\
30.0 \\
30.0 \\
30.0 \\
30.0\end{array}$ & $\begin{array}{r}10.0 \\
10.0 \\
7.0 \\
10.0 \\
5.0\end{array}$ & $\begin{array}{l}0.0 \\
0.0 \\
0.0 \\
0.0 \\
0.0\end{array}$ & $\begin{array}{l}0.0 \\
0.0 \\
0.0 \\
0.0 \\
0.0\end{array}$ & $\begin{array}{l}0.0 \\
0.0 \\
0.0 \\
0.0 \\
0.0\end{array}$ & $\begin{array}{l}30.00 \\
35.00 \\
35.00 \\
40.00 \\
40.00\end{array}$ & $\begin{array}{l}0.0 \\
0.0 \\
0.0 \\
0.0 \\
0.0\end{array}$ & $\begin{array}{l}0.0 \\
0.0 \\
0.0 \\
0.0 \\
0.0\end{array}$ & $\begin{array}{l}0.0 \\
0.0 \\
0.0 \\
0.0 \\
0.0\end{array}$ & $\begin{array}{l}0.0 \\
0.0 \\
0.0 \\
0.0 \\
0.0\end{array}$ \\
\hline $\begin{array}{l}7.1 .11 \\
7.1 .12 \\
7.1 .13 \\
7.1 .14 \\
7.2 .15\end{array}$ & $\begin{array}{l}31.0 \\
30.0 \\
20.0 \\
3.0 .0 \\
25.0\end{array}$ & $\begin{array}{r}10.0 \\
5.0 \\
10.0 \\
10.0 \\
10.0\end{array}$ & $\begin{array}{l}0.0 \\
0.0 \\
0.0 \\
0.0 \\
0.0\end{array}$ & $\begin{array}{l}0.0 \\
0.0 \\
0.0 \\
0.0 \\
0.0\end{array}$ & $\begin{array}{l}0.0 \\
0.0 \\
0.0 \\
0.0 \\
11.0\end{array}$ & $\begin{array}{l}50.0 .2 \\
50.00 \\
50.00 \\
60.00 \\
60.00\end{array}$ & $\begin{array}{l}0.0 \\
0.0 \\
0.0 \\
0.0 \\
0.0\end{array}$ & $\begin{array}{l}0.0 \\
0.0 \\
0.0 \\
0.0 \\
0.0\end{array}$ & $\begin{array}{l}0.0 \\
0.0 \\
0.0 \\
0.0 \\
0.0\end{array}$ & $\begin{array}{l}0.0 \\
0.0 \\
0.0 \\
0.0 \\
0.0\end{array}$ \\
\hline $\begin{array}{l}7.1 .10 \\
7.1 .17 \\
7.1 .18 \\
7.1 .19 \\
7.1 .20\end{array}$ & $\begin{array}{l}2.0 .0 \\
3.0 .0 \\
23.0 \\
23.0 \\
31.0\end{array}$ & $\begin{array}{l}13.0 \\
10.0 \\
10.0 \\
13.0 \\
10.0\end{array}$ & $\begin{array}{l}0.0 \\
0.0 \\
0.0 \\
0.0 \\
0.0\end{array}$ & $\begin{array}{l}0.0 \\
0.0 \\
0.01 \\
0.0 \\
0.0\end{array}$ & $\begin{array}{l}0.0 \\
0.0 \\
0.0 \\
0.0 \\
0.0\end{array}$ & $\begin{array}{l}60.00 \\
70.00 \\
70.00 \\
70.00 \\
70.00\end{array}$ & $\begin{array}{l}0.0 \\
0.0 \\
0.0 \\
0.0 \\
0.0\end{array}$ & $\begin{array}{l}0.0 \\
0.0 \\
0.0 \\
0.0 \\
0.0\end{array}$ & $\begin{array}{l}0.0 \\
0.0 \\
0.0 \\
0.0 \\
0.0\end{array}$ & $\begin{array}{l}0.0 \\
0.0 \\
0.0 \\
0.0 \\
0.0\end{array}$ \\
\hline $\begin{array}{l}7.1 .21 \\
7.1 .22 \\
7.1 .23 \\
7.1 .24 \\
7.1 .25\end{array}$ & $\begin{array}{l}3 j .0 \\
2 j .0 \\
2 j .0 \\
2 j . j \\
2 j .0\end{array}$ & $\begin{array}{r}10.0 \\
10.0 \\
10.0 \\
1.0 .0 \\
0.0\end{array}$ & $\begin{array}{l}0.0 \\
0.0 \\
0.0 \\
0.0 \\
0.0\end{array}$ & $\begin{array}{l}0.0 \\
0.5 \\
0.1 \\
0.0 \\
0.0\end{array}$ & $\begin{array}{l}0.0 \\
0.0 \\
0.0 \\
0.0 \\
0.0\end{array}$ & $\begin{array}{l}719.00 \\
70.06 \\
70.00 \\
70.00 \\
70.00\end{array}$ & $\begin{array}{l}10.00 \\
10.00 \\
10.00 \\
10.00 \\
20.00\end{array}$ & $\begin{array}{l}0.0 \\
0.0 \\
0.0 \\
0.0 \\
0.0\end{array}$ & $\begin{array}{l}0.0 \\
0.0 \\
0.0 \\
0.0 \\
0.0\end{array}$ & $\begin{array}{l}0.0 \\
0.0 \\
J .0 \\
0.0 \\
0.0\end{array}$ \\
\hline $\begin{array}{l}7.1 .26 \\
7.1 .27 \\
7.1 .24 \\
7.1 .29 \\
7.1 .30\end{array}$ & $\begin{array}{l}15.0 \\
10.0 \\
1.0 .0 \\
10.0 \\
10.0\end{array}$ & $\begin{array}{l}0.0 \\
0.0 \\
j .0 \\
j .0 \\
0.0\end{array}$ & $\begin{array}{l}0.0 \\
0.0 \\
0.0 \\
0.0 \\
0.0\end{array}$ & $\begin{array}{l}0.0 \\
0.0 \\
0.0 \\
0.0 \\
0.0\end{array}$ & $\begin{array}{c}0.00 \\
60.00 \\
70.00 \\
70.00 \\
80.00\end{array}$ & $\begin{array}{l}71.0100 \\
60.00 \\
60.20 \\
70.00 \\
70.20\end{array}$ & $\begin{array}{c}30.0 J \\
. .3 \\
0.3 \\
0.0 \\
0.0\end{array}$ & $\begin{array}{c}10.00 \\
0.0 \\
0.0 \\
0.0 \\
0.0\end{array}$ & $\begin{array}{l}3.0 \\
0.0 \\
0.0 \\
0.0 \\
0.0\end{array}$ & $\begin{array}{l}0.0 \\
0.0 \\
0.0 \\
0.0 \\
0.0\end{array}$ \\
\hline $\begin{array}{l}7.1 .31 \\
7.1 .32 \\
7.1 .33 \\
7.1 .34 \\
7.1 .35\end{array}$ & $\begin{array}{l}1 \text { J.J } \\
\text { J.u } \\
.0 .0 \\
j .0 \\
\text { J.u }\end{array}$ & $\begin{array}{l}0.0 \\
0.0 \\
0.0 \\
0.0 \\
0.0\end{array}$ & $\begin{array}{r}0.0 \\
100.0 \\
80.0 \\
70.0 \\
70.0\end{array}$ & $\begin{array}{l}0.0 \\
0.0 \\
0.0 \\
0.0 \\
0.0\end{array}$ & $\begin{array}{c}90.00 \\
0.0 \\
0.0 \\
j .0 \\
0.0\end{array}$ & $\begin{array}{c}I J .0 j \\
0.0 \\
u .11 \\
0.0 \\
1.0\end{array}$ & $\begin{array}{l}0.0 \\
10.03 \\
10.00 \\
10.00 \\
20.00\end{array}$ & $\begin{array}{l}0.0 \\
0.0 \\
0.0 \\
0.0 \\
1.0\end{array}$ & $\begin{array}{l}0.0 \\
0.0 \\
0.0 \\
0.0 \\
0.0\end{array}$ & $\begin{array}{l}0.0 \\
0.0 \\
0.0 \\
0.0 \\
0.0\end{array}$ \\
\hline $\begin{array}{l}7.1 .36 \\
7.1 .37 \\
7.1 .39 \\
7.1 .39 \\
7.1 .4 .\end{array}$ & $\begin{array}{l}3.0 \\
0.0 \\
j .0 \\
j .0 \\
1.0\end{array}$ & $\begin{array}{l}0.0 \\
0.0 \\
0.0 \\
0.0 \\
. .0\end{array}$ & $\begin{array}{r}60.0 \\
60.0 \\
0.0 \\
0.0 \\
0.0\end{array}$ & $\begin{array}{l}0.0 \\
0.0 \\
49.6 \\
50.2 \\
50.5\end{array}$ & $\begin{array}{c}0.0 \\
0.0 \\
50.00 \\
6.0 .00 \\
80.00\end{array}$ & $\begin{array}{l}0.0 \\
0.0 \\
0.0 \\
0.0 \\
0.0\end{array}$ & $\begin{array}{l}20.00 \\
20.00 \\
0.0 \\
0.0 \\
0.0\end{array}$ & $\begin{array}{l}0.0 \\
0.0 \\
0.0 \\
0.0 \\
0.0\end{array}$ & $\begin{array}{l}0.0 \\
0.0 \\
0.0 \\
0.0 \\
0.0\end{array}$ & $\begin{array}{l}0.0 \\
0.0 \\
0.0 \\
0.0 \\
0.0\end{array}$ \\
\hline
\end{tabular}




\section{TABLE XIII (Contd.)}

BASELING COUNTERCURRENT FLOW TEST GROUP 7.1

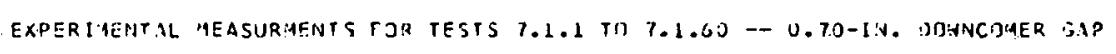

\begin{tabular}{|c|c|c|c|c|c|c|c|c|c|c|}
\hline $\begin{array}{c}\text { TEST } \\
\text { TO }\end{array}$ & $\begin{array}{c}R N=C A-1 \\
\left(S_{B} P M\right)\end{array}$ & $\begin{array}{c}R^{\prime} Y-C W-? \\
(G P: A)^{\prime}\end{array}$ & $\begin{array}{l}C T-(W-1 \\
(S P M)\end{array}$ & $\begin{array}{c}F T-C H-2 \\
(C, P: 1)\end{array}$ & $\begin{array}{c}P, i-1 \mid G-1 \\
(C F M)\end{array}$ & 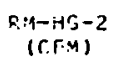 & $\begin{array}{c}2,4-i d r,-3 \\
(C F \cdot 1)\end{array}$ & $\begin{array}{l}\text { SII-HG-4 } \\
\text { (CFM) }\end{array}$ & $\begin{array}{c}F ?-(H G-1 \\
(G F M)\end{array}$ & $\begin{array}{l}\text { ST-HG-2 } \\
\text { (CFM) }\end{array}$ \\
\hline 7.1 .041 & נ.J & 0.0 & 0.0 & 39.9 & 0.0 & 0.3 & 0.10 & 0.0 & 14.02 & 0.0 \\
\hline 7.1 .42 & J.J & 0.0 & 0.0 & 30.1 & 0.0 & U.) & 0.0 & 0.0 & 17.44 & 0.0 \\
\hline $7.1 .0,3$ & נ.0. & 0.0 & 0.0 & 20.4 & 0.0 & 0.0 & 0.0 & 0.0 & 23.72 & 0.0 \\
\hline 7.1 .44 & J.j & 0.0 & 0.0 & 19.7 & 0.0 & ט.j & 0.0 & 0.0 & 23.85 & 0.0 \\
\hline $7.1 . .05$ & J.0. & 0.0 & $0 . \mathrm{C}$ & 15.0 & 0.0 & 0.0 & 0.0 & 0.0 & 28.21 & 0.0 \\
\hline 7.1 .46 & ง.s & 0.0 & 0.0 & 10.1 & 0.0 & j.e & $u . u$ & 0.0 & 48.08 & 0.0 \\
\hline 7.1 .47 & .00 & 3.0 & 0.3 & 3.2 & 0.0 & 0.0 & 0.0 & 3.0 & $42.5 \%$ & 0.0 \\
\hline 7.1 .40 & 0.0 & 0.0 & 129.0 & $n .0$ & 0.0 & $0 . j$ & $16.7 \mathrm{~J}$ & 0.0 & 0.0 & 0.0 \\
\hline 7.1 .99 & J. 0 & 5.0 & su. 0 & J.v & 0.0 & ग. & 20.10 & 0.0 & 0.0 & 0.0 \\
\hline 7.1 .50 & 0.0 & J.0 & 120.0 & J.J & 0.0 & $3 . j$ & $26.1,0$ & 0.0 & 0.0 & 0.0 \\
\hline 7.1 .51 & J.J & J. 0 & 90.0 & 0.0 & 50.00 & נ.J & 0.9 & 0.0 & 0.0 & 0.0 \\
\hline 7.1 .52 & 3.0 & 0.0 & 120.0 & נ. נ. & 50.00 & 0.0 & 0.0 & 0.0 & 0.0 & 0.0 \\
\hline 7.1 .33 & 1.0 & 0.0 & 90.0 & 0.0 & .66 .050 & 2.0 & 0.0 & 0.0 & 0.0 & 0.0 \\
\hline 7.1 .54 & 1.15 & 0.0 & 70.3 & J.0 & 0.0 & $\cdot ?$ & 0.0 & 0.0 & 20.64 & 0.0 \\
\hline 7.1 .55 & נ. . & 0.0 & 0.0 & 30.0 & 0.0 & ט.j & 0.0 & 0.3 & 27.02 & 0.0 \\
\hline 7.1 .50 & נ.J & 3.0 & i). 0 & $4 \%$. & 0.0 & 0.0 & 0.0 & 0.0 & 20.21 & 0.0 \\
\hline 7.1 .34 & 3.0 & 0.0 & 0.0 & 37.0 & 0.0 & 0.3 & 0.0 & 0.0 & 47.18 & 0.0 \\
\hline 7.1 .58 & 3.0 & 3.0 & J.0 & 50.0 & 0.0 & 0.0 & 0.0 & 0.0 & 47.05 & 0.0 \\
\hline 7.1 .59 & 3.0 & 0.0 & 0.0 & 5u.J & 0.0 & 0.0 & J.0 & 0.0 & 40.38 & 0.0 \\
\hline 7.1 .63 & ग.0 & 10.13 & 0.0 & 30.0 & 0.0 & U..u & 0.0 & 0.5 & $40.6 \%$ & 0.0 \\
\hline & & & & & & & & & & \\
\hline $\begin{array}{l}\text { TEST } \\
\text { NO }\end{array}$ & $\begin{array}{l}P-C d-1 \\
\text { (PSIG) }\end{array}$ & $\begin{array}{l}P-C W-2 \\
(P S I G)\end{array}$ & $\begin{array}{l}P-H E-1 \\
\text { (PSIG) }\end{array}$ & $\begin{array}{l}D-H ت-2 \\
\text { PSISI }\end{array}$ & $\begin{array}{l}0-115-3 \\
(P \leq 16)\end{array}$ & $\begin{array}{l}I F-C h-1 \\
(J E G F)\end{array}$ & 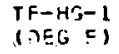 & $\begin{array}{l}T F-H G-2 \\
(D F S=)\end{array}$ & 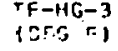 & $\begin{array}{l}\text { D-BAR? } \\
\text { (PSIA) }\end{array}$ \\
\hline 7.1 .1 & J.J & 12.0 & 1.1 .7 & 0.0 & 3.0 & 55.0 & 74.0 & 0.3 & 0.0 & 12.2 \\
\hline 7.1 .2 & 0.0 & 11.7 & 11.5 & v.u & 0.0 & 55.0 & 73.0 & 0.0 & 0.0 & 12.2 \\
\hline 7.1 .3 & 0.0 & 12.0 & 11.5 & 0.0 & 0.0 & 55.13 & 73. J & 0.0 & 0.0 & 12.2 \\
\hline 7.1 .4 & 3.0 & 12.0 & 10.7 & 0.0 & 0.0 & 55.18 & 73.0 & 0.00 & 0.0 & 12.2 \\
\hline 7.1 .5 & 0.0 & 12.0 & 10.7 & 0.0 & 0.0 & 55.1 & 78.0 & 0.5 & 0.0 & 12.2 \\
\hline & 3.0 & 2.2 .0 & 11.7 & J.) & 0.0 & ;5.0 & 78.0 & 0.0 & 2.0 & 12.2 \\
\hline 7.1 .7 & 3.0 & 11.5 & 11.2 & 0.0 & 0.0 & ل. & 13.0 & 0.0 & 0.0 & 12.2 \\
\hline 7.1 .3 & 0.0 & 12.2 & $11 . \mathrm{J}$ & u.u. & . 0.0 & 07.5 & 71.5 & J.0 & 0.0 & 12.5 \\
\hline $7.1 . y$ & 3.0 & 12.5 & 12.2 & 0.0 & 0.0 & 62.0 & 65.0 & 0.0 & 0.0 & 12.5 \\
\hline 7.1 .13 & 0.0 & 12.2 & 12.0 & 0.0 & 0.0 & 62.0 & 65.0 & 0.0 & 0.0 & 12.5 \\
\hline
\end{tabular}


TABLE XIII (Contd.)

BASELINE COUNTERCURRENT FLOW TEST GROUP 7.1

EXPER IMENTAL MEASURMENTS FOR TESTS 7.1 .1 TO $7.1 .60--0.70-1 \mathrm{~N}$. DOHNCOMER GAP

\begin{tabular}{|c|c|c|c|c|c|c|c|c|c|c|}
\hline $\begin{array}{l}\text { TEST } \\
\text { NO }\end{array}$ & $\begin{array}{l}P-C \cdot d-1 \\
(P S I G)\end{array}$ & $\begin{array}{l}P-C N-2 \\
|P S I G|\end{array}$ & $\begin{array}{l}\text { P-HG-I } \\
\text { |PSIGI }\end{array}$ & $\begin{array}{l}P-H G-2 \\
(P S(G)\end{array}$ & $\begin{array}{l}P-H(S ;-3 \\
(P S I G)\end{array}$ & $\begin{array}{l}\text { TF-CH-1 } \\
\text { (OEG F) }\end{array}$ & $\begin{array}{l}\text { TF-HG-1 } \\
\text { (DEG F) }\end{array}$ & $\begin{array}{l}T F-H G-2 \\
\text { (IDFG }\end{array}$ & $\begin{array}{l}\text { IF-HF }-3 \\
\text { (DEG F) }\end{array}$ & $\begin{array}{l}P \text { PAARח } \\
\text { (PSIA) }\end{array}$ \\
\hline $\begin{array}{l}7.1 .11 \\
7.1 .12 \\
7.1 .13 \\
7.1 .14 \\
7.1 .15\end{array}$ & $\begin{array}{l}0.0 \\
J .0 \\
0.0 \\
.00 \\
0.0\end{array}$ & $\begin{array}{l}11.7 \\
12.2 \\
12.2 \\
12.0 \\
12.7\end{array}$ & $\begin{array}{l}11.5 \\
12.2 \\
11.0 \\
12.2 \\
12.5\end{array}$ & $\begin{array}{l}0.0 \\
0.0 \\
0.0 \\
0.0 \\
0.0\end{array}$ & $\begin{array}{l}0.0 \\
0.0 \\
0.0 \\
0.0 \\
0.0\end{array}$ & $\begin{array}{l}59.0 \\
58.0 \\
58.0 \\
58.0 \\
58.0\end{array}$ & $\begin{array}{l}72.5 \\
73.0 \\
75.0 \\
72.0 \\
74.5\end{array}$ & $\begin{array}{l}0.0 \\
0.0 \\
0.0 \\
. .0 \\
0.0\end{array}$ & $\begin{array}{l}0.0 \\
0.0 \\
0.0 \\
0.0 \\
0.0\end{array}$ & $\begin{array}{l}12.5 \\
12.5 \\
12.5 \\
12.5 \\
12.5\end{array}$ \\
\hline $\begin{array}{l}7.1 .10 \\
7.1 .17 \\
7.1 .10 \\
7.2 .19 \\
7.1 .20\end{array}$ & $\begin{array}{l}J .0 \\
j .0 \\
\text { J.u } \\
\text { J.u }\end{array}$ & $\begin{array}{l}12.0 \\
12.5 \\
12.2 \\
12.0 \\
11.7\end{array}$ & $\begin{array}{l}12.0 \\
12.7 \\
12.0 \\
12.0 \\
12.2\end{array}$ & $\begin{array}{l}0.0 \\
0.0 \\
0.0 \\
0.0 \\
0.0\end{array}$ & $\begin{array}{l}u . u \\
0.0 \\
0.0 \\
0.0 \\
0.0\end{array}$ & $\begin{array}{l}54.0 \\
59.0 \\
58.0 \\
57.0 \\
57.0\end{array}$ & $\begin{array}{l}74 . J \\
76.5 \\
76.0 \\
77.0 \\
79.5\end{array}$ & $\begin{array}{l}0.0 \\
0.0 \\
0.0 \\
0.0 \\
.00\end{array}$ & $\begin{array}{l}0.0 \\
0.0 \\
0.0 \\
0.0 \\
0.0\end{array}$ & $\begin{array}{l}12.5 \\
12.4 \\
12.4 \\
12.4 \\
12.4\end{array}$ \\
\hline $\begin{array}{l}7.1 .21 \\
7.1 .22 \\
7.1 .23 \\
7.1 .24 \\
7.1 .23\end{array}$ & $\begin{array}{l}J . j \\
\text { J.u } \\
j .0 \\
. .0 \\
.0\end{array}$ & $\begin{array}{l}12.2 \\
12.5 \\
12.2 \\
11.5 \\
12.0\end{array}$ & $\begin{array}{l}12.0 \\
13.0 \\
12.0 \\
12.0 \\
11.5\end{array}$ & $\begin{array}{l}0.0 \\
0.0 \\
0.0 \\
0.0 \\
0.0\end{array}$ & $\begin{array}{l}0.0 \\
0.0 \\
0.0 \\
0.0 \\
0.0\end{array}$ & $\begin{array}{l}57.0 \\
57.0 \\
59.0 \\
53.0 \\
58.0\end{array}$ & $\begin{array}{l}76.0 \\
77.5 \\
78.0 \\
73.0 \\
78.0\end{array}$ & $\begin{array}{l}0.0 \\
0.0 \\
0.0 \\
0.0 \\
0.0\end{array}$ & $\begin{array}{l}0.0 \\
0.0 \\
0.0 \\
0.0 \\
0.0\end{array}$ & $\begin{array}{l}12.4 \\
12.4 \\
12.4 \\
12.4 \\
12.3\end{array}$ \\
\hline $\begin{array}{l}7.1 .25 \\
7.1 .21 \\
7.1 .21 \\
7.1 .29 \\
7.1 .5 \mathrm{j}\end{array}$ & 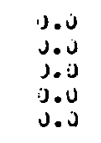 & $\begin{array}{l}10.0 \\
11.0 \\
11.0 \\
11.0 \\
12.0\end{array}$ & $\begin{array}{l}10.0 \\
11.0 \\
11.0 \\
12.0 \\
13.0\end{array}$ & $\begin{array}{l}\text { U.J } \\
\text { U.u } \\
\text { U.u } \\
\text { J.u } \\
0 . u\end{array}$ & $\begin{array}{l}0.0 \\
0.0 \\
0.0 \\
0.0 \\
0.3\end{array}$ & $\begin{array}{l}58.3 \\
53.0 \\
58.0 \\
53.0 \\
58.0\end{array}$ & $\begin{array}{l}77.0 \\
67.0 \\
07.0 \\
67.0 \\
67.0\end{array}$ & $\begin{array}{l}0.0 \\
0.0 \\
0.0 \\
0.0 \\
1.0\end{array}$ & $\begin{array}{l}0.0 \\
0.0 \\
0.0 \\
0.0 \\
.0 .0\end{array}$ & $\begin{array}{l}12.3 \\
12.3 \\
12.3 \\
12.3 \\
12.3\end{array}$ \\
\hline $\begin{array}{l}7.1 .31 \\
7.1 .32 \\
7.1 .33 \\
7.1 .34 \\
7.1 .35\end{array}$ & $\begin{array}{l}1.0 \\
11.7 \\
11.0 \\
10.8 \\
11.0\end{array}$ & $\begin{array}{r}11.0 \\
0.0 \\
3.0 \\
0.0 \\
0.0\end{array}$ & $\begin{array}{r}11.0 \\
9.4 \\
10.0 \\
13.6 \\
11.0\end{array}$ & $\begin{array}{l}0.0 \\
100.0 \\
105 . .5 \\
103.5 \\
101.5\end{array}$ & $\begin{array}{r}0.0 \\
33.5 \\
33.5 \\
33.5 \\
30.0\end{array}$ & $\begin{array}{l}53.0 \\
43.5 \\
44.5 \\
43.0 \\
43.0\end{array}$ & $\begin{array}{l}67 . J \\
73.5 \\
31.0 \\
19.0 \\
77.0\end{array}$ & $\begin{array}{r}0.0 \\
77.0 \\
7 \% .5 \\
73.5 \\
73.0\end{array}$ & $\begin{array}{r}9.0 \\
77.0 \\
96.5 \\
76.0 \\
73.5\end{array}$ & $\begin{array}{l}12.3 \\
12.3 \\
12.4 \\
12.4 \\
12.6\end{array}$ \\
\hline $\begin{array}{l}7.2 .30 \\
7.1 .37 \\
7.1 .30 \\
7.1 .39 \\
7.1 .40\end{array}$ & $\begin{array}{l}11.03 \\
10.7 \\
1 \% .5 \\
1.0 .5 \\
\text { lu.2 }\end{array}$ & $\begin{array}{l}0.0 \\
0.0 \\
0.0 \\
0.0 \\
0.0\end{array}$ & $\begin{array}{r}12.5 \\
10.6 \\
10.6 \\
9.0 \\
12.0\end{array}$ & $\begin{array}{l}99.0 \\
39.0 \\
95.0 \\
30.0 \\
15.5\end{array}$ & $\begin{array}{l}32.0 \\
32.0 \\
32.0 \\
32.0 \\
33.5\end{array}$ & $\begin{array}{l}42.5 \\
43.5 \\
40.0 \\
47.5 \\
41.5\end{array}$ & $\begin{array}{l}77.0 \\
77.5 \\
70.5 \\
71.0 \\
77.0\end{array}$ & $\begin{array}{l}74.0 \\
71.5 \\
79.5 \\
71.5 \\
79.0\end{array}$ & $\begin{array}{l}73.5 \\
74.0 \\
76.5 \\
78.0 \\
78.0\end{array}$ & $\begin{array}{l}12.4 \\
12.4 \\
12.4 \\
12.4 \\
12.4\end{array}$ \\
\hline $\begin{array}{l}7.1 .41 \\
7.1 .42 \\
7.1 .43 \\
7.1 .44 \\
7.1 .45\end{array}$ & $\begin{array}{l}1 \mathrm{~J} . J \\
1 \mathrm{~J} . \mathrm{J} \\
1 \mathrm{~J} .5 \\
1 . .2 \\
1.00\end{array}$ & $\begin{array}{l}0.0 \\
0.0 \\
0.0 \\
0.0 \\
0.0\end{array}$ & $\begin{array}{l}11.0 \\
10.5 \\
11.0 \\
11.5 \\
11.2\end{array}$ & $\begin{array}{l}36.0 \\
95.0 \\
34.0 \\
9.4 .0 \\
93.5\end{array}$ & $\begin{array}{l}33.5 \\
33.5 \\
33.5 \\
33.5 \\
33.5\end{array}$ & $\begin{array}{l}49.0 \\
47.0 \\
47.5 \\
47.5 \\
47.5\end{array}$ & $\begin{array}{l}81.0 \\
44.5 \\
87.5 \\
91.0 \\
93.4\end{array}$ & $\begin{array}{r}99.5 \\
95.5 \\
99.5 \\
103.5 \\
107.5\end{array}$ & $\begin{array}{r}8 \% .5 \\
88.5 \\
34.5 \\
97.0 \\
102.0\end{array}$ & $\begin{array}{l}12.4 \\
12.4 \\
12.4 \\
12.4 \\
12.4\end{array}$ \\
\hline $\begin{array}{l}7.1 .46 \\
7.1 .47 \\
7.1 .49 \\
7.1 .49 \\
7.1 .730\end{array}$ & $\begin{array}{l}11.7 \\
10.2 \\
12.2 \\
12.0 \\
11.5\end{array}$ & $\begin{array}{l}0.0 \\
0.0 \\
u .0 \\
j .0 \\
j .0\end{array}$ & $\begin{array}{l}11.4 \\
12.0 \\
10.0 \\
10.6 \\
10.5\end{array}$ & $\begin{array}{l}92.5 \\
91.0 \\
98.5 \\
90.5 \\
98.0\end{array}$ & $\begin{array}{l}33.5 \\
33.0 \\
32.5 \\
32.5 \\
32.5\end{array}$ & $\begin{array}{l}41.0 \\
51.5 \\
50.0 \\
47.5 \\
48.5\end{array}$ & $\begin{array}{r}49.5 \\
102.0 \\
00.5 \\
80.5 \\
79.5\end{array}$ & $\begin{array}{r}113.0 \\
115.5 \\
0.0 \\
0.0 \\
\text { J.J }\end{array}$ & $\begin{array}{r}106.5 \\
109.5 \\
9: .5 \\
80.0 \\
79.5\end{array}$ & $\begin{array}{l}12.4 \\
12.4 \\
12.4 \\
12.4 \\
12.4\end{array}$ \\
\hline
\end{tabular}


TABLE XIII (Contd.)

BASELINE COUNTERCURRENT FLOW TEST GROUP 7.1

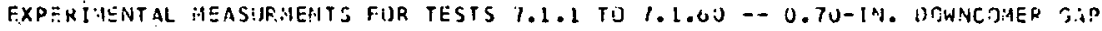

\begin{tabular}{|c|c|c|c|c|c|c|c|c|c|c|}
\hline $\begin{array}{l}T E S T \\
\text { NIJ }\end{array}$ & $\begin{array}{l}P-C H-1 \\
(P S I G)\end{array}$ & $\begin{array}{l}P-C \mathrm{CH}-2 \\
\text { (.DSICI }\end{array}$ & $\begin{array}{l}p-4 S-1 \\
(P S I S)\end{array}$ & $\begin{array}{l}P-H C-2 \\
(P S I G)\end{array}$ & $\begin{array}{l}P-140-3 \\
(P \leq 1 G)\end{array}$ & $\begin{array}{l}T F-C \cdot W-1 \\
(\cap E G \mid F)\end{array}$ & $\begin{array}{l}\text { IF-HS-1 } \\
(D E S T)\end{array}$ & 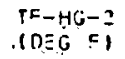 & $\begin{array}{l}\text { TE-HG-3 } \\
\text {. SES F }\end{array}$ & $\begin{array}{l}\text { O- } A A R R \\
\text { IPSIA }\end{array}$ \\
\hline $\begin{array}{l}7.1 .31 \\
1.1 .52 \\
7.1 .53 \\
1.1 .53 \\
7.1 .55\end{array}$ & $\begin{array}{r}11.7 \\
13.7 \\
12.1 \\
12.0 \\
9.7\end{array}$ & $\begin{array}{l}0.0 \\
0.0 \\
0.0 \\
0.0 \\
0.0\end{array}$ & $\begin{array}{l}10.3 \\
11.8 \\
11.7 \\
11.7 \\
1.1 .7\end{array}$ & $\begin{array}{r}96.5 \\
90.0 \\
95.5 \\
94.5 \\
101.0\end{array}$ & $\begin{array}{r}32.5 \\
32.5 \\
32.5 \\
0.0 \\
32.5\end{array}$ & $\begin{array}{l}47.0 \\
67.0 \\
30.3 \\
50.3 \\
47.0\end{array}$ & $\begin{array}{l}79.0 \\
79.0 \\
78.5 \\
80.1 \\
70.5\end{array}$ & $\begin{array}{r}0.3 \\
0.0 \\
0.0 \\
37.0 \\
64.5\end{array}$ & $\begin{array}{r}79.0 \\
79.5 \\
79.5 \\
1.0 .0 \\
0.0\end{array}$ & $\begin{array}{r}12.4 \\
12.4 \\
-12.4 \\
12.4 \\
12.4\end{array}$ \\
\hline $\begin{array}{l}7.1 .56 \\
7.1 .57 \\
7.1 .53 \\
7.1 .59 \\
7.1 .60\end{array}$ & $\begin{array}{l}13.0 \\
1 \% .0 \\
1.3 .7 \\
14.1 \\
1.3 .3\end{array}$ & $\begin{array}{l}10.0 \\
0.0 \\
0.0 \\
0.0 \\
0.0\end{array}$ & $\begin{array}{l}13.8 \\
15.0 \\
19.7 \\
16.4 \\
11.5\end{array}$ & $\begin{array}{r}100.5 \\
94.5 \\
9.3 .0 \\
93.5 \\
93.5\end{array}$ & $\begin{array}{r}32.5 \\
32.5 \\
0.0 \\
0.0 \\
0.0\end{array}$ & $\begin{array}{l}50.0 \\
50.5 \\
48.5 \\
41.5 \\
47.0\end{array}$ & $\begin{array}{r}69.0 \\
81.0 \\
92.0 \\
100.0 \\
106.0\end{array}$ & $\begin{array}{r}12.5 \\
94.5 \\
136.0 \\
115.0 \\
122.0\end{array}$ & $\begin{array}{l}0.0 \\
0.0 \\
0.0 \\
0.0 \\
0.0\end{array}$ & $\begin{array}{l}12.4 \\
12.4 \\
12.4 \\
12.4 \\
12.4\end{array}$ \\
\hline
\end{tabular}

\begin{tabular}{|c|c|c|c|c|c|c|c|c|c|}
\hline \multirow[b]{2}{*}{$\begin{array}{c}T E S T \\
N: J\end{array}$} & \multicolumn{3}{|c|}{ LLUER PLENUM FLON } & \multicolumn{3}{|c|}{ BYOASS FLIN } & \multirow[b]{2}{*}{ 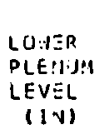 } & \multirow[b]{2}{*}{$\begin{array}{l}P-1 n-1 \\
\left.\text { (PS } 1 \sigma_{s}\right)\end{array}$} & \multirow[b]{2}{*}{$\begin{array}{l}T F-L P-1 \\
\text { (CES FI) }\end{array}$} \\
\hline & $\begin{array}{l}\text { TNITAL } \\
\text { NEIBHT } \\
\text { ILII }\end{array}$ & $\begin{array}{l}\text { FIIAL } \\
\text { WEIGHT } \\
\text { (LU) }\end{array}$ & $\begin{array}{l}\text { TIME OF } \\
\text { COLECTIIN } \\
\text { (SEC) }\end{array}$ & $\begin{array}{l}\text { THITIAL } \\
\text { WEIGHT } \\
\text { ILUI }\end{array}$ & $\begin{array}{l}\text { GIIALA } \\
\text { FEISHT } \\
\text { (1LU) }\end{array}$ & $\begin{array}{l}\text { TIME IF } \\
\text { COLLECTHON } \\
\text { (SEC) }\end{array}$ & & & \\
\hline & $\begin{array}{l}133.0 \\
274.3 \\
34.6 .5 \\
240.3 \\
230.3 \\
133.8\end{array}$ & $\begin{array}{l}276.3 \\
384.0 \\
493.0 \\
39.0 .3 \\
39.3 \\
237.5\end{array}$ & $\begin{array}{l}25.0 \\
20.0 \\
20.5 \\
20.0 \\
20.0\end{array}$ & $\begin{array}{r}86.3 \\
87 \% .3 \\
92.3 \\
105.0 \\
121.5\end{array}$ & $\begin{array}{r}87.3 \\
32.3 \\
105.0 \\
121.5 \\
151.5\end{array}$ & $\begin{array}{l}75.3 \\
75.3 \\
93.0 \\
75.0 \\
70.0\end{array}$ & $\begin{array}{l}12.0 \\
12.0 \\
12.0 \\
12.0 \\
12.0\end{array}$ & $\begin{array}{l}10.0 \\
10.0 \\
10.0 \\
10.0 \\
10.0\end{array}$ & $\begin{array}{l}56.0 \\
550.0 \\
55.0 \\
55.0 \\
56.0\end{array}$ \\
\hline & $\begin{array}{l}237.5 \\
336.0 .5 \\
133.5 \\
230.0 \\
353.0 .3\end{array}$ & $\begin{array}{l}364.3 \\
517.5 \\
230.3 \\
356.3 \\
497.8\end{array}$ & $\begin{array}{l}25.3 \\
30.0 \\
20.0 \\
25.0 \\
30.0\end{array}$ & $\begin{array}{l}151.5 \\
156.0 \\
33.0 \\
130.5 \\
139.3\end{array}$ & $\begin{array}{l}160.3 \\
199.3 \\
100.5 \\
139.3 \\
151.5\end{array}$ & $\begin{array}{l}70.0 \\
010.0 \\
60.01 \\
70.01 \\
70.0 \\
? 0.0\end{array}$ & $\begin{array}{l}12.0 \\
12.0 \\
12.0 \\
12.0 \\
12.0\end{array}$ & $\begin{array}{l}10.0 \\
10.0 \\
110.0 \\
10.10 \\
10.0\end{array}$ & $\begin{array}{l}56.0 \\
5500 \\
52.5 \\
53.0 \\
52.0\end{array}$ \\
\hline $\begin{array}{l}7.1 .11 \\
7.112 \\
7.1113 \\
7.1 .14 \\
7.1 .15\end{array}$ & $\begin{array}{l}173.0 \\
319.5 \\
133.0 \\
2560.3 \\
37 \% .0\end{array}$ & $\begin{array}{l}319.5 \\
477.8 \\
250.5 \\
374.8 \\
46 \% .5\end{array}$ & $\begin{array}{r}30.0 \\
20.0 \\
30.0 \\
25.0 \\
210.0\end{array}$ & $\begin{array}{l}151.5 \\
199.5 \\
145.5 \\
140.5 \\
209.0\end{array}$ & $\begin{array}{l}190.5 \\
225.3 \\
142.5 \\
208.0 \\
239.0\end{array}$ & $\begin{array}{l}70.0 \\
70.0 .0 \\
81.5 \\
7.0 .5 \\
6.5 .0\end{array}$ & $\begin{array}{l}12.0 \\
12: 3 \\
12: 0 \\
12: 0 \\
1 \therefore: 0\end{array}$ & $\begin{array}{l}10.0 \\
10.0 \\
10.0 \\
10.0 \\
10.0\end{array}$ & $\begin{array}{l}52.0 \\
552.5 \\
56.0 \\
550.0 \\
50.0\end{array}$ \\
\hline 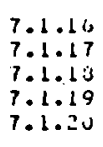 & 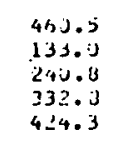 & $\begin{array}{l}342.5 \\
240.8 \\
333.8 \\
471.0 \\
500.0\end{array}$ & $\begin{array}{l}20.0 \\
25.0 \\
25.0 \\
35.0 \\
20.0 \\
20.0\end{array}$ & $\begin{array}{l}239.0 \\
33.5 \\
159.5 \\
224.3 \\
248.0\end{array}$ & $\begin{array}{r}445.0 \\
159.3 \\
224.8 \\
243.0 \\
313.3\end{array}$ & $\begin{array}{l}65.3 \\
5110.0 \\
55.0 \\
70.0 \\
35.00\end{array}$ & $\begin{array}{l}12.0 .0 \\
12.0 \\
12.0 \\
12.0 \\
12.0\end{array}$ & $\begin{array}{l}10.0 \\
1100 \\
0.00 \\
10.0 \\
10.0\end{array}$ & $\begin{array}{l}50.0 \\
50.0 \\
51.0 \\
56.0 \\
56.0\end{array}$ \\
\hline $\begin{array}{l}7.1 .21 \\
7.11 .22 \\
7.1 .23 \\
7.1 .24 \\
7.1 .21\end{array}$ & $\begin{array}{l}292.0 \\
313.0 \\
155.5 \\
237.3 \\
133.0\end{array}$ & $\begin{array}{r}373.0 \\
493.0 \\
237.3 \\
327.5 \\
216.0\end{array}$ & $\begin{array}{l}30.0 \\
40.0 \\
25.0 \\
30.0 \\
25.0\end{array}$ & $\begin{array}{r}313.3 \\
4955.0 \\
264.5 \\
323.5 \\
17.0\end{array}$ & 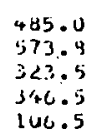 & $\begin{array}{l}10.0 \\
5100.0 \\
55.0 \\
55.0 \\
75.0\end{array}$ & $\begin{array}{l}12.3 \\
12.0 \\
12.0 \\
12.0 \\
12.0\end{array}$ & $\begin{array}{l}10.0 \\
110.0 \\
10.0 \\
10.0 \\
10.0\end{array}$ & $\begin{array}{l}56.0 \\
56.0 \\
56.0 \\
55.0 \\
56.0\end{array}$ \\
\hline
\end{tabular}


TABLE XIII (Contd.)

BASELINE COUNTERCURRENT FLOW TEST GROUP 7.1

EXPERIMENTAL MEASURMENTS FOR TESTS 7.1 .1 TO $7.1 .60--0.70-11 \%$. DOWNCUYER G.AP

\begin{tabular}{|c|c|c|c|c|c|c|c|c|c|}
\hline \multirow[b]{2}{*}{$\begin{array}{l}\text { TEST } \\
\text { NO }\end{array}$} & \multicolumn{3}{|c|}{ LOWER PLENUM FLON } & \multicolumn{3}{|c|}{ BYPASS FL'IH } & \multirow[b]{2}{*}{$\begin{array}{l}\text { LOWER } \\
\text { PLENIJM } \\
\text { LEVEL } \\
\text { IINI }\end{array}$} & \multirow[b]{2}{*}{$\begin{array}{l}P-L P-1 \\
\text { (PSIG) }\end{array}$} & \multirow[b]{2}{*}{$\begin{array}{l}T F-(P-1 \\
(D \leq G F)\end{array}$} \\
\hline & $\begin{array}{l}\text { INITLAL } \\
\text { MEISHT } \\
\text { (LY) }\end{array}$ & $\begin{array}{l}\text { FIHAL } \\
\text { WEIGHT } \\
\text { (LB) }\end{array}$ & $\begin{array}{l}\text { TIME OF } \\
\text { COLISCTICN } \\
\text { (SEC) }\end{array}$ & $\begin{array}{l}\text { INITIALL } \\
\text { WEIGHT } \\
\text { (LB) }\end{array}$ & $\begin{array}{l}\text { FIHAL } \\
\text { WEIGHIT } \\
\text { (LHI) }\end{array}$ & $\begin{array}{l}\text { TIME JJF } \\
\text { COLLECTIIJN } \\
\text { ISFC) }\end{array}$ & & & \\
\hline $\begin{array}{l}7.1 .26 \\
7.1 .27 \\
7.2 .28 \\
7.1 .29 \\
7.1 .3 .5\end{array}$ & $\begin{array}{l}218.0 \\
133.3 \\
132.0 \\
207.5 \\
229.0\end{array}$ & $\begin{array}{l}276.0 \\
182.0 \\
207.5 \\
229.0 \\
259.0\end{array}$ & $\begin{array}{l}30.0 \\
35.0 \\
20.0 \\
20.0 \\
35.0\end{array}$ & $\begin{array}{l}106.5 \\
237.8 \\
243.0 \\
252.5 \\
274.0\end{array}$ & $\begin{array}{l}12 E .0 \\
243.0 \\
252.5 \\
274.0 \\
298.5\end{array}$ & $\begin{array}{l}75.0 \\
82.0 \\
55.0 \\
65.0 \\
55.0\end{array}$ & $\begin{array}{l}12.0 \\
12.0 \\
12.0 \\
12.0 \\
12.0\end{array}$ & $\begin{array}{l}10.0 \\
10.0 \\
10.0 \\
10.0 \\
10.0\end{array}$ & $\begin{array}{l}56.0 \\
56.0 \\
56.0 \\
56.0 \\
56.0\end{array}$ \\
\hline $\begin{array}{l}7.1 .31 \\
7.1 .32 \\
7.1 .33 \\
7.1 .34 \\
7.1 .35\end{array}$ & $\begin{array}{l}25 \% .13 \\
143.5 \\
13 \% .0 \\
234.0 \\
26 \% .5\end{array}$ & $\begin{array}{l}283.0 \\
26.0 .0 \\
276.5 \\
375.0 \\
384.0\end{array}$ & $\begin{array}{l}20.0 \\
10.3 \\
15.0 \\
14.5 \\
15.0\end{array}$ & $\begin{array}{l}291.5 \\
108.5 \\
108.5 \\
134.5 \\
160.5\end{array}$ & $\begin{array}{l}329.0 \\
210.0 \\
157.0 \\
170.0 \\
219.5\end{array}$ & $\begin{array}{l}5 i 1.0 \\
60.0 \\
30.0 \\
30.0 \\
30.0\end{array}$ & $\begin{array}{l}12.0 \\
12.0 \\
12.0 \\
12.0 \\
12.0\end{array}$ & $\begin{array}{r}111.0 \\
7.5 \\
9.5 \\
7.7 \\
10.0\end{array}$ & $\begin{array}{l}56.0 \\
14.0 \\
45.0 \\
44.0 \\
13.5\end{array}$ \\
\hline $\begin{array}{l}7.1 .30 \\
7.1 .37 \\
7.1 .33 \\
7.1 .39 \\
7.1 .40\end{array}$ & $\begin{array}{l}197.0 \\
14.0 .5 \\
219.0 \\
1 \% 5.0 \\
1 \% 6.0\end{array}$ & $\begin{array}{l}323.0 \\
230.0 \\
293.5 \\
190.0 \\
230.0\end{array}$ & $\begin{array}{l}15.0 \\
14.5 \\
14.5 \\
15.0 \\
15.0\end{array}$ & $\begin{array}{l}219.5 \\
231.5 \\
327.0 \\
104.5 \\
201.5\end{array}$ & $\begin{array}{l}288.5 \\
327.0 \\
381.0 \\
231.5 \\
346.0\end{array}$ & $\begin{array}{l}45.0 \\
30.0 \\
30.0 \\
30.0 \\
30.0\end{array}$ & $\begin{array}{l}12.0 \\
12.0 \\
12.0 \\
12.0 \\
12.0\end{array}$ & $\begin{array}{l}10.5 \\
10.0 \\
10.0 \\
10.0 \\
10.0\end{array}$ & $\begin{array}{l}43.0 \\
44.0 \\
46.0 \\
49.0 \\
49.0\end{array}$ \\
\hline $\begin{array}{l}7 \cdot 1.41 \\
7.1 .42 \\
7.1 .43 \\
7.1 .44 \\
7.1 .45\end{array}$ & $\begin{array}{l}2136.5 \\
291.5 \\
151.5 \\
1117.5 \\
196.5\end{array}$ & $\begin{array}{l}266.5 \\
251.0 \\
187.5 \\
210.5 \\
216.5\end{array}$ & $\begin{array}{l}15.0 \\
15.0 \\
20.0 \\
15.0 \\
25.0\end{array}$ & $\begin{array}{l}214.0 \\
313.0 \\
335.5 \\
397.5 \\
395.0\end{array}$ & $\begin{array}{l}327.0 \\
355.5 \\
397.5 \\
439.0 \\
414.5\end{array}$ & $\begin{array}{l}30.0 \\
30.0 \\
30.0 \\
30.0 \\
15.0\end{array}$ & $\begin{array}{l}12.0 \\
12.0 \\
12.0 \\
12.0 \\
12.0\end{array}$ & $\begin{array}{r}9.7 \\
10.0 \\
10.0 \\
10.9 \\
10.2\end{array}$ & $\begin{array}{l}49.0 \\
48.0 \\
49.5 \\
49.5 \\
49.5\end{array}$ \\
\hline $\begin{array}{l}7.1 .40 \\
7.1 .47 \\
7.1 .48 \\
7.2 .49 \\
7.1 .30\end{array}$ & $\begin{array}{l}216.5 \\
235.5 \\
192.0 \\
153.0 \\
223.1\end{array}$ & $\begin{array}{l}235.5 \\
249.0 \\
401.0 \\
305.0 \\
363.0\end{array}$ & $\begin{array}{r}45.0 \\
120.0 \\
15.0 \\
15.0 \\
15.0\end{array}$ & $\begin{array}{l}\$ 14.5 \\
436.0 \\
104.5 \\
120.0 \\
131.0\end{array}$ & $\begin{array}{l}436.0 \\
472.0 \\
2114.5 \\
157.0 \\
232.5\end{array}$ & $\begin{array}{l}20.0 \\
60.0 \\
20.0 \\
1.5 .3 \\
15.0\end{array}$ & $\begin{array}{r}12.0 \\
12.0 \\
4.0 \\
4.0 \\
6.0\end{array}$ & $\begin{array}{l}10.0 \\
10.2 \\
10.5 \\
10.5 \\
10.0\end{array}$ & $\begin{array}{r}53.5 \\
59.0 \\
50.5 \\
48.0 \\
48.0\end{array}$ \\
\hline $\begin{array}{l}7.1 .51 \\
7.1 .52 \\
7.1 .53 \\
7.1 .54 \\
7.1 .55\end{array}$ & $\begin{array}{l}173.0 \\
141.5 \\
143.5 \\
161.5 \\
133.5\end{array}$ & $\begin{array}{l}316.0 \\
303.5 \\
211.5 \\
219.0 \\
161.5\end{array}$ & $\begin{array}{l}30.0 \\
30.0 \\
30.0 \\
30.0 \\
60.0\end{array}$ & $\begin{array}{r}144.5 \\
100.5 \\
200.0 \\
423.0 \\
38.5\end{array}$ & $\begin{array}{l}366.0 \\
446.0 \\
478.5 \\
652.5 \\
213.5\end{array}$ & $\begin{array}{l}30.0 \\
30.0 \\
30.0 \\
30.0 \\
30.0\end{array}$ & $\begin{array}{l}12.0 \\
12.0 \\
12.0 \\
12.0 \\
12.0\end{array}$ & $\begin{array}{l}10.2 \\
11.2 \\
11.0 \\
11.2 \\
10.0\end{array}$ & $\begin{array}{l}43.0 \\
47.0 \\
59.5 \\
51.5 \\
53.0\end{array}$ \\
\hline $\begin{array}{l}7.1 .56 \\
7.1 .57 \\
7.1 .50 \\
7.1 .57 \\
7.1 .601\end{array}$ & $\begin{array}{l}161.0 \\
179.0 \\
181.5 \\
192.0 \\
210.0\end{array}$ & $\begin{array}{l}195.0 \\
131.5 \\
192.0 \\
210.0 \\
2213.0\end{array}$ & $\begin{array}{r}60.0 \\
120.0 \\
60.0 \\
00.0 \\
120.0\end{array}$ & $\begin{array}{l}213.5 \\
240.5 \\
366.5 \\
374.0 \\
676.0\end{array}$ & $\begin{array}{l}406.5 \\
360: 5 \\
374.0 \\
476.5 \\
736.5\end{array}$ & $\begin{array}{l}30.0 \\
30: 0 \\
30.0 \\
15.0 \\
15.0\end{array}$ & $\begin{array}{l}12.0 \\
12.0 \\
12.0 \\
12.0 \\
12.0\end{array}$ & $\begin{array}{l}13.9 \\
11.2 \\
19.7 \\
15.7 \\
11.2\end{array}$ & $\begin{array}{r}50.0 \\
53.5 \\
55.0 \\
56.0 \\
55.0\end{array}$ \\
\hline
\end{tabular}




\section{TABLE XIV}

\section{BASELINE COUNTERCURRENT FLOW TEST GROUP 9.1}

TRANSDAPENT VESSEL TEST TATA FCR TESTS $9.1 .1 \mathrm{~T}$ T. 9.1 .98 L- 1.58-IN. DOWNCOLAR GAD

SUMMARY OF CALCULATED RESIJLTS

\begin{tabular}{|c|c|c|c|c|c|c|c|c|c|c|}
\hline $\begin{array}{c}\text { Nost } \\
\text { Not }\end{array}$ & $\begin{array}{l}\text { DOHNC JMEP. } \\
\text { AIR FL JN } \\
\text { (LA/SEC) }\end{array}$ & $\begin{array}{l}\text { 2TUNCOMER } \\
\text { AIS DE:IS ITY } \\
\text { (LO/FT } \$ 3)\end{array}$ & $\begin{array}{c}\text { LONEP PLENUM } \\
\text { PRESSURE } \\
\text { (PSIA) }\end{array}$ & $\begin{array}{l}\text { COLO LEG } \\
A I R F L O W \\
(L B / S E C)\end{array}$ & $\begin{array}{l}\text { TOWNCOMES } \\
\text { WATCP FLOWW } \\
\text { (LU/SEC) }\end{array}$ & $\begin{array}{l}\text { WATER FLOW } \\
\text { CANTINJITY }\end{array}$ & $\begin{array}{c}\text { J-AIR } \\
\text { DOWICOMEP } \\
\text { (FT/SEC) }\end{array}$ & $\begin{array}{l}J-W .9 T E R \\
\text { DDNNCUMER } \\
(E T / S E C)\end{array}$ & $\left(J G^{*}\right) * * 1 / 2$ & $(J L *) * * 1)$ \\
\hline 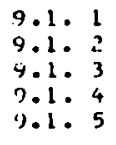 & $\begin{array}{l}0.4781 \\
0.4716 \\
0.5374 \\
0.5492 \\
0.3233\end{array}$ & $\begin{array}{l}0.137 ? \\
0.1539 \\
0.1398 \\
0.1594 \\
0.1229\end{array}$ & $\begin{array}{l}26.17 \\
29.17 \\
26.67 \\
30.17 \\
23.17\end{array}$ & $\begin{array}{l}0.0 \\
0.0 \\
0.0 \\
0.0 \\
0.0\end{array}$ & $\begin{array}{l}2.367 \\
2.392 \\
1.433 \\
1.733 \\
5.50 .5\end{array}$ & $\begin{array}{l}1.022 \\
1.034 \\
1.051 \\
1.023 \\
0.993\end{array}$ & $\begin{array}{l}14.76 \\
13.03 \\
16.34 \\
14.73 \\
11.36\end{array}$ & $\begin{array}{l}0.161 \\
0.204 \\
0.078 \\
0.118 \\
0.375\end{array}$ & $\begin{array}{l}0.4881 \\
0.4716 \\
0.5156 \\
0.5051 \\
0.4161\end{array}$ & $\begin{array}{l}0.2354 \\
0.2646 \\
0.1832 \\
0.2014 \\
0.3588\end{array}$ \\
\hline 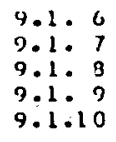 & $\begin{array}{l}0.379 z \\
0.1795 \\
0.0502 \\
0.4773 \\
0.5604\end{array}$ & $\begin{array}{l}0.1551 \\
0.1192 \\
0.1172 \\
0.1430 \\
0.1431\end{array}$ & $\begin{array}{l}29.30 \\
22.30 \\
22.30 \\
27.05 \\
27.30\end{array}$ & $\begin{array}{l}0.0 \\
0.0 \\
0.0 \\
0.0 \\
0.0\end{array}$ & $\begin{array}{r}5.003 \\
11.750 \\
18.700 \\
2.242 \\
1.329\end{array}$ & $\begin{array}{l}1.000 \\
1.010 \\
0.093 \\
1.030 \\
1.028\end{array}$ & $\begin{array}{r}10.40 \\
0.40 \\
1.79 \\
14.19 \\
16.65\end{array}$ & $\begin{array}{l}0.341 \\
0.800 \\
1.274 \\
0.153 \\
0.091\end{array}$ & $\begin{array}{l}0.4220 \\
0.3100 \\
0.1640 \\
0.4831 \\
0.5234\end{array}$ & $\begin{array}{l}0.3424 \\
0.5244 \\
0.6616 \\
0.2291 \\
0.1764\end{array}$ \\
\hline $\begin{array}{l}9.1 .11 \\
9.1 .12 \\
9.1 .13 \\
9.1 .14 \\
9.1 .15\end{array}$ & $\begin{array}{l}0.6559 \\
0.7282 \\
0.6571 \\
0.3687 \\
0.2525\end{array}$ & $\begin{array}{l}3.1524 \\
0.1641 \\
0.1453 \\
0.1092 \\
0.1179\end{array}$ & $\begin{array}{l}29.30 \\
31.30 \\
20.05 \\
20.55 \\
22.05\end{array}$ & $\begin{array}{l}0.0 \\
0.0 \\
0.0 \\
0.0 \\
0.0\end{array}$ & $\begin{array}{l}0.438 \\
0.280 \\
0.425 \\
3.900 \\
0.517\end{array}$ & $\begin{array}{l}1.037 \\
0.993 \\
1.041 \\
0.997 \\
0.968\end{array}$ & $\begin{array}{r}18.29 \\
18.96 \\
19.22 \\
14.35 \\
9.11\end{array}$ & $\begin{array}{l}0.030 \\
0.019 \\
0.029 \\
0.266 \\
0.444\end{array}$ & $\begin{array}{l}0.5574 \\
0.5766 \\
0.5646 \\
0.4542 \\
0.3697\end{array}$ & $\begin{array}{l}0.1012 \\
0.0810 \\
0.0997 \\
0.3021 \\
0.3905\end{array}$ \\
\hline $\begin{array}{l}9.1 .21 \\
9.1 .22 \\
9.1 .23 \\
9.1 .24 \\
9.1 .25\end{array}$ & $\begin{array}{l}0.2432 \\
0.2176 \\
0.1475 \\
0.1341 \\
0.1022\end{array}$ & $\begin{array}{l}0.1261 \\
0.1260 \\
0.1245 \\
0.1168 \\
0.1180\end{array}$ & $\begin{array}{l}23.84 \\
23.84 \\
23.34 \\
22.62 \\
22.37\end{array}$ & $\begin{array}{l}0.0 \\
0.0 \\
0.0 \\
0.0 \\
0.0\end{array}$ & $\begin{array}{r}6.520 \\
9.976 \\
11.400 \\
13.800 \\
16.600\end{array}$ & $\begin{array}{l}0.901 \\
0.707 \\
0.812 \\
0.996 \\
1.001\end{array}$ & $\begin{array}{l}8.29 \\
7.31 \\
5.03 \\
4.90 \\
3.66\end{array}$ & $\begin{array}{l}0.444 \\
0.690 \\
0.777 \\
0.940 \\
1.131\end{array}$ & $\begin{array}{l}0.3559 \\
0.3363 \\
0.2780 \\
0.2692 \\
0.2342\end{array}$ & $\begin{array}{l}0.3906 \\
0.4832 \\
0.5166 \\
0.5683 \\
0.6233\end{array}$ \\
\hline $\begin{array}{l}9.1 .26 \\
9.1 .27 \\
9.1 .28 \\
9.1 .29 \\
9.1 .30\end{array}$ & $\begin{array}{l}0.2001 \\
0.2583 \\
0.2526 \\
0.246 \mathrm{~J} \\
0.180 \%\end{array}$ & $\begin{array}{l}0.1175 \\
.11243 \\
0.1522 \\
0.1752 \\
0.1430\end{array}$ & $\begin{array}{l}22.12 \\
23.37 \\
28.132 \\
33.62 \\
26.87\end{array}$ & $\begin{array}{l}0.0 \\
0.0 \\
c .0 \\
0.0 \\
0.0\end{array}$ & $\begin{array}{r}6.983 \\
8.023 \\
9.283 \\
9.783 \\
12.650\end{array}$ & $\begin{array}{l}1.026 \\
0.973 \\
0.990 \\
0.965 \\
1.002\end{array}$ & $\begin{array}{l}9.41 \\
8.83 \\
7.05 \\
5.36 \\
5.36\end{array}$ & $\begin{array}{l}0.4 ? 6 \\
0.551 \\
0.632 \\
0.666 \\
0.862\end{array}$ & $\begin{array}{l}0.3745 \\
0.3681 \\
0.3460 \\
0.3284 \\
0.2970\end{array}$ & $\begin{array}{l}0.4043 \\
0.4350 \\
0.4662 \\
0.4786 \\
0.5442\end{array}$ \\
\hline $\begin{array}{l}9.1 .31 \\
9.1 .32 \\
9.1 .33 \\
9.1 .34 \\
9.1 .35\end{array}$ & $\begin{array}{l}0.4566 \\
0.1185 \\
0.1341 \\
0.1015 \\
0.1182\end{array}$ & $\begin{array}{l}0.1698 \\
0.1153 \\
0.1150 \\
0.1123 \\
0.1164\end{array}$ & $\begin{array}{l}31.87 \\
21.79 \\
21.79 \\
21.41 \\
22.16\end{array}$ & $\begin{array}{l}0.0 \\
0.0 \\
0.0 \\
0.0 \\
0.0\end{array}$ & $\begin{array}{l}3.733 \\
3.508 \\
5.133 \\
5.483 \\
5.383\end{array}$ & $\begin{array}{l}0.996 \\
1.048 \\
0.998 \\
1.019 \\
0.995\end{array}$ & $\begin{array}{r}11.50 \\
4.37 \\
4.98 \\
3.84 \\
4.32\end{array}$ & $\begin{array}{l}0.254 \\
0.375 \\
0.350 \\
0.374 \\
0.367\end{array}$ & $\begin{array}{l}0.4534 \\
0.2539 \\
0.2710 \\
0.2366 \\
0.2531\end{array}$ & $\begin{array}{l}0.2957 \\
0.3591 \\
0.3466 \\
0.3582 \\
0.3550\end{array}$ \\
\hline
\end{tabular}


TABLE XIV (Contd.)

BASELINE COUNTERCURRENT FLOW TEST GROUP 9.1

SIMMARY DF CALCULATEO RESULTS FOR TESTS 9.1 .1 TO $9.1 .98 \cdots \cdot 1.58$ IN. DOMNCOMER. GAP

\begin{tabular}{|c|c|c|c|c|c|c|c|c|c|c|}
\hline $\begin{array}{l}\text { TEST } \\
\text { NO }\end{array}$ & $\begin{array}{l}\text { DIWNCOMER } \\
\text { AIR FL FHA } \\
\text { (LB/SEC, }\end{array}$ & $\begin{array}{l}\text { DOWNCOSER } \\
\text { AIR OENSITY } \\
\text { (LB/FT*3) }\end{array}$ & $\begin{array}{l}\text { LOWER PLENUM } \\
\text { PRESSURE } \\
\text { (PSIA) }\end{array}$ & $\begin{array}{l}\text { COLD LEG } \\
\text { AIR FLNW } \\
(L B / S E C)\end{array}$ & $\begin{array}{l}\text { DOWNCOMEP. } \\
\text { WATEP FLOW } \\
\text { (LE/SEC) }\end{array}$ & $\begin{array}{l}\text { WATER FLOW } \\
\text { CONTINUITY }\end{array}$ & $\begin{array}{c}\text { J-AIR } \\
\text { OOWNCOMER } \\
(F T / S E C)\end{array}$ & $\begin{array}{l}\text { J-HATER } \\
\text { DOWNC NMER } \\
\text { (FT/SEC) }\end{array}$ & $(J G *) * * 1 / 2$ & $(\Omega l *) * * 1 / 2$ \\
\hline $\begin{array}{l}9.1 .41 \\
9.1 .42 \\
9.1 .43 \\
9.1 .44 \\
9.1 .45\end{array}$ & $\begin{array}{l}0.1784 \\
0.1778 \\
0.1766 \\
0.1748 \\
0.1765\end{array}$ & $\begin{array}{l}0.1130 \\
0.1130 \\
0.1175 \\
.11160 \\
0.1063\end{array}$ & $\begin{array}{l}21.48 \\
21.48 \\
22.29 \\
22.04 \\
20.29\end{array}$ & $\begin{array}{l}0.0 \\
0.0 \\
0.0 \\
0.0 \\
0.0\end{array}$ & $\begin{array}{l}4.108 \\
4.792 \\
5.667 \\
6.608 \\
7.867\end{array}$ & $\begin{array}{l}1.002 \\
0.999 \\
1.005 \\
0.995 \\
0.978\end{array}$ & $\begin{array}{l}6.71 \\
6.69 \\
6.39 \\
6.40 \\
7.06\end{array}$ & $\begin{array}{l}0.280 \\
0.326 \\
0.386 \\
0.450 \\
0.536\end{array}$ & $\begin{array}{l}0.3132 \\
0.3126 \\
0.3086 \\
0.3080 \\
0.3164\end{array}$ & $\begin{array}{l}0.3101 \\
0.3349 \\
0.3642 \\
0.3933 \\
0.4291\end{array}$ \\
\hline $\begin{array}{l}9.1 .46 \\
9.1 .47 \\
9.1 .48 \\
9.1 .49 \\
9.1 .50\end{array}$ & $\begin{array}{l}0.1766 \\
0.2554 \\
0.244 d \\
0.1771 \\
0.4749\end{array}$ & $\begin{array}{l}0.1118 \\
0.1157 \\
0.1404 \\
0.1271 \\
0.1589\end{array}$ & $\begin{array}{l}21.29 \\
22.79 \\
26.79 \\
23.78 \\
30.17\end{array}$ & $\begin{array}{l}0.0 \\
0.0 \\
0.0 \\
0.0 \\
0.0\end{array}$ & $\begin{array}{r}9.656 \\
4.117 \\
9.787 \\
13.900 \\
2.500\end{array}$ & $\begin{array}{l}0.994 \\
1.017 \\
0.984 \\
0.976 \\
1.016\end{array}$ & $\begin{array}{r}6.71 \\
9.07 \\
7.41 \\
5.32 \\
12.70\end{array}$ & $\begin{array}{l}0.653 \\
0.280 \\
0.667 \\
0.947 \\
0.170\end{array}$ & $\begin{array}{l}0.3125 \\
0.3694 \\
0.3475 \\
0.3031 \\
0.4694\end{array}$ & $\begin{array}{l}0.4754 \\
0.3104 \\
0.4787 \\
0.5704 \\
0.2419\end{array}$ \\
\hline $\begin{array}{l}9.1 .51 \\
9.1 .52 \\
9.1 .53 \\
9.1 .54 \\
9.1 .55\end{array}$ & $\begin{array}{l}0.2890 \\
0.1685 \\
0.1816 \\
0.0736 \\
0.1602\end{array}$ & $\begin{array}{l}0.1153 \\
0.1211 \\
3.1190 \\
0.1194 \\
3.1180\end{array}$ & $\begin{array}{l}21.92 \\
22.80 \\
22.30 \\
22.30 \\
22.30\end{array}$ & $\begin{array}{l}0.0 \\
0.0 \\
0.0 \\
0.0 \\
0.0\end{array}$ & $\begin{array}{r}3.125 \\
4.758 \\
8.067 \\
13.933 \\
7.522\end{array}$ & $\begin{array}{l}1.003 \\
0.995 \\
0.980 \\
0.979 \\
0.990\end{array}$ & $\begin{array}{r}10.61 \\
6.61 \\
6.49 \\
2.62 \\
5.77\end{array}$ & $\begin{array}{l}0.213 \\
0.324 \\
0.549 \\
0.949 \\
0.512\end{array}$ & $\begin{array}{l}0.3962 \\
0.3164 \\
0.3120 \\
0.1984 \\
0.2936\end{array}$ & $\begin{array}{l}0.2704 \\
0.3337 \\
0.4345 \\
0.5711 \\
0.4196\end{array}$ \\
\hline $\begin{array}{l}9.1 .56 \\
9.1 .57 \\
9.1 .59 \\
9.1 .59 \\
9.1 .60\end{array}$ & $\begin{array}{l}0.2522 \\
0.3750 \\
0.2474 \\
0.2469 \\
0.2454\end{array}$ & $\begin{array}{l}0.1180 \\
0.1173 \\
0.1176 \\
.11179 \\
0.1205\end{array}$ & $\begin{array}{l}22.30 \\
22.20 \\
22.35 \\
22.10 \\
22.55\end{array}$ & $\begin{array}{l}0.0 \\
0.0 \\
0.0 \\
0.0 \\
0.0\end{array}$ & $\begin{array}{l}5.35 n \\
2.817 \\
3.672 \\
4.650 \\
4.350\end{array}$ & $\begin{array}{l}0.997 \\
1.023 \\
0.973 \\
0.996 \\
1.022\end{array}$ & $\begin{array}{r}9.09 \\
13.59 \\
8.79 \\
8.90 \\
8.65\end{array}$ & $\begin{array}{l}0.365 \\
0.192 \\
0.250 \\
0.317 \\
0.296\end{array}$ & $\begin{array}{l}0.3684 \\
0.4500 \\
0.3637 \\
0.3646 \\
0.3615\end{array}$ & $\begin{array}{l}0.3540 \\
0.2568 \\
0.2932 \\
0.3299 \\
0.3191\end{array}$ \\
\hline $\begin{array}{l}9.1 .61 \\
9.1 .62 \\
9.1 .63 \\
9.1 .64 \\
9.1 .63\end{array}$ & $\begin{array}{l}0.1270 \\
0.1270 \\
0.1235 \\
0.278 \mathrm{~d} \\
0.3225\end{array}$ & $\begin{array}{l}0.1167 \\
0.1183 \\
0.1172 \\
0.1175 \\
0.1502\end{array}$ & $\begin{array}{l}21.80 \\
22.05 \\
21.80 \\
22.20 \\
29.95\end{array}$ & $\begin{array}{l}0.0 \\
0.0 \\
0.0 \\
0.0 \\
0.0\end{array}$ & $\begin{array}{r}5.100 \\
8.300 \\
9.200 \\
10.500 \\
7.225\end{array}$ & $\begin{array}{l}0.937 \\
0.996 \\
0.900 \\
1.002 \\
1.059\end{array}$ & $\begin{array}{r}4.52 \\
4.56 \\
4.66 \\
10.06 \\
8.56\end{array}$ & $\begin{array}{l}0.347 \\
0.565 \\
0.627 \\
0.715 \\
0.492\end{array}$ & $\begin{array}{l}0.2622 \\
0.2613 \\
0.2634 \\
0.3875 \\
0.3960\end{array}$ & $\begin{array}{l}0.3455 \\
0.4407 \\
0.4640 \\
0.4957 \\
0.4113\end{array}$ \\
\hline $\begin{array}{l}9.1 .66 \\
9.1 .67 \\
9.1 .68 \\
9.1 .69 \\
9.1 .70\end{array}$ & $\begin{array}{l}0.3167 \\
0.2436 \\
0.5067 \\
0.5326 \\
0.4926\end{array}$ & $\begin{array}{l}0.1509 \\
0.1200 \\
0.1533 \\
0.1864 \\
10.1721\end{array}$ & $\begin{array}{l}28.20 \\
22.45 \\
28.95 \\
35.42 \\
32.67\end{array}$ & $\begin{array}{l}0.0 \\
0.0 \\
0.0 \\
0.0 \\
0.0\end{array}$ & $\begin{array}{l}7.150 \\
0.933 \\
1.575 \\
3.350 \\
2.983\end{array}$ & $\begin{array}{l}1.045 \\
1.023 \\
1.022 \\
1.040 \\
1.011\end{array}$ & $\begin{array}{r}8.92 \\
8.63 \\
14.05 \\
11.46 \\
12.17\end{array}$ & $\begin{array}{l}0.497 \\
0.472 \\
0.107 \\
0.229 \\
0.203\end{array}$ & $\begin{array}{l}0.3883 \\
0.3606 \\
0.4893 \\
0.4641 \\
0.4686\end{array}$ & $\begin{array}{l}0.4091 \\
0.4028 \\
0.1920 \\
0.2801 \\
0.2643\end{array}$ \\
\hline $\begin{array}{l}9.1 .71 \\
9.1 .72 \\
9.1 .73 \\
9.2 .74 \\
9.1 .75\end{array}$ & $\begin{array}{l}0.5691 \\
0.5527 \\
0.5754 \\
0.5329 \\
0.4550\end{array}$ & $\begin{array}{l}0.1813 \\
1.1602 \\
0.1826 \\
0.1713 \\
0.1715\end{array}$ & $\begin{array}{l}34.42 \\
30.42 \\
34.67 \\
32.42 \\
32.42\end{array}$ & $\begin{array}{l}0.0 \\
0.0 \\
0.0 \\
0.0 \\
0.0\end{array}$ & $\begin{array}{l}2.017 \\
1.733 \\
1.783 . \\
1.483 \\
3.767 .\end{array}$ & $\begin{array}{l}1.039 \\
1.034 \\
1.039 \\
0.999 \\
1.021\end{array}$ & $\begin{array}{l}13.30 \\
14.66 \\
13.93 \\
14.55 \\
11.27\end{array}$ & $\begin{array}{l}0.137 \\
0.113 \\
0.121 \\
0.101 \\
0.257\end{array}$ & $\begin{array}{l}0.4969 \\
0.5053 \\
0.5090 \\
0.5112 \\
0.4507\end{array}$ & $\begin{array}{l}0.2173 \\
0.2014 \\
0.2044 \\
0.1864 \\
0.2970\end{array}$ \\
\hline $\begin{array}{l}9.1 .76 \\
9.1 .77 \\
9.1 .78 \\
9.1 .79 \\
9.1 .90\end{array}$ & $\begin{array}{l}0.5797 \\
0.4698 \\
0.4769 \\
0.4694 \\
0.4841\end{array}$ & $\begin{array}{l}0.1744 \\
0.1782 \\
0.1124 \\
0.1153 \\
0.1234\end{array}$ & $\begin{array}{l}33.17 \\
33.67 \\
21.49 \\
21.98 \\
24.48\end{array}$ & $\begin{array}{l}0.0 \\
0.0 \\
0.0 \\
0.0 \\
0.0\end{array}$ & $\begin{array}{l}1.700 \\
3.450 \\
1.329 \\
2.139 \\
2.378\end{array}$ & $\begin{array}{l}1.001 \\
0.994 \\
1.013 \\
1.049 \\
1.047\end{array}$ & $\begin{array}{l}14.13 \\
11.20 \\
18.04 \\
17.30 \\
16.02\end{array}$ & $\begin{array}{l}0.116 \\
0.235 \\
0.091 \\
0.146 \\
0.162\end{array}$ & $\begin{array}{l}0.5067 \\
0.4537 \\
0.5129 \\
0.5055 \\
0.4998\end{array}$ & $\begin{array}{l}0.1995 \\
0.2842 \\
0.1764 \\
0.2237 \\
0.2359\end{array}$ \\
\hline
\end{tabular}


TABLE XIV (Contd.)

BASELINE COUNTERCURRENT FLOW TEST GROUP 9.1

SIMMMARY OF CALCIJLATEN QESULIS FIRR TF.ST.S 9.1 .1 TO 9.1.78-- 1.58 IN. TOHNCIJMEP GAT

\begin{tabular}{|c|c|c|c|c|c|c|c|c|c|c|}
\hline $\begin{array}{l}\text { TEST } \\
\text { NO }\end{array}$ & $\begin{array}{l}\text { CHWNCIJYCR } \\
\text { AIP FLNW } \\
\text { (LB/SEC) }\end{array}$ & 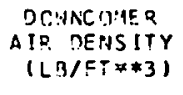 & $\begin{array}{l}\text { COWER PLENUM } \\
\text { PRESSURE } \\
\text { (PSIA) }\end{array}$ & $\begin{array}{l}\text { COLO LEG } \\
\text { AIR FLOH } \\
\text { (LE/SEC) }\end{array}$ & $\begin{array}{l}\text { DOWNCOMEP } \\
\text { WATER FLCNA } \\
\text { (LB/SEC) }\end{array}$ & $\begin{array}{l}\text { WATER FLNW } \\
\text { CONTINUITY }\end{array}$ & $\begin{array}{l}\text { J-A!P. } \\
\text { ODWVC UMEP } \\
\text { (FT/SEC) }\end{array}$ & $\begin{array}{l}\text { J-HATER } \\
\text { DOWNCOMER } \\
\text { (FT/SEC) }\end{array}$ & $(J 6 *) * * 1 / 2$ & $(\mathrm{JL} * 1 * 1 / 2$ \\
\hline $\begin{array}{l}9.1 .81 \\
9.1 .82 \\
9.1 .83 \\
9.1 .84 \\
9.1 .85\end{array}$ & $\begin{array}{l}0.3234 \\
0.3781 \\
0.1750 \\
0.2491 \\
J .3245\end{array}$ & $\begin{array}{l}0.1244 \\
0.1401 \\
0.122 .3 \\
0.1224 \\
0.1532\end{array}$ & $\begin{array}{l}23.73 \\
23.48 \\
23.29 \\
23.29 \\
29.28\end{array}$ & $\begin{array}{l}0.0 \\
0.0 \\
0.0 \\
c .0 \\
0.0\end{array}$ & $\begin{array}{r}5.367 \\
4.9 .42 \\
11.583 \\
6.756 \\
6.600\end{array}$ & $\begin{array}{l}1.103 \\
0.972 \\
0.999 \\
1.003 \\
0.999\end{array}$ & $\begin{array}{r}11.05 \\
10.77 \\
6.10 \\
8.65 \\
8.89\end{array}$ & $\begin{array}{l}0.366 \\
0.337 \\
0.789 \\
0.463 \\
0.450\end{array}$ & $\begin{array}{l}0.4117 \\
0.4255 \\
0.3046 \\
0.3629 \\
0.3903\end{array}$ & $\begin{array}{l}0.3544 \\
0.3401 \\
0.5207 \\
0.3976 \\
0.3931\end{array}$ \\
\hline $\begin{array}{l}9.1 .86 \\
9.1 .87 \\
9.1 .88 \\
9.1 .85 \\
9.1 .90\end{array}$ & $\begin{array}{l}0.4826 \\
0.4911 \\
0.5390 \\
0.3204 \\
0.5365\end{array}$ & $\begin{array}{l}.1249 \\
0.1240 \\
0.1252 \\
0.1176 \\
0.1355\end{array}$ & $\begin{array}{l}24.03 \\
24.17 \\
24.17 \\
22.17 \\
25.55\end{array}$ & $\begin{array}{l}0.0 \\
0.0 \\
0.0 \\
0.0 \\
0.0\end{array}$ & $\begin{array}{l}2.365 \\
1.762 \\
1.003 \\
4.267 \\
2.767\end{array}$ & $\begin{array}{l}1.034 \\
1.043 \\
1.084 \\
1.014 \\
1.036\end{array}$ & $\begin{array}{l}16.17 \\
16.83 \\
18.15 \\
11.91 \\
16.83\end{array}$ & $\begin{array}{l}0.161 \\
0.120 \\
0.069 \\
0.291 \\
0.188\end{array}$ & $\begin{array}{l}0.5005 \\
0.5078 \\
0.5296 \\
0.4214 \\
0.5192\end{array}$ & $\begin{array}{l}0.2353 \\
0.2031 \\
0.1536 \\
0.3160 \\
0.2545\end{array}$ \\
\hline $\begin{array}{l}9.1 .91 \\
9.1 .92 \\
9.1 .93 \\
9.1 .94 \\
9.1 .45\end{array}$ & $\begin{array}{l}0.4975 \\
0.2521 \\
0.1360 \\
0.5106 \\
0.2001\end{array}$ & $\begin{array}{l}0.1368 \\
0.1190 \\
0.1183 \\
0.1632 \\
0.1437\end{array}$ & $\begin{array}{l}25.80 \\
22.30 \\
22.09 \\
3 C .87 \\
28.12\end{array}$ & $\begin{array}{l}0.0 \\
0.0 \\
0.0 \\
0.0 \\
0.0\end{array}$ & $\begin{array}{r}2.300 \\
6.400 \\
12.333 \\
2.433 \\
11.075\end{array}$ & $\begin{array}{l}1.019 \\
1.063 \\
1.013 \\
1.032 \\
0.990\end{array}$ & $\begin{array}{r}15.46 \\
9.01 \\
4.84 \\
13.53 \\
5.72\end{array}$ & $\begin{array}{l}0.157 \\
0.436 \\
0.840 \\
0.166 \\
0.754\end{array}$ & $\begin{array}{l}0.4997 \\
0.3676 \\
0.2704 \\
0.4877 \\
0.3099\end{array}$ & $\begin{array}{l}0.2320 \\
0.3870 \\
0.5373 \\
0.2387 \\
0.5092\end{array}$ \\
\hline $\begin{array}{l}9.1 .90 \\
9.1 .97 \\
9.1 .98\end{array}$ & $\begin{array}{l}0.1315 \\
0.5081 \\
0.0756\end{array}$ & $\begin{array}{l}0.1243 \\
.01702 \\
.12000\end{array}$ & $\begin{array}{l}23.37 \\
32.12 \\
22.34\end{array}$ & $\begin{array}{l}0.0 \\
0.0 \\
0.0\end{array}$ & $\begin{array}{r}12.383 \\
2.967 \\
13.500\end{array}$ & $\begin{array}{l}1.001 \\
1.016 \\
0.981\end{array}$ & $\begin{array}{r}6.18 \\
12.69 \\
2.68\end{array}$ & $\begin{array}{l}0.344 \\
0.202 \\
0.947\end{array}$ & $\begin{array}{l}0.3082 \\
0.4773 \\
0.2009\end{array}$ & $\begin{array}{l}0.5384 \\
0.2636 \\
0.5704\end{array}$ \\
\hline
\end{tabular}


TABLE XIV (Contd.)

BASELINE COUNTERCURRENT FLOW TEST GROUP 9.1

EXPERIMENT AL MEASUP.MENTS FOR TESTS 9.1 .1 TO $9.1 .98--1.58-$ IN. DONNCOMER GAP

\begin{tabular}{|c|c|c|c|c|c|c|c|c|c|c|}
\hline $\begin{array}{c}\text { TEST } \\
\text { NO }\end{array}$ & $\begin{array}{c}R M-C W-1 \\
(G D M)\end{array}$ & $\begin{array}{l}\text { P.M-CH-2 } \\
\text { (GPM) }\end{array}$ & $\begin{array}{c}F T-C H-1 \\
(G P M)\end{array}$ & $\begin{array}{c}F T=C H-2 \\
(G P M)\end{array}$ & $\begin{array}{l}R H-H G-1 \\
(C F M)\end{array}$ & $\begin{array}{l}\text { RM-HG-2 } \\
(C F M)\end{array}$ & $\begin{array}{c}R M-H G-3 \\
(C F M)\end{array}$ & $\begin{array}{c}\text { RM-HG-4 } \\
(C F M)\end{array}$ & $\begin{array}{c}F T-H G-1 \\
\text { (CFM) }\end{array}$ & $\begin{array}{c}F T-H G-2 \\
\text { (CFM) }\end{array}$ \\
\hline 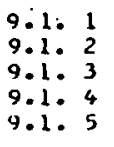 & $\begin{array}{l}0.10 \\
0.0 \\
0.0 \\
0.0 \\
0.0\end{array}$ & $\begin{array}{l}0.0 \\
0.0 \\
0.0 \\
0.0 \\
0.0\end{array}$ & $\begin{array}{r}0.0 \\
0.0 \\
0.0 \\
0.0 \\
10.0\end{array}$ & $\begin{array}{r}40.0 \\
50.0 \\
29.8 \\
39.9 \\
0.0\end{array}$ & $\begin{array}{l}0.0 \\
0.0 \\
0.0 \\
0.0 \\
0.0\end{array}$ & $\begin{array}{l}0.0 \\
0.0 \\
0.0 \\
0.0 \\
0.0\end{array}$ & $\begin{array}{l}0.0 \\
0.0 \\
0.0 \\
0.0 \\
0.0\end{array}$ & $\begin{array}{l}0.0 \\
0.0 \\
0.0 \\
0.0 \\
0.0\end{array}$ & $\begin{array}{l}60.26 \\
60.38 \\
82.44 \\
80.77 \\
40.38\end{array}$ & $\begin{array}{l}0.0 \\
0.0 \\
0.0 \\
0.0 \\
0.0\end{array}$ \\
\hline $\begin{array}{l}9.1 .6 \\
9.1: 7 \\
9.1 .8 \\
9.1 .9 \\
9.1 .10\end{array}$ & $\begin{array}{l}0.0 \\
0.0 \\
0.0 \\
0.0 \\
0.0\end{array}$ & $\begin{array}{l}0.0 \\
0.0 \\
0.0 \\
0.0 \\
0.0\end{array}$ & $\begin{array}{r}78.0 \\
130.0 \\
148.0 \\
0.0 \\
0.0\end{array}$ & $\begin{array}{r}0.0 \\
0.0 \\
0.0 \\
39.9 \\
30.6\end{array}$ & $\begin{array}{l}0.0 \\
0.0 \\
0.0 \\
0.0 \\
0.0\end{array}$ & $\begin{array}{l}0.0 \\
0.0 \\
0.0 \\
0.0 \\
0.0\end{array}$ & $\begin{array}{l}0.0 \\
0.0 \\
0 \% 0 \\
0.0 \\
0.0\end{array}$ & $\begin{array}{l}0.0 \\
0.0 \\
0.0 \\
0.0 \\
0.0\end{array}$ & $\begin{array}{c}39.49 \\
20.13 \\
0.0 \\
61.41 \\
72.44\end{array}$ & $\begin{array}{l}0.0 \\
0.0 \\
5.56 \\
0.0 \\
0.0\end{array}$ \\
\hline $\begin{array}{l}9.1 .11 \\
9.1 .12 \\
9.1 .13 \\
9.1 .14 \\
9.1 .15\end{array}$ & $\begin{array}{l}3.0 \\
0.0 \\
0.0 \\
0.0 \\
0.0\end{array}$ & $\begin{array}{l}0.0 \\
0.0 \\
0.0 \\
0.0 \\
0.0\end{array}$ & $\begin{array}{r}0.0 \\
0.0 \\
0.0 \\
0.0 \\
80.0\end{array}$ & $\begin{array}{r}20.2 \\
20.9 \\
20.1 \\
49.9 \\
0.0\end{array}$ & $\begin{array}{l}0.0 \\
0.0 \\
0.0 \\
0.0 \\
0.0\end{array}$ & $\begin{array}{l}0.0 \\
0.0 \\
0.0 \\
0.0 \\
0.0\end{array}$ & $\begin{array}{l}0.0 \\
0.0 \\
0.0 \\
0.0 \\
0.0\end{array}$ & $\begin{array}{l}0.0 \\
0.0 \\
0.0 \\
0.0 \\
0.0\end{array}$ & $\begin{array}{r}88.33 \\
101.41 \\
85.00 \\
44.49 \\
29.87\end{array}$ & $\begin{array}{l}0.0 \\
0.0 \\
0.0 \\
0.0 \\
0.0\end{array}$ \\
\hline $\begin{array}{l}9.1 .15 \\
9.1 .17 \\
9.1 .18 \\
9.1 .19 \\
9.1 .20\end{array}$ & $\begin{array}{l}0.0 \\
0.0 \\
0.0 \\
0.0 \\
0.0\end{array}$ & $\begin{array}{l}0.0 \\
0.0 \\
0.0 \\
0.0 \\
0.0\end{array}$ & $\begin{array}{r}130.0 \\
0.0 \\
0.0 \\
0.0 \\
0.0\end{array}$ & $\begin{array}{r}0.0 \\
29.9 \\
29.9 \\
.49 .9 \\
49.9\end{array}$ & $\begin{array}{l}0.0 \\
0.0 \\
0.0 \\
0.0 \\
0.0\end{array}$ & $\begin{array}{l}0.0 \\
0.0 \\
0.0 \\
0.0 \\
0.0\end{array}$ & $\begin{array}{l}0.0 \\
0.0 \\
0.0 \\
0.0 \\
0.0\end{array}$ & $\begin{array}{l}0.9 \\
0.9 \\
0.0 \\
0.0 \\
0.0\end{array}$ & $\begin{array}{l}14.74 \\
73.97 \\
81.03 \\
73.72 \\
56.03\end{array}$ & $\begin{array}{l}0.0 \\
0.0 \\
0.0 \\
0.0 \\
0.0\end{array}$ \\
\hline $\begin{array}{l}0.1 .21 \\
9.1 .22 \\
0.1 .23 \\
9.1 .24 \\
9.1 .25\end{array}$ & $\begin{array}{l}0.0 \\
0.0 \\
1.0 \\
0.0 \\
0.0\end{array}$ & $\begin{array}{l}0.0 \\
0.0 \\
0.0 \\
0.0 \\
0.0\end{array}$ & $\begin{array}{l}100.0 \\
150.0 \\
150.0 \\
150.0 \\
160.0\end{array}$ & $\begin{array}{l}0.0 \\
0.0 \\
0.0 \\
0.0 \\
0.0\end{array}$ & $\begin{array}{c}0.0 \\
80.00 \\
67.00 \\
0.0 \\
46.50\end{array}$ & $\begin{array}{l}0.0 \\
0.0 \\
0.0 \\
0.0 \\
0.0\end{array}$ & $\begin{array}{c}0.0 \\
20.00 \\
0.0 \\
0.0 \\
0.0\end{array}$ & $\begin{array}{l}0.0 \\
0.0 \\
0.0 \\
0.0 \\
0.0\end{array}$ & $\begin{array}{c}29.49 \\
0.0 \\
0.0 \\
14.49 \\
0.0\end{array}$ & $\begin{array}{l}0.0 \\
0.0 \\
0.0 \\
0.0 \\
0.0\end{array}$ \\
\hline $\begin{array}{l}9.1 .26 \\
9.1 .27 \\
9.1 .28 \\
9.1 .29 \\
9.1 .30\end{array}$ & $\begin{array}{l}0.0 . \\
0.0 \\
0.0 \\
0.0 \\
1.0\end{array}$ & $\begin{array}{l}0.0 \\
0.0 \\
0.0 \\
0.0 \\
0.0\end{array}$ & $\begin{array}{r}80.0 \\
101.0 \\
130.0 \\
1,50.0 \\
161.0\end{array}$ & $\begin{array}{l}0.0 \\
0.0 \\
0.0 \\
0.0 \\
0.0\end{array}$ & $\begin{array}{l}0.0 \\
0.0 \\
0.0 \\
0.0 \\
0.0\end{array}$ & $\begin{array}{l}0.0 \\
0.0 \\
0.0 \\
0.0 \\
0.0\end{array}$ & $\begin{array}{l}0.0 \\
0.0 \\
0.0 \\
0.0 \\
0.0\end{array}$ & $\begin{array}{l}0.0 \\
0.0 \\
0.0 \\
0.0 \\
0.0\end{array}$ & $\begin{array}{l}29.62 \\
29.74 \\
29.49 \\
29.10 \\
20.77\end{array}$ & $\begin{array}{l}0.0 \\
0.0 \\
0.0 \\
0.0 \\
0.0\end{array}$ \\
\hline $\begin{array}{l}9.1 .31 \\
9.1 .332 \\
9.1 .33 \\
9.1 .34 \\
9.1 .35\end{array}$ & $\begin{array}{l}3.0 \\
0.0 \\
2.0 \\
0.0 \\
0.0\end{array}$ & $\begin{array}{l}0.0 \\
0.0 \\
0.0 \\
0.0 \\
0.0\end{array}$ & $\begin{array}{l}69.0 \\
42.0 \\
40.0 \\
41.8 \\
41.8\end{array}$ & $\begin{array}{l}0.0 \\
0.0 \\
0.0 \\
0.0 \\
0.0\end{array}$ & $\begin{array}{c}0.0 \\
10.00 \\
10.00 \\
10.00 \\
10.00\end{array}$ & $\begin{array}{l}0.0 \\
0.0 \\
0.0 \\
0.0 \\
0.0\end{array}$ & $\begin{array}{c}0.0 \\
60.00 \\
70.00 \\
50.00 \\
60.00\end{array}$ & $\begin{array}{l}0.0 \\
0.0 \\
0.0 \\
0.0 \\
0.0\end{array}$ & $\begin{array}{c}56.03 \\
0.0 \\
0.0 \\
0.0 \\
0.0\end{array}$ & $\begin{array}{l}0.0 \\
0.0 \\
0.0 \\
0.0 \\
0.0\end{array}$ \\
\hline $\begin{array}{l}9.1 .36 \\
9.1 .37 \\
9.1 .38 \\
9.1 .39 \\
9.1 .40\end{array}$ & $\begin{array}{l}1.0 \\
3.0 \\
3.0 \\
0.3 \\
0.0\end{array}$ & $\begin{array}{l}0.0 \\
0.0 \\
0.0 \\
0.0 \\
0.0\end{array}$ & $\begin{array}{c}41.8 \\
0.0 \\
0.0 \\
0.0 \\
0.0\end{array}$ & $\begin{array}{r}0.0 \\
39.6 \\
19.7 \\
30.1 \\
40.0\end{array}$ & $\begin{array}{c}10.00 \\
0.0 \\
0.0 \\
0.0 \\
0.0\end{array}$ & $\begin{array}{l}0.0 \\
0.0 \\
0.0 \\
0.0 \\
0.0\end{array}$ & $\begin{array}{c}70.00 \\
0.0 \\
0.0 \\
0.0 \\
0.0\end{array}$ & $\begin{array}{l}0.0 \\
0.0 \\
0.0 \\
0.0 \\
0.0\end{array}$ & $\begin{array}{c}0.0 \\
59.36 \\
40.13 \\
40.26 \\
40.00\end{array}$ & $\begin{array}{l}0.0 \\
0.0 \\
0.0 \\
0.0 \\
0.0\end{array}$ \\
\hline
\end{tabular}


TABLE XIV (Contd.)

BASELINE COUNTERCURRENT FLOW TEST GROUP 9.1

EXPEFIMENTAL MEASJRMENTS FOR TESTS 9.1 .1 IN 9.1.98 -- 1.58-IN. DOWNCOMER GAP

\begin{tabular}{|c|c|c|c|c|c|c|c|c|c|c|}
\hline $\begin{array}{l}\text { TEST } \\
\text { NO }\end{array}$ & $\begin{array}{l}R: 1-C W-1 \\
(G \cap: 11)\end{array}$ & $\begin{array}{c}24-C ; A-2 \\
(G P,+1)\end{array}$ & $\begin{array}{c}F T-C: H-1 \\
(G P M)\end{array}$ & $\begin{array}{c}F T-C H-2 \\
(G P M)\end{array}$ & $\begin{array}{c}R M-H=-1 \\
(C F M)\end{array}$ & $\begin{array}{c}P . M-H S_{1}-2 \\
(C F M)\end{array}$ & $\begin{array}{c}R M-H H_{1}-3 \\
(C F M)\end{array}$ & $\begin{array}{c}R M-H G-4 \\
(C F M)\end{array}$ & $\begin{array}{l}F T-H F-1 \\
(C F M)\end{array}$ & $\begin{array}{c}F T-H G-2 \\
(C F M)\end{array}$ \\
\hline $\begin{array}{l}9.1 .41 \\
9.1 .42 \\
9.1 .43 \\
9.1 .44 \\
9.1 .45\end{array}$ & $\begin{array}{r}0.0 \\
3.0 \\
0.0 \\
.00 \\
.00\end{array}$ & $\begin{array}{l}0.0 \\
0.0 \\
0.0 \\
0.0 \\
0.0\end{array}$ & $\begin{array}{r}0.0 \\
0.0 \\
0.0 \\
10.0 \\
91.0\end{array}$ & $\begin{array}{r}30.0 \\
39.2 \\
55.0 \\
0.0 \\
0.0\end{array}$ & $\begin{array}{l}0.0 \\
0.0 \\
0.0 \\
0.0 \\
0.0\end{array}$ & $\begin{array}{l}0.0 \\
0.0 \\
0.0 \\
0.0 \\
0.0\end{array}$ & $\begin{array}{l}0.0 \\
0.0 \\
0.0 \\
0.0 \\
0.0\end{array}$ & $\begin{array}{l}0.0 \\
0.0 \\
0.0 \\
0.0 \\
0.0\end{array}$ & $\begin{array}{l}20.00 \\
20.26 \\
20.26 \\
20.26 \\
20.38\end{array}$ & $\begin{array}{l}0.0 \\
0.0 \\
0.0 \\
0.0 \\
0.0\end{array}$ \\
\hline $\begin{array}{l}9.1 .46 \\
9.1 .47 \\
9.1 .48 \\
9.1 .49 \\
9.1 .50\end{array}$ & $\begin{array}{l}0.0 \\
0.0 \\
0.0 \\
2.0 \\
3.0\end{array}$ & $\begin{array}{l}0.0 \\
0.0 \\
0.0 \\
0.0 \\
0.0\end{array}$ & $\begin{array}{r}109.0 \\
0.0 \\
120.0 \\
160.0 \\
0.0\end{array}$ & $\begin{array}{r}0.0 \\
39.9 \\
0.0 \\
0.0 \\
48.1\end{array}$ & $\begin{array}{l}0.0 \\
0.0 \\
0.0 \\
0.0 \\
0.0\end{array}$ & $\begin{array}{l}0.0 \\
0.0 \\
0.0 \\
0.0 \\
0.0\end{array}$ & $\begin{array}{l}0.0 \\
0.0 \\
0.1 \\
0.0 \\
0.0\end{array}$ & $\begin{array}{l}0.0 \\
0.0 \\
0.0 \\
0.0 \\
0.0\end{array}$ & $\begin{array}{l}20.26 \\
30.64 \\
30.00 \\
20.38 \\
55.38\end{array}$ & $\begin{array}{l}0.0 \\
0.0 \\
0.0 \\
0.0 \\
0.0\end{array}$ \\
\hline $\begin{array}{l}9.1 .51 \\
1.1 .52 \\
9.1 .53 \\
9.1 .54 \\
9.1 .55\end{array}$ & $\begin{array}{l}0.0 \\
j .0 \\
0.0 \\
j .0 \\
j .0\end{array}$ & $\begin{array}{l}J .0 \\
0.0 \\
0.0 \\
. .0 \\
0.0\end{array}$ & $\begin{array}{r}0.0 \\
0.0 \\
91.0 \\
120.0 \\
90.0\end{array}$ & $\begin{array}{r}30.0 \\
39.8 \\
0.0 \\
0.0 \\
0.0\end{array}$ & $\begin{array}{l}0.0 \\
0.0 \\
0.0 \\
0.0 \\
0.0\end{array}$ & $\begin{array}{l}0.0 \\
0.0 \\
0.0 \\
0.0 \\
0.0\end{array}$ & $\begin{array}{l}0.0 \\
0.0 \\
0.0 \\
0.0 \\
0.0\end{array}$ & $\begin{array}{r}0.0 \\
0.0 \\
0.0 \\
0.0 \\
0.0\end{array}$ & $\begin{array}{c}39.87 \\
20.00 \\
19.87 \\
0.0 \\
17.56\end{array}$ & $\begin{array}{l}0.0 \\
0.0 \\
0.0 \\
8.18 \\
0.0\end{array}$ \\
\hline $\begin{array}{l}9.1 .56 \\
9.1 .57 \\
9.1 .53 \\
9.1 .59 \\
9.1 .060\end{array}$ & $\begin{array}{l}J .0 \\
j .0 \\
.00 \\
0.0 \\
0.0\end{array}$ & $\begin{array}{l}0.0 \\
0.0 \\
0.0 \\
1.0 \\
0.0\end{array}$ & $\begin{array}{r}61.0 \\
0.0 \\
0.0 \\
0.0 \\
0.0\end{array}$ & $\begin{array}{r}0.0 \\
29.7 \\
29.9 \\
49.8 \\
40.0\end{array}$ & $\begin{array}{l}0.0 \\
0.0 \\
0.0 \\
0.0 \\
0.0\end{array}$ & $\begin{array}{l}0.0 \\
0.0 \\
0.0 \\
0.0 \\
0.0\end{array}$ & $\begin{array}{l}0.0 \\
0.0 \\
0.0 \\
0.0 \\
0.0\end{array}$ & $\begin{array}{l}0.0 \\
0.0 \\
0.0 \\
0.0 \\
0.0\end{array}$ & $\begin{array}{l}29.74 \\
43.85 \\
29.49 \\
29.23 \\
29.23\end{array}$ & $\begin{array}{l}0.0 \\
0.0 \\
0.0 \\
0.0 \\
0.0\end{array}$ \\
\hline $\begin{array}{l}9.1 .61 \\
9.1 .62 \\
9.1 .63 \\
4.1 .64 \\
9.1 .65\end{array}$ & $\begin{array}{l}0.0 \\
0.0 \\
j .0 \\
.00 \\
.00\end{array}$ & $\begin{array}{l}0.0 \\
0.0 \\
0.0 \\
10.0 \\
0.0\end{array}$ & $\begin{array}{r}0.0 \\
90.0 \\
130.0 \\
119.0 \\
100.0\end{array}$ & $\begin{array}{r}39.8 \\
0.0 \\
0.0 \\
0.0 \\
0.0\end{array}$ & $\begin{array}{c}0.0 \\
0.0 \\
0.0 \\
80.00 \\
0.0\end{array}$ & $\begin{array}{l}0.0 \\
0.0 \\
0.0 \\
0.0 \\
0.0\end{array}$ & $\begin{array}{c}0.0 \\
0.0 \\
0.0 \\
48.00 \\
0.0\end{array}$ & $\begin{array}{l}0.0 \\
0.0 \\
0.0 \\
0.0 \\
0.0\end{array}$ & $\begin{array}{c}14.62 \\
14.62 \\
14.74 \\
0.0 \\
38.59\end{array}$ & $\begin{array}{l}0.0 \\
0.0 \\
0.0 \\
0.0 \\
0.0\end{array}$ \\
\hline $\begin{array}{l}9.1 .60 \\
9.1 .67 \\
9.1 .68 \\
9.1 .64 \\
9.1 .70\end{array}$ & $\begin{array}{l}. .0 \\
.00 \\
.00 \\
.00 \\
.00\end{array}$ & $\begin{array}{l}0.0 \\
0.0 \\
0.0 \\
0.0 \\
0.0\end{array}$ & $\begin{array}{r}100.0 \\
81.0 \\
0.0 \\
70.0 \\
60.0\end{array}$ & $\begin{array}{r}0.0 \\
0.0 \\
40.4 \\
0.0 \\
0.0\end{array}$ & $\begin{array}{l}0.0 \\
0.0 \\
0.0 \\
0.0 \\
c .0\end{array}$ & $\begin{array}{l}0.0 \\
0.0 \\
0.0 \\
0.0 \\
0.0\end{array}$ & $\begin{array}{l}0.0 \\
0.0 \\
0.0 \\
0.0 \\
0.0\end{array}$ & $\begin{array}{l}0.0 \\
0.0 \\
0.0 \\
0.0 \\
0.0\end{array}$ & $\begin{array}{l}39.33 \\
29.23 \\
64.10 \\
56.15 \\
55.90\end{array}$ & $\begin{array}{l}0.0 \\
0.0 \\
0.0 \\
0.0 \\
0.0\end{array}$ \\
\hline $\begin{array}{l}9.1 .71 \\
9.1 .72 \\
9.1 .73 \\
9.1 .74 \\
3.1 .75\end{array}$ & $\begin{array}{l}\text { J.1. } \\
0.0 \\
j .0 \\
.00 \\
0.0\end{array}$ & $\begin{array}{l}0.0 \\
0.0 \\
0.0 \\
3.0 \\
0.0\end{array}$ & $\begin{array}{c}0.0 \\
0.0 \\
0.0 \\
0.0 \\
70.0\end{array}$ & $\begin{array}{r}49.0 \\
40.0 \\
47.8 \\
40.2 \\
0.0\end{array}$ & $\begin{array}{l}0.0 \\
0.0 \\
0.0 \\
0.0 \\
0.0\end{array}$ & $\begin{array}{l}0.0 \\
0.0 \\
0.0 \\
0.0 \\
0.0\end{array}$ & $\begin{array}{l}0.0 \\
0.0 \\
0.0 \\
0.0 \\
0.0\end{array}$ & $\begin{array}{l}0.0 \\
0.0 \\
0.0 \\
0.0 \\
0.0\end{array}$ & $\begin{array}{l}62.95 \\
63.59 \\
73.33 \\
73.21 \\
51.15\end{array}$ & $\begin{array}{l}0.0 \\
0.0 \\
0.0 \\
0.0 \\
0.0\end{array}$ \\
\hline $\begin{array}{l}9.1 .76 \\
9.1 .77 \\
9.1 .78 \\
9.1 .79 \\
9.1 .40\end{array}$ & $\begin{array}{l}J . J \\
0.0 \\
0.0 \\
j .0 \\
0.0\end{array}$ & $\begin{array}{l}0.0 \\
0.0 \\
0.0 \\
0.0 \\
0.0\end{array}$ & $\begin{array}{r}0.0 \\
69.0 \\
0.0 \\
0.0 \\
0.0\end{array}$ & $\begin{array}{r}45.0 \\
0.0 \\
10.0 \\
19.5 \\
30.3\end{array}$ & $\begin{array}{l}0.0 \\
0.0 \\
0.0 \\
0.0 \\
0.0\end{array}$ & $\begin{array}{l}0.0 \\
.0 .0 \\
0.0 \\
0.0 \\
0.0\end{array}$ & $\begin{array}{l}0.0 \\
0.0 \\
0.0 \\
0.0 \\
0.0\end{array}$ & $\begin{array}{l}0.0 \\
0.0 \\
0.0 \\
0.0 \\
0.0\end{array}$ & $\begin{array}{l}73.33 \\
55.90 \\
69.00 \\
59.62 \\
59.49\end{array}$ & $\begin{array}{l}0.0 \\
0.0 \\
0.0 \\
0.0 \\
0.0\end{array}$ \\
\hline
\end{tabular}


TABLE XIV (Contd.)

BASELINE COUNTERCURRENT FLOW TEST GROUP 9.1

EXPER IMENTAL MEASURMENTS FOR TESTS 9.1 .1 TO $9.1 .98-1$ 1.59-IN. DOWMCOMER GAP

\begin{tabular}{|c|c|c|c|c|c|c|c|c|c|c|}
\hline $\begin{array}{l}\text { TEST } \\
\text { NO }\end{array}$ & $\begin{array}{c}R M-C H-1 \\
(G P M)\end{array}$ & $\begin{array}{l}P M-C W-2 \\
(G, P M)\end{array}$ & $\begin{array}{l}F T-C H-1 \\
(G P M)^{-1}\end{array}$ & $\begin{array}{l}F T-C W-2 \\
(G P M)\end{array}$ & $\begin{array}{c}R M-H G-1 \\
(C F M)\end{array}$ & $\begin{array}{c}R M-H G-2 \\
-\quad(C F M)\end{array}$ & $\begin{array}{c}\text { RM-HG-3 } \\
(C F M)\end{array}$ & $\begin{array}{c}R M-H G=-4 \\
\text { (CFM) }\end{array}$ & $\begin{array}{c}F T-H G-1 \\
(C F M)\end{array}$ & $\begin{array}{c}F T-H G-2 \\
(C F M)\end{array}$ \\
\hline $\begin{array}{l}9.1 .81 \\
9.1 .82 \\
9.1 .83 \\
9.1 .84 \\
9.1 .85\end{array}$ & $\begin{array}{l}0.0 \\
0.0 \\
0.0 \\
0.0 \\
0.0\end{array}$ & $\begin{array}{l}0.0 \\
0.0 \\
0.0 \\
0.0 \\
0.0\end{array}$ & $\begin{array}{r}60.0 \\
69.0 \\
130.0 \\
80.0 \\
97.0\end{array}$ & $\begin{array}{l}0.0 \\
0.0 \\
0.0 \\
0.0 \\
0.0\end{array}$ & $\begin{array}{l}0.0 \\
0.0 \\
0.0 \\
0.0 \\
0.0\end{array}$ & $\begin{array}{l}0.0 \\
0.0 \\
0.0 \\
0.0 \\
0.0\end{array}$ & $\begin{array}{l}0.0 \\
0.0 \\
0.0 \\
0.0 \\
0.0\end{array}$ & $\begin{array}{l}0.0 \\
0.0 \\
0.0 \\
0.0 \\
0.0\end{array}$ & $\begin{array}{l}40.26 \\
39.74 \\
20.38 \\
30.26 \\
39.74\end{array}$ & $\begin{array}{l}0.0 \\
0.0 \\
0.0 \\
0.0 \\
0.0\end{array}$ \\
\hline $\begin{array}{l}9.1 .86 \\
9.1 .87 \\
9.1 .38 \\
9.1 .89 \\
9.1 .90\end{array}$ & $\begin{array}{l}0.0 \\
0.0 \\
0.0 \\
0.0 \\
.00\end{array}$ & $\begin{array}{l}0.0 \\
0.0 \\
0.0 \\
0.0 \\
0.0\end{array}$ & $\begin{array}{l}0.0 \\
0.0 \\
0.0 \\
0.0 \\
0.0\end{array}$ & $\begin{array}{l}35.9 \\
30.0 \\
20.0 \\
50.0 \\
30.3\end{array}$ & $\begin{array}{l}0.0 \\
0.0 \\
0.0 \\
0.0 \\
0.0\end{array}$ & $\begin{array}{l}0.0 \\
0.0 \\
0.0 \\
0.0 \\
0.0\end{array}$ & $\begin{array}{l}0.0 \\
0.0 \\
0.0 \\
0.0 \\
0.0\end{array}$ & $\begin{array}{l}0.0 \\
0.0 \\
0.0 \\
0.0 \\
0.0\end{array}$ & $\begin{array}{l}50.13 \\
59.36 \\
85.00 \\
40.51 \\
60.13\end{array}$ & $\begin{array}{l}0.0 \\
0.0 \\
0.0 \\
0.0 \\
0.0\end{array}$ \\
\hline $\begin{array}{l}9.1 .91 \\
9.1 .92 \\
9.1 .93 \\
9.1 .94 \\
9.1 .95\end{array}$ & $\begin{array}{l}0.0 \\
0.0 \\
0.0 \\
3.0 \\
0.0\end{array}$ & $\begin{array}{l}0.0 \\
0.0 \\
0.0 \\
0.0 \\
0.0\end{array}$ & $\begin{array}{r}0.0 \\
69.0 \\
129.0 \\
0.0 \\
151.0\end{array}$ & $\begin{array}{r}40.0 \\
0.0 \\
0.0 \\
50.0 \\
0.0\end{array}$ & $\begin{array}{l}0.0 \\
0.0 \\
0.0 \\
0.0 \\
0.0\end{array}$ & $\begin{array}{l}0.0 \\
0.0 \\
0.0 \\
0.0 \\
0.0\end{array}$ & $\begin{array}{l}0.0 \\
0.0 \\
0.0 \\
0.0 \\
0.0\end{array}$ & $\begin{array}{l}0.0 \\
0.0 \\
0.0 \\
0.0 \\
0.0\end{array}$ & $\begin{array}{l}59.74 \\
29.62 \\
14.74 \\
63.72 \\
21.92\end{array}$ & $\begin{array}{l}0.0 \\
0.0 \\
0.0 \\
0.0 \\
0.0\end{array}$ \\
\hline $\begin{array}{l}9.1 .96 \\
9.1 .97 \\
7.1 .98\end{array}$ & $\begin{array}{l}3.0 \\
0.0 \\
1.0\end{array}$ & $\begin{array}{l}0.0 \\
0.0 \\
0.0\end{array}$ & $\begin{array}{r}140.0 \\
59.0 \\
120.0\end{array}$ & $\begin{array}{l}0.0 \\
0.0 \\
0.0\end{array}$ & $\begin{array}{l}0.0 \\
0.0 \\
0.0\end{array}$ & $\begin{array}{l}0.0 \\
0.0 \\
0.0\end{array}$ & $\begin{array}{l}0.0 \\
0.0 \\
0.0\end{array}$ & $\begin{array}{l}0.0 \\
0.0 \\
0.0\end{array}$ & $\begin{array}{c}20.50 \\
63.9 ? \\
0.0\end{array}$ & $\begin{array}{l}0.0 \\
0.0 \\
8.19\end{array}$ \\
\hline $\begin{array}{l}\text { TEST } \\
\text { NO }\end{array}$ & $\begin{array}{l}D-C, N-1 \\
\text { (PSIS) }\end{array}$ & $\begin{array}{l}P-C W-2 \\
(r S 1 ;)\end{array}$ & $\begin{array}{l}\text { P-HG-1 } \\
\text { (PSIG) }\end{array}$ & $\begin{array}{l}D-H G-2 \\
(P S I S)\end{array}$ & $\begin{array}{l}\text { P-HG-3 } \\
\text { (PSIF, }\end{array}$ & $\begin{array}{l}T F-C W-1 \\
(O E G F)\end{array}$ & $\begin{array}{l}\text { TF-HF,- } \\
\text { (DEG F) }\end{array}$ & $\begin{array}{l}r=-H r_{3}-? \\
\left(D E S_{S}, F\right)\end{array}$ & $\begin{array}{l}T F-H G-3 \\
(O E G F)\end{array}$ & $\begin{array}{l}\text { P-BARO } \\
\text { (PSIA) }\end{array}$ \\
\hline $\begin{array}{ll}9.1 . & 1 \\
9.1 . & 2 \\
9.1 . & 3 \\
9.1 . & 4 \\
9.1 . & 5\end{array}$ & $\begin{array}{l}16.0 \\
13.5 \\
10.5 \\
2.0 .0 \\
14.2\end{array}$ & $\begin{array}{l}J .0 \\
0.0 \\
0.0 \\
0.0 \\
0.0\end{array}$ & $\begin{array}{l}15.0 \\
16.5 \\
15.5 \\
19.0 \\
11.5\end{array}$ & $\begin{array}{l}89.1 \\
89.0 \\
73.0 \\
77.0 \\
51.0\end{array}$ & $\begin{array}{l}27.0 \\
27.0 \\
30.0 \\
30.0 \\
30.0\end{array}$ & $\begin{array}{l}50.0 \\
50.0 \\
50.0 \\
50.0 \\
40.0\end{array}$ & $\begin{array}{r}93.0 \\
102.5 \\
116.0 \\
113.5 \\
98.9\end{array}$ & $\begin{array}{l}107.9 \\
117.0 \\
127.7 \\
130.0 \\
110.9\end{array}$ & $\begin{array}{l}100.5 \\
110.0 \\
122.5 \\
124.8 \\
105.5\end{array}$ & $\begin{array}{l}12.2 \\
12.2 \\
12.2 \\
12.2 \\
12.2\end{array}$ \\
\hline $\begin{array}{l}9.1 .6 \\
9.1 .9 \\
9.1 .9 \\
9.1 .9 \\
9.1 .1 \\
9.1 .1\end{array}$ & $\begin{array}{l}20.0 \\
11.00 \\
16.5 \\
17.0 \\
13.0\end{array}$ & $\begin{array}{l}0.0 \\
0.0 \\
0.0 \\
0.0 \\
0.0\end{array}$ & $\begin{array}{l}17.5 \\
10.5 \\
10.0 \\
15.0 \\
16.5\end{array}$ & $\begin{array}{r}100.0 \\
97.0 \\
99.0 \\
89.0 \\
89.0\end{array}$ & $\begin{array}{l}30.0 \\
30.0 \\
15.0 \\
30.0 \\
30.0\end{array}$ & $\begin{array}{l}50.0 \\
44.0 \\
44.0 \\
48.0 \\
49.0\end{array}$ & $\begin{array}{r}68.1 \\
79.0 \\
83.5 \\
105.0 \\
116.5\end{array}$ & $\begin{array}{r}66.0 \\
91.5 \\
94.0 \\
120.5 \\
129.0\end{array}$ & $\begin{array}{r}65.0 \\
85.0 \\
38.0 \\
113.0 \\
123.5\end{array}$ & $\begin{array}{l}12.3 \\
12.3 \\
12.3 \\
12.3 \\
12.3\end{array}$ \\
\hline $\begin{array}{l}9.1 .11 \\
9.1 .12 \\
9.1 .13 \\
9.1 .14 \\
9.1 .15\end{array}$ & $\begin{array}{l}19.1 \\
21.0 \\
17.5 \\
11.7 \\
13.5\end{array}$ & $\begin{array}{l}0.0 \\
0.0 \\
0.0 \\
0.0 \\
0.0\end{array}$ & $\begin{array}{l}17.5 \\
20.5 \\
16.7 \\
10.0 \\
11.4\end{array}$ & $\begin{array}{l}84.0 \\
83.0 \\
89.0 \\
94.0 \\
93.0\end{array}$ & $\begin{array}{l}30.0 \\
33.0 \\
32.0 \\
33.5 \\
33.5\end{array}$ & $\begin{array}{l}54.5 \\
49.0 \\
50.0 \\
47.0 \\
45.0\end{array}$ & $\begin{array}{r}110.0 \\
124.0 \\
117.0 \\
204.0 \\
90.5\end{array}$ & $\begin{array}{l}123.5 \\
237.0 \\
129.5 \\
117.0 \\
200.5\end{array}$ & $\begin{array}{r}117.0 \\
130.5 \\
123.5 \\
111.5 \\
95.5\end{array}$ & $\begin{array}{l}12.3 \\
12.3 \\
12.3 \\
12.3 \\
12.3\end{array}$ \\
\hline
\end{tabular}


TABLE XIV (Contd.)

BASELINE COUNTERCURRENT FLOW TEST GROUP 9.1

EXPERLMENTAL IEASURMENTS FOR TESTS 9.1 .1 TO $9.1 .98--1.53-1$ N. DOWNCOMER GAO

\begin{tabular}{|c|c|c|c|c|c|c|c|c|c|c|}
\hline $\begin{array}{c}\text { TSST } \\
\text { vo }\end{array}$ & $\begin{array}{l}P-C H-1 \\
(P S I C)\end{array}$ & $\begin{array}{l}D-C: N-2 \\
(P S I C)\end{array}$ & $\begin{array}{l}P-H G-1 \\
(P S I G)\end{array}$ & $\begin{array}{l}P-H F-2 \\
\text { (PSIG) }\end{array}$ & $\begin{array}{l}P-H G-3 \\
\text { (PSIG) }\end{array}$ & $\begin{array}{l}\text { TF-CH-1 } \\
\text { (DEG FI. }\end{array}$ & $\begin{array}{l}\text { TF-HG-I } \\
\text { (DEG }=\text { ) }\end{array}$ & $\begin{array}{l}T E-H G-2 \\
(D E G \quad C)\end{array}$ & $\begin{array}{l}\text { IF-HG-3 } \\
\text { (OEG FI }\end{array}$ & $\begin{array}{l}\text { P-SAR } \\
\text { (PSIA) }\end{array}$ \\
\hline $\begin{array}{l}9.1 .16 \\
9.1 .17 \\
9.1 .18 \\
9.1 .19 \\
9.1 .2 .5\end{array}$ & $\begin{array}{l}13.5 \\
14.7 \\
15.0 \\
14.5 \\
10.0\end{array}$ & $\begin{array}{l}0.0 \\
0.0 \\
0.0 \\
0.0 \\
0.0\end{array}$ & $\begin{array}{l}10.6 \\
16.5 \\
16.0 \\
16.0 \\
10.5\end{array}$ & $\begin{array}{r}100.0 \\
89.5 \\
89.0 \\
90.0 \\
91.0\end{array}$ & $\begin{array}{l}32.5 \\
32.0 \\
35.0 \\
35.5 \\
35.0\end{array}$ & $\begin{array}{l}51.5 \\
43.0 \\
48.5 \\
47.5 \\
48.5\end{array}$ & $\begin{array}{r}73.5 \\
108.0 \\
87.0 \\
100.5 \\
111.5\end{array}$ & $\begin{array}{r}72.0 \\
123.5 \\
102.5 \\
115.5 \\
125.0\end{array}$ & $\begin{array}{r}71.0 \\
116.5 \\
95.0 \\
108.5 \\
119.5\end{array}$ & $\begin{array}{l}12.3 \\
12.3 \\
12.3 \\
12.3 \\
12.3\end{array}$ \\
\hline $\begin{array}{l}5.1 .21 \\
9.1 .22 \\
9.1 .23 \\
9.1 .24 \\
9.1 .25\end{array}$ & $\begin{array}{l}7.0 \\
14.0 \\
14.5 \\
14.5 \\
15.0\end{array}$ & $\begin{array}{l}0.0 \\
0.0 \\
0.0 \\
0.0 \\
0.0\end{array}$ & $\begin{array}{l}11.7 \\
11.5 \\
12.0 \\
10.2 \\
10.3\end{array}$ & $\begin{array}{l}93.0 \\
44.5 \\
95.5 \\
97.5 \\
97.5\end{array}$ & $\begin{array}{l}35.0 \\
35.0 \\
35.0 \\
0.0 \\
33.5\end{array}$ & $\begin{array}{l}50.0 \\
48.0 \\
45.5 \\
54.0 \\
49.0\end{array}$ & $\begin{array}{r}104.0 \\
92.5 \\
91.5 \\
76.0 \\
74.5\end{array}$ & $\begin{array}{r}114.5 \\
106.5 \\
102.5 \\
74.0 \\
0.5\end{array}$ & $\begin{array}{r}110.5 \\
101.0 \\
88.5 \\
0.0 \\
73.0\end{array}$ & $\begin{array}{l}12.3 \\
12.3 \\
12.3 \\
12.4 \\
12.4\end{array}$ \\
\hline $\begin{array}{l}9.1 .26 \\
9.1 .27 \\
9.1 .28 \\
9.1 .29 \\
9.1 .30\end{array}$ & $\begin{array}{l}11.2 \\
13.2 \\
19.0 \\
26.2 \\
18.2\end{array}$ & $\begin{array}{l}1.0 \\
0.0 \\
0.0 \\
0.0 \\
0.0\end{array}$ & $\begin{array}{l}10.4 \\
12.0 \\
17.5 \\
22.5 \\
15.0\end{array}$ & $\begin{array}{l}95.0 \\
94.0 \\
93.5 \\
93.5 \\
94.0\end{array}$ & $\begin{array}{l}0.0 \\
0.0 \\
0.0 \\
0.0 \\
0.0\end{array}$ & $\begin{array}{l}47.5 \\
46.5 \\
46.5 \\
46.0 \\
46.5\end{array}$ & $\begin{array}{l}81.0 \\
82.0 \\
86.0 \\
90.0 \\
80.0\end{array}$ & $\begin{array}{r}90.0 \\
91.0 \\
96.0 \\
101.5 \\
91.0\end{array}$ & $\begin{array}{l}0.0 \\
0.0 \\
0.0 \\
0.0 \\
0.0\end{array}$ & $\begin{array}{l}12.4 \\
12.4 \\
12.4 \\
12.4 \\
12.4\end{array}$ \\
\hline $\begin{array}{l}9.1 .31 \\
9.1 .32 \\
9.1 .33 \\
9.1 .34 \\
9.1 .35\end{array}$ & $\begin{array}{r}2.0 .0 \\
0.5 \\
4.5 \\
9.0 \\
10.0\end{array}$ & $\begin{array}{l}0.0 \\
0.0 \\
0.0 \\
0.0 \\
0.0\end{array}$ & $\begin{array}{r}20.2 \\
9.2 \\
9.5 \\
8.5 \\
10.0\end{array}$ & $\begin{array}{l}89.0 \\
90.0 \\
90.0 \\
95.0 \\
95.0\end{array}$ & $\begin{array}{r}0.0 \\
15.0 \\
15.0 \\
15.0 \\
15.0\end{array}$ & $\begin{array}{l}47.5 \\
50.0 \\
54.0 \\
54.5 \\
53.5\end{array}$ & $\begin{array}{l}88.0 \\
84.0 \\
81.5 \\
84.5 \\
84.0\end{array}$ & $\begin{array}{l}99.5 \\
78.0 \\
89.0 \\
60.5 \\
83.5\end{array}$ & $\begin{array}{r}0.0 \\
75.0 \\
80.5 \\
77.5 \\
79.5\end{array}$ & $\begin{array}{l}12.4 \\
12.3 \\
12.3 \\
12.4 \\
12.4\end{array}$ \\
\hline $\begin{array}{l}2.1 .36 \\
9.1 .37 \\
4.1 .33 \\
9.1 .39 \\
9.1 .40\end{array}$ & $\begin{array}{r}9.0 \\
17.0 \\
10.0 \\
11.0 \\
10.0\end{array}$ & $\begin{array}{l}0.0 \\
0.0 \\
0.0 \\
0.0 \\
0.0\end{array}$ & $\begin{array}{r}8.5 \\
17.0 \\
9.0 \\
10.0 \\
5.2\end{array}$ & $\begin{array}{l}95.0 \\
88.0 \\
92.0 \\
92.0 \\
91.0\end{array}$ & $\begin{array}{l}15.0 \\
26.0 \\
27.0 \\
27.0 \\
27.0\end{array}$ & $\begin{array}{l}52.5 \\
54.5 \\
55.5 \\
54.0 \\
54.5\end{array}$ & $\begin{array}{r}84.9 \\
102.5 \\
103.5 \\
99.5 \\
106.5\end{array}$ & $\begin{array}{r}97.0 \\
115.5 \\
116.0 \\
113.0 \\
113.5\end{array}$ & $\begin{array}{r}81.5 \\
109.5 \\
110.5 \\
102.5 \\
113.5\end{array}$ & $\begin{array}{l}12.4 \\
12.5 \\
12.5 \\
12.5 \\
12.5\end{array}$ \\
\hline $\begin{array}{l}9.1 .41 \\
9.1 .42 \\
9.1 .43 \\
9.1 .44 \\
9.1 .45\end{array}$ & $\begin{array}{l}10.5 \\
10.0 \\
12.2 \\
12.5 \\
11.0\end{array}$ & $\begin{array}{l}0.0 \\
0.0 \\
0.0 \\
0.0 \\
0.0\end{array}$ & $\begin{array}{r}9.0 \\
9.0 \\
11.0 \\
10.2 \\
8.5\end{array}$ & $\begin{array}{l}95.0 \\
95.0 \\
95.0 \\
95.0 \\
93.0\end{array}$ & $\begin{array}{l}27.0 \\
27.0 \\
27.0 \\
27.0 \\
27.0\end{array}$ & $\begin{array}{l}52.5 \\
52.5 \\
52.5 \\
52.5 \\
55.0\end{array}$ & $\begin{array}{l}76.5 \\
80.5 \\
93.0 \\
86.0 \\
80.0\end{array}$ & $\begin{array}{l}82.0 \\
91.0 \\
93.5 \\
99.5 \\
87.0\end{array}$ & $\begin{array}{l}78.5 \\
85.0 \\
88.0 \\
92.5 \\
82.5\end{array}$ & $\begin{array}{l}12.5 \\
12.5 \\
12.3 \\
12.3 \\
12.3\end{array}$ \\
\hline $\begin{array}{l}9.1 .46 \\
9.1 .47 \\
9.1 .43 \\
9.1 .49 \\
9.1 .50\end{array}$ & $\begin{array}{l}13.0 \\
13.5 \\
19.5 \\
18.0 \\
29.0\end{array}$ & $\begin{array}{l}0.0 \\
0.0 \\
0.0 \\
0.0 \\
0.0\end{array}$ & $\begin{array}{r}0.0 \\
11: 5 \\
15.5 \\
12.0 \\
19.0\end{array}$ & $\begin{array}{l}94.0 \\
91.0 \\
91.0 \\
95.0 \\
90.0\end{array}$ & $\begin{array}{l}27.0 \\
27.0 \\
27.0 \\
27.0 \\
26.0\end{array}$ & $\begin{array}{l}53.5 \\
53.0 \\
54.5 \\
45.0 \\
52.0\end{array}$ & $\begin{array}{l}80.5 \\
85.0 \\
94.5 \\
83.0 \\
73.0\end{array}$ & $\begin{array}{r}98.5 \\
97.5 \\
109.5 \\
95.5 \\
76.0\end{array}$ & $\begin{array}{r}83.5 \\
91.0 \\
102.5 \\
89.5 \\
74.0\end{array}$ & $\begin{array}{l}12.3 \\
12.3 \\
12.3 \\
12.3 \\
12.2\end{array}$ \\
\hline $\begin{array}{l}9.1 .51 \\
9.1 .52 \\
9.1 .53 \\
9.1 .54 \\
9.1 .55\end{array}$ & $\begin{array}{l}12.2 \\
14.0 \\
14.0 \\
15.0 \\
14.0\end{array}$ & $\begin{array}{l}0.0 \\
0.0 \\
0.0 \\
0.0 \\
1.0\end{array}$ & $\begin{array}{l}10.5 \\
11.5 \\
10.5 \\
10.5 \\
10.0\end{array}$ & $\begin{array}{r}82.0 \\
100.0 \\
98.0 \\
98.0 \\
97.0\end{array}$ & $\begin{array}{l}30.0 \\
30.0 \\
30.0 \\
15.0 \\
30.0\end{array}$ & $\begin{array}{l}49.0 \\
46.5 \\
46.0 \\
44.0 \\
50.0\end{array}$ & $\begin{array}{r}113.5 \\
71.0 \\
74.0 \\
83.0 \\
77.0\end{array}$ & $\begin{array}{r}124.5 \\
76.0 \\
83.0 \\
91.5 \\
79.0\end{array}$ & $\begin{array}{r}120.5 \\
71.5 \\
77.5 \\
96.0 \\
77.0\end{array}$ & $\begin{array}{l}12.2 \\
12.3 \\
12.3 \\
12.3 \\
12.3\end{array}$ \\
\hline
\end{tabular}


TABLE XIV (Contd.)

BASELINE COUNTERCURRENT FLOW TEST GROUP 9.1

EXPERIMENTAL MEASURMENTS FOR TESTS 9.1 .1 TO $9.1 .98--1.58-1$. DOWNCOMER GAP

\begin{tabular}{|c|c|c|c|c|c|c|c|c|c|c|}
\hline $\begin{array}{l}\text { TEST } \\
\text { ND }\end{array}$ & $\begin{array}{l}P-C, W-1 \\
(P S(G)\end{array}$ & $\begin{array}{l}P-C W-2 \\
(P 5 I G)\end{array}$ & $\begin{array}{l}P-H G-1 \\
\text { (PSIG) }\end{array}$ & $\begin{array}{l}P-H G-2 \\
(P S I G)\end{array}$ & $\begin{array}{l}\text { P-HG-3 } \\
\text { (PSIG) }\end{array}$ & $\begin{array}{l}T F-C H-1 \\
\text { IOEG FI }\end{array}$ & $\begin{array}{l}\text { TF-HG-1 } \\
\text { (DEG F) }\end{array}$ & $\begin{array}{l}\text { TF-HG-2 } \\
\text { (DEG F) }\end{array}$ & $\begin{array}{l}T F-H G-3 \\
\text { (DEG F }\end{array}$ & $\begin{array}{l}\text { P-BARO } \\
\text { (PSIAI) }\end{array}$ \\
\hline $\begin{array}{l}9.1 .56 \\
9.1 .57 \\
9.1 .58 \\
9.1 .59 \\
9.1 .60\end{array}$ & $\begin{array}{l}13.2 \\
12.7 \\
13.0 \\
13.0 \\
13.0\end{array}$ & $\begin{array}{l}0.0 \\
0.0 \\
0.0 \\
0.0 \\
0.0\end{array}$ & $\begin{array}{l}10.5 \\
12.2 \\
12.0 \\
11.8 \\
12.2\end{array}$ & $\begin{array}{l}94.0 \\
95.5 \\
94.0 \\
93.5 \\
93.5\end{array}$ & $\begin{array}{l}30.0 \\
33.5 \\
33.5 \\
33.5 \\
33.5\end{array}$ & $\begin{array}{l}49.5 \\
50.0 \\
48.0 \\
45.0 \\
44.0\end{array}$ & $\begin{array}{l}89.0 \\
94.0 \\
98.0 \\
92.5 \\
94.5\end{array}$ & $\begin{array}{l}104.0 \\
107.0 \\
110.0 \\
103.5 \\
107.0\end{array}$ & $\begin{array}{r}96.5 \\
101.0 \\
104.5 \\
98.5 \\
101.0\end{array}$ & $\begin{array}{l}12.3 \\
12.3 \\
12.3 \\
12.3 \\
12.3\end{array}$ \\
\hline $\begin{array}{l}9.1 .61 \\
9.1 .62 \\
9.1 .63 \\
9.1 .64 \\
y .1 .65\end{array}$ & $\begin{array}{l}12.6 \\
14.2 \\
15.5 \\
12.7 \\
13.2\end{array}$ & $\begin{array}{l}0.0 \\
0.0 \\
0.0 \\
0.0 \\
0.0\end{array}$ & $\begin{array}{r}10.2 \\
11.0 \\
9.6 \\
10.0 \\
17.0\end{array}$ & $\begin{array}{r}95.5 \\
95.0 \\
95.0 \\
96.0 \\
92.5\end{array}$ & $\begin{array}{l}33.7 \\
33.0 \\
32.5 \\
33.5 \\
33.5\end{array}$ & $\begin{array}{l}43.0 \\
43.0 \\
42.0 \\
49.0 \\
44.0\end{array}$ & $\begin{array}{l}88.0 \\
87.0 \\
86.0 \\
83.5 \\
91.0\end{array}$ & $\begin{array}{r}99.0 \\
95.5 \\
94.0 \\
0.0 \\
103.5\end{array}$ & $\begin{array}{r}93.0 \\
91.5 \\
89.5 \\
80.5 \\
0.0\end{array}$ & $\begin{array}{l}12.3 \\
12.3 \\
12.3 \\
12.2 \\
12.2\end{array}$ \\
\hline $\begin{array}{l}9.1 .66 \\
9.1 .67 \\
9.1 .63 \\
9.1 .67 \\
9.1 .70\end{array}$ & $\begin{array}{l}17.5 \\
11.5 \\
17.0 \\
21.2 \\
20.0\end{array}$ & $\begin{array}{l}0.0 \\
0.0 \\
0.0 \\
0.0 \\
0.0\end{array}$ & $\begin{array}{l}16.5 \\
10.7 \\
18.4 \\
23.5 \\
20.8\end{array}$ & $\begin{array}{l}92.5 \\
93.5 \\
89.5 \\
97.0 \\
96.0\end{array}$ & $\begin{array}{r}33.5 \\
33.5 \\
33.0 \\
32.5 \\
0.0\end{array}$ & $\begin{array}{l}44.0 \\
44.0 \\
44.5 \\
52.0 \\
50.5\end{array}$ & $\begin{array}{r}96.5 \\
98.5 \\
106.0 \\
79.0 \\
80.0\end{array}$ & $\begin{array}{r}110.0 \\
110.5 \\
119.5 \\
90.0 \\
93.5\end{array}$ & $\begin{array}{l}0.0 \\
0.0 \\
0.0 \\
0.0 \\
0.0\end{array}$ & $\begin{array}{l}12.2 \\
12.2 \\
12.2 \\
12.4 \\
12.4\end{array}$ \\
\hline $\begin{array}{l}9.1 .71 \\
9.1 .72 \\
9.1 .73 \\
9.1 .74 \\
9.1 .75\end{array}$ & $\begin{array}{l}21.2 \\
17.5 \\
22.2 \\
19.5 \\
29.5\end{array}$ & $\begin{array}{l}0.0 \\
0.0 \\
0.0 \\
0.0 \\
0.0\end{array}$ & $\begin{array}{l}22.0 \\
18.5 \\
13.3 \\
20.5 \\
20.3\end{array}$ & $\begin{array}{l}96.5 \\
95.0 \\
92.0 \\
91.0 \\
91.5\end{array}$ & $\begin{array}{l}0.0 \\
0.0 \\
0.0 \\
0.0 \\
0.0\end{array}$ & $\begin{array}{l}49.5 \\
49.5 \\
47.5 \\
47.5 \\
49.0\end{array}$ & $\begin{array}{r}78.0 \\
85.0 \\
103.0 \\
112.0 \\
105.0\end{array}$ & $\begin{array}{r}82.0 \\
96.0 \\
115.5 \\
124.3 \\
117.0\end{array}$ & $\begin{array}{l}0.0 \\
0.0 \\
0.0 \\
0.0 \\
0.0\end{array}$ & $\begin{array}{l}12.4 \\
12.4 \\
12.4 \\
12.4 \\
12.4\end{array}$ \\
\hline $\begin{array}{l}9.1 .76 \\
9.1 .77 \\
9.1 .78 \\
9.1 .79 \\
9.1 .80\end{array}$ & $\begin{array}{l}23.5 \\
21.5 \\
10.5 \\
11.5 \\
10.5\end{array}$ & $\begin{array}{l}0.0 \\
0.0 \\
0.0 \\
0.0 \\
0.0\end{array}$ & $\begin{array}{l}21.2 \\
21.4 \\
10.0 \\
10.5 \\
13.5\end{array}$ & $\begin{array}{l}89.5 \\
93.5 \\
90.0 \\
90.0 \\
90.0\end{array}$ & $\begin{array}{r}0.0 \\
0.0 \\
15.0 \\
20.0 \\
25.0\end{array}$ & $\begin{array}{l}49.5 \\
48.0 \\
52.5 \\
52.0 \\
52.5\end{array}$ & $\begin{array}{r}108.0 \\
95.3 \\
103.0 \\
113.0 \\
94.0\end{array}$ & $\begin{array}{l}120.0 \\
107.0 \\
120.0 \\
125.5 \\
106.5\end{array}$ & $\begin{array}{r}0.0 \\
0.0 \\
106.5 \\
119.5 \\
100.0\end{array}$ & $\begin{array}{l}12.4 \\
12.4 \\
12.5 \\
12.5 \\
12.5\end{array}$ \\
\hline $\begin{array}{l}9.1 .81 \\
9.1 .82 \\
9.1 .83 \\
9.1 .84 \\
9.1 .85\end{array}$ & $\begin{array}{l}15.0 \\
13.5 \\
16.0 \\
14.0 . \\
21.0\end{array}$ & $\begin{array}{l}0.0 \\
0.0 \\
0.0 \\
0.0 \\
0.0\end{array}$ & $\begin{array}{l}12.5 \\
18.0 \\
11.5 \\
11.2 \\
18.0\end{array}$ & $\begin{array}{r}91.0 \\
100.0 \\
93.0 \\
91.0 \\
91.0\end{array}$ & $\begin{array}{l}27.0 \\
27.0 \\
27.0 \\
27.0 \\
27.0\end{array}$ & $\begin{array}{l}54.5 \\
55.0 \\
53.5 \\
52.5 \\
47.5\end{array}$ & $\begin{array}{r}106.5 \\
70.0 \\
81.0 \\
90.0 \\
95.0\end{array}$ & $\begin{array}{r}119.5 \\
72.0 \\
90.0 \\
104.5 \\
109.0\end{array}$ & $\begin{array}{r}113.5 \\
70.0 \\
84.5 \\
97.5 \\
102.5\end{array}$ & $\begin{array}{l}12.5 \\
12.5 \\
12.3 \\
12.3 \\
12.3\end{array}$ \\
\hline $\begin{array}{l}9.1 .86 \\
9.1 .97 \\
9.1 .88 \\
9.1 .84 \\
9.1 .90\end{array}$ & $\begin{array}{l}1 \% .5 \\
15.0 \\
13.5 \\
13.0 \\
10.0\end{array}$ & $\begin{array}{l}0.0 \\
0.0 \\
0.0 \\
0.0 \\
0.0\end{array}$ & $\begin{array}{l}13.5 \\
14.5 \\
12.5 \\
11.0 \\
15.0\end{array}$ & $\begin{array}{l}89.0 \\
90.0 \\
70.0 \\
91.0 \\
96.0\end{array}$ & $\begin{array}{l}27.0 \\
27.0 \\
30.0 \\
30.0 \\
32.0\end{array}$ & $\begin{array}{l}47.5 \\
57.0 \\
54.0 \\
48.0 \\
48.5\end{array}$ & $\begin{array}{r}99.0 \\
85.0 \\
110.0 \\
93.5 \\
76.0\end{array}$ & $\begin{array}{r}102.0 \\
95.5 \\
123.0 \\
110.8 \\
96.0\end{array}$ & $\begin{array}{r}105.0 \\
90.0 \\
117.0 \\
105.0 \\
81.0\end{array}$ & $\begin{array}{l}12.3 \\
12.2 \\
12.2 \\
12.2 \\
12.3\end{array}$ \\
\hline $\begin{array}{l}9.1 .91 \\
9.1 .92 \\
9.1 .93 \\
9.1 .94 \\
9.1 .45\end{array}$ & $\begin{array}{l}11.5 \\
13.5 \\
13.5 \\
13.7 \\
13.5\end{array}$ & $\begin{array}{l}0.0 \\
0.0 \\
0.0 \\
0.0 \\
0.0\end{array}$ & $\begin{array}{l}14.5 \\
12.0 \\
11.2 \\
19.5 \\
16.5\end{array}$ & $\begin{array}{l}93.5 \\
93.0 \\
98.0 \\
99.5 \\
93.0\end{array}$ & $\begin{array}{r}33.0 \\
33.5 \\
33.5 \\
0.0 \\
0.0\end{array}$ & $\begin{array}{l}47.0 \\
45.0 \\
43.5 \\
48.5 \\
49.5\end{array}$ & $\begin{array}{l}98.0 \\
87.0 \\
74.0 \\
86.0 \\
80.0\end{array}$ & $\begin{array}{r}111.5 \\
96.5 \\
79.0 \\
102.0 \\
94.0\end{array}$ & $\begin{array}{r}105.5 \\
92.5 \\
74.0 \\
0.0 \\
0.0\end{array}$ & $\begin{array}{l}12.3 \\
12.3 \\
12.3 \\
12.4 \\
12.4\end{array}$ \\
\hline
\end{tabular}


TABLE XIV (Contd.)

BASELINE COUNTERCURRENT FLOW TEST GROUP 9.1

EXPERI MENTAL MEASUPMENTS FOR TFSTS 9.1 .1 TO $9.1 .48--1.58-1$ N. DOWNCOMER GAP

\begin{tabular}{|c|c|c|c|c|c|c|c|c|c|c|}
\hline $\begin{array}{l}\text { TEST } \\
\text { NO }\end{array}$ & $\begin{array}{l}P-C(N-1) \\
(P S I G)\end{array}$ & $\begin{array}{l}P-(: V-2 \\
(P S I G)\end{array}$ & $\begin{array}{l}P-H G-1 \\
(P S I G)\end{array}$ & $\begin{array}{l}D-H C_{5}-2 \\
(P S I S)\end{array}$ & $\begin{array}{l}\text { P-HG, } \\
\text { (PSITI) }\end{array}$ & $\begin{array}{l}T F-C H-1 \\
(D E S F)\end{array}$ & $\begin{array}{l}\text { TF-HF, } 1 \\
(D E G F)\end{array}$ & $\begin{array}{l}T F-H G-2 \\
(D E G F)\end{array}$ & $\begin{array}{l}\text { IF-HG-3 } \\
\text { IDEG FI }\end{array}$ & $\begin{array}{l}\text { P-BAR } \\
\text { IPSIA }\end{array}$ \\
\hline $\begin{array}{l}9.1 .96 \\
9.1 .97 \\
9.1 .98\end{array}$ & $\begin{array}{l}14.5 \\
20.0 \\
12.7\end{array}$ & $\begin{array}{l}0.0 \\
0.0 \\
0.0\end{array}$ & $\begin{array}{l}20.0 \\
20.6 \\
10.0\end{array}$ & $\begin{array}{l}94.0 \\
88.0 \\
58.0\end{array}$ & $\begin{array}{r}0.0 \\
0.0 \\
10.5\end{array}$ & $\begin{array}{l}45.5 \\
47.0 \\
42.0\end{array}$ & $\begin{array}{l}84.0 \\
94.5 \\
76.5\end{array}$ & $\begin{array}{r}71.0 \\
108.5 \\
77.5\end{array}$ & $\begin{array}{r}0.0 \\
0.0 \\
74.5\end{array}$ & $\begin{array}{l}12.4 \\
12.4 \\
12.3\end{array}$ \\
\hline
\end{tabular}

\begin{tabular}{|c|c|c|c|c|c|c|c|c|c|}
\hline \multirow[b]{2}{*}{$\begin{array}{l}\text { TEST } \\
\text { YU }\end{array}$} & \multicolumn{3}{|c|}{$\begin{array}{l}\text { LOWFR DLENUM FLDW } \\
.\end{array}$} & \multicolumn{3}{|c|}{ BYPASS FL NW } & \multirow{2}{*}{$\begin{array}{l}\text { LOWER } \\
\text { PLENUM } \\
\text { LEVEL } \\
\text { (IN) }\end{array}$} & \multirow[b]{2}{*}{$\begin{array}{l}\text { P-LP-1 } \\
\text { PSI I }) \text { ( }\end{array}$} & \multirow[b]{2}{*}{$\begin{array}{l}\text { IF-LP-1 } \\
\text { IOEG FI }\end{array}$} \\
\hline & $\begin{array}{l}\text { INITIALL } \\
\text { NEISHT } \\
\text { (LH) }\end{array}$ & $\begin{array}{l}\text { FINAL } \\
\text { WEICHE } \\
\text { (LQI) }\end{array}$ & $\begin{array}{l}\text { TIMF AF } \\
\text { COL LECTICN } \\
\text { (SECI) }\end{array}$ & $\begin{array}{l}\text { INITIAL } \\
\text { WE I GHT } \\
\text { (LB) }\end{array}$ & $\begin{array}{l}\text { FENAL } \\
\text { WEIGHT } \\
\text { (LB) }\end{array}$ & $\begin{array}{l}\text { TIME OF } \\
\text { COLLLCTION } \\
\text { ISEC S }\end{array}$ & & & \\
\hline $\begin{array}{ll}9.1 .10 & 1 \\
9.1 . & 2 \\
9.1 .1 & 3 \\
9.1 .1 & 4 \\
9.1 . & 5\end{array}$ & $\begin{array}{l}325.0 \\
343.5 \\
239.5 \\
325.5 \\
211.0\end{array}$ & $\begin{array}{l}467.0 \\
523.0 \\
325.5 \\
429.5 \\
541.0\end{array}$ & $\begin{array}{l}60.0 \\
60.0 \\
60.0 \\
60.0 \\
60.0\end{array}$ & $\begin{array}{l}257.5 \\
330.0 \\
307.0 \\
390.0 \\
130.0\end{array}$ & $\begin{array}{l}457.0 \\
581.5 \\
482.5 \\
620.5 \\
380.0\end{array}$ & $\begin{array}{l}60.0 \\
60.0 \\
60.0 \\
60.0 \\
60.0\end{array}$ & $\begin{array}{l}18.0 \\
18.0 \\
18.0 \\
18.0 \\
18.0\end{array}$ & $\begin{array}{l}14.0 \\
17.0 \\
14.5 \\
18.0 \\
11.0\end{array}$ & $\begin{array}{l}53.0 \\
52.0 \\
55.0 \\
54.0 \\
49.0\end{array}$ \\
\hline 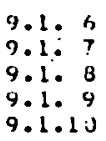 & $\begin{array}{l}154.0 \\
265.0 \\
140.5 \\
334.5 \\
173.0\end{array}$ & $\begin{array}{l}454.5 \\
500.0 \\
421.0 \\
469.0 \\
332.5\end{array}$ & $\begin{array}{r}60.0 \\
20.0 \\
15.0 \\
60.0 \\
120.0\end{array}$ & $\begin{array}{l}116.0 \\
483.0 \\
123.0 \\
460.0 \\
276.5\end{array}$ & $\begin{array}{l}466.0 \\
678.0 \\
226.5 \\
668.0 \\
551.0\end{array}$ & $\begin{array}{l}60.0 \\
30.0 \\
60.0 \\
60.0 \\
90.0\end{array}$ & $\begin{array}{l}18.0 \\
18.0 \\
18.0 \\
18.0 \\
18.0\end{array}$ & $\begin{array}{l}17.0 \\
10.0 \\
10.0 \\
14.7 \\
15.0\end{array}$ & $\begin{array}{l}50.0 \\
45.0 \\
45.0 \\
50.5 \\
55.0\end{array}$ \\
\hline $\begin{array}{l}9.1 .11 \\
9.1 .12 \\
9.1 .13 \\
9.1 .14 \\
9.1 .15\end{array}$ & $\begin{array}{l}242.5 \\
295.0 \\
197.5 \\
267.0 \\
250.5\end{array}$ & $\begin{array}{l}295.0 \\
337.0 \\
223.0 \\
442.5 \\
446.0\end{array}$ & $\begin{array}{r}120.0 \\
150.0 \\
60.0 \\
45.0 \\
30.0\end{array}$ & $\begin{array}{l}119.5 \\
209.0 \\
214.5 \\
296.0 \\
205.5\end{array}$ & $\begin{array}{l}305.0 \\
365.0 \\
363.5 \\
477.0 \\
333.0\end{array}$ & $\begin{array}{l}75.0 \\
60.0 \\
60.0 \\
60.0 \\
30.0\end{array}$ & $\begin{array}{l}18.0 \\
18.0 \\
18.0 \\
18.0 \\
18.0\end{array}$ & $\begin{array}{r}17.0 \\
19.5 \\
15.7 \\
8.2 \\
9 . ?\end{array}$ & $\begin{array}{l}59.0 \\
63.0 \\
61.0 \\
48.0 \\
45.0\end{array}$ \\
\hline $\begin{array}{l}9.1 .16 \\
9.1 .17 \\
9.1 .18 \\
9.1 .19 \\
9.1 .20\end{array}$ & $\begin{array}{l}152.0 \\
147.5 \\
13 \% .5 \\
22 \% .0 \\
34 \% .0\end{array}$ & $\begin{array}{l}383.0 \\
191.5 \\
220.0 \\
347.0 \\
437.0\end{array}$ & $\begin{array}{l}20.0 \\
30.0 \\
90.0 \\
60.0 \\
30.0\end{array}$ & $\begin{array}{l}101.5 \\
232.0 \\
102.5 \\
139.0 \\
211.5\end{array}$ & $\begin{array}{l}219.5 \\
365.0 \\
139.0 \\
211.5 \\
263.5\end{array}$ & $\begin{array}{l}20.0 \\
45.0 \\
11.0 \\
15.0 \\
20.0\end{array}$ & $\begin{array}{l}18.0 \\
18.0 \\
12.0 \\
12.0 \\
12.0\end{array}$ & $\begin{array}{r}9.7 \\
15.0 \\
15.0 \\
15.0 \\
9.7\end{array}$ & $\begin{array}{l}51.5 \\
49.0 \\
54.0 \\
49.5 \\
50.0\end{array}$ \\
\hline $\begin{array}{l}4.1 .21 \\
9.1 .22 \\
9.1 .23 \\
9.1 .24 \\
9.1 .25\end{array}$ & $\begin{array}{l}20 . j .0 \\
14 \% .5 \\
349.0 \\
143.5 \\
207.0\end{array}$ & $\begin{array}{l}423.0 \\
349.0 \\
520.0 \\
410.5 \\
456.0\end{array}$ & $\begin{array}{l}25.0 \\
20.5 \\
15.0 \\
20.0 \\
15.0\end{array}$ & $\begin{array}{l}263.5 \\
383.5 \\
479.0 \\
102.0 \\
152.5\end{array}$ & $\begin{array}{l}383.5 \\
479.0 \\
562.0 \\
241.5 \\
266.0\end{array}$ & $\begin{array}{l}20.0 \\
20.0 \\
15.0 \\
20.0 \\
20.0\end{array}$ & $\begin{array}{r}12.0 \\
4.0 \\
4.0 \\
4.0 \\
3.0\end{array}$ & $\begin{array}{l}11.5 \\
11.5 \\
11.0 \\
10.2 \\
10.0\end{array}$ & $\begin{array}{l}50.5 \\
48.5 \\
46.0 \\
54.0 \\
49.0\end{array}$ \\
\hline $\begin{array}{l}9.1 .26 \\
9.1 .27 \\
9.1 .28 \\
9.1 .29 \\
9.1 .30\end{array}$ & $\begin{array}{l}15 j .0 \\
159.0 \\
155.0 \\
155.5 \\
153.5\end{array}$ & $\begin{array}{l}364.5 \\
401.5 \\
433.5 \\
449.0 \\
533.0\end{array}$ & $\begin{array}{l}30.0 \\
30.0 \\
30.0 \\
30.0 \\
30.0\end{array}$ & $\begin{array}{l}166.0 \\
126.5 \\
130.5 \\
182.5 \\
119.0\end{array}$ & $\begin{array}{l}299.0 \\
294.0 \\
389.0 \\
533.0 \\
412.5\end{array}$ & $\begin{array}{l}30.0 \\
30.0 \\
30.0 \\
30.0 \\
30.0\end{array}$ & $\begin{array}{r}12.0 \\
12.0 \\
12.0 \\
3.0 \\
4.0\end{array}$ & $\begin{array}{r}9.7 \\
11.0 \\
16.2 \\
21.2 \\
14.5\end{array}$ & $\begin{array}{l}48.0 \\
47.5 \\
47.5 \\
46.5 \\
47.0\end{array}$ \\
\hline
\end{tabular}


TABLE XIV (Contd.)

BASELINE COUNTERCURRENT FLOW TEST GROUP 9.1

EXPCRIMENTAL MEA SURMENTS FOR TESTS 9.1 .1 TO 9.1.98 -- 1.58-IN. DOWNC OMER GAP

\begin{tabular}{|c|c|c|c|c|c|c|c|c|}
\hline \multicolumn{3}{|c|}{ COHCR PLENUM FLIH } & \multicolumn{3}{|c|}{ BYPASS FLDH } & \multirow[b]{2}{*}{$\begin{array}{l}\text { LOWER } \\
\text { PLENUM } \\
\text { LEVEL } \\
\text { (IN) }\end{array}$} & \multirow[b]{2}{*}{$\begin{array}{l}P-L P-1 \\
(P S I G)\end{array}$} & \multirow[b]{2}{*}{$\begin{array}{l}\text { TF-LP-1 } \\
\text { (DEG FI) }\end{array}$} \\
\hline $\begin{array}{l}\text { INITIAL } \\
\text { WEIGHT } \\
\text { (LO) }\end{array}$ & $\begin{array}{l}\text { FINAL } \\
\text { WEIGHT } \\
\text { (LB) }\end{array}$ & $\begin{array}{l}\text { TIME OF } \\
\text { COLLECTIICN } \\
\text { (SEC) }\end{array}$ & $\begin{array}{l}\text { IN IT I AL } \\
\text { WE I GHT } \\
\text { (LB) }\end{array}$ & $\begin{array}{l}\text { FINAL } \\
\text { WEIGHT } \\
\text { (IL BI }\end{array}$ & $\begin{array}{l}\text { TIME OF } \\
\text { COLLECTION } \\
\text { ISECI }\end{array}$ & & & \\
\hline $\begin{array}{l}145.5 \\
2914.8 \\
339.5 \\
139.0 \\
303.5\end{array}$ & $\begin{array}{l}257.5 \\
460.0 \\
493.5 \\
303.5 \\
465.0\end{array}$ & $\begin{array}{l}30.0 \\
30.0 \\
30.0 \\
30.0 \\
30.0\end{array}$ & $\begin{array}{r}308.5 \\
06.5 \\
123.0 \\
99.5 \\
126.0\end{array}$ & $\begin{array}{l}483.0 \\
123.0 \\
148.0 \\
126.0 \\
150.0\end{array}$ & $\begin{array}{l}30.0 \\
60.0 \\
60.0 \\
60.0 \\
60.0\end{array}$ & $\begin{array}{l}12.0 \\
12.0 \\
12.0 \\
12.0 \\
12.0\end{array}$ & $\begin{array}{r}19.5 \\
9.5 \\
9.5 \\
9.0 \\
9.7\end{array}$ & $\begin{array}{l}49.5 \\
50.0 \\
51.5 \\
54.5 \\
54.0\end{array}$ \\
\hline $\begin{array}{l}465.0 \\
143.0 \\
152.5 \\
141.0 \\
222.0\end{array}$ & $\begin{array}{l}618.0 \\
483.0 \\
479.5 \\
413.0 \\
535.5\end{array}$ & $\begin{array}{r}30.0 \\
120.0 \\
120.0 \\
90.0 \\
90.0\end{array}$ & $\begin{array}{l}150.0 \\
200.0 \\
120.0 \\
122.0 \\
263.0\end{array}$ & $\begin{array}{l}184.5 \\
449.0 \\
122.0 \\
336.5 \\
514.0\end{array}$ & $\begin{array}{r}60.0 \\
90.0 \\
240.0 \\
180.0 \\
120.0\end{array}$ & $\begin{array}{l}12.0 \\
18.0 \\
18.0 \\
18.0 \\
18.0\end{array}$ & $\begin{array}{r}9.7 \\
15.0 \\
7.5 \\
9.7 \\
8.5\end{array}$ & $\begin{array}{l}54.0 \\
56.5 \\
57.0 \\
54.0 \\
55.0\end{array}$ \\
\hline $\begin{array}{l}140.5 \\
251.5 \\
163.5 \\
180.5 \\
140.0\end{array}$ & $\begin{array}{l}387.0 \\
539.0 \\
500.5 \\
577.0 \\
494.0\end{array}$ & $\begin{array}{l}60.0 \\
60.0 \\
60.0 \\
60.0 \\
45.0\end{array}$ & $\begin{array}{l}109.0 \\
126.0 \\
244.0 \\
315.0 \\
103.0\end{array}$ & $\begin{array}{l}126.0 \\
244.00 \\
485.5 \\
684.0 \\
509.0\end{array}$ & $\begin{array}{r}240.0 \\
180.0 \\
120.0 \\
120.0 \\
90.0\end{array}$ & $\begin{array}{l}18.0 \\
18.0 \\
18.0 \\
18.0 \\
18.0\end{array}$ & $\begin{array}{r}9.0 \\
9.0 \\
10.0 \\
9.7 \\
9.0\end{array}$ & $\begin{array}{l}53.0 \\
53.0 \\
52.0 \\
53.0 \\
55.5\end{array}$ \\
\hline $\begin{array}{l}143.5 \\
14 J .5 \\
133.5 \\
133.5 \\
133.5\end{array}$ & $\begin{array}{l}578.0 \\
387.5 \\
575.0 \\
481.0 \\
439.0\end{array}$ & $\begin{array}{r}45.0 \\
60.0 \\
40.0 \\
25.0 \\
120.0\end{array}$ & $\begin{array}{r}135.0 \\
106.5 \\
392.0 \\
91.0 \\
100.5\end{array}$ & $\begin{array}{l}540.5 \\
288.0 \\
780.0 \\
286.5 \\
616.0\end{array}$ & $\begin{array}{r}75.0 \\
120.0 \\
60.0 \\
25.0 \\
120.0\end{array}$ & $\begin{array}{l}18.0 \\
18.0 \\
18.0 \\
18.0 \\
18.0\end{array}$ & $\begin{array}{l}9.9 \\
10.5 \\
14.5 \\
11.5 \\
18.0\end{array}$ & $\begin{array}{l}54.0 \\
54.0 \\
55.0 \\
45.0 \\
52.5\end{array}$ \\
\hline $\begin{array}{l}253.5 \\
143.0 \\
235.5 \\
282.0 \\
140.5\end{array}$ & $\begin{array}{l}447.0 \\
429.5 \\
470.5 \\
491.0 \\
479.0\end{array}$ & $\begin{array}{l}60.0 \\
60.0 \\
30.0 \\
15.0 \\
45.0\end{array}$ & $\begin{array}{l}444.0 \\
341.5 \\
405.0 \\
226.5 \\
116.5\end{array}$ & $\begin{array}{l}508.0 \\
409.0 \\
600.0 \\
370.0 \\
325.0\end{array}$ & $\begin{array}{l}60.0 \\
90.0 \\
45.0 \\
60.0 \\
60.0\end{array}$ & $\begin{array}{l}18.0 \\
18.0 \\
18.0 \\
18.0 \\
18.0\end{array}$ & $\begin{array}{l}9 . ? \\
10.5 \\
10.0 \\
10.0 \\
10.0\end{array}$ & $\begin{array}{l}51.0 \\
48.0 \\
46.0 \\
44.0 \\
50.0\end{array}$ \\
\hline $\begin{array}{l}293.0 \\
169.5 \\
327.5 \\
21 \% .0 \\
339.5\end{array}$ & $\begin{array}{l}534.0 \\
333.5 \\
445.0 \\
356.5 \\
435.0\end{array}$ & $\begin{array}{l}45.0 \\
60.0 \\
32.0 \\
30.0 \\
30.0\end{array}$ & $\begin{array}{l}325.0 \\
172.0 \\
411.0 \\
269.0 \\
318.0\end{array}$ & $\begin{array}{l}511.0 \\
277.5 \\
433.0 \\
404.0 \\
358.0\end{array}$ & $\begin{array}{l}60.0 \\
75.0 \\
60.0 \\
60.0 \\
30.0\end{array}$ & $\begin{array}{l}18.0 \\
18.0 \\
18.0 \\
18.0 \\
18.0\end{array}$ & $\begin{array}{r}10.0 \\
9.9 \\
10.2 \\
9.3 \\
10.2\end{array}$ & $\begin{array}{l}50.0 \\
51.0 \\
49.0 \\
46.0 \\
45.0\end{array}$ \\
\hline $\begin{array}{l}215.0 \\
302.0 \\
310.0 \\
141.0 \\
282.0\end{array}$ & $\begin{array}{l}368.0 \\
551.0 \\
314.0 \\
351.0 \\
426.5\end{array}$ & $\begin{array}{l}30.0 \\
30.0 \\
15.0 \\
20.0 \\
20.0\end{array}$ & $\begin{array}{l}309.5 \\
270.5 \\
324.5 \\
102.5 \\
110.5\end{array}$ & $\begin{array}{l}330.5 \\
403.5 \\
536.5 \\
224.0 \\
260.0\end{array}$ & $\begin{array}{l}60.0 \\
30.0 \\
30.0 \\
20.0 \\
20.0\end{array}$ & $\begin{array}{r}18.0 \\
19.0 \\
18.0 \\
5.0 \\
12.0\end{array}$ & $\begin{array}{r}9.5 \\
9.7 \\
7.5 \\
10.0 \\
17.7\end{array}$ & $\begin{array}{l}44.0 \\
43.0 \\
42.0 \\
48.5 \\
44.5\end{array}$ \\
\hline $\begin{array}{l}161.5 \\
173.5 \\
149.0 \\
174.5 \\
145.5\end{array}$ & $\begin{array}{l}304.5 \\
381.5 \\
243.5 \\
275.0 \\
235.0\end{array}$ & $\begin{array}{l}20.0 \\
30.0 \\
60.0 \\
30.0 \\
30.0\end{array}$ & $\begin{array}{l}116.0 \\
198.0 \\
127.5 \\
124.0 \\
129.0\end{array}$ & $\begin{array}{l}263.5 \\
335.5 \\
252.5 \\
327.0 \\
292.5\end{array}$ & $\begin{array}{l}20.0 \\
30.0 \\
30.0 \\
30.0 \\
30.0\end{array}$ & $\begin{array}{l}12.0 \\
12.0 \\
12.0 \\
12.0 \\
12.0\end{array}$ & $\begin{array}{l}16.0 \\
10.2 \\
16.7 \\
23.0 \\
20.2\end{array}$ & $\begin{array}{l}44.5 \\
45.0 \\
49.5 \\
53.0 \\
52.5\end{array}$ \\
\hline
\end{tabular}


TABLE XIV (Contd.)

BASELINE COUNTERCURRENT FLOW TEST GROUP 9.1

EXPERIMENTAL MFASURMENTS FOR TESTS 9.1 .1 TO $9.1 .98-2$ 1.58-1N. DDHNCDMEP GAP

\begin{tabular}{|c|c|c|c|c|c|c|c|c|c|}
\hline \multirow[b]{2}{*}{$\begin{array}{l}\text { TEST } \\
\text { !NO }\end{array}$} & \multicolumn{3}{|c|}{ LCNEP PLENIM FLOW } & \multicolumn{3}{|c|}{ BYPASS FLOH } & \multirow{2}{*}{$\begin{array}{l}\text { LOWER } \\
\text { PLENUM } \\
\text { LEVEL } \\
\text { (IN) }\end{array}$} & \multirow[b]{2}{*}{$\begin{array}{l}P-L P-1 \\
(P S I G)\end{array}$} & \multirow[b]{2}{*}{$\begin{array}{l}\text { TF-LP-1 } \\
\text { IDEG FI }\end{array}$} \\
\hline & $\begin{array}{l}\text { IN!TIAL } \\
\text { UEIGHT } \\
\text { (L3) }\end{array}$ & $\begin{array}{l}\text { FINAL } \\
\text { WEIGHT } \\
\text { (LBI }\end{array}$ & $\begin{array}{l}\text { TIME OF } \\
\text { COLLECTIINN } \\
\text { (SEC) }\end{array}$ & $\begin{array}{l}\text { INIITIAL } \\
\text { WEIGHT } \\
\text { (LB) }\end{array}$ & $\begin{array}{l}\text { FINAL } \\
\text { WEIGHT } \\
(L B)\end{array}$ & $\begin{array}{l}\text { TIME OF } \\
\text { COLLECTION } \\
\text { (SEC) }\end{array}$ & & & \\
\hline $\begin{array}{l}9.1 .71 \\
9.1 .72 \\
9.1 .73 \\
9.1 .74 \\
9.1 .75\end{array}$ & $\begin{array}{l}146.5 \\
193.5 \\
147.0 \\
186.5 \\
157.0\end{array}$ & $\begin{array}{l}207.0 \\
248.5 \\
200.5 \\
231.0 \\
270.0\end{array}$ & $\begin{array}{l}30.0 \\
30.0 \\
30.0 \\
30.0 \\
30.0\end{array}$ & $\begin{array}{l}165.0 \\
235.5 \\
216.0 \\
266.0 \\
119.0\end{array}$ & $\begin{array}{l}317.0 \\
356.0 \\
369.5 \\
389.0 \\
303.0\end{array}$ & $\begin{array}{l}30.0 \\
30.0 \\
30.0 \\
30.0 \\
30.0\end{array}$ & $\begin{array}{l}12.0 \\
12.0 \\
12.0 \\
12.0 \\
12.0\end{array}$ & $\begin{array}{l}22.0 \\
18.0 \\
22.2 \\
20.0 \\
20.0\end{array}$ & $\begin{array}{l}51.0 \\
52.5 \\
52.5 \\
54.0 \\
50.0\end{array}$ \\
\hline $\begin{array}{l}9.1 .75 \\
9.1 .77 \\
9.1 .78 \\
9.1 .74 \\
9.1 .80\end{array}$ & $\begin{array}{l}149.0 \\
141.5 \\
133.5 \\
293.0 \\
203.0\end{array}$ & $\begin{array}{l}200.0 \\
245.0 \\
293.0 \\
485.5 \\
414.0\end{array}$ & $\begin{array}{r}30.0 \\
30.0 \\
120.0 \\
90.0 \\
90.0\end{array}$ & $\begin{array}{r}173.0 \\
105.0 \\
98.0 \\
117.0 \\
224.0\end{array}$ & $\begin{array}{l}310.0 \\
287.5 \\
117.0 \\
286.5 \\
589.0\end{array}$ & $\begin{array}{r}30.0 \\
30.0 \\
240.0 \\
240.0 \\
.180 .0\end{array}$ & $\begin{array}{l}12.0 \\
12.0 \\
18.0 \\
18.0 \\
18.0\end{array}$ & $\begin{array}{r}20.7 \\
21.2 \\
9.0 \\
9.5 \\
12.0\end{array}$ & $\begin{array}{l}53.5 \\
50.0 \\
56.0 \\
54.5 \\
5.4 .5\end{array}$ \\
\hline $\begin{array}{l}9.1 .81 \\
9.1 .82 \\
9.1 .83 \\
9.1 .84 \\
9.1 .85\end{array}$ & $\begin{array}{l}236.0 \\
139.5 \\
157.5 \\
164.5 \\
141.0\end{array}$ & $\begin{array}{l}608.0 \\
435.0 \\
505.0 \\
468.5 \\
537.0\end{array}$ & $\begin{array}{l}60.0 \\
60.0 \\
30.0 \\
45.0 \\
60.0\end{array}$ & $\begin{array}{l}514.0 \\
100.0 \\
328.5 \\
199.0 \\
176.5\end{array}$ & $\begin{array}{l}859.0 \\
363.0 \\
716.5 \\
594.5 \\
486.0\end{array}$ & $\begin{array}{l}90.0 \\
60.0 \\
60.0 \\
90.0 \\
45.0\end{array}$ & $\begin{array}{l}13.0 \\
28.0 \\
18.0 \\
18.0 \\
18.0\end{array}$ & $\begin{array}{l}11.2 \\
16.0 \\
11.0 \\
11.0 \\
17.0\end{array}$ & $\begin{array}{l}55.0 \\
55.5 \\
54.0 \\
53.5 \\
49.0\end{array}$ \\
\hline $\begin{array}{l}9.1 .84 \\
9.1 .47 \\
9.1 .88 \\
9.1 .89 \\
9.1 .9 .1\end{array}$ & $\begin{array}{r}153: 0 \\
143.0 \\
337.0 \\
141.0 \\
97.0\end{array}$ & $\begin{array}{l}436.8 \\
325.0 \\
397.5 \\
397.0 \\
180.0\end{array}$ & $\begin{array}{r}120.0 \\
105.0 \\
60.0 \\
60.0 \\
30.0\end{array}$ & $\begin{array}{l}260.0 \\
102.5 \\
36 h .0 \\
109.0 \\
134.0\end{array}$ & $\begin{array}{l}595.0 \\
257.5 \\
485.0 \\
276.0 \\
182.0\end{array}$ & $\begin{array}{r}122.0 \\
60.0 \\
60.0 \\
60.0 \\
30.0\end{array}$ & $\begin{array}{l}18.0 \\
18.0 \\
18.0 \\
18.0 \\
18.0\end{array}$ & $\begin{array}{l}11.7 \\
12.0 \\
12.0 \\
10.0 \\
13.2\end{array}$ & $\begin{array}{l}51.0 \\
66.0 \\
57.0 \\
49.0 \\
49.0\end{array}$ \\
\hline $\begin{array}{l}9.1 .91 \\
9.1 .92 \\
9.1 .93 \\
9.1 .94 \\
9.1 .95\end{array}$ & $\begin{array}{l}140.0 \\
140.0 \\
282.0 \\
191.5 \\
142.0\end{array}$ & $\begin{array}{l}278.0 \\
338.0 \\
467.0 \\
264.5 \\
363.5\end{array}$ & $\begin{array}{l}60.0 \\
30.0 \\
15.0 \\
30.0 \\
20.0\end{array}$ & $\begin{array}{l}104.5 \\
143.5 \\
198.5 \\
132.0 \\
109.0\end{array}$ & $\begin{array}{l}307.0 \\
257.5 \\
286.0 \\
274.0 \\
303.0\end{array}$ & $\begin{array}{l}69.0 \\
30.0 \\
15.0 \\
30.0 \\
20.0\end{array}$ & $\begin{array}{r}18.0 \\
18.0 \\
10.0 \\
12.0 \\
4.0\end{array}$ & $\begin{array}{r}13.5 \\
10.0 \\
9.7 \\
18.5 \\
15.7\end{array}$ & $\begin{array}{l}49.0 \\
46.0 \\
44: 0 \\
50.5 \\
50.5\end{array}$ \\
\hline $\begin{array}{l}9.1 .96 \\
9.1 .97 \\
9.1 .95\end{array}$ & $\begin{array}{l}141.5 \\
143.5 \\
214.0\end{array}$ & $\begin{array}{l}513.0 \\
232.5 \\
422.5\end{array}$ & $\begin{array}{l}30.0 \\
30.0 \\
15.0\end{array}$ & $\begin{array}{l}108.5 \\
277.0 \\
255.0\end{array}$ & $\begin{array}{l}321.5 \\
438.0 \\
329.0\end{array}$ & $\begin{array}{l}30.0 \\
30.0 \\
30.0\end{array}$ & $\begin{array}{r}4.0 \\
12.0 \\
12.0\end{array}$ & $\begin{array}{l}11.0 \\
13.7 \\
10.0\end{array}$ & $\begin{array}{l}45.5 \\
49.5 \\
42.5\end{array}$ \\
\hline
\end{tabular}




\section{TABLE XV}

BYPASS FLOW TEST GROUP 5.5

TRANSPARENT VESSEL TEST DATA FOR TESTS 5.5 .1 TO $5.5 .23-0.53-$ IN. DOHNCOMER GAP SUMMARY OF CALCULATED RESULTS

\begin{tabular}{|c|c|c|c|c|c|c|c|c|c|c|}
\hline $\begin{array}{l}\text { TEST } \\
\text { NO }\end{array}$ & $\begin{array}{l}\text { DOHNCOMER } \\
\text { AIR FLOW } \\
(\mathrm{LB} / \mathrm{SEC})\end{array}$ & $\begin{array}{l}\text { DOW:NCOMER } \\
\text { AIR OENSITY } \\
\text { ILB/FT } * 3 \text { I }\end{array}$ & $\begin{array}{l}\text { LOWER PLENUM } \\
\text { PRESSURE } \\
\text { (PSIA) }\end{array}$ & $\begin{array}{l}\text { COLD LEG } \\
\text { AIR FLOW } \\
\text { (LB/SEC) }\end{array}$ & $\begin{array}{l}\text { DOWNCOMER } \\
\text { WATER FLOW } \\
\text { (LB/SEC) }\end{array}$ & $\begin{array}{l}\text { WATER FLOH } \\
\text { CONT INUITY }\end{array}$ & $\begin{array}{c}\text { J-AIR } \\
\text { OOWNCOMER } \\
\text { (FT/SEC) }\end{array}$ & $\begin{array}{l}\text { J-HATER } \\
\text { DOWNCDMER } \\
\text { (FT/SEC) }\end{array}$ & $(J G *) * 1 / 2$ & $(\mathrm{Jl} *) * 1 / 2$ \\
\hline $\begin{array}{l}5.5 .1 \\
5.5 .22 \\
5.5 .3 \\
5.5 .4 \\
5.5 .5\end{array}$ & $\begin{array}{l}0.0 \\
0.0 \\
0.0 \\
0.0 \\
0.0\end{array}$ & $\begin{array}{l}0.1196 \\
0.1164 \\
0.1235 \\
0.1171 \\
0.1185\end{array}$ & $\begin{array}{l}22.59 \\
22.09 \\
23.34 \\
22.34 \\
22.34\end{array}$ & $\begin{array}{l}0.0671 \\
0.0668 \\
0.0667 \\
0.0666 \\
0.1312\end{array}$ & $\begin{array}{l}4.596 \\
3.950 \\
3.433 \\
2.633 \\
3.450\end{array}$ & $\begin{array}{l}1.011 \\
0.966 \\
0.977 \\
1.041 \\
0.845\end{array}$ & $\begin{array}{l}0.0 \\
0.0 \\
0.0 \\
0.0 \\
0.0\end{array}$ & $\begin{array}{l}1.107 \\
0.951 \\
0.827 \\
0.634 \\
0.831\end{array}$ & $\begin{array}{l}0.0 \\
0.0 \\
0.0 \\
0.0 \\
0.0\end{array}$ & $\begin{array}{l}0.8109 \\
0.7517 \\
0.7009 \\
0.6138 \\
0.7026\end{array}$ \\
\hline $\begin{array}{l}5.5 .6 \\
5.5 .7 \\
5.5 .9 \\
5.5 .99 \\
5.5 .10\end{array}$ & $\begin{array}{l}0.0 \\
0.0 \\
0.0 \\
0.0 \\
0.0\end{array}$ & $\begin{array}{l}0.1196 \\
0.1170 \\
0.1190 \\
0.12011 \\
0.1170\end{array}$ & $\begin{array}{l}22.59 \\
22.20 \\
22.70 \\
22.70 \\
22.20\end{array}$ & $\begin{array}{l}0.1312 \\
0.1387 \\
0.1355 \\
0.1328 \\
0.1323\end{array}$ & $\begin{array}{l}3.200 \\
3.400 \\
2.458 \\
3.025 \\
3.317\end{array}$ & $\begin{array}{l}0.966 \\
1.003 \\
1.007 \\
1.004 \\
1.022\end{array}$ & $\begin{array}{l}0.0 \\
0.0 \\
0.0 \\
0.0 \\
0.0\end{array}$ & $\begin{array}{l}0.771 \\
0.819 \\
0.592 \\
0.728 \\
0.799\end{array}$ & $\begin{array}{l}0.0 \\
0.0 \\
0.0 \\
0.0 \\
0.0\end{array}$ & $\begin{array}{l}0.6766 \\
0.6974 \\
0.5930 \\
0.6579 \\
0.6888\end{array}$ \\
\hline $\begin{array}{l}5.5 .11 \\
5.5 .12 \\
5.5 .13 \\
5.5 .14 \\
5.5 .15\end{array}$ & $\begin{array}{l}0.0 \\
0.0 \\
0.0 \\
0.0 \\
0.0\end{array}$ & $\begin{array}{l}0.1223 \\
0.1223 \\
0.1175 \\
0.1199 \\
0.1197\end{array}$ & $\begin{array}{l}23.20 \\
23.20 \\
22.20 \\
22.70 \\
22.70\end{array}$ & $\begin{array}{l}0.1325 \\
0.1324 \\
0.1858 \\
0.1914 \\
0.1882\end{array}$ & $\begin{array}{l}3.458 \\
2.458 \\
2.267 \\
2.475 \\
3.342\end{array}$ & $\begin{array}{l}0.976 \\
0.994 \\
1.011 \\
1.001 \\
0.974\end{array}$ & $\begin{array}{l}0.0 \\
0.0 \\
0.0 \\
0.0 \\
0.0\end{array}$ & $\begin{array}{l}0.833 \\
0.592 \\
0.546 \\
0.596 \\
0.905\end{array}$ & $\begin{array}{l}0.0 \\
0.0 \\
0.0 \\
0.0 \\
0.0\end{array}$ & $\begin{array}{l}0.7034 \\
0.5931 \\
0.5695 \\
0.5951 \\
0.6914 .\end{array}$ \\
\hline $\begin{array}{l}5.5 .16 \\
5.5 .17 \\
5.5 .18 \\
5.5 .19 \\
5.5 .20\end{array}$ & $\begin{array}{l}0.0 \\
0.0 \\
0.0 \\
0.0 \\
0.0\end{array}$ & $\begin{array}{l}0.1157 \\
0.1150 \\
0.1216 \\
0.1172 \\
0.1211\end{array}$ & $\begin{array}{l}21.95 \\
21.92 \\
22.88 \\
22.13 \\
22.88 .\end{array}$ & $\begin{array}{l}0.1856 \\
0.2012 \\
0.0284 \\
0.0301 \\
0.0301\end{array}$ & $\begin{array}{l}2.467 \\
1.325 \\
4.333 \\
5.567 \\
3.767\end{array}$ & $\begin{array}{l}1.004 \\
1.010 \\
0.971 \\
0.973 \\
0.977\end{array}$ & $\begin{array}{l}0.0 \\
0.0 \\
0.0 \\
0.0 \\
0.0\end{array}$ & $\begin{array}{l}0.594 \\
0.319 \\
1.044 \\
1.341 \\
0.907\end{array}$ & $\begin{array}{l}0.0 \\
0.0 \\
0.0 \\
0.0 \\
0.0\end{array}$ & $\begin{array}{l}0.5940 \\
0.4354 \\
0.7874 \\
0.8924 \\
0.7341\end{array}$ \\
\hline $\begin{array}{l}5.5 .21 \\
5.5 .22 \\
5.5 .23\end{array}$ & $\begin{array}{l}0.0 \\
0.0 \\
0.0\end{array}$ & $\begin{array}{l}0.1189 \\
0.1190 \\
0.1224\end{array}$ & $\begin{array}{l}22.38 \\
22.67 \\
23.17\end{array}$ & $\begin{array}{l}0.0668 \\
0.1451 \\
0.2049\end{array}$ & $\begin{array}{l}4.283 \\
2.933 \\
2.811\end{array}$ & $\begin{array}{l}0.980 \\
0.983 \\
1.016\end{array}$ & $\begin{array}{l}0.0 \\
0.0 \\
0.0\end{array}$ & $\begin{array}{l}1.032 \\
0.706 \\
0.678\end{array}$ & $\begin{array}{l}0.0 \\
0.0 \\
0.0\end{array}$ & $\begin{array}{l}0.7828 \\
0.6478 \\
0.6348\end{array}$ \\
\hline
\end{tabular}


TABLE XV (Contd.)

BYPASS FLOW TEST GROUP 5.5

EXPERIMENTAL MEASURMENTS FOR TESTS 5.5 .1 TO $5.5 .23--0.53-1$. DOWNCOMER GAP

\begin{tabular}{|c|c|c|c|c|c|c|c|c|c|c|}
\hline $\begin{array}{l}\text { TEST } \\
\text { NO }\end{array}$ & $\begin{array}{c}R M-C W-1 \\
(G P M)\end{array}$ & $\begin{array}{c}R M-C H-2 \\
(G P M)\end{array}$ & $\begin{array}{c}F T-C H-1 \\
(G P M)\end{array}$ & $\begin{array}{c}F T-C H-2 \\
(G P M)\end{array}$ & $\begin{array}{c}R M-H G-1 \\
(C F M)\end{array}$ & $\begin{array}{c}R M-H G-2 \\
(C F M)\end{array}$ & $\begin{array}{l}\text { P.M-HG-3 } \\
(C F, Y)\end{array}$ & $\begin{array}{l}P . M-H G-4 \\
\text { (CFM) }\end{array}$ & $\begin{array}{l}F T-H G-1 \\
\text { (CFM) }\end{array}$ & $\begin{array}{l}F T-H G-2 \\
(C F M)\end{array}$ \\
\hline $\begin{array}{ll}5.5 . & 1 \\
5.5 . & 2 \\
5.5 .4 & 3 \\
5.5 .4 & 4 \\
5.5 . & \end{array}$ & $\begin{array}{l}0.0 \\
0.0 \\
0.0 \\
0.0 \\
0.0\end{array}$ & $\begin{array}{l}0.0 \\
0.0 \\
0.0 \\
0.0 \\
0.0\end{array}$ & $\begin{array}{l}0.0 \\
0.0 \\
0.0 \\
0.0 \\
0.0\end{array}$ & $\begin{array}{l}50.5 \\
39.8 \\
29.7 \\
20.0 \\
50.4\end{array}$ & $\begin{array}{l}0.0 \\
0.0 \\
0.0 \\
0.0 \\
0.0\end{array}$ & $\begin{array}{l}0.0 \\
0.0 \\
0.0 \\
0.0 \\
0.0\end{array}$ & $\begin{array}{l}0.0 \\
0.0 \\
0.0 \\
0.0 \\
u .0\end{array}$ & $\begin{array}{l}0.0 \\
0.0 \\
0.0 \\
0.0 \\
0.0\end{array}$ & $\begin{array}{l}0.0 \\
0.0 \\
0.0 \\
0.0 \\
0.0\end{array}$ & $\begin{array}{l}0.0 \\
0.0 \\
0.0 \\
0.0 \\
0.0\end{array}$ \\
\hline $\begin{array}{l}5.5 .0 \\
5.5 .9 \\
5.5 .3 \\
5.5 .9 \\
5.5 .10\end{array}$ & $\begin{array}{l}0.0 \\
0.0 \\
0.0 \\
0.0 \\
0.0\end{array}$ & $\begin{array}{l}0.0 \\
0.0 \\
0.0 \\
0.0 \\
0.0\end{array}$ & $\begin{array}{l}0.0 \\
0.0 \\
0.0 \\
0.0 \\
0.0\end{array}$ & $\begin{array}{l}39.8 \\
29.8 \\
19.8 \\
50.1 \\
40.2\end{array}$ & $\begin{array}{l}0.0 \\
0.0 \\
0.0 \\
0.0 \\
0.0\end{array}$ & $\begin{array}{l}0.0 \\
0.0 \\
0.0 \\
0.0 \\
0.0\end{array}$ & $\begin{array}{l}0.0 \\
0.0 \\
0.0 \\
0.0 \\
0.0\end{array}$ & $\begin{array}{l}0.0 \\
0.0 \\
0.0 \\
0.0 \\
0.0\end{array}$ & $\begin{array}{l}0.0 \\
0.0 \\
0.0 \\
0.0 \\
0.0\end{array}$ & $\begin{array}{l}0.0 \\
0.0 \\
0.0 \\
0.0 \\
0.0\end{array}$ \\
\hline $\begin{array}{l}5.5 .11 \\
5.5 .12 \\
5.5 .13 \\
5.5 .14 \\
5.5 .15\end{array}$ & $\begin{array}{l}0.0 \\
j .0 \\
j .0 \\
0.0 \\
j .0\end{array}$ & $\begin{array}{l}0.0 \\
0.0 \\
0.0 \\
0.0 \\
0.0\end{array}$ & $\begin{array}{l}0.0 \\
0.0 \\
0.0 \\
0.0 \\
0.0\end{array}$ & $\begin{array}{l}30.6 \\
20.2 \\
49.9 \\
40.0 \\
30.0\end{array}$ & $\begin{array}{l}0.0 \\
0.0 \\
0.0 \\
0.0 \\
0.0\end{array}$ & $\begin{array}{l}0.0 \\
0.0 \\
0.0 \\
0.0 \\
0.0\end{array}$ & $\begin{array}{l}0.0 \\
0.0 \\
0.0 \\
0.0 \\
0.0\end{array}$ & $\begin{array}{l}0.0 \\
0.0 \\
0.0 \\
0.0 \\
0.0\end{array}$ & $\begin{array}{l}0.0 \\
0.0 \\
0.0 \\
0.0 \\
0.0\end{array}$ & $\begin{array}{l}0.0 \\
0.0 \\
0.0 \\
0.0 \\
0.0\end{array}$ \\
\hline $\begin{array}{l}5.5 .16 \\
5.5 .17 \\
5.5 .18 \\
5.5 .19 \\
5.5 .20\end{array}$ & $\begin{array}{l}J .0 \\
0.0 \\
0.0 \\
0.0 \\
. .0\end{array}$ & $\begin{array}{l}0.0 \\
0.0 \\
0.0 \\
0.0 \\
0.0\end{array}$ & $\begin{array}{r}0.0 \\
0.0 \\
0.0 \\
60.0 \\
0.0\end{array}$ & $\begin{array}{r}19.9 \\
10.1 \\
40.1 \\
0.0 \\
30.2\end{array}$ & $\begin{array}{l}0.0 \\
0.0 \\
0.0 \\
0.0 \\
0.0\end{array}$ & $\begin{array}{l}0.0 \\
0.0 \\
0.0 \\
0.0 \\
0.0\end{array}$ & $\begin{array}{l}0.0 \\
0.0 \\
0.0 \\
0.0 \\
0.0\end{array}$ & $\begin{array}{l}0.0 \\
0.0 \\
0.0 \\
0.0 \\
0.0\end{array}$ & $\begin{array}{l}0.0 \\
0.0 \\
0.0 \\
0.0 \\
0.0\end{array}$ & $\begin{array}{l}0.0 \\
0.0 \\
0.0 \\
0.0 \\
0.0\end{array}$ \\
\hline $\begin{array}{l}5.5 .21 \\
5.5 .22 \\
5.5 .23\end{array}$ & $\begin{array}{l}j .0 \\
j .0 \\
0.0\end{array}$ & $\begin{array}{l}0.0 \\
0.0 \\
0.0\end{array}$ & $\begin{array}{l}70.0 \\
70.0 \\
70.0\end{array}$ & $\begin{array}{l}0.0 \\
0.0 \\
0.0\end{array}$ & $\begin{array}{l}0.0 \\
0.0 \\
0.0\end{array}$ & $\begin{array}{l}0.0 \\
0.0 \\
0.0\end{array}$ & $\begin{array}{l}0.0 \\
0.0 \\
0.0\end{array}$ & $\begin{array}{l}0.0 \\
0.0 \\
0.0\end{array}$ & $\begin{array}{l}0.0 \\
0.0 \\
0.0\end{array}$ & $\begin{array}{l}0.0 \\
0.0 \\
0.0\end{array}$ \\
\hline & & & & & & & & . & & \\
\hline $\begin{array}{l}\text { TEST } \\
\text { HO }\end{array}$ & $\begin{array}{l}P-C W-1 \\
\text { (PS|G) }\end{array}$ & $\begin{array}{l}P-C H-2 \\
\text { (PSIG }\end{array}$ & $\begin{array}{l}P-H G-i \\
(P S I G)\end{array}$ & $\begin{array}{l}\text { P-HG-2 } \\
\text { (PSIG) }\end{array}$ & $\begin{array}{l}\text { P- }-H G-3 \\
\text { (PSIG) }\end{array}$ & $\begin{array}{l}\text { TF-CH-1 } \\
\text { (DEG F) }\end{array}$ & $\begin{array}{l}\text { TF-HG-1 } \\
\text { (OEG F) }\end{array}$ & $\begin{array}{l}T F-H F \rightarrow 2 \\
(D E G F)\end{array}$ & $\begin{array}{l}\text { TF-HG-3 } \\
\text { (DES F) }\end{array}$ & $\begin{array}{l}P-B A R O \\
(P S \mid A)\end{array}$ \\
\hline $\begin{array}{ll}5.5 . & 1 \\
5.5 . & 2 \\
5.5 . & 3 \\
5.5 . & 4 \\
5.5 . & 5\end{array}$ & $\begin{array}{l}13.5 \\
11.5 \\
13.5 \\
11.2 \\
12.5\end{array}$ & $\begin{array}{l}0.0 \\
0.0 \\
0.0 \\
0.0 \\
0.0\end{array}$ & $\begin{array}{r}9.5 \\
10.0 \\
13.0 \\
10.0 \\
9.0\end{array}$ & $\begin{array}{r}95.0 \\
0.0 \\
0.0 \\
0.0 \\
0.0\end{array}$ & $\begin{array}{l}0.0 \\
0.0 \\
0.0 \\
0.0 \\
0.0\end{array}$ & $\begin{array}{l}50.0 \\
52.0 \\
51.5 \\
51.5 \\
49.0\end{array}$ & $\begin{array}{l}77.0 \\
76.5 \\
77.0 \\
77.5 \\
77.5\end{array}$ & $\begin{array}{l}77.0 \\
76.5 \\
76.5 \\
76.5 \\
76.0\end{array}$ & $\begin{array}{l}7.7 .0 \\
76.5 \\
76.5 \\
76.5 \\
77.0\end{array}$ & $\begin{array}{l}12.3 \\
12.3 \\
12.3 \\
12.3 \\
12.3\end{array}$ \\
\hline $\begin{array}{l}5.5 .6 \\
5.5 .7 \\
5.5 .8 \\
5.5 .9 \\
5.5 .10\end{array}$ & $\begin{array}{l}13.0 \\
12.0 \\
12.0 \\
14.5 \\
13.0\end{array}$ & $\begin{array}{l}0.0 \\
0.0 \\
0.0 \\
0.0 \\
0.0\end{array}$ & $\begin{array}{r}11.0 \\
9.0 \\
11.00 \\
11.0 \\
10.0\end{array}$ & $\begin{array}{l}0.0 \\
0.0 \\
0.0 \\
0.0 \\
0.0\end{array}$ & $\begin{array}{l}0.0 \\
0.0 \\
0.0 \\
0.0 \\
0.0\end{array}$ & $\begin{array}{l}49.5 \\
51.0 \\
53.0 \\
49.0 \\
51.0\end{array}$ & $\begin{array}{r}77.0 \\
77.0 \\
70.0 \\
0.0 \\
0.0\end{array}$ & $\begin{array}{l}77.0 \\
77.0 \\
76.0 \\
77.0 \\
77.0\end{array}$ & $\begin{array}{r}77.0 \\
77.0 \\
77.0 \\
0.0 \\
0.0\end{array}$ & $\begin{array}{l}12.3 \\
12.2 \\
12.2 \\
12.2 \\
12.2\end{array}$ \\
\hline
\end{tabular}


TABLE XV (Contd.)

BYPASS FLOW TEST GROUP 5.5

EXPERIMENTAL MEASURMENTS FOR TESTS 5.5.1 TO 5.5.23 -- 0.53-IN. OOHNCOMER GAP

\begin{tabular}{|c|c|c|c|c|c|c|c|c|c|c|}
\hline $\begin{array}{c}\text { TEST } \\
\text { NO }\end{array}$ & $\begin{array}{l}P-C H-1 \\
\text { (PSIG) }\end{array}$ & $\begin{array}{l}P-C H-2 \\
\text { (PSIG) }\end{array}$ & $\begin{array}{l}\text { P-HG-1 } \\
\text { (PSIG) }\end{array}$ & $\begin{array}{l}P \rightarrow H G-2 \\
\text { (PSIG) }\end{array}$ & $\begin{array}{l}\text { P-HG-3 } \\
\text { (PSIG) }\end{array}$ & $\begin{array}{l}T F-C H-1 \\
(D E G F)\end{array}$ & $\begin{array}{l}\text { IF-HG-1 } \\
\text { (DEG F) }\end{array}$ & $\begin{array}{l}\text { TF-HG-2 } \\
(D E G F)\end{array}$ & $\begin{array}{l}\text { IF-HG-3 } \\
\text { (DEG F) }\end{array}$ & $\begin{array}{l}P-B A R D \\
\text { (PSIA) }\end{array}$ \\
\hline $\begin{array}{l}5.5 .11 \\
5.5 .12 \\
5.5 .13 \\
5.5 .14 \\
5.5 .15\end{array}$ & $\begin{array}{l}13.0 \\
12.0 \\
16.0 \\
15.0 \\
14.0\end{array}$ & $\begin{array}{l}0.0 \\
0.0 \\
0.0 \\
0.0 \\
0.0\end{array}$ & $\begin{array}{l}10.5 \\
11.0 \\
10.0 \\
10.0 \\
11.0\end{array}$ & $\begin{array}{l}0.0 \\
0.0 \\
0.0 \\
0.0 \\
0.0\end{array}$ & $\begin{array}{l}0.0 \\
0.0 \\
0.0 \\
0.0 \\
0.0\end{array}$ & $\begin{array}{l}50.0 \\
52.0 \\
50.0 \\
51.0 \\
51.0\end{array}$ & $\begin{array}{l}0.0 \\
0.0 \\
0.0 \\
0.0 \\
0.0\end{array}$ & $\begin{array}{l}76.0 \\
16.0 \\
76.0 \\
76.0 \\
76.0\end{array}$ & $\begin{array}{l}0.0 \\
0.0 \\
0.0 \\
0.0 \\
0.0\end{array}$ & $\begin{array}{l}12.2 \\
12.2 \\
12.2 \\
12.2 \\
12.2\end{array}$ \\
\hline $\begin{array}{l}5.5 .16 \\
5.5 .17 \\
5.5 .18 \\
5.5 .19 \\
5.5 .21\end{array}$ & $\begin{array}{l}12.0 \\
11.0 \\
10.0 \\
10.5 \\
10.5\end{array}$ & $\begin{array}{l}0.0 \\
0.0 \\
0.0 \\
0.0 \\
0.0\end{array}$ & $\begin{array}{r}10.0 \\
9.8 \\
0.0 \\
0.0 \\
0.0\end{array}$ & $\begin{array}{l}0.0 \\
0.0 \\
0.0 \\
0.0 \\
0.0\end{array}$ & $\begin{array}{l}0.0 \\
0.0 \\
0.0 \\
0.0 \\
0.0\end{array}$ & $\begin{array}{l}52.0 \\
48.0 \\
48.0 \\
44.5 \\
50.0\end{array}$ & $\begin{array}{l}0.0 \\
0.0 \\
0.0 \\
0.0 \\
0.0\end{array}$ & $\begin{array}{r}76.0 \\
80.0 \\
0.0 \\
0.0 \\
0.0\end{array}$ & $\begin{array}{r}0.0 \\
.0 .0 \\
0.0 \\
0.0 \\
0.0\end{array}$ & $\begin{array}{l}12.2 \\
12.2 \\
12.4 \\
12.4 \\
12.4\end{array}$ \\
\hline $\begin{array}{l}5.5 .21 \\
5.5 .22 \\
5.5 .23\end{array}$ & $\begin{array}{l}11.0 \\
15.0 \\
17.5\end{array}$ & $\begin{array}{l}0.0 \\
0.0 \\
0.0\end{array}$ & $\begin{array}{l}0.0 \\
0.0 \\
0.0\end{array}$ & $\begin{array}{l}0.0 \\
0.0 \\
0.0\end{array}$ & $\begin{array}{l}0.0 \\
0.0 \\
0.0\end{array}$ & $\begin{array}{l}48.0 \\
50.0 \\
51.0\end{array}$ & $\begin{array}{l}0.0 \\
0.0 \\
0.0\end{array}$ & $\begin{array}{l}0.0 \\
0.0 \\
0.0\end{array}$ & $\begin{array}{l}0.0 \\
0.0 \\
0.0\end{array}$ & $\begin{array}{l}12.4 \\
12.4 \\
12.4\end{array}$ \\
\hline
\end{tabular}

\begin{tabular}{|c|c|c|c|c|c|c|c|c|c|}
\hline \multirow[b]{2}{*}{$\begin{array}{c}\text { TEST } \\
\text { NO }\end{array}$} & \multicolumn{3}{|c|}{ LOWER PLENUM FLOW } & \multicolumn{3}{|c|}{ BYPASS FLOH } & \multirow[b]{2}{*}{$\begin{array}{l}\text { LOWER } \\
\text { PLENUM } \\
\text { LEVEL } \\
\text { (IN) }\end{array}$} & \multirow[b]{2}{*}{$\begin{array}{l}P-(P-1 \\
(P S I G)\end{array}$} & \multirow[b]{2}{*}{$\begin{array}{l}\text { TF-LP-1 } \\
\text { (DEG F) }\end{array}$} \\
\hline & $\begin{array}{l}\text { INITIAL } \\
\text { HE I SHT } \\
\text { (LB) }\end{array}$ & $\begin{array}{l}\text { FINAL } \\
\text { WEIGHT } \\
\text { (LB) }\end{array}$ & $\begin{array}{l}\text { TIME OF } \\
\text { COLLECT ION } \\
\text { (SEC) }\end{array}$ & $\begin{array}{l}\text { INITIAL } \\
\text { WEIGHT } \\
\text { (LB) }\end{array}$ & $\begin{array}{l}\text { FINAL } \\
\text { WEIGHT } \\
\text { (LÁ) }\end{array}$ & $\begin{array}{l}\text { TIME OF } \\
\text { COLLECTION } \\
\text { (SEC) }\end{array}$ & & & \\
\hline $\begin{array}{ll}5.5 .1 & 1 \\
5.5 .12 \\
5.5 .13 \\
5.5 .4 \\
5.5 .5\end{array}$ & $\begin{array}{l}140.5 \\
220.0 \\
214.5 \\
196.0 \\
144.5\end{array}$ & $\begin{array}{l}231.5 \\
299.0 \\
266.0 \\
235.5 \\
213.5\end{array}$ & $\begin{array}{l}19.8 \\
20.0 \\
15.0 \\
15.0 \\
20.0\end{array}$ & $\begin{array}{l}107.0 \\
157.0 \\
185.0 \\
140.0 \\
146.5\end{array}$ & $\begin{array}{l}157.0 \\
185.0 \\
203.0 \\
198.0 \\
220.5\end{array}$ & $\begin{array}{l}20.0 \\
20.0 \\
30.0 \\
30.0 \\
30.0\end{array}$ & $\begin{array}{l}12.0 \\
12.0 \\
12.0 \\
12.0 \\
12.0\end{array}$ & $\begin{array}{r}10.2 \\
9.7 \\
11.0 \\
10.0 \\
10.0\end{array}$ & $\begin{array}{l}50.0 . \\
52.0 \\
50.0 \\
52.5 \\
49.0\end{array}$ \\
\hline $\begin{array}{l}5.5 .6 \\
5.5 .7 \\
5.5 .8 \\
5.5 .99 \\
5.5 .10\end{array}$ & $\begin{array}{l}213.5 \\
138.5 \\
342.5 \\
166.5 \\
.348 .0\end{array}$ & $\begin{array}{l}277.5 \\
.342 .5 \\
490.0 \\
340.0 \\
547.0\end{array}$ & $\begin{array}{l}20.0 \\
60.0 \\
60.0 \\
60.0 \\
60.0\end{array}$ & $\begin{array}{l}220.5 \\
101.0 \\
146.0 \\
175.5 \\
414.0\end{array}$ & $\begin{array}{l}263.5 \\
146.0 \\
165.0 \\
414.0 \\
558.0\end{array}$ & $\begin{array}{l}20.0 \\
60.0 \\
60.0 \\
60.0 \\
60.0\end{array}$ & $\begin{array}{l}12.0 \\
12.0 \\
12.0 \\
12.0 \\
12.0\end{array}$ & $\begin{array}{l}10.2 \\
10.0 \\
10.5 \\
10.5 \\
10.0\end{array}$ & $\begin{array}{l}50.0 \\
52.0 \\
55.0 \\
50.0 \\
52.0\end{array}$ \\
\hline $\begin{array}{l}5.5 .11 \\
5.5 .12 \\
5.5 .13 \\
5.5 .14 \\
5.5 .15\end{array}$ & $\begin{array}{l}183.0 \\
277.0 \\
140.0 \\
276.0 \\
209.5\end{array}$ & $\begin{array}{l}390.5 \\
424.5 \\
276.0 \\
424.5 \\
410.0\end{array}$ & $\begin{array}{l}60.0 \\
60.0 \\
60.0 \\
60.0 \\
60.0\end{array}$ & $\begin{array}{l}315.5 \\
251.5 \\
105.5 \\
319.0 \\
242.5\end{array}$ & $\begin{array}{l}377.5 \\
291.0 \\
319.0 \\
505.0 \\
308.0\end{array}$ & $\begin{array}{r}60.0 \\
120.0 \\
45.0 \\
60.0 \\
90.0\end{array}$ & $\begin{array}{l}12.0 \\
12.0 \\
12.0 \\
12.0 \\
12.0\end{array}$ & $\begin{array}{l}11.0 \\
11.0 \\
10.0 \\
10.5 \\
10.5\end{array}$ & $\begin{array}{l}52.0 \\
52.0 \\
50.0 \\
51.0 \\
52.0\end{array}$ \\
\hline $\begin{array}{l}5.5 .16 \\
5.5 .17 \\
5.5 .18 \\
5.5 .19 \\
5.5 .20\end{array}$ & $\begin{array}{l}374.0 \\
141.0 \\
141.5 \\
181.0 \\
226.5\end{array}$ & $\begin{array}{l}522.0 \\
220.5 \\
271.5 \\
354.0 \\
339.5\end{array}$ & $\begin{array}{l}60.0 \\
60.0 \\
30.0 \\
30.0 \\
30.0\end{array}$ & $\begin{array}{l}308.0 \\
106.0 \\
137.0 \\
162.5 \\
169.5\end{array}$ & $\begin{array}{l}345.5 \\
111.5 \\
169.5 \\
239.0 \\
189.5\end{array}$ & $\begin{array}{r}120.0 \\
60.0 \\
30.0 \\
30.0 \\
60.0\end{array}$ & $\begin{array}{l}12.0 \\
12.0 \\
12.0 \\
12.0 \\
12.0\end{array}$ & $\begin{array}{r}9.7 \\
3.7 \\
10.5 \\
9.7 \\
10.5\end{array}$ & $\begin{array}{l}52.0 \\
54.5 \\
48.0 \\
49.5 \\
50.0\end{array}$ \\
\hline
\end{tabular}


$\underline{\text { TABLE XV (Contd.) }}$

BYPASS FLOW TEST GROUP 5.5

EXPERIMENTAL MEASURMENTS FOR TESTS 5.5 .1 TO $5.5 .23=0.53-$ IN. DOHNCOMER GAP

\begin{tabular}{|c|c|c|c|c|c|c|c|c|c|}
\hline \multirow[b]{2}{*}{$\begin{array}{l}\text { TEST } \\
\text { ND }\end{array}$} & \multicolumn{2}{|c|}{ LOWER PLENUM } & \multicolumn{4}{|c|}{ BYPASS FLOW } & \multirow[b]{2}{*}{$\begin{array}{l}\text { LOWER } \\
\text { PLENUM } \\
\text { LEVEL } \\
\text { (IN) }\end{array}$} & \multirow[b]{2}{*}{$\begin{array}{l}P-L P-1 \\
(P S I G)\end{array}$} & \multirow[b]{2}{*}{$\begin{array}{l}T F-L P-1 \\
\text { (OEG F) }\end{array}$} \\
\hline & $\begin{array}{l}\text { INITIAL } \\
\text { WEIGHT } \\
\text { (LB) }\end{array}$ & $\begin{array}{l}\text { F INAL } \\
\text { WE IGHT } \\
\text { (LB) }\end{array}$ & $\begin{array}{l}\text { TIME OF } \\
\text { COLLECTIUN } \\
\text { (SEC) }\end{array}$ & $\begin{array}{l}\text { IN IT IALL } \\
\text { WE IGHT } \\
\text { (LB) }\end{array}$ & $\begin{array}{l}\text { FINAL } \\
\text { WEI GHT } \\
\text { ILBI }\end{array}$ & $\begin{array}{l}\text { TIME .OF } \\
\text { COLLECTION } \\
\text { (SEC) }\end{array}$ & & & \\
\hline $\begin{array}{l}5.5 .21 \\
5.5 .22 \\
5.5 .23\end{array}$ & $\begin{array}{l}144.5 \\
140.5 \\
228.5\end{array}$ & $\begin{array}{l}273.0 \\
228.5 \\
313.0\end{array}$ & $\begin{array}{l}30.0 \\
30.0 \\
30.0\end{array}$ & $\begin{array}{l}110.5 \\
106.0 \\
305.0\end{array}$ & $\begin{array}{l}268.0 \\
305.0 \\
517.0\end{array}$ & $\begin{array}{l}30.0 \\
30.0 \\
30.0\end{array}$ & $\begin{array}{l}12.0 \\
12.0 \\
12.0\end{array}$ & $\begin{array}{l}10.0 \\
10.2 \\
10.7\end{array}$ & $\begin{array}{l}48.0 \\
54.0 \\
51.0\end{array}$ \\
\hline
\end{tabular}

\begin{tabular}{|c|c|c|c|c|c|c|c|}
\hline $\begin{array}{l}\text { TEST } \\
\text { NO }\end{array}$ & $\begin{array}{c}P . M-C G-1 \\
(C F M)\end{array}$ & $\begin{array}{c}R M-C G-2 \\
(C F(Y) .\end{array}$ & $\begin{array}{l}F T-C G-1 \\
(C F M)\end{array}$ & $\begin{array}{l}\text { P-CG-1 } \\
\text { (PSIG) }\end{array}$ & $\begin{array}{l}P-C G-2 \\
(P S I G)\end{array}$ & $\begin{array}{l}\text { TF-CG-I } \\
\text { IOEG FI }\end{array}$ & $\begin{array}{l}\text { IF-CG-2 } \\
\text { (DEG F) }\end{array}$ \\
\hline $\begin{array}{l}5.5 .1 \\
5.5 .2 \\
5.5 .3 \\
5.5 .4 \\
5.5 .5\end{array}$ & $\begin{array}{l}0.0 \\
0.0 \\
0.0 \\
0.0 \\
0.0\end{array}$ & $\begin{array}{r}30.0 \\
30.0 \\
30.0 \\
30.0 \\
0.0\end{array}$ & $\begin{array}{r}0.0 \\
0.0 \\
0.0 \\
0.0 \\
14.7\end{array}$ & $\begin{array}{l}0.0 \\
96.0 \\
95.0 \\
95.0 \\
95.0\end{array}$ & $\begin{array}{r}35.5 \\
35.0 \\
35.0 \\
35.0 \\
0.0\end{array}$ & $\begin{array}{r}0.0 \\
0.0 \\
0.0 \\
0.0 \\
82.5\end{array}$ & $\begin{array}{r}76.0 \\
76.5 \\
77.5 \\
79.0 \\
0.0\end{array}$ \\
\hline $\begin{array}{l}5.5 .6 \\
5.5 .7 \\
5.5 .8 \\
5.5 .9 \\
5.5 .10\end{array}$ & $\begin{array}{l}0.0 \\
0.0 \\
0.0 \\
0.0 \\
0.0\end{array}$ & $\begin{array}{r}0.0 \\
0.0 \\
0.0 \\
60.0 \\
60.0\end{array}$ & $\begin{array}{r}14.9 \\
14.6 \\
14.7 \\
0.0 \\
0.0\end{array}$ & $\begin{array}{r}94.5 \\
100.0 \\
97.5 \\
96.0 \\
95.0\end{array}$ & $\begin{array}{l}0.0 \\
35.0 \\
35.0 \\
35.0 \\
35.0\end{array}$ & $\begin{array}{r}85.0 \\
72.0 \\
77.0 \\
0.0 \\
0.0\end{array}$ & $\begin{array}{r}0.0 \\
0.0 \\
0.0 \\
81.0 \\
80.0\end{array}$ \\
\hline $\begin{array}{l}3.5 .11 \\
5.5 .12 \\
5.5 .13 \\
5.5 .14 \\
3.5 .15\end{array}$ & $\begin{array}{l}0.0 \\
0.0 \\
0.0 \\
0.0 \\
0.0\end{array}$ & $\begin{array}{r}60.0 \\
60.0 \\
0.0 \\
0.0 \\
0.0\end{array}$ & $\begin{array}{r}0.0 \\
.0 .0 \\
21.4 \\
22.2 \\
21.9\end{array}$ & $\begin{array}{l}96.0 \\
95.0 \\
93.5 \\
93.5 \\
93.5\end{array}$ & $\begin{array}{l}35.0 \\
35.0 \\
35.0 \\
35.0 \\
35.0\end{array}$ & $\begin{array}{r}0.0 \\
0.0 \\
88.0 \\
91.0 \\
94.0\end{array}$ & $\begin{array}{r}83.0 \\
84.0 \\
0.0 \\
0.0 \\
0.0\end{array}$ \\
\hline $\begin{array}{l}5.5 .16 \\
5.5 .17 \\
5.5 .19 \\
5.5 .19 \\
5.5 .20\end{array}$ & $\begin{array}{l}0.0 \\
0.0 \\
0.0 \\
0.0 \\
0.0\end{array}$ & $\begin{array}{r}0.0 \\
0.0 \\
13.0 \\
13.5 \\
13.5\end{array}$ & $\begin{array}{r}21.7 \\
21.8 \\
0.0 \\
0.0 \\
0.0\end{array}$ & $\begin{array}{r}93.5 \\
98.0 \\
103.0 \\
102.5 \\
102.5\end{array}$ & $\begin{array}{l}35.0 \\
34.5 \\
35.0 \\
35.0 \\
35.1\end{array}$ & $\begin{array}{r}95.0 \\
77.0 \\
0.0 \\
76.5 \\
76.0\end{array}$ & $\begin{array}{r}0.0 \\
0.0 \\
78.0 \\
76.5 \\
76.0\end{array}$ \\
\hline $\begin{array}{l}5.5 .21 \\
5.5 .22 \\
5.5 .23\end{array}$ & $\begin{array}{l}0.0 \\
0.0 \\
0.0\end{array}$ & $\begin{array}{r}30.0 \\
0.0 \\
0.0\end{array}$ & $\begin{array}{r}0.0 \\
14.7 \\
21.0\end{array}$ & $\begin{array}{l}102.0 \\
103.0 \\
101.0\end{array}$ & $\begin{array}{r}34.8 \\
0.0 \\
0.0\end{array}$ & $\begin{array}{r}0.0 \\
67.5 \\
63.5\end{array}$ & $\begin{array}{r}74.0 \\
0.0 \\
0.0\end{array}$ \\
\hline
\end{tabular}


$\underline{\text { TABLE XVI }}$

BYPASS FLOW TEST GROUP 5.7

TFANSRAPENT VESSEL TFST DATA FRR TESTS 5.7 .1 TO 5.7. Y -- 0.53-IN. DONNCMMER GAP SIJM:AAPYY IE CALCULATED RCS!ULTS

\begin{tabular}{|c|c|c|c|c|c|c|c|c|c|c|}
\hline $\begin{array}{l}\text { TEST } \\
\text { NDS }\end{array}$ & $\begin{array}{l}\text { DOWNCOAFP } \\
\text { AIF FL RIW } \\
\text { (LB/SEC) }\end{array}$ & 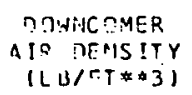 & $\begin{array}{l}\text { 1.OHEP, PLENUA } \\
\text { PRESSUP.E } \\
\text { (PSIA) }\end{array}$ & $\begin{array}{l}\text { COLD LEG } \\
A ! R \text { FLO'N } \\
\text { (LB/SEC) }\end{array}$ & $\begin{array}{l}\text { DOWNCOMER } \\
\text { WATER FLON } \\
\text { (LB/SEC) }\end{array}$ & $\begin{array}{l}\text { HATER FLLH } \\
\text { CONTINUITY }\end{array}$ & $\begin{array}{c}\text { J-AIR } \\
\text { DOWNCOMEP } \\
\text { (FT/SEC) }\end{array}$ & $\begin{array}{l}\text { J-WATER } \\
\text { DDHNCIIMER } \\
\text { IFT/SECI }\end{array}$ & $(J(, *) * * 1 / 2$ & $(J L *) * * 1$ \\
\hline $\begin{array}{l}5.7 \cdot 1 \\
5.7 \cdot 2 \\
5.7 \cdot 3 \\
5.7 \cdot 4 \\
5.7 \cdot 5\end{array}$ & $\begin{array}{l}0.0 \\
0.0 \\
0.0 \\
0.0 \\
0.0\end{array}$ & $\begin{array}{l}0.0635 \\
0.0642 \\
0.0641 \\
0.1198 \\
0.1134\end{array}$ & $\begin{array}{l}12.17 \\
12.17 \\
12.17 \\
22.67 \\
22.42\end{array}$ & $\begin{array}{l}0.0 \\
0.0 \\
0.0 \\
0.0666 \\
0.0666\end{array}$ & $\begin{array}{l}8.562 \\
6.700 \\
6.250 \\
5.550 \\
4.275\end{array}$ & $\begin{array}{l}0.997 \\
0.978 \\
1.004 \\
0.976 \\
0.386\end{array}$ & $\begin{array}{l}0.0 \\
0.0 \\
0.0 \\
0.0 \\
0.0\end{array}$ & $\begin{array}{l}2.086 \\
1.614 \\
1.505 \\
1.337 \\
1.030\end{array}$ & $\begin{array}{l}0.0 \\
0.0 \\
0.0 \\
0.0 \\
0.0\end{array}$ & $\begin{array}{l}1.1130 \\
0.9788 \\
0.9454 \\
0.8911 \\
0.7821\end{array}$ \\
\hline $\begin{array}{l}5.7 .6 \\
5.7 .7 \\
5.7: 8 \\
5.7 .9\end{array}$ & $\begin{array}{l}0.0 \\
0.0 \\
0.0 \\
0.0\end{array}$ & $\begin{array}{l}0.1157 \\
0.1183 \\
0.1169 \\
0.1307\end{array}$ & $\begin{array}{l}21.92 \\
22.42 \\
22.17 \\
22.42\end{array}$ & $\begin{array}{l}0.0664 \\
0.1322 \\
0.1311 \\
0.1313\end{array}$ & $\begin{array}{l}2.825 \\
4.158 \\
2.983 \\
1.333\end{array}$ & $\begin{array}{l}0.990 \\
0.996 \\
1.017 \\
1.016\end{array}$ & $\begin{array}{l}0.0 \\
0.0 \\
0.0 \\
0.0\end{array}$ & $\begin{array}{l}0.680 \\
1.001 \\
0.694 \\
0.321\end{array}$ & $\begin{array}{l}0.0 \\
0.0 \\
0.0 \\
0.0\end{array}$ & $\begin{array}{l}0.6357 \\
0.7713 \\
0.6423 \\
0.4368\end{array}$ \\
\hline
\end{tabular}


$\underline{\text { TABLE XVI (Contd.) }}$

BYPASS FLOW TEST GROUP 5.7

EXPERIYENTAL IEASURMENTS FOR TESTS 5.7.1 TO 5.7. 9 -- 0.53-IN. DOHNCOMER GAP

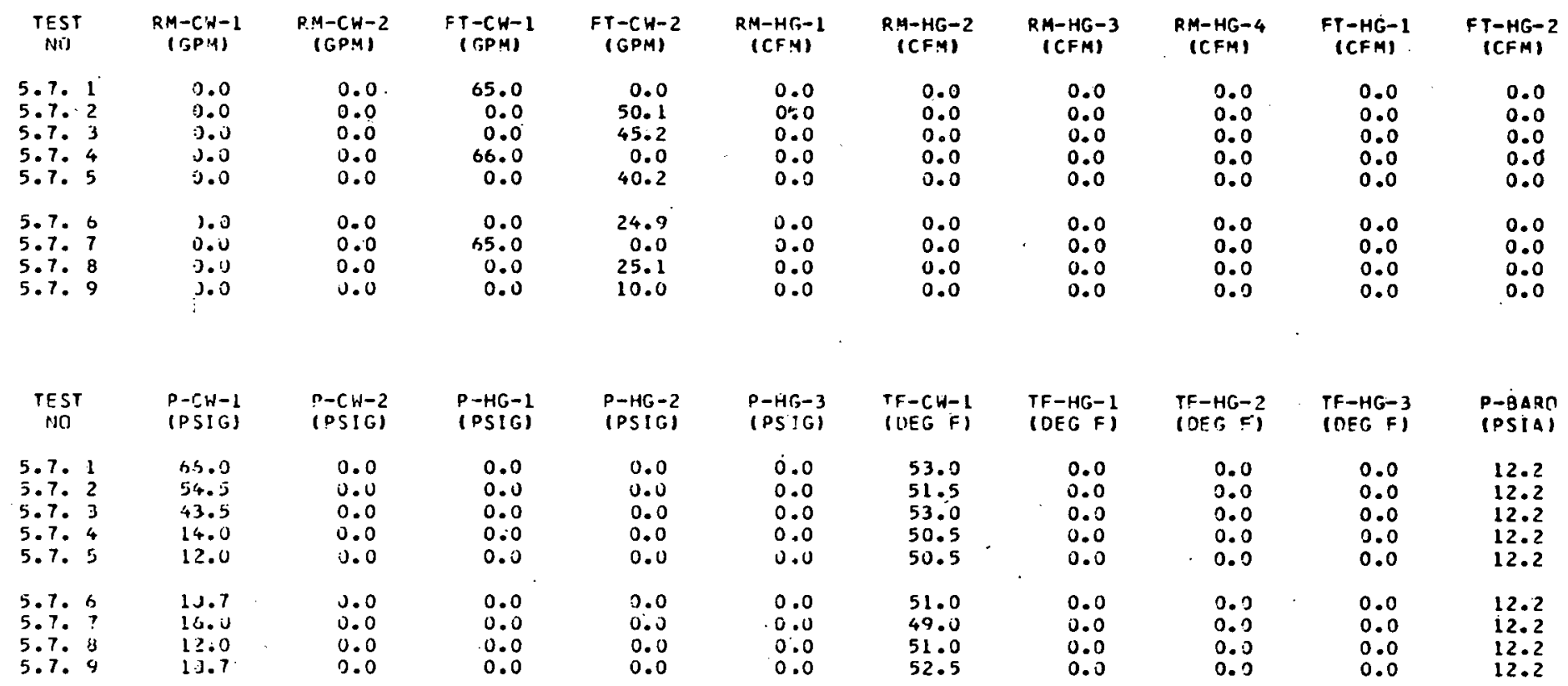

\begin{tabular}{|c|c|c|c|c|c|c|c|c|c|}
\hline \multirow[b]{2}{*}{$\begin{array}{c}T E S T \\
\text { NO }\end{array}$} & \multicolumn{3}{|c|}{ LIWWEF. PLENIJM FLOW } & \multicolumn{3}{|c|}{ BYPASS FLOW } & \multirow[b]{2}{*}{$\begin{array}{l}\text { L JWER } \\
\text { PLENUM } \\
\text { LEVFL } \\
\text { (INI) }\end{array}$} & \multirow[b]{2}{*}{$\begin{array}{l}P-L P-1 \\
(P S I G)\end{array}$} & \multirow[b]{2}{*}{$\begin{array}{l}\text { TF-LP-1 } \\
\text { IDEG F }\end{array}$} \\
\hline & $\begin{array}{l}\text { INITIAL } \\
\text { WE !GHT } \\
\text { ILG) }\end{array}$ & $\begin{array}{l}\text { CIHAL } \\
\text { WEIGHT } \\
\text { (L:B) }\end{array}$ & $\begin{array}{l}\text { TIME OF } \\
\text { COLLECTION } \\
\text { (SEC) }\end{array}$ & $\begin{array}{l}\text { IVITIAL } \\
\text { WEISHT } \\
\text { (LB) }\end{array}$ & $\begin{array}{l}\text { FINAL } \\
\text { WEIGHT } \\
\text { (LB) }\end{array}$ & $\begin{array}{l}\text { TIME OF } \\
\text { COLLECTIDN } \\
\text { (SEC) }\end{array}$ & & & \\
\hline $\begin{array}{l}5.7 \cdot 1 \\
5.7: 2 \\
5.7: 3 \\
5.7: 4 \\
5.7 .5\end{array}$ & $\begin{array}{l}145.5 \\
292.0 \\
287.0 \\
224.0 \\
249.5\end{array}$ & $\begin{array}{l}396.7 \\
426.0 \\
412.0 \\
335.0 \\
335.0\end{array}$ & $\begin{array}{l}29.0 \\
20.0 \\
20.0 \\
20.0 \\
20.0\end{array}$ & $\begin{array}{l}108.0 \\
129.0 \\
142.5 \\
150.5 \\
299.5\end{array}$ & $\begin{array}{l}129.0 \\
142.5 \\
150.5 \\
355.0 \\
373.5\end{array}$ & $\begin{array}{r}60.0 \\
120.0 \\
120.0 \\
60.0 \\
60.0\end{array}$ & $\begin{array}{l}12.0 \\
12.0 \\
12.0 \\
12.0 \\
12.0\end{array}$ & $\begin{array}{l}0.0 \\
0.0 \\
0.0 \\
10.5 \\
10.2\end{array}$ & $\begin{array}{l}57.5 \\
51.5 \\
53.5 \\
51.0 \\
51.0\end{array}$ \\
\hline $\begin{array}{l}5.7 .6 \\
5.7: 9 \\
5.709 \\
5.7 .9\end{array}$ & $\begin{array}{l}279.0 \\
141.0 \\
153.5 \\
215.0\end{array}$ & $\begin{array}{l}392.0 \\
220.0 \\
247.0 \\
295.0\end{array}$ & $\begin{array}{l}40.0 \\
19.0 \\
30.0 \\
60.0\end{array}$ & $\begin{array}{l}337.5 \\
104.0 \\
212.0 \\
252.0\end{array}$ & $\begin{array}{l}.371 .8 \\
246 . .5 \\
252.0 \\
261.5\end{array}$ & $\begin{array}{r}60.0 \\
30.0 \\
60.0 \\
120.0\end{array}$ & $\begin{array}{l}12.0 \\
12.0 \\
12.0 \\
12.0\end{array}$ & $\begin{array}{l}9.7 \\
10.2 \\
10.0 \\
10.2\end{array}$ & $\begin{array}{r}51.5 \\
49.5 \\
52.0 \\
3.0\end{array}$ \\
\hline
\end{tabular}




\section{TABLE XVI (Contd.)}

BYPASS FLOW TEST GROUP 5.7

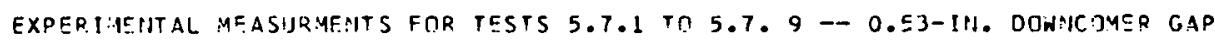

\begin{tabular}{|c|c|c|c|c|c|c|c|}
\hline $\begin{array}{c}\text { TEST } \\
N^{\prime} !\end{array}$ & $\begin{array}{c}P M-C, r_{j}-1 \\
\mid C r+m)\end{array}$ & $\begin{array}{l}f . y-C: 5-2 \\
(C F M)\end{array}$ & $\begin{array}{l}F T-C G-1 \\
(C F M)\end{array}$ & $\begin{array}{l}P-C G-1 \\
(P S I G)\end{array}$ & $\begin{array}{l}P-C G-2 \\
(P S I G)\end{array}$ & $\begin{array}{l}T F-C G-1 \\
(D \equiv C, F)\end{array}$ & $\begin{array}{l}\text { TF-CC,-2 } \\
(D E G \quad F)\end{array}$ \\
\hline 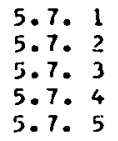 & $\begin{array}{l}0.0 \\
0.0 \\
0.0 \\
0.0 \\
0.0\end{array}$ & $\begin{array}{r}0.0 \\
0.0 \\
0.0 \\
30.0 \\
30.0\end{array}$ & $\begin{array}{l}0.0 \\
0.0 \\
0.0 \\
0.0 \\
0.0\end{array}$ & $\begin{array}{r}0.0 \\
0.0 \\
0.0 \\
25.5 \\
95.5\end{array}$ & $\begin{array}{r}0.0 \\
0.0 \\
0.00 \\
35.0 \\
35.0\end{array}$ & $\begin{array}{l}0.0 \\
0.0 \\
0.0 \\
0.0 \\
0.0\end{array}$ & $\begin{array}{r}0.0 \\
0.0 \\
0.0 \\
78.0 \\
78.0\end{array}$ \\
\hline $\begin{array}{l}5.7 .6 \\
5.7 .9 \\
5.7 .9 \\
5.7 .9\end{array}$ & $\begin{array}{l}0.0 \\
0.0 \\
j .0 \\
0.0\end{array}$ & $\begin{array}{l}30.0 \\
60.0 \\
60.0 \\
60.0\end{array}$ & $\begin{array}{l}0.0 \\
0.0 \\
0.0 \\
0.0\end{array}$ & $\begin{array}{l}96.0 \\
96.0 \\
96.0 \\
95.5\end{array}$ & $\begin{array}{l}35.0 \\
34.5 \\
34.0 \\
34.5\end{array}$ & $\begin{array}{l}0.0 \\
0.0 \\
0.0 \\
0.0\end{array}$ & $\begin{array}{l}80.0 \\
80.0 \\
83.0 \\
87.0\end{array}$ \\
\hline
\end{tabular}




\section{TABLE XVII}

BYPASS FLOW TEST GROUP 5.12

TRANSPARENT VESSEL TEST DATA FOR TESTS 5.12.1 TO 5.12.16 - 0.53-IN. DOMACOMER GAP SUMMARY OF CALCULATED RESULTS

TEST DOHNCOMER DOMNCOMER LOMER PLEMUM COLO LEG AIR FLOH AIR DENSITY PRESSURE

(LB/FI**3)

(PSIA)

(LB/SEC)

DOMNCOMER MATER FLOW J-ALR WATER FLOH CONTINUITY DOUNCDMER.

J-MATER

$(J G *) * * 1 / 2 \quad(J L *) * * 1 / 2$

0.0658

12.49
12.49

$5.12 \cdot 1 \frac{1}{5.12 .12} \quad 0.1271$

0.0662

$5.12 .3 \quad 0.1253$

5.12 .40 .1267

0.0663

12.49

0.0
0.0
0.0
0.0

5.12. 60.0

0.1186

22.24

5.12 .70 .0

0.1181

22.24

5.12 .990 .0

0.1195

22.49

5.12 .110 .0

0.1182

0.1267

22.74

$5.12 .12 \cdot 0.0$

$5.12 .13 \quad 0.0$

$5.12 .15 \quad 0.0$

$5.12 .16 \quad 0.0$

23.92

0.1189

22.42

22.17

0.1200

22.67

0.0673

0.0670

0.0670

0.1333

IFT/SEC

DOWNCOMER

$\begin{array}{llll}8.950 & 1.000 & 29.02 & 2.15\end{array}$

2.155
1.614

$0.7482 \quad 1.1313$

$0.7416 \quad 1.0194$

0.7394

0.0

1.0194
0.8733
0.9329

$\begin{array}{lll}5.333 & 0.963 & 28: 24 \\ 6.083 & 0.987 & 0.0\end{array}$

1.465

0.0

5.533

1.004

$\begin{array}{ll}4.650 & 0.930 \\ 3.317 & 1.016\end{array}$

0.0

1.333

$\begin{array}{lll}6 & 0.0 & 0.799\end{array}$

$\begin{array}{lll}5.033 & 0.0 & 1.212\end{array}$

0.0

0.0

0.8897

0.1319

3.167

1.108

0.0

0.6888

0.2738

2.167

0.995

3.733

0.973.

0.063

0.967

1.258

0.983

0.0
0.0

0.0

0.0

0.0

0.763

0.739

0.153

0.303

0.0

0.0

0.0

0.0

0.0

0.8486
0.8112

0.6731

0.6234
0.7308

0.6624

0.3010

0.4243 
TABLE XVII (Contd.)

BYPASS FLOW TEST GROUP 5.12

EXPERIMENTAL MEASURMENTS FOR TESTS 5.12 .1 TO $5.12 .16-0.53$-IN. DOHNCOMER GAP

\begin{tabular}{|c|c|c|c|c|c|c|c|c|c|c|}
\hline $\begin{array}{l}\text { TEST } \\
\text { NO }\end{array}$ & $\begin{array}{c}R M-C H-1 \\
(G P M)\end{array}$ & $\begin{array}{l}R M-C H-2 \\
(G P M)\end{array}$ & $\begin{array}{l}F T-C H-1 \\
(G P M) .\end{array}$ & $\begin{array}{c}F T-C H-2 \\
(G P M)\end{array}$ & $\begin{array}{l}R M-H G-1 \\
\text { (CFM) }\end{array}$ & $\begin{array}{c}R M-H G-2 \\
(C F M)^{2}\end{array}$ & $\begin{array}{l}\text { RM-HG -3 } \\
\text { (CFM) }\end{array}$ & $\begin{array}{c}\text { RM-HG -4 } \\
\text { (CFM) }\end{array}$ & $\begin{array}{c}F T-H G-1 \\
(C F M)\end{array}$ & $\begin{array}{l}F T-H G-2 \\
(C F M)\end{array}$ \\
\hline $\begin{array}{l}5.12 \cdot 1 \\
5.12 \cdot 2 \\
5.12 \cdot 3 \\
5.12 \cdot 4 \\
5.12 .5\end{array}$ & $\begin{array}{l}0.0 \\
0.0 \\
0.0 \\
0.0 \\
0.0\end{array}$ & $\begin{array}{l}0.0 \\
0.0 \\
0.0 \\
0.0 \\
0.0\end{array}$ & $\begin{array}{r}65.0 \\
50.0 \\
0.0 \\
0.0 \\
65.0\end{array}$ & $\begin{array}{r}0.0 \\
0.0 \\
59.5 \\
40.0 \\
0.0\end{array}$ & $\begin{array}{c}50.00 \\
50.00 \\
50.50 \\
52.00 \\
0.0\end{array}$ & $\begin{array}{l}0.0 \\
0.0 \\
0.0 \\
0.0 \\
0.0\end{array}$ & $\begin{array}{c}53.00 \\
51.00 \\
51.00 \\
49.00 \\
0.0\end{array}$ & $\begin{array}{l}0.0 \\
0.0 \\
0.0 \\
0.0 \\
0.0\end{array}$ & $\begin{array}{l}0.0 \\
0.0 \\
0.0 \\
0.0 \\
0.0\end{array}$ & $\begin{array}{l}0.0 \\
0.0 \\
0.0 \\
0.0 \\
0.0\end{array}$ \\
\hline $\begin{array}{l}5.12 \cdot 6 \\
5.12 .7 \\
5.12 .8 \\
5.12 .9 \\
5.12 .10\end{array}$ & $\begin{array}{l}0.0 \\
0.0 \\
0.0 \\
0.0 \\
0.0\end{array}$ & $\begin{array}{l}0.0 \\
0.0 \\
0.0 \\
0.0 \\
0.0\end{array}$ & $\begin{array}{r}0.0 \\
0.0 \\
0.0 \\
64.0 \\
0.0\end{array}$ & $\begin{array}{l}50.0 \\
40.1 \\
25.0 \\
0.0 \\
39.9\end{array}$ & $\begin{array}{l}0.0 \\
0.0 \\
0.0 \\
0.0 \\
0.0\end{array}$ & $\begin{array}{l}0.0 \\
0.0 \\
0.0 \\
0.0 \\
0.0\end{array}$ & $\begin{array}{l}0.0 \\
0.0 \\
0.0 \\
0.0 \\
0.0\end{array}$ & $\begin{array}{l}0.0 \\
0.0 \\
0.0 \\
0.0 \\
0.0\end{array}$ & $\begin{array}{l}0.0 \\
0.0 \\
0.0 \\
0.0 \\
0.0\end{array}$ & $\begin{array}{l}0.0 \\
0.0 \\
0.0 \\
0.0 \\
0.0\end{array}$ \\
\hline $\begin{array}{l}5.12 .11 \\
5.12 .12 \\
5.12 .13 \\
5.12 .14 \\
5.12 .15\end{array}$ & $\begin{array}{l}0.0 \\
0.0 \\
0.0 \\
0.0 \\
0.0\end{array}$ & $\begin{array}{l}0.0 \\
.0 .0 \\
0.0 \\
0.0 \\
0.0\end{array}$ & $\begin{array}{r}0.0 \\
60.0 \\
0.0 \\
0.0 \\
0.0\end{array}$ & $\begin{array}{r}24.9 \\
0.0 \\
40.0 \\
25.1 \\
5.0\end{array}$ & $\begin{array}{l}0.0 \\
0.0 \\
0.0 \\
0.0 \\
0.0\end{array}$ & $\begin{array}{l}0.0 \\
0.0 \\
0.0 \\
0.0 \\
0.0\end{array}$ & $\begin{array}{l}0.0 \\
0.0 \\
0.0 \\
0.0 \\
0.0\end{array}$ & $\begin{array}{l}0.0 \\
0.0 \\
0.0 \\
0.0 \\
0.0\end{array}$ & $\begin{array}{l}0.0 \\
0.0 \\
0.0 \\
0.0 \\
0.0\end{array}$ & $\begin{array}{l}0.0 \\
0.0 \\
0.0 \\
0.0 \\
0.0\end{array}$ \\
\hline 5.12 .16 & 0.0 & 0.0 & $\mathbf{0 . 0}$ & 10.0 & 0.0 & 0.0 & 0.0 & 0.0 & 0.0 & 0.0 \\
\hline $\begin{array}{c}\text { TEST } \\
\text { NO }\end{array}$ & $\begin{array}{l}P-C H-1 \\
(P S I G)\end{array}$ & $\begin{array}{l}\text { P-CH-2. } \\
\text { (PSIG) }\end{array}$ & $\begin{array}{l}\text { P-HG-1 } \\
|P S I G|\end{array}$ & $\begin{array}{l}\text { P-HG-2 } \\
\text { (PSIG) }\end{array}$ & $\begin{array}{l}\text { P-HG-3 } \\
\text { PSIGI }\end{array}$ & $\begin{array}{l}\text { TF-CW-1 } \\
\text { IDEG FI }\end{array}$ & $\begin{array}{l}\text { TF-HG-1 } \\
\text { IDEG FI }\end{array}$ & $\begin{array}{l}\text { TF-HG-2 } \\
\text { (DEG F) }\end{array}$ & $\begin{array}{l}\text { IF-MG-3 } \\
\text { (DEG F) }\end{array}$ & $\begin{array}{l}\text { P-BARO } \\
\text { SPSIAI }\end{array}$ \\
\hline $\begin{array}{l}5.12 \cdot 1 \\
5.12 \cdot 2 \\
5.12 \cdot 3 \\
5.12 \cdot 4 \\
5.12 \cdot 5\end{array}$ & $\begin{array}{r}0.0 \\
0.0 \\
0.0 \\
0.0 \\
12.5\end{array}$ & $\begin{array}{l}0.0 \\
0.0 \\
0.0 \\
0.0 \\
0.0\end{array}$ & $\begin{array}{l}0.0 \\
0.0 \\
0.0 \\
0.0 \\
0.0\end{array}$ & $\begin{array}{l}0.0 \\
0.0 \\
0.0 \\
0.0 \\
0.0\end{array}$ & $\begin{array}{l}0.0 \\
0.0 \\
0.0 \\
0.0 \\
0.0\end{array}$ & $\begin{array}{r}0.0 \\
0.0 \\
0.0 \\
0.0 \\
46.0\end{array}$ & $\begin{array}{r}0.0 \\
0.0 \\
0.0 \\
0.0 \\
75.0\end{array}$ & $\begin{array}{r}0.0 \\
0.0 \\
0.0 \\
0.0 \\
77.0\end{array}$ & $\begin{array}{r}0.0 \\
0.0 \\
0.0 \\
0.0 \\
77.5\end{array}$ & $\begin{array}{l}12.5 \\
12.5 \\
12.5 \\
12.5 \\
12.5\end{array}$ \\
\hline $\begin{array}{l}5.12 .9 \\
5.12,7 \\
5.12 .8 \\
5.12 .9 \\
5.12 .10\end{array}$ & $\begin{array}{l}12.0 \\
11.7 \\
11.0 \\
15.0 \\
13.0\end{array}$ & $\begin{array}{l}0.0 \\
0.0 \\
0.0 \\
0.0 \\
0.0\end{array}$ & $\begin{array}{l}0.0 \\
0.0 \\
0.0 \\
0.0 \\
0.0\end{array}$ & $\begin{array}{l}0.0 \\
0.0 \\
0.0 \\
0.0 \\
0.0\end{array}$ & $\begin{array}{l}0.0 \\
0.0 \\
0.0 \\
0.0 \\
0.0\end{array}$ & $\begin{array}{l}47.5 \\
46.0 \\
48.5 \\
48.0 \\
51.0\end{array}$ & $\begin{array}{l}0.0 \\
0.0 \\
0.0 \\
0.0 \\
0.0\end{array}$ & $\begin{array}{r}0.0 \\
0.0 \\
0.0 \\
77.0 \\
77.0\end{array}$ & $\begin{array}{l}0.0 \\
0.0 \\
0.0 \\
0.0 \\
0.0\end{array}$ & $\begin{array}{l}12.5 \\
12.5 \\
12.5 \\
12.5 \\
12.5\end{array}$ \\
\hline $\begin{array}{l}5.12 .11 \\
5.12 .12 \\
5.12 .13 \\
5.12 .14 \\
5.12 .15\end{array}$ & $\begin{array}{l}11.0 \\
21.0 \\
16.0 \\
14.0 \\
11.0\end{array}$ & $\begin{array}{l}0.0 \\
0.0 \\
0.0 \\
0.0 \\
0.0\end{array}$ & $\begin{array}{l}0.0 \\
0.0 \\
0.0 \\
0.0 \\
0.0\end{array}$ & $\begin{array}{l}0.0 \\
0.0 \\
0.0 \\
0.0 \\
0.0\end{array}$ & $\begin{array}{l}0.0 \\
0.0 \\
0.0 \\
0.0 \\
0.0\end{array}$ & $\begin{array}{l}51.0 \\
49.5 \\
49.0 \\
49.0 \\
50.0\end{array}$ & $\begin{array}{r}0.0 \\
77.0 \\
0.0 \\
0.0 \\
0.0\end{array}$ & $\begin{array}{r}77.0 \\
0.0 \\
0.0 \\
0.0 \\
0.0\end{array}$ & $\begin{array}{r}0.0 \\
7 T .0 \\
0.0 \\
0.0 \\
0.0\end{array}$ & $\begin{array}{l}12.5 \\
12.4 \\
12.4 \\
12.4 \\
12.4\end{array}$ \\
\hline
\end{tabular}


TABLE XVII (Contd.)

BYPASS FLOW TEST GROUP 5.12

XPERIMENTAL MEASURMENTS FOR TESTS 5.12 .1 TO 5.12 .16 --. 0.53-IN. DOHNCOMER GAP

\begin{tabular}{|c|c|c|c|c|c|c|c|c|c|c|}
\hline $\begin{array}{c}\text { TEST } \\
\text { NO }\end{array}$ & $\begin{array}{l}P-C H-1 \\
\text { (PSIG) }\end{array}$ & $\begin{array}{l}P-C H-2 \\
\text { (PSIGI }\end{array}$ & $\begin{array}{l}P+H G-1 \\
(P S I G)\end{array}$ & $\begin{array}{l}\text { P-HG-2 } \\
\text { (PSIG) }\end{array}$ & $\begin{array}{l}P-H G-3 \\
\text { (PSIG) }\end{array}$ & $\begin{array}{l}\text { TF-CW-1 } \\
\text { (DEG F) }\end{array}$ & $\begin{array}{l}\text { TF-HG- } 1 \\
\text { (OEG FI }\end{array}$ & $\begin{array}{l}\text { TF-HG-2 } \\
\text { IOEG FI }\end{array}$ & $\begin{array}{l}\text { TF-HG-3 } \\
\text { (DEG F) }\end{array}$ & $\begin{array}{l}\text { P-BARO } \\
\text { (PSIA) }\end{array}$ \\
\hline 5.12 .16 & 12.0 & 0.0 & 0.0 & 0.0 & 0.0 & 50.0 & 0.0 & 0.0 & 0.0 & 12.4 \\
\hline
\end{tabular}

\begin{tabular}{|c|c|c|c|c|c|c|c|c|c|}
\hline \multirow[b]{2}{*}{$\begin{array}{l}\text { TEST } \\
\text { NO }\end{array}$} & \multicolumn{3}{|c|}{ LOMER PLENUM FLOM } & \multicolumn{3}{|c|}{ BYPASS FLDW } & \multirow{2}{*}{$\begin{array}{l}\text { LOWER } \\
\text { PLENUM } \\
\text { LEVEL } \\
\text { (IN) }\end{array}$} & \multirow[b]{2}{*}{$\begin{array}{l}P-(P-1 \\
(P S I G)\end{array}$} & \multirow[b]{2}{*}{$\begin{array}{l}\text { TF- (P-1 } \\
\text { (DEG F) }\end{array}$} \\
\hline & $\begin{array}{l}\text { INIT IAL } \\
\text { WEIGHT } \\
\text { (LB) }\end{array}$ & $\begin{array}{l}\text { FINAL } \\
\text { MEIGHT } \\
\text { ILBI }\end{array}$ & $\begin{array}{l}\text { TIME OF } \\
\text { COLLECTION } \\
\text { ISECI }\end{array}$ & $\begin{array}{l}\text { INITIIAL } \\
\text { ME IGHT } \\
\text { (LB) }\end{array}$ & $\begin{array}{l}\text { FINAL } \\
\text { HEI GHT } \\
\text { IL BI }\end{array}$ & $\begin{array}{l}\text { TIME OF } \\
\text { COLLECTION } \\
\text { (SEC). }\end{array}$ & & & \\
\hline $\begin{array}{l}5.12 \cdot 1 \\
5.12 \cdot 2 \\
5.12 .3 \\
5.12 .4 \\
5.12 .5\end{array}$ & $\begin{array}{l}187.5 \\
456.0 \\
210.0 \\
428.0 \\
162.0\end{array}$ & $\begin{array}{l}456.0 \\
657.0 \\
428.0 \\
588.0 \\
344.5\end{array}$ & $\begin{array}{l}30.0 \\
30.0 \\
30.0 \\
30.0 \\
30.0\end{array}$ & $\begin{array}{l}252.5 \\
268.5 \\
300.5 \\
389.0 \\
363.5\end{array}$ & $\begin{array}{l}268.5 \\
300.5 \\
388.0 \\
390.0 \\
448.5\end{array}$ & $\begin{array}{r}180.0 \\
120.0 \\
75.0 \\
120.0 \\
30.0\end{array}$ & $\begin{array}{l}12.0 \\
12.0 \\
12.0 \\
12.0 \\
12.0\end{array}$ & $\begin{array}{l}0.0 \\
0.0 \\
0.0 \\
0.0 \\
9.7\end{array}$ & $\begin{array}{l}52.0 \\
49.0 \\
49.0 \\
48.0 \\
46.0\end{array}$ \\
\hline $\begin{array}{l}5.12 .6 \\
5.12 .7 \\
5.12 .8 \\
5.12 .9 \\
5.12 .10\end{array}$ & $\begin{array}{l}151.0 \\
215.0 \\
159.0 \\
141.0 \\
290.0\end{array}$ & $\begin{array}{l}317.0 \\
354.5 \\
258.5 \\
292.0 \\
428.0\end{array}$ & $\begin{array}{l}30.0 \\
30.0 \\
30.0 \\
30.0 \\
30.0\end{array}$ & $\begin{array}{l}207.0 \\
211.0 \\
118.0 \\
104.0 \\
219.0\end{array}$ & $\begin{array}{l}250.5 \\
243.5 \\
1.31 .0 \\
220.5 \\
242.5\end{array}$ & $\begin{array}{l}30.0 \\
60.0 \\
60.0 \\
30.0 \\
30.0\end{array}$ & $\begin{array}{l}12.0 \\
12.0 \\
12.0 \\
12.0 \\
12.0\end{array}$ & $\begin{array}{r}9.7 \\
10.5 \\
10.0 \\
10.0 \\
10.2\end{array}$ & $\begin{array}{l}48.0 \\
46.5 \\
49.5 \\
48.0 \\
51.0\end{array}$ \\
\hline $\begin{array}{l}5.12 .11 \\
5.12 .12 \\
5.12 .13 \\
5.12 .14 \\
5.12 .15\end{array}$ & $\begin{array}{l}325.0 \\
162.5 \\
230.0 \\
241.0 \\
196.5\end{array}$ & $\begin{array}{l}420.0 \\
244.0 \\
342.0 \\
333.0 \\
234.5\end{array}$ & $\begin{array}{l}30.0 \\
30.0 \\
30.0 \\
30.0 \\
60.0\end{array}$ & $\begin{array}{l}227.5 \\
127.5 \\
234.0 \\
245.5 \\
265.0\end{array}$ & $\begin{array}{l}245.5 \\
295.0 \\
289.5 \\
265.0 \\
267.5\end{array}$ & $\begin{array}{l}60.0 \\
30.0 \\
30.0 \\
60.0 \\
60.0\end{array}$ & $\begin{array}{l}12.0 \\
12.0 \\
12.0 \\
12.0 \\
12.0\end{array}$ & $\begin{array}{r}10.0 \\
11.5 \\
10.0 \\
10.0 \\
9.7\end{array}$ & $\begin{array}{l}53.5 \\
49.5 \\
49.5 \\
49.0 \\
50.0\end{array}$ \\
\hline 5.12 .16 & 234.5 & 310.0 & 60.0 & 267.5 & 274.0 & 60.0 & 12.0 & 10.2 & 50.0 \\
\hline
\end{tabular}

\begin{tabular}{|c|c|c|c|c|c|c|c|}
\hline $\begin{array}{c}\text { TEST } \\
\text { NO }\end{array}$ & $\begin{array}{c}\text { RM-CG-1 } \\
(C F M)\end{array}$ & $\begin{array}{c}R M-C G-2 \\
\text { (CFM) }\end{array}$ & $\begin{array}{c}F T-C G-1 \\
\text { (CFM) }\end{array}$ & $\begin{array}{l}P-C G-1 \\
\text { (PSIG) }\end{array}$ & $\begin{array}{l}\text { P-CG-2 } \\
\text { |PSIGI }\end{array}$ & $\begin{array}{l}\text { TF-CG-1 } \\
\text { (DEG F) }\end{array}$ & $\begin{array}{l}\text { TF-CG-2 } \\
\text { IDEG FI }\end{array}$ \\
\hline $\begin{array}{l}5.12 \cdot 1 \\
5.12 \cdot 2 \\
5.12 . \\
5.12 .4 \\
5.12 .5\end{array}$ & $\begin{array}{l}0.0 \\
0.0 \\
0.0 \\
0.0 \\
0.0\end{array}$ & $\begin{array}{r}0.0 \\
0.0 \\
0.0 \\
0.0 \\
30.0\end{array}$ & $\begin{array}{l}0.0 \\
0.0 \\
0.0 \\
0.0 \\
0.0\end{array}$ & $\begin{array}{r}0.0 \\
0.0 \\
0.0 \\
0.0 \\
102.0\end{array}$ & $\begin{array}{r}0.0 \\
0.0 \\
0.0 \\
0.0 \\
35.6\end{array}$ & $\begin{array}{l}0.0 \\
0.0 \\
0.0 \\
0.0 \\
0.0\end{array}$ & $\begin{array}{r}0.0 \\
0.0 \\
0.0 \\
0.0 \\
77.0\end{array}$ \\
\hline $\begin{array}{l}5.12 \cdot 6 \\
5.12 \cdot 7 \\
5.12 \cdot 8 \\
5.12 .9 \\
5.12 .10\end{array}$ & $\begin{array}{l}0.0 \\
0.0 \\
0.0 \\
0.0 \\
0.0\end{array}$ & $\begin{array}{r}30.0 \\
30.0 \\
30.0 \\
0.0 \\
0.0\end{array}$ & $\begin{array}{r}0.0 \\
0.0 \\
0.0 \\
14.7 \\
15.0\end{array}$ & $\begin{array}{l}99.5 \\
99.0 \\
98.5 \\
95.0 \\
94.0\end{array}$ & $\begin{array}{l}35.2 \\
35.2 \\
35.6 \\
34.4 \\
34.0\end{array}$ & $\begin{array}{r}0.0 \\
0.0 \\
0.0 \\
76.0 \\
79.0\end{array}$ & $\begin{array}{r}76.5 \\
76.0 \\
76.0 \\
0.0 \\
77.0\end{array}$ \\
\hline $\begin{array}{l}5.12 .11 \\
5.12 .12\end{array}$ & $\begin{array}{l}0.0 \\
0.0\end{array}$ & $\begin{array}{l}0.0 \\
0.0\end{array}$ & $\begin{array}{l}14.9 \\
29.5\end{array}$ & $\begin{array}{l}94.0 \\
98.0\end{array}$ & $\begin{array}{l}34.4 \\
34.0\end{array}$ & $\begin{array}{l}80.0 \\
75.0\end{array}$ & $\begin{array}{r}79.0 \\
0.0\end{array}$ \\
\hline
\end{tabular}


TABLE XVII (Contd.)

BYPASS FLOW TEST GROUP 5.12

EXPERIMENTAL MEASURMENTS FOR TESTS 5.12.1 TO 5.12.16-0.53-IN. OOWNCOMER GAP

$\begin{array}{crrrrrrr}\begin{array}{c}\text { TEST } \\ \text { NO }\end{array} & \begin{array}{c}\text { RM-CG-1 } \\ \text { (CFM) }\end{array} & \begin{array}{c}\text { RM-CG-2 } \\ \text { (CFM) }\end{array} & \begin{array}{c}\text { FT-CG-1 } \\ \text { (CFM) }\end{array} & \begin{array}{c}\text { P-CG-1 } \\ \text { (PSIG) }\end{array} & \begin{array}{c}P-C G-2 \\ \text { (PSIG) }\end{array} & \begin{array}{r}\text { TF-CG-1 } \\ \text { (DEG F) }\end{array} & \begin{array}{r}\text { TF-CG-2 } \\ \text { (DEG FI }\end{array} \\ 5.12 .13 & 0.0 & 0.0 & 29.9 & 97.0 & 34.0 & 84.0 & 0.0 \\ 5.12 .14 & 0.0 & 0.0 & 29.6 & 94.0 & 34.0 & 91.0 & 0.0 \\ 5.12 .15 & 0.0 & 0.0 & 29.5 & 95.0 & 34.0 & 93.0 & 0.0 \\ 5.12 .16 & 0.0 & 0.0 & 29.5 & 95.5 & 34.0 & 97.5 & 0.0\end{array}$




\section{TABLE XVIII}

BYPASS FLOW TEST GROUP 5.15

TRANSPARENT VESSEL TEST DATA FOR TESTS 5.15.1 TO 5.15.6- $0.53-1$. SUMmaRY OF CALCULATED RESULTS

\begin{tabular}{|c|c|c|c|c|c|c|c|c|c|c|}
\hline $\begin{array}{l}\text { TEST } \\
\text { ND }\end{array}$ & $\begin{array}{l}\text { DOWNCOMER } \\
\text { AIR FLOH } \\
\text { ILB/SEC }\end{array}$ & $\begin{array}{l}\text { DUHNCOMER } \\
\text { AIR DENSITY } \\
\text { (LB/FT**3) }\end{array}$ & $\begin{array}{l}\text { LUWER PLENUM } \\
\text { PRESSURE } \\
\text { (PSIA) }\end{array}$ & $\begin{array}{l}\text { COLD LEG } \\
\text { AIR FLOH } \\
(L B / S E C)\end{array}$ & $\begin{array}{l}\text { DOHNCOMER } \\
\text { WATER FLOH } \\
\text { (LB/SEC) }\end{array}$ & $\begin{array}{l}\text { WATER FLOW } \\
\text { CONTINUITY }\end{array}$ & $\begin{array}{c}J-A I R \\
\text { DOHNCSMER } \\
(F T / S E C)\end{array}$ & $\begin{array}{l}\text { J-IIATER } \\
\text { OOWNCIMMER } \\
\text { (FT/SEC) }\end{array}$ & $(J G *) * * 1 / 2$ & $(J L *) * * 1 / 2$ \\
\hline $\begin{array}{l}5.15 .1 \\
5.15 .2 \\
5.15 .3 \\
5.15 .4 \\
5.15 .5\end{array}$ & $\begin{array}{r}0.0 \\
0.0 \\
0.0 \\
0.0 \\
0.0\end{array}$ & $\begin{array}{l}0.1182 \\
0.1202 \\
0.11180 \\
0.11181 \\
0.0649\end{array}$ & $\begin{array}{l}22.25 \\
22.75 \\
22.25 \\
22.25 \\
1.2 .25\end{array}$ & $\begin{array}{l}0.0 \\
0.0662 \\
0.1446 \\
0.2514 \\
0.0\end{array}$ & $\begin{array}{r}10.050 \\
5.28 .3 \\
4.833 \\
2.750 \\
5.817\end{array}$ & $\begin{array}{l}0.997 \\
0.997 \\
1.005 \\
0.992 \\
0.933\end{array}$ & $\begin{array}{l}0.0 \\
0.0 \\
0.0 \\
0.0 \\
0.0\end{array}$ & $\begin{array}{l}2.420 \\
1.272 \\
1.164 \\
0.652 \\
1.401\end{array}$ & $\begin{array}{l}0.0 \\
0.0 \\
0.0 \\
0.0 \\
0.0\end{array}$ & $\begin{array}{l}1.1991 \\
0.8694 \\
0.8316 \\
0.6272 \\
0.9120\end{array}$ \\
\hline 5.15 .6 & 0.0 & 0.0051 & 12.25 & .0 .0 & 7.267 & $i .002$ & 0.0 & 1.750 & 0.0 & 1.0194 \\
\hline
\end{tabular}


TABLE XVII (Contd.)

BYPASS FLOW TEST GROUP 5.15

EXPERIMENTAL MEASURMENTS FOR TESTS 5.15 .1 TO 5.15. $6--0.53-1 \mathrm{~N}$. DOHNCOMER GAP

\begin{tabular}{|c|c|c|c|c|c|c|c|c|c|c|}
\hline $\begin{array}{l}\text { TEST } \\
\text { NO }\end{array}$ & $\begin{array}{l}\text { P. } M-C H-1 \\
(G P M)\end{array}$ & $\begin{array}{l}\text { RM-CH-2 } \\
\text { (GPMI }\end{array}$ & $\begin{array}{c}F T-C H-1 \\
(G P M)\end{array}$ & $\begin{array}{c}F T-C H-2 \\
(G P M)\end{array}$ & $\begin{array}{c}R M-H G-1 \\
(C F M)\end{array}$ & $\begin{array}{c}\text { RM-HG-2 } \\
\text { (CFM) }\end{array}$ & $\begin{array}{c}\text { RM-HG-3 } \\
\text { (CFM) }\end{array}$ & $\begin{array}{l}\text { RM-HG-4 } \\
\text { (CFM) }\end{array}$ & $\begin{array}{c}F T-H G-1 \\
(C F M)\end{array}$ & $\begin{array}{l}F T-H G-2 \\
\text { (CFMI }\end{array}$ \\
\hline $\begin{array}{l}5.15 .1 \\
5.15 .2 \\
5.15 .3 \\
5.15 .4 \\
5.15 .4\end{array}$ & $\begin{array}{l}0.0 \\
0.0 \\
0.0 \\
0.0 \\
0.0\end{array}$ & $\begin{array}{l}0.0 \\
0.0 \\
0.0 \\
0.0 \\
0.0\end{array}$ & $\begin{array}{r}75.0 \\
0.0 \\
0.0 \\
0.0 \\
100.0\end{array}$ & $\begin{array}{r}0.0 \\
39.9 \\
40.0 \\
39.9 \\
0.0\end{array}$ & $\begin{array}{l}0.0 \\
0.0 \\
0.0 \\
0.0 \\
0.0\end{array}$ & $\begin{array}{l}0.0 \\
0.0 \\
0.0 \\
0.0 \\
0.0\end{array}$ & $\begin{array}{l}0.0 \\
0.0 \\
0.0 \\
0.0 \\
0.0\end{array}$ & $\begin{array}{l}0.0 \\
0.0 \\
0.0 \\
0.0 \\
0.0\end{array}$ & $\begin{array}{l}0.0 \\
0.0 \\
0.0 \\
0.0 \\
0.0\end{array}$ & $\begin{array}{l}0.0 \\
0.0 \\
0.0 \\
0.0 \\
0.0\end{array}$ \\
\hline 5.15 .6 & 0.0 & 0.0 & 100.0 & 0.0 & 0.0 & 0.0 & 0.0 & 0.0 & 0.0 & 0.0 \\
\hline $\begin{array}{l}\text { TEST } \\
\text { ND }\end{array}$ & $\begin{array}{l}P-C H-1 \\
(P S I G)\end{array}$ & $\begin{array}{l}P-C H-2 \\
\text { (PSIG) }\end{array}$ & $\begin{array}{l}\text { P-HG-1 } \\
\text { (PSIG) }\end{array}$ & $\begin{array}{l}\text { P-HG-2 } \\
\text { (PSIG) }\end{array}$ & $\begin{array}{l}P-H G-3 \\
\text { (PSIGI }\end{array}$ & $\begin{array}{l}T F-C H-1 \\
\text { (DEG F) }\end{array}$ & $\begin{array}{l}\text { TF-HG-1 } \\
\text { (DEG F) }\end{array}$ & $\begin{array}{l}\text { TF-HG-2 } \\
\text { (DEG FI) }\end{array}$ & $\begin{array}{l}\text { IF-HG-3 } \\
\text { (OEG F) }\end{array}$ & $\begin{array}{l}\text { P-BARO } \\
\text { (PSIA) }\end{array}$ \\
\hline $\begin{array}{l}5.15 .1 \\
5.15 .2 \\
5.15 .3 \\
5.15 .4 \\
5.15 .5\end{array}$ & $\begin{array}{l}J .0 \\
9.5 \\
0.0 \\
\rightarrow .2 \\
0.0\end{array}$ & $\begin{array}{l}0.0 \\
0.0 \\
0.0 \\
0.0 \\
0.0\end{array}$ & $\begin{array}{l}0.0 \\
0.0 \\
0.0 \\
9.5 \\
0.0\end{array}$ & $\begin{array}{l}0.0 \\
0.0 \\
0.0 \\
0.0 \\
0.0\end{array}$ & $\begin{array}{l}0.0 \\
0.0 \\
0.0 \\
0.0 \\
0.0\end{array}$ & $\begin{array}{l}47.5 \\
50.0 \\
48.0 \\
48.0 \\
47.5\end{array}$ & $\begin{array}{r}0.0 \\
79.0 \\
0.0 \\
0.0 \\
0.0\end{array}$ & $\begin{array}{l}0.0 \\
0.0 \\
0.0 \\
0.0 \\
0.0\end{array}$ & $\begin{array}{r}0.0 \\
78.0 \\
0.0 \\
0.0 \\
0.0\end{array}$ & $\begin{array}{l}12.3 \\
12.3 \\
12.3 \\
12.3 \\
12.3\end{array}$ \\
\hline 5.15 .6 & 0.0 & 0.0 & 0.0 & 0.0 & 0.0 & 47.5 & 0.0 & 0.0 & 0.0 & 12.3 \\
\hline
\end{tabular}

\begin{tabular}{|c|c|c|c|c|c|c|c|c|c|}
\hline \multirow[b]{2}{*}{$\begin{array}{c}\text { TEST } \\
\text { ND }\end{array}$} & \multicolumn{3}{|c|}{ LOWER PLENUM FLOH } & \multicolumn{3}{|c|}{ BYPASS FLOW } & \multirow[b]{2}{*}{$\begin{array}{l}\text { LOWER } \\
\text { PLENUM } \\
\text { LEVEL } \\
\text { (IN) }\end{array}$} & \multirow[b]{2}{*}{$\begin{array}{l}P-(P-1 \\
(P S I G)\end{array}$} & \multirow[b]{2}{*}{$\begin{array}{l}\text { YF-LP-1 } \\
\text { (DEG F) }\end{array}$} \\
\hline & $\begin{array}{l}\text { INITIAL } \\
\text { HEIGHT } \\
\text { (LB) }\end{array}$ & $\begin{array}{l}\text { FINAL } \\
\text { WEIGHT } \\
\text { (LB) }\end{array}$ & $\begin{array}{l}\text { TIME OF } \\
\text { COLLECTICN } \\
\text { (SEC) }\end{array}$ & $\begin{array}{l}\text { INITIAL } \\
\text { WEIGHT } \\
\text { (LB) }\end{array}$ & $\begin{array}{l}\text { FINAL } \\
\text { WEIGHT } \\
\text { (LU) }\end{array}$ & $\begin{array}{l}\text { TIME OF } \\
\text { COLLECTION } \\
\text { (SEC) }\end{array}$ & & & \\
\hline $\begin{array}{ll}5.15 .1 & 1 \\
5.15 . & 2 \\
5.15 . & 3 \\
5.15 . & 4 \\
5.15 . & 5\end{array}$ & $\begin{array}{l}141.0 \\
267.0 \\
272.0 \\
169.5 \\
145.0\end{array}$ & $\begin{array}{l}442.5 \\
425.5 \\
417.0 \\
252.0 \\
319.5\end{array}$ & $\begin{array}{r}30.0 \\
30.0 \\
30.0 \\
30.0 \\
30.0\end{array}$ & $\begin{array}{l}103.0 \\
145.0 \\
129.5 \\
148.5 \\
116.5\end{array}$ & $\begin{array}{l}145.0 \\
160.0 \\
174.5 \\
231.0 \\
331.0\end{array}$ & $\begin{array}{r}120.0 \\
60.0 \\
60.0 \\
30.0 \\
30.0\end{array}$ & $\begin{array}{r}12.0 \\
10.0 \\
10.0 \\
10.0 \\
0.0\end{array}$ & $\begin{array}{r}10.0 \\
10.5 \\
10.0 \\
10.0 \\
0.0\end{array}$ & $\begin{array}{l}48.0 \\
51.0 \\
49.0 \\
48.5 \\
49.5\end{array}$ \\
\hline 5.15 .6 & 219.0 & 437.0 & 30.0 & 265.0 & $465.0^{\circ}$ & 30.0 & 0.0 & 0.0 & 48.0 \\
\hline
\end{tabular}

$\begin{array}{crrrrrrr}\begin{array}{c}\text { TEST } \\ \text { NO }\end{array} & \begin{array}{c}\text { RM-CG-1 } \\ \text { (CFM) }\end{array} & \begin{array}{c}\text { RM-CG-2 } \\ \text { (CFM) }\end{array} & \begin{array}{c}\text { FT-CG-1 } \\ \text { (CFM) }\end{array} & \begin{array}{r}\text { P-CG-1 } \\ \text { (PSIG) }\end{array} & \begin{array}{r}\text { P-CG-2 } \\ \text { (PSIG) }\end{array} & \begin{array}{r}\text { TF-CG-1 } \\ \text { (OEG.F) }\end{array} & \begin{array}{r}\text { TF-CF-2 } \\ \text { (OEGG F }\end{array} \\ 5.15 .1 & 0.0 & 0.0 & 0.0 & 0.0 & 0.0 & 0.0 & 0.0 \\ 5.15 .2 & 0.0 & 30.0 & 0.0 & 0.0 & 35.0 & 0.0 & 84.0 \\ 5.15 .3 & 0.0 & 0.0 & 14.9 & 95.5 & 0.0 & 84.5 & 0.0 \\ 5.15 .4 & 0.0 & 0.0 & 29.5 & 92.0 & 0.0 & 90.0 & 0.0 \\ 3.15 .5 & 0.0 & 0.0 & 0.0 & 0.0 & 0.0 & 0.0 & 0.0\end{array}$


TABLE XVII (Contd.)

BYPASS FLOW TEST GROUP 5.15

EXPERIMENTTAL MEASURMENTS FOR TESTS 5.15.1 TO $5.15 .6-0.53-1$ N. DOWNCOMER SAP

$\begin{array}{crrrrrrr}\begin{array}{c}\text { TEST } \\ \text { NO }\end{array} & \begin{array}{c}R M-C G-1 \\ \text { (CFM) }\end{array} & \begin{array}{c}\text { RM-CG-2 } \\ \text { (CFM) }\end{array} & \begin{array}{c}F T-C G-1 \\ \text { (CFM) }\end{array} & \begin{array}{c}P-C G-1 \\ \text { (PSIG) }\end{array} & \begin{array}{c}P-C G-2 \\ \text { (PSIG) }\end{array} & \begin{array}{c}\text { TF-CG-1 } \\ \text { (CEG F }\end{array} & \begin{array}{c}\text { TF-CG-2 } \\ \text { (DEG FI }\end{array} \\ 5.15 .6 & 0.0 & 0.0 & 0.0 & 0.0 & 0.0 & 0.0 & 0.0\end{array}$


TABLE XIX

BYPASS FLOW TEST GROUP 9.5

TRANSPARENT VESSEL TEST DATA FOR TESTS 9.5.1 TO 9.5.10 - 1.58-IN. DOHNCCIMER GAP SUMAary of CALCULATED RESULTS

\begin{tabular}{|c|c|c|c|c|c|c|c|c|c|c|}
\hline $\begin{array}{l}\text { TEST } \\
\text { NO }\end{array}$ & $\begin{array}{l}\text { DONNCOMER } \\
\text { AIR FLOH } \\
\text { iLB/SEC) }\end{array}$ & $\begin{array}{l}\text { DCWNCOMER } \\
\text { AIR OENSITY } \\
(L 8 / F T * 3)\end{array}$ & $\begin{array}{l}\text { LOWER PLENUM } \\
\text { PRESSURE } \\
\text { (PSIA) }\end{array}$ & $\begin{array}{l}\text { COLD LEG } \\
\text { AIR FLOH } \\
\text { (LB/SEC) }\end{array}$ & $\begin{array}{l}\text { DOWNCOMER } \\
\text { WATER FLOW } \\
\text { (LB/SEC) }\end{array}$ & $\begin{array}{l}\text { WATER FLOH } \\
\text { CONTINUITY }\end{array}$ & $\begin{array}{c}\text { J-AlR } \\
\text { OOWNCOMEP } \\
\text { (FT/SEC) }\end{array}$ & $\begin{array}{l}\text { J-WATER } \\
\text { DOWNC OMER } \\
\text { (FT/SEC) }\end{array}$ & $(J G *) * 1 / 2$ & $(\cdot J L *) * 1 / 2$ \\
\hline $\begin{array}{l}9.5 .1 \\
9.5 .2 \\
9.5 .3 \\
9.5 .4 \\
9.5 .5\end{array}$ & $\begin{array}{l}0.0 \\
0.0 \\
0.0 \\
0.0 \\
0.0\end{array}$ & $\begin{array}{l}0.1199 \\
0.1177 \\
0.1268 \\
0.1184 \\
0.1179\end{array}$ & $\begin{array}{l}22.70 \\
22.20 \\
23.70 \\
22.20 \\
22.20\end{array}$ & $\begin{array}{l}0.1277 \\
0.1278 \\
0.1249 \\
0.1313 \\
0.1276\end{array}$ & $\begin{array}{r}4.561 \\
9.825 \\
13.625 \\
3.550 \\
7.633\end{array}$ & $\begin{array}{l}0.989 \\
0.987 \\
0.975 \\
1.002 \\
0.968\end{array}$ & $\begin{array}{l}0.0 \\
0.0 \\
0.0 \\
0.0 \\
0.0\end{array}$ & $\begin{array}{l}0.311 \\
0.669 \\
0.329 \\
0.212 \\
j .520\end{array}$ & $\begin{array}{l}0.0 \\
0.0 \\
0.0 \\
0.0 \\
0.0\end{array}$ & $\begin{array}{l}0.3269 \\
0.4795 \\
0.5647 \\
0.2842 \\
0.4227\end{array}$ \\
\hline $\begin{array}{l}9.5 .6 \\
9.5 .7 \\
7.5 .8 \\
9.5 .99 \\
9.5 .10\end{array}$ & $\begin{array}{l}0.0 \\
0.0 \\
0.0 \\
0.0 \\
0.0\end{array}$ & $\begin{array}{l}0.1184 \\
0.1216 \\
0.1199 \\
0.1200 \\
0.1178\end{array}$ & $\begin{array}{l}22.20 \\
22.88 \\
22.63 \\
22.63 \\
22.13\end{array}$ & $\begin{array}{l}0.1267 \\
0.2080 \\
0.2061 \\
0.1995 \\
0.2917\end{array}$ & $\begin{array}{r}11.017 \\
3.517 \\
4.433 \\
9.117 \\
12.850\end{array}$ & $\begin{array}{l}0.994 \\
0.997 \\
0.980 \\
0.990 \\
0.997\end{array}$ & $\begin{array}{l}0.0 \\
0.0 \\
0.0 \\
0.0 \\
0.0\end{array}$ & $\begin{array}{l}0.750 \\
0.240 \\
0.302 \\
0.621 \\
0.875\end{array}$ & $\begin{array}{l}0.0 \\
0.0 \\
0.0 \\
0.0 \\
0.0\end{array}$ & $\begin{array}{l}0.5078 \\
0.2869 \\
0.3221 \\
0.4019 \\
0.5484\end{array}$ \\
\hline
\end{tabular}


$\underline{\text { TABLE XIX (Contd.) }}$

BYPASS FLOW TEST GROUP 9.5

EXPERIMENTAL MEASURMENTS FOR. TESTS 9.5.1 TO $4.5 .10=1.58-1 \mathrm{~N}$. DOWNCOMER GAP

\begin{tabular}{|c|c|c|c|c|c|c|c|c|c|c|}
\hline $\begin{array}{l}\text { TEST } \\
\text { NO }\end{array}$ & $\begin{array}{c}R M-C H-1 \\
(G P M)\end{array}$ & $\begin{array}{c}R M-C H-2 \\
(G P M)\end{array}$ & $\begin{array}{c}F T-C H-1 \\
(G P M)\end{array}$ & $\begin{array}{c}\mathrm{FT}-\mathrm{CH}-2 \\
(G P H)\end{array}$ & $\begin{array}{c}R M-H G-1 \\
(C F M)\end{array}$ & $\begin{array}{c}R M-H G-2 \\
(C F M)\end{array}$ & $\begin{array}{c}R M-H r_{3}=3 \\
(C F(1)\end{array}$ & $\begin{array}{l}\text { RHI-HG-A } \\
\text { (CFM) }\end{array}$ & $\begin{array}{c}F T-H G-1 \\
(C F M)\end{array}$ & $\begin{array}{c}F T-H G-2 \\
(C F M)\end{array}$ \\
\hline $\begin{array}{l}9.5 .1 \\
9.5 .5 \\
9.5 .5 \\
9.5 .4 \\
9.5 .5\end{array}$ & $\begin{array}{l}0.0 \\
0.0 \\
0.0 \\
0.0 \\
0.0\end{array}$ & $\begin{array}{l}0.0 \\
0.0 \\
0.0 \\
0.0 \\
0.0\end{array}$ & $\begin{array}{r}0.0 \\
89.0 \\
129.0 \\
0.0 \\
70.0\end{array}$ & $\begin{array}{r}40.0 \\
0.0 \\
0.0 \\
30.1 \\
0.0\end{array}$ & $\begin{array}{l}0.0 \\
0.0 \\
0.0 \\
0.0 \\
0.0\end{array}$ & $\begin{array}{l}0.0 \\
0.0 \\
0.0 \\
0.0 \\
0.0\end{array}$ & $\begin{array}{l}0.0 \\
0.0 \\
0.0 \\
0.0 \\
0.0\end{array}$ & $\begin{array}{l}0.0 \\
0.0 \\
0.0 \\
0.0 \\
0.0\end{array}$ & $\begin{array}{l}0.0 \\
0.0 \\
0.0 \\
0.0 \\
0.0\end{array}$ & $\begin{array}{l}0.0 \\
0.0 \\
0.0 \\
0.0 \\
0.0\end{array}$ \\
\hline $\begin{array}{l}9.5 .6 \\
9.5 .7 \\
9.5 .8 \\
9.5 .8 \\
9.5 .10\end{array}$ & $\begin{array}{l}0.0 \\
0.0 \\
0.0 \\
0.0 \\
0.0\end{array}$ & $\begin{array}{l}0.0 \\
0.0 \\
0.0 \\
0.0 \\
0.0\end{array}$ & $\begin{array}{r}100.0 \\
0.0 \\
0.0 \\
90.0 \\
130.0\end{array}$ & $\begin{array}{r}0.0 \\
30.2 \\
39.9 \\
0.0 \\
0.0\end{array}$ & $\begin{array}{l}0.0 \\
0.0 \\
0.0 \\
0.0 \\
0.0\end{array}$ & $\begin{array}{l}0.0 \\
0.0 \\
0.0 \\
0.0 \\
0.0\end{array}$ & $\begin{array}{l}0.0 \\
0.0 \\
0.0 \\
0.0 \\
0.0\end{array}$ & $\begin{array}{l}0.0 \\
0.0 \\
0.0 \\
0.0 \\
0.0\end{array}$ & $\begin{array}{l}0.0 \\
0.0 \\
0.0 \\
0.0 \\
0.0\end{array}$ & $\begin{array}{l}0.0 \\
0.0 \\
0.0 \\
0.0 \\
0.0\end{array}$ \\
\hline $\begin{array}{l}\text { TEST } \\
\text { NO }\end{array}$ & $\begin{array}{l}P-C H-1 \\
|P S I G|\end{array}$ & $\begin{array}{l}P-C H-2 \\
\text { (PSIG) }\end{array}$ & $\begin{array}{l}\text { P-HG-1 } \\
\text { (PSIG) }\end{array}$ & $\begin{array}{l}\text { P-HG-2 } \\
\text { (PSIG) }\end{array}$ & $\begin{array}{l}\text { P-HG-3 } \\
\text { (PSIL) }\end{array}$ & $\begin{array}{l}\text { IF-CW-1 } \\
\text { (DEG F) }\end{array}$ & $\begin{array}{l}\text { TF-HG-1 } \\
\text { (DEG H) }\end{array}$ & $\begin{array}{l}\text { TF-HG.-2 } \\
\text { (DESFI) }\end{array}$ & $\begin{array}{l}\text { TF-HG-3 } \\
\text { (DEG F) }\end{array}$ & $\begin{array}{l}\text { P-BARO } \\
\text { (PSIA) }\end{array}$ \\
\hline $\begin{array}{l}9.5 .1 \\
9.5 .2 \\
9.5 .3 \\
9.5 .4 \\
9.5 .5\end{array}$ & $\begin{array}{l}14.0 \\
17.0 \\
24.0 \\
13.0 \\
15.0\end{array}$ & $\begin{array}{l}0.0 \\
0.0 \\
0.0 \\
0.0 \\
0.0\end{array}$ & $\begin{array}{r}11.0 \\
0.0 \\
0.0 \\
11.0 \\
11.0\end{array}$ & $\begin{array}{r}95.0 \\
0.0 \\
0.0 \\
97.0 \\
90.0\end{array}$ & $\begin{array}{r}0.0 \\
0.0 \\
0.0 \\
76.0 \\
73.0\end{array}$ & $\begin{array}{l}51.0 \\
48.0 \\
44.5 \\
46.0 \\
47.0\end{array}$ & $\begin{array}{r}0.0 \\
0.0 \\
0.0 \\
110.0 \\
81.0\end{array}$ & $\begin{array}{r}0.0 \\
0.0 \\
0.0 \\
82: 0 \\
81.0\end{array}$ & $\begin{array}{r}0.0 \\
0.0 \\
0.0 \\
80.0 \\
80.0\end{array}$ & $\begin{array}{l}12.2 \\
12.2 \\
12.2 \\
12.2 \\
12.2\end{array}$ \\
\hline $\begin{array}{l}9.5 .6 \\
9.5 .7 \\
9.5 .8 \\
9.5 .9 \\
9.5 .10\end{array}$ & $\begin{array}{l}19.0 \\
13.0 \\
14.0 \\
21.0 \\
20.0\end{array}$ & $\begin{array}{l}0.0 \\
0.0 \\
0.0 \\
0.0 \\
0.0\end{array}$ & $\begin{array}{r}11.0 \\
10.7 \\
10.2 \\
10.0 \\
9.0\end{array}$ & $\begin{array}{r}96.0 \\
100.0 \\
0.0 \\
0.0 \\
0.0\end{array}$ & $\begin{array}{l}73.0 \\
31.0 \\
30.5 \\
30.0 \\
29.5\end{array}$ & $\begin{array}{l}46.0 \\
48.0 \\
48.5 \\
49.0 \\
47.0\end{array}$ & $\begin{array}{l}81.0 \\
80.5 \\
80.0 \\
80.0 \\
80.0\end{array}$ & $\begin{array}{r}80.0 \\
0.0 \\
0.0 \\
0.0 \\
0.0\end{array}$ & $\begin{array}{r}80.0 \\
0.0 \\
0.0 \\
0.0 \\
0.0\end{array}$ & $\begin{array}{l}12.2 \\
12: 4 \\
12.4 \\
12.4 \\
12.4\end{array}$ \\
\hline
\end{tabular}

LOWER PLENUM FLOH

\begin{tabular}{|c|c|c|c|c|c|c|c|c|c|}
\hline \multirow[b]{2}{*}{$\begin{array}{c}\text { TEST } \\
\text { NO }\end{array}$} & \multicolumn{3}{|c|}{ LOWER PLENUM FLOW } & \multicolumn{3}{|c|}{ BYPASS FLOW } & \multirow[b]{2}{*}{$\begin{array}{l}\text { LOWER } \\
\text { PLENIM } \\
\text { LEVEL } \\
\text { (IN) }\end{array}$} & \multirow[b]{2}{*}{$\begin{array}{l}\text { P-LP-1) } \\
(P S I G)\end{array}$} & \multirow[b]{2}{*}{$\begin{array}{l}\text { TF-LP- } \\
\text { IDEG F }\end{array}$} \\
\hline & $\begin{array}{l}\text { INITIAL } \\
\text { WEIUHT } \\
\text { (LB) }\end{array}$ & $\begin{array}{l}\text { FINAL } \\
\text { WEISHT } \\
\text { (LB) }\end{array}$ & $\begin{array}{l}\text { TIME OF } \\
\text { COLLECTICN } \\
\text { (SEC) }\end{array}$ & $\begin{array}{l}\text { INIT I AL } \\
\text { WE I GHTT } \\
\text { ILBI }\end{array}$ & $\begin{array}{l}\text { FINAL } \\
\text { HE IGHT } \\
(18)\end{array}$ & $\begin{array}{l}\text { TIME OF } \\
\text { CULLECTION } \\
\text { (SEC) }\end{array}$ & & & \\
\hline $\begin{array}{l}9.5 .1 \\
9.5 .1 \\
9.5 .13 \\
9.5 .4 \\
9.5 .5\end{array}$ & $\begin{array}{l}153.5 \\
202.5 \\
185.0 \\
142.0 \\
170.0\end{array}$ & $\begin{array}{l}290.5 \\
399.0 \\
457.5 \\
355.0 \\
399.0\end{array}$ & $\begin{array}{l}30.0 \\
20.0 \\
20.0 \\
60.0 \\
30.0\end{array}$ & $\begin{array}{l}147.0 \\
175.0 \\
246.5 \\
105.0 \\
143.5\end{array}$ & $\begin{array}{l}175.0 \\
246.5 \\
362.5 \\
143.5 \\
251.0\end{array}$ & $\begin{array}{l}30.0 \\
30.0 \\
30.0 \\
60.0 \\
60.0\end{array}$ & $\begin{array}{r}12.0 \\
5.0 \\
5.0 \\
12.0 \\
12.0\end{array}$ & $\begin{array}{l}10.5 \\
10.0 \\
11.5 \\
10.0 \\
10.0\end{array}$ & $\begin{array}{l}51.0 \\
49.0 \\
44.5 \\
46.0 \\
48.0\end{array}$ \\
\hline $\begin{array}{l}9.5 .6 \\
9.5 .7 \\
9.5 .8 \\
9.3 .9 \\
9.5 .10\end{array}$ & $\begin{array}{l}187.5 \\
160.5 \\
190.5 \\
100.0 \\
154.0\end{array}$ & $\begin{array}{l}518.0 \\
260.0 \\
329.5 \\
433.5 \\
411.0\end{array}$ & $\begin{array}{l}30.0 \\
30.0 \\
30.0 \\
30.0 \\
20.0\end{array}$ & $\begin{array}{l}188.0 \\
296.5 \\
261.0 \\
157.5 \\
114.0\end{array}$ & $\begin{array}{l}356.5 \\
316.5 \\
292.0 \\
255.5 \\
269.0\end{array}$ & $\begin{array}{l}60.0 \\
30.0 \\
31.0 \\
30.0 \\
30.0\end{array}$ & $\begin{array}{r}5.0 \\
12.0 \\
12.0 \\
3.0 \\
5.0\end{array}$ & $\begin{array}{r}10.0 \\
10.5 \\
10.2 \\
10.2 \\
9.7\end{array}$ & $\begin{array}{l}46.0 \\
48.0 \\
49.5 \\
49.0 \\
47.0\end{array}$ \\
\hline
\end{tabular}




\section{TABLE XIX (Contd.)}

BYPASS FLOW TEST GROUP 9.5

EXPERIMENTAL MEASURMENTS FOR TESTS 9.5 .1 TO $9.5 .10-1.58-1 \mathrm{~N}$. DDHNCOMER GAP

\begin{tabular}{|c|c|c|c|c|c|c|c|}
\hline $\begin{array}{l}\text { TEST } \\
\text { NO }\end{array}$ & $\begin{array}{c}R M-C G-1 \\
(C F M)\end{array}$ & $\begin{array}{c}R M-C G-2 \\
(C F M)\end{array}$ & $\begin{array}{c}F T-C G-1 \\
(C F M)\end{array}$ & $\begin{array}{l}P-C G-1 \\
(P S I G)\end{array}$ & $\begin{array}{l}P-C G-2 \\
(P S I G)\end{array}$ & $\begin{array}{l}\text { TF-CG-1 } \\
\text { (DEG F) }\end{array}$ & $\begin{array}{l}\text { TF-CG-2 } \\
\text { (DEG F) }\end{array}$ \\
\hline $\begin{array}{l}9.5 .1 \\
9.5 .22 \\
9.5 .3 \\
9.5 .4 \\
9.5 .5\end{array}$ & $\begin{array}{l}0.0 \\
0.0 \\
0.0 \\
0.0 \\
0.0\end{array}$ & $\begin{array}{l}0.0 \\
0.0 \\
0.0 \\
0.0 \\
0.0\end{array}$ & $\begin{array}{l}14.6 \\
14.7 \\
14.5 \\
14.9 \\
14.7\end{array}$ & $\begin{array}{l}96.0 \\
96.0 \\
96.0 \\
97.0 \\
96.0\end{array}$ & $\begin{array}{r}35.0 \\
35.0 \\
0.0 \\
35.0 \\
34.0\end{array}$ & $\begin{array}{r}97.0 \\
101.5 \\
104.5 \\
96.5 \\
101.5\end{array}$ & $\begin{array}{r}92.5 \\
98.0 \\
0.0 \\
93.5 \\
97.5\end{array}$ \\
\hline $\begin{array}{l}9.5 .6 \\
9.5 .7 \\
9.5 .8 \\
9.5 .89 \\
9.3 .10\end{array}$ & $\begin{array}{l}0.0 \\
0.0 \\
0.0 \\
0.0 \\
0.0\end{array}$ & $\begin{array}{l}0.0 \\
\dot{0.0} \\
0.0 \\
0.0 \\
0.0\end{array}$ & $\begin{array}{l}14.7 \\
22.1 \\
22.3 \\
22.2 \\
21.9\end{array}$ & $\begin{array}{r}96.0 \\
100.0 \\
98.5 \\
96.5 \\
95.0\end{array}$ & $\begin{array}{r}34.0 \\
0.0 \\
0.0 \\
0.0 \\
1.0\end{array}$ & $\begin{array}{r}106.5 \\
16.0 \\
80.0 \\
84.5 \\
92.5\end{array}$ & $\begin{array}{r}103.5 \\
0.0 \\
0.0 \\
0.0 \\
0.0\end{array}$ \\
\hline
\end{tabular}


flow on the bypass flow. Two types of tests were also performed in which the geometry of the upper annulus was modified to investigate the effect on bypass flow of changing the flow path between the cold leg vessel inlet and the cold leg vessel outlet. For the first type of tests, the hot leg simulators were removed from the upper annulus to provide a more direct flow path from the vessel cold leg inlet to the cold leg outlet. The purpose of these tests was to determine whether this unrestricted flow path resulted in additional bypass flow around the entrance to the downcomer. Test results from these tests are presented in Table XVI.

Additional modified upper annulus bypass flow tests were conducted in which an extension was placed on top of the normal transparent vessel upper annulus and the entire core barrel was raised 10 inches. Raising the core barrel lengthened the upper annulus region by 10 inches and effectively shortened the downcomer by the same amount. The hot leg simulators were appropriately moved on the core barrel to maintain their position in line with the inlet cold leg and the bypass leg. Lengthening the upper annulus provided a larger upper annulus vertical cross-sectional area in which the water entering from the cold leg could flow more easily over the top of the hot leg simulator and thereby reach the bypass leg more easily than with the shorter length upper annulus.

The purpose of these tests was to determine whether the potential for an increased flow through the upper annulus region would affect the bypass flow. Test results from these tests are presented in Table XVII.

Bypass flow tests were also performed to investigate the effect of the cold leg inlet flow velocity on the bypass flow phenomena. The cold leg flow area and, consequently, the velocity of the cold leg flow was varied by removing the cold leg nozzle insert at the cold leg inlet into the vessel and at the cold leg outlet. Removing the nozzle insert resulted in a flow area increase from $0.01227 \mathrm{ft}^{2}$ to $0.03758 \mathrm{ft}^{2}$ or about a factor of three increase. Results from these tests are presented in Table XVIII.

\section{MODIFIED UंPPER ANNULUS GEOMETRY TESTS}

Countercurrent flow tests were performed with several different upper annulus configurations. The objective of these tests was to provide data that could be compared with the baseline countercurrent flow data to determine whether geometry differences in the upper annulus or changes in the upper annulus inlet fluid velocity have significant influence on countercurrent flow phenomena. Changes in the upper annulus geometry were accomplished by varying the depth (the distance from the cold leg centerline to the beginning of the downcomer) and the height (the distance from the cold leg centerline to the baffle) of the upper annulus and by removing restrictions in the flow path between the cold leg inlet and the vessel outlet. The velocity of the fluid entering the upper annulus from the cold leg inlet and leaving the upper annulus through the vessel cold leg outlet was varied by removing the cold leg nozzle inserts. The results from these countercurrent flow tests with a modified upper annulus are presented in Tables XX through XXVI. 


\section{TABLE XX}

MODIFIED UPPER ANNULUS GEOMETRY TEST GROUP 5.4

TRANSPARENT VESSEL TEST TATA FOP. TESTS 5.4.1 TO 5.4.18-- 0.53-IN. ODWNCOMER GAP SUMMARY DF CALCULATED RESIJLTS

\begin{tabular}{|c|c|c|c|c|c|c|c|c|c|c|}
\hline $\begin{array}{l}\text { TEST } \\
\text { NO }\end{array}$ & $\begin{array}{l}\text { DOWNCOMCR. } \\
\text { AIR FLOW } \\
\text { (LB/SEC) }\end{array}$ & $\begin{array}{l}\text { DISWC TMER } \\
\text { AIR DENSITY } \\
\text { (LB/FT } 4+3)\end{array}$ & $\begin{array}{l}\text { LOWER PLENUM } \\
\text { PRE.SSURE } \\
\text { (PSIA) }\end{array}$ & $\begin{array}{l}\text { COLD LES } \\
\text { AIR FLOW } \\
(L B / S F C)\end{array}$ & $\begin{array}{l}\text { DOWHCOMER } \\
\text { WATER. FLOW } \\
\text { (LB/SEC) }\end{array}$ & $\begin{array}{l}\text { WATER FLON } \\
\text { CONTINUITY }\end{array}$ & $\begin{array}{c}\text { J-AIR } \\
\text { DUWNCDMER } \\
\text { (FT/SEC) }\end{array}$ & $\begin{array}{l}\text { J-IWATER } \\
\text { DOWNCOMER } \\
\text { (FT/SEC) }\end{array}$ & $(J G *) * * 1 / 2$ & $(J L *) * 1 / 2$ \\
\hline $\begin{array}{l}5.4 \cdot 1 \\
5.4 \cdot 2 \\
5.4 \cdot 3 \\
5.4 \cdot 3 \\
5.4 \cdot 5\end{array}$ & $\begin{array}{l}0.0076 \\
0.0152 \\
0.0222 \\
0.0222 \\
0.0178\end{array}$ & $\begin{array}{l}0.1164 \\
0.1160 \\
0.1164 \\
0.1169 \\
0.1169\end{array}$ & $\begin{array}{l}22.34 \\
22.34 \\
22.34 \\
22.34 \\
22.34\end{array}$ & $\begin{array}{l}0.0 \\
0.0 \\
0.0 \\
0.0 \\
0.0\end{array}$ & $\begin{array}{l}5.500 \\
5.410 \\
4.200 \\
4.850 \\
5.580\end{array}$ & $\begin{array}{l}0.989 \\
0.973 \\
0.984 \\
1.028 \\
1.003\end{array}$ & $\begin{array}{l}0.98 \\
1.96 \\
2.97 \\
2.86 \\
2.29\end{array}$ & $\begin{array}{l}1.325 \\
1.303 \\
1.011 \\
1.168 \\
1.344\end{array}$ & $\begin{array}{l}0.1584 \\
0.2243 \\
0.2713 \\
0.2710 \\
0.2426\end{array}$ & $\begin{array}{l}0.8870 \\
0.8798 \\
0.7752 \\
0.8330 \\
0.8935\end{array}$ \\
\hline $\begin{array}{l}5.4 .6 \\
5.4 .7 \\
5.4 .78 \\
5.4 .99 \\
5.4 .10\end{array}$ & $\begin{array}{l}0.0276 \\
0.0379 \\
0.0383 \\
0.0325 \\
0.0427\end{array}$ & $\begin{array}{l}0.1169 \\
0.1166 \\
0.1165 \\
0.1162 \\
0.1165\end{array}$ & $\begin{array}{l}22.34 \\
22.34 \\
22.35 \\
22.35 \\
22.35\end{array}$ & $\begin{array}{l}0.0 \\
0.0 \\
0.0 \\
0.0 \\
0.0\end{array}$ & $\begin{array}{r}3.860 \\
3.417 \\
3.553 \\
.3 .720 \\
3.012\end{array}$ & $\begin{array}{l}1.018 \\
0.994 \\
1.030 \\
1.005 \\
0.995\end{array}$ & $\begin{array}{l}3.81 \\
4.89 \\
4.95 \\
4.21 \\
5.51\end{array}$ & $\begin{array}{l}0.930 \\
0.823 \\
0.856 \\
0.896 \\
0.725\end{array}$ & $\begin{array}{l}0.3131 \\
0.3542 \\
0.3563 \\
0.3284 \\
0.3762\end{array}$ & $\begin{array}{l}0.7431 \\
0.6991 \\
0.7129 \\
0.7295 \\
0.6565\end{array}$ \\
\hline $\begin{array}{l}5.4 .11 \\
5.4 .12 \\
5.4 .13 \\
5.4 .14 \\
5.4 .15\end{array}$ & $\begin{array}{l}0.061 .3 \\
0.0783 \\
0.1057 \\
0.1343 \\
0.1671\end{array}$ & $\begin{array}{l}0.1165 \\
0.1165 \\
0.1162 \\
0.1162 \\
0.1156\end{array}$ & $\begin{array}{l}22.35 \\
22.35 \\
22.35 \\
22.35 \\
22.35\end{array}$ & $\begin{array}{l}0.0 \\
0.0 \\
0.0 \\
0.0 \\
0.0\end{array}$ & $\begin{array}{l}2.450 \\
1.960 \\
1.137 \\
0.579 \\
0.271\end{array}$ & $\begin{array}{l}1.012 \\
0.982 \\
1.025 \\
1.023 \\
1.009\end{array}$ & $\begin{array}{r}7.92 \\
10.11 \\
13.67 \\
17.43 \\
21.72\end{array}$ & $\begin{array}{l}0.590 \\
0.472 \\
0.274 \\
0.139 \\
0.065\end{array}$ & $\begin{array}{l}0.4507 \\
0.5093 \\
0.5921 \\
0.6685 \\
0.7452\end{array}$ & $\begin{array}{l}0.5920 \\
0.5295 \\
0.4034 \\
0.2877 \\
0.1971\end{array}$ \\
\hline $\begin{array}{l}5.4 .16 \\
5.4 .17 \\
5.4 .18\end{array}$ & $\begin{array}{l}0.1366 \\
0.2054 \\
0.2195\end{array}$ & $\begin{array}{l}0.1151 \\
0.1147 \\
0.1132\end{array}$ & $\begin{array}{l}22.35 \\
22.35 \\
22.35\end{array}$ & $\begin{array}{l}0.0 \\
0.0 \\
0.0\end{array}$ & $\begin{array}{l}0.161 \\
0.092 \\
0.050\end{array}$ & $\begin{array}{l}0.919 \\
0.999 \\
0.993\end{array}$ & $\begin{array}{l}24.36 \\
26.91 \\
29.14\end{array}$ & $\begin{array}{l}0.039 \\
0.022 \\
0.012\end{array}$ & $\begin{array}{l}0.7884 \\
0.8278 \\
0.8587\end{array}$ & $\begin{array}{l}0.1518 \\
0.1145 \\
0.0846\end{array}$ \\
\hline
\end{tabular}


TABLE XX (Contd.)

MODIFIED UPPER ANNULUS GEOMETRY TEST GROUP 5.4

EXPERINENT AL MEASURMENTS FOR TESTS 5.4.1 TO 5:4.18 - $0.53-$ IN. ONWNCOMER GAP

\begin{tabular}{|c|c|c|c|c|c|c|c|c|c|c|}
\hline $\begin{array}{c}\text { TEST } \\
\text { NO }\end{array}$ & $\begin{array}{c}\mathrm{RM}-\mathrm{CH}-1 \\
(G P M)\end{array}$ & $\begin{array}{c}\text { P. } \mathrm{M}-\mathrm{CW}-2 \\
(G, P M)\end{array}$ & $\begin{array}{c}F T-C H-1 \\
(G P M)\end{array}$ & $\begin{array}{c}F T-C W-2 \\
(G P: 1)\end{array}$ & $\begin{array}{c}\text { RM-HG-1 } \\
\text { (CF }(A) .\end{array}$ & $\begin{array}{c}R M-H G-2 \\
(C F M)\end{array}$ & $\begin{array}{c}R M-H G-3 \\
(C F M)\end{array}$ & $\begin{array}{c}R M-H(G-4 \\
(C F M)\end{array}$ & $\begin{array}{c}F T-H G-1 \\
(C F M)\end{array}$ & $\begin{array}{c}F T-H G-2 \\
(C F M)\end{array}$ \\
\hline $\begin{array}{ll}5.4 . & 1 \\
5.4 \cdot & 2 \\
5.4 \cdot & 3 \\
5.4 . & 4 \\
5.4 . & 5\end{array}$ & $\begin{array}{l}30.0 \\
30.0 \\
3 \% .0 \\
30.0 \\
30.0\end{array}$ & $\begin{array}{r}10.0 \\
10.0 \\
10.0 \\
4.0 \\
10.0\end{array}$ & $\begin{array}{l}0.0 \\
0.0 \\
0.0 \\
0.0 \\
0.0\end{array}$ & $\begin{array}{l}0.0 \\
0.0 \\
0.0 \\
0.0 \\
0.0\end{array}$ & $\begin{array}{l}0.0 \\
0.0 \\
0.0 \\
0.0 \\
0.0\end{array}$ & $\begin{array}{l}0.0 \\
0.0 \\
0.0 \\
0.0 \\
0.0\end{array}$ & $\begin{array}{l}0.0 \\
10.00 \\
15.00 \\
15.00 \\
12.00\end{array}$ & $\begin{array}{l}5.00 \\
0.0 \\
0.0 \\
0.0 \\
0.0\end{array}$ & $\begin{array}{l}0.0 \\
0.0 \\
0.0 \\
0.0 \\
0.0\end{array}$ & $\begin{array}{l}0.0 \\
0.0 \\
0.0 \\
0.0 \\
0.0\end{array}$ \\
\hline $\begin{array}{l}5.4 .6 \\
5.4 .7 \\
5.4 .8 \\
5.4 .8 \\
5.4 .10\end{array}$ & $\begin{array}{l}30.0 \\
30.0 \\
30.0 \\
30.0 \\
25.0\end{array}$ & $\begin{array}{r}10.0 \\
10.0 \\
0.0 \\
0.0 \\
0.0\end{array}$ & $\begin{array}{l}0.0 \\
0.0 \\
0.0 \\
0.0 \\
0.0\end{array}$ & $\begin{array}{l}0.0 \\
0.0 \\
0.0 \\
0.0 \\
0.0\end{array}$ & $\begin{array}{l}0.0 \\
0.0 \\
0.0 \\
0.0 \\
0.0\end{array}$ & $\begin{array}{l}0.0 \\
0.0 \\
25.00 \\
20.00 \\
30.00\end{array}$ & $\begin{array}{c}20.00 \\
25.00 \\
0.0 \\
0.0 \\
0.0\end{array}$ & $\begin{array}{l}0.0 \\
0.0 \\
0.0 \\
0.0 \\
0.0\end{array}$ & $\begin{array}{l}0.0 \\
0.0 \\
0.0 \\
0.0 \\
0.0\end{array}$ & $\begin{array}{l}0.0 \\
0.0 \\
0.0 \\
0.0 \\
0.0\end{array}$ \\
\hline $\begin{array}{l}5.4 .11 \\
5.4 .12 \\
5: 4.13 \\
5.4 .14 \\
5.4 .15\end{array}$ & $\begin{array}{r}20.0 \\
15.0 \\
15.0 \\
10.0 \\
5.0\end{array}$ & $\begin{array}{l}0.0 \\
0.0 \\
0.0 \\
0.0 \\
0.0\end{array}$ & $\begin{array}{l}0.0 \\
0.0 \\
0.0 \\
0.0 \\
0.0\end{array}$ & $\begin{array}{l}0.0 \\
0.0 \\
0.0 \\
0.0 \\
0.0\end{array}$ & $\begin{array}{c}0.0 \\
0.0 \\
0.0 \\
30.00 \\
60.00\end{array}$ & $\begin{array}{l}40.00 \\
50.00 \\
70.00 \\
60.00 \\
50.00\end{array}$ & $\begin{array}{l}0.0 \\
0.0 \\
0.0 \\
0.0 \\
0.0\end{array}$ & $\begin{array}{l}0.0 \\
0.0 \\
0.0 \\
0.0 \\
0.0\end{array}$ & $\begin{array}{l}0.0 \\
0.0 \\
0.0 \\
0.0 \\
0.0\end{array}$ & $\begin{array}{l}0.0 \\
0.0 \\
0.0 \\
0.0 \\
0.0\end{array}$ \\
\hline $\begin{array}{l}5.4 .16 \\
5.4 .17 \\
5.4 .18\end{array}$ & $\begin{array}{l}3.0 \\
3.0 \\
3.0\end{array}$ & $\begin{array}{l}0.0 \\
0.0 \\
0.0\end{array}$ & $\begin{array}{l}0.0 \\
0.0 \\
0.0\end{array}$ & $\begin{array}{l}0.0 \\
0.0 \\
0.0\end{array}$ & $\begin{array}{l}60.00 \\
70.00 \\
70.00\end{array}$ & $\begin{array}{l}60.00 \\
60.00 \\
70.00\end{array}$ & $\begin{array}{l}0.0 \\
0.0 \\
0.0\end{array}$ & $\begin{array}{l}0.0 \\
0.0 \\
0.0\end{array}$ & $\begin{array}{l}0.0 \\
0.00 \\
0.0\end{array}$ & $\begin{array}{l}0.0 \\
0.0 \\
0.0\end{array}$ \\
\hline $\begin{array}{l}\text { TEST } \\
\text { NO }\end{array}$ & $\begin{array}{l}P-C W-1 \\
(P S \mid G)\end{array}$ & $\begin{array}{l}P-C H-2 \\
\text { (PSIGI }\end{array}$ & $\begin{array}{l}P-H G-1 \\
\text { (PSIGI) }\end{array}$ & $\begin{array}{l}P-H G-2 \\
(P S I G)\end{array}$ & $\begin{array}{l}P-H G-3 \\
\text { (PSIG) }\end{array}$ & $\begin{array}{l}T F-C H-1 \\
(D E G \quad F)\end{array}$ & $\begin{array}{l}T F-H G-1 \\
(D E G F)\end{array}$ & $\begin{array}{l}\text { TF-HG-2 } \\
\text { (DEG F) }\end{array}$ & $\begin{array}{l}\text { TF-HG-3 } \\
\text { (DEG F) }\end{array}$ & $\begin{array}{l}\text { P-BARO } \\
\text { (PSIA) }\end{array}$ \\
\hline $\begin{array}{l}5.4 .1 \\
5.4 .2 \\
5.4 .3 \\
5.4 .4 \\
5.4 .5\end{array}$ & $\begin{array}{l}0.0 \\
0.0 \\
3.0 \\
0.0 \\
0.0\end{array}$ & $\begin{array}{r}9.5 \\
10.0 \\
9.0 \\
9.0 \\
9.0\end{array}$ & $\begin{array}{r}10.0 \\
10.0 \\
9.0 \\
9.0 \\
9.0\end{array}$ & $\begin{array}{l}0.0 \\
0.0 \\
0.0 \\
0.0 \\
0.0\end{array}$ & $\begin{array}{l}0.0 \\
0.0 \\
0.0 \\
0.0 \\
0.0\end{array}$ & $\begin{array}{l}58.0 \\
60.0 \\
58.0 \\
57.0 \\
57.0\end{array}$ & $\begin{array}{l}87.0 \\
86.0 \\
86.0 \\
86.0 \\
84.0\end{array}$ & $\begin{array}{l}0.0 \\
0.0 \\
0.0 \\
0.0 \\
0.0\end{array}$ & $\begin{array}{l}0.0 \\
0.0 \\
0.0 \\
0.0 \\
0.0\end{array}$ & $\begin{array}{l}12.3 \\
12.3 \\
12.3 \\
12.3 \\
12.3\end{array}$ \\
\hline $\begin{array}{l}5.4 .6 \\
5.4 .7 \\
5.4 .8 \\
5.4 .9 \\
5.4 .10\end{array}$ & $\begin{array}{l}0.0 \\
0.0 \\
0.0 \\
0.0 \\
0.0\end{array}$ & $\begin{array}{r}9.0 \\
9.0 \\
10.0 \\
11.0 \\
7.0\end{array}$ & $\begin{array}{r}9.0 \\
10.0 \\
10.0 \\
13.0 \\
7.0\end{array}$ & $\begin{array}{l}0.0 \\
0.0 \\
0.0 \\
0.0 \\
0.0\end{array}$ & $\begin{array}{l}0.0 \\
0.0 \\
0.0 \\
0.0 \\
0.0\end{array}$ & $\begin{array}{l}56.0 \\
57.0 \\
58.0 \\
59.0 \\
58.0\end{array}$ & $\begin{array}{l}85.0 \\
85.0 \\
77.0 \\
78.0 \\
81.0\end{array}$ & $\begin{array}{l}0.0 \\
0.0 \\
0.0 \\
0.0 \\
0.0\end{array}$ & $\begin{array}{l}0.0 \\
0.0 \\
0.0 \\
0.0 \\
0.0\end{array}$ & $\begin{array}{l}12.3 \\
12.3 \\
12.3 \\
12.3 \\
12.3\end{array}$ \\
\hline $\begin{array}{l}5.4 .11 \\
5.4 .12 \\
5.4 .13 \\
5.4 .14 \\
5.4 .15\end{array}$ & $\begin{array}{l}0.0 \\
0.0 \\
0.0 \\
0.0 \\
0.0\end{array}$ & $\begin{array}{r}8.0 \\
10.0 \\
2.0 \\
8.0 \\
10.0\end{array}$ & $\begin{array}{r}10.0 \\
11.0 \\
9.0 \\
9.0 \\
10.0\end{array}$ & $\begin{array}{l}0.0 \\
0.0 \\
0.0 \\
0.0 \\
0.0\end{array}$ & $\begin{array}{l}0.0 \\
0.0 \\
0.0 \\
0.0 \\
0.0\end{array}$ & $\begin{array}{l}58.0 \\
58.0 \\
58.0 \\
59.0 \\
59.0\end{array}$ & $\begin{array}{r}82.0 \\
87.0 \\
92.5 \\
92.5 \\
1.06 .0\end{array}$ & $\begin{array}{l}0.0 \\
0.0 \\
0.0 \\
0.0 \\
0.0\end{array}$ & $\begin{array}{l}0.0 \\
0.0 \\
0.0 \\
0.0 \\
0.0\end{array}$ & $\begin{array}{l}12.3 \\
12.3 \\
12.3 \\
12.3 \\
12.3\end{array}$ \\
\hline
\end{tabular}




\section{TABLE XX (Contd.)}

MODIFIED UPPER ANNULUS GEOMETRY TEST GROUP 5.4

FXPEPIMENTAL MEASURMENTS FOR TESTS 5.4.1 TO 5.4.18-- 0.53-IN. DOHNCOMEP GAP

\begin{tabular}{|c|c|c|c|c|c|c|c|c|c|c|}
\hline $\begin{array}{c}\text { TEST } \\
\text { NO }\end{array}$ & $\begin{array}{l}P-5 N-1 \\
\text { (PSIG) }\end{array}$ & $\begin{array}{l}P-C H-2 \\
\text { (PSIG) }\end{array}$ & $\begin{array}{l}P-H G-1 \\
(P S I G)\end{array}$ & $\begin{array}{l}P-H G-2 \\
\text { (PSIG) }\end{array}$ & $\begin{array}{l}\text { P-HG-3 } \\
\text { (PSIG) }\end{array}$ & $\begin{array}{l}\text { TF-CH-1 } \\
\text { (DEG F) }\end{array}$ & $\begin{array}{l}\text { TF-HS- } 1 \\
(D E G \text { F) }\end{array}$ & $\begin{array}{l}\text { TF-HG-2 } \\
(D F G \cdot F)\end{array}$ & $\begin{array}{l}\text { TF-HC-3 } \\
\text { (CFG F) }\end{array}$ & $\begin{array}{l}\text { P-BARO } \\
\text { (PSIA) }\end{array}$ \\
\hline $\begin{array}{l}5.4 .16 \\
5.4 .17 \\
5.4 .18\end{array}$ & $\begin{array}{r}3.0 \\
3.0 \\
1.0\end{array}$ & $\begin{array}{l}10.0 \\
10.0 \\
10.0\end{array}$ & $\begin{array}{l}11.0 \\
11.0 \\
11.0\end{array}$ & $\begin{array}{l}0.0 \\
0.0 \\
0.0\end{array}$ & $\begin{array}{l}0.0 \\
0.0 \\
0.0\end{array}$ & $\begin{array}{l}62.0 \\
79.0 \\
75.0\end{array}$ & $\begin{array}{r}106.5 \\
93.5 \\
106.0\end{array}$ & $\begin{array}{l}0.0 \\
0.0 \\
0.0\end{array}$ & $\begin{array}{l}0.0 \\
0.0 \\
0.0\end{array}$ & $\begin{array}{l}12.3 \\
12.3 \\
12.3\end{array}$ \\
\hline
\end{tabular}

\begin{tabular}{|c|c|c|}
\hline IIT: & F[MAL & \\
\hline $\begin{array}{l}\text { NEIGHT } \\
\text { (LS) }\end{array}$ & $\begin{array}{l}\text { WEIGHT } \\
\text { (LB) }\end{array}$ & $\begin{array}{l}\text { COLLECTICN } \\
\text { (SEC) }\end{array}$ \\
\hline
\end{tabular}

\begin{tabular}{l} 
BYPASS FLOH \\
\hline $\begin{array}{l}\text { FINAL } \\
\text { WEITIAL } \\
\text { (LB) }\end{array}$ WEIGHT COLLEC OFF \\
(LB) \\
ISEC)
\end{tabular}

LOWER

PLENUM

LEVEL

$P-L P-1$

(PSIG)

TF-LP-1

$132.5 \quad 270.0 \quad 25.0$

25.0
25.0

270.0

405.3

531.

30.0

20.0

95.0

95.0

45.0

95.0
95.0

95.0
165.0

165.0
165.5

165.5

40.0
55.0

55.0
60.0

165.5

264.5

165.5
264.5

25.0

526.5

564.0

240.5

30.0

30.4

461.5
132.5

333.5

25.0
20.0

5.4 .99
5.4 .10

333.5

393.8

90.0

123.5

380.5

123.5

144.8

164.8

40.0

12.0
12.0
12.0

$12.0 \quad 10.0$

10.0
10.0
10.0

10.0
10.0

THEG F

5.4 .11

$339.3 \quad 400.5$

$400.5 \quad 449.5$

$449.5 \quad 495.0$

25.0

25.0

5.4 .13

5.4 .14
5.4 .15

515.3

515.3
524.8

40.0

35.0 .

5.4 .16

$524.8 \quad 532.0$

$\begin{array}{ll}133.0 & 138.5 \\ 138.5 & 141.5\end{array}$

45.0
60.0
60.0

164.8

164.8
184.8
190.5

190.5

184.8

190.5

230.5

268.5

290.0

300.0

319.0
144.5

93.0
119.0

144.5

55.0
55.0

45.0

45.0

45.0

12.0

12.0

10.0

58.0

58.0

56.0

56.0

$\begin{array}{ll}12.0 & 10.0 \\ 12.0 & 10.0\end{array}$

$12.0 \quad 10.0$

56.0

57.0

58.0

59.0

$\begin{array}{lll}12.0 & 10.0 & 59.0 \\ 12.0 & 10.0 & 58.0\end{array}$

5.4 .18

141.5

55.0
65.0
40.0

65.0
40.0

45.0

12.0

12.0

10.0

58.0

10.0
10.0
10.0

58.0

59.0

59.0
62.0

45.0

12.0

10.0

62.0

45.0
80.0
70.0

12.0
12.0

10.10

10.0
10.0
10.0

64.0
66.0

66.0
73.0 
TABLE XXI

MODIFIED UPPER ANNULUS GËOMETRY TEST GROUP 5.8

TP.ANSPAPENT VESSEL TEST DATA FOR TESTS 5.8.1 T! 5.8.9-- $0.53-1$ N. DOWNCOMER GAP SUMMAFY OF CALCULATED RESIJLTS

\begin{tabular}{|c|c|c|c|c|c|c|c|c|c|c|}
\hline $\begin{array}{l}\text { TEST } \\
\text { NO }\end{array}$ & $\begin{array}{l}\text { D.OHNCOMER } \\
\text { A }(R \text { FLOWW } \\
\text { (LB/SECI }\end{array}$ & $\begin{array}{c}\text { DIUNNCi):1ER } \\
\text { AIR OEVSITY } \\
\text { (LB/FT } \$ 3 \text { ) }\end{array}$ & $\begin{array}{l}\text { LOHEP PLENISM } \\
\text { PRESSURE } \\
\text { (PSIA) }\end{array}$ & $\begin{array}{l}\text { COLD LEG } \\
\text { AIR FLOW } \\
\text { (LB/SEC) }\end{array}$ & $\begin{array}{l}\text { DOWNCOMER } \\
\text { WATER FLOH } \\
\text { (LS/SEC) }\end{array}$ & $\begin{array}{l}\text { WATEA FLOH } \\
\text { CONTINUITY }\end{array}$ & $\begin{array}{c}\text { J-AIR } \\
\text { DOWNCOMEP } \\
\text { (FT/SEC) }\end{array}$ & $\begin{array}{l}\text { J-HATEP. } \\
\text { DOHNICOMER } \\
\text { (FT/SEC) }\end{array}$ & $(J G *) * * 1 / 2$ & $(\mathrm{Jt} *) * * 1 / 2$ \\
\hline 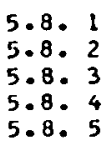 & $\begin{array}{l}0.0219 \\
0.0219 \\
0.0329 \\
0.0437 \\
0.0655\end{array}$ & $\begin{array}{l}0.11 .45 \\
0.1159 \\
0.1170 \\
0.1172 \\
0.1100\end{array}$ & $\begin{array}{l}21.67 \\
21.99 \\
22.24 \\
22.24 \\
21.99\end{array}$ & $\begin{array}{l}0.0 \\
0.0 \\
0.0 \\
0.0 \\
0.0\end{array}$ & $\begin{array}{l}4.086 \\
4.233 \\
3.541 \\
3.100 \\
2.217\end{array}$ & $\begin{array}{l}0.979 \\
1.035 \\
0.982 \\
1.045 \\
1.043\end{array}$ & $\begin{array}{l}2.87 \\
2.84 \\
4.23 \\
5.60 \\
8.48\end{array}$ & $\begin{array}{l}0.994 \\
1.019 \\
0.853 \\
0.747 \\
0.534\end{array}$ & $\begin{array}{l}0.2702 \\
0.2696 \\
0.3297 \\
0.3796 \\
0.4661\end{array}$ & $\begin{array}{l}0.7646 \\
0.7782 \\
0.7217 \\
0.6660 \\
0.5631\end{array}$ \\
\hline $\begin{array}{l}5.8 .6 \\
5.8 .7 \\
5.8 .8 \\
5.8 .9\end{array}$ & $\begin{array}{l}0.1090 \\
0.1961 \\
0.1463 \\
0.1569\end{array}$ & $\begin{array}{l}0.1131 \\
0.1185 \\
0.1166 \\
0.1157\end{array}$ & $\begin{array}{l}22.49 \\
22.49 \\
22.49 \\
22.24\end{array}$ & $\begin{array}{l}0.0 \\
0.0 \\
0.0 \\
0.0\end{array}$ & $\begin{array}{r}0.79 .2 \\
0.025 \\
.0 .233 \\
0.142\end{array}$ & $\begin{array}{l}1.020 \\
0.998 \\
1.016 \\
0.975\end{array}$ & $\begin{array}{l}13.87 \\
24.88 \\
18.85 \\
20.39\end{array}$ & $\begin{array}{l}0.191 \\
0.006 \\
0.056 \\
0.034\end{array}$ & $\begin{array}{l}0.5988 \\
0.8024 \\
0.6357 \\
0.7221\end{array}$ & $\begin{array}{l}0.3365 \\
0.0598 \\
0.1827 \\
0.1424\end{array}$ \\
\hline
\end{tabular}

18.85

0.7221

0.1424 


\section{TABLE XXI (Contd.)}

MODIFIED UPPER ANNULUS GEOMETRY TEST GROUP 5.8

EXPERIMENTAL MEASURMENTS FOR TESTS 5.8.1 TO 5.8.9-0.53-IN. JOMNCOMER GAP

\begin{tabular}{|c|c|c|c|c|c|c|c|c|c|c|}
\hline $\begin{array}{l}\text { TEST } \\
\text { NO }\end{array}$ & $\begin{array}{c}R M-C H-1 \\
(G P M)\end{array}$ & $\begin{array}{c}R M-C W-2 \\
(G P M)\end{array}$ & $\begin{array}{l}F T-C H-1 \\
(G P M)\end{array}$ & $\begin{array}{c}F T-C H-2 \\
(G P M)\end{array}$ & $\begin{array}{c}\text { RM-HG-1 } \\
\text { (CFM) }\end{array}$ & $\begin{array}{c}\text { RM-HG-2 } \\
\text { (CFM) }\end{array}$ & $\begin{array}{c}\text { RM-HG-3 } \\
\text { (CFM) }\end{array}$ & $\begin{array}{c}\text { RM-HG-4 } \\
\text { (CFM) }\end{array}$ & $\begin{array}{l}F T-H G-1 \\
(C F M)\end{array}$ & $\begin{array}{c}F T-H G-2 \\
\text { (CFM) }\end{array}$ \\
\hline $\begin{array}{l}5.8 .1 \\
5.8 .2 \\
5.8 .3 \\
5.8 .4 \\
5.8 .5\end{array}$ & $\begin{array}{l}0.0 \\
0.0 \\
0.0 \\
0.0 \\
0.0\end{array}$ & $\begin{array}{l}0.0 \\
0.0 \\
0.0 \\
0.0 \\
0.0\end{array}$ & $\begin{array}{r}60.0 \\
0.0 \\
0.0 \\
0.0 \\
0.0\end{array}$ & $\begin{array}{r}0.0 \\
45.0 \\
44.9 \\
34.9 \\
24.9\end{array}$ & $\begin{array}{l}0.0 \\
0.0 \\
0.0 \\
0.0 \\
0.0\end{array}$ & $\begin{array}{l}0.0 \\
0.0 \\
0.0 \\
0.0 \\
0.0\end{array}$ & $\begin{array}{l}10.00 \\
10.00 \\
15.00 \\
20.00 \\
30.00\end{array}$ & $\begin{array}{l}0.0 \\
0.0 \\
0.0 \\
0.0 \\
0.0\end{array}$ & $\begin{array}{l}0.0 \\
0.0 \\
0.0 \\
0.0 \\
0.0\end{array}$ & $\begin{array}{l}0.0 \\
0.0 \\
0.0 \\
0.0 \\
0.0\end{array}$ \\
\hline $\begin{array}{l}5.8 .6 \\
5.8 .7 \\
5.8 .8 \\
5.8 .9\end{array}$ & $\begin{array}{l}0.0 \\
0.0 \\
0.0 \\
0.0\end{array}$ & $\begin{array}{l}0.0 \\
0.0 \\
0.0 \\
0.0\end{array}$ & $\begin{array}{l}0.0 \\
0.0 \\
0.0 \\
0.0\end{array}$ & $\begin{array}{r}20.0 \\
10.1 \\
8.0 \\
5.0\end{array}$ & $\begin{array}{c}50.00 \\
90.00 \\
0.0 \\
0.0\end{array}$ & $\begin{array}{l}0.0 \\
0.0 \\
0.0 \\
0.0\end{array}$ & $\begin{array}{r}0.0 \\
0.0 \\
0.0 \\
.0 .0\end{array}$ & $\begin{array}{l}0.0 \\
0.0 \\
0.0 \\
0.0\end{array}$ & $\begin{array}{c}0.0 \\
0.0 \\
16.28 \\
17.82\end{array}$ & $\begin{array}{l}0.0 \\
0.0 \\
0.0 \\
0.0\end{array}$ \\
\hline $\begin{array}{l}\text { TEST } \\
\text { NO }\end{array}$ & $\begin{array}{l}P-C H-1 \\
(P S \mid G)\end{array}$ & $\begin{array}{l}\text { P-CW-2 } \\
\text { (PSIG) }\end{array}$ & $\begin{array}{l}P-H G-1 \\
\text { (PSIG) }\end{array}$ & $\begin{array}{l}\text { P-HG-2 } \\
(P S I G) .\end{array}$ & $\begin{array}{l}P-H G-3 \\
(P S I G)\end{array}$ & $\begin{array}{l}T F-C H-1 \\
(D E G F)\end{array}$ & $\begin{array}{l}\text { TF-HG-1 } \\
\text { (DEG F) }\end{array}$ & $\begin{array}{l}\text { TF-HG-2 } \\
\text { (DEF } F \text { ) }\end{array}$ & $\begin{array}{l}\text { TF-HG-3 } \\
\text { (DEG F) }\end{array}$ & $\begin{array}{l}\text { P-BARO } \\
\text { (PSIA) }\end{array}$ \\
\hline $\begin{array}{ll}5.8 . & 1 \\
5.8 . & 2 \\
5.8 . & 3 \\
5.8 . & 4 \\
5.8 . & 5\end{array}$ & $\begin{array}{r}9.7 \\
9.5 \\
10.2 \\
10.0 \\
9.5\end{array}$ & $\begin{array}{l}0.0 \\
0.0 \\
0.0 \\
0.0 \\
0.0\end{array}$ & $\begin{array}{r}9.0 \\
8.5 \\
10.0 \\
9.5 \\
8.0\end{array}$ & $\begin{array}{r}97.5 \\
100.0 \\
98.5 \\
97.5 \\
97.0\end{array}$ & $\begin{array}{l}33.5 \\
33.5 \\
33.5 \\
33.0 \\
33.0\end{array}$ & $\begin{array}{l}50 . C \\
52 . C \\
52.5 \\
51 . c \\
51 . C\end{array}$ & $\begin{array}{l}78.5 \\
78.0 \\
77.0 \\
75.0 \\
75.0\end{array}$ & $\begin{array}{l}75.0 \\
73.0 \\
73.0 \\
73.0 \\
74.0\end{array}$ & $\begin{array}{l}76.5 \\
75.0 \\
73.5 \\
73.0 \\
73.0\end{array}$ & $\begin{array}{l}12.2 \\
12.2 \\
12.2 \\
12.2 \\
12.2\end{array}$ \\
\hline $\begin{array}{l}5.8 \cdot 6 \\
5.8 \cdot 7 \\
5.8 \cdot 6 \\
5.8 .9\end{array}$ & $\begin{array}{l}9.2 \\
3.7 \\
9.5 \\
9.7\end{array}$ & $\begin{array}{l}0.0 \\
0.0 \\
0.0 \\
0.0\end{array}$ & $\begin{array}{l}10.2 \\
10.6 \\
10.0 \\
10.0\end{array}$ & $\begin{array}{l}95.5 \\
94.0 \\
97.0 \\
96.0\end{array}$ & $\begin{array}{l}33.0 \\
33.0 \\
33.0 \\
33.5\end{array}$ & $\begin{array}{l}51.0 \\
51.5 \\
59.5 \\
57.0\end{array}$ & $\begin{array}{l}74.0 \\
77.0 \\
80.5 \\
83.5\end{array}$ & $\begin{array}{l}78.0 \\
87.5 \\
97.0 \\
93.0\end{array}$ & $\begin{array}{l}74.0 \\
75.0 \\
83.5 \\
97.0\end{array}$ & $\begin{array}{l}12.2 \\
12.2 \\
12.2 \\
12.2\end{array}$ \\
\hline
\end{tabular}

\begin{tabular}{|c|c|c|c|c|c|c|c|c|c|}
\hline \multirow[b]{2}{*}{$\begin{array}{l}\text { TEST } \\
\text { NO }\end{array}$} & \multicolumn{3}{|c|}{ LDWEP. PLENUM FLOW } & \multicolumn{3}{|c|}{ BYPASS FL JW } & \multirow[b]{2}{*}{$\begin{array}{l}\text { LOWER } \\
\text { PLENUM } \\
\text { LEVEL } \\
\text { (IN) }\end{array}$} & \multirow[b]{2}{*}{$\begin{array}{l}P-(P-1 \\
(P S I G)\end{array}$} & \multirow[b]{2}{*}{$\begin{array}{l}T C-L P \rightarrow 1 \\
\text { (DEG F) }\end{array}$} \\
\hline & $\begin{array}{l}\text { INITIAL } \\
\text { NEISHT } \\
\text { (LS) }\end{array}$ & $\begin{array}{l}\text { FINAL } \\
\text { WE.IGHT } \\
\text { (LB) }\end{array}$ & $\begin{array}{l}\text { TIME DF } \\
\text { COLLECT ICN } \\
\text { (SEC) }\end{array}$ & $\begin{array}{l}\text { INITIAL } \\
\text { HEIGHT } \\
\text { (LB) }\end{array}$ & $\begin{array}{l}\text { FINAL } \\
\text { WEIGHT } \\
(L B)\end{array}$ & $\begin{array}{l}\text { TIME DF } \\
\text { COLLECTION } \\
\text { (SEC) }\end{array}$ & & & \\
\hline $\begin{array}{ll}5.8 . & 1 \\
5.8 . & 2 \\
5.8 . & 3 \\
5.8 . & 4 \\
5.8 . & 5\end{array}$ & $\begin{array}{l}141.5 \\
140.0 \\
163.0 \\
156.5 \\
171.5\end{array}$ & $\begin{array}{l}260.0 \\
267.0 \\
271.0 \\
249.5 \\
238.0\end{array}$ & $\begin{array}{l}29.0 \\
30.0 \\
30.5 \\
30.0 \\
30.0\end{array}$ & $\begin{array}{l}311.0 \\
102.0 \\
219.0 \\
220.5 \\
270.5\end{array}$ & $\begin{array}{l}556.0 \\
236.5 \\
374.5 \\
339.0 \\
354.5\end{array}$ & $\begin{array}{l}60.0 \\
60.0 \\
60.0 \\
60.0 \\
60.0\end{array}$ & $\begin{array}{l}12.0 \\
12.0 \\
12.0 \\
12.0 \\
12.0\end{array}$ & $\begin{array}{r}9.5 \\
9.7 \\
10.0 \\
10.0 \\
9.7\end{array}$ & $\begin{array}{l}51.0 \\
52.0 \\
53.0 \\
52.0 \\
51.5\end{array}$ \\
\hline $\begin{array}{l}5.8 .6 \\
5.8 .7 \\
5.8 .8 \\
5.8 .9\end{array}$ & $\begin{array}{l}143.0 \\
158.5 \\
159.5 \\
136.5\end{array}$ & $\begin{array}{l}190.5 \\
163.0 \\
186.5 \\
203.5\end{array}$ & $\begin{array}{r}60.0 \\
190.0 \\
120.0 \\
120.0\end{array}$ & $\begin{array}{l}127.5 \\
193.0 \\
275.5 \\
280.5\end{array}$ & $\begin{array}{l}250.5 \\
275.5 \\
329.0 \\
312.5\end{array}$ & $\begin{array}{l}60.0 \\
60.0 \\
61) .0 \\
60.0\end{array}$ & $\begin{array}{l}12.0 \\
12.0 \\
12.0 \\
12.0\end{array}$ & $\begin{array}{l}10.2 \\
10.2 \\
10.2 \\
10.0\end{array}$ & $\begin{array}{l}54.0 \\
52.5 \\
60.5 \\
59.0\end{array}$ \\
\hline
\end{tabular}


TABLE XXII

MODIFIED UPPER ANNUULUS GEOMETRY TEST GROUP 5.10

TRANSPARENT VESSEL TEST DATA FOR TESTS 5.10.1 TO 5.10.9-- 0.53-IN. DOWNCOMER GAP SUMMARY OF CALCULATEO RESULTS

\begin{tabular}{|c|c|c|c|c|c|c|c|c|c|c|}
\hline $\begin{array}{l}\text { TEST } \\
\text { NO }\end{array}$ & $\begin{array}{l}\text { DOWNCOAER } \\
\text { AIR FLOW } \\
\text { (LB/SEC) }\end{array}$ & $\begin{array}{l}\text { DOWNCOMER } \\
\text { AIR DENSITY } \\
\text { (LB/FT**3) }\end{array}$ & $\begin{array}{l}\text { LOWER PLENUM } \\
\text { PRESSURE } \\
\text { (PSIA) }\end{array}$ & $\begin{array}{l}\text { CCLD LEG } \\
\text { AIR FLOW } \\
\text { (LB/SEC) }\end{array}$ & $\begin{array}{l}\text { DOWNCOMER } \\
\text { WATER FLOW } \\
\text { (LB/SEC) }\end{array}$ & $\begin{array}{l}\text { WATER FLOW } \\
\text { CONTINUITY }\end{array}$ & $\begin{array}{c}\text { J-AIR } \\
\text { DUWNCUNER. } \\
\text { (FT/SEC) }\end{array}$ & $\begin{array}{l}\text { J-WATER } \\
\text { ODWNCIMER } \\
\text { (FT/SECI }\end{array}$ & $(J G *) * * 1 / 2$ & $(\mathrm{dL} * 1 * * 1 /$ \\
\hline $\begin{array}{l}5.10 \cdot 1 \\
5.10 \cdot 2 \\
5.10 \cdot 3 \\
5.10 \cdot 4 \\
5.10 \cdot 5\end{array}$ & $\begin{array}{l}0.0109 \\
0.0218 \\
0.0219 \\
0.0216 \\
0.0328\end{array}$ & $\begin{array}{l}0.1160 \\
0.1215 \\
0.1195 \\
0.1209 \\
0.1195\end{array}$ & $\begin{array}{l}21.90 \\
22.90 \\
22.40 \\
22.65 \\
22.40\end{array}$ & $\begin{array}{l}0.0 \\
0.0 \\
0.0 \\
0.0 \\
0.0\end{array}$ & $\begin{array}{l}5.879 \\
4.050 \\
4.733 \\
3.879 \\
3.483\end{array}$ & $\begin{array}{l}1.003 \\
0.915 \\
1.003 \\
0.974 \\
0.996\end{array}$ & $\begin{array}{l}1.41 \\
2.70 \\
2.75 \\
2.69 \\
4.13\end{array}$ & $\begin{array}{l}1.410 \\
0.975 \\
1.140 \\
0.934 \\
0.839\end{array}$ & $\begin{array}{l}0.1903 \\
0.2661 \\
0.2673 \\
0.2651 \\
0.3275\end{array}$ & $\begin{array}{l}0.9171 \\
0.7612 \\
0.8229 \\
0.7450 \\
0.7059\end{array}$ \\
\hline $\begin{array}{l}5.10 .6 \\
5.10 .7 \\
5.10 .8 \\
5.10 .9\end{array}$ & $\begin{array}{l}0.0438 \\
0.1092 \\
0.1746 \\
0.1593\end{array}$ & $\begin{array}{l}0.1207 \\
0.1177 \\
0.1190 \\
0.1187\end{array}$ & $\begin{array}{l}22.65 \\
22.15 \\
22.40 \\
22.40\end{array}$ & $\begin{array}{l}0.0 \\
0.0 \\
0.0 \\
0.0\end{array}$ & $\begin{array}{l}2.750 \\
0.858 \\
0.200 \\
0.250\end{array}$ & $\begin{array}{l}1.027 \\
0.990 \\
1.017 \\
1.021\end{array}$ & $\begin{array}{r}5.45 \\
13.94 \\
22.05 \\
20.18\end{array}$ & $\begin{array}{l}0.662 \\
0.207 \\
0.043 \\
0.060\end{array}$ & $\begin{array}{l}0.3774 \\
0.5997 \\
0.7563 \\
0.7230\end{array}$ & $\begin{array}{l}0.6272 \\
0.3504 \\
0.1692 \\
0.1891\end{array}$ \\
\hline
\end{tabular}


TABLE XXII (Contd.)

MODIFIED UPPER ANNULUS GEOMETRY TEST GROUP 5.10

EXPERIMENTAL MEASURMENTS FOR TESTS 5.10 .1 TO $5.10 .9-0.53-1 \mathrm{~N}$. DOHNCOMER GAP

\begin{tabular}{|c|c|c|c|c|c|c|c|c|c|c|}
\hline $\begin{array}{l}\text { TEST' } \\
\text { NO }\end{array}$ & $\begin{array}{c}R M-C H-1 \\
(G P M)\end{array}$ & $\begin{array}{c}R H-C H-2 \\
(G P M)\end{array}$ & $\begin{array}{c}F T-C H-1 \\
(G P M)\end{array}$ & $\begin{array}{c}F T-C H-2 \\
(G P M)\end{array}$ & $\begin{array}{c}\text { RM-HG-1 } \\
\text { (CFM) }\end{array}$ & $\begin{array}{c}\text { RM-HG-2 } \\
\text { (CFM) }\end{array}$ & $\begin{array}{c}\text { RM-HG-3 } \\
\text { (CFM) }\end{array}$ & $\begin{array}{c}\text { RM-HG-4 } \\
(C F M)\end{array}$ & $\begin{array}{l}F T-H G-1 \\
(C F M)\end{array}$ & $\begin{array}{c}F T-H G-2 \\
(C F M)\end{array}$ \\
\hline $\begin{array}{l}5.10 .1 \\
5.10 .2 \\
5.10 .3 \\
5.10 .4 \\
5.10 .5\end{array}$ & $\begin{array}{l}0.0 \\
0.0 \\
0.0 \\
0.0 \\
0.0\end{array}$ & $\begin{array}{l}0.0 \\
0.0 \\
0.0 \\
0.0 \\
0.0\end{array}$ & $\begin{array}{r}60.0 \\
60.0 \\
60.0 \\
0.0 \\
0.0\end{array}$ & $\begin{array}{r}0.0 \\
0.0 \\
0.0 \\
44.9 \\
44.9\end{array}$ & $\begin{array}{l}0.0 \\
0.0 \\
0.0 \\
0.0 \\
0.0\end{array}$ & $\begin{array}{l}0.0 \\
0.0 \\
0.0 \\
0.0 \\
0.0\end{array}$ & $\begin{array}{r}5.00 \\
10.00 \\
10.00 \\
10.00 \\
15.00\end{array}$ & $\begin{array}{l}0.0 \\
0.0 \\
0.0 \\
0.0 \\
0.0\end{array}$ & $\begin{array}{l}0.0 \\
0.0 \\
0.0 \\
0.0 \\
0.0\end{array}$ & $\begin{array}{l}0.0 \\
0.0 \\
0.0 \\
0.0 \\
0.0\end{array}$ \\
\hline $\begin{array}{l}5.10 .6 \\
5.10 .7 \\
5.10 .8 \\
5.10 .9\end{array}$ & $\begin{array}{l}0.0 \\
0.0 \\
0.0 \\
0.0\end{array}$ & $\begin{array}{l}0.0 \\
0.0 \\
0.0 \\
0.0\end{array}$ & $\begin{array}{l}0.0 \\
0.0 \\
0.0 \\
0.0\end{array}$ & $\begin{array}{r}35.0 \\
25.1 \\
15.0 \\
4.9\end{array}$ & $\begin{array}{c}0.0 \\
50.00 \\
80.00 \\
0.0\end{array}$ & $\begin{array}{l}0.0 \\
0.0 \\
0.0 \\
0.0\end{array}$ & $\begin{array}{c}20.00 \\
0.0 \\
0.0 \\
0.0\end{array}$ & $\begin{array}{l}0.0 \\
0.0 \\
0.0 \\
0.0\end{array}$ & $\begin{array}{c}0.0 \\
0.0 \\
0.0 \\
17.56\end{array}$ & $\begin{array}{l}0.0 \\
0.0 \\
0.0 \\
0.0\end{array}$ \\
\hline $\begin{array}{l}\text { TEST } \\
\text { NO }\end{array}$ & $\begin{array}{l}P-C H-1 \\
(P S I G) .\end{array}$ & $\begin{array}{l}P-C H-2 \\
(P S I G)\end{array}$ & $\begin{array}{l}\text { P-HG-1 } \\
\text { (PSIG) }\end{array}$ & $\begin{array}{l}\text { P-HG-2 } \\
(P S I G)\end{array}$ & $\begin{array}{l}P-H \mid G-3 \\
(P S I G)\end{array}$ & $\begin{array}{l}\text { TF-CW-I } \\
\text { IDEG }=1\end{array}$ & $\begin{array}{l}\text { TF-HG-1 } \\
\text { (DEG FI) }\end{array}$ & $\begin{array}{l}\text { TF-HG-2 } \\
\text { (DE G F I }\end{array}$ & $\begin{array}{l}\text { TF-HG-3 } \\
\text { (DEG F) }\end{array}$ & $\begin{array}{l}\text { P-BARO } \\
\text { (PSIA) }\end{array}$ \\
\hline $\begin{array}{l}5.10 \cdot 1 \\
5.10 .2 \\
5.10 .3 \\
5.10 .4 \\
5.10 .5\end{array}$ & $\begin{array}{r}9.5 \\
11.2 \\
10.0 \\
10.5 \\
10.0\end{array}$ & $\begin{array}{l}0.0 \\
0.0 \\
0.0 \\
0.0 \\
0.0\end{array}$ & $\begin{array}{r}9.0 \\
12.0 \\
10.0 \\
11.0 \\
10.0\end{array}$ & $\begin{array}{r}105.0 \\
103.0 \\
102.0 \\
100.0 \\
99.5\end{array}$ & $\begin{array}{l}33.0 \\
33.0 \\
33.0 \\
32.0 \\
33.0\end{array}$ & $\begin{array}{l}49.5 \\
48.0 \\
45.5 \\
45.0 \\
45.5\end{array}$ & $\begin{array}{l}79.0 \\
78.0 \\
78.0 \\
78.5 \\
77.0\end{array}$ & $\begin{array}{l}73.0 \\
72.5 \\
71.5 \\
70.5 \\
69.5\end{array}$ & $\begin{array}{l}75.0 \\
74.0 \\
73.5 \\
73.0 \\
72.5\end{array}$ & $\begin{array}{l}12.4 \\
12.4 \\
12.4 \\
12.4 \\
12.4\end{array}$ \\
\hline $\begin{array}{l}5.10 .6 \\
5.10 .7 \\
5.10 .8 \\
5.10 .9\end{array}$ & $\begin{array}{r}10.0 \\
9.0 \\
9.5 \\
9.5\end{array}$ & $\begin{array}{l}0.0 \\
0.0 \\
0.0 \\
0.0\end{array}$ & $\begin{array}{r}10.0 \\
9.5 \\
10.0 \\
9.5\end{array}$ & $\begin{array}{l}99.5 \\
98.0 \\
94.0 \\
96.5\end{array}$ & $\begin{array}{l}33.0 \\
33.0 \\
33.0 \\
33.5\end{array}$ & $\begin{array}{l}45.0 \\
46.0 \\
46.5 \\
48.0\end{array}$ & $\begin{array}{l}77.0 \\
74.5 \\
73.0 \\
74.0\end{array}$ & $\begin{array}{l}71.0 \\
69.5 \\
73.5 \\
80.0\end{array}$ & $\begin{array}{l}71.5 \\
74.5 \\
75.0 \\
75.0\end{array}$ & $\begin{array}{l}12.4 \\
12.4 \\
12.4 \\
12.4\end{array}$ \\
\hline
\end{tabular}

\begin{tabular}{|c|c|c|c|c|c|c|c|c|c|}
\hline \multirow[b]{2}{*}{$\begin{array}{c}\text { TEST } \\
\text { NI }\end{array}$} & \multicolumn{3}{|c|}{ LOWER PLENUM FLOH } & \multicolumn{3}{|c|}{ BYPASS FLOW } & \multirow[b]{2}{*}{$\begin{array}{l}\text { LOWER } \\
\text { PLEN'JM } \\
\text { LEVEL } \\
\text { IINI }\end{array}$} & \multirow[b]{2}{*}{$\begin{array}{l}P-L P-1 \\
\text { (PSIG) }\end{array}$} & \multirow[b]{2}{*}{$\begin{array}{l}\text { TF-LP-1 } \\
\text { (DEG F) }\end{array}$} \\
\hline & $\begin{array}{l}\text { INITIAL } \\
\text { WEIGHT } \\
\text { (LB) }\end{array}$ & $\begin{array}{l}\text { FINAL } \\
\text { MEIGHT } \\
\text { (LB) }\end{array}$ & $\begin{array}{l}\text { TIME OF } \\
\text { COLLECTION } \\
\text { ISEC I }\end{array}$ & $\begin{array}{l}\text { INITIIAL } \\
\text { WEIGHT } \\
\text { (LB) }\end{array}$ & $\begin{array}{l}\text { FINAL } \\
\text { WEIGHT } \\
(L 8)\end{array}$ & $\begin{array}{l}\text { TIME OF } \\
\text { COLLECIION } \\
\text { ISEC) }\end{array}$ & & & \\
\hline $\begin{array}{ll}5.10 .1 & 1 \\
5.10 . & 2 \\
5.10 . & 3 \\
5.10 . & 4 \\
5.10 . & 5\end{array}$ & $\begin{array}{l}138.0 \\
203.0 \\
254.5 \\
142.5 \\
2.17 .0\end{array}$ & $\begin{array}{l}308.5 \\
329.5 \\
396.5 \\
255.0 \\
321.5\end{array}$ & $\begin{array}{l}29.0 \\
30.0 \\
30.0 \\
29.0 \\
30.0\end{array}$ & $\begin{array}{l}126.5 \\
135.0 \\
236.0 \\
110.0 \\
176.0\end{array}$ & $\begin{array}{l}201.0 \\
242.5 \\
345.0 \\
176.0 \\
258.0\end{array}$ & $\begin{array}{l}30.0 \\
30.0 \\
30.0 \\
30.0 \\
30.0\end{array}$ & $\begin{array}{l}12.0 \\
12.0 \\
12.0 \\
12.0 \\
12.0\end{array}$ & $\begin{array}{l}9.5 \\
10.5 \\
10.0 \\
10.2 \\
10.0\end{array}$ & $\begin{array}{l}49.5 \\
49.5 \\
46.0 \\
45.5 \\
46.0\end{array}$ \\
\hline $\begin{array}{l}5.10 .6 \\
5.10 .7 \\
5.10 .8 \\
5.10 .9\end{array}$ & $\begin{array}{l}204.5 \\
156.0 \\
181.0 \\
207.0\end{array}$ & $\begin{array}{l}287.0 \\
207.5 \\
211.0 \\
237.0\end{array}$ & $\begin{array}{r}30.0 \\
60.0 \\
120.0 \\
120.0\end{array}$ & $\begin{array}{l}258.0 \\
185.0 \\
216.0 \\
280.0\end{array}$ & $\begin{array}{l}325.5 \\
340.5 \\
331.5 \\
307.0 .\end{array}$ & $\begin{array}{l}30.0 \\
60.0 \\
60.0 \\
60.0\end{array}$ & $\begin{array}{l}12.0 \\
12.0 \\
12.0 \\
12.0\end{array}$ & $\begin{array}{r}10.2 \\
9.7 \\
10.0 \\
10.0\end{array}$ & $\begin{array}{l}46.5 \\
48.0 \\
48.0 \\
49.5\end{array}$ \\
\hline
\end{tabular}


TABLE XXIII

MODIFIED UPPER ANNULUS GEOMETRY TEST GROUP 5.13

TRANSPARENT VESSEL TEST DATA FOR TESTS 5.13.1 TO 5.13.14 - 0.53-IN. DOHNCOMER GAP SUMMARY OF CALCULATED RESULTS

\begin{tabular}{|c|c|c|c|c|c|c|c|c|c|c|c|}
\hline & $\begin{array}{l}\text { TEST } \\
\text { NO }\end{array}$ & $\begin{array}{l}\text { DOHNCOMER } \\
\text { AIR FLOW } \\
\text { (LB/SEC) }\end{array}$ & $\begin{array}{l}\text { DOWNCOMER } \\
\text { AIR DENSITY } \\
\text { ILB/FT } * 3 \mid\end{array}$ & $\begin{array}{l}\text { LOWER PLENUM } \\
\text { PRESSURE } \\
\text { (PSIAI) }\end{array}$ & $\begin{array}{l}\text { COLO LEG } \\
\text { AIR FLOH } \\
\text { (LB/SEC) }\end{array}$ & $\begin{array}{l}\text { DOWNCOMER } \\
\text { HATER FLOW } \\
\text { (LB/SEC) }\end{array}$ & $\begin{array}{l}\text { HATER FLOW } \\
\text { CONT INUITY }\end{array}$ & $\begin{array}{c}\text { J-AIR } \\
\text { DOWNCOMER } \\
\text { (FT/SEC) }\end{array}$ & $\begin{array}{l}\text { J-YATER } \\
\text { OONNCOOMER } \\
\text { (FT/SEC) }\end{array}$ & $(J G *) * * 1 / 2$ & $(J L * 1) * 1 / 2$ \\
\hline - & $\begin{array}{l}5.13 \cdot 1 \\
5.13 \cdot 2 \\
5.13 \cdot 3 \\
5.13 \cdot 4 \\
5.13 \cdot 5\end{array}$ & $\begin{array}{l}0.0074 \\
0.0145 \\
0.0290 \\
0.0285 \\
0.0432\end{array}$ & $\begin{array}{l}0.1185 \\
0.1212 \\
0.1225 \\
0.1165 \\
0.1186\end{array}$ & $\begin{array}{l}22.17 \\
22.67 \\
22.92 \\
21.92 \\
22.42\end{array}$ & $\begin{array}{l}0.0 \\
0.0 \\
0.0 \\
0.0 \\
0.0\end{array}$ & $\begin{array}{l}5.567 \\
5.000 \\
3.650 \\
4.250 \\
3.267\end{array}$ & $\begin{array}{l}0.959 \\
1.017 \\
0.882 \\
1.034 \\
0.958\end{array}$ & $\begin{array}{l}0.94 \\
1.80 \\
3.55 \\
3.68 \\
5.47\end{array}$ & $\begin{array}{l}1.341 \\
1.204 \\
0.879 \\
1.023 \\
0.787\end{array}$ & $\begin{array}{l}0.1562 \\
0.2168 \\
0.3058 \\
0.3075 \\
0.3765\end{array}$ & $\begin{array}{l}0.8924 \\
0.8458 \\
0.7226 \\
0.7798 \\
0.6836\end{array}$ \\
\hline & $\begin{array}{l}5.13 .6 \\
5.13 .7 \\
5.13 .8 \\
5.13 .9 \\
5.13 .10\end{array}$ & $\begin{array}{l}0.0721 \\
0.0721 \\
0.1750 \\
0.0147 \\
0.0147\end{array}$ & $\begin{array}{l}0.1158 \\
0.1175 \\
0.119 .5 \\
0.1194 \\
0.1182\end{array}$ & $\begin{array}{l}21.79 \\
22.29 \\
22.79 \\
22.54 \\
22.29\end{array}$ & $\begin{array}{l}0.00 \\
0.0 \\
0.0 \\
0.0 \\
0.0\end{array}$ & $\begin{array}{l}2.000 \\
0.733 \\
0.092 \\
4.733 \\
4.633\end{array}$ & $\begin{array}{l}0.994 \\
1.058 \\
0.975 \\
1.017 \\
0.995\end{array}$ & $\begin{array}{r}9.35 \\
9.22 \\
22.0 .2 \\
1.85 \\
1.86\end{array}$ & $\begin{array}{l}0.482 \\
0.177 \\
0.022 \\
1.140 \\
1.116\end{array}$ & $\begin{array}{l}0.4893 \\
0.4875 \\
0.7565 \\
0.2190 \\
0.2196\end{array}$ & $\begin{array}{l}0.5349 \\
0.3239 \\
0.1145 \\
0.8229 \\
0.8142\end{array}$ \\
\hline & $\begin{array}{l}5.13 .11 \\
5.13 .12 \\
5.13 .13 \\
5.13 .14\end{array}$ & $\begin{array}{l}0.0219 \\
0.0733 \\
0.1170 \\
0.0291\end{array}$ & $\begin{array}{l}0.1186 \\
0.1201 \\
0.1196 \\
0.1175\end{array}$ & $\begin{array}{l}22.29 \\
22.54 \\
22.54 \\
22.04\end{array}$ & $\begin{array}{l}0.0 \\
0.0 \\
0.0 \\
0.0\end{array}$ & $\begin{array}{l}3.867 \\
1.917 \\
0.617 \\
3.633\end{array}$ & $\begin{array}{l}1.002 \\
1.022 \\
0.987 \\
0.987\end{array}$ & $\begin{array}{r}2.78 \\
9.17 \\
14.71 \\
3.73\end{array}$ & $\begin{array}{l}0.931 \\
0.462 \\
0.149 \\
0.875\end{array}$ & $\begin{array}{l}0.2681 \\
0.4888 \\
0.6184 \\
0.3099\end{array}$ & $\begin{array}{l}0.7438 \\
0.5237 \\
0.2970 \\
0.7210\end{array}$ \\
\hline
\end{tabular}


TABLE XXIII (Contd.)

MODIFIED UPPER ANNULUS GEOMETRY TEST GROUP 5.13

EXPERIMENTAL MEASURMENTS FOR TESTS 5.13.1 TO 5.13.14 -- 0.53 -IN. DOHNCOMER GAP

\begin{tabular}{|c|c|c|c|c|c|c|c|c|c|c|}
\hline $\begin{array}{l}\text { TEST } \\
\text { NO }\end{array}$ & $\begin{array}{c}R M-C H-1 \\
(G P M)\end{array}$ & $\begin{array}{c}R M-C H-2 \\
(G P M)\end{array}$ & $\begin{array}{c}F T-C H-1 \\
(G P M)\end{array}$ & $\begin{array}{c}F(-C W-2 \\
(G P M)\end{array}$ & $\begin{array}{c}R M-H G-1 \\
(C F M)\end{array}$ & $\begin{array}{c}R M-H G-2 \\
(C F M)\end{array}$ & $\begin{array}{c}\text { RM-HG-3 } \\
(C F M)\end{array}$ & $\begin{array}{c}\text { RM-HG-4 } \\
(C F M)\end{array}$ & $\begin{array}{c}F T-H G-1 \\
\text { (CFM) }\end{array}$ & $\begin{array}{c}F T-H G-2 \\
(C F M)\end{array}$ \\
\hline $\begin{array}{l}5.13 \cdot 1 \\
5.13 \cdot 2 \\
5.13 \cdot 3 \\
5.13 .4 \\
5.13 .5\end{array}$ & $\begin{array}{l}0.0 \\
0.0 \\
0.0 \\
0.0 \\
0.0\end{array}$ & $\begin{array}{l}0.0 \\
0.0 \\
0.0 \\
0.0 \\
0.0\end{array}$ & $\begin{array}{r}60.0 \\
60.0 \\
0.0 \\
0.0 \\
0.0\end{array}$ & $\begin{array}{r}0.0 \\
0.0 \\
45.0 \\
44.5 \\
34.5\end{array}$ & $\begin{array}{l}0.0 \\
0.0 \\
0.0 \\
0.0 \\
0.0\end{array}$ & $\begin{array}{l}0.0 \\
0.0 \\
0.0 \\
0.0 \\
0.0\end{array}$ & $\begin{array}{r}3.40 \\
6.60 \\
13.40 \\
13.20 \\
20.00\end{array}$ & $\begin{array}{l}0.0 \\
0.0 \\
0.0 \\
0.0 \\
0.0\end{array}$ & $\begin{array}{l}0.0 \\
0.0 \\
0.0 \\
0.0 \\
0.0\end{array}$ & $\begin{array}{l}0.0 \\
0.0 \\
0.0 \\
0.0 \\
0.0\end{array}$ \\
\hline $\begin{array}{l}5.13 .6 \\
5.13 .7 \\
5.13 .8 \\
5.13 .9 \\
5.13 .10\end{array}$ & $\begin{array}{l}0.0 \\
0.0 \\
0.0 \\
0.0 \\
0.0\end{array}$ & $\begin{array}{l}0.0 \\
0.0 \\
0.0 \\
0.0 \\
0.0\end{array}$ & $\begin{array}{r}0.0 \\
0.0 \\
0.0 \\
80.0 \\
90.0\end{array}$ & $\begin{array}{r}25.2 \\
15.1 \\
5.0 \\
0.0 \\
0.0\end{array}$ & $\begin{array}{c}33.50 \\
33.50 \\
80.00 \\
0.0 \\
0.0\end{array}$ & $\begin{array}{l}0.0 \\
0.0 \\
0.0 \\
0.0 \\
0.0\end{array}$ & $\begin{array}{l}0.0 \\
0.0 \\
0.0 \\
6.70 \\
6.70\end{array}$ & $\begin{array}{l}0.0 \\
0.0 \\
0.0 \\
0.0 \\
0.0\end{array}$ & $\begin{array}{l}0.0 \\
0.0 \\
0.0 \\
0.0 \\
0.0\end{array}$ & $\begin{array}{l}0.0 \\
0.0 \\
0.0 \\
0.0 \\
0.0\end{array}$ \\
\hline $\begin{array}{l}5.13 .11 \\
5.13 .12 \\
5.13 .13 \\
5.13 .14\end{array}$ & $\begin{array}{l}0.0 \\
0.0 \\
0.0 \\
0.0\end{array}$ & $\begin{array}{l}0.0 \\
0.0 \\
0.0 \\
0.0\end{array}$ & $\begin{array}{r}70.0 \\
0.0 \\
0.0 \\
60.0\end{array}$ & $\begin{array}{r}0.0 \\
50.1 \\
25.0 \\
0.0\end{array}$ & $\begin{array}{c}0.0 \\
33.50 \\
53.50 \\
0.0\end{array}$ & $\begin{array}{l}0.4 \\
0.0 \\
0.0 \\
0.0\end{array}$ & $\begin{array}{l}10.00 \\
0.0 \\
0.0 \\
13.30\end{array}$ & $\begin{array}{l}0.0 \\
0.0 \\
0.0 \\
0.0\end{array}$ & $\begin{array}{l}0.0 \\
0.0 \\
0.0 \\
0.0\end{array}$ & $\begin{array}{l}0.0 \\
0.0 \\
0.0 \\
0.0\end{array}$ \\
\hline $\begin{array}{l}\text { TEST } \\
\text { NO }\end{array}$ & $\begin{array}{l}P-C H-1 \\
\text { (PSIG) }\end{array}$ & $\begin{array}{l}P-C H-2 \\
\text { (PSIG) }\end{array}$ & $\begin{array}{l}P-H G-1 \\
\text { (PSIG) }\end{array}$ & $\begin{array}{l}P-H G-2 \\
\text { (PSIG) }\end{array}$ & $\begin{array}{l}P-H G-3 \\
(P S I G)\end{array}$ & $\begin{array}{l}\text { TF-Ch-1 } \\
(D E G F)\end{array}$ & $\begin{array}{l}\text { TF-HG-1 } \\
\text { (DEG F) }\end{array}$ & $\begin{array}{l}\text { TF-HG-2 } \\
\text { (DEG F) }\end{array}$ & $\begin{array}{l}\text { TF-HG-3 } \\
\text { (DEG F) }\end{array}$ & $\begin{array}{l}\text { P-BARO } \\
\text { (PSIA) }\end{array}$ \\
\hline $\begin{array}{l}5.13 \cdot 1 \\
5.13 \cdot 2 \\
5.13 \cdot 3 \\
5.13 \cdot 4 \\
5.13 .5\end{array}$ & $\begin{array}{r}9.5 \\
10.0 \\
9.7 \\
9.5 \\
10.5\end{array}$ & $\begin{array}{l}0.0 \\
0.0 \\
0.0 \\
0.0 \\
0.0\end{array}$ & $\begin{array}{l}10.5 \\
10.0 \\
11.0 \\
11.0 \\
10.5\end{array}$ & $\begin{array}{l}0.0 \\
0.0 \\
0.0 \\
0.0 \\
0.0\end{array}$ & $\begin{array}{l}33.5 \\
33.5 \\
32.0 \\
32.0 \\
32.0\end{array}$ & $\begin{array}{l}44.0 \\
45.0 \\
44.0 \\
48.0 \\
50.0\end{array}$ & $\begin{array}{l}78.0 \\
79.0 \\
78.0 \\
78.0 \\
77.0\end{array}$ & $\begin{array}{l}0.0 \\
0.0 \\
0.0 \\
0.0 \\
0.0\end{array}$ & $\begin{array}{l}80.0 \\
76.0 \\
74.0 \\
73.0 \\
74.0\end{array}$ & $\begin{array}{l}12.4 \\
12.4 \\
12.4 \\
12.4 \\
12.4\end{array}$ \\
\hline $\begin{array}{l}5.13 .6 \\
5.13 .7 \\
5.13 .8 \\
5.13 .9 \\
5.13 .10\end{array}$ & $\begin{array}{r}8.7 \\
9.2 \\
10.0 \\
9.7 \\
9.5\end{array}$ & $\begin{array}{l}0.0 \\
0.0 \\
0.0 \\
0.0 \\
0.0\end{array}$ & $\begin{array}{r}9.2 \\
9.6 \\
10.6 \\
10.3 \\
9.9\end{array}$ & $\begin{array}{l}0.0 \\
0.0 \\
0.0 \\
0.0 \\
0.0\end{array}$ & $\begin{array}{l}32.0 \\
32.0 \\
33.5 \\
33.5 \\
33.5\end{array}$ & $\begin{array}{l}47.5 \\
53.0 \\
55.0 \\
49.5 \\
48.5\end{array}$ & $\begin{array}{l}70.0 \\
76.0 \\
76.0 \\
78.0 \\
78.0\end{array}$ & $\begin{array}{l}0.0 \\
0.0 \\
0.0 \\
0.0 \\
0.0\end{array}$ & $\begin{array}{l}77.0 \\
77.0 \\
77.0 \\
76.0 \\
76.0\end{array}$ & $\begin{array}{l}12.3 \\
12.3 \\
12.3 \\
12.3 \\
12.3\end{array}$ \\
\hline $\begin{array}{l}5.13 .11 \\
5.13 .12 \\
5.13 .13 \\
5.13 .14\end{array}$ & $\begin{array}{l}9.7 \\
9.5 \\
9.5 \\
9.5\end{array}$ & $\begin{array}{l}0.0 \\
0.0 \\
0.0 \\
0.0\end{array}$ & $\begin{array}{r}9.7 \\
10.0 \\
10.0 \\
9.6\end{array}$ & $\begin{array}{l}0.0 \\
0.0 \\
0.0 \\
0.0\end{array}$ & $\begin{array}{l}33.5 \\
33.5 \\
33.5 \\
33.5\end{array}$ & $\begin{array}{l}47.0 \\
46.0 \\
48.0 \\
46.0\end{array}$ & $\begin{array}{l}78.0 \\
77.0 \\
77.0 \\
78.0\end{array}$ & $\begin{array}{l}0.0 \\
0.0 \\
0.0 \\
0.0\end{array}$ & $\begin{array}{l}76.0 \\
77.0 \\
7 ? .5 \\
76.0\end{array}$ & $\begin{array}{l}12.3 \\
12.3 \\
12.3 \\
12.3\end{array}$ \\
\hline
\end{tabular}


TABLE XXIII (Contd.)

MODIFIED UPPER ANNULUS GEOMETRY TEST GROUP 5.13

EXPERIMENTAL MEASURMENTS FOR TESTS 5.13 .1 T.0 5.13.14-- 0.53-IN. DOWNCOMER GAP

\begin{tabular}{|c|c|c|c|c|c|c|}
\hline \multirow[b]{2}{*}{$\begin{array}{c}\text { TEST } \\
\text { NO }\end{array}$} & \multicolumn{2}{|c|}{ LOHER PLENUM } & FLOW & \multicolumn{3}{|c|}{ BYPASS FLOW. } \\
\hline & $\begin{array}{l}\text { INITIAL } \\
\text { WEIGHT } \\
\text { (LB) }\end{array}$ & $\begin{array}{l}\text { FINAL } \\
\text { HEIGHT } \\
\text { (LB) }\end{array}$ & $\begin{array}{l}\text { TIME OF } \\
\text { COLLECT ION } \\
\text { (SEC) }\end{array}$ & $\begin{array}{l}\text { INITIAL } \\
\text { WE IGHT } \\
\text { (LO) }\end{array}$ & $\begin{array}{l}\text { FINAL } \\
\text { WEIGHT } \\
\text { (L'B) }\end{array}$ & $\begin{array}{l}\text { TIME OF } \\
\text { COLLECTION } \\
\text { (SEC) }\end{array}$ \\
\hline $\begin{array}{l}5.13 \cdot 1 \\
5.13 .2 \\
5.13 .2 \\
5.13 .4 \\
5.13 .5\end{array}$ & $\begin{array}{l}265.5 \\
141.5 \\
355.5 \\
287.5 \\
311.5\end{array}$ & $\begin{array}{l}432.5 \\
291.5 \\
465.0 \\
415.0 \\
409.5\end{array}$ & $\begin{array}{l}30.0 \\
30.0 \\
30.0 \\
30.0 \\
30.0\end{array}$ & $\begin{array}{l}120.0 \\
106.0 \\
1.73 .0 \\
142.5 \\
141.0\end{array}$ & $\begin{array}{l}193.0 \\
210.5 \\
229.0 \\
207.0 \\
181.0\end{array}$ & $\begin{array}{l}30.0 \\
30.0 \\
30.0 \\
30.0 \\
30.0\end{array}$ \\
\hline $\begin{array}{l}5.13 .6 \\
5.13 .7 \\
5.13 .8 \\
5.13 .9 \\
5.13 .10\end{array}$ & $\begin{array}{l}142.0 \\
202.0 \\
224.0 \\
143.0 \\
166.5\end{array}$ & $\begin{array}{l}202.0 \\
224.0 \\
235.0 \\
285.0 \\
305.5\end{array}$ & $\begin{array}{r}30.0 \\
30.0 \\
1.20 .0 \\
30.0 \\
30.0\end{array}$ & $\begin{array}{l}10.7 .5 \\
196.5 \\
241.0 \\
120.5 \\
247.0\end{array}$ & $\begin{array}{l}196.5 \\
241.0 \\
276.0 \\
318.0 \\
481.5\end{array}$ & $\begin{array}{l}60.0 \\
30.0 \\
60.0 \\
30.0 \\
30.0\end{array}$ \\
\hline $\begin{array}{l}5.13 .11 \\
5.13 .12 \\
5.13 .13 \\
5.13 .14\end{array}$ & $\begin{array}{l}144.5 \\
148.0 \\
149.5 \\
143.5\end{array}$ & $\begin{array}{l}260.5 \\
205.5 \\
168.0 \\
252.5\end{array}$ & $\begin{array}{l}30.0 \\
30.0 \\
30.0 \\
30.0\end{array}$ & $\begin{array}{l}249.5 \\
296.5 \\
452.5 \\
219.5\end{array}$ & $\begin{array}{l}426.0 \\
452.5 \\
537.0 \\
357.5\end{array}$ & $\begin{array}{l}30.0 \\
30.0 \\
30.0 \\
30.0\end{array}$ \\
\hline
\end{tabular}

LOWER

PLENUM P-LP-1 TF-LP-1

LEVEL

(PSIG) (DEG F)

5.13.
5.13.
5.13.

5.13.

5.13. 5

5.13 .6

5.13. 8

5.13. 9

5.13 .10

5.13 .11

5.13 .13

143.5

252.5

30.0

219.5

8.0

8.0
12.0

$\begin{array}{rr}9.7 & 45.0 \\ 10.2 & 45.0\end{array}$

$10.5 \quad 45.0$

$\begin{array}{rrr}12.0 & 9.5 & 48.0 \\ 12.0 & 10.0 & 50.0\end{array}$

5.13 .14

-

12.0

$-12.0$

$\begin{array}{lll}12.0 & 10.5 & 55.0 \\ 12.0 & 10.2 & 49.5\end{array}$

$12.0 \quad 10.0 \quad 49.0$

$12.0 \quad 10.0 \quad 47.5$

$12.0 \quad 10.2 \quad 46.5$

$\begin{array}{rrr}12.0 & 10.2 & 49.0 \\ 12.0 & 9.7 & 46.5\end{array}$ 


\section{TABLE XXIV}

MODIFIED UPPER ANNULUS GEOMETRY TEST GROUP 6.2

TRAMSPARENT VESSEL TEST DATA FOP. TESTS 6.2.1 TH 6.2.17 -- 0.63-INi. DOWNCOMER GAP SUMMARY DF CALCULATED RESULTS

\begin{tabular}{|c|c|c|c|c|c|c|c|c|c|c|}
\hline $\begin{array}{l}\text { TEST } \\
\text { NO }\end{array}$ & $\begin{array}{l}\text { ONWNCOAOAER } \\
\text { AIR FLDW } \\
\text { (LB/SEC) }\end{array}$ & $\begin{array}{l}\text { DOWNCD'AER } \\
\text { AIR DEVSITY } \\
\text { (LS/CT } \$ 3 \text { ) }\end{array}$ & $\begin{array}{l}\text { LOWER PLENUM } \\
\text { PRESSURE } \\
\text { (PSIA) }\end{array}$ & $\begin{array}{l}\text { CQLD LEG } \\
\text { AIR FLOW } \\
\text { (LB/SEC) }\end{array}$ & $\begin{array}{l}\text { DOWNCCMER } \\
\text { WATER FLOW } \\
\text { (LB/SEC) }\end{array}$ & $\begin{array}{l}\text { WATER FLOW } \\
\text { CINTINJITY }\end{array}$ & $\begin{array}{c}\text { J-AIR } \\
\text { DOWNCOMEP } \\
\text { (FT/SEC) }\end{array}$ & $\begin{array}{l}\text { J-WATER } \\
\text { DOHACOMEP. } \\
\text { (FT/SEC) }\end{array}$ & $(J G *) * 1 / 2$ & $(J L *) * 1 / 2$ \\
\hline $\begin{array}{l}6.2 \cdot 1 \\
6.2 \cdot 2 \\
6.2 \cdot 3 \\
6.2 \cdot 4 \\
6.2 \cdot 5\end{array}$ & $\begin{array}{l}0.0152 \\
0.0300 \\
0.0452 \\
0.0461 \\
0.0380\end{array}$ & $\begin{array}{l}0.1173 \\
0.1173 \\
0.1173 \\
0.1173 \\
0.1185\end{array}$ & $\begin{array}{l}22.39 \\
22.39 \\
22.39 \\
22.39 \\
22.39\end{array}$ & $\begin{array}{l}0.0 \\
0.0 \\
0.0 \\
0.0 \\
0.0\end{array}$ & $\begin{array}{l}5.550 \\
5.525 \\
5.650 \\
4.037 \\
5.573\end{array}$ & $\begin{array}{l}0.998 \\
0.993 \\
1.017 \\
0.994 \\
1.005\end{array}$ & $\begin{array}{l}1.61 \\
3.19 \\
4.80 \\
4.91 \\
4.00\end{array}$ & $\begin{array}{l}1.110 \\
1.105 \\
1.130 \\
0.807 \\
1.114\end{array}$ & $\begin{array}{l}0.1953 \\
0.2746 \\
0.3371 \\
0.3408 \\
0.3094\end{array}$ & $\begin{array}{l}0.7783 \\
0.7766 \\
0.7853 \\
0.7639 \\
0.7800\end{array}$ \\
\hline $\begin{array}{l}6.2 .6 \\
6.2 .7 \\
0.2 .9 \\
6.2 .9 \\
6.2 .10\end{array}$ & $\begin{array}{l}0.0527 \\
0.0538 \\
0.0630 \\
0.0954 \\
0.0630\end{array}$ & $\begin{array}{l}0.1176 \\
0.1173 \\
0.1176 \\
0.1173 \\
0.1173\end{array}$ & $\begin{array}{l}22.39 \\
22.39 \\
22.39 \\
22.39 \\
22.39\end{array}$ & $\begin{array}{l}0.0 \\
0.0 \\
0.0 \\
0.0 \\
0.0\end{array}$ & $\begin{array}{l}5.425 \\
3.569 \\
3.262 \\
2.300 \\
3.200\end{array}$ & $\begin{array}{l}0.980 \\
0.997 \\
0.994 \\
1.005 \\
0.994\end{array}$ & $\begin{array}{r}5.59 \\
5.72 \\
6.68 \\
10.15 \\
6.70\end{array}$ & $\begin{array}{l}1.085 \\
0.714 \\
0.652 \\
0.460 \\
0.640\end{array}$ & $\begin{array}{l}0.3640 \\
0.3691 \\
0.3979 \\
0.4902 \\
0.3983\end{array}$ & $\begin{array}{l}0.7695 \\
0.6241 \\
0.5968 \\
0.5011 \\
0.5910\end{array}$ \\
\hline $\begin{array}{l}6.2 .11 \\
6.2 .12 \\
0.2 .13 \\
6.2 .14 \\
0.2 .15\end{array}$ & $\begin{array}{l}0.0753 \\
0.1096 \\
0.1444 \\
0.1122 \\
0.1836\end{array}$ & $\begin{array}{l}0.1173 \\
0.1173 \\
0.1171 \\
0.1167 \\
0.1164\end{array}$ & $\begin{array}{l}22.39 \\
22.39 \\
22.39 \\
22.39 \\
22.39\end{array}$ & $\begin{array}{l}0.0 \\
0.0 \\
0.0 \\
0.0 \\
0.0\end{array}$ & $\begin{array}{l}2.800 \\
1.783 \\
1.058 \\
0.537 \\
0.431\end{array}$ & $\begin{array}{l}1.012 \\
1.014 \\
0.984 \\
0.973 \\
1.028\end{array}$ & $\begin{array}{r}8.06 \\
11.66 \\
15.38 \\
18.42 \\
19.69\end{array}$ & $\begin{array}{l}0.560 \\
0.357 \\
0.212 \\
0.107 \\
0.096\end{array}$ & $\begin{array}{l}0.4369 \\
0.5253 \\
0.6032 \\
0.6593 \\
0.6812\end{array}$ & $\begin{array}{l}0.5528 \\
0.4412 \\
0.3399 \\
0.2422 \\
0.2170\end{array}$ \\
\hline $\begin{array}{l}6.2 .16 \\
6.2 .17\end{array}$ & $\begin{array}{l}0.2021 \\
0.234 ?\end{array}$ & $\begin{array}{l}0.1160 \\
0.1155\end{array}$ & $\begin{array}{l}22.39 \\
22.39\end{array}$ & $\begin{array}{l}0.0 \\
0.0\end{array}$ & $\begin{array}{l}0.325 \\
0.169\end{array}$ & $\begin{array}{l}0.983 \\
1.076\end{array}$ & $\begin{array}{l}21.74 \\
25.36\end{array}$ & $\begin{array}{l}0.065 \\
0.034\end{array}$ & $\begin{array}{l}0.7153 \\
0.7719\end{array}$ & $\begin{array}{l}0.1883 \\
0.1357\end{array}$ \\
\hline
\end{tabular}


$\underline{\text { TABLE XXIV (Contd.) }}$

MODIFIED UPPER ANNULUS GEOMETRY TEST GROUP 6.2

EXPERIMENTAL MEASURMENTS FOR TESTS 6.2 .1 TO $6.2 .17+0.63-$ IN. DOHNCDMER GAP

\begin{tabular}{|c|c|c|c|c|c|c|c|c|c|c|}
\hline $\begin{array}{l}\text { TEST } \\
\text { NO }\end{array}$ & $\begin{array}{c}R M-C W-1 \\
(G P M)\end{array}$ & $\begin{array}{c}R^{4}-\mathrm{CH}-2 \\
(G P M)\end{array}$ & $\begin{array}{c}F T-C H-1 \\
(G P M)\end{array}$ & $\begin{array}{l}F T-(W-2 \\
(G P M)\end{array}$ & $\begin{array}{c}R M-H r_{;}-1 \\
(C F M)\end{array}$ & $\begin{array}{c}R M-H G-2 \\
(C F M)\end{array}$ & $\begin{array}{c}R M-H G-3 \\
(C F Y)\end{array}$ & $\begin{array}{c}R M-H G-4 \\
(C F M)\end{array}$ & $\begin{array}{c}F r-H G-1 \\
(C F M)\end{array}$ & $\begin{array}{l}F T-H G-2 \\
(C F M)\end{array}$ \\
\hline $\begin{array}{ll}6.2 \cdot 1 & 1 \\
6.2 \cdot & 2 \\
6.2 \cdot & 3 \\
6.2 .4 & 4 \\
6.2 .5 & \end{array}$ & $\begin{array}{l}30.0 \\
30.0 \\
30.0 \\
30.0 \\
30.0\end{array}$ & $\begin{array}{l}10.0 \\
10.0 \\
10.0 \\
10.0 \\
10.0\end{array}$ & $\begin{array}{l}0.0 \\
0.0 \\
0.0 \\
0.0 \\
0.0\end{array}$ & $\begin{array}{l}0.0 \\
0.0 \\
0.0 \\
0.0 \\
0.0\end{array}$ & $\begin{array}{l}0.0 \\
0.0 \\
0.0 \\
0.0 \\
0.0\end{array}$ & $\begin{array}{l}0.0 \\
0.0 \\
30.00 \\
30.00 \\
25.00\end{array}$ & $\begin{array}{c}10.00 \\
20.00 \\
0.0 \\
0.0 \\
0.0\end{array}$ & $\begin{array}{l}0.0 \\
0.0 \\
0.0 \\
0.0 \\
0.0\end{array}$ & $\begin{array}{l}0.0 \\
0.0 \\
0.0 \\
0.0 \\
0.0\end{array}$ & $\begin{array}{l}0.0 \\
0.0 \\
0.0 \\
0.0 \\
0.0\end{array}$ \\
\hline $\begin{array}{l}6.2 \cdot 6 \\
6.2 \cdot 7 \\
6.2 \cdot 9 \\
6.2 .9 \\
6.2 \cdot 10\end{array}$ & $\begin{array}{l}30.0 \\
30.0 \\
30.0 \\
32.0 \\
3.0\end{array}$ & $\begin{array}{r}10.0 \\
10.0 \\
10.0 \\
0.0 \\
0.0\end{array}$ & $\begin{array}{l}0.0 \\
0.0 \\
0.0 \\
0.0 \\
0.0\end{array}$ & $\begin{array}{l}0.0 \\
0.0 \\
0.0 \\
0.0 \\
0.0\end{array}$ & $\begin{array}{l}0.0 \\
0.0 \\
0.0 \\
0.0 \\
0.0\end{array}$ & $\begin{array}{l}35.00 \\
35.00 \\
40.00 \\
60.00 \\
40.00\end{array}$ & $\begin{array}{l}0.0 \\
0.0 \\
0.0 \\
0.0 \\
0.0\end{array}$ & $\begin{array}{l}0.0 \\
0.0 \\
0.0 \\
0.0 \\
0.0\end{array}$ & $\begin{array}{l}0.0 \\
0.0 \\
0.0 \\
0.0 \\
0.0\end{array}$ & $\begin{array}{l}0.0 \\
0.0 \\
0.0 \\
0.0 \\
0.0\end{array}$ \\
\hline $\begin{array}{l}6.2 .11 \\
6.2 .12 \\
6.2 .13 \\
6.2 .14 \\
6.2 .15\end{array}$ & $\begin{array}{r}25.0 \\
20.0 \\
12.0 \\
3.0 \\
3.0\end{array}$ & $\begin{array}{l}0.0 \\
0.0 \\
0.0 \\
0.0 \\
0.0\end{array}$ & $\begin{array}{l}0.0 \\
0.0 \\
0.0 \\
0.0 \\
0.0\end{array}$ & $\begin{array}{l}0.0 \\
0.0 \\
0.0 \\
0.0 \\
0.0\end{array}$ & $\begin{array}{l}0.0 \\
70.00 \\
90.00 \\
60.00 \\
60.00\end{array}$ & $\begin{array}{c}50.00 \\
0.0 \\
0.0 \\
50.00 \\
60.00\end{array}$ & $\begin{array}{l}0.0 \\
0.0 \\
0.0 \\
0.0 \\
0.0\end{array}$ & $\begin{array}{l}0.0 \\
0.0 \\
0.0 \\
0.0 \\
0.0\end{array}$ & $\begin{array}{l}0.0 \\
0.0 \\
0.0 \\
0.0 \\
0.0\end{array}$ & $\begin{array}{l}0.0 \\
0.0 \\
0.0 \\
0.0 \\
0.0\end{array}$ \\
\hline $\begin{array}{l}6.2 .16 \\
6.2 .17\end{array}$ & $\begin{array}{l}5.0 \\
3.0\end{array}$ & $\begin{array}{l}0.0 \\
0.0\end{array}$ & $\begin{array}{l}0.0 \\
0.0\end{array}$ & $\begin{array}{l}0.0 \\
0.0\end{array}$ & $\begin{array}{l}70.00 \\
80.00\end{array}$ & $\begin{array}{l}60.00 \\
70.00\end{array}$ & $\begin{array}{l}0.0 \\
0.0\end{array}$ & $\begin{array}{l}0.0 \\
0.0\end{array}$ & $\begin{array}{l}0.0 \\
0.0\end{array}$ & $\begin{array}{l}0.0 \\
0.0\end{array}$ \\
\hline $\begin{array}{l}\text { TEST } \\
\text { ND }\end{array}$ & $\begin{array}{l}P-C, N-1 \\
(P S I G)\end{array}$ & $\begin{array}{l}P-C W-2 \\
(P S I G)\end{array}$ & $\begin{array}{l}\text { P-HG-1 } \\
\text { (PSIG) }\end{array}$ & $\begin{array}{l}\text { P-HG-2 } \\
\text { (PSIG) }\end{array}$ & $\begin{array}{l}P-H G-3 \\
\text { (PSIG) }\end{array}$ & $\begin{array}{l}\text { TF-CH-1 } \\
\text { IDEG F) }\end{array}$ & $\begin{array}{l}\text { TF-HE- } \\
\text { (DEG F) }\end{array}$ & $\begin{array}{l}T F-H G-2 \\
(D E G F)\end{array}$ & $\begin{array}{l}\text { TF-HG-3 } \\
\text { (DEG F). }\end{array}$ & $\begin{array}{l}\text { P-BARC } \\
\text { IPSIAI }\end{array}$ \\
\hline $\begin{array}{ll}6.2 \cdot 1 & 1 \\
6.2 . & 2 \\
6.2 . & 3 \\
6.2 . & 4 \\
6.2 . & 5\end{array}$ & $\begin{array}{l}0.0 \\
0.0 \\
0.0 \\
0.0 \\
0.0\end{array}$ & $\begin{array}{r}9.7 \\
9.2 \\
9.0 \\
10.0 \\
9.7\end{array}$ & $\begin{array}{r}9.5 \\
9.0 \\
9.0 \\
10.0 \\
9.5\end{array}$ & $\begin{array}{l}0.0 \\
0.0 \\
0.0 \\
0.0 \\
0.0\end{array}$ & $\begin{array}{l}0.0 \\
0.0 \\
0.0 \\
0.0 \\
0.0\end{array}$ & $\begin{array}{l}55.0 \\
55.0 \\
55.0 \\
55.0 \\
55.0\end{array}$ & $\begin{array}{l}75.0 \\
75.0 \\
75.0 \\
76.0 \\
76.0\end{array}$ & $\begin{array}{l}0.0 \\
0.0 \\
0.0 \\
0.0 \\
0.0\end{array}$ & $\begin{array}{l}0.0 \\
0.0 \\
0.0 \\
0.0 \\
0.0\end{array}$ & $\begin{array}{l}12.4 \\
12.4 \\
12.4 \\
12.4 \\
12.4\end{array}$ \\
\hline $\begin{array}{l}6.2 .0 \\
6.2 .7 \\
6.2 .8 \\
6.2 .29 \\
6.2 .10\end{array}$ & $\begin{array}{l}J .0 \\
0.0 \\
j .0 \\
0.0 \\
\text { J. } 0\end{array}$ & $\begin{array}{r}9.5 \\
10.0 \\
9.5 \\
10.0 \\
10.0\end{array}$ & $\begin{array}{r}9.0 \\
10.0 \\
11.0 \\
11.0 \\
11.0\end{array}$ & $\begin{array}{l}0.0 \\
0.0 \\
0.0 \\
0.0 \\
0.0\end{array}$ & $\begin{array}{l}0.0 \\
0.0 \\
0.0 \\
0.0 \\
0.0\end{array}$ & $\begin{array}{l}55.0 \\
55.0 \\
54.0 \\
54.0 \\
55.0\end{array}$ & $\begin{array}{l}77.0 \\
78.0 \\
78.0 \\
77.0 \\
77.0\end{array}$ & $\begin{array}{l}0.0 \\
0.0 \\
0.0 \\
0.0 \\
0.0\end{array}$ & $\begin{array}{l}0.0 \\
0.0 \\
0.0 \\
0.0 \\
0.0\end{array}$ & $\begin{array}{l}12.4 \\
12.4 \\
12.4 \\
12.4 \\
12.4\end{array}$ \\
\hline $\begin{array}{l}6.2 .11 \\
6.2 .12 \\
6.2 .13 \\
6.2 .14 \\
6.2 .15\end{array}$ & $\begin{array}{l}.00 \\
.00 \\
0.0 \\
.00 \\
0.0\end{array}$ & $\begin{array}{r}10.0 \\
10.0 \\
10.0 \\
9.0 \\
9.0\end{array}$ & $\begin{array}{r}9.0 \\
10.0 \\
11.0 \\
11.0 \\
10.0\end{array}$ & $\begin{array}{l}0.0 \\
0.0 \\
0.0 \\
0.0 \\
0.0\end{array}$ & $\begin{array}{l}0.0 \\
0.0 \\
0.0 \\
0.0 \\
0.0\end{array}$ & $\begin{array}{l}55.0 \\
55.0 \\
55.0 \\
55.0 \\
56.0\end{array}$ & $\begin{array}{r}76.0 \\
78.0 \\
90.0 \\
96.5 \\
102.0\end{array}$ & $\begin{array}{l}0.0 \\
0.0 \\
0.0 \\
0.0 \\
0.0\end{array}$ & $\begin{array}{l}0.0 \\
0.0 \\
0.0 \\
0.0 \\
0.0\end{array}$ & $\begin{array}{l}12.4 \\
12.4 \\
12.4 \\
12.4 \\
12.4\end{array}$ \\
\hline
\end{tabular}




\section{TABLE XXIV (Contd.)}

MODIFIED UPPER ANNULUS GEOMETRY TEST GROUP 6.2

EXPEPIIIENTAL MEASURMENTS FOR TESTS 6.2 .1 TO $6.2 .17--0.63-1$. ONHNCOMER GAP

\begin{tabular}{|c|c|c|c|c|c|c|c|c|c|c|}
\hline $\begin{array}{l}\text { TEST } \\
\text { NO }\end{array}$ & $\begin{array}{l}\text { p-CW-1 } \\
\text { (PSIG) }\end{array}$ & $\begin{array}{l}P-C H-2 \\
\text { (PSIG) }\end{array}$ & $\begin{array}{l}\text { P-HG-1 } \\
\text { (PSIG) }\end{array}$ & $\begin{array}{l}P-H G-2 \\
\text { (PSIG) }\end{array}$ & $\begin{array}{l}\text { P-HG-3 } \\
\text { (PSIG) }\end{array}$ & $\begin{array}{l}T F-C W-1 \\
\text { IDEG FI }\end{array}$ & $\begin{array}{l}\text { TF-HG-1 } \\
(D E G F)\end{array}$ & $\begin{array}{l}\text { TF-HG-2 } \\
\text { (DEG F) }\end{array}$ & $\begin{array}{l}\text { TF-HG-3 } \\
\text { (DEG F) }\end{array}$ & $\begin{array}{l}\text { P-BARO } \\
\text { (PSIA) }\end{array}$ \\
\hline $\begin{array}{l}6.2 .16 \\
6.2 .17\end{array}$ & $\begin{array}{l}3.0 \\
1.0\end{array}$ & $\begin{array}{l}10.0 \\
10.0\end{array}$ & $\begin{array}{l}11.0 \\
11.0\end{array}$ & $\begin{array}{l}0.0 \\
0.0\end{array}$ & $\begin{array}{l}0.0 \\
0.0\end{array}$ & $\begin{array}{l}56.0 \\
57.0\end{array}$ & $\begin{array}{l}112.5 \\
113.5\end{array}$ & $\begin{array}{l}0.0 \\
0.0\end{array}$ & $\begin{array}{l}0.0 \\
0.0\end{array}$ & $\begin{array}{l}12.4 \\
12.4\end{array}$ \\
\hline
\end{tabular}

LOWTP. PLENUM FLOW

\begin{tabular}{|c|c|c|c|}
\hline $\begin{array}{l}\text { TEST } \\
\text {-ND }\end{array}$ & $\begin{array}{l}\text { INITIAL } \\
\text { WEIGHT } \\
\text { (LB) }\end{array}$ & $\begin{array}{l}\text { FINAL } \\
\text { WFIGHT } \\
\text { (LB) }\end{array}$ & $\begin{array}{l}\text { TIME OF } \\
\text { COLLECT ION } \\
\text { (SEC) }\end{array}$ \\
\hline
\end{tabular}

BYPASS FLOW

INITIAL FINAL TIME OF LOHER

INITIAL FINAL TIME OF PLENUM

(LB) HEIGHT COLLECTION LEVEL

$(P-(P-1)$

TF-LP-1
(DEG FI)

85.5

85.5

$\begin{array}{rr}85.5, & 45.0 \\ 85.5, \quad 35.5, & 75.0\end{array}$

$\begin{array}{lr}36.5 & 153.5\end{array}$

$153.5 \quad 155.0$

120.0
45.0

45.0
90.0

12.0

12.0

12.0

12.0

$155.0 \quad 156.5$

$156.5 \quad 245.5$

$245.5 \quad 334.0$

$334.0 \quad 428.5$

55.0

45.0

40.0

50.0
50.0

12.0

12.0

12.0

12.0

12.0

$141.5 \quad 181.0$

181.0

253.5

288.5

330.0

55.0

70.0

60.0

45.0

12.0

12.0

12.0
12.0
12.0

12.0

$\begin{array}{lllllll}6.2 .16 & 407.0 & 416.8 & 30.0 & 330.0 & 351.5 & 60.0 \\ 6.2 .17 & 416.8 & 423.5 & 40.0 & 351.5 & 365.5 & 50.0\end{array}$

$\begin{array}{lllllll}6.2 .16 & 407.0 & 416.8 & 30.0 & 330.0 & 351.5 & 60.0 \\ 6.2 .17 & 416.8 & 423.5 & 40.0 & 351.5 & 365.5 & 50.0\end{array}$

$\begin{array}{lllllll}6.2 .16 & 407.0 & 416.8 & 30.0 & 330.0 & 351.5 & 60.0 \\ 6.2 .17 & 416.8 & 423.5 & 40.0 & 351.5 & 365.5 & 50.0\end{array}$

$\begin{array}{lllllll}6.2 .16 & 407.0 & 416.8 & 30.0 & 330.0 & 351.5 & 60.0 \\ 6.2 .17 & 416.8 & 423.5 & 40.0 & 351.5 & 365.5 & 50.0\end{array}$

$\begin{array}{ll}12.0 & 10.0 \\ 12.0 & 10.0\end{array}$

10.0

10.0

10.0

10.0
10.0

55.0

55.0

55.0.
50.0

$10.0 \quad 54.0$

$10.0 \quad 54.0$
$10.0 \quad 55.0$

$10.0 \quad 55.0$

$10.0 \quad 55.0$

$10.0 \quad 55.0$

$10.0 \quad 55.0$

$10.0 \quad 55.0$

$10.0 \quad 56.0$

$10.0 \quad 53.0$

61.0 


\section{TABLE XXV}

MODIFTED UPPER ANNULUS GEOMETRY TTEST GROUP 9.2

TRANSPARENT VESSEL TESTY DATA FỌR. TESTS 9.2.1.TO 9.2.1.5 - - 1.58-1N. DONWNCOMER GAP SUMMARY, OF CALCIULATED, PEESULTS

\begin{tabular}{|c|c|c|c|c|c|c|c|c|c|c|}
\hline $\begin{array}{l}\text { TEST } \\
\text { NO }\end{array}$ & $\begin{array}{l}\text { DOWNCOMER } \\
\text { AIP. FLLI)W } \\
\text { (LB/SEC) }\end{array}$ & $\begin{array}{l}\text { TOHNCOMER } \\
\text { AIR DENSITY } \\
\text { (LG/ET *3) }\end{array}$ & $\begin{array}{l}\text { LOWER PLENUM } \\
\text { PPESSUPE } \\
\text { (PSIA) }\end{array}$ & $\begin{array}{l}\text { COLD. LEG } \\
\text { AIR FLOWW } \\
\text { (IIBSSEC) }\end{array}$ & $\begin{array}{c}\text { DOWNCOMER } \\
\text { HATER FLOW } \\
\text { (LS/SECI }\end{array}$ & $\begin{array}{l}\text { HATER,FLOW } \\
\text { CONTINUTYTY }\end{array}$ & $\begin{array}{c}\text { J-AIP } \\
\text { DOWNCOMEP. } \\
\text { (FT/SEC) }\end{array}$ & $\begin{array}{l}\text { J-WATEP } \\
\text { DOWNCBMEP. } \\
\text { (FT/SEC) }\end{array}$ & $(J G *) * * 1 / 2$ & $(J L *) * * 1 / 2$ \\
\hline $\begin{array}{l}9.2 \cdot 1 \\
9.202 \\
9.203 \\
9.204 \\
9.2 .5\end{array}$ & $\begin{array}{l}0.1753 \\
0.1456 \\
0.2600 \\
0.3770 \\
0.5066\end{array}$ & $\begin{array}{l}0.1188 \\
0.1196 \\
0.1183 \\
0.1192 \\
0.1270\end{array}$ & $\begin{array}{l}22.34 \\
22.34 \\
22.09 \\
22.34 \\
24.09\end{array}$ & $\begin{array}{r}0.0 \\
0.0 \\
0.0 \\
0.0 \\
0.0\end{array}$ & $\begin{array}{r}11.133 \\
10.533 \\
: 7.083 \\
4.000 \\
0.833\end{array}$ & $\begin{array}{r}0.989 \\
0.983 \\
10.008 \\
1.009 \\
1.074\end{array}$ & $\begin{array}{r}6.27 \\
5.17 \\
9.34 \\
13.45 \\
16.96\end{array}$ & $\begin{array}{r}0.758 \\
.0 .718 \\
0.493 \\
0.272 \\
0.057\end{array}$ & $\begin{array}{r}0.3066 \\
0.2790 \\
0.3739 \\
.0 .4494 \\
0.5127\end{array}$ & $\begin{array}{l}0.5105 \\
0.4965 \\
0.4072 \\
0.3060 \\
0.1397\end{array}$ \\
\hline
\end{tabular}


TABLE XXV (Contd.)

MODIFIED UPPER ANNULUS GEOMETRY TEST GROUP 9.2

EXPERIMENTAL MEASURMENTS FOR TESTS 9.2 .1 TO $9.2 .5--1.58-$ IN. DOWNCOMER GAP

\begin{tabular}{|c|c|c|c|c|c|c|c|c|c|c|}
\hline $\begin{array}{l}\text { TEST } \\
\text { NO }\end{array}$ & $\begin{array}{l}R M-C W-1 \\
(G P M)\end{array}$ & $\begin{array}{c}R M-C W-2 \\
(G P M)\end{array}$ & $\begin{array}{c}F T-C H-1 \\
(G P M)\end{array}$ & $\begin{array}{c}\mathrm{FT}-\mathrm{CH}-2 \\
(G P M)\end{array}$ & $\begin{array}{c}R M-H G-1 \\
((F M)\end{array}$ & $\begin{array}{c}R M-H G-2 \\
(C F M)\end{array}$ & $\begin{array}{c}R M-H G-3 \\
(C F M)\end{array}$ & $\begin{array}{c}R M-H G-4 \\
(C F M)\end{array}$ & $\begin{array}{c}F T-H G-1 \\
(C \subseteq M)\end{array}$ & $\begin{array}{c}F Y-H G-2 \\
(C F M)\end{array}$ \\
\hline $\begin{array}{l}9.2: 1 \\
9.202 \\
9.2 \cdot 3 \\
9.204 \\
9.2 \cdot 5\end{array}$ & $\begin{array}{l}0.0 \\
0.0 \\
0.0 \\
0.0 \\
0.0\end{array}$ & $\begin{array}{l}0.0 \\
0.0 \\
0.0 \\
0.0 \\
0.0\end{array}$ & $\begin{array}{r}120.0 \\
100.0 \\
79.0 \\
0.0 \\
0.0\end{array}$ & $\begin{array}{r}0.0 \\
0.0 \\
0.0 \\
50.0 \\
40.0\end{array}$ & $\begin{array}{c}80.00 \\
66.50 \\
0.0 \\
0.0 \\
0.0\end{array}$ & $\begin{array}{l}0.0 \\
0.0 \\
0.0 \\
0.0 \\
0.0\end{array}$ & $\begin{array}{l}0.0 \\
0.0 \\
0.0 \\
0.0 \\
0.0\end{array}$ & $\begin{array}{l}0.0 \\
0.0 \\
0.0 \\
0.0 \\
0.0\end{array}$ & $\begin{array}{c}0.0 \\
0.0 \\
29.62 \\
44.49 \\
63.72\end{array}$ & $\begin{array}{l}0.0 \\
0.0 \\
0.0 \\
0.0 \\
0.0\end{array}$ \\
\hline & & & & & & & & & & \\
\hline $\begin{array}{c}\text { TEST } \\
\text { NO }\end{array}$ & $\begin{array}{l}P-C N-1 \\
\text { (PSIG) }\end{array}$ & $\begin{array}{l}P-C H-2 \\
\text { (PSIG) }\end{array}$ & $\begin{array}{l}P-H G-1 \\
(P S I G)\end{array}$ & $\begin{array}{l}\text { P-HG-Z } \\
\text { (PSIGI }\end{array}$ & $\begin{array}{l}P-H G-3 \\
\text { (PSIG) }\end{array}$ & $\begin{array}{l}\text { TF-CW-1 } \\
\text { (DEG F) }\end{array}$ & $\begin{array}{l}\text { PF-HG-I } \\
\text { (DEG FI }\end{array}$ & $\begin{array}{l}T F-H G-2 \\
\text { IDEG FI }\end{array}$ & $\begin{array}{l}\text { TF-HG-3 } \\
\text { IDEG FI }\end{array}$ & $\begin{array}{l}\text { P-8ARO } \\
\text { (PSIAI) }\end{array}$ \\
\hline $\begin{array}{l}9.2 .1 \\
9.2 \cdot 2 \\
9.2 .3 \\
9.2 .4 \\
9.2 .5\end{array}$ & $\begin{array}{r}9.5 \\
10.0 \\
9.7 \\
10.0 \\
11.5\end{array}$ & $\begin{array}{l}0.0 \\
0.0 \\
0.0 \\
0.0 \\
0.0\end{array}$ & $\begin{array}{l}9.8 \\
10.2 \\
10.5 \\
11.2 \\
13.2\end{array}$ & $\begin{array}{r}0.0 \\
0.0 \\
95.5 \\
92.5 \\
90.0\end{array}$ & $\begin{array}{l}33.5 \\
33.5 \\
33.5 \\
33.0 \\
33.0\end{array}$ & $\begin{array}{l}47.0 \\
44.0 \\
44.0 \\
45.5 \\
47.5\end{array}$ & $\begin{array}{r}73.0 \\
76.0 \\
82.0 \\
84.0 \\
100.5\end{array}$ & $\begin{array}{r}0.0 \\
0.0 \\
92.5 \\
96.5 \\
119.0\end{array}$ & $\begin{array}{r}76.0 \\
76.5 \\
0.0 \\
0.0 \\
0.0\end{array}$ & $\begin{array}{l}12.3 \\
12.3 \\
12.3 \\
12.3 \\
12.3\end{array}$ \\
\hline
\end{tabular}

\begin{tabular}{|c|c|c|c|c|c|c|c|c|c|}
\hline \multirow[b]{2}{*}{$\begin{array}{l}\text { TEST } \\
\text { NO }\end{array}$} & \multicolumn{3}{|c|}{ LOWER PLENUM FLOH } & \multicolumn{3}{|c|}{ BYPASS FLOW } & \multirow[b]{2}{*}{$\begin{array}{l}\text { LOWER } \\
\text { PLENUM } \\
\text { LEVEL } \\
\text { (IN) }\end{array}$} & \multirow[b]{2}{*}{$\begin{array}{l}P-L P-1 \\
(P S I G)\end{array}$} & \multirow[b]{2}{*}{$\begin{array}{l}T F-(P-1) \\
\text { (OEG F) }\end{array}$} \\
\hline & $\begin{array}{l}\text { INITIAL } \\
\text { WEIGHT } \\
\text { (LB) }\end{array}$ & $\begin{array}{l}\text { FINAL } \\
\text { WEIS,HT } \\
\text { (LB) }\end{array}$ & $\begin{array}{l}\text { TIME OF } \\
\text { COLLECTIEN } \\
\text { (SEC) }\end{array}$ & $\begin{array}{l}\text { INITIAL } \\
\text { HEIGHT } \\
\text { (LB) }\end{array}$ & $\begin{array}{l}\text { FINAL } \\
\text { WEI GHT } \\
\text { (LB) }\end{array}$ & $\begin{array}{l}\text { TIME OF } \\
\text { COLLECTION } \\
\text { (SEC) }\end{array}$ & & & \\
\hline $\begin{array}{l}9.2 \cdot 1 \\
9.2 \cdot 2 \\
9.2 \cdot 3 \\
9.2 \cdot 4 \\
9.2 .5\end{array}$ & $\begin{array}{l}137.0 . \\
272.5 \\
197.0 \\
141.0 \\
252.5\end{array}$ & $\begin{array}{l}304.0 \\
430.5 \\
409.5 \\
261.0 \\
302.5\end{array}$ & $\begin{array}{l}15.0 \\
15.0 \\
30.0 \\
30.0 \\
60.0\end{array}$ & $\begin{array}{l}100.0 \\
180.5 \\
188.5 \\
102.5 \\
156.0\end{array}$ & $\begin{array}{l}180.5 \\
227.5 \\
308.0 \\
193.0 \\
464.5\end{array}$ & $\begin{array}{l}15.0 \\
15.0 \\
30.0 \\
30.0 \\
60.0\end{array}$ & $\begin{array}{r}5.0 \\
5.0 \\
12.0 \\
12.0 \\
12.0\end{array}$ & $\begin{array}{r}10.0 \\
10.0 \\
9.7 \\
10.0 \\
11.7\end{array}$ & $\begin{array}{l}47.5 \\
44.0 \\
44.0 \\
46.0 \\
52.0\end{array}$ \\
\hline
\end{tabular}




\section{TABLE XXVI}

MODIFIED UPPER ANNULUS GEOMETRY TEST GROUPP 9.6

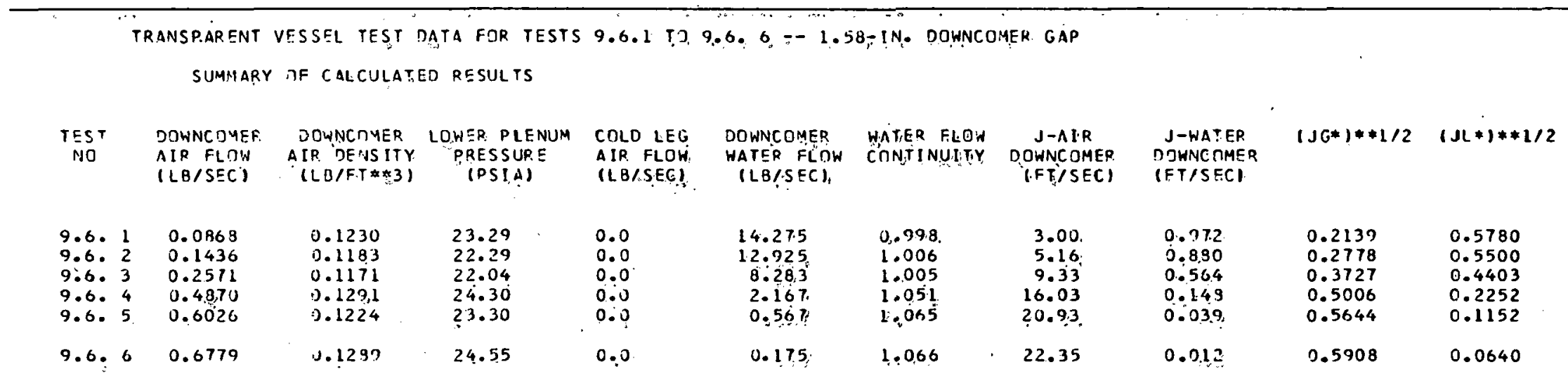


TABLE XXVI (Contd.)

MODIFIED UPPER ANNULUS GEOMETRY TEST GROUP 9.6

EXPERIMENTAL MEASURMENTS FOR TESTS 9.6.1 TO 9.6.6-1.58-IN. DOWNCOMER GAP

\begin{tabular}{|c|c|c|c|c|c|c|c|c|c|c|}
\hline $\begin{array}{l}\text { TEST } \\
\text { NO }\end{array}$ & $\begin{array}{c}R M-C W-1 \\
(G P M)\end{array}$ & $\begin{array}{c}R M-C W-2 \\
(G P M)\end{array}$ & $\begin{array}{c}F T-C W-1 \\
(G P M)\end{array}$ & $\begin{array}{c}F T-C H-2 \\
(G P M)\end{array}$ & $\begin{array}{c}R M-H C_{3}-1 \\
(C F M)\end{array}$ & $\begin{array}{l}\text { RM-HG-2 } \\
\text { (CFM) }\end{array}$ & $\begin{array}{c}\text { RM-HG-3 } \\
\text { (CFM) }\end{array}$ & $\begin{array}{c}R M-H(z-4 \\
(C \in M)\end{array}$ & $\begin{array}{c}F T-M G-1 \\
(C E M)\end{array}$ & $\begin{array}{c}F T-H G-2 \\
(C F M)\end{array}$ \\
\hline $\begin{array}{l}9.6 \cdot 1 \\
9.6 \cdot 12 \\
9.6 .3 \\
9.6 \cdot 4 \\
9.6 .5\end{array}$ & $\begin{array}{l}0.0 \\
0.0 \\
0.0 \\
0.0 \\
0.0\end{array}$ & $\begin{array}{l}0.0 \\
0.0 \\
0.0 \\
0.0 \\
0.0\end{array}$ & $\begin{array}{r}121.0 \\
129.0 \\
100.0 \\
60.0 \\
0.0\end{array}$ & $\begin{array}{r}0.0 \\
0.0 \\
0.0 \\
0.0 \\
30.3\end{array}$ & $\begin{array}{c}40.00 \\
66.50 \\
0.0 \\
0.0 \\
0.0\end{array}$ & $\begin{array}{l}0.0 \\
0.0 \\
0.0 \\
0.0 \\
0.0\end{array}$ & $\begin{array}{l}0.0 \\
0.0 \\
0.0 \\
0.0 \\
0.0\end{array}$ & $\begin{array}{l}0.0 \\
0.0 \\
0.0 \\
0.0 \\
0.0\end{array}$ & $\begin{array}{c}0.0 \\
0.0 \\
29.36 \\
56.03 \\
73.59\end{array}$ & $\begin{array}{l}0.0 \\
0.0 \\
0.0 \\
0.0 \\
0.0\end{array}$ \\
\hline 9.6 .6 & 0.0 & 0.0 & 0.0 & 20.0 & 0.0 & 0.0 & 0.0 & 0.0 & 88.59 & 0.0 \\
\hline $\begin{array}{l}\text { TEST } \\
\text { ND }\end{array}$ & $\begin{array}{l}P-C W-1 \\
(P S I G)\end{array}$ & $\begin{array}{l}\text { P-CH-2 } \\
\text { (PSIG) }\end{array}$ & $\begin{array}{l}P-H G-1 \\
\text { (PSIG) }\end{array}$ & $\begin{array}{l}\text { P-HG-2 } \\
\text { (PSIG) }\end{array}$ & $\begin{array}{l}\text { P-HG-3 } \\
\text { (PSIG) }\end{array}$ & $\begin{array}{l}T F-C H-1 \\
\text { (DES F ) }\end{array}$ & $\begin{array}{l}T F-H S-1 \\
(D E G \text { F) }\end{array}$ & $\begin{array}{l}\text { TF-HG-2 } \\
\text { (NEG F) }\end{array}$ & $\begin{array}{l}\text { TF-HG-3 } \\
\text { (DEG F) }\end{array}$ & $\begin{array}{l}\text { P-BARO } \\
\text { (PSIA) }\end{array}$ \\
\hline $\begin{array}{l}9.6 \cdot 1 \\
9.6 \cdot 2 \\
9.6 \cdot 3 \\
9.6 \cdot 4 \\
9.6 .5\end{array}$ & $\begin{array}{r}10.7 \\
10.0 \\
9.7 \\
13.0 \\
11.7\end{array}$ & $\begin{array}{l}0.0 \\
0.0 \\
0.0 \\
0.0 \\
0.0\end{array}$ & $\begin{array}{l}11.0 \\
10.5 \\
10.5 \\
14.5 \\
13.5\end{array}$ & $\begin{array}{r}100.0 \\
97.5 \\
94.0 \\
93.0 \\
30.0\end{array}$ & $\begin{array}{r}33.0 \\
32.5 \\
0.0 \\
0.0 \\
0.0\end{array}$ & $\begin{array}{l}51.0 \\
48.0 \\
47.0 \\
47.0 \\
49.5\end{array}$ & $\begin{array}{l}80.0 \\
77.0 \\
78.0 \\
80.0 \\
88.0\end{array}$ & $\begin{array}{r}0.0 \\
0.0 \\
86.0 \\
85.0 \\
102.0\end{array}$ & $\begin{array}{r}80.0 \\
79.0 \\
0.0 \\
0.0 \\
0.0\end{array}$ & $\begin{array}{l}12.3 \\
12.3 \\
12.3 \\
12.3 \\
12.3\end{array}$ \\
\hline 3.6 .6 & 11.5 & 0.0 & 15.6 & 85.0 & 0.0 & 50.0 & 97.0 & 112.0 & 0.0 & 12.3 \\
\hline
\end{tabular}

\begin{tabular}{|c|c|c|c|c|c|c|c|c|c|}
\hline \multirow[b]{2}{*}{$\begin{array}{c}\text { TEST } \\
\text { NO }\end{array}$} & \multicolumn{2}{|c|}{ LOHER PLENUM } & FLOW & \multicolumn{3}{|c|}{ BYPASS FLOW } & \multirow[b]{2}{*}{$\begin{array}{l}\text { LOWER } \\
\text { PLENUM } \\
\text { LEVEL } \\
\text { (IN) }\end{array}$} & \multirow[b]{2}{*}{$\begin{array}{l}P-1 \rho-1 \\
(0 S I r)\end{array}$} & \multirow[b]{2}{*}{$\begin{array}{l}T F-L P-1 \\
\text { (DES FI }\end{array}$} \\
\hline & $\begin{array}{l}\text { INITIAL } \\
\text { WEIGHT } \\
\text { (LB) }\end{array}$ & $\begin{array}{l}\text { F[NAL } \\
\text { WE[G:HT } \\
\text { (LB] }\end{array}$ & $\begin{array}{l}\text { TIME OF } \\
\text { COLLECTICN } \\
\text { ISECI I }\end{array}$ & $\begin{array}{l}\text { INIT ITAL } \\
\text { WE ISHT } \\
\text { (LB) }\end{array}$ & $\begin{array}{l}\text { FINAL } \\
\text { WEIGHT } \\
\text { (LB) }\end{array}$ & $\begin{array}{l}\text { TIME JF } \\
\text { COLLECTIOV } \\
\text { (SEC) }\end{array}$ & & & \\
\hline $\begin{array}{l}9.6 .1 \\
9.6 .2 \\
9.6 .3 \\
9.6 .4 \\
9.6 .5\end{array}$ & $\begin{array}{l}141.5 \\
165.5 \\
146.5 \\
144.0 \\
193.0\end{array}$ & $\begin{array}{l}427.0 \\
424.0 \\
395.0 \\
209.0 \\
210.0\end{array}$ & $\begin{array}{l}20.0 \\
20.0 \\
30.0 \\
30.0 \\
30.0\end{array}$ & $\begin{array}{l}115.5 \\
118.0 \\
271.5 \\
103.0 \\
244.5\end{array}$ & $\begin{array}{l}191.0 \\
271.5 \\
442.0 \\
301.0 \\
362.0\end{array}$ & $\begin{array}{l}30.0 \\
30.0 \\
30.0 \\
30.0 \\
30.0\end{array}$ & $\begin{array}{r}4.0 \\
4.0 \\
12.0 \\
12.0 \\
12.0\end{array}$ & $\begin{array}{r}11.0 \\
10.0 \\
9.7 \\
12.0 \\
11.0\end{array}$ & $\begin{array}{l}51.0 \\
48.5 \\
48.0 \\
48.0 \\
54.0\end{array}$ \\
\hline 9.6 .6 & 210.0 & 220.5 & 60.0 & 111.5 & 195.0 & 30.0 & 12.0 & 12.2 & 54.0 \\
\hline
\end{tabular}


The depth of the upper annulus was changed by lowering the filler piece to the bottom of the lower plenum which effectively decreased the length of the downcomer and increased the length of the upper annulus by about 34 inches. Tests conducted with this geometry are included in Test Groups 5.4 and 6.2. The geometry changes that resulted from increasing the height of the upper annulus, removing the hot leg simulators, and removing the nozzle inserts have been described in detail in the previous section on the bypass flow tests.

\section{SHORTENED CORE BARREL TESTS}

Tests were performed to investigate the effect on countercurrent flow of shortening the length of the downcomer. The downcomer was shortened from its nominal length of about 68 inches by shortening the length of the core barrel. For those tests with the shortened core barrel, the length of the downcomer was defined as the distance from the top of the downcomer filler piece to the bottom of the core barrel; or, if a filler piece was not used as part of the downcomer, the length was defined as the distance from the centerline of the cold leg pipe to the bottom of the core barrel. The shortened downcomers which used a filler piece were 34,24 , and 6 inches in length. The 34 -inch downcomer length resulted when the core barrel was cut in half. The 24- and 6-inch downcomer lengths were based on scaling the ratio of the Semiscale core barrel diameter divided by the downcomer length to this same ratio for the Loss-of-Fluid-Test (LOFT) reactor system and a typical pressurized water reactor (PWR), respectively. A 12-inch downcomer resulted when the core barrel cut for the 6-inch-length downcomer, which included a filler piece, was used in a downcomer configuration without a filler piece. Results from the shortened downcomer tests are included in Tables XXVII through XXXi.

\section{TWO-PHASE COLD LEG MIXTURE TESTS}

Tests were performed to investigate the effect on countercurrent flow of a two-phase mixture entering the upper annulus through the core barrel. A range of different cold leg air flow rates was used in an attempt to cover the wide range of steam-water mixtures that could be entering the Semiscale vessel during the ECC injection period of a blowdown test. The results from the two-phase cold leg mixture tests are presented in Tables XXXII through XXXVI.

\section{COMBINED EFFECTS TESTS}

Ten groups of tests were conducted with a combination of one or more of the following changes to the system geometry or to two-phase flow in the cold leg: downcomer length shortened, upper annulus depth and height changed, hot leg simulator removed, cold 
TABLE XXVII

SHORTENED CORE BARREL TEST GROUP 3.1

TRANSPARENT VESSEL TEST DATA FUR TESTS 3.1.1 TO 3.1.31--0.39-IN. OOWNCOMER gAP SUYMAPY TE CALCULATED RESULTS

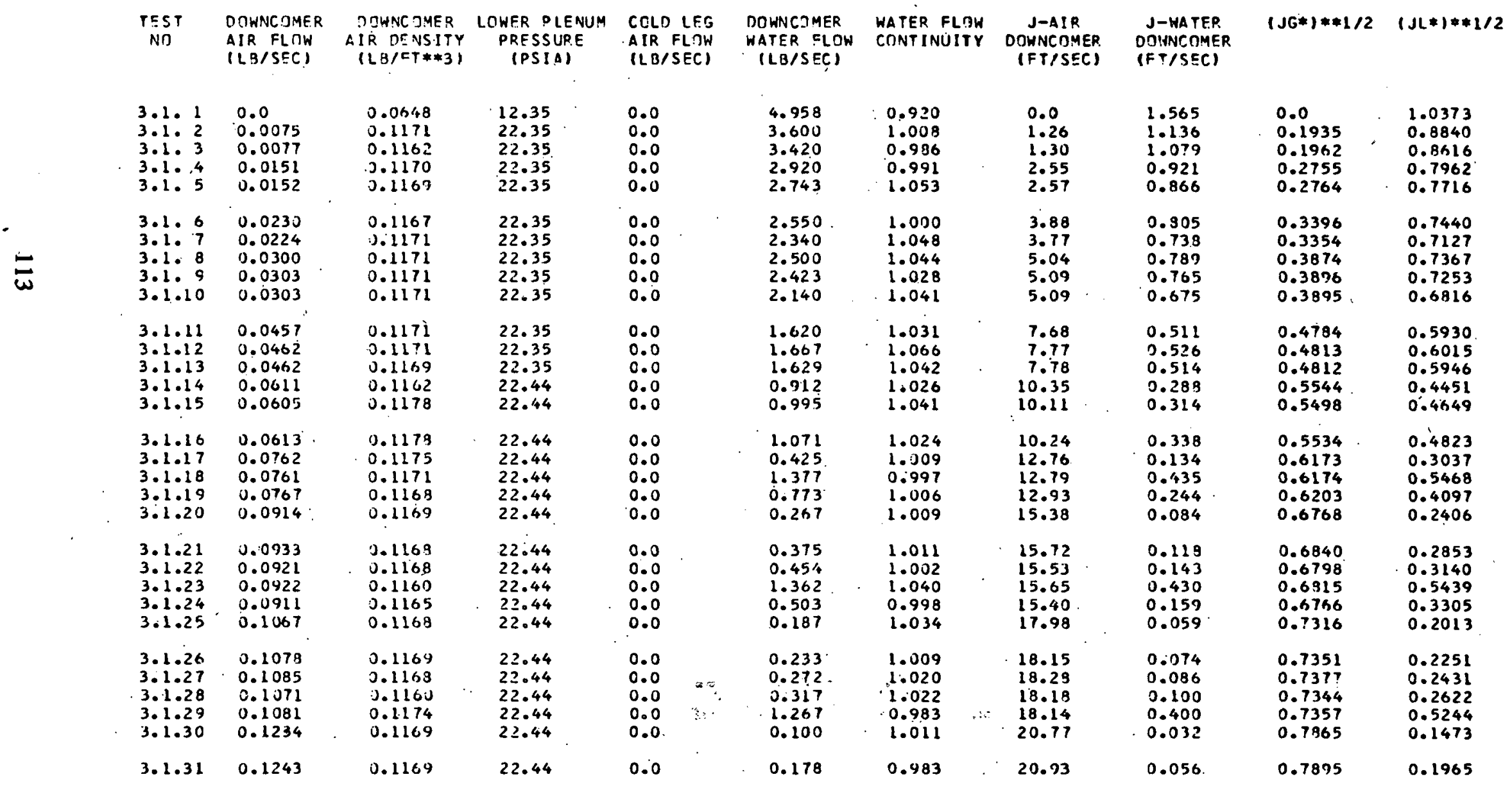




\begin{tabular}{|c|c|c|c|c|c|c|c|c|c|c|}
\hline$\cdots 1:$ & $\because \cdots:$ & $11=$ & $\because \quad$, & $20 ?$ & 9.16 & 0.893 & $20: 33$ & $1 \cdot 9=9$ & $\partial$ : the? & DIap? \\
\hline 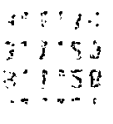 & 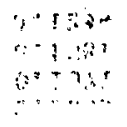 & 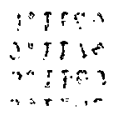 & 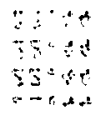 & $\begin{array}{l}0: 0 \\
0: 0 \\
0 \div 0 \\
0\end{array}$ & $\begin{array}{r}9500 \\
1005 \\
\text { TABLE XXVIII } \\
\end{array}$ & $\begin{array}{r}1+0.9 \\
0+283 \\
\text { (Contdis) } \\
\end{array}$ & 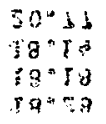 & 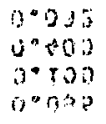 & $\begin{array}{l}0=1+8 ? \\
0 \times 82=1 \\
0=300 \\
0 \div 31\end{array}$ & 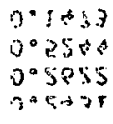 \\
\hline & . & . & & . & & . .. & $\therefore$ & $\cdot$ & 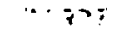 & 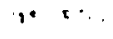 \\
\hline
\end{tabular}

SHORTENED CORE BARREL TEST GROUP 3.1

EXPER IMENTAL MEÁSURMENTS FÖR TESTS 3..1.i T'O 3.1.3i -- 0.39-IN' DOWNCOMER GAP

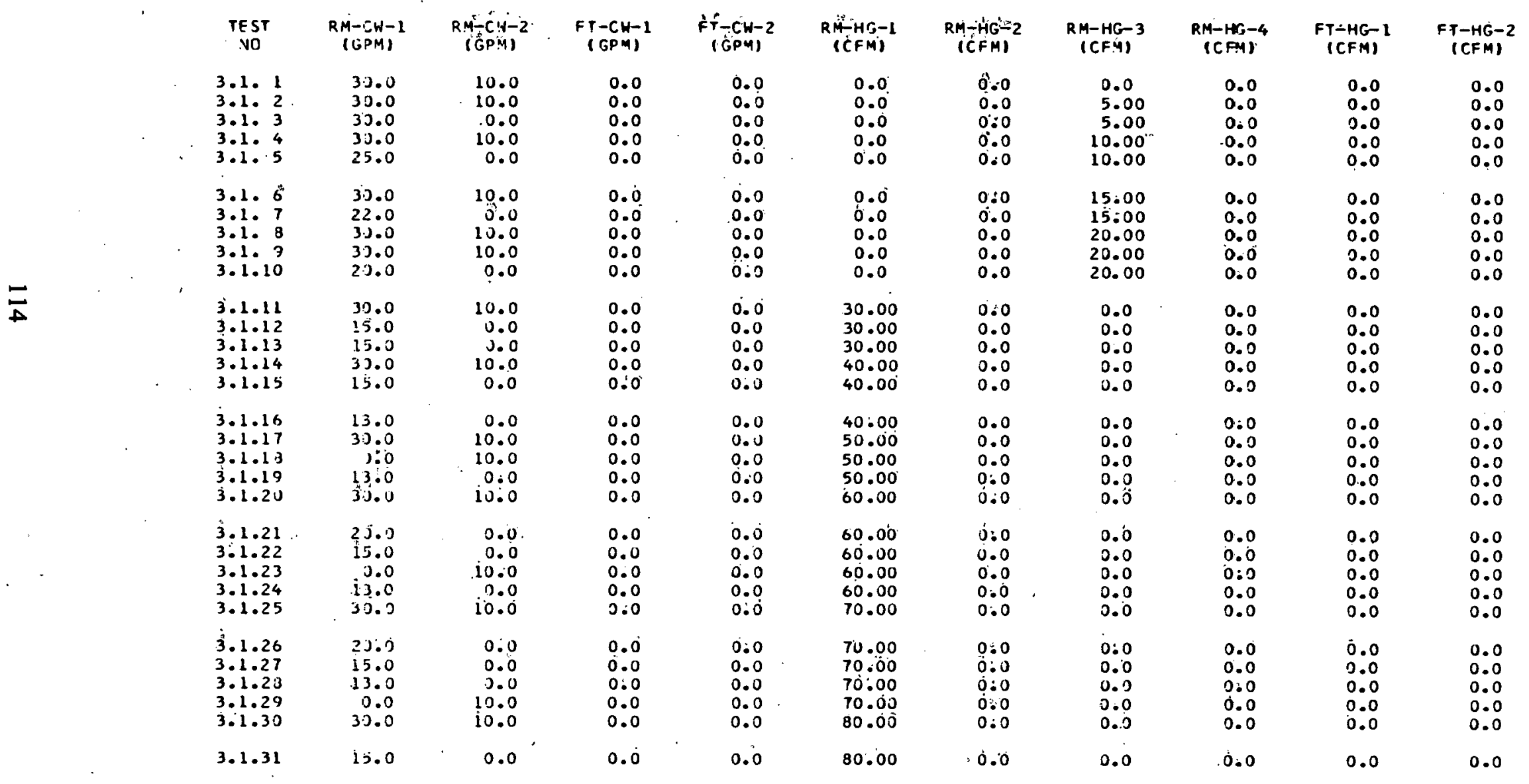




\section{TABLE XXVII. (Contd.)}

SHORTENED CORE BARREL TEST GROUP 3.1

EXPERI HENTAL MEASURMENTS FOR TESTS 3.1 .1 TO $3.1 .31-0.39-I N$. DONNCOMER GAP

\begin{tabular}{|c|c|c|c|c|c|c|c|c|c|c|}
\hline $\begin{array}{c}\text { TEST } \\
N^{\prime} J\end{array}$ & $\begin{array}{l}P-C W-1 \\
(P S I G)\end{array}$ & $\begin{array}{l}\dot{p}-C H-2 \\
(P S I G)\end{array}$ & $\begin{array}{l}P-H G-1 \\
(P S I G)\end{array}$ & $\begin{array}{l}\text { P-HG-2 } \\
\text { (PSIG) }\end{array}$ & $\begin{array}{l}\text { P-HG-3 } \\
\text { (PSIG) }\end{array}$ & $\begin{array}{l}T F-C H-1 \\
(D E G F)\end{array}$ & $\begin{array}{l}\text { TF-HF-1 } \\
(D E G F)\end{array}$ & $\begin{array}{l}\text { TF-HF,-2 } \\
\text { (DEG }=\text { I }\end{array}$ & $\begin{array}{l}\text { PF-HG-3 } \\
\text { (DEG F) }\end{array}$ & $\begin{array}{l}P-B A R D \\
(P S \mid A)\end{array}$ \\
\hline $\begin{array}{l}3.1: 1 \\
3.1 \cdot 2 \\
3.1 \cdot 2 \\
3.1: 4 \\
3.1 \cdot 5\end{array}$ & $\begin{array}{l}0.0 \\
0.0 \\
0.0 \\
0.0 \\
0.0\end{array}$ & $\begin{array}{r}0.0 \\
9.5 \\
10.0 \\
10.0 \\
10.0\end{array}$ & $\begin{array}{r}0.0 \\
9.0 \\
10.0 \\
9.5 \\
9.7\end{array}$ & $\begin{array}{l}0.0 \\
0.0 \\
0.0 \\
0.0 \\
0.0\end{array}$ & $\begin{array}{l}0.0 \\
0.0 \\
0.0 \\
0.0 \\
0.0\end{array}$ & $\begin{array}{l}53.0 \\
55.0 \\
57.5 \\
55.5 \\
55.0\end{array}$ & $\begin{array}{l}76.0 \\
76.0 \\
75.0 \\
75.0 \\
74.5\end{array}$ & $\begin{array}{l}0.0 \\
0.0 \\
0.0 \\
0.0 \\
0.0\end{array}$ & $\begin{array}{l}0.0 \\
0.0 \\
0.0 \\
0.0 \\
0.0\end{array}$ & $\begin{array}{l}12.3 \\
12.3 \\
12.3 \\
12.3 \\
12.3\end{array}$ \\
\hline $\begin{array}{l}3.1 \cdot 6 \\
3.1 \cdot 7 \\
3.1 .9 \\
3.1 .9 \\
3.1 .10\end{array}$ & $\begin{array}{r}0.0 \\
0.0 \\
0.0 \\
.0 .0 \\
.0 .0\end{array}$ & $\begin{array}{r}10.5 \\
10.0 \\
9.0 \\
9.5 \\
10.5\end{array}$ & $\begin{array}{r}10.0 \\
9.0 \\
9.0 \\
9.5 \\
9.5\end{array}$ & $\begin{array}{l}0.0 \\
0.0 \\
0.0 \\
0.0 \\
0.0\end{array}$ & $\begin{array}{l}0.0 \\
0.0 \\
0.0 \\
0.0 \\
0.0\end{array}$ & $\begin{array}{l}55.0 \\
55.0 \\
55.0 \\
55.0 \\
55.0\end{array}$ & $\begin{array}{l}75.0 \\
75.0 \\
74.0 \\
74.5 \\
75.0\end{array}$ & $\begin{array}{l}0.0 \\
0.0 \\
0.0 \\
0.0 \\
0.0\end{array}$ & $\begin{array}{l}0.0 \\
0.0 \\
0.0 \\
0.0 \\
0.0\end{array}$ & $\begin{array}{l}12.3 \\
12.3 \\
12.3 \\
12.3 \\
12.3\end{array}$ \\
\hline $\begin{array}{l}3.1 .11 \\
3.1 .12 \\
3.1 .13 \\
3.1 .14 \\
3.1 .15\end{array}$ & $\begin{array}{l}0.0 \\
0.0 \\
0.0 \\
0.0 \\
0.0\end{array}$ & $\begin{array}{l}9.0 \\
9.5 \\
9.0 \\
9.0 \\
9.0\end{array}$ & $\begin{array}{r}9.5 \\
10.0 \\
10.0 \\
9.5 \\
9.0\end{array}$ & $\begin{array}{l}0.0 \\
0.0 \\
0.0 \\
0.0 \\
0.0\end{array}$ & $\begin{array}{l}0.0 \\
0.0 \\
0.0 \\
0.0 \\
0.0\end{array}$ & $\begin{array}{l}54.0 \\
55.0 \\
55.5 \\
53.5 \\
54.0\end{array}$ & $\begin{array}{l}74.0 \\
73.0 \\
74.5 \\
77.0 \\
75.0\end{array}$ & $\begin{array}{l}0.0 \\
0.0 \\
0.0 \\
0.0 \\
0.0\end{array}$ & $\begin{array}{l}0.0 \\
0.0 \\
0.0 \\
0.0 \\
0.0\end{array}$ & $\begin{array}{l}12.3 \\
12.3 \\
12.3 \\
12.4 \\
12.4\end{array}$ \\
\hline $\begin{array}{l}3.1 .16 \\
3.1 .17 \\
3.1 .13 \\
3.1 .19 \\
3.1 .20\end{array}$ & $\begin{array}{l}0.0 \\
0.0 \\
0.0 \\
3.0 \\
.03\end{array}$ & $\begin{array}{l}9.0 \\
8.7 \\
3.7 \\
3.5 \\
8.5\end{array}$ & $\begin{array}{l}9.5 \\
9.0 \\
9.0 \\
9.5 \\
9.0\end{array}$ & $\begin{array}{l}0.0 \\
0.0 \\
0.0 \\
0.0 \\
0.0\end{array}$ & $\begin{array}{l}0.0 \\
0.0 \\
0.0 \\
0.0 \\
0.0\end{array}$ & $\begin{array}{l}55.0 \\
56.0 \\
57.5 \\
5.8 .0 \\
57.0\end{array}$ & $\begin{array}{l}73.0 \\
72.5 \\
74.0 \\
77.0 \\
79.0\end{array}$ & $\begin{array}{l}0.0 \\
0.0 \\
0.0 \\
0.0 \\
0.0\end{array}$ & $\begin{array}{l}0.0 \\
0.0 \\
0.0 \\
0.0 \\
0.0\end{array}$ & $\begin{array}{l}12.4 \\
12.4 \\
12.4 \\
12.4 \\
12.4\end{array}$ \\
\hline $\begin{array}{l}3.1 .21 \\
3.1 .22 \\
3.1 .23 \\
3.1 .24 \\
3.1 .25\end{array}$ & $\begin{array}{l}3.0 \\
3.0 \\
j .0 \\
0.0 \\
0.0\end{array}$ & $\begin{array}{l}10.0 \\
8.7 \\
0.5 \\
3.5 \\
3.5\end{array}$ & $\begin{array}{r}10.0 \\
9.5 \\
9.5 \\
9.0 \\
9.0\end{array}$ & $\begin{array}{r}0.0 \\
0.0 \\
-\quad 0.0 \\
0.0 \\
0.0\end{array}$ & $\begin{array}{l}0.0 \\
0.0 \\
0.0 \\
0.0 \\
0.0\end{array}$ & $\begin{array}{l}57.5 \\
57.5 \\
61.0 \\
57.5 \\
55.0\end{array}$ & $\begin{array}{l}80.5 \\
82.0 \\
80.5 \\
82.0 \\
85.0\end{array}$ & $\begin{array}{l}0.0 \\
0.0 \\
0.0 \\
0.0 \\
0.0\end{array}$ & $\begin{array}{l}0.0 \\
0.0 \\
0.0 \\
0.0 \\
0.0\end{array}$ & $\begin{array}{l}12.4 \\
12.4 \\
12.4 \\
12.4 \\
12.4\end{array}$ \\
\hline $\begin{array}{l}3.1 .26 \\
3.1 .27 \\
3.1 .28 \\
3.1 .29 \\
3.1 .30\end{array}$ & $\begin{array}{l}0.0 \\
J .0 \\
3.0 \\
0.0 \\
0.0\end{array}$ & $\begin{array}{l}9.0 \\
8.5 \\
8.5 \\
9.5 \\
8.5\end{array}$ & $\begin{array}{l}9.5 \\
9.5 \\
9.0 \\
9.2 \\
9.0\end{array}$ & $\begin{array}{l}0.0 \\
0.0 \\
0.0 \\
0.0 \\
0.0\end{array}$ & $\begin{array}{l}0.0 \\
0.0 \\
0.00 \\
0.00 \\
0.0\end{array}$ & $\begin{array}{l}55.5 \\
61.0 \\
56.5 \\
58.5 \\
54.5\end{array}$ & $\begin{array}{l}86.0 \\
79.0 \\
80.5 \\
76.5 \\
80.5\end{array}$ & $\begin{array}{l}0.0 \\
0.0 \\
0.0 \\
0.0 \\
0.0\end{array}$ & $\begin{array}{l}0.0 \\
0.0 \\
0.0 \\
0.0 \\
0.0\end{array}$ & $\begin{array}{l}12.4 \\
12.4 \\
12.4 \\
12.4 \\
12.4\end{array}$ \\
\hline 3.1 .31 & 0.0 & 9.0. & 9.5 & 0.0 & 0.00 & $55 \%$ & $: 84.0$ & 0.0 & 0.0 & 12.4 \\
\hline
\end{tabular}




\section{TABLE XXVII (Contd.)}

SHORTENED CORE BARREL TEST GROUP 3.1

EXPERIMENTAL MEASURMENTS FOR TESTS 3.1 .1 TO 3.1.31-- 0.39-IN. DOMNCOMER GAP

\begin{tabular}{|c|c|c|c|c|c|c|c|c|c|c|c|}
\hline & & & & LOWER PLENUM & FLOW & & YPASS FL & & & & \\
\hline & & $\begin{array}{l}\text { TEST } \\
\text { NO }\end{array}$ & $\begin{array}{l}\text { INITIAL } \\
\text { WEIGHT } \\
\text { (LB) }\end{array}$ & $\begin{array}{l}\text { FINAL } \\
\text { HEIGHT } \\
\text { (LB) }\end{array}$ & $\begin{array}{l}\text { TIME OF } \\
\text { COLLECTICN } \\
\text { (SECI }\end{array}$ & $\begin{array}{l}\text { IN ITIAL } \\
\text { WE IGHT } \\
\text { (LB) }\end{array}$ & $\begin{array}{l}\text { FINAL } \\
\text { WEIGHT } \\
\text { (LB) }\end{array}$ & $\begin{array}{l}\text { TIME OF } \\
\text { COLLECTION } \\
\text { (SEC) }\end{array}$ & $\begin{array}{l}\text { PLENUM } \\
\text { LEVEL } \\
\text { (IN) }\end{array}$ & $\begin{array}{l}P-(P-1 \\
(P S I G)\end{array}$ & $\begin{array}{l}\text { TF-LP-1 } \\
\text { (DEG FI }\end{array}$ \\
\hline & & $\begin{array}{ll}3.1 \cdot 1 \\
3.1 . & 2 \\
3.1 . & 3 \\
3.1 & 4 \\
3.1 & 5\end{array}$ & $\begin{array}{l}133.3 \\
232.0 \\
145.5 \\
231.0 \\
304.0\end{array}$ & $\begin{array}{l}282.0 \\
354.0 \\
231.0 \\
304.0 \\
400.0\end{array}$ & $\begin{array}{l}30.0 \\
20.0 \\
25.0 \\
25.0 \\
35.0\end{array}$ & $\begin{array}{r}57.5 \\
67.0 \\
163.3 \\
194.5 \\
311.0\end{array}$ & $\begin{array}{r}67.0 \\
147.3 \\
194.5 \\
311.0 \\
347.8\end{array}$ & $\begin{array}{l}60.0 \\
40.0 \\
45.0 \\
45.0 \\
40.0\end{array}$ & $\begin{array}{l}12.0 \\
12.0 \\
12.0 \\
12.0 \\
12.0\end{array}$ & $\begin{array}{r}0.0 \\
10.0 \\
10.0 \\
10.0 \\
10.0\end{array}$ & $\begin{array}{l}54.0 \\
55.0 \\
59.0 \\
55.5 \\
56.0\end{array}$ \\
\hline$\vec{a}$ & & $\begin{array}{l}3.1 .6 \\
3.1 .17 \\
3.1 .98 \\
3.1 .99 \\
3.1 .10\end{array}$ & $\begin{array}{l}135.0 \\
211.5 \\
270.0 \\
332.5 \\
395.5\end{array}$ & $\begin{array}{l}211.5 \\
270.0 \\
332.5 \\
395.5 \\
449.0\end{array}$ & $\begin{array}{l}30.0 \\
25.0 \\
25.0 \\
26.0 \\
25.0\end{array}$ & $\begin{array}{r}94.0 \\
214.5 \\
393.0 \\
492.3 \\
591.0\end{array}$ & $\begin{array}{l}214.5 \\
253.5 \\
492.3 \\
591.0 \\
625.0\end{array}$ & $\begin{array}{l}40.0 \\
45.0 \\
30.0 \\
30.0 \\
45.0\end{array}$ & $\begin{array}{l}12.0 \\
12.0 \\
12.0 \\
12.0 \\
12.0\end{array}$ & $\begin{array}{l}10.0 \\
10.0 \\
10.0 \\
10.0 \\
10.0\end{array}$ & $\begin{array}{l}57.0 \\
55.0 \\
55.0 \\
55.0 \\
55.0\end{array}$ \\
\hline & & $\begin{array}{l}3.1 .11 \\
3.1 .12 \\
3.1 .13 \\
3.1 .14 \\
3.1 .15\end{array}$ & $\begin{array}{l}449.0 \\
489.5 \\
285.0 \\
133.8 \\
170.3\end{array}$ & $\begin{array}{l}499.5 \\
539.5 \\
342.0 \\
170.3 \\
225.0\end{array}$ & $\begin{array}{l}25.0 \\
30.0 \\
35.0 \\
40.0 \\
55.0\end{array}$ & $\begin{array}{r}625.0 \\
789.5 \\
474.8 \\
80.3 \\
224.0\end{array}$ & $\begin{array}{l}789.5 \\
814.5 \\
502.0 \\
224.0 \\
271.0\end{array}$ & $\begin{array}{l}40.0 \\
45.0 \\
50.0 \\
30.0 \\
40.0\end{array}$ & $\begin{array}{l}12.0 \\
12.0 \\
12.0 \\
12.0 \\
12.0\end{array}$ & $\begin{array}{l}10.0 \\
10.0 \\
10.0 \\
10.0 \\
10.0\end{array}$ & $\begin{array}{l}55.0 \\
55.0 \\
56.0 \\
61.5 \\
54.0\end{array}$ \\
\hline & . & $\begin{array}{l}3.1 .16 \\
3.1 .17 \\
3.1 .18 \\
3.1 .19 \\
3.1 .20\end{array}$ & $\begin{array}{l}225.0 \\
300.0 \\
321.3 \\
397.0 \\
455.0\end{array}$ & $\begin{array}{l}300.0 \\
321.3 \\
397.0 \\
455.0 \\
475.0\end{array}$ & $\begin{array}{l}70.0 \\
50.0 \\
55.0 \\
75.0 \\
15.0\end{array}$ & $\begin{array}{l}271.0 \\
310.0 \\
491.5 \\
492.0 \\
544.3\end{array}$ & $\begin{array}{l}310.0 \\
491.5 \\
492.0 \\
544.3 \\
731.3\end{array}$ & $\begin{array}{l}50.0 \\
35.0 \\
60.0 \\
50.0 \\
35.0\end{array}$ & $\begin{array}{l}12.0 \\
12.0 \\
12.0 \\
12.0 \\
12.0\end{array}$ & $\begin{array}{l}10.0 \\
10.0 \\
10.0 \\
10.0 \\
10.0\end{array}$ & $\begin{array}{l}54.0 \\
55.5 \\
57.5 \\
58.5 \\
58.0\end{array}$ \\
\hline & & $\begin{array}{l}3.1 .21 \\
3.1 .22 \\
3.1 .23 \\
3.1 .24 \\
3.1 .25\end{array}$ & $\begin{array}{l}475.0 \\
501.3 \\
135.8 \\
217.5 \\
257.8\end{array}$ & $\begin{array}{l}501.3 \\
528.5 \\
217.5 \\
257.8 \\
271.8\end{array}$ & $\begin{array}{l}70.0 \\
60.0 \\
60.0 \\
80.0 \\
75.0\end{array}$ & $\begin{array}{l}731.3 \\
816.5 \\
103.3 \\
112.0 \\
177.0\end{array}$ & $\begin{array}{l}816.5 \\
873.8 \\
112.0 \\
177.0 \\
371.8\end{array}$ & $\begin{array}{r}35.0 \\
35.0 \\
105.0 \\
50.0 \\
35.0\end{array}$ & $\begin{array}{l}12.0 \\
12.0 \\
12.0 \\
12.0 \\
12.0\end{array}$ & $\begin{array}{l}10.0 \\
10.0 \\
10.0 \\
10.0 \\
10.0\end{array}$ & $\begin{array}{l}59.5 \\
58.5 \\
62.0 \\
60.0 \\
58.5\end{array}$ \\
\hline & ' & $\begin{array}{l}3.1 .26 \\
3.1 .27 \\
3.1 .23 \\
3.1 .29 \\
3.1 .30\end{array}$ & $\begin{array}{l}271.0 \\
135.5 \\
160.0 \\
189.0 \\
265.0\end{array}$ & $\begin{array}{l}285.8 \\
160.0 \\
188.5 \\
265.0 \\
274.0\end{array}$ & $\begin{array}{l}60.0 \\
90.0 \\
90.0 \\
60.0 \\
90.0\end{array}$ & $\begin{array}{r}371.8 \\
94.0 \\
196.0 \\
272.5 \\
280.0\end{array}$ & $\begin{array}{l}48.7 .5 \\
196.0 \\
272.5 \\
280.0 \\
390.5\end{array}$ & $\begin{array}{l}45.0 \\
55.0 \\
50.0 \\
75.0 \\
20.0\end{array}$ & $\begin{array}{l}12.0 \\
12.0 \\
12.0 \\
12.0 \\
12.0\end{array}$ & $\begin{array}{l}10.0 \\
10.0 \\
10.0 \\
10.0 \\
10.0\end{array}$ & $\begin{array}{l}58.0 \\
59.5 \\
62.0 \\
56.0 \\
58.0\end{array}$ \\
\hline & & 3.1 .31 & 274.0 & 290.0 & 90.0 & 390.5 & 493.5 & 55.0 & 12.0 & 10.0 & 59.0 \\
\hline
\end{tabular}




\section{TABLE XXVIII}

SHORTENED CORE BARREL TEST GROUP 4.4

TRANSPARENT VESSEL TEST OATA FOR TESTS 4.4 .1 TO $4.4 .11--0.49-$ IN. DGHNCOMER GAP SUMMARY OF CALCULATED RESULTS

\begin{tabular}{|c|c|c|c|c|c|c|c|c|c|c|}
\hline $\begin{array}{c}\text { TEST } \\
\text { NO }\end{array}$ & $\begin{array}{l}\text { DOWNC JMER } \\
\text { AIR FLOWW } \\
\text { (LB/SEC) }\end{array}$ & $\begin{array}{l}\text { DOWNCOMER } \\
\text { AIR OENSITY } \\
\text { (LB/FT**3) }\end{array}$ & $\begin{array}{l}\text { LDWER PLENUM } \\
\text { PRESSURE } \\
\text { (PSIA) }\end{array}$ & $\begin{array}{l}\text { COLD LEG } \\
\text { AIR FLOH } \\
(L B / S E C)\end{array}$ & $\begin{array}{l}\text { DOWNCOMER } \\
\text { WATER FLOW } \\
\text { (LB/SEC) }\end{array}$ & $\begin{array}{l}\text { HATER FLOW } \\
\text { CONT INUITY }\end{array}$ & $\begin{array}{c}\text { J-AIR } \\
\text { DOHNCOMER } \\
\text { (FT/SEC) }\end{array}$ & $\begin{array}{l}\text { J-WATER } \\
\text { DOWNCOMER } \\
\text { (FT/SEC) }\end{array}$ & $(J G *) * 1 / 2$ & $(J 6 *) * * 1 / 2$ \\
\hline $\begin{array}{l}4.4 \cdot 1 \\
4.4: 2 \\
4.4: 3 \\
4.4: 5 \\
4.4: 5\end{array}$ & $\begin{array}{l}0.0153 \\
0.0076 \\
0.0075 \\
0.0156 \\
0.0224\end{array}$ & $\begin{array}{l}0.1168 \\
0.1164 \\
0.1161 \\
0.1164 \\
0.1164\end{array}$ & $\begin{array}{l}22.33 \\
22.33 \\
22.33 \\
22.33 \\
22.33\end{array}$ & $\begin{array}{l}0.0 \\
0.0 \\
0.0 \\
0.0 \\
0.0\end{array}$ & $\begin{array}{l}3.813 \\
4.600 \\
4.200 \\
3.637 \\
3.125\end{array}$ & $\begin{array}{l}0.990 \\
1.000 \\
1.007 \\
1.005 \\
0.993\end{array}$ & $\begin{array}{l}2.03 \\
1.02 \\
1.00 \\
2.08 \\
2.99\end{array}$ & $\begin{array}{l}0.949 \\
1.145 \\
1.045 \\
0.905 \\
0.778\end{array}$ & $\begin{array}{l}0.2328 \\
0.1647 \\
0.1629 \\
0.2355 \\
0.2819\end{array}$ & $\begin{array}{l}0.7646 \\
0.8398 \\
0.8025 \\
0.7468 \\
0.6922\end{array}$ \\
\hline $\begin{array}{l}4.4 \cdot 6 \\
4.4 \cdot 7 \\
4.4 \cdot 9 \\
4.4 .99 \\
4.4 .10\end{array}$ & $\begin{array}{l}0.0299 \\
0.0469 \\
0.0763 \\
0.1053 \\
0.1379\end{array}$ & $\begin{array}{l}0.1166 \\
0.1164 \\
0.1166 \\
0.1166 \\
0.1164\end{array}$ & $\begin{array}{l}22.33 \\
22.33 \\
22.33 \\
22.33 \\
22.33\end{array}$ & $\begin{array}{l}0.0 \\
0.0 \\
0.0 \\
0.0 \\
0.0\end{array}$ & $\begin{array}{l}2.857 \\
2.310 \\
1.600 \\
0.817 \\
0.356\end{array}$ & $\begin{array}{l}1.006 \\
0.989 \\
2.248 \\
1.043 \\
0.996\end{array}$ & $\begin{array}{r}3.98 \\
6.15 \\
10.23 \\
14.02 \\
18.40\end{array}$ & $\begin{array}{l}0.711 \\
0.575 \\
0.399 \\
0.203 \\
0.089\end{array}$ & $\begin{array}{l}0.3255 \\
0.4043 \\
0.5219 \\
0.6110 \\
0.6996\end{array}$ & $\begin{array}{l}0.6619 \\
0.5951 \\
0.4953 \\
0.3539 \\
0.2337\end{array}$ \\
\hline 4.4 .11 & 0.1726 & 0.1164 & 22.33 & 0.0 & 0.156 & 0.984 & 23.04 & 0.039 & 0.7829 & 0.1548 \\
\hline
\end{tabular}


TABLE XXVIII (Contd.)

SHORTENED CORE BARREL TEST GROUP 4.4

EXPEPIMENTAL IEASURMENTS EOR TESTS 4.4 .1 TO $4.4 .11--0.49-I N$. DOHNCOMER GAP

\begin{tabular}{|c|c|c|c|c|c|c|c|c|c|c|}
\hline $\begin{array}{c}\text { TEST } \\
\text { NO }\end{array}$ & $\begin{array}{l}R M-C W-1 \\
(C, D \cdot 1)\end{array}$ & $\begin{array}{c}R . M-C(N-2 \\
(G P M)\end{array}$ & $\begin{array}{c}F T-C W-1 \\
(G P M)\end{array}$ & $\begin{array}{c}F T-C H-2 \\
(G P M)\end{array}$ & $\begin{array}{l}R M-H r,-1 \\
\cdot(C F M)\end{array}$ & $\begin{array}{c}P M-H G-2 \\
(C F M)\end{array}$ & $\begin{array}{c}Q M-H G-3 \\
(C F M)\end{array}$ & $\begin{array}{c}\text { RM-HG-4 } \\
\text { (CFM) }\end{array}$ & $\begin{array}{l}F T-H G-1 \\
\text { (CFM) }\end{array}$ & $\begin{array}{c}F T-H G-2 \\
(C F M)\end{array}$ \\
\hline $\begin{array}{l}4.4 .1 \\
4.4 .5 \\
4.4 .5 \\
4.4 .4 \\
4.4 .5\end{array}$ & $\begin{array}{l}30.0 \\
30.0 \\
30.0 \\
30.0 \\
28.0\end{array}$ & $\begin{array}{r}10.0 \\
10.0 \\
0.0 \\
0.0 \\
0.0\end{array}$ & $\begin{array}{l}0.0 \\
0.0 \\
0.0 \\
0.0 \\
0.0\end{array}$ & $\begin{array}{l}0.0 \\
0.0 \\
0.0 \\
0.0 \\
0.0\end{array}$ & $\begin{array}{l}0.0 \\
0.0 \\
0.0 \\
0.0 \\
0.0\end{array}$ & $\begin{array}{c}10.00 \\
0.0 \\
0.0 \\
0.0 \\
0.0\end{array}$ & $\begin{array}{l}0.0 \\
5.00 \\
5.00 \\
10.00 \\
15.00\end{array}$ & $\begin{array}{l}0.0 \\
0.0 \\
0.0 \\
0.0 \\
0.0\end{array}$ & $\begin{array}{l}0.0 \\
0.0 \\
0.0 \\
0.0 \\
0.0\end{array}$ & $\begin{array}{l}0.0 \\
0.0 \\
0.0 \\
0.0 \\
0.0\end{array}$ \\
\hline $\begin{array}{l}4.4 .6 \\
4.4 .7 \\
4.4 .78 \\
4.4 .09 \\
4.4 .10\end{array}$ & $\begin{array}{r}25.0 \\
22.0 \\
15.0 \\
10.0 \\
3.0\end{array}$ & $\begin{array}{l}0.0 \\
0.0 \\
0.0 \\
0.0 \\
0.0\end{array}$ & $\begin{array}{l}0.0 \\
0.0 \\
0.0 \\
0.0 \\
0.0\end{array}$ & $\begin{array}{l}0.0 \\
0.0 \\
0.0 \\
0.0 \\
0.0\end{array}$ & $\begin{array}{c}0.0 \\
0.0 \\
0.0 \\
20.00 \\
40.00\end{array}$ & $\begin{array}{l}20.00 \\
30.00 \\
50.00 \\
50.00 \\
50.00\end{array}$ & $\begin{array}{l}0.0 \\
0.0 \\
0.0 \\
0.0 \\
0.0\end{array}$ & $\begin{array}{l}0.0 \\
0.0 \\
0.0 \\
0.0 \\
0.0\end{array}$ & $\begin{array}{l}0.0 \\
0.0 \\
0.0 \\
0.0 \\
0.0\end{array}$ & $\begin{array}{l}0.0 \\
0.0 \\
0.0 \\
0.0 \\
0.0\end{array}$ \\
\hline 4.4 .11 & 4.0 & 0.0 & 0.0 & 0.0 & 60.00 & 50.00 & 0.0. & 0.0 & 0.0 & 0.0 \\
\hline $\begin{array}{c}\text { TEST } \\
\text { NO }\end{array}$ & $\begin{array}{l}P-C W-1 \\
(P S I G)\end{array}$ & $\begin{array}{l}P-C: N-2 \\
\text { (.PSI }(G)\end{array}$ & $\begin{array}{l}P-H G-1 \\
(P S I G)\end{array}$ & $\begin{array}{l}P-H G-2 \\
\text { (PSIG) }\end{array}$ & $\begin{array}{l}P-H S-3 \\
\text { (PSIG) }\end{array}$ & $\begin{array}{l}T F-C W-1 \\
(D E G F)\end{array}$ & $\begin{array}{l}\text { TF-HG- } 1 \\
(D E G F)\end{array}$ & $\begin{array}{l}T F-H G-2 \\
(\cap E G \quad F)\end{array}$ & $\begin{array}{l}\text { TF-HG-3 } \\
\text { (DES F) }\end{array}$ & $\begin{array}{l}\text { P-BARD } \\
\text { (PSIA) }\end{array}$ \\
\hline $\begin{array}{l}4.4 \cdot 1 \\
4.4 \cdot \frac{1}{2} \\
4.4 \cdot 3 \\
4.4 \cdot 4 \\
4.4 \cdot 5\end{array}$ & $\begin{array}{l}1.0 \\
j .0 \\
j .0 \\
j .0 \\
j .0\end{array}$ & $\begin{array}{r}10.0 \\
10.0 \\
9.2 \\
10.0 \\
9.0\end{array}$ & $\begin{array}{r}10.0 \\
10.0 \\
9.0 \\
11.0 \\
9.0\end{array}$ & $\begin{array}{l}0.0 \\
0.0 \\
0.0 \\
0.0 \\
0.0\end{array}$ & $\begin{array}{l}0.0 \\
0.0 \\
0.0 \\
0.0 \\
0.0\end{array}$ & $\begin{array}{l}56.0 \\
58.0 \\
59.0 \\
58.0 \\
58.0\end{array}$ & $\begin{array}{l}76.0 \\
77.0 \\
77.0 \\
77.0 \\
78.0\end{array}$ & $\begin{array}{l}0.0 \\
0.0 \\
0.0 \\
0.0 \\
0.0\end{array}$ & $\begin{array}{l}0.0 \\
0.0 \\
0.0 \\
0.0 \\
0.0\end{array}$ & $\begin{array}{l}12.3 \\
12.3 \\
12.3 \\
12.3 \\
12.3\end{array}$ \\
\hline $\begin{array}{l}4.4 .6 \\
4.4 .7 \\
4.4 .3 \\
4.4 .9 \\
4.4 .1 .]\end{array}$ & $\begin{array}{l}0.0 \\
0.0 \\
0.0 \\
0.0 \\
0.0\end{array}$ & $\begin{array}{r}9.0 \\
10.0 \\
9.7 \\
9.0 \\
9.7\end{array}$ & $\begin{array}{r}9.0 \\
10.0 \\
9.7 \\
9.0 \\
10.0\end{array}$ & $\begin{array}{l}0.0 \\
0.0 \\
0.0 \\
0.0 \\
0.0\end{array}$ & $\begin{array}{l}0.0 \\
0.0 \\
0.0 \\
0.0 \\
0.0\end{array}$ & $\begin{array}{l}57.0 \\
57.0 \\
57.0 \\
57.0 \\
57.0\end{array}$ & $\begin{array}{l}77.0 \\
77.0 \\
79.0 \\
82.0 \\
89.0\end{array}$ & $\begin{array}{l}0.0 \\
0.0 \\
0.0 \\
0.0 \\
0.0\end{array}$ & $\begin{array}{l}0.0 \\
0.0 \\
0.0 \\
0.0 \\
0.0\end{array}$ & $\begin{array}{l}12.3 \\
12.3 \\
12.3 \\
12.3 \\
12.3\end{array}$ \\
\hline 4.4 .11 & 3.0 & 10.0 & 11.0 & 0.0 & -0.0 & 58.0 & 92.5 & 0.0 & 0.0 & 12.3 \\
\hline
\end{tabular}


TABLE XXVIII (Contdi.)

SHORTENED CORE BẠREL TEST GROQUP 4.4

EXPERIMENTAL MEASURMENTS FOR TESTS 4.4 .1 TO: 4.4 .11 -- 0:49-1N. DONACOMER GAP.

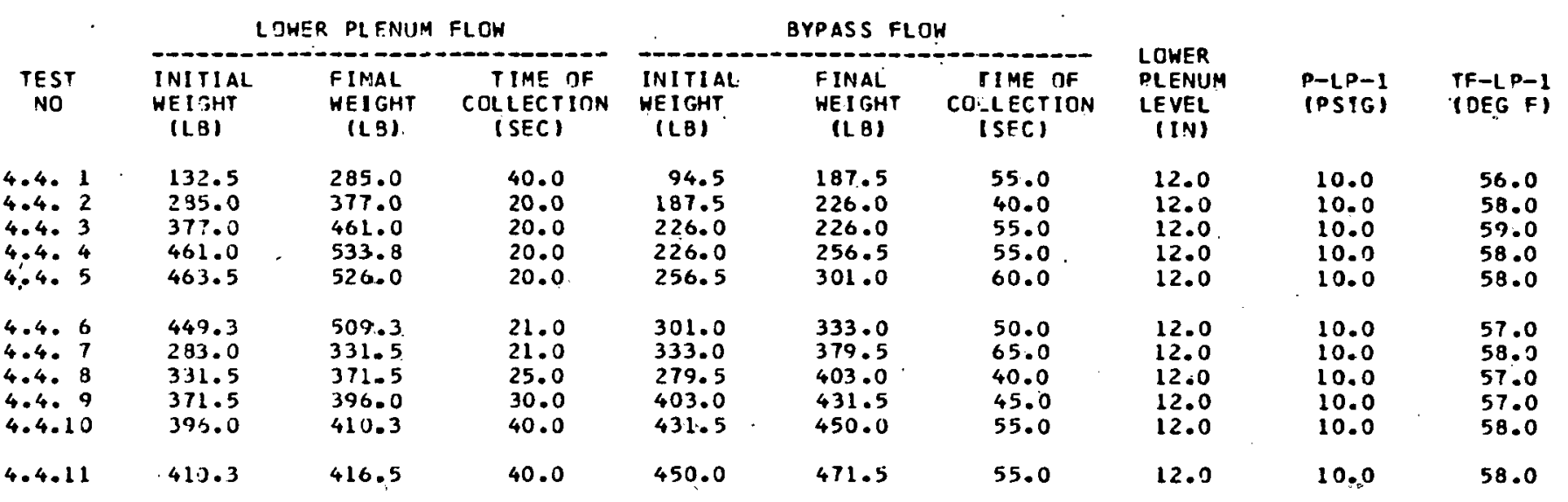


TABLE XXIX

SHORTENED CORE BARREL TEST GROUP 4.5

TRANSPARENT VESSEL TEST CATA FOP. TESTS 4.5 .1 TO $4.5 .12--0.49-1$ N. DOWNCOMER GAP SUMMARY JF CALCULATED RESULTS

\begin{tabular}{|c|c|c|c|c|c|c|c|c|c|c|}
\hline $\begin{array}{l}\text { TEST } \\
\text { NNO }\end{array}$ & $\begin{array}{l}\text { DOWNCOMER } \\
\text { AIR FLITW } \\
\text { (LB/SEC) }\end{array}$ & $\begin{array}{l}\text { DOWNCJMER } \\
\text { AIR DENSITY } \\
\text { (LB/FT } \$ 3)\end{array}$ & $\begin{array}{l}\text { LOWER PLENUM } \\
\text { PRESSURE } \\
\text { (PSIA) }\end{array}$ & $\begin{array}{l}\text { COLO LEG } \\
\text { AIR FLOW } \\
(L B / S E C)\end{array}$ & $\begin{array}{l}\text { DOWNCOMER } \\
\text { HATER FLOW } \\
\text { (LB/SEC) }\end{array}$ & $\begin{array}{l}\text { WATER FLDW } \\
\text { CONTINUITY }\end{array}$ & $\begin{array}{c}\text { J-AIR } \\
\text { DOHNOMER } \\
\text { (FT/SEC) }\end{array}$ & $\begin{array}{l}\text { J-WATER } \\
\text { DOINCOMEP. } \\
\text { (FT/SEC) }\end{array}$ & $(J g *) * 1 / 2$ & $(J L *) * 1 / 2$ \\
\hline $\begin{array}{l}4.5 \cdot 1 \\
4.5 \cdot 2 \\
4.5 \cdot 3 \\
4.5 \cdot 4 \\
4.5 \cdot 5\end{array}$ & $\begin{array}{l}0.0227 \\
0.0235 \\
0.0156 \\
0.0303 \\
0.0452\end{array}$ & $\begin{array}{l}0.1174 \\
0.1165 \\
0.1167 \\
0.1165 \\
0.1167\end{array}$ & $\begin{array}{l}22.39 \\
22.39 \\
22.39 \\
22.39 \\
22.39\end{array}$ & $\begin{array}{l}0.0 \\
0.0 \\
0.0 \\
0.0 \\
0.0\end{array}$ & $\begin{array}{l}3.375 \\
3.237 \\
3.712 \\
2.762 \\
2.407\end{array}$ & $\begin{array}{l}1.008 \\
1.018 \\
1.008 \\
0.996 \\
1.015\end{array}$ & $\begin{array}{l}3.01 \\
3.13 \\
2.03 \\
4.05 \\
6.01\end{array}$ & $\begin{array}{l}0.940 \\
0.806 \\
0.924 \\
0.388 \\
0.599\end{array}$ & $\begin{array}{l}0.2835 \\
0.2885 \\
0.2355 \\
0.3281 \\
0.4002\end{array}$ & $\begin{array}{l}0.7194 \\
0.7046 \\
0.7545 \\
0.6508 \\
0.6075\end{array}$ \\
\hline $\begin{array}{l}4.5 \cdot 6 \\
4.5 \cdot 7 \\
4.5 \cdot 8 \\
4.5 .99 \\
4.5 .10\end{array}$ & $\begin{array}{l}0.0603 \\
0.0767 \\
j .1097 \\
0.1395 \\
0.1533\end{array}$ & $\begin{array}{l}0.1165 \\
0.1156 \\
0.1165 \\
0.1167 \\
0.11165\end{array}$ & $\begin{array}{l}22.39 \\
22.39 \\
22.39 \\
22.39 \\
22.39\end{array}$ & $\begin{array}{l}0.0 \\
0.0 \\
0.0 \\
0.0 \\
0.0\end{array}$ & $\begin{array}{l}1.980 \\
1.556 \\
0.756 \\
0.400 \\
0.262\end{array}$ & $\begin{array}{l}0.998 \\
1.007 \\
1.023 \\
1.067 \\
1.025\end{array}$ & $\begin{array}{r}8.05 \\
10.31 \\
14.63 \\
18.56 \\
20.45\end{array}$ & $\begin{array}{l}0.493 \\
0.387 \\
0.189 \\
0.100 \\
0.065\end{array}$ & $\begin{array}{l}0.4627 \\
0.5227 \\
0.5241 \\
0.7032 \\
0.7377\end{array}$ & $\begin{array}{l}0.5510 \\
0.4884 \\
0.3404 \\
0.2477 \\
0.2006\end{array}$ \\
\hline $\begin{array}{l}4.5 .11 \\
4.5 .12\end{array}$ & $\begin{array}{l}0.1694 \\
0.1934\end{array}$ & $\begin{array}{l}0.1165 \\
0.1160\end{array}$ & $\begin{array}{l}22.39 \\
22.39\end{array}$ & $\begin{array}{l}0.0 \\
0.0\end{array}$ & $\begin{array}{l}0.169 \\
0.125\end{array}$ & $\begin{array}{l}1.018 \\
1.007\end{array}$ & $\begin{array}{l}22.59 \\
24.56\end{array}$ & $\begin{array}{l}0.042 \\
0.031\end{array}$ & $\begin{array}{l}0.7754 \\
0.8076\end{array}$ & $\begin{array}{l}0.1611 \\
0.1384\end{array}$ \\
\hline
\end{tabular}




\section{TABLE XXIX (Contd.)}

SHORTENED CORE BARREL TEST :GROTP 4.5

EXPERIMENTAL MEASURMENTS FOR TESTS 4.5 .1 TO $4.5 .12--0.49-1$ IN. DOWNCOMER GAP

\begin{tabular}{|c|c|c|c|c|c|c|c|c|c|c|}
\hline $\begin{array}{c}\text { TEST } \\
N S B\end{array}$ & $\begin{array}{c}R M-C W-1 \\
(G P M)\end{array}$ & $\begin{array}{c}\mathrm{R}(1-\mathrm{CH}-2 \\
(\mathrm{GPM})\end{array}$ & $\begin{array}{c}F T-C H-1 \\
(G P M)\end{array}$ & $\begin{array}{c}F T-C H-2 \\
(G P M)\end{array}$ & $\begin{array}{c}R M-H G-1 \\
(C F M)\end{array}$ & $\begin{array}{c}R M-H G-2 \\
\text { (CFM) }\end{array}$ & $\begin{array}{c}R M-H G-3 \\
(C F M)\end{array}$ & $\begin{array}{c}\text { RM-HG-4 } \\
(C F M)\end{array}$ & $\begin{array}{c}F T-H G-1 \\
(C F M)\end{array}$ & $\begin{array}{c}F T-H G-2 \\
(C F M)\end{array}$ \\
\hline $\begin{array}{l}4.5 \cdot 1 \\
4.5: 5 \\
4.5: 3 \\
4.5: 5 \\
4.5: 5\end{array}$ & $\begin{array}{l}30.0 \\
30.0 \\
3.0 .0 \\
25.0 \\
20.5\end{array}$ & $\begin{array}{r}10.0 \\
0.0 \\
0.0 \\
0.0 \\
0.0\end{array}$ & $\begin{array}{l}0.0 \\
0.0 \\
0.0 \\
0.0 \\
0.0\end{array}$ & $\begin{array}{l}0.0 \\
0.0 \\
0.0 \\
0.0 \\
0.0\end{array}$ & $\begin{array}{l}0.0 \\
0.0 \\
0.0 \\
0.0 \\
0.0\end{array}$ & $\begin{array}{l}15.00 \\
15.00 \\
10.00 \\
20.00 \\
30.00\end{array}$ & $\begin{array}{l}0.0 \\
0.0 \\
0.0 \\
0.0 \\
0.0\end{array}$ & $\begin{array}{l}0.0 \\
0.0 \\
0.0 \\
0.0 \\
0.0\end{array}$ & $\begin{array}{l}0.0 \\
0.0 \\
0.0 \\
0.0 \\
0.0\end{array}$ & $\begin{array}{l}0.0 \\
0.0 \\
0.0 \\
0.0 \\
0.0\end{array}$ \\
\hline $\begin{array}{l}4.5 .6 \\
4.5 .7 \\
4.5 .8 \\
4.5 .99 \\
4.5 .10\end{array}$ & $\begin{array}{r}20.0 \\
15.0 \\
10.0 \\
5.0 \\
5.0\end{array}$ & $\begin{array}{l}0.0 \\
0.0 \\
0.0 \\
0.0 \\
0.0\end{array}$ & $\begin{array}{l}0.0 \\
0.0 \\
0.0 \\
0.0 \\
0.0\end{array}$ & $\begin{array}{l}0.0 \\
0.0 \\
0.0 \\
0.0 \\
0.0\end{array}$ & $\begin{array}{l}0.0 \\
0.0 \\
70.00 \\
90.00 \\
70.00\end{array}$ & $\begin{array}{c}40.00 \\
50.00 \\
0.0 \\
0.0 \\
30.00\end{array}$ & $\begin{array}{l}0.0 \\
0.0 \\
0.0 \\
0.0 \\
0.0\end{array}$ & $\begin{array}{l}0.0 \\
0.0 \\
0.0 \\
0.0 \\
0.0\end{array}$ & $\begin{array}{l}0.0 \\
0.0 \\
0.0 \\
0.0 \\
0.0\end{array}$ & $\begin{array}{l}0.0 \\
0.0 \\
0.0 \\
0.0 \\
0.0\end{array}$ \\
\hline $\begin{array}{l}4.5 .11 \\
4.5 .12\end{array}$ & $\begin{array}{l}5.0 \\
5.0\end{array}$ & $\begin{array}{r}0.0 \\
0.0\end{array}$ & $\begin{array}{l}0.0 \\
0.0\end{array}$ & $\begin{array}{l}0.0 \\
0.0\end{array}$ & $\begin{array}{l}60.00 \\
60.00\end{array}$ & $\begin{array}{l}50.00 \\
60.00\end{array}$ & $\begin{array}{l}0.0 \\
0.0\end{array}$ & $\begin{array}{l}0.0 \\
0.0\end{array}$ & $\begin{array}{l}0.0 \\
0.0\end{array}$ & $\begin{array}{l}0.0 \\
0.0\end{array}$ \\
\hline $\begin{array}{l}\text { TEST } \\
\text { NO }\end{array}$ & $\begin{array}{l}P-C W-1 \\
(P S I G)\end{array}$ & $\begin{array}{l}P-C W-2 \\
\text { (PSIG) }\end{array}$ & $\begin{array}{l}P-H G-1 \\
(P S I G)\end{array}$ & $\begin{array}{l}P-H G-2 \\
(P S I G)\end{array}$ & $\begin{array}{l}P-H G-3 \\
(P S I G)\end{array}$ & $\begin{array}{l}\text { TF-CH-I } \\
\text { (DEG F) }\end{array}$ & $\begin{array}{l}T F-H G-1 \\
(D E G F)\end{array}$ & $\begin{array}{l}T F-H G-2 \\
(D E G \quad F)\end{array}$ & $\begin{array}{l}\text { TF-HG-3 } \\
\text { (DEG F }\end{array}$ & $\begin{array}{l}\text { P-BARR } \\
\text { (PSIA) }\end{array}$ \\
\hline $\begin{array}{l}4.5 .1 \\
4.5: 2 \\
4.5 .3 \\
4.5 .4 \\
4.5 .5\end{array}$ & $\begin{array}{l}0.0 \\
3.0 \\
1.0 \\
0.0 \\
0.0\end{array}$ & $\begin{array}{r}9.5 \\
10.0 \\
10.0 \\
9.5 \\
9.0\end{array}$ & $\begin{array}{r}9.5 \\
11.0 \\
11.0 \\
9.5 \\
9.0\end{array}$ & $\begin{array}{l}0.0 \\
0.0 \\
0.0 \\
0.0 \\
0.0\end{array}$ & $\begin{array}{l}0.0 \\
0.0 \\
0.0 \\
0.0 \\
0.0\end{array}$ & $\begin{array}{l}55.0 \\
58.0 \\
58.0 \\
59.0 \\
59.0\end{array}$ & $\begin{array}{l}75.0 \\
77.0 \\
77.0 \\
74.0 \\
75.0\end{array}$ & $\begin{array}{l}0.0 \\
0.0 \\
0.0 \\
0.0 \\
0.0\end{array}$ & $\begin{array}{l}0.0 \\
0.0 \\
0.0 \\
0.0 \\
0.0\end{array}$ & $\begin{array}{l}12.4 \\
12.4 \\
12.4 \\
12.4 \\
12.4\end{array}$ \\
\hline $\begin{array}{l}4.5 .6 \\
4.5: 7 \\
4.5: 9 \\
4.5 .99 \\
4.5 .10\end{array}$ & $\begin{array}{l}0.0 \\
0.0 \\
0.0 \\
1.0 \\
0.0\end{array}$ & $\begin{array}{l}3 . ? \\
8.0 \\
9.5 \\
3.5 \\
9.5\end{array}$ & $\begin{array}{r}9.0 \\
9.5 \\
10.0 \\
9.0 \\
9.5\end{array}$ & $\begin{array}{l}0.0 \\
0.0 \\
0.0 \\
0.0 \\
0.0\end{array}$ & $\begin{array}{l}0.0 \\
0.0 \\
0.0 \\
0.0 \\
0.0 \\
0.0\end{array}$ & $\begin{array}{l}58.0 \\
61.0 \\
58.0 \\
58.0 \\
59.0\end{array}$ & $\begin{array}{l}77.0 \\
76.0 \\
77.0 \\
83.0 \\
86.0\end{array}$ & $\begin{array}{l}0.0 \\
0.0 \\
0.0 \\
0.0 \\
0.0\end{array}$ & $\begin{array}{l}0.0 \\
0.0 \\
0.0 \\
0.0 \\
0.0\end{array}$ & $\begin{array}{l}12.4 \\
12.4 \\
12.4 \\
12.4 \\
12.4\end{array}$ \\
\hline $\begin{array}{l}4.5 .11 \\
4.5 .12\end{array}$ & $\begin{array}{l}0.0 \\
0.0\end{array}$ & $\begin{array}{l}9.7 \\
9.5\end{array}$ & $\begin{array}{r}10.0 \\
9.5 \\
9.5\end{array}$ & $\begin{array}{l}0.0 \\
0.0\end{array}$ & $\begin{array}{l}0.0 \\
0.0 \\
0.0\end{array}$ & $\begin{array}{l}59.0 \\
60.0\end{array}$ & $\begin{array}{l}91.5 \\
91.5\end{array}$ & $\begin{array}{l}0.0 \\
0.0\end{array}$ & $\begin{array}{l}0.0 \\
0.0\end{array}$ & $\begin{array}{l}12.4 \\
12.4\end{array}$ \\
\hline
\end{tabular}


TABLE XXIX (Contd.)

SHORTENED CORE BARREL TEST GROUP 4.5

EXPERINENTAL MEASURMENTS FDR TESTS 4.5 .1 TI $4.5 .12-0.49-1$ - D. DOHNCOMER GAP

\begin{tabular}{|c|c|c|c|c|c|c|c|c|c|}
\hline \multirow[b]{2}{*}{$\begin{array}{l}\text { TEST } \\
\text { ND }\end{array}$} & \multicolumn{3}{|c|}{ LUWER PLENUM RLOW } & \multicolumn{3}{|c|}{ BYPASS FLOW } & \multirow[b]{2}{*}{$\begin{array}{l}\text { LOWER } \\
\text { PLENUM } \\
\text { LEVEL } \\
\text { |IN| }\end{array}$} & \multirow[b]{2}{*}{$\begin{array}{l}P-L P-1 \\
(P S \mid G)\end{array}$} & \multirow[b]{2}{*}{$\begin{array}{l}T F-(P-1 \\
(D E G F)\end{array}$} \\
\hline & $\begin{array}{l}\text { INITIAL } \\
\text { HEIGHT } \\
\text { (LB) }\end{array}$ & $\begin{array}{l}\text { FINAL } \\
\text { HEIGHT } \\
\text { (LB) }\end{array}$ & $\begin{array}{l}\text { TIME OF } \\
\text { COLLECTION } \\
\text { (SEC) }\end{array}$ & $\begin{array}{l}\text { INITIAL } \\
\text { WEIGHT } \\
\text { (LB) }\end{array}$ & $\begin{array}{l}\text { FINAL } \\
\text { WEIGHT } \\
\text { IL BI }\end{array}$ & $\begin{array}{l}\text { TIME OF } \\
\text { COLLECTION } \\
\text { (SEC) }\end{array}$ & & & \\
\hline $\begin{array}{l}4.5 .1 \\
4.5 .2 \\
4.5 .3 \\
4.5 .5 \\
4.5 .5\end{array}$ & $\begin{array}{l}133.0 \\
207.5 \\
265.3 \\
377.3 \\
352.5\end{array}$ & $\begin{array}{l}200.5 \\
265.3 \\
339.5 \\
432.5 \\
436.8\end{array}$ & $\begin{array}{l}20.0 \\
20.0 \\
20.0 \\
20.0 \\
35.0\end{array}$ & $\begin{array}{r}91.0 \\
191.5 \\
242.0 \\
330.0 \\
365.0\end{array}$ & $\begin{array}{l}191.5 \\
242.0 \\
274.0 \\
365.0 \\
389.3\end{array}$ & $\begin{array}{l}45.0 \\
50.0 \\
65.0 \\
50.0 \\
50.0\end{array}$ & $\begin{array}{l}12.0 \\
12.0 \\
12.0 \\
12.0 \\
12.0\end{array}$ & $\begin{array}{l}10.0 \\
10.0 \\
10.0 \\
10.0 \\
10.0\end{array}$ & $\begin{array}{l}55.0 \\
59.0 \\
58.0 \\
59.0 \\
58.0\end{array}$ \\
\hline $\begin{array}{l}4.5 .6 \\
4.5 .7 \\
4.5 .8 \\
4.5 .99 \\
4.5 .10\end{array}$ & $\begin{array}{l}375.5 \\
133.0 \\
203.0 \\
237.0 \\
261.0\end{array}$ & $\begin{array}{l}425.0 \\
203.0 \\
237.0 \\
261.0 \\
276.8\end{array}$ & $\begin{array}{l}25.0 \\
45.0 \\
45.0 \\
60.0 \\
60.0\end{array}$ & $\begin{array}{r}389.3 \\
94.0 \\
124.0 \\
164.0 \\
184.5\end{array}$ & $\begin{array}{l}425.0 \\
124.0 \\
164.0 \\
184.5 \\
211.5\end{array}$ & $\begin{array}{l}45.0 \\
53.0 \\
60.0 \\
60.0 \\
60.0\end{array}$ & $\begin{array}{l}12.0 \\
12.0 \\
12.0 \\
12.0 \\
12.0\end{array}$ & $\begin{array}{l}10.0 \\
10.0 \\
10.0 \\
10.0 \\
10.0\end{array}$ & $\begin{array}{l}59.0 \\
63.0 \\
59.0 \\
58.0 \\
59.0\end{array}$ \\
\hline $\begin{array}{l}4.5 .11 \\
4.5 .12\end{array}$ & $\begin{array}{l}276.3 \\
287.8\end{array}$ & $\begin{array}{l}287.8 \\
295.3\end{array}$ & $\begin{array}{l}65.0 \\
60.0\end{array}$ & $\begin{array}{l}211.5 \\
246.5\end{array}$ & $\begin{array}{l}246.5 \\
281.0\end{array}$ & $\begin{array}{l}65.0 \\
60.0\end{array}$ & $\begin{array}{l}12.0 \\
12.0\end{array}$ & $\begin{array}{l}10.0 \\
10.0\end{array}$ & $\begin{array}{l}59.0 \\
61.0\end{array}$ \\
\hline
\end{tabular}




\section{TABLE XXX}

SHORTENED CORE BARREL TEST GROUP 4.6

TRANSPARENT VESSEL TEST DATA FOR TESTS 4.6.1 TO 4.6.48 -- 0.49-IN. DOUNCOMER GAP SUMMARY JF CALCULATED RESUL TS

\begin{tabular}{|c|c|c|c|c|c|c|c|c|c|c|}
\hline $\begin{array}{l}\text { TEST } \\
\text { NO }\end{array}$ & $\begin{array}{l}\text { OOHNC UMER } \\
\text { AIR FLOWH } \\
\text { LLB/SECI }\end{array}$ & 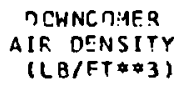 & $\begin{array}{l}\text { LOWEP PLENUM } \\
\text { PRESSURE } \\
\text { (PSIAI) }\end{array}$ & $\begin{array}{l}\text { COLD LEG } \\
\text { AIR FLLNW } \\
\text { (LB/SECI }\end{array}$ & $\begin{array}{l}\text { DOWNCOMER } \\
\text { HATER FLDH } \\
\text { (LB/SEC) }\end{array}$ & $\begin{array}{l}\text { WATER FLOWW } \\
\text { CONTINUITY }\end{array}$ & $\begin{array}{c}J-A I P \\
\text { OOWNCOMEP. } \\
\text { (FT/SEC) }\end{array}$ & $\begin{array}{l}\text { J-IAATER } \\
\text { DOWNCOMER } \\
\text { IFT/SEC) }\end{array}$ & $(J G * 1 * * 1 / 2$ & $(J L *) * 1 / 2$ \\
\hline $\begin{array}{l}4.6 \cdot 1 \\
4.6 .2 \\
4.6 .3 \\
4.6 .4 \\
4.6 .5\end{array}$ & $\begin{array}{l}0.0228 \\
0.0228 \\
0.0153 \\
0.0076 \\
0.0076\end{array}$ & $\begin{array}{l}0.1170 \\
0.1163 \\
0.1161 \\
0.1161 \\
0.1163\end{array}$ & $\begin{array}{l}22.31 \\
22.31 \\
22.31 \\
22.31 \\
22.31\end{array}$ & $\begin{array}{l}0.0 \\
0.0 \\
0.0 \\
0.0 \\
0.0\end{array}$ & $\begin{array}{l}3.313 \\
3.188 \\
3.688 \\
4.787 \\
4.600\end{array}$ & $\begin{array}{l}0.983 \\
0.990 \\
0.997 \\
0.987 \\
0.994\end{array}$ & $\begin{array}{l}3.03 \\
3.05 \\
2.05 \\
1.02 \\
1.01\end{array}$ & $\begin{array}{l}0.825 \\
0.793 \\
0.918 \\
1.132 \\
1.145\end{array}$ & $\begin{array}{l}0.2844 \\
0.2849 \\
0.2331 \\
0.1648 \\
0.1642\end{array}$ & $\begin{array}{l}0.7127 \\
0.6991 \\
0.7519 \\
0.8568 \\
0.8398\end{array}$ \\
\hline $\begin{array}{l}4.6 .6 \\
4.6 .7 \\
4.6 .8 \\
4.6 .99 \\
4.6 .10\end{array}$ & $\begin{array}{l}0.0309 \\
0.0456 \\
0.0761 \\
0.1059 \\
0.1067\end{array}$ & $\begin{array}{l}0.1152 \\
0.1163 \\
0.1163 \\
0.1163 \\
0.1163\end{array}$ & $\begin{array}{l}22.31 \\
22.31 \\
22.31 \\
22.31 \\
22.31\end{array}$ & $\begin{array}{l}0.0 \\
0.0 \\
0.0 \\
0.0 \\
0.0\end{array}$ & $\begin{array}{l}2.750 \\
2.330 \\
1.358 \\
1.321 \\
1.583\end{array}$ & $\begin{array}{l}1.014 \\
1.025 \\
1.025 \\
0.990 \\
1.044\end{array}$ & $\begin{array}{r}4.17 \\
6.09 \\
10.17 \\
14.14 \\
14.25\end{array}$ & $\begin{array}{l}0.635 \\
0.590 \\
0.338 \\
0.329 \\
0.394\end{array}$ & $\begin{array}{l}0.3322 \\
0.4023 \\
0.5200 \\
0.6133 \\
0.6156\end{array}$ & $\begin{array}{l}0.6494 \\
0.5977 \\
0.4564 . \\
0.4501 \\
0.4927\end{array}$ \\
\hline $\begin{array}{l}4.6 .11 \\
4.6 .12 \\
4.6 .13 \\
4.6 .14 \\
4.6 .15\end{array}$ & $\begin{array}{l}0.1364 \\
0.1665 \\
0.2004 \\
0.2325 \\
0.2075\end{array}$ & $\begin{array}{l}0.1163 \\
0.1163 \\
0.1162 \\
0.1161 \\
0.1210\end{array}$ & $\begin{array}{l}22.31 \\
22.31 \\
22.31 \\
22.31 \\
23.31\end{array}$ & $\begin{array}{l}0.0 \\
0.0 \\
0.0 \\
0.0 \\
0.0\end{array}$ & $\begin{array}{l}1.281 \\
0.817 \\
0.383 \\
0.461 \\
0.0\end{array}$ & $\begin{array}{l}1.044 \\
0.958 \\
0.983 \\
1.469 \\
1.013\end{array}$ & $\begin{array}{l}18.22 \\
22.24 \\
26.79 \\
31.11 \\
26.63\end{array}$ & $\begin{array}{l}0.319 \\
0.203 \\
0.035 \\
0.116 \\
0.0\end{array}$ & $\begin{array}{l}0.6960 \\
0.7690 \\
0.8439 \\
0.9091 \\
0.8499\end{array}$ & $\begin{array}{l}0.4432 \\
0.3539 \\
0.2424 \\
0.2675 \\
0.0\end{array}$ \\
\hline $\begin{array}{l}4.6 .16 \\
4.6 .17 \\
4.6 .18 \\
4.6 .19 \\
4.6 .20\end{array}$ & $\begin{array}{l}0.1989 \\
0.1632 \\
0.1647 \\
0.1362 \\
0.1343\end{array}$ & $\begin{array}{r}0.1157 \\
.1164 \\
.11164 \\
1.1163 \\
0.1163\end{array}$ & $\begin{array}{l}22.31 \\
22.31 \\
22.31 \\
22.31 \\
22.31\end{array}$ & $\begin{array}{l}0.0 \\
0.0 \\
0.0 \\
0.0 \\
0.0\end{array}$ & $\begin{array}{l}0.052 \\
0.096 \\
0.104 \\
0.239 \\
0.283\end{array}$ & $\begin{array}{l}0.997 \\
1.006 \\
0.996 \\
1.009 \\
1.001\end{array}$ & $\begin{array}{l}26.70 \\
21.78 \\
21.98 \\
18.19 \\
18.0 !\end{array}$ & $\begin{array}{l}0.013 \\
0.024 \\
0.026 \\
0.060 \\
0.071\end{array}$ & $\begin{array}{l}0.8416 \\
0.7612 \\
0.7647 \\
0.6954 \\
0.6921\end{array}$ & $\begin{array}{l}0.0897 \\
0.1212 \\
0.1264 \\
0.1915 \\
0.2084\end{array}$ \\
\hline $\begin{array}{l}4.6 .21 \\
4.6 .22 \\
4.6 .23 \\
4.6 .24 \\
4.6 .25\end{array}$ & $\begin{array}{l}0.2316 \\
0.1066 \\
0.0747 \\
0.0741 \\
0.0446\end{array}$ & $\begin{array}{l}0.1165 \\
0.1167 \\
0.1163 \\
0.1165 \\
0.1167\end{array}$ & $\begin{array}{l}22.31 \\
22.31 \\
22.31 \\
22.31 \\
22.31\end{array}$ & $\begin{array}{l}0.0 \\
0.0 \\
0.0 \\
0.0 \\
0.0\end{array}$ & $\begin{array}{l}0.533 \\
0.617 \\
1.111 \\
1.283 \\
2.350\end{array}$ & $\begin{array}{l}0.986 \\
1.006 \\
1.003 \\
1.01 .9 \\
1.011\end{array}$ & $\begin{array}{r}30.88 \\
14.19 \\
9.98 \\
9.89 \\
5.94\end{array}$ & $\begin{array}{l}0.133 \\
0.153 \\
0.277 \\
0.319 \\
0.585\end{array}$ & $\begin{array}{l}0.9067 \\
0.6149 \\
0.5151 \\
0.5128 \\
0.3977\end{array}$ & $\begin{array}{l}0.2860 \\
0.33075 \\
0.4128 \\
0.4436 \\
0.4003\end{array}$ \\
\hline $\begin{array}{l}4.0 .26 \\
4.6 .27 \\
4.4 .29 \\
4.6 .25 \\
4.6 .30\end{array}$ & $\begin{array}{l}0.1377 \\
0.1373 \\
0.1079 \\
0.1053 \\
0.0746\end{array}$ & $\begin{array}{l}0.1157 \\
0.1161 \\
0.1164 \\
0.1161 \\
0.1161\end{array}$ & $\begin{array}{l}22.31 \\
22.31 \\
22.31 \\
22.31 \\
22.32\end{array}$ & $\begin{array}{l}0.0 \\
0.0 \\
0.0 \\
0.0 \\
0.0\end{array}$ & $\begin{array}{l}0.211 \\
0.239 \\
0.418 \\
1.417 \\
1.258\end{array}$ & $\begin{array}{l}1.005 \\
1.014 \\
1.021 \\
1.050 \\
1.038\end{array}$ & $\begin{array}{r}18.49 \\
18.36 \\
14.40 \\
14.08 \\
9.99\end{array}$ & $\begin{array}{l}0.053 \\
0.059 \\
0.119 \\
0.353 \\
0.313\end{array}$ & $\begin{array}{l}0.7003 \\
0.6985 \\
0.6190 \\
0.6117 \\
0.5151\end{array}$ & $\begin{array}{l}0.1799 \\
0.1914 \\
0.2707 \\
0.4661 \\
0.4393\end{array}$ \\
\hline $\begin{array}{l}4.6 .31 \\
4.6 .32 \\
4.6 .33 \\
4.6 .34 \\
4.6 .35\end{array}$ & $\begin{array}{l}0.0291 \\
0.0302 \\
0.0149 \\
0.2007 \\
0.1983\end{array}$ & $\begin{array}{l}0.1164 \\
0.1165 \\
0.1165 \\
0.116 .3 \\
0.1147 .\end{array}$ & $\begin{array}{l}22.31 \\
22.31 \\
22.31 \\
22.31 \\
22.31\end{array}$ & $\begin{array}{l}0.0 \\
0.0 \\
0.0 \\
0.0 \\
0.0\end{array}$ & $\begin{array}{l}3.107 \\
2.908 \\
4.000 \\
0.421 \\
0.535\end{array}$ & $\begin{array}{l}1.002 \\
1.010 \\
0.977 \\
1.008 \\
0.932\end{array}$ & $\begin{array}{r}3.39 \\
4.02 \\
1.99 \\
26.87 \\
26.85\end{array}$ & $\begin{array}{l}0.773 \\
0.724 \\
0.996 \\
0.135 \\
0.133\end{array}$ & $\begin{array}{l}0.3215 \\
0.3272 \\
0.2303 \\
0.8449 \\
0.8421\end{array}$ & $\begin{array}{l}0.6902 \\
0.66678 \\
0.7832 \\
0.2540 \\
0.2863\end{array}$ \\
\hline $\begin{array}{l}4.6 .36 \\
4.6 .37 \\
4.6 .38 \\
4.6 .39 \\
4.6 .40\end{array}$ & $\begin{array}{l}0.1670 \\
0.1656 \\
0.1618 \\
0.1363 \\
0.1373\end{array}$ & $\begin{array}{l}0.1152 \\
0.1154 \\
0.1160 \\
0.1162 \\
0.1161\end{array}$ & $\begin{array}{l}22.31 \\
22.31 \\
22.31 \\
22.31 \\
22.31\end{array}$ & $\begin{array}{l}0.0 \\
0.0 \\
0.0 \\
0.0 \\
0.0\end{array}$ & $\begin{array}{l}0.161 \\
0.246 \\
0.637 \\
0.359 \\
1.350\end{array}$ & $\begin{array}{l}0.991 \\
1.021 \\
1.723 \\
1.016 \\
0.989 .\end{array}$ & $\begin{array}{l}22.51 \\
22.29 \\
21.66 \\
18.27 \\
18.37\end{array}$ & $\begin{array}{l}0.040 \\
0.061 \\
0.159 \\
0.089 \\
0.336\end{array}$ & $\begin{array}{l}0.7719 \\
0.7684 \\
0.7585 \\
0.6970 \\
0.6986\end{array}$ & $\begin{array}{l}0.1572 \\
0.1941 \\
0.3126 \\
0.2346 \\
0.4550\end{array}$ \\
\hline
\end{tabular}


TABLE XXX (Contd.)

SHORTENED CORE BARREL TEST GROUP 4.6

SUYMARY OF CALCULATED RESULTS FOR TESTS 4.6 .1 TO $4.6 .48--0.49$ IN. DOHNCOMER GAP

\begin{tabular}{|c|c|c|c|c|c|c|c|c|c|c|}
\hline $\begin{array}{l}\text { TEST } \\
\text { ND }\end{array}$ & $\begin{array}{l}\text { OJWNCOMEP } \\
\text { AIQ FI TW } \\
\text { (LB/SEC) }\end{array}$ & $\begin{array}{c}\text { THWNCRIMER } \\
\text { MIR DEIISITY } \\
\text { (LU,CT } \# 3)\end{array}$ & $\begin{array}{c}\text { LOHER PLENUM } \\
\text { PRESSUPE } \\
\text { (DSIAI }\end{array}$ & $\begin{array}{l}\text { CDLD LES } \\
\text { AIR FLTIN } \\
\text { (LI/SEC) }\end{array}$ & $\begin{array}{l}\text { DNWNCOOMEP } \\
\text { WATER FLOW } \\
\text { (LH/SEC) }\end{array}$ & $\begin{array}{l}\text { WATEP FLOH } \\
\text { CJNTINUITY }\end{array}$ & $\begin{array}{c}J-A I Q \\
\text { DOWNCAMEP. } \\
\text { (FT!SEC) }\end{array}$ & $\begin{array}{l}\text { J-HATER } \\
\text { ONWNETMER } \\
\text { (FT/SEC) }\end{array}$ & $(J G *) * 1 / 2$ & $(J L *) * 1 / 2$ \\
\hline $\begin{array}{l}4.6 .41 \\
4.6 .42 \\
4.6 .43 \\
4.6 .44 \\
4.6 .45\end{array}$ & $\begin{array}{l}0.1359 \\
0.1 .063 \\
0.1 .162 \\
0.1052 \\
0.0746\end{array}$ & $\begin{array}{l}0.1165 \\
3.1162 \\
0.1164 \\
3.1162 \\
0.1160\end{array}$ & $\begin{array}{l}22.31 \\
22.31 \\
22.31 \\
22.31 \\
22.31\end{array}$ & $\begin{array}{l}0.0 \\
0.0 \\
0.0 \\
0.0 \\
0.0\end{array}$ & $\begin{array}{l}0.469 \\
0.778 \\
0.787 \\
0.923 \\
1.700\end{array}$ & $\begin{array}{l}1.009 \\
1.026 \\
0.927 \\
1.035 \\
1.026\end{array}$ & $\begin{array}{r}18.13 \\
14.21 \\
14.18 \\
14.06 \\
9.98\end{array}$ & $\begin{array}{l}0.117 \\
0.194 \\
0.196 \\
0.230 \\
0.423\end{array}$ & $\begin{array}{l}0.6945 \\
0.6116 \\
0.6142 \\
0.6114 \\
0.5150\end{array}$ & $\begin{array}{l}0.2682 \\
0.3453 \\
0.3475 \\
0.3761 \\
0.5106\end{array}$ \\
\hline $\begin{array}{l}4.0 .46 \\
4.6 .47 \\
4.6 .48\end{array}$ & $\begin{array}{l}0.1952 \\
0.1956 \\
0.1975\end{array}$ & $\begin{array}{l}J .11 j a \\
0.1155 \\
.1154\end{array}$ & $\begin{array}{l}22.31 \\
22.31 \\
22.31\end{array}$ & $\begin{array}{l}0.0 \\
0.0 \\
0.0\end{array}$ & $\begin{array}{l}0.600 \\
0.392 \\
0.253\end{array}$ & $\begin{array}{l}1.018 \\
1.016 \\
1.272\end{array}$ & $\begin{array}{l}26.13 \\
26.31 \\
26.4 ?\end{array}$ & $\begin{array}{l}0.149 \\
0.097 \\
0.064\end{array}$ & $\begin{array}{l}0.8335 \\
0.8350 \\
0.8383\end{array}$ & $\begin{array}{l}0.3033 \\
0.2451 \\
0.1990\end{array}$ \\
\hline
\end{tabular}


$\underline{\text { TABLE XXX (Contd.) }}$

SHORTENED CORE BARREL TEST GROUP 4.6

EXPERIMENTAL ME ASURMENTS FOR TESTS 4.6 .1 TO $4.6 .48--0.49-$ IN. DONNCOMER GAP

\begin{tabular}{|c|c|c|c|c|c|c|c|c|c|c|}
\hline $\begin{array}{l}\text { TEST } \\
\text { NO }\end{array}$ & $\begin{array}{c}R M-C H-1 \\
(G P M)\end{array}$ & $\begin{array}{c}\text { RM-CH-2 } \\
\text { (GPM) }\end{array}$ & $\begin{array}{c}F T-C H-1 \\
(G P M)\end{array}$ & $\begin{array}{c}F T-C H-2 \\
(G P M)\end{array}$ & $\begin{array}{l}R M-H G-1 \\
(C F M)\end{array}$ & $\begin{array}{l}\text { RM-HG-2 } \\
\text { (CFM) }\end{array}$ & $\begin{array}{l}2 M-H G-3 \\
(C F, M)\end{array}$ & $\begin{array}{c}\text { RM-HG-4 } \\
(C F M)\end{array}$ & $\begin{array}{l}F T-H G-1 \\
(C F M)\end{array}$ & $\begin{array}{c}F T-H G-2 \\
(C F M)\end{array}$ \\
\hline $\begin{array}{l}4.6 .1 \\
4.6 .2 \\
4.6 .3 \\
4.6 .4 \\
4.6 .5\end{array}$ & $\begin{array}{l}30.0 \\
30.0 \\
30.0 \\
30.0 \\
30.0\end{array}$ & $\begin{array}{r}10.0 \\
0.0 \\
0.0 \\
10.0 \\
7.0\end{array}$ & $\begin{array}{l}0.0 \\
0.0 \\
0.0 \\
0.0 \\
0.0\end{array}$ & $\begin{array}{l}0.0 \\
0.0 \\
0.0 \\
0.0 \\
0.0\end{array}$ & $\begin{array}{l}0.0 \\
0.0 \\
0.0 \\
0.0 \\
0.0\end{array}$ & $\begin{array}{c}15.00 \\
15.00 \\
0.0 \\
0.0 \\
0.0\end{array}$ & $\begin{array}{c}0.0 \\
0.0 \\
10.00 \\
0.0 \\
0.0\end{array}$ & $\begin{array}{l}0.0 \\
0.0 \\
0.0 \\
5.00 \\
5.00\end{array}$ & $\begin{array}{l}0.0 \\
0.0 \\
0.0 \\
0.0 \\
0.0\end{array}$ & $\begin{array}{l}0.0 \\
0.0 \\
0.0 \\
0.0 \\
0.0\end{array}$ \\
\hline $\begin{array}{l}4.6 .6 \\
4.6 .7 \\
4.6 .8 \\
4.6 .67 \\
4.6 .10\end{array}$ & $\begin{array}{r}25.0 \\
20.0 \\
15.0 \\
10.0 \\
12.0\end{array}$ & $\begin{array}{l}0.0 \\
0.0 \\
0.0 \\
0.0 \\
0.0\end{array}$ & $\begin{array}{l}0.0 \\
0.0 \\
0.0 \\
0.0 \\
0.0\end{array}$ & $\begin{array}{l}0.0 \\
0.0 \\
0.0 \\
0.0 \\
0.0\end{array}$ & $\begin{array}{l}0.0 \\
0.0 \\
0.0 \\
0.0 \\
0.0\end{array}$ & $\begin{array}{l}20.00 \\
30.00 \\
50.00 \\
70.00 \\
70.00\end{array}$ & $\begin{array}{l}0.0 \\
0.0 \\
0.0 \\
0.0 \\
0.0\end{array}$ & $\begin{array}{l}0.0 \\
0.0 \\
0.0 \\
0.0 \\
0.0\end{array}$ & $\begin{array}{l}0.0 \\
0.0 \\
0.0 \\
0.0 \\
0.0\end{array}$ & $\begin{array}{l}0.0 \\
0.0 \\
0.0 \\
0.0 \\
0.0\end{array}$ \\
\hline $\begin{array}{l}4.6 .11 \\
4.6 .12 \\
4.6 .13 \\
4.6 .14 \\
4.6 .15\end{array}$ & $\begin{array}{r}10.0 \\
10.0 \\
5.0 \\
3.0 \\
3.0\end{array}$ & $\begin{array}{r}0.0 \\
0.0 \\
0.0 \\
5.0 \\
10.0\end{array}$ & $\begin{array}{l}0.0 \\
0.0 \\
0.0 \\
0.0 \\
0.0\end{array}$ & $\begin{array}{l}0.0 \\
0.0 \\
0.0 \\
0.0 \\
0.0\end{array}$ & $\begin{array}{l}30.00 \\
60.00 \\
60.00 \\
80.00 \\
60.00\end{array}$ & $\begin{array}{l}60.00 \\
50.00 \\
70.00 \\
70.00 \\
710.00\end{array}$ & $\begin{array}{l}0.0 \\
0.0 \\
0.0 \\
0.0 \\
0.0\end{array}$ & $\begin{array}{l}0.0 \\
0.0 \\
0.0 \\
0.0 \\
0.0\end{array}$ & $\begin{array}{l}0.0 \\
0.0 \\
0.0 \\
0.0 \\
0.0\end{array}$ & $\begin{array}{l}0.0 \\
0.0 \\
0.0 \\
0.0 \\
0.0\end{array}$ \\
\hline $\begin{array}{l}4.6 .16 \\
4.6 .17 \\
4.6 .18 \\
4.6 .19 \\
4.6 .20\end{array}$ & $\begin{array}{l}10.0 \\
30.0 \\
20.0 \\
30.0 \\
20.0\end{array}$ & $\begin{array}{r}0.0 \\
10.0 \\
0.0 \\
10.0 \\
0.0\end{array}$ & $\begin{array}{l}0.0 \\
0.0 \\
0.0 \\
0.0 \\
0.0\end{array}$ & $\begin{array}{l}0.0 \\
0.0 \\
0.0 \\
0.0 \\
0.0\end{array}$ & $\begin{array}{l}60.00 \\
50.00 \\
50.00 \\
20.00 \\
20.00\end{array}$ & $\begin{array}{l}70.00 \\
60.00 \\
60.00 \\
70.00 \\
70.00\end{array}$ & $\begin{array}{l}0.0 \\
0.0 \\
0.0 \\
0.0 \\
0.0\end{array}$ & $\begin{array}{l}0.0 \\
0.0 \\
0.0 \\
0.0 \\
0.0\end{array}$ & $\begin{array}{l}0.0 \\
0.0 \\
0.0 \\
0.0 \\
0.0\end{array}$ & $\begin{array}{l}0.0 \\
0.0 \\
0.0 \\
0.0 \\
0.0\end{array}$ \\
\hline $\begin{array}{l}4.6 .21 \\
4.6 .22 \\
4.6 .23 \\
4.0 .24 \\
4.6 .25\end{array}$ & $\begin{array}{l}30.0 \\
20.0 \\
3.0 .0 \\
2.0 .0 \\
30.0\end{array}$ & $\begin{array}{r}10.0 \\
0.0 \\
10.0 \\
0.0 \\
10.0\end{array}$ & $\begin{array}{r}0.0 \\
0.0 \\
0.0 \\
0.0 \\
0.0\end{array}$ & $\begin{array}{l}0.0 \\
0.0 \\
0.0 \\
0.0 \\
0.0\end{array}$ & $\begin{array}{l}0.0 \\
0.0 \\
0.0 \\
0.0 \\
0.0\end{array}$ & $\begin{array}{l}70.00 \\
70.00 \\
50.00 \\
50.00 \\
30.00\end{array}$ & $\begin{array}{l}0.0 \\
0.0 \\
0.0 \\
0.0 \\
0.0\end{array}$ & $\begin{array}{l}0.0 \\
0.0 \\
0.0 \\
0.0 \\
0.0\end{array}$ & $\begin{array}{l}0.0 \\
0.0 \\
0.0 \\
0.0 \\
0.0\end{array}$ & $\begin{array}{l}0.0 \\
0.0 \\
0.0 \\
0.0 \\
0.0\end{array}$ \\
\hline $\begin{array}{l}4.6 .26 \\
4.6 .27 \\
4.6 .28 \\
4.6 .29 \\
4.6 .30\end{array}$ & $\begin{array}{r}30.0 \\
3: 0.0 \\
30.0 \\
.0 .0 \\
30.0\end{array}$ & $\begin{array}{r}0.0 \\
10.0 \\
0.0 \\
10.0 \\
0.0\end{array}$ & $\begin{array}{l}0.0 \\
0.0 \\
0.0 \\
0.0 \\
0.0\end{array}$ & $\begin{array}{l}0.0 \\
0.0 \\
0.0 \\
0.0 \\
0.0\end{array}$ & $\begin{array}{c}30.00 \\
30.00 \\
0.0 \\
0.0 \\
0.0\end{array}$ & $\begin{array}{l}60.00 \\
60.00 \\
70.00 \\
70.00 \\
50.00\end{array}$ & $\begin{array}{l}0.0 \\
0.0 \\
0.0 \\
0.0 \\
0.0\end{array}$ & $\begin{array}{l}0.0 \\
0.0 \\
0.0 \\
0.0 \\
0.0\end{array}$ & $\begin{array}{l}0.0 \\
0.0 \\
0.0 \\
0.0 \\
0.0\end{array}$ & $\begin{array}{l}0.0 \\
0.0 \\
0.0 \\
0.0 \\
0.0\end{array}$ \\
\hline $\begin{array}{l}4.6 .31 \\
4.6 .32 \\
4.6 .33 \\
4.6 .34 \\
4.6 .35\end{array}$ & $\begin{array}{r}310.0 \\
30.0 \\
30.0 \\
3.0 \\
0.0\end{array}$ & $\begin{array}{r}10.0 \\
0.0 \\
10.0 \\
0.0 \\
6.0\end{array}$ & $\begin{array}{l}0.0 \\
0.0 \\
0.0 \\
0.0 \\
0.0\end{array}$ & $\begin{array}{l}0.0 \\
0.0 \\
0.0 \\
0.0 \\
0.0\end{array}$ & $\begin{array}{c}0.0 \\
0.0 \\
0.0 \\
60.00 \\
60.00\end{array}$ & $\begin{array}{c}20.00 \\
20.00 \\
0.00 \\
70.00 \\
70.00\end{array}$ & $\begin{array}{rl}0.0 & 0 \\
0.0 \\
10.00 \\
0.0 \\
0.0\end{array}$ & $\begin{array}{l}0.0 \\
0.0 \\
0.0 \\
0.0 \\
0.0\end{array}$ & $\begin{array}{l}0.0 \\
0.0 \\
0.0 \\
0.0 \\
0.0\end{array}$ & $\begin{array}{l}0.0 \\
0.0 \\
0.0 \\
0.0 \\
0.0\end{array}$ \\
\hline $\begin{array}{l}4.6 .36 \\
4.6 .37 \\
4.6 .38 \\
4.6 .39 \\
4.6 .40\end{array}$ & $\begin{array}{l}16.0 \\
12.0 \\
10.0 \\
16.0 \\
12.0\end{array}$ & $\begin{array}{l}0.0 \\
0.0 \\
0.0 \\
0.0 \\
0.0\end{array}$ & $\begin{array}{l}0.0 \\
0.0 \\
0.0 \\
0.0 \\
0.0\end{array}$ & $\begin{array}{l}0.0 \\
0.0 \\
0.0 \\
0.0 \\
0.0\end{array}$ & $\begin{array}{l}40.00 \\
50.00 \\
50.00 \\
20.00 \\
20.00\end{array}$ & $\begin{array}{l}70.00 \\
60.00 \\
60.00 \\
70.00 \\
70.00\end{array}$ & $\begin{array}{l}0.0 \\
0.0 \\
0.0 \\
0.0 \\
0.0\end{array}$ & $\begin{array}{l}0.0 \\
0.0 \\
0.0 \\
0.0 \\
0.0\end{array}$ & $\begin{array}{l}0.0 \\
0.0 \\
0.0 \\
0.0 \\
0.0\end{array}$ & $\begin{array}{l}0.0 \\
0.0 \\
0.0 \\
0.0 \\
0.0\end{array}$ \\
\hline
\end{tabular}


$\underline{\text { TABLE XXX (Contd.) }}$

SHORTENED CORE BARREL TEST GROUP 4.6

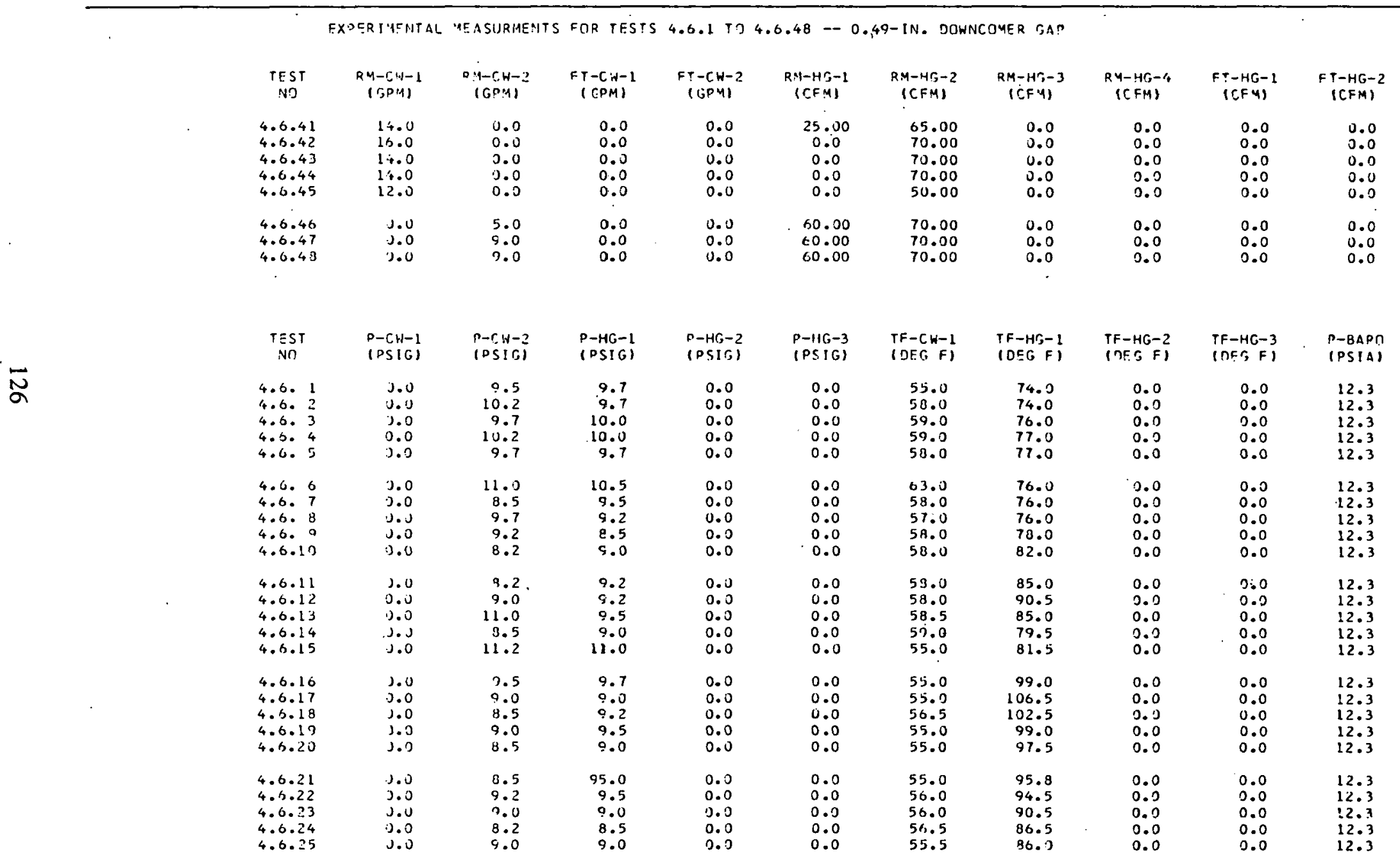


TABLE XXX (Contd.)

SHORTENED CORE BARREL TEST GROUP 4.6

EXPERIMFNTAL MEASIJRMENTS FOR TESTS 4.6.1 TO 4.6.48 -- 0.49-IN. OOWNCOMER GAP

\begin{tabular}{|c|c|c|c|c|c|c|c|c|c|c|}
\hline $\begin{array}{l}\text { TEST } \\
\text { NO }\end{array}$ & $\begin{array}{l}P-C H-1 \\
(P S I G)\end{array}$ & $\begin{array}{l}P-C H-2 \\
(P S I G)\end{array}$ & $\begin{array}{l}P-H G-1 \\
(P S I G)\end{array}$ & $\begin{array}{l}\mathrm{P}-\mathrm{HG}-2 \\
(P S I G)\end{array}$ & $\begin{array}{l}P-H G G-3 \\
(P S I G)\end{array}$ & $\begin{array}{l}T F-C H-1 \\
\text { (DEG F) }\end{array}$ & $\begin{array}{l}\text { TF-HG-1 } \\
\text { (DEG F) }\end{array}$ & $\begin{array}{l}T F-H G-2 \\
(D E G \quad F)\end{array}$ & $\begin{array}{l}\text { TF-HG-3 } \\
\text { (DES F) }\end{array}$ & $\begin{array}{l}\text { P-BARA } \\
\text { (PSIA) }\end{array}$ \\
\hline $\begin{array}{l}4.6 .26 \\
4.6 .27 \\
4.6 .28 \\
4.6 .29 \\
4.6 .30\end{array}$ & $\begin{array}{l}0.0 \\
0.0 \\
0.0 \\
0.0 \\
.00\end{array}$ & $\begin{array}{l}8.5 \\
9.2 \\
9.0 \\
8.0 \\
9.2\end{array}$ & $\begin{array}{l}9.2 \\
9.2 \\
9.5 \\
8.5 \\
8.7\end{array}$ & $\begin{array}{l}0.0 \\
0.0 \\
0.0 \\
0.0 \\
0.0\end{array}$ & $\begin{array}{l}0.0 \\
0.0 \\
0.0 \\
0.0 \\
0.0\end{array}$ & $\begin{array}{l}54.5 \\
56.0 \\
58.0 \\
59.5 \\
58.0\end{array}$ & $\begin{array}{l}74.5 \\
78.0 \\
81.5 \\
84.0 \\
85.0\end{array}$ & $\begin{array}{l}0.0 \\
0.0 \\
0.0 \\
0.0 \\
0.0\end{array}$ & $\begin{array}{l}0.0 \\
0.0 \\
0.0 \\
0.0 \\
0.0\end{array}$ & $\begin{array}{l}12.3 \\
12.3 \\
12.3 \\
12.3 \\
12.3\end{array}$ \\
\hline $\begin{array}{l}4.6 .31 \\
4.6 .32 \\
4.6 .33 \\
4.6 .34 \\
4.6 .35\end{array}$ & $\begin{array}{l}3.0 \\
0.0 \\
3.0 \\
. .0 \\
. .0\end{array}$ & $\begin{array}{l}8.2 \\
9.0 \\
9.0 \\
9.7 \\
9.0\end{array}$ & $\begin{array}{l}8.2 \\
9.7 \\
9.2 \\
9.5 \\
9.2\end{array}$ & $\begin{array}{l}0.0 \\
0.0 \\
0.0 \\
0.0 \\
0.0\end{array}$ & $\begin{array}{l}0.0 \\
0.0 \\
0.0 \\
0.0 \\
0.0\end{array}$ & $\begin{array}{l}57.0 \\
57.0 \\
57.0 \\
65.0 \\
60.5\end{array}$ & $\begin{array}{l}84.5 \\
84.0 \\
81.5 \\
83.0 \\
90.5\end{array}$ & $\begin{array}{l}0.0 \\
0.0 \\
0.0 \\
0.0 \\
0.0\end{array}$ & $\begin{array}{l}0.0 \\
0.0 \\
0.0 \\
0.0 \\
0.0\end{array}$ & $\begin{array}{l}12.3 \\
12.3 \\
12.3 \\
12.3 \\
12.3\end{array}$ \\
\hline $\begin{array}{l}4.6 .36 \\
4.6 .37 \\
4.6 .38 \\
4.6 .39 \\
4.6 .40\end{array}$ & $\begin{array}{l}3.0 \\
1.0 \\
J .0 \\
. .0 \\
.00\end{array}$ & $\begin{array}{l}9.0 \\
8.5 \\
8.0 \\
9.0 \\
9.5\end{array}$ & $\begin{array}{l}9.5 \\
9.0 \\
8.2 \\
9.5 \\
9.7\end{array}$ & $\begin{array}{l}0.0 \\
0.0 \\
0.0 \\
0.0 \\
0.0\end{array}$ & $\begin{array}{l}0.0 \\
0.0 \\
0.0 \\
0.0 \\
0.0\end{array}$ & $\begin{array}{l}57.0 \\
58.5 \\
56.5 \\
59.0 \\
56.5\end{array}$ & $\begin{array}{l}96.0 \\
90.5 \\
97.0 \\
94.0 \\
95.4\end{array}$ & $\begin{array}{l}0.0 \\
0.0 \\
0.0 \\
0.0 \\
0.0\end{array}$ & $\begin{array}{l}0.0 \\
0.0 \\
0.0 \\
0.0 \\
0.0\end{array}$ & $\begin{array}{l}12.3 \\
12.3 \\
12.3 \\
12.3 \\
12.3\end{array}$ \\
\hline $\begin{array}{l}4.6 .41 \\
4.6 .42 \\
4.6 .43 \\
4.60 .44 \\
4.6 .65\end{array}$ & $\begin{array}{l}3.0 \\
3.0 \\
3.0 \\
0.0 \\
0.0\end{array}$ & $\begin{array}{l}9.0 \\
8.7 \\
9.5 \\
3.7 \\
8.5\end{array}$ & $\begin{array}{l}9.5 \\
9.5 \\
9.5 \\
9.0 \\
9.0\end{array}$ & $\begin{array}{l}0.0 \\
0.0 \\
0.0 \\
0.0 \\
0.0\end{array}$ & $\begin{array}{l}0.0 \\
0.0 \\
0.0 \\
0.0 \\
0.0\end{array}$ & $\begin{array}{l}56.5 \\
57.0 \\
57.0 \\
57.5 \\
59.0\end{array}$ & $\begin{array}{l}97.5 \\
97.5 \\
98.5 \\
97.0 \\
92.0\end{array}$ & $\begin{array}{l}0.0 \\
0.0 \\
0.0 \\
0.0 \\
0.0\end{array}$ & $\begin{array}{l}0.0 \\
0.0 \\
0.0 \\
0.0 \\
0.0\end{array}$ & $\begin{array}{l}12.3 \\
12.3 \\
12.3 \\
12.3 \\
12.3\end{array}$ \\
\hline $\begin{array}{l}4.6 .46 \\
4.6 .47 \\
4.6 .48\end{array}$ & $\begin{array}{l}3.0 \\
3.0 \\
3.0\end{array}$ & $\begin{array}{l}8.5 \\
8.0 \\
9.2\end{array}$ & $\begin{array}{l}8.7 \\
9.0 \\
9.7\end{array}$ & $\begin{array}{l}0.0 \\
0.0 \\
0.0\end{array}$ & $\begin{array}{l}0.0 \\
0.0 \\
0.0\end{array}$ & $\begin{array}{l}58.5 \\
58.5 \\
59.0\end{array}$ & $\begin{array}{r}95.7 \\
59.5 \\
106.9\end{array}$ & $\begin{array}{l}0.0 \\
0.0 \\
0.0\end{array}$ & $\begin{array}{l}0.0 \\
0.0 \\
0.0\end{array}$ & $\begin{array}{l}12.3 \\
12.3 \\
12.3\end{array}$ \\
\hline
\end{tabular}

\begin{tabular}{|c|c|c|c|c|c|c|c|c|c|}
\hline \multirow[b]{2}{*}{$\begin{array}{c}\text { TEST } \\
\text { NO }\end{array}$} & \multicolumn{3}{|c|}{ LIJWER PLENUM FLOW } & \multicolumn{3}{|c|}{ BYPASS FLJW } & \multirow[b]{2}{*}{$\begin{array}{l}\text { LOWER } \\
\text { PLENUM } \\
\text { LEVEL } \\
\text { (IN) }\end{array}$} & \multirow[b]{2}{*}{$\begin{array}{l}P-(n-1) \\
(P S I G)\end{array}$} & \multirow[b]{2}{*}{$\begin{array}{l}T F-(P-1 \\
(D E S F)\end{array}$} \\
\hline & $\begin{array}{l}\text { INITIAL } \\
\text { WEIGHT } \\
\text { (LH) }\end{array}$ & $\begin{array}{l}\text { FINAL } \\
W E I G H T \\
\text { (LB) }\end{array}$ & $\begin{array}{l}\text { TIME NF } \\
\text { COLLECTICN } \\
\text { (SEC) }\end{array}$ & $\begin{array}{l}\text { INITIAL } \\
\text { HE IGIT } \\
\text { (LB) }\end{array}$ & $\begin{array}{l}\text { FINAL } \\
\text { WEIGHT } \\
\text { (L } 8)\end{array}$ & $\begin{array}{l}\text { TIME OF } \\
\text { CNLLECTION } \\
\text { (SFC) }\end{array}$ & & & \\
\hline $\begin{array}{ll}4.6 . & 1 \\
4.6 . & 2 \\
4.6 . & 3 \\
4.6 . & 4 \\
4.6 .5 & 5\end{array}$ & $\begin{array}{l}132.13 \\
193.0 \\
263.8 \\
330.5 \\
432.3\end{array}$ & $\begin{array}{l}199.0 \\
262.8 \\
336.5 \\
432.3 \\
524.3\end{array}$ & $\begin{array}{l}20.0 \\
20.0 \\
20.0 \\
20.0 \\
20.0\end{array}$ & $\begin{array}{r}90.5 \\
194.0 \\
241.0 \\
267.0 \\
302.0\end{array}$ & $\begin{array}{l}194.0 \\
241.0 \\
267.0 \\
302.0 \\
327.0\end{array}$ & $\begin{array}{l}49.0 \\
50.0 \\
55.0 \\
50.0 \\
50.0\end{array}$ & $\begin{array}{l}12.0 \\
12.0 \\
12.0 \\
12.0 \\
12.0\end{array}$ & $\begin{array}{l}10.0 \\
10.0 \\
10.0 \\
10.0 \\
10.0\end{array}$ & $\begin{array}{l}55.0 \\
59.0 \\
59.0 \\
59.0 \\
59.0\end{array}$ \\
\hline $\begin{array}{l}4.6 .6 \\
4.6 .7 \\
4.6 .8 \\
4.6 .8 \\
4.6 .10\end{array}$ & $\begin{array}{l}133.3 \\
202.0 \\
260.3 \\
301.0 \\
347.3\end{array}$ & $\begin{array}{l}202.0 \\
260.3 \\
301.0 \\
347.3 \\
418.5\end{array}$ & $\begin{array}{l}25.0 \\
25.0 \\
30.0 \\
35.0 \\
45.0\end{array}$ & $\begin{array}{r}90.0 \\
121.0 \\
147.0 \\
186.0 \\
189.0\end{array}$ & $\begin{array}{l}121.0 \\
147.0 \\
196.0 \\
189.0 \\
178.5\end{array}$ & $\begin{array}{l}40.0 \\
50.0 \\
50.0 \\
55.0: \\
60.0\end{array}$ & $\begin{array}{l}12.0 \\
12.0 \\
12.0 \\
12.0 \\
12.0\end{array}$ & $\begin{array}{l}10.0 \\
10.0 \\
10.0 \\
10.0 \\
10.0\end{array}$ & $\begin{array}{l}63.0 \\
58.0 \\
58.0 \\
58.0 \\
58.0\end{array}$ \\
\hline
\end{tabular}


$\underline{\text { TABLE XXX (Contd.) }}$

SHORTENED CORE BARREL TEST GROUP 4.6

EXPEPIAEUTAL YEASIJRMENTS FDR TESTS 4.6 .1 TO $4.6 .48-0.49-$ IN. OUINNCOMER GAP

\begin{tabular}{|c|c|c|c|c|c|c|c|c|}
\hline \multicolumn{3}{|c|}{ LEWTD. PLENU" CLOW } & \multicolumn{3}{|c|}{ RYPASS FLOH } & \multirow[b]{2}{*}{$\begin{array}{l}\text { LDNEF } \\
\text { PLEVIJM } \\
\text { LEVEL } \\
\text { IINI }\end{array}$} & \multirow[b]{2}{*}{$\begin{array}{l}P-L P-1 \\
\text { (DSIG) }\end{array}$} & \multirow[b]{2}{*}{$\begin{array}{l}T F-(P-1 \\
(D E G F)\end{array}$} \\
\hline $\begin{array}{l}\text { INI TIAL } \\
\text { WE ISHT } \\
\text { (LB) }\end{array}$ & $\begin{array}{l}\text { FINAL } \\
\text { HEISHT } \\
\text { (LS) }\end{array}$ & $\begin{array}{l}\text { TIME NF } \\
\text { COLLECTICN } \\
\text { (SEC) }\end{array}$ & $\begin{array}{l}\text { IN I I I AL } \\
\text { WE I GHT } \\
\text { (LB) }\end{array}$ & $\begin{array}{l}\text { FINAL } \\
\text { HFIGHT } \\
\text { (LI) }\end{array}$ & $\begin{array}{l}\text { TIME JF } \\
\text { COLLECTION } \\
\text { (SEC) }\end{array}$ & & & \\
\hline $\begin{array}{r}418.5 \\
443.0 \\
472.5 \\
484.0 \\
288.0\end{array}$ & $\begin{array}{l}469.8 \\
472.5 \\
484.0 \\
505.0 \\
283.0\end{array}$ & $\begin{array}{l}40.0 \\
30.0 \\
20.0 \\
45.0 \\
60.0\end{array}$ & $\begin{array}{l}198.5 \\
208.8 \\
234.5 \\
249.5 \\
290.5\end{array}$ & $\begin{array}{l}208.8 \\
234.5 \\
249.5 \\
280.0 \\
365.0\end{array}$ & $\begin{array}{l}50.0 \\
50.0 \\
50.0 \\
55.0 \\
15.0\end{array}$ & $\begin{array}{l}12.0 \\
12.0 \\
12.0 \\
12.0 \\
12.0\end{array}$ & $\begin{array}{l}10.0 \\
10.0 \\
10.0 \\
10.0 \\
11.0\end{array}$ & $\begin{array}{l}58.0 \\
59.0 \\
58.5 \\
59.0 \\
60.0\end{array}$ \\
\hline $\begin{array}{l}288.0 \\
293.3 \\
299.0 \\
305.3 \\
322.0\end{array}$ & $\begin{array}{l}293.3 \\
299.0 \\
305.3 \\
322.0 \\
339.0\end{array}$ & $\begin{array}{r}160.0 \\
60.0 \\
60.0 \\
70.0 \\
60.0\end{array}$ & $\begin{array}{l}365.0 \\
445.0 \\
527.5 \\
567.5 \\
675.0\end{array}$ & $\begin{array}{l}445.0 \\
527.0 \\
567.5 \\
675.0 \\
725.0\end{array}$ & $\begin{array}{l}60.0 \\
15.0 \\
15.0 \\
20.0 \\
20.0\end{array}$ & $\begin{array}{l}12.0 \\
12.0 \\
12.0 \\
12.0 \\
12.0\end{array}$ & $\begin{array}{l}10.0 \\
10.0 \\
10.0 \\
10.0 \\
10.0\end{array}$ & $\begin{array}{l}60.5 \\
57.5 \\
57.5 \\
59.0 \\
59.0\end{array}$ \\
\hline $\begin{array}{l}33 \% .0 \\
371.0 \\
403.0 \\
409.5 \\
447.0\end{array}$ & $\begin{array}{l}371.0 \\
403.0 \\
453.0 \\
447.0 \\
517.5\end{array}$ & $\begin{array}{l}60.0 \\
60.0 \\
45.0 \\
30.0 \\
30.0\end{array}$ & $\begin{array}{l}725.0 \\
739.0 \\
793.5 \\
777.0 \\
823.5\end{array}$ & $\begin{array}{l}829.0 \\
793.5 \\
927.5 \\
823.5 \\
938.0\end{array}$ & $\begin{array}{l}21.0 \\
25.0 \\
30.0 \\
30.0 \\
35.0\end{array}$ & $\begin{array}{l}12.0 \\
12.0 \\
12.0 \\
12.0 \\
12.0\end{array}$ & $\begin{array}{l}10.0 \\
10.0 \\
10.3 \\
10.0 \\
10.0\end{array}$ & $\begin{array}{l}57.0 \\
56.0 \\
58.0 \\
57.0 \\
56.0\end{array}$ \\
\hline $\begin{array}{l}132.0 \\
142.3 \\
153.0 \\
174.5 \\
217.0\end{array}$ & $\begin{array}{l}112.3 \\
153.0 \\
174.5 \\
217.0 \\
254.0\end{array}$ & $\begin{array}{l}45.0 \\
45.0 \\
45.0 \\
30.0 \\
30.0\end{array}$ & $\begin{array}{r}86.0 \\
185.5 \\
347.5 \\
442.0 \\
445.5\end{array}$ & $\begin{array}{r}135.5 \\
347.5 \\
442.0 \\
445.5 \\
.553 .0\end{array}$ & $\begin{array}{l}25.0 \\
30.0 \\
25.0 \\
80.0 \\
35.0\end{array}$ & $\begin{array}{l}12.0 \\
12.0 \\
12.0 \\
12.0 \\
12.0\end{array}$ & $\begin{array}{l}10.0 \\
10.0 \\
10.0 \\
10.0 \\
10.0\end{array}$ & $\begin{array}{l}60.5 \\
58.5 \\
57.5 \\
58.5 \\
59.0\end{array}$ \\
\hline $\begin{array}{l}254.8 \\
363.0 \\
424.5 \\
132.3 \\
153.0\end{array}$ & $\begin{array}{l}363.5 \\
450.3 \\
504.5 \\
158.0 \\
192.8\end{array}$ & $\begin{array}{l}35.0 \\
30.0 \\
20.0 \\
60.0 \\
65.0\end{array}$ & $\begin{array}{r}553.0 \\
639.3 \\
698.0 \\
93.5 \\
118.0\end{array}$ & $\begin{array}{l}639.3 \\
698.0 \\
762.5 \\
113.0 \\
126.5\end{array}$ & $\begin{array}{l}35.0 \\
45.0 \\
45.0 \\
35.0 \\
35.0\end{array}$ & $\begin{array}{l}12.0 \\
12.0 \\
12.0 \\
12.0 \\
12.0\end{array}$ & $\begin{array}{l}10.0 \\
10.0 \\
10.0 \\
10.0 \\
10.0\end{array}$ & $\begin{array}{l}57.5 \\
57.0 \\
57.0 \\
59.0 \\
.65 .0\end{array}$ \\
\hline $\begin{array}{l}192.8 \\
205.0 \\
214.8 \\
253.0 \\
272.8\end{array}$ & $\begin{array}{l}200.0 \\
214.8 \\
253.0 \\
272.8 \\
320.0\end{array}$ & $\begin{array}{l}45.0 \\
60.0 \\
60.0 \\
55.0 \\
35.0\end{array}$ & $\begin{array}{l}126.5 \\
198.0 \\
214.3 \\
267.5 \\
324.5\end{array}$ & $\begin{array}{l}198.0 \\
241.8 \\
267.5 \\
324.5 \\
338.0\end{array}$ & $\begin{array}{l}35.0 \\
30.0 \\
30.0 \\
30.0 \\
45.0\end{array}$ & $\begin{array}{l}12.0 \\
12.0 \\
12.0 \\
12.0 \\
12.0\end{array}$ & $\begin{array}{l}10.0 \\
10.0 \\
10.0 \\
10.0 \\
10.0\end{array}$ & $\begin{array}{l}62.5 \\
62.0 \\
59.0 \\
58.0 \\
59.5\end{array}$ \\
\hline $\begin{array}{l}325.0 \\
.350 .0 \\
395.0 \\
416.5 \\
449.3\end{array}$ & $\begin{array}{l}350.5 \\
385.0 \\
410.5 \\
441.3 \\
500.3\end{array}$ & $\begin{array}{l}65.0 \\
45.0 \\
40.0 \\
35.5 \\
30.0\end{array}$ & $\begin{array}{l}333.0 . \\
405.3 \\
473.0 \\
503.5 \\
557.0\end{array}$ & $\begin{array}{l}405.3 \\
473.0 \\
503.5 \\
557.0 \\
557.8\end{array}$ & $\begin{array}{l}45.0 \\
45.0 \\
30.0 \\
45.0 \\
60.0\end{array}$ & $\begin{array}{l}12.0 \\
12.0 \\
12.0 \\
12.0 \\
12.0\end{array}$ & $\begin{array}{l}10.0 \\
10.0 \\
10.0 \\
10.0 \\
10.0\end{array}$ & $\begin{array}{l}57.0 \\
53.0 \\
57.5 \\
59.0 \\
59.0\end{array}$ \\
\hline $\begin{array}{l}434.0 \\
461.0 \\
484.5\end{array}$ & $\begin{array}{l}461.0 \\
484.5 \\
500.0\end{array}$ & $\begin{array}{l}15.0 \\
60.0 \\
60.0\end{array}$ & $\begin{array}{l}380.3 \\
387.3 \\
440.0\end{array}$ & $\begin{array}{l}387.3 \\
440.0 \\
500.0\end{array}$ & $\begin{array}{l}65.0 \\
60.0 \\
45.0\end{array}$ & $\begin{array}{l}12.0 \\
12.0 \\
12.0\end{array}$ & $\begin{array}{l}10.0 \\
10.0 \\
10.0\end{array}$ & $\begin{array}{l}60.0 \\
61.5 \\
59.5\end{array}$ \\
\hline
\end{tabular}


TABLE XXXI

SHORTENED CORE BARREL TEST GROUP 8.1

TEAMSOARENT VESSEL TEST MATA FOR TESTS 8.1 .1 TO $8.1 .60-1.45-1 N$. OOWIICOMER GAP

SUMYARY OF CALCULATED RESIILTS

\begin{tabular}{|c|c|c|c|c|c|c|c|c|c|c|}
\hline $\begin{array}{l}\text { TEST } \\
\text { VI }\end{array}$ & $\begin{array}{l}\text { DTHNC TMER } \\
\text { AIR LLJH } \\
\text { ILI/SECS }\end{array}$ & $\begin{array}{l}\text { TI:NNC TMEP. } \\
\text { AIR DENSITY } \\
\text { (LI/FT } * 3 \text { ) }\end{array}$ & $\begin{array}{l}\text { LUWER PLENUM } \\
\text { PRESSUP.E } \\
\text { (PSIA) }\end{array}$ & $\begin{array}{l}\text { COLD LEG } \\
\text { AID FLOW } \\
\text { (LB/SEC) }\end{array}$ & $\begin{array}{l}\text { DUWNCOMER } \\
\text { WATEP FLOW } \\
\text { (LB/SEC) }\end{array}$ & $\begin{array}{l}\text { WATER FLOH } \\
\text { CONTINUITY }\end{array}$ & $\begin{array}{c}\text { J-AIR } \\
\text { DOWNCOMEP } \\
\text { (FT/SEC) }\end{array}$ & $\begin{array}{l}\text { J-HATCR } \\
\text { DOHNCOMER } \\
\text { (FT/SEC) }\end{array}$ & $(\mathrm{J}(, *) * * 1 / 2$ & $(J L *) * 1 / 2$ \\
\hline $\begin{array}{l}8.1: 1 \\
8.1: 2 \\
8.1: 5 \\
8.1: 4 \\
8.1 .5\end{array}$ & $\begin{array}{l}0.5203 \\
0.5055 \\
0.4979 \\
0.4980 \\
0.4937\end{array}$ & $\begin{array}{l}0.1738 \\
0.1740 \\
0.1765 \\
0.1763 \\
0.1737\end{array}$ & $\begin{array}{l}32.84 \\
32.94 \\
33.34 \\
33.34 \\
32.84\end{array}$ & $\begin{array}{l}0.0 \\
0.0 \\
0.0 \\
0.0 \\
0.0\end{array}$ & $\begin{array}{l}1.817 \\
2.450 \\
2.683 \\
2.500 \\
1.503\end{array}$ & $\begin{array}{l}1.026 \\
1.060 \\
1.004 \\
1.014 \\
1.123\end{array}$ & $\begin{array}{l}13.65 \\
13.24 \\
12.85 \\
12.87 \\
12.95\end{array}$ & $\begin{array}{l}0.133 \\
0.179 \\
0.196 \\
0.183 \\
0.110\end{array}$ & $\begin{array}{l}0.5088 \\
0.5012 \\
0.4956 \\
0.4958 \\
0.4955\end{array}$ & $\begin{array}{l}0.2183 \\
0.2535 \\
0.2653 \\
0.2561 \\
0.1989\end{array}$ \\
\hline $\begin{array}{l}8.1 .6 \\
8.1 .7 \\
8.1 .8 \\
8.1 .9 \\
8.1 .10\end{array}$ & $\begin{array}{l}0.5267 \\
0.5147 \\
0.512 .0 \\
0.5094 \\
0.4715\end{array}$ & $\begin{array}{l}0.1803 \\
J .1708 \\
.1701 \\
0.1703 \\
0.1030\end{array}$ & $\begin{array}{l}34.40 \\
32.40 \\
32.40 \\
32.40 \\
32.40\end{array}$ & $\begin{array}{l}0.0 \\
0.0 \\
c .0 \\
0.0 \\
0.0\end{array}$ & $\begin{array}{l}2.525 \\
2.467 \\
2.717 \\
2.600 \\
2.783\end{array}$ & $\begin{array}{l}1.027 \\
1.016 \\
0.986 \\
1.042 \\
0.993\end{array}$ & $\begin{array}{l}13.31 \\
13.73 \\
13.71 \\
13.60 \\
12.79\end{array}$ & $\begin{array}{l}0.194 \\
0.190 \\
0.199 \\
0.190 \\
0.203\end{array}$ & $\begin{array}{l}0.5071 \\
0.5080 \\
0.5072 \\
0.5053 \\
0.4883\end{array}$ & $\begin{array}{l}0.2574 \\
0.2544 \\
0.2670 \\
0.2612 \\
0.2702\end{array}$ \\
\hline $\begin{array}{l}8.1 .11 \\
8.1 .12 \\
8.1 .13 \\
8.1 .14 \\
8.1 .15\end{array}$ & $\begin{array}{l}0.4621 \\
0.4528 \\
0.4596 \\
0.4499 \\
0.4468\end{array}$ & $\begin{array}{l}0.1713 \\
0.1739 \\
0.1647 \\
0.1715 \\
0.1709\end{array}$ & $\begin{array}{l}32.40 \\
32.90 \\
32.40 \\
32.40 \\
32.40\end{array}$ & $\begin{array}{l}0.0 \\
0.0 \\
0.0 \\
0.0 \\
0.0\end{array}$ & $\begin{array}{l}3.383 \\
2.917 \\
2.714 \\
3.700 \\
3.950\end{array}$ & $\begin{array}{l}1.007 \\
1.016 \\
1.030 \\
0.971 \\
1.000\end{array}$ & $\begin{array}{l}12.29 \\
11.87 \\
12.42 \\
11.25 \\
11.92\end{array}$ & $\begin{array}{l}0.247 \\
0.213 \\
0.199 \\
0.270 \\
0.288\end{array}$ & $\begin{array}{l}0.4810 \\
0.4745 \\
0.4816 \\
0.4745 \\
0.4733\end{array}$ & $\begin{array}{l}0.2979 \\
0.2766 \\
0.2669 \\
0.3116 \\
0.3219\end{array}$ \\
\hline $\begin{array}{l}8.1 .16 \\
8.1 .17 \\
8.1 .18 \\
8.1 .19 \\
8.1 .20\end{array}$ & $\begin{array}{l}0.4445 \\
0.4933 \\
0.4429 \\
0.4421 \\
0.41779\end{array}$ & $\begin{array}{l}0.1705 \\
0.1703 \\
0.1703 \\
0.1703 \\
0.1707\end{array}$ & $\begin{array}{l}32.40 \\
32.40 \\
32.40 \\
32.40 \\
32.31\end{array}$ & $\begin{array}{l}0.0 \\
0.0 \\
0.0 \\
0.0 \\
0.0\end{array}$ & $\begin{array}{l}3.350 \\
2.783 \\
4.167 \\
4.350 \\
2.800\end{array}$ & $\begin{array}{l}1.018 \\
1.009 \\
1.008 \\
0.976 \\
1.009\end{array}$ & $\begin{array}{l}11.88 \\
13.20 \\
11.95 \\
11.79 \\
13.02\end{array}$ & $\begin{array}{l}0.245 \\
0.203 \\
0.304 \\
0.319 \\
0.204\end{array}$ & $\begin{array}{l}0.4723 \\
0.4978 \\
0.4716 \\
0.4709 \\
0.4947\end{array}$ & $\begin{array}{l}0.2965 \\
0.2702 \\
0.3306 \\
0.3378 \\
0.2710\end{array}$ \\
\hline $\begin{array}{l}8.1 .21 \\
8.1 .22 \\
8.1 .23 \\
8.1 .24 \\
8.1 .25\end{array}$ & $\begin{array}{l}0.4711 \\
0.4584 \\
0.6514 \\
0.6507 \\
0.6452\end{array}$ & $\begin{array}{l}0.1723 \\
0.1702 \\
0.1924 \\
0.1749 \\
0.1639\end{array}$ & $\begin{array}{l}32.81 \\
32.31 \\
34.81 \\
33.31 \\
31.31\end{array}$ & $\begin{array}{l}0.0 \\
0.0 \\
0.0 \\
0.0 \\
0.0\end{array}$ & $\begin{array}{l}3.317 \\
3.083 \\
1.244 \\
1.189 \\
1.073\end{array}$ & $\begin{array}{l}1.0110 \\
0.97 .1 \\
1.01 .5 \\
1.034 \\
1.030\end{array}$ & $\begin{array}{l}12.46 \\
12.27 \\
16.27 \\
16.95 \\
17.93\end{array}$ & $\begin{array}{l}0.242 \\
0.225 \\
0.091 \\
0.087 \\
0.079\end{array}$ & $\begin{array}{l}0.4850 \\
0.4799 \\
0.5622 \\
0.5678 \\
0.5747\end{array}$ & $\begin{array}{l}0.2950 \\
0.2844 \\
0.1807 \\
0.1766 \\
0.1682\end{array}$ \\
\hline $\begin{array}{l}8.1 .26 \\
8.1 .27 \\
8.1 .28 \\
8.1 .29 \\
8.1 .30\end{array}$ & $\begin{array}{l}0.6351 \\
0.6263 \\
0.6714 \\
0.4563 \\
0.4520\end{array}$ & $\begin{array}{l}0.1695 \\
0.1698 \\
0.1687 \\
0.1654 \\
0.1707\end{array}$ & $\begin{array}{l}32.31 \\
32.31 \\
32.31 \\
31.81 \\
32.31\end{array}$ & $\begin{array}{l}0.0 \\
0.0 \\
0.0 \\
0.0 \\
0.0\end{array}$ & $\begin{array}{l}1.15 \mathrm{~J} \\
1.21 .7 \\
0.68 .3 \\
3.183 \\
3.313\end{array}$ & $\begin{array}{l}1.031 \\
1.013 \\
.0 .995 \\
1.042 \\
0.0469\end{array}$ & $\begin{array}{l}17.07 \\
16.81 \\
18.14 \\
12.56 \\
12.07\end{array}$ & $\begin{array}{l}0.094 \\
0.089 \\
0.050 \\
0.232 \\
0.242\end{array}$ & $\begin{array}{l}0.5654 \\
0.5613 \\
0.5822 \\
0.4920 \\
0.4762\end{array}$ & $\begin{array}{l}0.1737 \\
0.1787 \\
0.1339 \\
0.2890 \\
0.2948\end{array}$ \\
\hline $\begin{array}{l}8.1 .31 \\
8.1 .32 \\
8.1 .33 \\
3.1 .34 \\
8.1 .35\end{array}$ & $\begin{array}{l}0.4516 \\
0.6343 \\
0.3514 \\
0.3529 \\
0.3603\end{array}$ & $\begin{array}{l}0.1683 \\
J .1605 \\
j .1709 \\
0.1697 \\
0.1747\end{array}$ & $\begin{array}{l}32.31 \\
32.31 \\
32.31 \\
32.31 \\
33.31\end{array}$ & $\begin{array}{l}0.0 \\
0.0 \\
0.0 \\
0.0 \\
0.0\end{array}$ & $\begin{array}{l}3.183 \\
0.608 \\
7.620 \\
6.283 \\
6.56 ?\end{array}$ & $\begin{array}{l}0.963 \\
0.951 \\
1.021 \\
1.005 \\
1.051\end{array}$ & $\begin{array}{r}12.19 \\
17.05 \\
9.37 \\
9.47 \\
9.39\end{array}$ & $\begin{array}{l}0.232 \\
0.044 \\
0.556 \\
0.459 \\
0.479\end{array}$ & $\begin{array}{l}0.4772 \\
0.5651 \\
0.4198 \\
0.4213 \\
0.4227\end{array}$ & $\begin{array}{l}0.2890 \\
0.1263 \\
0.4471 \\
0.4060 \\
0.4151\end{array}$ \\
\hline $\begin{array}{l}8.1 .36 \\
8.1 .37 \\
8.1 .33 \\
8.1 .39 \\
8.1 .45\end{array}$ & $\begin{array}{l}0.3573 \\
0.3547 \\
0.3553 \\
0.244 .3 \\
0.2511\end{array}$ & $\begin{array}{l}0.1761 \\
0.1703 \\
0.1754 \\
0.1759 \\
0.1709\end{array}$ & $\begin{array}{l}33.31 \\
32.31 \\
33.31 \\
33.31 \\
32.2 .5\end{array}$ & $\begin{array}{l}0.0 \\
0.0 \\
0.0 \\
0.0 \\
0.0\end{array}$ & $\begin{array}{r}5.412 \\
3.800 \\
6.650 \\
12.72 .5 \\
0.620\end{array}$ & $\begin{array}{l}1.023 \\
0.989 \\
1.023 \\
1.013 \\
1.002\end{array}$ & $\begin{array}{l}9.25 \\
9.47 \\
9.23 \\
6.33 \\
6.70\end{array}$ & $\begin{array}{l}0.395 \\
0.277 \\
0.496 \\
0.929 \\
0.702\end{array}$ & $\begin{array}{l}0.4203 \\
0.421 .6 \\
0.4193 \\
0.3 .47 .5 \\
0.354 .3\end{array}$ & $\begin{array}{l}0.3768 \\
0.3157 \\
0.41 .77 \\
0.5778 \\
0.50 .24\end{array}$ \\
\hline
\end{tabular}


TABLE XXXI (Contd.)

SHORTENED CORE BARREL TEST GROUP 8.1

SUMMARY OF CALCULATEU RESULTS FOR TESTS 8.1 .1 TO $8.1 .60-1.45$ IN. DOHNCOMER GAP

\begin{tabular}{|c|c|c|c|c|c|c|c|c|c|c|}
\hline $\begin{array}{l}\text { TEST } \\
\text { NO }\end{array}$ & $\begin{array}{l}\text { D IWNC QMER } \\
\text { AIP. FL TH } \\
\text { (LB/SEC) }\end{array}$ & $\begin{array}{c}\text { DOWNCOMER } \\
\text { AIR OENSITY } \\
\text { (LB/FT*3) }\end{array}$ & $\begin{array}{c}\text { LOWER PLENUM } \\
\text { PRESS SRE } \\
\text { (PSIA) }\end{array}$ & $\begin{array}{l}\text { COLD LEG } \\
\text { AIR FLOH } \\
\text { ILB/SECI }\end{array}$ & $\begin{array}{l}\text { DOWNCOMEP. } \\
\text { WATER FLOW } \\
\text { (LB/SEC) }\end{array}$ & $\begin{array}{l}\text { WATER FLOH } \\
\text { CONTINUITY }\end{array}$ & $\begin{array}{c}\text { J-AIR } \\
\text { DOWNCOMER } \\
\text { (FT/SEC) }\end{array}$ & $\begin{array}{l}\text { J-HATER } \\
\text { DOHNCONER } \\
\text { (FT/SEC) }\end{array}$ & (JG* $) * * 1 / 2$ & $(J L *) * 1$ \\
\hline $\begin{array}{l}8.1 .41 \\
8.1 .42 \\
8.1 .43 \\
8.1 .44 \\
8.1 .45\end{array}$ & $\begin{array}{l}0.4525 \\
0.4501 \\
0.4998 \\
0.5029 \\
0.5592\end{array}$ & $\begin{array}{l}0.1699 \\
0.1705 \\
0.1705 \\
0.1700 \\
0.1702\end{array}$ & $\begin{array}{l}32.25 \\
32.25 \\
32.25 \\
32.25 \\
32.25\end{array}$ & $\begin{array}{l}0.0 \\
0.0 \\
0.0 \\
c .0 \\
0.0\end{array}$ & $\begin{array}{l}3.267 \\
3.733 \\
2.700 \\
2.650 \\
1.867\end{array}$ & $\begin{array}{l}1.022 \\
0.997 \\
1.010 \\
1.011 \\
1.031\end{array}$ & $\begin{array}{l}12.14 \\
12.02 \\
13.35 \\
13.48 \\
14.97\end{array}$ & $\begin{array}{l}0.239 \\
0.273 \\
0.197 \\
0.193 \\
0.136\end{array}$ & $\begin{array}{l}0.4770 \\
0.4753 \\
0.5008 \\
0.5028 \\
0.5300\end{array}$ & $\begin{array}{l}0.2928 \\
0.3130 \\
0.2662 \\
0.2637 \\
0.2213\end{array}$ \\
\hline $\begin{array}{l}8.1 .40 \\
8.1 .47 \\
8.1 .48 \\
8.1 .49 \\
8.1 .50\end{array}$ & $\begin{array}{l}0.5537 \\
0.4892 \\
0.4857 \\
0.4853 \\
0.5649\end{array}$ & $\begin{array}{l}0.1702 \\
0.1694 \\
0.17100 \\
0.1702 \\
0.1861\end{array}$ & $\begin{array}{l}32.25 \\
32.25 \\
32.25 \\
32.25 \\
32.25\end{array}$ & $\begin{array}{l}0.0 \\
0.0 \\
0.0 \\
0.0 \\
0.0\end{array}$ & $\begin{array}{l}1.867 \\
2.567 \\
2.733 \\
3.000 \\
1.767\end{array}$ & $\begin{array}{l}1.031 \\
1.013 \\
1.024 \\
0.989 \\
1.027\end{array}$ & $\begin{array}{l}14.92 \\
13.16 \\
13.01 \\
12.99 \\
15.49\end{array}$ & $\begin{array}{l}0.136 \\
0.137 \\
0.200 \\
0.219 \\
0.129\end{array}$ & $\begin{array}{l}0.5274 \\
0.4963 \\
0.4941 \\
0.4938 \\
0.5359\end{array}$ & $\begin{array}{l}0.2213 \\
0.2595 \\
0.2678 \\
0.2805 \\
0.2153\end{array}$ \\
\hline $\begin{array}{l}8.1 .51 \\
8.1 .52 \\
8.1 .53 \\
8.1 .54 \\
8.1 .55\end{array}$ & $\begin{array}{l}0.5627 \\
j .5442 \\
0.5391 \\
0.5401 \\
0.5382\end{array}$ & $\begin{array}{l}0.1734 \\
0.1699 \\
0.1685 \\
0.1684 \\
0.1690\end{array}$ & $\begin{array}{l}32.25 \\
32.25 \\
32.25 \\
32.25 \\
32.25\end{array}$ & $\begin{array}{l}0.0 \\
0.0 \\
0.0 \\
0.0 \\
0.0\end{array}$ & $\begin{array}{l}1.967 \\
2.033 \\
2.300 \\
2.433 \\
2.033\end{array}$ & $\begin{array}{l}1.012 \\
1.048 \\
1.020 \\
1.015 \\
1.031\end{array}$ & $\begin{array}{l}15.05 \\
14.60 \\
14.57 \\
14.61 \\
14.51\end{array}$ & $\begin{array}{l}0.144 \\
0.148 \\
0.168 \\
0.178 \\
0.149\end{array}$ & $\begin{array}{l}0.5317 \\
0.5231 \\
0.5217 \\
0.5223 \\
0.5208\end{array}$ & $\begin{array}{l}0.2272 \\
0.2310 \\
0.2456 \\
0.2527 \\
0.2310\end{array}$ \\
\hline $\begin{array}{l}8.1 .56 \\
8.1 .57 \\
8.1 .58 \\
8.1 .59 \\
8.1 .60\end{array}$ & $\begin{array}{l}0.5391 \\
0.4751 \\
0.4762 \\
0.4693 \\
0.8091\end{array}$ & $\begin{array}{l}0.1680 \\
0.1090 \\
0.1700 \\
0.1695 \\
0.1693\end{array}$ & $\begin{array}{l}32.25 \\
32.25 \\
32.25 \\
32.25 \\
32.25\end{array}$ & $\begin{array}{l}0.0 \\
0.0 \\
0.0 \\
0.0 \\
0.0\end{array}$ & $\begin{array}{l}2.450 \\
3.033 \\
3.311 \\
3.533 \\
1.550\end{array}$ & $\begin{array}{l}1.028 \\
1.035 \\
1.025 \\
0.983 \\
1.047\end{array}$ & $\begin{array}{l}14.62 \\
12.91 \\
12.76 \\
12.61 \\
16.37\end{array}$ & $\begin{array}{l}0.17 .9 \\
0.221 \\
0.242 \\
.0 .258 \\
0.113\end{array}$ & $\begin{array}{l}0.5221 \\
0.4894 \\
0.4892 \\
0.4860 \\
0.5537\end{array}$ & $\begin{array}{l}0.2535 \\
0.2821 \\
0.2948 \\
0.3045 \\
0.2017\end{array}$ \\
\hline
\end{tabular}


TABLE XXXI (Contd.)

SHORTENED CORE BARREL TEST GROUP 8.1

EXPERIMEIJTAL MEASURHENTS COQ TESTS 8.1.1 TO 8.1.60-- 1.45-IN. DDWNEJMER GAP

\begin{tabular}{|c|c|c|c|c|c|c|c|c|c|c|}
\hline $\begin{array}{l}\text { TEST } \\
\text { MO }\end{array}$ & $\begin{array}{l}\text { R.M-CW-1 } \\
(I, P M)\end{array}$ & $\begin{array}{c}\mathrm{FM}^{\mathrm{M}}-\mathrm{CN}-2 \\
(\mathrm{r}, \mathrm{PM})\end{array}$ & $\begin{array}{c}F T-C H-1 \\
(G P M)\end{array}$ & $\begin{array}{c}F T-C: 1-2 \\
(G P: 1)\end{array}$ & $\begin{array}{c}R M-H r_{3}-1 \\
(C F M)\end{array}$ & $\begin{array}{c}R M-H G-2 \\
(C F M)\end{array}$ & $\begin{array}{c}R M-H F-3 \\
(C F M)\end{array}$ & $\begin{array}{c}\text { PY-HG-4 } \\
(C F Y) .\end{array}$ & $\begin{array}{c}F T-H F,-1 \\
(r F Y)\end{array}$ & $\begin{array}{c}F T-H G-2 \\
(C F M)\end{array}$ \\
\hline $\begin{array}{ll}3.1 \cdot 1 \\
9.1 \cdot 2 \\
8.1 \cdot 3 \\
8.1 .4 \\
3.1 .5\end{array}$ & $\begin{array}{l}0.0 \\
2.0 \\
0.0 \\
0.0 \\
.00\end{array}$ & $\begin{array}{l}0.0 \\
0.0 \\
0.0 \\
0.0 \\
0.0\end{array}$ & $\begin{array}{l}0.0 \\
0.0 \\
0.0 \\
0.0 \\
0.0\end{array}$ & $\begin{array}{l}49.8 \\
39.8 \\
29.9 \\
19.9 \\
10.0\end{array}$ & $\begin{array}{l}0.0 \\
0.0 \\
0.0 \\
0.0 \\
0.0\end{array}$ & $\begin{array}{l}0.0 \\
0.0 \\
0.0 \\
0.0 \\
0.0\end{array}$ & $\begin{array}{r}0.0 \\
0.0 \\
.0 .0 \\
0.0 \\
0.0\end{array}$ & $\begin{array}{l}0.0 \\
0.0 \\
0.0 \\
0.0 \\
0.0\end{array}$ & $\begin{array}{l}63.46 \\
63.46 \\
63.46 \\
63.46 \\
63.46\end{array}$ & $\begin{array}{l}0.0 \\
0.0 \\
0.0 \\
0.0 \\
0.0\end{array}$ \\
\hline $\begin{array}{ll}8.1 . & 6 \\
8.1 .9 \\
8.1 .9 \\
8.1 .9 \\
8.1 .1\end{array}$ & $\begin{array}{l}0.0 \\
0.0 \\
0.0 \\
0.0 \\
0.0\end{array}$ & $\begin{array}{l}0.0 \\
0.0 \\
0.0 \\
0.0 \\
0.0\end{array}$ & $\begin{array}{r}60.0 \\
0.0 \\
0.0 \\
0.0 \\
0.0\end{array}$ & $\begin{array}{r}0.0 \\
45.0 \\
35.0 \\
25.0 \\
50.0\end{array}$ & $\begin{array}{l}0.0 \\
0.0 \\
0.0 \\
0.0 \\
0.0\end{array}$ & $\begin{array}{l}0.0 \\
0.0 \\
0.0 \\
0.0 \\
0.0\end{array}$ & $\begin{array}{l}0.0 \\
0.0 \\
0.0 \\
0.0 \\
0.0\end{array}$ & $\begin{array}{l}0.0 \\
0.0 \\
0.0 \\
0.0 \\
0.0\end{array}$ & $\begin{array}{l}63.46 \\
63.46 \\
63.46 \\
63.46 \\
56.03\end{array}$ & $\begin{array}{l}0.0 \\
0.0 \\
0.0 \\
0.0 \\
0.0\end{array}$ \\
\hline $\begin{array}{l}8.1 .11 \\
8.1 .12 \\
8.1 .13 \\
8.1 .14 \\
9.1 .15\end{array}$ & $\begin{array}{l}3.0 \\
0.0 \\
0.0 \\
0.0 \\
0.0\end{array}$ & $\begin{array}{l}0.0 \\
0.0 \\
0.0 \\
0.0 \\
0.0\end{array}$ & $\begin{array}{r}0.0 \\
0.0 \\
0.0 \\
0.0 \\
0.0\end{array}$ & $\begin{array}{r}40.0 \\
30.0 \\
20.0 \\
0.0 \\
45.3\end{array}$ & $\begin{array}{l}0.0 \\
0.0 \\
0.0 \\
0.0 \\
0.0\end{array}$ & $\begin{array}{l}0.0 \\
0.0 \\
0.0 \\
0.0 \\
0.0\end{array}$ & $\begin{array}{l}0.0 \\
0.0 \\
0.0 \\
0.0 \\
0.0\end{array}$ & $\begin{array}{l}0.0 \\
0.0 \\
0.0 \\
0.0 \\
0.0\end{array}$ & $\begin{array}{l}56.03 \\
56.03 \\
56.03 \\
56.03 \\
56.03\end{array}$ & $\begin{array}{l}0.0 \\
0.0 \\
0.0 \\
0.0 \\
0.0\end{array}$ \\
\hline $\begin{array}{l}8.1 .16 \\
8.1 .1 \% \\
8.1 .18 \\
8.1 .19 \\
8.1 .20\end{array}$ & $\begin{array}{r}0.0 \\
3.0 \\
.00 \\
.00 \\
.00\end{array}$ & $\begin{array}{l}0.0 \\
0.0 \\
0.0 \\
0.0 \\
0.0\end{array}$ & $\begin{array}{r}0.0 \\
0.0 \\
0.0 \\
55.0 \\
60.0\end{array}$ & $\begin{array}{r}35.0 \\
50.0 \\
50.0 \\
0.0 \\
0.0\end{array}$ & $\begin{array}{l}0.0 \\
0.0 \\
0.0 \\
0.0 \\
0.0\end{array}$ & $\begin{array}{l}0.0 \\
0.0 \\
0.0 \\
0.0 \\
0.0\end{array}$ & $\begin{array}{l}0.0 \\
0.0 \\
0.0 \\
0.0 \\
0.0\end{array}$ & $\begin{array}{l}0.0 \\
0.0 \\
0.0 \\
0.0 \\
0.0\end{array}$ & $\begin{array}{l}56.03 \\
63.46 \\
56.03 \\
56.03 \\
56.03\end{array}$ & $\begin{array}{l}0.0 \\
0.0 \\
0.0 \\
0.0 \\
0.0\end{array}$ \\
\hline $\begin{array}{l}8.1 .21 \\
9.1 .22 \\
8.1 .23 \\
9.1 .24 \\
9.1 .25\end{array}$ & $\begin{array}{l}.00 \\
j .0 \\
0.0 \\
.00 \\
.00\end{array}$ & $\begin{array}{l}0.0 \\
j .0 \\
0.0 \\
0.0 \\
0.0\end{array}$ & $\begin{array}{r}70.0 \\
60.0 \\
0.0 \\
0.0 \\
0.0\end{array}$ & $\begin{array}{r}0.0 \\
0.0 \\
45.0 \\
40.0 \\
35.0\end{array}$ & $\begin{array}{l}0.0 \\
0.0 \\
0.0 \\
0.0 \\
0.0\end{array}$ & $\begin{array}{l}0.0 \\
0.0 \\
0.0 \\
0.0 \\
0.0\end{array}$ & $\begin{array}{l}0.0 \\
0.0 \\
0.0 \\
0.0 \\
0.0\end{array}$ & $\begin{array}{l}0.0 \\
0.0 \\
0.0 \\
0.0 \\
0.0\end{array}$ & $\begin{array}{l}56.03 \\
56.03 \\
81.67 \\
91.67 \\
81.67\end{array}$ & $\begin{array}{l}0.0 \\
0.0 \\
0.0 \\
0.0 \\
0.0\end{array}$ \\
\hline $\begin{array}{l}8.1 .26 \\
8.1 .27 \\
9.1 .23 \\
3.1 .29 \\
3.1 .30\end{array}$ & $\begin{array}{l}0.0 \\
0.0 \\
j .0 \\
j .0 \\
0.0\end{array}$ & $\begin{array}{l}0.0 \\
0.0 \\
0.0 \\
0.0 \\
0.0\end{array}$ & $\begin{array}{r}0.0 \\
0.0 \\
0.0 \\
65.0 \\
60.0\end{array}$ & $\begin{array}{r}30.0 \\
20.0 \\
10.0 \\
0.0 \\
0.0\end{array}$ & $\begin{array}{l}0.0 \\
0.0 \\
0.0 \\
0.0 \\
0.0\end{array}$ & $\begin{array}{l}0.0 \\
0.0 \\
0.0 \\
0.0 \\
0.0\end{array}$ & $\begin{array}{l}0.0 \\
0.0 \\
0.0 \\
0.0 \\
0.0\end{array}$ & $\begin{array}{l}0.0 \\
3.0 \\
0.0 \\
0.0 \\
0.0\end{array}$ & $\begin{array}{l}81.67 \\
81.67 \\
41.67 \\
56.03 \\
56.03\end{array}$ & $\begin{array}{l}0.0 \\
0.0 \\
0.0 \\
0.0 \\
0.0\end{array}$ \\
\hline $\begin{array}{l}8.1 .31 \\
8.1 .32 \\
8.1 .33 \\
3.1 .34 \\
9.1 .35\end{array}$ & $\begin{array}{l}3.0 \\
3.0 \\
3.0 \\
.00 \\
.00\end{array}$ & $\begin{array}{l}0.0 \\
0.0 \\
0.0 \\
0.0 \\
0.0\end{array}$ & $\begin{array}{r}55.0 \\
0.0 \\
90.0 \\
80.0 \\
100.0\end{array}$ & $\begin{array}{l}0.0 \\
5.0 \\
0.0 \\
0.0 \\
0.0\end{array}$ & $\begin{array}{l}0.0 \\
0.0 \\
0.0 \\
0.0 \\
0.0\end{array}$ & $\begin{array}{l}0.0 \\
0.0 \\
0.0 \\
0.0 \\
0.0\end{array}$ & $\begin{array}{l}0.0 \\
0.0 \\
0.0 \\
0.0 \\
0.0\end{array}$ & $\begin{array}{l}0.0 \\
0.0 \\
0.0 \\
0.0 \\
0.0\end{array}$ & $\begin{array}{l}56.03 \\
81.67 \\
44.23 \\
44.23 \\
44.23\end{array}$ & $\begin{array}{l}0.0 \\
0.0 \\
0.0 \\
0.0 \\
0.0\end{array}$ \\
\hline $\begin{array}{l}8.1 .36 \\
8.1 .37 \\
9.1 .33 \\
8.1 .39 \\
3.1 .4 .0\end{array}$ & $\begin{array}{l}3.0 \\
.00 \\
.00 \\
.00 \\
.00\end{array}$ & $\begin{array}{l}0.0 \\
0.0 \\
0.0 \\
0.0 \\
0.0\end{array}$ & $\begin{array}{r}60.0 \\
0.0 \\
85.0 \\
150.0 \\
112.0\end{array}$ & $\begin{array}{r}0.0 \\
40.0 \\
0.0 \\
0.0 \\
0.0\end{array}$ & $\begin{array}{l}0.0 \\
0.0 \\
0.0 \\
0.0 \\
0.0\end{array}$ & $\begin{array}{l}0.0 \\
0: 0 . \\
0.0 \\
0.0 \\
0.0\end{array}$ & $\begin{array}{l}0.0 \\
0.0 \\
0.0 \\
0.0 \\
0.0\end{array}$ & $\begin{array}{l}0.0 \\
0.0 \\
0.0 \\
0.0 \\
0.0\end{array}$ & $\begin{array}{l}44.23 \\
44.23 \\
44.23 \\
29.49 \\
29.49\end{array}$ & $\begin{array}{l}0.0 \\
0.0 \\
0.0 \\
0.0 \\
0.0\end{array}$ \\
\hline
\end{tabular}


TABLE XXXI (Contd.)

SHORTENED CORE BARREL TEST GROUP 8.1

EXPERTHENTAL MEASURMENTS FOR TESTS 8.1.1 TO 8.1.60 -- 1.45-IN. DOHNCOMER GAP

\begin{tabular}{|c|c|c|c|c|c|c|c|c|c|c|}
\hline $\begin{array}{l}\text { TEST } \\
\text { NO }\end{array}$ & $\begin{array}{c}R M-C: 1-1 \\
(G O M)\end{array}$ & $\begin{array}{c}R M-C H-2 \\
(C P M)\end{array}$ & $\begin{array}{c}F T-C H-1 \\
(G P M)\end{array}$ & $\begin{array}{c}\mathrm{FT}-\mathrm{CH}-2 \\
(G P)_{1}\end{array}$ & $\begin{array}{c}R M-H G-1 \\
(C F \cdot y)\end{array}$ & $\begin{array}{c}R Y-H G-2 \\
(C F M)\end{array}$ & $\begin{array}{c}\text { RM-HG-3 } \\
(C F M)\end{array}$ & $\begin{array}{c}R M-H G-4 \\
(C F M)\end{array}$ & $\begin{array}{l}F T-H G-1 \\
(C F M)\end{array}$ & $\begin{array}{c}F T-H G-2 \\
\text { (CFM) }\end{array}$ \\
\hline $\begin{array}{l}8.1 .41 \\
8.1 .42 \\
8.1 .43 \\
9.1 .44 \\
8.1 .45\end{array}$ & $\begin{array}{l}3.0 \\
j .0 \\
0.0 \\
0.0 \\
1.0\end{array}$ & $\begin{array}{l}0.0 \\
0.0 \\
0.0 \\
0.0 \\
0.0\end{array}$ & $\begin{array}{l}0.0 \\
0.0 \\
0.0 \\
0.0 \\
0.0\end{array}$ & $\begin{array}{l}50.0 \\
45.0 \\
40.0 \\
35.0 \\
40.0\end{array}$ & $\begin{array}{l}0.0 \\
0.0 \\
0.0 \\
0.0 \\
0.0\end{array}$ & $\begin{array}{l}0.0 \\
0.0 \\
0.0 \\
0.0 \\
0.0\end{array}$ & $\begin{array}{l}0.0 \\
0.0 \\
0.0 \\
0.0 \\
0.0\end{array}$ & $\begin{array}{l}0.0 \\
0.0 \\
0.0 \\
0.0 \\
0.0\end{array}$ & $\begin{array}{l}56.03 \\
56.03 \\
56.03 \\
56.03 \\
63.46\end{array}$ & $\begin{array}{l}0.0 \\
0.0 \\
0.0 \\
0.0 \\
0.0\end{array}$ \\
\hline $\begin{array}{l}8.1 .46 \\
8.1 .47 \\
8.1 .48 \\
3.1 .49 \\
8.1 .50\end{array}$ & $\begin{array}{l}1.0 \\
0.0 \\
0.0 \\
3.0 \\
.00\end{array}$ & $\begin{array}{l}0.0 \\
0.0 \\
0.0 \\
0.0 \\
0.0\end{array}$ & $\begin{array}{r}0.0 \\
0.0 \\
0.0 \\
0.0 \\
60.0\end{array}$ & $\begin{array}{r}45.0 \\
45.0 \\
50.0 \\
40.0 \\
0.0\end{array}$ & $\begin{array}{l}0.0 \\
0.0 \\
0.0 \\
0.0 \\
0.0\end{array}$ & $\begin{array}{l}0.0 \\
0.0 \\
0.0 \\
0.0 \\
0.0\end{array}$ & $\begin{array}{l}0.0 \\
0.0 \\
0.0 \\
0.0 \\
0.0\end{array}$ & $\begin{array}{l}0.0 \\
0.0 \\
0.0 \\
0.0 \\
0.0\end{array}$ & $\begin{array}{l}63.46 \\
56.03 \\
56.03 \\
56.03 \\
63.46\end{array}$ & $\begin{array}{l}0.0 \\
0.0 \\
0.0 \\
0.0 \\
0.0\end{array}$ \\
\hline $\begin{array}{l}8.1 .51 \\
3.1 .52 \\
3.1 .53 \\
9.1 .54 \\
8.1 .55\end{array}$ & $\begin{array}{l}0.0 \\
0.0 \\
0.0 \\
. .0 \\
0.0\end{array}$ & $\begin{array}{l}0.0 \\
0.0 \\
0.0 \\
0.0 \\
0.0\end{array}$ & $\begin{array}{r}55.0 \\
0.0 \\
0.0 \\
0.0 \\
0.0\end{array}$ & $\begin{array}{r}0.0 \\
50.0 \\
40.0 \\
30.0 \\
20.0\end{array}$ & $\begin{array}{l}0.0 \\
0.0 \\
0.0 \\
0.0 \\
0.0\end{array}$ & $\begin{array}{l}0.0 \\
0.0 \\
0.0 \\
0.0 \\
0.0\end{array}$ & $\begin{array}{l}0.0 \\
0.0 \\
0.0 \\
0.0 \\
0.0\end{array}$ & $\begin{array}{l}0.0 \\
0.0 \\
0.0 \\
0.0 \\
0.0\end{array}$ & $\begin{array}{l}63.46 \\
63.46 \\
63.46 \\
63.46 \\
63.46\end{array}$ & $\begin{array}{l}0.0 \\
0.0 \\
0.0 \\
0.0 \\
0.0\end{array}$ \\
\hline $\begin{array}{l}9.1 .56 \\
8.1 .57 \\
8.1 .58 \\
3.1 .59 \\
8.1 .60\end{array}$ & $\begin{array}{l}J .0 \\
. .0 \\
.00 \\
.0 \\
. .0\end{array}$ & $\begin{array}{l}J .0 \\
0.0 \\
0.0 \\
j .0 \\
0.0\end{array}$ & $\begin{array}{r}0.0 \\
60.0 \\
0.0 \\
0.0 \\
0.0\end{array}$ & $\begin{array}{r}35.0 \\
0.0 \\
50.0 \\
40.0 \\
45.0\end{array}$ & $\begin{array}{l}0.0 \\
0.0 \\
0.0 \\
0.0 \\
0.0\end{array}$ & $\begin{array}{l}0.0 \\
0.0 \\
0.0 \\
0.0 \\
0.0\end{array}$ & $\begin{array}{l}0.0 \\
0.0 \\
0.0 \\
0.0 \\
0.0\end{array}$ & $\begin{array}{l}0.0 \\
0.0 \\
0.0 \\
0.0 \\
0.0\end{array}$ & $\begin{array}{l}63.46 \\
56.03 \\
56.03 \\
56.03 \\
81.67\end{array}$ & $\begin{array}{l}0.0 \\
0.0 \\
0.0 \\
0.0 \\
0.0\end{array}$ \\
\hline $\begin{array}{c}\text { TEST } \\
\text { HO }\end{array}$ & $\begin{array}{l}\text { P-CH-1 } \\
\text { (PSI }(r,)\end{array}$ & $\begin{array}{l}D-C 4-2 \\
(P S I G)\end{array}$ & $\begin{array}{l}\text { P-HG-1 } \\
\text { (PSIG) }\end{array}$ & $\begin{array}{l}\text { P-HG-2 } \\
\text { (PSIG) }\end{array}$ & $\begin{array}{l}\text { P-HG-3 } \\
\text { (PSIG) }\end{array}$ & $\begin{array}{l}T F-C H-1 \\
(D F G F)\end{array}$ & $\begin{array}{l}T F-H S-1 \\
\text { IDEG FI }\end{array}$ & $\begin{array}{l}T F-H r,-2 \\
(n E r, F)\end{array}$ & $\begin{array}{l}\text { TF-HG-3 } \\
(D \leqslant G F)\end{array}$ & $\begin{array}{l}\text { P-BARD } \\
\text { (PSIA) }\end{array}$ \\
\hline $\begin{array}{l}8.1: 1 \\
3.1: 2 \\
8.1: 3 \\
8.1: 4 \\
3.1 .5\end{array}$ & $\begin{array}{l}29.0 \\
19.0 \\
19.0 \\
19.0 \\
13.5\end{array}$ & $\begin{array}{l}0.0 \\
0.0 \\
0.0 \\
0.0 \\
0.0\end{array}$ & $\begin{array}{l}21.0 \\
19.0 \\
19.0 \\
20.0 \\
18.0\end{array}$ & $\begin{array}{l}90.0 \\
9.0 .0 \\
89.5 \\
99.0 \\
89.0\end{array}$ & $\begin{array}{l}36.0 \\
35.0 \\
35.0 \\
35.0 \\
35.0\end{array}$ & $\begin{array}{l}46.0 \\
48.5 \\
48.5 \\
49.5 \\
48.0\end{array}$ & $\begin{array}{r}90.0 \\
103.0 \\
110.0 \\
109.0 \\
113.0\end{array}$ & $\begin{array}{l}101.0 \\
118.0 \\
124.0 \\
121.0 \\
126.0\end{array}$ & $\begin{array}{r}97.0 \\
111.0 \\
118.0 \\
116.0 \\
120.0\end{array}$ & $\begin{array}{l}12.3 \\
12.3 \\
12.3 \\
12.3 \\
12.3\end{array}$ \\
\hline $\begin{array}{ll}8.1 . & 0 \\
8.1 .7 \\
8.1 .8 \\
8.1 .9 \\
8.1 .10\end{array}$ & $\begin{array}{l}17.5 \\
13.5 \\
19.0 \\
13.0 \\
13.0\end{array}$ & $\begin{array}{l}0.0 \\
0.0 \\
0.0 \\
0.0 \\
0.0\end{array}$ & $\begin{array}{r}20.0 \\
19.0 \\
19.0 \\
19.0 \\
13.5\end{array}$ & $\begin{array}{l}90.0 \\
90.0 \\
90.0 \\
90.0 \\
92.0\end{array}$ & $\begin{array}{l}35.0 \\
35.0 \\
35.0 \\
35.0 \\
35.0\end{array}$ & $\begin{array}{l}50.0 \\
50.0 \\
51.5 \\
51.5 \\
50.0\end{array}$ & $\begin{array}{r}85.0 \\
94.0 \\
97.0 \\
100.0 \\
89.0\end{array}$ & $\begin{array}{r}95.0 \\
108.0 \\
111.0 \\
115.0 \\
79.0\end{array}$ & $\begin{array}{l}0.0 \\
0.0 \\
0.0 \\
0.0 \\
0.0\end{array}$ & $\begin{array}{l}12.4 \\
12.4 \\
12.4 \\
12.4 \\
12.4\end{array}$ \\
\hline
\end{tabular}


TABLE XXXI (Contd.)

SHORTENED CORE BARREL TEST GROU? 8.1

EXPERIIENTAL MEASURMENTS FOR TESTS 8.1 .1 TO B.1.60-- 1.45-IN. DOWMCUMER GAP

\begin{tabular}{|c|c|c|c|c|c|c|c|c|c|c|}
\hline $\begin{array}{l}\text { TEST } \\
\text { NO }\end{array}$ & $\begin{array}{l}P-C H-1 \\
\text { (PSIG) }\end{array}$ & $\begin{array}{l}P-C W-2 \\
\text { IPSIGI }\end{array}$ & $\begin{array}{l}P-H G-1 \\
(P S I G)\end{array}$ & $\begin{array}{l}P-4 G-2 \\
\left(051 r_{2}\right)\end{array}$ & $\begin{array}{l}P-H C_{5}-3 \\
(P S I G)\end{array}$ & $\begin{array}{l}\text { TF-CH-1 } \\
\text { (DE'G FI }\end{array}$ & $\begin{array}{l}\text { TF-HG-1 } \\
\text { (DEG F) }\end{array}$ & $\begin{array}{l}T F-H G-2 \\
\left(\cap F F_{G} F\right)\end{array}$ & $\begin{array}{l}\text { TF-HG-3 } \\
\text { (DEG F) }\end{array}$ & $\begin{array}{l}\text { P-BARח } \\
\text { (PSIA) }\end{array}$ \\
\hline $\begin{array}{l}8.1 .11 \\
9.1 .12 \\
3.1 .13 \\
8.1 .14 \\
8.1 .15\end{array}$ & $\begin{array}{l}13.5 \\
19.0 \\
19.5 \\
18.5 \\
13.0\end{array}$ & $\begin{array}{l}0.0 \\
0.0 . \\
0.0 \\
0.0 \\
0.0\end{array}$ & $\begin{array}{l}18.5 \\
19.0 \\
19.0 \\
18.5 \\
18.5\end{array}$ & $\begin{array}{l}91.0 \\
90.0 \\
89.0 \\
89.0 \\
39.0\end{array}$ & $\begin{array}{r}35.0 \\
0.0 \\
0.0 \\
0.0 \\
0.0\end{array}$ & $\begin{array}{l}50.0 \\
50.5 \\
54.5 \\
49.5 \\
51.5\end{array}$ & $\begin{array}{l}92.0 \\
97.0 \\
86.0 \\
98.0 \\
98.0\end{array}$ & $\begin{array}{r}104.0 \\
110.0 \\
96.0 \\
109.0 \\
112.0\end{array}$ & $\begin{array}{l}0.0 \\
0.0 \\
0.0 \\
0.0 \\
0.0\end{array}$ & $\begin{array}{l}12.4 \\
12.4 \\
12.4 \\
12.4 \\
12.4\end{array}$ \\
\hline $\begin{array}{l}8.1 .16 \\
8.1 .17 \\
8.1 .18 \\
8.1 .19 \\
8.1 .20\end{array}$ & $\begin{array}{l}19.0 \\
13.5 \\
13.0 \\
13.5 \\
13.0\end{array}$ & $\begin{array}{l}0.0 \\
0.0 \\
0.0 \\
0.0 \\
0.0\end{array}$ & $\begin{array}{l}18.5 \\
18.5 \\
18.5 \\
18.5 \\
19.0\end{array}$ & $\begin{array}{l}89.0 \\
98.0 \\
89.0 \\
09.0 \\
93.0\end{array}$ & $\begin{array}{l}0.0 \\
0.0 \\
0.0 \\
0.0 \\
0.0\end{array}$ & $\begin{array}{l}52.0 \\
51.0 \\
50.5 \\
50.5 \\
51.5\end{array}$ & $\begin{array}{r}100.0 \\
106.0 \\
104.0 \\
105.0 \\
79.0\end{array}$ & $\begin{array}{r}115.0 \\
121.0 \\
117.0 \\
118.0 \\
84.0\end{array}$ & $\begin{array}{l}0.0 \\
0.0 \\
0.0 \\
0.0 \\
0.0\end{array}$ & $\begin{array}{l}12.4 \\
12.4 \\
12.4 \\
12.4 \\
12.3\end{array}$ \\
\hline $\begin{array}{l}9.1 .21 \\
8.1 .22 \\
3.1 .23 \\
9.1 .24 \\
8.1 .25\end{array}$ & $\begin{array}{l}19.0 \\
13.5 \\
23.5 \\
19.0 \\
17.5 .\end{array}$ & $\begin{array}{l}0.0 \\
0.0 \\
0.0 \\
0.0 \\
0.0\end{array}$ & $\begin{array}{l}19.0 \\
19.0 \\
21.5 \\
19.5 \\
18.0\end{array}$ & $\begin{array}{l}92.0 \\
91.0 \\
90.0 \\
39.0 \\
89.5\end{array}$ & $\begin{array}{l}0.0 \\
0.0 \\
0.0 \\
0.0 \\
0.0\end{array}$ & $\begin{array}{l}52.0 \\
50.5 \\
50.0 \\
63.0 \\
52.5\end{array}$ & $\begin{array}{r}86.0 \\
94.0 \\
101.0 \\
97.0 \\
100.0\end{array}$ & $\begin{array}{r}98.0 \\
108.0 \\
117.0 \\
112.0 \\
114.0\end{array}$ & $\begin{array}{l}0.0 \\
0.0 \\
0.0 \\
0.0 \\
0.0\end{array}$ & $\begin{array}{l}12.3 \\
12.3 \\
12.3 \\
12.3 \\
12.3\end{array}$ \\
\hline $\begin{array}{l}9.1 .26 \\
8.1 .27 \\
8.1 .28 \\
8.1 .27 \\
8.1 .30\end{array}$ & $\begin{array}{l}19.5 \\
13.0 \\
13.9 \\
13.5 \\
13.5\end{array}$ & $\begin{array}{l}0.0 \\
0.0 \\
0.0 \\
0.0 \\
0.0\end{array}$ & $\begin{array}{l}19.0 \\
19.0 \\
19.0 \\
18.5 \\
18.5\end{array}$ & $\begin{array}{l}89.0 . \\
89.0 \\
91.0 \\
91.0 \\
91.0\end{array}$ & $\begin{array}{l}0.0 \\
0.0 \\
0.0 \\
0.0 \\
0.0\end{array}$ & $\begin{array}{l}50.5 \\
51.0 \\
60.0 \\
49.5 \\
50.5\end{array}$ & $\begin{array}{r}110.0 \\
117.0 \\
93.0 \\
99.0 \\
103.0\end{array}$ & $\begin{array}{l}126.0 \\
134.0 \\
105.0 \\
111.0 \\
116.0\end{array}$ & $\begin{array}{l}0.0 \\
0.0 \\
0.0 \\
0.0 \\
0.0\end{array}$ & $\begin{array}{l}12.3 \\
: 2.3 \\
12.3 \\
: 2.3 \\
12.3\end{array}$ \\
\hline $\begin{array}{l}8.1 .31 \\
3.1 .32 \\
8.1 .33 \\
8.1 .34 \\
3.1 .35\end{array}$ & $\begin{array}{l}13.5 \\
13.0 \\
2.1 .0 \\
19.0 \\
21.0\end{array}$ & $\begin{array}{l}0.0 \\
0.0 \\
0.0 \\
0.0 \\
0.0\end{array}$ & $\begin{array}{l}18.0 \\
19.0 \\
19.0 \\
18.4 \\
19.5\end{array}$ & $\begin{array}{l}90.0 \\
88.0 \\
91.0 \\
90.0 \\
90.0\end{array}$ & $\begin{array}{l}0.0 \\
0.0 \\
0.0 \\
0.0 \\
0.0\end{array}$ & $\begin{array}{l}54.0 \\
52.5 \\
50.5 \\
51.0 \\
48.5\end{array}$ & $\begin{array}{r}98.0 \\
107.0 \\
111.0 \\
104.0 \\
94.0\end{array}$ & $\begin{array}{l}111.0 \\
121.0 \\
125.0 \\
117.0 \\
105.0\end{array}$ & $\begin{array}{l}0.0 \\
0.0 \\
0.0 \\
0.0 \\
0.0\end{array}$ & $\begin{array}{l}12.3 \\
12.3 \\
12.3 \\
12.3 \\
12.3\end{array}$ \\
\hline $\begin{array}{l}8.1 .36 \\
9.1 .37 \\
8.1 .38 \\
8.1 .39 \\
8.1 .40\end{array}$ & $\begin{array}{l}13.5 \\
18.5 \\
23.0 \\
24.0 \\
21.0\end{array}$ & $\begin{array}{l}0.0 \\
0.0 \\
0.0 \\
0.0 \\
0.0\end{array}$ & $\begin{array}{l}19.5 \\
19.0 \\
19.5 \\
19.5 \\
19.0\end{array}$ & $\begin{array}{l}90.0 . \\
90.0 \\
90.0 \\
92.5 \\
93.0\end{array}$ & $\begin{array}{l}0.0 \\
0.0 \\
0.0 \\
0.0 \\
0.0\end{array}$ & $\begin{array}{l}49.0 \\
51.0 \\
51.0 \\
51.0 \\
49.5\end{array}$ & $\begin{array}{r}97.0 \\
101.0 \\
101.0 \\
98.0 \\
95.5\end{array}$ & $\begin{array}{r}109.0 \\
115.0 \\
113.0 \\
109.0 \\
96.0\end{array}$ & $\begin{array}{l}0.0 \\
0.0 \\
0.0 \\
0.0 \\
0.0\end{array}$ & $\begin{array}{l}12.3 \\
12.3 \\
12.3 \\
12.3 \\
12.3\end{array}$ \\
\hline $\begin{array}{l}8.1 .41 \\
8.1 .42 \\
8.1 .43 \\
9.1 .44 \\
8.1 .45\end{array}$ & $\begin{array}{l}19.0 \\
13.5 \\
19.5 \\
19.5 \\
13.5\end{array}$ & $\begin{array}{l}0.0 \\
0.0 \\
0.0 \\
0.0 \\
0.0\end{array}$ & $\begin{array}{l}19.0 \\
19.0 \\
19.0 \\
19.0 \\
19.0\end{array}$ & $\begin{array}{l}89.0 \\
99.0 \\
54.0 \\
97.0 \\
95.0\end{array}$ & $\begin{array}{l}0.0 \\
0.0 \\
0.0 \\
0.0 \\
0.0\end{array}$ & $\begin{array}{l}49.5 \\
49.5 \\
49.5 \\
50.5 \\
50.0\end{array}$ & $\begin{array}{l}91.0 \\
94.0 \\
94.0 \\
88.5 \\
85.0\end{array}$ & $\begin{array}{r}104.0 \\
107.0 \\
96.0 \\
87.5 \\
37.5\end{array}$ & $\begin{array}{l}0.0 \\
0.0 \\
0.0 \\
0.0 \\
0.0\end{array}$ & $\begin{array}{l}12.3 \\
12.3 \\
12.3 \\
12.3 \\
12.3\end{array}$ \\
\hline $\begin{array}{l}8.1 .46 \\
8.1 .47 \\
8.1 .48 \\
8.1 .47 \\
9.1 .50\end{array}$ & $\begin{array}{l}13.5 \\
13.5 \\
13.5 \\
13.5 \\
13.0\end{array}$ & $\begin{array}{l}0.0 \\
0.0 \\
0.0 \\
0.0 \\
0.0\end{array}$ & $\begin{array}{r}19.0 \\
19.0 \\
-19.0 \\
19.0 \\
19.0\end{array}$ & $\begin{array}{l}95.0 \\
94.5 \\
94.5 \\
95.0 \\
95.0\end{array}$ & $\begin{array}{l}0.0 \\
0.0 \\
0.0 \\
0.0 \\
0.0\end{array}$ & $\begin{array}{l}50.0 \\
52.5 \\
50.0 \\
50.5 \\
48.5\end{array}$ & $\begin{array}{l}86.5 \\
85.0 \\
86.5 \\
89.0 \\
78.0\end{array}$ & $\begin{array}{l}93.0 \\
90.0 \\
94.0 \\
97.0 \\
92.0\end{array}$ & $\begin{array}{l}0.0 \\
0.0 \\
0.0 \\
0.0 \\
0.0\end{array}$ & $\begin{array}{l}12.3 \\
12.3 \\
12.3 \\
12.3 \\
12.3\end{array}$ \\
\hline
\end{tabular}


TABLE XXXI (Contd.)

SHORTENED CORE BARREL TEST GROUP 8.1

EXPERI 1ENTAL MEASURMENTS FOR TESTS 8.1 .1 TO $8.1 .60-1$ - 1.45-IN. DOHNCOMER GAP

\begin{tabular}{|c|c|c|c|c|c|c|c|c|c|c|}
\hline $\begin{array}{l}\text { TEST } \\
\text { MO }\end{array}$ & $\begin{array}{l}P-C W-1 \\
(P S ! G)\end{array}$ & $\begin{array}{l}D-C H-2 \\
(P S I S)\end{array}$ & $\begin{array}{l}\text { P-HG-1 } \\
\text { (PSIG) }\end{array}$ & $\begin{array}{l}P-H G-2 \\
(P S I G)\end{array}$ & $\begin{array}{l}\text { P-HG-3 } \\
(P S I G \mid\end{array}$ & $\begin{array}{l}T F-C H-1 \\
(D E G F)\end{array}$ & $\begin{array}{l}T F-H C_{3}-1 \\
(D E G F)\end{array}$ & $\begin{array}{l}T F-H G-2 \\
(O E G F)\end{array}$ & $\begin{array}{l}\text { TF-HG-3 } \\
\text { (DEG F) }\end{array}$ & $\begin{array}{l}\text { P-BARO } \\
\text { (PSIA) }\end{array}$ \\
\hline $\begin{array}{l}8.1 .51 \\
8.1 .52 \\
8.1 .53 \\
8.1 .54 \\
8.1 .55\end{array}$ & $\begin{array}{l}13.0 \\
18.0 \\
18.0 \\
13.0 \\
18.0\end{array}$ & $\begin{array}{l}0.0 \\
0.0 \\
0.0 \\
0.0 \\
0.0\end{array}$ & $\begin{array}{l}19.0 \\
19.5 \\
19.0 \\
19.0 \\
18.5\end{array}$ & $\begin{array}{l}96.0 \\
94.5 \\
93.5 \\
93.5 \\
93.5\end{array}$ & $\begin{array}{l}0.0 \\
0.0 \\
0.0 \\
0.0 \\
0.0\end{array}$ & $\begin{array}{l}50.5 \\
51.5 \\
55.0 \\
53.5 \\
53.5\end{array}$ & $\begin{array}{l}81.0 \\
88.5 \\
90.0 \\
90.0 \\
92.0\end{array}$ & $\begin{array}{r}89.0 \\
100.0 \\
100.0 \\
99.0 \\
101.0\end{array}$ & $\begin{array}{l}0.0 \\
0.0 \\
0.0 \\
0.0 \\
0.0\end{array}$ & $\begin{array}{l}12.3 \\
12.3 \\
12.3 \\
12.3 \\
12.3\end{array}$ \\
\hline $\begin{array}{l}8.1 .56 \\
8.1 .57 \\
9.1 .58 \\
8.1 .59 \\
8.1 .60\end{array}$ & $\begin{array}{l}13.0 \\
13.0 \\
13.0 \\
17.5 \\
18.0\end{array}$ & $\begin{array}{l}0.0 \\
0.0 \\
0.0 \\
0.0 \\
0.0\end{array}$ & $\begin{array}{l}19.0 \\
18.5 \\
18.5 \\
18.5 \\
19.0\end{array}$ & $\begin{array}{l}93.5 \\
93.5 \\
34.5 \\
95.0 \\
84.0\end{array}$ & $\begin{array}{l}0.0 \\
0.0 \\
0.0 \\
0.0 \\
0.0\end{array}$ & $\begin{array}{l}54.5 \\
51.0 \\
51.5 \\
53.0 \\
52.5\end{array}$ & $\begin{array}{r}91.0 \\
92.0 \\
95.0 \\
103.0 \\
108.0\end{array}$ & $\begin{array}{l}100.0 \\
101.0 \\
105.0 \\
116.0 \\
120.5\end{array}$ & $\begin{array}{l}0.0 \\
0.0 \\
0.0 \\
0.0 \\
0.0\end{array}$ & $\begin{array}{l}12.3 \\
12.3 \\
12.3 \\
12.3 \\
12.3\end{array}$ \\
\hline
\end{tabular}

\begin{tabular}{|c|c|c|c|c|c|c|c|c|c|}
\hline \multirow[b]{2}{*}{$\begin{array}{l}T E S T \\
\text { NO }\end{array}$} & \multicolumn{3}{|c|}{ LONER OLENIJM FLOIA } & \multicolumn{3}{|c|}{ BYPASS FLOW } & \multirow[b]{2}{*}{$\begin{array}{l}\text { LOWER } \\
\text { PLENUM } \\
\text { LEVEL } \\
\text { (IN) }\end{array}$} & \multirow[b]{2}{*}{$\begin{array}{l}P-(P-1 \\
(P S I F)\end{array}$} & \multirow[b]{2}{*}{$\begin{array}{l}T F-L P-1 \\
|D E G F|\end{array}$} \\
\hline & $\begin{array}{l}\text { INIT IAL } \\
\text { JE!GHT } \\
\text { (ILA) }\end{array}$ & $\begin{array}{l}\text { FIMAL } \\
\text { WEIGHT } \\
\text { (LB) }\end{array}$ & $\begin{array}{l}\text { TIME OF } \\
\text { COLLECTICN } \\
\text { (SEC) }\end{array}$ & $\begin{array}{l}\text { INITIAL } \\
\text { WE IGHT } \\
\text { (LB) }\end{array}$ & $\begin{array}{l}\text { FINAL } \\
\text { WEIGHT } \\
\text { (LB) }\end{array}$ & $\begin{array}{c}\text { TIUE OF } \\
\text { COLLECTION } \\
\text { (SEC) }\end{array}$ & & & \\
\hline $\begin{array}{ll}8.1 . & 1 \\
9.1 .1 & 2 \\
8.1 . & 3 \\
8.1 .1 & 4 \\
8.1 . & 5\end{array}$ & $\begin{array}{l}142.0 \\
251.0 \\
324.5 \\
23 \div .0 \\
34 ? .5\end{array}$ & $\begin{array}{l}251.0 \\
324.5 \\
+05.0 \\
347.5 \\
438.0\end{array}$ & $\begin{array}{l}60.0 \\
30.0 \\
20.0 \\
45.0 \\
60.0\end{array}$ & $\begin{array}{l}103.5 \\
202.0 \\
364.5 \\
257.0 \\
280.0\end{array}$ & $\begin{array}{l}262.0 \\
364.5 \\
409.0 \\
280.0 \\
282.9\end{array}$ & $\begin{array}{l}30.0 \\
30.0 \\
30.0 \\
75.0 \\
60.0\end{array}$ & $\begin{array}{l}12.0 \\
12.0 \\
12.0 \\
12.0 \\
12.0\end{array}$ & $\begin{array}{l}20.5 \\
20.5 \\
21.0 \\
21.0 \\
20.5\end{array}$ & $\begin{array}{l}50.0 \\
49.5 \\
50.0 \\
50.5 \\
50.5\end{array}$ \\
\hline $\begin{array}{ll}8.1 . & 6 \\
8.1 . & 7 \\
8.1 . & 3 \\
8.1 .1 & 7 \\
8.1 .10\end{array}$ & $\begin{array}{l}142.5 \\
243.5 \\
311.5 \\
399.0 \\
141.5\end{array}$ & $\begin{array}{l}243.5 \\
317.5 \\
39 \% .0 \\
477.0 \\
225.0\end{array}$ & $\begin{array}{l}40.0 \\
30.0 \\
30.0 \\
30.0 \\
30.0\end{array}$ & $\begin{array}{l}116.0 \\
26 ? .0 \\
383.5 \\
446.0 \\
110.0\end{array}$ & $\begin{array}{l}267.0 \\
383.5 \\
446.0 \\
507.0 \\
233.5\end{array}$ & $\begin{array}{l}25.0 \\
30.0 \\
30.0 \\
60.0 \\
30.0\end{array}$ & $\begin{array}{l}12.0 \\
12.0 \\
12.0 \\
12.3 \\
12.0\end{array}$ & $\begin{array}{l}22.0 \\
20.0 \\
20.0 \\
20.0 \\
20.0\end{array}$ & $\begin{array}{l}55.0 \\
52.0 \\
54.0 \\
53.5 . \\
60.5\end{array}$ \\
\hline $\begin{array}{l}9.1 .11 \\
8.1 .12 \\
3.1 .13 \\
8.1 .14 \\
8.1 .15\end{array}$ & $\begin{array}{l}225.0 \\
326.5 \\
141.5 \\
236.5 \\
347.5\end{array}$ & $\begin{array}{l}326.5 \\
414.0 \\
1236.5 \\
347.5 \\
466.0\end{array}$ & $\begin{array}{l}30.0 \\
30.0 \\
35.0 \\
30.0 \\
30.0\end{array}$ & $\begin{array}{l}233.5 \\
120.5 \\
180.0 \\
199.0 \\
321.0\end{array}$ & $\begin{array}{l}300.0 \\
180.0 \\
189.0 \\
321.0 \\
390.0\end{array}$ & $\begin{array}{l}30.0 \\
45.0 \\
60.0 \\
30.0 \\
30.0\end{array}$ & $\begin{array}{l}12.0 \\
12.0 \\
12.0 \\
12.0 \\
12.0\end{array}$ & $\begin{array}{l}20.0 \\
20.5 \\
20.0 \\
20.0 \\
20.0\end{array}$ & $\begin{array}{l}50.5 \\
51.0 \\
53.5 \\
50.0 \\
52.0\end{array}$ \\
\hline $\begin{array}{l}8.1 .16 \\
8.1 .17 \\
8.1 .18 \\
8.1 .19 \\
0.1 .20\end{array}$ & $\begin{array}{l}353.5 \\
317.0 \\
132.5 \\
277.5 \\
142.5\end{array}$ & $\begin{array}{l}454.0 \\
400.5 \\
277.5 \\
403.0 \\
226.5\end{array}$ & $\begin{array}{l}30.0 \\
30.0 \\
30.0 \\
30.0 \\
30.0\end{array}$ & $\begin{array}{l}390.0 \\
265.0 \\
139.5 \\
253.0 \\
116.0\end{array}$ & $\begin{array}{l}438.0 \\
392.0 \\
253.0 \\
346.5 \\
284.5\end{array}$ & $\begin{array}{l}30.0 \\
30.0 \\
40.0 \\
30.0 \\
30.0\end{array}$ & $\begin{array}{l}12.0 \\
12.0 \\
12.0 \\
12.0 \\
12.0\end{array}$ & $\begin{array}{l}20.0 \\
20.0 \\
20.0 \\
20.0 \\
20.0\end{array}$ & $\begin{array}{l}53.0 \\
53.5 \\
53.5 \\
52.0 \\
51.0\end{array}$ \\
\hline $\begin{array}{l}8.1 .21 \\
8.1 .22 \\
8.1 .23 \\
8.1 .24 \\
8.1 .25\end{array}$ & $\begin{array}{l}226.5 \\
209.0 \\
144.5 \\
20: 5 \\
189.5\end{array}$ & $\begin{array}{l}326.0 \\
301.5 \\
200.5 \\
254.0 \\
237.5\end{array}$ & $\begin{array}{l}30.0 \\
30.0 \\
45.0 \\
45.0 \\
45.0\end{array}$ & $\begin{array}{l}294.5 \\
368.0 \\
260.0 \\
132.0 \\
117.0\end{array}$ & $\begin{array}{l}480.0 \\
518.5 \\
387.5 \\
246.0 \\
233.5\end{array}$ & $\begin{array}{l}30.0 \\
30.0 \\
25.0 \\
25.0 \\
30.0\end{array}$ & $\begin{array}{l}12.0 \\
12.0 \\
12.0 \\
12.0 \\
12.0\end{array}$ & $\begin{array}{l}20.5 \\
20.0 \\
22.5 \\
21.0 \\
19.0\end{array}$ & $\begin{array}{l}54.0 \\
52.5 \\
55.0 \\
54.0 \\
55.5\end{array}$ \\
\hline
\end{tabular}


TABLE XXXI (Contd.)

SHORTENED CORE BARREL TEST GROUP 8.1

EXPERIMENT AL MEASURMENTS FOR TESTS 8.1 .1 TU $8.1 .60--1.45-1$ N. DOWNCOMEP. G.AP

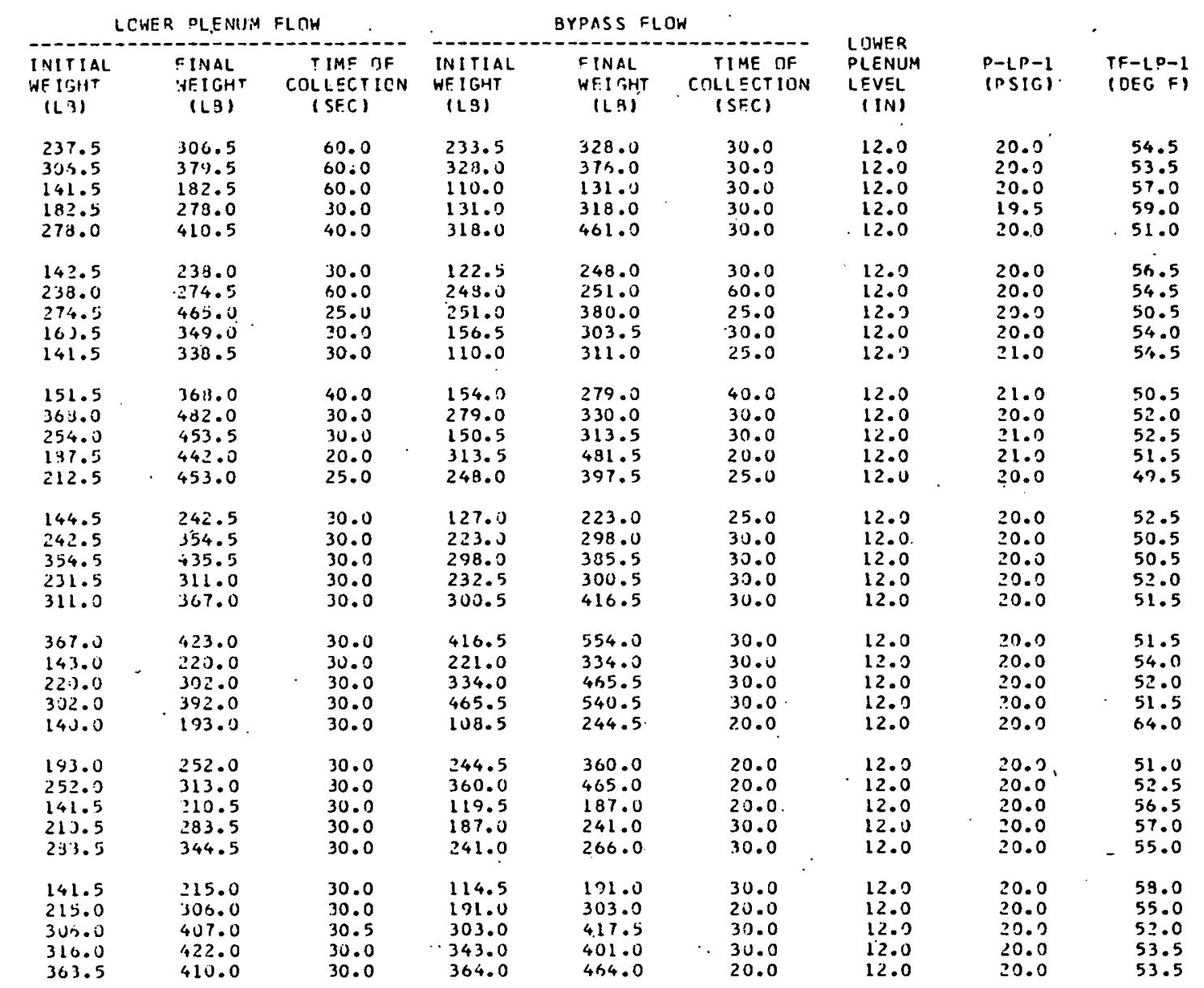




\section{TABLE XXXII}

TWO-PHASE COLD LEG MIXTURE TEST GROUP 1.3

TRANSPARENT VESSEL TEST DATA FOR TESTS 1.3 .1 TO $1.3 .65--0.35-1 \mathrm{~N}$. DOHNCOMER GAP SUMMARY OF CALCULATED RESULTS

\begin{tabular}{|c|c|c|c|c|c|c|c|c|c|c|}
\hline $\begin{array}{l}\text { TEST } \\
\text { NO }\end{array}$ & $\begin{array}{l}\text { DOWNCOMER } \\
\text { AIR FLDW } \\
(L B / S E C)\end{array}$ & $\begin{array}{l}\text { DOHNC JMER } \\
\text { AIR DENSITY } \\
\text { (LB/FT } \$ 3 \text { ) }\end{array}$ & $\begin{array}{c}\text { LOHER PLENUM } \\
\text { PRESSURE } \\
\text { (PSIA) }\end{array}$ & $\begin{array}{l}\text { COLD LEG } \\
\text { ALR FLOW } \\
\text { QLB/SECI }\end{array}$ & $\begin{array}{l}\text { DOWNCOMER } \\
\text { WATER FLOW } \\
\text { (LB/SEC) }\end{array}$ & $\begin{array}{l}\text { HATEP FLOH } \\
\text { CONTINUITY }\end{array}$ & $\begin{array}{c}\text { J-AIR } \\
\text { DOWNCDMER } \\
\text { (FT/SEC) }\end{array}$ & $\begin{array}{l}\text { J-HATER } \\
\text { DOHACOMER } \\
\text { IFT/SECI }\end{array}$ & $(J G *) * * 1 / 2$ & $(J L *) \neq * 1$ \\
\hline $\begin{array}{l}1.3 \cdot 1 \\
1 \cdot 3 \cdot 2 \\
1.3 \cdot 3 \\
1.3 \cdot 3 \\
1.3 .5\end{array}$ & $\begin{array}{l}0.1270 \\
0.1279 \\
0.1252 \\
0.1247 \\
0.0925\end{array}$ & $\begin{array}{l}0.1161 \\
0.1163 \\
0.1163 \\
0.1166 \\
0.1166\end{array}$ & $\begin{array}{l}22.32 \\
22.32 \\
22.32 \\
22.32 \\
22.32\end{array}$ & $\begin{array}{l}0.0485 \\
0.0481 \\
0.0476 \\
0.0467 \\
0.0468\end{array}$ & $\begin{array}{l}0.159 \\
0.165 \\
0.228 \\
0.161 \\
0.475\end{array}$ & $\begin{array}{l}1.021 \\
1.008 \\
0.995 \\
1.024 \\
1.005\end{array}$ & $\begin{array}{l}17.65 \\
17.74 \\
17.36 \\
17.26 \\
12.81\end{array}$ & $\begin{array}{l}0.041 \\
0.043 \\
0.059 \\
0.042 \\
0.123\end{array}$ & $\begin{array}{l}0.7441 \\
0.7464 \\
0.7383 \\
0.7366 \\
0.6345\end{array}$ & $\begin{array}{l}0.1731 \\
0.1762 \\
0.2070 \\
0.1741 \\
0.2989\end{array}$ \\
\hline $\begin{array}{l}1.3 .6 \\
1.3 .7 \\
1.3 .8 \\
1.3 .5 \\
1.3 .10\end{array}$ & $\begin{array}{l}0.0924 \\
0.0924 \\
0.0924 \\
0.0924 \\
0.0628\end{array}$ & $\begin{array}{l}0.1166 \\
0.1168 \\
0.1166 \\
0.1166 \\
.11168\end{array}$ & $\begin{array}{l}22.32 \\
22.32 \\
22.32 \\
22.32 \\
22.37\end{array}$ & $\begin{array}{l}0.0478 \\
0.0468 \\
0.0463 \\
0.0468 \\
0.0484\end{array}$ & $\begin{array}{l}0.594 \\
0.617 \\
0.535 \\
0.487 \\
1.256\end{array}$ & $\begin{array}{l}1.006 \\
0.985 \\
1.032 \\
1.064 \\
0.992\end{array}$ & $\begin{array}{r}12.80 \\
12.77 \\
12.80 \\
12.80 \\
8.68\end{array}$ & $\begin{array}{l}0.154 \\
0.159 \\
0.138 \\
0.126 \\
0.325\end{array}$ & $\begin{array}{l}0.6342 \\
0.6339 \\
0.6342 \\
0.6342 \\
0.5224\end{array}$ & $\begin{array}{l}0.3343 \\
0.3405 \\
0.3172 \\
0.3028 \\
0.4861\end{array}$ \\
\hline $\begin{array}{l}1.3 .11 \\
1.3 .12 \\
1.3 .13 \\
1.3 .14 \\
1.3 .15\end{array}$ & $\begin{array}{l}0.0625 \\
0.0532 \\
0.0635 \\
0.0311 \\
0.0308\end{array}$ & $\begin{array}{l}0.1168 \\
0.1168 \\
0.1163 \\
0.1169 \\
0.1168\end{array}$ & $\begin{array}{l}22.37 \\
22.37 \\
22.37 \\
22.37 \\
22.37\end{array}$ & $\begin{array}{l}0.0479 \\
0.0481 \\
0.0478 \\
0.0486 \\
0.0479\end{array}$ & $\begin{array}{l}1.261 \\
1.379 \\
1.209 \\
2.821 \\
2.409\end{array}$ & $\begin{array}{l}0.998 \\
1.013 \\
0.982 \\
1.016 \\
0.972\end{array}$ & $\begin{array}{l}8.64 \\
8.73 \\
8.81 \\
4.30 \\
4.26\end{array}$ & $\begin{array}{l}0.326 \\
0.356 \\
0.313 \\
0.730 \\
0.523\end{array}$ & $\begin{array}{l}0.5213 \\
0.5241 \\
0.5259 \\
0.3680 \\
0.3661\end{array}$ & $\begin{array}{l}0.4869 \\
0.5052 \\
0.4768 \\
0.7284 \\
0.6730\end{array}$ \\
\hline $\begin{array}{l}1.3 .16 \\
1.3 .17 \\
1.3 .18 \\
1.3 .19 \\
1.3 .20\end{array}$ & $\begin{array}{l}0.0312 \\
0.0309 \\
0.0156 \\
0.0155 \\
0.0154\end{array}$ & $\begin{array}{l}0.1168 \\
0.1166 \\
0.1170 \\
0.1170 \\
0.1170\end{array}$ & $\begin{array}{l}22.37 \\
22.37 \\
22.37 \\
22.37 \\
22.37\end{array}$ & $\begin{array}{l}0.0484 \\
0.0481 \\
0.0491 \\
0.0485 \\
0.0479\end{array}$ & $\begin{array}{l}2.586 \\
2.017 \\
3.450 \\
2.883 \\
.2 .286\end{array}$ & $\begin{array}{l}1.003 \\
0.997 \\
1.004 \\
0.9 \cdot 98 \\
0.913\end{array}$ & $\begin{array}{l}4.31 \\
4.28 \\
2.16 \\
2.13 \\
2.12\end{array}$ & $\begin{array}{l}0.569 \\
0.521 \\
0.892 \\
0.746 \\
0.591\end{array}$ & $\begin{array}{l}0.3681 \\
0.3670 \\
0.2606 \\
0.2592 \\
0.2585\end{array}$ & $\begin{array}{l}0.6973 \\
0.06158 \\
0.8055 \\
0.7364 \\
0.6556\end{array}$ \\
\hline $\begin{array}{l}1.3 .26 \\
1.3 .27 \\
1.3 .28 \\
1.3 .29 \\
1.3 .30\end{array}$ & $\begin{array}{l}0.0781 \\
0.0776 \\
0.0785 \\
0.1108 \\
0.1097\end{array}$ & $\begin{array}{l}0.1168 \\
0.1168 \\
0.1168 \\
0.1108 \\
0.1168\end{array}$ & $\begin{array}{l}22.37 \\
22.37 \\
22.37 \\
22.37 \\
22.37\end{array}$ & $\begin{array}{l}0.0474 \\
0.0468 \\
0.0471 \\
0.0481 \\
0.0476\end{array}$ & $\begin{array}{l}0.800 \\
0.906 \\
0.811 \\
0.271 \\
0.256\end{array}$ & $\begin{array}{l}0.996 \\
0.988 \\
0.999 \\
1.009 \\
0.994\end{array}$ & $\begin{array}{l}10.79 \\
10.73 \\
10.84 \\
15.31 \\
15.15\end{array}$ & $\begin{array}{l}0.207 \\
0.234 \\
0.210 \\
0.070 \\
0.066\end{array}$ & $\begin{array}{l}0.5825 \\
0.5810 \\
0.5841 \\
0.6941 \\
0.6904\end{array}$ & $\begin{array}{l}0.3879 \\
0.4127 \\
0.3906 \\
0.2257 \\
0.2192\end{array}$ \\
\hline $\begin{array}{l}1.3 .31 \\
1.3 .32 \\
1.3 .33 \\
1.3 .34 \\
1.3 .35\end{array}$ & $\begin{array}{l}0.1109 \\
0.1084 \\
0.1096 \\
0.1272 \\
0.1232\end{array}$ & $\begin{array}{l}0.1168 \\
0.1163 \\
0.1166 \\
0.1170 \\
0.1170\end{array}$ & $\begin{array}{l}22.37 \\
22.37 \\
22.37 \\
22.37 \\
22.37\end{array}$ & $\begin{array}{l}0.0479 \\
0.0471 \\
0.0474 \\
0.0998 \\
0.0958\end{array}$ & $\begin{array}{l}0.350 \\
0.342 \\
0.254 \\
0.237 \\
0.167\end{array}$ & $\begin{array}{l}1.038 \\
1.006 \\
1.024 \\
1.011 \\
1.013\end{array}$ & $\begin{array}{l}15.31 \\
15.03 \\
15.17 \\
17.55 \\
16.99\end{array}$ & $\begin{array}{l}0.091 \\
0.099 \\
0.066 \\
0.061 \\
0.043\end{array}$ & $\begin{array}{l}0.6941 \\
0.6870 \\
0.6904 \\
0.7434 \\
0.7315\end{array}$ & $\begin{array}{l}0.2566 \\
0.2535 \\
0.2186 \\
0.2112 \\
0.1770\end{array}$ \\
\hline $\begin{array}{l}1.3 .36 \\
1.3 .37 \\
1.3 .33 \\
1.3 .39 \\
1.3 .40\end{array}$ & $\begin{array}{l}0.1257 \\
0.1244 \\
0.1240 \\
0.0946 \\
0.0946\end{array}$ & $\begin{array}{l}0.1167 \\
0.1169 \\
0.1169 \\
0.1169 \\
0.1169\end{array}$ & $\begin{array}{l}22.43 \\
22.43 \\
22.43 \\
22.43 \\
22.43\end{array}$ & $\begin{array}{l}0.0964 \\
0.0959 \\
0.0937 \\
0.0979 \\
0.0974\end{array}$ & $\begin{array}{l}0.192 \\
0.118 \\
0.092 \\
0.495 \\
0.450\end{array}$ & $\begin{array}{l}1.011 \\
0.970 \\
0.948 \\
1.013 \\
0.993\end{array}$ & $\begin{array}{l}17.39 \\
17.17 \\
17.11 \\
13.06 \\
13.06\end{array}$ & $\begin{array}{l}0.050 \\
0.030 \\
0.024 \\
0.129 \\
0.116\end{array}$ & $\begin{array}{l}0.7395 \\
0.7352 \\
0.7339 \\
0.6412 \\
0.6412\end{array}$ & $\begin{array}{l}0.1899 \\
0.1489 \\
0.1313 \\
0.3051 \\
0.2909\end{array}$ \\
\hline
\end{tabular}


TABLE XXXII (Contd.)

TWO-PHASE COLD LEG MIXTURE TEST GROUP I.3

SIJMMARY gF CALCIJLATFE RE.SULTS FOR TESTS 1.3 .1 TO $1.3 .65-0.35$ IN. DOHNCOMER GAP

\begin{tabular}{|c|c|c|c|c|c|c|c|c|c|c|}
\hline $\begin{array}{l}\text { TEST } \\
\text { NO }\end{array}$ & $\begin{array}{l}\text { DOWACOMER } \\
\text { AIR FLOWW } \\
\text { (LB/SEC) }\end{array}$ & $\begin{array}{l}\text { D.JWNCOMER } \\
\text { AIR DENSITY } \\
\text { (LB/FT } 1 \text { I I }\end{array}$ & $\begin{array}{c}\text { LOWER PLENUM } \\
\text { PRESSURE } \\
\text { (PSIA) }\end{array}$ & $\begin{array}{l}\text { COLN LEG } \\
\text { AIR FLDW } \\
\text { (LS/SEC) }\end{array}$ & $\begin{array}{l}\text { DOWNCOMEP. } \\
\text { WATER FLUH } \\
\text { ILB/SECI }\end{array}$ & $\begin{array}{l}\text { WATFR FLחW } \\
\text { CONTINIJITY }\end{array}$ & $\begin{array}{c}\text { J-AID } \\
\text { DONCDMER } \\
\text { (FT/SECI }\end{array}$ & $\begin{array}{l}\text { J-WA TER } \\
\text { DOWNC TMER } \\
\text { IFT/SSC.I }\end{array}$ & $(J G *) * * 1 / 2$ & $(J L *) * * 1 / 2$ \\
\hline $\begin{array}{l}1.3 .41 \\
1.3 .42 \\
1.3 .43 \\
1.3 .44 \\
1.3 .45\end{array}$ & $\begin{array}{l}j .0946 \\
0.0941 \\
0.0450 \\
0.0623 \\
0.0616\end{array}$ & $\begin{array}{l}0.1169 \\
0.1169 \\
0.1169 \\
0.1171 \\
0.1171\end{array}$ & $\begin{array}{l}22.43 \\
22.43 \\
22.43 \\
22.43 \\
22.43\end{array}$ & $\begin{array}{l}0.0959 \\
0.0954 \\
0.0958 \\
0.0974 \\
0.0967\end{array}$ & $\begin{array}{l}0.486 \\
0.465 \\
0.464 \\
1.394 \\
1.225\end{array}$ & $\begin{array}{l}0.993 \\
1.000 \\
1.074 \\
0.995 \\
1.001\end{array}$ & $\begin{array}{r}13.06 \\
12.99 \\
13.12 \\
8.58 \\
8.49\end{array}$ & $\begin{array}{l}0.126 \\
0.120 \\
0.120 \\
0.360 \\
0.317\end{array}$ & $\begin{array}{l}0.6412 \\
0.6395 \\
0.6426 \\
0.5201 \\
0.5172\end{array}$ & $\begin{array}{l}0.3024 \\
0.2957 \\
0.2953 \\
0.5120 \\
0.4800\end{array}$ \\
\hline $\begin{array}{l}1.3 .46 \\
1.3 .47 \\
1.3 .48 \\
1.3 .49 \\
1.3 .50\end{array}$ & $\begin{array}{l}0.0023 \\
0.0616 \\
0.0309 \\
0.0310 \\
0.0314\end{array}$ & $\begin{array}{l}0.1173 \\
0.1173 \\
0.1173 \\
0.1173 \\
0.1173\end{array}$ & $\begin{array}{l}22.43 \\
22.43 \\
22.43 \\
22.43 \\
22.43\end{array}$ & $\begin{array}{l}0.0954 \\
0.0942 \\
0.0985 \\
0.1019 \\
0.1004\end{array}$ & $\begin{array}{l}1.275 \\
1.139 \\
2.106 \\
2.525 \\
2.142\end{array}$ & $\begin{array}{l}0.990 \\
1.020 \\
0.928 \\
0.987 \\
0.978\end{array}$ & $\begin{array}{l}8.57 \\
8.48 \\
4.25 \\
4.27 \\
4.31\end{array}$ & $\begin{array}{l}0.330 \\
0.295 \\
0.545 \\
0.553 \\
0.554\end{array}$ & $\begin{array}{l}0.5198 \\
0.5170 \\
0.3659 \\
0.3669 \\
0.3687\end{array}$ & $\begin{array}{l}0.4897 \\
0.4628 \\
0.6294 \\
0.6891 \\
0.6346\end{array}$ \\
\hline $\begin{array}{l}1.3 .51 \\
1.3 .52 \\
1.3 .53 \\
1.3 .54 \\
1.3 .55\end{array}$ & $\begin{array}{l}0.0312 \\
0.0153 \\
0.0153 \\
0.0154 \\
0.0459\end{array}$ & $\begin{array}{l}0.1173 \\
0.1173 \\
0.1173 \\
0.1173 \\
0.1173\end{array}$ & $\begin{array}{l}22.43 \\
22.43 \\
22.43 \\
22.43 \\
22.43\end{array}$ & $\begin{array}{l}0.0979 \\
0.1011 \\
0.1901 \\
0.0970 \\
0.0990\end{array}$ & $\begin{array}{l}1.942 \\
2.956 \\
2.392 \\
2.050 \\
1.909\end{array}$ & $\begin{array}{l}1.010 \\
1.011 \\
0.993 \\
0.991 \\
0.998\end{array}$ & $\begin{array}{l}4.29 \\
2.11 \\
2.11 \\
2.12 \\
6.32\end{array}$ & $\begin{array}{l}0.502 \\
0.764 \\
0.618 \\
0.530 \\
0.469\end{array}$ & $\begin{array}{l}0.3679 \\
0.2579 \\
0.2578 \\
0.2585 \\
0.4463\end{array}$ & $\begin{array}{l}0.6043 \\
0.7456 \\
0.6707 \\
0.6209 \\
0.5832\end{array}$ \\
\hline $\begin{array}{l}1.3 .56 \\
1.3 .57 \\
1.3 .58 \\
1.3 .59 \\
1.3 .60\end{array}$ & $\begin{array}{l}0.0467 \\
0.0472 \\
0.0792 \\
0.0784 \\
0.0772\end{array}$ & $\begin{array}{l}0.1173 \\
0.1173 \\
0.1169 \\
0.1169 \\
0.1109\end{array}$ & $\begin{array}{l}22.43 \\
22.43 \\
22.43 \\
22.43 \\
22.43\end{array}$ & $\begin{array}{l}0.0995 \\
0.0984 \\
0.1018 \\
0.0988 \\
0.0958\end{array}$ & $\begin{array}{l}1.983 \\
1.733 \\
0.862 \\
0.810 \\
0.794\end{array}$ & $\begin{array}{l}1.015 \\
1.001 \\
1.020 \\
0.993 \\
1.005\end{array}$ & $\begin{array}{r}6.42 \\
6.49 \\
10.94 \\
10.83 \\
10.66\end{array}$ & $\begin{array}{l}0.513 \\
0.449 \\
0.223 \\
0.209 \\
0.205\end{array}$ & $\begin{array}{l}0.4500 \\
0.4524 \\
0.5868 \\
0.5337 \\
0.5793\end{array}$ & $\begin{array}{l}0.6107 \\
0.5709 \\
0.4027 \\
0.3903 \\
0.3865\end{array}$ \\
\hline $\begin{array}{l}1.3 .61 \\
1.3 .62 \\
1.3 .63 \\
1.3 .64 \\
1.3 .65\end{array}$ & $\begin{array}{l}J .0773 . \\
0.1115 \\
0.1080 \\
3.1092 \\
0.1086\end{array}$ & $\begin{array}{l}0.1169 \\
0.1276 \\
0.1167 \\
0.1167 \\
0.1167\end{array}$ & $\begin{array}{l}22.43 \\
24.43 \\
22.43 \\
22.43 \\
22.43\end{array}$ & $\begin{array}{l}0.0948 \\
0.1019 \\
0.0964 \\
0.0958 \\
0.0937\end{array}$ & $\begin{array}{l}0.918 \\
0.229 \\
0.315 \\
0.279 \\
0.267\end{array}$ & $\begin{array}{l}1.041 \\
1.017 \\
1.031 \\
0.992 \\
1.019\end{array}$ & $\begin{array}{l}10.67 \\
14.11 \\
14.94 \\
15.10 \\
15.02\end{array}$ & $\begin{array}{l}0.212 \\
0.059 \\
0.091 \\
0.072 \\
0.069\end{array}$ & $\begin{array}{l}0.5736 \\
0.6811 \\
0.6854 \\
0.6832 \\
0.6873\end{array}$ & $\begin{array}{l}0.3923 \\
0.2076 \\
0.2434 \\
0.2286 \\
0.2239\end{array}$ \\
\hline
\end{tabular}


TABLE XXXII (Contd.)

TWO-PHASE COLD LEG MIXTURE TEST GROUP 1.3

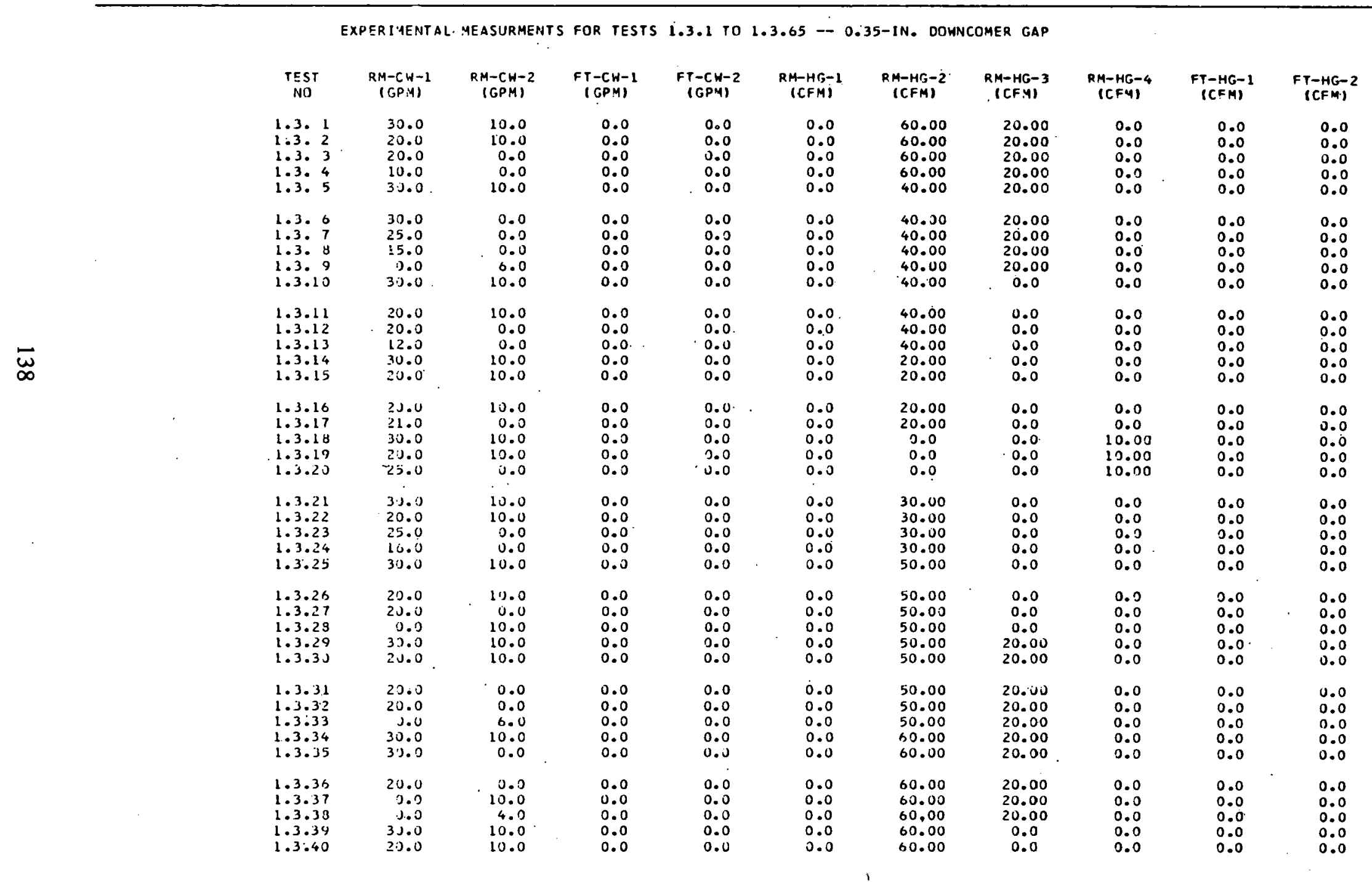


TABLE XXXII (Contd.)

TWO-PHASE COLD LEG MIXTURE TEST GROUP 1.3

EXDEFITENTAL MEASURMENTS FOQ TESTS 1.3 .1 TI 1.3.65 -- 0.35-IN. DOWNCOMER GAP

\begin{tabular}{|c|c|c|c|c|c|c|c|c|c|c|}
\hline $\begin{array}{c}T E S T \\
! N S\end{array}$ & $\begin{array}{c}R M-C H-1 \\
(G P \cdot 1)\end{array}$ & $\begin{array}{c}R M-C W-2 \\
(G P M)\end{array}$ & $\begin{array}{c}F T-(W-1 \\
(G P M) .\end{array}$ & $\begin{array}{c}F T-C H-2 \\
(G P M)\end{array}$ & $\begin{array}{c}R M-H r_{,}-1 \\
(C F M)\end{array}$ & $\begin{array}{l}R M-H G,-2 \\
(C, P M)\end{array}$ & $\begin{array}{c}P M-H G-3 \\
(C F Y)\end{array}$ & $\begin{array}{c}2 M-H G-4 \\
(C F M)\end{array}$ & $\begin{array}{c}F T-H G-1 \\
(C \subset M)\end{array}$ & $\begin{array}{c}F T-H r_{S}-2 \\
(C F M)\end{array}$ \\
\hline $\begin{array}{l}1.3 .41 \\
1.3 .42 \\
1.3 .43 \\
1.3 .44 \\
1.3 .45\end{array}$ & $\begin{array}{r}2.00 \\
3.0 \\
0.0 \\
30.0 \\
20.0\end{array}$ & $\begin{array}{r}0.0 \\
10.0 \\
5.0 \\
10.0 \\
10.0\end{array}$ & $\begin{array}{l}0.0 \\
0.0 \\
0.0 \\
0.0 \\
0.0\end{array}$ & $\begin{array}{l}0.0 \\
0.0 \\
0.0 \\
0.0 \\
0.0\end{array}$ & $\begin{array}{l}0.0 \\
0.0 \\
0.0 \\
0.0 \\
0.0\end{array}$ & $\begin{array}{l}60.00 \\
60.00 \\
60.00 \\
40.00 \\
40.00\end{array}$ & $\begin{array}{l}0.0 \\
0.0 \\
0.0 \\
0.0 \\
0.0\end{array}$ & $\begin{array}{l}0.0 \\
0.0 \\
0.0 \\
0.0 \\
0.0\end{array}$ & $\begin{array}{l}0.0 \\
0.0 \\
0.0 \\
0.0 \\
0.0\end{array}$ & $\begin{array}{l}0.0 \\
0.0 \\
0.0 \\
0.0 \\
0.0\end{array}$ \\
\hline $\begin{array}{l}1.3 .40 \\
1.3 .47 \\
1.3 .48 \\
1.3 .49 \\
1.3 .5 \mathrm{~J}\end{array}$ & $\begin{array}{l}20.0 \\
12.0 \\
30.0 \\
31.0 \\
20.0\end{array}$ & $\begin{array}{r}0.0 \\
0.0 \\
10.0 \\
10.0 \\
10.0\end{array}$ & $\begin{array}{l}0.0 \\
0.0 \\
0.0 \\
0.0 \\
0.0\end{array}$ & $\begin{array}{l}0.0 \\
0.0 \\
0.0 \\
0.0 \\
0.0\end{array}$ & $\begin{array}{l}0.0 \\
0.0 \\
0.0 \\
0.0 \\
0.0\end{array}$ & $\begin{array}{l}40.00 \\
40.00 \\
20.00 \\
20.00 \\
20.00\end{array}$ & $\begin{array}{l}0.0 \\
0.0 \\
0.0 \\
0.0 \\
0.0\end{array}$ & $\begin{array}{l}0.0 \\
0.0 \\
0.0 \\
0.0 \\
0.0\end{array}$ & $\begin{array}{l}0.0 \\
0.0 \\
0.0 \\
0.0 \\
0.0\end{array}$ & $\begin{array}{l}0.0 \\
0.0 \\
0.0 \\
0.0 \\
0.0\end{array}$ \\
\hline $\begin{array}{l}1.3 .51 \\
1.3 .52 \\
1.3 .53 \\
1.3 .54 \\
1.3 .55\end{array}$ & $\begin{array}{l}20.0 \\
30.0 \\
20.0 \\
20.0 \\
3.0 .0\end{array}$ & $\begin{array}{r}0.0 \\
10.0 \\
10.0 \\
0.0 \\
10.0\end{array}$ & $\begin{array}{l}0.0 \\
0.0 \\
0.0 \\
0.0 \\
0.0\end{array}$ & $\begin{array}{l}0.0 \\
0.0 \\
0.0 \\
0.0 \\
0.0\end{array}$ & $\begin{array}{l}0.0 \\
0.0 \\
0.0 \\
0.0 \\
0.0\end{array}$ & $\begin{array}{c}20.00 \\
10.00 \\
0.0 \\
0.0 \\
30.00\end{array}$ & $\begin{array}{c}0.0 \\
0.0 \\
10.00 \\
10.00 \\
0.0\end{array}$ & $\begin{array}{l}0.0 \\
0.0 \\
0.0 \\
0.0 \\
0.0\end{array}$ & $\begin{array}{l}0.0 \\
0.0 \\
0.0 \\
0.0 \\
0.0\end{array}$ & $\begin{array}{l}0.0 \\
0.0 \\
0.0 \\
0.0 \\
0.0\end{array}$ \\
\hline $\begin{array}{l}1.3 .56 \\
1.3 .57 \\
1.3 .58 \\
1.3 .59 \\
1.3 .63\end{array}$ & $\begin{array}{l}20.0 \\
21) .0 \\
30.0 \\
20.0 \\
2.0 .0\end{array}$ & $\begin{array}{r}10.0 \\
0.0 \\
10.0 \\
10.0 \\
0.0\end{array}$ & $\begin{array}{l}0.0 \\
0.0 \\
0.0 \\
0.0 \\
0.0\end{array}$ & $\begin{array}{l}0.0 \\
0.0 \\
0.0 \\
0.0 \\
0.0\end{array}$ & $\begin{array}{l}0.0 \\
0.0 \\
0.0 \\
0.0 \\
0.0\end{array}$ & $\begin{array}{l}30.00 \\
30.00 \\
50.00 \\
50.00 \\
50.00\end{array}$ & $\begin{array}{l}0.0 \\
0.0 \\
0.0 \\
0.0 \\
0.0\end{array}$ & $\begin{array}{l}0.0 \\
0.0 \\
0.0 \\
0.0 \\
0.0\end{array}$ & $\begin{array}{l}0.0 \\
0.0 \\
0.0 \\
0.0 \\
0.0\end{array}$ & $\begin{array}{l}0.0 \\
0.0 \\
0.0 \\
0.0 \\
0.0\end{array}$ \\
\hline $\begin{array}{l}1.3 .01 \\
1.3 .02 \\
1.3 .53 \\
1.3 .04 \\
1.3 .05\end{array}$ & $\begin{array}{l}j .0 \\
30.0 \\
20.0 \\
20.0 \\
0.0\end{array}$ & $\begin{array}{r}8.0 \\
10.0 \\
10.0 \\
0.0 \\
6.0\end{array}$ & $\begin{array}{l}0.0 \\
0.0 \\
0.0 \\
0.0 \\
0.0\end{array}$ & $\begin{array}{l}0.0 \\
0.0 \\
0.0 \\
0.0 \\
0.0\end{array}$ & $\begin{array}{l}0.0 \\
0.0 \\
0.0 \\
0.0 \\
0.0\end{array}$ & $\begin{array}{l}50.00 \\
50.00 \\
50.00 \\
50.00 \\
50.00\end{array}$ & $\begin{array}{l}0.0 \\
20.00 \\
20.00 \\
20.00 \\
20.00\end{array}$ & $\begin{array}{l}0.0 \\
0.0 \\
0.0 \\
0.0 \\
0.0\end{array}$ & $\begin{array}{l}0.0 \\
0.0 \\
0.0 \\
0.0 \\
0.0\end{array}$ & $\begin{array}{l}0.0 \\
0.0 \\
0.0 \\
0.0 \\
0.0\end{array}$ \\
\hline $\begin{array}{c}\text { TEST } \\
N, T\end{array}$ & $\begin{array}{l}D-C: 1-1 \\
(p S I \sigma)\end{array}$ & $\begin{array}{l}P-C(H-2 \\
(P S I G)\end{array}$ & $\begin{array}{l}\text { P-HG-1 } \\
\text { (PSIS) }\end{array}$ & $\begin{array}{l}\text { P-HG-2 } \\
\text { (PSIGI }\end{array}$ & $\begin{array}{l}P+H G-3 \\
(P \leq I G)\end{array}$ & $\begin{array}{l}T F-C H-1 \\
I D E G F \mid\end{array}$ & $\begin{array}{l}\text { TF-HG-1 } \\
\text { IDEG FI }\end{array}$ & $\begin{array}{l}T F-H G-2 \\
\text { (OFG F.) }\end{array}$ & $\begin{array}{l}\text { TF-HG-3 } \\
\text { (CES F) }\end{array}$ & $\begin{array}{l}P-B \Delta R \cap \\
\text { PSSIAI }\end{array}$ \\
\hline $\begin{array}{l}1.3 .1 \\
1.3 .2 \\
1.3 .3 \\
1.3 .4 \\
1.3 .5\end{array}$ & $\begin{array}{l}0.0 \\
. .0 \\
.00 \\
0.0 \\
0.0\end{array}$ & $\begin{array}{l}11.5 \\
11.2 \\
10.7 \\
10.0 \\
10.0\end{array}$ & $\begin{array}{l}11.0 \\
10.7 \\
10.0 \\
10.0 \\
10.0\end{array}$ & $\begin{array}{l}0.0 \\
0.0 \\
0.0 \\
0.0 \\
0.0\end{array}$ & $\begin{array}{l}0.0 \\
0.0 \\
0.0 \\
0.0 \\
0.0\end{array}$ & $\begin{array}{l}52.0 \\
55.0 \\
55.0 \\
56.0 \\
54.0\end{array}$ & $\begin{array}{l}75.0 \\
62.0 \\
68.0 \\
72.0 \\
74.0\end{array}$ & $\begin{array}{l}0.0 \\
0.0 \\
0.0 \\
0.0 \\
0.0\end{array}$ & $\begin{array}{l}0.0 \\
0.0 \\
0.0 \\
0.0 \\
0.0\end{array}$ & $\begin{array}{l}12.3 \\
12.3 \\
12.3 \\
12.3 \\
12.3\end{array}$ \\
\hline
\end{tabular}


TABLE XXXII (Contd.)

TWO-PHASE COLD LEG MIXTURE TEST GROUP 1.3

EXPERIMENTAL MEASURMENTS FOR TESTS 1.3 .1 TO $1.3 .65-0.35-1$ N. OOHNCOMER GAP

\begin{tabular}{|c|c|c|c|c|c|c|c|c|c|c|}
\hline $\begin{array}{c}\text { TEST } \\
\text { NO }\end{array}$ & $\begin{array}{l}P-C W-1 \\
(P S I G)\end{array}$ & $\begin{array}{l}P-C H-2 \\
(P S I G)\end{array}$ & $\begin{array}{l}\text { P-HG-1 } \\
\text { (PSIG) }\end{array}$ & $\begin{array}{l}\text { P-HG-2 } \\
\text { (PSIG) }\end{array}$ & $\begin{array}{l}P-H G-3 \\
(P S I G)\end{array}$ & $\begin{array}{l}\text { TF-CH-1. } \\
\text { IDEG FI }\end{array}$ & $\begin{array}{l}\text { TF-HG - } \\
\text { (DEG F) }\end{array}$ & $\begin{array}{l}\text { TF-HG-2 } \\
\text { IDES FI }\end{array}$ & $\begin{array}{l}T F-H G-3 \\
(O E G F)\end{array}$ & $\begin{array}{l}\text { P-BARO } \\
\text { TPSIAI }\end{array}$ \\
\hline $\begin{array}{l}1.3 .6 \\
1.3 .97 \\
1.3 .3 \\
1.3 .19 \\
1.3 .10\end{array}$ & $\begin{array}{l}0.0 \\
0.0 \\
3.0 \\
0.0 \\
0.0\end{array}$ & $\begin{array}{l}11.0 \\
10.0 \\
10.0 \\
10.0 \\
11.5\end{array}$ & $\begin{array}{l}10.0 \\
10.0 \\
10.0 \\
10.0 \\
10.7\end{array}$ & $\begin{array}{l}0.0 \\
0.0 \\
0.0 \\
0.0 \\
0.0\end{array}$ & $\begin{array}{l}0.0 \\
0.0 \\
0.0 \\
0.0 \\
0.0\end{array}$ & $\begin{array}{l}55.0 \\
54.0 \\
54.0 \\
55.0 \\
55.0\end{array}$ & $\begin{array}{l}75.0 \\
75.0 \\
75.0 \\
75.0 \\
75.0\end{array}$ & $\begin{array}{l}0.0 \\
0.0 \\
0.0 \\
0.0 \\
0.0\end{array}$ & $\begin{array}{l}0.0 \\
0.0 \\
0.0 \\
0.0 \\
0.0\end{array}$ & $\begin{array}{l}12.3 \\
12.3 \\
12.3 \\
12.3 \\
12.4\end{array}$ \\
\hline $\begin{array}{l}1.3 .11 \\
1.3 .12 \\
1.3 .13 \\
1.3 .14 \\
1.3 .15\end{array}$ & $\begin{array}{l}0.0 \\
0.0 \\
0.0 \\
0.0 \\
0.0\end{array}$ & $\begin{array}{r}11.0 \\
11.2 \\
11.0 \\
11.7 \\
.11 .0\end{array}$ & $\begin{array}{l}10.5 \\
11.0 \\
11.2 \\
10.7 \\
10.2\end{array}$ & $\begin{array}{l}0.0 \\
0.0 \\
0.0 \\
0.0 \\
0.0\end{array}$ & $\begin{array}{l}0.0 \\
0.0 \\
0.0 \\
0.0 \\
0.0\end{array}$ & $\begin{array}{l}55.0 \\
55.0 \\
57.0 \\
55.0 \\
55.0\end{array}$ & $\begin{array}{l}74.0 \\
74.0 \\
74.0 \\
75.0 \\
74.0\end{array}$ & $\begin{array}{l}0.0 \\
0.0 \\
0.0 \\
0.0 \\
0.0\end{array}$ & $\begin{array}{l}0.0 \\
0.0 \\
0.0 \\
0.0 \\
0.0\end{array}$ & $\begin{array}{l}12.4 \\
12.4 \\
12.4 \\
12.4 \\
12.4\end{array}$ \\
\hline $\begin{array}{l}1.3 .16 \\
1.3 .17 \\
1.3 .13 \\
1.3 .19 \\
1.3 .20\end{array}$ & $\begin{array}{l}0.0 \\
3.0 \\
3.0 \\
3.0 \\
3.0\end{array}$ & $\begin{array}{l}11.5 \\
11.2 \\
12.2 \\
11.5 \\
11.0\end{array}$ & $\begin{array}{l}10.7 \\
10.5 \\
11.0 \\
10.5 \\
10.2\end{array}$ & $\begin{array}{l}0.0 \\
0.0 \\
0.0 \\
0.0 \\
0.0\end{array}$ & $\begin{array}{l}0.0 \\
0.0 \\
0.0 \\
0.0 \\
0.0\end{array}$ & $\begin{array}{l}55.0 \\
56.0 \\
55.0 \\
53.0 \\
54.0\end{array}$ & $\begin{array}{l}74.0 \\
76.0 \\
76.0 \\
76.0 \\
76.0\end{array}$ & $\begin{array}{l}0.0 \\
0.0 \\
0.0 \\
0.0 \\
0.0\end{array}$ & $\begin{array}{l}0.0 \\
0.0 \\
0.0 \\
0.0 \\
0.0\end{array}$ & $\begin{array}{l}12.4 \\
12.4 \\
12.4 \\
12.4 \\
12.4\end{array}$ \\
\hline $\begin{array}{l}1.3 .21 \\
1.3 .22 \\
1.3 .23 \\
1.3 .24 \\
1.3 .25\end{array}$ & $\begin{array}{l}3.0 \\
3.0 \\
3.0 \\
3.0 \\
.00\end{array}$ & $\begin{array}{l}12.2 \\
11.7 \\
11.5 \\
11.2 \\
11.7\end{array}$ & $\begin{array}{l}10.7 \\
10.5 \\
10.7 \\
10.5 \\
10.7\end{array}$ & $\begin{array}{l}0.0 \\
0.0 \\
0.0 \\
0.0 \\
0.0\end{array}$ & $\begin{array}{l}0.0 \\
0.0 \\
0.0 \\
0.0 \\
0.0\end{array}$ & $\begin{array}{l}55.0 \\
55.0 \\
55.0 \\
55.0 \\
55.0\end{array}$ & $\begin{array}{l}76.0 \\
74.0 \\
74.0 \\
74.0 \\
74.0\end{array}$ & $\begin{array}{l}0.0 \\
0.0 \\
0.0 \\
0.0 \\
0.0\end{array}$ & $\begin{array}{l}0.0 \\
0.0 \\
0.0 \\
0.0 \\
0.0\end{array}$ & $\begin{array}{l}12.4 \\
12.4 \\
12.4 \\
12.4 \\
12.4\end{array}$ \\
\hline $\begin{array}{l}1.3 .26 \\
1.3 .27 \\
1.3 .28 \\
1.3 .29 \\
1.3 .30\end{array}$ & $\begin{array}{l}3.0 \\
3.0 \\
0.0 \\
0.0 \\
0.0\end{array}$ & $\begin{array}{l}10.5 \\
10.0 \\
10.2 \\
11.2 \\
10.7\end{array}$ & $\begin{array}{l}10.2 \\
10.0 \\
10.5 \\
11.0 \\
10.5\end{array}$ & $\begin{array}{l}0.0 \\
0.0 \\
0.0 \\
0.0 \\
0.0\end{array}$ & $\begin{array}{l}0.0 \\
0.0 \\
0.0 \\
0.0 \\
0.0\end{array}$ & $\begin{array}{l}55.0 \\
55.0 \\
55.0 \\
55.0 \\
55.0\end{array}$ & $\begin{array}{l}74.0 \\
74.0 \\
74.0 \\
74.0 \\
74.0\end{array}$ & $\begin{array}{l}0.0 \\
0.0 \\
0.0 \\
0.0 \\
0.0\end{array}$ & $\begin{array}{l}0.0 \\
0.0 \\
0.0 \\
0.0 \\
0.0\end{array}$ & $\begin{array}{l}12.4 \\
12.4 \\
12.4 \\
12.4 \\
12.4\end{array}$ \\
\hline $\begin{array}{l}1.3 .31 \\
1.3 .32 \\
1.3 .33 \\
1.3 .34 \\
1.3 .35\end{array}$ & $\begin{array}{l}0.0 \\
1.0 \\
0.0 \\
0.0 \\
0.0\end{array}$ & $\begin{array}{l}11.0 \\
10.2 \\
10.5 \\
13.0 \\
11.0\end{array}$ & $\begin{array}{r}11.0 \\
10.0 \\
10.5 \\
11.0 \\
9.5\end{array}$ & $\begin{array}{l}0.0 \\
0.0 \\
0.0 \\
0.0 \\
0.0\end{array}$ & $\begin{array}{l}0.0 \\
0.0 \\
0.0 \\
0.0 \\
0.0\end{array}$ & $\begin{array}{l}55.0 \\
54.0 \\
55.0 \\
55.0 \\
55.0\end{array}$ & $\begin{array}{l}74.0 \\
75.0 \\
75.0 \\
74.0 \\
74.0\end{array}$ & $\begin{array}{l}0.0 \\
0.0 \\
0.0 \\
0.0 \\
0.0\end{array}$ & $\begin{array}{l}0.0 \\
0.0 \\
0.0 \\
0.0 \\
0.0\end{array}$ & $\begin{array}{l}12.4 \\
12.4 \\
12.4 \\
12.4 \\
12.4\end{array}$ \\
\hline $\begin{array}{l}1.3 .36 \\
1.3 .37 \\
1.3 .38 \\
1.3 .39 \\
1.3 .40\end{array}$ & $\begin{array}{l}0.0 \\
0.0 \\
0.0 \\
0.0 \\
0.0\end{array}$ & $\begin{array}{l}11.2 \\
11.0 \\
10.0 \\
12.0 \\
11.7 .\end{array}$ & $\begin{array}{r}10.5 \\
10.0 \\
9.7 \\
10.5 \\
10.5\end{array}$ & $\begin{array}{l}0.0 \\
0.0 \\
0.0 \\
0.0 \\
0.0\end{array}$ & $\begin{array}{l}0.0 \\
0.0 \\
0.0 \\
0.0 \\
0.0\end{array}$ & $\begin{array}{l}55.0 \\
55.0 \\
56.0 \\
55.0 \\
55.0\end{array}$ & $\begin{array}{l}77.0 \\
77.0 \\
75.0 \\
76.0 \\
76.0\end{array}$ & $\begin{array}{l}0.0 \\
0.0 \\
0.0 \\
0.0 \\
0.0\end{array}$ & $\begin{array}{l}0.0 \\
0.0 \\
0.0 \\
0.0 \\
0.0\end{array}$ & $\begin{array}{l}12.4 \\
12.4 \\
12.4 \\
12.4 \\
12.4\end{array}$ \\
\hline $\begin{array}{l}1.3 .41 \\
1.3 .42 \\
1.3 .43 \\
1.3 .44 \\
1.3 .45\end{array}$ & $\begin{array}{l}0.0 \\
3.0 \\
0.0 \\
0.0 \\
0.0\end{array}$ & $\begin{array}{l}11.0 \\
10.7 \\
11.0 \\
12.0 \\
11.5\end{array}$ & $\begin{array}{l}10.5 \\
10.2 \\
10.7 \\
10.5 \\
10.0\end{array}$ & $\begin{array}{l}0.0 \\
0.0 \\
0.0 \\
0.0 \\
0.0\end{array}$ & $\begin{array}{l}0.0 \\
0.0 \\
0.0 \\
0.0 \\
0.0\end{array}$ & $\begin{array}{l}55.0 \\
55.0 \\
56.0 \\
59.0 \\
55.0\end{array}$ & $\begin{array}{l}76.0 \\
76.0 \\
77.0 \\
79.0 \\
79.0\end{array}$ & $\begin{array}{l}0.0 \\
0.0 \\
0.0 \\
0.0 \\
0.0\end{array}$ & $\begin{array}{l}0.0 \\
0.0 \\
0.0 \\
0.0 \\
0.0\end{array}$ & $\begin{array}{l}12.4 \\
12.4 \\
12.4 \\
12.4 \\
12.4\end{array}$ \\
\hline
\end{tabular}


TABLE XXXII (Contd.)

TWO-PHASE COLD LEG MIXTURE TEST GROUP 1.3

EXPERILANTAL MEASURMENTS FOR TESTS 1.3 .1 TO $1.3 .65-0.35-1$ N. DOHNCAMEZ GAP

\begin{tabular}{|c|c|c|c|c|c|c|c|c|c|c|}
\hline $\begin{array}{l}\text { TEST } \\
\text { iNO }\end{array}$ & $\begin{array}{l}P-C H-1 \\
(P S I G)\end{array}$ & $\begin{array}{l}\text { D-CW-2 } \\
\text { (DSIG) }\end{array}$ & $\begin{array}{l}P-H G-1 \\
(P S I G)\end{array}$ & $\begin{array}{l}P-H G,-2 \\
(P S I G)\end{array}$ & $\begin{array}{l}P-H T S-3 \\
(P S I G)\end{array}$ & $\begin{array}{l}T F-C W-1 \\
(D E G F)\end{array}$ & $\begin{array}{l}T F-H G-1 \\
(O \equiv G F)\end{array}$ & $\begin{array}{l}T F-H G-2 \\
(D E G, F)\end{array}$ & $\begin{array}{l}T E-H G-3 \\
(D E G F)\end{array}$ & $\begin{array}{l}\text { P-BARO } \\
\text { (PSIA) }\end{array}$ \\
\hline $\begin{array}{l}1.3 .40 \\
1.3 .47 \\
1.3 .48 \\
1.3 .49 \\
1.3 .53\end{array}$ & $\begin{array}{l}J .0 \\
u .0 \\
0.0 \\
j .0 \\
. .0\end{array}$ & $\begin{array}{l}10.7 \\
10.2 \\
12.2 \\
14.3 \\
13.2\end{array}$ & $\begin{array}{l}10.5 \\
10.0 \\
10.5 \\
10.7 \\
11.2\end{array}$ & $\begin{array}{l}0.0 \\
0.0 \\
0.0 \\
0.0 \\
0.0\end{array}$ & $\begin{array}{l}0.0 \\
0.0 \\
0.0 \\
0.0 \\
0.0\end{array}$ & $\begin{array}{l}55.0 \\
56.0 \\
54.0 \\
54.0 \\
55.0\end{array}$ & $\begin{array}{l}79.0 \\
79.0 \\
80.0 \\
81.0 \\
81.0\end{array}$ & $\begin{array}{l}0.0 \\
0.0 \\
0.0 \\
0.0 \\
0.0\end{array}$ & $\begin{array}{l}0.0 \\
0.0 \\
0.0 \\
0.0 \\
0.0\end{array}$ & $\begin{array}{l}12.4 \\
12.4 \\
12.4 \\
12.4 \\
12.4\end{array}$ \\
\hline $\begin{array}{l}1.3 .51 \\
1.3 .52 \\
1.3 .53 \\
1.3 .54 \\
1.3 .55\end{array}$ & $\begin{array}{l}3.0 \\
3.0 \\
3.0 \\
3.0 \\
3.0\end{array}$ & $\begin{array}{l}12.0 \\
13.5 \\
13.0 \\
11.5 \\
12.5\end{array}$ & $\begin{array}{l}11.0 \\
10.2 \\
10.2 \\
10.5 \\
10.0\end{array}$ & $\begin{array}{l}0.0 \\
0.0 \\
0.0 \\
0.0 \\
0.0\end{array}$ & $\begin{array}{l}0.0 \\
0.0 \\
0.0 \\
0.0 \\
0.0\end{array}$ & $\begin{array}{l}55.0 \\
53.0 \\
53.0 \\
54.0 \\
54.0\end{array}$ & $\begin{array}{l}80.0 \\
81.0 \\
82.0 \\
82.0 \\
82.0\end{array}$ & $\begin{array}{l}0.0 \\
0.0 \\
0.0 \\
0.0 \\
0.0\end{array}$ & $\begin{array}{l}0.0 \\
0.0 \\
0.0 \\
0.0 \\
0.0\end{array}$ & $\begin{array}{l}12.4 \\
12.4 \\
12.4 \\
12.4 \\
12.4\end{array}$ \\
\hline $\begin{array}{l}1.3 .56 \\
1.3 .57 \\
1.3 .58 \\
1.3 .59 \\
1.3 .00\end{array}$ & $\begin{array}{l}0.0 \\
0.0 \\
0.0 \\
0.0 \\
0.0\end{array}$ & $\begin{array}{l}12.7 \\
12.2 \\
14.0 \\
12.5 \\
11.0\end{array}$ & $\begin{array}{l}10.7 \\
11.2 \\
11.2 \\
10.7 \\
10.0\end{array}$ & $\begin{array}{l}0.0 \\
0.0 \\
0.0 \\
0.0 \\
0.0\end{array}$ & $\begin{array}{l}0.0 \\
0.0 \\
0.0 \\
0.0 \\
0.0\end{array}$ & $\begin{array}{l}54.0 \\
55.0 \\
55.0 \\
56.0 \\
56.0\end{array}$ & $\begin{array}{l}82.0 \\
82.0 \\
82.0 \\
82.0 \\
81.0\end{array}$ & $\begin{array}{l}0.0 \\
0.0 \\
0.0 \\
0.0 \\
0.0\end{array}$ & $\begin{array}{l}0.0 \\
0.0 \\
0.0 \\
0.0 \\
0.0\end{array}$ & $\begin{array}{l}12.4 \\
12.4 \\
12.4 \\
12.4 \\
12.4\end{array}$ \\
\hline $\begin{array}{l}1.3 .61 \\
1.3 .62 \\
1.3 .63 \\
1.3 .51 \\
1.3 .05\end{array}$ & $\begin{array}{l}0.0 \\
.0 . j \\
0.0 \\
0.0 \\
0.0\end{array}$ & $\begin{array}{l}10.5 \\
14.0 \\
11.2 \\
11.0 \\
10.0\end{array}$ & $\begin{array}{l}10.0 \\
11.5 \\
10.0 \\
10.5 \\
10.2\end{array}$ & $\begin{array}{l}0.0 \\
0.0 \\
0.0 \\
0.0 \\
0.0\end{array}$ & $\begin{array}{l}0.0 \\
0.0 \\
0.0 \\
0.0 \\
0.0\end{array}$ & $\begin{array}{l}56.0 \\
55.0 \\
55.0 \\
56.0 \\
56.0\end{array}$ & $\begin{array}{l}80.0 \\
80.0 \\
80.0 \\
80.0 \\
90.0\end{array}$ & $\begin{array}{l}0.0 \\
0.0 \\
0.0 \\
0.0 \\
0.0\end{array}$ & $\begin{array}{l}0.0 \\
0.0 \\
0.0 \\
0.0 \\
0.0\end{array}$ & $\begin{array}{l}12.4 \\
12.4 \\
12.4 \\
12.4 \\
12.4\end{array}$ \\
\hline
\end{tabular}

\begin{tabular}{|c|c|c|c|c|c|c|c|c|c|}
\hline $\begin{array}{l}T \equiv S T \\
\because V 0\end{array}$ & $\begin{array}{l}\text { INITISL } \\
\text { WEISHO } \\
\text { IL } 3 \text { ) }\end{array}$ & $\begin{array}{l}\text { FIHAL } \\
\text { WEISHT } \\
\text { (LB) }\end{array}$ & $\begin{array}{l}\text { TIME OF } \\
\text { COLLECTICN } \\
\text { (SEC) }\end{array}$ & $\begin{array}{l}\text { INITIAL } \\
\text { WE IGHT } \\
\text { (LB) }\end{array}$ & $\begin{array}{l}\text { F.INAL } \\
\text { WEIGHT } \\
\text { (LB) }\end{array}$ & $\begin{array}{l}\text { TIME OF } \\
\text { COLLECTION } \\
\text { (SEC) }\end{array}$ & $\begin{array}{l}\text { LOHER } \\
\text { PLENUM } \\
\text { LEVEL } \\
\text { (IN) }\end{array}$ & $\begin{array}{l}P-L P-1 \\
\quad P S I G)\end{array}$ & $\begin{array}{l}T F-L P \dot{1}=1 \\
\text { IDEG FI }\end{array}$ \\
\hline $\begin{array}{ll}1.3 . & 1 \\
1.3 .3 & 2 \\
1.3 .3 & 3 \\
1.3 .3 & 4 \\
1.3 . & 5\end{array}$ & $\begin{array}{l}133.3 \\
149.5 \\
143.8 \\
159.0 \\
165.3\end{array}$ & $\begin{array}{l}140.5 \\
148.8 \\
159.0 \\
160.3 \\
194.8\end{array}$ & $\begin{array}{l}45.5 \\
50.0 \\
45.0 \\
45.0 \\
60.0\end{array}$ & $\begin{array}{r}95.0 \\
233.0 \\
334.0 \\
397.5 \\
94.5\end{array}$ & $\begin{array}{l}233.0 \\
334.0 \\
397.5 \\
448.0 \\
273.5\end{array}$ & $\begin{array}{l}25.0 \\
25.0 \\
25.0 \\
40.0 \\
35.0\end{array}$ & $\begin{array}{l}12.0 \\
12.0 \\
12.0 \\
12.0 \\
12.0\end{array}$ & $\begin{array}{l}10.0 \\
10.0 \\
10.0 \\
10.0 \\
10.0\end{array}$ & $\begin{array}{l}59.0 \\
59.0 \\
58.0 \\
57.0 \\
57.0\end{array}$ \\
\hline $\begin{array}{l}1.3 .6 \\
1.3 .3 \\
1.3 .38 \\
1.3 .37 \\
1.3 .10\end{array}$ & $\begin{array}{l}194.8 \\
221.5 \\
13.0 \\
159.8 \\
133.0\end{array}$ & $\begin{array}{l}221.5 \\
249.3 \\
159.8 \\
179.3 \\
183.3\end{array}$ & $\begin{array}{l}45.0 \\
45.0 \\
50.0 \\
40.0 \\
40.0\end{array}$ & $\begin{array}{r}273.5 \\
381.5 \\
90.0 \\
138.5 \\
86.0\end{array}$ & $\begin{array}{l}381.5 \\
479.8 \\
138.5 \\
158.5 \\
192.5\end{array}$ & $\begin{array}{l}30.0 \\
35.0 \\
30.0 \\
50.0 \\
25.0\end{array}$ & $\begin{array}{l}12.0 \\
12.0 \\
12.0 \\
12.0 \\
12.0\end{array}$ & $\begin{array}{l}10.0 \\
10.0 \\
10.0 \\
10.0 \\
10.0\end{array}$ & $\begin{array}{l}57.0 \\
56.0 \\
57.0 \\
57.0 \\
57.0\end{array}$ \\
\hline $\begin{array}{l}1.3 .11 \\
1.3 .12 \\
1.3 .13 \\
1.3 .14 \\
1.3 .15\end{array}$ & $\begin{array}{l}133.3 \\
241.3 \\
289.5 \\
335.0 \\
45 \% .8\end{array}$ & $\begin{array}{l}241.3 \\
289.5 \\
356.0 \\
454.8 \\
527.0\end{array}$ & $\begin{array}{l}46.0 \\
35.0 \\
55.0 \\
35.0 \\
30.0\end{array}$ & $\begin{array}{l}192.5 \\
294.0 \\
351.5 \\
373.0 \\
472.0\end{array}$ & $\begin{array}{l}294.0 \\
351.5 \\
373.0 \\
472.0 \\
546.0\end{array}$ & $\begin{array}{l}35.0 \\
43.0 \\
50.0 \\
35.0 \\
45.0\end{array}$ & $\begin{array}{l}12.0 \\
12.0 \\
12.0 \\
12.0 \\
12.0\end{array}$ & $\begin{array}{l}10.0 \\
10.0 \\
10.0 \\
10.0 \\
10.0\end{array}$ & $\begin{array}{l}57.0 \\
57.0 \\
59.0 \\
57.0 \\
57.0\end{array}$ \\
\hline
\end{tabular}




\section{TABLE XXXII (Contd.)}

TWO-PHASE COLD LEG MIXTURE TEST GROUP 1.3

EXPEP.IMENTAL MEASURMENTS FOR TESTS 1.3 .1 TO 1.3.65:-- 0.35-IN. DOHNCOMER GAP

\begin{tabular}{|c|c|c|c|c|c|c|c|c|c|}
\hline \multirow[b]{2}{*}{$\begin{array}{l}\text { TEST } \\
\text { No }\end{array}$} & \multicolumn{3}{|c|}{ LCWER PLENiJ:A FLOW } & \multicolumn{3}{|c|}{ BYPASS FLOH } & \multirow[b]{2}{*}{$\begin{array}{l}\text { LOWER } \\
\text { PLENUM } \\
\text { LEVEL } \\
\text { (IN) }\end{array}$} & \multirow[b]{2}{*}{$\begin{array}{l}P-L P-1 \\
10 S|G|\end{array}$} & \multirow[b]{2}{*}{$\begin{array}{l}T F-L P-1 \\
(D F G F)\end{array}$} \\
\hline & $\begin{array}{l}\text { INITIAL } \\
\text { INEIGHT } \\
\text { ILS) }\end{array}$ & $\begin{array}{l}\text { FINAL } \\
\text { WE IGHT } \\
\text { (LS) }\end{array}$ & $\begin{array}{l}\text { TIME OF } \\
\text { COLLECTION } \\
\text { (SEC) }\end{array}$ & $\begin{array}{l}\text { INITIAL } \\
\text { WEIGHT } \\
\text { (LB) }\end{array}$ & $\begin{array}{l}\text { FINAL } \\
\text { WEIGHT } \\
\text { (L BI }\end{array}$ & $\begin{array}{l}\text { TIME OF } \\
\text { COLLECTION } \\
\text { (SFC) }\end{array}$ & & & \\
\hline $\begin{array}{l}1.3 .16 \\
1.3 .17 \\
1.3 .18 \\
1.3 .19 \\
1.3 .20\end{array}$ & $\begin{array}{l}463.0 \\
133.0 \\
223.8 \\
327.3 \\
413.8\end{array}$ & $\begin{array}{l}555.5 \\
223.8 \\
327.3 \\
413.8 \\
493.8\end{array}$ & $\begin{array}{l}35.0 \\
45.0 \\
30.0 \\
30.0 \\
35.0\end{array}$ & $\begin{array}{r}436.0 \\
89.3 \\
129.5 \\
193.5 \\
251.0\end{array}$ & $\begin{array}{l}492.0 \\
129.5 \\
193.5 \\
251.0 \\
304.0\end{array}$ & $\begin{array}{l}35.0 \\
45.0 \\
30.0 \\
45.0 \\
60.0\end{array}$ & $\begin{array}{l}12.0 \\
12.0 \\
12.0 \\
12.0 \\
12.0\end{array}$ & $\begin{array}{l}10.0 \\
10.0 \\
10.0 \\
10.0 \\
10.0\end{array}$ & $\begin{array}{l}57.0 \\
58.0 \\
56.0 \\
56.0 \\
56.0\end{array}$ \\
\hline $\begin{array}{l}1.3 .21 \\
1.3 .22 \\
1.3 .23 \\
1.3 .24 \\
1.3 .25\end{array}$ & $\begin{array}{l}133.3 \\
204.0 \\
235.3 \\
37: 0.0 \\
133.3\end{array}$ & $\begin{array}{l}204.0 \\
285.3 \\
370.0 \\
425.5 \\
173.3\end{array}$ & $\begin{array}{l}40.0 \\
45.0 \\
45.0 \\
35.0 \\
40.0\end{array}$ & $\begin{array}{l}304.3 \\
437.0 \\
552.0 \\
634.0 \\
121.5\end{array}$ & $\begin{array}{l}437.0 \\
552.0 \\
634.0 \\
663.0 \\
266.5\end{array}$ & $\begin{array}{l}35.0 \\
50.0 \\
50.0 \\
40.0 \\
30.0\end{array}$ & $\begin{array}{l}12.0 \\
12.0 \\
12.0 \\
12.0 \\
12.0\end{array}$ & $\begin{array}{l}10.0 \\
10.0 \\
10.0 \\
10.0 \\
10.0\end{array}$ & $\begin{array}{l}56.0 \\
59.0 \\
57.0 \\
57.0 \\
59.0\end{array}$ \\
\hline $\begin{array}{l}1.3 .20 \\
1.3 .27 \\
1.3 .28 \\
1.3 .24 \\
1.3 .30\end{array}$ & $\begin{array}{l}173.3 \\
205.3 \\
246.3 \\
282.5 \\
293.8\end{array}$ & $\begin{array}{l}205.3 \\
246.0 \\
282.5 \\
298.8 \\
310.3\end{array}$ & $\begin{array}{l}40.0 \\
45.0 \\
45.0 \\
60.0 \\
45.0\end{array}$ & $\begin{array}{l}266.5 \\
417.5 \\
492.0 \\
508.0 \\
668.0\end{array}$ & $\begin{array}{l}417.5 \\
482.0 \\
508.0 \\
668.0 \\
765.3\end{array}$ & $\begin{array}{l}45.0 \\
35.0 \\
45.0 \\
30.0 \\
25.0\end{array}$ & $\begin{array}{l}12.0 \\
12.0 \\
12.0 \\
12.0 \\
12.0\end{array}$ & $\begin{array}{l}10.0 \\
10.0 \\
10.0 \\
10.0 \\
10.0\end{array}$ & $\begin{array}{l}57.0 \\
57.0 \\
57.0 \\
57.0 \\
57.0\end{array}$ \\
\hline $\begin{array}{l}1.3 .31 \\
1.3 .32 \\
1.3 .33 \\
1.3 .34 \\
1.3 .35\end{array}$ & $\begin{array}{l}311.3 \\
133.0 \\
153.5 \\
168.8 \\
173.0\end{array}$ & $\begin{array}{l}331.3 \\
153.5 \\
168.5 \\
178.0 \\
185.8\end{array}$ & $\begin{array}{l}60.0 \\
60.0 \\
60.0 \\
39.0 \\
46.5\end{array}$ & $\begin{array}{r}765.3 \\
90.3 \\
200.8 \\
233.8 \\
341.5\end{array}$ & $\begin{array}{l}854.0 \\
200.8 \\
233.8 \\
341.5 \\
443.0\end{array}$ & $\begin{array}{l}35.0 \\
45.0 \\
55.0 \\
20.0 \\
25.0\end{array}$ & $\begin{array}{l}12.0 \\
12.0 \\
12.0 \\
12.0 \\
12.0\end{array}$ & $\begin{array}{l}10.0 \\
10.0 \\
10.0 \\
10.0 \\
10.0\end{array}$ & $\begin{array}{l}5 ? .0 \\
59.0 \\
58.0 \\
56.0 \\
56.0\end{array}$ \\
\hline $\begin{array}{l}1.3 .35 \\
1.3 .37 \\
1.3 .39 \\
1.3 .39 \\
1.3 .49\end{array}$ & $\begin{array}{l}147.3 \\
158.8 \\
167.0 \\
172.5 \\
197.3\end{array}$ & $\begin{array}{l}153.8 \\
167.0 \\
172.5 \\
197.3 \\
219.8\end{array}$ & $\begin{array}{l}60.0 \\
70.0 \\
60.0 \\
50.0 \\
50.0\end{array}$ & $\begin{array}{r}80.5 \\
146.0 \\
195.3 \\
210.5 \\
339.0\end{array}$ & $\begin{array}{l}146.0 \\
195.3 \\
210.5 \\
339.0 \\
431.3\end{array}$ & $\begin{array}{l}25.0 \\
40.0 \\
35.0 \\
25.0 \\
25.0\end{array}$ & $\begin{array}{l}12.0 \\
12.0 \\
12.0 \\
12.0 \\
12.0\end{array}$ & $\begin{array}{l}10.0 \\
10.0 \\
10.0 \\
10.0 \\
10.0\end{array}$ & $\begin{array}{l}59.0 \\
58.0 \\
58.0 \\
58.0 \\
59.0\end{array}$ \\
\hline $\begin{array}{l}1.3 .41 \\
1.3 .42 \\
1.3 .43 \\
1.3 .44 \\
1.3 .45\end{array}$ & $\begin{array}{l}21.9 .8 \\
245.5 \\
263.8 \\
196.0 \\
251.9\end{array}$ & $\begin{array}{l}246.5 \\
269.8 \\
295.3 \\
251.8 \\
300.8\end{array}$ & $\begin{array}{l}55.0 \\
50.0 \\
55.0 \\
40.0 \\
40.0\end{array}$ & $\begin{array}{l}431.3 \\
499.5 \\
536.5 \\
201.0 \\
325.3\end{array}$ & $\begin{array}{l}499.5 \\
536.5 \\
553.5 \\
325.3 \\
458.0\end{array}$ & $\begin{array}{r}30.0 \\
40.0 \\
60.0 \\
30.0 \\
45.0\end{array}$ & $\begin{array}{l}12.0 \\
12.0 \\
12.0 \\
12.0 \\
12.0\end{array}$ & $\begin{array}{l}10.0 \\
10.0 \\
10.0 \\
10.0 \\
10.0\end{array}$ & $\begin{array}{l}58.0 \\
59.0 \\
58.0 \\
57.0 \\
57.0\end{array}$ \\
\hline $\begin{array}{l}1.3 .46 \\
1.3 .47 \\
1.3 .43 \\
1.3 .49 \\
1.3 .50\end{array}$ & $\begin{array}{l}300.0 \\
351.0 \\
173.5 \\
133.0 \\
234.0\end{array}$ & $\begin{array}{l}351.8 \\
403.0 \\
282.8 \\
234.0 \\
298.3\end{array}$ & $\begin{array}{l}40.0 \\
45.0 \\
40.0 \\
40.0 \\
30.0\end{array}$ & $\begin{array}{r}458.0 \\
524.5 \\
120.0 \\
91.0 \\
194.3\end{array}$ & $\begin{array}{l}524.5 \\
547.0 \\
257.5 \\
194.8 \\
262.5\end{array}$ & $\begin{array}{l}45.0 \\
40.0 \\
45.0 \\
35: 0 \\
35.0\end{array}$ & $\begin{array}{l}12.0 \\
12.0 \\
12.0 \\
12.0 \\
12.0\end{array}$ & $\begin{array}{l}10.0 \\
10.0 \\
10.0 \\
10.0 \\
10.0\end{array}$ & $\begin{array}{l}56.0 \\
56.0 \\
56.0 \\
56.0 \\
56.0\end{array}$ \\
\hline $\begin{array}{l}1.3 .51 \\
1.3 .52 \\
1.3 .53 \\
1.3 .54 \\
1.3 .55\end{array}$ & $\begin{array}{l}294.3 \\
350.5 \\
345.5 \\
250.5 \\
261.5\end{array}$ & $\begin{array}{l}356.5 \\
474.8 \\
417.3 \\
329.3 \\
315.8\end{array}$ & $\begin{array}{l}30.0 \\
40.0 \\
30.0 \\
35.0 \\
30.0\end{array}$ & $\begin{array}{l}262.5 \\
301.5 \\
391.5 \\
443.3 \\
471.5\end{array}$ & $\begin{array}{l}301.5 \\
381.5 \\
443.3 \\
471.5 \\
565.0\end{array}$ & $\begin{array}{l}45.0 \\
30.0 \\
35.0 \\
40.0 \\
25.0\end{array}$ & $\begin{array}{l}12.0 \\
12.0 \\
12.0 \\
12.0 \\
12.0\end{array}$ & $\begin{array}{l}10.0 \\
10.0 \\
10.0 \\
10.0 \\
10.0\end{array}$ & $\begin{array}{r}56.0 \\
.56 .0 \\
56.0 \\
56.0 \\
56.0\end{array}$ \\
\hline
\end{tabular}


TABLE XXXII (Contd.)

TWO-PHASE COLD LEG MIXTURE TEST GROUP 1.3

EXPERIIENTAL MEASIJRMENTS FOR TESTS 1.3 .1 TO $1.3 .65--0.35-I N$. DOHNCUMER GAP

\begin{tabular}{|c|c|c|c|c|c|c|c|c|c|}
\hline \multirow[b]{2}{*}{$\begin{array}{c}\text { TEST } \\
\text { UAก }\end{array}$} & \multicolumn{3}{|c|}{ LJWEP PLENUM FLOH } & \multicolumn{3}{|c|}{ BYPASS FL TW } & \multirow{2}{*}{$\begin{array}{l}\text { LOWER } \\
\text { PLEN!JM } \\
\text { LEVEL } \\
\text { (IN) }\end{array}$} & \multirow[b]{2}{*}{$\begin{array}{l}P-(P-1 \\
(P S \mid G)\end{array}$} & \multirow[b]{2}{*}{$\begin{array}{l}\text { TF-LP-1 } \\
\text { (DEG F) }\end{array}$} \\
\hline & $\begin{array}{l}\text { INITIAL } \\
\text { WEIGHT } \\
\text { (LA) }\end{array}$ & $\begin{array}{l}\text { FINAL } \\
\text { WEIGHT } \\
\text { (LB) }\end{array}$ & $\begin{array}{l}\text { TIME RF } \\
\text { COL LECTION } \\
\text { (SFC) }\end{array}$ & $\begin{array}{l}\text { IN IT IAL } \\
\text { WE I GHT } \\
\text { (LB) }\end{array}$ & $\begin{array}{l}\text { FINAL } \\
\text { WEIGHT } \\
\text { IL क! }\end{array}$ & $\begin{array}{l}\text { TIME Gi } \\
\text { COLLECTINAS } \\
\text { (SEC) }\end{array}$ & & & \\
\hline $\begin{array}{l}1.3 .56 \\
1.3 .57 \\
1.3 .58 \\
1.3 .59 \\
1.3 .60\end{array}$ & $\begin{array}{l}241.5 \\
192.0 \\
244.0 \\
273.5 \\
319.0\end{array}$ & $\begin{array}{l}301.0 \\
244.0 \\
.273 .5 \\
319.0 \\
354.8\end{array}$ & $\begin{array}{l}30.0 \\
30.0 \\
40.0 \\
50.0 \\
45.0\end{array}$ & $\begin{array}{l}565.0 \\
375.8 \\
407.3 \\
503.5 \\
603.5\end{array}$ & $\begin{array}{l}055.0 \\
407.3 \\
503.5 \\
603.5 \\
663.5\end{array}$ & $\begin{array}{l}40.0 \\
30.0 \\
20.0 \\
30.0 \\
30.0\end{array}$ & $\begin{array}{l}12.0 \\
12.0 \\
12.0 \\
12.0 \\
12.0\end{array}$ & $\begin{array}{l}10.0 \\
10.0 \\
10.0 \\
10.0 \\
10.0\end{array}$ & $\begin{array}{l}56.0 \\
56.0 \\
58.0 \\
58.0 \\
59.0\end{array}$ \\
\hline $\begin{array}{l}1.3 .61 \\
1.3 .02 \\
1.3 .53 \\
1.3 .64 \\
1.3 .65\end{array}$ & $\begin{array}{l}354.8 \\
397.9 \\
413.5 \\
429.3 \\
441.8\end{array}$ & $\begin{array}{l}39 \% .8 \\
413.5 \\
429.3 \\
441.8 \\
453.8\end{array}$ & $\begin{array}{l}55.0 \\
60.0 \\
50.0 \\
45.0 \\
45.0\end{array}$ & $\begin{array}{l}663.5 \\
690.5 \\
643.5 \\
723.3 \\
785.3\end{array}$ & $\begin{array}{l}680.5 \\
789.0 \\
723.3 \\
785.3 \\
811.5\end{array}$ & $\begin{array}{l}50.0 \\
20.0 \\
20.0 \\
25.0 \\
45.0\end{array}$ & $\begin{array}{l}12.0 \\
12.0 \\
12.0 \\
12.0 \\
12.0\end{array}$ & $\begin{array}{l}10.0 \\
12.0 \\
10.0 \\
10.0 \\
10.0\end{array}$ & $\begin{array}{l}58.0 \\
57.0 \\
59.0 \\
59.0 \\
59.0\end{array}$ \\
\hline
\end{tabular}

\begin{tabular}{|c|c|c|c|c|c|c|c|}
\hline $\begin{array}{l}\text { TEST } \\
\text { NO }\end{array}$ & $\begin{array}{c}P M-C C-1 \\
(C F M)\end{array}$ & $\begin{array}{c}R \cdot Y-C(;-2 \\
(C F, 4)\end{array}$ & $\begin{array}{c}F T-C G-1 \\
(C F M)\end{array}$ & $\begin{array}{l}P-C G-1 \\
(P S I G)\end{array}$ & $\begin{array}{l}D-C G-2 \\
(P S I G)\end{array}$ & $\begin{array}{l}\text { TF-CG-1 } \\
\text { TDEG, FI }\end{array}$ & $\begin{array}{l}\text { TF-CG-2 } \\
(D E G F)\end{array}$ \\
\hline  & $\begin{array}{l}30.0 \\
30.0 \\
30.0 \\
30.0 \\
30.0\end{array}$ & $\begin{array}{l}0.0 \\
0.0 \\
0.0 \\
0.0 \\
0.0\end{array}$ & $\begin{array}{l}0.0 \\
0.0 \\
0.0 \\
0.0 \\
0.0\end{array}$ & $\begin{array}{l}0.0 \\
0.0 \\
0.0 \\
0.0 \\
0.0\end{array}$ & $\begin{array}{l}0.0 \\
0.0 \\
0.0 \\
0.0 \\
0.0\end{array}$ & $\begin{array}{l}0.0 \\
0.0 \\
0.0 \\
0.0 \\
0.0\end{array}$ & $\begin{array}{l}0.0 \\
0.0 \\
0.0 \\
0.0 \\
0.0\end{array}$ \\
\hline $\begin{array}{l}1.3 .9 \\
1.3 .9 \\
1.3 .9 \\
1.3 .99 \\
1.3 .10\end{array}$ & $\begin{array}{l}30.0 \\
310.0 \\
30.0 \\
30.0 \\
30.0\end{array}$ & $\begin{array}{l}0.0 \\
0.0 \\
0.0 \\
0.0 \\
0.0\end{array}$ & $\begin{array}{l}0.0 \\
0.0 \\
0.0 \\
0.0 \\
0.0\end{array}$ & $\begin{array}{l}0.0 \\
0.0 \\
0.0 \\
0.0 \\
0.0\end{array}$ & $\begin{array}{l}0.0 \\
0.0 \\
0.0 \\
0.0 \\
0.0\end{array}$ & $\begin{array}{l}0.0 \\
0.0 \\
0.0 \\
0.0 \\
0.0\end{array}$ & $\begin{array}{l}0.0 \\
0.0 \\
0.0 \\
0.0 \\
0.0\end{array}$ \\
\hline $\begin{array}{l}1.3 .11 \\
1.3 .12 \\
1.3 .13 \\
1.3 .14 \\
1.3 .13\end{array}$ & $\begin{array}{l}30.0 \\
30.0 \\
30.0 \\
30.0 \\
3.0 .0\end{array}$ & $\begin{array}{l}0.0 \\
0.0 \\
0.0 \\
0.0 \\
0.0\end{array}$ & $\begin{array}{l}0.0 \\
0.0 \\
0.0 \\
0.0 \\
0.0\end{array}$ & $\begin{array}{l}0.0 \\
0.0 \\
0.0 \\
0.0 \\
0.0\end{array}$ & $\begin{array}{l}0.0 \\
0.0 \\
0.0 \\
0.0 \\
0.0\end{array}$ & $\begin{array}{l}0.0 \\
0.0 \\
0.0 \\
0.0 \\
0.0\end{array}$ & $\begin{array}{l}0.0 \\
0.0 \\
0.0 \\
0.0 \\
0.0\end{array}$ \\
\hline $\begin{array}{l}1.3 .15 \\
1.3 .17 \\
1.3 .19 \\
1.3 .19 \\
1.3 .20\end{array}$ & $\begin{array}{l}30.0 \\
3.0 .0 \\
30.0 \\
30.0 \\
3.0 .0\end{array}$ & $\begin{array}{l}0.0 \\
0.0 \\
0.0 \\
0.0 \\
0.0\end{array}$ & $\begin{array}{r}0.0 \\
0.0 \\
0.0 \\
0.0 \\
0.0\end{array}$ & $\begin{array}{l}0.0 \\
0.0 \\
0.0 \\
0.0 \\
0.0\end{array}$ & $\begin{array}{l}0.0 \\
0.0 \\
0.0 \\
0.0 \\
0.0\end{array}$ & $\begin{array}{l}0.0 \\
0.0 \\
0.0 \\
0.0 \\
0.0\end{array}$ & $\begin{array}{l}0.0 \\
0.0 \\
0.0 \\
0.0 \\
0.0\end{array}$ \\
\hline $\begin{array}{l}1.3 .21 \\
1.3 .23 \\
1.3 .23 \\
1.3 .24 \\
1.3 .25\end{array}$ & $\begin{array}{l}30.0 \\
30.0 \\
.310 .0 \\
30.0 \\
30.0\end{array}$ & $\begin{array}{l}0.0 \\
0.0 \\
0.0 \\
0.0 \\
0.0\end{array}$ & $\begin{array}{l}0.0 \\
0.0 \\
0.0 \\
0.0 \\
0.0\end{array}$ & $\begin{array}{l}0.0 \\
0.0 \\
0.0 \\
0.0 \\
0.0\end{array}$ & $\begin{array}{l}0.0 \\
0.0 \\
0.0 \\
0.0 \\
0.0\end{array}$ & $\begin{array}{l}0.0 \\
0.0 \\
0.0 \\
0.0 \\
0.0\end{array}$ & $\begin{array}{l}0.0 \\
0.0 \\
0.0 \\
0.0 \\
0.0\end{array}$ \\
\hline
\end{tabular}


TABLE XXXII (Contd.)

TWO-PHASE COLD LEG MIXTURE TEST GROUP 1.3

EXPERIMENTAL MEASURMENTS FOR TESTS 1.3 .1 TO $1.3 .65-0.35$-IN. DOHNCOMER GAP

\begin{tabular}{|c|c|c|c|c|c|c|c|}
\hline $\begin{array}{l}\text { TEST } \\
\text { ND }\end{array}$ & $\begin{array}{c}R M-C G-1 \\
(C F M)\end{array}$ & $\begin{array}{c}R M-C G-2 \\
(C F M)\end{array}$ & $\begin{array}{l}\text { FT-CG-1 } \\
\text { (CFM) }\end{array}$ & $\begin{array}{l}P-C G-1 \\
\text { (PSIG) }\end{array}$ & $\begin{array}{l}P-C G-2 \\
\text { (PSIG) }\end{array}$ & $\begin{array}{l}T F-C G-1 \\
(D E G F)\end{array}$ & $\begin{array}{l}\text { TF-CG-2 } \\
\text { (DEG FI }\end{array}$ \\
\hline $\begin{array}{l}1.3 .26 \\
1.3 .27 \\
1.3 .28 \\
1.3 .29 \\
1.3 .30\end{array}$ & $\begin{array}{l}30.0 \\
30.0 \\
30.0 \\
30.0 \\
30.0 .\end{array}$ & $\begin{array}{l}0.0 \\
0.0 \\
0.0 \\
0.0 \\
0.0\end{array}$ & $\begin{array}{l}0.0 \\
0.0 \\
0.0 \\
0.0 \\
0.0\end{array}$ & $\begin{array}{l}0.0 \\
0.0 \\
0.0 \\
0.0 \\
0.0\end{array}$ & $\begin{array}{l}0.0 \\
0.0 \\
0.0 \\
0.0 \\
0.0\end{array}$ & $\begin{array}{l}0.0 \\
0.0 \\
0.0 \\
0.0 \\
0.0\end{array}$ & $\begin{array}{l}0.0 \\
0.0 \\
0.0 \\
0.0 \\
0.0\end{array}$ \\
\hline $\begin{array}{l}1.3 .31 \\
1.3 .32 \\
1.3 .33 \\
1.3 .34 \\
1.3 .35\end{array}$ & $\begin{array}{l}30.0 \\
30.0 \\
30.0 \\
60.0 \\
60.0\end{array}$ & $\begin{array}{l}0.0 \\
0.0 \\
0.0 \\
0.0 \\
0.0\end{array}$ & $\begin{array}{l}0.0 \\
0.0 \\
0.0 \\
0.0 \\
0.0\end{array}$ & $\begin{array}{l}0.0 \\
0.0 \\
0.0 \\
0.0 \\
0.0\end{array}$ & $\begin{array}{l}0.0 \\
0.0 \\
0.0 \\
0.0 \\
0.0\end{array}$ & $\begin{array}{l}0.0 \\
0.0 \\
0.0 \\
0.0 \\
0.0\end{array}$ & $\begin{array}{l}0.0 \\
0.0 \\
0.0 \\
0.0 \\
0.0\end{array}$ \\
\hline $\begin{array}{l}1.3 .36 \\
1.3 .37 \\
1.3 .38 \\
1.3 .39 \\
1.3 .40\end{array}$ & $\begin{array}{l}60.0 \\
60.0 \\
60.0 \\
60.0 \\
60.0\end{array}$ & $\begin{array}{l}0.0 \\
0.0 \\
0.0 \\
0.0 \\
0.0\end{array}$ & $\begin{array}{l}0.0 \\
0.0 \\
c .0 \\
0.0 \\
0.0\end{array}$ & $\begin{array}{l}0.0 \\
0.0 \\
0.0 \\
0.0 \\
0.0\end{array}$ & $\begin{array}{l}0.0 \\
0.0 \\
0.0 \\
0.0 \\
0.0\end{array}$ & $\begin{array}{l}0.0 \\
0.0 \\
0.0 \\
0.0 \\
0.0\end{array}$ & $\begin{array}{l}0.0 \\
0.0 \\
0.0 \\
0.0 \\
0.0\end{array}$ \\
\hline $\begin{array}{l}1.3 .41 \\
1.3 .42 \\
1.3 .43 \\
1.3 .44 \\
1.3 .45\end{array}$ & $\begin{array}{l}6.0 .0 \\
60.0 \\
60.0 \\
60.0 \\
60.0\end{array}$ & $\begin{array}{l}0.0 \\
0.0 \\
0.0 \\
0.0 \\
0.0\end{array}$ & $\begin{array}{l}0.0 \\
0.0 \\
0.0 \\
0.0 \\
0.0\end{array}$ & $\begin{array}{l}0.0 \\
j .0 \\
0.0 \\
0.0 \\
0.0\end{array}$ & $\begin{array}{l}0.0 \\
0.0 \\
0.0 \\
0.0 \\
0.0\end{array}$ & $\begin{array}{l}0.0 \\
0.0 \\
0.0 \\
0.0 \\
0.0\end{array}$ & $\begin{array}{l}0.0 \\
0.0 \\
0.0 \\
0.0 \\
0.0\end{array}$ \\
\hline $\begin{array}{l}1.3 .46 \\
1.3 .47 \\
1.3 .44 \\
1.3 .47 \\
1.3 .50\end{array}$ & $\begin{array}{l}60.0 \\
20.0 \\
60.0 \\
60.0 \\
6.0 .0\end{array}$ & $\begin{array}{l}0.0 \\
0.0 \\
0.0 \\
0.0 \\
0.0\end{array}$ & $\begin{array}{l}0.0 \\
0.0 \\
0.0 \\
0.0 \\
0.0\end{array}$ & $\begin{array}{l}0.0 \\
0.0 \\
0.0 \\
0.0 \\
0.0\end{array}$ & $\begin{array}{l}0.0 \\
0.0 \\
0.0 \\
0.0 \\
0.0\end{array}$ & $\begin{array}{l}0.0 \\
0.0 \\
0.0 \\
0.0 \\
0.0\end{array}$ & $\begin{array}{l}0.0 \\
0.0 \\
0.0 \\
0.0 \\
0.0\end{array}$ \\
\hline $\begin{array}{l}1.3 .51 \\
1.3 .52 \\
1.3 .53 \\
1.3 .54 \\
1.3 .55\end{array}$ & $\begin{array}{l}0.0 .0 \\
60.0 \\
60.0 \\
60.0 \\
60.0\end{array}$ & $\begin{array}{l}0.0 \\
0.0 \\
0.0 \\
0.0 \\
0.0\end{array}$ & $\begin{array}{l}0.0 \\
0.0 \\
0.0 \\
0.0 \\
0.0\end{array}$ & $\begin{array}{l}0.0 \\
0.0 \\
0.0 \\
0.0 \\
0.0\end{array}$ & $\begin{array}{l}0.0 \\
0.0 \\
0.0 \\
0.0 \\
0.0\end{array}$ & $\begin{array}{l}0.0 \\
0.0 \\
0.0 \\
0.0 \\
0.0\end{array}$ & $\begin{array}{l}0.0 \\
0.0 \\
0.0 \\
0.0 \\
0.0\end{array}$ \\
\hline $\begin{array}{l}1.3 .56 \\
1.3 .51 \\
0.3 .50 \\
03.51 \\
\therefore 3.60\end{array}$ & $\begin{array}{l}6.0 .0 \\
60.0 \\
69.0 \\
60.0 \\
63.0\end{array}$ & $\begin{array}{l}0.0 \\
0.0 \\
0.0 \\
0.0 \\
0.0\end{array}$ & $\begin{array}{l}0.0 \\
0.0 \\
0.0 \\
0.0 \\
0.0\end{array}$ & $\begin{array}{l}0.0 \\
0.0 \\
2.0 \\
0.0 \\
0.0\end{array}$ & $\begin{array}{l}0.0 \\
0.0 \\
0.0 \\
0.0 \\
0.0\end{array}$ & $\begin{array}{l}0.0 \\
0.0 \\
0.0 \\
0.0 \\
0.0\end{array}$ & $\begin{array}{l}0.0 \\
0.0 \\
0.0 \\
0.0 \\
0.0\end{array}$ \\
\hline $\begin{array}{l}1.3 .61 \\
1.3 .52 \\
1.3 .63 \\
1.3 .64 \\
1.3 .65\end{array}$ & $\begin{array}{l}63.0 \\
80.0 \\
60.0 \\
60.0 \\
60.0\end{array}$ & $\begin{array}{l}0.0 \\
0.0 \\
0.0 \\
0.0 \\
0.0\end{array}$ & $\begin{array}{l}0.0 \\
0.0 \\
0.0 \\
0.0 \\
0.0\end{array}$ & $\begin{array}{r}0.0 \\
0.0 \\
0.0 \\
0.0 \\
0.0\end{array}$ & $\begin{array}{l}0.0 \\
0.0 \\
0.0 \\
0.0 \\
0.0\end{array}$ & $\begin{array}{l}0.0 \\
0.0 \\
0.0 \\
0.0 \\
0.0\end{array}$ & $\begin{array}{l}0.0 \\
0.0 \\
0.0 \\
0.0 \\
0.0\end{array}$ \\
\hline
\end{tabular}


TABLE XXXIII

TWO-PHASE COLD LEG MIXTURE TEST GROUP 1.4

TPAMSPARENT VESSEL TEST DATA FOR TESTS 1.4.1 TO 1.4.40 -- 0.35-IN. ONWMCOMER GAP SIJMMARY DF CALCIJLATED RFSULTS

\begin{tabular}{|c|c|c|c|c|c|c|c|c|c|c|}
\hline $\begin{array}{l}\text { TEST } \\
\text { NO }\end{array}$ & $\begin{array}{l}\text { OOWNC UMAFR } \\
\text { AIR FLOW } \\
\text { (LB/SEC) }\end{array}$ & $\begin{array}{l}\text { PCHNCOMER } \\
\text { AIR DENSITY } \\
\text { (LB/FT*3) }\end{array}$ & $\begin{array}{l}\text { LIOWER PLENUM } \\
\text { PRESSURE } \\
\text { (PSIA) }\end{array}$ & $\begin{array}{l}\text { CCLD LEG } \\
\text { AIR FLOW } \\
\text { (LB/SEC) }\end{array}$ & $\begin{array}{l}\text { DOWNCOMER } \\
\text { WATER FLUH } \\
\text { (LB/SEC) }\end{array}$ & $\begin{array}{l}\text { WATER FLOH. } \\
\text { CONTINUITY }\end{array}$ & $\begin{array}{c}\text { J-AIR } \\
\text { OOWNCOMER } \\
\text { (FT/SEC) }\end{array}$ & $\begin{array}{l}\text { J-WA.TER } \\
\text { DOWNCDMER } \\
\text { (FT/SEC) }\end{array}$ & $(J G *) * * 1 / 2$ & $(J L * 1 * * 1 / 2$ \\
\hline $\begin{array}{l}1.4 .1 \\
1.4 .2 \\
1.4 .3 \\
1.4 .5 \\
1.4 .5\end{array}$ & $\begin{array}{l}0.0214 \\
0.0212 \\
0.0212 \\
0.0637 \\
0.0634\end{array}$ & $\begin{array}{l}0.2203 \\
0.2203 \\
0.2203 \\
0.2203 \\
0.2203\end{array}$ & $\begin{array}{l}42.28 \\
42.28 \\
42.28 \\
42.28 \\
42.28\end{array}$ & $\begin{array}{l}0.0658 \\
0.0655 \\
0.0659 \\
0.0659 \\
0.0653\end{array}$ & $\begin{array}{l}3.225 \\
2.760 \\
2.100 \\
2.010 \\
1.910\end{array}$ & $\begin{array}{l}1.022 \\
1.005 \\
0.970 \\
0.976 \\
0.983\end{array}$ & $\begin{array}{l}1.57 \\
1.55 \\
1.55 \\
4.67 \\
4.64\end{array}$ & $\begin{array}{l}0.834 \\
0.714 \\
0.543 \\
0.520 \\
0.494\end{array}$ & $\begin{array}{l}0.2602 \\
0.2591 \\
0.2591 \\
0.4494 \\
0.4481\end{array}$ & $\begin{array}{l}0.7791 \\
0.7207 \\
0.6287 \\
0.5151 \\
0.5996\end{array}$ \\
\hline $\begin{array}{l}1.4 .6 \\
1.4 .7 \\
1.4 .9 \\
1.4 .99 \\
1.4 .10\end{array}$ & $\begin{array}{l}0.0634 \\
0.0632 \\
0.0639 \\
0.1079 \\
0.1073\end{array}$ & $\begin{array}{l}0.2203 \\
0.2203 \\
0.2203 \\
0.2203 \\
0.2203\end{array}$ & $\begin{array}{l}42.28 \\
42.28 \\
42.28 \\
42.28 \\
42.28\end{array}$ & $\begin{array}{l}c .0652 \\
0.0644 \\
0.0651 \\
0.0659 \\
0.0644\end{array}$ & $\begin{array}{l}1.820 \\
1.586 \\
1.683 \\
1.075 \\
1.100\end{array}$ & $\begin{array}{l}0.973 \\
0.953 \\
1.037 \\
0.992 \\
1.261\end{array}$ & $\begin{array}{l}4.64 \\
4.63 \\
4.69 \\
7.90 \\
7.96\end{array}$ & $\begin{array}{l}0.471 \\
0.410 \\
0.435 \\
0.278 \\
0.284\end{array}$ & $\begin{array}{l}0.4481 \\
0.4474 \\
0.4500 \\
0.5845 \\
0.5929\end{array}$ & $\begin{array}{l}0.5853 \\
0.5463 \\
0.5629 \\
0.4498 \\
0.4550\end{array}$ \\
\hline $\begin{array}{l}1.4 .11 \\
1.4 .12 \\
1.4 .13 \\
1.4 .14 \\
1.4 .15\end{array}$ & $\begin{array}{l}0.1069 \\
0.1969 \\
0.1499 \\
0.1502 \\
0.1515\end{array}$ & $\begin{array}{l}0.2203 \\
0.2203 \\
0.2203 \\
0.2203 \\
0.2203\end{array}$ & $\begin{array}{l}42.28 \\
42.28 \\
42.28 \\
42.28 \\
42.28\end{array}$ & $\begin{array}{l}0.0644 \\
0.0648 \\
0.0652 \\
0.0652 \\
0.0653\end{array}$ & $\begin{array}{l}0.983 \\
1.056 \\
0.433 \\
0.432 \\
0.492\end{array}$ & $\begin{array}{l}0.960 \\
1.047 \\
0.989 \\
0.997 \\
1.049\end{array}$ & $\begin{array}{r}7.83 \\
7.83 \\
10.97 \\
11.00 \\
11.10\end{array}$ & $\begin{array}{l}0.254 \\
0.273 \\
0.112 \\
0.112 \\
0.127\end{array}$ & $\begin{array}{l}0.5820 \\
0.5820 \\
0.6889 \\
0.6898 \\
0.6927\end{array}$ & $\begin{array}{l}0.4302 \\
0.4457 \\
0.2856 \\
0.2851 \\
0.3044\end{array}$ \\
\hline $\begin{array}{l}1.4 .16 \\
1.4 .17 \\
1.4 .18 \\
1.4 .19 \\
1.4 .20\end{array}$ & $\begin{array}{l}0.1493 \\
0.1694 \\
0.1704 \\
0.1712 \\
0.1694\end{array}$ & $\begin{array}{l}0.2203 \\
0.2203 \\
0.2203 \\
0.2188 \\
0.2183\end{array}$ & $\begin{array}{l}42.28 \\
42.28 \\
42.28 \\
42.24 \\
42.24\end{array}$ & $\begin{array}{l}0.0643 \\
0.0648 \\
0.0652 \\
0.0649 \\
0.0635\end{array}$ & $\begin{array}{l}0.364 \\
0.325 \\
0.305 \\
0.271 \\
0.379\end{array}$ & $\begin{array}{l}0.945 \\
1.055 \\
1.008 \\
1.057 \\
1.020\end{array}$ & $\begin{array}{l}10.97 \\
12.41 \\
12.49 \\
12.62 \\
12.49\end{array}$ & $\begin{array}{l}0.094 \\
0.094 \\
0.079 \\
0.070 \\
0.098\end{array}$ & $\begin{array}{l}0.6889 \\
0.7326 \\
0.7347 \\
0.7376 \\
0.7336\end{array}$ & $\begin{array}{l}0.2616 \\
0.2473 \\
0.2396 \\
0.2258 \\
0.2671\end{array}$ \\
\hline $\begin{array}{l}1.4 .21 \\
1.4 .22 \\
1.4 .23 \\
1.4 .24 \\
1.4 .25\end{array}$ & $\begin{array}{l}0.171 .5 \\
0.1695 \\
0.1299 \\
0.1277 \\
0.1287\end{array}$ & $\begin{array}{l}0.2188 \\
0.2189 \\
0.2188 \\
0.2183 \\
0.2197\end{array}$ & $\begin{array}{l}42.24 \\
42.24 \\
42.24 \\
42.24 \\
42.24\end{array}$ & $\begin{array}{l}0.0646 \\
0.0642 \\
0.0655 \\
0.0647 \\
0.0646\end{array}$ & $\begin{array}{l}0.233 \\
0.221 \\
0.546 \\
0.522 \\
0.700\end{array}$ & $\begin{array}{l}0.964 \\
1.034 \\
0.935 \\
1.005 \\
0.973\end{array}$ & $\begin{array}{r}12.64 \\
12.50 \\
9.58 \\
9.41 \\
9.47\end{array}$ & $\begin{array}{l}0.060 \\
0.057 \\
0.141 \\
0.135 \\
0.181\end{array}$ & $\begin{array}{l}0.7382 \\
0.7340 \\
0.6424 \\
0.6370 \\
0.6394\end{array}$ & $\begin{array}{l}0.2096 \\
0.2039 \\
0.3205 \\
0.3135 \\
0.3630\end{array}$ \\
\hline $\begin{array}{l}1.4 .26 \\
1.4 .27 \\
1.4 .28 \\
1.4 .29 \\
1.4 .30\end{array}$ & $\begin{array}{l}0.1075 \\
0.1269 \\
0.1296 \\
0.0860 \\
0.0857\end{array}$ & $\begin{array}{l}0.2172 \\
0.2184 \\
0.2188 \\
. .2138 \\
0.2180\end{array}$ & $\begin{array}{l}42.24 \\
42.24 \\
42.24 \\
42.24 \\
42.24\end{array}$ & $\begin{array}{l}0.0644 \\
0.0645 \\
0.0653 \\
0.0659 \\
0.0658\end{array}$ & $\begin{array}{l}1.050 \\
0.625 \\
0.689 \\
1.400 \\
1.417\end{array}$ & $\begin{array}{l}1.009 \\
1.103 \\
0.971 \\
1.001 \\
1.002\end{array}$ & $\begin{array}{l}7.39 \\
9.52 \\
9.56 \\
.6 .34 \\
6.32\end{array}$ & $\begin{array}{l}0.272 \\
0.162 \\
0.178 \\
0.362 \\
0.366\end{array}$ & $\begin{array}{l}0.5856 \\
0.6403 \\
0.6419 \\
0.5227 \\
0.5219\end{array}$ & $\begin{array}{l}0.4445 \\
0.3430 \\
0.3601 \\
0.5133 \\
0.5164\end{array}$ \\
\hline $\begin{array}{l}1.4 .31 \\
1.4 .32 \\
1.4 .33 \\
1.4 .34 \\
1.4 .35\end{array}$ & $\begin{array}{l}0.0861 \\
0.0264 \\
0.0426 \\
0.0425 \\
0.0425\end{array}$ & $\begin{array}{l}0.2210 \\
0.2210 \\
0.2210 \\
0.2212 \\
0.2212\end{array}$ & $\begin{array}{l}42.24 \\
42.24 \\
42.24 \\
42.38 \\
42.38\end{array}$ & $\begin{array}{l}0.0659 \\
0.0654 \\
0.0657 \\
0.0656 \\
0.0652\end{array}$ & $\begin{array}{l}1.470 \\
1.300 \\
2.667 \\
2.417 \\
1.814\end{array}$ & $\begin{array}{l}1.001 . \\
0.989 \\
0.983 \\
1.024 \\
0.940\end{array}$ & $\begin{array}{l}6.29 \\
6.31 \\
3.11 \\
3.10 \\
3.10\end{array}$ & $\begin{array}{l}0.380 \\
0.334 \\
0.690 \\
0.625 \\
0.469\end{array}$ & $\begin{array}{l}0.5219 \\
0.5227 \\
0.3672 \\
0.3664 \\
0.3665\end{array}$ & $\begin{array}{l}0.5260 \\
0.4947 \\
0.7085 \\
0.6744 \\
0.5843\end{array}$ \\
\hline $\begin{array}{l}1.4 .36 \\
1.4 .37 \\
1.4 .38 \\
1.4 .39 \\
1.4 .40\end{array}$ & $\begin{array}{l}0.0421 \\
0.0431 \\
0.0107 \\
0.0107 \\
0.0106\end{array}$ & $\begin{array}{l}J .2204 \\
0.2200 \\
0.2200 \\
0.2208 \\
0.2212\end{array}$ & $\begin{array}{l}42.38 \\
42.38 \\
42.38 \\
42.38 \\
42.38\end{array}$ & $\begin{array}{l}0.0645 \\
0.0656 \\
0.0662 \\
0.0663 \\
0.0656\end{array}$ & $\begin{array}{l}1.922 \\
2.357 \\
3.385 \\
2.938 \\
2.330\end{array}$ & $\begin{array}{l}1.031 \\
0.969 \\
1.000 \\
0.996 \\
0.938\end{array}$ & $\begin{array}{l}3.08 \\
3.15 \\
0.79 \\
0.78 \\
0.78\end{array}$ & $\begin{array}{l}0.437 \\
0.610 \\
0.975 \\
0.760 \\
0.603\end{array}$ & $\begin{array}{l}0.3653 \\
0.3690 \\
0.1842 \\
0.1842 \\
0.1834\end{array}$ & $\begin{array}{l}0.6015 \\
0.6661 \\
0.7982 \\
0.7436 \\
0.6622\end{array}$ \\
\hline
\end{tabular}


TABLE XXXIII (Contd.)

TWO-PHASE COLD LEG MIXTURE TEST GROUP 1.4

EXPERIMENTAL MEASURMENTS FOR TESTS 1.4 .1 TO 1.4.40 -- $0.35-$ IN. DOHNCOMER GAP

\begin{tabular}{|c|c|c|c|c|c|c|c|c|c|c|}
\hline $\begin{array}{l}\text { TEST } \\
\text { NO }\end{array}$ & $\begin{array}{c}R M-C W-1 \\
(G P M)\end{array}$ & $\begin{array}{c}R M-C H-2 \\
(G P M)\end{array}$ & $\begin{array}{c}F T-C H-1 \\
(G P M)\end{array}$ & $\begin{array}{c}F T-C H-2 \\
(G P M)\end{array}$ & $\begin{array}{l}\text { RM-HG-1 } \\
\text { (CFM) }\end{array}$ & $\begin{array}{c}\text { RM-HG-2 } \\
(C F M)\end{array}$ & $\begin{array}{c}R M-H F-3 \\
(C F M)\end{array}$ & $\begin{array}{c}R M-H(-4)-4 \\
(C F M)\end{array}$ & $\begin{array}{l}F T-H G-1 \\
\text { (CFM) }\end{array}$ & $\begin{array}{l}F T-H G-2 \\
\text { (CFM) }\end{array}$ \\
\hline $\begin{array}{l}1.4 .1 \\
1.4 .2 \\
1.4 .3 \\
1.4 .4 \\
1.4 .5\end{array}$ & $\begin{array}{l}30.0 \\
20.0 \\
22.0 \\
29.0 \\
20.0\end{array}$ & $\begin{array}{r}10.0 \\
10.0 \\
0.0 \\
11.0 \\
10.0\end{array}$ & $\begin{array}{l}0.0 \\
0.0 \\
0.0 \\
0.0 \\
0.0\end{array}$ & $\begin{array}{l}0.0 \\
0.0 \\
0.0 \\
0.0 \\
0.0\end{array}$ & $\begin{array}{l}0.0 \\
0.0 \\
0.0 \\
0.0 \\
0.0\end{array}$ & $\begin{array}{c}0.0 \\
0.0 \\
0.0 \\
30.00 \\
30.00\end{array}$ & $\begin{array}{c}10.00 \\
10.00 \\
10.00 \\
0.0 \\
0.0\end{array}$ & $\begin{array}{l}0.0 \\
0.0 \\
0.0 \\
0.0 \\
0.0\end{array}$ & $\begin{array}{l}0.0 \\
0.0 \\
0.0 \\
0.0 \\
0.0\end{array}$ & $\begin{array}{l}0.0 \\
0.0 \\
0.0 \\
0.0 \\
0.0\end{array}$ \\
\hline $\begin{array}{l}1.4 .6 \\
1.4 .7 \\
1.4 .8 \\
1.4 .89 \\
1.4 .10\end{array}$ & $\begin{array}{l}25.0 \\
18.0 \\
19.0 \\
30.0 \\
20.0\end{array}$ & $\begin{array}{r}0.0 \\
0.0 \\
0.0 \\
10.0 \\
10.0\end{array}$ & $\begin{array}{l}0.0 \\
0.0 \\
0.0 \\
0.0 \\
0.0\end{array}$ & $\begin{array}{l}0.0 \\
0.0 \\
0.0 \\
0.0 \\
0.0\end{array}$ & $\begin{array}{l}0.0 \\
0.0 \\
0.0 \\
0.0 \\
0.0\end{array}$ & $\begin{array}{l}30.00 \\
30.00 \\
30.00 \\
50.00 \\
50.00\end{array}$ & $\begin{array}{l}0.0 \\
0.0 \\
0.0 \\
0.0 \\
0.0\end{array}$ & $\begin{array}{l}0.0 \\
0.0 \\
0.0 \\
0.0 \\
0.0\end{array}$ & $\begin{array}{l}0.0 \\
0.0 \\
0.0 \\
0.0 \\
0.0\end{array}$ & $\begin{array}{l}0.0 \\
0.0 \\
0.0 \\
0.0 \\
0.0\end{array}$ \\
\hline $\begin{array}{l}1.4 .11 \\
1.4 .12 \\
1.4 .13 \\
1.4 .14 \\
1.4 .15\end{array}$ & $\begin{array}{r}20.0 \\
9.0 \\
30.0 \\
20.0 \\
20.0\end{array}$ & $\begin{array}{r}0.0 \\
10.0 \\
10.0 \\
10.0 \\
0.0\end{array}$ & $\begin{array}{l}0.0 \\
0.0 \\
0.0 \\
0.0 \\
0.0\end{array}$ & $\begin{array}{l}0.0 \\
0.0 \\
0.0 \\
0.0 \\
0.0\end{array}$ & $\begin{array}{l}0.0 \\
0.0 \\
0.0 \\
0.0 \\
0.0\end{array}$ & $\begin{array}{l}50.00 \\
50.00 \\
70.00 \\
70.00 \\
70.00\end{array}$ & $\begin{array}{l}0.0 \\
0.0 \\
0.0 \\
0.0 \\
0.0\end{array}$ & $\begin{array}{l}0.0 \\
0.0 \\
0.0 \\
0.0 \\
0.0\end{array}$ & $\begin{array}{l}0.0 \\
0.0 \\
0.0 \\
0.0 \\
0.0\end{array}$ & $\begin{array}{l}0.0 \\
0.0 \\
0.0 \\
0.0 \\
0.0\end{array}$ \\
\hline $\begin{array}{l}1.4 .16 \\
1.4 .17 \\
1.4 .18 \\
1.4 .19 \\
1.4 .20\end{array}$ & $\begin{array}{r}0.0 \\
30.0 \\
30.0 \\
20.0 \\
20.0\end{array}$ & $\begin{array}{r}6.0 \\
10.0 \\
10.0 \\
10.0 \\
0.0\end{array}$ & $\begin{array}{l}0.0 \\
0.0 \\
0.0 \\
0.0 \\
0.0\end{array}$ & $\begin{array}{l}0.0 \\
0.0 \\
0.0 \\
0.0 \\
0.0\end{array}$ & $\begin{array}{l}0.0 \\
0.0 \\
0.0 \\
0.0 \\
0.0\end{array}$ & $\begin{array}{l}70.00 \\
50.00 \\
50.00 \\
50.00 \\
50.00\end{array}$ & $\begin{array}{c}0.0 \\
30.00 \\
30.00 \\
30.00 \\
30.00\end{array}$ & $\begin{array}{l}0.0 \\
0.0 \\
0.0 \\
0.0 \\
0.0\end{array}$ & $\begin{array}{l}0.0 \\
0.0 \\
0.0 \\
0.0 \\
0.0\end{array}$ & $\begin{array}{l}0.0 \\
0.0 \\
0.0 \\
0.0 \\
0.0\end{array}$ \\
\hline $\begin{array}{l}1.4 .21 \\
1.4 .22 \\
1.4 .23 \\
1.4 .24 \\
1.4 .25\end{array}$ & $\begin{array}{r}1.0 \\
3.10 \\
23.0 \\
23.0 \\
20.0\end{array}$ & $\begin{array}{r}10.0 \\
4.0 \\
12.0 \\
10.0 \\
0.0\end{array}$ & $\begin{array}{l}0.0 \\
0.0 \\
0.0 \\
0.0 \\
0.0\end{array}$ & $\begin{array}{l}0.0 \\
0.0 \\
0.0 \\
0.0 \\
0.0\end{array}$ & $\begin{array}{l}0.0 \\
0.0 \\
0.0 \\
0.0 \\
0.0\end{array}$ & $\begin{array}{l}50.00 \\
50.00 \\
60.00 \\
60.00 \\
60.00\end{array}$ & $\begin{array}{c}30.00 \\
30.00 \\
0.0 \\
0.0 \\
0.0\end{array}$ & $\begin{array}{l}0.0 \\
0.0 \\
0.0 \\
0.0 \\
0.0\end{array}$ & $\begin{array}{l}0.0 \\
0.0 \\
0.0 \\
0.0 \\
0.0\end{array}$ & $\begin{array}{l}0.0 \\
0.0 \\
0.0 \\
0.0 \\
0.0\end{array}$ \\
\hline $\begin{array}{l}1.4 .26 \\
1.4 .27 \\
1.4 .28 \\
1.4 .29 \\
1.4 .30\end{array}$ & $\begin{array}{r}12.0 \\
0.0 \\
12.0 \\
30.0 \\
20.0\end{array}$ & $\begin{array}{r}0.0 \\
0.0 \\
0.0 \\
10.0 \\
10.0\end{array}$ & $\begin{array}{l}0.0 \\
0.0 \\
0.0 \\
0.0 \\
0.0\end{array}$ & $\begin{array}{l}0.0 \\
0.0 \\
0.0 \\
0.0 \\
0.0\end{array}$ & $\begin{array}{l}0.0 \\
0.0 \\
0.0 \\
0.0 \\
0.0\end{array}$ & $\begin{array}{l}50.00 \\
60.00 \\
60.00 \\
40.00 \\
40.00\end{array}$ & $\begin{array}{l}0.0 \\
0.0 \\
0.0 \\
0.0 \\
0.0\end{array}$ & $\begin{array}{l}0.0 \\
0.0 \\
0.0 \\
0.0 \\
0.0\end{array}$ & $\begin{array}{l}0.0 \\
0.0 \\
0.0 \\
0.0 \\
0.0\end{array}$ & $\begin{array}{l}0.0 \\
0.0 \\
0.0 \\
0.0 \\
0.0\end{array}$ \\
\hline $\begin{array}{l}1.4 .31 \\
1.4 .32 \\
1.4 .33 \\
1.4 .34 \\
1.4 .35\end{array}$ & $\begin{array}{l}20.0 \\
12.0 \\
30.0 \\
20.0 \\
20.0\end{array}$ & $\begin{array}{r}0.0 \\
0.0 \\
10.0 \\
10.0 \\
0.0\end{array}$ & $\begin{array}{l}0.0 \\
0.0 \\
0.0 \\
0.0 \\
0.0\end{array}$ & $\begin{array}{l}0.0 \\
0.0 \\
0.0 \\
0.0 \\
0.0\end{array}$ & $\begin{array}{l}0.0 \\
0.0 \\
0.0 \\
0.0 \\
0.0\end{array}$ & $\begin{array}{l}40.00 \\
40.00 \\
20.00 \\
20.00 \\
20.00\end{array}$ & $\begin{array}{l}0.0 \\
0.0 \\
0.0 \\
0.0 \\
0.0\end{array}$ & $\begin{array}{l}0.0 \\
0.0 \\
0.0 \\
0.0 \\
0.0\end{array}$ & $\begin{array}{l}2.0 \\
0.0 \\
0.0 \\
0.0 \\
0.0\end{array}$ & $\begin{array}{l}0.0 \\
0.0 \\
0.0 \\
0.0 \\
0.0\end{array}$ \\
\hline $\begin{array}{l}1.4 .36 \\
1.4 .37 \\
1.4 .38 \\
1.4 .39 \\
1.4 .40\end{array}$ & $\begin{array}{l}20.0 \\
20.0 \\
21.0 \\
20.0 \\
24.0\end{array}$ & $\begin{array}{r}0.0 \\
10.0 \\
9.0 \\
10.0 \\
0.0\end{array}$ & $\begin{array}{l}0.0 \\
0.0 \\
0.0 \\
0.0 \\
0.0\end{array}$ & $\begin{array}{l}0.0 \\
0.0 \\
0.0 \\
0.0 \\
0.0\end{array}$ & $\begin{array}{l}0.0 \\
0.0 \\
0.0 \\
0.0 \\
0.0\end{array}$ & $\begin{array}{l}0.0 \\
0.0 \\
0.0 \\
0.0 \\
0.0\end{array}$ & $\begin{array}{c}20.00 \\
20.00 \\
0.0 \\
5.00 \\
0.0\end{array}$ & $\begin{array}{l}0.0 \\
0.0 \\
5.00 \\
0.0 \\
5.00\end{array}$ & $\begin{array}{l}0.0 \\
0.0 \\
0.0 \\
0.0 \\
0.0\end{array}$ & $\begin{array}{l}0.0 \\
0.0 \\
0.0 \\
0.0 \\
0.0\end{array}$ \\
\hline
\end{tabular}


TABLE XXXIII (Contd.)

TWO-PHASE COLD LEG MIXTURE TEST GROUP 1.4

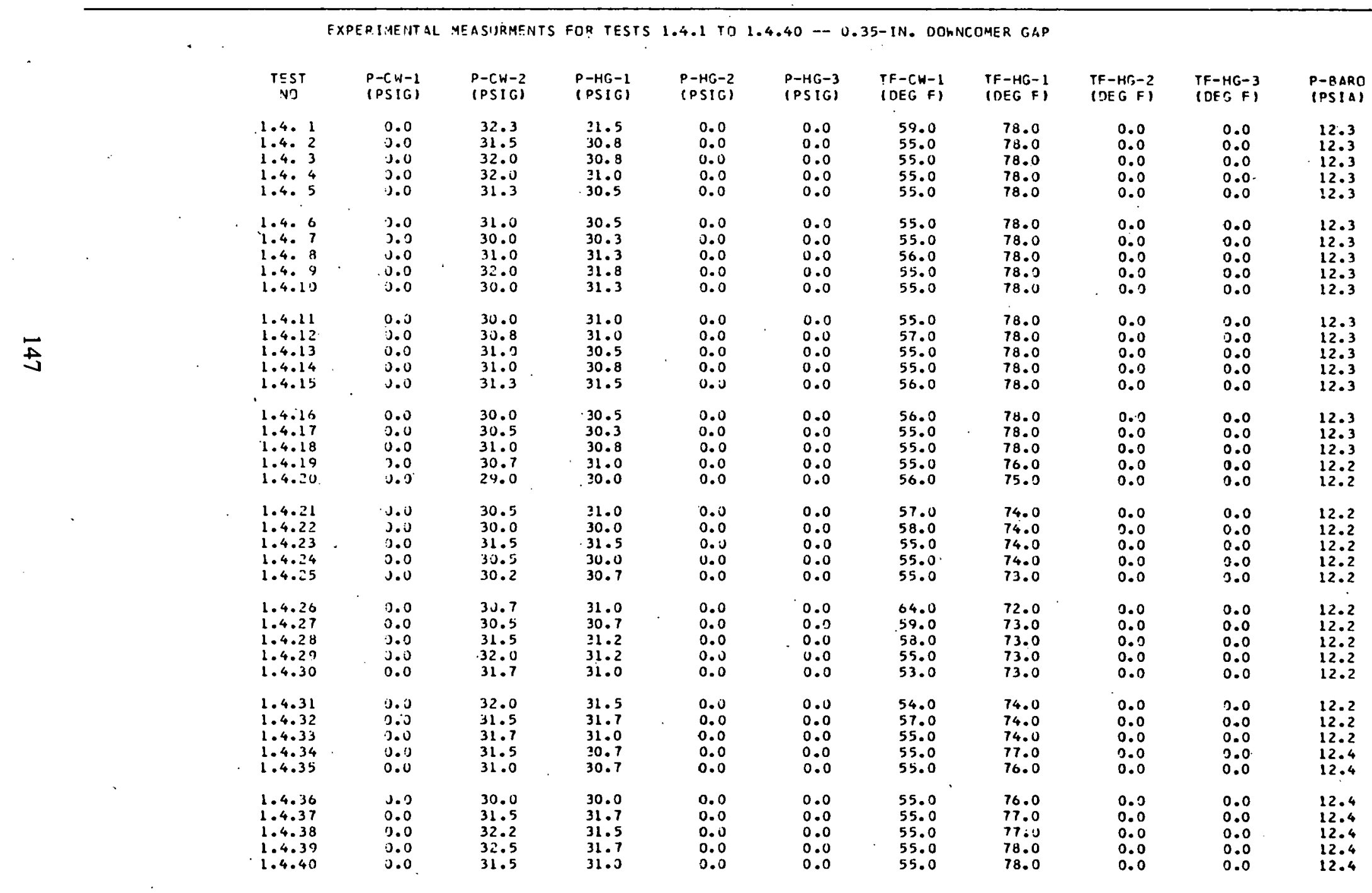


TABLE XXXIII (Contd.)

TWO-PHASE COLD LEG MIXTURE TEST GROUP 1.4

EXPERIMENTAL MEASURMENTS FOR TESTS 1.4 .1 TO $1.4 .40-0.35-$ IN. DOHNCOMER GAP

\begin{tabular}{|c|c|c|c|c|c|c|c|c|c|}
\hline \multirow[b]{2}{*}{$\begin{array}{c}\text { TEST } \\
N \mathbf{J}\end{array}$} & \multicolumn{3}{|c|}{ LOWER PLENUM FLOW } & \multicolumn{3}{|c|}{ BYPASS FLOH } & \multirow{2}{*}{$\begin{array}{l}\text { LQWER } \\
\text { PLENUM } \\
\text { LEVEL } \\
\text { (IN) }\end{array}$} & \multirow[b]{2}{*}{$\begin{array}{l}P-L P-1 \\
(P S I G)\end{array}$} & \multirow[b]{2}{*}{$\begin{array}{l}\text { TF-LP-1 } \\
\text { IDEG FI }\end{array}$} \\
\hline & $\begin{array}{l}\text { INITIAL } \\
\text { INI I SHT } \\
\text { (LB) }\end{array}$ & $\begin{array}{l}\text { FINAL: } \\
\text { WE IGHT } \\
\text { (LB) }\end{array}$ & $\begin{array}{l}\text { TIME OF } \\
\text { COLLECTIIIN } \\
\text { ISECI }\end{array}$ & $\begin{array}{l}\text { INITIAL } \\
\text { WEIGHT } \\
\text { (LB) }\end{array}$ & $\begin{array}{l}\text { FINAL } \\
\text { WEIGHT } \\
\text { (LBI }\end{array}$ & $\begin{array}{l}\text { TIME DF } \\
\text { COLLECTION } \\
\text { (SEC) }\end{array}$ & & & \\
\hline $\begin{array}{l}1.4 .1 \\
1.4 .2 \\
1.4 .3 \\
1.4 .4 \\
1.4 .5\end{array}$ & $\begin{array}{l}133.0 \\
229.8 \\
299.8 \\
372.3 \\
422.5\end{array}$ & $\begin{array}{l}229.8 \\
298.8 \\
372.3 \\
42.5 \\
470.5\end{array}$ & $\begin{array}{l}30.0 \\
25.0 \\
35.0 \\
25.0 \\
25.0\end{array}$ & $\begin{array}{l}75.0 \\
148.8 \\
191.8 \\
243.8 \\
380.8\end{array}$ & $\begin{array}{l}148.8 \\
191.8 \\
243.8 \\
380.5 \\
446.5\end{array}$ & $\begin{array}{l}30.0 \\
30.0 \\
60.0 \\
40.0 \\
30.0\end{array}$ & $\begin{array}{l}12.0 \\
12.0 \\
12.0 \\
12.0 \\
12.0\end{array}$ & $\begin{array}{l}30.0 \\
30.0 \\
30.0 \\
30.0 \\
30.0\end{array}$ & $\begin{array}{l}58.0 \\
58.0 \\
58.0 \\
58.0 \\
58.0\end{array}$ \\
\hline $\begin{array}{l}1.4 .6 \\
1.4 .7 \\
1.4 .89 \\
1.4 .99 \\
1.4 .13\end{array}$ & $\begin{array}{l}470.3 \\
46.3 .3 \\
133.0 \\
183.5 \\
226.5\end{array}$ & $\begin{array}{l}515.8 \\
523.8 \\
183.5 \\
226.5 \\
257.5\end{array}$ & $\begin{array}{l}25.0 \\
35.0 \\
30.0 \\
40.0 \\
30.0\end{array}$ & $\begin{array}{r}446.5 \\
426.0 \\
88.0 \\
129.0 \\
306.8\end{array}$ & $\begin{array}{l}516.8 \\
454.0 \\
129.0 \\
306.8 \\
431.5\end{array}$ & $\begin{array}{l}45.0 \\
35.0 \\
45.0 \\
40.0 \\
30.0\end{array}$ & $\begin{array}{l}12.0 \\
12.0 \\
12.0 \\
12.0 \\
12.0\end{array}$ & $\begin{array}{l}30.0 \\
30.0 \\
30.0 \\
30.0 \\
30.0\end{array}$ & $\begin{array}{l}58.0 \\
58.0 \\
58.0 \\
58.0 \\
58.0\end{array}$ \\
\hline $\begin{array}{l}1.4 .11 \\
1.4 .12 \\
1.4 .13 \\
1.4 .14 \\
1.4 .15\end{array}$ & $\begin{array}{l}259.5 \\
303.9 \\
170.3 \\
189.8 \\
213.5\end{array}$ & $\begin{array}{l}303.8 \\
341.8 \\
199.8 \\
213.5 \\
245.5\end{array}$ & $\begin{array}{l}45.0 \\
36.0 \\
45.0 \\
55.0 \\
65.0\end{array}$ & $\begin{array}{l}431.5 \\
499.0 \\
112.5 \\
264.5 \\
395.0\end{array}$ & $\begin{array}{l}499.0 \\
521.0 \\
264.5 \\
395.0 \\
492.0\end{array}$ & $\begin{array}{l}40.0 \\
55.0 \\
30.0 \\
35.0 \\
40.0\end{array}$ & $\begin{array}{l}12.0 \\
12.0 \\
12.0 \\
12.0 \\
12.0\end{array}$ & $\begin{array}{l}30.0 \\
30.0 \\
30.0 \\
30.0 \\
30.0\end{array}$ & $\begin{array}{l}58.0 \\
58.0 \\
58.0 \\
58.0 \\
58.0\end{array}$ \\
\hline $\begin{array}{l}1.4 .16 \\
1.4 .17 \\
1.4 .13 \\
1.4 .19 \\
1.4 .20\end{array}$ & $\begin{array}{l}245.5 \\
265.0 \\
281.3 \\
132.8 \\
149.0\end{array}$ & $\begin{array}{l}265.5 \\
281.3 \\
296.5 \\
149.0 \\
171.8\end{array}$ & $\begin{array}{l}55.0 \\
50.0 \\
50.0 \\
60.0 \\
60.0\end{array}$ & $\begin{array}{r}492.0 \\
517.5 \\
656.0 \\
84.5 \\
188.0\end{array}$ & $\begin{array}{l}517.5 \\
656.0 \\
841.5 \\
188.0 \\
.274 .0\end{array}$ & $\begin{array}{l}60.0 \\
25.0 \\
35.0 \\
25.0 \\
35.0\end{array}$ & $\begin{array}{l}12.0 \\
12.0 \\
12.0 \\
12.0 \\
12.0\end{array}$ & $\begin{array}{l}30.0 \\
30.0 \\
30.0 \\
30.0 \\
30.0\end{array}$ & $\begin{array}{l}59.0 \\
58.0 \\
58.0 \\
61.0 \\
61.0\end{array}$ \\
\hline $\begin{array}{l}1.4 .21 \\
1.4 .22 \\
1.4 .23 \\
1.4 .24 \\
1.4 .25\end{array}$ & $\begin{array}{l}171.8 \\
185.8 \\
199.0 \\
231.9 \\
253.3\end{array}$ & $\begin{array}{l}185.8 \\
199.0 \\
231.8 \\
255.8 \\
293.8\end{array}$ & $\begin{array}{l}60.0 \\
60.0 \\
60.0 \\
45.0 \\
55.0\end{array}$ & $\begin{array}{l}274.0 \\
312.8 \\
334.0 \\
457.3 \\
549.0\end{array}$ & $\begin{array}{l}312.8 \\
334.0 \\
457.3 \\
549.0 \\
639.3\end{array}$ & $\begin{array}{l}35.0 \\
60.0 \\
25.0 \\
25.0 \\
45.0\end{array}$ & $\begin{array}{l}12.0 \\
12.0 \\
12.0 \\
12.0 \\
12.0\end{array}$ & $\begin{array}{l}30.0 \\
30.0 \\
30.0 \\
30.0 \\
30.0\end{array}$ & $\begin{array}{l}61.0 \\
61.0 \\
61.0 \\
61.0 \\
59.0\end{array}$ \\
\hline $\begin{array}{l}1.4 .26 \\
1.4 .27 \\
1.4 .28 \\
1.4 .29 \\
1.4 .30\end{array}$ & $\begin{array}{l}133.0 \\
226.5 \\
264.0 \\
295.0 \\
337.0\end{array}$ & $\begin{array}{l}185.5 \\
264.0 \\
295.0 \\
337.0 \\
379.5\end{array}$ & $\begin{array}{l}50.0 \\
60.0 \\
45.0 \\
30.0 \\
30.0\end{array}$ & $\begin{array}{r}64.3 \\
92.8 \\
109.0 \\
146.3 \\
.271 .1\end{array}$ & $\begin{array}{r}92.8 \\
109.0 \\
146.3 \\
271.3 \\
354.0\end{array}$ & $\begin{array}{l}45.0 \\
55.0 \\
40.0 \\
30.0 \\
30.0\end{array}$ & $\begin{array}{l}12.0 \\
12.0 \\
12.0 \\
12.0 \\
12.0\end{array}$ & $\begin{array}{l}30.0 \\
30.0 \\
30.0 \\
30.0 \\
30.0\end{array}$ & $\begin{array}{l}65.0 \\
62.0 \\
61.0 \\
61.0 \\
61.0\end{array}$ \\
\hline $\begin{array}{l}1.4 .31 \\
1.4 .32 \\
1.4 .33 \\
1.4 .34 \\
1.4 .35\end{array}$ & $\begin{array}{l}377.5 \\
135.5 \\
187.5 \\
133.8 \\
242.5\end{array}$ & $\begin{array}{l}453.0 \\
187.5 \\
307.5 \\
242.5 \\
342.3\end{array}$ & $\begin{array}{l}50.0 \\
40.0 \\
45.0 \\
45.0 \\
55.0\end{array}$ & $\begin{array}{r}354.0 \\
96.5 \\
114.0 \\
93.3 \\
167.5\end{array}$ & $\begin{array}{l}400.0 \\
114.0 \\
240.0 \\
167.5 \\
207.5\end{array}$ & $\begin{array}{l}35.0 \\
50.0 \\
45.0 \\
40.0 \\
50.0\end{array}$ & $\begin{array}{l}12.0 \\
12.0 \\
12.0 \\
12.0 \\
12.0\end{array}$ & $\begin{array}{l}30.0 \\
30.0 \\
30.0 \\
30.0 \\
30.0\end{array}$ & $\begin{array}{l}56.0 \\
56.0 \\
56.0 \\
57.0 \\
57.0\end{array}$ \\
\hline $\begin{array}{l}1.4 .36 \\
1.4 .37 \\
1.4 .39 \\
1.4 .39 \\
1.4 .40\end{array}$ & $\begin{array}{l}342.3 \\
215.8 \\
310.0 \\
377.8 \\
435.5\end{array}$ & $\begin{array}{l}42 a .8 \\
310.0 \\
377.8 \\
436.5 \\
494.8\end{array}$ & $\begin{array}{l}45.0 \\
40.0 \\
20.0 \\
20.0 \\
25.0\end{array}$ & $\begin{array}{l}207.5 \\
250.0 \\
309.0 \\
374.3 \\
409.5\end{array}$ & $\begin{array}{l}250.0 \\
309.0 \\
374.3 \\
409.5 \\
467.5\end{array}$ & $\begin{array}{l}45.0 \\
35.0 \\
30.0 \\
30.0 \\
60.0 .\end{array}$ & $\begin{array}{l}12.0 \\
12.0 \\
12.9 \\
12.0 \\
12.0\end{array}$ & $\begin{array}{l}30.0 \\
30.0 \\
30.0 \\
30.0 \\
30.0\end{array}$ & $\begin{array}{l}59.0 \\
60.0 \\
60.0 \\
58.0 \\
57.0\end{array}$ \\
\hline
\end{tabular}




\section{TABLE XXXIII (Contd.)}

TWO-PHASE COLD LEG MIXTURE TEST GROUP 1.4

EXPERIYENTAL YEASIJRMENTS FOR TESTS 1.4 .1 TO $1.4 .40-0.35-I N$. OOWNCOMER GAP

\begin{tabular}{|c|c|c|c|c|c|c|c|}
\hline $\begin{array}{l}\text { TEST } \\
\text { HO }\end{array}$ & $\begin{array}{c}R M-E F-1 \\
(C F M)\end{array}$ & $\begin{array}{c}R .4-C C_{S}-2 \\
(C F M)\end{array}$ & $\begin{array}{l}F T-C G-1 \\
(C F M)\end{array}$ & $\begin{array}{l}P-C G-1 \\
(P S I G)\end{array}$ & $\begin{array}{l}P-C G-2 \\
(P S I G)\end{array}$ & $\begin{array}{l}T F-C G-1 \\
\text { (DEG FI }\end{array}$ & $\begin{array}{l}T F-C F-2 \\
(D E G F)\end{array}$ \\
\hline $\begin{array}{l}1.4 .1 \\
1.4 .2 \\
1.4 .3 \\
1.4 .4 \\
1.4 .3\end{array}$ & $\begin{array}{l}30.0 \\
30.0 \\
30.0 \\
30.0 \\
30.0\end{array}$ & $\begin{array}{l}0.0 \\
0.0 \\
0.0 \\
c .0 \\
0.0\end{array}$ & $\begin{array}{l}0.0 \\
0.0 \\
0.0 \\
0.0 \\
0.0\end{array}$ & $\begin{array}{l}0.0 \\
0.0 \\
0.0 \\
0.0 \\
0.0\end{array}$ & $\begin{array}{l}0.0 \\
0.0 \\
0.0 \\
0.0 \\
0.0\end{array}$ & $\begin{array}{l}0.0 \\
0.0 \\
0.0 \\
0.0 \\
0.0\end{array}$ & $\begin{array}{l}0.0 \\
0.0 \\
0.0 \\
0.0 \\
0.0\end{array}$ \\
\hline $\begin{array}{l}1.4 .6 \\
1.4 .9 \\
1.4 .9 \\
1.4 .9 \\
1.4 .1 .3\end{array}$ & $\begin{array}{l}30.0 \\
30.0 \\
30.0 \\
30.0 \\
30.0\end{array}$ & $\begin{array}{l}0.0 \\
0.0 \\
0.0 \\
0.0 \\
0.0\end{array}$ & $\begin{array}{l}0.0 \\
0.0 \\
0.0 \\
0.0 \\
0.0\end{array}$ & $\begin{array}{l}0.0 \\
0.0 \\
0.0 \\
0.0 \\
0.0\end{array}$ & $\begin{array}{l}0.0 \\
0.0 \\
0.0 \\
0.0 \\
0.0\end{array}$ & $\begin{array}{l}0.0 \\
0.0 \\
0.0 \\
0.0 \\
0.0\end{array}$ & $\begin{array}{l}0.0 \\
0.0 \\
0.0 \\
0.0 \\
0.0\end{array}$ \\
\hline $\begin{array}{l}1.4 .11 \\
1.4 .12 \\
1.4 .13 \\
1.4 .14 \\
1.4 .15\end{array}$ & $\begin{array}{l}30.0 \\
30.0 \\
30.0 \\
30.0 \\
30.0\end{array}$ & $\begin{array}{l}0.0 \\
0.0 \\
0.0 \\
0.0 \\
0.0\end{array}$ & $\begin{array}{l}0.0 \\
0.0 \\
0.0 \\
0.0 \\
0.0\end{array}$ & $\begin{array}{l}0.0 \\
0.0 \\
0.0 \\
0.0 \\
0.0\end{array}$ & $\begin{array}{l}0.0 \\
0.0 \\
0.0 \\
0.0 \\
0.0\end{array}$ & $\begin{array}{l}0.0 \\
0.0 \\
0.0 \\
0.0 \\
0.0\end{array}$ & $\begin{array}{l}0.0 \\
0.0 \\
0.0 \\
0.0 \\
0.0\end{array}$ \\
\hline $\begin{array}{l}1.4 .16 \\
1.4 .17 \\
1.4 .18 \\
1.4 .19 \\
1.4 .20\end{array}$ & $\begin{array}{l}30.0 \\
30.0 \\
30.0 \\
30.0 \\
30.0\end{array}$ & $\begin{array}{l}0.0 \\
0.0 \\
0.0 \\
0.0 \\
0.0\end{array}$ & $\begin{array}{l}0.0 \\
0.0 \\
0.0 \\
0.0 \\
0.0\end{array}$ & $\begin{array}{l}0.0 \\
0.0 \\
0.0 \\
0.0 \\
0.0\end{array}$ & $\begin{array}{l}0.0 \\
0.0 \\
0.0 \\
0.0 \\
0.0\end{array}$ & $\begin{array}{l}0.0 \\
0.0 \\
0.0 \\
0.0 \\
0.0\end{array}$ & $\begin{array}{l}0.0 \\
0.0 \\
0.0 \\
0.0 \\
0.0\end{array}$ \\
\hline $\begin{array}{l}1.4 .21 \\
1.4 .22 \\
1.4 .23 \\
1.4 .24 \\
1.4 .25\end{array}$ & $\begin{array}{l}30.0 \\
30.0 \\
30.0 \\
30.0 \\
30.0\end{array}$ & $\begin{array}{l}0.0 \\
0.0 \\
0.0 \\
0.0 \\
0.0\end{array}$ & $\begin{array}{l}0.0 \\
0.0 \\
0.0 \\
0.0 \\
0.0\end{array}$ & $\begin{array}{l}0.0 \\
0.0 \\
0.0 \\
0.0 \\
0.0\end{array}$ & $\begin{array}{l}0.0 \\
2.0 \\
0.0 \\
0.0 \\
0.0\end{array}$ & $\begin{array}{l}0.0 \\
0.0 \\
0.0 \\
0.0 \\
0.0\end{array}$ & $\begin{array}{l}0.0 \\
0.0 \\
0.0 \\
0.0 \\
0.0\end{array}$ \\
\hline $\begin{array}{l}1.4 .26 \\
1.4 .27 \\
1.4 .29 \\
1.4 .29 \\
1.4 .30\end{array}$ & $\begin{array}{l}30.0 \\
30.0 \\
30.0 \\
30.0 \\
30.0\end{array}$ & $\begin{array}{l}0.0 \\
0.0 \\
0.0 \\
0.0 \\
0.0\end{array}$ & $\begin{array}{l}0.0 \\
0.0 \\
0.0 \\
0.0 \\
0.0\end{array}$ & $\begin{array}{l}0.0 \\
0.0 \\
0.0 \\
0.0 \\
0.0\end{array}$ & $\begin{array}{l}0.0 \\
0.0 \\
0.0 \\
0.0 \\
0.0\end{array}$ & $\begin{array}{l}0.0 \\
0.0 \\
0.0 \\
0.0 \\
0.0\end{array}$ & $\begin{array}{l}0.0 \\
0.0 \\
0.0 \\
0.0 \\
0.0\end{array}$ \\
\hline $\begin{array}{l}1.4 .31 \\
1.4 .32 \\
1.4 .33 \\
1.4 .34 \\
1.4 .35\end{array}$ & $\begin{array}{l}30.0 \\
30.0 \\
30.0 \\
30.0 \\
30.0\end{array}$ & $\begin{array}{l}0.0 \\
0.0 \\
0.0 \\
0.0 \\
0.0\end{array}$ & $\begin{array}{l}0.0 \\
0.0 \\
0.0 \\
0.0 \\
0.0\end{array}$ & $\begin{array}{l}0.0 \\
0.0 \\
0.0 \\
0.0 \\
0.0\end{array}$ & $\begin{array}{l}0.0 \\
0.0 \\
0.0 \\
0.0 \\
0.0\end{array}$ & $\begin{array}{l}0.0 \\
0.0 \\
0.0 \\
0.0 \\
0.0\end{array}$ & $\begin{array}{l}0.0 \\
0.0 \\
0.0 \\
0.0 \\
0.0\end{array}$ \\
\hline $\begin{array}{l}1.4 .36 \\
1.4 .36 \\
1.4 .37 \\
1.4 .39 \\
1.4 .40\end{array}$ & $\begin{array}{l}30.0 \\
30.0 \\
30.3 \\
30.0 \\
30.0\end{array}$ & $\begin{array}{l}0.0 \\
0.0 \\
0.0 \\
0.0 \\
0.0\end{array}$ & $\begin{array}{l}0.0 \\
0.0 \\
0.0 \\
0.0 \\
0.0\end{array}$ & $\begin{array}{l}0.0 \\
0.0 \\
0.0 \\
0.0 \\
0.0\end{array}$ & $\begin{array}{l}0.0 \\
0.0 \\
0.0 \\
0.0 \\
0.0\end{array}$ & $\begin{array}{l}0.0 \\
0.0 \\
0.0 \\
0.0 \\
0.0\end{array}$ & $\begin{array}{l}0.0 \\
0.0 \\
0.0 \\
0.0 \\
0.0\end{array}$ \\
\hline
\end{tabular}




\section{TABLE XXXIV}

TWO-PHASE COLD LEG MIXTURE TEST GROUP 5.6

TPANSPARENT VESSEL TEST DATA EOR. TESTS 5.6.1 TO 5.6.17 - - 0.53-IN. DOWNCOMEP. GAP

SIMMARY TF CALCULATED RESULTS

\begin{tabular}{|c|c|c|c|c|c|c|c|c|c|c|}
\hline $\begin{array}{l}\text { TEST } \\
\text { NO }\end{array}$ & $\begin{array}{l}\text { DJWNC NAER } \\
\text { AIR FLUU: } \\
\text { ULB/SFCI }\end{array}$ & $\begin{array}{l}\text { (ONNCOMER } \\
\text { AR DFNSITY } \\
\text { (LB/FT*\#3) }\end{array}$ & $\begin{array}{l}\text { LOWEF PLENUM } \\
\text { PRESSURE } \\
\text { (PSIA) }\end{array}$ & $\begin{array}{l}\text { COLD LEG } \\
\text { AIR FLTW } \\
\text { ILU/SFCI }\end{array}$ & $\begin{array}{l}\text { DOWNCOMEF } \\
\text { WATER FLOW } \\
\text { (LB/SFC) }\end{array}$ & $\begin{array}{l}\text { WATER FLOW } \\
\text { COHTINUITY }\end{array}$ & $\begin{array}{c}\text { J-AIR } \\
\text { DOWNCQMFP } \\
\text { (FT/SEC) }\end{array}$ & $\begin{array}{l}\text { J-HATER } \\
\text { DOWACOMER } \\
\text { (FT/SEC) }\end{array}$ & $(J G * \mid * * 1 / 2$ & $(J L *) * * 1 / 2$ \\
\hline $\begin{array}{ll}5.6 .6 & 1 \\
5.6 . & 2 \\
5.6 .0 & 3 \\
5.6 .6 & 4 \\
5.6 . & 5\end{array}$ & $\begin{array}{l}0.0218 \\
0.0431 \\
0.1215 \\
0.1473 \\
0.0795\end{array}$ & $\begin{array}{l}0.1204 \\
0.1103 \\
0.1166 \\
0.1170 \\
0.1197\end{array}$ & $\begin{array}{l}22.70 \\
22.20 \\
22.20 \\
22.20 \\
22.67\end{array}$ & $\begin{array}{l}0.0667 \\
0.0667 \\
0.0667 \\
0.0667 \\
0.0670\end{array}$ & $\begin{array}{l}3.100 \\
2.233 \\
0.504 \\
0.221 \\
1.383\end{array}$ & $\begin{array}{l}1.018 \\
0.989 \\
1.010 \\
1.012 \\
1.002\end{array}$ & $\begin{array}{r}2.72 \\
5.54 \\
16.43 \\
18.98 \\
9.98\end{array}$ & $\begin{array}{l}0.747 \\
0.538 \\
0.121 \\
0.053 \\
0.333\end{array}$ & $\begin{array}{l}0.2662 \\
0.3774 \\
0.6496 \\
0.6989 \\
0.5096\end{array}$ & $\begin{array}{l}0.6660 \\
0.5653 \\
0.2685 \\
0.1777 \\
0.4449\end{array}$ \\
\hline $\begin{array}{l}5.6 .0 \\
5.6 .7 \\
5.6 .8 \\
5.6 .89 \\
5.6 .10\end{array}$ & $\begin{array}{l}0.1013 \\
0.1013 \\
0.1307 \\
0.0293 \\
0.0511\end{array}$ & $\begin{array}{l}0.1209 \\
0.1173 \\
1.1155 \\
0.1189 \\
0.1213\end{array}$ & $\begin{array}{l}22.92 \\
22.17 \\
22.67 \\
22.42 \\
22.92\end{array}$ & $\begin{array}{l}0.0672 \\
0.0672 \\
0.0663 \\
0.0671 \\
0.0670\end{array}$ & $\begin{array}{l}0.858 \\
0.917 \\
0.375 \\
2.933 \\
2.225\end{array}$ & $\begin{array}{l}0.939 \\
1.045 \\
1.035 \\
0.992 \\
1.008\end{array}$ & $\begin{array}{r}12.60 \\
12.98 \\
16.44 \\
3.70 \\
6.33\end{array}$ & $\begin{array}{l}0.207 \\
0.221 \\
0.090 \\
0.718 \\
0.536\end{array}$ & $\begin{array}{l}0.5739 \\
0.5782 \\
0.6537 \\
0.3098 \\
0.4073\end{array}$ & $\begin{array}{l}0.3504 \\
0.3621 \\
0.2316 \\
0.6533 \\
0.5642\end{array}$ \\
\hline $\begin{array}{l}5.6 .11 \\
5.5 .12 \\
5.0 .13 \\
5.6 .14 \\
5.6 .15\end{array}$ & $\begin{array}{l}0.0290 \\
0.0292 \\
0.0513 \\
0.1450 \\
0.1540\end{array}$ & $\begin{array}{l}0.1295 \\
0.1517 \\
0.1243 \\
0.1252 \\
0.1186\end{array}$ & $\begin{array}{l}24.42 \\
28.67 \\
23.67 \\
21.17 \\
22.42\end{array}$ & $\begin{array}{l}0.2745 \\
0.2709 \\
0.2662 \\
0.2663 \\
0.2535\end{array}$ & $\begin{array}{l}1.617 \\
2.367 \\
1.150 \\
0.333 \\
0.137\end{array}$ & $\begin{array}{l}1.005 \\
0.995 \\
1.022 \\
1.022 \\
1.017\end{array}$ & $\begin{array}{r}3.37 \\
2.89 \\
6.18 \\
17.40 \\
19.51\end{array}$ & $\begin{array}{l}0.389 \\
0.570 \\
0.277 \\
0.080 \\
0.033\end{array}$ & $\begin{array}{l}0.3018 \\
0.2909 \\
0.4052 \\
0.6805 \\
0.7109\end{array}$ & $\begin{array}{l}0.4809 \\
0.5820 \\
0.4056 \\
0.2184 \\
0.1403\end{array}$ \\
\hline $\begin{array}{l}5.6 .16 \\
5.6 .17\end{array}$ & $\begin{array}{l}0.0287 \\
0.1013\end{array}$ & $\begin{array}{l}0.1538 \\
0.1177\end{array}$ & $\begin{array}{l}29.17 \\
22.42\end{array}$ & $\begin{array}{l}0.2508 \\
0.2596\end{array}$ & $\begin{array}{l}2.711 \\
0.700\end{array}$ & $\begin{array}{l}1.009 \\
1.012\end{array}$ & $\begin{array}{r}2.81 \\
12.93\end{array}$ & $\begin{array}{l}0.653 \\
0.169\end{array}$ & $\begin{array}{l}0.2878 \\
0.5777\end{array}$ & $\begin{array}{l}0.6229 \\
0.3165\end{array}$ \\
\hline
\end{tabular}


$\underline{\text { TABLE XXXIV (Contd, ) }}$

TWO-PHASE COLD , LEG MIXTURE TEST GROUP 5.6

EXPERIMENTAL MEASURMENTS FOR TESTS 5.6.1 TO 5.6.17 -- 0.53-IN. DOHNCUMER GAP

\begin{tabular}{|c|c|c|c|c|c|c|c|c|c|c|}
\hline $\begin{array}{l}\text { TEST } \\
\text { NO }\end{array}$ & $\begin{array}{l}R ! H-C H-1 \\
\text { (GPMI) }\end{array}$ & $\begin{array}{c}\mathrm{RM}-\mathrm{CH}-2 \\
(G P M)\end{array}$ & $\begin{array}{c}F T-C H-1 \\
(G P M)\end{array}$ & $\begin{array}{c}F T-C H-2 \\
(G P M)\end{array}$ & $\begin{array}{c}\text { RM-HG-1 } \\
(C F M)\end{array}$ & $\begin{array}{c}\text { RM-HG-2 } \\
(C F M)\end{array}$ & $\begin{array}{c}R M-H G-3 \\
(C F M)\end{array}$ & $\begin{array}{c}\text { P.M-HG-4 } \\
(C \subseteq M)\end{array}$ & $\begin{array}{l}F T-H G-1 \\
\text { (CFM) }\end{array}$ & $\begin{array}{l}F T-H G-2 \\
\text { (CFM) }\end{array}$ \\
\hline $\begin{array}{l}5.6 .1 \\
5.6 .2 \\
5.6 .3 \\
5.6 .4 \\
5.6 .5\end{array}$ & $\begin{array}{l}0.0 \\
0.0 \\
0.0 \\
0.0 \\
0.0\end{array}$ & $\begin{array}{l}0.0 \\
0.0 \\
0.0 \\
0.0 \\
0.0\end{array}$ & $\begin{array}{l}0.0 \\
0.0 \\
0.0 \\
0.0 \\
0.0\end{array}$ & $\begin{array}{r}50.1 \\
40.1 \\
25.2 \\
9.9 \\
40.3\end{array}$ & $\begin{array}{c}0.0 \\
0.0 \\
60.00 \\
0.0 \\
36.50\end{array}$ & $\begin{array}{l}0.0 \\
0.0 \\
0.0 .0 \\
0.0 \\
0.0\end{array}$ & $\begin{array}{c}10.00 \\
20.00 \\
0.0 \\
0.0 \\
0.0\end{array}$ & $\begin{array}{l}0.0 \\
0.0 \\
0.0 \\
0.0 \\
0.0\end{array}$ & $\begin{array}{c}0.0 \\
0.0 \\
0.0 \\
16.28 \\
0.0\end{array}$ & $\begin{array}{l}0.0 \\
0.0 \\
0.0 \\
0.0 \\
0.0\end{array}$ \\
\hline $\begin{array}{l}5.6 .6 \\
5.6 .7 \\
5.6 .8 \\
5.6 .9 \\
5.6 .10\end{array}$ & $\begin{array}{l}0.0 \\
0.0 \\
0.0 \\
0.0 \\
0.0\end{array}$ & $\begin{array}{l}0.0 \\
0.0 \\
0.0 \\
0.0 \\
0.0\end{array}$ & $\begin{array}{r}0.0 \\
0.0 \\
0.0 \\
70.0 \\
60.0\end{array}$ & $\begin{array}{r}30.2 \\
34.9 \\
30.4 \\
0.0 \\
0.0\end{array}$ & $\begin{array}{c}46.50 \\
46.50 \\
60.00 \\
0.0 \\
0.0\end{array}$ & $\begin{array}{l}0.0 \\
0.0 \\
0.0 \\
0.0 \\
0.0\end{array}$ & $\begin{array}{c}0.0 \\
0.0 \\
0.0 \\
13.40 \\
23.40\end{array}$ & $\begin{array}{l}0.0 \\
0.0 \\
0.0 \\
0.0 \\
0.0\end{array}$ & $\begin{array}{l}0.0 \\
0.0 \\
0.0 \\
0.0 \\
0.0\end{array}$ & $\begin{array}{l}0.0 \\
0.0 \\
0.0 \\
0.0 \\
0.0\end{array}$ \\
\hline $\begin{array}{l}5.6 .11 \\
5.6 .12 \\
5.6 .13 \\
5.6 .14 \\
5.6 .15\end{array}$ & $\begin{array}{l}3.0 \\
1.0 \\
j .0 \\
0.0 \\
0.0\end{array}$ & $\begin{array}{l}0.0 \\
0.0 \\
0.0 \\
0.0 \\
0.0\end{array}$ & $\begin{array}{r}0.0 \\
71.0 \\
0.0 \\
0.0 \\
0.0\end{array}$ & $\begin{array}{r}49.7 \\
0.0 \\
40.0 \\
25.4 \\
10.0\end{array}$ & $\begin{array}{c}0.0 \\
0.0 \\
0.0 \\
66.60 \\
0.0\end{array}$ & $\begin{array}{l}0.0 \\
0.0 \\
0.0 \\
0.0 \\
0.0\end{array}$ & $\begin{array}{c}13.30 \\
13.30 \\
23.40 \\
0.0 \\
0.0\end{array}$ & $\begin{array}{l}0.0 \\
0.0 \\
0.0 \\
0.0 \\
0.0\end{array}$ & $\begin{array}{c}0.0 \\
0.0 \\
0.0 \\
0.0 \\
17.69\end{array}$ & $\begin{array}{l}0.0 \\
0.0 \\
0.0 \\
0.0 \\
0.0\end{array}$ \\
\hline $\begin{array}{l}5.6 .10 \\
5.6 .17\end{array}$ & $\begin{array}{l}0.0 \\
0.0\end{array}$ & $\begin{array}{l}0.0 \\
0.0\end{array}$ & $\begin{array}{r}81.0 \\
0.0\end{array}$ & $\begin{array}{r}0.0 \\
30.7\end{array}$ & $\begin{array}{c}0.0 \\
46.50\end{array}$ & $\begin{array}{l}0.0 \\
0.0\end{array}$ & $\begin{array}{c}13.30 \\
0.0\end{array}$ & $\begin{array}{l}0.0 \\
0.0\end{array}$ & $\begin{array}{l}0.0 \\
0.0\end{array}$ & $\begin{array}{l}0.0 \\
0.0\end{array}$ \\
\hline $\begin{array}{c}\text { TEST } \\
\text { NO }\end{array}$ & $\begin{array}{l}P-(W-1 \\
(P S \mid C)\end{array}$ & $\begin{array}{l}P-C W-2 \\
(P S I G)\end{array}$ & $\begin{array}{l}P-H G-1 \\
(P S I G)\end{array}$ & $\begin{array}{l}\text { P-HG-2 } \\
\text { (PSIG) }\end{array}$ & $\begin{array}{l}\text { P-HG-3 } \\
\text { (PSIOS) }\end{array}$ & $\begin{array}{l}T F-C W-1 \\
(D E G F)\end{array}$ & $\begin{array}{l}T F-H G-1 \\
(D \equiv G F)\end{array}$ & $\begin{array}{l}\text { TF-HG-2 } \\
(D E G, F)\end{array}$ & $\begin{array}{l}\text { TF-HG-3 } \\
\text { (DEG F) }\end{array}$ & $\begin{array}{l}\text { P-BARO } \\
\text { (PSIIA) }\end{array}$ \\
\hline $\begin{array}{ll}5.6 . & 1 \\
5.6 . & 2 \\
5.6 . & 3 \\
5.6 . & 4 \\
5.6 .5 & 5\end{array}$ & $\begin{array}{l}13.0 \\
12.0 \\
11.0 \\
11.0 \\
11.2\end{array}$ & $\begin{array}{l}0.0 \\
0.0 \\
0.0 \\
0.0 \\
0.0\end{array}$ & $\begin{array}{l}10.0 \\
10.0 \\
10.0 \\
10.0 \\
10.5\end{array}$ & $\begin{array}{r}98.0 \\
97.0 \\
94.0 \\
97.0 \\
0.0\end{array}$ & $\begin{array}{r}33.0 \\
32.0 \\
31.0 \\
33.0 \\
33.0\end{array}$ & $\begin{array}{l}49.0 \\
51.0 \\
50.0 \\
50.0 \\
50.0\end{array}$ & $\begin{array}{r}90.0 \\
78.0 \\
76.0 \\
77.0 \\
78.0\end{array}$ & $\begin{array}{l}74.0 \\
75.0 \\
80.0 \\
81.0 \\
71.0\end{array}$ & $\begin{array}{l}76.0 \\
75.0 \\
77.0 \\
77.0 \\
77: 0\end{array}$ & $\begin{array}{l}12.2 \\
12.2 \\
12.2 \\
12.2 \\
12.4\end{array}$ \\
\hline $\begin{array}{l}5.6 .9 \\
5.6 .9 \\
5.6 .9 \\
5.6 .99 \\
5.6 .10\end{array}$ & $\begin{array}{l}10.7 \\
10.0 \\
13.7 \\
11.5 \\
12.0\end{array}$ & $\begin{array}{l}0.0 \\
0.0 \\
0.0 \\
0.0 \\
0.0\end{array}$ & $\begin{array}{r}10.5 \\
9.5 \\
10.5 \\
9.5 \\
10.0\end{array}$ & $\begin{array}{l}100.0 \\
100.0 \\
99.0 \\
101.0 \\
100.0\end{array}$ & $\begin{array}{l}33.0 \\
33.0 \\
33.0 \\
33.0 \\
33.0\end{array}$ & $\begin{array}{r}49.0 \\
49.0 \\
49.0 \\
49.0 \\
50.0\end{array}$ & $\begin{array}{r}75.0 \\
75.0 \\
75.0 \\
77.0 \\
77.0\end{array}$ & $\begin{array}{l}71.5 \\
73.0 \\
75.0 \\
75.0 \\
75.0\end{array}$ & $\begin{array}{l}77.0 \\
77.0 \\
77.0 \\
74.0 \\
74.0\end{array}$ & $\begin{array}{l}12.4 \\
12.4 \\
12.4 \\
12.4 \\
12.4\end{array}$ \\
\hline $\begin{array}{l}5.6 .11 \\
5.6 .12 \\
5.6 .13 \\
5.6 .14 \\
5.6 .15\end{array}$ & $\begin{array}{l}19.0 \\
23.0 \\
13.5 \\
15.0 \\
10.7\end{array}$ & $\begin{array}{l}0.0 \\
0.0 \\
0.0 \\
0.0 \\
0.0\end{array}$ & $\begin{array}{r}12.4 \\
15.0 \\
10.5 \\
11.2 \\
9.6\end{array}$ & $\begin{array}{r}98.0 \\
98.0 \\
9 \% .0 \\
95.0 \\
95.0\end{array}$ & $\begin{array}{l}33.0 \\
33.5 \\
33.5 \\
33.0 \\
33.5\end{array}$ & $\begin{array}{l}48.0 \\
50.0 \\
51.0 \\
50.0 \\
55.0\end{array}$ & $\begin{array}{l}81.0 \\
80.0 \\
79.0 \\
77.0 \\
84.0\end{array}$ & $\begin{array}{r}75.0 \\
0.0 \\
0.0 \\
77.0 \\
75.0\end{array}$ & $\begin{array}{l}76.0 \\
76.0 \\
76.0 \\
78.0 \\
88.0\end{array}$ & $\begin{array}{l}12.4 \\
12.4 \\
12.4 \\
12.4 \\
12.4\end{array}$ \\
\hline
\end{tabular}


TABLE XXXIV (Contd.)

TWO-PHASE COLD LEG MIXTURE TEST GROUP 5.6

EXPERI MENTAL MEASURMENTS FOR TESTS 5.6.1 TO 5.6.17 -- U.53-1N. OOHNCOMER GAP

\begin{tabular}{|c|c|c|c|c|c|c|c|c|c|c|}
\hline $\begin{array}{l}\text { TEST } \\
\text { N!n }\end{array}$ & $\begin{array}{l}P \rightarrow C W-1 \\
\text { (PSISI) }\end{array}$ & $\begin{array}{l}\mathrm{P}-\mathrm{CH}-2 \\
(P S I G)\end{array}$ & $\begin{array}{l}\text { P-HGO-1 } \\
\text { IPSISI }\end{array}$ & $\begin{array}{l}P-H G-2 \\
(P S I G)\end{array}$ & $\begin{array}{l}P-H G-3 \\
(P S I F)\end{array}$ & $\begin{array}{l}T F-C W-1 \\
(D E G F)\end{array}$ & $\begin{array}{l}T F-H G-1 \\
(D E G F)\end{array}$ & $\begin{array}{l}T F \rightarrow H G-2 \\
\text { (nES F) }\end{array}$ & $\begin{array}{l}\text { TF-HG-3 } \\
(D E G F)\end{array}$ & $\begin{array}{l}\text { P-BARO } \\
\text { (PSIAI }\end{array}$ \\
\hline $\begin{array}{l}5.6 .16 \\
5.6 .17\end{array}$ & $\begin{array}{l}21.5 \\
13.5\end{array}$ & $\begin{array}{l}0.0 \\
0.0\end{array}$ & $\begin{array}{l}16.0 \\
10.0\end{array}$ & $\begin{array}{l}96.0 \\
97.0\end{array}$ & $\begin{array}{l}33.0 \\
33.5\end{array}$ & $\begin{array}{l}51.0 \\
52.0\end{array}$ & $\begin{array}{l}84.0 \\
83.0\end{array}$ & $\begin{array}{l}90.0 \\
92.5\end{array}$ & $\begin{array}{l}86.0 \\
83.0\end{array}$ & $\begin{array}{l}12.4 \\
12.4\end{array}$ \\
\hline
\end{tabular}

\begin{tabular}{|c|c|c|}
\hline & PLENUA & FLOW \\
\hline $\begin{array}{l}\text { INITIALL } \\
\text { WFISHT } \\
\text { (L) }\end{array}$ & $\begin{array}{l}\text { FINAL } \\
\text { WEIGHT } \\
\text { (LIS) }\end{array}$ & $\begin{array}{l}\text { TIME TE } \\
\text { COLLECTICN } \\
\text { (SEC) }\end{array}$ \\
\hline $\begin{array}{l}14 \% .0 \\
331.0 \\
153.0 \\
140.0 \\
14 \% .0\end{array}$ & $\begin{array}{l}331.0 \\
465.0 \\
316.0 \\
166.5 \\
22.8 .0\end{array}$ & $\begin{array}{r}60.0 \\
60.0 \\
125.0 \\
120.0 \\
60.0\end{array}$ \\
\hline $\begin{array}{l}143.0 \\
191.5 \\
211.5 \\
143.5 \\
222.5\end{array}$ & $\begin{array}{l}199.5 \\
246.5 \\
234.0 \\
232.5 \\
356.0\end{array}$ & $\begin{array}{l}60.0 \\
60.0 \\
60.0 \\
30.0 \\
60.0\end{array}$ \\
\hline $\begin{array}{l}14.3 .5 \\
231.5 \\
277.0 \\
141.0 \\
160.0\end{array}$ & $\begin{array}{l}23 ? .5 \\
308.5 \\
346.0 \\
160.0 \\
176.5\end{array}$ & $\begin{array}{r}60.0 \\
30.0 \\
60.0 \\
60.0 \\
120.0\end{array}$ \\
\hline $\begin{array}{l}17.3 .0 \\
300.0\end{array}$ & $\begin{array}{l}300.0 \\
331.5\end{array}$ & $\begin{array}{l}45.0 \\
45.0\end{array}$ \\
\hline
\end{tabular}

\begin{tabular}{|c|c|c|}
\hline \multicolumn{3}{|c|}{ BYPASS FLUW } \\
\hline $\begin{array}{l}\text { INI I I IAL } \\
\text { WE I GIIT } \\
\text { (LB) }\end{array}$ & 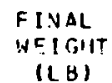 & $\begin{array}{l}\text { TIME UF. } \\
\text { COLLECTIINN } \\
\text { (SEC) }\end{array}$ \\
\hline
\end{tabular}

LOWER

$\begin{array}{llr}\text { PLENIUM } & P-L P-1 & \text { 'TF-LP-1 } \\ \text { LEVEL } & \text { (PSIG) } & \text { TDEG FI }\end{array}$

TEST

5.6.
5.6.

5.6 .

5.6 .

165.0
404.5

$404.5 \quad 604.5 \quad 60.5$

549.0

731.3

$313.5-175.0$

60.0

60.0
60.0

12.0

12.0
12.0

12.0
12.0
12.0

10.5

$324.5 \quad 525.0 \quad 60.0$

5.6.

5.6.

5.6 .9
5.6 .10

$3.01 \% .5$

591.5
508.5

500.5
307.0

30.0
30.0
30.0

421.5

30.0
30.0

12.0

10.0

$10.0 \quad 54.0$

$\begin{array}{ll}10.0 & 52.0 \\ 10.2 & 51.0\end{array}$

5.6.

104.5

$264.5 \quad 30.0$

264.5
447.0

102.5

488.0

583.3

239.0
376.0

30.0

5.6 .14
5.6 .15

5.6 .16

300.0

331.5

359.0

616.5

60.0

12.0

12.0
12.0

10.5

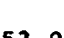

$3.7 \quad 50.0$

$\begin{array}{ll}10.2 & 52.0 \\ 10.0 & 49.0\end{array}$

$10.5 \quad 59.0$

12.0

49.0

12.0

12.0

16.2

$11.2 \quad 52.0$

$11.1 \quad 61.0$

$\begin{array}{lllll} & 30.0 & 12.0 & 16.7 & 52.0\end{array}$

\begin{tabular}{|c|c|c|c|c|c|c|c|}
\hline $\begin{array}{l}\text { TEST } \\
\text { NO }\end{array}$ & $\begin{array}{c}P . M-C G-1 \\
(C F: M)\end{array}$ & $\begin{array}{c}R M-C S-2 \\
(C F Y)\end{array}$ & $\begin{array}{c}F T=F G-1 \\
(C F M)\end{array}$ & $\begin{array}{l}P-C S-1 \\
(P S I G)\end{array}$ & $\begin{array}{l}P-C G-2 \\
(P S T G)\end{array}$ & $\begin{array}{l}T F-C F-1 \\
\text { (DEG F) }\end{array}$ & $\begin{array}{l}\text { TE-Cr-2 } \\
\text { IDEG FI }\end{array}$ \\
\hline $\begin{array}{l}5.6 .1 \\
5.6 .2 \\
5.6 .2 \\
5.6 .4 \\
5.6 .5\end{array}$ & $\begin{array}{l}0.0 \\
0.0 \\
0.0 \\
0.0 \\
0.0\end{array}$ & $\begin{array}{l}30.0 \\
30.0 \\
30.0 \\
30.0 \\
31.0\end{array}$ & $\begin{array}{l}c .0 \\
0.0 \\
0.0 \\
c .0 \\
0.0\end{array}$ & $\begin{array}{r}98.0 \\
97.0 \\
94.0 \\
97.0 \\
101.0\end{array}$ & $\begin{array}{l}35.0 \\
35.0 \\
35.0 \\
35.0 \\
34.5\end{array}$ & $\begin{array}{r}0.0 \\
0.0 \\
0.0 \\
0.0 \\
63.5\end{array}$ & $\begin{array}{l}76.0 \\
76.0 \\
76.0 \\
76.0 \\
67.5\end{array}$ \\
\hline $\begin{array}{ll}5.6 .0 \\
3.6 . \\
3.6 . & 1 \\
5.6 . & 4\end{array}$ & $\begin{array}{l}0.0 \\
0.0 \\
10.0 \\
0.0\end{array}$ & $\begin{array}{l}30.0 \\
30.0 \\
30.0 \\
30.0\end{array}$ & $\begin{array}{l}0.0 \\
0.0 \\
0.0 \\
0.0\end{array}$ & $\begin{array}{l}0.0 \\
0.0 \\
3.0 \\
0.0\end{array}$ & $\begin{array}{l}35.0 \\
35.0 \\
34.7 \\
35.0\end{array}$ & $\begin{array}{r}0.0 \\
0.0 \\
0.0 \\
73.0\end{array}$ & $\begin{array}{l}70.0 \\
71.0 \\
72.0 \\
72.0\end{array}$ \\
\hline
\end{tabular}




\section{TABLE XXXIV (Contd.)}

TWO-PHASE COLD - LEG MIXTURE TEST GROUP 5.6

EXPERIMENTAL MEASURMENTS FOR TESTS 5.6.1 TO 5.6.17 -- 0.53-IN. DOHNCOMER GAP

\begin{tabular}{|c|c|c|c|c|c|c|c|}
\hline $\begin{array}{l}\text { TEST } \\
\text { NI }\end{array}$ & $\begin{array}{c}R M-C G-1 \\
(C F M)\end{array}$ & $\begin{array}{c}\text { RM-CG-2 } \\
(C F M)\end{array}$ & $\begin{array}{c}F T-C G-1 \\
(C F M)\end{array}$ & $\begin{array}{l}P-C G-1 \\
(P S I G)\end{array}$ & $\begin{array}{l}P-C G-2 \\
(P S I G)\end{array}$ & $\begin{array}{l}\text { TF-CG-1 } \\
\text { (DEG F) }\end{array}$ & $\begin{array}{l}T F-C \dot{G}-2 \\
\text { (DEG } F \text { ) }\end{array}$ \\
\hline $\begin{array}{l}5.6 .10 \\
5.6 .11 \\
5.6 .12 \\
5.6 .13 \\
5.6 .14\end{array}$ & $\begin{array}{l}0.0 . \\
0.0 \\
0.0 \\
0.0 \\
0.0\end{array}$ & $\begin{array}{r}30.0 \\
0.0 \\
0.0 \\
0.0 \\
0.0\end{array}$ & $\begin{array}{r}0.0 \\
29.6 \\
29.6 \\
29.7 \\
29.9\end{array}$ & $\begin{array}{r}0.0 \\
98.0 \\
98.0 \\
97.0 \\
95.0\end{array}$ & $\begin{array}{r}35.0 \\
0.0 \\
0.0 \\
0.0 \\
0.0\end{array}$ & $\begin{array}{r}0.0 \\
76.0 \\
83.0 \\
90.0 \\
82.0\end{array}$ & $\begin{array}{r}73.0 \\
0.0 \\
0.0 \\
0.0 \\
0.0\end{array}$ \\
\hline $\begin{array}{l}5.6 .15 \\
5.6 .16 \\
5.6 .17\end{array}$ & $\begin{array}{l}0.0 \\
0.0 \\
0.0\end{array}$ & $\begin{array}{l}0.0 \\
0.0 \\
.0 .0\end{array}$ & $\begin{array}{l}29.5 \\
28.6 \\
29.6\end{array}$ & $\begin{array}{l}95.0 \\
96.0 \\
97.0\end{array}$ & $\begin{array}{l}0.0 \\
0.0 \\
0.0\end{array}$ & $\begin{array}{r}102.0 \\
96.0 \\
101.5\end{array}$ & $\begin{array}{l}0.0 \\
0.0 \\
0.0\end{array}$ \\
\hline
\end{tabular}




\section{TABLE XXXV}

\section{TWO-PHASE COLD LEG MIXURE TEST GROUP 7.2}

TRA.ISPAFENT VESSEL TEST CATA FOR TESTS 7.2.1 TO 7.2.10-- 0.70-IN. DOWNCOMER GAP S'JI:AARY OF CALC'JLATEO RESULTS

\begin{tabular}{|c|c|c|c|c|c|c|c|c|c|c|}
\hline $\begin{array}{c}\text { TeST } \\
\text { Hii }\end{array}$ & $\begin{array}{l}\text { D. JWNCJMER } \\
\text { AIR RLAW } \\
\text { ILU/SECI }\end{array}$ & $\begin{array}{l}\text { UCWACIUER } \\
\text { AIR DENSITY } \\
\text { (LB/FT } * * 3)\end{array}$ & $\begin{array}{l}\text { LOWCR PLENUM } \\
\text { PRESSUF.E } \\
\text { (PSIA) }\end{array}$ & $\begin{array}{l}\text { COLD LEF, } \\
\text { AIR FLIJN } \\
\text { LIISTEC.). }\end{array}$ & $\begin{array}{l}\text { ROWNCOMEP } \\
\text { WATER FLOW } \\
\text { (LS/STC) }\end{array}$ & $\begin{array}{l}\text { WATEE FLOH } \\
\text { CONTINUITY }\end{array}$ & $\begin{array}{c}\text { J-AIR } \\
\text { DOWNCAYEP } \\
\text { (FT/SEC) }\end{array}$ & $\begin{array}{l}\text { J-NATEP } \\
\text { DONCDYCR } \\
\text { (FT/SEC) }\end{array}$ & $(J G * \mid * * 1 / 2$ & $(J L * 1 * 1 / 2$ \\
\hline 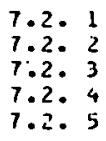 & $\begin{array}{l}0.0221 \\
0.0219 \\
0.0211 \\
0.0436 \\
0.0421\end{array}$ & $\begin{array}{l}.1192 \\
0.1200 \\
0.1212 \\
0.1220 \\
0.1209\end{array}$ & $\begin{array}{l}22.34 \\
22.55 \\
22.59 \\
22.59 \\
22.59\end{array}$ & $\begin{array}{l}0.0668 \\
0.0668 \\
0.0667 \\
0.0663 \\
0.0664\end{array}$ & $\begin{array}{r}10.467 \\
6.020 \\
9.533 \\
7.067 \\
6.60 \mathrm{~J}\end{array}$ & $\begin{array}{l}1.097 \\
0.436 \\
0.977 \\
0.960 \\
1.013\end{array}$ & $\begin{array}{l}1.57 \\
1.55 \\
1.54 \\
3.04 \\
2.96\end{array}$ & $\begin{array}{l}1.427 \\
0.321 \\
1.164 \\
0.964 \\
0.912\end{array}$ & $\begin{array}{l}0.1884 \\
0.1876 \\
0.1871 \\
0.2535 \\
0.2596\end{array}$ & $\begin{array}{l}0.8587 \\
0.6513 \\
0.7754 \\
0.7056 \\
0.6865\end{array}$ \\
\hline $\begin{array}{l}7.2 .9 \\
7.2 .97 \\
7.2 .9 \\
7.2 .99 \\
7.2 .10\end{array}$ & $\begin{array}{l}0.0861 \\
0.1291 \\
0.1560 \\
0.2125 \\
0.0438\end{array}$ & $\begin{array}{l}0.1208 \\
0.1220 \\
0.1130 \\
0.1120 \\
0.1184\end{array}$ & $\begin{array}{l}22.59 \\
22.84 \\
22.34 \\
22.09 \\
22.59\end{array}$ & $\begin{array}{l}0.0659 \\
0.0658 \\
0.0660 \\
0.0669 \\
0.0668\end{array}$ & $\begin{array}{l}5.172 \\
4.133 \\
2.350 \\
1.033 \\
7.237\end{array}$ & $\begin{array}{l}0.933 \\
1.014 \\
1.007 \\
0.974 \\
0.974\end{array}$ & $\begin{array}{r}6.07 \\
9.01 \\
11.21 \\
15.59 \\
3.15\end{array}$ & $\begin{array}{l}0.705 \\
0.564 \\
0.320 \\
0.141 \\
0.997 .\end{array}$ & $\begin{array}{l}0.3714 \\
0.4536 \\
0.5928 \\
0.5393 \\
0.2662\end{array}$ & $\begin{array}{l}0.6037 \\
0.5396 \\
0.4069 \\
0.2698 \\
0.7141\end{array}$ \\
\hline
\end{tabular}


TABLE XXXV (Contd.)

TWO-PHASE COLD LEG MIXTURE TEST GROUP 7.2

EXPERIMENTAL MEASURMENTS FOR TESTS 7.2 .1 TO 7.2 .10 - $0.70-$ IN. DOWNCOMER GAP

\begin{tabular}{|c|c|c|c|c|c|c|c|c|c|c|}
\hline $\begin{array}{l}\text { TEST } \\
\text { NO }\end{array}$ & $\begin{array}{c}R M-C H-l \\
(G P M)\end{array}$ & $\begin{array}{c}R 4-C H-2 \\
(S P M)\end{array}$ & $\begin{array}{c}F T-C H-1 \\
(G P M)\end{array}$ & $\begin{array}{c}F T-C H-2 \\
(G P M)\end{array}$ & $\begin{array}{c}\text { RM-HG-1 } \\
(C F M)\end{array}$ & $\begin{array}{c}R M-H G-2 \\
(C F M)\end{array}$ & $\begin{array}{c}R M-H G-3 \\
(C F M)\end{array}$ & $\begin{array}{l}\text { RM-HG-4 } \\
(C F M)\end{array}$ & $\begin{array}{l}F^{T}-H G-1 \\
(C F M)\end{array}$ & $\begin{array}{c}F T-H G-2 \\
(C F M)\end{array}$ \\
\hline $\begin{array}{ll}7.2 \cdot & 1 \\
7.2 .2 & 2 \\
7.2 .2 & 3 \\
7.2 .2 & 4 \\
7.2 . & 5\end{array}$ & $\begin{array}{l}0.0 \\
0.0 \\
0.0 \\
0.0 \\
0.0\end{array}$ & $\begin{array}{l}0.0 \\
0.0 \\
0.0 \\
0.0 \\
0.0\end{array}$ & $\begin{array}{r}101.0 \\
100.0 \\
89.0 \\
30.0 \\
39.0\end{array}$ & $\begin{array}{l}0.0 \\
0.0 \\
0.0 \\
0.0 \\
0.0\end{array}$ & $\begin{array}{l}0.0 \\
0.0 \\
0.0 \\
0.0 \\
0.0\end{array}$ & $\begin{array}{l}0.0 \\
0.0 \\
0.0 \\
0.0 \\
0.0\end{array}$ & $\begin{array}{l}10.00 \\
10.00 \\
10.00 \\
20.00 \\
20.00\end{array}$ & $\begin{array}{l}0.0 \\
0.0 \\
0.0 \\
0.0 \\
0.0\end{array}$ & $\begin{array}{l}0.0 \\
0.0 \\
0.0 \\
0.0 \\
0.0\end{array}$ & $\begin{array}{l}0.0 \\
0.0 \\
0.0 \\
0.0 \\
0.0\end{array}$ \\
\hline $\begin{array}{l}7.2 .6 \\
7.2 .9 \\
7.2 .8 \\
7.2 .9 \\
7.2 .10\end{array}$ & $\begin{array}{l}0.0 \\
0.0 \\
0.0 \\
0.0 \\
0.0\end{array}$ & $\begin{array}{l}0.0 \\
0.0 \\
0.0 \\
0.0 \\
0.0\end{array}$ & $\begin{array}{r}60.0 \\
60.0 \\
0.0 \\
0.0 \\
80.0\end{array}$ & $\begin{array}{r}0.0 \\
0.0 \\
30.2 \\
20.2 \\
0.0\end{array}$ & $\begin{array}{c}40.00 \\
60.00 \\
0.0 \\
0.0 \\
0.0\end{array}$ & $\begin{array}{l}0.0 \\
0.0 \\
0.0 \\
0.0 \\
0.0\end{array}$ & $\begin{array}{c}0.0 \\
0.0 \\
0.0 \\
0.0 \\
20.00\end{array}$ & $\begin{array}{l}0.0 \\
0.0 \\
0.0 \\
0.0 \\
0.0\end{array}$ & $\begin{array}{c}0.0 \\
0.0 \\
17.69 \\
23.33 \\
0.0\end{array}$ & $\begin{array}{l}0.0 \\
0.0 \\
0.0 \\
0.0 \\
0.0\end{array}$ \\
\hline & & & & & & & & . & & \\
\hline $\begin{array}{l}\text { TEST } \\
\text { NO }\end{array}$ & $\begin{array}{l}P-C W-1 \\
(P S I G)\end{array}$ & $\begin{array}{l}P-C W-2 \\
\text { PSIIG) }\end{array}$ & $\begin{array}{l}P-H r_{S}-1 \\
(P S I G)\end{array}$ & $\begin{array}{l}\text { P-HF-2 } \\
\text { PSIGI }\end{array}$ & $\begin{array}{l}\text { P-HG-3 } \\
\text { (PSIG) }\end{array}$ & $\begin{array}{l}T F-C H-1 \\
(D E G F)\end{array}$ & $\begin{array}{l}\text { TF-HG - } \\
\text { (DEG F) }\end{array}$ & $\begin{array}{l}T F-H C,-2 \\
(D E G, F)\end{array}$ & $\begin{array}{l}\text { TF-HG-3 } \\
\text { (DEG F) }\end{array}$ & $\begin{array}{l}P-B A P Q \\
(051 A)\end{array}$ \\
\hline $\begin{array}{ll}7.2 . & 1 \\
7.2 . & 2 \\
7.2 . & 3 \\
7.2 . & 4 \\
7.2 . & 5\end{array}$ & $\begin{array}{l}15.5 \\
17.3 \\
16.0 \\
15.0 \\
14.0\end{array}$ & $\begin{array}{l}0.0 \\
0.0 \\
0.0 \\
0.0 \\
0.0\end{array}$ & $\begin{array}{l}11.0 \\
12.0 \\
11.6 \\
12.0 \\
12: 0\end{array}$ & $\begin{array}{l}98.0 \\
58.5 \\
96.0 \\
95.5 \\
95.0\end{array}$ & $\begin{array}{l}34.0 \\
33.5 \\
33.5 \\
33.0 \\
30.0\end{array}$ & $\begin{array}{l}46.0 \\
47.5 \\
43.0 \\
43.5 \\
44.0\end{array}$ & $\begin{array}{l}79.0 \\
79.5 \\
79.5 \\
78.0 \\
78.0\end{array}$ & $\begin{array}{l}76.0 \\
76.5 \\
77.5 \\
78.5 \\
79.5\end{array}$ & $\begin{array}{l}76.5 \\
76.5 \\
76.5 \\
76.5 \\
76.0\end{array}$ & $\begin{array}{l}12.3 \\
12.3 \\
12.3 \\
12.3 \\
12.3\end{array}$ \\
\hline $\begin{array}{l}7.2 .6 \\
7.2 .7 \\
7.2 .8 \\
7.2 .89 \\
7.2 .10\end{array}$ & $\begin{array}{l}14.0 \\
13.5 \\
11.0 \\
11.0 \\
15.0\end{array}$ & $\begin{array}{l}0.0 \\
0.0 \\
0.0 \\
0.0 \\
0.0\end{array}$ & $\begin{array}{l}11.5 \\
11.5 \\
10.0 \\
11.0 \\
11.0\end{array}$ & $\begin{array}{l}94.5 \\
93.0 \\
95.0 \\
97.0 \\
98.0\end{array}$ & $\begin{array}{l}32.0 \\
32.0 \\
33.5 \\
33.5 \\
33.5\end{array}$ & $\begin{array}{l}45.0 \\
45.0 \\
46.0 \\
51.5 \\
51.0\end{array}$ & $\begin{array}{l}78.0 \\
79.0 \\
78.5 \\
75.0 \\
77.0\end{array}$ & $\begin{array}{l}82.0 \\
88.5 \\
85.5 \\
80.0 \\
79.0\end{array}$ & $\begin{array}{l}77.0 \\
77.5 \\
81.0 \\
76.5 \\
76.0\end{array}$ & $\begin{array}{l}12.3 \\
12.3 \\
12.3 \\
12.3 \\
12.3\end{array}$ \\
\hline
\end{tabular}

\begin{tabular}{|c|c|c|c|c|c|c|c|c|c|}
\hline \multirow[b]{2}{*}{$\begin{array}{c}\text { TEST } \\
\text { NO }\end{array}$} & \multicolumn{3}{|c|}{ LCWEP PLFNUM FL OW } & \multicolumn{3}{|c|}{ RYPASS FLOW } & \multirow{2}{*}{$\begin{array}{l}\text { LOWER } \\
\text { PLENIIY } \\
\text { LEVEL } \\
\text { IINI }\end{array}$} & \multirow[b]{2}{*}{$\begin{array}{l}p-(p-1) \\
(D \text { S } 16)\end{array}$} & \multirow[b]{2}{*}{$\begin{array}{l}\text { TE-LOD } \\
\text { IOES }\end{array}$} \\
\hline & $\begin{array}{l}\text { INITIAL } \\
\text { WEI:BHT } \\
\text { (LB) }\end{array}$ & $\begin{array}{l}\text { FINAL } \\
\text { WEIGHT } \\
\text { (LB) }\end{array}$ & $\begin{array}{l}\text { TIME TF } \\
\text { COLLECTICN } \\
\text { (SEC) }\end{array}$ & $\begin{array}{l}\text { INITIAL } \\
\text { WF IGHT } \\
\text { ILUI }\end{array}$ & $\begin{array}{l}\text { FINAL } \\
\text { WEIGHT } \\
\text { (L B) }\end{array}$ & $\begin{array}{l}\text { TIME OF } \\
\text { COLLECTIDN } \\
\text { ISECI }\end{array}$ & & & \\
\hline $\begin{array}{ll}7.2 . & 1 \\
7.2 . & 2 \\
7.2 .2 & 3 \\
7.2 . & 4 \\
7.2 . & 5\end{array}$ & $\begin{array}{l}137.0 \\
100.0 .0 \\
276.5 \\
333.10 \\
253.5\end{array}$ & $\begin{array}{l}290.0 \\
2.11 .5 \\
404.5 \\
444.0 \\
350.1\end{array}$ & $\begin{array}{l}15.0 \\
24.5 \\
15.0 \\
15.0 \\
14.5\end{array}$ & $\begin{array}{l}101.0 \\
203.5 \\
208.5 \\
200.0 \\
206.5\end{array}$ & $\begin{array}{l}211.0 \\
225.5 \\
315.0 \\
277.5 \\
266.5\end{array}$ & $\begin{array}{l}30.0 \\
30.0 \\
30.0 \\
17.0 \\
20.0\end{array}$ & $\begin{array}{r}12.0 \\
8.0 \\
8.0 \\
9.0 \\
8.0\end{array}$ & $\begin{array}{l}10.2 \\
10.2 \\
10.2 \\
10.2 \\
10.2\end{array}$ & $\begin{array}{r}46.0 \\
48.0 \\
43.5 \\
-40.0 \\
-44.5\end{array}$ \\
\hline $\begin{array}{l}7.2 .6 \\
7.2 .9 \\
7.2 .8 \\
7.2 .29 \\
7.2 .10\end{array}$ & $\begin{array}{l}190.0 \\
190.5 \\
140.0 \\
173.0 \\
204.0\end{array}$ & $\begin{array}{l}26 ! .5 \\
251.5 \\
197.0 \\
204.0 \\
417.5\end{array}$ & $\begin{array}{l}14.5 \\
15.0 \\
20.0 \\
30.0 \\
29.5\end{array}$ & $\begin{array}{l}266.5 \\
301.0 \\
104.5 \\
137.0 \\
188.0\end{array}$ & $\begin{array}{l}327.0 \\
387.5 \\
142.0 \\
188.0 \\
296.0\end{array}$ & $\begin{array}{l}20.0 \\
20.0 \\
20.0 \\
30.0 \\
30.0\end{array}$ & $\begin{array}{r}12.0 \\
12.0 \\
12.0 \\
12.0 \\
8.0\end{array}$ & $\begin{array}{r}10.2 \\
10.5 \\
10.0 \\
9.7 \\
10.2\end{array}$ & $\begin{array}{l}45.0 \\
45.5 \\
47.5 \\
54.0 \\
55.0\end{array}$ \\
\hline
\end{tabular}


TABLE XXXV (Contd.)

TWO-PHASE COLD LEG MIXTURE TEST GROUP 7.2

EXPERI 1T.YTAL HEASURMENTS FUP TESTS 7.2 .1 TI 7.2.10 -- 0.70-IN. DOWNCOMER G.AR

\begin{tabular}{|c|c|c|c|c|c|c|c|}
\hline $\begin{array}{l}\text { TSST } \\
\text { NO }\end{array}$ & $\begin{array}{c}F:(1-C)-1 \\
(S F U)\end{array}$ & $\begin{array}{c}R M-C S-2 \\
(C F M)\end{array}$ & $\begin{array}{c}\text { CT-CG } 1 \\
(C F M)\end{array}$ & $\begin{array}{l}\text { D-CG-1 } \\
\text { (PSIGI }\end{array}$ & $\begin{array}{l}\text { P-CG-2 } \\
(P S 1 C)\end{array}$ & $\begin{array}{l}T F-C G-1 \\
\text { ICEG FI }\end{array}$ & $\begin{array}{l}\text { IF-CG-2 } \\
\text { (EEG F) }\end{array}$ \\
\hline $\begin{array}{ll}7.2 . & 1 \\
7.2 .2 & 2 \\
7.2 .2 & 3 \\
7.2 .2 & 4 \\
7.2 . & 5\end{array}$ & $\begin{array}{l}0.0 \\
0.0 \\
0.0 \\
.00 \\
0.0\end{array}$ & $\begin{array}{l}30.0 \\
30.0 \\
30.0 \\
30.0 \\
30.0\end{array}$ & $\begin{array}{l}0.0 \\
0.0 \\
0.0 \\
c .0 \\
0.0\end{array}$ & $\begin{array}{l}0.0 \\
0.0 \\
0.0 \\
0.0 \\
0.0\end{array}$ & $\begin{array}{l}35.0 \\
35.0 \\
35.0 \\
34.5 \\
34.6\end{array}$ & $\begin{array}{l}0.0 \\
0.0 \\
0.0 \\
0.0 \\
0.0\end{array}$ & $\begin{array}{r}76.5 \\
-76.5 \\
77.5 \\
78.0 \\
78.5\end{array}$ \\
\hline $\begin{array}{l}7.2 .6 \\
7.2 .8 \\
7.2 .8 \\
7.2 .9 \\
7.2 .19\end{array}$ & $\begin{array}{l}0.0^{\circ} \\
. .0 \\
0.0 \\
0.0 \\
0.0\end{array}$ & $\begin{array}{l}30.0 \\
30.0 \\
30.0 \\
30.0 \\
30.0\end{array}$ & $\begin{array}{l}0.0 \\
0.0 \\
0.0 \\
0.0 \\
0.0\end{array}$ & $\begin{array}{l}0.0 \\
0.0 \\
0.0 \\
0.0 \\
0.0\end{array}$ & $\begin{array}{l}34.0 \\
34.0 \\
34.0 \\
35.0 \\
35.0\end{array}$ & $\begin{array}{l}0.0 \\
0.0 \\
0.0 \\
0.0 \\
0.0\end{array}$ & $\begin{array}{l}78.5 \\
80.0 \\
78.0 \\
74.5 \\
75.5\end{array}$ \\
\hline
\end{tabular}




\section{TABLE XXXVI}

TWO-PHASE COLD LEG MIXTURE TEST GROUP 9.4

TFAYYSPARENIT VISSFL TEST DATA FOR TESTS 9.4.1 TO $5.4 .30-1.58-1 N 0^{\circ}$ DIJANCOMER GAR.

SUMMAGY gF CALCULATFD PESULTS

\begin{tabular}{|c|c|c|c|c|c|c|c|c|c|c|}
\hline $\begin{array}{l}\text { TEST } \\
\text { NNO }\end{array}$ & $\begin{array}{l}\text { DJWNCOYIEP } \\
\text { AIR TLIJW } \\
\text { (LB/SECI }\end{array}$ & $\begin{array}{l}\text { DEINCOMEA } \\
\text { AIF DEUSITY } \\
\text { (LB/FT *3) }\end{array}$ & $\begin{array}{l}\text { LONER PLENUM } \\
\text { PRESSURE } \\
\text { (PSIA) }\end{array}$ & $\begin{array}{l}\text { COLD LES } \\
\text { AIR FLOWW } \\
\text { (LB/SEC) }\end{array}$ & $\begin{array}{l}\text { DOWNCOMEP } \\
\text { WATEP FLCW } \\
\text { (LB/SEC) }\end{array}$ & $\begin{array}{l}\text { WATFE FLTIA } \\
\text { CONTINUITY }\end{array}$ & $\begin{array}{c}\text { J-A!R } \\
\text { MTWNCOMER } \\
\text { (FT/SEC) }\end{array}$ & $\begin{array}{c}J-N A T E R \\
D \text { JWNCRUEP. } \\
\text { IFT/SECI }\end{array}$ & $(J G *) * * 1 / 2$ & $(J L *) * 1 / 2$ \\
\hline 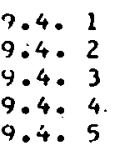 & $\begin{array}{l}0.1741 \\
0.3140 \\
0.2227 \\
0.3599 \\
0.4941\end{array}$ & $\begin{array}{l}0.1212 \\
0.1563 \\
0.1291 \\
0.1224 \\
0.1532\end{array}$ & $\begin{array}{l}22.70 \\
31.45 \\
24.45 \\
23.20 \\
20.75\end{array}$ & $\begin{array}{l}0.0664 \\
0.0664 \\
0.0665 \\
0.0665 \\
0.0662\end{array}$ & $\begin{array}{r}10.650 \\
6.833 \\
7.000 \\
4.267 \\
2.167\end{array}$ & $\begin{array}{l}1.003 \\
1.001 \\
1.077 \\
1.079 \\
1.040\end{array}$ & $\begin{array}{r}6.11 \\
8.03 \\
7.33 \\
12.46 \\
13.71\end{array}$ & $\begin{array}{l}0.725 \\
0.465 \\
0.477 \\
0.291 \\
0.1148\end{array}$ & $\begin{array}{l}0.3041 \\
0.3774 \\
0.3335 \\
0.4358 \\
0.4832\end{array}$ & $\begin{array}{l}0.4993 \\
0.4000 \\
0.4048 \\
0.3160 \\
0.2252\end{array}$ \\
\hline $\begin{array}{l}9.4 .6 \\
9.4 .77 \\
9.4 .3 \\
9.4 .17 \\
9.4 .10\end{array}$ & $\begin{array}{l}0.1116 \\
0.3156 \\
0.1741 \\
0.1878 \\
0.1325\end{array}$ & $\begin{array}{l}0.1116 \\
0.1235 \\
0.1132 \\
0.1155 \\
0.1180\end{array}$ & $\begin{array}{l}22.20 \\
23.20 \\
22.20 \\
21.70 \\
22.20\end{array}$ & $\begin{array}{l}0.0666 \\
0.0661 \\
0.0655 \\
0.0661 \\
0.0660\end{array}$ & $\begin{array}{l}7.450 \\
5.883 \\
4.045 \\
6.517 \\
3.150\end{array}$ & $\begin{array}{l}0.995 \\
0.985 \\
1.012 \\
0.976 \\
0.940\end{array}$ & $\begin{array}{r}6.15 \\
10.52 \\
6.26 \\
6.91 \\
6.54\end{array}$ & $\begin{array}{l}0.507 \\
0.401 \\
0.276 \\
0.414 \\
0.555\end{array}$ & $\begin{array}{l}0.3035 \\
0.4010 \\
0.3060 \\
0.3196 \\
0.3130\end{array}$ & $\begin{array}{l}0.4176 \\
0.3711 \\
0.3077 \\
0.3905 \\
0.4367\end{array}$ \\
\hline $\begin{array}{l}9.4 .11 \\
9.4 .12 \\
9.4 .13 \\
9.4 .114 \\
9.4 .15\end{array}$ & $\begin{array}{l}0.1301 \\
0.0364 \\
0.1269 \\
0.2471 \\
0.3084\end{array}$ & $\begin{array}{l}1.1319 \\
0.1183 \\
0.1152 \\
0.1311 \\
0.1242 .\end{array}$ & $\begin{array}{l}25.70 \\
22.40 \\
21.65 \\
24.65 \\
23.40\end{array}$ & $\begin{array}{l}0.0659 \\
0.0660 \\
0.0660 \\
0.0660 \\
0.0659\end{array}$ & $\begin{array}{r}11.000 \\
12.917 \\
12.733 \\
8.483 \\
5.917\end{array}$ & $\begin{array}{l}0.981 \\
0.987 \\
0.996 \\
0.985 \\
0.992\end{array}$ & $\begin{array}{r}5.67 \\
3.10 \\
4.68 \\
8.01 \\
10.55\end{array}$ & $\begin{array}{l}0.749 \\
0.993 \\
0.367 \\
0.579 \\
0.403\end{array}$ & $\begin{array}{l}0.3010 \\
0.2155 \\
0.2628 \\
0.3552 \\
0.4022\end{array}$ & $\begin{array}{l}0.5074 \\
0.5498 \\
0.5459 \\
0.4456 \\
0.3721\end{array}$ \\
\hline $\begin{array}{l}9.4 .16 \\
9.4 .17 \\
9.4 .13 \\
9.4 .19 \\
9.4 .20\end{array}$ & $\begin{array}{l}0.4163 \\
0.3642 \\
0.6523 \\
0.1489 \\
1.4783\end{array}$ & $\begin{array}{l}0.1493 \\
0.1473 \\
0.1474 \\
0.1315 \\
0.1493\end{array}$ & $\begin{array}{l}28.15 \\
28.15 \\
28.40 \\
24.70 \\
23.20\end{array}$ & $\begin{array}{l}0.0657 \\
0.0659 \\
0.0655 \\
0.1266 \\
0.1197\end{array}$ & $\begin{array}{c}3.917 \\
1.567 \\
0.617 \\
10.283 \\
2.883\end{array}$ & $\begin{array}{l}0.995 \\
1.057 \\
1.042 \\
0.575 \\
1.068\end{array}$ & $\begin{array}{r}11.85 \\
16.22 \\
28.81 \\
4.81 \\
13.56\end{array}$ & $\begin{array}{l}0.267 \\
0.107 \\
0.042 \\
0.700 \\
0.196\end{array}$ & $\begin{array}{l}0.41462 \\
0.5207 \\
0.5405 \\
0.2755 \\
0.4779\end{array}$ & $\begin{array}{l}0.3028 \\
0.1915 \\
0.1202 \\
0.4906 \\
0.2598\end{array}$ \\
\hline $\begin{array}{l}9.4 .21 \\
9.4 .22 \\
9.4 .23 \\
9.4 .24 \\
9.4 .25\end{array}$ & $\begin{array}{l}0.2443 \\
0.1237 \\
0.4139 \\
0.0858 \\
0.5094\end{array}$ & $\begin{array}{l}0.1564 \\
0.1372 \\
0.1622 \\
0.1331 \\
0.1504\end{array}$ & $\begin{array}{l}29.65 \\
25.90 \\
30.65 \\
25.15 \\
28.65\end{array}$ & $\begin{array}{l}0.2443 \\
0.2381 \\
0.2165 \\
0.0272 \\
0.2153\end{array}$ & $\begin{array}{r}7.467 \\
9.433 \\
4.767 \\
10.117 \\
2.250\end{array}$ & $\begin{array}{l}0.991 \\
1.002 \\
0.998 \\
0.980 \\
1.091\end{array}$ & $\begin{array}{r}6.64 \\
3.83 \\
10.98 \\
2.74 \\
14.39\end{array}$ & $\begin{array}{l}0.509 \\
0.643 \\
0.325 \\
0.639 \\
0.153\end{array}$ & $\begin{array}{l}0.3380 \\
0.2485 \\
0.4336 \\
1.2095 \\
0.4728\end{array}$ & $\begin{array}{l}0.4181 \\
0.4699 \\
0.3341 \\
0.4866 \\
0.2295\end{array}$ \\
\hline $\begin{array}{l}9.4 .26 \\
9.4 .27 \\
9.4 .28 \\
9.4 .29 \\
9.4 .30\end{array}$ & $\begin{array}{l}0.6016 \\
0.1447 \\
0.31222 \\
0.2331 \\
0.4713\end{array}$ & $\begin{array}{l}0.1649 \\
0.1325 \\
0.1541 \\
0.1424 \\
0.1641\end{array}$ & $\begin{array}{l}31.65 \\
24.95 \\
28.95 \\
26.70 \\
30.95\end{array}$ & $\begin{array}{l}0.2545 \\
0.2456 \\
0.2333 \\
0.2345 \\
0.2263\end{array}$ & $\begin{array}{l}0.933 \\
7.276 \\
6.033 \\
0.733 \\
2.933\end{array}$ & $\begin{array}{l}0.997 \\
0.963 \\
1.014 \\
0.970 \\
1.026\end{array}$ & $\begin{array}{r}17.35 \\
4.64 \\
8.33 \\
6.36 \\
12.21\end{array}$ & $\begin{array}{l}0.064 \\
0.476 \\
0.411 \\
0.459 \\
0.390\end{array}$ & $\begin{array}{l}0.54 .99 \\
0.2711 \\
0.3773 \\
0.3379 \\
0.4639\end{array}$ & $\begin{array}{l}0.1478 \\
0.4127 \\
0.3758 \\
0.3970 \\
0.2621\end{array}$ \\
\hline
\end{tabular}


TABLE XXXVI (Contd.)

TWO-PHASE COLD LEG MIXTURE TEST GROUP 9.4

EXPER IMENTAL MEASURMENTS FOR TESTS 9.4 .1 TO $9.4 .30--1.58-$ IN. DOWNCOMER GAP

\begin{tabular}{|c|c|c|c|c|c|c|c|c|c|c|}
\hline $\begin{array}{l}\text { TEST } \\
\text { NO }\end{array}$ & $\begin{array}{c}R M-C H-1 \\
(\text { GPH }\end{array}$ & $\begin{array}{c}R M-C H-2 \\
(G P M)\end{array}$ & $\begin{array}{c}F T-C H-1 \\
(G P M)\end{array}$ & $\begin{array}{c}F T-C i N-2 \\
(G P M)\end{array}$ & $\begin{array}{c}R M-H G-1 \\
(C F M)\end{array}$ & $\begin{array}{l}\text { P.M-HG-2 } \\
(C F M)\end{array}$ & $\begin{array}{c}R M-H F=3 \\
(C F Y)\end{array}$ & $\begin{array}{c}P M-H G-4 \\
(C F M)\end{array}$ & $\begin{array}{l}F T-H G-1 \\
(C F M)\end{array}$ & $\begin{array}{c}F T-H G-2 \\
\text { (CFM) }\end{array}$ \\
\hline $\begin{array}{l}9.4 \cdot 1 \\
9.4 \cdot 12 \\
9.4 \cdot 3 \\
9.4 \cdot 14 \\
9.4 \cdot 5\end{array}$ & $\begin{array}{l}0.0 \\
0.0 \\
0.0 \\
0.0 \\
0.0\end{array}$ & $\begin{array}{l}2.0 \\
0.0 \\
0.0 \\
0.0 \\
0.0\end{array}$ & $\begin{array}{r}119.0 \\
99.0 \\
79.0 \\
0.0 \\
0.0\end{array}$ & $\begin{array}{r}0.0 \\
0.0 \\
0.0 \\
49.9 \\
39.6\end{array}$ & $\begin{array}{c}80.00 \\
0.0 \\
0.0 \\
0.0 \\
0.0\end{array}$ & $\begin{array}{l}0.0 \\
0.0 \\
0.0 \\
0.0 \\
0.0\end{array}$ & $\begin{array}{l}0.0 \\
0.0 \\
0.0 \\
0.0 \\
0.0\end{array}$ & $\begin{array}{l}0.0 \\
0.0 \\
0.0 \\
0.0 \\
0.0\end{array}$ & $\begin{array}{c}0.0 \\
37.25 \\
29.62 \\
44.23 \\
63.21\end{array}$ & $\begin{array}{l}0.0 \\
0.0 \\
0.0 \\
0.0 \\
0.0\end{array}$ \\
\hline $\begin{array}{l}9.4 .6 \\
9.4 .7 \\
9.4 .8 \\
9.4 .99 \\
9.4 .10\end{array}$ & $\begin{array}{l}J .0 \\
0.0 \\
2.0 \\
0.0 \\
0.0\end{array}$ & $\begin{array}{l}0.0 \\
0.0 \\
0.0 \\
0.0 \\
0.0\end{array}$ & $\begin{array}{r}80.0 \\
69.0 \\
0.0 \\
70.0 \\
88.0\end{array}$ & $\begin{array}{r}0.0 \\
0.0 \\
40.0 \\
0.0 \\
0.0\end{array}$ & $\begin{array}{c}80.00 \\
0.0 \\
0.0 \\
0.0 \\
0.0\end{array}$ & $\begin{array}{l}0.0 \\
0.0 \\
0.0 \\
0.0 \\
0.0\end{array}$ & $\begin{array}{l}0.0 \\
0.0 \\
0.0 \\
0.0 \\
0.0\end{array}$ & $\begin{array}{l}0.0 \\
0.0 \\
0.0 \\
0.0 \\
0.0\end{array}$ & $\begin{array}{c}0.0 \\
38.03 \\
21.15 \\
21.41 \\
21.41\end{array}$ & $\begin{array}{l}0.0 \\
0.0 \\
0.0 \\
0.0 \\
0.0\end{array}$ \\
\hline $\begin{array}{l}9.4 .11 \\
9.4 .12 \\
9.4 .13 \\
9.4 .14 \\
9.4 .15\end{array}$ & $\begin{array}{l}3.0 \\
0.0 \\
3.0 \\
3.0 \\
0.0\end{array}$ & $\begin{array}{l}0.0 \\
0.0 \\
0.0 \\
0.0 \\
0.0\end{array}$ & $\begin{array}{r}1.30 .0 \\
121.0 \\
140.0 \\
100.0 \\
10.0\end{array}$ & $\begin{array}{l}0.0 \\
0.0 \\
0.0 \\
0.0 \\
0.0\end{array}$ & $\begin{array}{c}0.0 \\
40.00 \\
0.0 \\
0.0 \\
0.1\end{array}$ & $\begin{array}{l}0.0 \\
0.0 \\
0.0 \\
0.0 \\
0.0\end{array}$ & $\begin{array}{l}0.0 \\
0.0 \\
0.0 \\
0.0 \\
0.0\end{array}$ & $\begin{array}{l}0.0 \\
0.0 \\
0.0 \\
0.0 \\
0.0\end{array}$ & $\begin{array}{l}21.28 \\
0.0 \\
14.74 \\
29.74 \\
38.33\end{array}$ & $\begin{array}{l}0.0 \\
0.0 \\
0.0 \\
0.0 \\
0.0\end{array}$ \\
\hline $\begin{array}{l}9.4 .16 \\
5.4 .17 \\
9.4 .13 \\
9.4 .19 \\
5.4 .21\end{array}$ & $\begin{array}{l}3.0 \\
0.0 \\
3.0 \\
j .0 \\
3.0\end{array}$ & $\begin{array}{l}9.0 \\
0.0 \\
0.0 \\
0.0 \\
0.0\end{array}$ & $\begin{array}{r}60.0 \\
0.0 \\
0.0 \\
117.0 \\
0.0\end{array}$ & $\begin{array}{r}0.0 \\
30.4 \\
20.1 \\
0.0 \\
40.2\end{array}$ & $\begin{array}{l}0.0 \\
0.0 \\
0.0 \\
0.0 \\
0.0\end{array}$ & $\begin{array}{l}0.0 \\
0.0 \\
0.0 \\
0.0 \\
0.0\end{array}$ & $\begin{array}{l}0.0 \\
0.0 \\
0.0 \\
0.0 \\
0.0\end{array}$ & $\begin{array}{l}0.0 \\
0.0 \\
0.0 \\
0.0 \\
0.0\end{array}$ & $\begin{array}{l}56.79 \\
75.51 \\
38.46 \\
17.82 \\
62.31\end{array}$ & $\begin{array}{l}0.0 \\
0.0 \\
0.0 \\
0.0 \\
0.0\end{array}$ \\
\hline $\begin{array}{l}9.4 .21 \\
9.4 .22 \\
9.4 .23 \\
9.4 .24 \\
9.4 .25\end{array}$ & $\begin{array}{l}3.0 \\
3.0 \\
0.0 \\
j .0 \\
3.0\end{array}$ & $\begin{array}{l}0.0 \\
0.0 \\
0.0 \\
0.0 \\
0.0\end{array}$ & $\begin{array}{r}90.0 \\
105.0 \\
61.0 \\
115.0 \\
0.0\end{array}$ & $\begin{array}{r}0.0 \\
0.0 \\
0.0 \\
0.0 \\
29.5\end{array}$ & $\begin{array}{c}0.0 \\
0.0 \\
0.0 \\
40.00 \\
0.0\end{array}$ & $\begin{array}{l}0.0 \\
0.0 \\
0.0 \\
0.0 \\
0.0\end{array}$ & $\begin{array}{l}0.0 \\
0.0 \\
0.0 \\
0.0 \\
0.0\end{array}$ & $\begin{array}{l}0.0 \\
0.0 \\
0.0 \\
0.0 \\
0.0\end{array}$ & $\begin{array}{l}29.62 \\
14.97 \\
56.15 \\
0.0 \\
? 5.13\end{array}$ & $\begin{array}{l}0.0 \\
0.0 \\
0.0 \\
0.0 \\
0.0\end{array}$ \\
\hline $\begin{array}{l}9.4 .26 \\
9.4 .27 \\
9.4 .28 \\
9.4 .29 \\
9.4 .30\end{array}$ & $\begin{array}{l}J .0 \\
1.0 \\
j .0 \\
1.0 \\
3.0\end{array}$ & $\begin{array}{l}0.0 \\
j .0 \\
0.0 \\
0.0 \\
0.0\end{array}$ & $\begin{array}{r}0.0 \\
94.0 \\
70.0 \\
80.0 \\
0.0\end{array}$ & $\begin{array}{r}19.1 \\
0.0 \\
0.0 \\
0.0 \\
40.1\end{array}$ & $\begin{array}{l}0.0 \\
0.0 \\
0.0 \\
0.0 \\
0.0\end{array}$ & $\begin{array}{l}0.0 \\
0.0 \\
0.0 \\
0.0 \\
0.0\end{array}$ & $\begin{array}{l}0.0 \\
0.0 \\
0.0 \\
0.0 \\
0.0\end{array}$ & $\begin{array}{l}0.0 \\
0.0 \\
0.0 \\
0.0 \\
0.0\end{array}$ & $\begin{array}{l}76.79 \\
17.69 \\
38.33 \\
29.36 \\
63.59\end{array}$ & $\begin{array}{l}0.0 \\
0.0 \\
0.0 \\
0.0 \\
0.0\end{array}$ \\
\hline
\end{tabular}


TABLE XXXVI (Contd.)

TWO-PHASE COLD LEG MIXTURE TEST GROUP 9.4

EXPERI 1ENTAL MEASIJPMENTS FUD TESTS 9.4 .1 TO 9.4.30 -- 1.59-IN. DOWNCUMER GAP

\begin{tabular}{|c|c|c|c|c|c|c|c|c|c|c|}
\hline $\begin{array}{l}\text { TEST } \\
\text { NO }\end{array}$ & 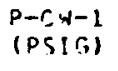 & $\begin{array}{l}P-C W-2 \\
(P S I r)\end{array}$ & $\begin{array}{l}P-H(S-1 \\
(P S I G)\end{array}$ & $\begin{array}{l}\text { P-HC,-2 } \\
\text { |PSIG) }\end{array}$ & $\begin{array}{l}\text { P-HG-3 } \\
\text { (PSIG) }\end{array}$ & $\begin{array}{l}\text { TF-C:N-1 } \\
\text { (OEG F) }\end{array}$ & $\begin{array}{l}T F-H G-1 \\
\text { IUEG FI }\end{array}$ & $\begin{array}{l}T F-H G-2 \\
(D E G F)\end{array}$ & $\begin{array}{l}\text { TF-HG-3 } \\
\text { (OEG F) }\end{array}$ & $\begin{array}{l}\text { P-BAPO } \\
\text { (PSIA) }\end{array}$ \\
\hline $\begin{array}{l}9.4 .11 \\
9.4 \cdot 2 \\
9.4 .3 \\
9.4 .5 \\
9.4 .5\end{array}$ & $\begin{array}{l}17.5 \\
2 \% .0 \\
10.2 \\
13.0 \\
11.5\end{array}$ & $\begin{array}{l}0.0 \\
0.0 \\
0.0 \\
0.0 \\
0.0\end{array}$ & $\begin{array}{l}10.5 \\
19.8 \\
12.3 \\
11.5 \\
17.5\end{array}$ & $\begin{array}{l}94.0 \\
92.0 \\
82.5 \\
90.0 \\
88.5\end{array}$ & $\begin{array}{l}33.5 \\
33.5 \\
33.5 \\
33.5 \\
33.0\end{array}$ & $\begin{array}{l}46.0 \\
50.0 \\
50.0 \\
48.0 \\
47.0\end{array}$ & $\begin{array}{r}84.0 \\
93.0 \\
94.0 \\
93.0 \\
106.0\end{array}$ & $\begin{array}{r}0.0 \\
106.5 \\
106.5 \\
105.0 \\
119.5\end{array}$ & $\begin{array}{r}91.5 \\
0.0 \\
0.0 \\
0.0 \\
0.0\end{array}$ & $\begin{array}{l}12.2 \\
12.2 \\
12.2 \\
12.2 \\
12.2\end{array}$ \\
\hline $\begin{array}{l}9.4 .6 \\
9.4 .7 \\
9.4 .7 \\
9.4 .79 \\
9.4 .10\end{array}$ & $\begin{array}{l}14.0 \\
14.5 \\
12.0 \\
13.0 \\
14.5\end{array}$ & $\begin{array}{l}0.0 \\
0.0 \\
0.0 \\
0.0 \\
0.0\end{array}$ & $\begin{array}{l}9.5 \\
12.0 \\
10.0 \\
10.0 \\
11.0\end{array}$ & $\begin{array}{l}96.0 \\
92.0 \\
94.0 \\
97.0 \\
96.0\end{array}$ & $\begin{array}{l}32.0 \\
33.0 \\
34.0 \\
34.0 \\
33.0\end{array}$ & $\begin{array}{l}52.0 \\
52.5 \\
46.0 \\
47.0 \\
44.5\end{array}$ & $\begin{array}{r}81.0 \\
110.5 \\
108.0 \\
88.0 \\
97.0\end{array}$ & $\begin{array}{r}94.0 \\
124.0 \\
120.5 \\
100.0 \\
111.0\end{array}$ & $\begin{array}{r}79.0 \\
117.0 \\
114.5 \\
94.0 \\
104.0\end{array}$ & $\begin{array}{l}12.2 \\
12.2 \\
12.2 \\
12.2 \\
12.2\end{array}$ \\
\hline $\begin{array}{l}9.4 .11 \\
9.4 .12 \\
9.4 .13 \\
9.4 .14 \\
9.4 .15\end{array}$ & $\begin{array}{l}21.0 \\
13.7 \\
19.0 \\
13.0 \\
14.0\end{array}$ & $\begin{array}{l}0.0 \\
0.0 \\
0.0 \\
0.0 \\
0.0\end{array}$ & $\begin{array}{l}14.0 \\
10.5 \\
10.0 \\
13.0 \\
12.0\end{array}$ & $\begin{array}{l}95.0 \\
95.0 \\
94.0 \\
91.0 \\
90.5\end{array}$ & $\begin{array}{r}34.0 \\
33.0 \\
0.0 \\
0.0 \\
0.0\end{array}$ & $\begin{array}{l}50.0 \\
50.5 \\
46.5 \\
46.5 \\
47.5\end{array}$ & $\begin{array}{l}97.0 \\
84.0 \\
85.0 \\
88.0 \\
99.0\end{array}$ & $\begin{array}{r}110.0 \\
92.0 \\
95.0 \\
98.5 \\
114.0\end{array}$ & $\begin{array}{r}104.0 \\
93.0 \\
0.0 \\
0.0 \\
0.0\end{array}$ & $\begin{array}{l}12.2 \\
12.1 \\
12.1 \\
12.1 \\
12.1\end{array}$ \\
\hline $\begin{array}{l}3.4 .16 \\
9.4 .17 \\
9.4 .13 \\
9.4 .19 \\
9.4 .20\end{array}$ & $\begin{array}{l}13.2 \\
16.5 \\
16.0 \\
22.0 \\
17.0\end{array}$ & $\begin{array}{l}0.0 \\
0.0 \\
j .0 \\
0.0 \\
0.0\end{array}$ & $\begin{array}{l}17.5 \\
17.0 \\
17.0 \\
12.0 \\
17.0\end{array}$ & $\begin{array}{l}83.5 \\
86.5 \\
83.0 \\
93.0 \\
88.0\end{array}$ & $\begin{array}{r}0.0 \\
0.0 \\
0.0 \\
33.0 \\
32.0\end{array}$ & $\begin{array}{l}46.0 \\
48.0 \\
52.0 \\
47.0 \\
45.0\end{array}$ & $\begin{array}{r}113.0 \\
120.0 \\
106.5 \\
94.5 \\
112.5\end{array}$ & $\begin{array}{l}127.5 \\
134.0 \\
120.5 \\
106.5 \\
127.5\end{array}$ & $\begin{array}{r}0.0 \\
0.0 \\
0.0 \\
100.5 \\
120.5\end{array}$ & $\begin{array}{l}12.1 \\
12.1 \\
12.1 \\
12.2 \\
12.2\end{array}$ \\
\hline $\begin{array}{l}9.4 .21 \\
9.4 .22 \\
9.4 .23 \\
9.4 .24 \\
9.4 .25\end{array}$ & $\begin{array}{l}23.0 \\
23.7 \\
24.5 \\
29.0 \\
13.0\end{array}$ & $\begin{array}{l}0.0 \\
0.0 \\
0.0 \\
0.0 \\
0.0\end{array}$ & $\begin{array}{l}18.0 \\
14.5 \\
19.6 \\
12.0 \\
16.5\end{array}$ & $\begin{array}{l}91.0 \\
92.0 \\
87.0 \\
86.0 \\
77.0\end{array}$ & $\begin{array}{r}0.0 \\
0.0 \\
0.0 \\
33.0 \\
0.0\end{array}$ & $\begin{array}{l}51.0 \\
49.0 \\
48.0 \\
50.0 \\
49.5\end{array}$ & $\begin{array}{r}86.0 \\
88.0 \\
121.0 \\
95.0 \\
116.5\end{array}$ & $\begin{array}{l}102.5 \\
103.5 \\
138.0 \\
113.5 \\
131.5\end{array}$ & $\begin{array}{r}0.0 \\
0.0 \\
0.0 \\
91.0 \\
0.0\end{array}$ & $\begin{array}{l}12.1 \\
12.1 \\
12.1 \\
12.1 \\
12.1\end{array}$ \\
\hline $\begin{array}{l}9.4 .26 \\
9.4 .27 \\
9.4 .28 \\
9.4 .29 \\
9.4 .30\end{array}$ & $\begin{array}{l}21.0 \\
23.7 \\
24.7 \\
24.5 \\
21.5\end{array}$ & $\begin{array}{l}0.0 \\
0.0 \\
0.0 \\
0.0 \\
0.0\end{array}$ & $\begin{array}{l}21.0 \\
12.8 \\
17.0 \\
14.7 \\
18.8\end{array}$ & $\begin{array}{l}95.0 \\
90.5 \\
89.0 \\
39.0 \\
85.5\end{array}$ & $\begin{array}{r}0.0 \\
33.5 \\
33.5 \\
33.5 \\
33.0\end{array}$ & $\begin{array}{r}51.5 \\
47.0 \\
46.0 \\
45.0 \\
46.0\end{array}$ & $\begin{array}{r}90.0 \\
92.8 \\
101.0 \\
99.5 \\
117.5\end{array}$ & $\begin{array}{r}99.5 \\
105.0 \\
117.5 \\
113.5 \\
133.0\end{array}$ & $\begin{array}{l}0.0 \\
0.0 \\
0.0 \\
0.0 \\
0.0\end{array}$ & $\begin{array}{l}12.1 \\
12.2 \\
12.2 \\
12.2 \\
12.2\end{array}$ \\
\hline
\end{tabular}


TABLE XXXVI (Contd.)

TWO-PHASE COLD LEG MIXTURE TEST GROUP 9.4

EXPFP.IMENTAL MEASURIAENTS FOR TESTS 9.4 .1 TO $9.4 .30-1.58-I N$. DOWNCOMER GAP

\begin{tabular}{|c|c|c|c|c|c|c|c|c|c|}
\hline \multicolumn{4}{|c|}{ LOTHER PLENUM FLOW } & \multicolumn{3}{|c|}{ BYPASS FLIJH } & \multirow[b]{2}{*}{$\begin{array}{l}\text { LOWER } \\
\text { PLEN!JM } \\
\text { LEVEL } \\
\text { (IN) }\end{array}$} & \multirow[b]{2}{*}{$\begin{array}{l}P-\perp P-1 \\
(P S I G)\end{array}$} & \multirow[b]{2}{*}{$\begin{array}{l}T F-L P-1 \\
\text { (DEG FI }\end{array}$} \\
\hline $\begin{array}{l}\text { TEST } \\
\text { NO }\end{array}$ & $\begin{array}{l}\text { INITIIAL } \\
\text { WEIGHT } \\
\text { (LS) }\end{array}$ & $\begin{array}{l}\text { FINAL } \\
\text { WEIGHT } \\
\text { (LB) }\end{array}$ & $\begin{array}{l}\text { TIME OF } \\
\text { COLLECTICN } \\
\text { I.SEC) }\end{array}$ & $\begin{array}{l}\text { INITI IAL } \\
\text { WE IGHT } \\
\text { (LSI }\end{array}$ & $\begin{array}{l}\text { FINAL } \\
\text { WEIGHT } \\
\text { (LB) }\end{array}$ & $\begin{array}{l}\text { TIME DF } \\
\text { COLLECTIION } \\
\text { (SEC) }\end{array}$ & & & \\
\hline $\begin{array}{l}9.4 .1 \\
9.4 .2 \\
9.4 .3 \\
9.4 .5 \\
9.4 .5\end{array}$ & $\begin{array}{l}141.0 \\
198.0 \\
167.0 \\
142.0 \\
241.0\end{array}$ & $\begin{array}{l}354.0 \\
300.5 \\
377.0 \\
270.0 \\
371.0\end{array}$ & $\begin{array}{l}20.0 \\
15.0 \\
30.0 \\
30.0 \\
60.0\end{array}$ & $\begin{array}{l}102.0 \\
121.5 \\
139.0 \\
106.0 \\
116.0\end{array}$ & $\begin{array}{l}221.0 \\
330.0 \\
284.0 \\
188.0 \\
223.0\end{array}$ & $\begin{array}{l}20.0 \\
30.0 \\
30.0 \\
30.0 \\
30.0\end{array}$ & $\begin{array}{l}12.0 \\
12.0 \\
12.0 \\
12.0 \\
12.0\end{array}$ & $\begin{array}{l}10.5 \\
19.2 \\
12.2 \\
11.0 \\
16.7\end{array}$ & $\begin{array}{l}45.5 \\
50.5 \\
51.0 \\
50.0 \\
50.0\end{array}$ \\
\hline $\begin{array}{l}9.4 .10 \\
9.4 .7 \\
9.4 .8 \\
9.4 .99 \\
9.4 .10\end{array}$ & $\begin{array}{l}140.5 \\
297.0 \\
220.0 \\
141.0 \\
152.5\end{array}$ & $\begin{array}{l}364.0 \\
463.5 \\
398.0 \\
336.5 \\
397.0\end{array}$ & $\begin{array}{l}30.0 \\
30.0 \\
44.0 \\
30.0 \\
30.0\end{array}$ & $\begin{array}{r}32.5 \\
117.0 \\
128.0 \\
102.0 \\
121.5\end{array}$ & $\begin{array}{l}201.0 \\
224.0 \\
199.0 \\
191.5 \\
240.5\end{array}$ & $\begin{array}{l}30.0 \\
30.0 \\
45.0 \\
30.0 \\
30.0\end{array}$ & $\begin{array}{l}12.0 \\
12.0 \\
12.0 \\
12.0 \\
12.0\end{array}$ & $\begin{array}{r}10.0 \\
11.0 \\
10.0 \\
9.5 \\
10.0\end{array}$ & $\begin{array}{l}45.0 \\
47.0 \\
47.0 \\
47.0 \\
45.0\end{array}$ \\
\hline $\begin{array}{l}9.4 .11 \\
9.4 .12 \\
9.4 .113 \\
9.4 .14 \\
9.4 .15\end{array}$ & $\begin{array}{l}192.0 \\
141.0 \\
164.0 \\
143.5 \\
164.0\end{array}$ & $\begin{array}{l}412.0 \\
528.5 \\
546.0 \\
403.0 \\
341.5\end{array}$ & $\begin{array}{l}20.0 \\
30.0 \\
30.0 \\
30.0 \\
30.0\end{array}$ & $\begin{array}{l}138.5 \\
104.0 \\
118.0 \\
112.5 \\
125.5\end{array}$ & $\begin{array}{l}340.5 \\
256.0 \\
312.0 \\
269.0 \\
237.5\end{array}$ & $\begin{array}{l}30.0 \\
30.0 \\
30.0 \\
30.0 \\
30.0\end{array}$ & $\begin{array}{r}5.0 \\
4.0 \\
4.0 \\
12.0 \\
12.0\end{array}$ & $\begin{array}{r}13.5 \\
10.2 \\
9.5 \\
12.5 \\
11.2\end{array}$ & $\begin{array}{l}54.0 \\
51.0 \\
47.0 \\
47.5 \\
49.5\end{array}$ \\
\hline $\begin{array}{l}9.4 .16 \\
9.4 .17 \\
9.4 .18 \\
9.4 .13 \\
9.4 .20\end{array}$ & $\begin{array}{l}144.0 \\
141.5 \\
147.5 \\
142.5 \\
149.5\end{array}$ & $\begin{array}{l}261.5 \\
235.5 \\
166.0 \\
451.0 \\
236.0\end{array}$ & $\begin{array}{l}30.0 \\
60.0 \\
30.0 \\
30.0 \\
30.0\end{array}$ & $\begin{array}{l}237.5 \\
107.0 \\
216.0 \\
105.0 \\
110.5\end{array}$ & $\begin{array}{l}369.0 \\
194.0 \\
285.0 \\
272.5 \\
.203 .0\end{array}$ & $\begin{array}{l}30.0 \\
30.0 \\
30.0 \\
30.0 \\
30.0\end{array}$ & $\begin{array}{r}12.0 \\
12.0 \\
12.0 \\
5.0 \\
12.0\end{array}$ & $\begin{array}{l}16.0 \\
16.0 \\
16.2 \\
12.5 \\
16.0\end{array}$ & $\begin{array}{l}49.0 \\
54.0 \\
60.0 \\
47.0 \\
48.0\end{array}$ \\
\hline $\begin{array}{l}9.4 .21 \\
9.4 .22 \\
9.4 .23 \\
9.4 .24 \\
9.4 .25\end{array}$ & $\begin{array}{l}138.5 \\
159.0 \\
166.0 \\
158.0 \\
143.5\end{array}$ & $\begin{array}{l}362.5 \\
442.0 \\
309.0 \\
461.5 \\
211.0\end{array}$ & $\begin{array}{l}30.0 \\
30.0 \\
30.0 \\
30.0 \\
30.0\end{array}$ & $\begin{array}{r}99.0 \\
125.5 \\
125.0 \\
125.0 \\
160.5\end{array}$ & $\begin{array}{l}247.0 \\
281.5 \\
236.0 \\
291.5 \\
227.5\end{array}$ & $\begin{array}{l}30.0 \\
30.0 \\
30.0 \\
30.0 \\
30.0\end{array}$ & $\begin{array}{r}12.0 \\
12.0 \\
12.0 \\
6.0 \\
12.0\end{array}$ & $\begin{array}{l}17.5 \\
13.7 \\
13.5 \\
13.0 \\
16.5\end{array}$ & $\begin{array}{l}51.5 \\
49.5 \\
50.0 \\
50.0 \\
54.0\end{array}$ \\
\hline $\begin{array}{l}9.4 .26 \\
9.4 .27 \\
9.4 .28 \\
9.4 .29 \\
9.4 .30\end{array}$ & $\begin{array}{l}158.0 \\
141.5 \\
200.0 \\
143.5 \\
251.5\end{array}$ & $\begin{array}{l}185.0 \\
352.5 \\
381.0 \\
345.5 \\
339.5\end{array}$ & $\begin{array}{l}30.0 \\
29.0 \\
30.0 \\
30.0 \\
30.0\end{array}$ & $\begin{array}{l}190.0 \\
104.0 \\
122.5 \\
109.5 \\
180.5\end{array}$ & $\begin{array}{l}217.0 \\
223.0 \\
237.5 \\
231.0 \\
264.0\end{array}$ & $\begin{array}{l}15.0 \\
30.0 \\
30.0 \\
30.0 \\
30.0\end{array}$ & $\begin{array}{l}12.0 \\
12.0 \\
12.0 \\
12.0 \\
12.0\end{array}$ & $\begin{array}{l}19.5 \\
12.7 \\
16.7 \\
14.5 \\
18.7\end{array}$ & $\begin{array}{l}58.0 \\
48.0 \\
47.0 \\
46.0 \\
49.0\end{array}$ \\
\hline
\end{tabular}


TABLE XXXVI (Contd.)

TWO-PHASE COLD LEG MIXTURE TEST GROUP 9.4

EXPERIMENT AL IIEASUPMENTS FOP TESTS 9.4.1 TO 9.4.30-- 1.59-IN. DOHNCUMER GAP

\begin{tabular}{|c|c|c|c|c|c|c|c|}
\hline $\begin{array}{l}\text { TEST } \\
\text { ND }\end{array}$ & $\begin{array}{l}R \cdot 1-C G-1 \\
(C F M)\end{array}$ & $\begin{array}{c}P M-55-2 \\
(C F: M)\end{array}$ & $\begin{array}{c}F T-C G-1 \\
(C F M)\end{array}$ & $\begin{array}{l}P-C G-1 \\
(P S I G)\end{array}$ & $\begin{array}{l}P-C G-2 \\
(P S \operatorname{Sg} \mid\end{array}$ & $\begin{array}{l}\text { TF-CG-1 } \\
\text { (DEG FI) }\end{array}$ & $\begin{array}{l}\text { TF-CG-2 } \\
(D E G F)\end{array}$ \\
\hline 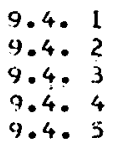 & $\begin{array}{l}0.0 \\
0.0 \\
0.0 \\
0.0 \\
.0 .0\end{array}$ & $\begin{array}{l}30.0 \\
30.0 \\
30.0 \\
30.0 \\
30.0\end{array}$ & $\begin{array}{l}0.0 \\
0.0 \\
0.0 \\
0.0 \\
0.0\end{array}$ & $\begin{array}{l}0.0 \\
0.0 \\
0.0 \\
0.0 \\
0.0\end{array}$ & $\begin{array}{l}34.8 \\
35.0 \\
35.1 \\
35.1 \\
34.9\end{array}$ & $\begin{array}{r}80.0 \\
0.0 \\
0.0 \\
0.0 \\
33.0\end{array}$ & $\begin{array}{l}78.5 \\
80.0 \\
91.0 \\
91.0 \\
82.5\end{array}$ \\
\hline $\begin{array}{l}9.4 .6 \\
9.4 .77 \\
9.4 .8 \\
3.4 .77 \\
9.4 .19\end{array}$ & $\begin{array}{l}0.0 \\
0.0 \\
0.0 \\
0.0 \\
0.3\end{array}$ & $\begin{array}{l}30.0 \\
30.0 \\
30.0 \\
30.0 \\
30.0\end{array}$ & $\begin{array}{l}0.0 \\
0.0 \\
0.0 \\
0.0 \\
0.0\end{array}$ & $\begin{array}{r}3.0 \\
0.0 \\
94.0 \\
97.0 \\
96.0\end{array}$ & $\begin{array}{l}35.0 \\
35.0 \\
35.0 \\
35.0 \\
35.0\end{array}$ & $\begin{array}{l}81.0 \\
90.0 \\
99.5 \\
84.0 \\
91.5\end{array}$ & $\begin{array}{l}78.0 \\
85.0 \\
95.5 \\
86.0 \\
88.0\end{array}$ \\
\hline $\begin{array}{l}9.4 .11 \\
9.4 .12 \\
9.4 .13 \\
9.4 .14 \\
9.4 .15\end{array}$ & $\begin{array}{l}0.0 \\
0.0 \\
0.0 \\
0.0 \\
0.0\end{array}$ & $\begin{array}{l}30.0 \\
30.0 \\
30.0 \\
30.0 \\
30.0\end{array}$ & $\begin{array}{l}0.0 \\
0.0 \\
0.0 \\
0.0 \\
0.0\end{array}$ & $\begin{array}{r}95.0 \\
0.0 \\
0.0 \\
0.0 \\
0.0\end{array}$ & $\begin{array}{l}35.0 \\
35.0 \\
34.9 \\
34.8 \\
34.8\end{array}$ & $\begin{array}{r}93.5 \\
0.0 \\
0.0 \\
0.0 \\
0.0\end{array}$ & $\begin{array}{l}90.0 \\
97.5 \\
36.0 \\
85.0 \\
86.5\end{array}$ \\
\hline $\begin{array}{l}9.4 .16 \\
9.4 .17 \\
9.4 .19 \\
9.4 .119 \\
9.4 .20\end{array}$ & $\begin{array}{l}0.0 \\
0.0 \\
0.0 \\
0.0 \\
0.0\end{array}$ & $\begin{array}{r}30.0 \\
30.0 \\
30.0 \\
0.0 \\
0.0\end{array}$ & $\begin{array}{r}0.0 \\
0.0 \\
0.0 \\
.14 .9 \\
14.5\end{array}$ & $\begin{array}{r}0.0 \\
0.0 \\
0.0 \\
93.0 \\
88.0\end{array}$ & $\begin{array}{l}34.7 \\
34.8 \\
34.8 \\
34.0 \\
35.0\end{array}$ & $\begin{array}{r}0.0 \\
0.0 \\
0.0 \\
96.0 \\
100.0\end{array}$ & $\begin{array}{l}98.0 \\
38.0 \\
92.5 \\
90.5 \\
95.5\end{array}$ \\
\hline $\begin{array}{l}9.4 .21 \\
9.4 .22 \\
9.4 .23 \\
9.4 .24 \\
9.4 .25\end{array}$ & $\begin{array}{l}0.0 \\
0.0 \\
0.0 \\
0.0 \\
0.0\end{array}$ & $\begin{array}{l}0.0 \\
0.0 \\
0.0 \\
0.0 \\
0.0\end{array}$ & $\begin{array}{l}27.4 \\
28.7 \\
28.2 \\
28.6 \\
30.3\end{array}$ & $\begin{array}{r}91.0 \\
92.0 \\
87.0 \\
0.0 \\
77.0\end{array}$ & $\begin{array}{l}0.0 \\
0.0 \\
0.0 \\
0.0 \\
0.0\end{array}$ & $\begin{array}{r}96.5 \\
105.0 \\
121.0 \\
114.0 \\
103.5\end{array}$ & $\begin{array}{l}0.0 \\
0.0 \\
0.0 \\
0.0 \\
0.0\end{array}$ \\
\hline $\begin{array}{l}9.4 .26 \\
9.4 .27 \\
9.4 .23 \\
9.4 .20 \\
9.4 .30\end{array}$ & $\begin{array}{l}3.0 \\
3.0 \\
0.0 \\
0.0 \\
0.0\end{array}$ & $\begin{array}{l}0.0 \\
0.0 \\
0.0 \\
0.0 \\
0.0\end{array}$ & $\begin{array}{l}29.4 \\
29.9 \\
29.2 \\
29.4 \\
29.7\end{array}$ & $\begin{array}{l}95.0 \\
910.5 \\
87.0 \\
89.0 \\
85.5\end{array}$ & $\begin{array}{l}0.0 \\
0.0 \\
0.0 \\
0.0 \\
0.0\end{array}$ & $\begin{array}{r}96.0 \\
102.0 \\
110.5 \\
110.0^{2} \\
116.5\end{array}$ & $\begin{array}{l}0.0 \\
0.0 \\
0.0 \\
0.0 \\
0.0\end{array}$ \\
\hline
\end{tabular}


leg nozzle removed, and two-phase flow entering the vessel through the cold leg. The objective of these combined effects tests was to investigate the sensitivity of the system countercurrent flow to several coinciding changes in geometry of cold leg flow conditions. Tables XXXVII through XLVI contain the data for these ten tests and Table I includes a listing of the tests and the respective changes to the normal configuration.

\section{SPECIAL TESTS}

Following is a brief discussion of countercurrent flow tests which did not have objectives consistent with the classifications discussed previously because either specially designed equipment was required or special operating procedures were used.

\subsection{One-Dimensional Inlet Test Group}

A limited number of countercurrent flow tests were performed with the upper annulus portion of the transparent vessel, including the cold leg and bypass leg, removed and replaced with an inlet water distributor that was designed to induce flow parallel to the downcomer axis and to distribute the water uniformly around the top of the downcomer. The purpose of these tests was to provide countercurrent flow data for a uniform inlet flow distribution that could be compared with countercurrent flow data obtained for the normal inlet to indicate the effect on countercurrent flow of the nonuniform distribution of water to the downcomer entrance that results from injection normal to the downcomer.

The design for the one-dimensional inlet flow distributor was discussed in Section II-1. The test procedure for these tests included the following steps:

(1) Downcomer air flow was initiated at a predetermined value.

(2) Water flow was gradually increased until a level above the top of weir entrance was established.

(3) The system was allowed to run until the height of the water level above the weir entrance stabilized.

(4) The water flow was increased slightly and the preceding step was repeated; water flow was gradually increased in steps until a water flow rate was reached at which the height of the water level above the weir entrance did not stabilize but was continually increasing.

(5) The water flow was decreased slightly until the water level stabilized.

(6). The necessary measurements were recorded. 
TABLE XXXVII

COMBINED EFFECTS TEST GROUP 3.2

TRANSPARENT VESSEL TEST DATA FIR TESTS 3.2.1 TO 3.2.25 -- 0.39-IN. DOWNCOMER GAP SUMAMARY IJF CALCISLATED RESIJLTS

\begin{tabular}{|c|c|c|c|c|c|c|c|c|c|c|}
\hline $\begin{array}{l}\text { TEST } \\
\text { NO }\end{array}$ & $\begin{array}{l}\text { DONNCJYER } \\
\text { AIR FL:TW } \\
\text { (L9/SEC) }\end{array}$ & $\begin{array}{l}\text { DOWNCOMER } \\
\text { AIR UENSITY } \\
\text { ILG/FT*3) }\end{array}$ & $\begin{array}{l}\text { LUWER PLENUM } \\
\text { PRESSURE } \\
\text { (PSIA) }\end{array}$ & $\begin{array}{l}\text { COLD LEG } \\
\text { A IP. FLOW } \\
(L \& / S E C)\end{array}$ & $\begin{array}{l}\text { UOWNCOMER } \\
\text { HATER FLOW } \\
\text { (LB/SEC) }\end{array}$ & $\begin{array}{l}\text { WATER FLOW } \\
\text { CONTI NIJI TY }\end{array}$ & $\begin{array}{c}\text { J-AIR } \\
\text { DOWNC JMER } \\
\text { (FT/SEC) }\end{array}$ & $\begin{array}{l}\text { J-HATER } \\
\text { DIJWNCOMER } \\
\text { IFT/SEC) }\end{array}$ & $1 J G * 1 * * 1 / 2$ & $(J L * 1 * 1)$ \\
\hline $\begin{array}{l}3.2 \cdot 1 \\
3.2 \cdot 2 \\
3.2 \cdot 3 \\
3.2 \cdot 4 \\
3.2 .5\end{array}$ & $\begin{array}{l}0.0 \\
0.0 \\
0.0 \\
0.0 \\
0.0\end{array}$ & $\begin{array}{l}0.5175 \\
J .1168 \\
0.11174 \\
0.1173 \\
j .1172\end{array}$ & $\begin{array}{l}22.46 \\
22.46 \\
22.44 \\
22.44 \\
22.44\end{array}$ & $\begin{array}{l}0.0958 \\
0.0958 \\
0.0474 \\
0.0484 \\
0.0479\end{array}$ & $\begin{array}{l}2.420 \\
2.767 \\
3.700 \\
3.050 \\
2.375\end{array}$ & $\begin{array}{l}1.003 \\
1.003 \\
1.010 \\
0.987 \\
0.985\end{array}$ & $\begin{array}{l}0.0 \\
0.00 \\
0.0 \\
0.0 \\
0.0\end{array}$ & $\begin{array}{l}0.764 \\
0.973 \\
1.193 \\
0.962 \\
0.769\end{array}$ & $\begin{array}{l}0.0 \\
0.0 \\
0.0 \\
0.0 \\
0.0\end{array}$ & $\begin{array}{l}0.7248 \\
0.7750 \\
0.9059 \\
0.9137 \\
0.7180\end{array}$ \\
\hline $\begin{array}{l}3.2 \cdot 0 \\
3.2 .7 \\
3.2 .8 \\
3.2 .8 \\
3.2 .10\end{array}$ & $\begin{array}{l}0.0075 \\
0.0976 \\
0.0153 \\
0.0155 \\
0.0227\end{array}$ & $\begin{array}{l}0.1180 \\
0.1171 \\
0.1177 \\
0.1172 \\
0.1176\end{array}$ & $\begin{array}{l}22.46 \\
22.46 \\
22.46 \\
22.46 \\
22.46\end{array}$ & $\begin{array}{l}0.0470 \\
0.0469 \\
0.0475 \\
0.0468 \\
0.0469\end{array}$ & $\begin{array}{l}3.275 \\
2.800 \\
2.587 \\
2.412 \\
2.063\end{array}$ & $\begin{array}{l}0.997 \\
0.989 \\
0.987 \\
1.023 \\
1.006\end{array}$ & $\begin{array}{l}1.25 \\
1.2 .7 \\
2.56 \\
2.61 \\
3.81\end{array}$ & $\begin{array}{l}1.033 \\
0.834 \\
0.817 \\
0.761 \\
0.651\end{array}$ & $\begin{array}{l}0.1932 \\
0.1946 \\
0.2768 \\
0.2788 \\
0.3373\end{array}$ & $\begin{array}{l}0.8432 \\
0.7796 \\
0.7495 \\
0.7237 \\
0.6691\end{array}$ \\
\hline $\begin{array}{l}3.2 .11 \\
3.2 .12 \\
3.2 .13 \\
3.2 .14 \\
3.2 .15\end{array}$ & $\begin{array}{l}0.0225 \\
0.0310 \\
0.0303 \\
0.0462 \\
0.0457\end{array}$ & $\begin{array}{l}0.1175 \\
0.11772 \\
0.1172 \\
0.1163 \\
0.1175\end{array}$ & $\begin{array}{l}22.46 \\
22.46 \\
22.46 \\
22.46 \\
22.46\end{array}$ & $\begin{array}{l}0.0479 \\
0.0479 \\
0.0458 \\
0.0480 \\
0.0474\end{array}$ & $\begin{array}{l}2.010 \\
1.787 \\
1.675 \\
1.200 \\
1.125\end{array}$ & $\begin{array}{l}1.020 \\
1.036 \\
1.032 \\
1.000 \\
1.041\end{array}$ & $\begin{array}{l}3.11 \\
5.21 \\
5.09 \\
7.82 \\
7.66\end{array}$ & $\begin{array}{l}0.634 \\
0.564 \\
0.529 \\
0.379 \\
0.355\end{array}$ & $\begin{array}{l}0.3354 \\
0.3941 \\
0.3897 \\
0.4820 \\
0.4782\end{array}$ & $\begin{array}{l}0.6606 \\
0.6229 \\
0.6030 \\
0.5104 \\
0.4942\end{array}$ \\
\hline $\begin{array}{l}3.2 .16 \\
3.2 .17 \\
3.2 .18 \\
3.2 .19 \\
3.2 .20\end{array}$ & $\begin{array}{l}0.0610 \\
0.0617 \\
0.0713 \\
0.0754 \\
0.0909\end{array}$ & $\begin{array}{l}0.1172 \\
0.1176 \\
0.1174 \\
0.1174 \\
0.1171\end{array}$ & $\begin{array}{l}22.46 \\
22.46 \\
22.46 \\
22.46 \\
22.46 .\end{array}$ & $\begin{array}{l}0.0469 \\
0.0469 \\
0.0469 \\
0.0447 \\
0.0469\end{array}$ & $\begin{array}{l}0.833 \\
0.750 \\
0.479 \\
0.469 \\
0.389\end{array}$ & $\begin{array}{l}1.020 \\
1.055 \\
1.004 \\
0.995 \\
1.036\end{array}$ & $\begin{array}{l}10.25 \\
10.33 \\
12.37 \\
12.65 \\
15.28\end{array}$ & $\begin{array}{l}0.263 \\
0.237 \\
0.151 \\
0.148 \\
0.123\end{array}$ & $\begin{array}{l}0.5529 \\
0.5553 \\
0.6220 \\
0.06143 \\
0.6748\end{array}$ & $\begin{array}{l}0.4253 \\
0.4035 \\
0.3223 \\
0.3190 \\
0.2906\end{array}$ \\
\hline
\end{tabular}


TABLE XXVII (Contd.)

COMBINED EFFECTS TEST GROUP 3.2

EXPERIMENTAL MEASURMENTS FOR TESTS 3.2.1 TO 3.2.25 -- $0.39-$ IN. DOHNCOMER GAP

\begin{tabular}{|c|c|c|c|c|c|c|c|c|c|c|}
\hline $\begin{array}{l}\text { TEST } \\
\text { NO }\end{array}$ & $\begin{array}{c}R M-C H-1 \\
(G P M)\end{array}$ & $\begin{array}{c}R M-C H-2 \\
(G P M)\end{array}$ & $\begin{array}{c}F T-C H-1 \\
(G P M)\end{array}$ & $\begin{array}{c}\mathrm{FT}-\mathrm{CH}-2 \\
(\mathrm{GPH})\end{array}$ & $\begin{array}{c}R M-H G-1 \\
(C F M)\end{array}$ & $\begin{array}{c}R M-H G-2 \\
(C F M)\end{array}$ & $\begin{array}{c}\text { RM-HG-3 } \\
\text { (CFM) }\end{array}$ & $\begin{array}{l}\text { R/LHG-4 } \\
\text { (CFM) }\end{array}$ & $\begin{array}{c}\text { FT-HG-1 } \\
\text { (CFM) }\end{array}$ & $\begin{array}{l}F T-H G-2 \\
\text { (CFM) }\end{array}$ \\
\hline $\begin{array}{ll}3.2 .1 & 1 \\
3.2 . & 2 \\
3.2 . & 3 \\
3.2 . & 4 \\
3.2 . & 5\end{array}$ & $\begin{array}{l}20.0 \\
30.00 \\
30.0 \\
30.0 \\
20.0\end{array}$ & $\begin{array}{r}0.0 \\
10.0 \\
10.0 \\
0.0 \\
0.0\end{array}$ & $\begin{array}{l}0.0 \\
0.0 \\
0.0 \\
0.0 \\
0.0\end{array}$ & $\begin{array}{l}0.0 \\
0.0 \\
0.0 \\
0.0 \\
0.0\end{array}$ & $\begin{array}{l}0.0 \\
0.0 \\
0.0 \\
0.0 \\
0.0\end{array}$ & $\begin{array}{l}0.0 \\
0.0 \\
0.0 \\
0.0 \\
0.0\end{array}$ & $\begin{array}{l}0.0 \\
0.0 \\
0.0 \\
0.0 \\
0.0\end{array}$ & $\begin{array}{l}0.0 \\
0.0 \\
0.0 \\
0.0 \\
0.0\end{array}$ & $\begin{array}{l}0.0 \\
0.0 \\
0.0 \\
0.0 \\
0.0\end{array}$ & $\begin{array}{l}0.0 \\
0.0 \\
0.0 \\
0.0 \\
0.0\end{array}$ \\
\hline $\begin{array}{l}3.2 .6 \\
3.2 .7 \\
3.2 .8 \\
3.2 .9 \\
3.2 .10\end{array}$ & $\begin{array}{l}30.0 \\
28.0 \\
33.0 \\
24.0 \\
30.0\end{array}$ & $\begin{array}{r}10.0 \\
0.0 \\
10.0 \\
0.0 \\
10.0\end{array}$ & $\begin{array}{l}0.0 \\
0.0 \\
0.0 \\
0.0 \\
0.0\end{array}$ & $\begin{array}{l}0.0 \\
0.0 \\
0.0 \\
0.0 \\
0.0\end{array}$ & $\begin{array}{l}0.0 \\
0.0 \\
0.0 \\
0.0 \\
0.0\end{array}$ & $\begin{array}{l}0.0 \\
0.00 \\
0.0 \\
0.0 \\
0.0\end{array}$ & $\begin{array}{r}5.00 \\
5.00 \\
10.00 \\
10.00 \\
15.00\end{array}$ & $\begin{array}{l}0.0 \\
0.0 \\
0.0 \\
0.0 \\
0.0\end{array}$ & $\begin{array}{l}0.0 \\
0.0 \\
0.0 \\
0.0 \\
0.0\end{array}$ & $\begin{array}{l}0.0 \\
0.0 \\
0.0 \\
0.0 \\
0.0\end{array}$ \\
\hline $\begin{array}{l}3.2 .11 \\
3.2 .12 \\
3.2 .13 \\
3.2 .14 \\
3.2 .15\end{array}$ & $\begin{array}{l}23.0 \\
33.0 \\
15.0 \\
3.0 .0 \\
12.0\end{array}$ & $\begin{array}{r}0.0 \\
10.0 \\
0.0 \\
10.0 \\
0.0\end{array}$ & $\begin{array}{l}0.0 \\
0.0 \\
0.0 \\
0.0 \\
0.0\end{array}$ & $\begin{array}{l}0.0 \\
0.0 \\
0.0 \\
0.0 \\
0.0\end{array}$ & $\begin{array}{c}0.0 \\
0.0 \\
0.0 \\
30.00 \\
30.00\end{array}$ & $\begin{array}{l}0.0 \\
0.0 \\
0.0 \\
0.0 \\
0.0\end{array}$ & $\begin{array}{c}15.00 \\
20.00 \\
20.00 \\
0.0 \\
0.0\end{array}$ & $\begin{array}{l}0.0 \\
0.0 \\
0.0 \\
0.0 \\
0.0\end{array}$ & $\begin{array}{l}0.0 \\
0.0 \\
0.0 \\
0.0 \\
0.0\end{array}$ & $\begin{array}{l}0.0 \\
0.0 \\
0.0 \\
0.0 \\
0.0\end{array}$ \\
\hline $\begin{array}{l}3.2 .16 \\
3.2 .17 \\
3.2 .18 \\
3.2 .17 \\
3.2 .20\end{array}$ & $\begin{array}{l}30.0 \\
10.0 \\
30.0 \\
3.0 \\
30.0\end{array}$ & $\begin{array}{r}10.0 \\
0.0 \\
10.0 \\
8.0 \\
10.0\end{array}$ & $\begin{array}{l}0.0 \\
0.0 \\
0.0 \\
0.0 \\
0.0\end{array}$ & $\begin{array}{l}0.0 \\
0.0 \\
0.0 \\
0.0 \\
0.0\end{array}$ & $\begin{array}{l}40.00 \\
40.00 \\
50.00 \\
50.00 \\
60.00\end{array}$ & $\begin{array}{l}0.0 \\
0.01 \\
0.0 \\
0.0 \\
0.0\end{array}$ & $\begin{array}{l}0.0 \\
0.0 \\
0.0 \\
0.0 \\
0.0\end{array}$ & $\begin{array}{l}0.0 \\
0.0 \\
0.0 \\
0.0 \\
0.0\end{array}$ & $\begin{array}{l}0.0 \\
0.0 \\
0.0 \\
0.0 \\
0.0\end{array}$ & $\begin{array}{l}0.0 \\
0.0 \\
0.0 \\
0.0 \\
0.0\end{array}$ \\
\hline $\begin{array}{l}3.2 .21 \\
3.2 .22 \\
3.2 .23 \\
3.2 .24 \\
3.2 .25\end{array}$ & $\begin{array}{r}0.0 \\
30.0 \\
3.0 \\
30.0 \\
20.0\end{array}$ & $\begin{array}{r}6.0 \\
10.0 \\
5.0 \\
10.0 \\
0.0\end{array}$ & $\begin{array}{l}0.0 \\
0.0 \\
0.0 \\
0.0 \\
0.0\end{array}$ & $\begin{array}{l}0.0 \\
0.0 \\
0.0 \\
0.0 \\
0.0\end{array}$ & $\begin{array}{c}60.00 \\
70.00 \\
70.00 \\
0.0 \\
0.0\end{array}$ & $\begin{array}{l}0.0 \\
0.0 \\
0.0 \\
0.0 \\
0.0\end{array}$ & $\begin{array}{l}0.0 \\
0.0 \\
0.0 \\
5.00 \\
5.00\end{array}$ & $\begin{array}{l}0.0 \\
0.0 \\
0.0 \\
0.0 \\
0.0\end{array}$ & $\begin{array}{l}0.0 \\
0.0 \\
0.0 \\
0.0 \\
0.0\end{array}$ & $\begin{array}{l}0.0 \\
0.0 \\
0.0 \\
0.0 \\
0.0\end{array}$ \\
\hline$\underset{\text { NOST }}{\text { TeST }}$ & $\begin{array}{l}P-C W-1 \\
|P S| G \mid\end{array}$ & $\begin{array}{l}P-C H-2 \\
(P S I G)\end{array}$ & $\begin{array}{l}\text { P-HG-1 } \\
\text { (PSIG) }\end{array}$ & $\begin{array}{l}P-H G-2 \\
(P S I G)\end{array}$ & $\begin{array}{l}P-H G-3 \\
(P S(G)\end{array}$ & $\begin{array}{l}T F-C H-1 \\
(D E G F)\end{array}$ & $\begin{array}{l}\text { TF }-1+15-1 \\
\text { (DEG F) }\end{array}$ & $\begin{array}{l}\text { TF-HF, } \\
\text { IDES FI }\end{array}$ & $\begin{array}{l}\text { TF-HC-3 } \\
\text { (DEG F) }\end{array}$ & $\begin{array}{l}\text { P-BARO } \\
\text { (PSIA) }\end{array}$ \\
\hline $\begin{array}{ll}3.2 . & 1 \\
3.2 .2 & 2 \\
3.2 . & 3 \\
3.2 .2 & 4 \\
3.2 . & 5\end{array}$ & $\begin{array}{l}0.0 \\
0.0 \\
0.0 \\
0.0 \\
0.0\end{array}$ & $\begin{array}{l}11.0 \\
11.0 \\
10.5 \\
11.5 \\
11.00\end{array}$ & $\begin{array}{r}9.0 \\
10.0 \\
9.5 \\
10.5 \\
9.0\end{array}$ & $\begin{array}{l}0.0 \\
0.0 \\
0.0 \\
0.0 \\
0.0\end{array}$ & $\begin{array}{l}0.0 \\
0.0 \\
0.0 \\
0.0 \\
0.0\end{array}$ & $\begin{array}{l}56.0 \\
36.5 \\
55.5 \\
56.5 \\
57.0\end{array}$ & $\begin{array}{l}80.5 \\
81.0 \\
83.5 \\
76.5 \\
77.5\end{array}$ & $\begin{array}{l}0.0 \\
0.0 \\
0.0 \\
0.0 \\
0.0\end{array}$ & $\begin{array}{l}0.0 \\
0.0 \\
0.0 \\
0.0 \\
0.0\end{array}$ & $\begin{array}{l}12.5 \\
12.5 \\
12.4 \\
12.4 \\
12.4\end{array}$ \\
\hline
\end{tabular}




\section{TABLE XXVII (Contd.)}

COMBINED PFFECTS TEST GROUP 3.2

EXPERIYENTAL MEASURMENTS FOR TESTS 3.2.1 TOI 3.2.25 -- 0.39-IN. DOWNCOMER BAP

\begin{tabular}{|c|c|c|c|c|c|c|c|c|c|c|}
\hline $\begin{array}{l}\text { TEST } \\
\text { NO }\end{array}$ & $\begin{array}{l}P-C H-1 \\
(P S I G)\end{array}$ & $\begin{array}{l}P-C, H-2 \\
|P S| G \mid\end{array}$ & $\begin{array}{l}\text { P-HG-1 } \\
\text { (PSIG) }\end{array}$ & $\begin{array}{l}\text { P-HG-2 } \\
(P S I G S)\end{array}$ & $\begin{array}{l}P-H G-3 \\
\text { (PSIG) }\end{array}$ & $\begin{array}{l}T F-C H-1 \\
(D E G F)\end{array}$ & $\begin{array}{l}\text { TF-HG-1 } \\
\text { (OEG F) }\end{array}$ & $\begin{array}{l}\text { TF-HC, } \\
\text { IDEG FI }\end{array}$ & $\begin{array}{l}\text { TF-HG-3 } \\
\text { (DEG F) }\end{array}$ & $\begin{array}{l}\text { P-BARR } \\
\text { IPSIA }\end{array}$ \\
\hline $\begin{array}{l}3.2 .0 \\
3.2 .7 \\
3.2 .08 \\
3.2 .9 \\
3.2 .10\end{array}$ & $\begin{array}{l}0.0 \\
j .0 \\
0.0 \\
j .0 \\
j .0\end{array}$ & $\begin{array}{l}10.0 \\
10.0 \\
10.5 \\
10.0 \\
10.0\end{array}$ & $\begin{array}{r}9.0 \\
9.5 \\
10.0 \\
10.5 \\
9.5\end{array}$ & $\begin{array}{l}0.0 \\
0.0 \\
0.0 \\
. .0 \\
0.0\end{array}$ & $\begin{array}{l}0.0 \\
0.0 \\
0.0 \\
0.0 \\
0.0\end{array}$ & $\begin{array}{l}53.0 \\
55.0 \\
55.0 \\
57.0 \\
55.5\end{array}$ & $\begin{array}{l}78.0 \\
77.0 \\
76.5 \\
75.0 \\
76.0\end{array}$ & $\begin{array}{l}0.0 \\
0.0 \\
0.0 \\
0.0 \\
0.0\end{array}$ & $\begin{array}{l}0.0 \\
0.0 \\
0.0 \\
0.0 \\
0.0\end{array}$ & $\begin{array}{l}12.5 \\
12.5 \\
12.5 \\
12.5 \\
12.5\end{array}$ \\
\hline $\begin{array}{l}3.2 .11 \\
3.2 .12 \\
3.2 .13 \\
3.2 .14 \\
3.2 .115\end{array}$ & $\begin{array}{l}3.0 \\
3.0 \\
.00 \\
3.0 \\
0.0\end{array}$ & $\begin{array}{r}11.0 \\
11.0 \\
9.0 \\
11.0 \\
10.5\end{array}$ & $\begin{array}{r}9.0 \\
10.5 \\
9.5 \\
10.0 \\
9.5\end{array}$ & $\begin{array}{l}0.0 \\
0.0 \\
0.0 \\
0.0 \\
0.0\end{array}$ & $\begin{array}{l}0.0 \\
0.0 \\
0.0 \\
0.0 \\
0.0\end{array}$ & $\begin{array}{l}56.0 \\
55.5 \\
57.0 \\
55.0 \\
56.0\end{array}$ & $\begin{array}{l}76.0 \\
76.0 \\
76.0 \\
76.0 \\
76.0\end{array}$ & $\begin{array}{l}0.0 \\
0.0 \\
0.0 \\
0.0 \\
0.0\end{array}$ & $\begin{array}{l}0.0 \\
0.0 \\
0.0 \\
0.0 \\
0.0\end{array}$ & $\begin{array}{l}12.5 \\
12.5 \\
12.5 \\
12.5 \\
12.5\end{array}$ \\
\hline $\begin{array}{l}3.2 .16 \\
3.2 .17 \\
3.2 .18 \\
3.2 .19 \\
3.2 .23\end{array}$ & $\begin{array}{l}0.0 \\
0.0 \\
10.0 \\
0.0 \\
0.0\end{array}$ & $\begin{array}{r}10.0 \\
10.0 \\
10.0 \\
3.0 \\
10.0\end{array}$ & $\begin{array}{r}9.5 \\
10.0 \\
10.0 \\
9.0 \\
9.0\end{array}$ & $\begin{array}{l}0.0 \\
0.0 \\
0.0 \\
0.0 \\
0.0\end{array}$ & $\begin{array}{l}0.0 \\
0.0 \\
0.0 \\
0.0 \\
0.0\end{array}$ & $\begin{array}{l}55.0 \\
55.5 \\
55.5 \\
57.0 \\
56.0\end{array}$ & $\begin{array}{l}78.0 \\
79.0 \\
81.0 \\
84.0 \\
85.0\end{array}$ & $\begin{array}{l}0.0 \\
0.0 \\
0.0 \\
0.0 \\
0.0\end{array}$ & $\begin{array}{l}0.0 \\
0.0 \\
0.0 \\
0.0 \\
0.0\end{array}$ & $\begin{array}{l}12.5 \\
12.5 \\
12.5 \\
12.5 \\
12.5\end{array}$ \\
\hline $\begin{array}{l}3.2 .21 \\
3.2 .22 \\
3.2 .23 \\
3.2 .24 \\
3.2 .25\end{array}$ & $\begin{array}{l}0.0 \\
0.0 \\
0.0 \\
1.0 \\
0.0\end{array}$ & $\begin{array}{r}8.5 \\
9.0 \\
8.5 \\
11.0 \\
9.5\end{array}$ & $\begin{array}{l}9.0 \\
9.5 \\
9.0 \\
9.5 \\
9.0\end{array}$ & $\begin{array}{l}0.0 \\
0.0 \\
0.0 \\
0.0 \\
0.0\end{array}$ & $\begin{array}{l}0.0 \\
0.0 \\
0.0 \\
0.0 \\
0.0\end{array}$ & $\begin{array}{l}56.0 \\
56.0 \\
50.0 \\
56.0 \\
56.0\end{array}$ & $\begin{array}{l}86.0 \\
88.5 \\
91.0 \\
76.5 \\
79.0\end{array}$ & $\begin{array}{l}0.0 \\
0.0 \\
0.0 \\
0.0 \\
0.0\end{array}$ & $\begin{array}{l}0.0 \\
0.0 \\
0.0 \\
0.0 \\
0.0\end{array}$ & $\begin{array}{l}12.5 \\
12.5 \\
12.5 \\
12.5 \\
12.5\end{array}$ \\
\hline
\end{tabular}

\begin{tabular}{|c|c|c|c|c|c|c|c|c|c|}
\hline \multirow[b]{2}{*}{$\begin{array}{l}\text { TESTT } \\
\text { NO }\end{array}$} & \multicolumn{2}{|r|}{ LOWER PLENUM } & FLOW & \multicolumn{3}{|c|}{ BYPASS FLUW } & \multirow[b]{2}{*}{$\begin{array}{l}\text { LONER } \\
\text { PLENUM } \\
\text { LEVEL } \\
\text { IINI }\end{array}$} & \multirow[b]{2}{*}{$\begin{array}{l}P-L P-1 \\
\text { (PSIGI) }\end{array}$} & \multirow[b]{2}{*}{$\begin{array}{l}\text { IF } L P P-1 \\
\text { IOEG F }\end{array}$} \\
\hline & $\begin{array}{l}\text { INITIAL } \\
\text { WEIGHT } \\
\text { (LB). }\end{array}$ & $\begin{array}{l}\text { FINAL } \\
\text { :NEIGHI } \\
\text { (LB) }\end{array}$ & $\begin{array}{l}\text { TIME DF } \\
\text { COLLECTICN } \\
\text { ISECI }\end{array}$ & $\begin{array}{l}\text { INITIAL } \\
\text { WE IGHT } \\
\text { (LB) }\end{array}$ & $\begin{array}{l}\text { FINAL } \\
\text { HEI GHT } \\
\text { (LB) }\end{array}$ & $\begin{array}{l}\text { TIME JF } \\
\text { COLLECTIJN } \\
\text { ISEC) }\end{array}$ & & & \\
\hline $\begin{array}{ll}3.2 \cdot 1 \\
3.2 .2 \\
3.2 . & 3 \\
3.2 .2 & 4 \\
3.2 .5\end{array}$ & $\begin{array}{l}213.5 \\
135.5 \\
290.0 \\
384.5 \\
443.5\end{array}$ & $\begin{array}{l}279.0 \\
218.5 \\
384.5 \\
445.5 \\
516.8\end{array}$ & $\begin{array}{l}25.0 \\
30.0 \\
25.0 \\
20.0 \\
30.0\end{array}$ & $\begin{array}{l}160.8 \\
104.5 \\
493.5 \\
576.3 \\
629.5\end{array}$ & $\begin{array}{l}175.5 \\
160.8 \\
576.3 \\
629.5 \\
653.3\end{array}$ & $\begin{array}{l}40.0 \\
20.0 \\
45.0 \\
50.0 \\
65.0\end{array}$ & $\begin{array}{l}12.0 \\
12.0 \\
12.0 \\
12.0 \\
12.0\end{array}$ & $\begin{array}{l}10.0 \\
10.0 \\
10.0 \\
10.0 \\
10.0\end{array}$ & $\begin{array}{l}56.0 \\
59.0 \\
56.0 \\
56.5 \\
57.0\end{array}$ \\
\hline $\begin{array}{l}3.2 .0 \\
3.2 .9 \\
3.2 .8 \\
3.2 .9 \\
3.2 .10\end{array}$ & $\begin{array}{l}134.3 \\
232.5 \\
316.5 \\
36.5 \\
419.5\end{array}$ & $\begin{array}{l}232.5 \\
316.5 \\
368.3 \\
416.5 \\
.457 .8\end{array}$ & $\begin{array}{l}30.0 \\
30.0 \\
20.0 \\
20.0 \\
20.0\end{array}$ & $\begin{array}{r}90.5 \\
170.0 \\
222.5 \\
338.5 \\
373.5\end{array}$ & $\begin{array}{l}170.0 \\
222.5 \\
338.5 \\
373.5 \\
479.5\end{array}$ & $\begin{array}{l}35.0 \\
50.0 \\
40.0 \\
35.0 \\
30.0\end{array}$ & $\begin{array}{l}12.0 \\
12.0 \\
12.0 \\
12.0 \\
12.0\end{array}$ & $\begin{array}{l}10.0 \\
10.0 \\
10.0 \\
10.0 \\
10.0\end{array}$ & $\begin{array}{l}53.5 \\
55.0 \\
55.0 \\
57.0 \\
55.5\end{array}$ \\
\hline $\begin{array}{l}3.2 .11 \\
3.2 .12 \\
3.2 .13 \\
3.2 .14 \\
3.2 .15\end{array}$ & $\begin{array}{l}437.8 \\
442.8 \\
478.5 \\
135.8 \\
183.8\end{array}$ & $\begin{array}{l}508.0 \\
478.5 \\
512.0 \\
183.8 \\
217.5\end{array}$ & $\begin{array}{l}25.0 \\
20.0 \\
20.0 \\
40.4 \\
30.0\end{array}$ & $\begin{array}{r}479.5 \\
592.5 \\
672.5 \\
94.5 \\
203.5\end{array}$ & $\begin{array}{l}512.5 \\
672.0 \\
693.5 \\
203.5 \\
231.0\end{array}$ & $\begin{array}{l}40.0 \\
20.0 \\
45.0 \\
25.0 \\
45.0\end{array}$ & $\begin{array}{l}12.0 \\
12.0 \\
12.0 \\
12.0 \\
12.0\end{array}$ & $\begin{array}{l}10.0 \\
10.0 \\
10.0 \\
10.0 \\
10.0\end{array}$ & $\begin{array}{l}56.0 \\
57.0 \\
57.0 \\
611.0 \\
56.0\end{array}$ \\
\hline
\end{tabular}


TABLE XXVII (Contd.)

COMBINED EFFECTS TEST GROUP 3.2

EXPERIMENTAL MEASURMENTS FOR TESTS 3.2.1 TO 3.2.25 -- 0.39-IN. DOMNCOMER GAP

\begin{tabular}{|c|c|c|c|c|c|c|c|c|c|}
\hline \multirow[b]{2}{*}{$\begin{array}{l}\text { TEST } \\
\text { NO }\end{array}$} & \multicolumn{3}{|c|}{$\begin{array}{l}\text { LOWER PLENUM FLOH } \\
\text { L }\end{array}$} & \multicolumn{3}{|c|}{ BYPASS FLOH } & \multirow[b]{2}{*}{$\begin{array}{l}\text { LUWER } \\
\text { PLENIIM } \\
\text { LEVEL } \\
\text { (IN) }\end{array}$} & \multirow[b]{2}{*}{$\begin{array}{l}P-(P-1) \\
(P S I G)\end{array}$} & \multirow[b]{2}{*}{$\begin{array}{l}\text { IF-LP-1 } \\
\text { IOEG FI }\end{array}$} \\
\hline & $\begin{array}{l}\text { INITIAL } \\
\text { WE I GHT } \\
\text { (LB) }\end{array}$ & $\begin{array}{l}\text { FINAL } \\
\text { WEIGHT } \\
\text { (LB) }\end{array}$ & $\begin{array}{l}\text { T IME OF } \\
\text { COLLECTICN } \\
\text { (SEC) }\end{array}$ & $\begin{array}{l}\text { INITIAL } \\
\text { WEIGHT } \\
\text { (LB) }\end{array}$ & $\begin{array}{l}\text { FINAL } \\
\text { WEIGHT } \\
\text { (LB) }\end{array}$ & $\begin{array}{l}\text { TIME OF } \\
\text { COLLECTION } \\
\text { ISEC) }\end{array}$ & & & \\
\hline $\begin{array}{l}3.2: 16 \\
3.2 .17 \\
3.2 .18 \\
3.2 .19 \\
3.2 .20\end{array}$ & $\begin{array}{l}217.5 \\
242.5 \\
272.5 \\
289.3 \\
308.0\end{array}$ & $\begin{array}{l}242.5 \\
272.5 \\
289.3 \\
308.0 \\
325.5\end{array}$ & $\begin{array}{l}30.0 \\
40.0 \\
35.0 \\
40.0 \\
45.0\end{array}$ & $\begin{array}{l}231.0 \\
352.0 \\
384.3 \\
563.0 \\
588.5\end{array}$ & $\begin{array}{l}352.0 \\
384.3 \\
563.0 \\
588.5 \\
696.0\end{array}$ & $\begin{array}{l}25.0 \\
45.0 \\
35.0 \\
40.0 \\
20.0\end{array}$ & $\begin{array}{l}12.0 \\
12.0 \\
12.0 \\
12.0 \\
12.0\end{array}$ & $\begin{array}{l}10.0 \\
10.0 \\
10.0 \\
10.0 \\
10.0\end{array}$ & $\begin{array}{l}57.0 \\
55.5 \\
56.5 \\
56.5 \\
57.5\end{array}$ \\
\hline $\begin{array}{l}3.2 .21 \\
3.2 .22 \\
3.2 .23 \\
3.2 .24 \\
3.2 .25\end{array}$ & $\begin{array}{l}325.5 \\
339.5 \\
354.3 \\
279.0 \\
337.5\end{array}$ & $\begin{array}{l}339.5 \\
354.3 \\
365.8 \\
337.5 \\
409.0\end{array}$ & $\begin{array}{l}45.0 \\
65.0 \\
60.0 \\
25.0 \\
30.0\end{array}$ & $\begin{array}{l}696.0 \\
716.5 \\
822.0 \\
175.5 \\
240.5\end{array}$ & $\begin{array}{r}716.5 \\
822.0 \\
846.0 \\
240.5 \\
257.5\end{array}$ & $\begin{array}{l}40.0 \\
20.0 \\
50.0 \\
20.0 \\
40.0\end{array}$ & $\begin{array}{l}12.0 \\
12.0 \\
12.0 \\
12.0 \\
12.0\end{array}$ & $\begin{array}{l}10.0 \\
10.0 \\
10.0 \\
10.0 \\
10.0\end{array}$ & $\begin{array}{l}58.0 \\
57.5 \\
57.5 \\
56.0 \\
56.0\end{array}$ \\
\hline
\end{tabular}

\begin{tabular}{|c|c|c|c|c|c|c|c|}
\hline $\begin{array}{l}\text { TEST } \\
\text { NO }\end{array}$ & $\begin{array}{l}R M-C G-1 \\
(C F M)\end{array}$ & $\begin{array}{c}R M-C G-2 \\
(C F M)\end{array}$ & $\begin{array}{c}F T-C G-1 \\
(C F M)\end{array}$ & $\begin{array}{l}P-C G-1 \\
|P S| G \mid\end{array}$ & $\begin{array}{l}\text { P-CG-2 } \\
\text { (PSIGI }\end{array}$ & $\begin{array}{l}\text { TF-CG-1 } \\
\text { (DEG F) }\end{array}$ & $\begin{array}{l}\text { TF-CG-2 } \\
(D E G F)\end{array}$ \\
\hline $\begin{array}{l}3.2 \cdot 1 \\
3.2 \cdot 2 \\
3.2 \cdot 3 \\
3.2 .4 \\
3.2 .5\end{array}$ & $\begin{array}{l}60.0 \\
60.0 \\
30.0 \\
30.0 \\
30.0\end{array}$ & $\begin{array}{l}0.0 \\
0.0 \\
0.0 \\
0.0 \\
0.0\end{array}$ & $\begin{array}{l}0.0 \\
0.0 \\
0.0 \\
0.0 \\
0.0\end{array}$ & $\begin{array}{l}0.0 \\
0.0 \\
0.0 \\
0.0 \\
0.0\end{array}$ & $\begin{array}{l}0.0 \\
0.0 \\
0.0 \\
0.0 \\
0.0\end{array}$ & $\begin{array}{l}0.0 \\
0.0 \\
0.0 \\
0.0 \\
0.0\end{array}$ & $\begin{array}{l}0.0 \\
0.0 \\
0.0 \\
0.0 \\
0.0\end{array}$ \\
\hline $\begin{array}{l}3.2 .6 \\
3.2 .9 \\
3.2 .3 \\
3.2 .9 \\
3.2 .10\end{array}$ & $\begin{array}{l}30.0 \\
30.0 \\
30.0 \\
30.0 \\
30.0\end{array}$ & $\begin{array}{l}0.0 \\
0.0 \\
0.0 \\
0.0 \\
0.0\end{array}$ & $\begin{array}{l}0.0 \\
0.0 \\
0.0 \\
0.0 \\
0.0\end{array}$ & $\begin{array}{l}0.0 \\
0.0 \\
0.0 \\
0.0 \\
0.0\end{array}$ & $\begin{array}{l}0.0 \\
0.0 \\
0.0 \\
0.0 \\
0.0\end{array}$ & $\begin{array}{l}0.0 \\
0.0 \\
0.0 \\
0.0 \\
0.0\end{array}$ & $\begin{array}{l}0.0 \\
0.0 \\
0.0 \\
0.0 \\
0.0\end{array}$ \\
\hline $\begin{array}{l}3.2 .11 \\
3.2 .12 \\
3.2 .13 \\
3.2 .14 \\
3.2 .15\end{array}$ & $\begin{array}{l}30.0 \\
30.0 \\
30.0 \\
30.0 \\
30.0\end{array}$ & $\begin{array}{l}0.0 \\
0.0 \\
0.0 \\
0.0 \\
0.0\end{array}$ & $\begin{array}{l}0.0 \\
0.0 \\
0.0 \\
0.0 \\
0.0\end{array}$ & $\begin{array}{l}0.0 \\
0.0 \\
0.0 \\
0.0 \\
0.0\end{array}$ & $\begin{array}{l}0.0 \\
0.0 \\
0.0 \\
0.0 \\
0.0\end{array}$ & $\begin{array}{l}0.0 \\
0.0 \\
0.0 \\
0.0 \\
0.0\end{array}$ & $\begin{array}{l}0.0 \\
0.0 \\
0.0 \\
0.0 \\
0.0\end{array}$ \\
\hline $\begin{array}{l}3.2 .16 \\
3.2 .17 \\
3.2 .18 \\
3.2 .19 \\
3.2 .20\end{array}$ & $\begin{array}{l}30.0 \\
30.0 \\
30.0 \\
30.0 \\
30.0\end{array}$ & $\begin{array}{l}0.0 \\
0.0 \\
0.0 \\
0.0 \\
0.0\end{array}$ & $\begin{array}{l}0.0 \\
0.0 \\
0.0 \\
0.0 \\
0.0\end{array}$ & $\begin{array}{l}0.0 \\
0.0 \\
0.0 \\
0.0 \\
0.0\end{array}$ & $\begin{array}{l}0.0 \\
0.0 \\
0.0 \\
0.0 \\
0.0\end{array}$ & $\begin{array}{l}0.0 \\
0.0 \\
0.0 \\
0.0 \\
0.0\end{array}$ & $\begin{array}{l}0.0 \\
0.0 \\
0.0 \\
0.0 \\
0.0\end{array}$ \\
\hline $\begin{array}{l}3.2 .21 \\
3.2 .22 \\
3.2 .23 \\
3.2 .24 \\
3.2 .25\end{array}$ & $\begin{array}{r}30.0 \\
30.0 \\
30.0 \\
60.0 \\
60.0\end{array}$ & $\begin{array}{l}0.0 \\
0.0 \\
0.0 \\
0.0 \\
0.0\end{array}$ & $\begin{array}{l}0.0 \\
0.0 \\
0.0 \\
0.0 \\
0.0\end{array}$ & $\begin{array}{l}0.0 \\
0.0 \\
0.0 \\
0.0 \\
0.0\end{array}$ & $\begin{array}{l}0.0 \\
0.0 \\
0.0 \\
0.0 \\
0.0\end{array}$ & $\begin{array}{l}0.0 \\
0.0 \\
0.0 \\
0.0 \\
0.0\end{array}$ & $\begin{array}{l}0.0 \\
0.0 \\
0.0 \\
0.0 \\
0.0\end{array}$ \\
\hline
\end{tabular}




\section{TABLE XXXVIII}

COMBINED EFFECTS TEST GROUP 4.3

TRANSPARENT VESSCL TEST DATA FOR TESTS 4.3.1 TIO 4.3. $5--0.49-I N$. DOHNCOMFR GAP SUMMARY OF CALCULATEN RESULTS

\begin{tabular}{|c|c|c|c|c|c|c|c|c|c|c|}
\hline $\begin{array}{c}T E S T \\
\text { No }\end{array}$ & $\begin{array}{l}\text { DOWNCOAER } \\
\text { AIR FLONW } \\
\text { (LB/SEC) }\end{array}$ & $\begin{array}{l}\text { DCWNCDMEP } \\
\text { A!R DENSITY } \\
\left.\text { (L B/FT: } \$ 3^{\circ}\right)\end{array}$ & $\begin{array}{l}\text { LUWER PLENUM } \\
\text { PKESSURE } \\
\text { (PSIA) }\end{array}$ & $\begin{array}{l}\text { COLD LES, } \\
\text { AIR FLOW } \\
\text { (LS/SFC) }\end{array}$ & $\begin{array}{l}\text { DOWNCDMEP. } \\
\text { WATER FLOW } \\
\text { (LB/SEC) }\end{array}$ & $\begin{array}{l}\text { WATER FLOH } \\
\text { CONTINUITY }\end{array}$ & $\begin{array}{c}\text { J-AIR } \\
\text { DWWCAMEP. } \\
\text { (FT/SEC.) }\end{array}$ & $\begin{array}{l}\text { J-WATER } \\
\text { DOWNC JMER } \\
\text { (FT/SCCI }\end{array}$ & $(J G *) * 1 / 2$ & $(1 L *) * * 1 /$ \\
\hline $\begin{array}{l}4.3 \cdot 1 \\
4.3 \cdot 3 \\
4.3 \cdot 3 \\
4.3 \cdot 3 \\
4.3 .5\end{array}$ & $\begin{array}{l}0.0299 \\
0.0460 \\
0.0526 \\
0.0619 \\
0.0089\end{array}$ & $\begin{array}{l}0.1170 \\
0.1161 \\
0.1162 \\
0.1164 \\
0.1164\end{array}$ & $\begin{array}{l}22.33 \\
22.33 \\
22.33 \\
22.33 \\
22.33\end{array}$ & $\begin{array}{l}0.0 \\
0.0 \\
0.0 \\
0.0 \\
0.0\end{array}$ & $\begin{array}{l}5.352 \\
5.548 \\
5.429 \\
5.525 \\
5.362\end{array}$ & $\begin{array}{l}0.962 \\
1.001 \\
0.986 \\
1.007 \\
0.996\end{array}$ & $\begin{array}{l}3.96 \\
6.16 \\
7.03 \\
8.27 \\
9.20\end{array}$ & $\begin{array}{l}1.332 \\
1.391 \\
1.351 \\
1.375 \\
1.335\end{array}$ & $\begin{array}{l}0.3252 \\
0.4045 \\
0.4323 \\
0.4690 \\
0.44946\end{array}$ & $\begin{array}{l}0.9059 \\
0.9223 \\
0.9123 \\
0.9204 \\
0.9068\end{array}$ \\
\hline
\end{tabular}


TABLE XXXVIII (Contd.)

COMBINED EFFECTS TEST GROUP 4.3

EXPERIMENTAL MEASURMENTS FOR TESTS 4.3 .1 TO $4.3 .5-0.49-$ IN. DOHNCOMER GAP

\begin{tabular}{|c|c|c|c|c|c|c|c|c|c|c|}
\hline $\begin{array}{l}\text { TEST } \\
\text { NO }\end{array}$ & $\begin{array}{c}R M-C H-I \\
(G P M)\end{array}$ & $\begin{array}{c}R M-C W-2 \\
(G P M)\end{array}$ & $\begin{array}{c}F T-C M-1 \\
(G P M)\end{array}$ & $\begin{array}{c}F T-C H-2 \\
(G P M)\end{array}$ & $\begin{array}{c}R M-H G-1 \\
(C F M)\end{array}$ & $\begin{array}{c}\text { RM-HG-2 } \\
\text { (CFM) }\end{array}$ & $\begin{array}{c}R M-H G-3 \\
(C F M)\end{array}$ & $\begin{array}{c}\text { RM-HG-4 } \\
\text { (CFM) }\end{array}$ & $\begin{array}{c}F T-H G-1 \\
(C F M)\end{array}$ & $\begin{array}{l}F T-H G-2 \\
\text { (CFM) }\end{array}$ \\
\hline $\begin{array}{l}4.3 \cdot 1 \\
4.3 \cdot 2 \\
4.3 \cdot 3 \\
4.3 \cdot 4 \\
4.3 \cdot 5\end{array}$ & $\begin{array}{l}30.0 \\
30.0 \\
30.0 \\
30.0 \\
30.0\end{array}$ & $\begin{array}{l}10.0 \\
10.0 \\
10.0 \\
10.0 \\
10.0\end{array}$ & $\begin{array}{l}0.0 \\
0.0 \\
0.0 \\
0.0 \\
0.0\end{array}$ & $\begin{array}{l}0.0 \\
0.0 \\
0.0 \\
0.0 \\
0.0\end{array}$ & $\begin{array}{l}0.0 \\
0.0 \\
0.0 \\
0.0 \\
0.0\end{array}$ & $\begin{array}{l}20.00 \\
30.00 \\
35.00 \\
40.00 \\
45.00\end{array}$ & $\begin{array}{l}0.0 \\
0.0 \\
0.0 \\
0.0 \\
0.0\end{array}$ & $\begin{array}{l}0.0 \\
0.0 \\
0.0 \\
0.0 \\
0.0\end{array}$ & $\begin{array}{l}0.0 \\
0.0 \\
0.0 \\
0.0 \\
0.0\end{array}$ & $\begin{array}{l}0.0 \\
0.0 \\
0.0 \\
0.0 \\
0.0\end{array}$ \\
\hline $\begin{array}{l}\text { TEST } \\
\text { VO }\end{array}$ & $\begin{array}{l}P-C W-1 \\
\text { (PSIGI }\end{array}$ & $\begin{array}{l}P-C H-2 \\
(P S I G)\end{array}$ & $\begin{array}{l}P-H G-1 \\
(P S I G)\end{array}$ & $\begin{array}{l}P-H G-2 \\
(P S I G)\end{array}$ & $\begin{array}{l}P-H G-3 \\
\text { (PSIG) }\end{array}$ & $\begin{array}{l}\text { TF-CH-1 } \\
\text { (DEG FI }\end{array}$ & $\begin{array}{l}\text { TF-HS-I } \\
\text { (DEG F) }\end{array}$ & $\begin{array}{l}\text { TE-HG-2 } \\
\text { (DEG FI }\end{array}$ & $\begin{array}{l}\text { TF-HG-3 } \\
\text { (DEG F) }\end{array}$ & $\begin{array}{l}\text { P-BARn } \\
\text { (PSIA) }\end{array}$ \\
\hline $\begin{array}{l}4 \cdot 3 \cdot 1 \\
4 \cdot 3 \cdot 2 \\
4 \cdot 3 \cdot 3 \\
4 \cdot 3 \cdot 4 \\
4 \cdot 3 \cdot 5\end{array}$ & $\begin{array}{l}0.0 \\
0.0 \\
0.0 \\
0.0 \\
0.0\end{array}$ & $\begin{array}{l}9.7 \\
10.5 \\
11.7 \\
11.0 \\
10.2\end{array}$ & $\begin{array}{r}9.0 \\
10.0 \\
9.0 \\
10.2 \\
9.7\end{array}$ & $\begin{array}{l}0.0 \\
0.0 \\
0.0 \\
0.0 \\
0.0\end{array}$ & $\begin{array}{l}0.0 \\
0.0 \\
0.0 \\
0.0 \\
0.0\end{array}$ & $\begin{array}{l}55.0 \\
59.0 \\
59.0 \\
58.0 \\
58.0\end{array}$ & $\begin{array}{l}77.0 \\
77.0 \\
77.0 \\
77.0 \\
80.0\end{array}$ & $\begin{array}{l}0.0 \\
0.0 \\
0.0 \\
0.0 \\
0.0\end{array}$ & $\begin{array}{l}0.0 \\
0.0 \\
0.0 \\
0.0 \\
0.0\end{array}$ & $\begin{array}{l}12.3 \\
12.3 \\
12.3 \\
12.3 \\
12.3\end{array}$ \\
\hline
\end{tabular}

\begin{tabular}{|c|c|c|c|c|c|c|c|c|c|}
\hline \multirow[b]{2}{*}{$\begin{array}{l}\text { TEST } \\
\text { NO }\end{array}$} & \multicolumn{2}{|c|}{ LOWEO PLENUM } & FLOW & \multicolumn{3}{|c|}{ BYPASS FLOH } & \multirow[b]{2}{*}{$\begin{array}{l}\text { LOWER } \\
\text { PLEN'JM } \\
\text { LEVEL } \\
\text { (IN) }\end{array}$} & \multirow[b]{2}{*}{$\begin{array}{l}\text { P-LP-1 } \\
(P S I G)\end{array}$} & \multirow[b]{2}{*}{$\begin{array}{l}\text { TF- LP- } \\
\text { (DEG F) }\end{array}$} \\
\hline & $\begin{array}{l}\text { INITIAL } \\
\text { WEIGHT } \\
\text { (LS) }\end{array}$ & $\begin{array}{l}\text { FINAL } \\
\text { WEIGHT } \\
\text { (LS) }\end{array}$ & $\begin{array}{l}\text { TIME OF } \\
\text { COLLECTICN } \\
\text { (SEC) }\end{array}$ & $\begin{array}{l}\text { INITI IAL } \\
\text { WEIGHT } \\
\text { (LB) }\end{array}$ & $\begin{array}{l}\text { FINAL } \\
\text { WE I GHT } \\
\text { IL B! }\end{array}$ & $\begin{array}{l}\text { TIME OF } \\
\text { COLLECTION } \\
\text { (SEC) }\end{array}$ & & & \\
\hline $\begin{array}{l}4.3 \cdot 1 \\
4.3 \cdot 2 \\
4.3 \cdot 3 \\
4.3 \cdot 4 \\
4.3 \cdot 5\end{array}$ & $\begin{array}{l}132.3 \\
250.5 \\
177.0 \\
368.8 \\
477.3\end{array}$ & $\begin{array}{l}250.5 \\
367.0 \\
402.3 \\
479.3 \\
586.5\end{array}$ & $\begin{array}{l}22.0 \\
21.0 \\
41.5 \\
20.0 \\
20.0\end{array}$ & $\begin{array}{r}90.8^{\circ} \\
90.3 \\
92.5 \\
97.0 \\
104.0^{\circ}\end{array}$ & $\begin{array}{r}90.8 \\
92.5 \\
97.0 \\
104.0 \\
112.5\end{array}$ & $\begin{array}{r}85.0 \\
100.0 \\
80.0 \\
90.0 \\
70.0\end{array}$ & $\begin{array}{l}12.0 \\
12.0 \\
12.0 \\
12.0 \\
12.0\end{array}$ & $\begin{array}{l}10.0 \\
10.0 \\
10.0 \\
10.0 \\
10.0\end{array}$ & $\begin{array}{l}55.0 \\
59.0 \\
58.5 \\
58.0 \\
58.0\end{array}$ \\
\hline
\end{tabular}




\section{TABLE XXXIX}

COMBINED EFFECTS TEST GROUP 4.7

RANSPAPENT VESSEL TEST DATA FDR TESTS 4.7.1 TU 4.7.25 -- 0.49-IN. DONICOYER GAP SUPYARY JF CALCULATED RESULTS

\begin{tabular}{|c|c|c|c|c|c|c|c|c|c|c|}
\hline $\begin{array}{c}T E S T \\
N ! S\end{array}$ & $\begin{array}{l}\text { DMINNCOMER } \\
\text { AIF. FLIIW } \\
\text { (LA/SEC) }\end{array}$ & $\begin{array}{l}\text { DOWNCOIEP } \\
\text { AIR DENSITY } \\
\text { (LIS/FT } \$ 3)\end{array}$ & $\begin{array}{c}\text { LOWEP PLENUM } \\
\text { PRESSURE } \\
\text { (PSIA) }\end{array}$ & $\begin{array}{l}\text { COLD LEG } \\
A I R \text { FL.JW } \\
\text { (LB/SEC) }\end{array}$ & $\begin{array}{l}\text { DOWNCOACR } \\
\text { WATEP. FLDWN } \\
\text { (LO/SEC) }\end{array}$ & $\begin{array}{l}\text { HATER FLLW } \\
\text { CONTINUITY }\end{array}$ & $\begin{array}{c}J-A I R \\
\text { DIAISTMEP } \\
(F T / S E C)\end{array}$ & $\begin{array}{l}\text { J-HATEP, } \\
\text { DONFICDMEP. } \\
\text { (FT/SEC) }\end{array}$ & $\left(J G^{ \pm}\right) \neq * 1 / 2$ & $(\mathrm{JL} * 1 * 1 / 2$ \\
\hline $\begin{array}{l}4.7 \cdot 1 \\
4.7 \cdot 2 \\
4.7 \cdot 3 \\
4.7 \cdot 4 \\
4.7 .5\end{array}$ & $\begin{array}{l}0.0070 \\
0.0075 \\
0.0152 \\
0.0151 \\
0.0223\end{array}$ & $\begin{array}{l}0.1160 \\
.01161 \\
0.1153 \\
.01239 \\
.01159\end{array}$ & $\begin{array}{l}22.25 \\
22.25 \\
22.25 \\
22.25 \\
22.25\end{array}$ & $\begin{array}{l}0.0991 \\
0.0937 \\
0.0973 \\
0.0931 \\
0.0973\end{array}$ & $\begin{array}{l}2.640 \\
2.780 \\
2.280 \\
2.410 \\
2.083\end{array}$ & $\begin{array}{l}1.005 \\
0.963 \\
1.025 \\
1.017 \\
1.017\end{array}$ & $\begin{array}{l}1.01 \\
1.01 \\
2.04 \\
1.32 \\
3.05\end{array}$ & $\begin{array}{l}0.657 \\
0.692 \\
0.568 \\
0.609 \\
0.519\end{array}$ & $\begin{array}{l}0.1639 \\
0.1642 \\
0.2329 \\
0.2255 \\
0.2349\end{array}$ & $\begin{array}{l}0.6362 \\
0.6529 \\
0.5913 \\
0.6079 \\
0.5652\end{array}$ \\
\hline $\begin{array}{l}4.7 .6 \\
4.7 .7 \\
4.7 .8 \\
4.7 .9 \\
4.7 .10\end{array}$ & $\begin{array}{l}0.0477 \\
0.0394 \\
0.029 .9 \\
0.046 \mathrm{~J} \\
0.0446\end{array}$ & $\begin{array}{l}0.0641 \\
0.1159 \\
0.1149 \\
0.1150 \\
0.1163\end{array}$ & $\begin{array}{l}12.43 \\
22.25 \\
22.25 \\
22.25 \\
22.25\end{array}$ & $\begin{array}{l}0.0703 \\
0.0993 \\
0.0923 \\
0.0976 \\
0.0903\end{array}$ & $\begin{array}{l}2.208 \\
1.800 \\
1.950 \\
1.408 \\
1.742\end{array}$ & $\begin{array}{l}1.020 \\
1.001 \\
1.045 \\
1.020 \\
1.056\end{array}$ & $\begin{array}{r}11.46 \\
4.07 \\
4.02 \\
6.18 \\
5.96\end{array}$ & $\begin{array}{l}0.550 \\
0.449 \\
0.485 \\
0.351 \\
0.434\end{array}$ & $\begin{array}{l}0.4767 \\
0.3299 \\
0.3260 \\
0.4046 \\
0.3981\end{array}$ & $\begin{array}{l}0.5813 \\
0.5254 \\
0.5468 \\
0.464 ? \\
0.5168\end{array}$ \\
\hline $\begin{array}{l}4.7 .11 \\
4.7 .12 \\
4.7 .13 \\
4.7 .14 \\
4.7 .15\end{array}$ & $\begin{array}{l}0.0456 \\
0.0453 \\
0.0625 \\
0.00^{\prime} 144 \\
0.0636\end{array}$ & $\begin{array}{l}0.1161 \\
0.1164 \\
0.1162 \\
0.1153 \\
0.1147\end{array}$ & $\begin{array}{l}22.25 \\
22.25 \\
22.25 \\
22.25 \\
22.25\end{array}$ & $\begin{array}{l}0.0984 \\
0.0954 \\
0.0434 \\
0.0899 \\
0.0937\end{array}$ & $\begin{array}{l}1.850 \\
1.767 \\
1.129 \\
1.557 \\
1.567\end{array}$ & $\begin{array}{l}1.005 \\
1.026 \\
1.019 \\
1.048 \\
1.026\end{array}$ & $\begin{array}{l}6.10 \\
6.27 \\
8.35 \\
7.96 \\
8.14\end{array}$ & $\begin{array}{l}0.463 \\
0.440 \\
0.281 \\
0.388 \\
0.390\end{array}$ & $\begin{array}{l}0.4026 \\
0.4019 \\
0.4712 \\
0.4596 \\
0.4647\end{array}$ & $\begin{array}{l}0.5338 \\
0.5205 \\
0.4160 \\
0.4888 \\
0.4901\end{array}$ \\
\hline $\begin{array}{l}4.7 .16 \\
4.7 .17 \\
4.7 .18 \\
4.7 .19 \\
4.7 .20\end{array}$ & $\begin{array}{l}0.0609 \\
0.0613 \\
0.0761 \\
0.0749 \\
0.0921\end{array}$ & $\begin{array}{l}0.1159 \\
0.1156 \\
0.1162 \\
0.1162 \\
0.1159\end{array}$ & $\begin{array}{l}22.25 \\
22.25 \\
22.25 \\
22.25 \\
22.25\end{array}$ & $\begin{array}{l}0.0963 \\
0.0974 \\
0.0994 \\
0.0911 \\
0.0984\end{array}$ & $\begin{array}{l}1.367 \\
1.119 \\
0.971 \\
1.186 \\
0.750\end{array}$ & $\begin{array}{l}1.015 \\
1.004 \\
1.026 \\
1.0033 \\
1.025\end{array}$ & $\begin{array}{r}8.16 \\
8.24 \\
10.25 \\
10.02 \\
12.33\end{array}$ & $\begin{array}{l}0.340 \\
0.278 \\
0.242 \\
0.295 \\
0.19 ?\end{array}$ & $\begin{array}{l}0.4655 \\
0.4674 \\
0.5221 \\
0.5160 \\
0.5723\end{array}$ & $\begin{array}{l}0.4578 \\
0.4142 \\
0.3859 \\
0.4264 \\
0.3391\end{array}$ \\
\hline $\begin{array}{l}4.7 .21 \\
4.7 .22 \\
4.7 .23 \\
4.7 .24 \\
4.7 .25\end{array}$ & $\begin{array}{l}0.0303 \\
0.1110 \\
0.1056 \\
0.1326 \\
0.1241\end{array}$ & $\begin{array}{l}0.1153 \\
.11235 \\
0.1153 \\
j .1315 \\
0.1161\end{array}$ & $\begin{array}{l}22.25 \\
23.75 \\
22.25 \\
25.19 \\
22.19\end{array}$ & $\begin{array}{l}0.0911 \\
0.1003 \\
0.0906 \\
0.1024 \\
0.0921\end{array}$ & $\begin{array}{l}0.350 \\
0.511 \\
0.550 \\
0.317 \\
0.342\end{array}$ & $\begin{array}{l}1.011 \\
1.021 \\
1.043 \\
1.032 \\
1.067\end{array}$ & $\begin{array}{l}12.11 \\
13.95 \\
14.16 \\
15.66 \\
16.61\end{array}$ & $\begin{array}{l}0.236 \\
0.127 \\
0.137 \\
0.079 \\
0.095\end{array}$ & $\begin{array}{l}0.5670 \\
0.6194 \\
0.6131 \\
0.6656 \\
0.6643\end{array}$ & $\begin{array}{l}0.3817 \\
0.2800 \\
0.2904 \\
0.2204 \\
0.2289\end{array}$ \\
\hline
\end{tabular}


TABLE XXXIX (Contd.)

COMBINED EFFECTS TEST GROUP 4.7

EXPERIMENTAL MEASURMENTS FOR TESTS 4.7 .1 IN $4.7 .25--0.49-$ IN. DOWNCOMER GAP

\begin{tabular}{|c|c|c|c|c|c|c|c|c|c|c|}
\hline $\begin{array}{l}\text { TEST } \\
\text { NO }\end{array}$ & $\begin{array}{c}R M-C+N-1 \\
(G P M)\end{array}$ & $\begin{array}{l}R M-C W-2 \\
(G P M)\end{array}$ & $\begin{array}{l}F T-C H-1 \\
(G P M)\end{array}$ & $\begin{array}{c}F T-C H-2 \\
(G P M)\end{array}$ & $\begin{array}{l}\text { RM-HG-1 } \\
(C F M)\end{array}$ & $\begin{array}{c}\text { RM-HG-2 } \\
\text { (CFM) }\end{array}$ & $\begin{array}{c}\text { RM-HG-3 } \\
\text { (CFM) }\end{array}$ & $\begin{array}{c}\text { RM-HG-4 } \\
\text { (CFM) }\end{array}$ & $\begin{array}{c}F T-H G-1 \\
(C F M)\end{array}$ & $\begin{array}{l}F T-H G-2 \\
(C F M)\end{array}$ \\
\hline $\begin{array}{l}4.7 .1 \\
4.7 .7 \\
4.7 .3 \\
4.7 .54 \\
4.7 .5\end{array}$ & $\begin{array}{l}30.0 \\
25.0 \\
30.0 \\
20.0 \\
30.0\end{array}$ & $\begin{array}{r}10.0 \\
0.0 \\
10.0 \\
0.0 \\
10.0\end{array}$ & $\begin{array}{l}0.0 \\
0.0 \\
0.0 \\
0.0 \\
0.0\end{array}$ & $\begin{array}{l}0.0 \\
0.0 \\
0.0 \\
0.0 \\
0.0\end{array}$ & $\begin{array}{l}0.0 \\
0.0 \\
0.0 \\
0.0 \\
0.0\end{array}$ & $\begin{array}{l}0.0 \\
0.0 \\
0.0 \\
0.0 \\
0.0\end{array}$ & $\begin{array}{r}5.00 \\
5.00 \\
10.00 \\
10.00 \\
15.00\end{array}$ & $\begin{array}{l}0.0 \\
0.0 \\
0.0 \\
0.0 \\
0.0\end{array}$ & $\begin{array}{l}0.0 \\
0.0 \\
0.0 \\
0.0 \\
0.0\end{array}$ & $\begin{array}{l}0.0 \\
0.0 \\
0.00 \\
0.0 \\
0.0\end{array}$ \\
\hline $\begin{array}{l}4.7 .6 \\
4.7 .7 \\
4.7 .8 \\
4.7 .89 \\
4.7 .10\end{array}$ & $\begin{array}{l}13.0 \\
32.0 \\
15.0 \\
30.0 \\
13.0\end{array}$ & $\begin{array}{r}0.0 \\
10.0 \\
0.0 \\
10.0 \\
0.0\end{array}$ & $\begin{array}{l}0.0 \\
0.0 \\
0.0 \\
0.0 \\
0.0\end{array}$ & $\begin{array}{l}0.0 \\
0.0 \\
0.0 \\
0.0 \\
0.0\end{array}$ & $\begin{array}{l}0.0 \\
0.0 \\
0.0 \\
0.0 \\
0.0\end{array}$ & $\begin{array}{l}0.0 \\
0.0 \\
0.0 \\
0.0 \\
0.0\end{array}$ & $\begin{array}{l}15.00 \\
20.00 \\
20.00 \\
30.00 \\
30.00\end{array}$ & $\begin{array}{l}0.0 \\
0.0 \\
0.0 \\
0.0 \\
0.0\end{array}$ & $\begin{array}{l}0.0 \\
0.0 \\
0.0 \\
0.0 \\
0.0\end{array}$ & $\begin{array}{l}0.0 \\
0.0 \\
0.0 \\
0.0 \\
0.0\end{array}$ \\
\hline $\begin{array}{l}4.711 \\
4.7 .12 \\
4.7 .13 \\
4.7 .14 \\
4.7 .15\end{array}$ & $\begin{array}{l}20.0 \\
30.0 \\
30.0 \\
12.0 \\
20.0\end{array}$ & $\begin{array}{r}0.0 \\
0.0 \\
10.0 \\
0.0 \\
0.0\end{array}$ & $\begin{array}{l}0.0 \\
0.0 \\
0.0 \\
0.0 \\
0.0\end{array}$ & $\begin{array}{l}0.0 \\
0.0 \\
0.0 \\
0.0 \\
0.0\end{array}$ & $\begin{array}{c}0.0 \\
0.0 \\
40.00 \\
40.00 \\
40.00\end{array}$ & $\begin{array}{l}0.0 \\
0.0 \\
0.0 \\
0.0 \\
0.0\end{array}$ & $\begin{array}{c}30.00 \\
30.00 \\
0.0^{\circ} \\
0.0 \\
0.0\end{array}$ & $\begin{array}{l}0.0 \\
0.0 \\
0.0 \\
0.0 \\
0.0\end{array}$ & $\begin{array}{l}0.0 \\
0.0 \\
0.0 \\
0.0 \\
0.0\end{array}$ & $\begin{array}{l}0.0 \\
u .0 \\
0.0 \\
0.0 \\
0.0\end{array}$ \\
\hline $\begin{array}{l}4.7 .16 \\
4.7 .11 \\
4.7 .10 \\
4.7 .19 \\
4.7 .20\end{array}$ & $\begin{array}{l}30.0 \\
30.0 \\
30.0 \\
10.0 \\
30.0\end{array}$ & $\begin{array}{r}0.0 \\
10.0 \\
10.0 \\
3.0 \\
10.0\end{array}$ & $\begin{array}{l}0.0 \\
0.0 \\
0.0 \\
0.0 \\
0.0\end{array}$ & $\begin{array}{l}0.0 \\
0.0 \\
0.0 \\
0.0 \\
0.0\end{array}$ & $\begin{array}{l}40.00 \\
40.00 \\
50.00 \\
50.00 \\
60.00\end{array}$ & $\begin{array}{l}0.0 \\
0.0 \\
0.0 \\
0.0 \\
0.0\end{array}$ & $\begin{array}{l}0.0 \\
0.0 \\
0.0 \\
0.0 \\
0.0\end{array}$ & $\begin{array}{l}0.0 \\
0.0 \\
0.0 \\
0.0 \\
0.0\end{array}$ & $\begin{array}{l}0.0 \\
0.0 \\
0.0 \\
0.0 \\
0.0\end{array}$ & $\begin{array}{l}0.0 \\
0.0 \\
0.0 \\
0.0 \\
0.0\end{array}$ \\
\hline $\begin{array}{l}4.7 .21 \\
4.7 .222 \\
4.77 .23 \\
4.7 .24 \\
4.7 .25\end{array}$ & $\begin{array}{r}3.0 \\
3.0 .0 \\
3.0 \\
3 \% .0 \\
0.0\end{array}$ & $\begin{array}{r}10.0 \\
10.0 \\
6.0 \\
10.0 \\
5.0\end{array}$ & $\begin{array}{l}0.0 \\
0.0 \\
0.0 \\
0.0 \\
0.0\end{array}$ & $\begin{array}{l}0.0 \\
0.0 \\
0.0 \\
0.0 \\
0.0\end{array}$ & $\begin{array}{l}60.00 \\
70.00 \\
70.00 \\
80.00 \\
80.00\end{array}$ & $\begin{array}{l}0.0 \\
0.0 \\
0.0 \\
0.0 \\
0.0\end{array}$ & $\begin{array}{l}0.0 \\
0.0 \\
0.0 \\
0.0 \\
0.0\end{array}$ & $\begin{array}{l}0.0 \\
0.0 \\
0.0 \\
0.0 \\
0.0\end{array}$ & $\begin{array}{l}0.0 \\
0.0 \\
0.0 \\
0.0 \\
0.0\end{array}$ & $\begin{array}{l}0.0 \\
0.00 \\
0.0 \\
0.0 \\
0.0\end{array}$ \\
\hline $\begin{array}{l}\text { TEST } \\
\text { NU }\end{array}$ & $\begin{array}{l}p-c, n-1 \\
(p 2 i c)\end{array}$ & $\begin{array}{l}P-C: 1)-2 \\
\text { (PSIGI }\end{array}$ & $\begin{array}{l}P-H C-1 \\
\mid P S I G I\end{array}$ & $\begin{array}{l}P-H G-2 \\
(P \subseteq I G)\end{array}$ & $\begin{array}{l}P-H G-3 \\
(P S I G)\end{array}$ & $\begin{array}{l}\text { TF-CW-1 } \\
(\text { IE.G F) }\end{array}$ & $\begin{array}{l}T F-H_{F}-1 \\
(D E G F)\end{array}$ & $\begin{array}{l}\text { TF-HIS-2 } \\
\text { (DES F) }\end{array}$ & $\begin{array}{l}T F-4 G,-3 \\
(O E G, F)\end{array}$ & $\begin{array}{l}\text { P-BARO } \\
\text { IPSIAI }\end{array}$ \\
\hline $\begin{array}{l}4.7 .1 \\
4.7 .2 \\
4.7 .3 \\
4.7 .4 \\
4.7 .5\end{array}$ & $\begin{array}{l}.00 \\
.00 \\
.00 \\
3.0 \\
0.0\end{array}$ & $\begin{array}{r}7.5 \\
10.2 \\
12.0 \\
10.0 \\
12.0\end{array}$ & $\begin{array}{r}9.7 \\
9.7 \\
10.0 \\
9.5 \\
10.0\end{array}$ & $\begin{array}{l}0.0 \\
0.0 \\
0.0 \\
0.0 \\
0.0\end{array}$ & $\begin{array}{l}0.0 \\
0.0 \\
0.0 \\
0.0 \\
0.0\end{array}$ & $\begin{array}{l}55.5 \\
58.0 \\
59.0 \\
53.5 \\
53.0\end{array}$ & $\begin{array}{l}78.0 \\
77.0 \\
78.0 \\
77.5 \\
79.5\end{array}$ & $\begin{array}{l}0.0 \\
0.0 \\
0.0 \\
0.0 \\
0.0\end{array}$ & $\begin{array}{l}0.0 \\
0.0 \\
0.0 \\
0.0 \\
0.0\end{array}$ & $\begin{array}{l}12.3 \\
12.3 \\
12.3 \\
12.3 \\
12.3\end{array}$ \\
\hline
\end{tabular}


TABLE XXXIX (Contd.)

COMBINED EFFECTS TEST GROUP 4.7

EOERIMTNTAL MEASURMENTS FOR TESTS 4.7 .1 TI $4.7 .25--$ 0.44-IN. DOWNCOMER GAP

\begin{tabular}{|c|c|c|c|c|c|c|c|c|c|c|}
\hline $\begin{array}{l}\text { TEST } \\
\text { MT }\end{array}$ & $\begin{array}{l}P-C N-1 \\
(P \leq 1 G)\end{array}$ & $\begin{array}{l}P-C H-? \\
(D S I C)\end{array}$ & $\begin{array}{l}P-H G-1 \\
(P S I G)\end{array}$ & $\begin{array}{l}P-H G-2 \\
(P S I G)\end{array}$ & $\begin{array}{l}P-H C,-3 \\
(D S I G)\end{array}$ & $\begin{array}{l}T F-C W-1 \\
(D E C, F)\end{array}$ & $\begin{array}{l}T F-H G-1 \\
(U E G F)\end{array}$ & $\begin{array}{l}\text { TF-HG-2 } \\
\text { IJER, CI }\end{array}$ & $\begin{array}{l}\text { TF- }+15-3 \\
\text { (DFr, } C \text { ) }\end{array}$ & $\begin{array}{l}\text { P-BARח } \\
\text { (PSIAI) }\end{array}$ \\
\hline $\begin{array}{l}4.7 .6 \\
4.7 .7 \\
4.7 .9 \\
4.7 .79 \\
4.7 .10\end{array}$ & $\begin{array}{l}3.0 \\
3.0 \\
3.0 \\
.00 \\
.00\end{array}$ & $\begin{array}{r}10.3 \\
13.0 \\
9.7 \\
12.5 \\
9.0\end{array}$ & $\begin{array}{r}95.0 \\
10.0 \\
9.0 \\
10.2 \\
9.0\end{array}$ & $\begin{array}{l}0.0 \\
0.0 \\
0.0 \\
0.0 \\
0.0\end{array}$ & $\begin{array}{l}0.0 \\
0.0 \\
0.0 \\
0.0 \\
0.0\end{array}$ & $\begin{array}{l}59.5 \\
57.0 \\
61.5 \\
65.5 \\
60.5\end{array}$ & $\begin{array}{l}80.0 \\
81.0 \\
79.0 \\
78.0 \\
7 \% .0\end{array}$ & $\begin{array}{l}0.0 \\
0.0 \\
0.0 \\
0.0 \\
0.0\end{array}$ & $\begin{array}{l}3.0 \\
0.0 \\
0.0 \\
0.0 \\
0.0\end{array}$ & $\begin{array}{r}2.4 \\
12.3 \\
12.3 \\
12.3 \\
12.3\end{array}$ \\
\hline $\begin{array}{l}4.7 .11 \\
4.7 .12 \\
4.7 .13 \\
4.7 .14 \\
4.7 .15\end{array}$ & $\begin{array}{l}J .0 \\
.00 \\
3.0 \\
j .0 \\
0.0\end{array}$ & $\begin{array}{l}11.5 \\
11.0 \\
12.5 \\
-3.5 \\
10.2\end{array}$ & $\begin{array}{r}10.0 \\
10.0 \\
11.0 \\
8.7 \\
9.5\end{array}$ & $\begin{array}{l}0.0 \\
0.0 \\
0.0 \\
0.0 \\
0.0\end{array}$ & $\begin{array}{l}0.0 \\
0.0 \\
0.0 \\
0.0 \\
0.0\end{array}$ & $\begin{array}{l}56.5 \\
56.0 \\
57.0 \\
59.5 \\
53.0\end{array}$ & $\begin{array}{l}81.0 \\
03.0 \\
83.5 \\
84.5 \\
80.0\end{array}$ & $\begin{array}{l}0.0 \\
0.0 \\
0.0 \\
0.0 \\
0.0\end{array}$ & $\begin{array}{l}0.0 \\
0.0 \\
0.0 \\
0.0 \\
0.0\end{array}$ & $\begin{array}{l}12.3 \\
12.3 \\
12.3 \\
12.3 \\
12.3\end{array}$ \\
\hline $\begin{array}{l}4.7 .16 \\
4.7 .17 \\
4.7 .18 \\
4.7 .10 \\
4.7 .20\end{array}$ & $\begin{array}{l}3.0 \\
3.0 \\
3.0 \\
.0 .0 \\
3.0\end{array}$ & $\begin{array}{r}11.5 \\
12.0 \\
13.0 \\
9.0 \\
12.5\end{array}$ & $\begin{array}{r}9.7 \\
10.0 \\
10.0 \\
9.0 \\
10.0\end{array}$ & $\begin{array}{l}0.0 \\
0.0 \\
0.0 \\
0.0 \\
0.0\end{array}$ & $\begin{array}{l}0.0 \\
0.0 \\
0.0 \\
0.0 \\
0.0\end{array}$ & $\begin{array}{l}57.0 \\
57.0 \\
56.0 \\
57.0 \\
57.0\end{array}$ & $\begin{array}{l}81.5 \\
80.0 \\
84.5 \\
85.5 \\
90.0\end{array}$ & $\begin{array}{l}0.3 \\
0.0 \\
0.0 \\
0.0 \\
0.0\end{array}$ & $\begin{array}{l}0.0 \\
0.0 \\
0.0 \\
0.0 \\
0.0\end{array}$ & $\begin{array}{l}12.3 \\
12.3 \\
12.3 \\
12.3 \\
12.3\end{array}$ \\
\hline $\begin{array}{l}4.7 .21 \\
4.7 .22 \\
4.7 .23 \\
4.7 .24 \\
4.7 .25\end{array}$ & $\begin{array}{l}J .0 \\
0.0 \\
.00 \\
3.0 \\
0.0\end{array}$ & $\begin{array}{r}9.0 \\
13.5 \\
3.7 \\
14.5 \\
9.5\end{array}$ & $\begin{array}{r}9.2 \\
11.5 \\
9.2 \\
12.5 \\
9.5\end{array}$ & $\begin{array}{l}0.0 \\
0.0 \\
0.0 \\
0.0 \\
0.0\end{array}$ & $\begin{array}{l}0.0 \\
0.0 \\
0.0 \\
0.0 \\
0.0\end{array}$ & $\begin{array}{l}57.5 \\
57.3 \\
57.0 \\
54.5 \\
56.5\end{array}$ & $\begin{array}{l}92.7 \\
95.0 \\
97.5 \\
74.0 \\
79.5\end{array}$ & $\begin{array}{l}0.0 \\
0.0 \\
0.0 \\
0.0 \\
0.0\end{array}$ & $\begin{array}{l}0.0 \\
0.0 \\
0.0 \\
0.0 \\
0.0\end{array}$ & $\begin{array}{l}12.3 \\
12.3 \\
12.3 \\
12.2 \\
12.2\end{array}$ \\
\hline
\end{tabular}

\begin{tabular}{|c|c|c|c|c|c|c|c|c|c|}
\hline \multirow[b]{2}{*}{$\begin{array}{l}\text { TEST } \\
\text { NDO }\end{array}$} & \multicolumn{3}{|c|}{ LOWER PLENUM FLOW } & \multicolumn{3}{|c|}{ BYPASS FI $\mathrm{OH}$} & \multirow{2}{*}{$\begin{array}{l}\text { L DWFR } \\
\text { PLEMUY } \\
\text { LEVEL } \\
\text { (1N) }\end{array}$} & \multirow[b]{2}{*}{ 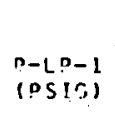 } & \multirow[b]{2}{*}{$\begin{array}{l}\text { TF- } L P-1 \\
\text { IDES, F }\end{array}$} \\
\hline & $\begin{array}{l}\text { IHITIALL } \\
\text { WEI I,!IT } \\
\text { (LS): }\end{array}$ & $\begin{array}{l}\text { FINAL } \\
\text { AEIGHT } \\
\text { (LB) }\end{array}$ & $\begin{array}{l}\text { TIME OF } \\
\text { COLLECTICN } \\
\text { (SEC) }\end{array}$ & $\begin{array}{l}\text { IN IT I AL } \\
\text { WE I SHIT } \\
\text { (I B B }\end{array}$ & $\begin{array}{l}\text { FINAL } \\
\text { WFI ISHT } \\
(L \nLeftarrow)\end{array}$ & $\begin{array}{l}\text { TIME OF } \\
\text { COLLECTION } \\
\text { (SEC) }\end{array}$ & & & \\
\hline $\begin{array}{l}4.7: 1 \\
4.7 .02 \\
4.7 .5 \\
4.7 .5 \\
4.7 .5\end{array}$ & $\begin{array}{l}133.5 \\
199.5 \\
269.0 \\
326.0 \\
336.3\end{array}$ & $\begin{array}{l}199.5 \\
269.0 \\
326.0 \\
386.3 \\
433.8\end{array}$ & $\begin{array}{l}25.0 \\
25.0 \\
25.0 \\
25.0 \\
25.2\end{array}$ & $\begin{array}{r}90.8 \\
164.5 \\
131.5 \\
267.0 \\
279.5\end{array}$ & $\begin{array}{l}164.5 \\
181.5 \\
267.0 \\
279.5 \\
404.5\end{array}$ & $\begin{array}{l}25.0 \\
30.0 \\
25.0 \\
30.0 \\
35.0\end{array}$ & $\begin{array}{l}12.0 \\
12.0 \\
12.0 \\
12.0 \\
12.0\end{array}$ & $\begin{array}{l}10.0 \\
10.0 \\
10.0 \\
10.0 \\
10.0\end{array}$ & $\begin{array}{r}55.0 \\
57.5 \\
53.5 \\
5.8 \\
53.0\end{array}$ \\
\hline $\begin{array}{l}4.7 .6 \\
4.7 .7 \\
4.7 .8 \\
4.7 .9 \\
4.7 .10\end{array}$ & $\begin{array}{l}439.8 \\
503.0 \\
133.5 \\
201.9 \\
244.0\end{array}$ & $\begin{array}{l}305.0 \\
550.0 \\
201.8 \\
244.0 \\
296.3\end{array}$ & $\begin{array}{l}30.0 \\
25.0 \\
35.0 \\
30.0 \\
30.0\end{array}$ & $\begin{array}{r}404.5 \\
420.0 \\
95.5 \\
107.0 \\
235.0\end{array}$ & $\begin{array}{l}420.0 \\
533.0 \\
107.0 \\
235.0 \\
245.0\end{array}$ & $\begin{array}{l}45.0 \\
30.0 \\
50.0 \\
30.0 \\
60.0\end{array}$ & $\begin{array}{r}12.0 \\
12.0 \\
12.0 \\
12.0 \\
12.0\end{array}$ & $\begin{array}{l}10.0 \\
10.0 \\
10.0 \\
10.0 \\
10.0\end{array}$ & $\begin{array}{l}58.5 \\
58.0 \\
62.5 \\
53.5 \\
56.5\end{array}$ \\
\hline $\begin{array}{l}4.7 .11 \\
4.7 .12 \\
4.9 .713 \\
4.7 .14 \\
4.7 .15\end{array}$ & $\begin{array}{l}290.3 \\
352.0 \\
405.0 \\
414.5 \\
461.0\end{array}$ & $\begin{array}{l}352.0 \\
405.0 \\
444.5 \\
49 \% .0 \\
508.0\end{array}$ & $\begin{array}{l}20.0 \\
30.0 \\
35.0 \\
35.0 \\
30.0\end{array}$ & $\begin{array}{l}245.0 \\
282.5 \\
333.0 \\
496.5 \\
5=17.0\end{array}$ & $\begin{array}{l}282.5 \\
383.0 \\
496.5 \\
507.0 \\
558.5\end{array}$ & $\begin{array}{l}40.0 \\
40.0 \\
25.0 \\
55.0 \\
40.0\end{array}$ & $\begin{array}{l}12.0 \\
12.0 \\
12.0 \\
12.0 \\
12.0\end{array}$ & $\begin{array}{l}10.0 \\
10.0 \\
10.0 \\
10.0 \\
10.0\end{array}$ & $\begin{array}{l}57.5 \\
56.0 \\
57.00 \\
58.5 \\
59.0\end{array}$ \\
\hline
\end{tabular}


TABLE XXXIX (Contd.)

COMBINED EFFECTS TEXT GROUP 4:7

EXPERIMENITAL MEASURMENTS FOR TESTS 4.7.1 TO 4.7.25

\begin{tabular}{|c|c|c|c|c|c|c|c|c|c|}
\hline \multirow[b]{2}{*}{$\begin{array}{l}\text { TEST } \\
\text { NO }\end{array}$} & \multicolumn{3}{|c|}{ LCWER PLENUM FLOW } & \multicolumn{3}{|c|}{ BYPASS FLOW } & \multirow{2}{*}{$\begin{array}{l}\text { LOWER } \\
\text { PLENUM } \\
\text { LEVEL } \\
\text { IINI }\end{array}$} & \multirow[b]{2}{*}{$\begin{array}{l}P-(P-1 \\
(P S \mid G)\end{array}$} & \multirow[b]{2}{*}{$\begin{array}{l}\text { TF-LP-1 } \\
\text { (DEG FI) }\end{array}$} \\
\hline & $\begin{array}{l}\text { INITIAL } \\
\text { HEIGHT } \\
\text { (LB) }\end{array}$ & $\begin{array}{l}\text { FINAL } \\
\text { WEIGHT } \\
\text { (LB) }\end{array}$ & $\begin{array}{l}\text { TIME OF } \\
\text { COLLECTICN } \\
\text { (SEC) }\end{array}$ & $\begin{array}{l}\text { INITIAL } \\
\text { WE!GHT } \\
\text { (LBI }\end{array}$ & $\begin{array}{l}\text { FINAL } \\
\text { HEIGHT } \\
\text { (LB) }\end{array}$ & $\begin{array}{l}\text { TIME DF } \\
\text { COLLECTION } \\
\text { (SEC) }\end{array}$ & & & \\
\hline $\begin{array}{l}4.7 .16 \\
4.7 .17 \\
4.7 .18 \\
4.7 .19 \\
4.7 .20\end{array}$ & $\begin{array}{l}463.3 \\
183.0 \\
227.8 \\
261.8 \\
303.3\end{array}$ & $\begin{array}{l}509.3 \\
227.8 \\
261.8 \\
303.3 \\
329.5\end{array}$ & $\begin{array}{l}30.0 \\
40.0 \\
35.0 \\
35.0 \\
35.0\end{array}$ & $\begin{array}{l}558.5 \\
102.5 \\
236.5 \\
378.5 \\
396.0\end{array}$ & $\begin{array}{l}644.5 \\
236.5 \\
378.5 \\
396.0 \\
544.5\end{array}$ & $\begin{array}{l}30.0 \\
30.0 \\
30.0 \\
60.0 \\
30.0\end{array}$ & $\begin{array}{l}12.0 \\
12.0 \\
12.0 \\
12.0 \\
12.0\end{array}$ & $\begin{array}{l}10.0 \\
10.0 \\
10.0 \\
10.0 \\
10.0\end{array}$ & $\begin{array}{l}58.0 \\
59.5 \\
57.0 \\
57.0 \\
58.0\end{array}$ \\
\hline $\begin{array}{l}4.7 .21 \\
4.7 .22 \\
4.7 .23 \\
4.7 .24 \\
4.7 .25\end{array}$ & $\begin{array}{l}329.5 \\
367.5 \\
390.5 \\
132.8 \\
151.8\end{array}$ & $\begin{array}{l}367.5 \\
390.5 \\
415.3 \\
151.8 \\
172.3\end{array}$ & $\begin{array}{l}40.0 \\
45.0 \\
45.0 \\
60.0 \\
60.0\end{array}$ & $\begin{array}{r}544.5 \\
565.0 \\
720.0 \\
83.0 \\
191.5\end{array}$ & $\begin{array}{l}565.0 \\
720.0 \\
736.0 \\
191.5 \\
209.5\end{array}$ & $\begin{array}{l}45.0 \\
30.0 \\
50.0 \\
20.0 \\
45.0\end{array}$ & $\begin{array}{l}12.0 \\
12.0 \\
12.0 \\
12.0 \\
12.0\end{array}$ & $\begin{array}{l}10.0 \\
11.5 \\
10.0 \\
13.0 \\
10.0\end{array}$ & $\begin{array}{l}58.5 \\
58.5 \\
59.5 \\
57.0 \\
56.0\end{array}$ \\
\hline
\end{tabular}

\begin{tabular}{|c|c|c|c|c|c|c|c|}
\hline $\begin{array}{l}\text { TEST } \\
\text { NO }\end{array}$ & $\begin{array}{c}R M-r \cdot r-1 \\
(C E M)\end{array}$ & $\begin{array}{c}R M-C_{G}-2 \\
(C F M)\end{array}$ & $\begin{array}{c}F T-C S-1 \\
(C F M)\end{array}$ & $\begin{array}{l}P-C S-1 \\
\text { (PSIG) }\end{array}$ & $\begin{array}{l}P-(C,-2 \\
\text { (PSIG) }\end{array}$ & $\begin{array}{l}\text { TE-CG-1 } \\
\text { (DEG F) }\end{array}$ & $\begin{array}{l}r f-C r-2 \\
(n \in S, F)\end{array}$ \\
\hline $\begin{array}{l}4.7 .1 \\
4.7 .22 \\
4.7 .3 \\
4.7 .4 \\
4.7 .5\end{array}$ & $\begin{array}{l}60.0 \\
60.0 \\
60.0 \\
60.0 \\
6.3 .0\end{array}$ & $\begin{array}{l}0.0 \\
0.0 \\
0.0 \\
0.0 \\
0.0\end{array}$ & $\begin{array}{l}0.0 \\
0.0 \\
0.0 \\
0.0 \\
0.0\end{array}$ & $\begin{array}{l}0.0 \\
0.0 \\
0.0 \\
0.0 \\
0.0\end{array}$ & $\begin{array}{l}0.0 \\
0.0 \\
0.0 \\
0.0 \\
0.0\end{array}$ & $\begin{array}{l}0.0 \\
0.0 \\
0.0 \\
0.0 \\
0.0\end{array}$ & $\begin{array}{l}0.0 \\
0.0 \\
0.0 \\
0.0 \\
0.0\end{array}$ \\
\hline $\begin{array}{l}4.7 .6 \\
4.7 .7 \\
4.7 .9 \\
4.7 .4 \\
4.7 .19\end{array}$ & $\begin{array}{l}50.0 \\
60.3 \\
20.0 \\
60.0 \\
30.0\end{array}$ & $\begin{array}{l}0.0 \\
0.0 \\
0.0 \\
0.0 \\
0.0\end{array}$ & $\begin{array}{l}0.0 \\
0.0 \\
0.0 \\
0.0 \\
0.0\end{array}$ & $\begin{array}{l}0.0 \\
0.0 \\
0.0 \\
0.0 \\
0.0\end{array}$ & $\begin{array}{l}3.0 \\
0.0 \\
0.0 \\
0.0 \\
0.0\end{array}$ & $\begin{array}{l}0.0 \\
0.0 \\
0.0 \\
0.0 \\
0.0\end{array}$ & $\begin{array}{l}0.0 \\
0.0 \\
0.0 \\
0.0 \\
0.0\end{array}$ \\
\hline $\begin{array}{l}4.7 .11 \\
4.7 .112 \\
6.7 .13 \\
4.7 .14 \\
6.7 .15\end{array}$ & $\begin{array}{l}60.3 \\
60.0 \\
60.3 \\
60.0 \\
60.0\end{array}$ & $\begin{array}{l}0.0 \\
0.0 \\
0.0 \\
0.0 \\
0.0\end{array}$ & $\begin{array}{l}0.0 \\
0.0 \\
0.0 \\
0.0 \\
0.0\end{array}$ & $\begin{array}{l}J .0 \\
0.0 \\
0.0 \\
0.0 \\
0.0\end{array}$ & $\begin{array}{l}0.0 \\
0.0 \\
0.0 \\
0.0 \\
0.0\end{array}$ & $\begin{array}{l}0.0 \\
0.0 \\
0.0 \\
0.0 \\
0.0\end{array}$ & $\begin{array}{l}0.0 \\
0.0 \\
0.0 \\
0.0 \\
0.0\end{array}$ \\
\hline $\begin{array}{l}4.7 .16 \\
4.7 .17 \\
4.7 .13 \\
4.7 .19 \\
4.7 .20\end{array}$ & $\begin{array}{l}60.1 \\
60.0 \\
63.0 \\
60.0 \\
60.0\end{array}$ & $\begin{array}{l}0.0 \\
0.0 \\
0.0 \\
0.0 \\
0.0\end{array}$ & $\begin{array}{l}0.0 \\
0.0 \\
0.0 \\
0.0 \\
0.0\end{array}$ & $\begin{array}{l}0.0 \\
0.0 \\
0.0 \\
0.0 \\
0.0\end{array}$ & $\begin{array}{l}0.0 \\
0.0 \\
0.0 \\
0.0 \\
0.0\end{array}$ & $\begin{array}{l}0.0 \\
0.0 \\
0.0 \\
0.0 \\
0.0\end{array}$ & $\begin{array}{l}0.0 \\
0.0 \\
0.0 \\
0.0 \\
0.0\end{array}$ \\
\hline $\begin{array}{l}4.7 .21 \\
4.7 .22 \\
4.7 .23 \\
4.7 .24 \\
+.7 .25\end{array}$ & $\begin{array}{l}60.0 \\
60.0 \\
60.0 \\
60.3 \\
60.0\end{array}$ & $\begin{array}{l}0.0 \\
0.0 \\
0.0 \\
0.0 \\
0.0\end{array}$ & $\begin{array}{l}0.0 \\
0.0 \\
0.0 \\
0.0 \\
0.0\end{array}$ & $\begin{array}{l}0.0 \\
0.0 \\
3.0 \\
J .0 \\
0.0\end{array}$ & $\begin{array}{l}0.0 \\
0.0 \\
0.0 \\
0.0 \\
0.0\end{array}$ & $\begin{array}{l}0.0 \\
0.0 \\
0.0 \\
0.0 \\
0.0\end{array}$ & $\begin{array}{l}0.0 \\
0.0 \\
0.0 \\
0.0 \\
0.0\end{array}$ \\
\hline
\end{tabular}


TABLE XI

COMBINED EFFECTS TEST GROUP 4.8

TRAISPPAPENT VESSEL TEST OATA FOR TESTS 4.8 .1 TO $4.8 .34-0.49-1 N$. DOWNCOMER GAP SUMMARY JF GALCIJATEN RESILLTS

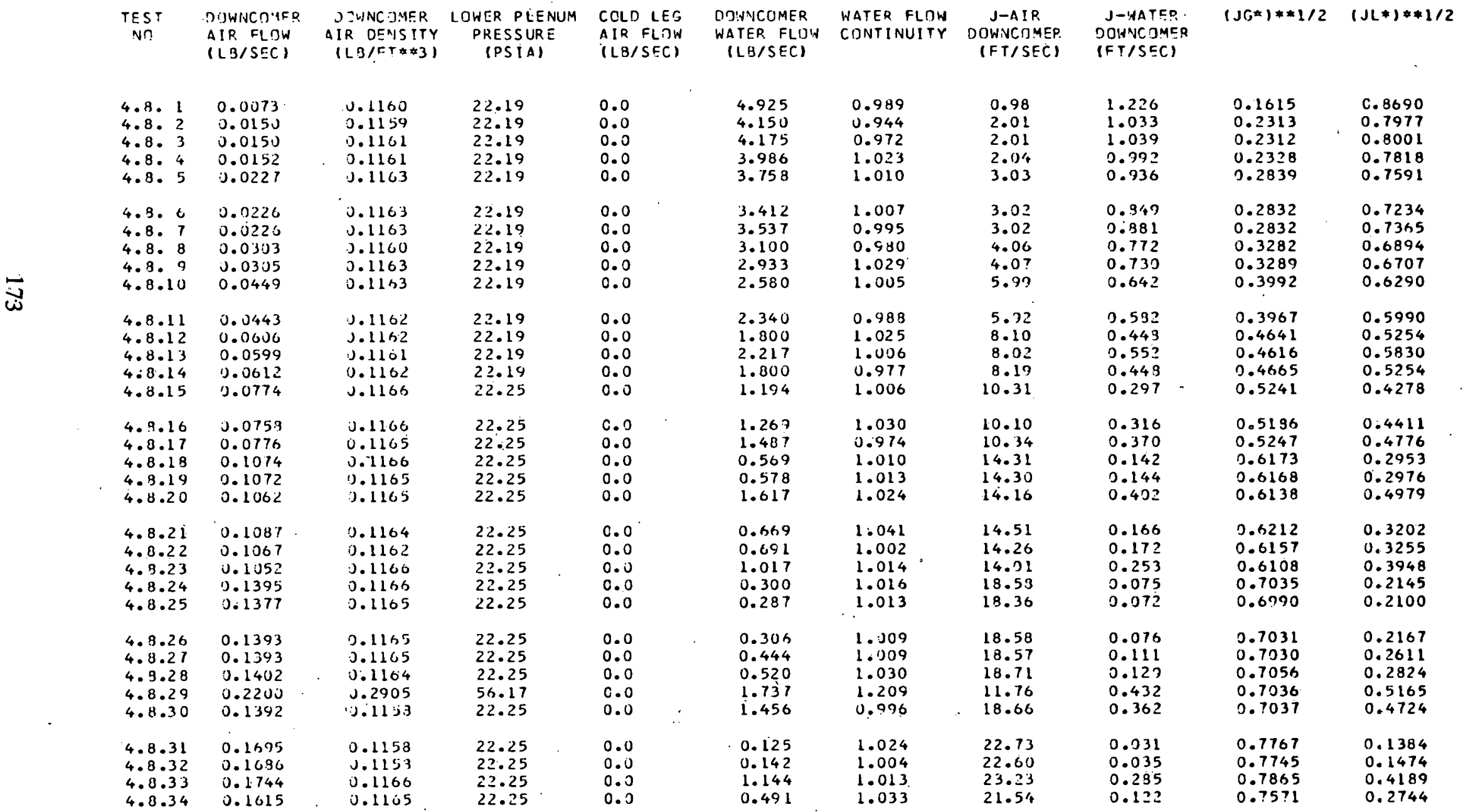


TABLE XL (Contd.)

COMBINED EFFECTS TEST GROUP 4.8

EXPERIMENTAL MEASURMENTS FOR TESTS 4.8 .1 TO $4.8 .34-0.49$-IN. DONNCOMER GAP

\begin{tabular}{|c|c|c|c|c|c|c|c|c|c|c|}
\hline $\begin{array}{c}\text { TEST } \\
\text { NO }\end{array}$ & $\begin{array}{c}R M-C W-1 \\
(G P M)\end{array}$ & $\begin{array}{c}\text { R.M-CW-Z } \\
(G P M)\end{array}$ & $\begin{array}{c}F Y-C H-1 \\
(G P M)\end{array}$ & $\begin{array}{c}F T-C H-2 \\
(G P M)\end{array}$ & $\begin{array}{c}\text { RM-HG-1 } \\
\text { (CFM) }\end{array}$ & $\begin{array}{c}R M-H G-2 \\
(C F M)\end{array}$ & $\begin{array}{c}R M-H G-3 \\
\text { (CFM) }\end{array}$ & $\begin{array}{c}\text { RM-HG-4 } \\
\text { (CFM) }\end{array}$ & $\begin{array}{c}F_{T-H G}-1 \\
\text { (CFM) }\end{array}$ & $\begin{array}{c}F T-H G-2 \\
\text { (CFM) }\end{array}$ \\
\hline $\begin{array}{l}4.8 \cdot 1 \\
4.8 \cdot 2 \\
4.8 \cdot 3 \\
4.8 \cdot 4 \\
4.8 .5\end{array}$ & $\begin{array}{l}30.0 \\
30.0 \\
30.0 \\
32.0 \\
30.0\end{array}$ & $\begin{array}{r}10.0 \\
10.0 \\
10.0 \\
0.0 \\
10.0\end{array}$ & $\begin{array}{l}0.0 \\
0.0 \\
0.0 \\
0.0 \\
0.0\end{array}$ & $\begin{array}{l}0.0 \\
0.0 \\
0.0 \\
0.0 \\
0.0\end{array}$ & $\begin{array}{l}0.0 \\
0.0 \\
0.0 \\
0.0 \\
0.0\end{array}$ & $\begin{array}{l}0.0 \\
0.0 \\
0.0 \\
0.0 \\
0.0\end{array}$ & $\begin{array}{l}5.00 \\
10.00 \\
10.00 \\
10.00 \\
15.00\end{array}$ & $\begin{array}{l}0.0 \\
0.0 \\
0.0 \\
0.0 \\
0.0\end{array}$ & $\begin{array}{l}0.0 \\
0.0 \\
0.0 \\
0.0 \\
0.0\end{array}$ & $\begin{array}{l}0.0 \\
0.0 \\
0.0 \\
0.0 \\
0.0\end{array}$ \\
\hline $\begin{array}{l}4.8 \cdot 6 \\
4.8 \cdot 7 \\
4.8 \cdot 8 \\
4.8 \cdot 9 \\
4.8 .10\end{array}$ & $\begin{array}{l}30.0 \\
35.0 \\
30.0 \\
26.0 \\
30.0\end{array}$ & $\begin{array}{r}0.0 \\
5.0 \\
10.0 \\
0.0 \\
10.0\end{array}$ & $\begin{array}{l}0.0 \\
0.0 \\
0.0 \\
0.0 \\
0.0\end{array}$ & $\begin{array}{l}0.0 \\
0.0 \\
0.0 \\
0.0 \\
0.0\end{array}$ & $\begin{array}{c}0.0 \\
0.0 \\
0.0 \\
0.0 \\
30.00\end{array}$ & $\begin{array}{l}0.0 \\
0.0 \\
0.0 \\
0.0 \\
0.0\end{array}$ & $\begin{array}{c}15.00 \\
15.00 \\
20.00 \\
20.00 \\
0.0\end{array}$ & $\begin{array}{l}0.0 \\
0.0 \\
0.0 \\
0.0 \\
0.0\end{array}$ & $\begin{array}{l}0.0 \\
0.0 \\
0.0 \\
0.0 \\
0.0\end{array}$ & $\begin{array}{l}0.0 \\
0.0 \\
0.0 \\
0.0 \\
0.0\end{array}$ \\
\hline $\begin{array}{l}4.8 .11 \\
4.8 .12 \\
4.8 .13 \\
4.3 .14 \\
4.8 .15\end{array}$ & $\begin{array}{l}22.0 \\
30.0 \\
16.0 \\
25.0 \\
30.0\end{array}$ & $\begin{array}{r}0.0 \\
10.0 \\
0.0 \\
0.0 \\
10.0\end{array}$ & $\begin{array}{l}0.0 \\
0.0 \\
0.0 \\
0.0 \\
0.0\end{array}$ & $\begin{array}{l}0.0 \\
0.0 \\
0.0 \\
0.0 \\
0.0\end{array}$ & $\begin{array}{l}0.0 \\
40.00 \\
40.00 \\
40.00 \\
50.00\end{array}$ & $\begin{array}{c}30.00 \\
0.0 \\
0.0 \\
0.0 \\
0.0\end{array}$ & $\begin{array}{l}0.0 \\
0.0 \\
0.0 \\
0.0 \\
0.0\end{array}$ & $\begin{array}{l}0.0 \\
0.0 \\
0.0 \\
0.0 \\
0.0\end{array}$ & $\begin{array}{l}0.0 \\
0.0 \\
0.0 \\
0.0 \\
0.0\end{array}$ & $\begin{array}{l}0.0 \\
0.0 \\
0.0 \\
0.0 \\
0.0\end{array}$ \\
\hline $\begin{array}{l}4.8 .16 \\
4.8 .17 \\
4.8 .18 \\
4.8 .19 \\
4.8 .20\end{array}$ & $\begin{array}{l}25.0 \\
15.0 \\
30.0 \\
25.0 \\
12.0\end{array}$ & $\begin{array}{r}0.0 \\
0.0 \\
10.0 \\
0.0 \\
0.0\end{array}$ & $\begin{array}{l}0.0 \\
0.0 \\
0.0 \\
0.0 \\
0.0\end{array}$ & $\begin{array}{l}0.0 \\
0.0 \\
0.0 \\
0.0 \\
0.0\end{array}$ & $\begin{array}{l}50.00 \\
50.00 \\
70.00 \\
70.00 \\
70.00\end{array}$ & $\begin{array}{l}0.0 \\
0.0 \\
0.0 \\
0.0 \\
0.0\end{array}$ & $\begin{array}{l}0.0 \\
0.0 \\
0.0 \\
0.0 \\
0.0\end{array}$ & $\begin{array}{l}0.0 \\
0.0 \\
0.0 \\
0.0 \\
0.0\end{array}$ & $\begin{array}{l}0.0 \\
0.0 \\
0.0 \\
0.0 \\
0.0\end{array}$ & $\begin{array}{l}0.0 \\
0.0 \\
0.0 \\
0.0 \\
0.0\end{array}$ \\
\hline $\begin{array}{l}4.9 .21 \\
4.9 .22 \\
4.8 .23 \\
4.9 .24 \\
4.9 .25\end{array}$ & $\begin{array}{l}2.5 .0 \\
16.0 \\
14.0 \\
30.0 \\
25.0\end{array}$ & $\begin{array}{l}0.0 \\
0.0 \\
0.0 \\
10.0 \\
.0 .0\end{array}$ & $\begin{array}{l}0.0 \\
0.0 \\
0.0 \\
0.0 \\
0.0\end{array}$ & $\begin{array}{l}0.0 \\
0.0 \\
0.0 \\
0.0 \\
0.0\end{array}$ & $\begin{array}{l}70.00 \\
70.00 \\
70.00 \\
90.00 \\
90.00\end{array}$ & $\begin{array}{l}0.0 \\
0.0 \\
0.0 \\
0.0 \\
0.0\end{array}$ & $\begin{array}{l}0.0 \\
0.0 \\
0.0 \\
0.0 \\
0.0\end{array}$ & $\begin{array}{l}0.0 \\
0.0 \\
0.0 \\
0.0 \\
0.0\end{array}$ & $\begin{array}{l}0.0 \\
0.0 \\
0.0 \\
0.0 \\
0.0\end{array}$ & $\begin{array}{l}0.0 \\
0.0 \\
0.0 \\
0.0 \\
0.0\end{array}$ \\
\hline $\begin{array}{l}4.3 .26 \\
4.3 .27 \\
4.3 .28 \\
4.8 .24 \\
4.3 .30\end{array}$ & $\begin{array}{l}20.0 \\
16.0 \\
14.0 \\
12.0 \\
12.0\end{array}$ & $\begin{array}{l}0.0 \\
0.0 \\
0.0 \\
0.0 \\
0.0\end{array}$ & $\begin{array}{l}0.0 \\
0.0 \\
0.0 \\
0.0 \\
0.0\end{array}$ & $\begin{array}{l}0.0 \\
0.0 \\
0.0 \\
0.0 \\
0.0\end{array}$ & $\begin{array}{l}90.00 \\
90.00 \\
90.00 \\
90.00 \\
90.00\end{array}$ & $\begin{array}{l}0.0 \\
0.0 \\
0.0 \\
0.0 \\
0.0\end{array}$ & $\begin{array}{l}0.0 \\
0.0 \\
0.0 \\
0.0 \\
0.0\end{array}$ & $\begin{array}{l}0.0 \\
0.0 \\
0.0 \\
0.0 \\
0.0\end{array}$ & $\begin{array}{l}0.0 \\
0.0 \\
0.0 \\
0.0 \\
0.0\end{array}$ & $\begin{array}{l}0.0 \\
0.0 \\
0.0 \\
0.0 \\
0.0\end{array}$ \\
\hline $\begin{array}{l}4.3 .31 \\
4.3 .32 \\
4.3 .33 \\
4.8 .34\end{array}$ & $\begin{array}{l}30.0 \\
20.0 \\
10.0 \\
12.0\end{array}$ & $\begin{array}{r}10.0 \\
0.0 \\
0.0 \\
0.0\end{array}$ & $\begin{array}{l}0.0 \\
0.0 \\
0.0 \\
0.0\end{array}$ & $\begin{array}{l}0.0 \\
0.0 \\
0.0 \\
0.0\end{array}$ & $\begin{array}{l}90.00 \\
90.00 \\
90.00 \\
90.00\end{array}$ & $\begin{array}{l}0.0 \\
0.0 \\
0.0 \\
0.0\end{array}$ & $\begin{array}{l}20.00 \\
20.00 \\
20.00 \\
20.00\end{array}$ & $\begin{array}{l}0.0 \\
0.0 \\
0.0 \\
0.0\end{array}$ & $\begin{array}{l}0.0 \\
0.0 \\
0.0 \\
0.0\end{array}$ & $\begin{array}{l}0.0 \\
0.0 \\
0.0 \\
0.0\end{array}$ \\
\hline
\end{tabular}


TABLE XL (Contd.)

COMBINED EFFECTS TEST GROUP 4.8

EXPEPIMFNTAL MEASIJPMENTS FIJR TESTS 4.3 .1 TO $4.8 .34-0.49-$ IN. DOWNCDMER GAP

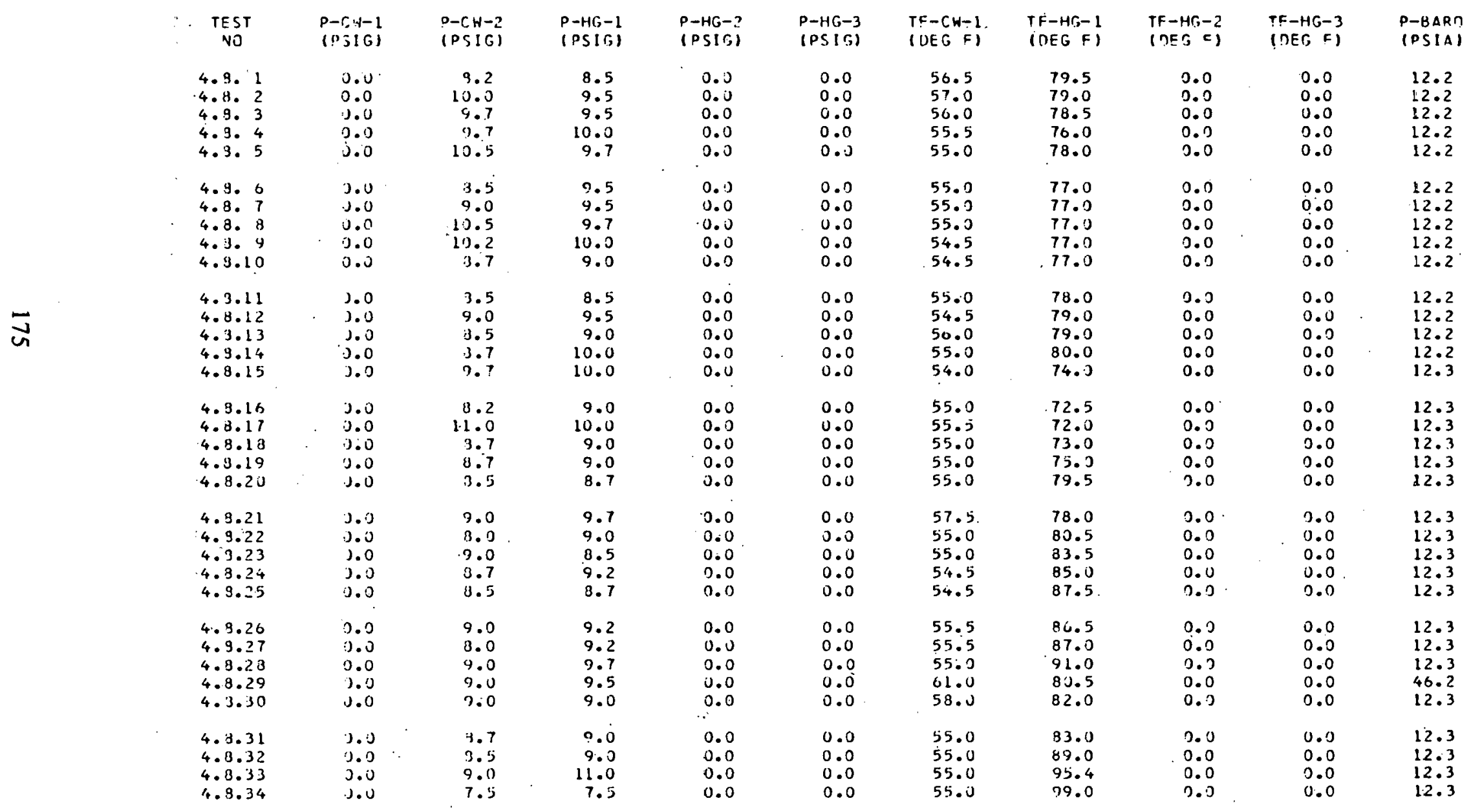


TABLE XL (Contd.)

COMBINED EFFECTS TEST GROUP 4.8

EXPERIMENTAL MEASURMENTS FOR TESTS 4.8 .1 TO $4.8 .34--0.49-$ IN. DOHNCOMER GAP

\begin{tabular}{|c|c|c|c|c|c|c|c|c|c|}
\hline & & R PLENUM & FLOW & & YPASS FL & & & & \\
\hline $\begin{array}{l}\text { TEST } \\
\text { NO }\end{array}$ & $\begin{array}{l}\text { INIT IAL } \\
\text { WE IGHT } \\
\text { (LB) }\end{array}$ & $\begin{array}{l}\text { FINAL } \\
\text { HEIGHT } \\
\text { ILBI }\end{array}$ & $\begin{array}{l}\text { TIME OF } \\
\text { COLLECTICN } \\
\text { (SEC) }\end{array}$ & $\begin{array}{l}\text { INITIAL } \\
\text { WE IGHT } \\
\text { (LB) }\end{array}$ & $\begin{array}{l}\text { FINAL } \\
\text { WEIGHT } \\
\text { (L B) }\end{array}$ & $\begin{array}{l}\text { TIME OF } \\
\text { COLLECTION } \\
\text { (SEC) }\end{array}$ & $\begin{array}{l}\text { PLENUM } \\
\text { LEVEL } \\
\text { (IN) }\end{array}$ & $\begin{array}{l}P-(P-1) \\
\text { (PSIG) }\end{array}$ & $\begin{array}{l}T F-L P-1 \\
(D E G \quad F)\end{array}$ \\
\hline $\begin{array}{ll}4.8 .8 & 1 \\
4.3 . & 2 \\
4.8 .8 & 3 \\
4.8 .8 & 4 \\
4.3 . & 5\end{array}$ & $\begin{array}{l}133.3 \\
281.0 \\
405.5 \\
132.8 \\
272.3\end{array}$ & $\begin{array}{l}281.0 \\
405.5 \\
530.8 \\
272.3 \\
385.0\end{array}$ & $\begin{array}{l}30.0 \\
30.0 \\
30.0 \\
35.0 \\
30.0\end{array}$ & $\begin{array}{r}95.5 \\
121.5 \\
176.5 \\
94.5 \\
122.5\end{array}$ & $\begin{array}{l}121.5 \\
176.5 \\
232.0 \\
122.5 \\
187.5\end{array}$ & $\begin{array}{l}45.0 \\
50.0 \\
45.0 \\
50.0 \\
35.0\end{array}$ & $\begin{array}{l}12.0 \\
12.0 \\
12.0 \\
12.0 \\
12.0\end{array}$ & $\begin{array}{l}10.0 \\
10.0 \\
10.0 \\
10.0 \\
10.0\end{array}$ & $\begin{array}{l}56.5 \\
57.0 \\
56.0 \\
56.0 \\
55.0\end{array}$ \\
\hline $\begin{array}{r}4.8 .6 \\
4.8 .8 \\
4.8 .8 \\
4.8 .89 \\
4.8 .10\end{array}$ & $\begin{array}{l}385.0 \\
453.3 \\
150.0 \\
212.0 \\
300.0\end{array}$ & $\begin{array}{l}453.3 \\
524.0 \\
212.0 \\
300.0 \\
364.5\end{array}$ & $\begin{array}{l}20.0 \\
20.0 \\
20.0 \\
30.0 \\
25.0\end{array}$ & $\begin{array}{r}187.5 \\
219.0 \\
98.0 \\
192.0 \\
231.3\end{array}$ & $\begin{array}{l}219.0 \\
284.3 \\
192.0 \\
231.3 \\
321.5\end{array}$ & $\begin{array}{l}40.0 \\
50.0 \\
40.0 \\
50.0 \\
30.0\end{array}$ & $\begin{array}{l}12.0 \\
12.0 \\
12.0 \\
12.0 \\
12.0\end{array}$ & $\begin{array}{l}10.0 \\
10.0 \\
10.0 \\
10.0 \\
10.0\end{array}$ & $\begin{array}{l}55.0 \\
55.0 \\
56.5 \\
55.0 \\
55.0\end{array}$ \\
\hline $\begin{array}{r}4.8 .11 \\
4.0 .12 \\
4.8 .13 \\
4.8 .14 \\
4.8 .15\end{array}$ & $\begin{array}{l}364.5 \\
423.0 \\
468.0 \\
433.5 \\
132.5\end{array}$ & $\begin{array}{l}423.0 \\
463.0 \\
534.5 \\
525.5 \\
180.3\end{array}$ & $\begin{array}{l}25.0 \\
25.0 \\
30.0 \\
25.0 \\
40.0\end{array}$ & $\begin{array}{r}321.5 \\
342.0 \\
459.0 \\
460.0 \\
91.0\end{array}$ & $\begin{array}{l}342.0 \\
459.0 \\
460.0 \\
487.5 \\
223.0\end{array}$ & $\begin{array}{l}30.0 \\
30.0 \\
45.0 \\
30.0 \\
30.0\end{array}$ & $\begin{array}{l}12.0 \\
12.0 \\
12.0 \\
12.0 \\
12.0\end{array}$ & $\begin{array}{l}10.0 \\
10.0 \\
10.0 \\
10.0 \\
10.0\end{array}$ & $\begin{array}{l}55.5 \\
55.5 \\
56.0 \\
55.5 \\
55.0\end{array}$ \\
\hline $\begin{array}{r}4.8 .16 \\
4.8 .17 \\
4.3 .18 \\
4.8 .19 \\
4.9 .20\end{array}$ & $\begin{array}{l}190.3 \\
231.0 \\
291.5 \\
313.3 \\
339.3\end{array}$ & $\begin{array}{l}231.0 \\
290.5 \\
313.3 \\
339.3 \\
412.0\end{array}$ & $\begin{array}{l}40.0 \\
40.0 \\
40.0 \\
45.0 \\
45.0\end{array}$ & $\begin{array}{l}223.0 \\
315.5 \\
340.0 \\
491.5 \\
594.5\end{array}$ & $\begin{array}{l}315.5 \\
340.0 \\
491.5 \\
594.5 \\
600.0\end{array}$ & $\begin{array}{l}40.0 \\
45.0 \\
30.0 \\
35.0 \\
60.0\end{array}$ & $\begin{array}{l}12.0 \\
12.0 \\
12.0 \\
12.0 \\
12.0\end{array}$ & $\begin{array}{l}10.0 \\
10.0 \\
10.0 \\
10.0 \\
10.0\end{array}$ & $\begin{array}{l}55.0 \\
55.5 \\
55.0 \\
55.5 \\
55.5\end{array}$ \\
\hline $\begin{array}{r}4.9 .21 \\
4.3 .22 \\
4.0 .23 \\
4.8 .24 \\
4.8 .25\end{array}$ & $\begin{array}{l}133.8 \\
160.5 \\
193.5 \\
229.0 \\
241.0\end{array}$ & $\begin{array}{l}160.5 \\
198.5 \\
229.0 \\
241.0 \\
252.5\end{array}$ & $\begin{array}{l}40.0 \\
55.0 \\
30.0 \\
40.0 \\
40.0\end{array}$ & $\begin{array}{l}104.5 \\
193.5 \\
255.0 \\
288.5 \\
449.0\end{array}$ & $\begin{array}{l}193.5 \\
255.0 \\
288.5 \\
449.0 \\
546.0\end{array}$ & $\begin{array}{l}40.0 \\
40.0 \\
35.0 \\
30.0 \\
30.0\end{array}$ & $\begin{array}{l}12.0 \\
12.0 \\
12.0 \\
12.0 \\
12.0\end{array}$ & $\begin{array}{l}10.0 \\
10.0 \\
10.0 \\
10.0 \\
10.0\end{array}$ & $\begin{array}{l}56.0 \\
57.0 \\
55.0 \\
55.0 \\
55.5\end{array}$ \\
\hline $\begin{array}{r}4.5 .26 \\
4.3 .27 \\
4.3 .20 \\
4.8 .29 \\
4.9 .30\end{array}$ & $\begin{array}{l}253.5 \\
364.8 \\
234.8 \\
152.5 \\
222.0\end{array}$ & $\begin{array}{l}264.8 \\
234.8 \\
310.8 \\
222.0 \\
207.5\end{array}$ & $\begin{array}{l}40.0 \\
45.0 \\
50.0 \\
40.0 \\
45.0\end{array}$ & $\begin{array}{l}546.0 \\
634.0 \\
688.0 \\
107.0 \\
121.0\end{array}$ & $\begin{array}{l}634.0 \\
688.0 \\
140.0 \\
121.0 \\
130.0\end{array}$ & $\begin{array}{l}35.2 \\
30.0 \\
35.0 \\
50.0 \\
45.0\end{array}$ & $\begin{array}{l}12.0 \\
12.0 \\
12.0 \\
12.0 \\
12.0\end{array}$ & $\begin{array}{l}10.0 \\
10.0 \\
10.0 \\
10.0 \\
10.0\end{array}$ & $\begin{array}{l}55.5 \\
55.5 \\
56.0 \\
62.0 \\
58.5\end{array}$ \\
\hline $\begin{array}{l}4.8 .31 \\
4.3 .32 \\
4.3 .33 \\
4.3 .34\end{array}$ & $\begin{array}{l}247.5 \\
245.0 \\
303.3 \\
353.0\end{array}$ & $\begin{array}{r}295.0 \\
303.5 \\
355.0 \\
382.0\end{array}$ & $\begin{array}{l}60.0 \\
60.0 \\
45.0 \\
55.0\end{array}$ & $\begin{array}{l}130.3 \\
269.5 \\
375.5 \\
394.0\end{array}$ & $\begin{array}{l}269.5 \\
375.5 \\
394.0 \\
431.0\end{array}$ & $\begin{array}{l}25.0 \\
40.0 \\
70.0 \\
30.0\end{array}$ & $\begin{array}{l}12.0 \\
12.0 \\
12.0 \\
12.0\end{array}$ & $\begin{array}{l}10.0 \\
10.0 \\
10.0 \\
10.0\end{array}$ & $\begin{array}{l}58.5 \\
58.5 \\
55.0 \\
55.5\end{array}$ \\
\hline
\end{tabular}




\section{TABLE XLI}

COMBINED EFFECTS TEST GROUP 4.9

TRANSPAPENT VESSEL TEST DATA FIJR TESTS 4.9.1 TO 4.9.21-- 0.49-IN. DOMNCOMER GAP SUMMARY UF CALCULATED RESULTS

\begin{tabular}{|c|c|c|c|c|c|c|c|c|c|c|}
\hline $\begin{array}{l}\text { TEST } \\
\text { NO }\end{array}$ & $\begin{array}{l}\text { DJWNCOMER } \\
\text { AIR FLUW } \\
\text { ILISISECI }\end{array}$ & $\begin{array}{l}\text { D DWNC TYEQ } \\
\text { AIF OENSITY } \\
\text { (LYIFT } \$ 3 \text { ) }\end{array}$ & $\begin{array}{l}\text { LOWEP. PLENUM } \\
\text { PRESSUPE } \\
\text { (PSIA) }\end{array}$ & $\begin{array}{l}\text { CCLO LEG } \\
\text { AIR FLOW } \\
\text { (LB/SEC) }\end{array}$ & $\begin{array}{l}\text { DOWNCOMER } \\
\text { HATER FLUWW } \\
\text { (LB/SSC) }\end{array}$ & $\begin{array}{l}\text { WATER FLJW } \\
\text { CONTINUITY }\end{array}$ & $\begin{array}{c}\text { J-A!? } \\
\text { DOWNCSMEP. } \\
\text { (FT/SEC) }\end{array}$ & $\begin{array}{l}J-H A T E R \\
\text { NOWEIC JMER } \\
(E T / S E C)\end{array}$ & $\left(J r_{*} *\right) * 1 / 2$ & $(J L *) * * 1 / 2$ \\
\hline 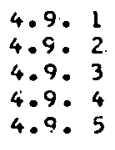 & $\begin{array}{l}0.0 \\
0.0075 \\
0.0075 \\
0.0153 \\
0.0152\end{array}$ & $\begin{array}{l}0.1166 \\
0.1164 \\
0.1165 \\
0.1165 \\
0.1164\end{array}$ & $\begin{array}{l}22.21 \\
22.21 \\
22.21 \\
22.21 \\
22.21\end{array}$ & $\begin{array}{l}0.0976 \\
0.0965 \\
0.0955 \\
0.0496 \\
0.0955\end{array}$ & $\begin{array}{l}4.275 \\
3.740 \\
3.575 \\
3.350 \\
3.095\end{array}$ & $\begin{array}{l}1.064 \\
0.938 \\
1.012 \\
1.027 \\
0.997\end{array}$ & $\begin{array}{l}0.0 \\
1.00 \\
1.00 \\
2.04 \\
2.02\end{array}$ & $\begin{array}{l}1.064 \\
0.931 \\
0.390 \\
0.834 \\
0.770\end{array}$ & $\begin{array}{l}0.0 \\
0.1635 \\
0.1636 \\
0.2332 \\
0.2320\end{array}$ & $\begin{array}{l}0.3096 \\
0.7573 \\
0.7404 \\
0.7167 \\
0.6389\end{array}$ \\
\hline 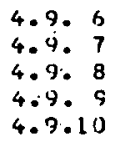 & $\begin{array}{l}0.0231 \\
0.0223 \\
0.0314 \\
0.0299 \\
0.0457\end{array}$ & $\begin{array}{l}0.1162 \\
0.1164 \\
0.1165 \\
0.1163 \\
0.1166\end{array}$ & $\begin{array}{l}22.21 \\
22.21 \\
22.21 \\
22.21 \\
22.21\end{array}$ & $\begin{array}{l}0.0935 \\
0.0934 \\
0.0935 \\
0.0935 \\
0.0988\end{array}$ & $\begin{array}{l}2.840 \\
2.767 \\
2.600 \\
2.480 \\
2.067\end{array}$ & $\begin{array}{l}0.946 \\
1.005 \\
0.980 \\
1.0113 \\
1.006\end{array}$ & $\begin{array}{l}3.09 \\
2.99 \\
4.18 \\
3.98 \\
6.09\end{array}$ & $\begin{array}{l}0.707 \\
0.699 \\
0.647 \\
0.617 \\
0.514\end{array}$ & $\begin{array}{l}0.2866 \\
0.2915 \\
0.3336 \\
0.3257 \\
0.4025\end{array}$ & $\begin{array}{l}0.6599 \\
0.6513 \\
0.6314 \\
0.6167 \\
0.5629\end{array}$ \\
\hline $\begin{array}{l}4.9 .11 \\
4.9 .12 \\
4.9 .11 \\
4.9 .14 \\
4.9 .15\end{array}$ & $\begin{array}{l}0.0456 \\
0.0459 \\
0.0769 \\
0.0762 \\
0.0613\end{array}$ & $\begin{array}{l}0.1165 \\
0.1146 \\
0.1157 \\
0.11164 \\
0.1164\end{array}$ & $\begin{array}{l}22.21 \\
22.21 \\
22.21 \\
22.21 \\
22.21\end{array}$ & $\begin{array}{l}0.0965 \\
0.0939 \\
0.0965 \\
0.0934 \\
0.0936\end{array}$ & $\begin{array}{l}2.050 \\
1.929 \\
1.417 \\
1.071 \\
1.68 .8\end{array}$ & $\begin{array}{l}1.076 \\
0.494 \\
1.017 \\
1.025 \\
0.986\end{array}$ & $\begin{array}{r}6.08 \\
6.22 \\
10.32 \\
10.16 \\
8.19\end{array}$ & $\begin{array}{l}0.510 \\
0.480 \\
0.353 \\
0.267 \\
0.420\end{array}$ & $\begin{array}{l}0.4022 \\
0.4051 \\
0.5232 \\
0.5200 \\
0.4654\end{array}$ & $\begin{array}{l}0.5607 \\
0.5438 \\
0.4661 \\
0.4053 \\
0.5087\end{array}$ \\
\hline $\begin{array}{l}4.9 .16 \\
4.0 .1 .7 \\
4.9 .18 \\
4.9 .19 \\
4.9 .20\end{array}$ & $\begin{array}{l}0.0608 \\
0.0762 \\
0.1098 \\
0.1068 \\
0.1496\end{array}$ & $\begin{array}{l}0.1164 \\
0.1163 \\
0.1215 \\
0.1164 \\
0.1374\end{array}$ & $\begin{array}{l}22.21 \\
22.21 \\
23.21 \\
22.21 \\
26.21\end{array}$ & $\begin{array}{l}0.0934 \\
0.0954 \\
0.0986 \\
0.0934 \\
0.1034\end{array}$ & $\begin{array}{l}1.453 \\
1.121 \\
0.737 \\
0.563 \\
0.387\end{array}$ & $\begin{array}{l}1.018 \\
1.003 \\
0.999 \\
1.028 \\
1.023\end{array}$ & $\begin{array}{r}8.12 \\
10.18 \\
14.03 \\
14.25 \\
16.92\end{array}$ & $\begin{array}{l}0.363 \\
0.279 \\
0.184 \\
0.149 \\
0.096\end{array}$ & $\begin{array}{l}0.4647 \\
0.5202 \\
0.6176 \\
0.6157 \\
0.6973\end{array}$ & $\begin{array}{l}0.4729 \\
0.4147 \\
0.3363 \\
0.2937 \\
0.2438\end{array}$ \\
\hline 4.9 .21 & 0.1369 & 0.1102 & 22.21 & 0.0913 & 0.239 & 1.066 & 13.29 & 0.059 & 0.6972 & 0.1014 \\
\hline
\end{tabular}


TABLE XLI (Contd.)

COMBINED EFFECTS TEST GROUP 4.9

EXPER IMENTAL MEASURMENTS FOR. TESTS 4.9 .1 TO $4.9 .21--0.49-$ IN. DOHNCOMER GAP

\begin{tabular}{|c|c|c|c|c|c|c|c|c|c|c|}
\hline $\begin{array}{l}\text { TEST } \\
\text { NO }\end{array}$ & $\begin{array}{c}R M-C H-1 \\
(G P M)\end{array}$ & $\begin{array}{c}R: 1-C W-2 \\
(G P M)\end{array}$ & $\begin{array}{c}F T-(W-1 \\
(G P M)\end{array}$ & $\begin{array}{c}F T-C H-2 \\
(G P M M)\end{array}$ & $\begin{array}{c}\text { RM-HG-1 } \\
\text { (CFM) }\end{array}$ & $\begin{array}{c}R M-H F-2 \\
(C F M)\end{array}$ & $\begin{array}{c}R M-H G-3 \\
(C F M)\end{array}$ & $\begin{array}{c}\text { RH-HG-4 } \\
\text { (CFM) }\end{array}$ & $\begin{array}{c}F^{\top}-H G-1 \\
\text { (CFM) }\end{array}$ & $\begin{array}{l}F Y-H G-2 \\
\text { (CFM) }\end{array}$ \\
\hline $\begin{array}{ll}4.9 . & 1 \\
4.9 . & 2 \\
4.9 . & 3 \\
4.9 . & 4 \\
4.9 . & 5\end{array}$ & $\begin{array}{l}30.0 \\
3.0 .0 \\
30.0 \\
30.0 \\
30.0\end{array}$ & $\begin{array}{r}10.0 \\
10.0 \\
3.0 \\
10.0 \\
0.0\end{array}$ & $\begin{array}{l}0.0 \\
0.0 \\
0.0 \\
0.0 \\
0.0\end{array}$ & $\begin{array}{l}0.0 \\
0.0 \\
0.0 \\
0.0 \\
0.0\end{array}$ & $\begin{array}{l}0.0 \\
0.0 \\
0.0 \\
0.0 \\
0.0\end{array}$ & $\begin{array}{l}0.0 \\
0.0 \\
0.0 \\
0.0 \\
0.0\end{array}$ & $\begin{array}{c}0.0 \\
5.00 \\
5.00 \\
10.00 \\
10.00\end{array}$ & $\begin{array}{l}0.0 \\
0.0 \\
0.0 \\
0.0 \\
0.0\end{array}$ & $\begin{array}{l}0.0 \\
0.0 \\
0.0 \\
0.0 \\
0.0\end{array}$ & $\begin{array}{l}0.0 \\
0.0 \\
0.0 \\
0.0 \\
0.0\end{array}$ \\
\hline $\begin{array}{l}4.9 .6 \\
4.9 .7 \\
4.9 .8 \\
4.9 .79 \\
4.9 .10\end{array}$ & $\begin{array}{l}30.0 \\
25.0 \\
30.0 \\
22.0 \\
30.0\end{array}$ & $\begin{array}{r}10.0 \\
0.0 \\
10.0 \\
0.0 \\
10.0\end{array}$ & $\begin{array}{l}0.0 \\
0.0 \\
0.0 \\
0.0 \\
0.0\end{array}$ & $\begin{array}{l}0.0 \\
0.0 \\
0.0 \\
0.0 \\
0.0\end{array}$ & $\begin{array}{l}0.0 \\
0.0 \\
0.0 \\
0.0 \\
0.0\end{array}$ & $\begin{array}{l}0.0 \\
0.0 \\
0.0 \\
0.0 \\
0.0\end{array}$ & $\begin{array}{l}15.00 \\
15.00 \\
20.00 \\
20.00 \\
30.00\end{array}$ & $\begin{array}{l}0.0 \\
0.0 \\
0.0 \\
0.0 \\
0.0\end{array}$ & $\begin{array}{l}0.0 \\
0.0 \\
0.0 \\
0.0 \\
0.0\end{array}$ & $\begin{array}{l}0.0 \\
0.0 \\
0.0 \\
0.0 \\
0.0\end{array}$ \\
\hline $\begin{array}{l}4.9 .11 \\
4.9 .12 \\
4.9 .13 \\
4.9 .14 \\
4.9 .15\end{array}$ & $\begin{array}{l}18.0 \\
12.0 \\
3.3 .0 \\
13.0 \\
30.0\end{array}$ & $\begin{array}{r}0 . c \\
0.0 \\
10.0 \\
0.0 \\
10.0\end{array}$ & $\begin{array}{l}0.0 \\
0.0 \\
0.0 \\
0.0 \\
0.0\end{array}$ & $\begin{array}{l}0.0 \\
0.0 \\
0.0 \\
0.0 \\
0.0\end{array}$ & $\begin{array}{c}0.0 \\
0.0 \\
50.00 \\
50.00 \\
40.00\end{array}$ & $\begin{array}{l}0.0 \\
0.0 \\
0.0 \\
0.0 \\
0.0\end{array}$ & $\begin{array}{c}30.00 \\
30.00 \\
0.00 \\
0.0 \\
0.0\end{array}$ & $\begin{array}{l}0.0 \\
0.0 \\
0.0 \\
0.0 \\
0.0\end{array}$ & $\begin{array}{l}0.0 \\
0.0 \\
0.0 \\
0.0 \\
0.0\end{array}$ & $\begin{array}{l}0.0 \\
0.0 \\
0.0 \\
0.0 \\
0.0\end{array}$ \\
\hline $\begin{array}{l}4.9 .16 \\
4.9 .17 \\
4.9 .18 \\
4.9 .19 \\
4.9 .20\end{array}$ & $\begin{array}{l}15.0 \\
15.0 \\
30.0 \\
10.0 \\
30.0\end{array}$ & $\begin{array}{r}0.0 \\
0.0 \\
10.0 \\
0.0 \\
10.0\end{array}$ & $\begin{array}{l}0.0 \\
0.0 \\
0.0 \\
0.0 \\
0.0\end{array}$ & $\begin{array}{l}0.0 \\
0.0 \\
0.0 \\
0.0 \\
0.0\end{array}$ & $\begin{array}{l}40.00 \\
50.00 \\
70.00 \\
70.00 \\
90.00\end{array}$ & $\begin{array}{l}0.0 \\
0.0 \\
0.0 \\
0.0 \\
0.0\end{array}$ & $\begin{array}{l}0.0 \\
0.0 \\
0.0 \\
0.0 \\
0.0\end{array}$ & $\begin{array}{l}0.0 \\
0.0 \\
0.0 \\
0.0 \\
0.0\end{array}$ & $\begin{array}{l}0.0 \\
0.0 \\
0.0 \\
0.0 \\
0.0\end{array}$ & $\begin{array}{l}0.0 \\
0.0 \\
0.0 \\
0.0 \\
0.0\end{array}$ \\
\hline 4.9 .21 & 6.0 & 0.0 & 0.0 & 0.0 & 90.00 & 0.0 & 0.0 & 0.0 & 0.0 & 0.0 \\
\hline $\begin{array}{c}\text { TSST } \\
\text { NO }\end{array}$ & $\begin{array}{l}P-C W-1 \\
(P S I G)\end{array}$ & $\begin{array}{l}P-C W-2 \\
(P S I G)\end{array}$ & $\begin{array}{l}P-H G-1 \\
(P S \mid G)\end{array}$ & $\begin{array}{l}P-H G-2 \\
(P S I G)\end{array}$ & $\begin{array}{l}P-H G-3 \\
\text { (PSIG) }\end{array}$ & $\begin{array}{l}T F-C H-1 \\
(O E G F)\end{array}$ & $\begin{array}{l}T F-H G-1 \\
(D E G F)\end{array}$ & $\begin{array}{l}\text { TE-MG-2 } \\
(D E G F)\end{array}$ & $\begin{array}{l}\text { TF-Mr-3 } \\
\text { (DFG F) }\end{array}$ & $\begin{array}{l}\text { P-BARO } \\
\text { (PSIAI) }\end{array}$ \\
\hline $\begin{array}{ll}4.9 . & 1 \\
4.9 . & 2 \\
4.9 . & 3 \\
4.9 . & 4 \\
4.9 . & 5\end{array}$ & $\begin{array}{l}0.0 \\
0.0 \\
0.0 \\
0.0 \\
0.0\end{array}$ & $\begin{array}{l}12.0 \\
11.5 \\
11.0 \\
12.5 \\
11.0\end{array}$ & $\begin{array}{r}10.0 \\
9.5 \\
9.5 \\
10.2 \\
9.7\end{array}$ & $\begin{array}{l}0.0 \\
0.0 \\
0.0 \\
0.0 \\
0.0\end{array}$ & $\begin{array}{l}0.0 \\
0.0 \\
0.0 \\
0.0 \\
0.0\end{array}$ & $\begin{array}{l}54.0 \\
54.5 \\
54.0 \\
54.0 \\
54.5\end{array}$ & $\begin{array}{l}76.5 \\
77.5 \\
76.0 \\
76.5 \\
76.5\end{array}$ & $\begin{array}{l}0.0 \\
0.0 \\
0.0 \\
0.0 \\
0.0\end{array}$ & $\begin{array}{l}0.0 \\
0.0 \\
0.0 \\
0.0 \\
0.0\end{array}$ & $\begin{array}{l}12.2 \\
12.2 \\
12.2 \\
12.2 \\
12.2\end{array}$ \\
\hline $\begin{array}{l}4.9 .6 \\
4.9 .97 \\
4.9 .8 \\
4.9 .8 \\
4.9 .10\end{array}$ & $\begin{array}{l}0.0 \\
0.0 \\
0.0 \\
0.0 \\
0.0\end{array}$ & $\begin{array}{l}12.5 \\
10.0 \\
12.5 \\
10.0 \\
12.5\end{array}$ & $\begin{array}{r}10.5 \\
9.0 \\
11.0 \\
9.0 \\
10.0\end{array}$ & $\begin{array}{l}0.0 \\
0.0 \\
0.0 \\
0.0 \\
0.0\end{array}$ & $\begin{array}{l}0.0 \\
0.0 \\
0.0 \\
0.0 \\
0.0\end{array}$ & $\begin{array}{l}54.5 \\
54.5 \\
55.0 \\
53.0 \\
52.0\end{array}$ & $\begin{array}{l}77.0 \\
78.0 \\
70.0 \\
72.0 \\
77.5\end{array}$ & $\begin{array}{l}0.0 \\
0.0 \\
0.0 \\
0.0 \\
0.0\end{array}$ & $\begin{array}{l}0.0 \\
0.0 \\
0.0 \\
0.0 \\
0.0\end{array}$ & $\begin{array}{l}12.2 \\
12.2 \\
12.2 \\
12.2 \\
12.2\end{array}$ \\
\hline
\end{tabular}


TABLE XLI (Contd.)

COMBINED EFFECTS TEST GROIP 4.9

EXPERIMENTAL MFASIJPMENTS FOR TESTS 4.9 .1 TO $4.9 .21-0.49-1$ - D. DOHNCOMER GAP

\begin{tabular}{|c|c|c|c|c|c|c|c|c|c|c|}
\hline $\begin{array}{l}\text { TEST } \\
\text { NOT }\end{array}$ & $\begin{array}{l}P-C W-1 \\
\text { (PSIGI) }\end{array}$ & $\begin{array}{l}D-C W-2 \\
(P S I G)\end{array}$ & $\begin{array}{l}P-H G-1 \\
(P S I G)\end{array}$ & $\begin{array}{l}P-H G-2 \\
(P S I G)\end{array}$ & $\begin{array}{l}\mathrm{P}-\mathrm{HG}-3 \\
(\mathrm{OS} I(6)\end{array}$ & $\begin{array}{l}T F-C H-1 \\
(D E G F)\end{array}$ & $\begin{array}{l}\text { TF-HG- } \\
\text { (DEG F) }\end{array}$ & $\begin{array}{l}\text { YE-HG-2 } \\
\text { (DES F) }\end{array}$ & $\begin{array}{l}\text { TC-HG-3 } \\
\text { IDET F.) }\end{array}$ & $\begin{array}{l}P-B A R \cap \\
\text { IPSIAI }\end{array}$ \\
\hline $\begin{array}{r}4.9 .11 \\
4.9 .12 \\
4.9 .13 \\
4.9 .14 \\
4.9 .15\end{array}$ & $\begin{array}{l}0.0 \\
0.0 \\
0.0 \\
0.0 \\
0.0\end{array}$ & $\begin{array}{l}11.5 \\
10.5 \\
11.5 \\
10.0 \\
12.5\end{array}$ & $\begin{array}{r}10.0 \\
10.2 \\
10.0 \\
9.7 \\
10.2\end{array}$ & $\begin{array}{l}0.0 \\
0.0 \\
0.0 \\
0.0 \\
0.0\end{array}$ & $\begin{array}{l}0.0 \\
0.0 \\
0.0 \\
0.0 \\
0.0\end{array}$ & $\begin{array}{l}54.0 \\
61.0 \\
54.0 \\
55.0 \\
54.0\end{array}$ & $\begin{array}{l}80.0 \\
79.0 \\
81.0 \\
85.0 \\
86.0\end{array}$ & $\begin{array}{l}0.0 \\
0.0 \\
0.0 \\
0.0 \\
0.0\end{array}$ & $\begin{array}{l}0.0 \\
0.0 \\
0.0 \\
0.0 \\
0.0\end{array}$ & $\begin{array}{l}12.2 \\
12.2 \\
12.2 \\
12.2 \\
12.2\end{array}$ \\
\hline $\begin{array}{r}4.9 .16 \\
4.9 .17 \\
4.9 .18 \\
4.9 .19 \\
4.9 .20\end{array}$ & $\begin{array}{l}0.0 \\
0.0 \\
0.0 \\
0.0 \\
0.0\end{array}$ & $\begin{array}{l}10.0 \\
11.0 \\
12.5 \\
10.0 \\
13.0\end{array}$ & $\begin{array}{r}10.0 \\
10.0 \\
11.0 \\
9.7 \\
13.5\end{array}$ & $\begin{array}{l}0.0 \\
0.0 \\
0.0 \\
0.0 \\
0.0\end{array}$ & $\begin{array}{l}0.0 \\
0.0 \\
0.0 \\
0.0 \\
0.0\end{array}$ & $\begin{array}{l}55.0 \\
55.0 \\
54.0 \\
55.0 \\
54.5\end{array}$ & $\begin{array}{l}88.0 \\
90.5 \\
95.0 \\
96.5 \\
99.5\end{array}$ & $\begin{array}{l}0.0 \\
0.0 \\
0.0 \\
0.0 \\
0.0\end{array}$ & $\begin{array}{l}0.0 \\
0.0 \\
0.0 \\
0.0 \\
0.0\end{array}$ & $\begin{array}{l}12.2 \\
12.2 \\
12.2 \\
12.2 \\
12.2\end{array}$ \\
\hline 4.9 .21 & 1.0 & 9.0 & 9.0 & 0.0 & 0.0 & 54.0 & 100.0 & 0.0 & 0.0 & 12.2 \\
\hline
\end{tabular}

LOHEP PLENUM FLOH

\begin{tabular}{|c|c|c|c|}
\hline $\begin{array}{c}\text { TEST } \\
\text { NO }\end{array}$ & $\begin{array}{l}\text { INITIALL } \\
\text { WEIGHT }\end{array}$ & $\begin{array}{l}\text { FINAL } \\
\text { HEIGHT } \\
\text { (L } 9 \text { ) }\end{array}$ & $\begin{array}{l}\text { TIME OF } \\
\text { COLLECTICN } \\
\text { (SEC) }\end{array}$ \\
\hline
\end{tabular}

\begin{tabular}{l} 
BYPASS FL OH \\
\hdashline INITIAL \\
FINAL
\end{tabular}

INITIAL FINAL TIME DF

WEIGHT HEISHT COLLECTION

93.0
134.0
195.5

4.9.

$4 \cdot 9 \cdot 4$
$4 \cdot 9.5$

4.9. 0

4.9 .7
4.9 .9

$4.9: 9$

4.9 .10

4.9 .11

4.9 .12

4.7 .13

4.9 .114
4.9 .15

4.9 .1

4.9 .16
4.7 .17

4.9 .13
4.9 .19

.4 .9 .19

4.9 .21

219.0

384.0

13). 5

.210 .5

293.5

371.5
.433 .5

495.5

495.5
135.5
203.0

243.5

219.0
312.5

384.0

\begin{tabular}{l}
$412.9-20.0$ \\
\hline
\end{tabular}

210.5

293.5

371.5
433.5
495.5

25.0

546.8
203.0.

203.0
245.5

283.0

350.5

35.).5

394.3
433.5

433.5

485.5

$39 \% .3$

433.5

463.0
495.5

501.0

5)1.0

311.9
195.5
227.5

227.5
280.5

134.0
195.5

195.5
227.5

286.5

325.0

181.0

210.0

281.3
304.0

304.0
393.0

412.3
117.5

117.5
287.0
319.0

319.0
470.8

\begin{abstract}
424.0
\end{abstract}
494.0
528.0

048.5

674.5
754.0

783.3

\section{0}

25.0
35.0
30.0
25.0
40.0

25.0
40.0

30.0

40.0
25.0

25.0
40.0

25.0

30.0

45.0

40.0
30.0
40.0

35.9

35.9
35.0

25.0
30.0

30.0
15.0

45.0

45.0

LOWER

PLENHMM

(IN)

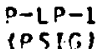

TE-LP-1
(DES F)

$\begin{array}{lll}12.0 & 10.0 & 54.0 \\ 12.0 & 10.0 & 55.0\end{array}$

$12.0 \quad 10.0 \quad 54.5$

$\begin{array}{lll}12.0 & 10.0 & 54.5 \\ 12.0 & 10.0 & 55.0\end{array}$

$12.0 \quad 10.0$

$\begin{array}{ll}12.0 & 10.0 \\ 12.0 & 10.0\end{array}$

$12.0 \quad 10.0$

54.0
55.0

54.5

53.5
54.0

$12.0 \quad 10.0$

12.0 .0110 .0

54.5

63.0

55.0

12.0

10.0

55.0
55.5

55.5

55.0

55.0

56.0 
TABLE XLI (Contd.)

COMBINED EFFECTS TEST GROUP 4.9

EXPER IMENTAL MEASURMENTS FOR TESTS 4.9.1 TO 4.9.21 -- 0.49-IN. DOWNCOMER GAP

\begin{tabular}{|c|c|c|c|c|c|c|c|}
\hline $\begin{array}{c}\text { TEST } \\
\text { NO }\end{array}$ & $\begin{array}{l}R M-C G-1 \\
(C F M)\end{array}$ & $\begin{array}{c}R M-C G-2 \\
\text { (CFM) }\end{array}$ & $\begin{array}{c}F T-C G-1 \\
(C F M)\end{array}$ & $\begin{array}{l}\text { P-CG-1 } \\
\text { (PSIG) }\end{array}$ & $\begin{array}{l}P-C G-2 \\
(P S I G)\end{array}$ & $\begin{array}{l}\text { TF-CG-1 } \\
\text { (DEG FI }\end{array}$ & $\begin{array}{l}\text { TF-CG-2 } \\
\text { (DEG F) }\end{array}$ \\
\hline $\begin{array}{l}4.9 .1 \\
4.9 .2 \\
4.9 .93 \\
4.9 .9 \\
4.9 .5\end{array}$ & $\begin{array}{l}60.0 \\
60.0 \\
60.0 \\
60.0 \\
60.0\end{array}$ & $\begin{array}{l}0.0 \\
0.0 \\
0.0 \\
0.0 \\
0.0\end{array}$ & $\begin{array}{l}0.0 \\
0.0 \\
0.0 \\
0.0 \\
0.0\end{array}$ & $\begin{array}{l}0.0 \\
0.0 . \\
0.0 \\
0.0 \\
0.0\end{array}$ & $\begin{array}{l}0.0 \\
0.0 \\
0.0 \\
0.0 \\
0.0\end{array}$ & $\begin{array}{l}0.0 \\
0.0 \\
0.0 \\
0.0 \\
0.0\end{array}$ & $\begin{array}{l}0.0 \\
0.0 \\
0.0 \\
0.0 \\
0.0\end{array}$ \\
\hline $\begin{array}{l}4.9 .9 \\
4.9 .7 \\
4.9 .9 \\
4.9 .7 \\
4.9 .10\end{array}$ & $\begin{array}{l}60.0 \\
60.0 \\
60.0 \\
60.0 \\
60.0\end{array}$ & $\begin{array}{l}0.0 \\
Q .0 \\
0.0 \\
0.0 \\
0.0\end{array}$ & $\begin{array}{l}0.0 \\
0.0 \\
0.0 \\
0.0 \\
0.0\end{array}$ & $\begin{array}{l}0.0 \\
0.0 \\
0.0 \\
0.0 \\
0.0\end{array}$ & $\begin{array}{l}0.0 \\
0.0 \\
0.0 \\
0.0 \\
0.0\end{array}$ & $\begin{array}{l}0.0 \\
0.0 \\
0.0 \\
0.0 \\
0.0\end{array}$ & $\begin{array}{l}0.0 \\
0.0 \\
0.0 \\
0.0 \\
0.0\end{array}$ \\
\hline $\begin{array}{l}4.9 .11 \\
4.9 .12 \\
4.9 .13 \\
4.9 .14 \\
4.9 .15\end{array}$ & $\begin{array}{l}60.0 \\
60.0 \\
60.0 \\
60.0 \\
60.0\end{array}$ & $\begin{array}{l}0.0 \\
0.0 \\
0.0 \\
0.0 \\
0.0\end{array}$ & $\begin{array}{l}0.0 \\
0.0 \\
0.0 \\
0.0 \\
0.0\end{array}$ & $\begin{array}{l}0.0 \\
0.0 \\
0.0 \\
0.0 \\
0.0\end{array}$ & $\begin{array}{l}0.0 \\
0.0 \\
0.0 \\
0.0 \\
0.0\end{array}$ & $\begin{array}{l}0.0 \\
0.0 \\
0.0 \\
0.0 \\
0.0\end{array}$ & $\begin{array}{l}0.0 \\
0.0 \\
0.0 \\
0.0 \\
0.0\end{array}$ \\
\hline $\begin{array}{l}4.9 .16 \\
4.9 .17 \\
4.9 .18 \\
4.9 .19 \\
4.9 .20\end{array}$ & $\begin{array}{l}60.0 \\
60.0 \\
60.0 \\
60.0 \\
60.0\end{array}$ & $\begin{array}{l}0.0 \\
0.0 \\
0.0 \\
0.0 \\
0.0\end{array}$ & $\begin{array}{l}0.0 \\
0.0 \\
0.0 \\
0.0 \\
0.0\end{array}$ & $\begin{array}{l}0.0 \\
0.0 \\
0.0 \\
0.0 \\
0.0\end{array}$ & $\begin{array}{l}0.0 \\
0.0 \\
0.0 \\
0.0 \\
0.0\end{array}$ & $\begin{array}{l}0.0 \\
0.0 \\
0.0 \\
0.0 \\
0.0\end{array}$ & $\begin{array}{l}0.0 \\
0.0 \\
0.0 \\
0.0 \\
0.0\end{array}$ \\
\hline 4.9 .21 & 60.0 & 0.0 & 0.0 & 0.0 & 0.0 & 0.0 & 0.0 \\
\hline
\end{tabular}




\section{TABLE XIII}

COMBINED EFFECTS TEST GROUP $\mathbf{5 . 9}$

TRANSPARENT VESSEL TEST DATA FOR TESTS 5.9.1 TO 5.9.11 -- 0.53-IN. DOHNCOMER GAP SUMMARY OF CALCULATED RESULTS

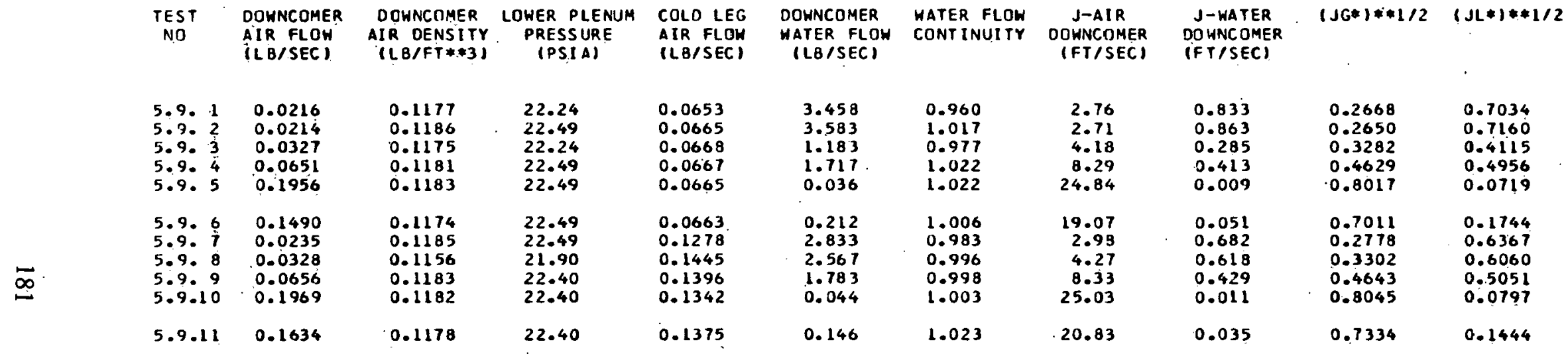


TABLE XLII (Contd.)

COMBINED EFFECTS TEST GROUP 5.9

EXPERIMENTAL MEASURMENTS FOR TESTS 5.9 .1 TO $5.9 .11-0.53-$ IN. DOWNCONER GAP

$$
\text { RM-CH-1 }
$$

$\begin{array}{cc}\text { TEST } & R M-C H-1 \\ \text { NO } & \begin{array}{c}\text { (GPM) } \\ 5.9 .1\end{array} \\ 5.9 .2 & 0.0 \\ 5.9 .3 & 0.0 \\ 5.9 .4 & 0.0 \\ 5.9 .5 & 0.0 \\ 5.9 .6 & 0.0 \\ 5.9 .7 & 0.0 \\ 5.9 .8 & 0.0 \\ 5.9 .9 & 0.0 \\ 5.9 .10 & 0.0 \\ 5.9 .11 & 0.0 \\ 5.11 & 0.0\end{array}$

RH-CH-2
(GPM)
0.0

FT $-\mathrm{CH}-1$
$(\mathrm{GPM})$

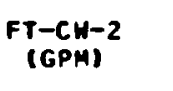
RM-HG-1 (CFM)
$R M-H G-2$ (CFM)

RH-HG-
(CFM]

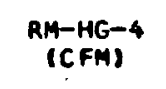

FT-HG-1 (CFM)

FT-HG-2

$60.0 \quad 0.0$

0.0
0.0

0.0
0.0
0.0

90.00

0.0
0.0
0.0

$\begin{array}{ll}0.0 & 10.00 \\ 0.0 & 10.00\end{array}$

15.00

$\begin{array}{ccc}0.0 & 30.00 & 0.0 \\ 0.0 & 0.0 & 0.0\end{array}$

0.0
0.0
0.0

0.0
0.0

$0.0 \quad 0.0$

$0.0 \quad 25.0$

10.0

0.0

0.0
61.0

61.0
0.0

5.0
0.0

45.2
25.1

0.0
0.0
0.0

0.0

90.00

0.0

0.0
10.00

10.00
15.00

$\begin{array}{ll}0.0 & 15.00 \\ 0.0 & 30.00 \\ 0.0 & 0.0\end{array}$

0.0

0.0
0.0
0.0

0.0
0.0

0.0

0.0

0.0

0.0

5.1

0.0

0.0

0.0

0.0

17.69
0.0

$\begin{array}{ll}0.69 & 0.0 \\ 0.0 & 0.0\end{array}$

$0.0 \quad 0.0$

$\begin{array}{ll}0.0 & 0.0 \\ 0.0 & 0.0\end{array}$

0.0

0.0

17.82

0.0

$\begin{array}{cc}\text { TEST } & \begin{array}{c}P-C Y-1 \\ \text { NO }\end{array} \\ \text { (PSIG) } \\ 5.9 .1 & 12.5 \\ 5.9 .12 & 12.5 \\ 5.9 .3 & 11.0 \\ 5.9 .4 & 12.0 \\ 5.9 .5 & 10.0 \\ 5.9 .6 & 10.0 \\ 5.9 .7 & 15.5 \\ 5.9 .8 & 13.0 \\ 5.9 .9 & 11.5 \\ 5.9 .10 & 11.0 \\ 5.9 .11 & 10.5\end{array}$

$P-C H-2$
IPSIG
0.0
0.0
0.0
0.0
0.0
0.0
0.0
0.0
0.0
0.0
0.0

P-HG-1
(PSIG)

P-HG-2
(PSIG)

P-HG-3

TF-CW-1

TF-HG-

TF-HG-

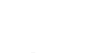

TF-HG-3
(DEG F)

P-BARO

$10.0 \quad 96.0$

33.0

$$
\begin{aligned}
& 50.0 \\
& 52.0
\end{aligned}
$$
50.0
52.0
50.5
50.5
51.5

IDEG F

IOEG F

84.0
82.0

81.0

78.5
77.5

12.2

$95.5 \quad 33.5$

$\begin{array}{lll}10.0 & 95.0 & 33.0 \\ 10.0 & 92.0 & 33.0\end{array}$

51.5

79.0

$\begin{array}{rrr}10.0 & 93.0 & 0.0 \\ 11.0 & 94.0 & 33.0 \\ 9.0 & 101.0 & 33.0\end{array}$

$\begin{array}{rrr}10.0 & 93.0 & 0.0 \\ 11.0 & 94.0 & 33.0 \\ 9.0 & 101.0 & 33.0\end{array}$

$9.0 \quad 101.0$

99.5
95.5

52.0
52.5

52.5
51.0

50.0
51.0

87.0

81.0
72.0

33.0
33.0

72.0
70.0

11.0

98.0

$33 \cdot 5$

51.5

73.0

0.0
0.0

102.5

88.5
67.5

72.5

0.0
0.0

0.0
70.0

70.0

69.5

$80.5 \quad 76.5$

12.2

12.2

12.2
12.2

12.2

12.2

12.1

12.1

. 
TABLE XLII (Contd.)

COMBINED EFFECTS TEST GROJP 5.9

EXPER IMENTAL MEASURMENTS FOR TESTS 5.9.1 TO 5.9.11 -- 0.53-1N. OOHNCOMER GAP

\begin{tabular}{|c|c|c|}
\hline & PLENU & FLOH \\
\hline $\begin{array}{l}\text { INIT IAL } \\
\text { ME I GHT } \\
\text { (LB) }\end{array}$ & $\begin{array}{l}\text { FINAL } \\
\text { HEIGHT } \\
\text { (LB) }\end{array}$ & $\begin{array}{l}\text { TIME OF } \\
\text { COLLECTION } \\
\text { (SEC) }\end{array}$ \\
\hline
\end{tabular}

\begin{tabular}{|c|c|c|}
\hline \multicolumn{3}{|c|}{ BYPASS FLOH } \\
\hline $\begin{array}{l}\text { IN I TIAL } \\
\text { HE I GHT } \\
\text { (LB) }\end{array}$ & $\begin{array}{l}\text { FINAL } \\
\text { WEIGHT } \\
\text { (LB) }\end{array}$ & $\begin{array}{l}\text { TIME OF } \\
\text { COLLECTION } \\
\text { (SEC) }\end{array}$ \\
\hline
\end{tabular}

LOHER

$\begin{array}{lll}\text { PENUM } & P-L P-1 & \text { TF-LP-1 } \\ \text { LEVE } & \text { (PSIG) } & \text { (OEG F) }\end{array}$

TEST
NO

LLB

411.0

60.0

(2B)

(IN)

IPSIGI IOEG FI

5.9 .2

203.5
272.5

380.0

30.0

216.0

$489.0 \quad 60.0$

12.0

$\begin{array}{lr}10.0 & 50.0 \\ 10.2 & 52.0 \\ 10.0 & 51.0\end{array}$

$\begin{array}{llll}5.9 .3 & 202.5 & 238.0 & 30.0\end{array}$

$\begin{array}{rrrr}5.9 .4 & 141.5 & 193.0 & 30.0 \\ 5.9 .5 & 143.5 & 150.0 & 180.0\end{array}$

345.5
150.5

298.0

30.0

30.0
60.0

12.0

$10.2 \quad 52.0$

150.0

175.5

120.0

160.0

243.5

12.0

$10.2 \quad 54.0$

5.9. $6 \quad 150.0$

5.9 .7

$5.9 .10 \quad 270.5$

$227.0 \quad 30.0$

$272.5 \quad 60.0$

113.0

305.5

60.0

60.0

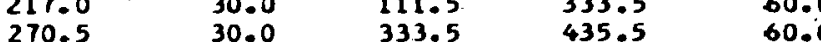

$278.5 \cdot 180.0$

435.5

516.5

60.0

12.0

$10.2 \quad 53.0$

5.9 .11

278.5

$296.0 \quad 120.0$

516.5

551.0

60.0

12.0
12.0

12.0

$10.2 \quad 57.0$

$10.2 \quad 52.5$

\begin{tabular}{|c|c|c|c|c|c|c|c|}
\hline $\begin{array}{l}\text { TEST } \\
\text {.NO }\end{array}$ & $\begin{array}{c}R M-C G-1 \\
(C F M)\end{array}$ & $\begin{array}{c}R M-C G-2 \\
\text { (CFM) }\end{array}$ & $\begin{array}{c}F T-C G-1 \\
(C F M)\end{array}$ & $\begin{array}{l}P-C G-1 \\
(P S I G)\end{array}$ & $\begin{array}{l}P-C G-2 \\
(P S I G)\end{array}$ & $\begin{array}{l}\text { TF-CG-1 } \\
\text { (OEG F) }\end{array}$ & $\begin{array}{l}\text { TF-CG-2 } \\
\text { (DEG F) }\end{array}$ \\
\hline $\begin{array}{ll}5.9 . & 1 \\
5.9 . & 2 \\
5.9 . & 3 \\
5.9 . & 4 \\
5.9 . & 5\end{array}$ & $\begin{array}{l}0.0 \\
0.0 \\
0.0 \\
0.0 \\
0.0\end{array}$ & $\begin{array}{l}30.0 \\
30.0 \\
30.0 \\
30.0 \\
30.0\end{array}$ & $\begin{array}{l}0.0 \\
0.0 \\
0.0 \\
0.0 \\
0.0\end{array}$ & $\begin{array}{l}96.0 \\
96.0 \\
95.5 \\
95.0 \\
92.0\end{array}$ & $\begin{array}{l}35.0 \\
35.0 \\
35.4 \\
35.2 \\
35.0\end{array}$ & $\begin{array}{l}0.0 \\
0.0 \\
0.0 \\
0.0 \\
0.0\end{array}$ & $\begin{array}{r}100.0 \\
79.5 \\
80.0 \\
79.0 \\
79.5\end{array}$ \\
\hline $\begin{array}{l}5.9 .6 \\
5.9 .7 \\
5.9 .8 \\
5.9 .9 \\
5.9 .10\end{array}$ & $\begin{array}{l}0.0 \\
0.0 \\
0.0 \\
0.0 \\
0.0\end{array}$ & $\begin{array}{r}30.0 \\
0.0 \\
0.0 \\
0.0 \\
0.0\end{array}$ & $\begin{array}{l}0.0 \\
14.7 \\
15.0 \\
14.7 \\
14.7\end{array}$ & $\begin{array}{r}93.0 \\
94.0 \\
101.0 \\
99.5 \\
95.5\end{array}$ & $\begin{array}{l}35.2 \\
34.6 \\
35.0 \\
35.2 \\
34.5\end{array}$ & $\begin{array}{r}0.0 \\
91.5 \\
68.5 \\
70.5 \\
72.0\end{array}$ & $\begin{array}{l}84.5 \\
99.0 \\
66.5 \\
68.5 \\
70.0\end{array}$ \\
\hline 5.9 .11 & 0.0 & 0.0 & 14.9 & 98.0 & 35.0 & 76.0 & 73.0 \\
\hline
\end{tabular}


TABLE XIIII

COMBINED EFFECTS TEST GROUP 5.11

TRANSPARENT VESSEL TEST DATA FOR TESTS 5.11 .1 TO $5.11 .12 \cdots 0.53-$ IN. DOHNCOMER GAP SUMMARY OF CALCULATED RESULTS

\begin{tabular}{|c|c|c|c|c|c|c|c|c|c|c|c|}
\hline & $\begin{array}{l}\text { TEST } \\
\text { NO }\end{array}$ & $\begin{array}{l}\text { DOWNCOMER } \\
\text { AIR FLOH } \\
(L B / S E C)\end{array}$ & $\begin{array}{l}\text { DOHNCOMER } \\
\text { AIR DENSITY } \\
\text { (LB/FT } * 3 \text { ) }\end{array}$ & $\begin{array}{l}\text { LOWER PLENUM } \\
\text { PRESSURE } \\
\text { (PSIA) }\end{array}$ & $\begin{array}{l}\text { COLD LEG } \\
\text { AIR FLOW } \\
\text { (LB/SEC) }\end{array}$ & $\begin{array}{l}\text { DOWNCOMER } \\
\text { HATER FLOW } \\
\text { (LB/SEC) }\end{array}$ & $\begin{array}{l}\text { WATER FLOW } \\
\text { CONTINUITY }\end{array}$ & $\begin{array}{c}\text { J-AIR } \\
\text { DOWNCOMER } \\
\text { (FT/SEC) }\end{array}$ & $\begin{array}{l}\text { J-HATER } \\
\text { DOWNC DMER } \\
\text { (FT/SEC) }\end{array}$ & $(J G *) * * 1 / 2$ & $(J l *) * 1 / 2$ \\
\hline & $\begin{array}{l}5.11 \cdot 1 \\
5.11: 2 \\
5.11: 3 \\
5.11: 4 \\
5.11 \cdot 5\end{array}$ & $\begin{array}{l}0.0110 \\
0.0109 \\
0.0330 \\
0.0440 \\
0.1097\end{array}$ & $\begin{array}{l}0.1168 \\
0.1203 \\
0.1190 \\
0.1199 \\
0.1181\end{array}$ & $\begin{array}{l}21.90 \\
22.65 \\
22.40 \\
22.65 \\
22.40\end{array}$ & $\begin{array}{l}0.0670 \\
0.0673 \\
0.0673 \\
0.0672 \\
0.0673\end{array}$ & $\begin{array}{l}4.450 \\
4.817 \\
3.150 \\
2.667 \\
0.917\end{array}$ & $\begin{array}{l}0.941 \\
1.007 \\
1.008 \\
1.020 \\
1.014\end{array}$ & $\begin{array}{r}1.41 \\
1.37 \\
4.17 \\
5.52 \\
13.96\end{array}$ & $\begin{array}{l}1.072 \\
1.160 \\
0.759 \\
0.642 \\
0.221\end{array}$ & $\begin{array}{l}0.1905 \\
0.1887 \\
0.3288 \\
0.3790 \\
0.6007\end{array}$ & $\begin{array}{l}0.7979 \\
0.8301 \\
0.6713 \\
0.6177 \\
0.3621\end{array}$ \\
\hline & $\begin{array}{l}5.11 \cdot 6 \\
5.11 \cdot 7 \\
5.11 \cdot 8 \\
5.11 \cdot 9 \\
5.11 \cdot 10\end{array}$ & $\begin{array}{l}0.1744 \\
0.1537 \\
0.0110 \\
0.0110 \\
0.0412\end{array}$ & $\begin{array}{l}0.1194 \\
0.1176 \\
0.1217 \\
0.1241 \\
0.1203\end{array}$ & $\begin{array}{l}22.65 \\
22.40 \\
22.90 \\
23.40 \\
22.65\end{array}$ & $\begin{array}{l}0.0666 \\
0.0664 \\
0.2567 \\
0.2526 \\
0.2492\end{array}$ & $\begin{array}{l}0.171 \\
0.283 \\
2.517 \\
2.642 \\
1.827\end{array}$ & $\begin{array}{l}1.010 \\
0.991 \\
0.992 \\
1.002 \\
1.011\end{array}$ & $\begin{array}{r}21.95 \\
19.64 \\
1.35 \\
1.33 \\
5.15\end{array}$ & $\begin{array}{l}0.041 \\
0.068 \\
0.606 \\
0.636 \\
0.440\end{array}$ & $\begin{array}{l}0.7553 \\
0.7117 \\
0.1884 \\
0.1875 \\
0.3664\end{array}$ & $\begin{array}{l}0.1563 \\
0.2013 \\
0.6000 \\
0.6148 \\
0.5112\end{array}$ \\
\hline & $\begin{array}{l}5.11 .11 \\
5.11 .12\end{array}$ & $\begin{array}{l}0.1727 \\
0.1524\end{array}$ & $\begin{array}{l}0.1164 \\
0.1169\end{array}$ & $\begin{array}{l}22.24 \\
22.24\end{array}$ & $\begin{array}{l}0.2626 \\
0.2534\end{array}$ & $\begin{array}{l}0.183 \\
0.271\end{array}$ & $\begin{array}{l}1.022 \\
1.008\end{array}$ & $\begin{array}{l}22.29 \\
19.59\end{array}$ & $\begin{array}{l}0.044 \\
0.065\end{array}$ & $\begin{array}{l}0.7562 \\
0.7097\end{array}$ & $\begin{array}{l}0.1620 \\
0.1968\end{array}$ \\
\hline
\end{tabular}


TABLE XLIII (Contd.)

COMBINED EFFECTS TEST GROUP 5.11

EXPERIMENTAL MEASURMENTS FOR TESTS 5.11 .1 TO $5.11 .12--0.53-1 \mathrm{~N}$. ODHNCOMER GAP

\begin{tabular}{|c|c|c|c|c|c|c|c|c|c|c|}
\hline $\begin{array}{l}\text { TEST } \\
\text { NO }\end{array}$ & $\begin{array}{c}R M-C W-1 \\
(G P M)\end{array}$ & $\begin{array}{c}R M-C H-2 \\
(G P M)\end{array}$ & $\begin{array}{c}F T-C H-1 \\
(G P M)\end{array}$ & $\begin{array}{c}\mathrm{FT}-\mathrm{CH}-2 \\
(G P M)\end{array}$ & $\begin{array}{c}R M-H G-1 \\
(C F M)\end{array}$ & $\begin{array}{l}\text { RM-HG-2 } \\
(C F M)\end{array}$ & $\begin{array}{c}\text { RM-HG-3 } \\
\text { (CFM) }\end{array}$ & $\begin{array}{c}R M-M G-4 \\
(C F M)\end{array}$ & $\begin{array}{c}F T-H G-1 \\
(C F M)\end{array}$ & $\begin{array}{c}F T-H G-2 \\
(C F M)\end{array}$ \\
\hline $\begin{array}{l}5.11 \cdot 1 \\
5.11 \cdot 2 \\
5.11 \cdot 3 \\
5.11 \cdot 4 \\
5.11 \cdot 5\end{array}$ & $\begin{array}{l}0.0 \\
0.0 \\
0.0 \\
0.0 \\
0.0\end{array}$ & $\begin{array}{l}0.0 \\
0.0 \\
0.0 \\
0.0 \\
0.0\end{array}$ & $\begin{array}{r}\epsilon 0.0 \\
60.0 \\
0.0 \\
0.0 \\
0.0\end{array}$ & $\begin{array}{r}0.0 \\
0.0 \\
45.0 \\
35.0 \\
25.1\end{array}$ & $\begin{array}{c}0.0 \\
0.0 \\
0.0 \\
0.0 \\
50.00\end{array}$ & $\begin{array}{l}0.0 \\
0.0 \\
0.0 \\
0.0 \\
0.0\end{array}$ & $\begin{array}{r}5.00 \\
5.00 \\
15.00 \\
20.00 \\
0.0\end{array}$ & $\begin{array}{l}0.0 \\
0.0 \\
0.0 \\
0.0 \\
0.0\end{array}$ & $\begin{array}{l}0.0 \\
0.0 \\
0.0 \\
0.0 \\
0.0\end{array}$ & $\begin{array}{l}0.0 \\
0.0 \\
0.0 \\
0.0 \\
0.0\end{array}$ \\
\hline $\begin{array}{l}5.11 \cdot 6 \\
5.11 \cdot 7 \\
5.11 \cdot 8 \\
5.11 .9 \\
5.11 .10\end{array}$ & $\begin{array}{l}0.0 \\
0.0 \\
0.0 \\
0.0 \\
0.0\end{array}$ & $\begin{array}{l}0.0 \\
0.0 \\
0.0 \\
0.0 \\
0.0\end{array}$ & $\begin{array}{r}0.0 \\
0.0 \\
60.0 \\
60.0 \\
0.0\end{array}$ & $\begin{array}{r}15.2 \\
.5 .0 \\
0.0 \\
0.0 \\
35.0\end{array}$ & $\begin{array}{c}80.00 \\
0.0 \\
0.0 \\
0.0 \\
0.0\end{array}$ & $\begin{array}{l}0.0 \\
0.0 \\
0.0 \\
0.0 \\
0.0\end{array}$ & $\begin{array}{l}0.0 \\
0.0 \\
5.00 \\
5.00 \\
20.00\end{array}$ & $\begin{array}{l}0.0 \\
0.0 \\
0.0 \\
0.0 \\
0.0\end{array}$ & $\begin{array}{c}0.0 \\
17.56 \\
0.0 \\
0.0 \\
0.0\end{array}$ & $\begin{array}{l}0.0 \\
0.0 \\
0.0 \\
0.0 \\
0.0\end{array}$ \\
\hline $\begin{array}{l}5.11 .11 \\
5.11 .12\end{array}$ & $\begin{array}{l}0.0 \\
0.0\end{array}$ & $\begin{array}{l}0.0 \\
0.0\end{array}$ & $\begin{array}{l}0.0 \\
0.0\end{array}$ & $\begin{array}{r}15.1 \\
5.0\end{array}$ & $\begin{array}{c}80.00 \\
0.0\end{array}$ & $\begin{array}{l}0.0 \\
0.0\end{array}$ & $\begin{array}{l}0.0 \\
0.0\end{array}$ & $\begin{array}{l}0.0 \\
0.0\end{array}$ & $\begin{array}{c}0.0 \\
17.69\end{array}$ & $\begin{array}{l}0.0 \\
0.0\end{array}$ \\
\hline & & & & & . & & & & & \\
\hline $\begin{array}{l}\text { TEST } \\
\text { NO }\end{array}$ & $\begin{array}{l}P-C H-1 \\
\text { (PSIG) }\end{array}$ & $\begin{array}{l}P-C H-2 \\
(P S I G)\end{array}$ & $\begin{array}{l}\text { P-HG-1 } \\
\text { (PSIG) }\end{array}$ & $\begin{array}{l}P-H G-2 \\
(P S I G)\end{array}$ & $\begin{array}{l}P-H G-3 \\
\text { (PSIG) }\end{array}$ & $\begin{array}{l}T F-C H-1 \\
(D E G F)\end{array}$ & $\begin{array}{l}T F-H G-1 \\
\text { (OEG F) }\end{array}$ & $\begin{array}{l}\text { IF-HG-2 } \\
\text { (DEG F) }\end{array}$ & $\begin{array}{l}\text { TF-HG-3 } \\
\text { (DEG F) }\end{array}$ & $\begin{array}{l}\text { P-BARO } \\
\text { (PSIAI) }\end{array}$ \\
\hline $\begin{array}{l}5.11 \cdot 1 \\
5.11 .2 \\
5.11 .3 \\
5.11 .4 \\
5.11 .5\end{array}$ & $\begin{array}{l}12.0 \\
13.0 \\
11.5 \\
11.5 \\
10.7\end{array}$ & $\begin{array}{l}0.0 \\
0.0 \\
0.0 \\
0.0 \\
0.0\end{array}$ & $\begin{array}{r}9.0 \\
10.0 \\
9.5 \\
10.5 \\
10.5\end{array}$ & $\begin{array}{r}102.0 \\
99.0 \\
97.5 \\
97.0 \\
95.0\end{array}$ & $\begin{array}{l}33.5 \\
33.0 \\
33.5 \\
33.5 \\
33.5\end{array}$ & $\begin{array}{l}45.0 \\
47.0 \\
48.0 \\
49.0 \\
50.0\end{array}$ & $\begin{array}{l}70.0 \\
76.0 \\
76.0 \\
77.0 \\
75.0\end{array}$ & $\begin{array}{l}75.0 \\
72.5 \\
72.0 \\
72.0 \\
76.0\end{array}$ & $\begin{array}{l}75.0 \\
73.0 \\
72.0 \\
72.0 \\
75.0\end{array}$ & $\begin{array}{l}12.4 \\
12.4 \\
12.4 \\
12.4 \\
12.4\end{array}$ \\
\hline $\begin{array}{l}5.11 \cdot 6 \\
5.11 \cdot 7 \\
5.11 \cdot 8 \\
5.11 .9 \\
5.11 .10\end{array}$ & $\begin{array}{r}10.2 \\
9.5 \\
2.0 .0 \\
20.0 \\
16.0\end{array}$ & $\begin{array}{l}0.0 \\
0.0 \\
0.0 \\
0.0 \\
0.0\end{array}$ & $\begin{array}{l}10.0 \\
10.0 \\
11.0 \\
11.0 \\
10.0\end{array}$ & $\begin{array}{l}90.0 \\
94.0 \\
93.0 \\
93.0 \\
92.0\end{array}$ & $\begin{array}{l}33.0 \\
33.5 \\
33.5 \\
33.5 \\
28.0\end{array}$ & $\begin{array}{l}51.0 \\
52.0 \\
48.0 \\
49.0 \\
48.0\end{array}$ & $\begin{array}{l}76.0 \\
78.0 \\
77.0 \\
76.0 \\
74.0\end{array}$ & $\begin{array}{l}83.0 \\
87.0 \\
78.0 \\
77.0 \\
77.0\end{array}$ & $\begin{array}{l}70.0 \\
81.0 \\
77.0 \\
76.0 \\
74.0\end{array}$ & $\begin{array}{l}12.4= \\
12.4 \\
12.4 \\
12.4 \\
12.4\end{array}$ \\
\hline $\begin{array}{l}5.11 .11 \\
5.11 .12\end{array}$ & $\begin{array}{l}12.0 \\
10.2\end{array}$ & $\begin{array}{l}0.0 \\
0.0\end{array}$ & $\begin{array}{l}95.0 \\
10.2\end{array}$ & $\begin{array}{l}95.0 \\
93.0\end{array}$ & $\begin{array}{l}32.0 \\
33.5\end{array}$ & $\begin{array}{l}51.0 \\
50.5\end{array}$ & $\begin{array}{l}75.0 \\
79.0\end{array}$ & $\begin{array}{l}85.0 \\
91.0\end{array}$ & $\begin{array}{l}76.0 \\
84.0\end{array}$ & $\begin{array}{l}12.5 \\
12.5\end{array}$ \\
\hline
\end{tabular}


TABLE XLIII (Contd.)

COMBINED EFFECTS TEST GROUP 5.11

EXPERIMENTAL MEASURMENTS FOR TESTS 5.11.1 TO $5.11 .12-0.53-1$ - 0 . DOWNCOMER GAP

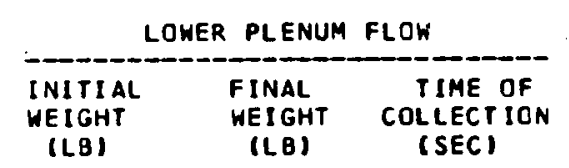

\begin{tabular}{|c|c|c|}
\hline \multicolumn{3}{|c|}{ BYPASS FLOW } \\
\hline $\begin{array}{l}\text { INITIAL } \\
\text { HE IGHT } \\
\text { (LB) }\end{array}$ & $\begin{array}{l}\text { FINAL } \\
\text { WEI I GHT } \\
\text { (L B) }\end{array}$ & $\begin{array}{l}\text { TINE OF } \\
\text { COLLECTION } \\
\text { (SEC) }\end{array}$ \\
\hline
\end{tabular}

LOWER

(IN)

$\begin{array}{ll}P-(P-1 & \text { TF-LP-1 } \\ \text { (PSIG) } & \text { (DEG FI }\end{array}$

141.5

275.0

(SEC)

103.5

205.5

SEC)

5.11.

5.11. $3 \quad 248.0$

$\begin{array}{ll}275.0 & 30.0 \\ 419.5 & 30.0\end{array}$

205.5

313.0

30.0

12.0

30.0

12.0

9.546 .0

5.11. 5

$\begin{array}{llll}248.0 & 342.5 & 30.0 & 151.5 \\ 143.5 & 304.0 & 60.0 & 111.0\end{array}$

$30.0 \quad 157.5$

252.0

60.0

$12.0 \quad 10.0$

$10.0 \quad 48.0$

$\begin{array}{lll}10.2 & 50.0\end{array}$

$5.11 .6 \quad 158.5 \quad 179.0$

5.11 .

158.5
144.5

179.0
195.5

$120.0 \quad 170.5$

273.5

60.0

$12.0 \quad 10.0$

52.0

5.11 .8

142.5

180.0

170.5
141.0

288.0

218.0

$30.0 \quad 107.5$

.165 .5

5.11 .10

146.5

283.5 .

$60.0 \quad 350.5$

60.0

12.0

453.0

693.5

60.0

60.0

12.0

10.2

52.0

5.11 .1

138.0
160.0

160.0

$\begin{array}{ll}120.0 & 109.0 \\ 120.0 & 226.5\end{array}$

392.5

60.0

$10.5 \quad 54.0$

5.11 .12

192.5

$226 \cdot 5$
$252 \cdot 5$

60.0
60.0

12.0

10.2

49.0

48.0

\begin{tabular}{|c|c|c|c|c|c|c|c|}
\hline $\begin{array}{c}\text { TEST } \\
\text { NO }\end{array}$ & $\begin{array}{c}R M-C G-1 \\
\text { (CFM) }\end{array}$ & $\begin{array}{c}R M-C G-2 \\
(C F M)\end{array}$ & $\begin{array}{l}F T-C G-1 \\
(C F M)\end{array}$ & $\begin{array}{l}P-C G-1 \\
\text { (PSIG) }\end{array}$ & $\begin{array}{l}P-C G-2 \\
(P S I G)\end{array}$ & $\begin{array}{l}\text { TF-CG-1 } \\
\text { (DEG F) }\end{array}$ & $\begin{array}{l}\text { TF-CG-2 } \\
\text { (DEG F) }\end{array}$ \\
\hline $\begin{array}{l}11.1 \\
11.2 \\
11.3 \\
11.4 \\
11.5\end{array}$ & $\begin{array}{l}0.0 \\
0.0 \\
0.0 \\
0.0 \\
0.0\end{array}$ & $\begin{array}{l}30.0 \\
30.0 \\
30.0 \\
30.0 \\
30.0\end{array}$ & $\begin{array}{l}0.0 \\
0.0 \\
0.0 \\
0.0 \\
0.0\end{array}$ & $\begin{array}{r}102.0 \\
99.0 \\
97.5 \\
97.0 \\
95.0\end{array}$ & $\begin{array}{l}35.0 \\
35.2 \\
35.2 \\
35.2 \\
35.4\end{array}$ & $\begin{array}{l}0.0 \\
0.0 \\
0.0 \\
0.0 \\
0.0\end{array}$ & $\begin{array}{l}73.0 \\
71.0 \\
71.0 \\
72.0 \\
73.0\end{array}$ \\
\hline $\begin{array}{l}11.6 \\
11.97 \\
11.8 \\
11.99 \\
11.10\end{array}$ & $\begin{array}{l}0.0 \\
0.0 \\
0.0 \\
0.0 \\
0.0\end{array}$ & $\begin{array}{r}30.0 \\
30.0 \\
0.0 \\
0.0 \\
0.0\end{array}$ & $\begin{array}{r}0.0 \\
0.0 \\
29.6 \\
29.4 \\
29.5\end{array}$ & $\begin{array}{l}90.0 \\
94.0 \\
93.0 \\
93.0 \\
92.0\end{array}$ & $\begin{array}{r}34.5 \\
34.4 \\
0.0 \\
0.0 \\
0.0\end{array}$ & $\begin{array}{r}0.0 \\
0.0 \\
87.0 \\
91.0 \\
35.8\end{array}$ & $\begin{array}{r}74.0 \\
76.0 \\
0.0 \\
0.0 \\
0.0\end{array}$ \\
\hline $\begin{array}{l}11.11 \\
11.12\end{array}$ & $\begin{array}{l}0.0 \\
0.0\end{array}$ & $\begin{array}{l}0.0 \\
0.0\end{array}$ & $\begin{array}{l}29.5 \\
29.6\end{array}$ & $\begin{array}{l}95.0 \\
93.0\end{array}$ & $\begin{array}{l}0.0 \\
0.0\end{array}$ & $\begin{array}{l}83.0 \\
94.5\end{array}$ & $\begin{array}{l}0.0 \\
0.0\end{array}$ \\
\hline
\end{tabular}




\section{TABLE XLIV}

COMBINED EFFECTS TEST GROUP 5.14

TRANSPARENT VESSEL TEST DATA FOR TESTS 5.14.1 TO 5.14.14 -- 0.53-IN. DQWNCOMER GAP SUMMARY OF CALCULATED RESULTS

\begin{tabular}{|c|c|c|c|c|c|c|c|c|c|c|}
\hline $\begin{array}{l}\text { TEST } \\
\text { NO }\end{array}$ & $\begin{array}{l}\text { DOWNCOMER } \\
\text { AIR FLOH } \\
(L B / S E C)\end{array}$ & $\begin{array}{l}\text { DOHNCOMER } \\
\text { AIR DENSITY } \\
\text { (LB/FT } * 3 \text { I }\end{array}$ & $\begin{array}{l}\text { LOHER PLENUM } \\
\text { PRESSURE } \\
\text { (PSIA) }\end{array}$ & $\begin{array}{l}\text { CCLD LEG } \\
\text { AIR FLDH } \\
(L B / S E C)\end{array}$ & $\begin{array}{l}\text { DOWNCOMER } \\
\text { HATER FLOH } \\
\text { (LB/SEC) }\end{array}$ & $\begin{array}{l}\text { HATER FLQW } \\
\text { CONTINUITY }\end{array}$ & $\begin{array}{c}\text { J-AIR } \\
\text { DOWNCOMER } \\
\text { (FT/SEC) }\end{array}$ & $\begin{array}{l}\text { J-WATER } \\
\text { DOHNCOMER } \\
\text { (FT/SEC) }\end{array}$ & $(J G * 1 * * 1 / 2$ & $(J l *) * 1 / 2$ \\
\hline $\begin{array}{l}5.14 \cdot 1 \\
5.14 \cdot 2 \\
5.14 \cdot 3 \\
5.14 \cdot 4 \\
5.14 \cdot 5\end{array}$ & $\begin{array}{l}0.0074 \\
0.0074 \\
0.0219 \\
0.0286 \\
0.1169\end{array}$ & $\begin{array}{l}0.1192 \\
0.1211 \\
0.1229 \\
0.1218 \\
0.1171\end{array}$ & $\begin{array}{l}22.29 \\
22.79 \\
2.3 .04 \\
22.79 \\
22.29\end{array}$ & $\begin{array}{l}0.0667 \\
0.0663 \\
0.0664 \\
0.0662 \\
0.0663\end{array}$ & $\begin{array}{l}5.583 \\
6.167 \\
4.683 \\
3.831 \\
0.600\end{array}$ & $\begin{array}{l}0.949 \\
1.007 \\
0.982 \\
0.948 \\
0.971\end{array}$ & $\begin{array}{r}0.94 \\
0.92 \\
2.67 \\
3.53 \\
15.01\end{array}$ & $\begin{array}{l}1.345 \\
1.485 \\
1.128 \\
0.922 \\
0.144\end{array}$ & $\begin{array}{l}0.1559 \\
0.1553 \\
0.2654 \\
0.3043 \\
0.6215\end{array}$ & $\begin{array}{l}0.8937 \\
0.9393 \\
0.8186 \\
0.7403 \\
0.2930\end{array}$ \\
\hline $\begin{array}{l}5.14 .6 \\
5.14 .7 \\
5.14 .8 \\
5.14 .9 \\
5.14 .10\end{array}$ & $\begin{array}{l}0.1749 \\
0.0072 \\
0.0286 \\
0.1170 \\
0.1748\end{array}$ & $\begin{array}{l}0.1168 \\
0.1164 \\
0.1175 \\
0.1186 \\
0.1155\end{array}$ & $\begin{array}{l}22.29 \\
22.00 \\
22.25 \\
22.50 \\
22.00\end{array}$ & $\begin{array}{l}0.0662 \\
0.1366 \\
0.1361 \\
0.1303 \\
0.1280\end{array}$ & $\begin{array}{l}0.104 \\
4.283 \\
3.450 \\
0.633 \\
0.033\end{array}$ & $\begin{array}{l}1.003 \\
0.975 \\
0.979 \\
1.007 \\
0.913\end{array}$ & $\begin{array}{r}22.49 \\
0.93 \\
3.66 \\
14.82 \\
22.73\end{array}$ & $\begin{array}{l}0.025 \\
1.032 \\
0.831 \\
0.153 \\
0.008\end{array}$ & $\begin{array}{l}0.7603 \\
0.1549 \\
0.3072 \\
0.5196 \\
0.7623\end{array}$ & $\begin{array}{l}0.1221 \\
0.7828 \\
0.7025 \\
0.3010 \\
0.0691\end{array}$ \\
\hline $\begin{array}{l}5.14 .11 \\
5.14 .12 \\
5.14 .13 \\
5.14 .14\end{array}$ & $\begin{array}{l}0.0072 \\
0.0292 \\
0.1170 \\
0.1748\end{array}$ & $\begin{array}{l}0.1206 \\
0.1191 \\
0.1168 \\
0.1182\end{array}$ & $\begin{array}{l}22.75 \\
22.50 \\
22.25 \\
22.50\end{array}$ & $\begin{array}{l}0.2499 \\
0.2476 \\
0.2422 \\
0.2379\end{array}$ & $\begin{array}{l}2.867 \\
2.200 \\
0.667 \\
0.117\end{array}$ & $\begin{array}{l}1.027 \\
1.000 \\
1.052 \\
1.074\end{array}$ & $\begin{array}{r}0.90 \\
3.69 \\
15.05 \\
22.23\end{array}$ & $\begin{array}{l}0.690 \\
0.530 \\
0.161 \\
0.028\end{array}$ & $\begin{array}{l}0.1531 \\
0.3095 \\
0.6219 \\
0.7580\end{array}$ & $\begin{array}{l}0.6404 \\
0.5610 \\
0.3088 \\
0.1292\end{array}$ \\
\hline
\end{tabular}


TABLE XLIV (Contd.)

COMBINED EFFECTS TEST GROUP 5.14

EXPERIMENTAL MEASURMENTS FOR TESTS 5.14.1 TO 5.14.14-- 0.53 -IN. DOMNCOMER GAP

\begin{tabular}{|c|c|c|c|c|c|c|c|c|c|c|c|}
\hline & $\begin{array}{l}\text { TEST } \\
\text { NO }\end{array}$ & $\begin{array}{c}R H-C H-1 \\
(G P M)\end{array}$ & $\begin{array}{c}R M-C H=2 \\
(G P M)\end{array}$ & $\begin{array}{c}F T-C H-1 \\
(G P M)\end{array}$ & $\begin{array}{c}F T-C H-2 \\
(G P H)\end{array}$ & $\begin{array}{c}R M-H G-1 . \\
(C F M)\end{array}$ & $\begin{array}{c}\text { RM-HG-2 } \\
(C F M)\end{array}$ & $\begin{array}{c}R M-H G-3 . \\
\text { (CFM) }\end{array}$ & $\begin{array}{c}\text { RM-HG-4 } \\
\text { (CFM) }\end{array}$ & $\begin{array}{c}\text { FT-HG-1 } \\
\text { (CFM) }\end{array}$ & $\begin{array}{c}F^{T}+\mathrm{HG}-2 \\
(C F M)^{-2}\end{array}$ \\
\hline & $\begin{array}{l}5.14 \cdot 1 \\
5.14 \cdot 2 \\
5.14 \cdot 3 \\
5.14 \cdot 4 \\
5.14 .5\end{array}$ & $\begin{array}{l}0.0 \\
0.0 \\
0.0 \\
0.0 \\
0.0\end{array}$ & $\begin{array}{l}0.0 \\
0.0 \\
0.0 \\
0.0 \\
0.0\end{array}$ & $\begin{array}{r}59.0 \\
60.0 \\
0.0 \\
0.0 \\
0.0\end{array}$ & $\begin{array}{r}0.0 \\
0.0 \\
45.1 \\
35.2 \\
15.1\end{array}$ & $\begin{array}{c}0.0 \\
0.0 \\
0.0 \\
0.0 \\
53.50\end{array}$ & $\begin{array}{l}0.0 \\
0.0 \\
0.0 \\
0.0 \\
0.0\end{array}$ & $\begin{array}{r}3.40 \\
3.40 \\
10.00 \\
13.30 \\
0.0\end{array}$ & $\begin{array}{l}0.0 \\
0.0 \\
0.0 \\
0.0 \\
0.0\end{array}$ & $\begin{array}{l}0.0 \\
0.0 \\
0.0 \\
0.0 \\
0.0\end{array}$ & $\begin{array}{l}0.0 \\
0.0 \\
0.0 \\
0.0 \\
0.0\end{array}$ \\
\hline & $\begin{array}{l}5.14 .6 \\
5.14 .7 \\
5.14 .8 \\
5.14 .99 \\
5.14 .10\end{array}$ & $\begin{array}{l}0.0 \\
0.0 \\
0.0 \\
0.0 \\
0.0\end{array}$ & $\begin{array}{l}0.0 \\
0.0 \\
0.0 \\
0.0 \\
0.0\end{array}$ & $\begin{array}{r}0.0 \\
60.0 \\
0.0 \\
0.0 \\
0.0\end{array}$ & $\begin{array}{r}4.9 \\
0.0 \\
35.2 \\
15.1 \\
5.1\end{array}$ & $\begin{array}{c}80.00 \\
0.0 \\
0.0 \\
53.50 \\
80.00\end{array}$ & $\begin{array}{l}0.0 \\
0.0 \\
0.0 \\
0.0 \\
0.0\end{array}$ & $\begin{array}{c}0.0 \\
3.33 \\
13.30 \\
0.0 \\
0.0\end{array}$ & $\begin{array}{l}0.0 \\
0.0 \\
0.0 \\
0.0 \\
0.0\end{array}$ & $\begin{array}{l}0.0 \\
0.0 \\
0.0 \\
0.0 \\
0.0\end{array}$ & $\begin{array}{l}0.0 \\
0.0 \\
0.0 \\
0.0 \\
0.0\end{array}$ \\
\hline$\vec{\infty}$ & $\begin{array}{l}5.14 .11 \\
5.14 .12 \\
5.14 .13 \\
5.14 .14\end{array}$ & $\begin{array}{l}0.0 \\
0.0 \\
0.0 \\
0.0\end{array}$ & $\begin{array}{l}0.0 \\
0.0 \\
0.0 \\
0.0\end{array}$ & $\begin{array}{r}60.0 \\
0.0 \\
0.0 \\
0.0\end{array}$ & $\begin{array}{r}0.0 \\
35.0 \\
14.7 \\
5.0\end{array}$ & $\begin{array}{c}0.0 \\
0.0 \\
53.50 \\
80.00\end{array}$ & $\begin{array}{l}0.0 \\
0.0 \\
0.0 \\
0.0\end{array}$ & $\begin{array}{c}3.30 \\
13.40 \\
0.0 \\
0.0\end{array}$ & $\begin{array}{l}0.0 \\
0.0 \\
0.0 \\
0.0\end{array}$ & $\begin{array}{l}0.0 \\
0.0 \\
0.0 \\
0.0\end{array}$ & $\begin{array}{l}0.0 \\
0.0 \\
0.0 \\
0.0\end{array}$ \\
\hline & $\begin{array}{c}\text { TEST } \\
\text { NO }\end{array}$ & $\begin{array}{l}P-C H-1 \\
\text { (PSIG) }\end{array}$ & $\begin{array}{l}P-C H-2 \\
(P S I G)\end{array}$ & $\begin{array}{l}\text { P-HG-I } \\
\text { (PSIG) }\end{array}$ & $\begin{array}{l}\text { P-HG-2 } \\
\text { (PSIG) }\end{array}$ & $\begin{array}{l}\text { P-HG-3 } \\
\text { (PSIGI }\end{array}$ & $\begin{array}{l}\text { TF-CH-I } \\
(D E G F)\end{array}$ & $\begin{array}{l}\text { TF-HG- } \\
\text { (DEG F) }\end{array}$ & $\begin{array}{l}\text { TF-HG-2 } \\
\text { (DEG F) }\end{array}$ & $\begin{array}{l}\text { TF-HG-3 } \\
\text { IOEG FI }\end{array}$ & $\begin{array}{l}\text { P-BARO } \\
\text { (PSIAI) }\end{array}$ \\
\hline & $\begin{array}{l}5.14 \cdot \cdot 1 \\
5.14 \cdot 2 \\
5.14 \cdot 3 \\
5.14 \cdot 4 \\
5.14 .5\end{array}$ & $\begin{array}{r}10.0 \\
11.0 \\
10.5 \\
9.7 \\
9.5\end{array}$ & $\begin{array}{l}0.0 \\
0.0 \\
0.0 \\
0.0 \\
0.0\end{array}$ & $\begin{array}{l}10.5 \\
10.5 \\
11.5 \\
10.0 \\
10.0\end{array}$ & $\begin{array}{l}0.0 \\
0.0 \\
0.0 \\
0.0 \\
0.0\end{array}$ & $\begin{array}{l}33.5 \\
33.5 \\
33.5 \\
32.0 \\
33.5\end{array}$ & $\begin{array}{l}45.0 \\
46.0 \\
45.0 \\
45.0 \\
53.0\end{array}$ & $\begin{array}{l}81.0 \\
81.0 \\
79.0 \\
80.0 \\
78.0\end{array}$ & $\begin{array}{l}0.0 \\
0.0 \\
0.0 \\
0.0 \\
0.0\end{array}$ & $\begin{array}{l}79.0 \\
79.0 \\
78.0 \\
78.0 \\
78.0\end{array}$ & $\begin{array}{l}12.3 \\
12.3 \\
12.3 \\
12.3 \\
12.3\end{array}$ \\
\hline & $\begin{array}{l}5.14 .6 \\
5.14 .7 \\
5.14 .8 \\
5.14 .9 \\
5.14 .10\end{array}$ & $\begin{array}{l}9.7 \\
9.2 \\
9.7 \\
9.5 \\
9.5\end{array}$ & $\begin{array}{l}0.0 \\
0.0 \\
0.0 \\
0.0 \\
0.0\end{array}$ & $\begin{array}{r}10.4 \\
9.5 \\
10.0 \\
10.2 \\
9.8\end{array}$ & $\begin{array}{l}0.0 \\
0.0 \\
0.0 \\
0.0 \\
0.0\end{array}$ & $\begin{array}{r}33.5 \\
33.0 \\
32.0 \\
33.5 \\
-33.5\end{array}$ & $\begin{array}{l}52.0 \\
49.0 \\
50.0 \\
52.0 \\
54.0\end{array}$ & $\begin{array}{l}78.0 \\
79.5 \\
78.0 \\
78.0 \\
79.0\end{array}$ & $\begin{array}{l}0.0 \\
0.0 \\
0.0 \\
0.0 \\
0.0\end{array}$ & $\begin{array}{l}78.0 \\
77.0 \\
76.0 \\
77.0 \\
78.0\end{array}$ & $\begin{array}{l}12.3 \\
12.3 \\
12.3 \\
12.3 \\
12.3\end{array}$ \\
\hline & $\begin{array}{l}5.14 .11 \\
5.14 .12 \\
5.14 .13 \\
5.14 .14\end{array}$ & $\begin{array}{r}10.2 \\
10.0 \\
9.7 \\
9.7\end{array}$ & $\begin{array}{l}0.0 \\
0.0 \\
0.0 \\
0.0\end{array}$ & $\begin{array}{l}10.0 \\
10.4 \\
10.4 \\
10.5\end{array}$ & $\begin{array}{l}0.0 \\
0.0 \\
0.0 \\
0.0\end{array}$ & $\begin{array}{l}33.5 \\
33.5 \\
33.5 \\
33.5\end{array}$ & $\begin{array}{l}48.0 \\
49.0 \\
53.0 \\
55.0\end{array}$ & $\begin{array}{l}79.0 \\
79.0 \\
78.0 \\
80.0\end{array}$ & $\begin{array}{l}0.0 \\
0.0 \\
0.0 \\
0.0\end{array}$ & $\begin{array}{l}79.5 \\
79.0 \\
77.0 \\
78.0\end{array}$ & $\begin{array}{l}12.3 \\
12.3 \\
12.3 \\
12.3\end{array}$ \\
\hline
\end{tabular}


TABLE XLIV (Contd.)

COMBINED EFFECTS TEST GROUP 5.14 .

EXPERIMENTAL MEASURMENTS FOR TESTS 5.14 .1 TO 5.14.14 -- 0.53-IN. DOUNCOMER GAP

\begin{tabular}{|c|c|c|c|c|c|c|c|c|c|}
\hline \multirow[b]{2}{*}{$\begin{array}{l}\text { TEST } \\
\text { NO }\end{array}$} & \multirow{2}{*}{$\begin{array}{l}\text { INITIAL } \\
\text { WEIGHT } \\
\text { (LB) }\end{array}$} & LOMER PLENUM & FLOH & \multicolumn{3}{|c|}{ BYPASS FLOW } & \multirow{2}{*}{$\begin{array}{l}\text { LOWER } \\
\text { PLENUM } \\
\text { LEVEL } \\
\text { (IN) }\end{array}$} & \multirow[b]{2}{*}{$\begin{array}{l}\text { P-LP-1 } \\
\text { (PSIG) }\end{array}$} & \multirow[b]{2}{*}{$\begin{array}{l}\text { TF-LP-1 } \\
\text { IOEG FI }\end{array}$} \\
\hline & & $\begin{array}{l}\text { FINAL } \\
\text { WE (GHT } \\
\text { (LS) }\end{array}$ & $\begin{array}{l}\text { TIME OF } \\
\text { COLLECTION } \\
\text { (SEC) }\end{array}$ & $\begin{array}{l}\text { INITIAL } \\
\text { HEIGHT } \\
\text { (LB) }\end{array}$ & $\begin{array}{l}\text { FINAL } \\
\text { WEIGHT } \\
\text { ILBI }\end{array}$ & $\begin{array}{l}\text { TIME OF } \\
\text { COLLECTION } \\
\text { (SEC) }\end{array}$ & & & \\
\hline $\begin{array}{l}5.14 .1 \\
5.14 . \\
5.14 .3 \\
5.14 .5 \\
5.14 .5\end{array}$ & $\begin{array}{l}141.5 \\
163.5 \\
175.5 \\
144.5 \\
141.5\end{array}$ & $\begin{array}{l}309.0 \\
348.5 \\
316.0 \\
257.5 \\
186.5\end{array}$ & $\begin{array}{l}30.0 \\
30.0 \\
30.0 \\
29.5 \\
75.0\end{array}$ & $\begin{array}{l}104.0 \\
149.0 \\
128.5 \\
112.0 \\
114.0\end{array}$ & $\begin{array}{l}170.0 \\
216.0 \\
172.5 \\
161.0 \\
200.0\end{array}$ & $\begin{array}{l}30.0 \\
30.0 \\
30.0 \\
30.0 \\
00.0\end{array}$ & $\begin{array}{r}8.0 \\
2.0 \\
12.0 \\
12.0 \\
12.0\end{array}$ & $\begin{array}{l}10.0 \\
10.5 \\
10.7 \\
10.5 \\
10.0\end{array}$ & $\begin{array}{l}45.0 \\
48.0 \\
46.0 \\
45.0 \\
54.0\end{array}$ \\
\hline $\begin{array}{l}5.14 .6 \\
5.14 .7 \\
5.14 .8 \\
5.14 .9 \\
5.14 .10\end{array}$ & $\begin{array}{l}186.5 \\
145.0 \\
259.5 \\
164.0 \\
147.0\end{array}$ & $\begin{array}{l}199.0 \\
273.5 \\
363.0 \\
202.0 \\
149.0\end{array}$ & $\begin{array}{r}120.0 \\
30.0 \\
30.0 \\
60.0 \\
60.0\end{array}$ & $\begin{array}{l}170.5 \\
110.0 \\
125.0 \\
117.0 \\
181.0\end{array}$ & $\begin{array}{l}205.5 \\
225.5 \\
165.0 \\
206.0 \\
217.0\end{array}$ & $\begin{array}{l}50.0 \\
30.0 \\
30.0 \\
50.0 \\
59.0\end{array}$ & $\begin{array}{l}12.0 \\
12.0 \\
12.0 \\
12.0 \\
12.0\end{array}$ & $\begin{array}{r}10.0 \\
9.7 \\
10.0 \\
10.2 \\
9.7\end{array}$ & $\begin{array}{l}55.0 \\
50.0 \\
51.0 \\
52.0 \\
54.0\end{array}$ \\
\hline $\begin{array}{l}5.14 .11 \\
5.14 .12 \\
5.14 .13 \\
5.14 .14\end{array}$ & $\begin{array}{l}149.0 \\
217.0 \\
143.0 \\
165.0\end{array}$ & $\begin{array}{l}235.0 \\
283.0 \\
183.0 \\
172.0\end{array}$ & $\begin{array}{l}30.0 \\
30.0 \\
60.0 \\
60.0\end{array}$ & $\begin{array}{l}106.0 \\
218.0 \\
113.0 \\
202.0\end{array}$ & $\begin{array}{l}277.0 \\
298.0 \\
202.0 \\
240.0\end{array}$ & $\begin{array}{l}30.0 \\
30.0 \\
60.0 \\
60.0\end{array}$ & $\begin{array}{l}12.0 \\
12.0 \\
12.0 \\
12.0\end{array}$ & $\begin{array}{l}10.5 \\
10.2 \\
10.0 \\
10.2\end{array}$ & $\begin{array}{l}49.0 \\
50.0 \\
54.0 \\
54.0\end{array}$ \\
\hline
\end{tabular}

\begin{tabular}{|c|c|c|c|c|c|c|c|}
\hline $\begin{array}{l}\text { TEST } \\
\text { NO }\end{array}$ & $\begin{array}{c}R M-C G-1 \\
\text { (CFM) }\end{array}$ & $\begin{array}{c}R H-C G-2 \\
\text { (CFM) }\end{array}$ & $\begin{array}{c}F T-C G-1 \\
(C F M) .\end{array}$ & $\begin{array}{l}\text { P-CG-1 } \\
\text { (PSIG) }\end{array}$ & $\begin{array}{l}\text { P-CG-2 } \\
\text { (PSIG) }\end{array}$ & $\begin{array}{l}\text { TF-CG-1 } \\
\text { (DEG F) }\end{array}$ & $\begin{array}{l}\text { TF-CG-2 } \\
(D E G F)\end{array}$ \\
\hline $\begin{array}{l}5.14 .1 \\
5.14 .2 \\
5.14 .3 \\
5.14 .4 \\
5.14 .5\end{array}$ & $\begin{array}{l}0.0 \\
0.0 \\
0.0 \\
0.0 \\
0.0\end{array}$ & $\begin{array}{l}30.0 \\
30.0 \\
30.0 \\
30.0 \\
30.0\end{array}$ & $\begin{array}{l}0.0 \\
0.0 \\
0.0 \\
0.0 \\
0.0\end{array}$ & $\begin{array}{l}0.0 \\
0.0 \\
0.0 \\
0.0 \\
0.0\end{array}$ & $\begin{array}{l}35.0 \\
34.5 \\
34.6 \\
34.6 \\
34.5\end{array}$ & $\begin{array}{l}0.0 \\
0.0 \\
0.0 \\
0.0 \\
0.0\end{array}$ & $\begin{array}{l}77.0 \\
78.0 \\
78.0 \\
80.0 \\
78.0\end{array}$ \\
\hline $\begin{array}{l}5.14 .6 \\
5.14 .7 \\
5.14 .8 \\
5.14 .99 \\
5.14 .10\end{array}$ & $\begin{array}{l}0.0 \\
0.0 \\
0.0 \\
0.0 \\
0.0\end{array}$ & $\begin{array}{r}30.0 \\
0.0 \\
0.0 \\
0.0 \\
0.0\end{array}$ & $\begin{array}{r}0.0 \\
14.5 \\
14.7 \\
14.7 \\
14.9\end{array}$ & $\begin{array}{r}0.0 \\
99.5 \\
97.5 \\
94.0 \\
92.0\end{array}$ & $\begin{array}{r}34.5 \\
0.0 \\
0.0 \\
0.0 \\
0.0\end{array}$ & $\begin{array}{l}.0 .0 \\
73.0 \\
75.0 \\
81.0 \\
85.0\end{array}$ & $\begin{array}{r}79.5 \\
0.0 \\
0.0 \\
0.0 \\
0.0\end{array}$ \\
\hline $\begin{array}{l}5.14 .11 \\
5.14 .12 \\
5.14 .13 \\
5.14 .14\end{array}$ & $\begin{array}{l}0.0 \\
0.0 \\
0.0 \\
0.0\end{array}$ & $\begin{array}{l}0.0 \\
0.0 \\
0.0 \\
0.0\end{array}$ & $\begin{array}{l}29.4 \\
29.4 \\
29.4 \\
29.6\end{array}$ & $\begin{array}{l}92.0 \\
92.0 \\
90.0 \\
89.0\end{array}$ & $\begin{array}{l}0.0 \\
0.0 \\
0.0 \\
0.0\end{array}$ & $\begin{array}{r}91.0 \\
96.0 \\
97.5 \\
107.0\end{array}$ & $\begin{array}{l}0.0 \\
0.0 \\
0.0 \\
0.0\end{array}$ \\
\hline
\end{tabular}




\section{TABLE LXV}

\section{COMBINED EFFECTS TEST GROUP 9.3}

TFANSPAF ENT VESSEL TEST CATA FCP TESTS 9.3.1 TO 9.3. - - 1.58-IN. ODWNCOMER GAP

SUMMARY TF CALCIJLATED RESULTS

\begin{tabular}{|c|c|c|c|c|c|c|c|c|c|c|}
\hline $\begin{array}{l}\text { hest } \\
\text { no }\end{array}$ & $\begin{array}{l}\text { COWNCOYEE: } \\
\text { AIP. FL'IW } \\
\text { ILB/SECI }\end{array}$ & $\begin{array}{l}\text { D JWNCOMEP } \\
\text { AIP. TEUISITY } \\
\text { (LB/FT } \$ 3 \text { I }\end{array}$ & $\begin{array}{l}\text { LOWEE PLENUM } \\
\text { PRESSIJPE } \\
\text { (PSIA) }\end{array}$ & $\begin{array}{l}\text { COLD LEG } \\
\text { AIR FLOW } \\
\text { (LB/SCC) }\end{array}$ & $\begin{array}{l}\text { COWNCRMER } \\
\text { WATER FLTH } \\
\text { (LB/SEC) }\end{array}$ & $\begin{array}{l}\text { WATER FLIIW } \\
\text { CONTINUITY }\end{array}$ & $\begin{array}{l}\text { J-AIR } \\
\text { DOWNCDMER } \\
\text { (FT/SEC) }\end{array}$ & $\begin{array}{l}\text { J-HATER } \\
\text { DOHNCOMER } \\
\text { (FT/SEC) }\end{array}$ & $(J G *) * * 1 / 2$ & $(J L * 1 * * 1 / 2$ \\
\hline $\begin{array}{l}9.3 \cdot 1 \\
9.3 \cdot 2 \\
9.3 \cdot 3 \\
9.3 \cdot 4 \\
9.3 \cdot 5\end{array}$ & $\begin{array}{l}0.1722 \\
0.1436 \\
0.2468 \\
0.3547 \\
0.4742\end{array}$ & $\begin{array}{l}0.1165 \\
0.1183 \\
0.1195 \\
0.1174 \\
0.1374\end{array}$ & $\begin{array}{l}21.84 \\
22.09 \\
22.34 \\
22.09 \\
26.09\end{array}$ & $\begin{array}{l}0.0667 \\
0.0665 \\
0.0055 \\
0.0665 \\
0.0661\end{array}$ & $\begin{array}{r}10.820 \\
9.675 \\
6.800 \\
3.717 \\
0.817\end{array}$ & $\begin{array}{r}0.999 \\
1.012 \\
0.989 \\
.1 .013 \\
1.060\end{array}$ & $\begin{array}{r}6.29 \\
5.16 \\
8.78 \\
12.73 \\
14.67\end{array}$ & $\begin{array}{l}0.737 \\
0.053 \\
0.463 \\
0.253 \\
0.056\end{array}$ & $\begin{array}{l}0.3054 \\
0.2778 \\
0.3633 \\
0.4394 \\
0.4864\end{array}$ & $\begin{array}{l}0.5032 \\
0.4759 \\
0.3989 \\
0.2949 \\
0.1383\end{array}$ \\
\hline $\begin{array}{l}9.3 .6 \\
9.3 .7 \\
9.3 .9 \\
9.3 .9\end{array}$ & $\begin{array}{l}0.1403 \\
0.1213 \\
0.3199 \\
0.4905\end{array}$ & $\begin{array}{r}0.1160 \\
0.1210 \\
0.1219 \\
0.1562\end{array}$ & $\begin{array}{l}21.84 \\
22.59 \\
22.95 \\
29.70\end{array}$ & $\begin{array}{l}0.2441 \\
0.2426 \\
0.2059 \\
0.2259\end{array}$ & $\begin{array}{r}10.333 \\
8.761 \\
3.667 \\
1.533\end{array}$ & $\begin{array}{l}1.013 \\
0.990 \\
1.047 \\
1.066\end{array}$ & $\begin{array}{r}5.13 \\
4.26 \\
13.59 \\
13.34\end{array}$ & $\begin{array}{l}0.704 \\
0.597 \\
0.250 \\
0.104\end{array}$ & $\begin{array}{l}0.2761 \\
0.2539 \\
0.4544 \\
0.4790\end{array}$ & $\begin{array}{l}0.4918 \\
0.4530 \\
0.2929 \\
0.1895\end{array}$ \\
\hline
\end{tabular}


TABLE LXV (Contd.)

COMBINED EFFECTS TEST GROUP 9.3

EXPERIMENTAL MEASURMENTS FOR TESTS 9.3 .1 TO 9.3. 9.-- 1.58-IN. DOWNCOMER GAP

\begin{tabular}{|c|c|}
\hline $\begin{array}{l}\text { TEST } \\
\text { ND }\end{array}$ & $\begin{array}{c}R M-C H-1 \\
(G P M)\end{array}$ \\
\hline $\begin{array}{l}9.3 .1 \\
9.3 .2 \\
9.3 .3 \\
9.3 .4 \\
9.3 .5\end{array}$ & $\begin{array}{l}0.0 \\
0.0 \\
0.0 \\
0.0 \\
0.0\end{array}$ \\
\hline $\begin{array}{l}9.3 .6 \\
9.3 .7 \\
9.3 .8 \\
9.3 .9\end{array}$ & $\begin{array}{l}0.0 \\
0.0 \\
0.0 \\
0.0\end{array}$ \\
\hline
\end{tabular}

$F T-C H-1$
$(G P M)$

$F T-C H-2$
$(G P M)$

119.0

119.0
99.0

80.0

0.0

0.0
0.0

0.0
0.0
0.0
49.7

(CFM)

R.M-HG-2 (CFM)
(CFG-2

80.00

66.50

0.0
0.0

0.0

0.0
0.0

0.0
0.0
0.0
0.0

0.0
0.0

119.0

100.0
0.0

0.0

0.0
0.0
50.1

0.0
50.1
40.6

0.0

0.0
0.0
0.0

0.0

0.0
0.0
0.0

$2 M-H G-3$
(CFM)

P.M-HG -4
(CFM)

CT- $\mathrm{HG}-1$
(CFM)

FT-HG-2

0.0
0.0

0.0
0.0
0.0

0.0

0.0

0.0
0.0

0.0

0.0

0.0
29.49

44.23

64.23

17.44

14.8

44.36
63.85

.

\begin{tabular}{|c|c|c|c|c|c|c|c|c|c|}
\hline $\begin{array}{l}\text { TEST } \\
\text { ND }\end{array}$ & $\begin{array}{l}P-C W-1 \\
(P S I G)\end{array}$ & $\begin{array}{l}P-C H-2 \\
(P S \mid G)\end{array}$ & $\begin{array}{l}\text { P-HG-I } \\
\text { (PSIG) }\end{array}$ & $\begin{array}{l}P-H G-2 \\
(P S I G)\end{array}$ & $\begin{array}{l}P-. H G-3 \\
\text { (PSIG) }\end{array}$ & $\begin{array}{l}\text { TF-CW-1 } \\
\text { (OESG F) }\end{array}$ & $\begin{array}{l}T F-H G-1 \\
(D E G \quad F)\end{array}$ & $\begin{array}{l}T E-H G=2 \\
(D E G F)\end{array}$ & $\begin{array}{l}T F-H G-3 \\
(D E R G F)\end{array}$ \\
\hline $\begin{array}{l}9.3 .1 \\
9 \cdot 3 \cdot 2 \\
9.3 \cdot 3 \\
9.3 .4 \\
9.3 .5\end{array}$ & $\begin{array}{r}9.7 \\
10.0 \\
10.0 \\
9.7 \\
13.0\end{array}$ & $\begin{array}{l}0.0 \\
0.0 \\
0.0 \\
0.0 \\
0.0\end{array}$ & $\begin{array}{r}10.0 \\
10.3 \\
10.5 \\
9.8 \\
14.5\end{array}$ & $\begin{array}{l}95.5 \\
95.0 \\
92.5 \\
90.5 \\
83.5\end{array}$ & $\begin{array}{l}33.0 \\
33.0 \\
33.5 \\
33.0 \\
33.0\end{array}$ & $\begin{array}{l}46.0 \\
89.5 \\
44.0 \\
45.0 \\
46.0\end{array}$ & $\begin{array}{r}94.0 \\
92.0 \\
91.0 \\
96.0 \\
109.5\end{array}$ & $\begin{array}{l}110.0 \\
104.5 \\
103.5 \\
110.5 \\
124.0\end{array}$ & $\begin{array}{r}89.5 \\
86.0 \\
0.0 \\
0.0 \\
0.0\end{array}$ \\
\hline \multirow[t]{3}{*}{$\begin{array}{l}9.3: 5 \\
9.3 .7 \\
3.3 .8 \\
5.3 .9\end{array}$} & $\begin{array}{l}10.0 \\
1.5: 7 \\
11.0 \\
15.0\end{array}$ & $\begin{array}{l}0.0 \\
0.0 \\
0.0 \\
0.0\end{array}$ & $\begin{array}{r}9.2 \\
10.5 \\
13.7 \\
16.8\end{array}$ & $\begin{array}{l}91.0 \\
91.0 \\
94.0 \\
36.0\end{array}$ & $\begin{array}{l}33.5 \\
33.5 \\
33.5 \\
33.0\end{array}$ & $\begin{array}{l}45.0 \\
40.0 \\
47.5 \\
49.0\end{array}$ & $\begin{array}{r}101.5 \\
96.0 \\
76.0 \\
98.0\end{array}$ & $\begin{array}{r}115.5 \\
110.0 \\
83.5 \\
115.0\end{array}$ & $\begin{array}{l}0.0 \\
0.00 \\
0.00 \\
0.0\end{array}$ \\
\hline & ' & & & & & - & & & \\
\hline & \multicolumn{3}{|c|}{ L MINEF PLENIUY FLIIW } & \multicolumn{3}{|c|}{ BYPASS FLOW } & & & \\
\hline $\begin{array}{l}\text { TEST } \\
\text { NO }\end{array}$ & $\begin{array}{l}\text { INITIAL } \\
\text { WEISIIT } \\
\text { (Lis) }\end{array}$ & $\begin{array}{l}F \text { INAL } \\
\text { 'UEIFHT } \\
\text { (LB) }\end{array}$ & $\begin{array}{l}\text { TIME TF } \\
\text { CULLECTION } \\
\text { (IEC) }\end{array}$ & $\begin{array}{l}\text { INITIAL } \\
\text { WE:GHT } \\
\text { (LB) }\end{array}$ & $\begin{array}{l}\text { FINAL } \\
\text { WEIGHT } \\
\text { (L B] }\end{array}$ & $\begin{array}{l}\text { TIME AF } \\
\text { COLLECTION } \\
\text { (SFCI }\end{array}$ & $\begin{array}{l}\text { PLENUM } \\
\text { LFVEL } \\
\text { IINI }\end{array}$ & $\begin{array}{l}P-(P-1 \\
\text { (PSIG) }\end{array}$ & $\begin{array}{l}\text { TE-LP-1 } \\
\text { (DES F) }\end{array}$ \\
\hline $\begin{array}{l}9.3 \cdot 1 \\
9.3 .2 \\
9.3 .3 \\
9.3 .34 \\
9.3 .5\end{array}$ & $\begin{array}{l}145.5 \\
163.5 \\
214.0 \\
141.0 \\
252.0\end{array}$ & $\begin{array}{l}416.0 \\
354.0 \\
418.0 \\
252.5 \\
301.0\end{array}$ & $\begin{array}{l}25.0 \\
20.0 \\
30.0 \\
30.0 \\
60.0\end{array}$ & $\begin{array}{l}222.0 \\
106.5 \\
251.5 \\
104.0 \\
202.5\end{array}$ & $\begin{array}{l}364.5 \\
251.5 \\
377.0 \\
202.5 \\
353.0\end{array}$ & $\begin{array}{l}25.0 \\
20.0 \\
30.0 \\
30.0 \\
30.0\end{array}$ & $\begin{array}{r}5.0 \\
5.0 \\
12.0 \\
12.0 \\
12.0\end{array}$ & $\begin{array}{r}9.5 \\
3.7 \\
10.0 \\
7.7 \\
13 . ?\end{array}$ & $\begin{array}{l}46.0 \\
44.0 \\
44.5 \\
45.5 \\
52.5\end{array}$ \\
\hline $\begin{array}{l}9.3 .6 \\
9.3 .7 \\
9.3 .8 \\
9.3 .9\end{array}$ & $\begin{array}{l}180.0 \\
195.0 \\
156.0 \\
266.0\end{array}$ & $\begin{array}{l}490.0 \\
458.0 \\
256.0 \\
358.0\end{array}$ & $\begin{array}{l}30.0 \\
30.0 \\
30.0 \\
60.0\end{array}$ & $\begin{array}{l}237.5 \\
259.5 \\
163.5 \\
272.5\end{array}$ & $\begin{array}{l}430.5 \\
409.5 \\
272.5 \\
407.0\end{array}$ & $\begin{array}{l}30.0 \\
30.0 \\
30.0 \\
30.0\end{array}$ & $\begin{array}{r}5.0 \\
12.0 \\
12.0 \\
12.0\end{array}$ & $\begin{array}{r}9.5 \\
10.2 \\
10.7 \\
17.5\end{array}$ & $\begin{array}{r}45.5 \\
.44 .0 \\
48.0 \\
53.0\end{array}$ \\
\hline
\end{tabular}


TABLE LXV (Contd.)

COMBINED EFFECTS TEST. GROUP 9.3

EXPERIMENTAL MEASURMENTS FOQ TESTS 9.3 .1 TO 9.3. 9 -- 1.58-IN. DOWNCOMER GAP

\begin{tabular}{|c|c|c|c|c|c|c|c|}
\hline $\begin{array}{c}\text { TEST } \\
\text { NO }\end{array}$ & $\begin{array}{c}R M-C G-1 \\
(C F M)\end{array}$ & $\begin{array}{c}R M-C G-2 \\
(C F M)\end{array}$ & $\begin{array}{c}F T-C G-1 \\
(C F M)\end{array}$ & $\begin{array}{l}P-C G-1 \\
\text { (PSIGI) }\end{array}$ & $\begin{array}{l}P-C G-2 \\
(P S I C)\end{array}$ & $\begin{array}{l}\text { TF-CG-1 } \\
\text { (CEG F) }\end{array}$ & $\begin{array}{l}\text { TF-CG-2 } \\
\text { (DEG F) }\end{array}$ \\
\hline $\begin{array}{l}9.3 .1 \\
9.3 .2 \\
9.3 .3 \\
9.3 .4 \\
9.3 .5\end{array}$ & $\begin{array}{l}0.0 \\
0.0 \\
0.0 \\
0.0 \\
0.0\end{array}$ & $\begin{array}{l}30.0 \\
30.0 \\
30.0 \\
30.0 \\
30.0\end{array}$ & $\begin{array}{l}0.0 \\
0.0 \\
0.0 \\
0.0 \\
0.0\end{array}$ & $\begin{array}{l}0.0 \\
0.0 \\
0.0 \\
0.0 \\
0.0\end{array}$ & $\begin{array}{l}35.0 \\
35.0 \\
35.0 \\
35.0 \\
34.6\end{array}$ & $\begin{array}{l}0.0 \\
0.0 \\
0.0 \\
0.0 \\
0.0\end{array}$ & $\begin{array}{l}78.0 \\
80.0 \\
80.0 \\
81.0 \\
92.5\end{array}$ \\
\hline $\begin{array}{l}9.3 .6 \\
9.3 .7 \\
9.3 .8 \\
9.3 .9\end{array}$ & $\begin{array}{l}0.0 \\
0.0 \\
0.0 \\
0.0\end{array}$ & $\begin{array}{l}0.0 \\
0.0 \\
0.0 \\
0.0\end{array}$ & $\begin{array}{l}29.9 \\
29.7 \\
29.9 \\
29.4\end{array}$ & $\begin{array}{l}91.0 \\
91.0 \\
94.0 \\
86.0\end{array}$ & $\begin{array}{l}0.0 \\
0.0 \\
0.0 \\
0.0\end{array}$ & $\begin{array}{r}109.0 \\
110.0 \\
77.0 \\
114.0\end{array}$ & $\begin{array}{l}0.0 \\
0.0 \\
0.0 \\
0.0\end{array}$ \\
\hline
\end{tabular}




\section{TABLE XIVI}

COMBINED EFFECTS TEST GROUP 9.7

TRANSPARENT VESSEL TEST DATA FOR TESTS 9.7 .1 TO $9.7 .11-1.58-$ IN. DOHNCOMER GAP SUMMARY OF CALCULATED RESULTS

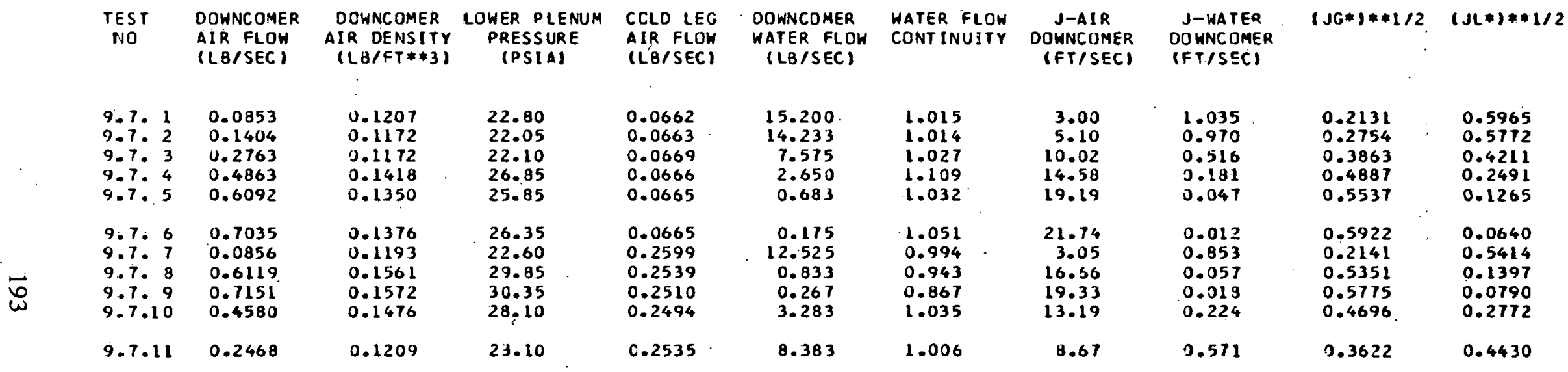




\section{TABLE XIVI (Contd.)}

COMBINED EFFECTS TEST GROUP 9.7

EXPERIMENTAL MEASURMENTS FOR TESTS 9.7.1 TO $9.7 .11--1.58-1$ - DONNCOMER GAP

\begin{tabular}{|c|c|c|c|c|c|c|c|c|c|c|}
\hline $\begin{array}{c}\text { TEST } \\
\text { NO }\end{array}$ & $\begin{array}{c}R P-C H-1 \\
(G P M)\end{array}$ & $\begin{array}{c}\mathrm{R} H-C H-2 \\
(\mathrm{GPM})\end{array}$ & $\begin{array}{c}F T-C H-1 \\
(G P M)\end{array}$ & $\begin{array}{c}F T-C H-2 \\
(G P M)\end{array}$ & $\begin{array}{c}R M-H G-1 \\
(C F M)\end{array}$ & $\begin{array}{c}R M-H G-2 \\
|C F M|\end{array}$ & $\begin{array}{c}R M-H G-3 \\
(C F M)\end{array}$ & $\begin{array}{c}R A-H G-4 \\
\text { (CFM) }\end{array}$ & $\begin{array}{c}F T-H G-1 \\
\text { (CFM) }\end{array}$ & $\begin{array}{c}F T-H G-2 \\
(C F M)\end{array}$ \\
\hline $\begin{array}{l}9.7 .1 \\
9.7 .2 \\
9.7 .3 \\
9.7 .4 \\
9.7 .5\end{array}$ & $\begin{array}{l}0.0 \\
0.0 \\
0.0 \\
0.0 \\
0.0\end{array}$ & $\begin{array}{l}0.0 \\
0.0 \\
0.0 \\
0.0 \\
0.0\end{array}$ & $\begin{array}{r}150.0 \\
150.0 \\
101.0 \\
60.0 \\
0.0\end{array}$ & $\begin{array}{r}0.0 \\
0.0 \\
0.0 \\
0.0 \\
30.1\end{array}$ & $\begin{array}{c}40.00 \\
66.50 \\
0.0 \\
0.0 \\
0.0\end{array}$ & $\begin{array}{l}0.0 \\
0.0 \\
0.0 \\
0.0 \\
0.0\end{array}$ & $\begin{array}{l}0.0 \\
0.0 \\
0.0 \\
0.0 \\
0.0\end{array}$ & $\begin{array}{l}0.0 \\
0.0 \\
0.0 \\
0.0 \\
0.0\end{array}$ & $\begin{array}{c}0.0 \\
0.0 \\
29.62 \\
57.31 \\
73.72\end{array}$ & $\begin{array}{l}0.0 \\
0.0 \\
0.0 \\
0.0 \\
0.0\end{array}$ \\
\hline $\begin{array}{l}9.7 .6 \\
9.7 .7 \\
9.7 .8 \\
9.7 .99 \\
9.7 .10\end{array}$ & $\begin{array}{l}0.0 \\
0.0 \\
0.0 \\
0.0 \\
0.0\end{array}$ & $\begin{array}{l}0.0 \\
0.0 \\
0.0 \\
0.0 \\
0.0\end{array}$ & $\begin{array}{r}0.0 \\
139.0 \\
0.0 \\
0.0 \\
60.0\end{array}$ & $\begin{array}{r}19.9 \\
0.0 \\
30.0 \\
20.1 \\
0.0\end{array}$ & $\begin{array}{c}0.0 \\
40.00 \\
0.0 \\
0.0 \\
0.0\end{array}$ & $\begin{array}{l}0.0 \\
0.0 \\
0.0 \\
0.0 \\
0.0\end{array}$ & $\begin{array}{l}0.0 \\
0.0 \\
0.0 \\
0.0 \\
0.0\end{array}$ & $\begin{array}{l}0.0 \\
0.0 \\
0.0 \\
0.0 \\
0.0\end{array}$ & $\begin{array}{c}88.72 \\
0.0 \\
73.21 \\
88.72 \\
55.77\end{array}$ & $\begin{array}{l}0.0 \\
0.0 \\
0.0 \\
0.0 \\
0.0\end{array}$ \\
\hline 9.7 .11 & 0.0 & 0.0 & 100.0 & 0.0 & 0.0 & 0.0 & 0.0 & 0.0 & 29.62 & 0.0 \\
\hline $\begin{array}{c}\text { TEST } \\
\text { NO }\end{array}$ & $\begin{array}{l}P-C H-1 \\
(P S I G)\end{array}$ & $\begin{array}{l}P-C H-2 \\
\text { (PSIG) }\end{array}$ & $\begin{array}{l}\text { P-HG-1 } \\
\text { (PSIG) }\end{array}$ & $\begin{array}{l}P-H G-2 \\
\text { (PSIG) }\end{array}$ & $\begin{array}{l}\text { P-HG-3 } \\
\text { (PSIG) }\end{array}$ & $\begin{array}{l}\text { TF-CH-1 } \\
\text { (DEG F) }\end{array}$ & $\begin{array}{l}\text { TF-HG- } 1 \\
\text { (DEG F) }\end{array}$ & $\begin{array}{l}\text { TF-HG-2 } \\
\text { IOEG FI }\end{array}$ & $\begin{array}{l}\text { TF-HG-3 } \\
\text { (DEG F) }\end{array}$ & $\begin{array}{l}\text { P-BARO } \\
\text { (PSIA) }\end{array}$ \\
\hline $\begin{array}{l}9.7 .1 \\
9.702 \\
9.703 \\
9.7 .4 \\
9.7 .5\end{array}$ & $\begin{array}{r}10.7 \\
10.0 \\
9.2 \\
13.0 \\
13.0\end{array}$ & $\begin{array}{l}0.0 \\
0.0 \\
0.0 \\
0.0 \\
0.0\end{array}$ & $\begin{array}{r}10.5 \\
10.0 \\
9.0 \\
14.0 \\
14.6\end{array}$ & $\begin{array}{r}0.0 \\
0.0 \\
98.0 \\
96.5 \\
93.0\end{array}$ & $\begin{array}{l}32.0 \\
31.0 \\
32.5 \\
32.5 \\
32.5\end{array}$ & $\begin{array}{l}50.0 \\
48.0 \\
49.0 \\
49.0 \\
51.0\end{array}$ & $\begin{array}{r}87.0 \\
87.0 \\
75.0 \\
100.5 \\
101.5\end{array}$ & $\begin{array}{r}0.0 \\
0.0 \\
72.0 \\
117.0 \\
113.5\end{array}$ & $\begin{array}{r}87.0 \\
85.0 \\
0.0 \\
110.0 \\
0.0\end{array}$ & $\begin{array}{l}12.3 \\
12.3 \\
12.3 \\
12.3 \\
12.3\end{array}$ \\
\hline $\begin{array}{l}9.7 \cdot 6 \\
9.7-7 \\
9.7 .8 \\
9.709 \\
9.7 .10\end{array}$ & $\begin{array}{l}13.2 \\
11.7 \\
17.2 \\
17.2 \\
15.0\end{array}$ & $\begin{array}{l}0.0 \\
0.0 \\
0.0 \\
0.0 \\
0.0\end{array}$ & $\begin{array}{l}14.7 \\
10.5 \\
18.2 \\
18.8 \\
16.6\end{array}$ & $\begin{array}{l}90.5 \\
96.0 \\
93.0 \\
93.0 \\
95.0\end{array}$ & $\begin{array}{l}32.0 \\
33.0 \\
32.5 \\
32.5 \\
32.5\end{array}$ & $\begin{array}{l}50.5 \\
51.5 \\
49.5 \\
50.0 \\
51.5\end{array}$ & $\begin{array}{r}108.5 \\
94.5 \\
96.0 \\
113.0 \\
117.0\end{array}$ & $\begin{array}{r}123.5 \\
0.0 \\
107.0 \\
128.0 \\
128.0\end{array}$ & $\begin{array}{r}0.0 \\
95.5 \\
0.0 \\
0.0 \\
0.0\end{array}$ & $\begin{array}{l}12.3 \\
12.3 \\
12.3 \\
12.3 \\
12.3\end{array}$ \\
\hline 9.7 .11 & 11.2 & 0.0 & 13.0 & 96.0 & 32.5 & 51.0 & 114.5 & 125.0 & 0.0 & 12.3 \\
\hline
\end{tabular}


TABLE XLVI (Contd.)

COMBINED EFFECTS TEST GROUP 9.7

EXPERIMENTAL MEASURMENTS FOR TESTS 9.7.1 TO 9.7.11 -- $1.58-1$ IN. DONNCOMER GAP

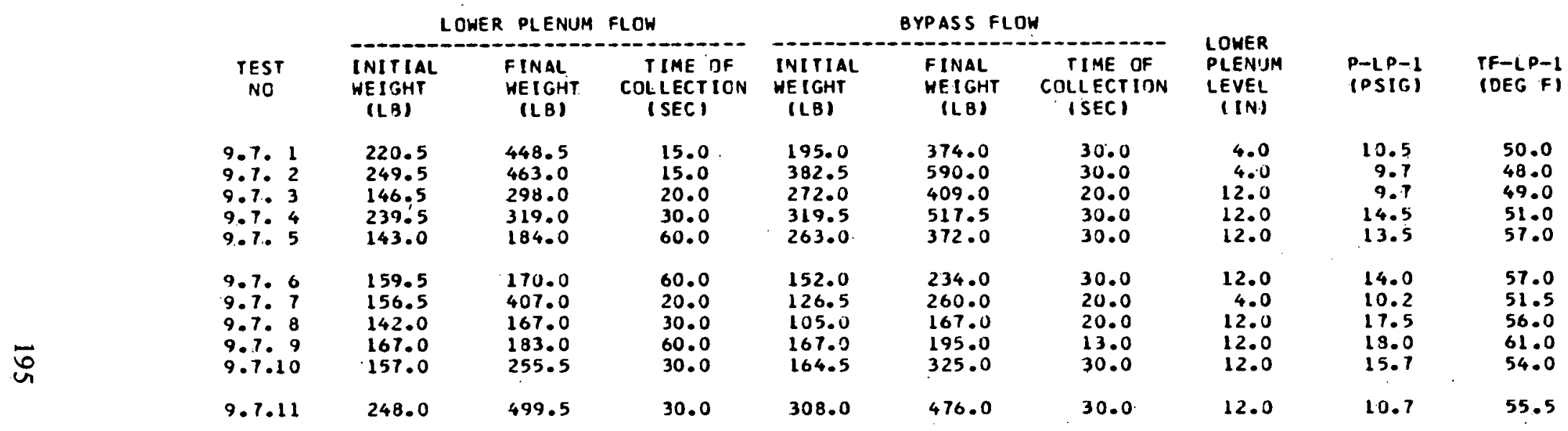

\begin{tabular}{|c|c|c|c|c|c|c|c|}
\hline $\begin{array}{c}\text { TEST } \\
\text { NO }\end{array}$ & $\begin{array}{c}R M-C G-1 \\
(C F M)\end{array}$ & $\begin{array}{c}R M-C G-2 \\
(C F M)\end{array}$ & $\begin{array}{c}F T-C G-1 \\
(C F M)\end{array}$ & $\begin{array}{l}P-C G-1 \\
\text { (PSIG) }\end{array}$ & $\begin{array}{l}P-C G-2 \\
\text { (PSIG) }\end{array}$ & $\begin{array}{l}\text { TF-CG-1 } \\
(D E G F)\end{array}$ & $\begin{array}{l}\text { IF-CG-2 } \\
\text { (DEG F) }\end{array}$ \\
\hline $\begin{array}{l}9.7 \cdot 1 \\
9.7 .2 \\
9.7 \cdot 3 \\
9.704 \\
9.7 .5\end{array}$ & $\begin{array}{l}0.0 \\
0.0 \\
0.0 \\
0.0 \\
0.0\end{array}$ & $\begin{array}{l}30.0 \\
30.0 \\
30.0 \\
30.0 \\
30.0\end{array}$ & $\begin{array}{l}0.0 \\
0.0 \\
0.0 \\
0.0 \\
0.0\end{array}$ & $\begin{array}{r}0.0 \\
0.0 \\
98.0 \\
96.5 \\
93.0\end{array}$ & $\begin{array}{l}34.5 \\
34.8 \\
35.1 \\
35.0 \\
34.9\end{array}$ & $\begin{array}{l}0.0 \\
0.0 \\
0.0 \\
0.0 \\
0.0\end{array}$ & $\begin{array}{l}79.0 \\
80.5 \\
76.0 \\
79.0 \\
80.0\end{array}$ \\
\hline $\begin{array}{r}9.7 .6 \\
9.7 .7 \\
9.7 .8 \\
9.7 .99 \\
9.7 .10\end{array}$ & $\begin{array}{l}0.0 \\
0.0 \\
0.0 \\
0.0 \\
0.0\end{array}$ & $\begin{array}{r}30.0 \\
0.0 \\
0.0 \\
0.0 \\
0.0\end{array}$ & $\begin{array}{l}0.0 \\
29.6 \\
29.5 \\
29.7 \\
29.2\end{array}$ & $\begin{array}{l}90.5 \\
96.0 \\
93.0 \\
93.0 \\
95.0\end{array}$ & $\begin{array}{r}35.0 \\
0.0 \\
0.0 \\
0.0 \\
0.0\end{array}$ & $\begin{array}{r}0.0 \\
95.5 \\
90.5 \\
101.5 \\
106.0\end{array}$ & $\begin{array}{r}81.0 \\
0.0 \\
0.0 \\
0.0 \\
0.0\end{array}$ \\
\hline 9.7 .11 & 0.0 & 0.0 & 29.6 & 96.0 & 0.0 & 109.5. & 0.0 \\
\hline
\end{tabular}


Results from tests in the one-dimensional inlet test group are shown in Table XLVII.

\subsection{Increasing and Decreasing Air Flow at Conśtant Water Flow}

Tests were performed to investigate the influence on countercurrent flow of maintaining a constant water flow rate and consistently increasing the air flow. The objective of these tests was to check for hysteresis effects in the method of establishment of a test. Some evidence existed in the literature that a slightly different countercurrent water flow at a given air flow would occur depending on whether the air flow was increased from a lower air flow or decreased from a higher air flow.

The test procedures for these tests included the following steps:

(1) A constant water flow of about $45 \mathrm{gpm}$ was established.

(2) The air flow was increased from a volumetric flow of $5 \mathrm{cfm}$ to a volumetric flow of about $125 \mathrm{cfm}$ in eleven increments. Vessel pressure was atmospheric for tests up to air flows of about $60 \mathrm{cfm}$. Above $60 \mathrm{cfm}$, the valve on the vessel outlet was throttled to maintain pressure varying from 3 to $8.5 \mathrm{psig}$.

(3) At $125 \mathrm{gpm}$, a second test was conducted by holding the water flow constant at $45 \mathrm{cfm}$ and decreasing the air flow to $55 \mathrm{cfm}$ in five increments. For these tests, the pressure and air flows were matched closely to the pressure and flows used during the tests in which the air flows were increasing.

Results from tests with increasing and decreasing air flow are presented in Tables XLVIII and XLIX, respectively.

\subsection{Double Annulus Tests}

A series of tests was performed to investigate the influence on countercurrent flow of the presence of two parallel downcomer gaps in which the air and water flows could interact. This downcomer configuration was tested because two parallel downcomers are crèated in some reactor systems by a cylindrical thermal shield which is supported within the downcomer. The objective of these tests was to determine whether the parallel flow paths would cause more water to be delivered to the lower plenum because of large water flows in one downcomer and large air flows in the other downcomer. Results from these tests are presented in Table L.

Parallel downcomers were created by modifying Filler Piece A (6.30-inch ID) to allow flow in the 0.70 -inch radial gap between the filler piece and the vessel as well as the normal downcomer flow path (0.53-inch radial gap) between the filler piece and the 5.24-inch-OD core barrel.' Modifications to the filler piece included machining the support rings to within a short distance of the support pins. Details of the support ring modification are shown in Figure 6 . The procedures for conducting these tests followed those of the single downcomer countercurrent flow tests. 


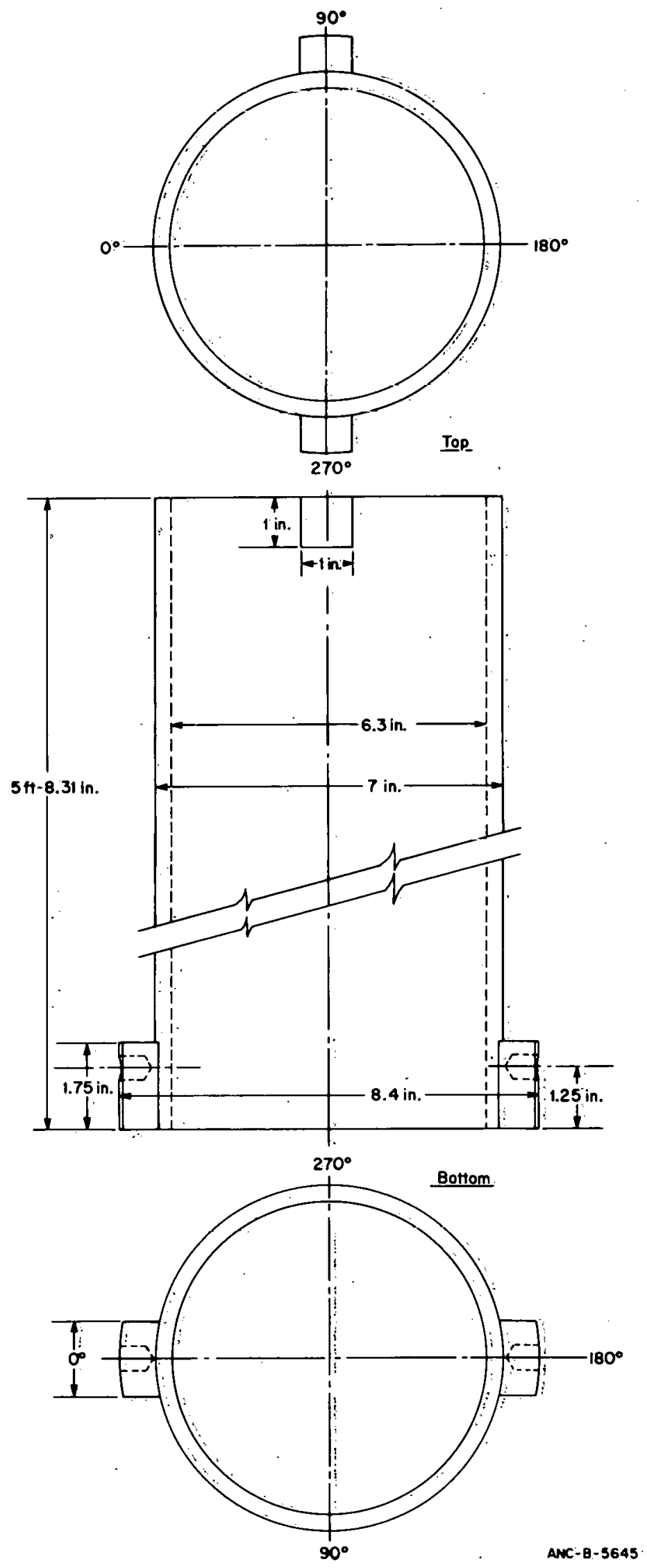

Fig. 6 Filler piece flange configuration for double annulus tests. 
TABLE XIVII

SPECIAL TEST GROUP 4.10 - ONE-DIMENSIONAL INLET

TRANSPARENT VESSEL TEST DATA FOR TESTS 4.10.1 TO 4.10. 7 -- 0.49-IN. DOWNCOMER GAP SUMMARY OF CALCULATEO RESULTS

\begin{tabular}{|c|c|c|c|c|c|c|c|c|c|c|}
\hline $\begin{array}{c}\text { TEST } \\
\text { NO }\end{array}$ & $\begin{array}{l}\text { DOHNCOMER } \\
\text { AIR FLIJW } \\
\text { (LB/SEC) }\end{array}$ & $\begin{array}{l}\text { DOWNCOMER } \\
\text { AIR DENSITY } \\
\text { (LO/FT**3) }\end{array}$ & $\begin{array}{l}\text { LOWER PLENUM } \\
\text { PRESSURE } \\
\text { (PSIA) }\end{array}$ & $\begin{array}{l}\text { CCLO LEG } \\
\text { ALR FLOW } \\
(L B / S E C)\end{array}$ & $\begin{array}{l}\text { DOWNCOMER } \\
\text { WATER FLOH } \\
\text { (LB/SEC) }\end{array}$ & $\begin{array}{l}\text { WATER FLOH } \\
\text { CONTINUITY }\end{array}$ & $\begin{array}{c}\text { J-AIR } \\
\text { DOUNCOMER } \\
\text { (FT/SEC) }\end{array}$ & $\begin{array}{l}\text { J-HATER } \\
\text { OOHNCOMER } \\
\text { (FT/SEC) }\end{array}$ & $(J G *) * * 1 / 2$ & $(J L * 1 * 1 / 2$ \\
\hline $\begin{array}{l}4.10 \cdot 1 \\
4.10 \cdot 2 \\
4.10 \cdot 3 \\
4.10 .4 \\
4.10 .5\end{array}$ & $\begin{array}{l}0.0430 \\
0.0709 \\
0.0709 \\
0.1074 \\
0.1074\end{array}$ & $\begin{array}{l}0.0711 \\
0.0709 \\
0.0709 \\
0.0707 \\
0.0708\end{array}$ & $\begin{array}{l}13.48 \\
13.48 \\
13.48 \\
13.48 \\
13.48\end{array}$ & $\begin{array}{l}0.0 \\
0.0 \\
0.0 \\
0.0 \\
0.0\end{array}$ & $\begin{array}{l}1.958 \\
1.111 \\
1.192 \\
0.692 \\
0.642\end{array}$ & $\begin{array}{l}0.988 \\
1.009 \\
1.353 \\
1.000 \\
1.000\end{array}$ & $\begin{array}{r}9.39 \\
15.52 \\
15.52 \\
23.61 \\
23.57\end{array}$ & $\begin{array}{l}0.487 \\
0.278 \\
0.297 \\
0.172 \\
0.160\end{array}$ & $\begin{array}{l}0.4417 \\
0.5677 \\
0.5677 \\
0.6995 \\
0.6991\end{array}$ & $\begin{array}{l}0.5479 \\
0.4137 \\
0.4274 \\
0.3256 \\
0.3136\end{array}$ \\
\hline $\begin{array}{l}4.10 .6 \\
4.10 .7\end{array}$ & $\begin{array}{l}0.1303 \\
0.1303\end{array}$ & $\begin{array}{l}0.0705 \\
0.0705\end{array}$ & $\begin{array}{l}13.48 \\
13.48\end{array}$ & $\begin{array}{l}0.0 \\
0.0\end{array}$ & $\begin{array}{l}0.300 \\
0.375\end{array}$ & $\begin{array}{l}0.973 \\
0.994\end{array}$ & $\begin{array}{l}28.71 \\
28.71\end{array}$ & $\begin{array}{l}0.075 \\
0.093\end{array}$ & $\begin{array}{l}0.7709 \\
0.7709\end{array}$ & $\begin{array}{l}0.2144 \\
0.2397\end{array}$ \\
\hline
\end{tabular}


TABLE XLVII (Contd.)

SPECIAL TEST GROUP 4.10 - ONE-DIMENSIONAL INLET

EXṔERIMENTAL MEASURMENTS FOR TESTS 4.10.1 TO 4.10. $7-0.49-1$. OOMNCOMER GAP

\begin{tabular}{|c|c|c|c|c|c|c|c|c|c|c|}
\hline $\begin{array}{c}\text { TEST } \\
\text { NO }\end{array}$ & $\begin{array}{c}R M-C H-1 \\
(G \dot{P} M)\end{array}$ & $\begin{array}{c}R M-C H-2 \\
(G P M)\end{array}$ & $\begin{array}{c}F T-C H-1 \\
(G P M)\end{array}$ & $\begin{array}{c}F T-C H-2 \\
(G P M)\end{array}$ & $\begin{array}{c}R M-H G-1 \\
\text { (CFM) }\end{array}$ & $\begin{array}{c}R M-H G-2 \\
(C F M)\end{array}$ & $\begin{array}{c}\text { RH-HG }-3 \\
(C F M)\end{array}$ & $\begin{array}{c}R M-H G-4 \\
(C F M)\end{array}$ & $\begin{array}{c}F T-H G-1 \\
(C F M)\end{array}$ & $\begin{array}{c}F T-H G-2 \\
(C F M)\end{array}$ \\
\hline $\begin{array}{l}4.10 .1 \\
4.10 .2 \\
4.10 .3 \\
4.10 .4 \\
4.10 .5\end{array}$ & $\begin{array}{l}0.0 \\
0.0 \\
0.0 \\
0.0 \\
0.0\end{array}$ & $\begin{array}{l}0.0 \\
0.0 \\
0.0 \\
0.0 \\
0.0\end{array}$ & $\begin{array}{l}0.0 \\
0.0 \\
0.0 \\
0.0 \\
0.0\end{array}$ & $\begin{array}{r}14.3 \\
8.0 \\
6.3 \\
5.0 \\
4.6\end{array}$ & $\begin{array}{l}20.00 \\
33.00 \\
33.00 \\
50.00 \\
50.00\end{array}$ & $\begin{array}{l}0.0 \\
0.0 \\
0.0 \\
0.0 \\
0.0\end{array}$ & $\begin{array}{l}0.0 \\
0.0 \\
0.0 \\
0.0 \\
0.0\end{array}$ & $\begin{array}{l}0.0 \\
0.0 \\
0.0 \\
0.0 \\
0.0\end{array}$ & $\begin{array}{l}0.0 \\
0.0 \\
0.0 \\
0.0 \\
0.0\end{array}$ & $\begin{array}{l}0.0 \\
0.0 \\
0.0 \\
Q .0 \\
0.0\end{array}$ \\
\hline $\begin{array}{l}4.10 .6 \\
4.10 .7\end{array}$ & $\begin{array}{l}0.0 \\
0.0\end{array}$ & $\begin{array}{l}0.0 \\
0.0\end{array}$ & $\begin{array}{l}0.0 \\
0.0\end{array}$ & $\begin{array}{l}2.2 \\
2.7\end{array}$ & $\begin{array}{l}60.00 \\
60.00\end{array}$ & $\begin{array}{l}0.0 \\
0.0\end{array}$ & $\begin{array}{l}0.0 \\
0.0\end{array}$ & $\begin{array}{l}0.0 \\
0.0\end{array}$ & $\begin{array}{l}0.0 \\
0.0\end{array}$ & $\begin{array}{l}0.0 \\
0.0\end{array}$ \\
\hline $\begin{array}{c}\text { TES̀T } \\
\text { NÕ }\end{array}$ & $\begin{array}{l}P-C H-1 \\
(P S I G)\end{array}$ & $\begin{array}{l}P-C H-2 \\
(P S I G)\end{array}$ & $\begin{array}{l}P-H G-1 \\
(P S I G)\end{array}$ & $\begin{array}{l}P-H G-2 \\
(P S I G)\end{array}$ & $\begin{array}{l}P-H G-3 \\
|P S I G|\end{array}$ & $\begin{array}{l}\text { TF-CN-1 } \\
\text { (DEG F) }\end{array}$ & $\begin{array}{l}T F-H G-1 \\
(D E G F)\end{array}$ & $\begin{array}{l}\text { TF-HG-i } \\
\text { (DEG F) }\end{array}$ & $\begin{array}{l}\text { TF-HG-3 } \\
\text { (DEG F) }\end{array}$ & $\begin{array}{l}\text { P-BARO } \\
\text { (PSIAA) }\end{array}$ \\
\hline $\begin{array}{l}4.10 .1 \\
4.10 .2 \\
4.10 .23 \\
4.10 .4 \\
4.10 .5\end{array}$ & $\begin{array}{l}0.0 \\
0.0 \\
0.0 \\
0.0 \\
0.0\end{array}$ & $\begin{array}{l}0.0 \\
0.0 \\
0.0 \\
0.0 \\
0.0\end{array}$ & $\begin{array}{l}0.0 \\
0.0 \\
0.0 \\
0.0 \\
0.0\end{array}$ & $\begin{array}{l}0.0 \\
0.0 \\
0.0 \\
0.0 \\
0.0\end{array}$ & $\begin{array}{l}32.0 \\
32.0 \\
32.0 \\
32.0 \\
32.0\end{array}$ & $\begin{array}{l}0.0 \\
0.0 \\
0.0 \\
0.0 \\
0.0\end{array}$ & $\begin{array}{l}80.0 \\
80.0 \\
80.0 \\
80.0 \\
80.0\end{array}$ & $\begin{array}{l}0.0 \\
0.0 \\
0.0 \\
0.0 \\
0.0\end{array}$ & $\begin{array}{l}81.0 \\
81.0 \\
81.0 \\
81.0 \\
81.0\end{array}$ & $\begin{array}{l}12.5 \\
12.5 \\
12.5 \\
12.5 \\
12.5\end{array}$ \\
\hline $\begin{array}{l}4.10 \cdot 6 \\
4.10 .7\end{array}$ & $\begin{array}{l}0.0 \\
0.00\end{array}$ & $\begin{array}{l}0.0 \\
0.0\end{array}$ & $\begin{array}{l}0.0 \\
0.0\end{array}$ & $\begin{array}{l}0.0 \\
0.0\end{array}$ & $\begin{array}{l}33.0 \\
33.0\end{array}$ & $\begin{array}{l}0.0 \\
0.0\end{array}$ & $\begin{array}{l}80.0 \\
80.0\end{array}$ & $\begin{array}{l}0.0 \\
0.0\end{array}$ & $\begin{array}{l}81.0 \\
81.0\end{array}$ & $\begin{array}{l}12.5 \\
12.5\end{array}$ \\
\hline
\end{tabular}

\begin{tabular}{|c|c|c|c|c|c|c|c|c|c|}
\hline & & $\begin{array}{l}\text { R PLENUM } \\
-\end{array}$ & $\begin{array}{l}\text { FLOW } \\
-\end{array}$ & & YPASS F & 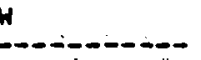 & LOWER & & \\
\hline $\begin{array}{c}\text { TEST } \\
\text { NÓ }\end{array}$ & $\begin{array}{l}\text { INITIAL } \\
\text { WEIGHT } \\
\text { (LB) }\end{array}$ & $\begin{array}{l}\text { FINAL } \\
\text { WEIGHT } \\
\text { (LB) }\end{array}$ & $\begin{array}{l}\text { TIME OF } \\
\text { COLLECTION } \\
\text { ( SEC) }\end{array}$ & $\begin{array}{l}\text { INITIAL } \\
\text { WEIGHT } \\
\text { (LB) }\end{array}$ & $\begin{array}{l}\text { FINAL } \\
\text { WEIGHT } \\
\text { (L B) }\end{array}$ & $\begin{array}{l}\text { TIME OF } \\
\text { COLLECTION } \\
\text { ISEC }\end{array}$ & $\begin{array}{l}\text { PLENUM } \\
\text { LEVEL } \\
\text { (INI) }\end{array}$ & $\begin{array}{l}p-i p-1 \\
\text { (PSirj) }\end{array}$ & $\begin{array}{l}T F-L P-1 \\
\text { (DEG F) }\end{array}$ \\
\hline $\begin{array}{l}4.1001 \\
4.10 .52 \\
4.10 .3 \\
4.10 .54 \\
4.1005\end{array}$ & $\begin{array}{l}326.0 \\
443.5 \\
510.0 \\
581.0 \\
272.0\end{array}$ & $\begin{array}{l}443.5 \\
510.5 \\
581.5 \\
622.5 \\
310.5\end{array}$ & $\begin{array}{l}60.0 \\
60.0 \\
60.0 \\
60.0 \\
60.0\end{array}$ & $\begin{array}{l}0.0 \\
0.0 \\
0.0 \\
0.0 \\
0.0\end{array}$ & $\begin{array}{l}0.0 \\
0.0 \\
0.0 \\
0.0 \\
0.0 \\
0.0\end{array}$ & $\begin{array}{l}0.0 \\
0.0 \\
0.0 \\
0.0 \\
0.0\end{array}$ & $\begin{array}{l}12.0 \\
12.0 \\
12.0 \\
12.0 \\
12.0\end{array}$ & $\begin{array}{l}1.0 \\
1.0 \\
1.0 \\
1.0 \\
1.0\end{array}$ & $\begin{array}{l}52.0 \\
53: 0 \\
53.0 \\
55.0 \\
54: 0\end{array}$ \\
\hline
\end{tabular}




\section{TABLE XIVIII}

SPECIAL TEST GROUP 5.2 - INCREASING AIR FLOW

TRANSPARENT VESSEL TEST DATA FOR TESTS 5.2 .1 TO $5.2 .13-0.53-$ IN. DOHNCOMER GAP SUMMARY OF CALCULATEO RESULTS

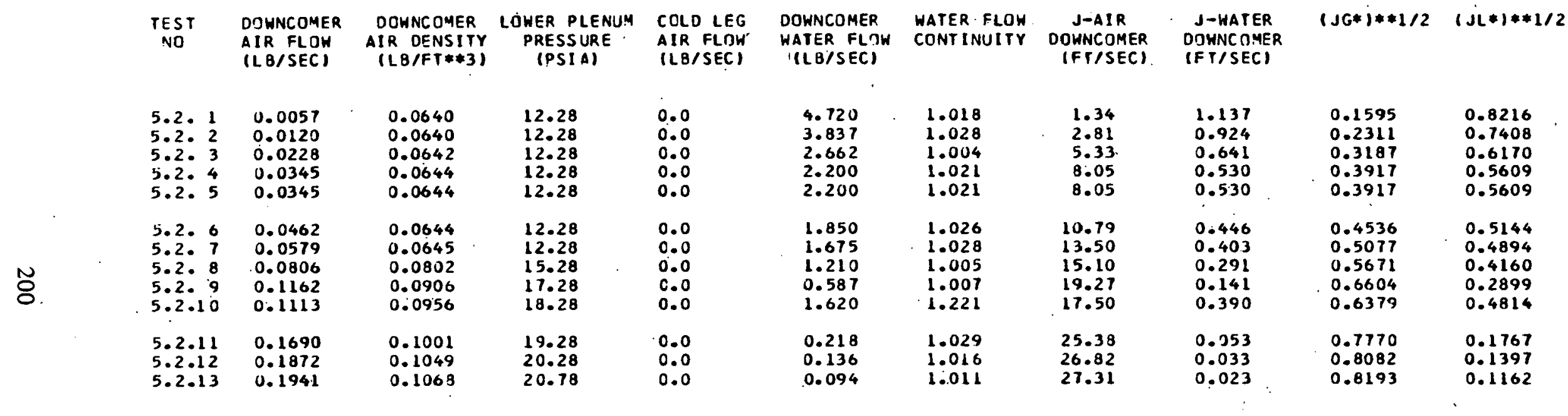


TABLE XLVIII (Contd.)

SPECIAL TEST GROUP 5.2 - INCREASING AIR FLOW

EXPERIMENTAL MEASURMENTS FOR TESTS 5.2 .1 TO 5.2.13 - 0.53-IN. DOWNCOMER GAP

\begin{tabular}{|c|c|c|c|c|c|c|c|c|c|c|c|}
\hline & $\begin{array}{c}\text { TEST } \\
\text { NO }\end{array}$ & $\begin{array}{c}R M-C H-1 \\
(G P M)\end{array}$ & $\begin{array}{c}R M-C H-2 \\
(G P M)\end{array}$ & $\begin{array}{c}F T-C W-1 \\
(G P M)\end{array}$ & $\begin{array}{c}F T-C H-2 \\
(G P M \mid\end{array}$ & $\begin{array}{c}R M-H G-1 \\
(C F M)\end{array}$ & $\begin{array}{c}\text { RM-HG-2 } \\
\text { (CFM) }\end{array}$ & $\begin{array}{c}R M-H G-3 \\
(C F M)\end{array}$ & $\begin{array}{c}\text { RM-HG-4 } \\
\text { (CFM) }\end{array}$ & $\begin{array}{c}F T-H G-1 \\
(C F M)\end{array}$ & $\begin{array}{c}F T-H G-2 \\
\text { (CFM) }\end{array}$ \\
\hline & $\begin{array}{ll}5.2 \cdot 1 & 1 \\
5.2 \cdot & 2 \\
5.2 \cdot & 3 \\
5.2 \cdot & 4 \\
5.2 \cdot & 5\end{array}$ & $\begin{array}{l}32.0 \\
32.0 \\
32.0 \\
32.0 \\
32.0\end{array}$ & $\begin{array}{l}12.5 \\
12.5 \\
12.5 \\
12.5 \\
12.5\end{array}$ & $\begin{array}{l}0.0 \\
0.0 \\
0.0 \\
0.0 \\
0.0\end{array}$ & $\begin{array}{l}0.0 \\
0.0 \\
0.0 \\
0.0 \\
0.0\end{array}$ & $\begin{array}{c}0.0 \\
0.0 \\
0.0 \\
30.00 \\
30.00\end{array}$ & $\begin{array}{l}0.0 \\
0.0 \\
0.0 \\
0.0 \\
0.0\end{array}$ & $\begin{array}{c}5.00 \\
10.50 \\
20.00 \\
0.0 \\
0.0\end{array}$ & $\begin{array}{l}0.0 \\
0.0 \\
0.0 \\
0.0 \\
0.0\end{array}$ & $\begin{array}{l}0.0 \\
0.0 \\
0.0 \\
0.0 \\
0.0\end{array}$ & $\begin{array}{l}0.0 \\
0.0 \\
0.0 \\
0.0 \\
0.0\end{array}$ \\
\hline & $\begin{array}{l}5.2 \cdot 6 \\
5.2 \cdot 7 \\
5.2 \cdot 8 \\
5.2 .9 \\
5.2 .10\end{array}$ & $\begin{array}{l}32.0 \\
32.0 \\
32.0 \\
32.0 \\
32.0\end{array}$ & $\begin{array}{l}12.5 \\
12.5 \\
12.5 \\
12.5 \\
12.5\end{array}$ & $\begin{array}{l}0.0 \\
0.0 \\
0.0 \\
0.0 \\
0.0\end{array}$ & $\begin{array}{l}0.0 \\
0.0 \\
0.0 \\
0.0 \\
0.0\end{array}$ & $\begin{array}{l}40.00 \\
50.00 \\
59.00 \\
80.00 \\
90.00\end{array}$ & $\begin{array}{l}0.0 \\
0.0 \\
0.0 \\
0.0 \\
0.0\end{array}$ & $\begin{array}{l}0.0 \\
0.0 \\
0.0 \\
0.0 \\
0.0\end{array}$ & $\begin{array}{l}0.0 \\
0.0 \\
0.0 \\
0.0 \\
0.0\end{array}$ & $\begin{array}{l}0.0 \\
0.0 \\
0.0 \\
0.0 \\
0.0\end{array}$ & $\begin{array}{l}0.0 \\
0.0 \\
0.0 \\
0.0 \\
0.0\end{array}$ \\
\hline$\stackrel{N}{0}$ & $\begin{array}{l}5.2 .11 \\
5.2 .12 \\
5.2 .13\end{array}$ & $\begin{array}{l}32.0 \\
32.0 \\
32.0\end{array}$ & $\begin{array}{l}12.5 \\
12.5 \\
12.5\end{array}$ & $\begin{array}{l}0.0 \\
0.0 \\
0.0\end{array}$ & $\begin{array}{l}0.0 \\
0.0 \\
0.0\end{array}$ & $\begin{array}{l}90.00 \\
90.00 \\
90.00\end{array}$ & $\begin{array}{l}20.00 \\
30.00 \\
35.00\end{array}$ & $\begin{array}{l}0.0 \\
0.0 \\
0.0\end{array}$ & $\begin{array}{l}0.0 \\
0.0 \\
0.0\end{array}$ & $\begin{array}{l}0.0 \\
0.0 \\
0.0\end{array}$ & $\begin{array}{l}0.0 \\
0.0 \\
0.0\end{array}$ \\
\hline & $\begin{array}{c}\text { TEST } \\
\text { NO }\end{array}$ & $\begin{array}{l}P-C W-1 \\
\text { (PSIG) }\end{array}$ & $\begin{array}{l}P-C H-2 \\
\text { (PSIG) }\end{array}$ & $\begin{array}{l}P-H G-1 \\
\text { (PSIG) }\end{array}$ & $\begin{array}{l}\text { P-HG-2 } \\
\text { (PSIG) }\end{array}$ & $\begin{array}{l}\text { P-HG-3 } \\
\text { (PS } \mid G \text { ) }\end{array}$ & $\begin{array}{l}\text { TF-CH-l } \\
\text { (DEG F) }\end{array}$ & $\begin{array}{l}\text { TF-HG-1 } \\
\text { (DEG F) }\end{array}$ & $\begin{array}{l}\text { TF-HG-2 } \\
(D E G \text { F) }\end{array}$ & $\begin{array}{l}\text { TF-HG-3 } \\
\text { (DEG FI }\end{array}$ & $\begin{array}{l}\text { P-BARO } \\
\text { (PSIA) }\end{array}$ \\
\hline & $\begin{array}{l}5.2 \cdot 1 \\
5.2 \cdot 2 \\
5.2 \cdot 3 \\
5.2 \cdot 4 \\
5.2 \cdot 5\end{array}$ & $\begin{array}{l}0.0 \\
0.0 \\
0.0 \\
0.0 \\
0.0\end{array}$ & $\begin{array}{l}0.0 \\
0.0 \\
0.0 \\
0.0 \\
0.0\end{array}$ & $\begin{array}{l}0.0 \\
0.0 \\
0.0 \\
0.0 \\
0.0\end{array}$ & $\begin{array}{l}0.0 \\
0.0 \\
0.0 \\
0.0 \\
0.0\end{array}$ & $\begin{array}{l}0.0 \\
0.0 \\
0.0 \\
0.0 \\
0.0\end{array}$ & $\begin{array}{l}58.0 \\
58.0 \\
57.0 \\
55.0 \\
55.0\end{array}$ & $\begin{array}{l}71.0 \\
71.0 \\
71.0 \\
71.0 \\
71.0\end{array}$ & $\begin{array}{l}0.0 \\
0.0 \\
0.0 \\
0.0 \\
0.0\end{array}$ & $\begin{array}{l}0.0 \\
0.0 \\
0.0 \\
0.0 \\
0.0\end{array}$ & $\begin{array}{l}12.3 \\
12.3 \\
12.3 \\
12.3 \\
12.3\end{array}$ \\
\hline & $\begin{array}{l}5.2 \cdot 6 \\
5.2 \cdot 7 \\
5.2 .8 \\
5.2 .9 \\
5.2 .10\end{array}$ & $\begin{array}{l}0.0 \\
0.0 \\
0.0 \\
0.0 \\
0.0\end{array}$ & $\begin{array}{l}0.0 \\
0.0 \\
4.8 \\
6.5 \\
7.0\end{array}$ & $\begin{array}{l}0.0 \\
0.0 \\
5.0 \\
7.0 \\
1.0\end{array}$ & $\begin{array}{l}0.0 \\
0.0 \\
0.0 \\
0.0 \\
0.0\end{array}$ & $\begin{array}{l}0.0 \\
0.0 \\
0.0 \\
0.0 \\
0.0\end{array}$ & $\begin{array}{l}55.0 \\
55.0 \\
54.0 \\
55.0 \\
54.0\end{array}$ & $\begin{array}{r}72.0 \\
77.0 \\
83.0 \\
92.0 \\
100.0\end{array}$ & $\begin{array}{l}0.0 \\
0.0 \\
0.0 \\
0.0 \\
0.0\end{array}$ & $\begin{array}{l}0.0 \\
0.0 \\
0.0 \\
0.0 \\
0.0\end{array}$ & $\begin{array}{l}12.3 \\
12.3 \\
12.3 \\
12.3 \\
12.3\end{array}$ \\
\hline & $\begin{array}{l}5.2 .11 \\
5.2 .12 \\
5.2 .13\end{array}$ & $\begin{array}{l}0.0 \\
0.0 \\
0.0\end{array}$ & $\begin{array}{r}9.0 \\
10.0 \\
10.8\end{array}$ & $\begin{array}{l}10.0 \\
11.0 \\
11.0\end{array}$ & $\begin{array}{l}0.0 \\
0.0 \\
0.0\end{array}$ & $\begin{array}{l}0.0 \\
0.0 \\
0.0\end{array}$ & $\begin{array}{l}54.0 \\
48.0 \\
54.0\end{array}$ & $\begin{array}{l}108.5 \\
114.0 \\
119.0\end{array}$ & $\begin{array}{l}0.0 \\
0.0 \\
0.0\end{array}$ & $\begin{array}{l}0.0 \\
0.0 \\
0.0\end{array}$ & $\begin{array}{l}12.3 \\
12.3 \\
12.3\end{array}$ \\
\hline
\end{tabular}


TABLE XLVIII (Contd.)

SPECIAL TEST GROUP 5.2 - INCREASING AIR FLOW

EXPERIMENTAL MEASURMENTS FOR TESTS 5.2 .1 TO $5.2 .13--0.53-$ IN. DOHNCOMER GAP

\begin{tabular}{|c|c|c|c|c|c|c|c|c|c|c|}
\hline & \multirow[b]{2}{*}{$\begin{array}{l}\text { TEST } \\
\text { NO }\end{array}$} & \multicolumn{2}{|c|}{ LOWER PLENUM } & \multicolumn{2}{|l|}{ FLOH } & \multicolumn{2}{|c|}{ BYPASS FLOW } & \multirow[b]{2}{*}{$\begin{array}{l}\text { LOWER } \\
\text { PLENUH } \\
\text { LEVEL } \\
\text { (IN) }\end{array}$} & \multirow[b]{2}{*}{$\begin{array}{l}P-L P-1 \\
(P S I G)\end{array}$} & \multirow[b]{2}{*}{$\begin{array}{l}\text { TF- LP- } 1 \\
\text { (CEG FI }\end{array}$} \\
\hline & & $\begin{array}{l}\text { INITIAL } \\
\text { WEIGHT } \\
\text { ILBI }\end{array}$ & $\begin{array}{l}\text { FINAL } \\
\text { HEIGHT } \\
\text { (LB) }\end{array}$ & $\begin{array}{l}\text { TIME OF } \\
\text { COLLECTION } \\
\text { ISEC) }\end{array}$ & $\begin{array}{l}\text { INITIAL } \\
\text { WEIGHT } \\
\text { (LB) }\end{array}$ & $\begin{array}{l}\text { FINAL } \\
\text { VIEIGHT } \\
\text { (L. B] }\end{array}$ & $\begin{array}{l}\text { TIME OF } \\
\text { COLLECTION } \\
\text { ISEC) }\end{array}$ & & & \\
\hline \multirow{3}{*}{ N } & $\begin{array}{ll}5.2 \cdot 1 \\
5.2 \cdot 2 \\
5.2 \cdot 3 \\
5.2 \cdot 4 \\
5.2 \cdot 5\end{array}$ & $\begin{array}{l}133.0 \\
228.0 \\
304.8 \\
358.0 \\
358.0\end{array}$ & $\begin{array}{l}251.0 \\
304.8 \\
358.0 \\
402.0 \\
402.0\end{array}$ & $\begin{array}{l}25.0 \\
20.0 \\
20.0 \\
20.0 \\
20.0\end{array}$ & $\begin{array}{r}87.0 \\
126.5 \\
177.0 \\
248.0 \\
248.0\end{array}$ & $\begin{array}{l}126.5 \\
177.0 \\
248.0 \\
351.0 \\
351.0\end{array}$ & $\begin{array}{l}25.0 \\
20.0 \\
20.0 \\
25.0 \\
25.0\end{array}$ & $\begin{array}{l}12.0 \\
12.0 \\
12.0 \\
12.0 \\
12.0\end{array}$ & $\begin{array}{l}0.0 \\
0.0 \\
0.0 \\
0.0 \\
0.0\end{array}$ & $\begin{array}{l}58.0 \\
58.0 \\
56.0 \\
55.0 \\
55.0\end{array}$ \\
\hline & $\begin{array}{l}5.2 \cdot 6 \\
5.2 \cdot 7 \\
5.2 .8 \\
5.2 .9 \\
5.2 .10\end{array}$ & $\begin{array}{l}356.0 \\
324.5 \\
358.0 \\
388.3 \\
400.0\end{array}$ & $\begin{array}{l}393.0 \\
358.0 \\
388.3 \\
400.0 \\
440.5\end{array}$ & $\begin{array}{l}20.0 \\
20.0 \\
25.0 \\
20.0 \\
25.0\end{array}$ & $\begin{array}{l}351.0 \\
441.0 \\
534.8 \\
685.0 \\
671.0\end{array}$ & $\begin{array}{l}441.0 \\
534.8 \\
685.0 \\
826.0 \\
849.0\end{array}$ & $\begin{array}{l}20.0 \\
20.0 \\
30.0 \\
25.0 \\
30.0\end{array}$ & $\begin{array}{l}12.0 \\
12.0 \\
12.0 \\
12.0 \\
12.0\end{array}$ & $\begin{array}{l}0.0 \\
0.0 \\
3.0 \\
5.0 \\
6.0\end{array}$ & $\begin{array}{l}55.0 \\
54.0 \\
54.0 \\
55.0 \\
56.0\end{array}$ \\
\hline & $\begin{array}{l}5.2 .11 \\
5.2 .12 \\
5.2 .13\end{array}$ & $\begin{array}{l}410.5 \\
371.5 \\
379.0\end{array}$ & $\begin{array}{l}422.5 \\
379.0 \\
383.3\end{array}$ & $\begin{array}{l}55.0 \\
55.0 \\
45.0\end{array}$ & $\begin{array}{l}678.0 \\
667.5 \\
623.0\end{array}$ & $\begin{array}{l}801.0 \\
790.5 \\
777.0\end{array}$ & $\begin{array}{l}20.0 \\
20.0 \\
25.0\end{array}$ & $\begin{array}{l}12.0 \\
12.0 \\
12.0\end{array}$ & $\begin{array}{l}7.0 \\
8.0 \\
9.5\end{array}$ & $\begin{array}{l}60.0 \\
62.0 \\
65.0\end{array}$ \\
\hline
\end{tabular}


TABLE XLIX

SPECIAL TEST GROUP 5.3 - DECREASING AIR FLOW

TRANSPARENT VESSEL TEST DATA FOR TESTS 5.3 .1 TO $5.3 .6--0.53$-IN. DOWNCOMER GAP SUMMARY OF CALCULATED RESULTS

\begin{tabular}{|c|c|c|c|c|c|c|c|c|c|c|}
\hline $\begin{array}{l}\text { TES:T } \\
\text { ND }\end{array}$ & $\begin{array}{l}\text { DOHNCOMER } \\
\text { AIR FLOW } \\
\text { (LB/SEC) }\end{array}$ & $\begin{array}{l}\text { DOWNCOMER } \\
\text { AIR DENSITY } \\
\text { (LB/FT**3) }\end{array}$ & $\begin{array}{l}\text { LOWER PLENUM } \\
\text { PRESS URE } \\
\text { (PSIA) }\end{array}$ & $\begin{array}{l}\text { COLD LEG } \\
\text { AIR FLOH } \\
(L B / S E C)\end{array}$ & $\begin{array}{l}\text { DOWNCOMER } \\
\text { WATER FLOH } \\
\text { (LB/SEC) }\end{array}$ & $\begin{array}{l}\text { WATER FLOW } \\
\text { CONTINJITY }\end{array}$ & $\begin{array}{c}\text { J-AlR } \\
\text { DOHNCDMER } \\
\text { (FT/SEC) }\end{array}$ & $\begin{array}{l}\text { J-HATER } \\
\text { DOWNCGMER } \\
\text { (FT/SEC) }\end{array}$ & $(J G *) * 1 / 2$ & $(J L *) * 1 / 2$ \\
\hline $\begin{array}{l}5.3 \cdot 1 \\
5.3 \cdot 2 \\
5.3 \cdot 3 \\
5.3 \cdot 4 \\
5.3 \cdot 5\end{array}$ & $\begin{array}{l}0.1922 \\
0.1815 \\
0.1633 \\
0.1285 \\
0.0772\end{array}$ & $\begin{array}{l}0.1120 \\
0.1103 \\
0.1090 \\
0.1005 \\
0.0855\end{array}$ & $\begin{array}{l}22.28 \\
21.78 \\
21.28 \\
19.28 \\
16.28\end{array}$ & $\begin{array}{l}0.0 \\
0.0 \\
0.0 \\
0.0 \\
0.0\end{array}$ & $\begin{array}{l}0.087 \\
0.117 \\
0.167 \\
0.380 \\
1.400\end{array}$ & $\begin{array}{l}1.004 \\
1.017 \\
1.001 \\
0.999 \\
1.014\end{array}$ & $\begin{array}{l}25.80 \\
24.73 \\
22.52 \\
19.22 \\
13.57\end{array}$ & $\begin{array}{l}0.021 \\
0.028 \\
0.040 \\
0.092 \\
0.337\end{array}$ & $\begin{array}{l}0.8057 \\
0.7858 \\
0.7477 \\
0.6769 \\
0.5461\end{array}$ & $\begin{array}{l}0.1119 \\
0.1292 \\
0.1544 \\
0.2331 \\
0.4475\end{array}$ \\
\hline 5.3 .6 & 0.0709 & 0.0801 & 15.28 & 0.0 & 1.517 & 1.008 & 13.30 & 0.365 & 0.5320 & 0.4657 \\
\hline
\end{tabular}


TABLE XLIX (Contd.)

SPECIAL TEST GROUP 5.3 - DECREASING AIR FLOW

EXPERIMENTAL MEASURMENTS FOR TESTS 5.3 .1 TO 5.3.6 - $0.53-$ IN. DONNCOMER GAP

\begin{tabular}{|c|c|c|c|c|c|c|c|c|c|c|}
\hline $\begin{array}{l}\text { TEST } \\
\text { NO }\end{array}$ & $\begin{array}{c}R M-C H-1 \\
(G P M)\end{array}$ & $\begin{array}{c}R M-C H-2 \\
(G P M)\end{array}$ & $\begin{array}{c}F T-C H-1 \\
(G P M)\end{array}$ & $\begin{array}{c}F T-C H-2 \\
(G P M)\end{array}$ & $\begin{array}{c}R M-H G-1 \\
(C F M U)\end{array}$ & $\begin{array}{c}\text { RM-HG-2 } \\
\text { (CFM) }\end{array}$ & $\begin{array}{c}\text { RM-HG-3 } \\
(C F M)\end{array}$ & $\begin{array}{c}R M-H G-4 \\
\text { (CFM) }\end{array}$ & $\begin{array}{c}F T-H G-1 \\
\text { (CFM) }\end{array}$ & $\begin{array}{c}F T-H G-2 \\
(C F M)^{2}\end{array}$ \\
\hline $\begin{array}{l}5.3 .1 \\
5.3 .2 \\
5.3 .3 \\
5.3 .4 \\
5.3 .5\end{array}$ & $\begin{array}{l}32.0 \\
32.0 \\
32.0 \\
32.0 \\
32.0\end{array}$ & $\begin{array}{l}12.5 \\
12.5 \\
12.5 \\
12.5 \\
12.5\end{array}$ & $\begin{array}{l}0.0 \\
0.0 \\
0.0 \\
0.0 \\
0.0\end{array}$ & $\begin{array}{l}0.0 \\
0.0 \\
0.0 \\
0.0 \\
0.0\end{array}$ & $\begin{array}{l}90.00 \\
90.00 \\
90.00 \\
90.00 \\
60.00\end{array}$ & $\begin{array}{c}35.00 \\
30.00 \\
20.00 \\
0.0 \\
0.0\end{array}$ & $\begin{array}{l}0.0 \\
0.0 \\
0.0 \\
0.0 \\
0.0\end{array}$ & $\begin{array}{l}0.0 \\
0.0 \\
0.0 \\
0.0 \\
0.0\end{array}$ & $\begin{array}{l}0.0 \\
0.0 \\
0.0 \\
0.0 \\
0.0\end{array}$ & $\begin{array}{l}0.0 \\
0.0 \\
0.0 \\
0.0 \\
0.0\end{array}$ \\
\hline $5 \cdot 3 \cdot 6$ & 32.0 & 12.5 & 0.0 & 0.0 & 55.00 & 0.0 & 0.0 & 0.0 & 0.0 & 0.0 \\
\hline $\begin{array}{c}\text { TEST } \\
\text { NO }\end{array}$ & $\begin{array}{l}P-C H-1 \\
\text { (PSIG) }\end{array}$ & $\begin{array}{l}P-C W-2 \\
\text { (PSIG) }\end{array}$ & $\begin{array}{l}P-H G-1 \\
\text { (PSIG) }\end{array}$ & $\begin{array}{l}\text { P-HG-2 } \\
\text { (PSIG) }\end{array}$ & $\begin{array}{l}\text { P-HG-3 } \\
\text { (PSIG) }\end{array}$ & $\begin{array}{l}\text { TF-CH-1 } \\
\text { (DEG F) }\end{array}$ & $\begin{array}{l}\text { TF-HG-I } \\
\text { (DEG FI) }\end{array}$ & $\begin{array}{l}\text { TF-HG-2 } \\
(D E G \text { F) }\end{array}$ & $\begin{array}{l}\text { TF-HG-3 } \\
\text { (DEG F) }\end{array}$ & $\begin{array}{l}\text { P-BARO } \\
\text { (PSIA) }\end{array}$ \\
\hline $\begin{array}{l}5.3 .1 \\
5.3 .2 \\
5.3 .3 \\
5.3 .4 \\
5.3 .5\end{array}$ & $\begin{array}{l}0.0 \\
.0 .0 \\
0.0 \\
0.0 \\
0.0\end{array}$ & $\begin{array}{r}10.5 \\
10.0 \\
3.0 \\
7.0 \\
4.5\end{array}$ & $\begin{array}{r}11.0 \\
10.0 \\
9.0 \\
7.0 \\
4.0\end{array}$ & $\begin{array}{l}0.0 \\
0.0 \\
0.0 \\
0.0 \\
0.0\end{array}$ & $\begin{array}{l}0.0 \\
0.0 \\
0.0 \\
0.0 \\
0.0\end{array}$ & $\begin{array}{l}54.0 \\
54.0 \\
54.0 \\
54.0 \\
54.0\end{array}$ & $\begin{array}{l}130.5 \\
128.5 \\
126.0 \\
124.5 \\
120.0\end{array}$ & $\begin{array}{l}0.0 \\
0.0 \\
0.0 \\
0.0 \\
0.0\end{array}$ & $\begin{array}{l}0.0 \\
0.0 \\
0.0 \\
0.0 \\
0.0\end{array}$ & $\begin{array}{l}12.3 \\
12.3 \\
12.3 \\
12.3 \\
12.3\end{array}$ \\
\hline 5.3 .6 & 0.0 & 4.0 & 4.0 & 0.0 & 0.0 & 54.0 & 113.5 & 0.0 & 0.0 & 12.3 \\
\hline
\end{tabular}

\begin{tabular}{|c|c|c|c|c|c|c|c|c|c|}
\hline \multirow[b]{2}{*}{$\begin{array}{c}\text { TEST } \\
\text { NO }\end{array}$} & \multicolumn{2}{|r|}{ LOHER PLENUY } & FLOW & \multicolumn{3}{|c|}{ BYPASS FLOH } & \multirow{2}{*}{$\begin{array}{l}\text { LOWER } \\
\text { PLENIIM } \\
\text { LEVEL } \\
\text { IIN) }\end{array}$} & \multirow[b]{2}{*}{$\begin{array}{l}P-L P-1 \\
\text { (PSIG) }\end{array}$} & \multirow[b]{2}{*}{$\begin{array}{l}\text { TF-LP-1 } \\
\text { (DEG F) }\end{array}$} \\
\hline & $\begin{array}{l}\text { INI TI AL } \\
\text { WE I GHT } \\
\text { (LB) }\end{array}$ & $\begin{array}{l}\text { FINAL } \\
\text { WEIGHT } \\
\text { (LB) }\end{array}$ & $\begin{array}{l}\text { TIME OF } \\
\text { COLLECTICN } \\
\text { (SEC) }\end{array}$ & $\begin{array}{l}\text { INITIAL } \\
\text { WE IGHT } \\
\text { (LB) }\end{array}$ & $\begin{array}{l}\text { FINAL } \\
\text { WEIGHT } \\
(L B)\end{array}$ & $\begin{array}{l}\text { TIME OF } \\
\text { COLLECTION } \\
\text { (SEC) }\end{array}$ & & & \\
\hline $\begin{array}{l}5 \cdot 3 \cdot 1 \\
5 \cdot 3 \cdot 2 \\
5 \cdot 3 \cdot 3 \\
5 \cdot 3 \cdot 4 \\
5 \cdot 3 \cdot 5\end{array}$ & $\begin{array}{l}133.8 \\
139.0 \\
146.0 \\
156.0 \\
175.0\end{array}$ & $\begin{array}{l}139.0 \\
146.0 \\
156.0 \\
175.0 \\
217.0\end{array}$ & $\begin{array}{l}60.0 \\
60.0 \\
60.0 \\
50.0 \\
30.0\end{array}$ & $\begin{array}{l}102.0 \\
224.5 \\
348.0 \\
468.5 \\
584.5\end{array}$ & $\begin{array}{l}224.5 \\
348.0 \\
468.5 \\
584.5 \\
682.0\end{array}$ & $\begin{array}{l}20.0 . \\
20.0 \\
20.0 \\
20.0 \\
20.0\end{array}$ & $\begin{array}{l}12.0 \\
12.0 \\
12.0 \\
12.0 \\
12.0\end{array}$ & $\begin{array}{r}10.0 \\
9.5 \\
9.0 \\
7.0 \\
4.0\end{array}$ & $\begin{array}{l}77.0 \\
73.0 \\
67.0 \\
58.0 \\
54.0\end{array}$ \\
\hline
\end{tabular}




\section{TABLE L}

SPECIAL TEST GROUP 7.3 - DOUBLE ANNULUS

TRANSPARENT VESSEL TEST DATA FOR TESTS 7.3 .1 TO $7.3 .21-1.23-1 \mathrm{~N}$. DOHNCOMER GAP SUMMARY UF CALCULATED RESULTS

\begin{tabular}{|c|c|c|c|c|c|c|c|c|c|c|}
\hline $\begin{array}{l}\text { TEST } \\
\text { NO }\end{array}$ & $\begin{array}{l}\text { OJWNCOMER } \\
\text { AIR FLOW } \\
\text { (LB/SEC) }\end{array}$ & $\begin{array}{l}\text { DOWNCOMER } \\
\text { AIR DENS ITY } \\
\text { (LB/FT } \$ 3)\end{array}$ & $\begin{array}{c}\text { LOHER PLENUM } \\
\text { PRESSUPE } \\
\text { (PSIA) }\end{array}$ & $\begin{array}{l}\text { COLO LEG } \\
\text { AIF FLOW } \\
(L B / S E C)\end{array}$ & $\begin{array}{l}\text { DOWNCOMER } \\
\text { WATER. FLUW } \\
\text { (LB/SEC) }\end{array}$ & $\begin{array}{l}\text { WATER FLOW } \\
\text { CDNTINUITY }\end{array}$ & $\begin{array}{c}\text { J-AIR } \\
\text { DOWNCOMER } \\
\text { (FT/SEC) }\end{array}$ & $\begin{array}{l}\text { J-MATER } \\
\text { ODWNCOMER } \\
\text { IFT/SECI }\end{array}$ & $(J G *) * * 1 / 2$ & $(J L *) * 1 / 2$ \\
\hline $\begin{array}{l}7.3 \cdot 1 \\
7 \cdot 3 \cdot 2 \\
7.3 \cdot 3 \\
7.3 \cdot 4 \\
7.3 .5\end{array}$ & $\begin{array}{l}0.5590 \\
0.5325 . \\
0.4059 \\
0.3999 \\
0.3052\end{array}$ & $\begin{array}{l}0.1812 \\
0.1008 \\
0.1910 \\
0.1616 \\
0.1944\end{array}$ & $\begin{array}{l}34.45 \\
30.45 \\
35.45 \\
30.45 \\
36.45\end{array}$ & $\begin{array}{l}0.0 \\
0.0 \\
0.0 \\
0.0 \\
0.0\end{array}$ & $\begin{array}{l}1.533 \\
1.533 \\
2.500 \\
2.150 \\
3.725\end{array}$ & $\begin{array}{l}1.015 \\
1.008 \\
0.989 \\
1.010 \\
1.004\end{array}$ & $\begin{array}{r}16.77 \\
18.00 \\
11.55 \\
13.45 \\
8.53\end{array}$ & $\begin{array}{l}0.134 \\
0.134 \\
0.218 \\
0.197 \\
0.325\end{array}$ & $\begin{array}{l}0.5935 \\
0.5968 \\
0.4991 \\
0.5165 \\
0.4309\end{array}$ & $\begin{array}{l}0.2282 \\
0.2282 \\
0.2914 \\
0.2702 \\
0.3557\end{array}$ \\
\hline $\begin{array}{l}7.3 .6 \\
7.3 .7 \\
7.3 .88 \\
7.3 .5 \\
7.3 .10\end{array}$ & $\begin{array}{l}0.3072 \\
0.4085 \\
0.3041 \\
0.4111 \\
0.6209\end{array}$ & $\begin{array}{l}0.1720 \\
0.1790 \\
0.1726 \\
0.1355 \\
0.1846\end{array}$ & $\begin{array}{l}32.45 \\
33.70 \\
32.45 \\
34.98 \\
34.98\end{array}$ & $\begin{array}{l}0.0 \\
c .0 \\
c .0 \\
c .0 \\
c .0\end{array}$ & $\begin{array}{l}3.333 \\
2.067 \\
3.283 \\
2.125 \\
1.142\end{array}$ & $\begin{array}{l}1.111 \\
0.928 \\
0.995 \\
0.997 \\
1.038\end{array}$ & $\begin{array}{r}9.68 \\
12.40 \\
9.53 \\
12.63 \\
18.28\end{array}$ & $\begin{array}{l}0.290 \\
0.180 \\
0.286 \\
0.195 \\
0.099\end{array}$ & $\begin{array}{l}0.4454 \\
0.5089 \\
0.4431 \\
0.5182 \\
0.6226\end{array}$ & $\begin{array}{l}0.3364 \\
0.2649 \\
0.3339 \\
0.2686 \\
0.1969\end{array}$ \\
\hline $\begin{array}{l}7.3 .11 \\
7.3 .12 \\
7.3 .13 \\
7.3 .14 \\
7.3 .15\end{array}$ & $\begin{array}{l}0.6243 \\
0.2165 \\
0.1520 \\
0.1134 \\
0.0857\end{array}$ & $\begin{array}{l}0.1004 \\
0.1736 \\
0.1590 \\
j .1330 \\
J .1070\end{array}$ & $\begin{array}{l}31.48 \\
32.48 \\
29.69 \\
24.98 \\
19.98\end{array}$ & $\begin{array}{l}0.0 \\
c .0 \\
0.0 \\
c .0 \\
0.0\end{array}$ & $\begin{array}{r}0.829 \\
5.978 \\
8.111 \\
9.371 \\
11.700\end{array}$ & $\begin{array}{l}1.021 \\
1.022 \\
1.011 \\
1.002 \\
0.915\end{array}$ & $\begin{array}{r}21.16 \\
6.78 \\
5.2 .15 \\
4.61 \\
4.35\end{array}$ & $\begin{array}{l}0.072 \\
0.521 \\
0.707 \\
0.816 \\
1.019\end{array}$ & $\begin{array}{l}0.6466 \\
0.3733 \\
0.3198 \\
0.2883 \\
0.2650\end{array}$ & $\begin{array}{l}0.1677 \\
0.4505 \\
0.5248 \\
0.5640 \\
0.6301\end{array}$ \\
\hline $\begin{array}{l}7.3 .16 \\
7.3 .17 \\
7.3 .18 \\
7.3 .19 \\
7.3 .20\end{array}$ & $\begin{array}{l}0.2202 \\
0.6244 \\
0.1576 \\
0.1300 \\
0.0848\end{array}$ & $\begin{array}{l}0.1975 \\
0.1721 \\
0.1430 \\
0.1195 \\
0.1190\end{array}$ & $\begin{array}{l}30.98 \\
32.48 \\
20.98 \\
22.37 \\
22.37\end{array}$ & $\begin{array}{l}c .0 \\
0.0 \\
c .0 \\
c .0 \\
c .0\end{array}$ & $\begin{array}{r}6.130 \\
0.447 \\
7.267 \\
8.310 \\
11.400\end{array}$ & $\begin{array}{l}0.993 \\
1.022 \\
1.001 \\
1.004 \\
1.027\end{array}$ & $\begin{array}{r}6.23 \\
19.73 \\
5.97 \\
5.91 \\
3.95\end{array}$ & $\begin{array}{l}0.531 \\
0.082 \\
0.633 \\
0.740 \\
0.993\end{array}$ & $\begin{array}{l}0.3696 \\
0.6354 \\
0.3340 \\
0.3175 \\
0.2564\end{array}$ & $\begin{array}{l}0.4552 \\
0.1793 \\
0.4967 \\
0.5371 \\
0.6220\end{array}$ \\
\hline
\end{tabular}


TABLE L (Contd.)

SPECIAL TEST GROUP 7.3 - DOUBLE ANNULUS

EXPERIMENTAL MEASURMENTS FOR TESTS 7.3.1 TO 7.3.21 - 0.63-IN. DOMNCOMER GAP

\begin{tabular}{|c|c|c|c|c|c|c|c|c|c|c|}
\hline $\begin{array}{l}\text { TEST } \\
\text { NO }\end{array}$ & $\begin{array}{c}\text { RH-CH-I } \\
(G P H)\end{array}$ & $\begin{array}{c}\mathrm{RM}-\mathrm{CH}-2 \\
(\mathrm{GPM})\end{array}$ & $\begin{array}{c}F T-C W-1 \\
(G P M)\end{array}$ & $\begin{array}{l}\mathrm{FT}-\mathrm{CH}-2 \\
(G P M)\end{array}$ & $\begin{array}{c}R M-H G-1 \\
(C F M)\end{array}$ & $\begin{array}{c}\text { RM-HG-2 } \\
(C F M)\end{array}$ & $\begin{array}{c}R+-H G-3 \\
\text { (CFM) }\end{array}$ & $\begin{array}{c}\text { RM-HG-4 } \\
(C F Y)\end{array}$ & $\begin{array}{c}F T-H G-1 \\
(C F M)\end{array}$ & $\begin{array}{c}F T-H G-2 \\
(C F M)\end{array}$ \\
\hline $\begin{array}{l}7.3 .1 \\
7.3 .2 \\
7.3 .3 \\
7.3 .4 \\
7.3 .5\end{array}$ & $\begin{array}{l}0.0 \\
0.0 \\
0.0 \\
0.0 \\
0.0\end{array}$ & $\begin{array}{l}0.0 \\
0.0 \\
0.0 \\
0.0 \\
0.0\end{array}$ & $\begin{array}{r}0.0 \\
0.0 \\
80.0 \\
60.0 \\
110.0\end{array}$ & $\begin{array}{r}48.0 \\
40.0 \\
0.0 \\
0.0 \\
0.0\end{array}$ & $\begin{array}{l}0.0 \\
0.0 \\
0.0 \\
0.0 \\
0.0\end{array}$ & $\begin{array}{l}0.0 \\
0.0 \\
0.0 \\
0.0 \\
0.0\end{array}$ & $\begin{array}{l}0.0 \\
0.0 \\
0.0 \\
0.0 \\
0.0\end{array}$ & $\begin{array}{l}0.0 \\
0.0 \\
0.0 \\
0.0 \\
0.0\end{array}$ & $\begin{array}{l}65.77 \\
65.26 \\
50.51 \\
50.13 \\
37.05\end{array}$ & $\begin{array}{l}0.0 \\
0.0 \\
0.0 \\
0.0 \\
0.0\end{array}$ \\
\hline $\begin{array}{l}7.3 .6 \\
7.3 .9 \\
7.3 .9 \\
7.3 .99 \\
7.3 .10\end{array}$ & $\begin{array}{l}0.0 \\
0.0 \\
0.0 \\
0.0 \\
0.0\end{array}$ & $\begin{array}{l}0.0 \\
0.0 \\
0.0 \\
0.0 \\
0.0\end{array}$ & $\begin{array}{r}90.0 \\
70.0 \\
90.0 \\
70.0 \\
0.0\end{array}$ & $\begin{array}{r}0.0 \\
0.0 \\
0.0 \\
0.0 \\
40.4\end{array}$ & $\begin{array}{l}0.0 \\
0.0 \\
0.0 \\
0.0 \\
0.0\end{array}$ & $\begin{array}{l}0.0 \\
0.0 \\
0.0 \\
0.0 \\
0.0\end{array}$ & $\begin{array}{l}0.0 \\
0.0 \\
0.0 \\
0.0 \\
0.0\end{array}$ & $\begin{array}{l}0.0 \\
0.0 \\
0.0 \\
0.0 \\
0.0\end{array}$ & $\begin{array}{l}36.79 \\
50.26 \\
37.18 \\
50.13 \\
79.36\end{array}$ & $\begin{array}{l}0.0 \\
0.0 \\
0.0 \\
0.0 \\
0.0\end{array}$ \\
\hline $\begin{array}{l}7.3 .11 \\
7.3 .12 \\
7.3 .13 \\
7.3 .14 \\
7.3 .15\end{array}$ & $\begin{array}{l}0.0 \\
0.0 \\
0.0 \\
0.0 \\
0.0\end{array}$ & $\begin{array}{l}0.0 \\
0.0 \\
0.0 \\
0.0 \\
0.0\end{array}$ & $\begin{array}{r}0.0 \\
130.0 \\
153.0 \\
150.0 \\
150.0\end{array}$ & $\begin{array}{r}29.9 \\
0.0 \\
0.0 \\
0.0 \\
0.0\end{array}$ & $\begin{array}{c}0.0 \\
0.0 \\
0.0 \\
0.0 \\
40.00\end{array}$ & $\begin{array}{l}0.0 \\
0.0 \\
0.0 \\
0.0 \\
0.0\end{array}$ & $\begin{array}{l}0.0 \\
0.0 \\
0.0 \\
0.0 \\
0.0\end{array}$ & $\begin{array}{l}0.0 \\
0.0 \\
0.0 \\
0.0 \\
0.0\end{array}$ & $\begin{array}{c}81.28 \\
26.79 \\
19.23 \\
13.21 \\
0.0\end{array}$ & $\begin{array}{l}0.0 \\
0.0 \\
0.0 \\
0.0 \\
0.0\end{array}$ \\
\hline $\begin{array}{l}7.3 .16 \\
7.3 .17 \\
7.3 .18 \\
7.3 .19 \\
7.3 .20\end{array}$ & $\begin{array}{l}0.0 \\
0.0 \\
0.0 \\
0.0 \\
0.0\end{array}$ & $\begin{array}{l}0.0 \\
0.0 \\
0.0 \\
0.0 \\
0.0\end{array}$ & $\begin{array}{r}150.0 \\
0.0 \\
130.0 \\
130.0 \\
129.0\end{array}$ & $\begin{array}{r}0.0 \\
34.7 \\
0.0 \\
0.0 \\
0.0\end{array}$ & $\begin{array}{c}0.0 \\
0.0 \\
0.0 \\
60.00 \\
40.00\end{array}$ & $\begin{array}{l}0.0 \\
0.0 \\
0.0 \\
0.0 \\
0.0\end{array}$ & $\begin{array}{l}0.0 \\
0.0 \\
0.0 \\
0.0 \\
0.0\end{array}$ & $\begin{array}{l}0.0 \\
0.0 \\
0.0 \\
0.0 \\
0.0\end{array}$ & $\begin{array}{c}26.79 \\
82.31 \\
19.10 \\
0.0 \\
0.0\end{array}$ & $\begin{array}{l}0.0 \\
0.0 \\
0.0 \\
0.0 \\
0.0\end{array}$ \\
\hline 7.3 .21 & 0.0 & 0.0 & 98.0 & 0.0 & 0.0 & 0.0 & 0.0 & 0.0 & 31.56 & 0.0 \\
\hline $\begin{array}{l}\text { TEST } \\
\text { NO }\end{array}$ & $\begin{array}{l}\text { P-CH-L } \\
(P S I G)\end{array}$ & $\begin{array}{l}P-C H-2 \\
\text { PSSIGI }\end{array}$ & $\begin{array}{l}\text { P-HG-1 } \\
\text { (PSIG) }\end{array}$ & $\begin{array}{l}\text { P-HG-2 } \\
\text { (PSIG) }\end{array}$ & $\begin{array}{l}\text { P-HG-3 } \\
\text { (PSIG) }\end{array}$ & $\begin{array}{l}\text { TF-CH-1 } \\
\text { IDEG FI }\end{array}$ & $\begin{array}{l}\text { TF-HG-1 } \\
\text { (DEG E) }\end{array}$ & $\begin{array}{l}\text { TF-HG-2 } \\
\text { (DEGF) }\end{array}$ & $\begin{array}{l}\text { TF-HG-3 } \\
\text { IOEG FI }\end{array}$ & $\begin{array}{l}\text { P-BARO } \\
\text { (PSIA) }\end{array}$ \\
\hline $\begin{array}{l}7.3 .1 \\
7.3 .2 \\
7.3 .3 \\
7.3 .4 \\
7.3 .5\end{array}$ & $\begin{array}{l}21.5 \\
17.7 \\
24.5 \\
18.0 \\
25.7\end{array}$ & $\begin{array}{l}0.0 \\
0.0 \\
0.0 \\
0.0 \\
0.0\end{array}$ & $\begin{array}{l}22.3 \\
18.8 \\
24.2 \\
18.0 \\
24.7\end{array}$ & $\begin{array}{l}92.5 \\
91.5 \\
91.5 \\
90.5 \\
92.0\end{array}$ & $\begin{array}{l}0.0 \\
0.0 \\
0.0 \\
0.0 \\
0.0\end{array}$ & $\begin{array}{l}50.0 \\
48.5 \\
45.5 \\
45.5 \\
45.0\end{array}$ & $\begin{array}{r}84.0 \\
92.0 \\
108.0 \\
108.0 \\
99.5\end{array}$ & $\begin{array}{r}95.5 \\
113.0 \\
122.0 \\
120.5 \\
110.5\end{array}$ & $\begin{array}{l}0.0 \\
0.0 \\
0.0 \\
0.0 \\
0.0\end{array}$ & $\begin{array}{l}12.4 \\
12.4 \\
12.4 \\
12.4 \\
12.4\end{array}$ \\
\hline $\begin{array}{l}7.3 .6 \\
7.3 .7 \\
7.3 .8 \\
7.3 .9 \\
7.3 .10\end{array}$ & $\begin{array}{l}21.0 \\
21.5 \\
21.0 \\
22.5 \\
22.5\end{array}$ & $\begin{array}{l}0.0 \\
0.0 \\
0.0 \\
0.0 \\
0.0\end{array}$ & $\begin{array}{l}20.8 \\
21.0 \\
20.0 \\
22.5 \\
23.0\end{array}$ & $\begin{array}{l}91.5 \\
90.0 \\
92.0 \\
92.0 \\
89.0\end{array}$ & $\begin{array}{l}0.0 \\
0.0 \\
0.0 \\
0.0 \\
0.0\end{array}$ & $\begin{array}{l}46.0 \\
45.5 \\
46.0 \\
48.0 \\
49.0\end{array}$ & $\begin{array}{r}91.5 \\
95.5 \\
102.5 \\
78.0 \\
107.0\end{array}$ & $\begin{array}{r}100.0 \\
107.0 \\
114.5 \\
86.5 \\
123.5\end{array}$ & $\begin{array}{l}0.0 \\
0.0 \\
0.0 \\
0.0 \\
0.0\end{array}$ & $\begin{array}{l}12.4 \\
12.4 \\
12.4 \\
12.5 \\
12.5\end{array}$ \\
\hline
\end{tabular}




\section{TABLE L (Contd.)}

SPECIAL TEST GROUP 7.3 - DOUBLE ANNULUS

EXPER IMENTAL MEASURMENTS FOR TESTS 7.3.1 TO 7.3 .21 - 0.63-IN. DONNCOMER GAP

\begin{tabular}{|c|c|c|c|c|c|c|c|c|c|c|}
\hline $\begin{array}{l}\text { TEST } \\
\text { NO }\end{array}$ & $\begin{array}{l}P-C H-1 \\
(P S I G)\end{array}$ & $\begin{array}{l}P-C H-2 \\
\text { (PSIGI }\end{array}$ & $\begin{array}{l}P-H G-1 \\
(P S I G)\end{array}$ & $\begin{array}{l}\text { P-HG-2 } \\
\text { (PSIG) }\end{array}$ & $\begin{array}{l}\text { P-HG-3 } \\
\text { (PSIG) }\end{array}$ & $\begin{array}{l}\text { TF-CH-1 } \\
\text { (DEG F) }\end{array}$ & $\begin{array}{l}\text { TF-HG- } \\
\text { (DEG FI }\end{array}$ & $\begin{array}{l}\text { TF-HG-2 } \\
\text { (OEG F) }\end{array}$ & $\begin{array}{l}\text { TF-HG-3 } \\
\text { IOEG FI }\end{array}$ & $\begin{array}{l}\text { P-BARO } \\
\text { (PSIA) }\end{array}$ \\
\hline $\begin{array}{l}7.3 .11 \\
7.3 .12 \\
7.3 .13 \\
7.3 .14 \\
7.3 .15\end{array}$ & $\begin{array}{l}18.0 \\
23.0 \\
21.5 \\
17.0 \\
10.0\end{array}$ & $\begin{array}{l}0.0 \\
0.0 \\
0.0 \\
0.0 \\
0.0\end{array}$ & $\begin{array}{r}20.0 \\
21.0 \\
17.4 \\
13.0 \\
7.5\end{array}$ & $\begin{array}{l}88.0 \\
93.5 \\
89.0 \\
94.5 \\
94.5\end{array}$ & $\begin{array}{r}0.0 \\
0.0 \\
0.0 \\
0.0 \\
32.0\end{array}$ & $\begin{array}{l}48.5 \\
45.0 \\
44.0 \\
44.0 \\
44.0\end{array}$ & $\begin{array}{r}113.5 \\
118.0 \\
106.0 \\
92.5 \\
85.0\end{array}$ & $\begin{array}{r}128.5 \\
130.0 \\
117.5 \\
100.5 \\
94.5\end{array}$ & $\begin{array}{r}0.0 \\
0.0 \\
0.0 \\
0.0 \\
84.0\end{array}$ & $\begin{array}{l}12.5 \\
12.5 \\
12.5 \\
12.5 \\
12.5\end{array}$ \\
\hline $\begin{array}{l}7.3 .16 \\
7.3 .17 \\
7.3 .18 \\
7.3 .19 \\
7.3 .20\end{array}$ & $\begin{array}{l}28.5 \\
20.0 \\
17.5 \\
14.0 \\
13.5\end{array}$ & $\begin{array}{l}0.0 \\
0.0 \\
0.0 \\
0.0 \\
0.0\end{array}$ & $\begin{array}{l}25.0 \\
21.0 \\
15.0 \\
11.0 \\
11.0\end{array}$ & $\begin{array}{r}93.0 \\
85.5 \\
94.0 \\
0.0 \\
0.0\end{array}$ & $\begin{array}{r}0.0 \\
0.0 \\
0.0 \\
32.5 \\
32.5\end{array}$ & $\begin{array}{l}45.0 \\
44.0 \\
46.5 \\
47.5 \\
44.4\end{array}$ & $\begin{array}{r}90.0 \\
103.5 \\
105.5 \\
75.5 \\
101.5\end{array}$ & $\begin{array}{r}102.0 \\
121.0 \\
120.5 \\
80.8 \\
117.5\end{array}$ & $\begin{array}{r}0.0 \\
0.0 \\
0.0 \\
76.9 \\
100.0\end{array}$ & $\begin{array}{l}12.5 \\
12.5 \\
12.5 \\
12.4 \\
12.4\end{array}$ \\
\hline 7.3 .21 & 23.5 & 0.0 & 22.5 & 92.0 & 35.0 & 43.5 & 106.0 & 116.5 & 112.0 & 12.4 \\
\hline
\end{tabular}

\begin{tabular}{|c|c|c|c|c|c|c|c|c|c|}
\hline \multirow[b]{2}{*}{$\begin{array}{c}\text { TEST } \\
\text { NO }\end{array}$} & \multicolumn{3}{|c|}{ LOMER PLENUM FLOH } & \multicolumn{3}{|c|}{ BYPASS FLOH } & \multirow{2}{*}{$\begin{array}{l}\text { LOWER } \\
\text { PLENUM } \\
\text { LEVEL } \\
\text { (INI }\end{array}$} & \multirow[b]{2}{*}{$\begin{array}{l}P-L P-1 \\
(P S I G)\end{array}$} & \multirow[b]{2}{*}{$\begin{array}{l}\text { IF- LP-1 } \\
\text { (CEG FI }\end{array}$} \\
\hline & $\begin{array}{l}\text { INITIAL } \\
\text { HE IGHT } \\
\text { (LB) }\end{array}$ & $\begin{array}{l}\text { FINAL } \\
\text { WEIGHT } \\
\text { ILBI }\end{array}$ & $\begin{array}{c}\text { TIME OF } \\
\text { COLLECT ICN } \\
\text { (SEC) }\end{array}$ & $\begin{array}{l}\text { INITIALL } \\
\text { HEIGHT } \\
\text { (LB) }\end{array}$ & $\begin{array}{l}\text { F INAL } \\
\text { WEIGHT } \\
(L B)\end{array}$ & $\begin{array}{l}\text { TIME OF } \\
\text { COLLECTION } \\
\text { (SEC) }\end{array}$ & & & \\
\hline $\begin{array}{l}7.3 .1 \\
7.3 . \\
7.3 .5 \\
7.3 .4 \\
7.3 .5\end{array}$ & $\begin{array}{l}140.5 \\
186.5 \\
232.5 \\
307.5 \\
143.5\end{array}$ & $\begin{array}{l}186.5 \\
232.5 \\
307.5 \\
372.0 \\
218.0\end{array}$ & $\begin{array}{l}30.0 \\
30.0 \\
30.0 \\
30.0 \\
20.0\end{array}$ & $\begin{array}{l}103.5 \\
182.0 \\
263.5 \\
391.0 \\
219.0\end{array}$ & $\begin{array}{l}182.0 \\
263.5 \\
391.0 \\
516.5 \\
393.5\end{array}$ & $\begin{array}{l}15.0 \\
20.0 \\
15.0 \\
20.0 \\
15.0\end{array}$ & $\begin{array}{l}12.0 \\
12.0 \\
12.0 \\
12.0 \\
12.0\end{array}$ & $\begin{array}{l}22.0 \\
18.0 \\
23.5 \\
18.0 \\
24.0\end{array}$ & $\begin{array}{l}53.0 \\
51.0 \\
48.0 \\
48.5 \\
46.0\end{array}$ \\
\hline $\begin{array}{l}7.3 .6 \\
7.3 .9 \\
7.3 .8 \\
7.3 .99 \\
7.3 .10\end{array}$ & $\begin{array}{l}162.0 \\
262.0 \\
186.5 \\
140.5 \\
268.0\end{array}$ & $\begin{array}{l}262.0 \\
324.0 \\
285.0 \\
268.0 \\
336.5\end{array}$ & $\begin{array}{l}30.0 \\
30.0 \\
30.0 \\
60.0 \\
60.0\end{array}$ & $\begin{array}{l}111.5 \\
270.0 \\
375.0 \\
130.0 \\
354.5\end{array}$ & $\begin{array}{l}270.0 \\
479.0 \\
650.0 \\
354.5 \\
495.0\end{array}$ & $\begin{array}{l}15.0 \\
30.0 \\
30.0 \\
30.0 \\
30.0\end{array}$ & $\begin{array}{l}12.0 \\
12.0 \\
12.0 \\
12.0 \\
12.0\end{array}$ & $\begin{array}{l}20.0 \\
21.2 \\
20.0 \\
22.5 \\
22.5\end{array}$ & $\begin{array}{l}47.5 \\
48.0 \\
47.5 \\
49.0 \\
51.5\end{array}$ \\
\hline $\begin{array}{l}7.3 .11 \\
7.3 .12 \\
7.3 .13 \\
7.3 .16 \\
7.3 .15\end{array}$ & $\begin{array}{l}163.5 \\
142.0 \\
183.5 \\
146.0 \\
156.0\end{array}$ & $\begin{array}{l}221.5 \\
411.0 \\
548.5 \\
474.0 \\
448.5\end{array}$ & $\begin{array}{l}70.0 \\
45.0 \\
45.0 \\
35.0 \\
25.0\end{array}$ & $\begin{array}{l}335.0 \\
114.5 \\
283.5 \\
210.5 \\
368.5\end{array}$ & $\begin{array}{l}437.5 \\
427.0 \\
618.5 \\
614.0 \\
627.5\end{array}$ & $\begin{array}{l}30.0 \\
25.0 \\
25.0 \\
35.0 \\
30.0\end{array}$ & $\begin{array}{r}12.0 \\
12.0 \\
12.0 \\
12.0 \\
4.0\end{array}$ & $\begin{array}{r}18.0 \\
20.0 \\
17.2 \\
12.5 \\
7.5\end{array}$ & $\begin{array}{l}53.0 \\
45.0 \\
44.0 \\
44.0 \\
44.0\end{array}$ \\
\hline $\begin{array}{l}7.3 .16 \\
7.3 .17 \\
7.3 .18 \\
7.3 .19 \\
7.3 .20\end{array}$ & $\begin{array}{l}225.0 \\
141.5 \\
212.5 \\
144.5 \\
141.5\end{array}$ & $\begin{array}{l}408.0 \\
212.5 \\
430.5 \\
484.5 \\
369.5\end{array}$ & $\begin{array}{l}30.0 \\
75.0 \\
30.0 \\
40.0 \\
20.0\end{array}$ & $\begin{array}{l}400.0 \\
123.0 \\
242.5 \\
107.5 \\
104.0\end{array}$ & $\begin{array}{l}692.0 \\
242.5 \\
567.0 \\
348.5 \\
244.5\end{array}$ & $\begin{array}{l}20.0 \\
30.0 \\
30.0 \\
25.0 \\
20.0\end{array}$ & $\begin{array}{r}12.0 \\
12.0 \\
12.0 \\
12.0 \\
4.0\end{array}$ & $\begin{array}{l}24.5 \\
20.0 \\
14.5 \\
10.0 \\
10.0\end{array}$ & $\begin{array}{l}45.5 \\
49.5 \\
47.0 \\
45.5 \\
44.8\end{array}$ \\
\hline 7.3 .21 & 369.5 & 525.0 & 45.0 & 244.5 & 555.0 & 30.0 & 12.0 & 21.2 & 45.0 \\
\hline
\end{tabular}




\subsection{Longitudinal Flow Restrictor Tests}

Countercurrent flow tests were performed with flow restrictors attached to the core barrel. These flow restrictors ran longitudinally along the core barrel and protruded out into the 1.58-inch radial downcomer gap about 1.0 inch. Figure 7 shows a sketch of the azimuthal location and relative dimensions of the. flow restrictors and the downcomer gap. Two groups of tests were conducted. In the first test group (Test Group 9.8), the restrictors ran nearly the full length of the vessel from the core barrel flange at the top to near the bottom of the lower plenum. For the second group of tests, the restrictors were shortened at the upper end such that they began at the top of the downcomer and ran the entire length of the downcomer. This latter configuration left the upper annulus region unrestricted.

The objective of these two test groups was to determine whether the longitudinal restrictors affected the lower plenum delivery rate and the two-phase flow patterns occurring in the downcomer. These tests were considered to be demonstration tests of the countercurrent flow phenomena that might be expected to occur in the LOFT downcomer as a result of the instrumentation stalks that run longitudinally down the core barrel and protrude into the downcomer gap. The tests were only demonstrational because many of the pertinent transparent vessel dimensions were not scaled to the equivalent LOFT dimensions.

Results from the longitudinal flow restrictor tests are shown in Tables LI and LII.

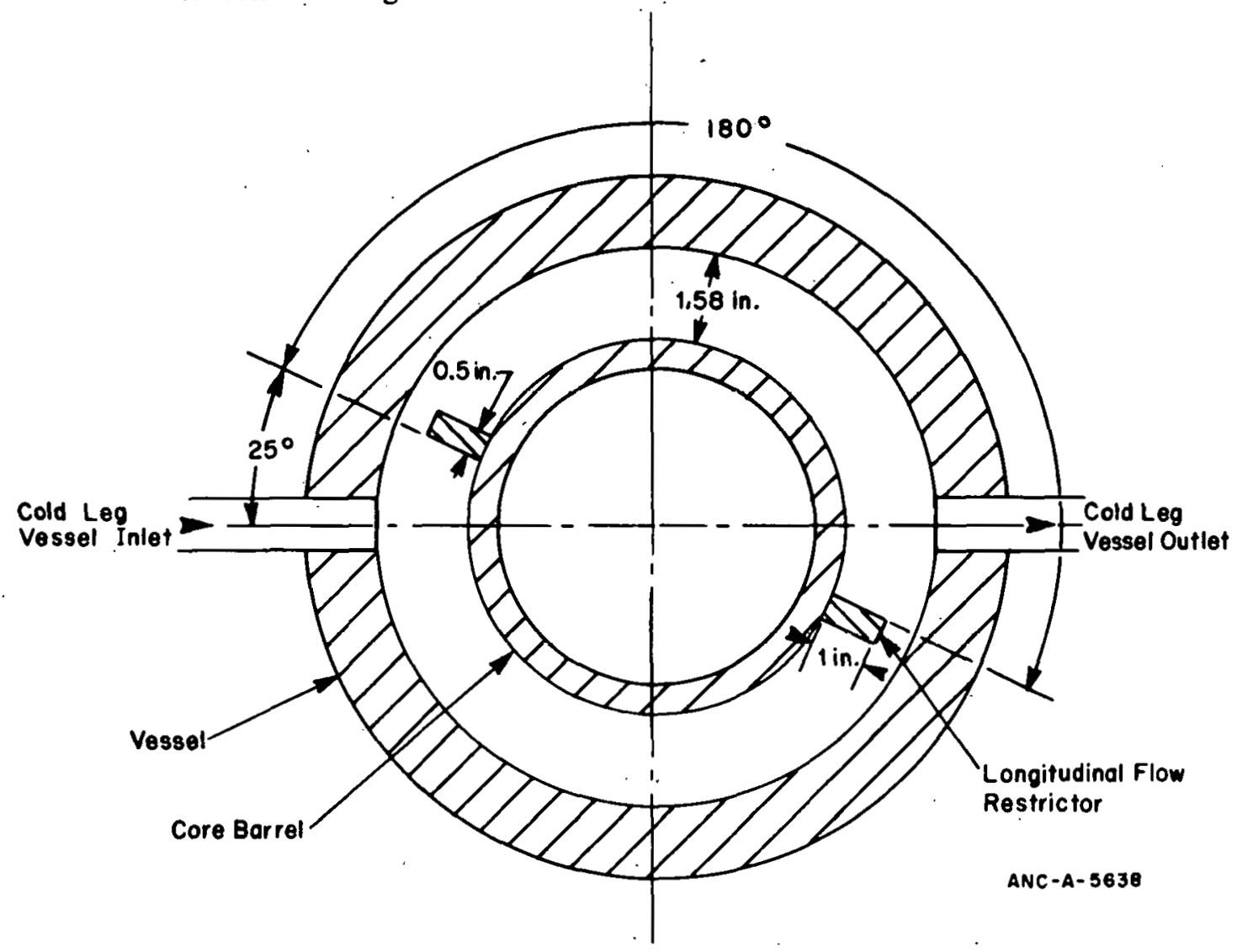

Fig. 7 Location and dimensions of longitudinal flow restrictors. 


\section{TABLE LI}

SPECIAL TEST GROUP 9.8 - LONGITUDINAI FLOW RESTRICTORS

TRANSPARENT VESSEL TEST DATA FOR TESTS 9.8 .1 TO $9.8 .20--1.58-$ IN. DOWNCOMER GAP SUMMARY OF CALCULATED RESULTS

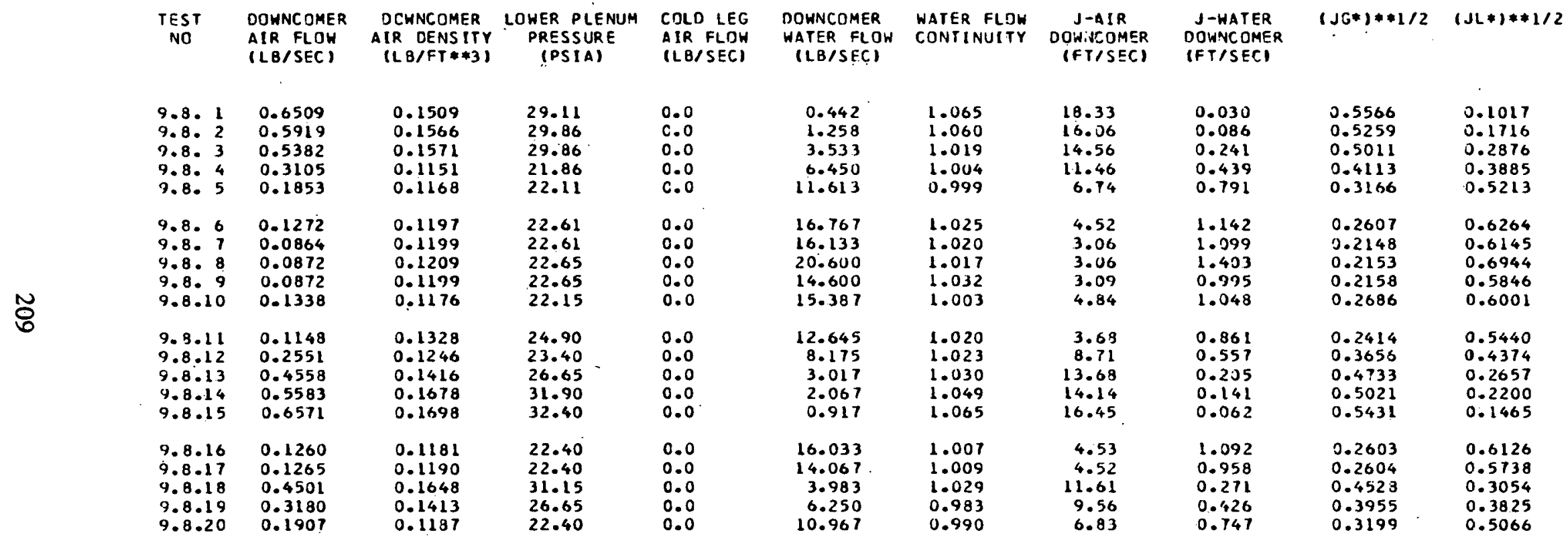


TABLE LI (Contd.)

SPECIAL TEST GROUP 9.8 - LONGITUDINAL FLOW RESTRICTORS

EXPER IMENTAL MEASURMENTS FOR TESTS 9.8.1 TO 9.8.20 -- 1.58-IN. DONNCOMER GAP

\begin{tabular}{|c|c|c|c|c|c|c|c|c|c|c|}
\hline $\begin{array}{l}\text { TEST } \\
\text { NO }\end{array}$ & $\begin{array}{c}R H-C H-1 \\
|G P M|\end{array}$ & ${ }_{(G P M)}^{R H-C H-2}$ & $\begin{array}{c}F T-C H-1 \\
\text { (GPM) }\end{array}$ & $\begin{array}{c}F T-C H-2 \\
(G P M)\end{array}$ & $\begin{array}{c}R M-H G-1 \\
(C F M)\end{array}$ & $\begin{array}{c}R M-H G-2 \\
(C F M)^{2}\end{array}$ & $\begin{array}{c}\text { RM-HG-3 } \\
\text { (CFM) }\end{array}$ & $\begin{array}{c}\text { RM-HG-4 } \\
\text { (CFM) }\end{array}$ & $\begin{array}{l}F T-H G-1 \\
(C F M)\end{array}$ & $\begin{array}{l}F T-H G-2 \\
\text { (CFM) }\end{array}$ \\
\hline $\begin{array}{l}9.8 .1 \\
9.8 .22 \\
9.8 .3 \\
9.8 .4 \\
9.8 .5\end{array}$ & $\begin{array}{l}0.0 \\
0.0 \\
0.0 \\
0.0 \\
0.0\end{array}$ & $\begin{array}{l}0.0 \\
0.0 \\
0.0 \\
0.0 \\
0.0\end{array}$ & $\begin{array}{r}0.0 \\
0.0 \\
0.0 \\
70.0 \\
130.0\end{array}$ & $\begin{array}{r}20.1 \\
30.0 \\
49.9 \\
0.0 \\
0.0\end{array}$ & $\begin{array}{l}0.0 \\
0.0 \\
0.0 \\
0.0 \\
0.0\end{array}$ & $\begin{array}{l}0.0 \\
0.0 \\
0.0 \\
0.0 \\
0.0\end{array}$ & $\begin{array}{l}0.0 \\
0.0 \\
0.0 \\
0.0 \\
0.0\end{array}$ & $\begin{array}{l}0.0 \\
0.0 \\
0.0 \\
0.0 \\
0.0\end{array}$ & $\begin{array}{l}88.46 \\
73.72 \\
66.28 \\
38.21 \\
22.44\end{array}$ & $\begin{array}{l}0.0 \\
0.0 \\
0.0 \\
0.0 \\
0.0\end{array}$ \\
\hline $\begin{array}{l}9.8 .6 \\
9.8 .7 \\
9.8 .8 \\
9.8 .89 \\
9.8 .10\end{array}$ & $\begin{array}{l}0.0 \\
0.0 \\
0.0 \\
0.0 \\
0.0\end{array}$ & $\begin{array}{l}0.0 \\
0.0 \\
0.0 \\
0.0 \\
0.0\end{array}$ & $\begin{array}{l}150.0 \\
160.0 \\
180.0 \\
110.0 \\
141.0\end{array}$ & $\begin{array}{l}0.0 \\
0.0 \\
0.0 \\
0.0 \\
0.0\end{array}$ & $\begin{array}{c}0.0 \\
40.00 \\
40.00 \\
40.00 \\
0.0\end{array}$ & $\begin{array}{l}0.0 \\
0.0 \\
0.0 \\
0.0 \\
0.0\end{array}$ & $\begin{array}{l}0.0 \\
0.0 \\
0.0 \\
0.0 \\
0.0\end{array}$ & $\begin{array}{l}0.0 \\
0.0 \\
0.0 \\
0.0 \\
0.0\end{array}$ & $\begin{array}{c}14.74 \\
0.0 \\
0.0 \\
0.0 \\
14.74\end{array}$ & $\begin{array}{l}0.0 \\
0.0 \\
0.0 \\
0.0 \\
0.0\end{array}$ \\
\hline $\begin{array}{l}9.8 .11 \\
9.9 .12 \\
9.8 .13 \\
9.8 .14 \\
9.8 .15\end{array}$ & $\begin{array}{l}0.0 \\
0.0 \\
0.0 \\
0.0 \\
0.0\end{array}$ & $\begin{array}{l}0.0 \\
0.0 \\
0.0 \\
0.0 \\
0.0\end{array}$ & $\begin{array}{r}151.0 \\
100.0 \\
0.0 \\
0.0 \\
0.0\end{array}$ & $\begin{array}{r}0.0 \\
0.0 \\
50.3 \\
49.0 \\
30.3\end{array}$ & $\begin{array}{l}0.0 \\
0.0 \\
0.0 \\
0.0 \\
0.0\end{array}$ & $\begin{array}{l}0.0 \\
0.0 \\
0.0 \\
0.0 \\
0.0\end{array}$ & $\begin{array}{l}0.0 \\
0.0 \\
0.0 \\
0.0 \\
0.0\end{array}$ & $\begin{array}{l}0.0 \\
0.0 \\
0.0 \\
0.0 \\
0.0\end{array}$ & $\begin{array}{l}22.44 \\
29.49 \\
56.03 \\
71.67 \\
82.44\end{array}$ & $\begin{array}{l}0.0 \\
0.0 \\
0.0 \\
0.0 \\
0.0\end{array}$ \\
\hline $\begin{array}{l}9.8 .16 \\
9.8 .17 \\
9.8 .18 \\
9.8 .19 \\
9.8 .20\end{array}$ & $\begin{array}{l}0.0 \\
0.0 \\
0.0 \\
0.0 \\
0.0\end{array}$ & $\begin{array}{l}0.0 \\
0.0 \\
0.0 \\
0.0 \\
0.0\end{array}$ & $\begin{array}{r}149.0 \\
130.0 \\
70.0 \\
89.0 \\
100.0\end{array}$ & $\begin{array}{l}0.0 \\
0.0 \\
0.0 \\
0.0 \\
0.0\end{array}$ & $\begin{array}{l}0.0 \\
0.0 \\
0.0 \\
0.0 \\
0.0\end{array}$ & $\begin{array}{l}0.0 \\
0.0 \\
0.0 \\
0.0 \\
0.0\end{array}$ & $\begin{array}{l}0.0 \\
0.0 \\
0.0 \\
0.0 \\
0.0\end{array}$ & $\begin{array}{l}0.0 \\
0.0 \\
0.0 \\
0.0 \\
0.0\end{array}$ & $\begin{array}{l}14.74 \\
14.87 \\
55.77 \\
38.33 \\
.22 .31\end{array}$ & $\begin{array}{l}0.0 \\
0.0 \\
0.0 \\
0.0 \\
0.0\end{array}$ \\
\hline $\begin{array}{l}\text { TEST } \\
\text { NO }\end{array}$ & $\begin{array}{l}P-C H-1 \\
(P S I G)\end{array}$ & $\begin{array}{l}P-C H-2 \\
\text { (PSIG) }\end{array}$ & $\begin{array}{l}\text { P-HG-1 } \\
(P S I G)\end{array}$ & $\begin{array}{l}\text { P-HG-2 } \\
\text { (PSIG) }\end{array}$ & $\begin{array}{l}\text { P-HG - } 3 \\
\text { (PS IG) }\end{array}$ & $\begin{array}{l}\text { TF-CH-1 } \\
\text { (DEG F) }\end{array}$ & $\begin{array}{l}\text { TF-HG-1 } \\
(D E G \quad F)\end{array}$ & $\begin{array}{l}\text { TF-HC-2 } \\
\text { IDEG FI }\end{array}$ & $\begin{array}{l}\text { TF-HG-3 } \\
(D E G F)\end{array}$ & $\begin{array}{l}\text { P-BARO } \\
\text { (PSIA) }\end{array}$ \\
\hline $\begin{array}{l}9.8 \cdot 1 \\
9.8 \cdot 2 \\
9.8 \cdot 3 \\
9.8 \cdot 4 \\
9.8 .5\end{array}$ & $\begin{array}{r}15.5 \\
17.0 \\
19.0 \\
9.7 \\
12.5\end{array}$ & $\begin{array}{l}0.0 \\
0.0 \\
0.0 \\
0.0 \\
0.0\end{array}$ & $\begin{array}{r}18.0 \\
18.0 \\
19.0 \\
9.6 \\
9.8\end{array}$ & $\begin{array}{l}83.0 \\
90.0 \\
89.0 \\
92.5 \\
93.0\end{array}$ & $\begin{array}{l}33.0 \\
33.0 \\
33.0 \\
33.0 \\
33.5\end{array}$ & $\begin{array}{l}50.5 \\
49.5 \\
50.5 \\
51.5 \\
50.5\end{array}$ & $\begin{array}{l}109.0 \\
100.5 \\
103.5 \\
106.5 \\
103.0\end{array}$ & $\begin{array}{l}123.0 \\
113.5 \\
101.5 \\
120.5 \\
114.0\end{array}$ & $\begin{array}{l}116.0 \\
108.0 \\
110.5 \\
113.5 \\
108.5\end{array}$ & $\begin{array}{l}12.4 \\
12.4 \\
12.4 \\
12.4 \\
12.4\end{array}$ \\
\hline $\begin{array}{r}9.8 .6 \\
9.8 .7 \\
9.8 .8 \\
9.8 .9 \\
9.8 .10\end{array}$ & $\begin{array}{l}14.7 \\
14.0 \\
12.7 \\
12.7 \\
14.0\end{array}$ & $\begin{array}{l}0.0 \\
0.0 \\
0.0 \\
0.0 \\
0.0\end{array}$ & $\begin{array}{l}10.7 \\
10.5 \\
11.5 \\
10.6 \\
10.1\end{array}$ & $\begin{array}{l}95.0 \\
96.0 \\
78.0 \\
98.0 \\
96.0\end{array}$ & $\begin{array}{l}33.5 \\
33.0 \\
33.0 \\
33.0 \\
33.5\end{array}$ & $\begin{array}{l}50.0 \\
49.0 \\
70.0 \\
50.0 \\
48.0\end{array}$ & $\begin{array}{l}91.5 \\
86.5 \\
76.0 \\
76.5 \\
75.5\end{array}$ & $\begin{array}{r}120.0 \\
100.0 \\
70.0 \\
0.0 \\
77.5\end{array}$ & $\begin{array}{l}96.5 \\
86.0 \\
76.5 \\
76.5 \\
76.5\end{array}$ & $\begin{array}{l}12.4 \\
12.4 \\
12.4 \\
12.4 \\
12.4\end{array}$ \\
\hline
\end{tabular}


TABLE LI (Contd.)

SPECIAL TEST GROUP 9.8 - LONGITUDINAL FLOW RESTRICTORS

EXPERIMENTAL MEASURMENTS FOR TESTS 9.8 .1 TO $9.8 .20-1.58-I N$. DOHNCOMER GAP

\begin{tabular}{|c|c|c|c|c|c|c|c|c|c|c|}
\hline $\begin{array}{l}\text { TEST } \\
\text { NO }\end{array}$ & $\begin{array}{l}P-C H-1 \\
\text { (PSIG) }\end{array}$ & $\begin{array}{l}\text { P-CH-2 } \\
\text { IPSIGI }\end{array}$ & $\begin{array}{l}P-H G-1 \\
(P S I G)\end{array}$ & $\begin{array}{l}P-H G-2 \\
(P S I G)\end{array}$ & $\begin{array}{l}P-H G-3 \\
(P S I G)\end{array}$ & $\begin{array}{l}\text { IF-CW-1 } \\
\text { IOEG FI }\end{array}$ & $\begin{array}{l}T F-H G-1 \\
\text { (DEG F) }\end{array}$ & $\begin{array}{l}\text { TF-HG-2 } \\
\text { IOEG FI }\end{array}$ & $\begin{array}{l}\text { TF-HG-3 } \\
\text { (DEG F) }\end{array}$ & $\begin{array}{l}\text { P-SARO } \\
\text { (PSIA) }\end{array}$ \\
\hline $\begin{array}{r}9.8 .11 \\
9.8 .12 \\
9.8 .13 \\
9.8 .14 \\
9.8 .15\end{array}$ & $\begin{array}{l}17.5 \\
13.5 \\
14.5 \\
19.7 \\
19.7\end{array}$ & $\begin{array}{l}0.0 \\
0.0 \\
0.0 \\
0.0 \\
0.0\end{array}$ & $\begin{array}{l}13.2 \\
12.2 \\
15.4 \\
21.0 \\
21.0\end{array}$ & $\begin{array}{l}49.5 \\
93.0 \\
89.5 \\
89.0 \\
91.0\end{array}$ & $\begin{array}{r}33.5 \\
33.5 \\
33.5 \\
0.0 \\
0.0\end{array}$ & $\begin{array}{l}46.0 \\
47.0 \\
47.0 \\
50.0 \\
49.5\end{array}$ & $\begin{array}{r}77.0 \\
80.0 \\
-90.0 \\
111.5 \\
110.0\end{array}$ & $\begin{array}{r}34.0 \\
88.0 \\
103.5 \\
125.5 \\
123.5\end{array}$ & $\begin{array}{r}76.5 \\
76.5 \\
76.5 \\
0.0 \\
0.0\end{array}$ & $\begin{array}{l}12.4 \\
12.4 \\
12.4 \\
12.4 \\
12.4\end{array}$ \\
\hline $\begin{array}{l}9.8 .16 \\
9.8 .17 \\
9.8 .18 \\
9.8 .19 \\
9.8 .20\end{array}$ & $\begin{array}{l}14.0 \\
13.0 \\
19.2 \\
14.7 \\
11.5\end{array}$ & $\begin{array}{l}0.0 \\
0.0 \\
0.0 \\
0.0 \\
0.0\end{array}$ & $\begin{array}{l}10.0 \\
10.6 \\
19.0 \\
15.0 \\
10.2\end{array}$ & $\begin{array}{l}97.0 \\
94.0 \\
99.5 \\
91.5 \\
94.0\end{array}$ & $\begin{array}{l}0.0 \\
0.0 \\
0.0 \\
0.0 \\
0.0\end{array}$ & $\begin{array}{l}51.0 \\
47.0 \\
48.5 \\
48.0 \\
49.0\end{array}$ & $\begin{array}{r}104.0 \\
94.0 \\
97.5 \\
94.5 \\
91.5\end{array}$ & $\begin{array}{l}116.0 \\
102.5 \\
108.0 \\
103.5 \\
100.0\end{array}$ & $\begin{array}{l}0.0 \\
0.0 \\
0.0 \\
0.0 \\
0.0\end{array}$ & $\begin{array}{l}12.4 \\
12.4 \\
12.4 \\
12.4 \\
12.4\end{array}$ \\
\hline
\end{tabular}

\begin{tabular}{|c|c|c|c|}
\hline & & $R$ PLENUM & FLOW \\
\hline$\underset{N O}{T E S T}$ & $\begin{array}{l}\text { INITIALL } \\
\text { WEIGHT } \\
\text { (LB) }\end{array}$ & $\begin{array}{l}\text { FINAL } \\
\text { WEIGHT } \\
\text { (LBI }\end{array}$ & $\begin{array}{l}\text { TIME OF } \\
\text { COLLECT ICI } \\
\text { (SEC) }\end{array}$ \\
\hline $\begin{array}{l}9.8 .1 \\
9.8 .2 \\
9.8 . \\
9.8 .4 \\
9.8 .5\end{array}$ & $\begin{array}{l}174.5 \\
141.5 \\
141.5 \\
156.0 \\
202.5\end{array}$ & $\begin{array}{l}201.0 \\
217.0 \\
247.5 \\
349.5 \\
562.5\end{array}$ & $\begin{array}{l}60.0 \\
60.0 \\
30.0 \\
30.0 \\
31.0\end{array}$ \\
\hline $\begin{array}{l}9.8 .6 \\
9.8: 7 \\
9.8 .8 \\
9.8 .9 \\
9.8 .10\end{array}$ & $\begin{array}{l}142.5 \\
146.0 \\
140.0 \\
232.0 \\
155.0\end{array}$ & $\begin{array}{l}394.0 \\
398.0 \\
449.0 \\
378.0 \\
393.5\end{array}$ & $\begin{array}{l}15.0 \\
15.0 \\
15.0 \\
10.0 \\
15.5\end{array}$ \\
\hline $\begin{array}{l}9.8 .11 \\
9.8 .12 \\
9.8 .13 \\
9.8 .14 \\
9.8 .15\end{array}$ & $\begin{array}{l}170.0 \\
147.5 \\
161.5 \\
161.5 \\
141.0\end{array}$ & $\begin{array}{l}366.0 \\
311.0 \\
252.0 \\
223.5 \\
168.5\end{array}$ & $\begin{array}{l}15.5 \\
20.0 \\
30.0 \\
30.0 \\
30.0\end{array}$ \\
\hline $\begin{array}{l}9.8 .16 \\
9.8 .17 \\
9.8 .18 \\
9.3 .19 \\
9.8 .20\end{array}$ & $\begin{array}{l}162.0 \\
145.0 \\
151.5 \\
141.5 \\
151.5\end{array}$ & $\begin{array}{l}402.5 \\
356.0 \\
270.0 \\
329.0 \\
480.5\end{array}$ & $\begin{array}{l}15.0 \\
15.0 \\
30.0 \\
30.0 \\
30.0\end{array}$ \\
\hline
\end{tabular}

\begin{tabular}{|c|c|c|c|}
\hline $\begin{array}{l}\text { IN I IIAL } \\
\text { HE IGHT } \\
\text { (LB) }\end{array}$ & $\begin{array}{l}\text { FINAL } \\
\text { WEIGHT } \\
\text { (LB) }\end{array}$ & $\begin{array}{l}\text { TIME OF } \\
\text { COLLECTION } \\
\text { ISEC) }\end{array}$ & $\begin{array}{l}\text { PLENUM } \\
\text { LEVEL } \\
\text { (IN) }\end{array}$ \\
\hline $\begin{array}{l}104.0 \\
108.5 \\
104.5 \\
176.0 \\
194.5\end{array}$ & $\begin{array}{l}180.0 \\
206.5 \\
210.5 \\
275.5 \\
378.0\end{array}$ & $\begin{array}{l}30.0 \\
31.0 \\
30.0 \\
30.0 \\
30.0\end{array}$ & $\begin{array}{r}12.0 \\
12.0 \\
12.0 \\
12.0 \\
5.0\end{array}$ \\
\hline $\begin{array}{l}111.5 \\
250.0 \\
105.0 \\
110.0 \\
132.5\end{array}$ & $\begin{array}{l}250.0 \\
447.0 \\
250.5 \\
145.5 \\
260.5\end{array}$ & $\begin{array}{r}30.0 \\
30.0 \\
30.0 \\
-30.0 \\
30.0\end{array}$ & $\begin{array}{l}4.0 \\
2.0 \\
3.0 \\
5.0 \\
4.0\end{array}$ \\
\hline $\begin{array}{l}260.5 \\
268.5 \\
278.0 \\
336.0 \\
108.0\end{array}$ & $\begin{array}{l}523.5 \\
389.5 \\
403.5 \\
488.5 \\
215.0\end{array}$ & $\begin{array}{l}30.0 \\
20.0 \\
30.0 \\
30.0 \\
30.0\end{array}$ & $\begin{array}{l}5.0 \\
12.0 \\
12.0 \\
12.0 \\
12.0\end{array}$ \\
\hline $\begin{array}{l}215.0 \\
164.5 \\
263.5 \\
109.5 \\
124.5\end{array}$ & $\begin{array}{l}359.5 \\
289.5 \\
444.5 \\
287.0 \\
208.5\end{array}$ & $\begin{array}{l}30.0 \\
30.0 \\
30.0 \\
30.0 \\
30.0\end{array}$ & $\begin{array}{r}3.0 \\
4.0 \\
12.0 \\
12.0 \\
12.0\end{array}$ \\
\hline
\end{tabular}

P-LP -

TF-LP-I

(PSIG) (OEG FI

$\begin{array}{rr}16.7 & 60.5 \\ 17.5 & 54.5 \\ 17.5 & 53.0 \\ 9.5 & 52.5 \\ 9.7 & 51.0 \\ 10.2 & 50.0 \\ 10.2 & 49.0 \\ 10.2 & 45.5 \\ 10.2 & 50.0 \\ 9.7 & 48.5 \\ 12.5 & 46.0 \\ 11.0 & 47.0 \\ 14.2 & 48.0 \\ 19.5 & 53.0 \\ 20.0 & 55.0 \\ 10.0 & 52.0 \\ 10.0 & 48.0 \\ 19.7 & 50.0 \\ 14.2 & 49.0 \\ 10.0 & 49.5\end{array}$


TABLE LII

SPECIAL TEST GROUP 9.9 - SHORTENED LONGITUDINAL FLOW RESTRICTORS

TRANSPARENT VESSEL TEST DATA FOR TESTS 9.9.1 TO $9.9 .14--1.58-$ IN. DOMNCOMER GAP

SUMMARY OF CALCULATEO RESULTS

\begin{tabular}{|c|c|c|c|c|c|c|c|c|c|c|}
\hline $\begin{array}{c}\text { TEST } \\
\text { NO }\end{array}$ & $\begin{array}{l}\text { DOWNCOMER } \\
\text { AIR FLOW } \\
(L B / S E C)\end{array}$ & $\begin{array}{l}\text { DOHNCOMER } \\
\text { AIR DENSITY } \\
\text { (LB/FT*3) }\end{array}$ & $\begin{array}{l}\text { LOHER PLENUM } \\
\text { PRESSURS } \\
\text { (PSIA) }\end{array}$ & $\begin{array}{l}\text { COLD LEG } \\
\text { AIR FLOW } \\
\text { (LB/SEC) }\end{array}$ & $\begin{array}{l}\text { DOWNCOMER } \\
\text { WATER FLOH } \\
\text { (LB/SEC) }\end{array}$ & $\begin{array}{l}\text { HATER FLOH } \\
\text { CONTINUITY }\end{array}$ & $\begin{array}{c}\text { J-AIR } \\
\text { DOWNCDMER } \\
\text { (FT/SEC) }\end{array}$ & $\begin{array}{l}\text { J-WATER } \\
\text { DOWNCOMER } \\
\text { (FT/SEC) }\end{array}$ & $(J G *) * 1 / 2$ & $(J L *) * * 1 / 2$ \\
\hline 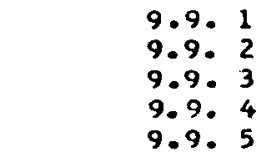 & $\begin{array}{l}0.3656 \\
0.5765 \\
0.5512 \\
0.1312 \\
0.1410\end{array}$ & $\begin{array}{l}0.1384 \\
0.1662 \\
0.1717 \\
0.1197 \\
0.1190\end{array}$ & $\begin{array}{l}26.16 \\
31.66 \\
32.66 \\
22.61 \\
22.66\end{array}$ & $\begin{array}{l}0.0 \\
0.0 \\
0.0 \\
0.0 \\
0.0\end{array}$ & $\begin{array}{r}5.100 \\
1.533 \\
2.183 \\
12.133 \\
13.700\end{array}$ & $\begin{array}{l}1.022 \\
0.812 \\
1.045 \\
0.990 \\
1.003\end{array}$ & $\begin{array}{r}11.23 \\
14.74 \\
13.65 \\
4.66 \\
5.04\end{array}$ & $\begin{array}{l}0.347 \\
0.104 \\
0.149 \\
0.827 \\
0.933\end{array}$ & $\begin{array}{l}0.4263 \\
0.5114 \\
0.4960 \\
0.2648 \\
0.2749\end{array}$ & $\begin{array}{l}0.3455 \\
0.1895 \\
0.2261 \\
0.5329 \\
0.5663\end{array}$ \\
\hline $\begin{array}{l}9.9 .6 \\
9.9 .7 \\
9.9 .8 \\
9.9 .19 \\
9.9 .10\end{array}$ & $\begin{array}{l}0.2056 \\
0.2687 \\
0.4944 \\
0.5830 \\
0.7045\end{array}$ & $\begin{array}{l}0.1518 \\
0.1297 \\
0.1583 \\
0.1903 \\
0.1770\end{array}$ & $\begin{array}{l}28.66 \\
24.41 \\
29.91 \\
34.41 \\
33.91\end{array}$ & $\begin{array}{l}0.0 \\
0.0 \\
0.0 \\
0.0 \\
0.0\end{array}$ & $\begin{array}{r}11.100 \\
8.000 \\
2.603 \\
1.833 \\
0.717\end{array}$ & $\begin{array}{l}1.001 \\
1.005 \\
1.019 \\
1.035 \\
1.018\end{array}$ & $\begin{array}{r}5.76 \\
8.81 \\
13.28 \\
13.74 \\
16.92\end{array}$ & $\begin{array}{l}0.756 \\
0.545 \\
0.177 \\
0.125 \\
0.049\end{array}$ & $\begin{array}{l}0.3124 \\
0.3714 \\
0.4794 \\
0.5039 \\
0.5565\end{array}$ & $\begin{array}{l}0.5098 \\
0.4327 \\
0.2469 \\
0.2072 \\
0.1295\end{array}$ \\
\hline $\begin{array}{l}9.9 .11 \\
9.9 .12 \\
9.9 .13 \\
9.9 .14\end{array}$ & $\begin{array}{l}0.1960 \\
0.1308 \\
0.3253 \\
0.3131\end{array}$ & $\begin{array}{l}0.1212 \\
0.1200 \\
0.1427 \\
0.1855\end{array}$ & $\begin{array}{l}22.91 \\
22.66 \\
26.91 \\
34.91\end{array}$ & $\begin{array}{l}0.0 \\
0.0 \\
0.0 \\
0.0\end{array}$ & $\begin{array}{r}8.775 \\
12.400 \\
6.650 \\
7.875\end{array}$ & $\begin{array}{l}1.011 \\
1.001 \\
1.006 \\
1.021\end{array}$ & $\begin{array}{l}6.87 \\
4.63 \\
9.69 \\
7.18\end{array}$ & $\begin{array}{l}0.598 \\
0.845 \\
0.453 \\
0.536\end{array}$ & $\begin{array}{l}0.3226 \\
0.2642 \\
0.3991 \\
0.3667\end{array}$ & $\begin{array}{l}0.4532 \\
0.5387 \\
0.3946 \\
0.4294\end{array}$ \\
\hline
\end{tabular}


TABLE LII (Contd.)

SPECIAL TEST GROUP 9.9 - SHORTENED LONGITUDINAL FLOW RESTTRICTORS

EXPERIMENTAL MEASURMENTS FOR TESTS 9.9 .1 TO $9.9 .14--1.58-1$ IN. CONNCOMER GAP

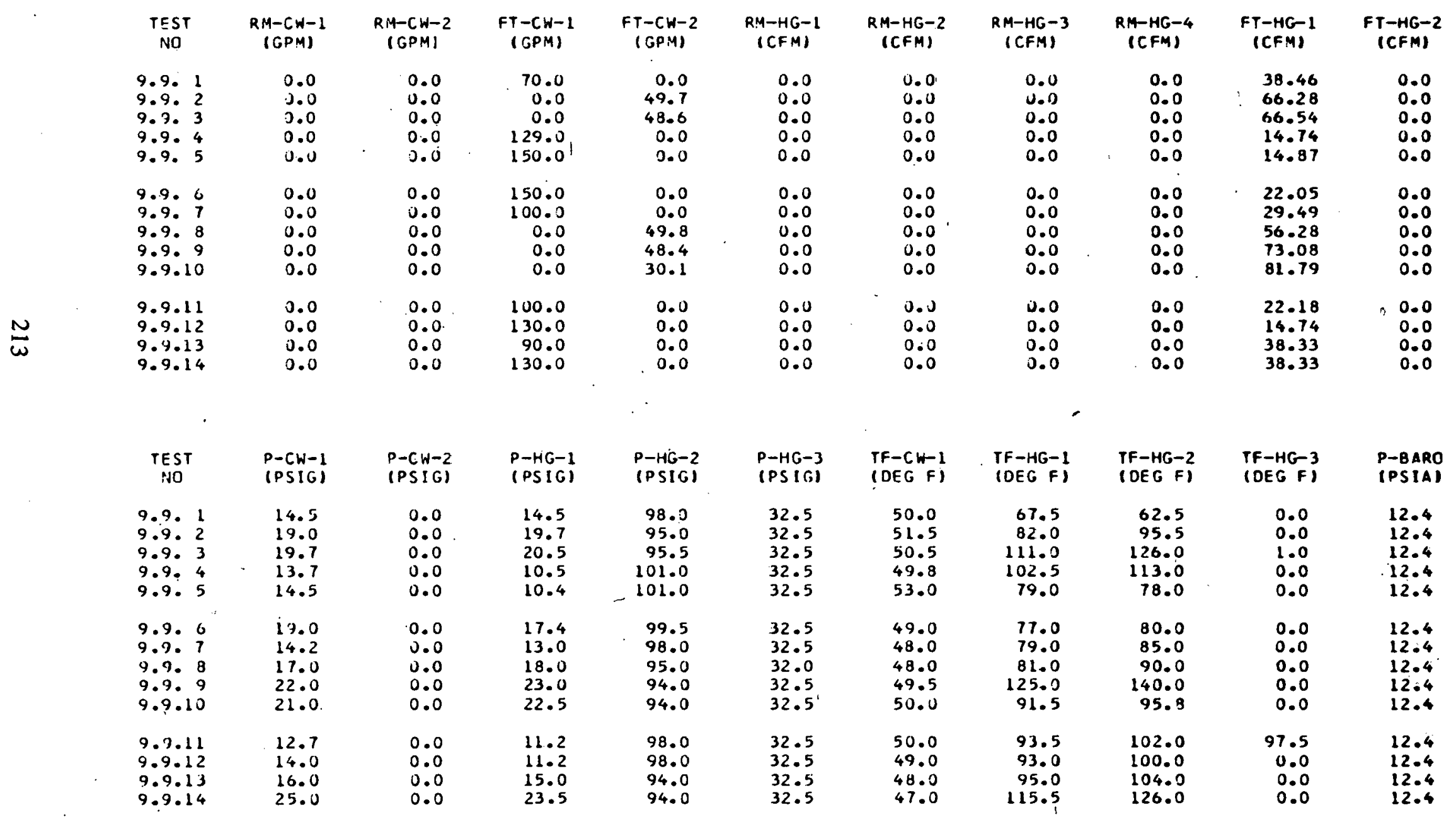


TABLE LII (Contd.)

SPECIAL TEST GROUP 9.9 - SHORTENED LONGITUDINAL FLOW RESTRICTORS

EXPERIMENTAL MEASURMENTS FOR TESTS 9.9 .1 TO $9.9 .14-1.58-$ IN. DOWNCOMER GAP

\begin{tabular}{|c|c|c|c|c|c|c|c|c|c|}
\hline \multirow[b]{2}{*}{$\begin{array}{c}\text { TEST } \\
\text { ND }\end{array}$} & \multicolumn{2}{|c|}{ LOWER PLENUM } & FLOH & \multicolumn{3}{|c|}{ BYPASS FLOW } & \multirow{2}{*}{$\begin{array}{l}\text { LOWER } \\
\text { PLENUM } \\
\text { LEVEL } \\
\text { (IN) }\end{array}$} & \multirow[b]{2}{*}{$\begin{array}{l}P-L P-1 \\
\text { (PSIG) }\end{array}$} & \multirow[b]{2}{*}{$\begin{array}{l}\text { TF-LP-1 } \\
\text { (DEG F) }\end{array}$} \\
\hline & $\begin{array}{l}\text { INITI IAL } \\
\text { WEI GHT } \\
\text { (LB) }\end{array}$ & $\begin{array}{l}\text { FINAL } \\
\text { WEIGHT } \\
\text { (LB) }\end{array}$ & $\begin{array}{l}\text { TIME OF } \\
\text { COLLECTION } \\
\text { (SEC) }\end{array}$ & $\begin{array}{l}\text { INITIAL } \\
\text { WE IGHT } \\
\text { (LB) }\end{array}$ & $\begin{array}{l}\text { FINAL } \\
\text { WEIGHT } \\
\text { (LB) }\end{array}$ & $\begin{array}{l}\text { TIME OF } \\
\text { COLLECTION } \\
\text { (SEC) }\end{array}$ & & & \\
\hline 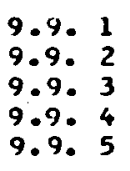 & $\begin{array}{l}160.5 \\
251.0 \\
164.0 \\
213.0 \\
141.0\end{array}$ & $\begin{array}{l}313.5 \\
297.0 \\
229.5 \\
395.0 \\
346.5\end{array}$ & $\begin{array}{l}30.0 \\
30.0 \\
30.0 \\
15.0 \\
15.0\end{array}$ & $\begin{array}{l}127.5 \\
262.0 \\
302.5 \\
377.0 \\
105.5\end{array}$ & $\begin{array}{l}273.0 \\
384.5 \\
449.0 \\
545.5 \\
250.0\end{array}$ & $\begin{array}{l}30.0 \\
30.0 \\
30.0 \\
30.0 \\
20.0\end{array}$ & $\begin{array}{r}12.0 \\
12.0 \\
12.0 \\
4.0 \\
4.0\end{array}$ & $\begin{array}{l}13.7 \\
19.2 \\
20.2 \\
10.2 \\
10.2\end{array}$ & $\begin{array}{l}50.0 \\
54.0 \\
53.5 \\
50.0 \\
54.0\end{array}$ \\
\hline $\begin{array}{l}9.9 .11 \\
9.9 .12 \\
9.9 .13 \\
9.9 .14\end{array}$ & $\begin{array}{l}144.5 \\
203.5 \\
167.5 \\
144.5\end{array}$ & $\begin{array}{l}320.0 \\
394.5 \\
367.0 \\
302.0\end{array}$ & $\begin{array}{l}20.0 \\
15.0 \\
30.0 \\
20.0\end{array}$ & $\begin{array}{l}183.0 \\
269.5 \\
291.0 \\
219.0\end{array}$ & $\begin{array}{l}341.5 \\
440.0 \\
469.0 \\
430.5\end{array}$ & $\begin{array}{l}30.0 \\
30.0 \\
30.0 \\
20.0\end{array}$ & $\begin{array}{r}8.0 \\
4.0 \\
12.0 \\
12.0\end{array}$ & $\begin{array}{l}10.5 \\
10.2 \\
14.5 \\
22.5\end{array}$ & $\begin{array}{l}50.0 \\
49.5 \\
49.0 \\
48.0\end{array}$ \\
\hline
\end{tabular}




\section{REFERENCES}

1. S. A. Naff, R. S. Alder, P. A. Pinson, Experiment Data Report for Semiscale System Countercurrent Flow Tests, ANCR-1151 (June 1974).

2. S. A. Naff and P. A. Pinson, 1-1/2-Loop Semiscale Isothermal Test Program Program and System Description in Support of Experiment Data Reports; ANCR1143 (February 1974).

3. R. S. Alder, E. M. Feldman, D. J. Olson, P. A. Pinson, Experiment Data Report for 1-1/2-Loop Semiscale System Isothermal Tests 1001, 1002, and 1003, ANCR-1148 (May 1974).

4. R. S. Alder, E. M. Feldman, P. A. Pinson, Experiment Data Report for 1-1/2-Loop Semiscale System Isothermal Tests 1004 and 1006, ANCR-1144 (March 1974).

5. R. S. Alder, E. M. Feldman, P. A. Pinson, Experiment Data Report for 1-1/2-Loop Semiscale System Isothermal Tests 1008 and 1010, ANCR-1145 (March 1974).

6. R. S. Alder, E. M. Feldman, P. A. Pinson, Experiment Data Report for 1-1/2-Loop Semiscale System Isothermal Test 1011, ANCR-1 146 (March 1974).

7. E. M. Feldman and P. A. Pinson, Experiment Data Report for 1-1/2-Loop Semiscale System Isothermal Test 1014, ANCR-1147 (March 1974).

8. E. M. Feldman, Experiment Data Report for 1-1/2-Loop Semiscale System Isothermal Test 1009, ANCR-1 152 (June 1974).

9. E. M. Feldman and P. A. Pinson, Experiment Data Report for Semiscale Isothermal Test Program Hot-Wall Tests, ANCR-1149 (May 1974).

10. G. B. Wallis, One-Dimensional Two-Phase Flow, New York: McGraw-Hill Book Company, Inc., 1969. 


\section{THIS PAGE}

\section{WAS INTENTIONALLY LEFT BLANK}


APPENDIX A

DATA ERROR ANALYSIS 


\section{THIS PAGE \\ WAS INTENTIONALLY \\ LEFT BLANK}




\section{APPENDIX A}

\section{DATA ERROR ANALYSIS}

An error analysis was performed to evaluate the total probable and total maximum errors in the air and water volumetric fluxes for several representative tests from the transparent vessel test program. This error analysis was based on the following assumptions:

(1) Each input to the total probable or total maximum error is normally distributed.

(2) The maximum error limits (reported in Table III of the main body of the text) for each measurement device assumes $95 \%$ of the area of a normal distribution is between the maximum error limits.

(3) The inputs to the total probable or total maximum error are independent.

(4) Measurements were not adversely affected by nonhomogeneous flow.

An abbreviated Taylor series expansion may be used to obtain a first order approximation to the total probable error $[\mathrm{A}-1]$ in $\mathrm{J}_{\mathrm{g}}^{*}$ and $\mathrm{J}_{1}^{*}$. This Taylor series expansion can be more clearly presented if $\mathrm{J}_{\mathrm{g}}^{*}$ is assigned to be the independent variable $\mathrm{y}$ and $\mathrm{J}_{1}^{*}$ is the dependent variable $\mathrm{x}$. With this definition, the Taylor series expansion which defines the error in $\mathrm{J}_{\mathrm{g}}^{*}$ is.

$$
\left(\frac{d y}{y}\right)^{2}=\Sigma\left(\frac{\partial y}{\partial z_{i}}\right)^{2}\left(\frac{d z_{i}}{y}\right)^{2}
$$

and the error in $\mathrm{J}_{1}^{*}$ is

$$
\left(\frac{d x}{x}\right)^{2}=\sum\left(\frac{\partial x}{\partial z_{i}}\right)^{2}\left(\frac{d z_{i}}{x}\right)^{2}
$$

where

$$
\mathrm{i}=1,2,3, \ldots \mathrm{n} \text {. }
$$

The experimentally measured variables, $z_{1}, z_{2}, \ldots z_{j}$, when combined in a mathematical relationship (discussed later), define either $\mathrm{J}_{1}^{*}$ or $\mathrm{J}_{\mathrm{g}}^{*}$. The error computed by the preceding method is referred to in this text as the probable error. 
From a statistical viewpoint, the quantity actually being propagated in the preceding equation is two times the coefficient of variance or "the relative error". Stated mathematically:

$$
\frac{\partial y}{\partial z_{i}} \frac{\mathrm{d} z_{i}}{y} \simeq \frac{2 \sigma_{i}}{\hat{z}_{i}}
$$

where $\sigma_{i}$ is the variance associated with $z_{i}$ and $\hat{z}_{i}$ is the true value. With the preceding definition:

$$
\frac{d y}{\hat{y}}=\frac{2 u y}{\hat{y}}
$$

where $\sigma_{\mathrm{y}}$ is the variance of $\mathrm{J}_{\mathrm{g}}^{*}$ from the combined measurements which define $\mathrm{J}_{\mathrm{g}}^{*}$. In a similar manner, $\sigma_{\mathrm{x}}$ can be defined.

In addition to the probable error, a maximum error was computed. The maximum error is defined as the error that would result if all the instruments which combine to make $\mathrm{J}_{\mathrm{g}}^{*}$ and $\mathrm{J}_{1}^{*}$ read in such a way that the total error is the sum of the individual inaccuracies. Mathematically stated:

$$
\begin{aligned}
& \left.\frac{\mathrm{dy}}{\mathrm{y}} \quad\right|_{\max }=\Sigma\left|\frac{\partial \mathrm{y}}{\partial z_{i}} \frac{\mathrm{d} z_{i}}{\mathrm{y}}\right| \\
& \left.\frac{\mathrm{dx}}{\mathrm{x}} \quad\right|_{\max }=\Sigma\left|\frac{\partial x_{1}}{\partial z_{i}} \cdot \frac{\mathrm{d} z_{i}}{\mathrm{dx}}\right| .
\end{aligned}
$$

When the relationship between two variables is considered, one variable is usually expected to be more precisely determined than the other, or the uncertainty of measurement can be solely attributed to the dependent variable $y$; that is:

$$
\frac{d x}{x}<<\frac{d y}{y}
$$


This type of relationship is not always the case. The error in the dependent and independent variables can be of the same order of magnitude. In such a case, the method suggested in Reference $A-1$ is to assign all of the error to the dependent variable in the following manner:

$$
\sigma_{j}=\sqrt{\left(\frac{d x}{x}\right)^{2}+\left(\frac{d y}{y}\right)^{2}}
$$

where $\sigma_{\mathrm{j}}$ is the new uncertainty assigned to the dependent variable.

The uncertainties in $\mathrm{J}_{\mathrm{g}}^{*}$ and $\mathrm{J}_{1}^{*}$ for the steady state countercurrent flow tests were of the same order of magnitude; therefore, the uncertainties in $\mathrm{J}_{\mathrm{g}^{*}}^{*}$ and $\mathrm{J}_{1}^{*}$ were combined by the technique mentioned and assigned to the dependent variable $\mathrm{J}_{\mathrm{g}}$.

\section{DISCUSSION}

The data from the transparent vessel tests were used to calculate the following nondimensional volumetric fluxes:

$$
\begin{aligned}
& J_{g}^{*}=J_{g}\left(\frac{\rho_{g}}{g D\left(\rho_{1}-\rho_{g}\right)}\right)^{1 / 2} \\
& J_{f}^{*}=J_{f}\left(\frac{\rho_{1}}{g D\left(\rho_{1}-\rho_{g}\right)}\right)^{1 / 2}
\end{aligned}
$$

where $\mathrm{D}$ is the hydraulic diameter of the downcomer

$$
\mathrm{D}=\mathrm{D}_{0}-\mathrm{D}_{i}
$$

To find the error in $\mathrm{J}_{\mathrm{g}}^{*} 1 / 2$ and $\mathrm{J}_{\mathrm{g}}^{*} 1 / 2$, total differentials must be obtained; that is:

$$
{ }_{\mathrm{g}} \sqrt{\mathrm{J}^{*}}=1 / 2 \frac{\mathrm{dJ}_{\mathrm{g}}^{*}}{\sqrt{\mathrm{J}_{\mathrm{g}}^{*}}}
$$


Division by $\sqrt{\mathrm{J}_{\mathrm{g}}^{*}}$ results in

$$
\frac{d \sqrt{J_{g}^{*}}}{\sqrt{J_{g}^{*}}}=1 / 2 \frac{\mathrm{dJ}_{g}^{*}}{J_{g}^{*}} .
$$

The error in $\mathrm{d} \mathrm{J}_{\mathrm{g}}^{*} 1 / 2 / \mathrm{J}_{\mathrm{g}}^{*} 1 / 2$ is one-half the error in $\mathrm{d} \mathrm{J}_{\mathrm{g}}^{*} / \mathrm{J}_{\mathrm{g}}^{*}$. Thus, for ease of presentation, $\mathrm{d}_{\mathbf{g}}^{*} / \mathrm{J}_{\mathrm{g}}^{*}$ is developed.

The magnitude of errors in the gas volumetric flux may be estimated by determining the total differential in terms of basic measured quantities. From Equation (A-1), the dimensionless volumetric gas flux is a function of $\mathrm{J}_{\mathrm{g}}, \mathrm{D}, \rho_{\mathrm{g}}$, and $\rho_{1}$. The total differential of $\mathrm{J}_{\mathrm{g}}^{*}$ is

$$
\begin{aligned}
\mathrm{dJ}_{\mathrm{g}}^{*} & \frac{\partial\left(\mathrm{J}_{\mathrm{g}}^{*}\right)}{\partial \mathrm{J}_{\mathrm{g}}} \mathrm{dJ} \mathrm{g}+\frac{\partial\left(\mathrm{J}_{\mathrm{g}}^{*}\right)}{\partial \mathrm{D}_{\mathrm{o}}} \mathrm{dD}_{\mathrm{o}}+\frac{\partial\left(\mathrm{J}_{\mathrm{g}}^{*}\right)}{\partial \mathrm{D}_{\mathrm{I}}} \mathrm{dD_{i }} \\
& +\frac{\partial\left(\mathrm{J}_{\mathrm{g}}^{*}\right)}{\partial \rho_{g}} \mathrm{~d} \rho_{g}+\frac{\partial\left(\mathrm{J}_{\mathrm{g}}^{*}\right)}{\partial \rho_{1}} \mathrm{~d} \rho_{1}
\end{aligned}
$$

The partial differentials in Equation (A-3) are

$$
\begin{aligned}
& \frac{\partial\left(\mathrm{J}_{\mathrm{g}}^{*}\right)}{\partial \mathrm{J}_{\mathrm{g}}}=\left(\frac{\rho_{g}}{\mathrm{~g} \mathrm{D}\left(\rho_{1}-\rho_{g}\right)}\right)^{1 / 2} \mathrm{dJ} g \\
& \frac{\partial J_{g}^{*}}{\partial D_{0}}=-1 / 2 J_{g}\left(\frac{\rho_{g}}{g D\left(\rho_{1}-\rho_{g}\right)}\right)^{1 / 2} \frac{\mathrm{dD}_{0}}{D} \\
& \frac{\partial \mathrm{J}_{\mathrm{g}}^{*}}{\partial \mathrm{D}_{i}}=1 / 2 \mathrm{~J}_{g}\left(\frac{\rho_{g}}{g \mathrm{D}\left(\rho_{1}-\rho_{g}\right)}\right)^{1 / 2} \frac{\mathrm{dD}_{i}}{\mathrm{D}} \\
& \frac{\partial \mathrm{J}_{\mathrm{g}}^{*}}{\partial \rho_{\mathrm{g}}}=1 / 2 \mathrm{~J}_{\mathrm{g}}\left(\frac{\rho_{\mathrm{g}}}{\mathrm{gD}\left(\rho_{1}-\rho_{\mathrm{g}}\right)}\right)^{1 / 2}\left(1+\frac{\rho_{\mathrm{g}}}{\rho_{\mathrm{l}}-\rho_{\mathrm{g}}}\right) \frac{\mathrm{d} \rho_{\mathrm{g}}}{\rho_{\mathrm{g}}}
\end{aligned}
$$




$$
\frac{\partial J_{g}^{*}}{\partial \rho_{1}}=-1 / 2 \quad J_{g}\left(\frac{\rho_{g}}{g D\left(\rho_{1}-\rho_{g}\right)}\right)^{1 / 2}\left(\frac{\rho_{1}}{\rho_{1}-\rho_{g}}\right) \frac{d \rho_{1}}{\rho_{1}}
$$

Substituting Equations (A-4) through (A-8) into Equation (A-3) and dividing by $\mathrm{J}$ * from Equation (A-1) gives

$$
\begin{gathered}
\frac{\mathrm{dJ}_{\mathrm{g}}^{*}}{\mathrm{~J}_{\mathrm{g}}^{*}}=\frac{\mathrm{dJ} \mathrm{g}}{\mathrm{J}}-1 / 2\left(\frac{\mathrm{dD}}{\mathrm{D}}-\frac{\mathrm{d} \mathrm{D}_{\mathrm{i}}}{\mathrm{D}}\right)+1 / 2\left(1+\frac{\rho_{\mathrm{g}}}{\rho_{1}-\rho_{g}}\right) \frac{\mathrm{d} \rho_{g}}{\rho_{g}} \\
-1 / 2\left(\frac{\rho_{1}}{\rho_{1}-\rho_{g}}\right) \frac{\mathrm{d} \rho_{1}}{\rho_{1}} .
\end{gathered}
$$

The terms $\mathrm{d} \mathrm{J} / \mathrm{g} / \mathrm{g}, \mathrm{d} \rho_{\mathrm{g}} / \rho_{\mathrm{g}}$, and $\mathrm{d} \rho_{1} / \rho_{1}$ in Equation (A-9) require further definition in terms of measurable parameters. The basic definition of $\mathrm{J}_{\mathrm{g}}$

$$
\mathrm{J}_{\mathrm{g}}=\frac{\mathrm{Q}_{\mathrm{g}}}{\mathrm{A}}
$$

where

$\mathrm{Q}_{\mathrm{g}}=$ the volumetric flow of the air in the downcomer

A $=$ the total cross-sectional area of the downcomer

can be differentiated to give

$$
\frac{\mathrm{dJ}_{\mathrm{g}}}{\mathrm{J}_{\mathrm{g}}}=\frac{\mathrm{dQ}_{\mathrm{g}}}{\mathrm{Q}_{\mathrm{g}}}-\frac{\mathrm{dA}}{\mathrm{A}}
$$

A direct measurement of the volumetric flow in the downcomer was not possible due to the complexity of the geometry in the downcomer and lower plenum regions. The volumetric flow of air in the downcomer was derived from volumetric flow measurements in the system piping using a mass balance. The measured flow was therefore corrected with the ratio of the air specific volume in the lower plenum divided by the air specific volume at the measurement location. Using the perfect gas law to define the specific volumes at the 
measurement location and the lower plenum in terms of the measured pressure and temperature yields

$$
\frac{d Q_{g}}{Q_{g}}=\frac{d T_{L}}{T_{L}}-\frac{d_{L}}{P_{L}}-\frac{d T_{m}}{T_{m}}+\frac{d P_{m}}{P_{m}}+\frac{d Q_{m}}{Q_{m}}
$$

where the subscripts $\mathrm{L}$ and $\mathrm{m}$ refer to the lower plenum and the air measurement location, respectively.

The area term in Equation (A-10) can be evaluated by considering the following possible causes of errors in downcomer flow area:

(1) Error in misalignment of core barrel and filler piece

(2) Dependence of A on wear and scaling

(3) Variation of A with position in the annulus

(4) Dependence of A on temperature.

The area of the annulus is dependent on the filler piece inside diameter and the core barrel outside diameter. Neither the area of the filler piece inside diameter nor the area of the core barrel outside diameter is dependent on the position of the core barrel within the vessel, and therefore, the annulus area is not dependent on position of the core barrel within the vessel. The vessel liner was measured after the test was completed. No indication of wear and only negligible scaling was observed. From measurements of the vessel liner and tolerances for construction of the core barrel, the dependence of A on position in the annulus is determined. By using the following definition for annulus area:

$$
A=\frac{\pi}{4}\left(D_{0}^{2}-D_{i}^{2}\right)
$$


Lhe following results:

$$
\frac{\mathrm{dA}}{\mathrm{A}}=\frac{2 \mathrm{D}_{\mathrm{o}} \mathrm{dD}_{\mathrm{o}}-2 \mathrm{D}_{\mathrm{i}} \mathrm{dD}_{\mathrm{i}}}{\mathrm{D}_{\mathrm{o}}^{2}-\mathrm{D}_{i}^{2}}
$$

In computing $\mathrm{dA} / \mathrm{A}$, the term $\mathrm{dD}_{\mathrm{o}}$ was taken to be

$$
1 / 2\left[D_{0}(\max )-D_{0}(\min )\right] .
$$

The same technique was employed for $\mathrm{dD}_{\mathrm{i}}$ using the tolerances given for the construction of the core barrel.

The dimensions of the vessel and vessel internals were measured at room temperature, but some tests were conducted with the water substantially below this temperature. An equation describing the effect of temperature on cross-sectional area can be derived using the coefficient of thermal expansion for the plexiglass filler piece or vessel and for the aluminum core barrel considering the change in diameter with temperature

$$
\frac{\mathrm{dA}(\mathrm{T})}{\mathrm{dA}}=\frac{2 \mathrm{D}_{\mathrm{o}}{ }^{2}{ }_{\mathrm{a}_{\mathrm{p}}} \Delta \mathrm{T}-2 \mathrm{D}_{i}{ }^{2} \alpha_{a} \Delta \mathrm{T}}{\mathrm{D}_{\mathrm{o}}{ }^{2}-\mathrm{D}_{\mathrm{i}}{ }^{2}}
$$

This equation shows that this component of error is small because for the case for which $\alpha_{\mathrm{p}}=\alpha_{\mathrm{a}}$, the equation reduces to

$$
\frac{\mathrm{dA}(\mathrm{T})}{\mathrm{dA}}=2 \alpha \Delta \mathrm{T}
$$

Since both $\alpha_{\mathrm{a}}$ and $\alpha_{\mathrm{p}}$ are small and $\Delta \mathrm{T}$ is not larger than about $40^{\circ} \mathrm{F}$, the contribution of temperature to the error in annulus area would be negligible. The corresponding effect of the temperature on the diameter terms in Equation (A-9) would also be negligible for the.. same reasons as discussed for the flow area . 
Substitution of Equations (A-12) and (A-11) into Equation (A-10) leads to

$$
\begin{gathered}
\frac{\mathrm{dJ}_{\mathrm{g}}}{\mathrm{J}_{\mathrm{g}}}=\frac{\mathrm{dT}_{\mathrm{L}}}{\mathrm{T}_{\mathrm{L}}}-\frac{\mathrm{dP}_{\mathrm{L}}}{\mathrm{P}_{\mathrm{L}}}-\frac{\mathrm{dT}}{\mathrm{T}_{\mathrm{m}}}+\frac{\mathrm{dP}_{\mathrm{m}}}{\mathrm{P}_{\mathrm{m}}}+\frac{\mathrm{dQ}_{\mathrm{m}}}{\mathrm{Q}_{\mathrm{m}}} \\
-\frac{2 \mathrm{D}_{\mathrm{o}} \mathrm{dD}_{0}-2 \mathrm{D}_{i} \mathrm{dD}_{i}}{\mathrm{D}_{0}^{2}-\mathrm{D}_{i}^{2}} .
\end{gathered}
$$

The term $\mathrm{d} \rho_{\mathrm{g}} / \rho_{\mathrm{g}}$ in Equation (A-9) can be defined by differentiating the perfect gas law to give (in terms of the lower plenum properties)

$$
\frac{d \rho_{g}}{\rho_{g}}=\frac{d P_{L}}{\rho_{L}}-\frac{d T_{L}}{\rho_{L}}
$$

The term $\mathrm{d} \rho_{1} / \rho_{1}$ in Equation (A-9) can be derived assuming the liquid is incompressible and that changes in $\rho_{1}$ are caused only by temperature changes so that

$$
\frac{\mathrm{d} \rho_{1}}{\rho_{1}}=\frac{\mathrm{d} \rho_{1}}{\rho \mathrm{T}_{1}} \frac{\mathrm{dT}}{\rho_{1}}
$$

but from the property table for water

$$
\frac{\mathrm{d} \rho_{1}}{\mathrm{dT}}=2.5 \times 10^{-3} 1 \mathrm{~b}_{\mathrm{m}} / \mathrm{ft}^{3}{ }^{{ }^{\circ} \mathrm{F}} .
$$

for the range of temperatures used during testing; therefore, this contribution to the total error would be so small as to be negligible . 
Substitution of Equations (A-13) and (A-14) along with $\mathrm{d} \rho_{1} / \rho_{\mathrm{g}} \approx 0$ into Equation (A-9) results in

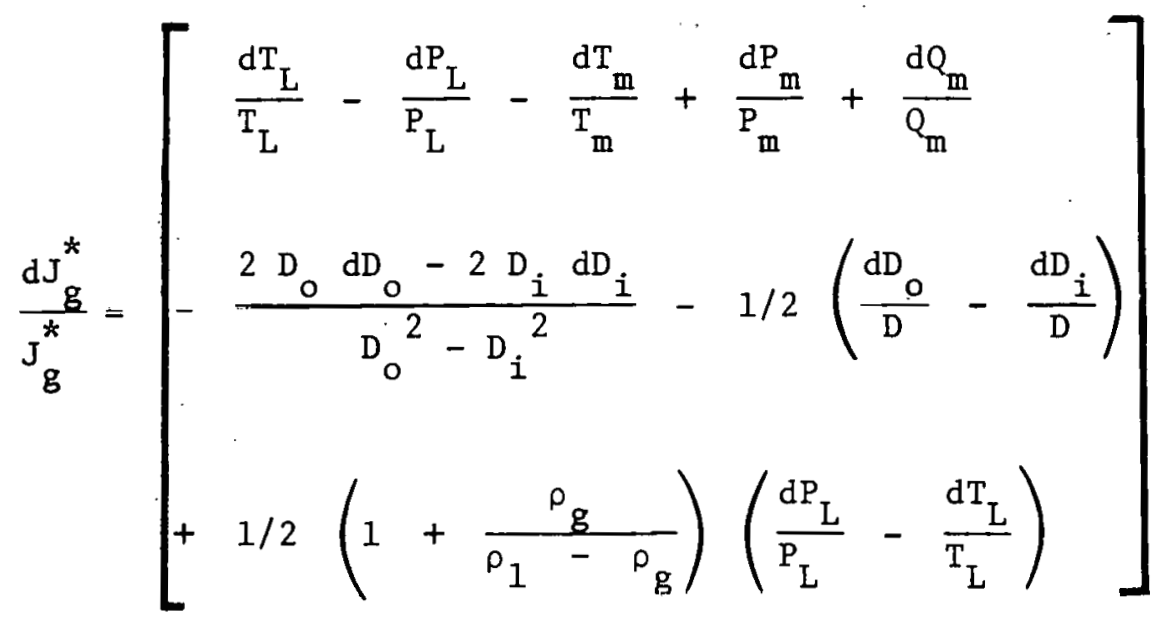

This equation can now be used to define the maximum and probable errors for the gas volumetric flux.

The errors in the liquid volumetric flux can be evaluated using a method similar to that used for the air volumetric flux. From Equation (A-1), the liquid volumetric flux can be differentiated and the partial derivations evaluated to give

$$
\begin{aligned}
\frac{\mathrm{dJ}_{1}^{*}}{\mathrm{~J}_{1}^{*}}=\frac{\mathrm{dJ}_{1}}{\mathrm{~J}_{1}}-1 / 2 & {\left[\frac{\mathrm{dD}_{0}}{\mathrm{D}_{0}}-\frac{\mathrm{dD}_{\mathrm{i}}}{\mathrm{D}_{\mathrm{i}}}\right]+\left(1-\frac{\rho_{1}}{\rho_{1}-\rho_{\mathrm{g}}}\right) \frac{\mathrm{d} \rho_{1}}{\rho_{1}} } \\
+ & 1 / 2\left(\frac{\rho_{\mathrm{g}}}{\rho_{1}-\rho_{\mathrm{g}}}\right) \frac{\mathrm{d} \rho_{\mathrm{g}}}{\rho_{\mathrm{g}}}
\end{aligned}
$$

The term $\mathrm{dJ}_{1} / \mathrm{J}_{1}$ must be evaluated in terms of measured quantities. Through use of a method similar to the derivation of Equation (A-10):

$$
\frac{\mathrm{dJ}_{1}}{\mathrm{~J}_{1}}=\frac{\mathrm{dQ}_{1}}{\mathrm{Q}_{1}}-\frac{\mathrm{dA}}{\mathrm{A}}
$$


The dA/A terms have already been evaluated and are given by Equation (A-13).

Use of the weigh tank measurements for liquid flow in the downcomer results in a volumetric flow measurement defined by

$$
Q_{1}=\frac{M}{t \rho_{1}}
$$

where

$$
\begin{aligned}
& \mathrm{M}=\text { the measured mass from the weigh tanks } \\
& \mathrm{t} \quad=\quad \text { the collection time: }
\end{aligned}
$$

Differentiating and dividing by $\mathrm{Q}_{1}$ gives

$$
\frac{d Q_{1}}{Q_{1}}=\frac{d M}{M}-\frac{d t}{t}-\frac{d \rho_{1}}{\rho_{1}}
$$

but $\mathrm{d} \rho_{1} / \rho_{1}$ has been shown to be negligible, so the preceding equation along with Equation (A-13) can be substituted into Equation (A-17) which along with Equation (A-14) can be substituted into Equation (A-16) to give

$$
\frac{d J_{1}^{*}}{J_{1}}=\left[\begin{array}{c}
\frac{d M}{M}-\frac{d t}{t}-\frac{2 D_{0} D_{o}-2 D_{i} d_{i}}{D_{0}^{2}-D_{i}^{2}} \\
-1 / 2\left(\frac{d_{0}}{D}-\frac{d D_{i}}{D}\right) \\
+\left(\frac{\rho_{g}}{\rho_{1}-\rho_{g}}\right)\left(\frac{d P_{L}}{P_{L}}-\frac{d T_{L}}{T_{L}}\right)
\end{array}\right]
$$


Equation (A-18) can now be used to define the maximum and probable errors for the liquid volumetric flux.

By taking all nonmutually exclusive error terms in Equations (A-15) and (A-18), positive, maximum error Equations (A-19) and (A-20) are obtained.

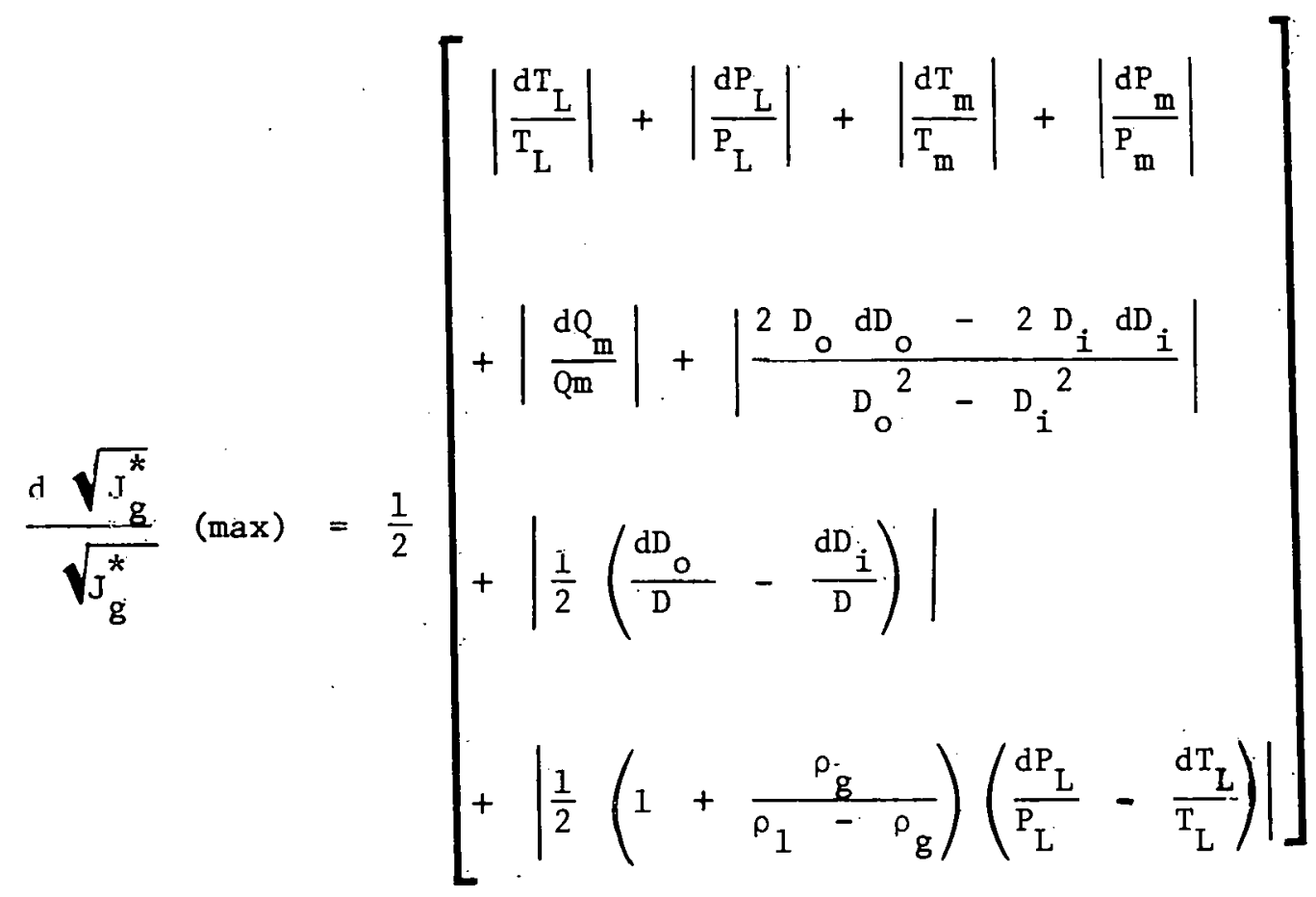

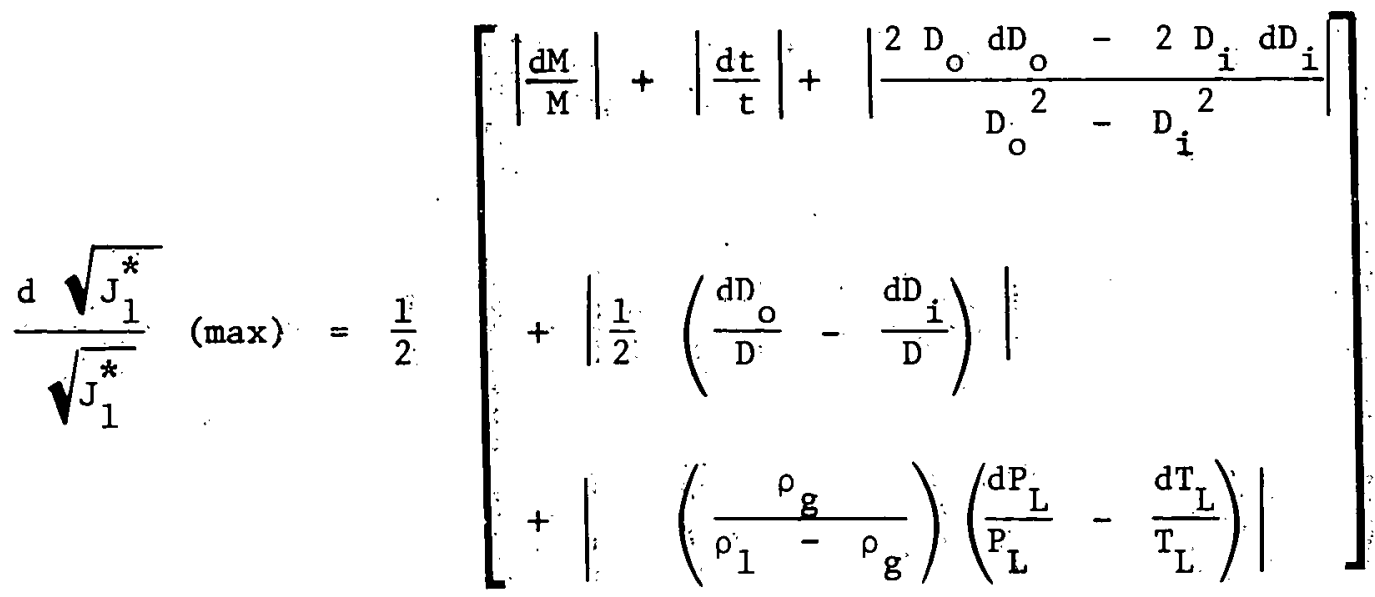


The probable errors for these quantities are expressed by

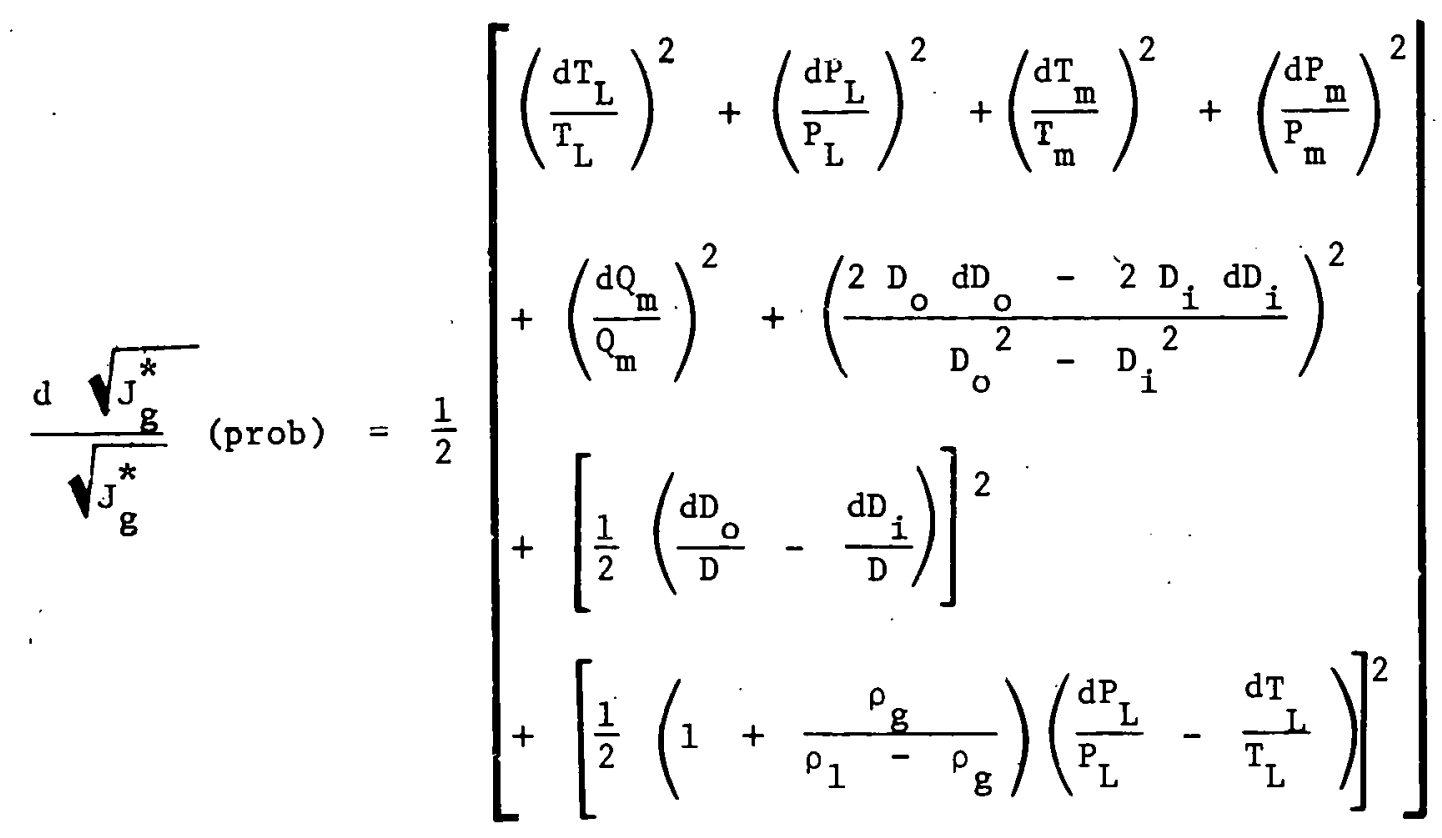

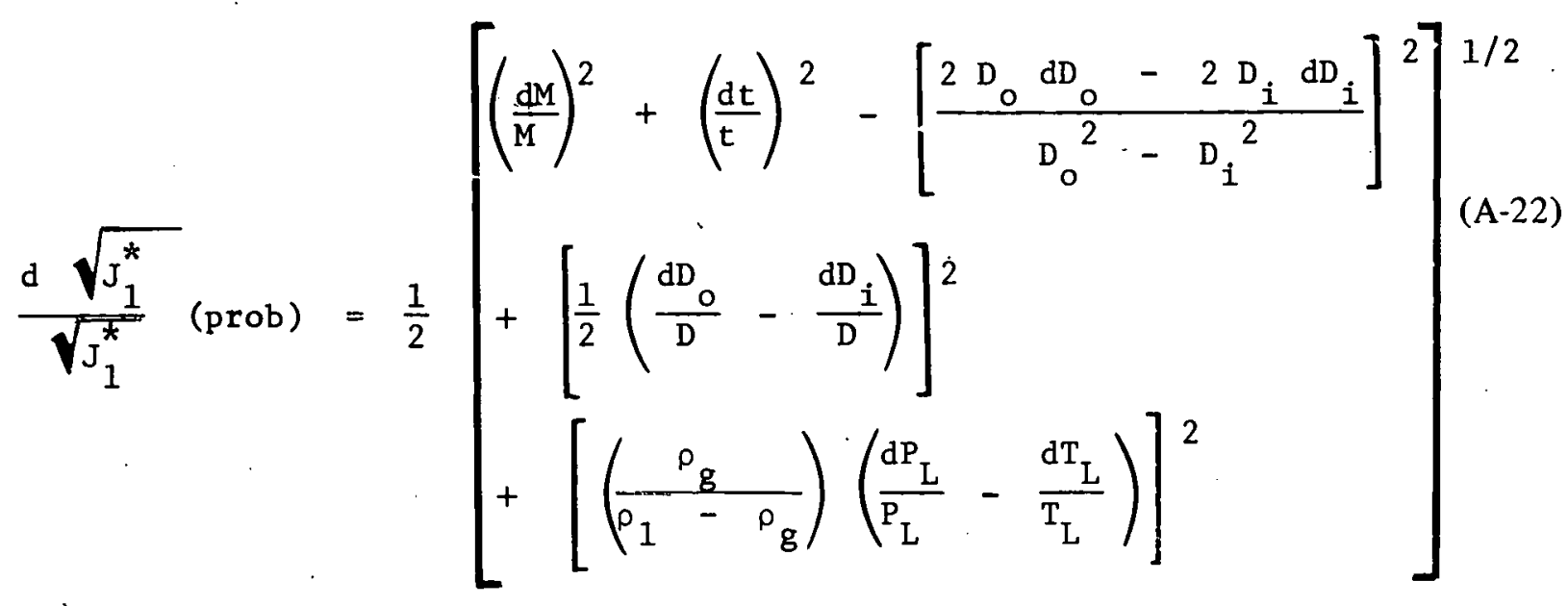




\section{RESULTS}

The equations for the dimensionless volumetric fluxes defined in Equation (A-1) have been reduced to basic measured quantities so that the maximum and probable errors can be calculated. Table A-I summarizes the maximum and probable errors for selected transparent vessel tests. An examination of the distribution of the components that make up the maximum errors indicates that errors in the pressure and turbine flowmeter measurements are the largest contributors to the errors in the calculated volumetric fluxes.

TABLE A-I

PROBABLE AND MAXIMUM ERRORS

\begin{tabular}{|c|c|c|c|c|c|c|c|c|}
\hline \multirow[b]{2}{*}{ Test } & \multirow[b]{2}{*}{$\sqrt{j_{g}{ }^{k}}$} & \multirow{2}{*}{$\sqrt{j_{1}{ }^{*}}$} & \multicolumn{3}{|c|}{ Probable Error (\%) } & \multicolumn{3}{|c|}{ Maximum Error (\%) } \\
\hline & & & $d \sqrt{j_{g}{ }^{*}} / \sqrt{f_{g}^{*}}$ & $d \sqrt{j_{1}{ }^{*}} / \sqrt{j_{1}{ }^{*}}$ & Total $[\mathrm{a}]$ & $\mathrm{d} \sqrt{j_{\mathrm{g}}{ }^{*}} / \sqrt{j_{\mathrm{g}}{ }^{*}}$ & $d \sqrt{j_{1}^{*}} / \sqrt{j_{1}^{*}}$ & Total ${ }^{[a]}$ \\
\hline 7.1 .32 & 0.1887 & 0.9175 & 4.4 & 2.7 & 5.2 & 9.8 & 5.1 & 11.04 \\
\hline 7.1 .36 & 0.269 & 0.7693 & 4.0 & 2.1 & 4.5 & 8.9 & 4.3 & 9.4 \\
\hline 7.1 .38 & 0.4165 & 0.6016 & 4.8 & 2.7 & 5.5 & 8.8 & 5.2 & 10.2 \\
\hline 7.1 .42 & 0.4948 & 0.4520 & 9.2 & 3.7 & 9.9 & 14.5 & 6.5 & 15.9 \\
\hline .7 .1 .40 & 0.5314 & 0.3996 & 3.8 & 4.4 & 5.8 & 8.6 & 7.4 & 11.3 \\
\hline 7.1 .45 & 0.6167 & 0.2374 & 6.0 & 6.0 & 8.5 & 1.2 & 10.5 & 15.2 \\
\hline 7.1 .47 & 0.7468 & 0.0890 & $4 ., 4$ & 4.8 & 6.5 & 8.8 & 14.7 & 17.1 \\
\hline
\end{tabular}

\section{REFERENCE}

A-1. P. R. Bevington, Data Reduction and Error Analysis for the Physical Sciences, New York: McǴraw-Hill Book Company, Inc., 1969. 


\section{THIS PAGE}

\section{WAS INTENTIONALLY \\ LEFT BLANK}




\section{DISTRIBUTION RECORD FOR ANCR-1163}

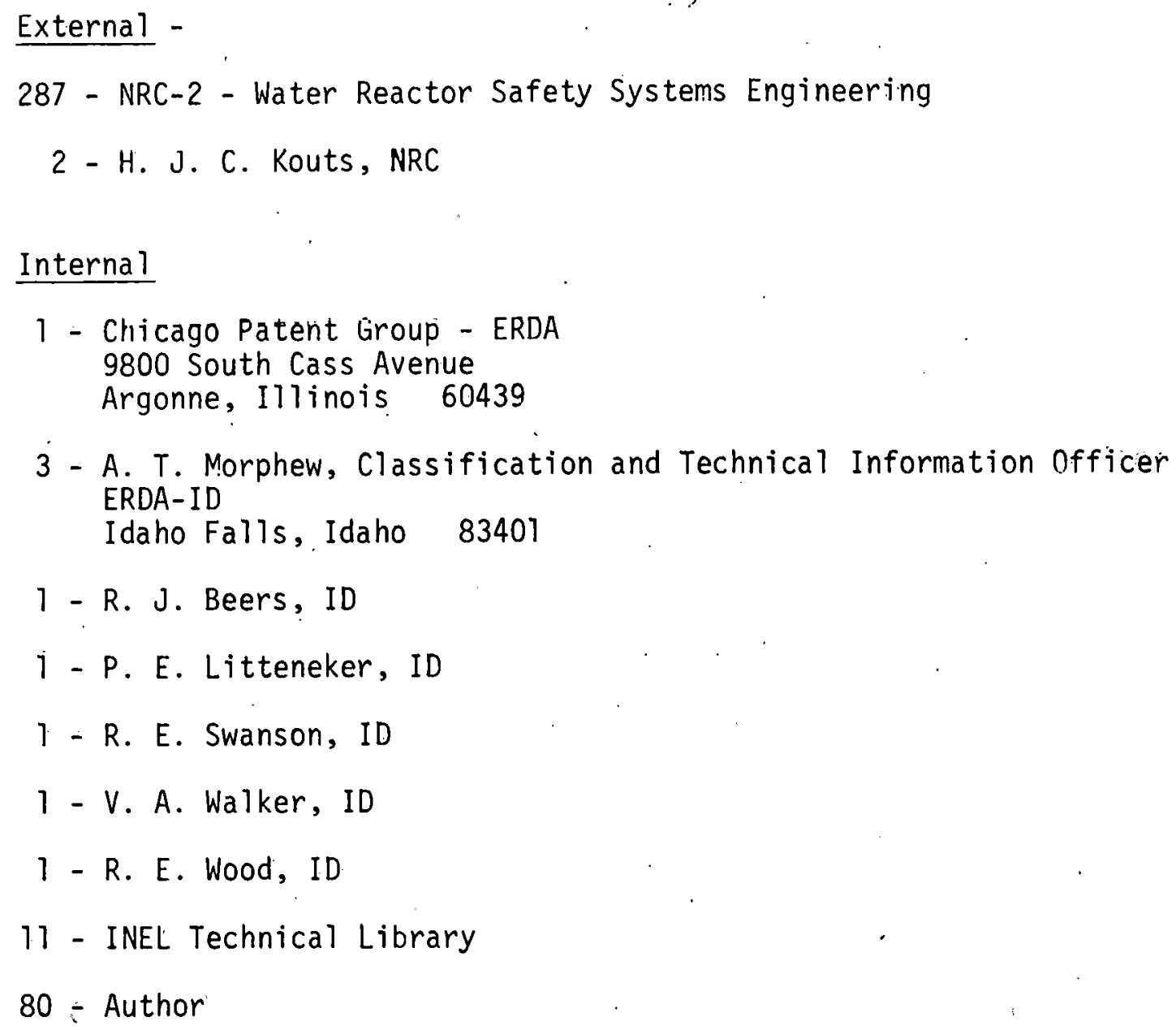


